\title{
Physical Activity and the Health of Wheelchair Users: A Systematic Review in Multiple Sclerosis, Cerebral Palsy, and Spinal Cord Injury
}


Number 241

\section{Physical Activity and the Health of Wheelchair Users: A Systematic Review in Multiple Sclerosis, Cerebral Palsy, and Spinal Cord Injury}

\section{Prepared for:}

Agency for Healthcare Research and Quality

U.S. Department of Health and Human Services

5600 Fishers Lane

Rockville, MD 20857

www.ahrq.gov

Contract No. 290-2015-00009-I

Prepared by:

Pacific Northwest Evidence-based Practice Center

Portland, OR

Investigators:

Shelley S. Selph, M.D., M.P.H.

Andrea C. Skelly, Ph.D., M.P.H.

Ngoc Wasson, M.P.H.

Joseph R. Dettori, Ph.D., M.P.H., M.P.T.

Erika D. Brodt, B.S.

Erik Ensrud, M.D.

Diane Elliot, M.D., FACSM

Kristin M. Dissinger, P.T., D.P.T.

Erica Hart, M.S.T.

Shelby Kantner, B.A.

Elaine Graham, M.L.S.

Mark Junge, B.A.

Tracy Dana, M.L.S.

Marian McDonagh, Pharm.D.

AHRQ Publication No. 21(22)-EHC017

October 2021 
This report is based on research conducted by the Pacific Northwest Evidence-based Practice Center (EPC) under contract to the Agency for Healthcare Research and Quality (AHRQ), Rockville, MD (Contract No. 290-2015-00009-I). The report was commissioned and funded by the National Institutes of Health (NIH) Office of Disease Prevention to inform a Pathways to Prevention Workshop. The findings and conclusions in this document are those of the authors, who are responsible for its contents; the findings and conclusions do not necessarily represent the views of AHRQ or NIH. Therefore, no statement in this report should be construed as an official position of NIH, AHRQ, or the U.S. Department of Health and Human Services.

\section{None of the investigators have any affiliations or financial involvement that conflicts with the material presented in this report.}

The information in this report is intended to help healthcare decision makers - patients and clinicians, health system leaders, and policymakers, among others-make well-informed decisions and thereby improve the quality of healthcare services. This report is not intended to be a substitute for the application of clinical judgment. Anyone who makes decisions concerning the provision of clinical care should consider this report in the same way as any medical reference and in conjunction with all other pertinent information, i.e., in the context of available resources and circumstances presented by individual patients.

This report is made available to the public under the terms of a licensing agreement between the author and the Agency for Healthcare Research and Quality. This report may be used and reprinted without permission except those copyrighted materials that are clearly noted in the report. Further reproduction of those copyrighted materials is prohibited without the express permission of copyright holders.

AHRQ or U.S. Department of Health and Human Services endorsement of any derivative products that may be developed from this report, such as clinical practice guidelines, other quality enhancement tools, or reimbursement or coverage policies, may not be stated or implied.

AHRQ appreciates appropriate acknowledgment and citation of its work. Suggested language for acknowledgment: This work was based on an evidence report, Physical Activity and the Health of Wheelchair Users: A Systematic Review in Multiple Sclerosis, Cerebral Palsy, and Spinal Cord Injury, by the Evidence-based Practice Center Program at the Agency for Healthcare Research and Quality (AHRQ).

Suggested citation: Selph SS, Skelly AC, Wasson N, Dettori JR, Brodt ED, Ensrud E, Elliot D, Dissinger KM, Hart E, Kantner S, Graham E, Junge M, Dana T, McDonagh M. Comparative Effectiveness Review No. 241. Physical Activity and the Health of Wheelchair Users: A Systematic Review of Evidence in Multiple Sclerosis, Cerebral Palsy, and Spinal Cord Injury. (Prepared by the Pacific Northwest Evidence-based Practice Center under Contract No. 290-2015-00009-I.) AHRQ Publication No. 21(22)-EHC017. Rockville, MD: Agency for Healthcare Research and Quality; October 2021.

DOI: https://doi.org/10.23970/AHRQEPCCER241. Posted final reports are located on the Effective Health Care Program search page. 


\section{Preface}

The Agency for Healthcare Research and Quality (AHRQ), through its Evidence-based Practice Centers (EPCs), sponsors the development of evidence reports and technology assessments to assist public- and private-sector organizations in their efforts to improve the quality of healthcare in the United States.

The National Institutes of Health (NIH) Office of Disease Prevention requested this report from the EPC Program at AHRQ to inform a Pathways to Prevention Workshop. The NIH Office of Disease Prevention provided the funding for this report through an Interagency Agreement with AHRQ. AHRQ assigned this report to the following EPC: Pacific Northwest Evidencebased Practice Center (Contract Number 290-2015-00009-I).

The report was presented at the NIH Office of Disease Prevention's Pathways to Prevention Workshop public meeting - "Can Physical Activity Improve the Health of Wheelchair Users?"December 1, 2020, to December 3, 2020.

The reports and assessments provide organizations with comprehensive, evidence-based information on common medical conditions and new healthcare technologies and strategies. For the NIH Pathways to Prevention program, the EPC reports identify research gaps in the selected scientific area, identify methodological and scientific weaknesses, suggest research needs, and move the field forward through an unbiased, evidence-based assessment of the available literature. The EPCs systematically review the relevant scientific literature on topics assigned to them by AHRQ and conduct additional analyses when appropriate prior to developing their reports and assessments.

To bring the broadest range of experts into the development of evidence reports and health technology assessments, AHRQ encourages the EPCs to form partnerships and enter into collaborations with other medical and research organizations. The EPCs work with these partner organizations to ensure that the evidence reports and technology assessments they produce will become building blocks for healthcare quality improvement projects throughout the Nation. The reports undergo peer review and public comment prior to their release as a final report.

AHRQ expects that the EPC evidence reports and technology assessments, when appropriate, will inform individual health plans, providers, and purchasers as well as the healthcare system as a whole by providing important information to help improve healthcare quality.

If you have comments on this evidence report, they may be sent by mail to the Task Order Officer named below at: Agency for Healthcare Research and Quality, 5600 Fishers Lane, Rockville, MD 20857, or by email to epc@ahrq.hhs.gov.

David Meyers, M.D.

Acting Director

Agency for Healthcare Research and Quality

Christine Chang, M.D., M.P.H.

Acting Director

Evidence-based Practice Center Program

Center for Evidence and Practice

Improvement

Agency for Healthcare Research and Quality
Arlene S. Bierman, M.D., M.S.

Director

Center for Evidence and Practice

Improvement

Agency for Healthcare Research and Quality

David W. Niebuhr, M.D., M.P.H., M.Sc.

Task Order Officer

Center for Evidence and Practice

Improvement

Agency for Healthcare Research and Quality 


\section{Acknowledgments}

The authors gratefully acknowledge the following individuals for their contributions to this project: David Niebuhr, M.D., M.P.H., M.Sc., AHRQ Task Order Officer; Keisha Shropshire, M.P.H., Public Health Analyst, P2P Coordinator, NIH Office of Disease Prevention (ODP) Working Group and NIH partners; Meera Viswanathan (Associate Editor); Christine Chang, M.D., M.P.H., Acting Director, Evidence-based Practice Center Program, Center for Evidence and Practice Improvement, AHRQ; Technical Expert Panel members; Peer Reviewers; Leah Williams, B.S., publications editor; Roger Chou, M.D., FACP, Pacific Northwest EPC Director.

\section{Technical Expert Panel}

In designing the study questions and methodology at the outset of this report, the EPC consulted several technical and content experts. Broad expertise and perspectives were sought. Divergent and conflicted opinions are common and perceived as healthy scientific discourse that results in a thoughtful, relevant systematic review. Therefore, in the end, study questions, design, methodologic approaches, and/or conclusions do not necessarily represent the views of individual technical and content experts.

Technical Experts must disclose any financial conflicts of interest greater than $\$ 5,000$ and any other relevant business or professional conflicts of interest. Because of their unique clinical or content expertise, individuals with potential conflicts may be retained. The TOO and the EPC work to balance, manage, or mitigate any potential conflicts of interest identified.

The list of Technical Experts who provided input to this report follows:

Sheila Stahl Butler, P.T.

Physical Therapist

Director of Rehabilitation Services

Forward Stride

Beaverton, OR

Mark Nash, Ph.D., FACSM*

Professor, Departments of Neurological Surgery and Rehabilitation Medicine

Principal Investigator, the Miami Project to Cure Paralysis

Director of Research, Department of Physical Medicine \& Rehabilitation, University of Miami

Miller School of Medicine Lois Pope Life Center

Miami, FL

Mark D. Peterson, Ph.D., M.S., FACSM

Associate Professor, University of Michigan-Medicine Department of Physical

Medicine and Rehabilitation, Neuroscience Graduate Program

Ann Arbor, MI

Rebecca Romero, M.D., FAAN*

Assistant Professor of Neurology

Associate Program Director Neurology 
Director of Multiple Sclerosis Clinic

University of Texas Health

San Antonio, TX

Wei-Han Tan, M.D.*

Director of Clinical Quality, Spinal Cord Injury, VA North Texas HCS

Assistant Professor, UT Southwestern Medical Center, Department of Physical Medicine and

Rehabilitation

Dallas, TX

*Provided input on Draft Report. 


\section{Peer Reviewers}

Prior to publication of the final evidence report, EPCs sought input from independent Peer Reviewers without financial conflicts of interest. However, the conclusions and synthesis of the scientific literature presented in this report do not necessarily represent the views of individual reviewers.

Peer Reviewers must disclose any financial conflicts of interest greater than $\$ 5,000$ and any other relevant business or professional conflicts of interest. Because of their unique clinical or content expertise, individuals with potential nonfinancial conflicts may be retained. The TOO and the EPC work to balance, manage, or mitigate any potential nonfinancial conflicts of interest identified.

The list of Peer Reviewers follows:

Cheri Blauwet, M.D.

Director, Kelley Adaptive Sports Research Institute \& Assistant Professor, Harvard Medical School, Harvard University Cambridge, MA
Sherilyn Driscoll, M.D.

Associate Professor of Physical Medicine and Rehabilitation, Department of Physical Medicine \& Rehabilitation and Department of Pediatric and Adolescent Medicine Mayo Clinic

Rochester, MN

Amanda Montague, Ed.M.

Vice President of Education \& Healthcare Relations

Multiple Sclerosis Association of America Western Regional Office

Seattle, WA 


\section{Physical Activity and the Health of Wheelchair Users: A Systematic Review in Multiple Sclerosis, Cerebral Palsy, and Spinal Cord Injury}

\section{Structured Abstract}

Objectives. Although the health benefits of physical activity are well described for the general population, less is known about the benefits and harms of physical activity in people dependent upon, partially dependent upon, or at risk for needing a wheelchair. This systematic review summarizes the evidence for physical activity in people with multiple sclerosis, cerebral palsy, and spinal cord injury regardless of current use or nonuse of a wheelchair.

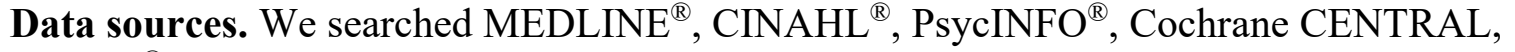
Embase $^{\circledR}$, and Rehabilitation and Sports Medicine Source from 2008 through November 2020, reference lists, and clinical trial registries.

Review methods. Predefined criteria were used to select randomized controlled trials, quasiexperimental nonrandomized trials, and cohort studies that addressed the benefits and harms of observed physical activity (at least 10 sessions on 10 different days of movement using more energy than rest) in participants with multiple sclerosis, cerebral palsy, and spinal cord injury. Individual study quality (risk of bias) and the strength of bodies of evidence for key outcomes were assessed using prespecified methods. Dual review procedures were used. Effects were analyzed by etiology of impairment and physical activity modality, such as treadmill, aquatic exercises, and yoga, using qualitative, and when appropriate, quantitative synthesis using random effects meta-analyses.

Results. We included 146 randomized controlled trials, 15 quasiexperimental nonrandomized trials, and 7 cohort studies (168 studies in 197 publications). More studies enrolled participants with multiple sclerosis (44\%) than other conditions, followed by cerebral palsy (38\%) and spinal cord injury (18\%). Most studies were rated fair quality (moderate risk of bias). The majority of the evidence was rated low strength.

- In participants with multiple sclerosis, walking ability may be improved with treadmill training and multimodal exercise regimens that include strength training; function may be improved with treadmill training, balance exercises, and motion gaming; balance is likely improved with postural control exercises (which may also reduce risk of falls) and may be improved with aquatic exercises, robot-assisted gait training, treadmill training, motion gaming, and multimodal exercises; activities of daily living may be improved with aquatic therapy; sleep may be improved with aerobic exercises; aerobic fitness may be improved with multimodal exercises; and female sexual function may be improved with aquatic exercise.

- In participants with cerebral palsy, balance may be improved with hippotherapy and motion gaming, and function may be improved with cycling, treadmill training, and hippotherapy. 
- In participants with spinal cord injury, evidence suggested that activities of daily living may be improved with robot-assisted gait training.

- When randomized controlled trials were pooled across types of exercise, physical activity interventions were found to improve walking in multiple sclerosis and likely improve balance and depression in multiple sclerosis. Physical activity may improve function and aerobic fitness in people with cerebral palsy or spinal cord injury. When studies of populations with multiple sclerosis and cerebral palsy were combined, evidence indicated dance may improve function.

- Evidence on long-term health outcomes was not found for any analysis groups. For intermediate outcomes such as blood pressure, lipid profile, and blood glucose, there was insufficient evidence from which to draw conclusions. There was inadequate reporting of adverse events in many trials.

Conclusions. Physical activity was associated with improvements in walking ability, general function, balance (including fall risk), depression, sleep, activities of daily living, female sexual function, and aerobic capacity, depending on population enrolled and type of exercise utilized. No studies reported long-term cardiovascular or metabolic disease health outcomes. Future trials could alter these findings; further research is needed to examine health outcomes, and to understand the magnitude and clinical importance of benefits seen in intermediate outcomes. 


\section{Contents}

Evidence Summary ............................................................................................................................ ES-1

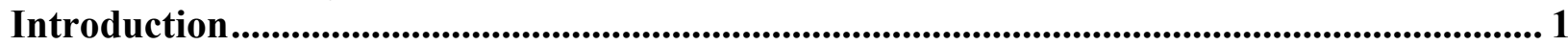

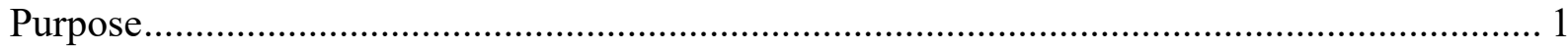

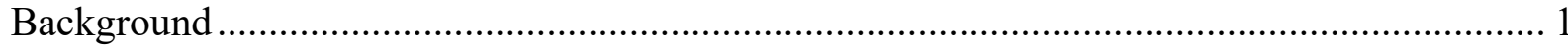

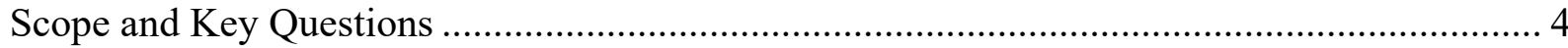

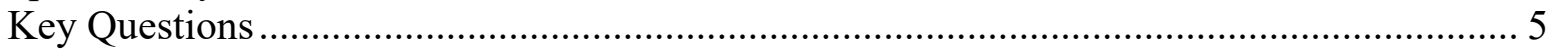

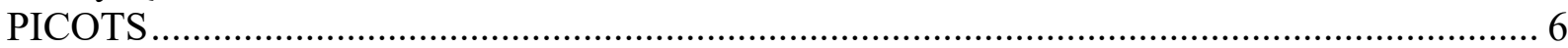

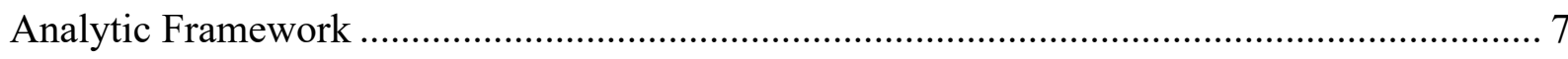

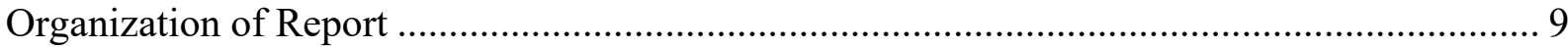

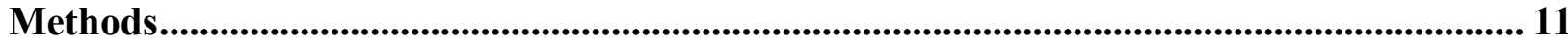

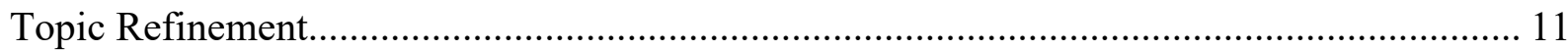

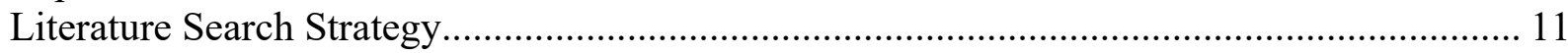

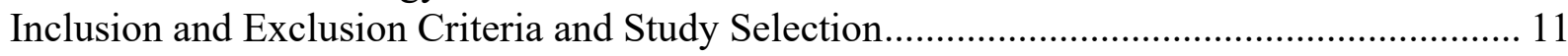

Data Abstraction and Data Management ...................................................................... 15

Quality (Risk of Bias) Assessment of Individual Studies...................................................... 15

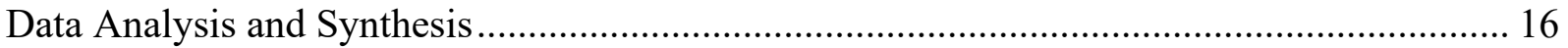

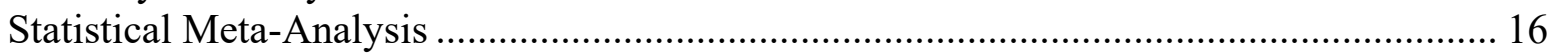

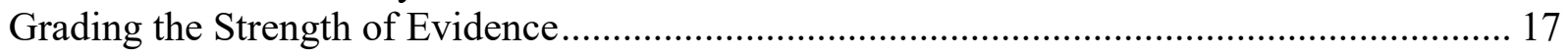

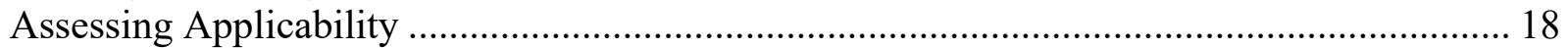

Peer Review and Public Commentary ............................................................................ 18

Results ................................................................................................................................................ 19

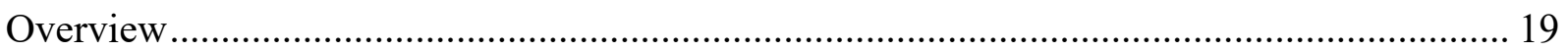

Results of Literature Searches ..................................................................................... 19

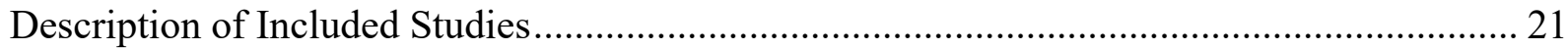

KQ1: Prevention of Obesity, Diabetes, and Cardiovascular Conditions ................................... 26

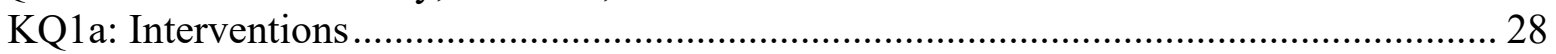

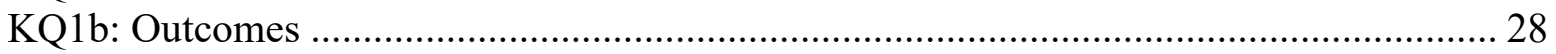

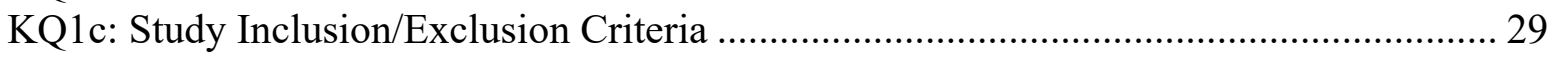

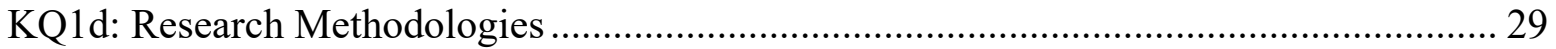

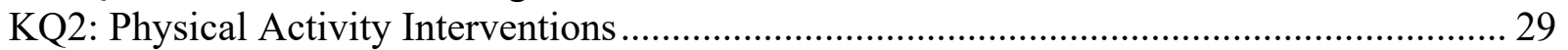

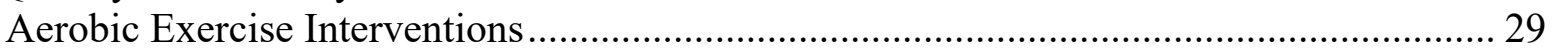

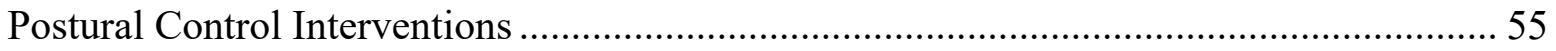

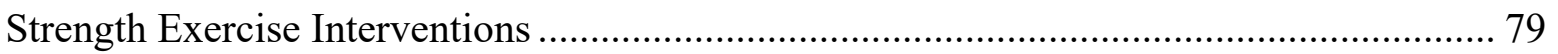

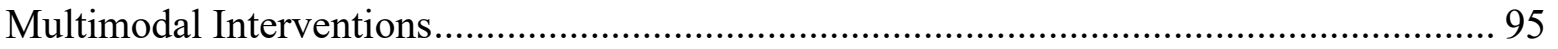

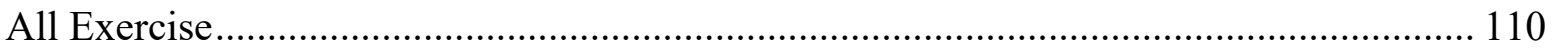

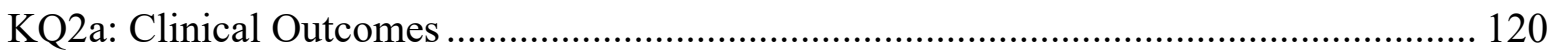

KQ2b: Intermediate Outcomes ................................................................................ 128

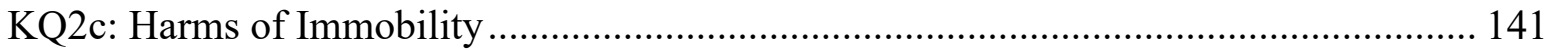

KQ2d: Risk of Adverse Outcomes due to MS, CP, SCI................................................. 142

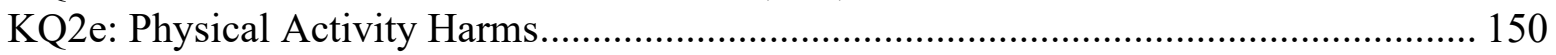

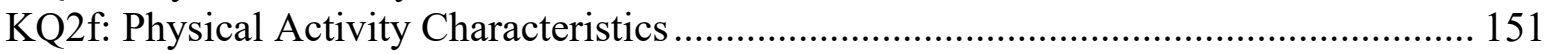

KQ3: Patient Factors and Physical Activity ………….................................................. 152

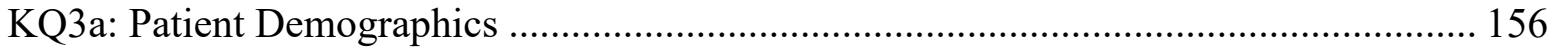

KQ3b: Variations by Condition and Intervention......................................................... 157

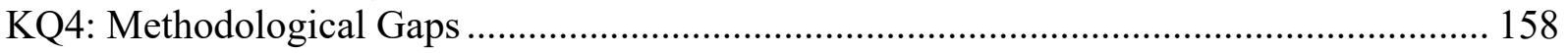




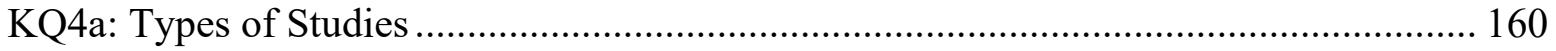

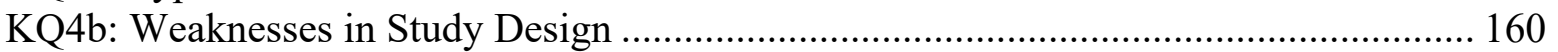

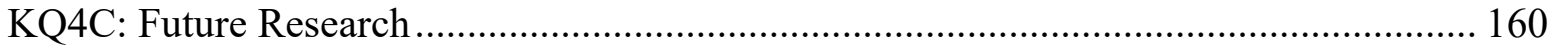

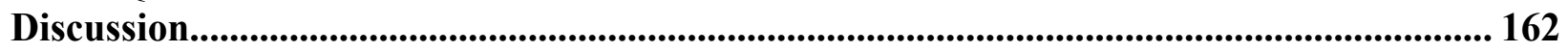

Key Findings and Strength of Evidence ………………….............................................. 162

Findings in Relationship to What Is Already Known....................................................... 164

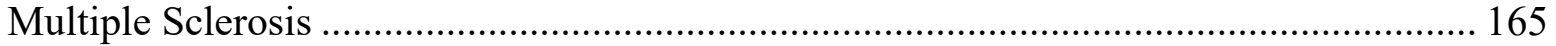

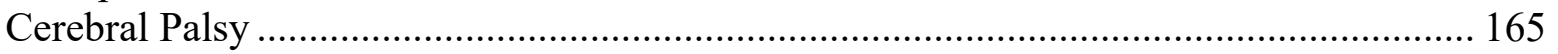

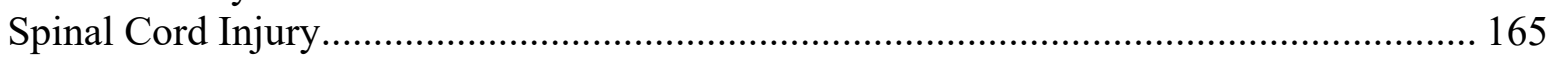

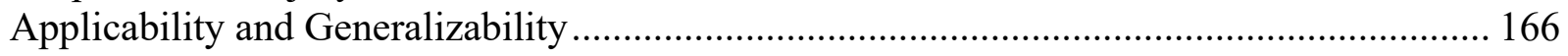

Limitations of the Evidence Base ...................................................................................... 166

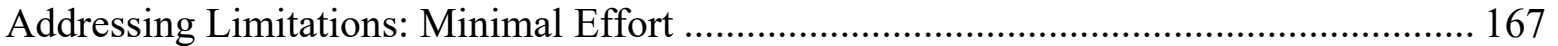

Addressing Limitations: Moderate to Large Effort ……................................................ 168

Addressing Limitations: Large Effort ............................................................................. 168

Implications for Clinical and Policy Decision Making........................................................ 168

Implications for All Providers ..................................................................................... 169

Implications for Primary Care Providers with MS, CP, and SCI Patients......................... 169

Implications for Primary Care Providers With Patients With Disabilities Other than MS, CP,

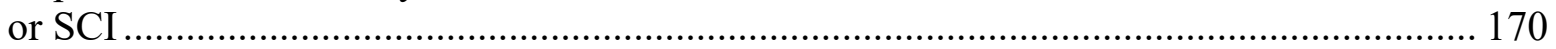

Implications for Physical Activity during a Pandemic .................................................... 170

Limitations of the Systematic Review Process .................................................................. 171

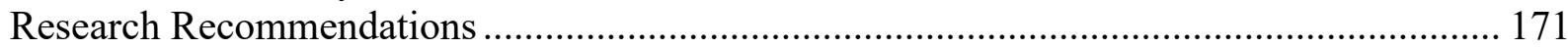

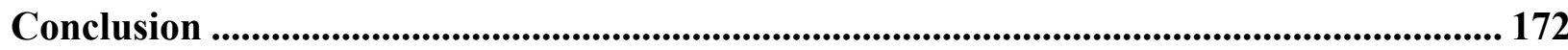

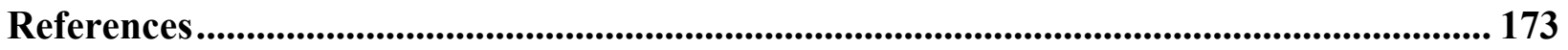

Abbreviations and Acronyms .................................................................................................. 194

\section{Tables}

Table A. Effects of physical activity interventions compared with usual care......................... ES-2

Table 1. Characteristics, causes, and prevalence of multiple sclerosis, cerebral palsy, and spinal

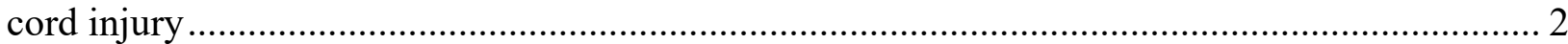

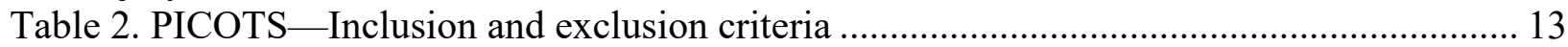

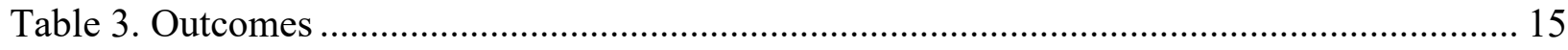

Table 4. Included studies by intervention category and population ${ }^{\mathrm{a}}$....................................... 23

Table 5. Included trials with intermediate outcomes related to cardiovascular disease, diabetes,

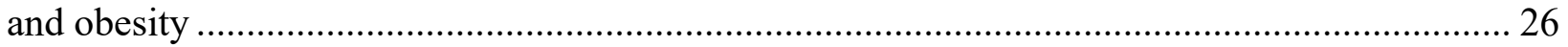

Table 6. Studies with intermediate outcomes related to cardiovascular disease, diabetes, and

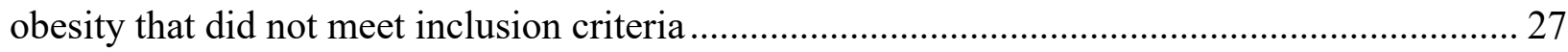

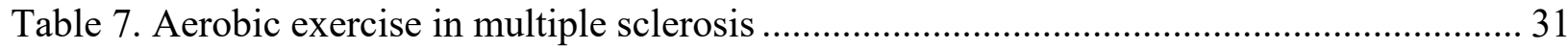

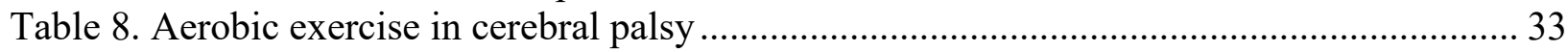

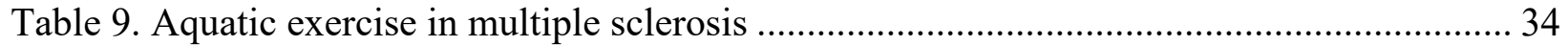

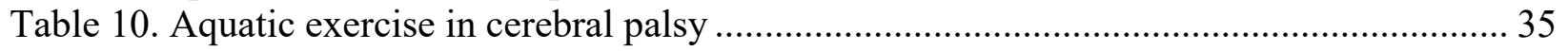

Table 11. Cycling exercise in multiple sclerosis ........................................................................ 37

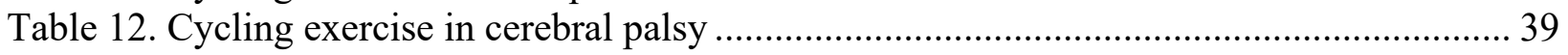

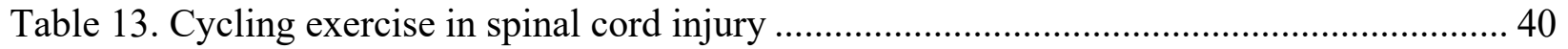

Table 14. Robot-assisted gait training in multiple sclerosis .................................................... 42

Table 15. Robot-assisted gait training in cerebral palsy ……........................................................ 44 
Table 16. Robot-assisted gait training in spinal cord injury ................................................ 47

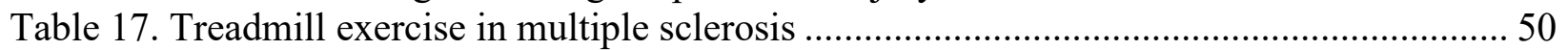

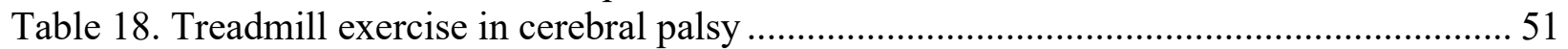

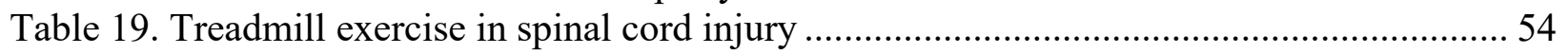

Table 20. Balance training in multiple sclerosis.................................................................. 57

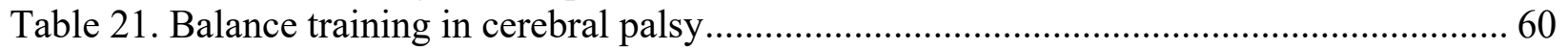

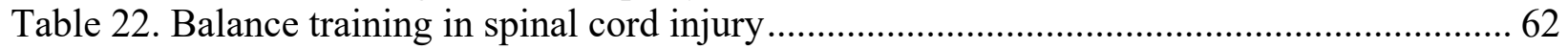

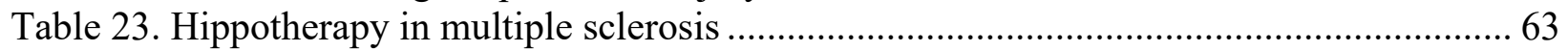

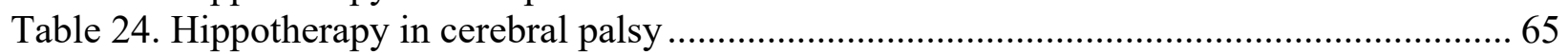

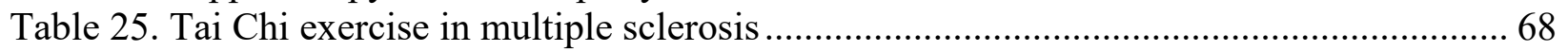

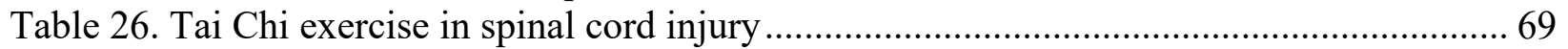

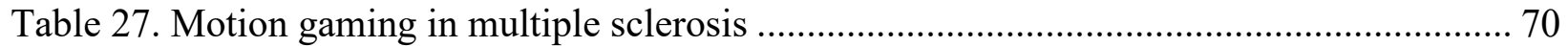

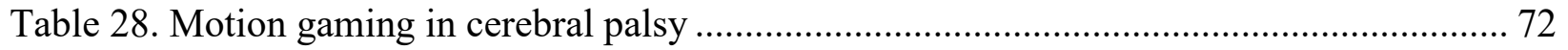

Table 29. Motion gaming in spinal cord injury ................................................................ 74

Table 30. Whole body vibration exercise in multiple sclerosis ........................................... 75

Table 31. Whole body vibration exercise in cerebral palsy .................................................. 76

Table 32. Whole body vibration exercise in spinal cord injury ............................................ 76

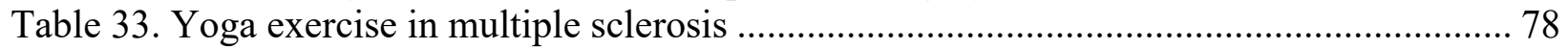

Table 34. Muscle strength exercise in multiple sclerosis ............................................... 81

Table 35. Muscle strength exercise in cerebral palsy ........................................................ 91

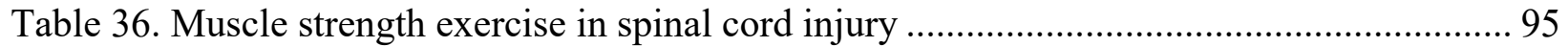

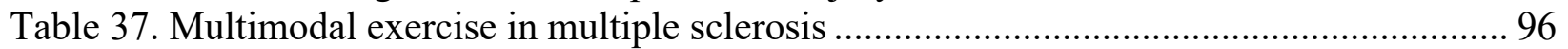

Table 38. Multimodal exercise in cerebral palsy ................................................................. 106

Table 39. Multimodal strength exercise in spinal cord injury .............................................. 109

Table 40. Effects of exercise on depression in participants with multiple sclerosis................. 121

Table 41. Effects of exercise on depression and anxiety in spinal cord injury ......................... 126

Table 42. Intermediate outcomes of physical activity in participants with multiple sclerosis ... 128

Table 43. Intermediate outcomes of physical activity in participants with cerebral palsy ........ 133

Table 44. Intermediate outcomes of physical activity in participants with spinal cord injury ... 135

Table 45. Impact of physical activity interventions on spasticity in participants with multiple

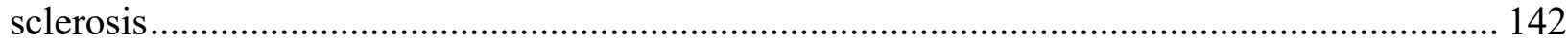

Table 46. Impact of physical activity interventions on spasticity in children with cerebral palsy

Table 47. Impact of physical activity interventions on spasticity in participants with spinal cord

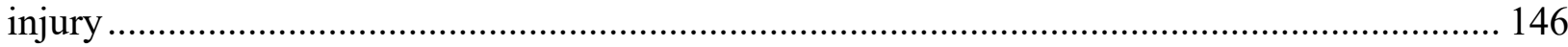

Table 48. Within-study subgroup analyses of effects of exercise in participants with MS, SCI, or CP.

Table 49. Effects of physical activity interventions compared with usual care....................... 162

\section{Figures}

Figure 1. Analytic framework for physical activity and the health of wheelchair users with multiple sclerosis, cerebral palsy, and spinal cord injury .................................................... 8

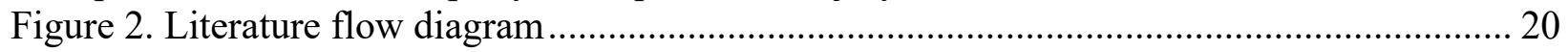

Figure 3. Overview of included studies by population and intervention ${ }^{\mathrm{a}}$................................ 22

Figure 4. Wheelchair user and exercise number of studies: interventions and population ${ }^{\mathrm{a}}$......... 24

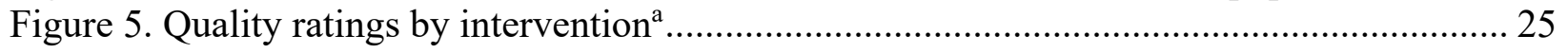


Figure 6. Insomnia Sleep Index in multiple sclerosis trials ................................................. 31

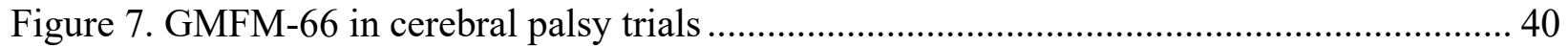

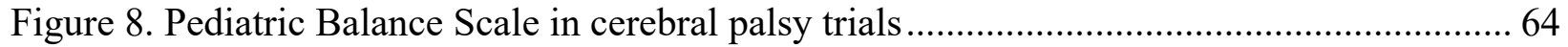

Figure 9. Muscle strength exercise versus usual care in multiple sclerosis: 6MWT immediately

following intervention....................................................................................... 86

Figure 10. Muscle strength exercise versus usual care in multiple sclerosis: 2MWT immediately

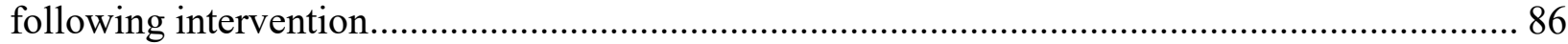

Figure 11. Muscle strength exercise versus usual care in multiple sclerosis: 10MWT immediately

following intervention and during the short-term followup ................................................ 87

Figure 12. Muscle strength exercise versus usual care in multiple sclerosis: MSWS-12

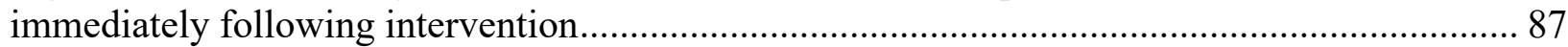

Figure 13. Muscle strength exercise versus usual care in multiple sclerosis: 25FWT immediately

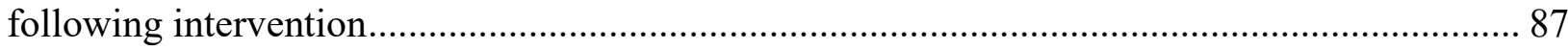

Figure 14. Muscle strength exercise versus usual care in multiple sclerosis: TUG immediately

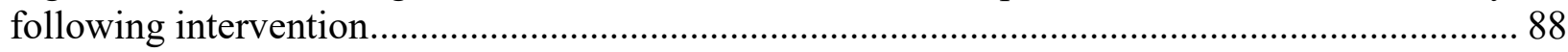

Figure 15. Muscle strength exercise versus usual care in multiple sclerosis: SSST immediately

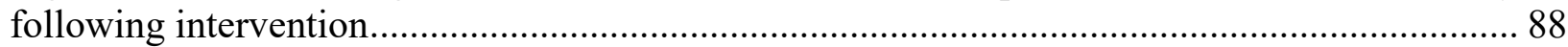

Figure 16. Muscle strength exercise versus usual care in multiple sclerosis: MSQOL/SF-36 MCS

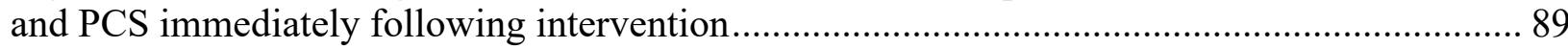

Figure 17. Muscle strength exercise versus usual care in multiple sclerosis: ABCS immediately

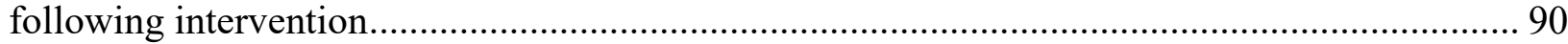

Figure 18. Muscle strength exercise versus usual care in multiple sclerosis: BBS immediately

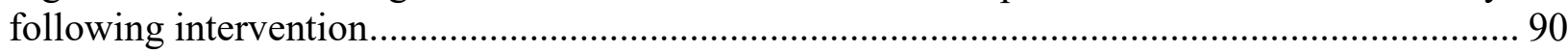

Figure 19. Muscle strength exercise versus usual care in multiple sclerosis: depression

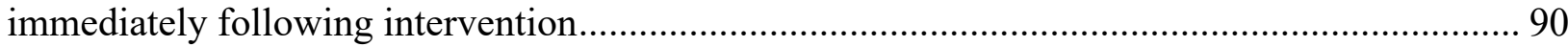

Figure 20. Muscle strength exercise versus usual care in cerebral palsy: 10MWT immediately

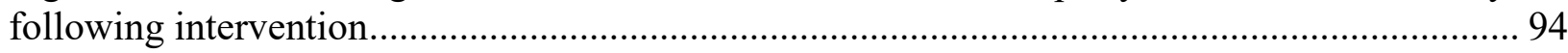

Figure 21. Multimodal exercise versus usual care in multiple sclerosis: 6MWT.................... 102

Figure 22. Multimodal exercise versus usual care in multiple sclerosis: 10MWT.................. 103

Figure 23. Multimodal exercise versus usual care in multiple sclerosis: TUG ....................... 103

Figure 24. Multimodal exercise versus usual care in multiple sclerosis: MSQOL MCS and

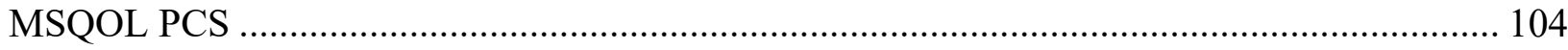

Figure 25. Multimodal exercise versus usual care in multiple sclerosis: BBS ........................ 105

Figure 26. Multimodal exercise versus usual care in multiple sclerosis: EDSS ...................... 105

Figure 27. Multimodal exercise versus usual care in cerebral palsy: $1 \mathrm{MWT}^{\mathrm{a}}$........................ 108

Figure 28. Multimodal exercise versus usual care in cerebral palsy: GMFM-66.................... 108

Figure 29. 6MWT meta-analysis of all randomized controlled trials versus no treatment/usual

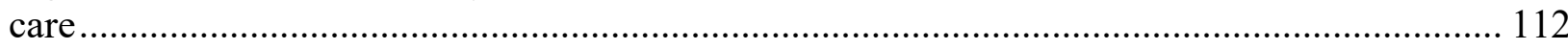

Figure 30. 10MWT meta-analysis of all randomized controlled trials versus no treatment/usual

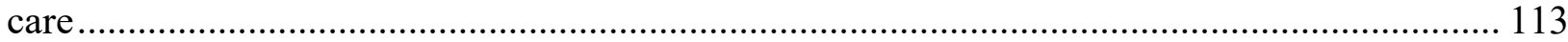

Figure 31. MSWS-12 meta-analysis of all RCTs versus no treatment/usual care................... 114

Figure 32. GMFM-66 meta-analysis of all randomized controlled trials versus no treatment/usual

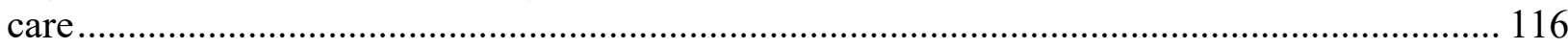

Figure 33. GMFM-66-D meta-analysis of all randomized controlled trials versus no

intervention/usual care 
Figure 34. GMFM-66-E meta-analysis of all randomized controlled trials versus no

intervention/usual care....

Figure 35. Timed Up and Go Test versus no interventin/usual care

Figure 36. BBS meta-analysis of all randomized controlled trials versus no intervention/usual

care

Figure 37. Effect of exercise versus usual care on depression scores in multiple sclerosis

\section{Appendixes}

Appendix A. Literature Search Strategies

Appendix B. Included Studies List

Appendix C. Excluded Studies List

Appendix D. Included and Excluded Study Details and Systematic Reviews Evaluated

Appendix E. Criteria for Assessing Quality and Internal Validity of Individual Studies

Appendix F. Evidence Tables

Appendix G. Quality Assessment

Appendix H. Strength of Evidence 


\section{Evidence Summary}

\section{Main Points}

- We found physical activity to be associated with improvements in walking ability, general function, balance (including fall risk), depression, sleep, activities of daily living, aerobic capacity, and female sexual function, depending on population and type of activity.

- No studies reported long-term cardiovascular or metabolic disease health outcomes.

- Evidence was also limited by heterogeneity in interventions and control groups and by small sample sizes; evidence in spinal cord injury was limited by the small number of trials.

- Evidence was lacking for many prioritized outcomes.

- Adverse effects of the interventions were inadequately reported in many studies.

\section{Background and Purpose}

The benefits of regular physical activity (movement using more energy than rest) for the general population include reduced risk of heart disease, stroke, type 2 diabetes, dementia, depression, falls with injuries among the elderly, and breast, colon, endometrial, esophageal, kidney, stomach, and lung cancer. ${ }^{1}$ Although routine physical activity combining aerobic exercise with strength and balance training is recommended for people with physical disabilities, ${ }^{2}$ less is known about the specific benefits and potential harms for this diverse population. In particular, the various populations using wheelchairs as a result of their physical disabilities is broad and poorly captured in the literature on physical activity. This review includes three diverse conditions commonly associated with wheelchair use: multiple sclerosis, cerebral palsy, and spinal cord injury. The three populations were chosen as representative of those using a wheelchair or those who might benefit from using a wheelchair in the future. While there are differences in etiology and pathophysiology, a common denominator is the involvement of the corticospinal tracts of the central nervous system, which results in impaired central control and/or coordination of the peripheral muscles. This may lead to paralysis or reduced extremity muscle force and increased spasticity, which can greatly affect general mobility or coordinated movement such as posture and gait.

\section{Methods}

We employed methods consistent with those outlined in the Agency for Healthcare Research and Quality Evidence-based Practice Center Program Methods Guidance (https://effectivehealthcare.ahrq.gov/topics/cer-methods-guide/overview), and these are described in the full report. Our searches covered publication dates from 2008 to November 2020. (See Appendix A of the full report for search strategies.)

\section{Results}

We included 168 studies in 197 publications $(\mathrm{n}=7,511)$, comprising of 146 randomized controlled trials, 15 quasiexperimental nonrandomized trials, and 7 cohort studies. More studies enrolled participants with multiple sclerosis (44\%) than other conditions, followed by cerebral palsy (38\%) and spinal cord injury (18\%). 


\section{Key Question 1: Prevention of Cardiovascular Conditions, Diabetes, and Obesity}

No included study ( $\mathrm{n}=168$ ) or study excluded at the full-text level provided evidence on the prevention of cardiovascular conditions (e.g., myocardial infarction, stroke, development of hypertension) or the development of diabetes or obesity.

\section{Key Question 2: Benefits and Harms}

Compared with no physical activity or usual care, physical activity improved walking ability, function, balance, sleep, activities of daily living, cardiovascular fitness as measure with $\mathrm{VO}_{2}$ peak, female sexual function (e.g., desire, lubrication, pain), and depression in participants with multiple sclerosis. Physical activity improved balance, function, and $\mathrm{VO}_{2}$ peak in trials that enrolled participants with cerebral palsy. The evidence in spinal cord injury was sparse. Physical activity improved activities of daily living, function, and $\mathrm{VO}_{2}$ peak in participants with spinal cord injury. All studies focused on benefits of physical activity, with inadequate reporting of adverse events in many studies. However, physical activity was associated with increased episodes of autonomic dysreflexia in spinal cord injury. Table A summarizes the strength of evidence on effects of physical activity interventions compared with usual care and general exercise effect across interventions compared with usual care.

\section{Key Question 3: Patient Factors Affecting Benefits and Harms}

In patients with incomplete spinal cord injury, having better function and more recent injury at baseline was associated with better response to aerobic interventions ( 2 randomized controlled trials). Other subgroup analyses ( 3 randomized controlled trials) did not find evidence of variation in effects based on baseline function or spasticity in children with cerebral palsy (total body vibration), or based on weight category in multiple sclerosis patients (cycling). There were no differences across cerebral palsy trials in walking outcomes when stratified by age group (children, adolescents, and adults).

Table A. Effects of physical activity interventions compared with usual care ${ }^{a}$

\begin{tabular}{|l|c|c|c|}
\hline $\begin{array}{l}\text { Intervention } \\
\text { Category }\end{array}$ & $\begin{array}{c}\text { Multiple Sclerosis } \\
\text { Studies } \\
\text { Intervention }\end{array}$ & $\begin{array}{c}\text { Cerebral Palsy } \\
\text { Studies } \\
\text { (Direction of Finding) }\end{array}$ & $\begin{array}{c}\text { Spinal Cord Injury } \\
\text { Studies }\end{array}$ \\
\hline $\begin{array}{l}\text { Aerobic Exercise } \\
\text { Dance (1 RCT in MS and } \\
\text { 1 RCT in CP) }\end{array}$ & $\begin{array}{c}\text { Low } \\
\text { (Direction of Finding) }\end{array}$ & $\begin{array}{c}\text { Strength of Evidence } \\
\text { (Direction of Finding) }\end{array}$ \\
\hline $\begin{array}{l}\text { Aerobic Exercise } \\
\text { Aerobics }\end{array}$ & $\begin{array}{c}\text { Low } \\
\text { (function improvement) }\end{array}$ & $\begin{array}{c}\text { Low } \\
\text { (function improvement) }\end{array}$ & Insufficient \\
\hline $\begin{array}{l}\text { Aerobic Exercise } \\
\text { Aquatics }\end{array}$ & $\begin{array}{c}\text { Low } \\
\text { (balance, ADL } \\
\text { improvement, female } \\
\text { sexual function) }\end{array}$ & Insufficient & Insufficient \\
\hline $\begin{array}{l}\text { Aerobic Exercise } \\
\text { Cycling }\end{array}$ & $\begin{array}{c}\text { Low } \\
\text { (no clear benefit on } \\
\text { walking) }\end{array}$ & Insfficient & Insufficient \\
\hline Aerobic Exercise & Low & Low & Insufficient \\
\hline
\end{tabular}




\begin{tabular}{|c|c|c|c|}
\hline $\begin{array}{l}\text { Intervention } \\
\text { Category } \\
\text { Intervention }\end{array}$ & $\begin{array}{l}\text { Multiple Sclerosis } \\
\text { Studies } \\
\text { Strength of Evidence } \\
\text { (Direction of Finding) }\end{array}$ & $\begin{array}{c}\text { Cerebral Palsy } \\
\text { Studies } \\
\text { Strength of Evidence } \\
\text { (Direction of Finding) }\end{array}$ & $\begin{array}{l}\text { Spinal Cord Injury } \\
\text { Studies } \\
\text { Strength of Evidence } \\
\text { (Direction of Finding) }\end{array}$ \\
\hline $\begin{array}{l}\text { Robot-Assisted Gait } \\
\text { Training }\end{array}$ & $\begin{array}{c}\text { (balance improvement) } \\
\text { Low } \\
\text { (no clear benefit in } \\
\text { function) }\end{array}$ & & $\begin{array}{c}\text { (ADL improvement) } \\
\text { Low } \\
\text { (no clear benefit on function }\end{array}$ \\
\hline $\begin{array}{l}\text { Aerobic Exercise } \\
\text { Treadmill }\end{array}$ & $\begin{array}{l}\text { Low } \\
\text { (walking, function, and } \\
\text { balance improvement) }\end{array}$ & $\begin{array}{c}\text { Low } \\
\text { (function improvement) }\end{array}$ & Insufficient \\
\hline $\begin{array}{l}\text { Postural Control } \\
\text { Balance Exercises }\end{array}$ & $\begin{array}{c}\text { Moderate } \\
\text { (balance improvement) }\end{array}$ & Insufficient & Insufficient \\
\hline $\begin{array}{l}\text { Postural Control } \\
\text { Balance Exercises }\end{array}$ & $\begin{array}{c}\text { Low } \\
\text { (fall risk improvement) }\end{array}$ & Insufficient & Insufficient \\
\hline $\begin{array}{l}\text { Postural Control } \\
\text { Balance Exercises }\end{array}$ & $\begin{array}{c}\text { Low } \\
\text { (function improvement) }\end{array}$ & Insufficient & Insufficient \\
\hline $\begin{array}{l}\text { Postural Control } \\
\text { Hippotherapy }\end{array}$ & Insufficient & $\begin{array}{l}\text { Low } \\
\text { (balance and function } \\
\text { improvement) }\end{array}$ & Insufficient \\
\hline $\begin{array}{l}\text { Postural Control } \\
\text { Tai Chi }\end{array}$ & Insufficient & Insufficient & Insufficient \\
\hline $\begin{array}{l}\text { Postural Control } \\
\text { Motion Gaming }\end{array}$ & $\begin{array}{l}\text { Low } \\
\text { (function, balance } \\
\text { improvement) }\end{array}$ & $\begin{array}{c}\text { Low } \\
\text { (balance improvement) }\end{array}$ & Insufficient \\
\hline $\begin{array}{l}\text { Postural Control } \\
\text { Whole Body Vibration }\end{array}$ & Insufficient & Insufficient & Insufficient \\
\hline $\begin{array}{l}\text { Postural Control } \\
\text { Yoga }\end{array}$ & $\begin{array}{c}\text { Low } \\
\text { (no clear benefit on } \\
\text { function) }\end{array}$ & Insufficient & Insufficient \\
\hline $\begin{array}{l}\text { Strength Interventions } \\
\text { Muscle Strength } \\
\text { Exercise }\end{array}$ & $\begin{array}{c}\text { Low } \\
\text { (no clear benefit on } \\
\text { walking, function, } \\
\text { balance, quality of life, } \\
\text { spasticity) }\end{array}$ & $\begin{array}{l}\text { Low } \\
\text { (no clear benefit on } \\
\text { walking and function) }\end{array}$ & Insufficient \\
\hline $\begin{array}{l}\text { Multimodal Exercise } \\
\text { Progressive Resistance } \\
\text { or Strength Exercise } \\
\text { Plus Aerobic and/or } \\
\text { Balance Exercise }\end{array}$ & $\begin{array}{c}\text { Low } \\
\text { (walking, balance, } \mathrm{VO}_{2} \\
\text { improvement) }\end{array}$ & $\begin{array}{c}\text { Low } \\
\text { (no clear benefit on } \\
\text { function, quality of life) }\end{array}$ & Insufficient \\
\hline All Types of Exercise & $\begin{array}{c}\text { High } \\
\text { (walking improvement) }\end{array}$ & $\begin{array}{c}\text { Low } \\
\text { (function) }\end{array}$ & $\begin{array}{c}\text { Low } \\
\text { (function) }\end{array}$ \\
\hline
\end{tabular}




\begin{tabular}{|c|c|c|c|}
\hline $\begin{array}{l}\text { Intervention } \\
\text { Category } \\
\text { Intervention }\end{array}$ & $\begin{array}{l}\text { Multiple Sclerosis } \\
\text { Studies } \\
\text { Strength of Evidence } \\
\text { (Direction of Finding) }\end{array}$ & $\begin{array}{l}\text { Cerebral Palsy } \\
\text { Studies } \\
\text { Strength of Evidence } \\
\text { (Direction of Finding) }\end{array}$ & $\begin{array}{l}\text { Spinal Cord Injury } \\
\text { Studies } \\
\text { Strength of Evidence } \\
\text { (Direction of Finding) }\end{array}$ \\
\hline & $\begin{array}{c}\text { Moderate } \\
\text { (balance, depression } \\
\text { improvement, no clear } \\
\text { benefit on function) }\end{array}$ & $\begin{array}{c}\text { Low } \\
\left(\mathrm{VO}_{2} \text { improvement) }\right.\end{array}$ & $\begin{array}{c}\text { Low } \\
\mathrm{(VO}_{2} \text { improvement, } \\
\text { increased episodes of } \\
\text { autonomic dysreflexiac }^{\mathrm{c}} \text { no } \\
\text { clear benefit on depression) }\end{array}$ \\
\hline
\end{tabular}

Abbreviations: $\mathrm{ADL}=$ activities of daily living; $\mathrm{CP}=$ cerebral palsy; $\mathrm{MS}=$ multiple sclerosis; $\mathrm{RCT}=$ randomized controlled trial

a Strength of evidence color shading: blue $=$ high strength of evidence, green=moderate, yellow $=$ low, white $=$ insufficient

${ }^{\mathrm{b}}$ Strength of evidence based on combining the two populations, multiple sclerosis and cerebral palsy.

${ }^{\mathrm{c}}$ Whole-body exercise versus exercise limited to upper body

\section{Limitations}

\section{Key Question 4: Methodological Weaknesses or Gaps}

Conclusions that can be drawn from the evidence on physical activity in patients with multiple sclerosis, cerebral palsy, and spinal cord injury are limited by small sample sizes; few trials (in spinal cord injury); inadequate descriptions of population characteristics, control group activities, and intensity of physical activity; incomplete data analysis; inadequate reporting of adverse events; and relatively few trials considered to be high quality (low risk of bias). The addition of larger, well-conducted randomized controlled trials of longer duration and including all disability levels would greatly strengthen the evidence base and may alter the current conclusions.

\section{Implications and Conclusions}

Physical activity was associated with improvements in walking ability, general function, balance (including fall risk), depression, aerobic capacity, activities of daily living, female sexual function, and sleep, depending on population and type of physical activity. No studies reported long-term cardiovascular or metabolic disease health outcomes. Future trials could alter these findings, and further research is needed to examine health outcomes to understand the magnitude and clinical importance of benefits seen in intermediate outcomes.

\section{References}

1. U.S. Department of Health and Human Services. Physical Activity Guidelines for Americans, 2nd edition. Washington, D.C.: U.S. Department of Health and Human Services; 2018.

https://health.gov/paguidelines/secondedition/pdf/Physical_Activity_Guidelines_2 nd_edition.pdf Accessed Sept. 15, 2019.
2. UK Chief Medical Officers' Physical Activity Guidelines Care UKDoHS; Sept. 7, 2019.

https://assets.publishing.service.gov.uk/gove rnment/uploads/system/uploads/attachment data/file/829841/uk-chief-medical-officersphysical-activity-guidelines.pdf Accessed Sept. 15, 2019. 


\section{Introduction}

\section{Purpose}

This systematic review summarizes the current evidence on the health effects of physical activity interventions in people with multiple sclerosis (MS), cerebral palsy (CP), and spinal cord injury (SCI). These three diverse conditions were chosen to represent individuals using a wheelchair or individuals who may benefit from using a wheelchair in the future. ("Wheeled mobility device" is sometimes used to encompass manual wheelchairs, motorized wheelchairs, and motorized scooters; this report uses the term wheelchair in this broad sense.) The review is focused on four Key Questions developed by the National Institutes of Health to inform a Pathways to Prevention Workshop. It is anticipated that the evidence synthesis on the health effects of physical activity intervention in people with multiple sclerosis, cerebral palsy, and spinal cord injury will be of ongoing interest to primary and specialty care providers, health researchers, policymakers, and other stakeholders.

\section{Background}

For the general population, the health benefits of regular physical activity are wellrecognized, as highlighted in 2008 and 2018 reports to the Department of Health and Human Services from the Physical Activity Guidelines Advisory Committee. ${ }^{1,2}$ In addition to a reduced risk of death, greater amounts of regular moderate-to-vigorous physical activity reduces the risk of many of the most common and expensive diseases or conditions in the United States. Heart disease, stroke, hypertension, type 2 diabetes, dementia, depression, postpartum depression, excessive weight gain, falls with injuries among the elderly, and breast, colon, endometrial, esophageal, kidney, stomach, and lung cancer are all less common among individuals who are or become more physically active. ${ }^{2}$ Physical activity may also help reduce the natural progression of disability in certain populations. ${ }^{3}$ In 2016 one in four noninstitutionalized U.S. adults $(25.7 \%$, representing an estimated 61.4 million people) reported having a physical and/or cognitive disability, and mobility was the most prevalent disability type (13.7\% of the total). ${ }^{4}$ Newly released physical activity guidelines suggest adults with disability benefit from similar amounts of physical activity and muscle strengthening as the general population, although there may be some risk of injury for populations who are not accustomed to exercise. ${ }^{5}$ The U.S. Department of Health and Human Services indicates that routine physical activity programs combining aerobic exercise with muscle strength and balance training improve fitness, function, and quality of life for individuals with physical disabilities. ${ }^{2}$ Less is known regarding specific health benefits of physical activity in people who use a wheelchair.

The various populations using wheelchairs are broad and poorly captured in the literature on physical activity, making a systematic review of all "wheelchair users" unfeasible. Additionally, some individuals may only need the use of a wheelchair some of the time- to cover longer distances or when experiencing a disease flare-up, for example. In order to generate a meaningful result from representative populations that reflect relatively consistent examples of why and how wheelchairs are being used, the analysis is focused on a broad but representative sample of potential wheelchair users - individuals with MS, CP, and SCI. Wheelchair users with these conditions have diverse underlying physiologic mechanisms, demographic profiles, respective physical limitations, and potential outcomes from regular physical activity. Understanding those 
differences assists in interpreting the literature relating to exercise among these diverse groups (Table 1).

Table 1. Characteristics, causes, and prevalence of multiple sclerosis, cerebral palsy, and spinal cord injury

\begin{tabular}{|c|c|c|c|}
\hline $\begin{array}{l}\text { Causes, } \\
\text { Prevalence, } \\
\text { and } \\
\text { Characteristics }\end{array}$ & Cerebral Palsy & Multiple Sclerosis & Spinal Cord Injury \\
\hline Etiology & $\begin{array}{l}\text { Traumatic injury to a } \\
\text { developing brain before, } \\
\text { during, or after birth }\end{array}$ & $\begin{array}{l}\text { Progressive autoimmune } \\
\text { disease of the central } \\
\text { nervous system with variable } \\
\text { disease patterns; } 10 \% \\
\text { primary progressive and } \\
\text { others progressive after initial } \\
\text { relapse and remitting course }\end{array}$ & $\begin{array}{l}\text { Usually traumatic cord injury } \\
\text { (motor vehicle accidents, } \\
\text { falls, violence, sports); } \\
\text { nervous system above the } \\
\text { lesion is intact }\end{array}$ \\
\hline Prevalence & $\begin{array}{l}1.5 \text { to more than } 4 \text { per } 1,000 \\
\text { live births; males } 30 \% \\
\text { greater than females; } \\
764,000 \text { children and adults } \\
\text { living with CP in the United } \\
\text { States }^{6}\end{array}$ & $\begin{array}{l}\text { Nearly } 1 \text { million people in the } \\
\text { United States have MS; } \\
\text { average age onset } 30 \text { years } \\
\text { old and females } 2 \text { to } 3 \text { times } \\
\text { males }^{7}\end{array}$ & $\begin{array}{l}\text { Estimated } 282,000 \text { in the } \\
\text { United States with } \mathrm{SCl} \text {; recent } \\
\text { evidence puts the average } \\
\text { age } 43 \text { years old; } 78 \% \text { male }^{8,9}\end{array}$ \\
\hline Mobility & $\begin{array}{l}40 \% \text { limitations in walking } \\
\text { and } 30 \% \text { use walkers or } \\
\text { wheelchairs }\end{array}$ & $\begin{array}{l}\text { Mobility limitations generally } \\
\text { occur later in disease course; } \\
\text { after } 45 \text { years of disease, on } \\
\text { average } 76 \% \text { of individuals } \\
\text { require ambulatory aid and } \\
52 \% \text { bilateral assistance }{ }^{10}\end{array}$ & $\begin{array}{l}\text { Variable and depends on } \\
\text { level and completeness of } \\
\text { injury; generally stable after } \\
\text { injury and initial rehabilitation }\end{array}$ \\
\hline $\begin{array}{l}\text { Associated } \\
\text { morbidity }\end{array}$ & $\begin{array}{l}40 \% \text { of children with CP have } \\
\text { intellectual disability, } 35 \% \\
\text { epilepsy, and more than } 15 \% \\
\text { had vision impairment }\end{array}$ & $\begin{array}{l}\text { Sequela of immune } \\
\text { suppression including urinary } \\
\text { and respiratory infections, } \\
\text { seizures, other autoimmune } \\
\text { diseases, visual } \\
\text { abnormalities, ataxia }^{11}\end{array}$ & $\begin{array}{l}\text { Respiratory complications, } \\
\text { thromboembolism, autonomic } \\
\text { dysreflexia, orthostatic } \\
\text { hypotension, bladder } \\
\text { dysfunction, neurogenic } \\
\text { bowel, spasticity, pain, } \\
\text { pressure ulcers }^{12}\end{array}$ \\
\hline $\begin{array}{l}\text { Usual intent of } \\
\text { physical activity }\end{array}$ & $\begin{array}{l}\text { Increase mobility and overall } \\
\text { level of function as } \\
\text { component multimodality } \\
\text { efforts during childhood } \\
\text { development }\end{array}$ & $\begin{array}{l}\text { Maintain mobility and } \\
\text { attenuate limitations of } \\
\text { progressive disease; } \\
\text { because those with MS often } \\
\text { have normal life expectancies } \\
\text { the benefits of exercise for } \\
\text { the general population would } \\
\text { also apply }\end{array}$ & $\begin{array}{l}\text { Maximize functional abilities; } \\
\text { recreation; because long-term } \\
\text { sequela SCI better } \\
\text { prevented/managed, longer } \\
\text { term health benefits of regular } \\
\text { exercise also are relevant }\end{array}$ \\
\hline
\end{tabular}

Abbreviations: $\mathrm{CP}=$ cerebral palsy; $\mathrm{MS}$ = multiple sclerosis; $\mathrm{SCI}=$ spinal cord injury

SCI, MS, and CP have very different physiologic mechanisms (brain vs. spinal cord, degenerative vs. not) and demographic profiles (male vs. female predominance, childhood vs. adult onset). CP is usually present at birth. While the brain injury involved in CP can in general be relatively static, its early onset has effects on musculoskeletal development with functional sequela. In contrast, MS and SCI most often have onset after skeletal maturation is complete. MS can affect any central nervous system function, including vision, and can be progressive for many years. SCI does not affect motor or sensory systems above the level of the spinal cord lesion, sparing cerebral function, and the nervous system injury is usually static after the acute period.

While they are distinctly different in general etiology and pathophysiology, a common denominator for all three conditions is the involvement of the corticospinal tracts of the central nervous system, which results in an impaired central control and/or coordination of the 
peripheral muscles. This may lead to paralysis or reduced extremity muscle force and increased spasticity, which can greatly affect general mobility or coordinated movement such as posture and gait. The consequences on ambulation of this corticospinal tract injury exist along a functional spectrum, from fully ambulatory despite motor involvement, to a wide range of overlap of independent ambulation with intermittent wheelchair use, to full-time wheelchair use. Having MS, CP, or SCI is typically permanent and may result in decades of being sedentary if engaging in physical activity is not made a priority. The potential benefits in these populations may be even greater than in able-bodied people who are still mobile and who achieve some benefit of activity through performing ordinary activities in daily life such as pushing a grocery cart around a store, fixing dinner, or carrying a child up the stairs.

Many users of wheelchairs encounter psychological and physical barriers as well as limitations of access to preventive healthcare and appropriate physical activity programs intended to maintain healthy weight or body composition and physical fitness. The preventive benefits of regular exercise are particularly relevant for people with disabilities, who experience accelerated risk for the conditions known to be attenuated by regular exercise, such as obesity or increased body fat, ${ }^{13-15}$ dyslipidemia, ${ }^{16,17}$ and cardiovascular events such as myocardial infarction, ${ }^{17,18}$ stroke, ${ }^{18-20}$ and death. ${ }^{8,18}$ Increased risk for morbidity and mortality may be due, in part, to the specific disease that limits mobility or leads to the use of a wheelchair, the treatment for the disease (e.g., steroids used to treat MS), and/or a sedentary lifestyle.

The National Academies of Sciences, Engineering, and Medicine's 2017 report on the use of assistive technologies to enhance activity recommends that individuals who require wheeled and seated mobility devices receive regular evaluations of their physical condition. ${ }^{21}$ Evaluation should include at least annual assessments of the functioning and fitting of the devices, ergonomics and safety, ability to use the device, underlying disorder and secondary health conditions, functional needs, and the individual's satisfaction. Access to appropriate care can facilitate education, linkage to activity resources, and encouragement of physical activity to help mitigate these risks.

People with disabilities face a number of barriers to exercise. Skill at using a wheelchair, fatigue, fear of falling, pain, heat sensitivity, negative bias/stigma, ${ }^{22}$ and conflicting information from providers have been listed as barriers to exercise among those with MS. ${ }^{23-27}$ Those with CP cite the need for caregiver support, prohibitive cost, and their medical condition as barriers to regular physical activity. ${ }^{28}$ Additionally, it can be challenging to find physical activities that a child with quadriplegic CP can do to improve strength or aerobic conditioning when motor control is insufficient. For individuals with SCI, concern about autonomic dysfunction, blood pressure and temperature regulation during exercise, may limit exercise participation, ${ }^{29,30}$ contributing to decreasing fitness levels with increasing time since injury. ${ }^{21}$ All wheelchair users are limited by lack of access to facilities, lack of transportation, and insufficient Americans with Disabilities Act compliance at community fitness centers. ${ }^{31-33}$ Individuals who infrequently need a wheelchair may not be completely comfortable with their wheelchair skills and therefore may not be active enough in participating in wheelchair sports or physical activities. ${ }^{34}$ Special equipment such as robot-assisted gait training (RAGT) or body weight support treadmill devices can be prohibitively difficult for people outside of major urban areas to access.

A review of Canadian community-based physical activity and wheelchair mobility programs points out a clear need for more programs, particularly those that assess long-term impact. ${ }^{32}$ Longer time since injury is associated with lower fitness levels in SCI with paraplegia. ${ }^{35}$ 
Decreased strength and muscle mass associated with aging increases risk for shoulder injury, and elderly wheelchair users need specific interventions to preserve mobility. ${ }^{36}$

Physical activity has been shown to improve body composition, ${ }^{37-39}$ cognition, ${ }^{40}$ glucose metabolism, ${ }^{39,41,42}$ and lipid profiles,${ }^{39,43}$ and to decrease risk of morbidity and mortality in nondisabled people. ${ }^{38,44}$ Physical activity could similarly benefit those with disabilities. Recently published SCI guidelines recommend moderate to vigorous intensity aerobic exercise at least twice weekly and strength exercise for each major functioning muscle group twice weekly. ${ }^{45}$ Verschuren et al. recommend aerobic sessions and strength training twice weekly for individuals with $\mathrm{CP},{ }^{46}$ while Halabchi et al. recommend aerobic exercises, strength training, and daily flexibility and stretching exercises for individuals with $\mathrm{MS} .{ }^{47}$ In the past, exercise was not recommended for individuals with MS due to fear of worsening of symptoms. ${ }^{48}$ However, more recent evidence suggests that physical activity improves health outcomes in people with disabilities (including people with MS), and the updated 2018 Physical Activity Guidelines for Americans now recommend between 2.5 to 5 hours of moderate aerobic exercise weekly, or over 1 hour to 2.5 hours of vigorous aerobic exercise weekly, plus muscle strengthening activities, for people with physical disabilities. ${ }^{2}$ These guidelines suggest that children ages 3 through 5 years engage in physical activity throughout the day for normal growth and development and that school-aged children and adolescents receive 60 minutes of moderate to vigorous-intensity aerobic activity, 60 minutes of muscle-strengthening activity, and 60 minutes of bonestrengthening activity at least 3 days a week. The guidelines do not offer recommendations regarding physical activity in children or adolescents with chronic disease or physical disability.

\section{Scope and Key Questions}

This systematic review summarizes and synthesizes current research on the specific benefits and potential harms of physical activity for people with MS, CP, and SCI, regardless of current use of a wheelchair. This topic was nominated by the Director of the National Center for Medical Rehabilitation Research, and supported by the National Institute of Child Health and Human Development, the National Institute of Neurological Disorders and Stroke, the National Institutes of Health Office of Disease Prevention, and the National Institutes of Health Medical Rehabilitation Coordinating Committee, which has representatives from 20 Institutes and Centers, along with other federal partners for a Pathways to Prevention (P2P) workshop to assess the benefits and harms of physical activity on the physical and mental health of adults, children, and adolescents using a wheelchair, or who may benefit from using a wheelchair in the future. In considering studies related to physical activity among three representative populations who consistently use, sometimes use, or who may, at some point in their lives, need to use a wheelchair as a result of neurological conditions of MS, CP, and SCI, we prioritized certain outcomes. These included long-term health outcomes of: cardiovascular mortality; myocardial infarction; stroke; development of diabetes; and new or increased need for a wheelchair. Other prioritized immediate health outcomes included: pulmonary function tests; $\mathrm{VO}_{2}$ peak; hemoglobin A1c (HbAlc); bowel, bladder, and sexual function; decubitus ulcers; development of obesity; body mass index; weight; depression; quality of life; falls; function; autonomic dysreflexia; and spasticity. We evaluated outcomes of diverse physical activity interventions, inclusion/exclusion criteria, and research methodologies to identify future research needs. The outcomes of pain and cognition were not included because it is expected that the magnitude of the literature involved would indicate that these topics should be separate reviews. Our overarching objective was to understand the specific benefits and potential harms of physical 
activity for those currently using or those who may benefit from using a wheelchair in the future and to identify domains for future research focus - ultimately improving health and quality of life.

\section{Key Questions}

Key Question 1: What is the evidence base on physical activity interventions to prevent obesity, diabetes, and cardiovascular conditions, including evidence on harms of the interventions in people with multiple sclerosis, cerebral palsy, or spinal cord injury who are at risk for or currently using a wheeled mobility device?
a. What interventions have been studied?
b. What outcomes have been studied?
c. What inclusion/exclusion criteria have been used in studies?
d. What other research methodologies (control/comparison group design, length of intervention, research setting) have been used?

Key Question 2: What are the benefits and harms of physical activity interventions for people with multiple sclerosis, cerebral palsy, or spinal cord injury who are at risk for or currently using a wheeled mobility device?

a. Does physical activity improve clinical outcomes such as cardiovascular disease, diabetes, overweight or obesity, mental health, or sexual function?

b. Does physical activity improve intermediate outcomes such as physical fitness, obesity, or bone density?

c. Does physical activity reduce the harms of immobility, such as incidence of decubitus ulcer, urinary tract infection, bowel dysfunction, or autonomic dysfunction?

d. Does physical activity decrease the risk for adverse outcomes of disorders associated with wheeled mobility device use, such as spasticity, autonomic dysreflexia, or muscle contractures?

e. What are the harms of physical activity, such as injuries that are associated with wheeled mobility device use (e.g., falls, tips, overuse injuries)?

f. Do the benefits or harms of physical activity vary by the location of the intervention (e.g., home, community, clinic), amount of training or instruction (e.g., no training, some training, all physical activity sessions with training), or level of supervision (e.g., inpatient, telehealth)?

Key Question 3: What are the patient factors that may affect the benefits and harms of physical activity in patients with multiple sclerosis, cerebral palsy, or spinal cord injury who are at risk for or currently using a wheeled mobility device?

a. Do the benefits and harms of physical activity vary by age, sex, or race/ethnicity?

b. Do the benefits and harms of physical activity vary by primary disease or injury that led to wheelchair use? 
Key Question 4: What are methodological weaknesses or gaps that exist in the evidence to determine benefits and harms of physical activity in patients with multiple sclerosis, cerebral palsy, or spinal cord injury who are at risk for or currently using a wheeled mobility device?

a. What types of studies supported conclusions in Key Questions 2 and 3 ?

b. What are the major weaknesses in study designs?

c. What would improve ability of future research to address the Key Questions?

\section{PICOTS}

The Methods section provides details on the Populations, Interventions, Comparators, Outcomes, Timing, Settings, and Study Designs (PICOTS) inclusion and exclusion criteria. An overview of the PICOTS for this review follows.

\section{Populations}

- Include for Key Question (KQ) 1, KQ2, and KQ3: Patients with MS, CP, or SCI; in studies of mixed populations, at least 80 percent will be individuals with MS, CP, and/or SCI. All ages included.

- Exclude: Other populations.

\section{Interventions}

- Include for all KQs: Any gross motor intervention with a defined period of directed physical activity that is expected to increase energy expenditure. Intervention must have a minimum of 10 sessions on 10 different days of activity in a supervised individual or group setting. Include: aerobic exercise, strength training, standing, balance, flexibility, and combination interventions.

- Exclude: Unobserved physical activity; parent or caregiver observed interventions; interventions that do not target the whole body (e.g., interventions to improve reaching or to improve the function of one joint, partial body vibration); single studies of one intervention.

\section{Comparators}

- Include for all KQs: Between-group comparisons with no physical activity or other types of physical activity or a behavioral intervention with a physical activity outcome.

- Exclude: Comparisons to other active comparators such as drug therapy; pre-post studies with only one group of participants.

\section{Outcomes}

- For KQ1: Outcome measures, physical activity interventions, inclusion/exclusion criteria, and research methodologies related to prevention of obesity, diabetes, cardiovascular 
conditions, or harms; types of studies or bodies of studies supporting conclusions for KQs 2 and 3.

- For KQ2 and KQ3: Benefits and harms of physical activity including: (a) clinical outcomes such as cardiovascular mortality, myocardial infarction, stroke, diabetes, mental health, obesity/overweight, and sexual function; (b) intermediate outcomes such as physical fitness, HbA1c, bone density, and resting heart rate; and (c) subgroup differences based on location of intervention (e.g., home, community, clinic), level of instruction or training (e.g., no training, some training, all physical activity sessions with training), and level of supervision (e.g., inpatient, telehealth).

- For KQ4: Major weakness in study design, items that improve the ability to address the KQs.

- Exclude: Outcomes not used to make clinical decisions (e.g., estradiol level, muscle thickness).

\section{Timing}

- Include for all KQs: At least 10 sessions of physical activity spread out over no fewer than 10 days.

- Exclude: Acute spinal cord trauma stabilization period, immediate postoperative period (e.g., after surgeries to improve musculoskeletal function in CP).

\section{Setting}

- Include for all KQs: Any U.S. or U.S.-applicable study, including clinic, home (provided physical activity is observed by healthcare or research staff), or community setting (e.g., gym or athletic class).

- Exclude: Non-U.S.-applicable setting.

\section{Study Designs}

- Include for all KQs: Clinical trials and observational studies (cohort studies and casecontrol studies).

- Include for all KQs: Studies with the following minimum sample sizes analyzed: MS $(\mathrm{n}=30), \mathrm{CP}(\mathrm{n}=20), \mathrm{SCI}(\mathrm{n}=20)$.

- Include for all KQs: Studies published since 2008; systematic reviews published since 2014.

- Include, if needed, due to lack of clinical trials or controlled observational studies: Prepost studies.

- Exclude: Case report, case series, and cross-sectional studies.

\section{Analytic Framework}

The analytic framework (Figure 1) illustrates the relationship between the KQs and the outcomes for this review. The figure indicates the questions associated with intermediate outcomes, descriptions of the evidence base, clinical outcomes, and harms. The complete 
PICOTS criteria for inclusion and exclusion of studies in this review appear in the Methods section.

Figure 1. Analytic framework for physical activity and the health of wheelchair users with multiple sclerosis, cerebral palsy, and spinal cord injury

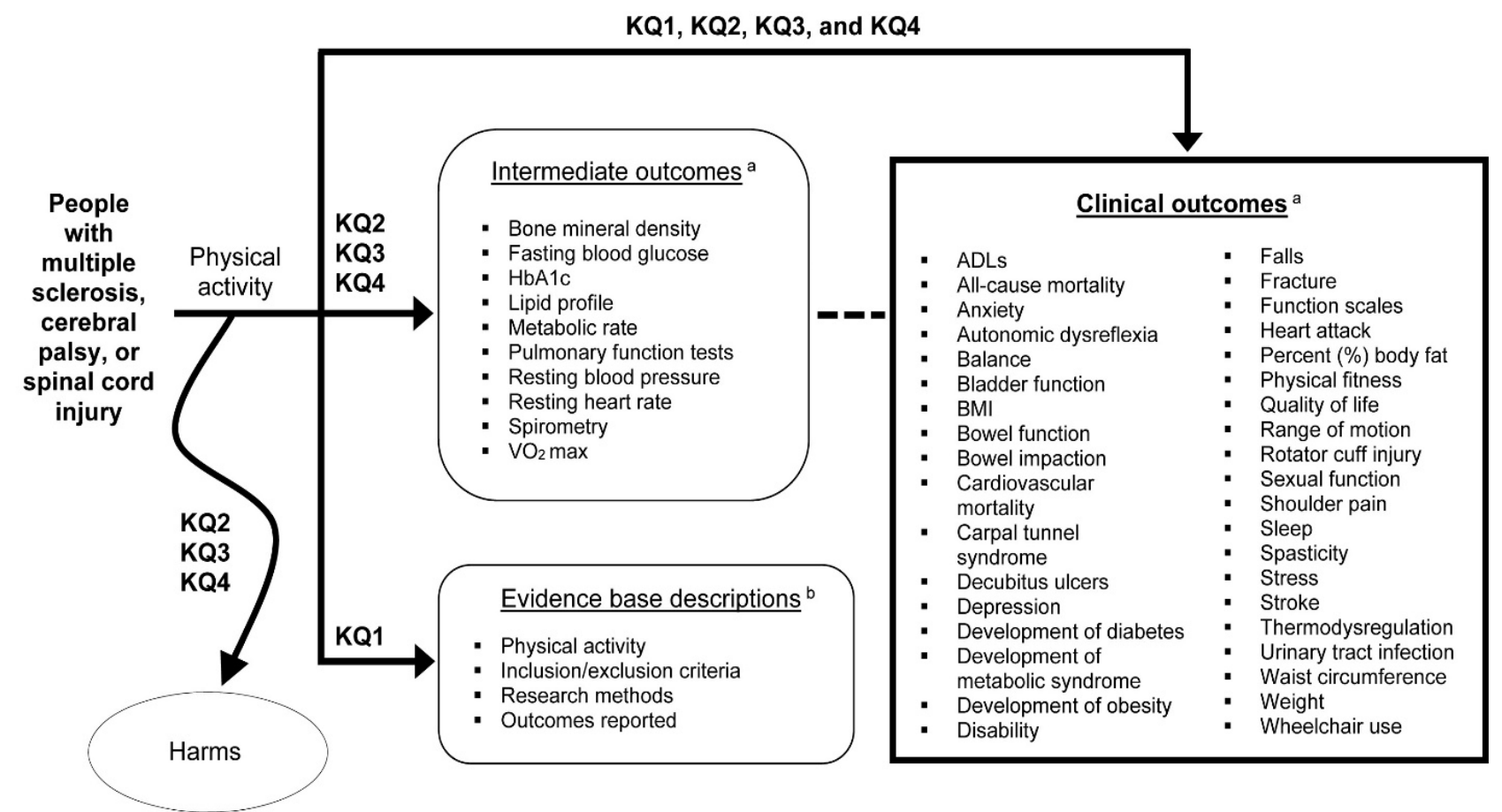

Abbreviations: $\mathrm{ADL}=$ activities of daily living; $\mathrm{BMI}=$ body mass index; $\mathrm{HbA} 1 \mathrm{c}=$ hemoglobin $\mathrm{A} 1 \mathrm{c} ; \mathrm{KQ}=\mathrm{Key} \mathrm{Question}_{\mathrm{VO}} \mathrm{VO}_{2}$ $\max =$ maximal oxygen uptake

${ }^{a}$ Outcomes are specified in the Methods section

${ }^{\mathrm{b}}$ Studies that evaluate prevention of obesity, diabetes, cardiovascular conditions, and harms 


\section{Organization of Report}

This report is organized by sections. Each represents either a main section of the report (i.e., Introduction, Methods, Results, Discussion, and Conclusion) or a Key Question.

Key Question 1 provides an overview of the evidence base of included studies as well as identification of studies that did not meet the inclusion criteria of this report.

For Key Question 2 we present the results of the benefits and harms of physical activity interventions for clinical outcomes of interest (Figure 1), subdivided by intervention categories of aerobic exercise, postural control, strength interventions, and multimodal interventions. Interventions specific to each of these categories are indicated with a brief description of the subtype of exercise, key points specific to that intervention, and detailed results are organized by the specific population of MS, CP, and SCI. Studies that reported on only one or more intermediate outcomes (Figure 1) are reported separately.

The Key Question 2 intervention categories include:

\section{Aerobic exercise interventions:}

- Aerobics

- Aquatics

- Cycling

- Hand cycling

- RAGT

- Treadmill

\section{Postural control interventions:}

- Balance exercise

- Hippotherapy

- Tai Chi

- Motion gaming

- Whole body vibration

- Yoga

\section{Strength interventions:}

- Muscle strength exercise

\section{Multimodal interventions:}

- Progressive resistance or strengthening exercise plus aerobics and/or postural control interventions

For Key Question 2 the general effects of exercise were also assessed:

\section{All exercise interventions:}

- Interventions with sufficient outcomes data to be analyzed independent of population or intervention category 
Key Question 3 evaluates patient factors that may affect the benefits and harms of physical activity, and Key Question 4 reports the methodological weaknesses or gaps in the evidence base. 


\section{Methods}

This Comparative Effectiveness Review follows the methods suggested in the AHRQ Methods Guide for Effectiveness and Comparative Effectiveness Reviews (hereafter the "EPC Methods Guide"). ${ }^{49}$ All methods were determined a priori and the protocol was published on the Agency for Healthcare Research and Quality website (https://effectivehealthcare.ahrq.gov/sites/default/files/pdf/wheelchair-users-amendedprotocol.pdf). The protocol for this review was also submitted to the PROSPERO systematic review registry (CRD42019130060).

\section{Topic Refinement}

Prior to conducting this review, the Evidence-based Practice Center (EPC) refined the preliminary Key Questions and PICOTS (Populations, Interventions, Comparators, Outcomes, Timing, Studies, Settings) with the AHRQ Task Order Officer (TOO), representatives from National Institutes of Health (NIH), Key Informants and the Technical Expert Panel (TEP). NIH assisted in evaluating proposed changes based on the preliminary literature review and input from the stakeholders as well as prioritization of outcomes.

\section{Literature Search Strategy}

We searched MEDLINE ${ }^{\circledR}$, CINAHL $^{\circledR}$, PsycINFO ${ }^{\circledR}$, Cochrane CENTRAL, Embase ${ }^{\circledR}$, and Rehabilitation and Sports Medicine Source. We also searched ClinicalTrials.gov to capture gray literature. These databases were broad enough to capture the study types, the populations (multiple sclerosis [MS], cerebral palsy [CP], and spinal cord injury [SCI]), and physical activities studied. The full search strategies are in Appendix A. We reviewed reference lists of systematic reviews for includable literature. In addition, TEP members were asked to provide suggestions about unpublished literature. We limited the search to studies published since 2008 and systematic reviews since 2014. An updated literature search was conducted in November 2020. Authors of three studies were contacted for information (no additional information was provided). ${ }^{50-54}$

The criteria for selection of studies to be included in the review were pre-established (Table 2) and used to determine eligibility for inclusion and exclusion of abstracts according to the EPC Methods Guide. ${ }^{49}$ Two team members trained in systematic review methodology reviewed titles and abstracts for potential eligibility. ${ }^{55}$ Excluded abstracts were dual reviewed. We retrieved the full text of articles for all abstracts selected by at least one reviewer as potentially eligible for inclusion in the review, and two team members independently reviewed the full-text articles. Disagreements on eligibility were resolved by consensus.

\section{Inclusion and Exclusion Criteria and Study Selection}

The criteria for inclusion and exclusion of studies were designed to identify outcomes that answer the Key Questions and are based on the PICOTS (Table 2).

The populations for this review are people who have MS, CP, or SCI. Limiting the population to these three groups was designed to capture a broad, diverse population of those who need or who may need the assistance of a wheelchair. Study designs indicated in Table 2 were included. We included studies from countries with a very high or high score on the Human Development Index because studies from these countries are more likely to generate results 
similar to a study conducted in the United States. Pre-post studies that otherwise meet inclusion criteria were considered for inclusion in the absence of higher-quality evidence.

Interventions with a defined period of observed physical activity (movement using more energy than rest) with a minimum of 10 sessions of activity on 10 days or more in a supervised or group setting were included. Prioritized outcomes for which we assessed the strength of evidence include: cardiovascular mortality; myocardial infarction; stroke; pulmonary function tests; $\mathrm{VO}_{2}$ max or peak; development of diabetes; hemoglobin A1c (HbA1c); bowel, bladder, and sexual function; decubitus ulcers; development of obesity; body mass index (BMI); weight; depression; quality of life; time to and amount of wheelchair use; falls; general function; autonomic dysreflexia; and spasticity (Table 3). The strength of evidence was as also assessed for balance. We did not include interventions that were reported in only one study.

Given the publication of the initial 2008 Physical Activity Guidelines ${ }^{1}$ (updated with a second edition in $2018^{5}$ ) and the large number of potentially relevant publications for this review, we searched for studies published since 2008 and systematic reviews published since 2014. Systematic reviews were used only to identify additional studies because the populations, inclusion criteria, interventions, and timing of the studies in the systematic reviews differed in study eligibility from the inclusion criteria for this report. We examined reference lists from systematic reviews and if specific studies reported in the systematic reviews met our inclusion criteria based on the Key Questions and PICOTS (Table 2) those studies were dual reviewed and included in the report.

These decisions regarding study design, study size, publication date range, and prioritization of outcomes were developed in collaboration with the NIH Pathways 2 Prevention Working Group and reviewed with a panel of technical experts. 
Table 2. PICOTS-inclusion and exclusion criteria

\begin{tabular}{|c|c|c|}
\hline PICOTS & Inclusion & Exclusion \\
\hline Populations & $\begin{array}{l}\text { Patients using a wheelchair or those who may benefit from using a } \\
\text { wheelchair in the future due to MS, CP, or SCl. All ages included. }\end{array}$ & $\begin{array}{ll}- & \text { Other populations } \\
\text { - } & \text { Studies of mixed } \\
\text { populations with }<80 \% \\
\mathrm{MS}, \mathrm{CP}, \mathrm{SCl}\end{array}$ \\
\hline Interventions & $\begin{array}{l}\text { Any gross motor intervention with a defined period of directed } \\
\text { physical activity that is expected to increase energy expenditure. } \\
\text { Intervention must have a minimum of } 10 \text { sessions of activity on } 10 \\
\text { days or more in a supervised or group setting. Include aerobic } \\
\text { exercise, strength training, standing, balance, flexibility, and } \\
\text { combination interventions. } \\
\text { Included activities (not exhaustive, additional activities may qualify): } \\
\text { Balance/flexibility } \\
\text { - Stretching/flexibility } \\
\text { - Yoga or Pilates } \\
\text { - Martial arts (e.g., Tai Chi) } \\
\text { Physical/aerobic exercise } \\
\text { - } \quad \text { Arm ergometry } \\
\text { - } \quad \text { Wycling (stationary, recumbent, or arm) } \\
\text { - } \quad \text { Functional electronic stimulation } \\
\text { - Robot-assisted gait training } \\
\text { - } \quad \text { Swimming } \\
\text { - Aquatherapy } \\
\text { - Group exercise } \\
\text { - Team sports } \\
\text { - Treadmill (including with body weight support) } \\
\text { Strength/resistance training } \\
\text { - } \quad \text { Resistance bands } \\
\text { - Weight lifting }\end{array}$ & $\begin{array}{ll}\text { - } & \text { Interventions with }<10 \\
\text { - } & \text { sessions } \\
\text { - } & \text { pnterventions over a } \\
\text { - } & \text { Unobserved physical } \\
\text { activity } & \\
\text { - } \quad \text { Family- or caregiver-only } \\
\text { observed physical activity } \\
\text { - Patient-recalled physical } \\
\text { activity } \\
\text { - Postoperative physical } \\
\text { activity } \\
\text { Intervention focused on } \\
\text { improving reaching } \\
\text { - Interventions without } \\
\text { whole body effect (e.g., } \\
\text { targeting one joint) } \\
\text { Intervention reported in } \\
\text { only one study }\end{array}$ \\
\hline Comparators & $\begin{array}{l}\text { Comparisons to no physical activity or other types of physical } \\
\text { activity or behavioral counseling. }\end{array}$ & - $\quad$ All other active controls \\
\hline Outcomes & $\begin{array}{l}\text { Cardiovascular } \\
\text { - Cardiovascular mortality, myocardial infarction, stroke, all-cause } \\
\text { mortality, resting heart rate, resting blood pressure, lipid profile } \\
\text { Respiratory } \\
\text { - Pulmonary function tests, } \mathrm{VO}_{2} \text { max/peak, spirometry } \\
\text { Endocrine } \\
\text { - Development of diabetes, } \mathrm{HbA} 1 \mathrm{c} \text {, fasting blood glucose, } \\
\quad \text { development of metabolic syndrome, metabolic rate } \\
\text { Gastrointestinal } \\
\text { Bowel function, bowel impaction } \\
\text { Genitourinary } \\
\text { B Bladder function, urinary tract infection } \\
\text { Musculoskeletal } \\
\text { - Fracture, bone mineral density, muscle strength, rotator cuff } \\
\quad \text { injury, shoulder pain, range of motion } \\
\text { Reproductive } \\
\text { - Sexual function }\end{array}$ & $\begin{array}{ll}-\quad \text { Outcomes not used to } \\
\text { make clinical decisions } \\
\text { (e.g., estradiol level) } \\
\text { Other outcomes (e.g., } \\
\text { head pitch and roll, } \\
\text { kinematic variables, } \\
\text { stepping kinematics, } \\
\text { reaching, muscle } \\
\text { thickness, muscle quality, } \\
\text { blood flow restriction, } \\
\text { premotoneuronal control) } \\
\text { Hospitalization or length of } \\
\text { stay } \\
\text { Cognition } \\
\text { Pain other than shoulder } \\
\text { pain }\end{array}$ \\
\hline
\end{tabular}




\begin{tabular}{|c|c|c|}
\hline PICOTS & Inclusion & Exclusion \\
\hline $\begin{array}{l}\text { Outcomes } \\
\text { (continued) }\end{array}$ & $\begin{array}{l}\text { Integumentary } \\
\bullet \quad \text { Decubitus ulcers } \\
\text { Body composition } \\
-\quad \text { Weight, BMI, development of obesity, waist circumference, \% } \\
\text { body fat } \\
\text { Mental health } \\
\text { Gepression, quality of life, anxiety, stress, sleep } \\
\text { General function } \\
\text { - Walking, falls, wheelchair use, function scales, disability, ADL, } \\
\text { balance, physical fitness } \\
\text { Neurological } \\
\text { - Autonomic dysreflexia, spasticity, thermodysregulation, carpal } \\
\quad \text { tunnel syndrome }\end{array}$ & \\
\hline Timing & $\begin{array}{l}\text { At least } 10 \text { days with at least one session of physical activity per } \\
\text { day. }\end{array}$ & $\begin{array}{l}\text { - } \quad \text { Acute } \mathrm{SCl} \text {, undergoing } \\
\text { stabilization } \\
\text { - Immediate postoperative } \\
\text { period }\end{array}$ \\
\hline Setting & $\begin{array}{l}\text { Any setting, including, clinic, home, or community setting (e.g., gym } \\
\text { or athletic class). Physical activity occurring in the home must still be } \\
\text { observed by medical, research, or athletic staff. }\end{array}$ & $\begin{array}{l}\text { Non-U.S. applicable } \\
\text { studies }\end{array}$ \\
\hline $\begin{array}{l}\text { Study } \\
\text { Designs }\end{array}$ & $\begin{array}{ll} & \text { Randomized controlled trials published since } 2008 \\
\text { - } & \text { Controlled observational studies published since } 2008 \\
\text { - } & \text { Systematic reviews published since } 2014 \text { to review for } \\
\text { additional studies meeting inclusion criteria } \\
\text { - } \quad \text { Potentially include pre-post studies in the absence of clinical } \\
\text { trials and controlled observational studies } \\
\text { Studies with the following sample sizes: MS ( } n \geq 30), C P(n \geq 20) \text {, } \\
\text { SCI }(n \geq 20) \text {. }\end{array}$ & $\begin{array}{ll}- & \text { All other study designs } \\
\text { (e.g., case series and } \\
\text { case reports) } \\
\text { Studies published before } \\
2008 \\
\text { - Systematic reviews } \\
\text { published prior to } 2015\end{array}$ \\
\hline
\end{tabular}

Abbreviations: $\mathrm{ADL}=$ activities of daily living; $\mathrm{BMI}=$ body mass index; $\mathrm{CP}=$ cerebral palsy; HbA1c $=$ hemoglobin $\mathrm{A} 1 \mathrm{c} ; \mathrm{MS}=$ multiple sclerosis; $\mathrm{SCI}=$ spinal cord injury; $\mathrm{VO}_{2} \max =$ maximal oxygen uptake; $\mathrm{VO}_{2}$ peak = highest value of $\mathrm{VO}_{2}$ attained 
Table 3. Outcomes

\begin{tabular}{|c|c|c|}
\hline System & Prioritized Outcomes & Other Outcomes \\
\hline Cardiovascular & $\begin{array}{ll}\text { - } & \text { Cardiovascular mortality } \\
\text { - } & \text { Myocardial infarction } \\
\text { - } & \text { Stroke }\end{array}$ & $\begin{array}{ll}\text { - } & \text { All-cause mortality } \\
\text { - } & \text { Lipid profile } \\
\text { - } & \text { Resting blood pressure } \\
\text { - } & \text { Resting heart rate }\end{array}$ \\
\hline Respiratory & $\begin{array}{ll}\text { - } & \text { Pulmonary function tests } \\
\text { - } & \mathrm{VO}_{2} \mathrm{max} / \mathrm{peak}\end{array}$ & - $\quad$ Spirometry \\
\hline Endocrine & $\begin{array}{ll}\text { - } & \text { Development of diabetes } \\
\text { - } & \mathrm{HbA} 1 \mathrm{c} \\
\end{array}$ & $\begin{array}{ll}- & \text { Development of metabolic syndrome } \\
\text { - } & \text { Fasting blood glucose } \\
\text { - } & \text { Metabolic rate } \\
\end{array}$ \\
\hline Gastrointestinal & - Bowel function & - Impaction \\
\hline Genitourinary & - $\quad$ Bladder function & - Urinary tract infection \\
\hline Musculoskeletal & None prioritized & $\begin{array}{ll}\text { - } & \text { Bone mineral density } \\
\text { - } & \text { Fracture } \\
\text { - } & \text { Muscle strength } \\
\text { - } & \text { Range of motion } \\
\text { - } & \text { Rotator cuff injury } \\
\text { - } & \text { Shoulder pain } \\
\end{array}$ \\
\hline Reproductive & - Sexual function & None \\
\hline Integumentary & - Decubitus ulcers & None \\
\hline $\begin{array}{l}\text { Body } \\
\text { Composition }\end{array}$ & $\begin{array}{ll}\text { - } & \text { Body mass index } \\
\text { - } & \text { Development of obesity } \\
\text { - } & \text { Weight } \\
\end{array}$ & $\begin{array}{l}\text { - } \quad \text { Percent body fat } \\
\text { - } \quad \text { Waist circumference }\end{array}$ \\
\hline Mental Health & $\begin{array}{l}\text { - } \quad \text { Depression } \\
\text { - } \quad \text { Quality of life }\end{array}$ & $\begin{array}{ll} & \text { Anxiety } \\
- & \text { Sleep } \\
- & \text { Stress } \\
\end{array}$ \\
\hline General Function & $\begin{array}{ll}\text { - } & \text { Falls } \\
\text { - } & \text { Function scales } \\
\text { - } & \text { Wheelchair use } \\
\end{array}$ & $\begin{array}{ll}\text { - } & \text { Activities of daily living } \\
\text { - } & \text { Balance } \\
\text { - } & \text { Disability } \\
\text { - } & \text { Physical fitness } \\
\end{array}$ \\
\hline Neurological & $\begin{array}{l}\text { - } \quad \text { Autonomic dysreflexia } \\
\text { - } \quad \text { Spasticity }\end{array}$ & $\begin{array}{l}\text { - } \quad \text { Carpal tunnel syndrome } \\
\text { - Thermodysregulation }\end{array}$ \\
\hline
\end{tabular}

Abbreviations: $\mathrm{HbA1c}=$ hemoglobin $\mathrm{A} 1 \mathrm{c} ; \mathrm{VO}_{2} \max =$ maximal oxygen uptake; $\mathrm{VO}_{2}$ peak = highest value of $\mathrm{VO}_{2}$ attained

\section{Data Abstraction and Data Management}

Data was abstracted from studies meeting the inclusion criteria (Table 2, and Appendix B). We abstracted data on study design, year, setting, country, sample size, eligibility criteria, population, clinical characteristics, (e.g., age, sex, race, MS, CP, or SCI), current versus potential wheelchair users, interventions and comparators, characteristics of the intervention (e.g., number of sessions, level of training of session supervisor), and outcomes (e.g., body mass index, resting heart rate, 6-Minute Walk Test (6MWT), depression scale scores, balance scale scores), and funding. Abstracted study data was verified for accuracy and completeness by a second team member. A record of studies excluded at the full-text level with reasons for exclusion is included in Appendix C. Definitions and characteristics of the included and excluded studies and details of the systematic reviews evaluated for potential includable studies are in Appendix D.

\section{Quality (Risk of Bias) Assessment of Individual Studies}

We assessed the risk of bias of randomized controlled trials (RCTs), quasiexperimental studies (nonrandomized studies), and cohort studies following the EPC Methods Guide ${ }^{56}$ using 
study design-specific criteria adapted from the U.S. Preventive Services Task Force ${ }^{57}$ and the Cochrane Collaboration. ${ }^{58}$ For RCTs, we evaluated factors such as randomization and allocation concealment methods, attrition, use of intent-to-treat methods, and blinding. For nonrandomized studies, we assessed factors such as participant selection methods; attrition; accuracy of methods for measuring exposures, outcomes, and confounders; and appropriateness of methods to address potential confounding.

Studies rated "good" have the least risk of bias, and their results are considered valid. Goodquality studies include clear descriptions of the population, setting, interventions, and comparison groups; a valid method for allocation of participants to treatment; low dropout rates and clear reporting of dropouts; appropriate means for preventing bias; and appropriate measurement of outcomes.

Studies rated "fair" may be susceptible to some bias, though not enough to invalidate the results. These studies may not meet all the criteria for a rating of good quality, but no flaw is likely to cause major bias. The study may be missing information, making it difficult to assess limitations and potential problems. The fair-quality category is broad, and studies with this rating will vary in their strengths and weaknesses. The results of some fair-quality studies are likely to be valid, while others may be only possibly valid.

Studies rated "poor" have significant flaws that imply biases of various types that may invalidate the results. They may have a serious or "fatal" flaw in design, analysis, or reporting; large amounts of missing information; discrepancies in reporting; or serious problems in the delivery of the intervention. The results of these studies will be at least as likely to reflect flaws in the study design as the true difference between the compared interventions.

Each included study was independently dual-reviewed for quality by two EPC team members and disagreements resolved by consensus. Criteria for assessing the quality and external validity of studies is provided in Appendix E.

\section{Data Analysis and Synthesis}

The findings are summarized in evidence tables indicating the study characteristics and outcome results and study quality ratings, and are included in summary tables of the key findings. Findings are organized by the intervention categories: aerobic exercise (including aquatics, cycling, dance, and robot-assisted gait training [RAGT]), postural control (including postural control exercises, hippotherapy, Tai Chi, motion games, whole body vibration [WBV], and yoga) as well as strength exercises (including; muscle strengthening exercise and multimodal exercise with strength as a major component). Results for each of these categories are reported by etiology of disability (i.e., MS, CP, SCI). Evidence from the included studies is in Appendix $\mathrm{F}$, and Appendix $\mathrm{G}$ provides the quality ratings for individual studies.

\section{Statistical Meta-Analysis}

We conducted quantitative synthesis involving pooling of study findings in meta-analyses as appropriate (i.e., when studies are homogeneous enough to provide meaningful combined estimates) to summarize data from multiple studies and to obtain more precise and accurate estimates of effects. The difference between each intervention's mean change from baseline to followup, or the mean difference (MD), was the primary effect size. Standardized mean differences were calculated when the scale of units varied within an outcome. Methods for calculating the standard deviation of the change scores followed the recommendations given in The Cochrane Handbook 7.7. ${ }^{58}$ Meta-analyses were conducted using STATA 14.0 and 14.2 
(StataCorp, College Station Texas). In the case of nonconvergence with Profile Likelihood, or when there was no meaningful difference between analyses using Profile Likelihood and Dersimonian and Laird, the Dersimonian and Laird estimates were reported. The I-squared $\left(\mathrm{I}^{2}\right)$ statistic was used to assess statistical heterogeneity. When statistical heterogeneity is present (i.e., $\mathrm{I}^{2}>30 \%$ ), an attempt to understand the heterogeneity through stratification of data and/or sensitivity analysis was conducted.

When pooled studies varied in quality and included poor-quality RCTs, a sensitivity analysis was conducted by removing studies rated poor quality. Quasiexperimental and cohort studies were not included in meta-analyses involving RCTs due to the difference in study design and the relatively poor quality of these studies. Pooled analysis focused on prioritized outcomes for which there was sufficient data.

Due to the large number of potential outcomes, quantitative synthesis focused on those outcomes previously prioritized for strength of evidence rating (Table 3) with the addition of the Berg Balance Scale, which was not a prioritized outcome but was the outcome with the most evidence.

\section{Grading the Strength of Evidence}

The strength of evidence for each Key Question was initially assessed by one researcher and verified by a second reviewer for each outcome by using the approach described in the EPC

Methods Guide. ${ }^{56}$ To ensure consistency and validity of the evaluation, the grades reviewed for:

- Study limitations (low, medium, or high level of study limitations)

- Consistency (consistent, inconsistent, or unknown/not applicable)

- Directness (direct or indirect)

- Precision (precise or imprecise)

- Reporting bias (suspected or not detected)

The strength of evidence was assigned an overall grade of high, moderate, low, or insufficient according to a four-level scale by evaluating and weighing the combined results of the above domains:

- High: Very confident that the estimate of effect lies close to the true effect for this outcome. The body of evidence has few or no deficiencies. The findings are stable, meaning another study would not change the conclusions.

- Moderate: Moderately confident that the estimate of effect lies close to the true effect for this outcome. The body of evidence has some deficiencies. The findings are likely to be stable, but some doubt remains.

- Low: Limited confidence that the estimate of effect lies close to the true effect for this outcome. The body of evidence has major or numerous deficiencies (or both). Additional evidence is needed before concluding either that the findings are stable or that the estimate of effect is close to the true effect.

- Insufficient: No evidence, unable to estimate an effect, or have no confidence in the estimate of effect for this outcome. No evidence is available or the body of evidence has unacceptable deficiencies, precluding reaching a conclusion. 
Individual strength of evidence domains are indicated in summary tables with ratings for the strength of evidence. Ratings for strength of evidence were assigned for prioritized outcomes only and focus on concepts when possible (e.g., an overall rating for depression rather than individual ratings for each depression scale). Strength of evidence ratings were assigned by study population (i.e., MS, CP, SCI). Appendix H describes the strength of evidence for each Key Question.

\section{Assessing Applicability}

Applicability was assessed in accordance with the Methods Guide, ${ }^{56}$ which is based on the PICOTS framework. Applicability addresses the extent to which outcomes associated with an intervention are likely to be similar across different participants and settings in clinical practice based on the populations, interventions, comparisons, and outcomes evaluated in the studies. For example, exclusion of adults in CP trials may render findings that are not applicable to all CP patients seen in clinical practice. Results from trials of elite wheelchair athletes may not be applicable to the average wheelchair user. Factors that may affect applicability, which we have identified a priori include eligibility criteria and participant factors (e.g., age, gender, age at injury or diagnosis, duration of injury or diagnosis, baseline fitness level, degree of dependence on the use of a wheelchair, etiology of disability or potential disability), intervention factors (e.g., dose and duration of the intervention, degree of physical activity supervision), comparisons and rate in the comparison group (e.g., no physical activity, other physical activity), outcomes (e.g., clinical health outcomes, intermediate outcomes, validated or unvalidated outcomes), setting (e.g., home, community, research lab), and study design features (e.g., RCT vs. non-RCT, study location). We used this information to assess the situations in which the evidence is most relevant and to evaluate applicability to real-world clinical practice in typical U.S. settings, summarizing applicability assessments qualitatively.

\section{Peer Review and Public Commentary}

Clinical and methodological experts were invited to provide external peer review of this systematic review. Comments and editorial review were also be provided by the AHRQ TOO and an EPC Program Associate Editor. The peer-reviewed draft report was posted on the AHRQ website for 4 weeks to elicit public comment. We revised the text as needed and address all relevant reviewer comments in an associated disposition of comments report with the authors' individual responses. The final report of the review will be posted on AHRQ's website. 


\section{Results}

\section{Overview}

Findings are presented in order of the Key Questions (KQ). For KQ1 we identified studies within the entire literature base (including studies that met inclusion criteria and those that did not) with results on prevention of obesity, diabetes, and cardiovascular conditions in participants with multiple sclerosis (MS), cerebral palsy (CP), or spinal cord injury (SCI). For KQ2 on the benefits and harms of physical activity interventions, we reported results by type of intervention: aerobics, postural control, strength, and multimodal exercise, and by population. We assessed the strength of evidence (SOE) for prioritized outcomes and described other outcomes. For KQ3 we examined patient factors that may affect the benefits and harms of physical activity, and for KQ4 we reported the methodological weaknesses or gaps in the evidence base.

We synthesized results qualitatively and quantitatively. We did meta-analysis for studies that had criteria similar enough to provide meaningful combined estimates. Meta-analyses included the 6-Minute Walk Test (6MWT), 10-Meter Walk Test (10MWT), Timed Up and Go Test (TUG), Gross Motor Function Measure (GMFM), MS Walking Scale, and Berg Balance Scale (BBS), among others.

Acronyms and abbreviations used are listed at the end of the report.

\section{Results of Literature Searches}

Searches identified 19,247 potentially relevant abstracts with 10, 242 nonduplicated abstracts. From the abstracts, 1,392 full-text articles were dual reviewed by two researchers, and 168 studies in 197 publications $(n=7,511)$ met the inclusion criteria. We included 146 randomized controlled trials (RCTs), 15 quasiexperimental studies, and 7 cohort studies.

Results of the literature search are indicated in the literature flow diagram (Figure 2) which shows the number of excluded abstracts and the number of excluded articles, as well as exclusion reasons at the full-text level. The list of included studies is in Appendix B and excluded full-text studies list is in Appendix C. Full-text review resulted in inclusion of 74 studies ( 85 publications) for MS, 63 studies (73 publications) for CP, and 31 studies (39 publications) for SCI. We evaluated the lists of studies included in 116 different systematic reviews judged to be relevant to our review topic to identify additional publications of studies that might be eligible for inclusion in our review (Appendix D). We identified no studies of mixed populations that met inclusion criteria.

At the full-text review level there were 1,195 studies excluded. Primary reasons for exclusion included ineligible interventions and inadequate sample sizes. The majority of excluded studies did not meet our inclusion criteria due to sample size (328 studies with inadequate sample sizes) or intervention requirements (322 interventions did not have adequate duration or number of interventions, the exercise was unobserved, or otherwise did not meet criteria for inclusion). Evidence tables and quality assessment tables for all included studies are in Appendix F and Appendix G. 
Figure 2. Literature flow diagram

Abstracts of potentially relevant articles identified through MEDLINE $®$, CINAHL $®$, PsycINFO $($,

Cochrane CENTRAL, Embase, and Rehabilitation and Sports Medicine Source $\quad(n=19,247)$

Excluded duplicates $(n=9,005)$

Abstracts of potentially relevant articles reviewed for Key Questions $\quad(n=10,242)$
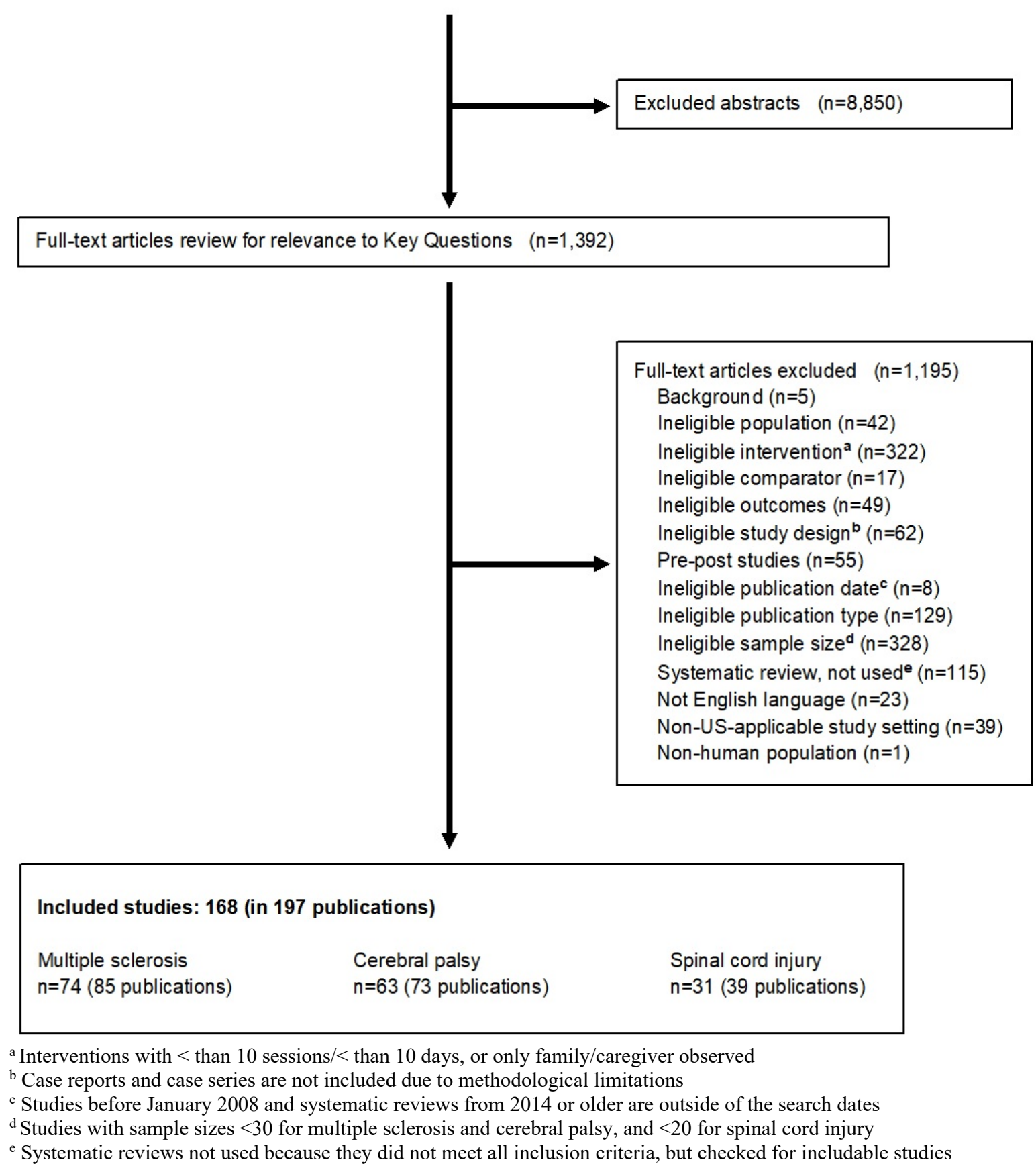


\section{Description of Included Studies}

A general overview of the included studies for each population by intervention categories of aerobics exercise, postural control, strength, and multimodal interventions is in Figure 3, which indicates the percentage of each type of exercise studied for people with MS, CP, and SCI. Table 4 shows the studies with primary outcomes from the 168 studies (in 197 publications) that met inclusion criteria and the details of the included studies. A visual summary of the interventions by each population (Figure 4) indicates that in patients with MS, the most frequent interventions were muscle strengthening, multimodal exercises, and cycling. For $\mathrm{CP}$, treadmill and hippotherapy were frequent interventions, and for SCI, robot-assisted gait training (RAGT), treadmill training, hand cycling, and multimodal interventions were used most often. The mean number of participants per study was 45 (range 20 to 242) with only three studies having a sample size of 100 or more. Studies in MS and CP tended to enroll participants with less disability (average study mean Expanded Disability Status [EDSS] score in MS 3.6 standard deviation (SD) 1.77, with little evidence in participants with EDSS scores of 6.5 or higher; GMFCS in CP typically I to III (average study mean Gross Motor Function Classification System [GMFCS] I-IV) 2.40, SD 0.87). Studies in SCI enrolled a wider spectrum of disability. The mean number of exercise sessions and the mean duration of exercise in MS was 25 sessions over 9 weeks, in CP 28 sessions over 10 weeks, and in SCI 68 sessions over 17 weeks. Studies were conducted most often in Iran (26 studies), Turkey (19 studies), the United States (15 studies), Italy (12 studies), and South Korea (12 studies). The remaining studies were conducted in numerous countries with fewer studies per location. Most studies were conducted in an (usually unspecified) outpatient setting (51\%) or in an inpatient hospital or rehabilitation center (14\%). Some studies were conducted in more than one location; 18 percent did not report study setting. Most studies were funded by a government entity $(25 \%)$ or by a nonprofit $(13 \%)$. Twenty-nine percent of studies did not comment on funding and 15 percent reported that no funding was received. No study reported on the use of a wheeled scooter (as opposed to a wheelchair).

The distribution of quality ratings by intervention is shown in Figure 5. Two-thirds of the studies were fair quality $(n=113)$, one-fourth were poor quality $(n=42)$, and only 8 percent $(n=13)$ were considered good quality. 
Figure 3. Overview of included studies by population and intervention ${ }^{a}$

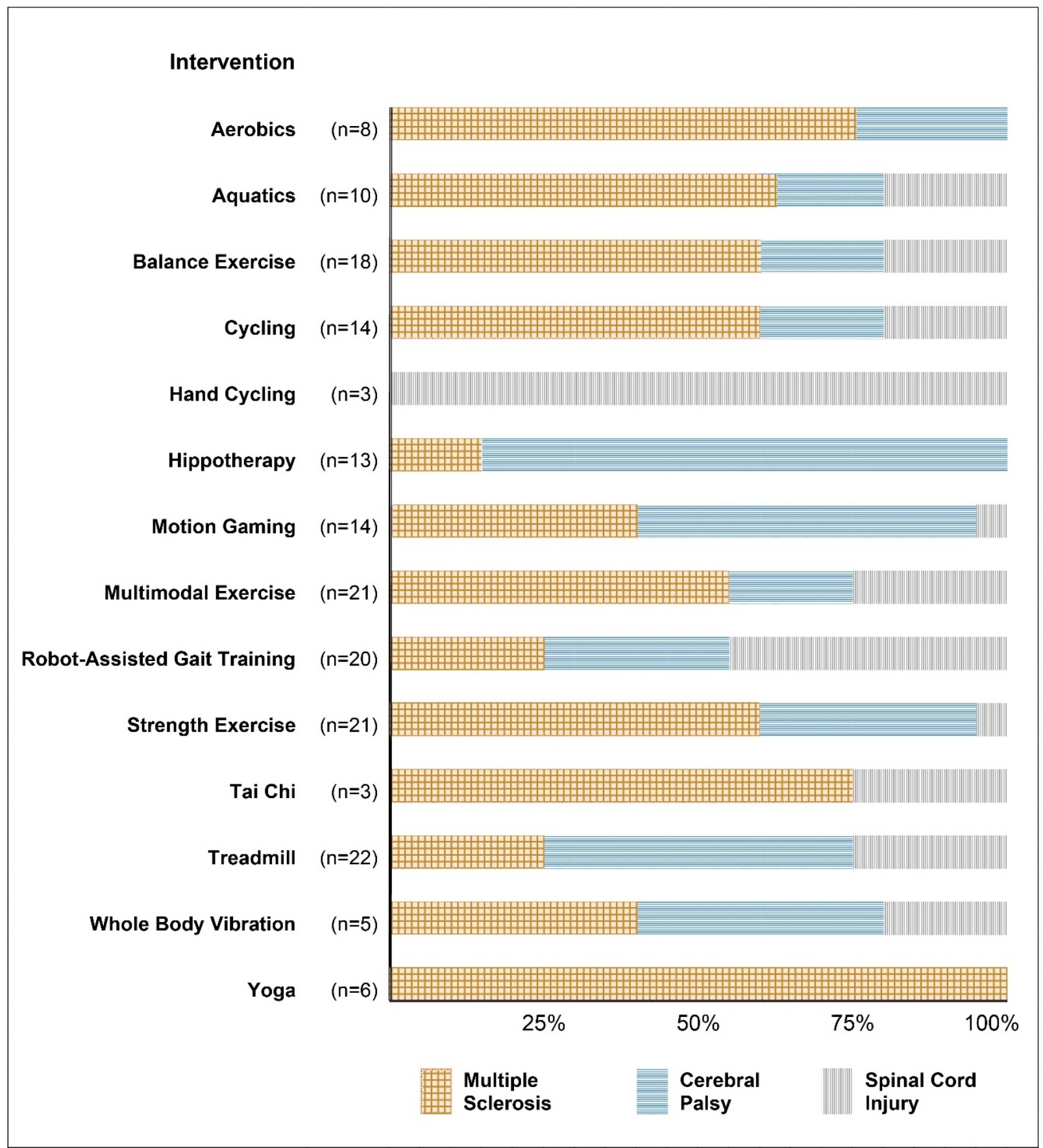

${ }^{a}$ Studies with multiple interventions appear more than once. 
Table 4. Included studies by intervention category and population ${ }^{a}$

\begin{tabular}{|c|c|c|c|c|c|}
\hline Category & Intervention & $\begin{array}{l}\text { Multiple Sclerosis } \\
n=74 \\
\text { (85 Publications) }\end{array}$ & $\begin{array}{l}\text { Cerebral Palsy } \\
n=63 \\
\text { (73 Publications) }\end{array}$ & $\begin{array}{l}\text { Spinal Cord Injury } \\
n=31 \\
\text { (39 Publications) }\end{array}$ & $\begin{array}{l}\text { Total Studies } \\
n=168 \\
\text { (197 Publications) }\end{array}$ \\
\hline $\begin{array}{l}\text { Aerobic } \\
\text { Exercise }\end{array}$ & Aerobics & $\begin{array}{l}4 \mathrm{RCTs}^{54,59-61} \\
2 \text { Quasiexperimental } \\
\text { studies }^{62,63}\end{array}$ & $2 \mathrm{RCTs}^{64,65}$ & No studies & $\begin{array}{l}n=8 \\
6 \text { RCTs } \\
2 \text { Quasiexperimental } \\
\text { studies }\end{array}$ \\
\hline $\begin{array}{l}\text { Aerobic } \\
\text { Exercise }\end{array}$ & Aquatics & $6 \mathrm{RCTs}^{66-72}$ & $\begin{array}{l}1 \mathrm{RCT}^{73} \\
1 \text { Cohort }^{74}\end{array}$ & $2 \mathrm{RCTs}^{75,76}$ & $\begin{array}{l}\mathrm{n}=10 \\
9 \text { RCTs } \\
1 \text { Cohort study }\end{array}$ \\
\hline $\begin{array}{l}\text { Aerobic } \\
\text { Exercise }\end{array}$ & Cycling & $\begin{array}{l}7 \mathrm{RCTs}^{53,77-83} \\
1 \text { Quasiexperimental } \\
\text { study }^{84}\end{array}$ & \begin{tabular}{|l|}
2 RCTs $^{85-87}$ \\
1 \\
Quasiexperimental \\
study $^{88}$
\end{tabular} & $\begin{array}{l}1 \mathrm{RCT}^{89} \\
1 \text { Cohort study }^{90} \\
1 \\
\text { Quasiexperimental } \\
\text { study }^{91}\end{array}$ & $\begin{array}{l}\mathrm{n}=14 \\
10 \text { RCTs } \\
3 \text { Quasiexperimental } \\
\text { studies } \\
1 \text { Cohort study }\end{array}$ \\
\hline $\begin{array}{l}\text { Aerobic } \\
\text { Exercise }\end{array}$ & $\begin{array}{l}\text { Hand } \\
\text { Cycling }\end{array}$ & No studies & No studies & $\begin{array}{l}2 \text { RCTs }^{89,92} \\
1 \text { Cohort study }\end{array}$ & $\begin{array}{l}\mathrm{n}=3 \text { studies } \\
2 \text { RCTs } \\
1 \text { Cohort study }\end{array}$ \\
\hline $\begin{array}{l}\text { Aerobic } \\
\text { Exercise }\end{array}$ & \begin{tabular}{|l|} 
Robot- \\
Assisted \\
Gait Training
\end{tabular} & 5 RCTs ${ }^{94-98}$ & \begin{tabular}{|l|}
5 RCTs $^{99-104}$ \\
1 \\
Quasiexperimental \\
study $^{105}$ \\
1 Cohort study $^{106}$ \\
\end{tabular} & $8 \mathrm{RCTs}^{107-116}$ & $\begin{array}{l}\mathrm{n}=20 \text { studies } \\
18 \text { RCTs } \\
1 \text { Quasiexperimental study } \\
1 \text { Cohort study }\end{array}$ \\
\hline $\begin{array}{l}\text { Aerobic } \\
\text { Exercise }\end{array}$ & Treadmill & $4 \mathrm{RCTs}^{117-120}$ & $\begin{array}{l}10 \text { RCTs }^{121-130} \\
2 \\
\text { Quasiexperimental } \\
\text { studies }^{131,132}\end{array}$ & 6 RCTs $^{113,133-140}$ & $\begin{array}{l}\mathrm{n}=22 \\
20 \mathrm{RCTs} \\
2 \text { Quasiexperimental } \\
\text { studies }\end{array}$ \\
\hline $\begin{array}{l}\text { Postural } \\
\text { Control }\end{array}$ & $\begin{array}{l}\text { Balance } \\
\text { Exercises }\end{array}$ & 12 RCTs $^{61,83,141-151}$ & $\begin{array}{l}\mathrm{RCT}^{152} \\
2 \\
\text { Quasiexperimental } \\
\text { studies }^{153,154} \\
1 \text { Cohort study }\end{array}$ & $2 \mathrm{RCT}^{156,157}$ & $\begin{array}{l}\mathrm{n}=18 \\
15 \text { RCTs } \\
2 \text { Quasiexperimental } \\
\text { studies } \\
1 \text { Cohort study }\end{array}$ \\
\hline $\begin{array}{l}\text { Postural } \\
\text { Control }\end{array}$ & Hippotherapy & 2 RCTs $^{158-160}$ & $\begin{array}{l}\text { RCTs } \\
2 \\
\text { Quasiexperimental } \\
\text { studies }^{169,170} \\
1 \text { Cohort study }^{171} \\
\end{array}$ & No studies & $\begin{array}{l}\mathrm{n}=13 \text { studies } \\
10 \text { RCTs } \\
2 \text { Quasiexperimental } \\
\text { studies } \\
1 \text { Cohort study }\end{array}$ \\
\hline $\begin{array}{l}\text { Postural } \\
\text { Control }\end{array}$ & Tai Chi & 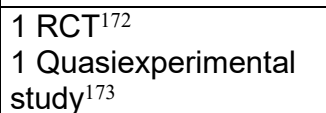 & No studies & $1 \mathrm{RCT}^{174}$ & $\begin{array}{l}n=3 \text { studies } \\
2 \text { RCTs } \\
1 \text { Quasiexperimental study }\end{array}$ \\
\hline $\begin{array}{l}\text { Postural } \\
\text { Control }\end{array}$ & \begin{tabular}{|l|} 
Motion \\
Gaming \\
\end{tabular} & 6 RCTs $^{51,83,151,175-177}$ & $7 \mathrm{RCTs}^{50,178-183}$ & $1 \mathrm{RCT}^{184}$ & $\begin{array}{l}\mathrm{n}=14 \text { studies } \\
14 \text { RCTs }\end{array}$ \\
\hline $\begin{array}{l}\text { Postural } \\
\text { Control }\end{array}$ & $\begin{array}{l}\text { Whole Body } \\
\text { Vibration }\end{array}$ & $2 \mathrm{RCTs}^{185,186}$ & 2 RCTs $^{187,188}$ & 1 RCT 189 & $\begin{array}{l}\mathrm{n}=5 \text { studies } \\
5 \text { RCTs }\end{array}$ \\
\hline $\begin{array}{l}\text { Postural } \\
\text { Control }\end{array}$ & Yoga & 6 RCTs R4,120,190-197 $^{54}$ & No studies & No studies & $\begin{array}{l}\mathrm{n}=6 \text { studies } \\
6 \text { RCTs }\end{array}$ \\
\hline $\begin{array}{l}\text { Strength } \\
\text { Exercise }\end{array}$ & $\begin{array}{l}\text { Muscle } \\
\text { Strength } \\
\text { Exercises }\end{array}$ & $\begin{array}{l}11 \mathrm{RCTs}^{52,68,69,83,149,198-} \\
206 \\
1 \text { Quasiexperimental } \\
\text { study }^{62}\end{array}$ & \begin{tabular}{|l}
7 RCTs $^{207-216}$ \\
1 \\
Quasiexperimental \\
study $^{217}$
\end{tabular} & $1 \mathrm{RCT}^{218,219}$ & $\begin{array}{l}\mathrm{n}=21 \text { studies } \\
19 \text { RCTs } \\
2 \text { Quasiexperimental study }\end{array}$ \\
\hline $\begin{array}{l}\text { Multimodal } \\
\text { Exercise }\end{array}$ & $\begin{array}{l}\text { PRE or } \\
\text { strength } \\
\text { exercise } \\
\text { plus aerobic } \\
\text { or balance } \\
\end{array}$ & $\begin{array}{l}12 \text { RCTs }^{220-232} \\
1 \text { Quasiexperimental } \\
\text { study }^{233}\end{array}$ & $5 \mathrm{RCTs}^{234-243}$ & $\begin{array}{l}3 \text { RCTs }^{244-249} \\
1 \text { Cohort study }\end{array}$ & $\begin{array}{l}\mathrm{n}=21 \text { studies } \\
19 \text { RCTs } \\
1 \text { Cohort study } \\
1 \text { Quasiexperimental study }\end{array}$ \\
\hline
\end{tabular}

Abbreviations: PRE = progressive resistance exercise; RCT = randomized controlled trial

${ }^{\text {a }}$ Studies with multiple interventions appear more than once on the table. Studies with only an intermediate outcome(s) appear in

Tables 42, 43, 44, and 46. 
Figure 4. Wheelchair user and exercise number of studies: interventions and population ${ }^{a}$

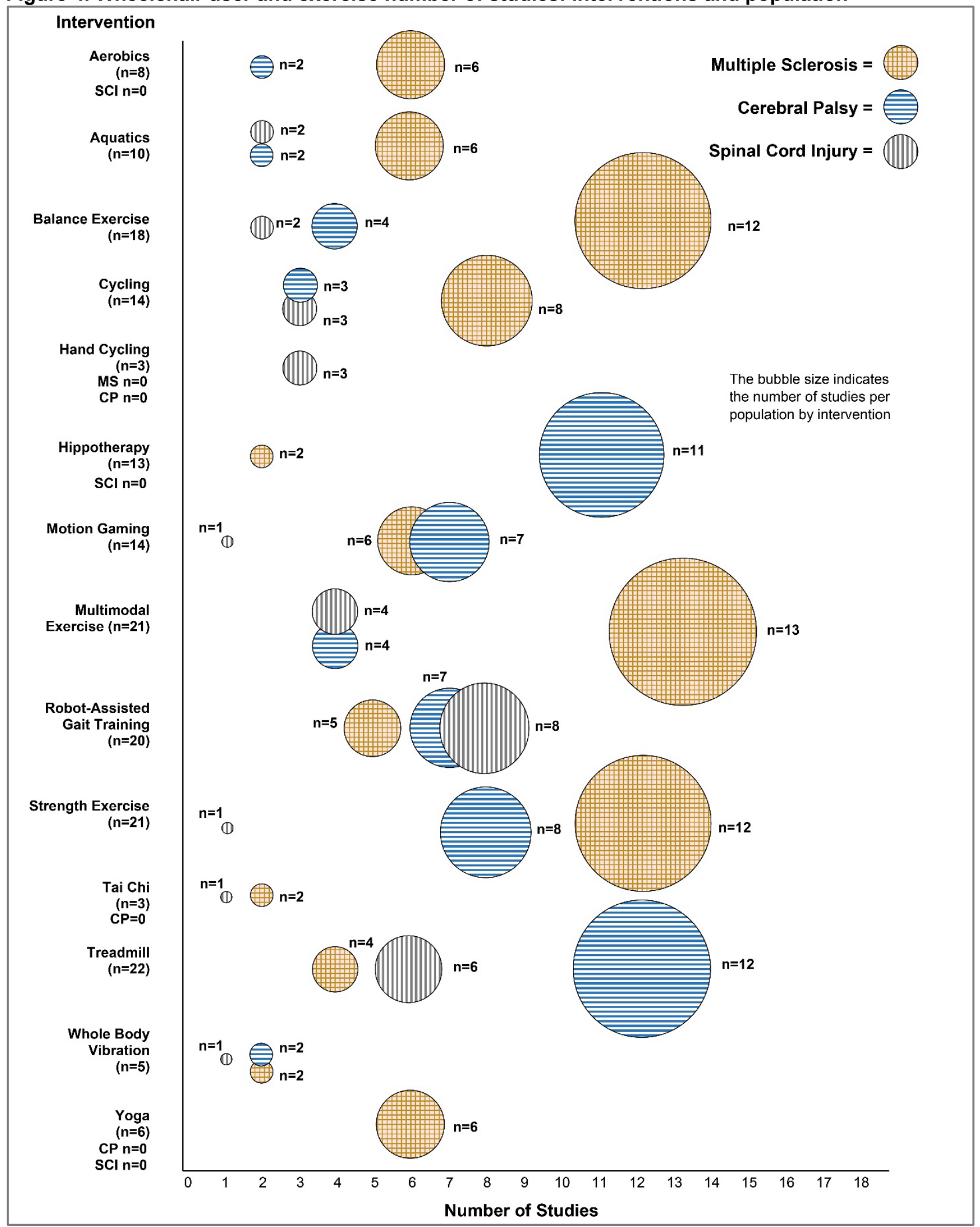

Abbreviations: $\mathrm{CP}=$ Cerebral palsy; $\mathrm{MS}=$ Multiple Sclerosis; $\mathrm{SCI}=$ Spinal cord injury

${ }^{\text {a }}$ Studies with multiple interventions appear more than once. 
Figure 5. Quality ratings by interventiona

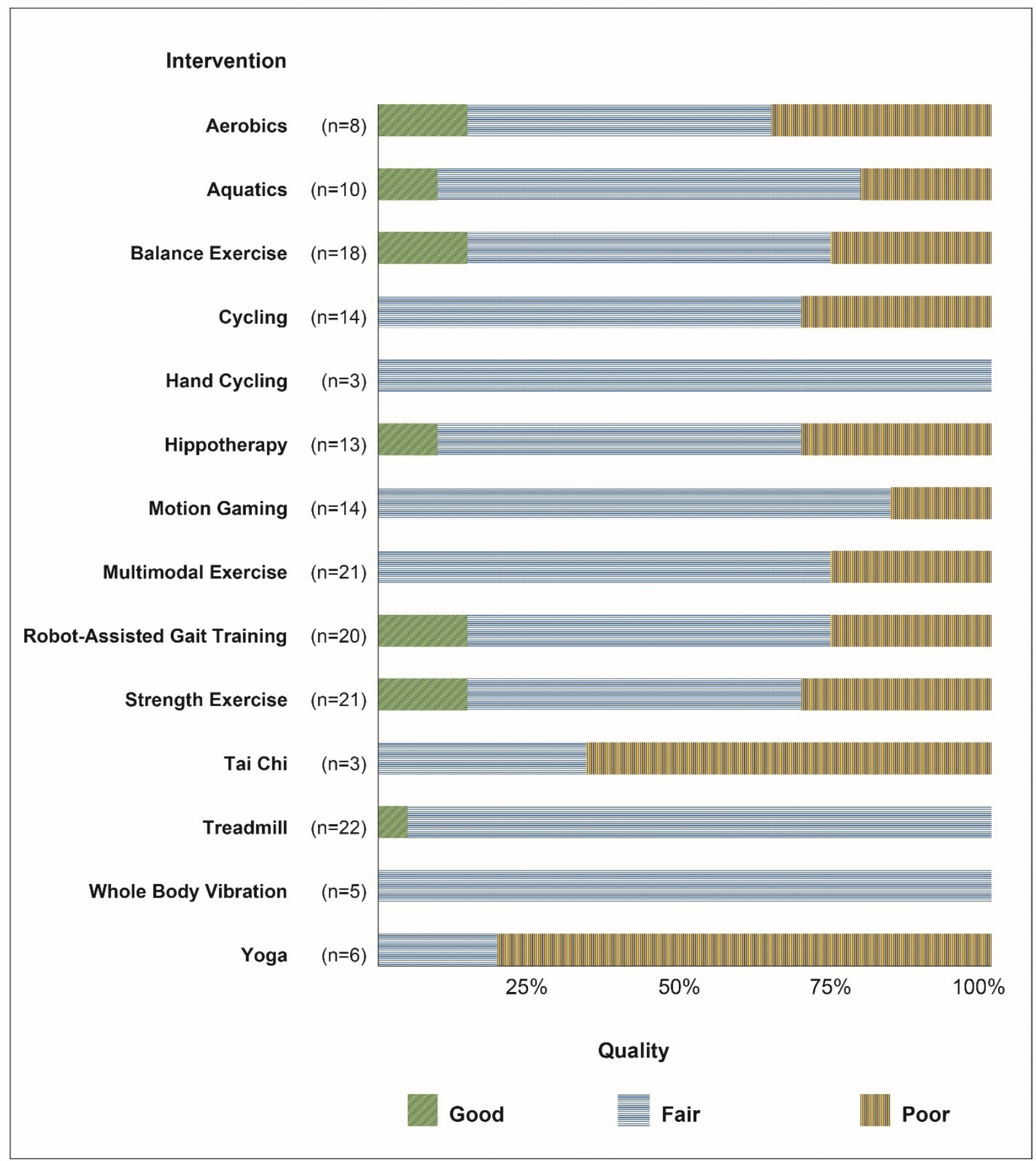

a Studies with multiple interventions appear more than once. 


\section{KQ1: Prevention of Obesity, Diabetes, and Cardiovascular Conditions}

This KQ identifies existing research that links physical activity to the prevention of longterm health outcomes such as myocardial infarction, stroke, and the development of diabetes or obesity, regardless of whether that study met inclusion criteria. No studies on the effects of physical activity in participants with MS, CP, or SCI reported long-term cardiovascular or longterm metabolic disease health outcomes, although some studies reported intermediate outcomes such as blood pressure, lipid profile, and blood glucose. Table 5 identifies the studies that met inclusion criteria for this review. Table 6 identifies the studies that did not meet inclusion criteria but nonetheless reported intermediate obesity, diabetes, and/or cardiovascular outcomes. The results of the included studies are discussed in KQ2. Studies not meeting inclusion criteria for this review may help identify gaps in the evidence and inform future research needs.

Table 5. Included trials with intermediate outcomes related to cardiovascular disease, diabetes, and obesity

\begin{tabular}{|c|c|c|c|c|c|}
\hline $\begin{array}{l}\text { Study } \\
\text { (Author, } \\
\text { Year) }\end{array}$ & $\begin{array}{l}\text { Outcome } \\
\text { Measure }\end{array}$ & $\begin{array}{l}\text { Condition } \\
\text { (MS, CP, } \\
\text { SCI) }\end{array}$ & $\begin{array}{l}\text { Intervention } \\
\text { (Treatment Duration) }\end{array}$ & $\begin{array}{l}\text { Study Inclusion/ } \\
\text { Exclusion Criteria }\end{array}$ & $\begin{array}{l}\text { Study } \\
\text { Design }\end{array}$ \\
\hline $\begin{array}{l}\text { Akkurt, } \\
2017^{89}\end{array}$ & $\begin{array}{l}\text { BMI, weight, } \\
\text { fat, lipids }\end{array}$ & $\mathrm{SCl}$ & $\begin{array}{l}\text { Upper extremity } \\
\text { cycling vs. general } \\
\text { exercises (12 weeks) }\end{array}$ & $\begin{array}{l}\text { Age } 15-65 \text {, traumatic } \\
\text { lesion, less than } 2 \text { hours } \\
\text { per week of physical } \\
\text { activity, CV disease }\end{array}$ & RCT \\
\hline $\begin{array}{l}\text { Faramarzi, } \\
2020^{230}\end{array}$ & Lipids, fat & MS & $\begin{array}{l}\text { Resistance exercises } \\
+ \text { endurance exercise } \\
+ \text { balance exercises + } \\
\text { Pilates + Stretching } \\
\text { (12 weeks) }\end{array}$ & $\begin{array}{l}\text { Female, Age } 18 \text { to } 50 \text {, less } \\
\text { than } 2 \text { hours per week of } \\
\text { physical activity, no history } \\
\text { of cardiovascular, kidney or } \\
\text { other chronic disease }\end{array}$ & RCT \\
\hline $\begin{array}{l}\text { Gervasoni, } \\
2014^{117}\end{array}$ & Resting HR & MS & $\begin{array}{l}\text { Treadmill + usual } \\
\text { therapy vs. usual } \\
\text { therapy ( } 2 \text { weeks) }\end{array}$ & $\begin{array}{l}\text { Stand for } 30 \text { seconds, walk } \\
\text { for } 6 \text { minutes/no cardiac, } \\
\text { pulmonary, or metabolic } \\
\text { disease }\end{array}$ & RCT \\
\hline $\begin{array}{l}\text { Giangregorio, } \\
2012^{134}\end{array}$ & $\begin{array}{l}\text { BMI, weight, } \\
\text { fat }\end{array}$ & $\mathrm{SCl}$ & $\begin{array}{l}\text { Treadmill walking with } \\
\text { FES vs. aerobic and } \\
\text { resistance training (16 } \\
\text { weeks) }\end{array}$ & $\begin{array}{l}\text { Traumatic incomplete } \\
\mathrm{SCl} / \text { no cardiac pacemaker, } \\
\text { ulcer at harness site, } \\
\text { orthostatic hypotension, } \\
\text { unstable AD }\end{array}$ & RCT \\
\hline $\begin{array}{l}\text { Jones, } \\
2014 a / b^{246,247}\end{array}$ & $\begin{array}{l}\text { BMI, weight, } \\
\text { fat, glucose }\end{array}$ & $\mathrm{SCl}$ & $\begin{array}{l}\text { Activity-based therapy } \\
\text { vs. waitlist ( } 24 \text { weeks) }\end{array}$ & $\begin{array}{l}\text { Age } \geq 18, \text { motor incomplete } \\
\text { SCl }\end{array}$ & RCT \\
\hline $\begin{array}{l}\text { Marandi, } \\
2013^{68,69}\end{array}$ & $\begin{array}{l}\text { BMI, weight, } \\
\text { fat }\end{array}$ & MS & $\begin{array}{l}\text { Aquatics vs. Pilates } \\
\text { (12 weeks) }\end{array}$ & EDSS $<4.5$ & RCT \\
\hline $\begin{array}{l}\text { Mogharnasi, } \\
2019^{219}\end{array}$ & $\begin{array}{l}\text { BMI, weight, } \\
\text { fat, lipids }\end{array}$ & SClk & $\begin{array}{l}\text { Upper body resistance } \\
\text { training vs. usual care } \\
\text { (8 weeks) }\end{array}$ & $\begin{array}{l}\text { Obese, paraplegic, male, } \\
\text { traumatic lesions, could sit } \\
\text { and maintain upper body } \\
\text { balance/no braces, } \\
\text { crutches or walkers }\end{array}$ & RCT \\
\hline $\begin{array}{l}\text { Sandroff, } \\
2017^{221}\end{array}$ & $\begin{array}{l}\text { BMl, weight, } \\
\text { fat }\end{array}$ & MS & $\begin{array}{l}\text { Resistance + aerobics } \\
+ \text { balance vs. toning } \\
\text { and stretching ( } 24 \\
\text { weeks) }\end{array}$ & $\begin{array}{l}\text { Age 18-64, EDSS } 4.0-6.0 \text {, } \\
\text { engaging in low level of } \\
\text { physical activity, low risk for } \\
\text { exercise contraindication }\end{array}$ & RCT \\
\hline
\end{tabular}




\begin{tabular}{|c|c|c|c|c|c|}
\hline $\begin{array}{l}\text { Study } \\
\text { (Author, } \\
\text { Year) }\end{array}$ & $\begin{array}{l}\text { Outcome } \\
\text { Measure }\end{array}$ & $\begin{array}{l}\text { Condition } \\
\text { (MS, CP, } \\
\text { SCI) }\end{array}$ & $\begin{array}{l}\text { Intervention } \\
\text { (Treatment Duration) }\end{array}$ & $\begin{array}{l}\text { Study Inclusion/ } \\
\text { Exclusion Criteria }\end{array}$ & $\begin{array}{l}\text { Study } \\
\text { Design }\end{array}$ \\
\hline $\begin{array}{l}\text { Slaman, } \\
2014^{235}\end{array}$ & $\begin{array}{l}\text { BMI, weight, } \\
\text { fat, lipids }\end{array}$ & $\mathrm{CP}$ & $\begin{array}{l}\text { Strength + aerobic } \\
\text { training + counseling } \\
\text { on physical activity and } \\
\text { sports participation vs. } \\
\text { usual care (12 weeks) }\end{array}$ & $\begin{array}{l}\text { Age 16-24, GMFCS I-IV, } \\
\text { spastic CP/no disabilities } \\
\text { other than CP that affect } \\
\text { physical activity, } \\
\text { contraindications to } \\
\text { maximal exercise, severe } \\
\text { cognitive disorder }\end{array}$ & RCT \\
\hline $\begin{array}{l}\text { Totosy de } \\
\text { Zepetnek, } \\
2015^{249}\end{array}$ & $\begin{array}{l}\text { Resting BP, } \\
\text { HR, HbA1c, } \\
\text { lipids, BMI }\end{array}$ & $\mathrm{SCl}$ & $\begin{array}{l}\text { Progressive resistance } \\
+ \text { aerobic training ( } 16 \\
\text { weeks) }\end{array}$ & $\begin{array}{l}\text { Age 18-65, no progressive } \\
\text { loss of neurologic function } \\
\text { within past } 6 \text { months }\end{array}$ & RCT \\
\hline $\begin{array}{l}\text { Wens, } \\
2015^{224}\end{array}$ & $\begin{array}{l}\text { Resting HR, } \\
\text { BMI, weight, } \\
\text { fat, glucose }\end{array}$ & MS & $\begin{array}{l}\text { Strength }+ \text { high- } \\
\text { intensity interval } \\
\text { training vs. strength + } \\
\text { high-intensity } \\
\text { continuous aerobic } \\
\text { training vs. sedentary } \\
\text { control ( } 12 \text { weeks) }\end{array}$ & $\begin{array}{l}\text { Age } \geq 18 \text { years/no diabetes } \\
\text { or other chronic disease } \\
(\mathrm{CV}, \text { pulmonary, and/or } \\
\text { renal) }\end{array}$ & RCT \\
\hline
\end{tabular}

Abbreviations: $\mathrm{AD}=$ autonomic dysreflexia; $\mathrm{BMI}=$ body mass index; $\mathrm{BP}=$ blood pressure; $\mathrm{CP}=$ cerebral palsy; $\mathrm{CV}=$ cardiovascular; EDSS = Expanded Disability Status Scale; FES = functional electrical stimulation; GMFCS = Gross Motor Function Classification System; HbA1c = hemoglobin A1c; HR = heart rate; MS = multiple sclerosis; $\mathrm{RCT}=$ randomized controlled trial; $\mathrm{SCI}=$ spinal cord injury

Table 6. Studies with intermediate outcomes related to cardiovascular disease, diabetes, and obesity that did not meet inclusion criteria

\begin{tabular}{|c|c|c|c|c|c|}
\hline $\begin{array}{l}\text { Study } \\
\text { (Author, Year) }\end{array}$ & $\begin{array}{l}\text { Outcome } \\
\text { Measure }\end{array}$ & $\begin{array}{l}\text { Condition } \\
\text { (MS, CP, } \\
\text { SCI) }\end{array}$ & $\begin{array}{l}\text { Intervention } \\
\text { (Treatment Duration) }\end{array}$ & $\begin{array}{l}\text { Study Inclusion/ } \\
\text { Exclusion Criteria }\end{array}$ & $\begin{array}{l}\text { Study } \\
\text { Design }\end{array}$ \\
\hline $\begin{array}{l}\text { Brochetti, } \\
2018^{251}\end{array}$ & $\begin{array}{l}\text { Weight, waist } \\
\text { circumference }\end{array}$ & $\mathrm{SCl}$ & $\begin{array}{l}\text { Weight management } \\
\text { program: working on } \\
\text { healthy eating, exercise } \\
\text { and lifestyle ( } 12 \text { weeks) }\end{array}$ & $\begin{array}{l}1 \text { year post-SCI with } \\
\text { chronic weight issue or } \\
\text { obesity diagnosis }\end{array}$ & Pre-Post \\
\hline $\begin{array}{l}\text { de Groot, } \\
2018^{252}\end{array}$ & $\begin{array}{l}\text { Hip circum- } \\
\text { ference, fat }\end{array}$ & $\mathrm{SCl}$ & $\begin{array}{l}\text { Competitive self-guided } \\
\text { training for Dutch } \\
\text { cycling race } \\
\text { HandbikeBattle ( } 12 \\
\text { weeks) }\end{array}$ & $\begin{array}{l}\text { Passed medical } \\
\text { screening; no } \\
\text { contraindications to } \\
\text { exercise }\end{array}$ & Pre-Post \\
\hline $\begin{array}{l}\text { de Rossi, } \\
2014^{253}\end{array}$ & LV function & $\mathrm{SCl}$ & None & $\begin{array}{l}\text { Tetraplegic rugby } \\
\text { players; paraplegic } \\
\text { basketball players; } \\
\text { sedentary SCl; no } \\
\text { diabetes, smoking, CV } \\
\text { or pulmonary disease, } \\
\text { no cancer, no } \\
\text { hypertension or } \\
\text { hyperlipidemia }\end{array}$ & $\begin{array}{l}\text { Cross- } \\
\text { sectional }\end{array}$ \\
\hline $\begin{array}{l}\text { Gibbons, } \\
2016^{254,255} \\
2 \text { studies }\end{array}$ & $\begin{array}{l}\text { Cardiac } \\
\text { structure and } \\
\text { function }\end{array}$ & $\mathrm{SCl}$ & $\begin{array}{l}\text { FES rowing: FES } \\
\text { trained vs. untrained } \\
\text { FES rowing: } \\
\text { FES naïve (length } \\
\text { varied based on } \\
\text { progression) }\end{array}$ & $\begin{array}{l}\text { Age } 26-56 \text {, at least } 12 \\
\text { months post-SCI } \\
\text { between C4 and T10, } \\
\text { ASIA A or B, otherwise } \\
\text { healthy; FES trained } \\
\text { and non-FES trained }\end{array}$ & $\begin{array}{l}\text { Cross- } \\
\text { sectional } \\
\text { Pre-Post }\end{array}$ \\
\hline Hubli, $2014^{256}$ & $\begin{array}{l}\text { Arterial } \\
\text { stiffness, BP, } \\
\text { HR } \\
\end{array}$ & $\mathrm{SCl}$ & None & $\begin{array}{l}\text { Elite hand cyclists; } \\
\text { nonathletes }\end{array}$ & $\begin{array}{l}\text { Cross- } \\
\text { sectional }\end{array}$ \\
\hline
\end{tabular}




\begin{tabular}{|c|c|c|c|c|c|}
\hline $\begin{array}{l}\text { Study } \\
\text { (Author, Year) }\end{array}$ & $\begin{array}{l}\text { Outcome } \\
\text { Measure }\end{array}$ & $\begin{array}{l}\text { Condition } \\
\text { (MS, CP, } \\
\text { SCI) }\end{array}$ & $\begin{array}{l}\text { Intervention } \\
\text { (Treatment Duration) }\end{array}$ & $\begin{array}{l}\text { Study Inclusion/ } \\
\text { Exclusion Criteria }\end{array}$ & $\begin{array}{l}\text { Study } \\
\text { Design }\end{array}$ \\
\hline $\begin{array}{l}\text { Jorissen, } \\
2018^{257}\end{array}$ & Lipids & MS & $\begin{array}{l}\text { Medium vs. high- } \\
\text { intensity CV training } \\
\text { (12 weeks) }\end{array}$ & $\begin{array}{l}\text { No hyperlipidemia, CV } \\
\text { disease, diabetes, } \\
\text { cholesterol modifying } \\
\text { drugs }\end{array}$ & $\mathrm{RCT}$ \\
\hline $\begin{array}{l}\text { Keytsman, } \\
2017^{258}\end{array}$ & $\begin{array}{l}\text { Glucose, } \\
\text { HOMA, fat, } \\
\text { BP, lipids }\end{array}$ & MS & $\begin{array}{l}\text { High-intensity interval } \\
\text { and resistance training } \\
(12 \text { weeks) }\end{array}$ & Age $\geq 18 ;$ No EDSS $>6$ & Pre-Pos \\
\hline Lauglo, $2016^{259}$ & $\begin{array}{l}\text { Weight, BMI, } \\
\text { fat }\end{array}$ & $\mathrm{CP}$ & $\begin{array}{l}\text { High-intensity walking } \\
\text { or running on treadmill } \\
\text { ( } 6 \text { to } 12 \text { weeks) }\end{array}$ & Age 10-17, GMFCS I-IV & Pre-Pos \\
\hline $\begin{array}{l}\text { Magnani, } \\
2016^{260}\end{array}$ & $\begin{array}{l}\text { Hemo- } \\
\text { dynamic data }\end{array}$ & MS & $\begin{array}{l}\text { Aerobic and strength } \\
\text { training vs. no exercise } \\
\text { control ( } 6 \text { months) }\end{array}$ & $\begin{array}{l}\text { Age 18-65; no chronic } \\
\text { cardio-pulmonary } \\
\text { diseases }\end{array}$ & RCT \\
\hline Myers, $2012^{261}$ & $\begin{array}{l}\text { Weight, } \\
\text { HOMA }\end{array}$ & $\mathrm{SCl}$ & $\begin{array}{l}\text { Visits by dietitian, } \\
\text { physical therapist, } \\
\text { exercise physiologist ( } 2 \\
\text { years) }\end{array}$ & $\begin{array}{l}\text { Male veteran at high risk } \\
\text { for CVD, } \geq 20 \% 10 \text { year } \\
\text { risk of CVD; no CVD }\end{array}$ & Pre-Pos \\
\hline $\begin{array}{l}\text { Zabay Neiro, } \\
2018^{262}\end{array}$ & BMI, BP & MS & $\begin{array}{l}\text { Walking with classes } \\
\text { on healthy diet, rest, } \\
\text { and physical activity vs. } \\
\text { usual care (12 weeks) }\end{array}$ & EDSS score $<6.0$ & RCT \\
\hline Orban, $2019^{263}$ & Fat & MS & $\begin{array}{l}\text { Aerobic exercise vs. } \\
\text { guided stretching } \\
\text { control ( } 8 \text { weeks) }\end{array}$ & $\begin{array}{l}\text { Age } 18-65, \text { RRMS, fully } \\
\text { ambulatory; no } \\
\text { uncontrolled CV or } \\
\text { pulmonary disease }\end{array}$ & $\begin{array}{l}\text { Matched } \\
\text { cohort }\end{array}$ \\
\hline
\end{tabular}

Abbreviations: ASIA = American Spinal Injury Association Impairment Scale; BMI = body mass index; BP = blood pressure; $\mathrm{CV}=$ cardiovascular; $\mathrm{CVD}=$ cardiovascular disease; EDSS = Expanded Disability Status Scale; FES = functional electrical stimulation; GMFCS = Gross Motor Function Classification System; HR = heart rate; HOMA = homeostatic model assessment; $\mathrm{LV}=$ left ventricular function; $\mathrm{MS}=$ multiple sclerosis; $\mathrm{RCT}=$ randomized controlled trial; RRMS = relapsing-remitting multiple sclerosis; SCI = spinal cord injury

\section{KQ1a: Interventions}

There is no evidence for any intervention studied on the prevention of cardiovascular conditions, diabetes, or obesity. Interventions paired with relevant intermediate outcomes of cardiac structure and function, heart rate, blood pressure, lipid profile, glucose levels or metabolism, body weight, body mass index (BMI), fat mass, or other measures of body composition are numerous. These interventions include aerobic exercise (e.g., aerobic exercises, treadmill walking or running, cycling, and aquatics), strength exercise (e.g., resistance training and Pilates), and postural control training. Physical activity interventions include multimodal therapies (e.g., strength plus aerobic training and/or balance exercises), training at different levels of effort (e.g., high-intensity interval and resistance training and medium-intensity aerobic training), and training with a guidance component (e.g., walking, with classes on healthy diet, rest, and physical activity). Interventions have also been self-guided, focused on weight management, or did not involve a specified physical activity (e.g., intervention focused on visits by exercise professionals plus a dietitian).

\section{KQ1b: Outcomes}

Only intermediate outcomes have been studied. Studies have evaluated blood pressure, heart rate, blood sugar, homeostatic model assessment (HOMA) (an estimate of insulin resistance), lipid profile, body weight, BMI, fat mass, percent body fat, waist and hip circumference, echocardiographic images of cardiac structure and function, measures of central arterial stiffness, 
systemic vascular resistance, and other hemodynamic variables (e.g., stroke volume and ventricular filling rate).

\section{KQ1c: Study Inclusion/Exclusion Criteria}

Most studies in MS and SCI, when an age was specified, required participants to be 18 years or older (Tables 5 and 6). One study required veterans to be male ${ }^{261}$ and another required participants to be female. ${ }^{230}$ Two studies enrolled obese participants. ${ }^{146,219}$ Several studies limited participants to those without known cardiometabolic disease. Sometimes study eligibility parameters were placed around degree of impairment (EDSS $<6$, wheelchair use only, GMFCS IIV). Three studies enrolled elite athletes. ${ }^{252,253,256}$ Several studies in SCI enrolled only participants with traumatic SCI (vs. atraumatic SCI) ${ }^{89,134,219}$ or required American Spinal Injury Association Impairment Scale (ASIA) scores of A or B. ${ }^{254,255}$

\section{KQ1d: Research Methodologies}

Comparison and control groups of studies that assess intermediate outcomes for cardiovascular conditions, diabetes, and obesity have varied. The most frequent control group was usual care and no exercise/waitlist control. Other comparison groups included general exercise, aerobic plus resistance training, functional electrical stimulation (FES)-untrained and FES-naïve rowing. One study compared aquatics with Pilates; ${ }^{68,69}$ another compared strength plus high-intensity interval training with strength plus high-intensity continuous aerobic training. ${ }^{224}$ The duration of the intervention was most often 12 weeks (53\%), which was also the median treatment duration (range 2 weeks to 2 years). The research setting was rarely specified in study publications.

\section{KQ2: Physical Activity Interventions}

This KQ presents the results of included studies subdivided by intervention categories. These include results for aerobic exercise (aerobics, aquatics, cycling, hand cycling, RAGT, and treadmill), postural control interventions (balance exercise, hippotherapy, Tai Chi, whole body vibration [WBV], and yoga), strength interventions (muscle strength exercises), and multimodal interventions (progressive resistance or strengthening exercise combined with aerobic or postural control interventions). Results for interventions with enough data to analyze outcomes independent of population or intervention category are also provided.

\section{Aerobic Exercise Interventions}

Aerobic exercise is exercise that raises heart rate and blood pressure through cardiovascular conditioning. Examples of aerobic exercise include running, fast walking, cycling, swimming laps, group exercise classes like Zumba ${ }^{\circledR}$, fast sports like basketball, soccer, lacrosse, or football, and certain types of dance with a fast rhythm such a mambo, east-coast swing, or Viennese waltz.

\section{Aerobics}

Some aerobic programs incorporate music as a fitness motivator or use cadence to help participants keep in time or rhythm. Two dance programs met inclusion criteria and are 
discussed below. This section also includes aerobic exercise studies that did not solely involve use of a treadmill, robot assistance, cycling, or a swimming pool, as studies employing these methods are presented in other sections under aerobic exercise. One study in this section that did not fit neatly into another section involved used of a treadmill, cycling, and/or walking/jogging as the aerobic activity of participant choice. ${ }^{61}$

\section{Key Points}

- Two fair-quality RCTs, one in participants with MS and one in participants with CP, provided evidence that function improved with dance compared with controls (SOE: low).

- One poor- and one fair-quality MS trial found aerobic exercise associated with improved sleep in pooled analysis (SOE: low).

- Evidence was insufficient for improvement in function with aerobic exercises not centered on music (SOE: insufficient).

\section{Detailed Synthesis}

One good-quality RCT $(n=42),{ }^{65}$ four fair-quality RCTs $(n=164),{ }^{54,59,61,64}$ one poor-quality $\operatorname{RCT}(n=30)^{60}$ and two poor-quality, nonrandomized, quasiexperimental studies $(n=67)^{62,63}$ (total $\mathrm{n}=303$ ) evaluated aerobic exercise versus no treatment, ${ }^{54}$ usual care, ${ }^{63,65}$ attention control, ${ }^{61}$ traditional kinesiotherapy exercises, ${ }^{64}$ home exercises,${ }^{59,60}$ and pilates. ${ }^{62}$ Types of aerobic exercise included movement to music or dance, ${ }^{54,64}$ and aerobic exercises, running, ${ }^{65}$ stair stepping, ${ }^{60}$ or calisthenics. ${ }^{59,62,63}$ One RCT allowed participants to either walk or jog on or off a treadmill or to use an exercise bicycle for aerobic activity. ${ }^{61}$ Study quality ratings were downgraded due to unclear methods of randomization and concealment of the allocation, differences between randomized groups based on prognostic characteristics, and high attrition.

\section{Aerobics-Multiple Sclerosis}

Six studies with durations of $6,{ }^{60,63} 8,{ }^{59,61,62}$ and 12 weeks ${ }^{54,59}$ enrolled 235 participants with MS (Table 7). The mean participant age across trials ranged from 35.5 to 48.5 years and the study proportion female ranged from 50 to 100 percent. Only one trial reported race and was 47.5 percent nonwhite. ${ }^{54}$ No trial described the participant's degree of ambulation or need for wheelchair. Mean EDSS scores ranged from 2.0 to 3.1.

One fair-quality RCT $(n=55)$ in MS patients found improved function with movement to music that involved three 60-minute sessions per week for 36 sessions and targeted strength, cardiorespiratory endurance, and balance. ${ }^{54}$ Each session was choreographed and led by a dance instructor and accompanied by music. Outcomes that demonstrated improvement were TUG and 6MWT, although neither Movement to Music nor waitlist controls showed statistically significant improvement on 5 Times Sit-to-Stand. Average attendance was 53.7 percent with a mean of seven make-up classes offered.

Two RCTs $(n=77)$ provided low-strength evidence of improved pooled sleep scores on the Insomnia Sleep Index (mean difference [MD] -3.34, 95\% CI 5.65 to $1.03, \mathrm{I}^{2}=58 \%$ ) with aerobic exercise compared with home exercise or attention control ${ }^{60,61}$ (Figure 6). One trial also reported improved sleep time. ${ }^{60}$ 
Figure 6. Insomnia Sleep Index in multiple sclerosis trials

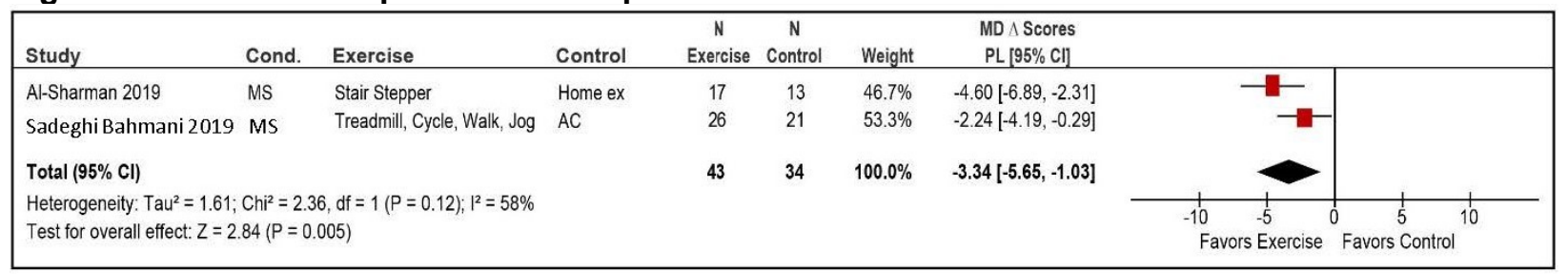

Abbreviatons: $\Delta=$ change; $\mathrm{AC}=$ attention control; $\mathrm{CI}=$ confidence interval; $\mathrm{MD}=$ mean difference; $\mathrm{MS}=$ multiple sclerosis; $\mathrm{PL}$ $=$ profile likelihood

One trial ${ }^{61}$ and the two quasiexperimental studies found no clear evidence of differences between aerobic exercise and comparisons groups on function, balance, or quality of life. ${ }^{61-63}$

One study reported that adverse events in the Movement to Music group were one each muscle strain, stress fracture, and knee pain, compared with none reported in the control group. ${ }^{54}$ The remaining studies did not address adverse events or harms.

Table 7. Aerobic exercise in multiple sclerosis

\begin{tabular}{|c|c|c|c|}
\hline $\begin{array}{l}\text { Author, Year } \\
\text { Intervention } \\
\text { Study Design } \\
\text { Study Quality }\end{array}$ & $\begin{array}{l}\text { Intervention } \\
\text { and Comparison }\end{array}$ & Population & Results \\
\hline $\begin{array}{l}\text { Al-Sharman, } \\
2019^{60} \\
\text { Aerobics } \\
\text { RCT } \\
\text { Poor }\end{array}$ & $\begin{array}{l}\text { A. Moderate- } \\
\text { intensity exercise } \\
\text { with stair stepper, } \\
18 \text { sessions over } 6 \\
\text { weeks }(n=17) \\
\begin{array}{l}\text { B. Home exercises } \\
(n=13)\end{array}\end{array}$ & $\begin{array}{l}\text { A vs. } B \\
\text { Age: } 39 \text { vs. } 32 \\
\text { Female: } 76 \% \text { vs. } 77 \% \\
\text { EDSS: } 2.1 \text { vs. } 1.9\end{array}$ & $\begin{array}{l}\text { A vs. B, mean (SD), } p \text {-value is between groups: } \\
\text { PSQI: } 8.0(3.8) \text { to } 4.6(2.3) \text { vs. } 8.9(4.3) \text { to } 7.1 \\
\text { (3.2), } p<0.001 \\
\frac{\text { ISI: } 12.8(5.3) \text { to } 6.6(4.08) \text { vs. } 10.3(3.3) \text { to } 8.7}{(5.1), p=0.04} \\
\text { Total Sleep Time: } 333.38(84.6) \text { to } 372.4(59.4) \\
\text { vs. } 325.9(84.5) \text { to } 320(54), p=0.05\end{array}$ \\
\hline $\begin{array}{l}\text { Aydin, } 2014^{59} \\
\text { Aerobics } \\
\text { RCT } \\
\text { Fair }\end{array}$ & $\begin{array}{l}\text { A. Callisthenic } \\
\text { exercises (in } \\
\text { clinic): } 60 \\
\text { sessions, over } 12 \\
\text { weeks, }(n=16) \\
\text { B. Callisthenic } \\
\text { exercises (home- } \\
\text { based): } 60 \\
\text { sessions, over } 12 \\
\text { weeks, }(n=20)\end{array}$ & $\begin{array}{l}\text { A vs. B } \\
\text { Age: } 32.6 \text { vs. } 33 \\
\text { Female: } 56 \% \text { vs. } 55 \% \\
\text { EDSS: } 3.6 \text { vs. } 3.4\end{array}$ & $\begin{array}{l}\text { A vs. B, mean (SD) } \\
\text { 10MWT: } \\
10.81(2.15) \text { vs. } 9.95(1.92), p=0.211 \text { (baseline) } \\
9.47(1.56) \text { vs. } 9.02(1.78), p=0.386 \\
\text { (postintervention) } \\
\text { Pre-post exercise intra-group comparison: } \\
\text { Difference1.34 (1.26) vs. } 0.93(1.12), p=0.442 \\
\text { MusiQoL: } \\
63.69(17.00) \text { vs. } 59.75 \text { (14.06), } p=0.293 \\
\text { (baseline) } \\
76.00(18.81) \text { vs. } 69.00 \text { (15.11), } p=0.119 \\
\text { (postintervention) } \\
\text { Pre-post exercise intra-group comparison: } \\
\text { Difference12.31 (7.45) vs. 9.25 (6.99), } p=0.146 \\
\text { BBS: } \\
47.56(6.57) \text { vs. } 48.95 \text { (5.38) (baseline) } \\
50.94(4.97) \text { vs. } 50.40 \text { (5.27) (postintervention), } \\
p=0.031\end{array}$ \\
\hline $\begin{array}{l}\text { Kara, } 2017^{62} \\
\text { Aerobics } \\
\text { Quasiexperimental } \\
\text { Poor }\end{array}$ & $\begin{array}{l}\text { A. Aerobic } \\
\text { exercise } 16 \\
\text { sessions over } 8 \\
\text { weeks }(n=28) \\
\text { B. Pilates } 16 \\
\text { sessions over } 8 \\
\text { weeks }(n=9)\end{array}$ & $\begin{array}{l}\text { A vs. } B \\
\text { Age: } 43 \text { vs. } 50 \\
\text { Female: } 65 \% \text { vs. } 67 \% \\
\text { EDSS: } 3.2 \text { vs. } 2.85\end{array}$ & $\begin{array}{l}\text { A vs. B mean difference between groups: } \\
\text { TUG right: } \\
-0.47,95 \% \mathrm{Cl}-2.98 \text { to } 2.04, p=0.71 \\
\text { TUG left: } \\
-3.07,95 \% \mathrm{Cl}-6.34 \text { to } 0.20), p=0.07 \\
\text { BBS: } \\
-0.67,95 \% \mathrm{Cl}-10.56 \text { to } 9.22, p=0.89\end{array}$ \\
\hline
\end{tabular}




\begin{tabular}{|c|c|c|c|}
\hline $\begin{array}{l}\text { Author, Year } \\
\text { Intervention } \\
\text { Study Design } \\
\text { Study Quality }\end{array}$ & $\begin{array}{l}\text { Intervention } \\
\text { and Comparison }\end{array}$ & Population & Results \\
\hline $\begin{array}{l}\text { Keser, } 2011^{63} \\
\text { Aerobic exercise } \\
\text { Quasiexperimental } \\
\text { Poor }\end{array}$ & $\begin{array}{l}\text { A. Calisthenics, } 18 \\
\text { sessions over } 6 \\
\text { weeks }(n=15) \\
\text { B. Neuro- } \\
\text { rehabilitation } 18 \\
\text { sessions over } 6 \\
\text { weeks }(n=15)\end{array}$ & $\begin{array}{l}\text { A vs. B } \\
\text { Age: } 36 \text { vs. } 35 \\
\text { Female: } 53 \% \text { vs. } 47 \% \\
\text { EDSS: } 2.9 \text { vs. } 2.8\end{array}$ & 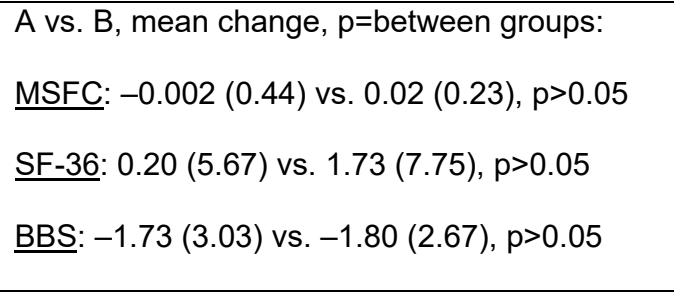 \\
\hline $\begin{array}{l}\text { Sadeghi Bahmani, } \\
2019^{61} \\
\text { Aerobics } \\
\text { RCT } \\
\text { Fair }\end{array}$ & $\begin{array}{l}\text { A. Endurance } \\
\text { training (treadmill, } \\
\text { cycling, walking, } \\
\text { jogging), } 24 \\
\text { sessions over } 8 \\
\text { weeks ( } n=26 \text { ) } \\
\text { B. Attention } \\
\text { control, } 24 \\
\text { sessions over } 8 \\
\text { weeks }(n=21)\end{array}$ & $\begin{array}{l}\text { A vs. B } \\
\text { Age: } 38 \text { vs. } 38 \\
\text { Female: } 100 \% \\
\text { EDSS: } 2.46 \text { vs. } 2.02\end{array}$ & $\begin{array}{l}\text { A vs. } B, \text { mean }(S D), p=\text { between groups: } \\
\text { EDSS: } 2.46(1.50) \text { to } 2.27(1.64) \text { vs. } 2.02(1.84) \\
\text { to } 1.98(1.70), p>0.05 \\
\frac{\text { ISI: } 11.62(5.23) \text { to } 8.81(5.41) \text { vs. } 1.71(5.43) \text { to }}{11.14(5.39), p>0.05}\end{array}$ \\
\hline $\begin{array}{l}\text { Young, } 2019^{54} \\
\text { Aerobic exercise } \\
\text { RCT } \\
\text { Fair }\end{array}$ & $\begin{array}{l}\text { A. Movement to } \\
\text { Music, } 36 \text { sessions } \\
\text { over } 12 \text { weeks } \\
(n=27) \\
\begin{array}{l}\text { B. Waitlist control } \\
(n=28)\end{array}\end{array}$ & $\begin{array}{l}\text { A vs. B } \\
\text { Age: } 50 \text { vs. } 47 \\
\text { Female: } 81 \% \text { vs. } 86 \% \\
\text { White: } 44 \text { vs. } 61 \% \\
\text { PDDS: } \\
\text { PDDS } 0: 30 \% \text { vs. } 21 \% \\
\text { PDDS } 3: 15 \% \text { vs. } 14 \% \\
\text { PDDS } 6: 11 \% \text { vs. } 11 \% \\
\end{array}$ & $\begin{array}{l}\text { A vs. B mean difference between groups: } \\
\text { TUG: }-1.89,95 \% \mathrm{Cl}-3.30 \text { to }-0.48, p=0.01 \\
\underline{6 \mathrm{MWT}}: 40.98,95 \% \mathrm{Cl} 2.21 \text { to } 79.75, p=0.04 \\
\frac{5 \mathrm{x} \text { Sit-to-Stand: }}{\mathrm{p}=0.38}-1.00,95 \% \mathrm{Cl}-2.58 \text { to } 0.55 \text {, }\end{array}$ \\
\hline
\end{tabular}

Abbreviations: 5x = five times; 6MWT =6-Minute Walk Test; 10MWT = 10-Minute Walk Test; BMI = body mass index; BBS ISI = Insomnia Severity Index; MusiQoL = Multiple Sclerosis International Quality of Life Scale; PSQI = Pittsburgh Sleep Quality Index; SF-36; Short Form; MSFC = Multiple Sclerosis Functional Composite; $n=$ number; PDDS = Patient Determined Disease Steps; $\mathrm{RCT}=$ randomized controlled trial; $\mathrm{SD}=$ standard deviation; $\mathrm{TUG}=$ Timed $\mathrm{Up}$ and Go Test

\section{Aerobics-Cerebral Palsy}

One good-quality trial found no improvement in running speed or mobility with a running program compared with usual care in adolescents (mean age 12 years, $\mathrm{n}=42$ ) with $\mathrm{CP} .{ }^{65}$ All adolescents except one had scores on the Gross Motor Classification System of I or II. Changes in walking measures were not assessed (Table 8).

Another trial ${ }^{64}$ enrolled young adult participants (mean age 17.5 years, $n=26$ ) with CP. Fiftyeight percent were female, and race was not reported. The proportions who could ambulate well or who used a wheelchair part time were also not reported. However, mean scores on the International Classification of Functioning, Disability and Health (ICF) indicated a severe to a complete problem walking a long distance such as a kilometer.

Dance, in this trial, involved 24 one-hour sessions that covered range of motion, motor coordination, space-time orientation, temporal coordination, proprioception, and skill and agility. Kinesiotherapy exercises consisted of range of motion, motor coordination, space-time orientation, proprioception, and skill and agility. Dance was conducted in a space with parallel bars, mirror, and sound equipment whereas the kinesiotherapy exercises were conducted as part of physical therapy (PT). Dance was associated with an improvement in function, disability, and health as measured by the Functional Independence Measure (FIM) and the ICF compared with traditional kinesiotherapy exercises. Harms and adverse events were not addressed. 
Table 8. Aerobic exercise in cerebral palsy

\begin{tabular}{|c|c|c|c|}
\hline $\begin{array}{l}\text { Author, Year } \\
\text { Intervention } \\
\text { Study Design } \\
\text { Study Quality }\end{array}$ & $\begin{array}{l}\text { Intervention } \\
\text { and Comparison }\end{array}$ & Population & Results \\
\hline $\begin{array}{l}\text { Gibson, } 2018^{65} \\
\text { Aerobics } \\
\text { RCT } \\
\text { Good }\end{array}$ & $\begin{array}{l}\text { A. Running and } \\
\text { running exercises, } \\
48 \text { sessions over } \\
12 \text { weeks }(n=21) \\
\text { B. Usual care } \\
(n=21)\end{array}$ & $\begin{array}{l}\text { A vs. B } \\
\text { Age: } 12.4 \text { vs. } 12.5 \\
\text { Female: } 33 \% \text { vs. } 38 \% \\
\text { GMFCS I: } 57 \% \text { vs. } 60 \% \\
\text { GMFCS II: } 38 \% \text { vs. } 40 \% \\
\text { GMFCS III: } 5 \% \text { vs. } 0 \%\end{array}$ & $\begin{array}{l}\text { A vs. B, mean difference between groups: } \\
\text { Shuttle Run Test (min): } 0.9,95 \% \mathrm{Cl}-0.3 \text { to } 2.2 \text {, } \\
p=0.142 \\
\text { HiMat: } 0.8,95 \% \mathrm{Cl}-2.7 \text { to } 4.3, \mathrm{p}=0.651 \\
\frac{10 \times 5 \text { sprint }(\mathrm{sec})}{p=0.535}-1.3,95 \% \mathrm{Cl}-5.4 \text { to } 2.8,\end{array}$ \\
\hline $\begin{array}{l}\text { Teixeira- } \\
\text { Machado, } \\
2018^{64}\end{array}$ & $\begin{array}{l}\text { A. Dance exercise } \\
24 \text { sessions over } \\
12 \text { weeks }(n=13)\end{array}$ & $\begin{array}{l}\text { A vs. B } \\
\text { Age: } 18 \text { vs. } 17.07 \\
\text { Female: } 54 \% \text { vs. } 62 \% \\
\text { GMFCS II: } 46 \% \text { vs. } 23 \%\end{array}$ & $\begin{array}{l}\text { A vs. B mean change scores: } \\
\text { FIM: } 1.7 \text { vs. } 0.03, p<0.001\end{array}$ \\
\hline $\begin{array}{l}\text { Aerobic } \\
\text { exercise } \\
\text { RCT }\end{array}$ & $\begin{array}{l}\text { B. Kinesiotherapy } \\
\text { exercises } 24 \\
\text { sessions over } 12 \\
\text { weeks }(n=13)\end{array}$ & $\begin{array}{l}\text { GMFCS III: } 23 \% \text { vs. } 38 \% \\
\text { GMFCS IV: } 23 \% \text { vs. } 31 \% \\
\text { GMFCS V: } 8 \% \text { vs. } 8 \%\end{array}$ & ICF: -44.56 vs. $14.90, p<0.001$ \\
\hline Fair & & & \\
\hline
\end{tabular}

Abbreviations: FIM = Functional Independence Measure; GMFCS = Gross Motor Function Classification System; HiMAT = High-level Mobility Assessment Tool; ICF = International Classification of Functioning; RCT= randomized controlled trial

Both trials that involved dancing or dance moves in participants with $\mathrm{MS}^{54}$ or $\mathrm{CP}^{64}$ found improvement in functioning with dancing (SOE: low), whereas the two poor-quality, nonrandomized studies ${ }^{62,63}$ found no evidence of improvement in function with aerobic exercises.

\section{Aerobics-Spinal Cord Injury}

No studies were identified.

\section{Aquatics}

Aquatic exercise has advantages of providing body weight support for the exerciser while also increasing resistance against limb movement. Participants may be kept upright during aquatic exercise with the use of flotation devices.

\section{Key Points}

- Balance may be improved with aquatic exercises when compared with usual care in adults with MS (SOE: low).

- Evidence was too limited to draw conclusions about aquatic exercise for those with CP (SOE: insufficient).

- There were no studies of aquatics in SCI.

\section{Detailed Synthesis}

Five RCTs ${ }^{66-69,71,73}$ and one cohort study ${ }^{74}$ of aquatic exercise met inclusion criteria $(n=231)$. These included three RCTs ${ }^{66-69}$ and one cohort study ${ }^{74}$ of aquatic exercise versus usual care and one RCT of aquatic exercise compared with cycling and strength exercises. ${ }^{73}$ One trial met criteria for good quality, ${ }^{71}$ one RCT was rated poor quality, ${ }^{68,69}$ and the remainder were rated fair quality. ${ }^{66,67,73,74}$ Trials were downgraded due to unclear randomization and concealment of the allocation, groups not similar at baseline on prognostic variables, unclear blinding of outcome 
assessors, and lack of intent-to-treat analysis. The most frequently reported outcomes were related to function (e.g., GMFM, Wee-Functional Independence Measure for children [WeeFIM], 6MWT).

\section{Aquatics-Multiple Sclerosis}

Four trials $(n=175)^{66-69,71}$ enrolled participants with MS (Table 9). The mean age of participants ranged from 19 to 50 years; three trials enrolled only female participants and the fourth had predominately female enrollees. ${ }^{71}$ Race was not reported in these trials, but three studies took place in Iran and one in Spain. ${ }^{71}$ In three trials enrolled participants could ambulate at least 100 meters overground, but in one trial most participants needed consistent assistance to walk from 5 meters to 100 meters. $^{71}$

Table 9. Aquatic exercise in multiple sclerosis

\begin{tabular}{|c|c|c|c|}
\hline $\begin{array}{l}\text { Author, Year } \\
\text { Intervention } \\
\text { Study Design } \\
\text { Study Quality }\end{array}$ & $\begin{array}{l}\text { Intervention } \\
\text { and Comparison }\end{array}$ & Population & Results \\
\hline $\begin{array}{l}\text { Castro-Sanchez, } \\
2012^{71} \\
\text { Aerobic Exercise } \\
\text { RCT } \\
\text { Good }\end{array}$ & $\begin{array}{l}\text { A. Ai-Chi aqua } \\
\text { therapy with Tai- } \\
\text { Chi music, } 40 \\
\text { sessions over } 20 \\
\text { weeks ( } n=36 \text { ) } \\
\text { B. Relaxation } \\
\text { exercises on } \\
\text { exercise mat } \\
\text { without music, } 40 \\
\text { sessions over } 20 \\
\text { weeks ( } n=37 \text { ) }\end{array}$ & $\begin{array}{l}\text { A vs. B } \\
\text { Age: } 46 \text { vs. } 50 \\
\text { Female: } 72 \% \text { vs. } 65 \% \\
\text { EDSS: } 6.3 \text { vs. } 5.9 \\
\text { PPMS: } 17 \% \text { vs. } 24 \% \\
\text { SPMS: } 25 \% \text { vs. } 32 \%\end{array}$ & $\begin{array}{l}\text { A vs. B, median (SD), p-value=between groups: } \\
\text { MSIS-29 Physical: } 48(15.91) \text { to } 41 \text { (12.37) vs. } 46 \\
\text { (18.34) to } 45(17.14), p=0.014 \\
\frac{\text { MSIS-29 Psychological: } 34(29.47) \text { to } 21(15.73)}{\text { vs. } 30(23.53) \text { to } 25(19.36), p=0.023} \\
\text { Barthel Index: } 91(7.12) \text { to } 86 \text { (9.23) vs. } 87 \\
\text { (10.34) to } 88 \text { (8.92), p>0.05 } \\
\text { Differences in MSIS-29 maintained at } 30 \text { weeks }\end{array}$ \\
\hline $\begin{array}{l}\text { Kargarfard, } \\
2018^{66} \\
\text { Aerobic Exercise } \\
\text { RCT } \\
\text { Fair }\end{array}$ & $\begin{array}{l}\text { A. Aquatic } \\
\text { exercise, } 24 \\
\text { sessions over } 8 \\
\text { weeks }(n=17) \\
\text { B. Waitlist control } \\
\text { group }(n=15)\end{array}$ & $\begin{array}{l}\text { A vs. B } \\
\text { Age: } 36.5 \text { vs. } 36.2 \\
\text { Female: } 100 \% \\
\text { EDSS } 3.4 \text { vs. } 3.7\end{array}$ & $\begin{array}{l}\text { A vs. B, mean change scores: } \\
\text { 6MWT: }-52 \text { vs. } 29, p<0.001 \\
\text { Sit to Stand: } 4.2 \text { vs. }-5.9, p<0.001 \\
\text { BBS: }-1.6 \text { vs. } 2.1, p<0.001\end{array}$ \\
\hline $\begin{array}{l}\text { Kooshiar, } 2015^{67} \\
\text { Aerobic Exercise } \\
\text { RCT } \\
\text { Fair }\end{array}$ & $\begin{array}{l}\text { A. Aquatic } \\
\text { exercise, } 24 \\
\text { sessions over } 8 \\
\text { weeks }(n=20) \\
\begin{array}{l}\text { B. Usual care } \\
(n=20)\end{array}\end{array}$ & $\begin{array}{l}\text { A vs. B } \\
\text { Age: } 29.24(<46 \text { years }) \\
\text { Female: } 100 \% \\
\text { EDSS: } 2.5 \\
\text { RRMS: } 75.7 \% \\
\text { PPMS: } 16.2 \% \\
\text { SPMS: } 8.1 \%\end{array}$ & $\begin{array}{l}\text { A vs. } B, \text { mean change scores: } \\
\text { MQLIM: }-16.93 \text { vs. }-1.04, p<0.001\end{array}$ \\
\hline $\begin{array}{l}\text { Marandi, } \\
2013^{68,69} \\
\text { Aerobic Exercise } \\
\text { RCT } \\
\text { Poor }\end{array}$ & $\begin{array}{l}\text { A. Aquatics: } 36 \\
\text { sessions over } 12 \\
\text { weeks }(n=15) \\
\begin{array}{l}\text { B. Usual care } \\
(n=15)\end{array}\end{array}$ & $\begin{array}{l}\text { A vs. B } \\
\text { Age: Unclear } \\
\text { Female: } 100 \% \\
\text { Ambulatory: } 100 \% \\
\text { EDSS: }<4.5\end{array}$ & $\begin{array}{l}\text { A vs. B, Six Spot Step Test: Adjusted mean } \\
\text { difference between groups: } \\
\text { Right leg dynamic balance: }-5.88 \text { (SE 1.4), } \\
\begin{array}{l}\mathrm{p}<0.001 \\
\text { Left leg dynamic balance: }\end{array}-6.23 \text { (SE 1.2), } \\
\mathrm{p}<0.001\end{array}$ \\
\hline
\end{tabular}

Abbreviations: 6MWT=6-Minute Walk Test; BBS=Berg Balance Scale; EDSS = Expanded Disability Status Scale; MQLIM=Multicultural Quality of Life Index; MSIS-29 = Multiple Sclerosis Impact Scale; PPMS = primary progressive multiple sclerosis; RCT = randomized controlled trial; RRMS = relapsing-remitting multiple sclerosis; $\mathrm{SCI}=$ spinal cord injury; SPMS = secondary progressive multiple sclerosis; $\mathrm{SE}=$ standard error 
Two trials found low-strength evidence for improved balance with aquatic exercises compared with usual care or attention control, ${ }^{66,68}$ as measured with the Berg Balance Scale, the Six Spot Step test, and dynamic balance.

The trial rated good quality found low-strength evidence for improvement in activities of daily living (ADL) with aquatic exercises compared with relaxation exercises out of water (on mat) ${ }^{71}$ Scores on the Multiple Sclerosis Impact Scale-29 (MSIS-29) (physical and psychological) were better with Ai-Chi aqua therapy and the differences were maintained at 30 weeks (10 weeks postintervention), while scores on the Barthel Index favored aquatics, but the difference did not reach statistical significance. ${ }^{71}$

Of the Iranian trials, one found significant improvement with aquatic exercise in the 6MWT and Sit to Stand compared with usual care $(p<0.001 \text { for both outcomes })^{66}$ and another trial found significant improvement in quality of life assessed with the Multicultural Quality of Life Index (MQLIM) compared with usual care $(\mathrm{p}<0.001) .{ }^{67}$ No study addressed harms or adverse events.

Two additional trials of aquatics report depression outcomes and are found in table 40, KQ2a. ${ }^{70,72}$

\section{Aquatics-Cerebral Palsy}

One RCT $(n=32)^{73}$ and one cohort study $(n=24)^{74}$ enrolled children and adolescents with CP (Table 10). The mean age of participants ranged from 4 to 17 years with the proportion female 47 percent. Race was not reported in these trials. Baseline EDSS scores ranged from 1 to 4 . All children were able to walk short distances either with or without assist devices.

Table 10. Aquatic exercise in cerebral palsy

\begin{tabular}{|c|c|c|c|}
\hline $\begin{array}{l}\text { Author, Year } \\
\text { Intervention } \\
\text { Study Design } \\
\text { Study Quality }\end{array}$ & $\begin{array}{l}\text { Intervention } \\
\text { and Comparison }\end{array}$ & Population & Outcomes \\
\hline $\begin{array}{l}\text { Adar, } 2017^{73} \\
\text { Aerobic } \\
\text { exercise } \\
\text { RCT } \\
\text { Fair }\end{array}$ & $\begin{array}{l}\text { A. Aquatic } \\
\text { exercise, } 30 \\
\text { sessions over } 6 \\
\text { weeks ( } n=17) \\
\text { B. Land-based } \\
\text { exercise, } 30 \\
\text { sessions over } 6 \\
\text { weeks ( } n=15)\end{array}$ & $\begin{array}{l}\text { A vs. B } \\
\text { Age: } 10.1 \text { vs. } 9.3 \\
\text { Female: } 53 \% \text { vs. } 40 \% \\
\text { Spastic diplegia: } 65 \% \\
\text { vs. } 67 \% \\
\text { Hemiplegia: } 35 \% \text { vs. } \\
33 \% \\
\text { GMFCS: Median } 2 \text { vs. } \\
2\end{array}$ & $\begin{array}{l}\text { A vs. B, mean change scores: } \\
\text { TUG: }-0.13(0.14) \text { vs. }-0.16(0.13), p=0.664 \\
\text { GMFM-88: } 0.05(0.05) \text { vs. } 0.05(0.03), p=0.451 \\
\frac{\text { WeeFIM motor: } 0.04(0.04) \text { vs. } 0.06(0.06), p=0.860}{\frac{\text { WeeFIM total: }}{p=0.287}-0.13(0.14) \text { vs. }-0.16(0.13),}\end{array}$ \\
\hline $\begin{array}{l}\text { Lai, } 2015^{74} \\
\text { Taiwan } \\
\text { Aerobic } \\
\text { exercise } \\
\text { Cohort study } \\
\text { Fair }\end{array}$ & $\begin{array}{l}\text { A. Aquatic therapy, } \\
24 \text { sessions over } \\
12 \text { weeks, rehab } \\
\text { exercises, } 24-36 \\
\text { sessions over } 12 \\
\text { weeks ( } n=11) \\
\text { B. Rehab } \\
\text { exercises, } 24-36 \\
\text { sessions over } 12 \\
\text { weeks }(n=13)\end{array}$ & $\begin{array}{l}\text { A vs. B } \\
\text { Age: } 7.6 \text { vs. } 6.6 \\
\text { Female: } 64 \% \text { vs. } 31 \% \\
\text { Diplegia: } 27 \% \text { vs. } \\
46 \% \\
\text { Quadriplegia } 45 \% \text { vs. } \\
31 \% \\
\text { Hemiplegia } 27 \% \text { vs. } \\
23 \% \\
\text { GMFCS: } 2.7 \text { vs. } 2.6\end{array}$ & $\begin{array}{l}\text { A vs. B, mean difference between groups: } \\
\text { GMFM-66: } 5.0 \text { vs. } 0.7, p=0.007 \\
\text { CPQoL scales for Social, Functioning, Participation, } \\
\text { Emotional, Access, Pain and Disability, and Family } \\
\text { Health: All NS }\end{array}$ \\
\hline
\end{tabular}

Abbreviations: CPQoL = Cerebral Palsy Quality of Life scale; FIM = Functional Independence Measure; GMFCS $=$ Gross Motor Function Classification System; GMFM-66 = Gross Motor Function Measure 66; GMFM-88 = Gross Motor Function Measure 88; NS = not significant; RCT $=$ randomized control trial; $\mathrm{TUG}=$ Timed Up and Go Test; WeeFIM = Wee-Functional Independence Measure for children

One trial in children with CP found no differences between 6 weeks of aquatic therapy and land-based exercises on the functional outcomes of TUG and GMFM- 88 tests. ${ }^{73}$ One cohort 
study that enrolled children with CP compared aquatic therapy plus traditional rehabilitation over 12 weeks found a significant effect on GMFM-66 scores $(p=0.007)$ compared with traditional rehabilitation, but did not show a significant effect on quality of life as measured with the Cerebral Palsy Quality of Life (CPQoL) scales. ${ }^{74}$ Harms and adverse events were not addressed.

\section{Aquatics-Spinal Cord Injury}

No studies were identified.

\section{Cycling}

Stationary cycling has been studied as means for aerobic or endurance training. This exercise method has multiple attributes, including relatively easy access in a study setting, and the potential to be used as an exercise method at home. Another advantage of cycling is the possibility of exercising the lower and/or upper extremities. Two SCI trials ${ }^{89,93}$ utilized upper extremity cycling, and one MS trial ${ }^{53}$ used both upper and lower extremity cycling. All other trials utilized lower extremity cycling.

\section{Key Points}

- There was no clear benefit in function (primarily walking ability) versus control interventions with leg cycling in participants with MS (SOE: low); evidence on quality of life was too limited to draw conclusions (SOE: insufficient).

- Leg cycling interventions were associated with improvement in function in participants with CP over control interventions (SOE: low); evidence for quality of life was lacking (SOE: insufficient).

- Evidence for function and quality of life in participants with SCI was limited (SOE: insufficient).

- Evidence for hand cycling was insufficient.

\section{Detailed Synthesis}

Ten RCTs, ${ }^{53,77-83,85-87,89}$ one quasiexperimental nonrandomized study, ${ }^{84}$ and one cohort study $^{90}(\mathrm{n}=596)$ involving cycling interventions met inclusion criteria. These included five $\mathrm{RCTs}^{53,77,83,85-87}$ and one quasiexperimental study ${ }^{84}$ of cycling versus usual care; one RCT, ${ }^{89}$ one cohort study, ${ }^{90}$ and one quasiexperimental study ${ }^{84}$ of cycling versus active rehabilitation; one RCT of cycling using visual feedback compared with home exercise ${ }^{81}$ one RCT of cycling versus MS-nurse consultations, ${ }^{78}$ one trial of cycling versus vestibular rehabilitation versus waitlist control, ${ }^{79,80}$ and one trial of intermittent versus static cycling. ${ }^{82}$

Seven RCTs $\mathrm{s}^{53,77-82}$ and one quasiexperimental trial ${ }^{84}$ enrolled participants with MS (Table 11), two RCTs ${ }^{85-87}$ were conducted in participants with CP (Table 12), and one RCT ${ }^{89}$ and one cohort study ${ }^{90}$ were conducted in participants with SCI (Table 13). Two RCTs and one cohort study was rated poor quality and the remaining trials were rated fair quality. Methodological limitations for downgrading studies included unclear methods of the allocation, baseline differences in prognostic variables between randomized arms, and high attrition. The most frequently reported outcomes were related to function (e.g., GMFM, 6MWT) and quality of life (e.g., SF-36, World Health Organization Quality of Life scale [WHOQOL]). 


\section{Cycling-Multiple Sclerosis}

Seven RCTs ${ }^{53,77,78,80-83}$ and one quasiexperimental study ${ }^{84}(n=459)$ enrolled participants with MS (Table 11). Mean ages of participants in the trials ranged from 32 to 52 years with a range of 58 to 93 percent female. Race was not reported in these trials. All studies enrolled participants who could ambulate with assistance or better.

Table 11. Cycling exercise in multiple sclerosis

\begin{tabular}{|c|c|c|c|}
\hline $\begin{array}{l}\text { Author, Year } \\
\text { Intervention } \\
\text { Study Design } \\
\text { Study Quality } \\
\end{array}$ & $\begin{array}{l}\text { Intervention } \\
\text { and Comparison }\end{array}$ & Population & Results \\
\hline $\begin{array}{l}\text { Baquet, } 2018^{77} \\
\text { Aerobic exercise } \\
\text { RCT } \\
\text { Fair }\end{array}$ & $\begin{array}{l}\text { A. Bicycle ergometry, } \\
24-36 \text { sessions over } 12 \\
\text { weeks }(n=34) \\
\text { B. Waitlist control group } \\
(n=34)\end{array}$ & $\begin{array}{l}\text { A vs. B } \\
\text { Age: } 38.2 \text { vs. } 39.6 \\
\text { Female: } 62 \% \text { vs. } \\
74 \% \\
\text { EDSS: } 1.7 \text { vs. } 1.8 \\
\text { RRMS: } 100 \%\end{array}$ & $\begin{array}{l}\text { A vs. B mean difference between groups: } \\
\text { 6MWT: } 4.0,95 \% \mathrm{Cl}-36.5 \text { to } 44.5, p=0.85 \\
\text { 25 foot walk: }-0.1,95 \% \mathrm{Cl}-0.4 \text { to } 0.2, p=0.49 \\
\text { MSWS- } 12:-0.3,95 \% \mathrm{Cl}-2.1 \text { to } 1.6, p=0.78 \\
\text { HAQUAMS: }-0.4,95 \% \mathrm{Cl}-4.5 \text { to } 3.7, p=0.84\end{array}$ \\
\hline $\begin{array}{l}\text { Collett, } 2011^{82} \\
\text { Aerobic exercise } \\
\text { RCT } \\
\text { Poor }\end{array}$ & $\begin{array}{l}\text { A. Combined intermittent } \\
\text { and continuous static } \\
\text { cycling, } 24 \text { sessions } \\
\text { over } 12 \text { weeks }(n=20) \\
\text { B. Intermittent static } \\
\text { cycling, } 24 \text { sessions } \\
\text { over } 12 \text { weeks }(n=21) \\
\text { C. Continuous static } \\
\text { cycling, } 24 \text { sessions } \\
\text { over } 12 \text { weeks }(n=20)\end{array}$ & $\begin{array}{l}\text { A vs. B vs. C } \\
\text { Age: } 55 \text { vs. } 50 \text { vs. } 52 \\
\text { Female: } 53 \% \text { vs. } \\
78 \% \text { vs. } 80 \% \\
\text { Ambulatory: } 100 \%\end{array}$ & $\begin{array}{l}\text { Change postintervention: no data provided } \\
\text { 2MWT, SF-36 total, TUG: All NS }\end{array}$ \\
\hline $\begin{array}{l}\text { Heine, } 2017^{78} \\
\text { Aerobic exercise } \\
\text { RCT } \\
\text { Fair }\end{array}$ & $\begin{array}{l}\text { A. Leg cycling, } 48 \\
\text { sessions over } 16 \text { weeks } \\
(n=43) \\
\text { B. MS nurse } \\
\text { consultation, } 3 \\
\text { consultations over } 16 \\
\text { weeks }(n=46)\end{array}$ & $\begin{array}{l}\text { A vs. B } \\
\text { Age: } 43.1 \text { vs. } 48.2 \\
\text { Female: } 74 \% \text { vs. } \\
72 \% \\
\text { Ambulatory: } 100 \% \\
\text { EDSS: } 2.5 \text { vs. } 3.0 \\
\text { RRMS: } 72 \% \text { vs. } 74 \% \\
\text { SPMS: } 7 \% \text { vs. } 11 \% \\
\text { PPMS: } 21 \% \text { vs. } 15 \%\end{array}$ & $\begin{array}{l}\text { A vs. B, mean difference (SE) between } \\
\text { groups: } \\
\text { IPA autonomy indoors: }-0.11(0.088), p=0.203 \\
\left.\frac{\text { IPA family role: }-0.082}{\text { IPA autonomy outdoors: }}-0.1222\right), p=0.502(0.125), \\
\text { IPA.438 } \\
\text { IPA Social Relations: }-0.138(0.092), p=0.135 \\
\text { IPA Work/education: } 0.225(0.167), p=0.181\end{array}$ \\
\hline $\begin{array}{l}\text { Hebert, } 2011^{80} \\
\text { Aerobic Exercise } \\
\text { RCT } \\
\text { Fair }\end{array}$ & $\begin{array}{l}\text { A. Bicycle ergometry, } 12 \\
\text { sessions for } 6 \text { weeks } \\
(n=12) \\
\text { B. Vestibular rehab } \\
(n=13) \\
\text { C. Waitlist control }(n=13)\end{array}$ & $\begin{array}{l}\text { A vs. B vs. C } \\
\text { Age: } 46.8 \text { vs. } 42.6 \\
\text { vs. } 50.2 \\
\text { Female: } 75 \% \text { vs. } \\
85 \% \text { vs. } 85 \% \\
\text { Ambulatory: } 100 \%\end{array}$ & $\begin{array}{l}\text { Mean difference between groups: } \\
\text { 6MWT: } \\
\text { A vs. } \mathrm{B}: 39.1,95 \% \mathrm{Cl}-105 \text { to } 183, p=1.00 \\
\text { A vs. C: } 62.7,95 \% \mathrm{Cl}-81 \text { to } 2.7, p=1.00 \\
\text { B vs. C: } 23.6,95 \% \mathrm{Cl}-117 \text { to } 165, p=1.00\end{array}$ \\
\hline $\begin{array}{l}\text { Hochsprung, } \\
2017^{81} \\
\text { Aerobic exercise } \\
\text { RCT } \\
\text { Poor }\end{array}$ & $\begin{array}{l}\text { A. Visual biofeedback } \\
\text { cycling training, } 12 \\
\text { sessions over } 12 \text { weeks } \\
\text { plus home exercise } \\
\text { program ( } n=30) \\
\text { B. Home exercise } \\
\text { program }(n=31)\end{array}$ & $\begin{array}{l}\text { A vs. B } \\
\text { Female: } 66 \% \text { vs. } \\
50 \% \\
\text { Ambulatory: } 100 \% \\
\text { RRMS: } 37 \% \text { vs. } 52 \% \\
\text { PPMS: } 20 \% \text { vs. } 26 \% \\
\text { SPMS: } 43 \% \text { vs. } 23 \% \\
\end{array}$ & $\begin{array}{l}\text { A vs. B mean change scores: } \\
\frac{\text { FAP: }}{3.036(p=0.002)} \text { vs. }-1.06(p=0.289) \\
\text { No comparison between groups provided }\end{array}$ \\
\hline
\end{tabular}




\begin{tabular}{|c|c|c|c|}
\hline $\begin{array}{l}\text { Author, Year } \\
\text { Intervention } \\
\text { Study Design } \\
\text { Study Quality }\end{array}$ & $\begin{array}{l}\text { Intervention } \\
\text { and Comparison }\end{array}$ & Population & Results \\
\hline $\begin{array}{l}\text { Negaresh, } 2019^{53} \\
\text { Aerobic exercise } \\
\text { RCT } \\
\text { Fair }\end{array}$ & $\begin{array}{l}\text { A. Normal BMI cycling } \\
\text { UE/LE, } 24 \text { sessions over } \\
8 \text { weeks }(n=18) \\
\text { B. Normal BMI control } \\
(n=15) \\
\text { C. Overweight cycling } \\
\text { UE/LE, } 24 \text { sessions over } \\
8 \text { weeks ( } n=17) \\
\text { D. Overweight control } \\
(n=13)\end{array}$ & $\begin{array}{l}\text { A vs. B vs. C vs. D } \\
\text { Age: } 31.2 \text { vs. } 29.1 \\
\text { vs. } 32.1 \text { vs. } 2.1 \\
\text { Female: } 64 \% \text { vs. } \\
64 \% \text { vs. } 64 \% \text { vs. } \\
69 \% \\
\text { EDSS: }<4 \\
\text { RRMS: } 100 \%\end{array}$ & $\begin{array}{l}\text { A vs. B vs. C vs. D, mean difference between } \\
\text { groups (scores are estimates from graph): } \\
\text { TUG: }-3.8 \text { vs. }-0.1 \text { vs. }-2.5 \text { vs. } 0, p=0.001 \\
\text { Interaction between weight and exercise } \\
p=0.52\end{array}$ \\
\hline $\begin{array}{l}\text { Niwald, } 2017^{84} \\
\text { Aerobic exercise } \\
\text { Quasiexperimental } \\
\text { Fair }\end{array}$ & $\begin{array}{l}\text { A. Cycle ergometry, } 60 \\
\text { sessions over } 4 \text { weeks } \\
\text { plus } 480 \text { minutes of } \\
\text { rehab exercises over } 4 \\
\text { weeks ( } n=21 \text { ) } \\
\text { B. } 480 \text { minutes of rehab } \\
\text { exercises } 480 \text { over } 4 \\
\text { weeks }(n=32)\end{array}$ & $\begin{array}{l}\text { A vs. B } \\
\text { Age: } 57 \text { vs. } 60 \\
\text { Female: } 62 \% \text { vs. } \\
65 \% \\
\text { Race: NR } \\
\text { Ambulatory: } 100 \% \\
\text { EDSS: } 6.33 \text { vs. } 6.20\end{array}$ & $\begin{array}{l}\text { A vs. B, mean difference between groups: } \\
\text { EDDS: } 0.01,95 \% \mathrm{Cl}-0.61 \text { to } 1.29, \mathrm{p}=0.48 \\
\text { WHOQOL-Bref Physical: } 1.45,95 \% \mathrm{Cl}-0.72 \\
\text { to } 3.62, \mathrm{p}=0.19 \\
\text { WHOQOL-Bref Psychological: } 3.05,95 \% \mathrm{Cl} \\
1.30 \text { to } 4.80 \text { to, } \mathrm{p}=0.001 \\
\text { WHOQOL-Bref Social: } 0.60,95 \% \mathrm{Cl}-0.64 \text { to } \\
1.84, \mathrm{p}=0.34 \\
\text { WHOQOL-Bref Environmental: } 2.56,95 \% \mathrm{Cl} \\
0.20 \text { to } 4.92, \mathrm{p}=0.03\end{array}$ \\
\hline $\begin{array}{l}\text { Tollar, } 2020^{83} \\
\text { Aerobic exercise } \\
\text { RCT } \\
\text { Fair } \\
\end{array}$ & $\begin{array}{l}\text { A. Stationary cycling, } 25 \\
\text { sessions over } 5 \text { weeks } \\
(n=14) \\
\text { B. Usual PT, } 25 \\
\text { sessions over } 5 \text { weeks } \\
(n=12)\end{array}$ & $\begin{array}{l}\text { A vs. B } \\
\text { Age: } 48.1 \text { vs. } 44.4 \\
\text { Female: } 93 \% \text { vs. } \\
92 \% \\
\text { EDSS median: } 5.0 \\
\text { vs. } 5.0 \\
\text { RRMS: } 64 \% \text { vs. } 67 \% \\
\end{array}$ & $\begin{array}{l}\text { A vs. B, mean difference between groups: } \\
\text { MSIS-29: }-6.3(8.07) \text { vs. } 1.0(3.46), p=0.008 \\
\text { 6MWT: } 32.1(44.58) \text { vs. } 6.3(49.27), p=0.174 \\
\text { BBS: } 2.5(2.62) \text { vs. }-0.2(2.62), p=0.015 \\
\text { EQ-5 Sum score:-1.4 (1.7) vs. } 0.0(1.13), \\
p=0.023\end{array}$ \\
\hline
\end{tabular}

Abbreviations: 2MWT = 2-Minute Walk Test; 6MWT = 6-Minute Walk Test, BBB = Berg Balance Scale; BMI = body mass index; $\mathrm{CI}=$ confidence interval; EDSS = Expanded Disability Status Scale; FAP = Functional Ambulation Profile; IPA = Impact on Participation and Autonomy; LE = lower extremity; MS = multiple sclerosis; MSWS-12 = Multiple Sclerosis Walking Scale12; MSIS-29 = Multiple Sclerosis Impact Scale; HAQUAMS = Hamburg Quality of Life Questionnaire in Multiple Sclerosis questionnaire; $\mathrm{NR}=$ not reported; $\mathrm{NS}=$ not significant; $\mathrm{PPMS}=$ primary progressive multiple sclerosis; $\mathrm{PT}=$ physical therapy; $\mathrm{RCT}$ = randomized controlled trial; RRMS = relapsing-remitting multiple sclerosis; SE = standard error; SF-36; Short Form-36; SPMS = secondary progressive multiple sclerosis; TUG $=$ Timed Up and Go Test; UE $=$ upper extremity; WHOQOL $=$ World Health Organization Quality of Life scale

These studies provided low SOE of no clear difference in function (primarily walking outcomes) between cycling and usual rehabilitation/no intervention. One fair-quality RCT did find a difference in change from baseline BMI across four groups (two intervention arms and two control arms, $\mathrm{p}=0.001$ ), but pairwise comparisons were not provided. ${ }^{53}$ Change in ADLs as measured with the Impact of Participation and Autonomy questionaire ${ }^{80}$ was insufficient from which to draw conclusions as was the evidence for quality of life on the Hamburg Quality of Life Questionnaire in Multiple Sclerosis. ${ }^{77}$ A poor-quality RCT, without a usual care/no intervention arm, found no differences between three cycling intervention groups (combined intermittent continuous static cycling vs. intermittent static cycling vs. continuous static cycling) on the SF36 , a quality of life measure, or on function outcomes (i.e., 2-Minute Walk Test [2MWT], TUG). ${ }^{82}$

Of the seven studies, five did not address harms or adverse events. An RCT of only active groups reported that three participants in the combined exercise group experienced adverse events (tachycardia, leg pain, and exacerbation of knee injury), while four participants in the intermittent group left the study due to adverse events (pain with cycling, exacerbation of MS 
symptoms, and loss of consciousness during cycling), and no adverse events were reported in the continuous exercise group. ${ }^{82}$ Another RCT evaluated the risk of experiencing an MS relapse in patients with relapsing-remitting MS as a potential adverse event, but found lower risk in the aerobic training group (OR [odds ratio] $0.28,95 \%$ CI 0.10 to $0.79, p=0.016$ ) in favor of aerobic training. ${ }^{78}$ The remaining study reported that no participant experienced any adverse event. ${ }^{53}$

\section{Cycling-Cerebral Palsy}

Two cycling RCTs ${ }^{85-87}(\mathrm{n}=85)$ enrolled participants with CP (Table 12). Mean ages of participants were 11 years and 13.9 years in the trial and mean proportion female was 53 and 60 percent. One RCT reported the proportion of nonwhite participants as 48 percent. ${ }^{85,86}$ One trial enrolled participants who had markedly limited or no ambulation (GMFCS IV or V) ${ }^{87}$ and one RCT enrolled only ambulatory participants (GMFCS I-III). ${ }^{85,86}$

Table 12. Cycling exercise in cerebral palsy

\begin{tabular}{|c|c|c|c|}
\hline $\begin{array}{l}\text { Author, Year } \\
\text { Intervention } \\
\text { Study Design } \\
\text { Study Quality }\end{array}$ & $\begin{array}{l}\text { Intervention } \\
\text { and Comparison }\end{array}$ & Population & Results \\
\hline $\begin{array}{l}\text { Bryant, } 2013^{87} \\
\text { Aerobic } \\
\text { exercise } \\
\text { RCT } \\
\text { Fair }\end{array}$ & $\begin{array}{l}\text { A. Static bike } \\
\text { group, } 18 \text { sessions } \\
\text { over } 6 \text { weeks } \\
(n=11) \\
\text { B: No intervention } \\
\text { control }(n=12)\end{array}$ & $\begin{array}{l}\text { A vs. B } \\
\text { Age: } 14.3 \text { vs. } 13.8 \\
\text { Female: } 45 \% \text { vs. } 58 \% \\
\text { Race: NR } \\
\text { Ambulatory: } 0 \% \\
\text { Wheelchair user: } 100 \% \\
\text { Bilateral CP: } 100 \% \\
\text { GMFCS: } 4.3 \text { vs. } 4.4\end{array}$ & $\begin{array}{l}\text { A vs. B mean difference between groups: } \\
\text { GMFM-66: } 0.70,95 \% \mathrm{Cl}-1.43 \text { to } 2.83, p=0.52 \\
\text { GMFM-88-D: } 5.4,95 \% \mathrm{Cl} 1.23 \text { to } 9.57, p=0.01 \\
\text { GMFM-88-E: } 2.3,95 \% \mathrm{Cl} 0.20 \text { to } 4.40, p=0.03\end{array}$ \\
\hline $\begin{array}{l}\text { Demuth, 201286 } \\
\text { Fowler, 201085 } \\
\text { Aerobic } \\
\text { exercise } \\
\text { RCT } \\
\text { Fair }\end{array}$ & $\begin{array}{l}\text { A. Stationary } \\
\text { cycling, } 30 \\
\text { sessions over } 12 \\
\text { weeks ( } n=31) \\
\text { B. No intervention } \\
\text { control }(n=31)\end{array}$ & $\begin{array}{l}\text { A vs. B } \\
\text { Age: } 10.7 \text { vs. } 11.2 \\
\text { Female: } 42 \% \text { vs. } 65 \% \\
\text { Race: African-American: } \\
16 \% \text { vs. } 10 \% \\
\text { White: } 58 \% \text { vs. } 48 \% \\
\text { Asian: } 3 \% \text { vs. } 16 \% \\
\text { Other: } 23 \% \text { vs. } 26 \% \\
\text { Ambulatory: } 100 \% \\
\text { GMFCS: } 2.0 \text { vs. } 2.3\end{array}$ & $\begin{array}{l}\text { A vs. B } \\
\text { GMFM- } 66 \text { : } \\
\text { Change from baseline: } 1.2,95 \% \mathrm{Cl} 0.5 \text { to } 1.8 \\
\text { vs. } 0.5,95 \% \mathrm{Cl}-0.2 \text { to } 1.3 \text {, between groups } \\
\mathrm{p}=0.23 \\
600 \text {-Yard Walk-Run Test: } \\
\text { Change from baseline: } 5.6,95 \% \mathrm{Cl} 1.6 \text { to } 9.5 \\
\text { vs. } 2.5,95 \% \mathrm{Cl}-1.1 \text { to } 6.0, \mathrm{p}=0.24 \\
\text { Peds Quality of Life Total Score: } \\
\text { Mean difference between groups: } \\
3.5,95 \% \mathrm{Cl}-2.0 \text { to } 8.8, p=0.21\end{array}$ \\
\hline
\end{tabular}

Abbreviations: $\mathrm{CI}=$ confidence interval; $\mathrm{CP}=$ cerebral palsy; GMFCS $=$ Gross Motor Function Classification System; GMFM$66=$ Gross Motor Function Measure-66 items, GMFM-88 = Gross Motor Function Measure-88 items; GMFM-88-D = Gross Motor Function Measure 88 (standing); GMFM-88-E = Gross Motor Function Measure 88 (walking, running, jumping); NR = not reported; RCT = randomized controlled trial

One RCT found an improvement with cycling on the GMFM-88-D and GMFM-88-E subscales that focused on standing (D subscale) and walking, running, and jumping (E subscale) ${ }^{87}$ Pooled analysis of GMFM-66 favored cycling over control condition, but did not reach statistical significance $\left(\mathrm{MD}-0.70,95 \% \text { confidence interval }[\mathrm{CI}]-1.60 \text { to } 0.20, \mathrm{I}^{2}=0 \%\right)^{85,87}$ (Figure 7). Similarly, performance on the 600-yard walk-run test favored cycling but was not significant. ${ }^{85}$ These studies provided low-strength evidence that cycling may improve gross motor function when compared with no intervention ${ }^{85}$ or usual care ${ }^{87}$ in children with CP. 
Figure 7. GMFM-66 in cerebral palsy trials

\begin{tabular}{|c|c|c|c|c|c|c|c|c|c|c|c|}
\hline Study & Cond. & Exercise & Control & $\begin{array}{c}\mathrm{N} \\
\text { Exercise }\end{array}$ & $\begin{array}{c}\mathrm{N} \\
\text { Control }\end{array}$ & Weight & $\begin{array}{l}\text { MD } \triangle \text { Scores } \\
\text { PL }[95 \% \mathrm{Cl}]\end{array}$ & & & & \\
\hline Bryant 2013 & $\mathrm{CP}$ & Stationary Cycling & No Intervention & 11 & 11 & $17.8 \%$ & $-0.70[-2.83,1.43]$ & & & & \\
\hline Demuth 2012/Fowler 2010 & $\mathrm{CP}$ & Stationary Cycling & No Intervention & 29 & 29 & $82.2 \%$ & $-0.70[-1.69,0.29]$ & & & & \\
\hline \multicolumn{4}{|l|}{ Total $(95 \% \mathrm{Cl})$} & 40 & 40 & $100.0 \%$ & $-0.70[-1.60,0.20]$ & & & & \\
\hline \multirow{2}{*}{\multicolumn{8}{|c|}{$\begin{array}{l}\text { Heterogeneity: } \text { Tau }^{2}=0.00 ; \mathrm{Chi}^{2}=0.00, \mathrm{df}=1(P=1.00) ; I^{2}=0 \% \\
\text { Test for overall effect: } Z=1.53(P=0.13)\end{array}$}} & -4 & -2 & 2 & 4 \\
\hline & & & & & & & & & Favors Exercise & Favors Control & \\
\hline
\end{tabular}

Abbreviations: $\Delta=$ change; $\mathrm{CI}=$ confidence interval; $\mathrm{CP}=$ cerebral palsy; GMFM-66 = Gross Motor Function Measure 66; MD $=$ mean difference

One RCT found no statistically significant difference in quality of life between cycling and no intervention on the Pediatric Quality of Life Inventory total score (insufficient evidence). ${ }^{86}$

Although no overall harms or adverse events were reported by treatment group, one RCT reported that two participants withdrew from the treadmill group (one due to gastric problems and one to recurrence of hip pain). ${ }^{87}$ The other RCT reported that there were mild adverse events (falls, soreness, muscle cramping, pain, fatigue, skin rash, colds, flu, tooth loss, headache, stomach ache, tonsillectomy) but did not specify the study group. ${ }^{86}$

\section{Cycling-Spinal Cord Injury}

One $\mathrm{RCT}^{89}$ and one cohort study ${ }^{90}$ enrolled participants $(\mathrm{n}=78)$ with SCI (Table 13). The mean age of participants was 35 and 36 years with 12 and 16 percent female. Racial background was not reported in these studies. Ambulatory status and wheelchair use were also not reported.

Table 13. Cycling exercise in spinal cord injury

\begin{tabular}{|c|c|c|c|}
\hline $\begin{array}{l}\text { Author, Year } \\
\text { Intervention } \\
\text { Study Design } \\
\text { Study Quality }\end{array}$ & $\begin{array}{l}\text { Intervention } \\
\text { and Comparison }\end{array}$ & Population & Results \\
\hline $\begin{array}{l}\text { Akkurt, } 2017^{89} \\
\text { Aerobic exercise } \\
\text { RCT } \\
\text { Fair }\end{array}$ & $\begin{array}{l}\text { A. Arm ergometer, } 36 \\
\text { sessions over } 12 \\
\text { weeks plus } 120 \\
\text { sessions general } \\
\text { exercises over } 12 \\
\text { weeks ( } n=17) \\
\text { B. General exercises, } \\
120 \text { sessions over } 12 \\
\text { weeks }(n=16)\end{array}$ & $\begin{array}{l}\text { A vs. B } \\
\text { Age: } 33 \text { vs. } 37 \\
\text { Female: } 5 \% \text { vs. } 19 \% \\
\text { Ambulatory: } 41 \% \text { vs. } \\
50 \% \\
\text { Wheelchair user: } \\
59 \% \text { vs. } 50 \% \\
\text { Paraplegia: } 100 \% \text { vs. } \\
94 \%\end{array}$ & $\begin{array}{l}\text { A vs. B, mean change scores: } \\
\text { FIM: } 0.5 \text { vs. }-0.5, p=1.00 \\
\text { CHART-sf, } p>0.05 \\
\text { WHOQOL-Bref, } p>0.05\end{array}$ \\
\hline $\begin{array}{l}\text { Sadowsky, } \\
2013^{90} \\
\text { Aerobic exercise } \\
\text { Cohort study } \\
\text { Poor }\end{array}$ & $\begin{array}{l}\text { A. FES cycle } \\
\text { ergometry, } 3 \text { sessions } \\
\text { per week over a } \\
\text { mean of } 120 \text { weeks } \\
(n=25) \\
\text { B. Rehabilitation care, } \\
\text { not specified }(n=20)\end{array}$ & $\begin{array}{l}\text { A vs. B } \\
\text { Age: } 37.2 \text { vs. } 34.6 \\
\text { Female: } 12 \% \text { vs. } \\
20 \% \\
\text { Quadriplegia: } 52 \% \\
\text { vs. } 75 \%\end{array}$ & $\begin{array}{l}\text { A vs. B, mean change scores: } \\
\text { Total FIM: } 80 \% \text { vs. } 60 \%, p<0.001 \\
\text { With significant improvement with FES in } \\
\text { subscales: self-care, sphincter control, transfer, } \\
\text { and locomotion } \\
\text { SF-36: total and composite scores NR } \\
\text { Significant improvement in physical function and } \\
\text { role limit physical with FES, no difference in } \\
\text { mental health subscales }\end{array}$ \\
\hline
\end{tabular}

Abbreviations: CHART $=$ Craig Handicap and Assessment Reporting Technique; FES $=$ functional electrical stimulation; FIM = Functional Independence Measure; NR = not reported; SF-36 = Short-Form 36; RCT = randomized controlled trial; WHOQOL = World Health Organization Quality of Life scale

One fair-quality RCT showed no significant improvement in function, quality of life, or ADL with upper extremity cycling versus general exercises in patients with $\mathrm{SCI}^{89}$ (rated insufficient for all outcomes). One poor-quality cohort study reported that lower extremity cycling resulted in significant improvement in mean FIM total score compared with usual care, but this study has 
substantial risk of bias based on study quality and nonrandomized design. ${ }^{90}$ Harms and adverse events were not addressed in either study.

One additional trial of the reports the effects of hand cycling on asymptomatic bacteria is discussed in KQ2b. ${ }^{92}$

\section{Robot-Assisted Gait Training}

Robot-assisted gait training is a form of physical activity/gait training in which a motorized, computer-controlled orthotic device provides a guidance force to the lower extremities, usually coupled with body weight support, enabling a person with limited ambulation the ability to walk greater distances and with potentially increased speed and safety, without necessarily requiring physical assistance of a physical therapist or caregiver. The robotic device places the lower extremity in improved alignment and form throughout the gait cycle, which can improve gait mechanics overground after the training. RAGT's value as a form of aerobic exercise for patients with ambulation impairment may be limited by the cost and access of the device to most people with MS, CP, or SCI.

\section{Key Points}

- Evidence suggested that RAGT training may improve balance in patients with MS versus usual care (SOE: low); there was low-strength evidence of no clear benefit of RAGT on function (SOE: low).

- When compared with overground or treadmill walking, there was low-strength evidence of no clear benefit with RAGT on function, balance, or quality of life in MS (SOE: low).

- Evidence for the effectiveness of RAGT on function and balance in CP was insufficient due to the poor quality of the trials (SOE: insufficient).

- Evidence from RCTs suggested that RAGT may improve function in patients with SCI in head-to-head studies (SOE: low) but the evidence was less clear in three trials versus usual care (SOE: low); there was low-strength evidence that RAGT training may improve ADL in people with SCI (SOE: low).

\section{Detailed Synthesis}

Seventeen RCTs $(n=810),{ }^{94-104,107,108,110-112,114-116}$ one quasiexperimental study $(n=44),{ }^{105}$ and one cohort study ${ }^{106}(\mathrm{n}=24)$ evaluated RAGT interventions. These included eight RAGT studies versus usual care, ${ }^{95,98,101,102,104,106,112,115,116}$ one versus no intervention, ${ }^{114}$ two RCTs versus overground gait training, ${ }^{107,108}$ and eight RCTs versus other interventions such as aquatics, taskoriented physical therapy (TOP), and nonrobotic treadmill training. Five RCTs enrolled participants with MS (Table 14), seven RCTs enrolled children and adolescents with CP (Table 15), one RCT enrolled adolescents and adults, ${ }^{104}$ and the remaining RAGT studies were in adults, and ten RCTs were conducted in participants with SCI (Table 16).

Three studies ${ }^{96-98}$ met criteria for good quality, eleven ${ }^{94,95,99,100,103,107,108,110-112,115,116}$ for fair

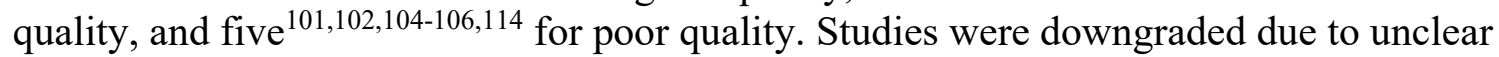
methods of selection and concealment of the allocation, differences between groups in prognostic patient characteristics, and lack of intent-to-treat analysis. Almost all of the trials used the Lokomat ${ }^{\circledR}$ (manufactured by Hocoma) ${ }^{264}$ as their RAGT device. The most frequently reported outcomes were gait parameters such as gait speed, walking endurance, and measures of overall physical function such as EDSS and GMFM. 


\section{Robot-Assisted Gait Training-Multiple Sclerosis}

Five RAGT trials enrolled 252 participants with MS ${ }^{94-98}$ (Table 13). Mean trial age of participants ranged from 42 to 56 years and the proportion female ranged from 52 to 68 percent. The length of the interventions ranged from 4 to 8 weeks, 3 to 5 sessions per week.

Participants' EDSS scores ranged from 4.0 to 7 , encompassing a wide range of walking ability at baseline. One RCT ${ }^{96}$ examined the effect of RAGT on less impaired participants with RRMS (relapsing-remitting multiple sclerosis) with EDSS of 4.0 to 5.5, indicating ability to ambulate 100 to 500 meters without aid, while the other three RCTs ${ }^{94,95,97}$ enrolled only participants with EDSS scores between 6.0 and 7.5 (minimal ability to walk, from a few steps to 100 meters total with the use of an assistive device).

Table 14. Robot-assisted gait training in multiple sclerosis

\begin{tabular}{|c|c|c|c|}
\hline $\begin{array}{l}\text { Author, Year } \\
\text { Intervention } \\
\text { Study Design } \\
\text { Study Quality }\end{array}$ & $\begin{array}{l}\text { Intervention and } \\
\text { Comparison }\end{array}$ & Population & Results \\
\hline $\begin{array}{l}\text { Calabro, } 2017^{96} \\
\text { Aerobic exercise } \\
\text { RCT } \\
\text { Good }\end{array}$ & $\begin{array}{l}\text { A. Lokomat-Pros } \\
\text { (RAGT + VR), } 40 \\
\text { sessions over } 8 \\
\text { weeks }(n=20) \\
\\
\text { B. Lokomat-Nanos } \\
(\text { RAGT), } 40 \text { sessions } \\
\text { over } 8 \text { weeks }(n=20)\end{array}$ & $\begin{array}{l}\text { A vs. } B \\
\text { Age: } 44 \text { vs. } 41 \\
\text { Female: } 65 \% \text { vs. } 60 \% \\
\text { EDSS: } 4.40 \text { vs. } 4.75\end{array}$ & $\begin{array}{l}\text { A vs. B, mean difference between groups: } \\
\text { TUG: }-0.064,95 \% \mathrm{Cl}-0.408 \text { to } 0.536, p=0.3 \\
\text { FIM: }-0.054,95 \% \mathrm{Cl}-1.73 \text { to } 2.839, p=0.5 \\
\text { BBS: }-0.019,95 \% \mathrm{Cl}-2.403 \text { to } 2.365, p=0.8\end{array}$ \\
\hline $\begin{array}{l}\text { Pompa, } 2017^{94} \\
\text { Aerobic exercise } \\
\text { RCT } \\
\text { Fair }\end{array}$ & $\begin{array}{l}\text { A. RAGT, } 12 \\
\text { sessions over } 4 \\
\text { weeks }(n=21) \\
\text { B. Conventional } \\
\text { Walking Training, } 12 \\
\text { sessions over } 4 \\
\text { weeks }(n=22)\end{array}$ & $\begin{array}{l}\text { A vs. } B \\
\text { Age: } 47 \text { vs. } 50 \\
\text { Female: } 48 \% \text { vs. } 55 \% \\
\text { PPMS: } 0 \% \text { vs. } 13.6 \% \\
\text { EDSS: } 6.62 \text { vs. } 6.50\end{array}$ & $\begin{array}{l}\text { A vs. B, mean difference between groups: } \\
\text { 2MWT: } 6.07,95 \% \mathrm{Cl}-6.51 \text { to } 18.65, p=0.34 \\
\text { FAC: } \\
\text { O.66, } 95 \% \mathrm{Cl}-0.07 \text { to } 1.39, p=0.08 \\
\text { Rivermead Mobility Index: } \\
0.73,95 \% \mathrm{Cl}-0.85 \text { to } 2.31, p=0.37 \\
\text { EDSS: } 0.14,95 \% \mathrm{Cl}-0.13 \text { to } 0.41, p=0.30 \\
\text { mBl: } 3.99,95 \% \mathrm{Cl}-6.69 \text { to } 14.67, p=0.46\end{array}$ \\
\hline $\begin{array}{l}\text { Russo, } 2018^{95} \\
\text { Aerobic exercise } \\
\text { RCT } \\
\text { Fair }\end{array}$ & $\begin{array}{l}\text { A. RAGT, } 18 \\
\text { sessions over } 6 \\
\text { weeks then } 36 \\
\text { sessions of } \\
\text { rehabilitation } \\
\text { exercises over } 12 \\
\text { weeks ( }=30) \\
\text { B. Rehabilitation } \\
\text { exercises, } 54 \\
\text { sessions over } 18 \\
\text { weeks ( } n=15)\end{array}$ & $\begin{array}{l}\text { A vs. } B \\
\text { Age: } 42 \text { vs. } 41 \\
\text { Female: } 53 \% \text { vs. } 67 \%\end{array}$ & 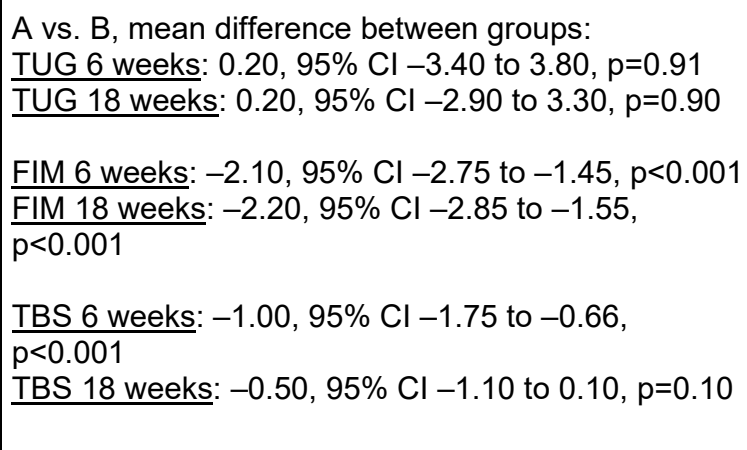 \\
\hline $\begin{array}{l}\text { Straudi, } 2016^{97} \\
\text { Aerobic exercise } \\
\text { RCT } \\
\text { Good }\end{array}$ & $\begin{array}{l}\text { A. RAGT, } 12 \\
\text { sessions over } 6 \\
\text { weeks ( } n=27) \\
\\
\text { B. Conventional } \\
\text { physiotherapy, } 12 \\
\text { sessions over } 6 \\
\text { weeks }(n=25)\end{array}$ & $\begin{array}{l}\text { A vs. } B \\
\text { Age: } 52 \text { vs. } 54 \\
\text { Female: } 63 \% \text { vs. } 68 \% \\
\text { EDSS: } 6.43 \text { vs. } 6.46 \\
\text { PPMS: } 33 \% \text { vs. } 28 \% \\
\text { SPMS: } 67 \% \text { vs. } 72 \%\end{array}$ & $\begin{array}{l}\text { A vs. B, mean change scores: } \\
\text { TUG: } 2.66(13.79) \text { vs. }-3.96(10.50), p=0.95 \\
\underline{\text { 6MWT: } 23.22(32.23) \text { vs. }-0.75(26.40), p=0.01} \\
\text { SF 36-PCS: } 1.67(7.74) \text { vs. } 1.84(6.77), p=0.99 \\
\underline{\text { SF 36-MCS: }} 5.37(9.58) \text { vs. } 1.60(9.41), p=0.14 \\
\text { BBS: } 3.24(4.99) \text { vs. } 0.87(6.45), p=0.19\end{array}$ \\
\hline
\end{tabular}




\begin{tabular}{|c|c|c|c|}
\hline $\begin{array}{l}\text { Author, Year } \\
\text { Intervention } \\
\text { Study Design } \\
\text { Study Quality }\end{array}$ & $\begin{array}{l}\text { Intervention and } \\
\text { Comparison }\end{array}$ & Population & Results \\
\hline $\begin{array}{l}\text { Straudi, } 2019^{98} \\
\text { Aerobic exercise } \\
\text { RCT } \\
\text { Good }\end{array}$ & $\begin{array}{l}\text { A. RAGT, } 12 \\
\text { sessions over } 4 \\
\text { weeks }(n=36) \\
\text { B. Overground } \\
\text { walking, } 12 \text { sessions } \\
\text { over } 4 \text { weeks }(n=36)\end{array}$ & $\begin{array}{l}\text { A vs. } B \\
\text { Age: } 56 \text { vs. } 55 \\
\text { Female: } 67 \% \text { vs. } 69 \% \\
\text { EDSS: } 6.5 \text { vs. } 6.5 \\
\text { PPMS: } 50 \% \text { vs. } 45 \% \\
\text { SPMS: } 50 \% \text { vs. } 55 \%\end{array}$ & $\begin{array}{l}\text { A vs. B, mean difference between groups: } \\
\text { 6MWT: } 4,95 \% \mathrm{Cl}-10 \text { to } 18, p=0.86 \\
\text { 25FWT: } 0,95 \% \mathrm{Cl}-0.06 \text { to } 0.05, p=0.98 \\
\text { TUG: } 7.8,-0.2 \text { to } 15.8, p=0.25 \\
\text { BBS: } 0,95 \% \mathrm{Cl}-2 \text { to } 2, p=0.91 \\
\text { MSIS-29 motor: }-3,95 \% \mathrm{Cl}-9 \text { to } 3, p=0.31 \\
\text { MSIS-29 psychological: }-2,95 \% \mathrm{Cl}-5 \text { to } 1, p=0.22 \\
\text { SF-36 PCS: }-1,95 \% \mathrm{Cl}-4 \text { to } 3, p=0.13 \\
\text { SF-36 MCS: } 1,95 \% \mathrm{Cl}-2 \text { to } 4, p=0.94\end{array}$ \\
\hline
\end{tabular}

Abbreviations: 2MWT = 2-Minute Walk Test; 6MWT =6-Minute Walk Test; 25FWT = 25-Foot Walk Test; BBS = Berg Balance Scale; CI = confidence interval; EDSS = Expanded Disability Status Scale; FAC = functional ambulation category; FIM = Functional Independence Measure; MSIS-29 = Multiple Sclerosis Impact Scale; PPMS = primary progressive multiple sclerosis; RAGT = robot-assisted gait training; RCT = randomized controlled trial; SF-36 = Short-Form 36; SF 36-MCS = ShortForm 36 Mental Component Summary; SF 36-PCS = Short-Form 36 Physical Component Summary; SPMS = secondary progressive multiple sclerosis; TBS = Tinetti Balance Scale; TUG = Timed Up and Go Test; VR = virtual reality

These studies indicated that use of RAGT can improve balance versus usual care without RAGT (SOE: low). There was low-strength evidence of no clear benefit of RAGT versus usual care on function as assessed with the TUG test,${ }^{95,97}$ while evidence for walking ability, ${ }^{94,97}$ quality of life, ${ }^{97}$ and $\mathrm{ADL}^{94}$ was too limited to draw conclusions (SOE: insufficient).

One RCT enrolled participants with MS and compared RAGT with virtual reality versus RAGT alone. ${ }^{96}$ In this RCT, there were no differences between groups on the TUG, FIM, and the BBS, indicating that virtual reality added to 40 sessions of RAGT did not improve balance, time up and go, or ADL compared with RAGT without virtual reality. However, this finding needs confirmation with other trials (SOE: insufficient).

Two head-to-head trials $(\mathrm{n}=95)$ compared RAGT with treadmill or overground walking ${ }^{97,98}$ and found low-strength evidence of no clear difference between treatment groups on function, balance, or quality of life.

Four RCTs did not address harms or adverse events. One RCT reported that no participant withdrew from the study due to an adverse event. ${ }^{96}$

\section{Robot-Assisted Gait Training-Cerebral Palsy}

Five RAGT trials, ${ }^{99-104}$ one quasiexperimental study, ${ }^{105}$ and one cohort study ${ }^{106}$ enrolled 220 participants with CP (Table 15). The trial mean age of participants ranged from 8 to 21 years (one trial did not report age but inclusion criteria were between 6 and 14 years ${ }^{103}$ ) with the mean proportion female ranging between 39 and 50 percent. GMFCS functional categories ranged from I-IV, with one study ${ }^{101}$ enrolling only GMFCS level II participants. 
Table 15. Robot-assisted gait training in cerebral palsy

\begin{tabular}{|c|c|c|c|}
\hline $\begin{array}{l}\text { Author, Year } \\
\text { Intervention } \\
\text { Study Design } \\
\text { Study Quality }\end{array}$ & $\begin{array}{l}\text { Intervention and } \\
\text { Comparison }\end{array}$ & Population & Outcomes \\
\hline $\begin{array}{l}\text { Aras, } 2019^{103} \\
\text { Aerobic } \\
\text { exercise } \\
\text { RCT } \\
\text { Fair }\end{array}$ & $\begin{array}{l}\text { A. RAGT, } 20 \\
\text { sessions over } 4 \\
\text { weeks ( } n=10) \\
\text { B. Partial body- } \\
\text { weight supported } \\
\text { treadmill training, } \\
20 \text { sessions over } \\
4 \text { weeks ( } n=10) \\
\text { C. Anti-gravity } \\
\text { treadmill training, } \\
20 \text { sessions over } \\
4 \text { weeks }(n=9)\end{array}$ & $\begin{array}{l}\text { A vs. B } \\
\text { Age: NR } \\
\text { Female: } 40 \% \text { vs. } 40 \% \\
\text { vs. } 33.3 \% \\
\text { GMFCS II: } 90 \% \text { vs. } \\
70 \% \text { vs. } 88.9 \% \\
\text { Hemiplegic: } 30 \% \text { vs. } \\
30 \% \text { vs. } 33.3 \%\end{array}$ & $\begin{array}{l}\text { A vs. B vs. C, mean change (SD): } \\
\text { 6MWT: } 39.6(40.4) \text { vs. } 37.6(20.2) \text { vs. } 48.3(25.1) \\
\text { p>0.05 for all pairwise comparisons } \\
\text { 6MWT (3-month followup): } 45.2(44.4) \text { vs. } 48.6(37.8) \\
\text { vs. } 58.2(22.9), p>0.05 \text { for all pairwise comparisons } \\
\text { GMFM-D: } 3.6(2.5) \text { vs. } 4.6(4.6) \text { vs. } 3.5(2.5), p>0.05 \\
\text { for all pairwise comparisons } \\
\text { GMFM-D (3-month followup): } 3.6(2.5) \text { vs. } 4.6(4.6) \text { vs. } \\
\text { 3.5 (2.5), p>0.05 for all pairwise comparisons } \\
\text { GMFM-E: } 2.4(2.0) \text { vs. } 2.6(1.7) \text { vs. } 3.7(1.9), p>0.05 \\
\text { for all pairwise comparisons } \\
\text { GMFM-E (3-month followup): } 2.6(1.8) \text { vs. } 2.6(1.7) \text { vs. } \\
3.7(1.9), p>0.05 \text { for all pairwise comparisons }\end{array}$ \\
\hline $\begin{array}{l}\text { Klobucka, } \\
2020^{104} \\
\text { Aerobic } \\
\text { exercise } \\
\text { RCT } \\
\text { Poor }\end{array}$ & $\begin{array}{l}\text { A. RAGT, } 20 \\
\text { sessions over } 4 \text { to } \\
6 \text { weeks }(n=21) \\
\text { B. Conventional } \\
\text { therapy }(n=26)\end{array}$ & $\begin{array}{l}\text { A vs. B } \\
\text { Age: } 18.3 \text { vs. } 23.4 \\
\text { Female: } 48 \% \text { vs. } 39 \% \\
\text { GMFCS I: } 4.8 \% \text { vs. } 0 \% \\
\text { GMFCS II: } 14.3 \% \text { vs. } \\
15.4 \% \\
\text { GMFCS III: } 42.9 \% \text { vs. } \\
46.2 \% \\
\text { GMFCS IV: } 38.1 \% \text { vs. } \\
38.5 \% \\
\text { Mechanical wheelchair: } \\
23.8 \% \text { vs. } 53.8 \% \\
\text { Electric wheelchair: } 0 \% \\
\text { vs. } 15.3 \%\end{array}$ & $\begin{array}{l}\text { A vs. B, mean change scores, } p=\text { between groups: } \\
\text { Total GMFM: MD } 9.43,95 \% \mathrm{Cl} 6.989 \text { to } 11.891 \text { vs. MD } \\
0.80,95 \% \mathrm{Cl} 0.154 \text { to } 1.446, p<0.001 \\
\frac{\text { GMFM D: MD } 8.30,95 \% \mathrm{Cl} 4.699 \text { to } 11.901 \text { vs. MD }}{1.09,95 \% \mathrm{Cl}-0.438 \text { to } 2.619, \mathrm{p}<0.001} \\
\text { GMFM E: MD } 9.32,95 \% \mathrm{Cl} 5.329 \text { to } 13.310 \text { vs. MD } \\
0.53,95 \% \mathrm{Cl}-0.208 \text { to } 1.268, \mathrm{p}<0.001\end{array}$ \\
\hline $\begin{array}{l}\text { Peri, } 2017^{105} \\
\text { Aerobic } \\
\text { exercise } \\
\text { Quasiexperimen } \\
\text { tal } \\
\text { Poor }\end{array}$ & $\begin{array}{l}\text { A. RAGT plus } \\
\text { TOP (20 sessions } \\
\text { each over } 10 \\
\text { weeks ( } n=10) \\
\text { B. Personalized } \\
\text { RAGT plus TOP, } \\
20 \text { sessions each } \\
\text { over } 4 \text { weeks } \\
(n=12) \\
\text { C. TOP } 40 \\
\text { sessions over } 10 \\
\text { weeks ( } n=10) \\
\text { D. RAGT } 40 \\
\text { sessions over } 10 \\
\text { weeks ( }=12 \text { ) }\end{array}$ & $\begin{array}{l}\text { A vs. B vs. C vs. D } \\
\text { Age: } 6.8 \text { vs. } 10.8 \text { vs. } \\
9.3 \text { vs. } 8 \\
\text { Female: } 60 \% \text { vs. } 42 \% \\
\text { vs. } 50 \% \text { vs. } 50 \% \\
\text { Spastic bilateral CP: } \\
100 \% \\
\text { Ambulatory: } 100 \% \text { with } \\
\text { or without aid }\end{array}$ & 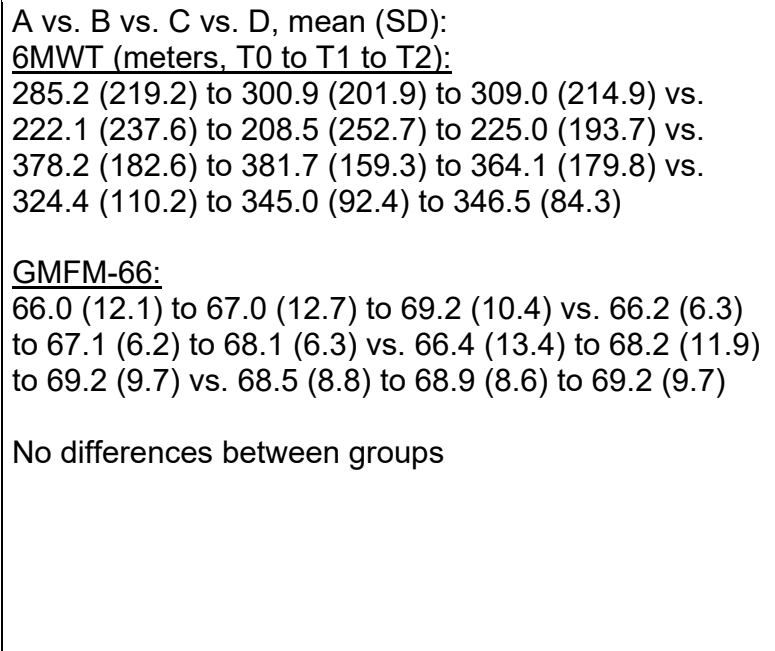 \\
\hline
\end{tabular}




\begin{tabular}{|c|c|c|c|}
\hline $\begin{array}{l}\text { Author, Year } \\
\text { Intervention } \\
\text { Study Design } \\
\text { Study Quality }\end{array}$ & $\begin{array}{l}\text { Intervention and } \\
\text { Comparison }\end{array}$ & Population & Outcomes \\
\hline $\begin{array}{l}\text { Yazici, } 2019^{106} \\
\text { Aerobic } \\
\text { exercise } \\
\text { Cohort } \\
\text { Poor }\end{array}$ & $\begin{array}{l}\text { A. RAGT, } 36 \\
\text { sessions over } 12 \\
\text { weeks ( } n=12 \text { ) } \\
\text { B. Physiotherapy } \\
\text { assumed, } 36 \\
\text { sessions over } 12 \\
\text { weeks assumed } \\
(n=12)\end{array}$ & $\begin{array}{l}\text { A vs. B } \\
\text { Age: } 8.8 \text { vs. } 9.5 \\
\text { Female: } 50 \% \text { vs. } 50 \% \\
\text { GMFCS I or II: } 100 \%\end{array}$ & $\begin{array}{l}\text { A vs. B, mean or median (SD), MD calculated as if all } \\
\text { are means, } p=\text { between groups } \\
\text { 6MWT: } 409.58(49.1) \text { to } 475.17(47.7) \text { vs. } 437.00 \\
\text { (55.0) to } 459.17(53.75) \text {; MD } 43.42,95 \% \mathrm{Cl} 19.64 \text { to } \\
67.21, p<0.001 \\
\text { GMFM-88: } 253.00(8.81) \text { to } 256.17(8.23) \text { vs. } 253.67 \\
\text { (7.70) to } 255.25(7.94), M D 1.59,95 \% \mathrm{Cl}-2.19 \text { to } \\
\text { 5.37, p=0.410 } \\
\text { GMFM-88-D: } 36.08(2.27) \text { to } 36.92(1.73) \text { vs. } 36.75 \\
(2.22) \text { to } 37.42(1.98), M D 0.17,95 \% \mathrm{Cl}-0.79 \text { to } 1.13 \text {, } \\
\text { p=0.729 } \\
\text { GMFM-88-E: } 64.00(6.90) \text { to } 66.25(6.78) \text { vs. } 64.08 \\
(6.43) \text { to } 64.92(6.72), M D 1.14,95 \% \mathrm{Cl}-1.69 \text { to } 4.51 \text {, } \\
\text { p=0.373 } \\
\text { BBS: } 50.08(2.43) \text { to } 52.08(2.68) \text { vs. } 50.25(2.93) \text { to } \\
51.00(3.30), M D 1.25,95 \% \mathrm{Cl}-0.07 \text { to } 2.57, p=0.064\end{array}$ \\
\hline $\begin{array}{l}\text { Wallard, 2017 } \\
\text { Wallard, 2018 } \\
\text { Aerobic } \\
\text { exercise } \\
\text { RCT } \\
\text { Poor }\end{array}$ & $\begin{array}{l}\text { A. RAGT, } 20 \\
\text { sessions over } 4 \\
\text { weeks ( } n=14) \\
\text { B. Usual care, } 20 \\
\text { sessions over } 4 \\
\text { weeks ( } n=16)\end{array}$ & $\begin{array}{l}\text { A vs. B } \\
\text { Age: } 8.3 \text { vs. } 9.6 \\
\text { Female: } 43 \% \text { vs. } 56 \% \\
\text { Ambulatory: } 100 \% \\
\text { Ambulatory without } \\
\text { aids: } 57 \% \text { vs. } 63 \% \\
\text { GMFCS II: } 100 \%\end{array}$ & $\begin{array}{l}\text { A vs. B, mean difference between groups: } \\
\text { GMFM-66-D: } 4.73,95 \% \mathrm{Cl}-6.14 \text { to } 15.60, p=0.39 \\
\text { GMFM-66-E: } 7.54,95 \% \mathrm{Cl}-2.64 \text { to } 17.42, p=0.15\end{array}$ \\
\hline $\begin{array}{l}\text { Wu, 2017b } \\
\text { (effects of) } \\
\text { Aerobic } \\
\text { exercise } \\
\text { RCT } \\
\text { Fair }\end{array}$ & $\begin{array}{l}\text { A. RAGT } \\
\text { (resistive force), } \\
18 \text { sessions over } \\
6 \text { weeks }(n=11) \\
\\
\begin{array}{l}\text { B. Treadmill } \\
\text { training, } 18 \\
\text { sessions over } 6 \\
\text { weeks }(n=12)\end{array}\end{array}$ & $\begin{array}{l}\text { A vs. B } \\
\text { Age: } 11.3 \text { vs. } 10.5 \\
\text { Female: } 45 \% \text { vs. } 33 \% \\
\text { Race: nonwhite: } 54.5 \% \\
\text { vs. } 58 \% \\
\text { GMFCS I: } 9 \% \text { vs. } 17 \% \\
\text { GMFCS II: } 55 \% \text { vs. } \\
25 \% \\
\text { GMFCS III: } 27 \% \text { vs. } \\
42 \% \\
\text { GMFCS IV: } 9 \% \text { vs. } \\
17 \%\end{array}$ & $\begin{array}{l}\text { A vs. } B \text {, mean difference between groups: } \\
\text { GMFM-66 total: }-5.1,95 \% \mathrm{Cl} 13.62 \text { to } 3.42, p=0.24 \\
\text { GMFM-66-D: } 3.6,95 \% \mathrm{Cl}-5.40 \text { to } 12.60, p=0.43 \\
\text { GMFM-66-E: } 0.2,95 \% \mathrm{Cl}-17.79 \text { to } 19.19, p=0.98 \\
\text { PODCl self: } 7.5,95 \% \mathrm{Cl}-10.48 \text { to } 25.48, p=0.41 \\
\text { PODCl parent: } 5.5,95 \% \mathrm{Cl}-8.96 \text { to } 19.96, p=0.46\end{array}$ \\
\hline $\begin{array}{l}\text { Wu, 2017a } a^{100} \\
\text { Aerobic } \\
\text { exercise } \\
\text { RCT } \\
\text { Fair }\end{array}$ & $\begin{array}{l}\text { A. RAGT with } \\
\text { resistance, } 18 \\
\text { sessions over } 6 \\
\text { weeks }(n=12) \\
\text { B. RAGT with } \\
\text { assistance, } 18 \\
\text { sessions over } 6 \\
\text { weeks }(n=11)\end{array}$ & $\begin{array}{l}\text { A vs. B } \\
\text { Age: } 10.6 \text { vs. } 10.8 \\
\text { Female: } 50 \% \text { vs. } 45 \% \\
\text { GMFCS I: } 8 \% \text { vs. } 0 \% \\
\text { GMFCS II: } 42 \% \text { vs. } \\
45 \% \\
\text { GMFCS III: } 42 \% \text { vs. } \\
36 \% \\
\text { GMFCS IV: } 8 \% \text { vs. } \\
18 \%\end{array}$ & $\begin{array}{l}\text { A vs. B, mean difference between groups: } \\
\text { 6MWT: } 49.8,95 \% \mathrm{Cl}-49.85 \text { to } 149.45, p=0.33 \\
\text { GMFM-66 total: } 0.10,95 \% \mathrm{Cl}-7.74 \text { to } 7.94, p=0.98 \\
\text { GMFM-66-D: } 0.10,95 \% \mathrm{Cl}-8.55 \text { to } 8.75, p=0.98 \\
\text { GMFM-66-E: } 0.10,95 \% \mathrm{Cl}-16.32 \text { to } 16.52, p=0.99 \\
\text { PODCl self: }-3.5,95 \% \mathrm{Cl}-20.80,13.80, p=0.69 \\
\text { PODCl parent: } 9.7,95 \% \mathrm{Cl}-6.29 \text { to } 25.69, p=0.23\end{array}$ \\
\hline
\end{tabular}

Abbreviations: 6MWT = 6-Minute Walk Test; $\mathrm{CI}=$ confidence interval; GMFCS = Gross Motor Function Classification System; GMFM-66 = Gross Motor Function Measure 66; GMFM-66-D = Gross Motor Function Measure 66 (standing); GMFM-66-E = Gross Motor Function Measure 66 (walking, running, jumping); GMFM-88 = Gross Motor Function Measure 88; GMFM-88-D $=$ Gross Motor Function Measure 88 (standing); GMFM-88-E = Gross Motor Function Measure 88 (walking, running, jumping); $\mathrm{MD}=$ mean difference; PODCI = Pediatric Outcomes Data Collection Instrument; RAGT = robot-assisted gait training; RCT = randomized controlled trial; $\mathrm{SD}=$ standard deviation; $\mathrm{TOP}=$ task-oriented physical therapy 
The RAGT studies in the CP population enrolled children who were generally limited community ambulators. The control groups received conventional PT, TOP, or treadmill training. There was little difference on function outcomes, regardless of comparator. One RCT ${ }^{100}$ used a specific device that produced a resistive force during treadmill training (as opposed to the Lokomat ${ }^{\mathbb{Q}}$, which gives a guidance force to the patient). Between group differences were not significant, and the experimental group did not have statistically significant improvement in function from baseline, except for the 6MWT. One small $\mathrm{RCT}^{104}$ that enrolled adolescents and adults found improved GMFM- 88 scores with RAGT compared with usual care, but due to the poor-quality rating of this trial and two other studies that assessed GMFM and found no difference ${ }^{101,102,106}$ this evidence was considered insufficient to draw conclusions regarding the benefit of RAGT on function in CP.

Another trial by the same author ${ }^{99}$ assessed RAGT versus treadmill training on function as measured with the GMFM-66 and also did not find a difference between groups. A trial comparing RAGT with partial body-weight supported treadmill training and with anti-gravity treadmill training found no differences between groups on walking or function as assessed with the 6MWT, the GMFM-D and GMFM-E measures postintervention or at 3-month followup. ${ }^{103}$

A poor quality cohort study found improvement on the 6MWT with RAGT compared with physiotherapy, but no difference on the GMFM-88. ${ }^{106}$ Finally, one quasiexperimental study ${ }^{105}$ found no significant difference in function compared with TOP. However, due to the low quality of the included trials, along with small samples sizes, the evidence was considered insufficient to determine whether RAGT is beneficial in children with $\mathrm{CP}$ on function and balance.

None of the five studies included for primary outcomes addressed harms or adverse events.

\section{Robot-Assisted Gait Training-Spinal Cord Injury}

Seven RAGT RCTs enrolled 406 participants with SCI. ${ }^{107,108,110-112,114-116}$ The study mean age of participants ranged from 35 to 50 years and the proportion female ranged from 0 to 38 percent. One study reported racial breakdown and was 47 percent White and 36 percent Hispanic. Number of training sessions ranged from 12 to 60 over the course of 4 to 12 weeks. Most of the study participants were limited ambulators. 
Table 16. Robot-assisted gait training in spinal cord injury

\begin{tabular}{|c|c|c|c|}
\hline $\begin{array}{l}\text { Author, Year } \\
\text { Intervention } \\
\text { Study Design } \\
\text { Study Quality }\end{array}$ & $\begin{array}{l}\text { Intervention and } \\
\text { Comparison }\end{array}$ & Population & Outcomes \\
\hline $\begin{array}{l}\text { Duffell, } 2014^{114} \\
\text { Aerobic exercise } \\
\text { RCT } \\
\text { Poor }\end{array}$ & $\begin{array}{l}\text { A. RAGT, } 12 \text { sessions } \\
\text { over } 4 \text { weeks }(n=23) \\
\text { B. No intervention } \\
(n=29)\end{array}$ & $\begin{array}{l}\text { A vs. B } \\
\text { Age: NR } \\
\text { Female: NR } \\
\text { Incomplete: } 100 \%\end{array}$ & 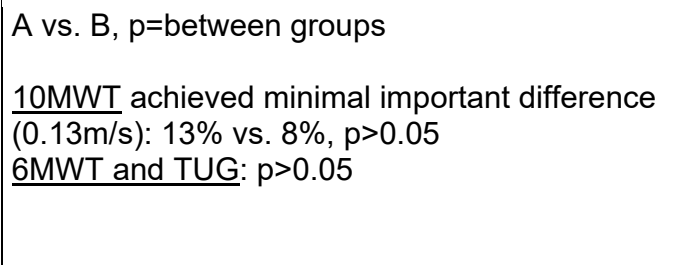 \\
\hline $\begin{array}{l}\text { Esclarin-Ruz, } \\
2014^{107} \\
\text { Aerobic exercise } \\
\text { RCT } \\
\text { Fair }\end{array}$ & $\begin{array}{l}\text { A. RAGT overground, } \\
40 \text { sessions over } 8 \\
\text { weeks }(n=44) \\
\text { B. Overground } \\
\text { therapy without } \\
\text { RAGT, } 40 \text { sessions } \\
\text { over } 8 \text { weeks }(n=44)\end{array}$ & $\begin{array}{l}\text { A vs. B } \\
\text { Age UMN injury: } 43.6 \\
\text { vs. } 44.9 \\
\text { Age LMN injury: } 36.4 \\
\text { vs. } 42.7 \\
\text { Female UMN: } 29 \% \text { vs. } \\
29 \% \\
\text { Female LMN: } 30 \% \text { vs. } \\
29 \%\end{array}$ & $\begin{array}{l}\text { A vs. B, mean (SD): } \\
\text { 10MWT: UMN: } 0.48(0.25) \text { to } 0.54(0.31) \text { vs. } \\
0.36(0.25) \text { to } 0.39(0.31), \text { LMN: } 0.24(0.11) \text { to } \\
0.46(0.25) \text {, vs. } 0.28(0.27) \text { to } 0.45(0.25), \\
\text { p=0.09 } \\
\text { 6MWT: UMN: } 122.3(49.2) \text { to } 187.48(103.78) \\
\text { vs. } 93.3(53.1) \text { to } 119.41(89.25), \text { LMN: } 82.7 \\
(45.5) \text { to } 157.54(89.51) \text { vs. } 94.3(75.1) \text { to } \\
\text { 145.62 (125.15), p=0.047, favors RAGT } \\
\text { FIM/Motor: UMN: } 5(2.7) \text { to } 8.95(2.96) \text { vs. } 4.9 \\
\text { (4.1) to 7.05 (2.62), LMN: } 6(2.9) \text { to } 8.9(2.61) \\
\text { vs. } 5(2.8) \text { to } 8.67(2.65), p=0.09 \\
\text { WISCl-II: UMN: } 5.9(4.5) \text { to } 13.47(5.65) \text { vs. } 4.9 \\
(4.1) \text { to } 11.04(5.09), \text { LMN: } 6(3.2) \text { to } 12.45 \\
(4.17) \text { vs. } 5(3.7) \text { to } 10.8(4.54), p=0.10 \\
\text { LEMS: UMN: } 30(10.4) \text { to } 38.33(10.6) \text { vs. } 27 \\
\text { (10.9) to } 32.28(11.04) \text { vs. LMN: } 21(10.3) \text { to } \\
\text { 27.15 (10.8) vs. } 20(9.9) \text { to } 22.57(10.8), p<0.01 \\
\text { favors RAGT }\end{array}$ \\
\hline $\begin{array}{l}\text { Field-Fote, } \\
2011^{108} \\
\text { Kressler, } 2013^{110} \\
\text { Aerobic exercise } \\
\text { RCT } \\
\text { Fair }\end{array}$ & $\begin{array}{l}\text { A. Treadmill BWS } \\
\text { training with manual } \\
\text { assistance, } 60 \\
\text { sessions over } 12 \\
\text { weeks (n=17) } \\
\text { B. Treadmill BWS } \\
\text { training with electrical } \\
\text { stimulation, } \\
60 \text { sessions over } 12 \\
\text { weeks ( } n=18 \text { ) } \\
\text { C. Overground BWS } \\
\text { training with electrical } \\
\text { stimulation, } 60 \\
\text { sessions over } 12 \\
\text { weeks ( } n=15 \text { ) } \\
\\
\text { D. RAGT treadmill } \\
\text { BWS training with } \\
\text { robot assistance, } 60 \\
\text { sessions over } 12 \\
\text { weeks ( } n=14 \text { ) }\end{array}$ & $\begin{array}{l}\text { A vs. B } \\
\text { Age: } 39.3 \text { vs. } 38.5 \text { vs. } \\
42.2 \text { vs. } 45 \\
\text { Female: } 17.7 \% \text { vs. } \\
22.2 \% \text { vs. } 13.9 \% \text { vs. } \\
18 \% \\
\text { White: } 58.8 \% \text { vs. } \\
44.4 \% \text { vs. } 40.0 \% \text { vs. } \\
42.9 \% \\
\text { Hispanic: } 29.4 \% \text { vs. } \\
38.9 \% \text { vs. } 40 \% \text { vs. } \\
35.7 \% \\
\text { African American: } \\
11.8 \% \text { vs. } 16.7 \% \text { vs. } \\
20 \% \text { vs. } 21.4 \%\end{array}$ & $\begin{array}{l}\text { Mean difference between groups: } \\
\text { 2MWT: } \\
\text { A vs. B: }-3.0,95 \% \mathrm{Cl}-17.91 \text { to } 11.91, p=0.69 \\
\text { A vs. } \mathrm{C}:-13.4,95 \% \mathrm{Cl}-36.82 \text { to } 10.02, p=0.26 \\
\text { A vs. D: }-0.4,95 \% \mathrm{Cl}-12.19 \text { to } 11.39, p=0.95 \\
\text { B vs. } \mathrm{C}:-10.4,95 \% \mathrm{Cl}-34.21 \text { to } 13.41, p=0.39 \\
\text { B vs. D: } 2.6,95 \% \mathrm{Cl}-9.93 \text { to } 15.13, p=0.68 \\
\text { C vs. D: } 13.0,95 \% \mathrm{Cl}-8.99 \text { to } 34.99, p=0.25 \\
\text { Time X Group Interaction } p<0.001 \\
\text { A vs. B vs. C vs. D, mean difference (SD): } \\
\text { 2MWT: } 0.8(7.7) \text { vs. } 3.8(6.3) \text { vs. } 14.2(15.2) \\
\text { vs.1.2 (5.1), favors e-stim } \\
\text { Velocity changed scores averaged across } \\
\text { speeds: Group X Time Interaction } p=0.004, \\
\text { favors e-stim } \\
\text { A vs. B: NR, NS } \\
\text { A vs. C: } 3.66(0.74) \text { vs. } 4.36 \text { (0.74), } p=0.15 \\
\text { A vs. D: NR, NS } \\
\text { B vs. C: NR, NS } \\
\text { B vs. D: } 4.13(0.74) \text { vs. } 3.33(0.76), p=0.009 \\
\text { C vs. D: } 4.36(0.74) \text { vs. } 3.33 \text { (0.76), } p=0.001\end{array}$ \\
\hline
\end{tabular}




\begin{tabular}{|c|c|c|c|}
\hline $\begin{array}{l}\text { Author, Year } \\
\text { Intervention } \\
\text { Study Design } \\
\text { Study Quality }\end{array}$ & $\begin{array}{l}\text { Intervention and } \\
\text { Comparison }\end{array}$ & Population & Outcomes \\
\hline $\begin{array}{l}\text { Kumru, } 2016^{111} \\
\text { Aerobic exercise } \\
\text { RCT } \\
\text { Fair }\end{array}$ & $\begin{array}{l}\text { A. RAGT with rTMS, } \\
20 \text { sessions over } 4 \\
\text { weeks, then RAGT } \\
(n=15) \\
\text { B. RAGT with sham } \\
\text { rTMS, } 20 \text { sessions } \\
\text { over } 4 \text { weeks }(n=16)\end{array}$ & $\begin{array}{l}\text { A vs. B } \\
\text { Age: } 51 \text { vs. } 49 \\
\text { Female: } 33 \% \text { vs. } 13 \% \\
\text { Cervical or thoracic: } \\
100 \% \\
\text { Cervical: } 53 \% \text { vs. } 38 \%\end{array}$ & $\begin{array}{l}\text { A vs. } B, p=\text { between groups: } \\
\text { Change in number able to perform 10MWT } \\
\text { between groups: } 4 \text { vs. } 2, p=0.09 \\
\text { Change in WISCI-II between groups, } p>0.05 \\
\text { Change in UEMS between groups, } p=0.02 \\
\text { Change in } \underline{\text { LEMS }} \text { between groups, } p=0.001\end{array}$ \\
\hline $\begin{array}{l}\text { Midik, } 2020^{116} \\
\text { Aerobic exercise } \\
\text { RCT } \\
\text { Fair }\end{array}$ & $\begin{array}{l}\text { A. RAGT plus } \\
\text { conventional rehab, } \\
25 \text { sessions over } 5 \\
\text { weeks }(n=15) \\
\text { B. Conventional rehab } \\
\text { only, } 25 \text { sessions over } \\
5 \text { weeks }(n=15)\end{array}$ & $\begin{array}{l}\text { A vs. B } \\
\text { Age: } 35.4 \text { vs. } 37.9 \\
\text { Female: } 0 \% \\
\text { AIS C: } 40 \% \text { vs. } 67 \% \\
\text { AIS D: } 60 \% \text { vs. } 33 \%\end{array}$ & $\begin{array}{l}\text { A vs. } B \text {, mean change (SE), } p=\text { between groups: } \\
\text { WISCI: } 3.9(0.8) \text { vs. } 2.5(0.5), p=0.178 \\
\text { SCIM: } 9.9(2.5) \text { vs. } 7.0(1.3), p=0.326 \\
\text { LEMS: } 1.8(0.4) \text { vs. } 0.6(0.2), p=0.061 \\
\text { At } 3 \text { month followup, change from baseline: } \\
\text { WISC: } 4.3(1.0) \text { vs. } 2.5(0.5), p=0.139 \\
\underline{\text { SCIM: }} 16.5(3.2) \text { vs. } 7.6(1.5), p=0.127 \\
\underline{\text { LEMS: }} 2.1(0.5) \text { vs. } 0.6(0.2), p=0.049\end{array}$ \\
\hline $\begin{array}{l}\text { Shin, } 2014^{112} \\
\text { Aerobic exercise } \\
\text { RCT } \\
\text { Fair }\end{array}$ & $\begin{array}{l}\text { A. RAGT, } 12 \text { sessions } \\
\text { over } 4 \text { weeks plus } \\
\text { usual physiotherapy, } \\
28 \text { sessions over } 4 \\
\text { weeks ( } n=27) \\
\\
\text { B. Conventional } \\
\text { overground training, } \\
40 \text { sessions over } 4 \\
\text { weeks }(n=26)\end{array}$ & $\begin{array}{l}\text { A vs. } B \\
\text { Age: } 43 \text { vs. } 48 \\
\text { Female: } 26 \% \text { vs. } 46 \% \\
\text { Cervical: } 52 \% \text { vs. } 62 \% \\
\text { Months since injury: } \\
3.3 \text { vs. } 2.7\end{array}$ & 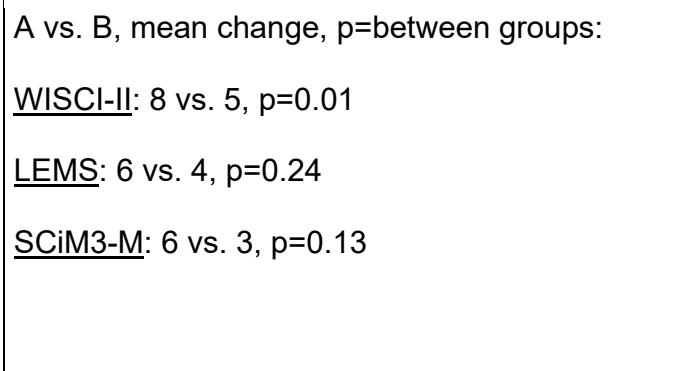 \\
\hline $\begin{array}{l}\text { Yildirim, } 2019^{115} \\
\text { Aerobic exercise } \\
\text { RCT } \\
\text { Fair }\end{array}$ & $\begin{array}{l}\text { A. RAGT, } 16 \text { sessions } \\
\text { over } 8 \text { weeks }+ \\
\text { conventional therapy } \\
(n=44) \\
\text { B. Conventional } \\
\text { therapy }(n=44)\end{array}$ & $\begin{array}{l}\text { A vs. B } \\
\text { Age: } 32 \text { vs. } 37 \\
\text { Female: } 39 \% \text { vs. } 36 \% \\
\text { Tetraplegia: } 20 \% \text { vs. } \\
16 \% \\
\text { ASIA Complete: } 48 \% \\
\text { vs. } 41 \%\end{array}$ & $\begin{array}{l}\text { A vs. B, median (IQR), p-value=between } \\
\text { groups: } \\
\frac{\text { FIM: } 69(31) \text { to } 85(35) \text { vs. } 67(36) \text { to } 77(24),}{p=0.022} \\
\frac{\text { WISCI II: }}{p=0.011}\end{array}$ \\
\hline
\end{tabular}

Abbreviations: 2MWT = 2-Minute Walk Test; 6MWT = 6-Minute Walk Test; 10MWT = 10-Meter Walk Test; ASIA = American Spinal Injury Association Impairment Scale; BWS = body weight supported; CI = confidence interval; FIM = Functional Independence Measure; IQR = interquartile range; LEMS = Lower Extremity Motor Score; LMN = lower motor neuron; NR = not reported; NS = not significant; rTMS = transcranial magnetic stimulation; RAGT = robot-assisted gait training; RCT = randomized controlled trial; $\mathrm{SD}=$ standard deviation; TUG = Timed Up and Go Test; UEMS = Upper Extremity Motor Score; $\mathrm{UMN}=$ upper motor neuron; SCiM3-M = Spinal Cord Independence Measurement III mobility section; WISCI-II = Walking Index for Spinal Cord Injury

RAGT showed positive results in function for participants with SCI in head-to-head studies comparing RAGT with treadmill training or overground walking (SOE: low), but evidence was insufficient for comparisons with usual care due to inconsistent results. ${ }^{107,108,110,112}$ There was also low-strength evidence that RAGT may improve ADL in SCI based on two RCTs. ${ }^{107,115}$ There was insufficient evidence to determine the benefit of transcranial magnetic stimulation (rTMS) in conjunction with RAGT, but the trial was small. ${ }^{111}$

An RCT of overground therapy with and without RAGT stratified results according to upper motor neuron (UMN) versus lower motor neuron (LMN) SCI participants. ${ }^{107}$ Both UMN and LMN injured individuals improved significantly more on the 6MWT and Lower Extremity 
Motor Score (LEMS) with RAGT. A study that compared RAGT with treadmill training, overground training, and treadmill plus $\mathrm{FES}^{108}$ did not find RAGT significantly different to the other groups in function outcomes.

Six studies did not address harms or adverse events. One RCT reported that three individuals left the study ( 2 in the RAGT plus rTMS group due to repeated urinary tract infection and severe spasticity; 1 in the sham rTMS group due to severe spasticity). ${ }^{111}$ Other adverse events experienced in the rTMS group were twitching of facial muscles and headache.

One additional trial of reports the effects of RAGT on bowel dysfunction and is discussed in KQ2c. ${ }^{113}$

\section{Treadmill}

A motorized treadmill is a common means for aerobic or endurance training. Its speed and elevation can be manipulated to provide a wide range of training intensities. Handrails are used for support and stability, and for those unable to walk more than short distances, it can be modified to add a harness that provides partial body weight support. Specialized treadmills accommodate upper body training by self-propelling a wheelchair on a treadmill.

\section{Key Points}

- Among ambulatory individuals with MS, there was evidence that treadmill training may improve balance and function, including walking (SOE: low).

- When compared with usual care, there was low-strength evidence that treadmill training may improve function in CP (SOE: low).

- There was low-strength evidence of no clear benefit of treadmill training on walking in $\mathrm{CP}$ when compared with overground walking (SOE: low).

- Among study participants with SCI, there was low-strength evidence of no clear benefit of treadmill training compared with structured PT or aerobic plus strength training on function, including walking (SOE: low).

\section{Detailed Synthesis}

Seventeen RCTs, ${ }^{117-130,133-138}$ and two quasiexperimental, nonrandomized studies ${ }^{131,132}$ $(\mathrm{n}=583)$ using treadmill training met inclusion criteria.

These included six RCTs and one quasiexperimental trial of treadmill training versus usual care in $\mathrm{CP},{ }^{121,126,129,132} \mathrm{MS},{ }^{117,120}$ and SCI$;{ }^{133}$ three comparisons of treadmill versus overground walking in CP, ${ }^{123,127,128}$ two RCTs where treadmill training was compared with strength training in $\mathrm{CP}^{125}$ and $\mathrm{MS} ;{ }^{118}$ and one treadmill training with direct-current stimulation of the motor cortex compared with treadmill training with sham stimulation study in participants with CP. ${ }^{130}$

An additional five RCTs and one quasiexperimental trial had unique comparisons, where treadmill training was compared with different alternative training methods or unique modifications of standard treadmill walking ( $\mathrm{SCI},{ }^{133,138} \mathrm{CP},{ }^{122,131,134-137} \mathrm{MS}^{119}$ ). Thus, few studies used similar treadmill training and comparator/control training for the same condition. One trial $^{128}$ met criteria for good quality and the remainder were rated fair quality.

The most frequently reported outcomes were related to walking parameters (e.g., 6MWT, 10MWT), balance (e.g., BBS), combined gait and balance measures such as TUG, and standardized functional constructs combining several measures, such as the GMFM. The GMFM was used both to classify $\mathrm{CP}$ participants and as an outcome variable. 


\section{Treadmill-Multiple Sclerosis}

Four trials ${ }^{117-120}$ enrolled individuals $(\mathrm{n}=119)$ with MS (Table 17). The mean age of participants across trials ranged from 33 to 53 years with a range of 40 to 100 percent female. Each trial enrolled ambulatory individuals, and participants needed to be able to walk a minimum of 6 meters ${ }^{117}$ or 10 meters, ${ }^{118,119}$ with or without assistance.

Table 17. Treadmill exercise in multiple sclerosis

\begin{tabular}{|c|c|c|c|}
\hline $\begin{array}{l}\text { Author, Year } \\
\text { Intervention } \\
\text { Study Design } \\
\text { Study Quality }\end{array}$ & $\begin{array}{l}\text { Intervention } \\
\text { and Comparison }\end{array}$ & Population & Results \\
\hline $\begin{array}{l}\text { Ahmadi, } 2013^{120} \\
\text { Aerobic } \\
\text { exercise } \\
\text { RCT } \\
\text { Fair }\end{array}$ & $\begin{array}{l}\text { A. Treadmill, } 24 \\
\text { sessions over } 8 \text { weeks } \\
(n=10) \\
\begin{array}{l}\text { B. Waitlist control } \\
(n=10)\end{array}\end{array}$ & $\begin{array}{l}\text { A vs. B } \\
\text { Age: } 37 \text { vs. } 37 \\
\text { Female: } 100 \% \\
\text { EDSS: } 2.40 \text { vs. } 2.25\end{array}$ & $\begin{array}{l}\text { A vs. } B \text {, mean (SD), p-value between } \\
\text { groups: } \\
\text { 10MWT: } 8.68(1.93) \text { to } 7.07(1.03) \text { vs. } \\
9.16(1.88) \text { to } 9.47(1.92), p=0.001 \\
\text { 2MWT: } 120.40(20.29) \text { to } 139.90(20.78) \\
\text { vs. } 121.50(27.73) \text { to } 119.05(27.12), \\
\text { p=0.001 } \\
\text { BBS: } 46.20(6.32) \text { to } 53.80(2.34) \text { vs. } \\
44.50(9.43) \text { to } 41.70(8.48), p=0.001\end{array}$ \\
\hline $\begin{array}{l}\text { Gervasoni, } \\
2014^{117} \\
\text { Aerobic } \\
\text { exercise } \\
\text { RCT } \\
\text { Fair }\end{array}$ & $\begin{array}{l}\text { A. } 30 \text { minutes } \\
\text { conventional therapy + } \\
15 \text { minutes treadmill } \\
\text { training, } 12 \text { sessions } \\
\text { over } 2 \text { weeks }(n=15) \\
\text { B. } 45 \text { minutes } \\
\text { conventional therapy, } \\
12 \text { sessions over } 2 \\
\text { weeks }(n=15)\end{array}$ & $\begin{array}{l}\text { A vs. B } \\
\text { Age: } 49.6 \text { vs. } 45.7 \\
\text { Female: } 40 \% \\
\text { Able to walk } 6 \text { meters } \\
\text { with or without assist } \\
\text { device } \\
\text { RRMS: } 47.6 \% \\
\text { PPMS: } 19.0 \% \\
\text { SPMS: } 33.3 \% \\
\text { EDSS (median): } 5.5\end{array}$ & $\begin{array}{l}\text { A vs. } B, \text { mean change, } p=\text { between } \\
\text { groups } \\
\text { DGI: } 2.16 \text { vs. } 2.07, p=0.51 \\
\text { BBS: } 4.01 \text { vs. } 3.15, p=0.33\end{array}$ \\
\hline $\begin{array}{l}\text { Jonsdottir, } \\
2018^{118} \\
\text { Aerobic } \\
\text { exercise } \\
\text { RCT } \\
\text { Fair }\end{array}$ & $\begin{array}{l}\text { A. Treadmill walking, } 20 \\
\text { sessions over } 4 \text { weeks } \\
(n=26) \\
\text { B. Strength training, 16- } \\
20 \text { sessions over } 4 \\
\text { weeks }(n=12)\end{array}$ & $\begin{array}{l}\text { A vs. B } \\
\text { Age: } 51.4 \text { vs. } 56.7 \\
\text { Female: } 48 \% \text { vs. } 29 \% \\
\text { EDSS: } 5.5 \text { vs. } 5.6 \\
\text { RRMS: } 85 \% \text { vs. } 58 \% \\
\text { PPMS: } 8 \% \text { vs. } 17 \% \\
\text { SPMS: } 8 \% \text { vs. } 25 \%\end{array}$ & $\begin{array}{l}\text { A vs. } B, \text { mean difference between } \\
\text { groups: } \\
\text { TUG: }-2.83,95 \% \mathrm{Cl}-4.7 \text { to }-0.9, \\
\text { p=0.009 } \\
\text { DGI: } 0.2,95 \% \mathrm{Cl}-1.95 \text { to } 2.27, p=0.87 \\
\text { 2MWT: } 28.3,95 \% \mathrm{Cl} 13.04 \text { to } 43.60 \text {, } \\
\text { p<0.001 } \\
\text { SF-12 mental: }-3.0,95 \% \mathrm{Cl}-9.43 \text { to } \\
3.38, p=0.34 \\
\text { SF-12 physical: } 1.8,95 \% \mathrm{Cl}-2.08 \text { to } \\
\text { B.59, } p=0.36 \\
\text { BBS: } 1.1,95 \% \mathrm{Cl}-1.4 \text { to } 3.7, p=0.39\end{array}$ \\
\hline $\begin{array}{l}\text { Samaei, } 2016^{119} \\
\text { Aerobic } \\
\text { exercise } \\
\text { RCT } \\
\text { Fair }\end{array}$ & $\begin{array}{l}\text { A. Downhill treadmill } \\
\text { training, } 12 \text { sessions } \\
\text { over } 4 \text { weeks }(n=16) \\
\text { B. Uphill treadmill } \\
\text { training, } 12 \text { sessions } \\
\text { over } 4 \text { weeks }(n=15)\end{array}$ & $\begin{array}{l}\text { A vs. B } \\
\text { Age: } 33.9 \text { vs. } 32.1 \\
\text { Female: } 82 \% \text { vs. } 82 \% \\
\text { Ambulatory: } 100 \%\end{array}$ & $\begin{array}{l}\text { A vs. B, mean change between groups: } \\
\text { 25FWT: } 8.7(2.4) \text { to } 6.1(1.8) \text { vs. } 7.9(1.1) \\
\text { to } 7.0(1.6), p=0.001 \\
\text { 2MWT: } 120.01(23.6) \text { to } 160.1(35.7) \text { vs. } \\
\text { 132.6 }(32.3) \text { to } 147.5(29.8), p<0.001 \\
\text { TUG: } 9.8(1.7) \text { to } 7.5(1.8) \text { vs. } 9.4(2.3) \text { to } \\
8.9(0.9), p=0.041 \\
\text { GNDS: } 35.4(9.1) \text { to } 21.8(5.3) \text { vs. } 32.1 \\
\text { (8.6) to } 27.5(6.1), p=0.012 \\
\text { Modified Riverman Mobility Index: } 10.6 \\
\text { (3.2) to } 14.3(2.7) \text { vs.10.5 (2.3) to } 11.9 \\
\text { (2.1), p=0.005 }\end{array}$ \\
\hline
\end{tabular}

Abbreviations: 2MWT = 2-Minute Walk Test; 10MWT = 10-Meter Walk Test; 25FWT = 25-Foot Walk Test; BBS = Berg Balance Scale; CI = confidence interval; DGI = Dynamic Gait Index; EDSS = Expanded Disability Status Scale; GNDS = Guy's Neurological Disability Scale; PPMS = primary progressive multiple sclerosis; RCT = randomized controlled trial; RRMS = relapsing-remitting multiple sclerosis; SF-12 = Short Form (12) Health Survey; SPMS = secondary progressive multiple sclerosis; $\mathrm{TUG}=$ Timed Up and Go Test 
The single trial using 2 weeks of training ${ }^{117}$ did not find a benefit of treadmill walking. However, 2 weeks is less than the typical duration of exercise training studies. Combining with the longer duration study of 8 weeks ${ }^{118,120}$ provided low-strength evidence for improved function, including walking with treadmill training. Two trials also provided low-strength evidence for improved balance versus usual care or waitlist control. ${ }^{171,120}$ There was insufficient evidence to determine whether downhill treadmill training is superior to uphill training, ${ }^{119}$ or whether treadmill training is better than strength training, due to small sample sizes and only a single study evaluating each comparison. ${ }^{118}$

Each trial's treadmill sessions were closely supervised and the treadmill speeds adjusted to maintain appropriate intensities and safety while participants trained. Three RCTs did not address harms or adverse events; the fourth RCT reported that participants experienced only muscle and general fatigue that resolved in a few hours after exercising. ${ }^{118}$

\section{Treadmill-Cerebral Palsy}

The greatest number of treadmill trials were with children and adolescents with CP. Twelve trials $^{121-125,127,128,130-132}$ enrolled children $(\mathrm{n}=127)$ or adolescents $(\mathrm{n}=102)$ with CP (Table 18). Two RCTs ${ }^{126,129}$ assessed 146 adults with CP (mean age 27 years and $52 \%$ to $56 \%$ male) (Table $18)$.

The trials with adults, ${ }_{126,129}$ three of the four trials with adolescents, ${ }^{121,131,132}$ and three of the six trials with children ${ }^{123,124,130}$ enrolled ambulatory individuals and assessed treadmill walking without body weight support. Other trials ${ }^{122,125,127,128}$ used partial body weight support as an intervention component.

Table 18. Treadmill exercise in cerebral palsy

\begin{tabular}{|c|c|c|c|}
\hline $\begin{array}{l}\text { Author, Year } \\
\text { Intervention } \\
\text { Study Design } \\
\text { Study Quality }\end{array}$ & $\begin{array}{l}\text { Intervention } \\
\text { and Comparison }\end{array}$ & Population & Results \\
\hline $\begin{array}{l}\text { Aviram, } 2017 \\
131 \\
\text { Aerobic exercise } \\
\text { Quasiexperimental } \\
\text { Fair }\end{array}$ & $\begin{array}{l}\text { A. Treadmill walking, } \\
30 \text { sessions over } 3 \\
\text { months }(n=43) \\
\text { B. Group resistance } \\
\text { training, } 30 \text { sessions } \\
\text { over } 3 \text { months }(n=52)\end{array}$ & $\begin{array}{l}\text { A vs. B } \\
\text { Age: } 43 \text { vs. } 52 \\
\text { Female: } 21 \% \text { vs. } 48 \% \\
\text { GMFCS II: } 72 \% \text { vs. } 75 \% \\
\text { GMFCS III: } 28 \% \text { vs. } 25 \%\end{array}$ & $\begin{array}{l}\text { A vs. B, mean (SE) change from baseline and } 6 \\
\text { months postintervention; } p \text {-values are between } \\
\text { groups } \\
\text { 6MWT: } 20.9(4.0) \text { vs. } 27.9(6.7), p=0.31 \\
\text { TUG: }-2.82(0.51) \text { vs. } 3.52(0.60), p=0.014 \\
\text { GMFM-66: } 1.98(0.40) \text { vs. } 3.10(0.44), p=0.001 \\
\text { GMFM-66-D: } 5.53(1.61) \text { vs. } 8.36(1.24), p=0.013 \\
\text { GMFM-66-E: } 4.80(1.33) \text { vs. } 7.21(0.96), p=0.81 \\
\text { 10MWT-self-paced: } 0.272(0.045) \text { vs. } 0.276 \\
\text { (0.049), p=0.41 } \\
\text { 10MWT-fast: } 0.387(0.070) \text { vs. } 0.374(0.069),\end{array}$ \\
\hline $\begin{array}{l}\text { Bahrami, } 2019^{129} \\
\text { Aerobic exercise } \\
\text { RCT } \\
\text { Fair }\end{array}$ & 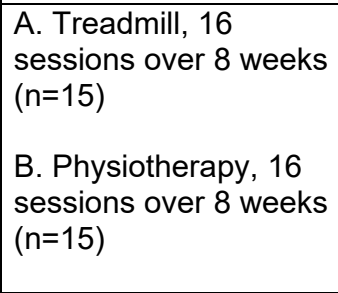 & $\begin{array}{l}\text { A vs. B } \\
\text { Age: } 30 \text { vs. } 25 \\
\text { Female: } 47 \% \text { vs. } 40 \% \\
\text { GMFCS I: } 47 \% \text { vs. } 53 \% \\
\text { GMFCS II: } 13 \% \text { vs. } 13 \% \\
\text { GMFCS III: } 40 \% \text { vs. } 33 \%\end{array}$ & $\begin{array}{l}\text { A vs. B, mean (SD); percentage change score, } \\
p=\text { between groups } \\
\text { 10MWT: } 22.46 \% \text { change vs. } 1.28 \% \text { change, } \\
\text { p<0.05 } \\
\text { 6MWT: } 23.68 \% \text { change vs. } 16.54 \% \text { change, } \\
\text { W }>0.05 \\
8.94 \% \text { change, } p>0.05\end{array}$ \\
\hline
\end{tabular}




\begin{tabular}{|c|c|c|c|}
\hline $\begin{array}{l}\text { Author, Year } \\
\text { Intervention } \\
\text { Study Design } \\
\text { Study Quality }\end{array}$ & $\begin{array}{l}\text { Intervention } \\
\text { and Comparison }\end{array}$ & Population & Results \\
\hline $\begin{array}{l}\text { Chrysagis, } 2012^{121} \\
\text { Aerobic exercise } \\
\text { RCT } \\
\text { Fair }\end{array}$ & $\begin{array}{l}\text { A. Treadmill training, } \\
36 \text { sessions over } 12 \\
\text { weeks }(n=11) \\
\text { B. Conventional PT, } 36 \\
\text { sessions over } 12 \\
\text { weeks }(n=11)\end{array}$ & $\begin{array}{l}\text { A vs. B } \\
\text { Age: } 15.90 \text { vs. } 16.09 \\
\text { Female: } 45 \% \text { vs. } 36 \% \\
\text { Ambulatory: } 100 \% \\
\text { GMFM-D+E: } 67.81 \text { vs. } \\
64.45\end{array}$ & 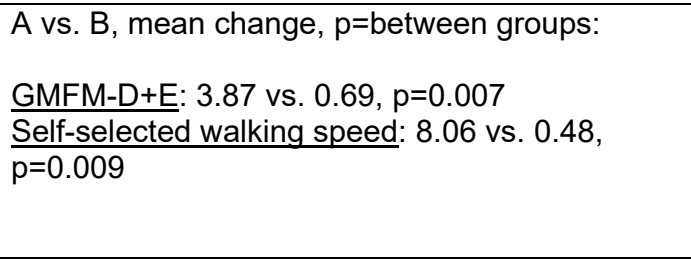 \\
\hline $\begin{array}{l}\text { Duarte Nde, } \\
2014^{130} \\
\text { Aerobic exercise } \\
\text { RCT } \\
\text { Fair } \\
\text { May share } \\
\text { participants with } \\
\text { Grecco, } 2014\end{array}$ & $\begin{array}{l}\text { A. Treadmill + tDCS, } \\
10 \text { sessions over } 2 \\
\text { weeks }(n=12) \\
\\
\text { B. Treadmill + sham } \\
\text { tDCS, } 10 \text { sessions } \\
\text { over } 2 \text { weeks, }(n=12)\end{array}$ & $\begin{array}{l}\text { A vs. B } \\
\text { Age: } 8 \text { vs. } 8 \\
\text { Female: NR } \\
\text { GMFCS I: } 25 \% \text { vs. } 17 \% \\
\text { GMFCS II: } 50 \% \text { vs. } 57 \% \\
\text { GMFCS III: } 25 \% \text { vs. } 25 \%\end{array}$ & $\begin{array}{l}\text { A vs. B, mean (SD), } p \text {-value=between groups: } \\
\text { PBS: } 40.5(9.4) \text { to } 45.3(7.9) \text { vs. } 39.1(9.8) \text { to } 39.7 \\
\text { (8.4); MD } 4.2,95 \% \mathrm{Cl}-2.88 \text { to } 11.28, p=0.245 \\
\text { PEDI self-care: } 46.1(10) \text { to } 48.0(9.5) \text { vs. } 45.0 \\
\text { (9.2) to } 45.5(9.3) ; M D 1.4,95 \% \mathrm{Cl}-6.21 \text { to } 9.01 \text {, } \\
\text { p=0.718 } \\
\text { PEDI mobility: } 38.0(8.5) \text { to } 41.7(7.4) \text { vs. } 38.3 \\
\text { (7.4) to } 39.5(7.6) ; M D 2.5,95 \% \mathrm{Cl}-3.71 \text { to } 8.71 \text {, } \\
p=0.430\end{array}$ \\
\hline $\begin{array}{l}\text { Emara, } 2016^{122} \\
\text { Aerobic exercise } \\
\text { RCT } \\
\text { Fair }\end{array}$ & $\begin{array}{l}\text { A. Treadmill walking, } \\
36 \text { sessions over } 12 \\
\text { weeks }(n=10) \\
\text { B. Overground walking } \\
\text { with spider cage, } 36 \\
\text { sessions over } 12 \\
\text { weeks }(n=10)\end{array}$ & $\begin{array}{l}\text { A vs. B } \\
\text { Age: } 6.6 \text { vs. } 6.9 \\
\text { Female: } 70 \% \text { vs. } 60 \% \\
\text { Spastic diplegic CP: } 100 \% \\
\text { GMFCS III: } 100 \%\end{array}$ & $\begin{array}{l}\text { A vs. B, mean difference between groups: } \\
\text { 10MWT: } 0.4(0.04) \text { to } 0.5(0.04) \text { vs. } 0.4(0.03) \text { to } \\
0.6(0.04), p=0.12 \\
\text { 5XSit-to-Stand: } 21.5(1.3) \text { to } 18.9(1.0) \text { vs. } 21.7 \\
\text { (1.5) to } 17.7(0.8), p=0.26 \\
\text { GMFM-88-D: } 12.5(1.6) \text { to } 15.8(1.5) \text { vs. } 12.0 \\
\text { (0.7) to } 19.2(2.1), p=0.02 \\
\text { GMFM-88-E: } 10.9(1.3) \text { to } 14.8(1.5) \text { vs. } 10.4 \\
\text { (0.8) to } 17.2(2.1), p=0.05\end{array}$ \\
\hline $\begin{array}{l}\text { Grecco, } 2014^{123} \\
\text { Aerobic exercise } \\
\text { RCT } \\
\text { Fair } \\
\text { May share } \\
\text { participants with } \\
\text { Duarte Nde, } \\
2014^{130}\end{array}$ & 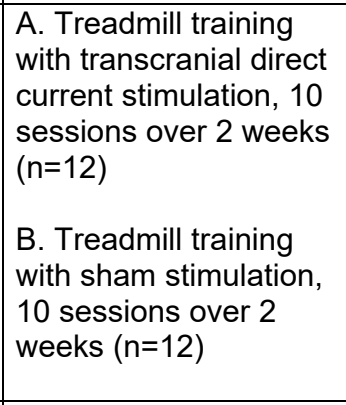 & $\begin{array}{l}\text { A vs. B } \\
\text { Age: } 7.8 \text { vs. } 8.0 \\
\text { Female: } 75 \% \text { vs. } 67 \% \\
\text { GMFCS II: } 67 \% \text { vs. } 67 \% \\
\text { GMFCS III: } 33 \% \text { vs. } 33 \%\end{array}$ & $\begin{array}{l}\text { A vs. B, mean difference between groups: } \\
\text { 6MWT: MD 1996.6 (133.1 to } 266.0) \text { vs. } 111.8 \\
\text { (27.1 to 196.4), p<0.05 } \\
\text { GMFM-88-D: MD } 11.5 \text { (-1.6 to } 24.7) \text { vs. MD } 3.7 \\
\text { (-2.3 to 9.8), p>0.05 } \\
\text { GMFM-88-E: MD } 0.8 \text { (-1.5 to } 3.2) \text { vs. MD } 1.0 \text { (- } \\
0.1 \text { to } 2.1), p>0.05\end{array}$ \\
\hline $\begin{array}{l}\text { Grecco } 2013^{124} \\
\text { Aerobic exercise } \\
\text { RCT } \\
\text { Fair }\end{array}$ & $\begin{array}{l}\text { A. Treadmill walking, } \\
14 \text { sessions over } 7 \\
\text { weeks }(n=16) \\
\text { B. Overground } \\
\text { walking, } 14 \text { sessions } \\
\text { over } 7 \text { weeks }(n=17)\end{array}$ & $\begin{array}{l}\text { A vs. B } \\
\text { Age: } 6.8 \text { vs. } 6.0 \\
\text { Female: } 63 \% \text { vs. } 47 \% \\
\text { GMFCS I: } 31 \% \text { vs. } 47 \% \\
\text { GMFCS II: } 50 \% \text { vs. } 41 \% \\
\text { GMFCS III: } 19 \% \text { vs. } 12 \%\end{array}$ & $\begin{array}{l}\text { A vs. } B \text {, mean change, } p=\text { between groups: } \\
\text { 6MWT: } 149.7 \text { vs. } 44.8, p<0.001 \\
\text { TUG: }-6.4 \text { vs. }-2.0, p=0.004 \\
\text { GMFM-88-D: } 23.9 \text { vs. } 8.1, p<0.001 \\
\text { GMFM-88-E: } 20.1 \text { vs. } 8.2, p<0.001 \\
\text { PEDI: } 11.0 \text { vs. } 4.0, p=0.035 \\
\text { BBS: } 11.8 \text { vs. } 3.3, p<0.001\end{array}$ \\
\hline $\begin{array}{l}\text { Johnston, } 2011^{125} \\
\text { Aerobic exercise } \\
\text { RCT } \\
\text { Fair }\end{array}$ & $\begin{array}{l}\text { A. Partial BWS } \\
\text { treadmill training with } \\
20 \text { sessions over } 2 \\
\text { weeks, then } 50 \\
\text { sessions at home over } \\
10 \text { weeks ( } n=14) \\
\text { B. Individualized } \\
\text { strength-based PT, } 20 \\
\text { sessions over } 2 \\
\text { weeks, then } 50 \\
\text { session at home over } \\
10 \text { weeks }(n=12) \\
\end{array}$ & $\begin{array}{l}\text { A vs. B } \\
\text { Age: } 9.6 \text { vs. } 9.5 \\
\text { Female: } 50 \% \text { vs. } 42 \% \\
\text { GMFCS II: } 7 \% \text { vs. } 8 \% \\
\text { GMFCS III: } 64 \% \text { vs. } 50 \% \\
\text { GMFCS IV: } 29 \% \text { vs. } 42 \% \\
\text { Diplegic CP: } 57 \% \text { vs. } 33 \% \\
\text { Triplegic CP: } 0 \% \text { vs. } 17 \% \\
\text { Quadriplegic CP: } 43 \% \text { vs. } \\
50 \%\end{array}$ & 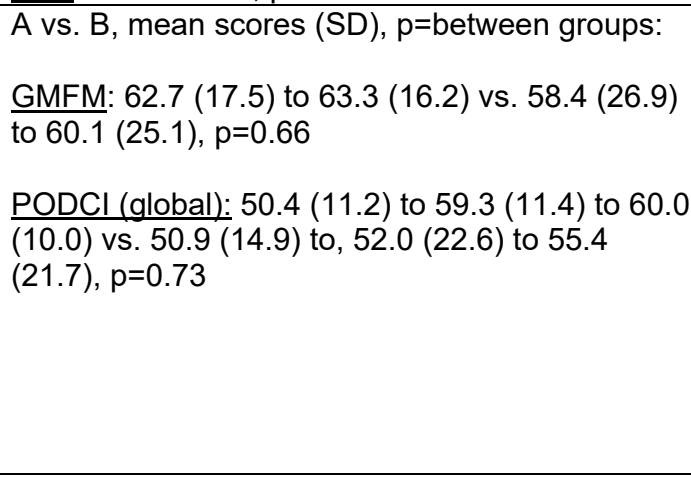 \\
\hline
\end{tabular}




\begin{tabular}{|c|c|c|c|}
\hline $\begin{array}{l}\text { Author, Year } \\
\text { Intervention } \\
\text { Study Design } \\
\text { Study Quality }\end{array}$ & $\begin{array}{l}\text { Intervention } \\
\text { and Comparison }\end{array}$ & Population & Results \\
\hline $\begin{array}{l}\text { Kim, } 2015^{126} \\
\text { Aerobic exercise } \\
\text { RCT } \\
\text { Fair }\end{array}$ & $\begin{array}{l}\text { A. Treadmill walking, } \\
20 \text { sessions over } 1-2 \\
\text { months plus PT }(n=14) \\
\text { B. PT }(n=7)\end{array}$ & $\begin{array}{l}\text { A vs. B } \\
\text { Age: } 28.6 \text { vs. } 24.4 \\
\text { Female: } 50 \% \text { vs. } 43 \% \\
\text { Ambulatory without gait } \\
\text { aid: } 100 \%\end{array}$ & $\begin{array}{l}\text { A vs. } B, \text { mean difference between groups: } \\
\text { 6MWT on treadmill: } 5.71,95 \% \mathrm{Cl}-53.22 \text { to } \\
64.64, p=0.85 \\
\frac{6 \mathrm{MWT} \text { on overground walking: } 24.07,95 \% \mathrm{Cl}}{-46.80 \text { to } 94.94, p=0.51}\end{array}$ \\
\hline $\begin{array}{l}\text { Nsenga Leunkeu, } \\
2012^{132} \\
\text { Aerobic exercise } \\
\text { Quasiexperimental } \\
\text { Fair }\end{array}$ & $\begin{array}{l}\text { A. Treadmill walking, } \\
24 \text { sessions over } 8 \\
\text { weeks, }(n=12) \\
\text { B. No training, }(n=12)\end{array}$ & $\begin{array}{l}\text { A vs. B } \\
\text { Age: } 14.2 \text { vs. } 14.2 \\
\text { Female: } 50 \% \text { vs. } 50 \% \\
\text { Hemiplegic CP: } 83 \% \text { vs. } \\
83 \% \\
\text { GMFCS I: } 67 \% \text { vs. } 67 \% \\
\text { GMFCS II: } 33 \% \text { vs. } 33 \%\end{array}$ & $\begin{array}{l}\text { A vs. B, mean change: } \\
\text { (estimates from bar graph) } \\
\text { 6MWT: } 480 \text { to } 601 \text { vs. } 450 \text { to } 450 \text {, no difference } \\
\text { in baseline values, significant difference in } \\
\text { postintervention values favoring treatment }\end{array}$ \\
\hline $\begin{array}{l}\text { Swe, } 2015^{128} \\
\text { Aerobic exercise } \\
\text { RCT } \\
\text { Good }\end{array}$ & $\begin{array}{l}\text { A. Partial BWS } \\
\text { treadmill walking, } 16 \\
\text { sessions over } 8 \text { weeks } \\
(n=15) \\
\text { B. Overground } \\
\text { walking, } 16 \text { sessions } \\
\text { over } 8 \text { weeks }(n=15)\end{array}$ & $\begin{array}{l}\text { A vs. B } \\
\text { Age: } 13.03 \text { vs. } 13.37 \\
\text { Female: } 33 \% \text { vs. } 33 \% \\
\text { GMFCS II: } 67 \% \text { vs. } 53 \% \\
\text { GMFCS III: } 33 \% \text { vs. } 47 \% \\
\text { 6MWT: } 233.33 \text { vs. } 205.00\end{array}$ & $\begin{array}{l}\text { A vs. B, mean difference between groups: } \\
\text { 6MWT: }-17.00,95 \% \mathrm{Cl}-89.77 \text { to } 55.77, p=0.65 \\
\text { 10MWT: }-0.013,95 \% \mathrm{Cl}-0.23,0.21, p=0.91 \\
\frac{\text { GMFM-88-D: }}{p=0.67}-2.94,95 \% \mathrm{Cl}-16.42 \text { to } 10.64 \text {, } \\
\frac{\text { GMFM-88-E: }}{p=0.75}-2.8,95 \% \mathrm{Cl}-20.02 \text { to } 14.42,\end{array}$ \\
\hline $\begin{array}{l}\text { Willoughby, } \\
2010^{127} \\
\text { Aerobic exercise } \\
\text { RCT } \\
\text { Fair }\end{array}$ & $\begin{array}{l}\text { A. Partial BWS } \\
\text { treadmill training, } 18 \\
\text { sessions over } 9 \text { weeks } \\
(n=12) \\
\text { B. Overground } \\
\text { walking, } 18 \text { sessions } \\
\text { over } 9 \text { weeks }(n=14)\end{array}$ & $\begin{array}{l}\text { A vs. B } \\
\text { Age: } 10.35 \text { vs. } 11.24 \\
\text { Female: } 50 \% \text { vs. } 36 \% \\
\text { GMFCS III: } 42 \% \text { vs. } 21 \% \\
\text { GMFCS IV: } 58 \% \text { vs. } 79 \%\end{array}$ & $\begin{array}{l}\text { A vs. } B \text {, mean (SD), } p=\text { between groups: } \\
\frac{10 M W T:}{118.36} \text { (89.89) to } 135.82 \text { (95.65), } p=0.097\end{array}$ \\
\hline
\end{tabular}

Abbreviations: $5 \mathrm{x}$ = five times; 6MWT = 6-Minute Walk Test; 10MWT = 10-Meter Walk Test; BBS = Berg Balance Scale ; $\mathrm{BWS}=$ body weight supported; $\mathrm{CI}=$ confidence interval; $\mathrm{CP}=$ cerebral palsy; GMFCS = Gross Motor Function Classification System; GMFM = Gross Motor Function Measure; GMFM-66-D = Gross Motor Function Measure 66 (standing); GMFM-66-E $=$ Gross Motor Function Measure 66 (walking, running, jumping); GMFM-88-D = Gross Motor Function Measure 88 (standing); GMFM-88-E = Gross Motor Function Measure 88 (walking, running, jumping); $\mathrm{MD}=$ mean difference; NR = not reported; PBS $=$ Pediatric Balance Scale; PEDI $=$ Pediatric Evaluation Disability Inventory; PODCI $=$ Pediatric Outcomes Data Collection Instrument; $\mathrm{PT}=$ physical therapy; $\mathrm{RCT}=$ randomized controlled trial; $\mathrm{SD}=$ standard deviation; $\mathrm{SE}=$ standard error; $\mathrm{tDCS}=$ transcranial direct current stimulation; TUG $=$ Timed Up and Go Test; WHOQOL = World Health Organization Quality of Life scale

These trials provided low-strength evidence of benefit on function with treadmill training in $\mathrm{CP}$ compared with usual care, and low-strength evidence of no clear benefit of treadmill training when compared with overground walking. When trials enrolling participants of various ages are combined, ${ }^{122,124,127,128}$ the evidence does not suggest a benefit of treadmill training when compared with various comparator interventions.

When treadmill training combined with transcranial direct current stimulation to the child's motor cortex was compared with treadmill training with sham stimulation, performance on the 6MWT was improved significantly, although GMFM-88-D and GMFM-88-E scores were not. ${ }^{123}$ In another publication with the same trial registry number, there was no association between treadmill training with or without transcranial stimulation on balance or ADL. ${ }^{130}$

Two trials in children with CP evaluated ADL on the Pediatric Outcomes Data Collection Instrument ${ }^{125}$ and the Pediatric Evaluation Disability Inventory ${ }^{124}$ and found mixed results. 
Activities of daily living were improved with treadmill training versus overground walking in younger children (mean age 6) ) $^{124}$ but not versus individualized strength-based PT in older children (mean age 9). ${ }^{125}$ One trial reported balance as measured with the BBS and found BBS scores improved with treadmill training versus overground walking in younger children. ${ }^{24}$ Trials in children were too varied and results were too inconsistent to draw conclusions regarding treadmill training versus other interventions in children with CP.

Six trials did not address harms or adverse events. ${ }^{121,122,124,130-132}$ One RCT reported that there were no adverse events. ${ }^{128}$ One RCT indicated that there were no reports of adverse events with transcranial stimulation during treadmill training but did not comment on other potential adverse events. ${ }^{123}$ Another RCT reported that no injury occurred that was due to the intervention. ${ }^{129}$ One trial reported that four children in the experimental group and one in the control group dropped out due to unexpected surgery or botulinum toxin administration and that one child dropped out due to back pain with walking. ${ }^{127}$ Another trial reported that two children developed knee or leg pain and one child developed a blister underneath the ankle-foot arthrosis. ${ }^{125}$

\section{Treadmill-Spinal Cord Injury}

Three trials ${ }^{133-139}$ enrolled individuals $(n=89)$ with SCI (Table 19). Trial mean age of participants ranged from 37 to 55 years with an average mean proportion female between 14 and 30 percent. The disability of participants varied. One trial ${ }^{138}$ enrolled individuals who could walk at least 5 meters with braces or walking aids, while another trial ${ }^{133}$ only required that participants be able to independently advance one leg.

Table 19. Treadmill exercise in spinal cord injury

\begin{tabular}{|c|c|c|c|}
\hline $\begin{array}{l}\text { Author, Year } \\
\text { Intervention } \\
\text { Study Design } \\
\text { Study Quality }\end{array}$ & $\begin{array}{l}\text { Intervention } \\
\text { and } \\
\text { Comparison }\end{array}$ & Population & Results \\
\hline $\begin{array}{l}\text { Alexeeva, } \\
2011^{133} \\
\text { Aerobic } \\
\text { exercise } \\
\text { RCT } \\
\text { Fair }\end{array}$ & $\begin{array}{l}\text { A. BWS treadmill } \\
\text { training, max } 39 \\
\text { sessions over } 13 \\
\text { weeks ( } n=9 \text { ) } \\
\text { B. BWS track } \\
\text { training, max } 39 \\
\text { sessions over } 13 \\
\text { weeks ( } n=14) \\
\text { C. Structured PT, } \\
\text { max } 39 \text { sessions } \\
\text { over } 13 \text { weeks } \\
(n=12)\end{array}$ & $\begin{array}{l}\text { A vs. B vs. } C \\
\text { Age: } 43 \text { vs. } 36 \text { vs. } \\
35 \\
\text { Female: } 11 \% \text { vs. } \\
14 \% \text { vs. } 17 \% \\
\text { Cervical: } 89 \% \text { vs. } \\
57 \% \text { vs. } 58 \%\end{array}$ & $\begin{array}{l}\text { A vs. B vs. C: mean (SD), } p=\text { across all groups: } \\
\text { 10MWT (m/s): } 0.30(0.26) \text { to } 0.46(0.40) \text { vs. } 0.22(0.20) \text { to } 0.44 \\
\text { (0.33) vs. } 0.41(0.34) \text { to } 0.51(0.36), p>0.05 \\
\text { TBS: } 9.8(5.4) \text { to } 19.4(5.0) \text { vs. } 10.5(3.4) \text { to } 11.9(2.5) \text { vs. } 10.1(3.6) \\
\text { to } 12.9(2.7), p<0.05, \text { post-hoc group } C \\
\text { improving }(p<0.001) \text { and B improving ( } p<0.01) \text { but not } A(p=0.23) \\
\text { SAWS: } 39.3((8.3) \text { to } 35.2(8.7) \text { vs. } 35.9(6.9) \text { to } 32.4(7.6) \text { vs. } 36.6 \\
\text { (9.9) to } 29.0(7.9), p>0.05\end{array}$ \\
\hline
\end{tabular}




\begin{tabular}{|c|c|c|c|}
\hline $\begin{array}{l}\text { Author, Year } \\
\text { Intervention } \\
\text { Study Design } \\
\text { Study Quality }\end{array}$ & $\begin{array}{l}\text { Intervention } \\
\text { and } \\
\text { Comparison }\end{array}$ & Population & Results \\
\hline $\begin{array}{l}\text { Giangregorio, } \\
2012^{134} \\
\text { Hitzig, } 2013^{135} \\
\text { Kapadia, } \\
2014^{136} \\
\text { Craven, } \\
2017^{137} \\
\text { Aerobic } \\
\text { exercise } \\
\text { RCT } \\
\text { Fair }\end{array}$ & $\begin{array}{l}\text { A. BWS treadmill } \\
\text { walking with } \\
\text { FES, } 48 \text { sessions } \\
\text { over } 16 \text { weeks } \\
(n=17) \\
\\
\text { B. Aerobic and } \\
\text { resistance } \\
\text { training, } 48 \\
\text { sessions over } 16 \\
\text { weeks }(n=17)\end{array}$ & $\begin{array}{l}\text { A vs. B } \\
\text { Age: } 56.6 \text { vs. } 54.1 \\
\text { Female: } 18 \% \text { vs. } \\
29 \% \\
\text { Tetraplegia: } 82 \% \\
\text { vs. } 71 \% \\
\text { UEMS: } 38.3 \text { vs. } \\
37.5 \\
\text { LEMS: } 30.4 \text { vs. } \\
27.9 \\
\text { C2-T12: } 100 \% \\
\text { AIS C or D: } 100 \%\end{array}$ & $\begin{array}{l}\text { A vs. B, mean (SD), pre, post, and } 8 \text { months after intervention: } \\
\text { 10MWT: } 42.8(46.2) \text { to } 35.2(40.8) \text { to } 42.2(67.7) \text { vs. } 49.1(41.7) \text { to } \\
\text { 28.7 (8.3) to } 35.1(18.8), p=0.829 \\
\text { 6MWT: } 187.9(123.4) \text { to } 217.1(134.4) \text { to } 232.5(138.9) \text { vs. } 79.4 \\
\text { (83.9) to } 130(46.0) \text { to } 126.4(63.8), p=0.096 \\
\text { TUG: } 43.6(25.5) \text { to } 33.0(15.7) \text { to } 32.2(19.1) \text { vs. } 61.6(36.2) \text { to } \\
49.5(21.9) \text { to } 51.3(19.6), p=0.138 \\
\text { FIM: } 4.7(1.82) \text { to } 5.19(1.80) \text { to } 5.19(1.83) \text { vs. } 4.18(2.14) \text { to } 4.82 \\
\text { (1.66) to } 5.09(2.98), p=0.115 \\
\text { CHART Mobility subscale: } 79.81(21.00) \text { to } 85.28(13.81) \text { to } 86.36 \\
\text { (14.44) vs. } 82.09(19.31) \text { to } 84.27(11.89) \text { to } 88.45(15.25), p=0.840 \\
\text { CHART Social subscale: } 89.94(13.12) \text { to } 90.31(18.02) \text { to } 88.69 \\
\text { (17.10) vs. } 72.73(24.00) \text { to } 89.64(12.63) \text { to } 73.73(31.15), p=0.065 \\
\text { CHART Physical subscale: } 92.35(11.75) \text { to } 93.72(8.02) \text { to } 93.81 \\
\text { (6.16) vs. } 97.94 \text { (2.49) to } 94.99(7.30) \text { to } 93.85(5.01), p=0.214\end{array}$ \\
\hline $\begin{array}{l}\text { Yang, } 2014^{138} \\
\text { Aerobic } \\
\text { Exercise } \\
\text { RCT } \\
\text { (Crossover) } \\
\text { Fair }\end{array}$ & $\begin{array}{l}\text { A. BWS (if } \\
\text { needed) treadmill } \\
\text { walking, } 40 \\
\text { sessions over } 8 \\
\text { weeks }(n=10) \\
\\
\text { B. Precision track } \\
\text { walking training, } \\
40 \text { sessions over } \\
8 \text { weeks }(n=10)\end{array}$ & $\begin{array}{l}\text { A vs. B } \\
\text { Age: } 48 \text { vs. } 44 \\
\text { Female: } 30 \% \text { vs. } \\
30 \% \\
\text { Able to walk } \geq 5 \\
\text { meters with walking } \\
\text { aid or braces: } 100 \% \\
\text { Cervical: } 50 \%\end{array}$ & $\begin{array}{l}\text { A vs. B, mean change, } p=\text { between groups: } \\
\text { 6MWT: } 29 \text { vs. } 10, p=0.045 \\
\text { 10MWT (self-selected): } 0.070 \text { vs. } 0.025, p>0.05 \\
\text { 10MWT (fast): } 0.075 \text { vs. } 0.12, p>0.05 \\
\text { SCIFAP: }-75 \text { vs. }-42, p>0.05 \\
\frac{\text { WISCI (self-selected): } 0.08 \text { vs. } 0.85, p>0.05}{\text { WISCI (max): } 0.04 \text { vs. } 0.08, p>0.05}\end{array}$ \\
\hline
\end{tabular}

Abbreviations: 6MWT = 6-Minute Walk Test; 10MWT = 10-Meter Walk Test; AIS = ASIA Impairment Scale; BWS = body weight supported; CHART = Craig Handicap and Assessment Reporting Technique; FES = functional electrical stimulation; FIM

= Functional Independence Measure; LEMS = Lower Extremity Motor Score; PT = physical therapy; RCT = randomized controlled trial; SAWS = Satisfaction with Abilities and Well-Being Scale; SD = standard deviation; TBS = Tinetti Balance Scale; TUG = Timed Up and Go Test; UEMS = Upper Extremity Motor Score; WISCI = Walking Index for Spinal Cord Injury

All three trials provided low-strength evidence for no clear benefit of treadmill training versus all comparators on function, including walking (SOE: low). Evidence was insufficient to draw conclusions on the effects of treadmill training on quality of life or balance in study participants with SCI.

One RCT did not address harms or adverse events. ${ }^{133}$ One $\mathrm{RCT}^{138}$ reported a single participant withdrew due to wrist pain and that no other adverse events that were related to training occurred; one RCT reported adverse events that were considered a result of the intervention (bruising, blistering, fall on treadmill, and pain in heel/ankle and hip/groin) and loss of consciousness, muscle strain, swollen knees, elbow pain, and dizziness in the control group. ${ }^{134}$

One additional trial of reports the effects of RAGT on bowel dysfunction and is discussed in KQ2c. ${ }^{113}$

\section{Postural Control Interventions}

\section{Balance Exercises}

Balance training involves muscle-strengthening exercises to improve stability and coordination. Balance exercises may involve specific methods (e.g., Cawthorne-Cooksey exercises, Frenkel exercises, core stability, dual tasking, sensory strategies [CoDuSe] exercises) or individual or group standard balance exercises or traditional rehabilitation with a balance 
focus. These exercises are designed to improve postural control and walking, or other functional outcomes, and/or to decrease falls and near falls.

\section{Key Points}

- Balance exercises were associated with improved balance in MS compared with usual care or waitlist controls (SOE: moderate), a lower risk of falls (SOE: low), and lowstrength evidence of improved function (SOE: low).

- Due to poor-quality studies, evidence for the effects of balance exercises in CP was rated insufficient to draw conclusions (SOE: insufficient).

- There was also limited evidence for the effects of balance training in SCI (SOE: insufficient).

\section{Detailed Synthesis}

Fifteen RCTs, ${ }^{61,83,141-149,151,152,156,157}$ two quasiexperimental nonrandomized studies, ${ }^{153-155}$ and one cohort study ${ }^{155}$ involving 751 participants evaluated balance training. Fifteen studies compared balance training interventions with usual care, waitlist, or attention control, ${ }^{61,83,141,143,145-149,151-157}$ and one study ${ }^{142}$ compared balance training alone and in combination with lumbar stabilization exercises or task-oriented training exercises. Twelve studies enrolled people with MS, ${ }^{61,83,141-143,145-149,151}$ four studies enrolled people with CP, ${ }^{152-155}$ and two studies were conducted in participants with SCI. ${ }^{156,157}$ Among the trials, three met criteria for good quality, ${ }^{145,146,148}$ and eleven were rated fair quality. ${ }^{61,83,141-143,147,149,151,152,156,157}$ The three quasiexperimental nonrandomized studies were rated poor quality due to unclear enrollment methods, unbalanced study group allocation, and/or lack of clear adjustment for clinical or demographic confounders. ${ }^{153-155}$ The most frequently reported outcomes were related to balance and function.

\section{Balance Exercise-Multiple Sclerosis}

Twelve trials enrolled people $(n=640)$ with MS (Table 20). ${ }^{61,83,141-143,145-149,151}$ The trial mean age of participants ranged from 32 to 59 years with a mean proportion female from 59 to 100 percent. Race/ethnicity was not reported in any of the trials. Mean baseline EDSS score ranged from 2.4 to 6.1 .

BBS scores were evaluated in eight trials (Table 20). ${ }^{83,141-146,151}$ Seven trials $(n=332)$ comparing balance training with usual care found balance training consistently associated with significant improvements in BBS scores relative to usual care (MD -4.14, 95\% CI -5.57 to $\left.-2.70, \mathrm{I}^{2}=79 \%\right){ }^{83,141,143-146,151}$ Two other RCTs measured balance with the Mini Balance Evaluation System Test (MiniBEST) and found similar results (MD 2.40, 95\% CI 1.10 to 3.70, $\left.\mathrm{I}^{2}=51 \%\right) .{ }^{148,149}$ One study reported improvements in static and dynamic balance with balance exercises. ${ }^{147}$ These studies provide moderate-strength evidence of improved balance with balance exercises in MS compared with usual care or waitlist controls.

Two RCTs reported falls or near falls as an outcome, with both reporting significantly fewer falls in the intervention groups than the control groups, based on patient diaries. ${ }^{141,143}$ Comparing the average number of falls and near falls before, during, and after the intervention in one study showed they significantly decreased over time (falls: $4.18,2.17,1.68$, before-after $\mathrm{p}=0.0011$; near falls: $23.2,18.0,8.64$, before-after $\mathrm{p}=0.0038)$. The other RCT found that the likelihood of having an unexpected fall was lower with balance training than with usual care $(p=0.005) .{ }^{141}$ These studies provide low-strength evidence of improvement in fall risk with balance training. 
A trial $(n=42)$ comparing balance training alone with balance training combined with lumbar stabilization or task-oriented training found the combination therapies improved balance more than balance training alone (mean change from baseline 3.57 [balance training alone] versus 5.78 [balance training with lumbar stabilization] versus 5.57 [balance training with task-oriented training]), but the difference between groups was not statistically significant (SOE: insufficient). ${ }^{142}$

Seven studies provided low-strength evidence of benefit of balance exercises on function. Subjective assessment of walking was improved with balance training in four studies $(\mathrm{n}=248$, MD $-4.66,95 \%$ CI -6.65 to $\left.-2.67, \mathrm{I}^{2}=0 \%\right) .{ }^{143,144,149,150}$ Objective measures of walking (e.g., 6MWT, Functional Gait Assessment) largely favored balance exercises compared with usual care, although individual results were not always statistically significant. ${ }^{83,143,144,147,149-151}$ One trial reported improved scores on the MSIS-29 with balance exercises versus usual PT. ${ }^{83}$ However, there was no improvement in TUG with balance exercises in pooled analysis of three trials ( $\mathrm{n}=127$, MD $0.45,95 \% \mathrm{CI}-1.92$ to $\left.2.82, \mathrm{I}^{2}=54 \%\right) .{ }^{143,144,151}$

One fair-quality RCT $(n=45)$ found no difference between balance exercises and attention control on sleep as measured by the Insomnia Sleep Index but future trials are needed to confirm these findings. ${ }^{61}$ (SOE: insufficient). Quality of life, based on Multiple Sclerosis Quality of Life (MSQOL)-54 score, was not significantly different between balance training with conventional rehabilitation one trial $(\mathrm{n}=80){ }^{141}$ (SOE: insufficient).

Six RCTs did not address harms or adverse events. ${ }^{61,83,146-148,151}$ Of the studies that did report on adverse events, one reported no falls or adverse events during the study, and another reported two falls during CoDuSe training. ${ }^{143,145,149}$ Two other studies reported that there were no adverse events related to training. ${ }^{141,142}$

Table 20. Balance training in multiple sclerosis

\begin{tabular}{|c|c|c|c|}
\hline $\begin{array}{l}\text { Author, Year } \\
\text { Intervention } \\
\text { Study Design } \\
\text { Study Quality }\end{array}$ & $\begin{array}{l}\text { Intervention } \\
\text { and Comparison }\end{array}$ & Population & Results \\
\hline $\begin{array}{l}\text { Afrasiabifar, } \\
2018^{145} \\
\text { Postural control } \\
\text { RCT } \\
\text { Good }\end{array}$ & $\begin{array}{l}\text { A. Cawthorne- } \\
\text { Cooksey exercise: } 36 \\
\text { sessions over } 12 \\
\text { weeks }(n=24) \\
\text { B. Frenkel exercises, } \\
\text { number of sessions } \\
\begin{array}{l}\text { NR, over } 12 \text { weeks } \\
(n=23)\end{array} \\
\text { C. Usual care }(n=25)\end{array}$ & $\begin{array}{l}\text { A vs. B vs. C } \\
\text { Age: } 32.4 \text { vs. } 32 \text { vs. } 33.6 \\
\text { Female: } 83 \% \text { vs. } 74 \% \text { vs. } \\
76 \% \\
\text { RRMS: } 96 \% \text { vs. } 96 \% \text { vs. } \\
92 \% \\
\text { PPMS+SPMS: } 4 \% \text { vs. } \\
4 \% \text { vs. } 8 \%\end{array}$ & $\begin{array}{l}\text { A vs. B vs. C, mean change from baseline } \\
\text { (SD): } \\
\text { BBS: } 8.9 \text { (SD } 1.8 \text { ) vs. } 2.3 \text { (SD } 0.9 \text { ) vs. }-1.2 \\
\text { (SD } 1.05) \\
\text { BBS: mean difference between-groups: } \\
\text { A vs. B: } 5.9,95 \% \mathrm{Cl} 1.9 \text { to } 9.9, p=0.001 \\
\text { A vs. C: } 10.7,95 \% \mathrm{Cl} 6.8 \text { to } 14.6, p=0.001 \\
\text { B vs. C: } 4.8,95 \% \mathrm{Cl} 0.9 \text { to } 8.8, p=0.01\end{array}$ \\
\hline $\begin{array}{l}\text { Amiri, } 2019^{147} \\
\text { Postural control } \\
\text { RCT } \\
\text { Fair }\end{array}$ & $\begin{array}{l}\text { A. Core stability } \\
\text { training, } 30 \text { sessions } \\
\text { over } 10 \text { weeks }(n=35) \\
\text { B. Conventional } \\
\text { treatment }(n=34)\end{array}$ & $\begin{array}{l}\text { A vs. B } \\
\text { Age: } 32 \text { vs. } 31 \\
\text { Female: } 100 \% \\
\text { EDSS: } 3.58 \text { vs. } 3.74 \\
\text { RRMS: } 100 \%\end{array}$ & $\begin{array}{l}\text { Significant interaction between time and } \\
\text { group according to baseline EDSS score for } \\
\text { core muscle function (i.e., core endurance } \\
\text { and core strength tests) and static and } \\
\text { dynamic stability }(p<0.05)\end{array}$ \\
\hline
\end{tabular}




\begin{tabular}{|c|c|c|c|}
\hline $\begin{array}{l}\text { Author, Year } \\
\text { Intervention } \\
\text { Study Design } \\
\text { Study Quality }\end{array}$ & $\begin{array}{l}\text { Intervention } \\
\text { and Comparison }\end{array}$ & Population & Results \\
\hline $\begin{array}{l}\text { Arntzen, } 2019^{148} \\
\text { Arntzen, } 2020^{150} \\
\text { Postural control } \\
\text { RCT } \\
\text { Good }\end{array}$ & $\begin{array}{l}\text { A. GroupCoreDIST, } 18 \\
\text { sessions over } 6 \text { weeks } \\
+ \text { home exercises } \\
(n=39) \\
\text { B. Usual care }(n=40)\end{array}$ & $\begin{array}{l}\text { A vs. B } \\
\text { Age: } 52 \text { vs. } 48 \\
\text { Female: } 69 \% \text { vs. } 73 \% \\
\text { EDSS: } 2.45 \text { vs. } 2.28 \\
\text { RRMS: } 82 \% \text { vs. } 90 \% \\
\text { PPMS: } 13 \% \text { vs. } 5 \% \\
\text { SPMS: } 5 \% \text { vs. } 5 \%\end{array}$ & $\begin{array}{l}\text { A vs. B, mean difference between groups: } \\
\text { MiniBEST: MD } 1.91,95 \% \mathrm{Cl} 1.07 \text { to } 2.76 \text {, } \\
\text { p<0.001 } \\
\text { 2MWT at } 7 \text { weeks: MD } 16.7,95 \% \mathrm{Cl} 8.15 \text { to } \\
25.25 \\
2 \mathrm{MWT} \text { at } 30 \text { weeks: MD } 16.38,95 \% \mathrm{Cl} 7.65 \\
\text { to } 25.12 \\
10 \mathrm{MWT} \text { at } 7 \text { weeks: MD } 0.48,95 \% \mathrm{Cl} 0.11 \\
\text { to } 0.85 \\
10 \mathrm{MWT} \text { at } 30 \text { weeks: MD } 0.33,95 \% \mathrm{Cl} \\
-0.04 \text { to } 0.71 \\
\text { MSWS-12 at } 7 \text { weeks: MD } 9.77,95 \% \mathrm{Cl} \\
3.19 \text { to } 16.35 \\
\text { MSWS-12 at } 30 \text { weeks: } \mathrm{MD} 3.87,95 \% \mathrm{Cl} \\
-2.80 \text { to } 10.54\end{array}$ \\
\hline $\begin{array}{l}\text { Brichetto, } 2015^{146} \\
\text { Postural control } \\
\text { RCT } \\
\text { Good }\end{array}$ & $\begin{array}{l}\text { A. Personalized rehab } \\
\text { (tailored to sensory } \\
\text { impairment), } 12 \\
\text { sessions over } 4 \text { weeks } \\
(n=16) \\
\text { B. Traditional rehab } \\
\text { (visual rehab for } \\
\text { balance disorders), } 12 \\
\text { sessions over } 4 \text { weeks } \\
\text { (n=16) }\end{array}$ & $\begin{array}{l}\text { A vs. B } \\
\text { Age: } 50.1 \text { vs. } 51.0 \\
\text { Female: } 69 \% \text { vs. } 75 \% \\
\text { RRMS: } 56 \% \text { vs. } 63 \% \\
\text { SPMS: } 31 \% \text { vs. } 25 \% \\
\text { PPMS: } 13 \% \text { vs. } 13 \% \\
\text { EDSS: } 3.7 \text { vs. } 3.7\end{array}$ & $\begin{array}{l}\text { A vs. } B, \text { mean (SD), } p=\text { between groups: } \\
\text { BBS: } 46.5(3.6) \text { to } 52.8(2.8) \text { vs. } 45.8(6.6) \\
\text { to } 47.8(6.1), p<0.001\end{array}$ \\
\hline $\begin{array}{l}\text { Callesen, } 2019^{149} \\
\text { Postural control } \\
\text { RCT } \\
\text { Fair }\end{array}$ & $\begin{array}{l}\text { A. Balance and motor } \\
\text { control training, } 20 \\
\text { sessions over } 10 \\
\text { weeks }(n=28) \\
\text { B. Waitlist control } \\
(n=18)\end{array}$ & $\begin{array}{l}\text { A vs. B } \\
\text { Age: } 51 \text { vs. } 56 \\
\text { Female: } 82 \% \text { vs. } 80 \% \\
\text { EDSS: } 4 \text { vs. } 3.5 \\
\text { RRMS: } 75 \% \text { vs. } 65 \% \\
\text { SPMS: } 14 \% \text { vs. } 15 \% \\
\text { PPMS: } 11 \% \text { vs. } 20 \%\end{array}$ & 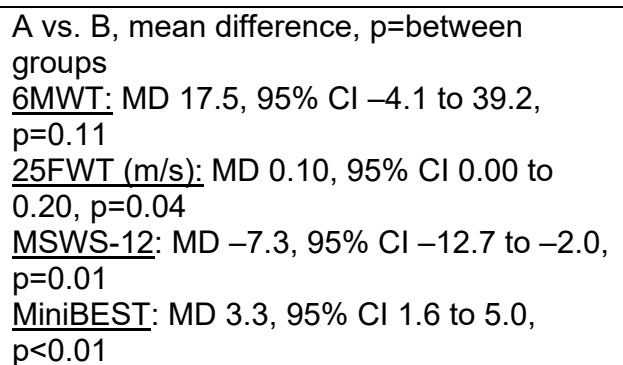 \\
\hline $\begin{array}{l}\text { Carling, } 2017^{143} \\
\text { Postural control } \\
\text { RCT } \\
\text { Fair }\end{array}$ & $\begin{array}{l}\text { A. Group CoDuSe, } 14 \\
\text { sessions over } 7 \text { weeks } \\
(n=23) \\
\text { B. Waitlist (Late start) } \\
\text { controls }(n=25)\end{array}$ & $\begin{array}{l}\text { A vs. B } \\
\text { Age: } 62 \text { vs. } 55 \\
\text { Female: } 76 \% \text { vs. } 62 \% \\
\text { EDSS: } 6.16 \text { vs. } 6.06 \\
\text { RRMS: } 0 \% \text { vs. } 23 \% \\
\text { SPMS: } 68 \% \text { vs. } 58 \% \\
\text { PPMS: } 32 \% \text { vs. } 19 \%\end{array}$ & $\begin{array}{l}\text { A vs. B, mean change (SE): } \\
\text { BBS: } 3.65(1.44), p=0.015 \\
\text { TUG: } 4.41(3.17), p=0.17 \\
\text { 2MWT: }-3.24(3.37), p=0.34 \\
\text { Sit-to-Stand: } 0.2492 .12), p=0.17 \\
\text { 10MWT: } 1.49(3.84), p=0.70 \\
\text { Falls Efficiency Scale: }-1.66(2.39), p=0.49 \\
\text { MSWS-12: }-7.21(3.60), p=0.051 \\
\text { Falls: }-1.24(1.66), p<0.001 \\
\text { Near Falls: }-8.24(14.78), p=0.002\end{array}$ \\
\hline $\begin{array}{l}\text { Forsberg, } 2016^{144} \\
\text { Postural control } \\
\text { RCT } \\
\text { Fair }\end{array}$ & $\begin{array}{l}\text { A. Group CoDuSe, } 14 \\
\text { sessions over } 7 \text { weeks } \\
(n=35) \\
\text { B. No intervention } \\
(n=38)\end{array}$ & $\begin{array}{l}\text { A vs. B } \\
\text { Age: } 52 \text { vs. } 56 \\
\text { Female: } 80 \% \text { vs. } 82 \% \\
\text { EDSS } 6.0 \text { or less: } 100 \% \\
\text { RRMS: } 57 \% \text { vs. } 34 \% \\
\text { PPMS: } 11 \% \text { vs. } 13 \% \\
\text { SPMS: } 31 \% \text { vs. } 53 \%\end{array}$ & 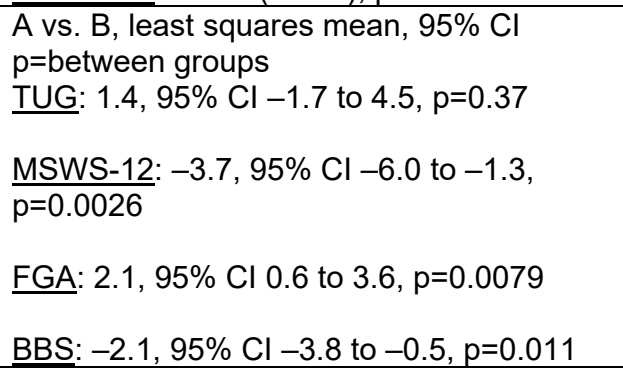 \\
\hline
\end{tabular}




\begin{tabular}{|c|c|c|c|}
\hline $\begin{array}{l}\text { Author, Year } \\
\text { Intervention } \\
\text { Study Design } \\
\text { Study Quality }\end{array}$ & $\begin{array}{l}\text { Intervention } \\
\text { and Comparison }\end{array}$ & Population & Results \\
\hline $\begin{array}{l}\text { Gandolfi, } 2015^{141} \\
\text { Postural control } \\
\text { RCT } \\
\text { Fair }\end{array}$ & $\begin{array}{l}\text { A. Balance training } \\
\text { (sensory integration), } \\
15 \text { sessions over } 5 \\
\text { weeks ( } n=39) \\
\text { B. Conventional } \\
\text { rehabilitation, } 15 \\
\text { sessions over } 5 \text { weeks } \\
(n=41)\end{array}$ & $\begin{array}{l}\text { A vs. B } \\
\text { Age: } 47.21 \text { vs. } 49.56 \\
\text { Female: } 72 \% \text { vs. } 76 \% \\
\text { EDSS (median): } 3.00 \text { vs. } \\
3.66 \\
\text { RRMS: } 100 \%\end{array}$ & 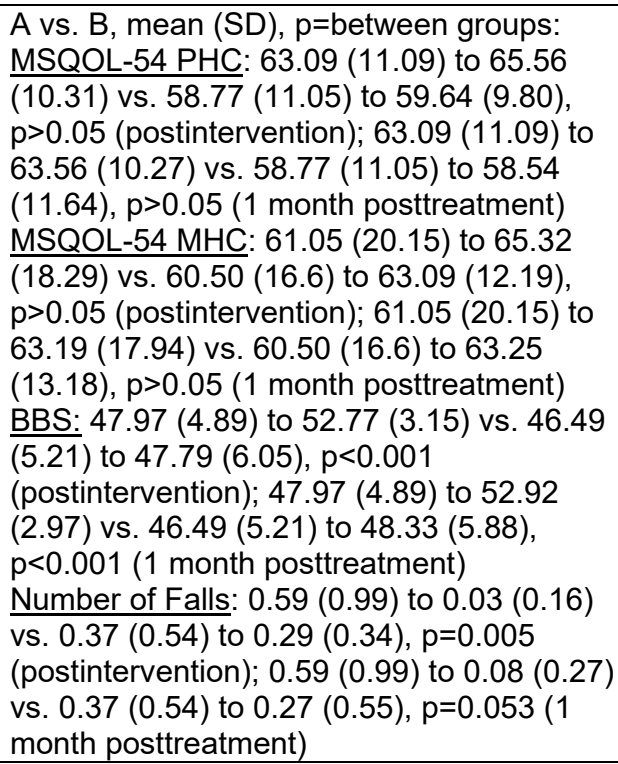 \\
\hline $\begin{array}{l}\text { Ozkul, } 2020^{151} \\
\text { Postural control } \\
\text { RCT } \\
\text { Fair }\end{array}$ & 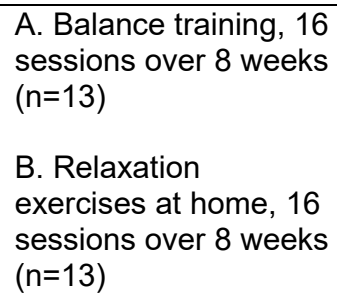 & $\begin{array}{l}\text { A vs. B } \\
\text { Age: } 34 \text { vs. } 34 \\
\text { Female: } 85 \% \text { vs. } 77 \% \\
\text { EDSS median: } 1 \text { vs. } 2 \\
\text { Number of relapses: } 2 \text { vs. } \\
2\end{array}$ & $\begin{array}{l}\text { Pre-post median (IQR): } \\
\text { BBS: } 47(44,56) \text { to } 52(46,56) \text { vs. } 55(53, \\
56) \text { to } 56(53.5,56), p>0.05 \\
\text { TUG: } 7.3(6.7,8.5) \text { to } 7.3(6,7.9) \text { vs. } 6.9 \\
(6.5,7.5) \text { to } 7.4(6.4,7.7), p<0.017\end{array}$ \\
\hline $\begin{array}{l}\text { Sadeghi } \\
\text { Bahmani, 201961 } \\
\text { Postural control } \\
\text { RCT } \\
\text { Fair }\end{array}$ & $\begin{array}{l}\text { A. Balance and } \\
\text { coordination } \\
\text { exercises, } 24 \text { sessions } \\
\text { over } 8 \text { weeks }(n=24) \\
\text { B. Attention control, } 24 \\
\text { sessions over } 8 \text { weeks } \\
(n=21)\end{array}$ & $\begin{array}{l}\text { A vs. B } \\
\text { Age: } 39 \text { vs. } 38 \\
\text { Female: } 100 \% \\
\text { EDSS: } 3.38 \text { vs. } 2.02\end{array}$ & $\begin{array}{l}\text { A vs. } B, \text { mean (SD), } p=\text { between groups: } \\
\text { EDSS: } 3.38(1.87) \text { to } 3.10(1.86) \text { vs. } 2.02 \\
\text { (1.84) to } 1.98(1.70), p>0.05 \\
\frac{\text { ISI: } 13.46(5.81) \text { to } 10.13(4.92) \text { vs. } 1.71}{(5.43) \text { to } 11.14(5.39), p>0.05}\end{array}$ \\
\hline $\begin{array}{l}\text { Salci, } 2017^{142} \\
\text { Postural control } \\
\text { RCT } \\
\text { Fair }\end{array}$ & $\begin{array}{l}\text { A. Balance training, } 18 \\
\text { sessions over } 6 \text { weeks } \\
(n=14) \\
\text { B. Lumbar stabilization } \\
\text { plus balance training, } \\
18 \text { sessions over } 6 \\
\text { weeks ( } n=14) \\
\text { C. Task-oriented } \\
\text { training (individualized } \\
\text { exercises) plus } \\
\text { balance training, } 18 \\
\text { sessions over } 6 \text { weeks } \\
(n=14)\end{array}$ & $\begin{array}{l}\text { A vs. B vs. C } \\
\text { Age: } 35.36 \text { vs. } 37.29 \text { vs. } \\
34.36 \\
\text { Female: } 43 \% \text { vs. } 62 \% \text { vs. } \\
71 \% \\
\text { Ambulatory: } 100 \% \\
\text { EDSS (median): } 3.5 \text { vs. } \\
3.5 \text { vs. } 3.5 \\
\text { RRMS: } 79 \% \text { vs. } 79 \% \text { vs. } \\
86 \% \\
\text { PPMS: } 7 \% \text { vs. } 7 \% \text { vs. } 0 \% \\
\text { SPMS: } 14 \% \text { vs. } 14 \% \text { vs. } \\
14 \%\end{array}$ & $\begin{array}{l}\text { A vs. B vs. C, mean change (SD), } \\
p=\text { between groups: } \\
\text { 2MWT: } 10.75 \text { (SD 9.97) vs. } 25.55 \text { (SD } \\
\text { 16.90) vs. } 18.69 \text { (SD 14.24) } \\
\text { A vs. B: } p=0.08 ; A \text { vs. C: } p=0.085 ; \text { B vs. C: } \\
p=0.265 \\
\text { BBS: } 3.57 \text { (SD } 2.20) \text { vs. } 5.78 \text { (SD } 3.40) \text { vs. } \\
5.57 \text { (SD } 3.73) ; p=>0.05 \text { for all comparisons }\end{array}$ \\
\hline
\end{tabular}




\begin{tabular}{|c|c|c|c|}
\hline $\begin{array}{l}\text { Author, Year } \\
\text { Intervention } \\
\text { Study Design } \\
\text { Study Quality }\end{array}$ & $\begin{array}{l}\text { Intervention } \\
\text { and Comparison }\end{array}$ & Population & Results \\
\hline $\begin{array}{l}\text { Tollar, } 2020^{85} \\
\text { Postural control } \\
\text { RCT } \\
\text { Fair }\end{array}$ & $\begin{array}{l}\text { A. Balance training, } 25 \\
\text { sessions over } 5 \text { weeks } \\
(n=14) \\
\text { B. Usual PT, } 25 \\
\text { sessions over } 5 \text { weeks } \\
(n=12)\end{array}$ & $\begin{array}{l}\text { A vs. B } \\
\text { Age: } 46.9 \text { vs. } 44.4 \\
\text { Female: } 86 \% \text { vs. } 92 \% \\
\text { EDSS median: } 5.0 \text { vs. } \\
5.0 \\
\text { RRMS: } 64 \% \text { vs. } 67 \%\end{array}$ & $\begin{array}{l}\text { A vs. B, mean difference between groups: } \\
\text { MSIS-29: }-6.3(4.36) \text { vs. } 1.0(3.46), p=0.008 \\
\text { 6MWT: } 19.2(35.40) \text { vs. } 6.3(49.27), \\
p=0.174 \\
\text { BBS: } 3.9(2.25) \text { vs. }-0.2(2.62), p=0.015 \\
\text { EQ-5 Sum score:-0.6 (1.15) vs. } 0.0(1.13), \\
p=0.023\end{array}$ \\
\hline
\end{tabular}

Abbreviations: 2MWT = 2-Minute Walk Test; 6MWT =6-Minute Walk Test; 10MWT = 10-Meter Walk Test; 25FWT = 25-Foot Walk Test; BBS = Berg Balance Scale; CoDuSe = core stability, dual tasking, sensory strategies; EDSS=Expanded Disability Status Scale; EQ-5 = Euro Quality of Life; FGA = Functional Gait Assessment; ISI = Insomnia Severity Index; IQR = interquartile range; MiniBEST = Mini Balance Evaluation Systems Test; MSIS-29 = Multiple Sclerosis Impact Scale; MSQOL=Multiple Sclerosis Quality of Life; MSWS-12 = Multiple Sclerosis Walking Scale-12; NR = not reported; PPMS = primary progressive multiple sclerosis; $\mathrm{PT}=$ physical therapy; $\mathrm{RRMS}=$ relapsing-remitting multiple sclerosis; $\mathrm{RCT}=$ randomized controlled trial; $\mathrm{SD}=$ standard deviation; $\mathrm{SE}=$ standard error; $\mathrm{SPMS}=$ secondary progressive multiple sclerosis; TUG $=$ Timed Up and Go Test

\section{Balance Exercise-Cerebral Palsy}

One RCT, ${ }^{152}$ two quasiexperimental studies, ${ }^{153,154}$ and one cohort study ${ }^{155}$ enrolled people $(\mathrm{n}=117)$ with CP (Table 21). Three studies ${ }^{152-154}$ enrolled children and one ${ }^{155}$ enrolled young adults. In the studies conducted in children, mean age was 10 years (range 8 to 11 years) and 40 percent were female (range $32 \%$ to 50\%). In the study conducted in adults, the mean age was 22 years and 46 percent were female. None of the studies reported race/ethnicity. Ambulatory status was not reported in any of the studies. Three studies reported baseline function using the GMFCS. ${ }^{152-154}$ One study primarily enrolled people with Level I GMFCS classification ${ }^{154}$ and the other two studies enrolled people classified Level III or higher. ${ }^{152,153}$ One fair-quality trial ${ }^{152}$ $(\mathrm{n}=28)$ and 2 poor-quality quasiexperimental studies $(\mathrm{n}=66)^{153,154}$ provided evidence on the effects of balance exercises versus usual care on function in CP. Due to the high risk of bias in this body of evidence, evidence was considered insufficient to draw conclusions (SOE: insufficient). Similarly, evidence was insufficient to draw conclusions on the effects of balance training exercises on balance ${ }^{152,154}$ and ADL. ${ }^{152,153,155}$

Harms and adverse events were not addressed in any of the studies of balance training in people with $\mathrm{CP} .{ }^{152-155}$

\section{Table 21. Balance training in cerebral palsy}

\begin{tabular}{|c|c|c|c|}
\hline $\begin{array}{l}\text { Author, Year } \\
\text { Intervention } \\
\text { Study Design } \\
\text { Study Quality }\end{array}$ & $\begin{array}{l}\text { Intervention } \\
\text { and Comparison }\end{array}$ & Population & Results \\
\hline $\begin{array}{l}\text { Bleyenheuft, } \\
2017^{153} \\
\text { Postural control } \\
\text { Quasiexperimental } \\
\text { Poor }\end{array}$ & $\begin{array}{l}\text { A. Hand-arm } \\
\text { bimanual intensive } \\
\text { therapy including } \\
\text { lower extremity, } \\
\text { thirteen } 6.5 \text { hours } \\
\text { per day over } 13 \\
\text { days }(n=10) \\
\text { B. Usual PT, } 2 \\
\text { weeks }(n=10)\end{array}$ & $\begin{array}{l}\text { A vs. B } \\
\text { Age: } 10.5 \text { vs. } 11.4 \\
\text { Female: } 40 \% \text { vs. } 50 \% \\
\text { GMFCS II: } 20 \% \text { vs. } 20 \% \\
\text { GMFCS III: } 70 \% \text { vs. } 70 \% \\
\text { GMFCS IV: } 10 \% \text { vs. } 10 \%\end{array}$ & $\begin{array}{l}\text { A vs. B, mean (SD); } p=\text { interaction of } 2 \\
\text { interventions } X 3 \text { time points (baseline, } \\
\text { postintervention and } 3 \text { months postintervention): } \\
\text { GMFM-66: } 55(5.9) \text { to } 58(6.2) \text { to } 62 \text { (6.4) vs. } 55 \\
\text { (8.7) to } 56(7.6) \text { to } 57(6.6), p<0.001 \\
\text { 6MWT: } 190(108.5) \text { to } 226(100.8) \text { to } 236(105.1) \\
\text { vs. } 194(101.1) \text { to } 180(111.1) \text { to } 182(101.1), \\
\text { p=0.026 } \\
\text { PEDI: } 52(12.4) \text { to } 57(11.5) \text { to } 60(10.7) \text { vs. } 51 \\
\text { (14.6) to } 51(15.3) \text { to } 51(15.8), p=0.001 \\
\text { PBS: } 33 \text { (17.5) to } 43(20.1) \text { to } 42(21.3) \text { vs. } 30 \\
\text { (23.9) to } 27(22.2) \text { to } 26(23.2), p=0.002\end{array}$ \\
\hline
\end{tabular}




\begin{tabular}{|c|c|c|c|}
\hline $\begin{array}{l}\text { Author, Year } \\
\text { Intervention } \\
\text { Study Design } \\
\text { Study Quality }\end{array}$ & $\begin{array}{l}\text { Intervention } \\
\text { and Comparison }\end{array}$ & Population & Results \\
\hline $\begin{array}{l}\text { Curtis, } 2018^{152} \\
\text { Postural control } \\
\text { RCT } \\
\text { Fair }\end{array}$ & $\begin{array}{l}\text { A. Trunk control } \\
\text { training: } 120 \\
\text { sessions over } 24 \\
\text { weeks }(n=14) \\
\begin{array}{l}\text { B. Usual care } \\
(n=14)\end{array}\end{array}$ & $\begin{array}{l}\text { A vs. B } \\
\text { Age: } 8 \text { vs. } 8 \\
\text { Female: } 21 \% \text { vs. } 50 \% \\
\text { Spastic: } 50 \% \text { vs. } 64 \% \\
\text { Dyskinetic: } 50 \% \text { vs. } 36 \% \\
\text { GMFCS III: } 14 \% \text { vs. } 21 \% \\
\text { GMFCS IV: } 29 \% \text { vs. } 14 \% \\
\text { GMFCS V: } 57 \% \text { vs. } 64 \%\end{array}$ & $\begin{array}{l}\text { A vs. B, mean difference, } p=\text { between groups: } \\
\text { GMFM-66: } 1.1,95 \% \mathrm{Cl}-2.2 \text { to } 4.4, p>0.05 \\
\text { (postintervention); } 0.1,95 \% \mathrm{Cl}-3.6 \text { to } 3.3, p>0.05 \\
\text { (12-month followup) } \\
\text { SATCo: mean between group difference at end of } \\
\text { treatment and at posttreatment followup: } p>0.05 \\
\text { PEDI Self Care, PEDI Mobility, PEDI Mobility } \\
\text { Caregiver Assistance: mean between group } \\
\text { difference at end of treatment and at posttreatment } \\
\text { followup: } p>0.05\end{array}$ \\
\hline $\begin{array}{l}\text { Kim, } 2017^{155} \\
\text { Postural control } \\
\text { Social } \\
\text { activity/exercise } \\
\text { (Boccia) } \\
\text { Cohort study } \\
\text { Poor }\end{array}$ & $\begin{array}{l}\text { A. Group boccia, } \\
12 \text { sessions over } 6 \\
\text { weeks }(n=11) \\
\text { B. Usual care } \\
(n=12)\end{array}$ & $\begin{array}{l}\text { A vs. B } \\
\text { Age: } 22.36 \text { vs. } 21.83 \\
\text { Female: } 45 \% \text { vs. } 42 \%\end{array}$ & $\begin{array}{l}\text { A vs. } B \text {, mean (SD), } p=\text { between groups: } \\
\text { Modified Barthel Index, mean change from } \\
\text { baseline: } 2.82(S D 1.25) \text { vs. } 1.58 \text { (SD } 1.38) \\
p<0.05 ; \text { MD } 1.24,95 \% \text { Cl } 0.09 \text { to } 2.34, p=0.04\end{array}$ \\
\hline $\begin{array}{l}\text { Lorentzen, } 2015^{154} \\
\text { Postural control } \\
\text { Quasiexperimental } \\
\text { Poor }\end{array}$ & $\begin{array}{l}\text { A. Interactive, } \\
\text { home-based } \\
\text { computer training, } \\
140 \text { sessions over } \\
20 \text { weeks }(n=34) \\
\text { B. Usual care } \\
(n=12)\end{array}$ & $\begin{array}{l}\text { A vs. } B \\
\text { Age: } 10.9 \text { vs. } 11.3 \\
\text { Female: } 32 \% \text { vs. } 42 \% \\
\text { GMFCS I: } 97 \% \text { vs. } 92 \% \\
\text { GMFCS II: } 3 \% \text { vs. } 8 \%\end{array}$ & $\begin{array}{l}\text { A. vs. B, mean (SD), } p=\text { between groups: } \\
\text { Sit-to-stand, number of cycles performed: } 20.0 \\
(0.9) \text { vs, } 15.1(0.9), p=0.04 \\
\text { Left leg lateral step up, number of steps: } 23.5(1.4) \\
\text { vs. } 17.8(2.2), p=0.004 \\
\text { Right leg lateral step up, number of steps: } 22.1 \\
\text { (1.4) vs. } 18.0(2.0), p<0.001 \\
\text { Romberg Balance Test center of gravity } \\
\text { maintenance area (mm2): } 462.2(62.5) \text { vs. } 314.6 \\
(104.9), p=0.18\end{array}$ \\
\hline
\end{tabular}

Abbreviations: 6MWT = 6-Minute Walk Test; CI = confidence interval; GMFCS = Gross Motor Function Classification System; GMFM = Gross Motor Function Measure; GMFM-66 = Gross Motor Function Measure 66; MD = mean difference; PBS = Pediatric Balance Scale; PEDI = Pediatric Evaluation of Disability Inventory; PT = physical therapy; RCT $=$ randomized controlled trial; SATCo=Segmental Assessment of Trunk Control; SD = standard deviation

\section{Balance Exercise-Spinal Cord Injury}

There was insufficient evidence on the benefits and harms of balance training in SCI. One RCT compared the effects of Cawthorne/Cooksey exercises versus conventional therapy on balance in Iranian male veterans with SCI $(\mathrm{n}=20)$ (Table 22). ${ }^{156}$ All participants had L3 to L4 injury. A second trial in SCI patients of various ages with CSI compared dual task exercises to a control group. ${ }^{157}$ Both trials reported improved balance as assessed with the BBS with balance exercises ( $\mathrm{n}=60$, MD $4.53,95 \%$ CI 2.61 to $6.46, \mathrm{I}^{2}=0 \%$ ). The second trial also reported improved coordination and walking speed in the intervention group compared with the control group. ${ }^{157}$ 
Table 22. Balance training in spinal cord injury

\begin{tabular}{|c|c|c|c|}
\hline $\begin{array}{l}\text { Author, Year } \\
\text { Intervention } \\
\text { Study Design } \\
\text { Study Quality }\end{array}$ & $\begin{array}{l}\text { Intervention } \\
\text { and Comparison }\end{array}$ & Population & Results \\
\hline $\begin{array}{l}\text { Hota, } 2020^{157} \\
\text { Postural control } \\
\text { RCT } \\
\text { Fair }\end{array}$ & $\begin{array}{l}\text { A. Dual task } \\
\text { exercises for } \\
\text { upper and lower } \\
\text { limbs, } 24 \text { sessions } \\
\text { over } 4 \text { weeks } \\
(n=20) \\
\text { B. Control group - } \\
\text { details NR, }(n=20)\end{array}$ & $\begin{array}{l}\text { A vs. B } \\
\text { Age } 11-25: 40 \% \text { vs. } 30 \% \\
\text { Age } 26-40: 25 \% \text { vs. } 45 \% \\
\text { Age } 41-55: 25 \% \text { vs. } 25 \% \\
\text { Age } 56-70: 10 \% \text { vs. } 0 \% \\
\text { Female: } 10 \% \text { vs. } 10 \%\end{array}$ & $\begin{array}{l}\text { A vs. B, mean (SD): } \\
\text { BBS: MD } 4.55,95 \% \mathrm{Cl} 2.16 \text { to } 6.94 \\
\frac{\text { Motor Assessment Scale: }}{1.09 \text { to } 6.55, p=0.006} 3.82,95 \% \mathrm{Cl}\end{array}$ \\
\hline $\begin{array}{l}\text { Norouzi, } 2019^{156} \\
\text { Postural control } \\
\text { RCT } \\
\text { Fair }\end{array}$ & $\begin{array}{l}\text { A. Cawthorne/ } \\
\text { Cooksey } \\
\text { exercises, } 12 \\
\text { sessions over } 4 \\
\text { weeks }(n=10) \\
\\
\text { B. Usual care, } 4 \\
\text { sessions over } 4 \\
\text { weeks }(n=10)\end{array}$ & $\begin{array}{l}\text { A vs. B } \\
\text { Age: NR } \\
\text { Female: } 0 \% \\
\text { L3-L4: } 100 \%\end{array}$ & $\begin{array}{l}\text { A vs. B, mean (SD), p-value=between groups } \\
\text { BBS: } 38.36(6.01) \text { to } 48.39(4.01) \text { vs. } 37.67 \\
\text { (6.07) to } 43.20(4.05), M D 4.5,95 \% \mathrm{Cl}-0.17 \text { to } \\
9.17, p=0.059\end{array}$ \\
\hline
\end{tabular}

Abbreviations: $\mathrm{BBS}=$ Berg Balance Scale; $\mathrm{CI}=$ confidence interval; $\mathrm{MD}=$ mean difference; $\mathrm{NR}=$ not reported; $\mathrm{RCT}=$ randomized controlled trial; $\mathrm{SD}=$ standard deviation

\section{Hippotherapy}

Hippotherapy is a type of therapeutic horseback riding that uses the movement of a horse (or a simulated horse) in conjunction with a physical and/or occupational therapist to improve function and stability and other outcomes in people with neuromuscular disease or disability. ${ }^{265}$

\section{Key Points}

- Although data from two fair-quality trials favored hippotherapy over usual care on walking, short-term quality of life, and balance in adults with MS, no firm conclusions can be drawn (SOE: insufficient).

- Low-strength evidence found hippotherapy associated with improved function and balance in CP (SOE: low).

- There were no studies of hippotherapy in SCI.

\section{Detailed Synthesis}

Nine RCTs, ${ }^{158,160-162,164-168}$ two quasiexperimental nonrandomized studies, ${ }^{169,170}$ and one cohort study ${ }^{171}$ involving 567 participants evaluated hippotherapy. Five studies compared hippotherapy versus usual care, ${ }^{158,162,165,169,170}$ four studies compared hippotherapy versus no hippotherapy (either waitlist or inactive hippotherapy simulator), ${ }^{160,161,168,171}$ one trial compared hippotherapy versus home aerobic exercise, ${ }^{164}$ one RCT compared hippotherapy versus outdoor recreation, ${ }^{167}$ and one RCT examined the effects of hippotherapy versus a hippotherapy simulator. ${ }^{166}$ Two RCTs enrolled participants with MS (Table 23), ${ }^{158,160}$ and the remaining 11 studies were in participants with CP (Table 24).

Among the trials, one met criteria for good quality, ${ }^{164}$ six for fair quality, ${ }^{158,160-162,167,168}$ and one was rated poor quality and deemed to have high risk of bias due to unclear reporting of randomization method, allocation concealment, blinding of outcome assessors, and high loss to followup. ${ }^{166}$ One quasiexperimental study was rated fair quality ${ }^{169}$ and one quasiexperimental 
study ${ }^{170}$ and one cohort study ${ }^{171}$ were rated poor quality due to unclear enrollment methods and lack of clear adjustment for prognostic clinical or demographic confounders. The most

frequently reported outcomes were related to function (e.g., GMFM) ${ }^{160-162,164,165,167,169,171}$ and balance (e.g. BBS, Pediatric Balance Scale [PBS]). ${ }^{158,164,166,169-171}$

\section{Hippotherapy-Multiple Sclerosis}

Two trials of hippotherapy enrolled patients with MS, one (MS-HIPPO) with a usual care control group, and the other with a waitlist control group (Table 23). Both trials enrolled more females with similar ages but EDSS scores were higher in one trial, indicating greater disability. ${ }^{158}$

The MS-HIPPO trial found that weekly hippotherapy was associated with significantly greater improvement in quality of life based on MSQoL-54 mental and physical health scores compared with usual care (mean difference $12.0,95 \%$ CI 6.2 to 17.7 and $14.4,95 \%$ CI 7.5 to 21.3). ${ }^{158}$ Balance, measured by BBS score, was also significantly better with hippotherapy versus usual care after 12 weeks (mean difference $2.33,95 \%$ CI 0.03 to 4.63 ) using imputed (last observation carried forward) data. ${ }^{158}$ Subgroup analysis found hippotherapy associated with greater improvement in BBS score in participants with EDSS 5 or greater (vs. usual care, MD $5.1,95 \%$ CI 2.3 to 7.9 ) and in participants age 50 years or older (MD 4.8, 95\% CI 1.8 to 7.8 ) than in those with EDSS less than 5 at baseline (MD $-0.8,95 \% \mathrm{CI}-4.2$ to 2.5 ) or less than age 50 years (MD $1.8,95 \% \mathrm{CI}-1.3$ to 4.9$)$. The other hippotherapy study in patients with MS (n=33) found improved walking distance on the $6 \mathrm{MWT}$ and walking speed on the $25 \mathrm{FWT} .{ }^{160}$ Data from these trials provides insufficient evidence from which to draw firm conclusions.

One trial did not address adverse events, ${ }^{160}$ while the other trial reported similar numbers of participants with any adverse event (43\% vs. $41 \%$; $=0.82)$ and serious adverse events $(3 \%$ vs. $5 \% ; p=0.69)$ in hippotherapy and control groups. Participants in the hippotherapy group were more likely to experience unspecified accidents versus control $(13 \%$ vs. $3 \% ; p=0.14) .{ }^{158}$

Table 23. Hippotherapy in multiple sclerosis

\begin{tabular}{|c|c|c|c|}
\hline $\begin{array}{l}\text { Author, Year } \\
\text { Intervention } \\
\text { Study Design } \\
\text { Study Quality }\end{array}$ & $\begin{array}{l}\text { Intervention } \\
\text { and Comparison }\end{array}$ & Population & Results \\
\hline $\begin{array}{l}\text { Moraes, } 2020^{160} \\
\text { Postural control } \\
\text { RCT } \\
\text { Fair }\end{array}$ & $\begin{array}{l}\text { A. Hippotherapy, } \\
16 \text { sessions over } \\
8 \text { weeks }(n=17) \\
\text { B. Waitlist control } \\
(n=16)\end{array}$ & $\begin{array}{l}\text { A vs. B } \\
\text { Age: } 45.5 \text { vs. } 48.4 \\
\text { Female: } 94 \% \text { vs. } \\
94 \% \\
\text { EDSS, median: } 2.0 \\
\text { vs. } 1.75 \\
\text { RRMS: } 100 \%\end{array}$ & $\begin{array}{l}\text { A vs. B, mean (SD): } \\
\text { 6MWT: } 459.06(118.34) \text { to } 503.59(126.38) \text { vs. } \\
513.00(101.97) \text { to } 497.13(88.88), p<0.001 \\
\text { 25FWT: } 6.37(1.70) \text { to } 5.36(1.43) \text { vs. } 5.82(1.29) \text { to } \\
5.84(1.08), p<0.001\end{array}$ \\
\hline $\begin{array}{l}\text { Vermohlen, } \\
2018^{158} \\
\text { Postural control } \\
\text { RCT } \\
\text { Fair }\end{array}$ & $\begin{array}{l}\text { A. Hippotherapy } \\
\text { plus standard } \\
\text { care, } 12 \text { sessions } \\
\text { over } 12 \text { weeks } \\
(n=32) \\
\text { B. Control group } \\
\text { (standard care), } \\
12 \text { weeks }(n=38)\end{array}$ & $\begin{array}{l}\text { A vs. B } \\
\text { Age (median): } 50 \\
\text { vs. } 51 \\
\text { Female: } 90 \% \text { vs. } \\
73 \% \\
\text { EDSS: } 5.4 \text { vs. } 5.3\end{array}$ & $\begin{array}{l}\text { A vs. B, mean difference, } \mathrm{p}=\text { between groups: } \\
\text { MSQoL-54 mental health subscale score: } 14.4,95 \% \\
\mathrm{Cl} 7.5 \text { to } 21.3, p<0.001 \\
\text { MSQoL-54 physical health subscale score: } 12.0 \text {, } \\
95 \% \mathrm{Cl}: 6.2 \text { to } 17.7, p<0.001 \\
\text { BBS: } 2.33,95 \% \mathrm{Cl}: 0.03 \text { to } 4.63, p=0.047\end{array}$ \\
\hline
\end{tabular}

Abbreviations: 6MWT = 6-Minute Walk Test; 25FWT = 25-Foot Walk Test; BBS = Berg Balance Scale; $\mathrm{CI}=$ confidence interval; EDSS = Expanded Disability Status Scale; MSQoL = Multiple Sclerosis Quality of Life; RCT = randomized controlled trial; $\mathrm{SD}=$ standard deviation 


\section{Hippotherapy-Cerebral Palsy}

Seven RCTs, ${ }^{161,162,164-168}$ two quasiexperimental nonrandomized studies, ${ }^{169,170}$ and one cohort study ${ }^{171}$ enrolled children ( $\left.\mathrm{n}=464\right)$ with CP (Table 24). The trial mean age ranged from 6 to 11 years with a trial mean of 29 to 58 percent female. None of the studies reported race/ethnicity. Two trials ${ }^{169,170}$ specified that they enrolled ambulatory participants but participants' ambulatory status was not reported in the remaining seven studies. Ten studies reported baseline function using the GMFCS. ${ }^{161-165,167-171}$ GMFCS classification at baseline varied widely among the studies (Table 24). Three studies enrolled only participants classified GMFCS Level I or II. ${ }^{168-170}$

Evidence on functional outcomes with hippotherapy in children with CP was based on seven studies (Table 24) and provided low-strength evidence of a benefit of hippotherapy on function versus control groups. ${ }^{161,162,164,165,167,169,171}$ The largest $(n=92)$ and only good-quality trial found significantly higher GMFM-66 scores after 8 weeks (16 sessions) of hippotherapy compared with at-home exercise; GMFM-88 and subscale scores D and E (standing and walking, running, jumping) were also improved with hippotherapy. ${ }^{164}$

The effect of hippotherapy on balance/sitting ability was assessed in four fair- and two poorquality studies, using the PBS ${ }^{164,166,169}$ or Sitting Assessment Scale. ${ }^{161,168,170}$ Most trials compared hippotherapy with usual care or, in one case, a hippotherapy simulator. ${ }^{161}$ These trials provided low-strength evidence for improved balance scores on the PBS in pooled analysis (MD $-3.14,95 \% \mathrm{CI}-5.28$ to $-1.18, \mathrm{I}^{2}=0 \%$ ) (Figure 8 ). Sitting ability was assessed in three studies and showed a benefit in two, ${ }^{168,170}$ but one of the two studies was rated poor quality and in the other study the control group had worse baseline disability (SOE: insufficient).

\section{Figure 8. Pediatric Balance Scale in cerebral palsy trials}

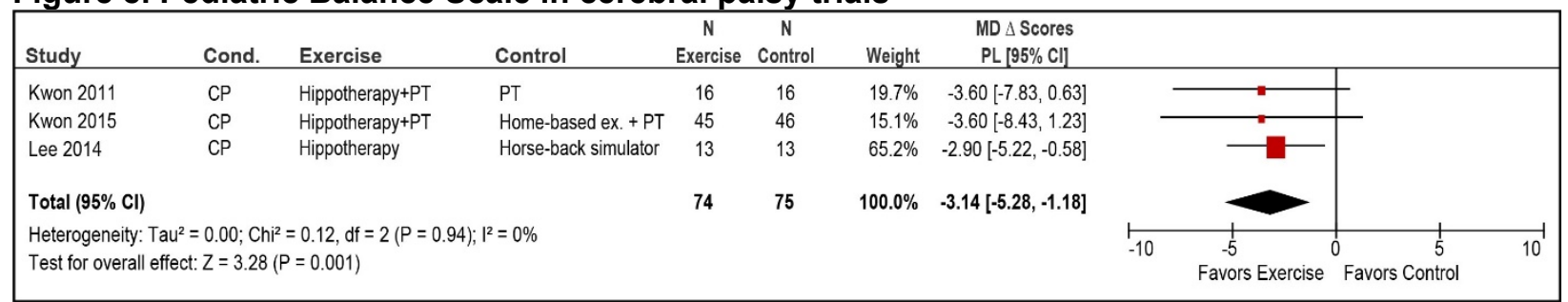

Abbreviations: $\Delta$ = change; $\mathrm{CI}=$ confidence interval; $\mathrm{CP}=$ cerebral palsy; $\mathrm{MD}=$ mean difference; $\mathrm{PL}=$ profile likelihood; $\mathrm{PT}=$ physical therapy

One fair-quality RCT $(\mathrm{n}=24)$ reported improvement in positive feeling and self-esteem scores on the WHOQOL instrument with hippotherapy compared with outdoor recreation, whereas negative feeling scores were similar between groups (Table 24). ${ }^{167}$ The only other evidence on the effect of hippotherapy on quality of life was from a poor-quality, crossover trial of 73 children with CP. ${ }^{165}$ The trial found no difference between hippotherapy and conventional physiotherapy on the Child Health Questionnaire-28 psychosocial or physical subscale scores or on KIDSCREEN-27 parental scale scores. These studies provided insufficient evidence to draw a conclusion on the effects of hippotherapy on quality of life in children with $\mathrm{CP}$.

One cohort study ${ }^{171}(\mathrm{n}=55)$ found ADL as assessed with the Pediatric Evaluation Disability Inventory (PEDI) to favor hippotherapy over waitlist control, but the study had high risk of bias and the data were too limited to draw conclusions.

Six studies did not address harms or adverse events. The good-quality trial reported one fall in the hippotherapy group versus no falls in the control group (2\% [1/46] vs. $0 \%$ [0/46]; OR 3.01, $95 \%$ CI 0.12 to 77 ). A poor-quality crossover study also reported one fracture as a result of a fall from a horse during hippotherapy. ${ }^{165}$ 
One additional trial that reports the effects of hippotherapy on spasticity is found in Table 46, KQ2d. ${ }^{163}$

Table 24. Hippotherapy in cerebral palsy

\begin{tabular}{|c|c|c|c|}
\hline $\begin{array}{l}\text { Author, Year } \\
\text { Intervention } \\
\text { Study Design } \\
\text { Study Quality }\end{array}$ & $\begin{array}{l}\text { Intervention } \\
\text { and Comparison }\end{array}$ & Population & Results \\
\hline $\begin{array}{l}\text { Deutz, } 2018^{165} \\
\text { Postural control } \\
\text { RCT } \\
\text { Poor }\end{array}$ & $\begin{array}{l}\text { A. Hippotherapy, } 16 \\
\text { to } 32 \text { sessions over } \\
16 \text { to } 20 \text { weeks plus } \\
\text { usual physiotherapy } \\
\text { ( } n=35) \\
\text { B. Usual } \\
\text { physiotherapy over } \\
16 \text { to } 20 \text { weeks } \\
\text { ( } n=38) \\
\text { Crossover study }\end{array}$ & $\begin{array}{l}\text { A vs. B } \\
\text { Age: } 9.29 \text { vs. } 8.87 \\
\text { Female: } 34 \% \text { vs. } 45 \% \\
\text { GMFCS II: } 29 \% \text { vs. } 45 \% \\
\text { GMFCS III: } 20 \% \text { vs. } 26 \% \\
\text { GMFCS IV: } 51 \% \text { vs. } 29 \%\end{array}$ & 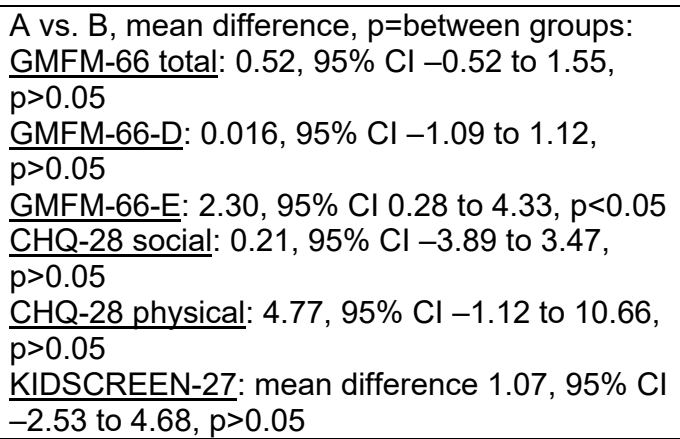 \\
\hline $\begin{array}{l}\text { Herrero, } 2012^{161} \\
\text { Postural control } \\
\text { RCT } \\
\text { Fair }\end{array}$ & $\begin{array}{l}\text { A. Hippotherapy } \\
\text { simulator ON, } 10 \\
\text { sessions over } 10 \\
\text { weeks }(n=19) \\
\text { B. Hippotherapy } \\
\text { simulator OFF, } 10 \\
\text { sessions over } 10 \\
\text { week }(n=19)\end{array}$ & $\begin{array}{l}\text { A vs. B } \\
\text { Age: } 9.95 \text { vs. } 9.05 \\
\text { Female: } 26 \% \text { vs. } 32 \% \\
\text { GMFCS I: } 11 \% \text { vs. } 11 \% \\
\text { GMFCS II: } 11 \% \text { vs. } 5 \% \\
\text { GMFCS III: } 16 \% \text { vs. } 11 \% \\
\text { GMFCS IV: } 16 \% \text { vs. } 21 \% \\
\text { GMFCS V: } 47 \% \text { vs. } 53 \%\end{array}$ & $\begin{array}{l}\text { A vs. B, mean difference, } \mathrm{p}=\text { between groups } \\
\text { GMFM total: } 0.27,95 \% \mathrm{Cl}-0.07 \text { to } 0.62, \mathrm{p}>0.05 \\
\text { GMFM total, } 22 \text { weeks: } 0.25,95 \% \mathrm{Cl}-0.10 \text { to } \\
0.60, \mathrm{p}>0.05 \\
\text { GMFM total: Proportion with improvement from } \\
\text { baseline, } 10 \text { weeks: }(11 / 19) \text { vs. }(8 / 19) ; \text { OR } 1.89 \\
\text { (95\% Cl } 0.5 \text { to } 6.9), p>0.05 \\
\text { GMFM total: Proportion with improvement from } \\
\text { baseline, } 22 \text { weeks: }(10 / 19) \text { vs. }(12 / 19) ; \text { OR } \\
0.65(95 \% \text { Cl } 0.18 \text { to } 2.37), p>0.05 \\
\text { Sitting Assessment Scale: } 0.26(0.65) \text { vs. }-0.21 \\
\text { (0.92), p>0.05 }\end{array}$ \\
\hline $\begin{array}{l}\text { Kwon, 2011'169 } \\
\text { Postural control } \\
\text { Quasiexperimental } \\
\text { Fair }\end{array}$ & $\begin{array}{l}\text { A. Hippotherapy, } 16 \\
\text { sessions over } 8 \\
\text { weeks plus usual } \\
\text { PT, } 16 \text { sessions } \\
\text { over } 8 \text { weeks }(n=16) \\
\text { B. Usual PT, } 16 \\
\text { sessions over } 8 \\
\text { weeks }(n=16)\end{array}$ & $\begin{array}{l}\text { A vs. B } \\
\text { Age: } 6.4 \text { vs. } 6.1 \\
\text { Female: } 31 \% \text { vs. } 38 \% \\
\text { Ambulatory: } 100 \% \\
\text { GMFCS I: } 25 \% \text { vs. } 25 \% \\
\text { GMFCS II: } 75 \% \text { vs. } 75 \%\end{array}$ & $\begin{array}{l}\text { A vs. B, mean (SD), } p=\text { between groups: } \\
\text { GMFM-66: } 70.4(7.4) \text { to } 73.7(8.3) \text { vs. } 69.8(8.7) \\
\text { to } 70.1(8.1), p=0.003 \\
\text { GMFM-88: } 89.4(7.3) \text { to } 91.1(6.7) \text { vs. } 88.0(8.3) \\
\text { to } 88.3(8.4), p=0.054 \\
\text { GMFM-88-D: } 83.2(15.5) \text { to } 83.3(10.9) \text { vs. } 79.6 \\
\text { (15.5) to } 79.3(16.6), p=0.826 \\
\text { GMFM-88-E: } 67.2(17.5) \text { to } 74.6(19.3) \text { vs. } 65.3 \\
\text { (20.0) vs. } 66.9(20.1), p=0.042 \\
\text { PBS: } 41.7(8.8) \text { to } 45.8(8.6) \text { vs. } 41.0(10.4) \text { to } \\
1.5(10.6), p=0.004\end{array}$ \\
\hline $\begin{array}{l}\text { Kwon, } 2015^{164} \\
\text { Balance } \\
\text { RCT } \\
\text { Good }\end{array}$ & $\begin{array}{l}\text { A. Hippotherapy, } 16 \\
\text { sessions over } 8 \\
\text { weeks plus usual PT } \\
(n=46) \\
\text { B. Home-based } \\
\text { aerobic exercise, } 16 \\
\text { sessions over } 8 \\
\text { weeks plus usual PT } \\
(n=46)\end{array}$ & $\begin{array}{l}\text { A vs. B } \\
\text { Age: } 5.7 \text { vs. } 5.9 \\
\text { Female: } 56 \% \text { vs. } 37 \% \\
\text { GMFCS I: } 27 \% \text { vs. } 26 \% \\
\text { GMFCS II: } 27 \% \text { vs. } 26 \% \\
\text { GMFCS III: } 24 \% \text { vs. } 26 \% \\
\text { GMFCS IV: } 22 \% \text { vs. } 22 \% \\
\text { Spastic: } 91 \% \text { vs. } 93 \% \\
\text { Unilateral: } 9 \% \text { vs. } 13 \%\end{array}$ & $\begin{array}{l}\text { A vs. B, mean (SD), } p=\text { between groups: } \\
\text { GMFM-66: } 60.8(14.9) \text { to } 63.5 \text { (15.8) vs. } 61.4 \\
\text { (14.8) to } 61.8(15.0), p<0.01 \\
\text { GMFM-88: } 72.7(19.2) \text { to } 75.7(18.3) \text { vs. } 73.9 \\
\text { (17.9) to } 74.3(18.1), p<0.01 \\
\text { GMFM-88-D: } 54.1(34.2) \text { to } 59.7(32.5) \text { vs. } 55.5 \\
\text { (32.2) to } 54.9(33.2), p<0.01 \\
\text { GMFM-88-E: } 41.0(34.1) \text { to } 45.1(35.4) \text { vs. } 42.0 \\
\text { (33.2) to } 43.0(33.0), p<0.01 \\
\text { PBS: } 25.1(18.9) \text { to } 28.9(18.8) \text { vs. } 26.9(18.3) \\
\text { to } 27.1(18.3), p<0.01\end{array}$ \\
\hline
\end{tabular}




\begin{tabular}{|c|c|c|c|}
\hline $\begin{array}{l}\text { Author, Year } \\
\text { Intervention } \\
\text { Study Design } \\
\text { Study Quality }\end{array}$ & $\begin{array}{l}\text { Intervention } \\
\text { and Comparison }\end{array}$ & Population & Results \\
\hline $\begin{array}{l}\text { Lee, } 2014^{166} \\
\text { Postural control } \\
\text { RCT } \\
\text { Poor }\end{array}$ & $\begin{array}{l}\text { A. Hippotherapy, } 36 \\
\text { sessions over } 12 \\
\text { weeks }(n=13) \\
\text { B. Horseback } \\
\text { riding simulator, } 36 \\
\text { sessions over } 12 \\
\text { weeks }(n=13)\end{array}$ & $\begin{array}{l}\text { A vs. B } \\
\text { Age: } 10.8 \text { vs. } 10.0 \\
\text { Female: } 38 \% \text { vs. } 31 \% \\
\text { Walk > } 10 \text { meters } \\
\text { independently: } 100 \%\end{array}$ & $\begin{array}{l}\text { A vs. } B, \text { mean (SD), } p=\text { between groups } \\
\frac{\text { PBS: } 35.6(3.8) \text { to } 41.2(4.7) \text { vs. } 35.8(4.7) \text { to }}{38.5(5.3), p>0.05}\end{array}$ \\
\hline $\begin{array}{l}\text { Matusiak- } \\
\text { Wieczorek, } \\
2016^{170} \\
\text { Postural control } \\
\text { Quasiexperimental } \\
\text { Poor }\end{array}$ & $\begin{array}{l}\text { A. Hippotherapy, } 12 \\
\text { sessions over } 12 \\
\text { weeks }(n=19) \\
\text { B. Maintain current } \\
\text { activities }(n=20)\end{array}$ & $\begin{array}{l}\text { A vs. B } \\
\text { Age: } 8.42 \text { vs. } 8.3 \\
\text { Female: } 47 \% \text { vs. } 45 \% \\
\text { Ambulatory: } 100 \% \\
\text { Hemiplegia: } 68 \% \text { vs. } \\
75 \% \\
\text { GMFCS I: } 63 \% \text { vs. } 55 \% \\
\text { GMFCS II: } 37 \% \text { vs. } 45 \%\end{array}$ & $\begin{array}{l}\text { A vs. B, mean (SD) } \\
\text { Sitting Assessment Scale: } 14.42(4.39) \text { to } 15.63 \\
\text { (3.65) vs.15.50 (3.14) to } 15.75(3.19), p=0.010\end{array}$ \\
\hline $\begin{array}{l}\text { Matusiak- } \\
\text { Wieczorek, } \\
2020^{168} \\
\text { Postural control } \\
\text { RCT } \\
\text { Fair }\end{array}$ & $\begin{array}{l}\text { A. Hippotherapy, } 24 \\
\text { sessions over } 12 \\
\text { weeks }(n=15) \\
\text { B. Hippotherapy, } 12 \\
\text { sessions over } 12 \\
\text { weeks ( } n=15) \\
\text { C. No hippotherapy } \\
(n=15)\end{array}$ & $\begin{array}{l}\text { A vs. B vs. C } \\
\text { Age: } 7.93 \text { vs. } 7.60 \text { vs. } \\
8.13 \\
\text { Female: } 40 \% \text { vs. } 47 \% \\
\text { vs. } 47 \% \\
\text { GMFCS I: } 67 \% \text { vs. } 80 \% \\
\text { vs. } 47 \% \\
\text { GMFCS II: } 33 \% \text { vs. } 20 \% \\
\text { vs. } 53 \%\end{array}$ & $\begin{array}{l}\text { A vs. B vs. C, mean (SD), } p=\text { between groups } \\
\text { Sitting Assessment Scale: } 10.93 \text { (3.97) to } 13.13 \\
\text { (3.46) vs. } 15.93(4.17) \text { to } 17.27 \text { (2.76) vs. } 14.87 \\
\text { (3.27) to } 15.13(3.36) \\
\text { A vs. C: MD } 1.93,95 \% \text { Cl } 0.94 \text { to } 2.92, p<0.001 \\
\text { B vs. C: MD } 1.06,95 \% \text { Cl } 0.61 \text { to } 1.51, p<0.001 \\
\text { A vs. B: MD } 0.87,95 \% \text { Cl } 0.06 \text { to } 1.69, p=0.036\end{array}$ \\
\hline $\begin{array}{l}\text { Mutoh, } 2019^{167} \\
\text { Postural control } \\
\text { RCT } \\
\text { Fair }\end{array}$ & $\begin{array}{l}\text { A. Hippotherapy, } 48 \\
\text { sessions over } 48 \\
\text { weeks }(n=12) \\
\text { B. Outdoor } \\
\text { recreation } 48 \\
\text { sessions over } 48 \\
\text { weeks }(n=12)\end{array}$ & $\begin{array}{l}\text { A vs. B } \\
\text { Age: } 8 \text { vs. } 9 \\
\text { Female: } 58 \% \text { vs. } 50 \% \\
\text { GMFCS II: } 42 \% \text { vs. } 42 \% \\
\text { GMFCS III: } 58 \% \text { vs. } 58 \%\end{array}$ & $\begin{array}{l}\text { A vs. B, mean (SD), } p=\text { between groups } \\
\text { GMFM-66: } 56.6(9.2) \text { to } 62.8(10.8) \text { vs. } 57.4 \\
\text { (7.9) to } 57.9(9.2), p<0.05 \\
\text { GMFM-66-E: } 45.4(7.0) \text { to } 49.7(7.6) \text { vs. } 46.0 \\
\text { (6.3) to } 46.5(6.6), p<0.05 \\
\text { 5MWT (m/min): } 31.9(10.7) \text { to } 38.8(13.5) \text { vs. } \\
31.1(11.3) \text { to } 32.3(11.6), p<0.05 \\
\text { WHOQOL (positive feelings): } 3.1(1) \text { to } 4.1(1) \\
\text { vs. 3.1 (0.9) to } 3.4(1), p<0.05 \\
\text { WHOQOL (self-esteem): } 2.9(1.2) \text { to } 4.0(0.7) \\
\text { vs. 3.3 (1.1) to } 3.7(0.7), p<0.05 \\
\text { WHOQOL (negative feelings): } 2.9(0.8) \text { to } 2.8 \\
\text { (0.7) vs. } 2.8(0.8) \text { to } 2.8(0.8), p>0.05\end{array}$ \\
\hline $\begin{array}{l}\text { Park, } 2014^{171} \\
\text { Postural control } \\
\text { Cohort } \\
\text { Poor }\end{array}$ & $\begin{array}{l}\text { A. Hippotherapy, } 16 \\
\text { sessions over } 8 \\
\text { weeks }(n=34) \\
\text { B. Waitlist control } \\
(n=21)\end{array}$ & $\begin{array}{l}\text { A vs. B } \\
\text { Age: } 6.68 \text { vs. } 7.76 \\
\text { Female: } 56 \% \text { vs. } 52 \% \\
\text { Bilateral CP: } 94 \% \text { vs. } \\
90 \% \\
\text { GMFCS I: } 24 \% \text { vs. } 29 \% \\
\text { GMFCS II: } 32 \% \text { vs. } 19 \% \\
\text { GMFCS III: } 15 \% \text { vs. } 29 \% \\
\text { GMFCS IV: } 29 \% \text { vs. } 24 \%\end{array}$ & $\begin{array}{l}\text { A vs. B, mean (SD) change from baseline, } \\
p=\text { between groups: } \\
\text { GMFM-66: } 2.93(3.95) \text { vs. } 1.25 \text { (1.99), } p<0.05 \\
\text { PEDI: } 10.89(11.94) \text { vs. } 2.00 \text { (4.93), } p<0.05\end{array}$ \\
\hline
\end{tabular}




\begin{tabular}{|c|c|c|c|}
\hline $\begin{array}{l}\text { Author, Year } \\
\text { Intervention } \\
\text { Study Design } \\
\text { Study Quality }\end{array}$ & $\begin{array}{l}\text { Intervention } \\
\text { and Comparison }\end{array}$ & Population & Results \\
\hline $\begin{array}{l}\text { Silva e Borges, } \\
2011^{162} \\
\text { Postural control } \\
\text { RCT } \\
\text { Fair }\end{array}$ & $\begin{array}{l}\text { A. Riding simulator, } \\
12 \text { sessions over } 6 \\
\text { weeks }(n=20) \\
\text { B. Usual PT, } 12 \\
\text { sessions over } 6 \\
\text { weeks }(n=20)\end{array}$ & $\begin{array}{l}\text { A vs. B } \\
\text { Age: } 5.65 \text { vs. } 5.77 \\
\text { Female: } 60 \% \text { vs. } 55 \% \\
\text { GMFCS II: } 20 \% \\
\text { GMFCS III: } 40 \% \\
\text { GMFCS IV: } 35 \% \\
\text { GMFCS V: } 5 \%\end{array}$ & $\begin{array}{l}\text { A vs. } B, p=\text { between groups: } \\
\text { GMFCS reclassification indicating improved } \\
\text { function: } 25 \%(5 / 20) \text { vs. } 10 \%(2 / 20), p=0.24\end{array}$ \\
\hline
\end{tabular}

Abbreviations: $\mathrm{CI}=$ confidence interval; $\mathrm{CP}=$ cerebral palsy; GMFCS = Gross Motor Function Classification System; GMFM = Gross Motor Function Measure; GMFM-66 = Gross Motor Function Measure 66; GMFM-66-D = Gross Motor Function Measure 66 (standing); GMFM-66-E = Gross Motor Function Measure 66 (walking, running, jumping); GMFM-88 = Gross Motor Function Measure 88; GMFM-88-D = Gross Motor Function Measure 88 (standing); GMFM-88-E = Gross Motor Function Measure 88 (walking, running, jumping); PBS = Pediatric Balance Scale; PEDI = Pediatric Evaluation Disability Inventory; PT = physical therapy; RCT = randomized controlled trial; $\mathrm{SD}=$ standard deviation; WHOQOL $=$ World Health Organization Quality of Life scale

\section{Hippotherapy—Spinal Cord Injury}

No studies were identified.

\section{Tai Chi}

Tai Chi is a form of Chinese martial arts exercise with focused movements and deep breathing that combines balance, core strength, flexibility, and meditation. It can be performed in a standing or seated position and is practiced to improve balance, flexibility, and mindfulness meditation.

\section{Key Points}

- There was insufficient evidence to determine the effect of Tai Chi on quality of life, balance, or other outcomes in participants with MS (SOE: insufficient).

- No Tai Chi studies of participants with CP were identified.

- There was insufficient evidence to determine the effect of wheelchair Tai Chi on quality of life or other outcomes in participants with SCI (SOE: insufficient).

\section{Detailed Synthesis}

Two RCTs ${ }^{172,174}$ and one quasiexperimental study, ${ }^{173}$ with a combined total of 106 participants, evaluated Tai Chi. These included two studies of Tai Chi versus usual care ${ }^{173,174}$ and one trial ${ }^{172}$ of Tai Chi plus psychological classes and PT versus psychological classes and PT without Tai Chi. Two studies ${ }^{172,173}$ enrolled participants with MS (Table 25), and one trial was conducted in participants with SCI. ${ }^{174}$ (Table 26). No studies met criteria for good quality, one trial was rated fair quality, ${ }^{174}$ and two were rated poor quality and deemed to have high risk of bias due to unclear randomization and treatment allocation concealment and a lack of comparability between groups at baseline. Reported outcomes were related to quality of life (e.g., WHOQOL scale), balance (e.g., BBS), and depression (Center for Epidemiological Studies Depression Scale). 


\section{Tai Chi-Multiple Sclerosis}

One poor-quality $\mathrm{RCT}^{172}$ and one poor-quality quasiexperimental study ${ }^{173}$ enrolled participants $(\mathrm{n}=66)$ with MS (Table 25). Trial mean participant ages were 35 and 43 years, mean proportion female was 69 and 100 percent, and no trials reported race. Both studies enrolled participants who could ambulate.

The RCT found twice-weekly Tai Chi plus usual care obtained similar results as usual care on balance, ${ }^{172}$ whereas the quasiexperimental trial found twice-weekly Tai Chi compared with usual care resulted in improved balance and quality of life. ${ }^{173}$ However, the two balance measures were very different and provided insufficient evidence to draw conclusions on the effects of Tai Chi on these outcomes (SOE: insufficient).

Neither study adequately addressed harms or adverse events. The RCT reported a participant in the Tai Chi intervention group withdrew due to unspecified health issues. ${ }^{172}$

Table 25. Tai Chi exercise in multiple sclerosis

\begin{tabular}{|c|c|c|c|}
\hline $\begin{array}{l}\text { Author, Year } \\
\text { Intervention } \\
\text { Study Design } \\
\text { Study Quality }\end{array}$ & $\begin{array}{l}\text { Intervention } \\
\text { and Comparison }\end{array}$ & Population & Results \\
\hline $\begin{array}{l}\text { Azimzadeh, } \\
2015^{172} \\
\text { Postural control } \\
\text { RCT } \\
\text { Poor }\end{array}$ & $\begin{array}{l}\text { A. Tai Chi plus } \\
\text { usual care, } 24 \\
\text { sessions over } 12 \\
\text { weeks ( } n=16) \\
\begin{array}{l}\text { B. Usual care } \\
(n=18)\end{array}\end{array}$ & $\begin{array}{l}\text { A vs. } B \\
\text { Age: } 37.5 \text { vs. } 33 \\
\text { Female: } 100 \% \\
\text { Ambulatory: } 100 \%\end{array}$ & $\begin{array}{l}\text { A vs. B, mean (SD) } \\
\text { BBS: } 52.25(3.39) \text { to } 53.94(2.23) \text { vs. } 53.22(2.23) \\
\text { to } 53.61(2.14) ; \mathrm{MD} 1.39,95 \% \mathrm{Cl}-0.39 \text { to } 3.17 \\
p=0.13\end{array}$ \\
\hline $\begin{array}{l}\text { Burschka, } \\
2014^{173} \\
\text { Postural control } \\
\text { Quasiexperime } \\
\text { ntal } \\
\text { Poor }\end{array}$ & $\begin{array}{l}\text { A. Tai Chi, } 48 \\
\text { sessions } 6 \text { months } \\
(n=15) \\
\begin{array}{l}\text { B. Usual care } \\
(n=17)\end{array}\end{array}$ & $\begin{array}{l}\text { A vs. B } \\
\text { Age: } 42 \text { vs. } 43 \\
\text { Female: } 66 \% \text { vs. } 71 \% \\
\text { Ambulatory: } 100 \% \\
\text { RRMS: } 93 \% \text { vs. } 76 \% \\
\text { SPMS: } 0 \% \text { vs. } 24 \% \\
\text { CIS: } 7 \% \text { vs. } 0 \%\end{array}$ & $\begin{array}{l}\text { A vs. B, mean (SD), } p=\text { between groups: } \\
\text { CES-D: } 12.21(6.66) \text { to } 7.67(5.12) \text { vs. } 13.87 \\
\text { (10.82) to } 16.13(11.99), p<0.05 \\
\text { QLS } 7 \text { item, } 1-7 \text { rating scale, maximum score } 420 \\
\text { points): } 215(25.55) \text { to } 232.57(25.62) \text { vs. } 204.46 \text { to } \\
193.81(36.20), p<0.01 \\
\text { Balance }(14 \text { Balance tasks, measured } 1=\text { achieved } \\
\text { task, } 0=\text { =ailed task): } 8.00(2.83) \text { to } 9.33(2.26) \text { vs. } \\
6.88(4.09) \text { to } 6.53(4.49), p<0.05\end{array}$ \\
\hline
\end{tabular}

Abbreviations: BBS = Berg Balance Scale; CES-D = Center for Epidemiologic Studies Depression Scale; CI = confidence interval; CIS = Clinically Isolated Syndrome; $\mathrm{MD}=$ mean difference; QLS=Questionnaire of Life Satisfaction; RCT = randomized controlled trial; RRMS = relapsing-remitting multiple sclerosis; SPMS = secondary progressive multiple sclerosis; $\mathrm{SD}=$ standard deviation

\section{Tai Chi-Cerebral Palsy}

No studies of Tai Chi in participants with CP were identified.

\section{Tai Chi-Spinal Cord Injury}

One RCT enrolled 40 adults with SCI (Table 26). ${ }^{174}$ The mean age of participants in the trial was 40 and the mean proportion female was 23 percent. All were wheelchair users. The mean baseline rating on the American Spinal Injury Association Impairment Scale was 7.5 (range 2 to 8). This trial found that treatment with wheelchair Tai Chi resulted in improved quality of life scores on the psychological subscale of the WHOQOL-BREF, but there were no differences between wheelchair Tai Chi and usual care on the physical, social, and environmental subscales (SOE: insufficient).

This study reports that there no adverse events occurred during this trial. 
Table 26. Tai Chi exercise in spinal cord injury

\begin{tabular}{|c|c|c|c|}
\hline $\begin{array}{l}\text { Author, Year } \\
\text { Intervention } \\
\text { Study Design } \\
\text { Study Quality }\end{array}$ & $\begin{array}{l}\text { Intervention } \\
\text { and Comparison }\end{array}$ & Population & Results \\
\hline $\begin{array}{l}\text { Qi, 2018b } \\
\text { Postural } \\
\text { control } \\
\text { RCT } \\
\text { Fair }\end{array}$ & $\begin{array}{l}\text { A. Wheelchair Tai } \\
\text { Chi, } 60 \text { sessions } \\
\text { over } 6 \text { weeks } \\
(n=20) \\
\text { B. Usual care } \\
\text { control, }(n=20)\end{array}$ & $\begin{array}{l}\text { A vs. B } \\
\text { Age: } 38.3 \text { vs. } 43.05 \\
\text { Female: } 25 \% \text { vs. } 20 \% \\
\text { Wheelchair user: } 100 \% \\
\text { C6-T1: } 15 \% \text { vs. } 20 \% \\
\text { T2-T5: } 25 \% \text { vs. } 30 \% \\
\text { T6-T12: } 40 \% \text { vs. } 35 \% \\
\text { Below L1: } 20 \% \text { vs. } 15 \%\end{array}$ & $\begin{array}{l}\text { A vs. B, mean (SD), } p=\text { between groups: } \\
\text { WHOQOL-BREF (physical): } 11.40(1.25) \text { to } 11.80 \\
\text { (1.33) vs. } 10.94(1.15) \text { to } 11.09(1.29), p=0.08 \\
\text { WHOQOL-BREF (psychological): } 10.95(1.57) \text { to } \\
12.23(1.65) \text { vs. } 10.87(1.08) \text { to } 11.20(1.33), p=0.01 \\
\text { WHOQOL-BREF (social): } 10.93(1.60) \text { to } 12.40 \\
(1.79) \text { vs. } 10.53(1.29) \text { to } 11.27(1.47), p=0.07 \\
\text { WHOQOL-BREF (environmental): } 10.00(1.72) \text { to } \\
10.65 \text { (1.58) vs. } 9.67 \text { (1.51) to } 10.09(1.77), p=0.28\end{array}$ \\
\hline
\end{tabular}

Abbreviations: RCT = randomized controlled trial; $\mathrm{SD}=$ standard deviation; WHOQOL-Bref = World Health Organization Quality of Life Assessment-BREF

\section{Motion Gaming}

Motion gaming or active video gaming is defined as technology-driven activities that require the game user to be physically active to play the game. ${ }^{266}$

\section{Key Points}

- Four RCTs provided low-strength evidence of improvement in function and balance with motion gaming compared with usual care in trial participants with MS (SOE: low).

- Four RCTs provided low-strength evidence of improved balance with motion gaming compared with use of a mouse, conventional balance exercises, or usual physical activity in participants with $\mathrm{CP}$ (SOE: low).

- One RCT found improved dynamic balance with the T-shirt test in SCI patients with motion gaming versus conventional rehabilitation, but additional evidence is needed to confirm this finding (SOE: insufficient).

\section{Detailed Synthesis}

Fourteen RCTs examined the effects of motion gaming on function, quality of life, and balance..$^{50,51,83,151,175-184}$ Twelve trials met criteria for fair quality ${ }^{50,51,83,151,175-178,181-184}$ and two ${ }^{179,180}$ were rated poor quality and deemed to have high risk of bias due to unclear methods of patient selection and concealment of the allocation, differences in prognostic patient factors between groups at baseline, and high attrition.

\section{Motion Gaming-Multiple Sclerosis}

Six RCTs enrolled 240 adult participants with MS, ${ }^{51,83,151,175-177}$ with a mean age of participants between 32 and 50 years and between 64 and 90 percent female (Table 27). These trials assessed effects on balance compared with no intervention or conventional balance training. 
Table 27. Motion gaming in multiple sclerosis

\begin{tabular}{|c|c|c|c|}
\hline $\begin{array}{l}\text { Author, Year } \\
\text { Intervention } \\
\text { Study Design } \\
\text { Study Quality }\end{array}$ & $\begin{array}{l}\text { Intervention } \\
\text { and Comparison }\end{array}$ & Population & Results \\
\hline $\begin{array}{l}\text { Kalron, } 2016^{51} \\
\text { Postural control } \\
\text { RCT } \\
\text { Fair }\end{array}$ & $\begin{array}{l}\text { A. Balance training using } \\
\text { Caren Integrated Virtual } \\
\text { Reality System with 3D } \\
\text { visual, sound and } \\
\text { proprioception, } 12 \\
\text { sessions over } 6 \text { weeks } \\
(n=15) \\
\text { B. Static postural control, } \\
\text { weight shifting and } \\
\text { perturbation exercises, } 12 \\
\text { sessions over } 6 \text { weeks } \\
(n=15)\end{array}$ & $\begin{array}{l}\text { A vs. B } \\
\text { Age: } 47.3 \text { vs. } 43.9 \\
\text { Female: } 67 \% \text { vs. } 60 \% \\
\text { EDSS: } 4.5 \text { vs. } 3.9\end{array}$ & $\begin{array}{l}\text { A vs. B, mean (SD), } p=\text { between groups: } \\
\text { BBS: } 46.8(9.6) \text { to } 47.9(6.4) \text { vs. } 43.3(7.1) \text { to } \\
44.6(4.9), p=0.56 \\
\text { Four Square Step Test: } 16.2(7.0) \text { to } 12.7(6.4) \\
\text { vs. } 14.2(7.1) \text { to } 11.7(5.9), p=0.361 \\
\text { FES-l: } 36.4(9 / 7) \text { to } 29.4(7.8) \text { vs. } 32.9(10.3) \text { to } \\
28.6(5.8), p=0.021\end{array}$ \\
\hline $\begin{array}{l}\text { Khalil, } 2018^{176} \\
\text { Postural control } \\
\text { RCT } \\
\text { Fair }\end{array}$ & $\begin{array}{l}\text { A. Nintendo Wii balance } \\
\text { board and VR scenarios } \\
\text { with tasks to complete, } 12 \\
\text { sessions over } 6 \text { weeks } \\
(n=16) \\
\text { B. Balance training at } \\
\text { home, } 18 \text { sessions over } 6 \\
\text { weeks ( } n=16)\end{array}$ & $\begin{array}{l}\text { A vs. B } \\
\text { Age: } 39.9 \text { vs. } 34.9 \\
\text { Female: } 75 \% \text { vs. } 63 \% \\
\text { EDSS: } 2.9 \text { vs. } 3.1 \\
\text { RRMS: } 100 \%\end{array}$ & $\begin{array}{l}\text { A vs. B, mean difference between groups: } \\
\text { TUG: } 0.04,95 \% \mathrm{Cl}-2.24 \text { to } 2.32, \mathrm{p}=0.97 \\
\text { 10MWT: } 8.48,95 \% \mathrm{Cl}-5.16 \text { to } 22.12, p=0.21 \\
\text { 3MWT: }-7.11,95 \% \mathrm{Cl}-34.18 \text { to } 19.95, p=0.59 \\
\text { SF-36 PCS: }-11.62,95 \% \mathrm{Cl}-22.27 \text { to }-0.99, \\
\text { p=0.03 } \\
\text { SF-36 MCS: }-13.60,95 \% \mathrm{Cl}-23.66 \text { to }-3.55, \\
\text { FES-I: } 3.86,95 \% \mathrm{Cl}-0.062 \text { to } 8.34, p=0.08 \\
\text { BBS: }-4.52,95 \% \mathrm{Cl}-7.90 \text { to }-1.09, p=0.01\end{array}$ \\
\hline $\begin{array}{l}\text { Nilsagard, } \\
2013^{175} \\
\text { Postural control } \\
\text { RCT } \\
\text { Fair }\end{array}$ & $\begin{array}{l}\text { A. Play games using } \\
\text { Nintendo Wii Fit Plus }{ }^{\circledR} \\
\text { Balance Board for } \\
\text { balance, yoga, strength } \\
\text { and aerobics, } 12 \text { sessions } \\
\text { over } 6 \text { weeks }(n=42) \\
\text { B. No balance exercise } \\
\text { during routine PT }(n=42)\end{array}$ & $\begin{array}{l}\text { A vs. B } \\
\text { Age: } 50.0 \text { vs. } 49.4 \\
\text { Female: } 76 \% \text { vs. } 76 \% \\
\text { Able to walk } 100 \mathrm{~m} \text { : } \\
100 \% \\
\text { RRMS: } 62 \% \text { vs. } 67 \% \\
\text { SPMS: } 31 \% \text { vs. } 31 \% \\
\text { PPMS: } 7 \% \text { vs. } 2 \% \\
\text { No assist device } \\
\text { indoors: } 76 \% \text { vs. } 88 \% \\
\text { No assist device } \\
\text { outdoors: } 52 \% \text { vs. } 50 \% \\
\end{array}$ & $\begin{array}{l}\text { A vs. B, mean (SD) change at followup, } \\
\text { p=between groups: } \\
\text { TUG: }-0.8(2.4) \text { vs. } 0.1(2.1), p=0.10 \\
\text { 25footWT: }-0.3(1.1) \text { vs. }-0.1(1.4), p=0.51 \\
\text { DGl: } 1.78(2.3) \text { vs. } 1.0(2.0), p=0.21 \\
\text { MS Walking Scale: }-5.9(11.5) \text { vs. }-3.95(18.1), \\
p=0.76 \\
\text { Four Square Step Test: }-1.6(2.1) \text { vs. }-2.0(6.6) \\
p=0.64\end{array}$ \\
\hline $\begin{array}{l}\text { Ozkul, } 2020^{151} \\
\text { Postural control } \\
\text { RCT } \\
\text { Fair }\end{array}$ & $\begin{array}{l}\text { A. Immersive virtual } \\
\text { reality, } 16 \text { sessions over } 8 \\
\text { weeks }(n=13) \\
\text { B. Relaxation exercises at } \\
\text { home, } 16 \text { sessions over } 8 \\
\text { weeks }(n=13)\end{array}$ & $\begin{array}{l}\text { A vs. B } \\
\text { Age: } 29 \text { vs. } 34 \\
\text { Female: } 69 \% \text { vs. } 77 \% \\
\text { EDSS median: } 1 \text { vs. } 2 \\
\text { Number of relapses: } 3 \\
\text { vs. } 2\end{array}$ & $\begin{array}{l}\text { Pre-post median (IQR): } \\
\text { BBS: } 52(42.5,56) \text { to } 54(44.5,56) \text { vs. } 55(53 \text {, } \\
56) \text { to } 56(53.5,56), p>0.05 \\
\frac{\text { TUG: } 7.6(6.9,8) \text { to } 6.3(5.7,7.2) \text { vs. } 6.9(6.5 \text {, }}{7.5) \text { to } 7.4(6.4,7.7), p<0.017}\end{array}$ \\
\hline $\begin{array}{l}\text { Tollar, } 2020^{83} \\
\text { Postural control } \\
\text { RCT } \\
\text { Fair }\end{array}$ & $\begin{array}{l}\text { A. Xbox } 360, \text { Adventure } \\
\text { video game, } 25 \text { sessions } \\
\text { over } 5 \text { weeks }(n=14) \\
\text { B. Usual PT, } 25 \text { sessions } \\
\text { over } 5 \text { weeks }(n=12)\end{array}$ & $\begin{array}{l}\text { A vs. } \mathrm{B} \\
\text { Age: } 48.2 \text { vs. } 44.4 \\
\text { Female: } 86 \% \text { vs. } 92 \% \\
\text { EDSS median: } 5.0 \text { vs. } \\
5.0 \\
\text { RRMS: } 50 \% \text { vs. } 67 \%\end{array}$ & $\begin{array}{l}\text { A vs. B, mean difference between groups: } \\
\text { MSIS-29: }-10.8(6.09) \text { vs. } 1.0(3.46), p<0.001 \\
\text { 6MWT: } 57.4(52.09) \text { vs. } 6.3(49.27), p=0.017 \\
\text { BBS: } 6.1(3.52) \text { vs. }-0.2(2.62), p<0.001 \\
\text { EQ-5 Sum score:- }-2.3(1.44) \text { vs. } 0.0(1.13), \\
\text { p<0.001 }\end{array}$ \\
\hline
\end{tabular}




\begin{tabular}{|c|c|c|c|}
\hline $\begin{array}{l}\text { Author, Year } \\
\text { Intervention } \\
\text { Study Design } \\
\text { Study Quality }\end{array}$ & $\begin{array}{l}\text { Intervention } \\
\text { and Comparison }\end{array}$ & Population & Results \\
\hline $\begin{array}{l}\text { Yazgan, } \\
2020^{177} \\
\text { Postural control } \\
\text { RCT } \\
\text { Fair }\end{array}$ & $\begin{array}{l}\text { A. Nintendo Wii Fit, } 16 \\
\text { sessions over } 8 \text { weeks } \\
(n=15) \\
\text { B. Balance Trainer motion } \\
\text { gaming, } 16 \text { sessions over } \\
8 \text { weeks }(n=12) \\
\text { C. Waitlist control }(n=15)\end{array}$ & $\begin{array}{l}\text { A vs. B vs. C } \\
\text { Age: } 47.5 \text { vs. } 43.1 \text { vs. } \\
40.7 \\
\text { Female: } 86.7 \% \text { vs. } \\
100 \% \text { vs. } 86.7 \% \\
\text { EDSS: } 4.16 \text { vs. } 3.83 \text { vs. } \\
4.06 \\
\text { RRMS: } 73.3 \% \text { vs. } \\
66.7 \% \text { vs. } 93.3 \%\end{array}$ & $\begin{array}{l}\text { A vs. } C \text {, mean change scores: } \\
\text { BBS: } 5.8 \text { vs. } 0.93, p<0.05 \\
\text { TUG: }-1.54 \text { vs; } 0.05, p<0.05 \\
\text { 6MWT: } 42.71 \text { vs. } 7.59 p<0.05 \\
\text { MusiQoL: } 12.61 \text { vs. }-0.19, p<0.05 \\
\text { B vs. C, mean change scores: } \\
\text { BBS: } 2.66 \text { vs. } 0.93, p<0.05 \\
\text { TUG: }-0.64 \text { vs; } 0.05, p<0.05 \\
\text { 6MWT: } 23.25 \text { vs. } 7.59 p>0.05 \\
\text { MusiQoL: } 5.32 \text { vs. }-0.19, p<0.05 \\
\text { A vs. C, mean change scores: } p<0.05 \text { in favor of } \\
\text { group A for BBS and MusiQoL }\end{array}$ \\
\hline
\end{tabular}

Abbreviations: 3MWT = 3-Minute Walk Test; 6MWT = 6-Minute Walk Test; 10MWT = 10-Meter Walk Test; BBS = Berg Balance Scale; CI = confidence interval; DGI = Dynamic Gait Index; EDSS = Expanded Disability Status Scale; EQ-5 = EuroQuality of Life; FES-I = Falls Efficacy Scale-International; IQR = interquartile range; MS = multiple sclerosis; MSIS-29 = Multiple Sclerosis Impact Scale; MusiQoL = Multiple Sclerosis International Quality of Live Questionnaire; SF-36 MCS = Short-Form 36 Mental Component Summary; SF-36 PCS = Short-Form 36 Physical Component Score PPMS = primary progressive multiple sclerosis; $\mathrm{PT}=$ physical therapy; $\mathrm{RCT}=$ randomized controlled trial; RRMS = relapsing-remitting multiple sclerosis; SD = standard deviation; SPMS = secondary progressive multiple sclerosis; SF-36 = Short Form-36; TUG = Timed Up and Go Test; VR = virtual reality

Four RCTs ( $\mathrm{n}=177)$ provided low-strength evidence of improvement with motion gaming versus usual care on function in MS as measured with the 6MWT ( 2 studies, $\mathrm{n}=68, \mathrm{MD}-30.90$, $95 \% \mathrm{CI}-49.55$ to $-12.25, \mathrm{I}^{2}=14 \%$ ) and the TUG ( 3 studies, $\mathrm{n}=152, \mathrm{MD}-1.06,95 \% \mathrm{CI}-1.43$ to $\left.-0.69, \mathrm{I}^{2}=0 \%\right){ }^{51,83,151,175-177}$ Three studies provided low-strength evidence of improvement with motion gaming on balance ( $\mathrm{n}=94, \mathrm{MD}-3.43,95 \% \mathrm{CI}-6.30$ to $\left.-0.57, \mathrm{I}^{2}=89 \%\right){ }^{83,151,177}$ Evidence was insufficient to draw conclusions regarding the effects of motion gaming compared with different balance exercises on function, balance, and quality of life.

Three trials did not mention adverse events, ${ }^{83,151,177}$ one trial reported that there were no harms or adverse events,${ }^{51}$ one trial reported that there were no serious adverse events but did not report adverse events considered not serious, ${ }^{176}$ and one trial reported 10 falls in the exercise group compared with 14 in the control group - none occurring during balance exercises, and no other adverse events. ${ }^{175}$

\section{Motion Gaming-Cerebral Palsy}

Seven RCTs assessed 237 children with CP (Table 28). ${ }^{50,178-183}$ For these trials, mean participant age ranged between 7 and 11 years old, and the proportion of girls ranged between 27 and 100 percent. Four trials primarily focused on upper extremity movements ${ }^{50,179-181}$ while two used a balance system ${ }^{178,182}$ and one used a virtual dance game. ${ }^{183}$ 
Table 28. Motion gaming in cerebral palsy

\begin{tabular}{|c|c|c|c|}
\hline $\begin{array}{l}\text { Author, Year } \\
\text { Intervention } \\
\text { Study Design } \\
\text { Study Quality }\end{array}$ & $\begin{array}{l}\text { Intervention } \\
\text { and Comparison }\end{array}$ & Population & Results \\
\hline $\begin{array}{l}\text { Acar, } 2016^{179} \\
\text { Postural control } \\
\text { RCT } \\
\text { Poor }\end{array}$ & $\begin{array}{l}\text { A. Nintendo Wii gaming } \\
\text { plus neuro-developmental } \\
\text { treatment, } 12 \text { sessions } \\
\text { over } 6 \text { weeks }(n=15) \\
\text { B. Neurodevelopmental } \\
\text { treatment, } 12 \text { sessions } \\
\text { over } 6 \text { weeks }(n=15)\end{array}$ & $\begin{array}{l}\text { A vs. B } \\
\text { Age: } 9.5 \text { vs. } 9.7 \\
\text { Female: } 47 \% \text { vs. } 60 \% \\
\text { GMFCS I: } 40 \% \text { vs. } 40 \% \\
\text { GMFCS II: } 60 \% \text { vs. } 60 \% \\
\text { Spastic hemiparesis: } \\
100 \%\end{array}$ & $\begin{array}{l}\text { A vs. B, mean (SD), p=between groups } \\
\text { WeeFIM: } 46.0(8.23) \text { to } 46.751(7.51) \text { vs. } \\
\text { 48.3 (7.27) to } 48.0(7.14), p>0.05 \\
\text { QUEST (dissociated movement): } 80.1 \\
\text { (7.73) to } 85.6(8.54) \text { vs. } 81.4(10.70) \text { to } 86.4 \\
\text { (8.78), p>0.05 } \\
\text { QUEST (grasp): } 42.2(18.76) \text { to } 47.1(16.64) \\
\text { vs. 53.0 (16.45) to } 55.7(15.30), p>0.05 \\
\text { QUEST (weight bearing): } 60.2 \text { to } 72.7 \\
\text { (19.60) vs. } 75.4(19.97) \text { to } 77.3(15.43), \\
\text { p>0.05 } \\
\text { QUEST (extension): } 72.9(14.78) \text { to } 77.0 \\
(12.05) \text { vs. } 71.0(23.53) \text { to } 74.0(23.36), \\
\text { p>0.05 }\end{array}$ \\
\hline $\begin{array}{l}\text { El-Shamy, } \\
2018^{181} \\
\text { Postural control } \\
\text { RCT } \\
\text { Fair }\end{array}$ & $\begin{array}{l}\text { A. Arm exoskeletal + virtual } \\
\text { reality } 36 \text { sessions over } 12 \\
\text { weeks }(n=15) \\
\text { B. Conventional therapy, } \\
36 \text { sessions over } 12 \text { weeks } \\
(n=15)\end{array}$ & $\begin{array}{l}\text { A vs. B } \\
\text { Age: } 7 \text { vs. } 7 \\
\text { Female } 40 \% \text { vs. } 27 \% \\
\text { Mobile Ability } \\
\text { Classification } \\
\text { I: } 33 \% \text { vs. } 40 \% \\
\text { II: } 53 \% \text { vs. } 40 \% \\
\text { III: } 13 \% \text { vs. } 20 \%\end{array}$ & $\begin{array}{l}\text { A vs. } B \text {, mean (SD), } p=\text { between groups } \\
\text { QUEST total: } 61.9(2) \text { to } 84.6(2.7) \text { vs. } 62.3 \\
\begin{array}{l}(1.8) \text { to } 79.1 \\
\text { p<0.05 }\end{array}\end{array}$ \\
\hline $\begin{array}{l}\text { Hsieh, } 2018^{50} \\
\text { Postural control } \\
\text { RCT } \\
\text { Fair }\end{array}$ & $\begin{array}{l}\text { A. PC gaming using arm } \\
\text { and trunk, } 60 \text { sessions } \\
\text { over } 12(n=20) \\
\text { B. PC gaming using } \\
\text { mouse, } 60 \text { sessions over } \\
12 \text { weeks }(n=20)\end{array}$ & $\begin{array}{l}\text { A vs. B } \\
\text { Age: } 7.3 \text { vs. } 7.4 \\
\text { Female: } 30 \% \text { vs. } 25 \% \\
\text { Quadriplegia: } 55 \% \text { vs. } \\
60 \% \\
\text { Diplegia: } 20 \% \text { vs. } 15 \% \\
\text { Athetoid: } 10 \% \text { vs. } 10 \% \\
\text { Ataxic: } 15 \% \text { vs. } 15 \%\end{array}$ & 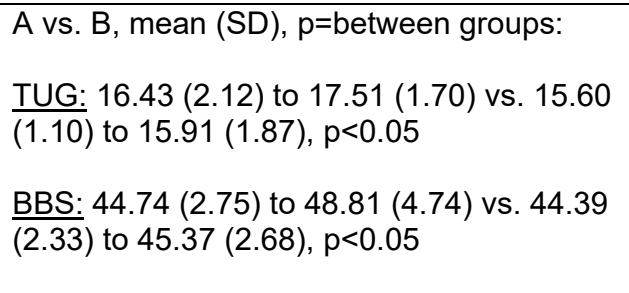 \\
\hline $\begin{array}{l}\text { Hsieh, } 2020^{182} \\
\text { Postural control } \\
\text { RCT } \\
\text { Fair }\end{array}$ & $\begin{array}{l}\text { A. PC gaming using } \\
\text { balance board, } 36 \text { sessions } \\
\text { over } 12 \text { weeks }(n=28) \\
\text { B. PC gaming using } \\
\text { mouse, } 36 \text { sessions over } \\
12 \text { weeks }(n=28)\end{array}$ & $\begin{array}{l}\text { A vs. B } \\
\text { Age: } 7.9 \text { vs. } 8.1 \\
\text { Female: } 32 \% \text { vs. } 31.5 \% \\
\text { GMFCS I: } 53.5 \% \text { vs. } 50 \% \\
\text { GMFCS II: } 28.6 \% \text { vs. } \\
32.1 \% \\
\text { GMFCS III: } 17.9 \% \text { vs. } \\
17.9 \% \\
\text { Deplegic: } 57.1 \% \text { vs. } \\
42.9 \%\end{array}$ & $\begin{array}{l}\text { A vs. B, mean (SD) } \\
\text { 2MWT: } 103.4(16.6) \text { to } 120.1(20.2) \text { vs. } \\
101.4(23.1) \text { to } 106.1(22.8), p=0.002 \\
\text { PBS-total: } 29.9(5.3) \text { to } 35.8(5.5) \text { vs. } 32.3 \\
\text { (7.5) to } 34.4(5.9), p=0.002\end{array}$ \\
\hline $\begin{array}{l}\text { Pourazar, } \\
2020^{183} \\
\text { Postural control } \\
\text { RCT } \\
\text { Fair }\end{array}$ & $\begin{array}{l}\text { A. Virtual reality Microsoft } \\
\text { Xbox } 360 \text { Kinect, } 20 \\
\text { sessions over } 6 \text { weeks } \\
(n=10) \\
\text { B. Encouraged to do } \\
\text { typical physical activity at } \\
\text { home }(n=10)\end{array}$ & $\begin{array}{l}\text { A vs. B } \\
\text { Age: } 9.2 \text { vs. } 9.6 \\
\text { Female: } 100 \% \\
\text { GMFCS I: } 50 \% \text { vs. } 60 \% \\
\text { GMFCS II: } 20 \% \text { vs. } 30 \% \\
\text { GMFCS III: } 30 \% \text { vs. } 10 \%\end{array}$ & $\begin{array}{l}\text { Dynamic balance was improved in the } \\
\text { anterior, posterolateral, and posteromedial } \\
\text { directions with virtual reality dance game } \\
\text { compare with the control group, } p=0.001 \text { all } \\
\text { comparisons }\end{array}$ \\
\hline
\end{tabular}




\begin{tabular}{|c|c|c|c|}
\hline $\begin{array}{l}\text { Author, Year } \\
\text { Intervention } \\
\text { Study Design } \\
\text { Study Quality }\end{array}$ & $\begin{array}{l}\text { Intervention } \\
\text { and Comparison }\end{array}$ & Population & Results \\
\hline $\begin{array}{l}\text { Tarakci, } \\
2016^{178} \\
\text { Postural control } \\
\text { RCT } \\
\text { Fair }\end{array}$ & $\begin{array}{l}\text { A. Nintendo Wii-Fit } \\
\text { balanced gaming, } 24 \\
\text { sessions over } 12 \text { weeks } \\
(n=15) \\
\text { B. Conventional balance } \\
\text { training, } 24 \text { sessions over } \\
12 \text { weeks }(n=15)\end{array}$ & $\begin{array}{l}\text { A vs. B } \\
\text { Age: } 10.5 \text { vs. } 10.5 \\
\text { Female: } 33 \% \text { vs. } 40 \% \\
\text { Hemiplegic: } 47 \% \text { vs. } 47 \% \\
\text { Diplegic: } 47 \% \text { vs. } 33 \% \\
\text { Dyskinetic: } 7 \% \text { vs. } 20 \% \\
\text { Assist devices: } 0 \% \text { vs. } \\
20 \%\end{array}$ & 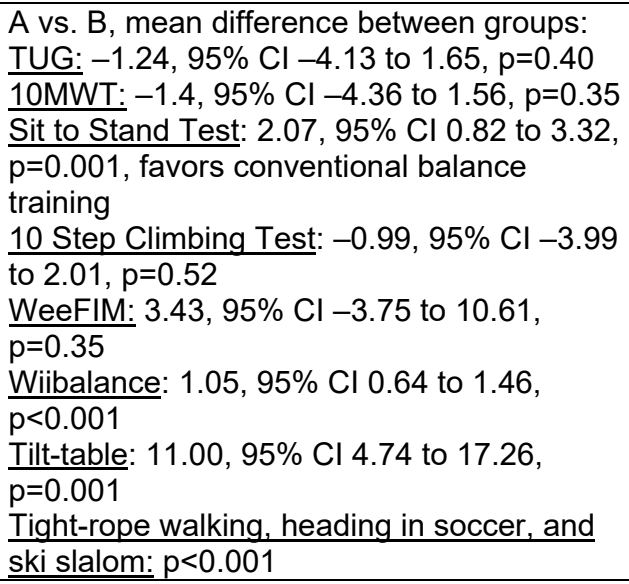 \\
\hline $\begin{array}{l}\text { Zoccolillo, } \\
2015^{180} \\
\text { Postural control } \\
\text { RCT } \\
\text { Poor }\end{array}$ & $\begin{array}{l}\text { A. Microsoft Xbox with } \\
\text { Kinect (3D motion capture) } \\
\text { gaming plus neuro- } \\
\text { developmental treatment, } \\
16 \text { sessions over } 8 \text { weeks } \\
(n=15) \\
\text { B. Neurodevelopmental } \\
\text { treatment, } 16 \text { sessions } \\
\text { over } 8 \text { weeks }(n=16)\end{array}$ & $\begin{array}{l}\text { No demographics by } \\
\text { group } \\
\text { Age: } 6.89 \\
\text { Female: NR } \\
\text { GMFM-88: } 84.6\end{array}$ & $\begin{array}{l}\text { A vs. } B, \text { mean (SD), } p=\text { between groups: } \\
\text { QUEST: } 76(21) \text { to } 81(20) \text { vs. } 74(20) \text { to } 78 \\
(20), p>0.05\end{array}$ \\
\hline
\end{tabular}

Abbreviations: 10MWT = 10-Meter Walk Test; BBS = Berg Balance Scale; CI = confidence interval; FIM = Functional Independence Measure; GMFCS = Gross Motor Function Classification System; GMFM-88 = Gross Motor Function Measure 88; $\mathrm{MD}=$ mean difference; NR = not reported; QUEST $=$ Quality of Upper Extremity Skills Test RCT $=$ randomized controlled trial; $\mathrm{SD}=$ standard deviation; TUG = Timed Up and Go Test; WeeFIM = Wee-Functional Independence Measure for children

Four fair-quality $\mathrm{RCTs}^{50,178,182,183}$ provided low-strength evidence of improved balance with motion gaming, although the specific gaming interventions studied varied (Table 28). Changes in other outcomes (TUG, 10MWT, 10-Step Walking Test) after treatment were not different between groups, were inconsistent, or favored conventional balance training (SOE: insufficient).

Three trials of children with CP evaluated upper extremity function with interventions using a Wii ${ }^{179,181}$ and a Microsoft Xbox. ${ }^{180}$ Two trials did not show an advantage over traditional neurodevelopmental PT, ${ }^{179,180}$ while the third $(\mathrm{n}=30)$ found the quality of upper extremity movement improved with virtual reality plus an exoskeleton versus conventional rehabilitation. ${ }^{181}$ Due to low quality of included studies and small sample sizes, the evidence for improved quality of upper extremity movements is too limited to draw conclusions (SOE: insufficient).

Six RCTs did not address harms or adverse events. One RCT reported that there were no adverse events during the study. ${ }^{223}$

\section{Motion Gaming-Spinal Cord Injury}

One RCT $(\mathrm{n}=26)$ demonstrated reduced time to put on a t-shirt, as a measure of dynamic balance, after Nintendo Wii training, in conjunction with conventional therapy compared with conventional therapy alone, but additional evidence is needed to confirm these results (SOE: insufficient) (Table 29). ${ }^{184}$

This study did not address adverse events or harms. 
Table 29. Motion gaming in spinal cord injury

\begin{tabular}{|c|c|c|c|}
\hline $\begin{array}{l}\text { Author, Year } \\
\text { Intervention } \\
\text { Study Design } \\
\text { Study Quality }\end{array}$ & $\begin{array}{l}\text { Intervention } \\
\text { and Comparison }\end{array}$ & Population & Results \\
\hline $\begin{array}{l}\text { Tak, } 2015^{184} \\
\text { Postural control } \\
\text { RCT } \\
\text { Fair }\end{array}$ & $\begin{array}{l}\text { A. Nintendo Wii, } 18 \\
\text { sessions over } 6 \text { weeks } \\
+ \text { conventional } \\
\text { rehabilitation }(n=13) \\
\text { B. Conventional } \\
\text { rehabilitation }(n=13)\end{array}$ & $\begin{array}{l}\text { A vs. B } \\
\text { Age: } 50 \text { vs. } 43 \\
\text { Cervical: } 31 \% \text { vs. } 38 \% \\
\text { ASIA (A): } 77 \% \text { vs. } 77 \% \\
\text { ASIA (B): } 23 \% \text { vs. } 23 \%\end{array}$ & $\begin{array}{l}\text { A vs. B mean (SD), } p=\text { between groups } \\
\text { T-shirt test (s): } 29.5(10.95) \text { to } 22.60 \\
(8.28) \text { vs. } 23.59(11.35) \text { to } 22.15 \\
(12.28), p<0.05\end{array}$ \\
\hline
\end{tabular}

Abbreviations: ASIA = American Spinal Injury Association Impairment Scale; RCT = randomized controlled trial; $\mathrm{SD}=$ standard deviation

\section{Whole Body Vibration}

Whole body vibration is performed in a PT setting using a vibration board upon which a person can stand, sit, or lie for a set period of time, either passively or while performing active movements, depending on the ability of a participant to move on the vibration board.

There is not a standard frequency or amplitude level for vibration therapy, and more research is needed to establish what vibration frequency is most beneficial for this form of exercise.

\section{Key Points}

- Evidence for WBV on function, balance, and quality of life in patients with MS is too sparse to draw conclusions (SOE: insufficient).

- Two trials provided insufficient evidence on the effect on walking with WBV in children with CP (SOE: insufficient).

- One trial provided insufficient evidence for improved function in patients with SCI (SOE: insufficient).

\section{Detailed Synthesis}

Five RCTs evaluated the effects of WBV in participants with MS, ${ }^{185,186} \mathrm{CP},{ }^{187,188}$ and SCI. ${ }^{189}$ All trials met criteria for fair quality.

\section{Whole Body Vibration-Multiple Sclerosis}

Two RCTs ( $\mathrm{n}=93$ ) studied WBV exercise in participants with MS (Table 30). ${ }^{185,186}$ All RCTs had intervention groups who performed exercises on the vibration board with a vibration level of $20 \mathrm{~Hz}^{186}$ or $30-40 \mathrm{~Hz} \cdot{ }^{185}$ One RCT had the participants perform active exercises on the vibration board, while the control group performed the exercises on a stable surface. ${ }^{185}$ The second RCT had a no intervention control group. ${ }^{186}$

One RCT provided insufficient evidence to draw conclusions on the effects of WBV versus usual care on function, including walking. ${ }^{185}$ One RCT found improvements in quality of life using the MSQoL-54 instrument ${ }^{186}$ but additional evidence is needed to confirm this finding (SOE: insufficient).

None of the trials addressed harms or adverse events. 
Table 30. Whole body vibration exercise in multiple sclerosis

\begin{tabular}{|c|c|c|c|}
\hline \begin{tabular}{|l} 
Author, Year \\
Intervention \\
Study Design \\
Study Quality
\end{tabular} & $\begin{array}{l}\text { Intervention and } \\
\text { Comparison }\end{array}$ & Population & Results \\
\hline $\begin{array}{l}\text { Abbasi, } 2019^{186} \\
\text { Postural control } \\
\text { RCT } \\
\text { Fair }\end{array}$ & $\begin{array}{l}\text { A. WBV, } 18 \text { sessions } \\
\text { over } 6 \text { weeks }(n=22) \\
\begin{array}{l}\text { B. No intervention } \\
(n=24)\end{array}\end{array}$ & $\begin{array}{l}\text { A vs. B } \\
\text { Age: } 37 \text { vs. } 39 \\
\text { Female: } 5 \% \text { vs. } 17 \% \\
\text { EDSS: } 1.54 \text { vs. } 1.55\end{array}$ & $\begin{array}{l}\text { A vs. B, median (IQR) followup baseline } \\
\text { scores, } p=\text { between groups: } \\
\text { MSQOL-54 (PCS): } 4.20(1.73,8.40) \text { vs. - } \\
1.26(-3.28,0), p<0.001 \\
\frac{\text { MSQOL-54 (MCS): } 5.96(2.71,11.89) \text { vs. - }}{0.17(-2.20,0.07), p<0.001}\end{array}$ \\
\hline $\begin{array}{l}\text { Claerbout, } \\
2012^{185} \\
\text { Postural control } \\
\text { RCT } \\
\text { Fair }\end{array}$ & $\begin{array}{l}\text { A. WBV, } 10 \text { sessions } \\
\text { over } 3 \text { weeks plus } \\
\text { conventional therapy } \\
(n=16) \\
\text { B. Whole body light } \\
\text { vibration, } 10 \text { sessions } \\
\text { over } 3 \text { weeks plus } \\
\text { conventional therapy } \\
(n=14) \\
\text { C. Conventional therapy } \\
(n=17)\end{array}$ & $\begin{array}{l}\text { A vs. B vs. C } \\
\text { Age: } 39.1 \text { vs. } 43.8 \text { vs. } 47.6 \\
\text { Female: } 28.6 \% \text { vs. } 22.2 \% \text { vs. } \\
64.7 \% \\
\text { EDSS: } 5.3 \text { vs. } 5.1 \text { vs. } 5.2\end{array}$ & 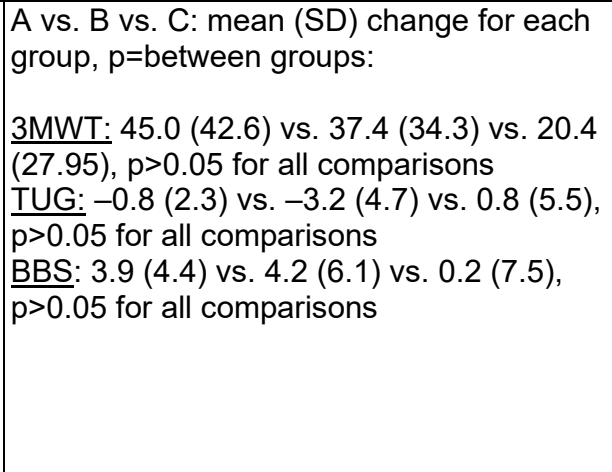 \\
\hline
\end{tabular}

Abbreviations: 3MWT = 3-Minute Walk Test; 6MWT = 6-Minute Walk Test; BBS = Berg Balance Scale; EDSS = Expanded Disability Status Scale; IQR = interquartile range; MSQoL = Multiple Sclerosis Quality of Life; RCT = randomized controlled trial; RRMS = relapsing-remitting multiple sclerosis; $\mathrm{SD}=$ standard deviation; TUG $=$ Timed Up and Go Test; WBV = whole body vibration

\section{Whole Body Vibration-Cerebral Palsy}

Two RCTs ${ }^{187,188,267}(\mathrm{n}=50)$ studied WBV as an intervention for children with CP, providing insufficient evidence on improvement in walking with WBV (Table 31) due to heterogeneous outcomes reported and inconsistency in direction of findings. One ${ }^{187}$ found improved walking speed with vibration therapy where the participants stood or squatted on a vibration board and re

ceived varying frequencies of vibration from $5-25 \mathrm{~Hz}$. The second trial ${ }^{188}$ found that walking distance was significantly improved in the control group that stood on the nonvibrating device compared with the group that received 18 minutes of standing WBV (20 to $24 \mathrm{~Hz}$ ) per training day. ${ }^{188}$

The trials did not address harms or adverse events. 
Table 31. Whole body vibration exercise in cerebral palsy

\begin{tabular}{|c|c|c|c|}
\hline $\begin{array}{l}\text { Author, Year } \\
\text { Intervention } \\
\text { Study Design } \\
\text { Study Quality }\end{array}$ & $\begin{array}{l}\text { Intervention and } \\
\text { Comparison }\end{array}$ & Population & Results \\
\hline $\begin{array}{l}\text { Ahmadizadeh, } \\
2020188 \\
\text { Postural control } \\
\text { RCT } \\
\text { Fair }\end{array}$ & $\begin{array}{l}\text { A. WBV + stretching, } 18 \\
\text { sessions over } 6 \text { weeks } \\
(n=10) \\
\text { B. Stretching only, } 16 \\
\text { sessions over } 6 \text { weeks } \\
(n=10)\end{array}$ & $\begin{array}{l}\text { A vs. B } \\
\text { Age: } 6.9 \text { vs. } 8.1 \\
\text { Hemiplegic: } 30 \% \text { vs. } 60 \% \\
\text { Diplegic: } 60 \% \text { vs. } 40 \% \\
\text { Quadrapletic: } 10 \% \text { vs. } 0 \%\end{array}$ & $\begin{array}{l}\text { A vs. } B \text {, mean (SD): } \\
\text { 6MWT: } 158.8(100.24) \text { to } 189.45(115.47) \\
\text { vs. } 194(78.82) \text { to } 271.5(60.81), p=0.04\end{array}$ \\
\hline $\begin{array}{l}\text { Lee, } 2013^{187} \\
\text { Postural control } \\
\text { RCT } \\
\text { Fair }\end{array}$ & $\begin{array}{l}\text { A. WBV + PT, } 24 \\
\text { sessions of vibration } \\
\text { over } 8 \text { weeks }(n=15) \\
\text { B. PT }(n=15)\end{array}$ & $\begin{array}{l}\text { A vs. } B \\
\text { Age: } 10.00 \text { vs. } 9.66 \\
\text { Female: } 60 \% \text { vs. } 40 \% \\
\text { Ambulatory: } 100 \% \\
\text { GMFM: } 78.4 \text { vs. } 79.53\end{array}$ & $\begin{array}{l}\text { A vs. } B, \text { mean }(S D), p=\text { between groups: } \\
\text { Walking speed (meters/second): } 0.37(0.04) \\
\text { to } 0.48(0.06) \text { vs. } 0.39(0.05) \text { to } 0.40(0.05), \\
p=0.001\end{array}$ \\
\hline
\end{tabular}

Abbreviations: 6MWT = 6-Minute Walk Test; GMFM = Gross Motor Function Measure; PT = physical therapy; RCT = randomized controlled trial; $\mathrm{SD}=$ standard deviation; $\mathrm{WBV}=$ whole body vibration

\section{Whole Body Vibration-Spinal Cord Injury}

One $\mathrm{RCT}^{189}(\mathrm{n}=28)$ studied the effect of WBV on adults with incomplete cervical SCI. Participants were a mean of 14 months post-SCI (Table 32). All participants were ambulatory at baseline and received 80 treatments over 8 weeks in an inpatient rehabilitation setting. The intervention group performed repetitions of a semi-squatting position on a vibration board at $30 \mathrm{~Hz}$, while the control group received a sham treatment where they performed the movements on a nonvibrating board. Both groups also received conventional PT. While favoring WBV over usual care on improvement in function, additional studies are needed to confirm this finding (SOE: insufficient).

This trial reported that there were no serious adverse events at $30 \mathrm{~Hz}$ but did not address nonserious adverse events.

Table 32. Whole body vibration exercise in spinal cord injury

\begin{tabular}{|c|c|c|c|}
\hline $\begin{array}{l}\text { Author, Year } \\
\text { Intervention } \\
\text { Study Design } \\
\text { Study Quality }\end{array}$ & $\begin{array}{l}\text { Intervention and } \\
\text { Comparison }\end{array}$ & Population & Results \\
\hline $\begin{array}{l}\text { In, } 2018^{189} \\
\text { Postural control } \\
\text { RCT } \\
\text { Fair }\end{array}$ & $\begin{array}{l}\text { A. WBV plus PT, } 80 \\
\text { sessions over } 8 \text { weeks } \\
(n=14) \\
\text { B. Sham WBV plus PT, } \\
80 \text { sessions over } 8 \\
\text { weeks }(n=14)\end{array}$ & $\begin{array}{l}\text { A vs. B } \\
\text { Age: } 46.1 \text { vs. } 49.9 \\
\text { Female: } 36 \% \text { vs. } 29 \% \\
\text { Ambulatory: } 100 \% \\
\text { C6-C7: } 100 \%\end{array}$ & $\begin{array}{l}\text { A vs. B, mean (SD), } p=\text { between groups: } \\
\text { 10MWT: } 29.3(9.0) \text { to } 25.8(8.1) \text { vs. } 28.8 \\
\text { (7.2) to } 27.5(6.3), p=0.005 \\
\text { TUG: } 13.7(3.1) \text { to } 11.4(2.8) \text { vs. } 14.7(4.5) \\
\text { to } 13.7(4.1), p=0.016\end{array}$ \\
\hline
\end{tabular}

Abbreviations: 10MWT = 10-Meter Walk Test; PT = physical therapy; RCT = randomized controlled trial; $\mathrm{SD}=\mathrm{standard}$ deviation; TUG = Timed Up and Go Test; $\mathrm{WBV}=$ whole body vibration 


\section{Yoga}

Originating from ancient India, yoga is a group of physical, mental, and spiritual practices or disciplines. Goals of yoga practices include improving physical and mental health and in the United States yoga often involves meditation, breathing techniques, and holding specific physical postures.

\section{Key Points}

- There was low-strength evidence of no clear benefit of yoga compared with usual care on function in MS (SOE: low).

- Evidence was insufficient to draw conclusions among MS study participants on the effects of yoga versus a variety of active controls on balance, function, or quality of life (SOE: insufficient).

- There were no studies of yoga in people with CP or SCI.

\section{Detailed Synthesis}

Six RCTs, reported in nine publications, ${ }^{54,120,190-197}$ enrolled and randomized participants to yoga versus a comparison group. The types of yoga included Hatha (postures, breathing techniques, and meditation), ${ }^{191,194-196}$ an Iyengar approach (focus on alignment) to Hatha Yoga, ${ }^{54}$ or was not predefined or well-delineated. ${ }^{192,193,197}$ Comparison groups included waitlist control, ${ }^{54}$ no intervention, ${ }^{192}$ walking, ${ }^{191,193-196}$ movement to music, ${ }^{54}$ PT-led exercise, ${ }^{120,192,193}$ fitness instructor-led exercise, ${ }^{192,193}$ group physiotherapy, ${ }^{197}$ and one-on-one physiotherapy. ${ }^{197}$ Two RCTs were rated fair quality, ${ }^{54,120}$ and the remaining were rated poor quality due to methodological limitations that included unclear methods of randomization, allocation concealment and blinding, lack of intent-to-treat analysis, and high attrition. All trials were in participants with MS. Type of MS was reported in one RCT only, ${ }^{192,193}$ including 45 percent RRMS, 16 percent SPMS (secondary progressive multiple sclerosis), 10 percent PPMS (primary progressive multiple sclerosis), and 2 percent considered benign MS. All trials involved 8, 10, or 12 weeks of yoga.

\section{Yoga-Multiple Sclerosis}

Six RCTs enrolled 648 participants with MS with a weighted mean age of participants of 46.8 years (range 31.6 to 51.4 years) and a weighted mean proportion female of 79.6 percent (range $72.3 \%$ to $100 \%$ ). Three trials enrolled only females (Table 33). Race was only reported in the U.S. study ${ }^{54}$ and was 54 percent White, 44 percent Black, and 2 percent other. One study was conducted in participants needing bilateral support for ambulation who may need a wheelchair for longer distances, ${ }^{197}$ and one trial enrolled participants with minimal gait impairment. ${ }^{192,193}$ Scores on the Patient Determined Disease Steps (PDDS) $(0=$ normal, $8=$ bedridden $)$ tended toward lower disability $(32 \%$ PDDS $=0)$ in one RCT. ${ }^{54}$

Four trials provided low-strength evidence of no clear benefit on function with yoga compared with usual care in MS. ${ }^{54,120,192,193,197}$ All other findings were supported by limited evidence and/or evidence that was considered to have a high risk of bias (SOE: insufficient). These included balance and quality of life in trials comparing yoga with dance, ${ }^{54}$ aerobics, ${ }^{194-196}$ physiotherapist-led exercises, ${ }^{192,193}$ fitness instructor-led exercises, ${ }^{192,193}$ group exercises, ${ }^{197}$ and one-on-one exercises. ${ }^{197}$ Also included was function in the trial comparing yoga with physiotherapist- and fitness instructor-led exercises ${ }^{192,193}$ and in the trial comparing yoga with group and one-on-one exercises. ${ }^{197}$ 
Three trials did not address harms or adverse events. One RCT reported one adverse event (stroke) in the yoga group versus no adverse events in the control group. ${ }^{54}$ Another trial excluded deaths from the analysis but did not report the incidence of death. ${ }^{196}$

One additional trial reports the effects of yoga on depression and is discussed in KQ2a. ${ }^{70}$

Table 33. Yoga exercise in multiple sclerosis

\begin{tabular}{|c|c|c|c|}
\hline $\begin{array}{l}\text { Author, Year } \\
\text { Intervention } \\
\text { Study Design } \\
\text { Study Quality }\end{array}$ & $\begin{array}{l}\text { Intervention } \\
\text { and Comparison }\end{array}$ & Population & Results \\
\hline $\begin{array}{l}\text { Ahmadi, } 2013^{120} \\
\text { Postural control } \\
\text { RCT } \\
\text { Fair }\end{array}$ & $\begin{array}{l}\text { A. Yoga, } 24 \\
\text { sessions over } 8 \\
\text { weeks }(n=11) \\
\begin{array}{l}\text { B. Waitlist control } \\
(n=10)\end{array}\end{array}$ & $\begin{array}{l}\text { A vs. B } \\
\text { Age: } 32 \text { vs. } 37 \\
\text { Female: } 100 \% \\
\text { EDSS: } 2.00 \text { vs. } 2.25\end{array}$ & $\begin{array}{l}\text { A vs. B, mean (SD), } p \text {-value between groups: } \\
\text { 10MWT (sec): } 8.78 \text { to } 8.13 \text { vs. } 9.16 \text { to } 9.47 \text {, } \\
\text { p<0.001 } \\
\frac{2 M W T:}{121.50}(27.73) \text { to } 119.05(27.12), p=0.11 \\
\text { BBS: } 47.72(6.78) \text { to } 53.81(3.40) \text { vs. } 44.50 \\
(8.48) \text { to } 41.70(8.48), p=0.07\end{array}$ \\
\hline $\begin{array}{l}\text { Doulatabad, } \\
2012^{190} \\
\text { Najafidoulatabad, } \\
2014^{191} \\
\text { Postural control } \\
\text { RCT } \\
\text { Poor }\end{array}$ & $\begin{array}{l}\text { A. Yoga, } 24 \\
\text { sessions over } 12 \\
\text { weeks }(n=30) \\
\text { B. No intervention } \\
\text { over } 12 \text { weeks } \\
(n=30)\end{array}$ & $\begin{array}{l}\text { A vs. B } \\
\text { Age: } 31.6(18 \text { to } 45) \\
\text { Female: } 100 \%\end{array}$ & $\begin{array}{l}\text { A vs. } B, \text { mean difference between groups; } \\
\text { mean (SD), p-value within groups } \\
\frac{\text { MSQoL-54: } 2.6,95 \% \text { Cl } 1.64 \text { to } 3.56}{p<0.001} \\
\text { Sexual satisfaction: } \\
\text { A: baseline } 1.8(2.0) \text { to } 1.4(1.5), p=0.001 \\
\text { B: } 2.1(1.2) \text { to } 2.1(1.2), p>0.05\end{array}$ \\
\hline $\begin{array}{l}\text { Garrett, 2013a193 } \\
\text { Garrett, 2013b } \\
\text { Postural control } \\
\text { RCT } \\
\text { Poor }\end{array}$ & $\begin{array}{l}\text { A. Physiotherapist- } \\
\text { led exercise, } 10 \\
\text { sessions over } 10 \\
\text { weeks }(n=80)^{a} \\
\text { B. Yoga, } 10 \\
\text { sessions over } 10 \\
\text { weeks ( } n=77) \\
\text { C. Fitness } \\
\text { instructor-led } \\
\text { exercise, } 10 \\
\text { sessions over } 10 \\
\text { weeks ( } n=86)^{a} \\
\text { D. Usual care } \\
(n=71)\end{array}$ & $\begin{array}{l}\text { A vs. B vs. C vs. D } \\
\text { Age: } 51.7 \text { vs. } 49.6 \text { vs. } 50.3 \\
\text { vs. } 48.8 \\
\text { Female: } 79 \% \text { vs. } 70 \% \text { vs. } \\
68 \% \text { vs. } 87 \% \\
\text { Wheelchair user: } 0 \% \\
\text { RRMS: } 55 \% \text { vs. } 60 \% \text { vs. } \\
49 \% \text { vs. } 55 \% \\
\text { SPMS: } 14 \% \text { vs. } 11 \% \text { vs. } \\
19 \% \text { vs. } 20 \% \\
\text { PPMS: } 7 \% \text { vs. } 13 \% \text { vs. } \\
13 \% \text { vs. } 6 \% \\
\text { Benign: } 0 \% \text { vs. } 2 \% \text { vs. } 5 \% \\
\text { vs. } 2 \%\end{array}$ & $\begin{array}{l}\text { B vs. D, median (SIQR), } p=\text { between groups: } \\
\text { 6MWT: } 268 \text { (222) to } 285 \text { (152) vs. } 250 \text { (206) } \\
\text { to } 315 \text { ( } 232), p=0.73 \\
\text { MSIS-29 (physical): } 33.4(20.0) \text { to } 29.4(19.4) \\
\text { vs. } 29.6 \text { (23.0) to } 29.9(20.7), p=0.12 \\
\text { MSIS-29 (psychological): } 33.3 \text { (33.3) to } 25.9 \\
\text { (33.3) vs. } 22.2(24.1) \text { to } 18.5 \text { (38.9), } p=0.04\end{array}$ \\
\hline $\begin{array}{l}\text { Hasanpour- } \\
\text { Dehkordi, } 2014^{196} \\
\text { Hasanpour- } \\
\text { Dehkordi, } 2016^{195} \\
\text { Hasanpour- } \\
\text { Dehkordi, } 2016 \\
(2)^{194} \\
\text { Postural control } \\
\text { RCT } \\
\text { Poor }\end{array}$ & $\begin{array}{l}\text { A. Yoga, } 36 \\
\text { sessions over } 12 \\
\text { weeks }(n=20) \\
\text { B. Aerobics, } 36 \\
\text { sessions over } 12 \\
\text { weeks }(n=20) \\
\text { C. Usual care } \\
\text { control }(n=21)\end{array}$ & $\begin{array}{l}\text { A vs. B vs. C } \\
\text { Age: } 31.9 \\
\text { Female: } 98 \%\end{array}$ & $\begin{array}{l}\text { A vs. B vs. C mean difference, } p=\text { between } \\
\text { groups on } S F-36 Q 0 L: \\
\text { C vs. A: } 1106.41, p<0.001 \\
\text { B vs. A: } 229.32, p=0.07 \\
\text { C vs. B: } 877.10, p<0.001\end{array}$ \\
\hline
\end{tabular}




\begin{tabular}{|c|c|c|c|}
\hline $\begin{array}{l}\text { Author, Year } \\
\text { Intervention } \\
\text { Study Design } \\
\text { Study Quality }\end{array}$ & $\begin{array}{l}\text { Intervention } \\
\text { and Comparison }\end{array}$ & Population & Results \\
\hline $\begin{array}{l}\text { Hogan, } 2014^{197} \\
\text { Postural control } \\
\text { RCT } \\
\text { Poor }\end{array}$ & $\begin{array}{l}\text { A. Group PT, } 10 \\
\text { sessions over } 10 \\
\text { weeks ( } n=48) \\
\text { B. 1-on-1 PT, } 10 \\
\text { sessions over } 10 \\
\text { weeks ( } n=35) \\
\text { C. Yoga ( } n=13) \\
\begin{array}{l}\text { D. Usual care } \\
(n=15)\end{array}\end{array}$ & $\begin{array}{l}\text { A vs. B vs. C vs. D } \\
\text { Age: } 57 \text { vs. } 52 \text { vs. } 58 \text { vs. } \\
49 \\
\text { Female: } 63 \% \text { vs. } 57 \% \text { vs. } \\
62 \% \text { vs. } 87 \% \\
\text { RRMS: } 27 \% \text { vs. } 20 \% \text { vs. } \\
31 \% \text { vs. } 33 \% \\
\text { SPMS: } 42 \% \text { vs. } 46 \% \text { vs. } \\
38 \% \text { vs. } 33 \% \\
\text { PPMS: } 17 \% \text { vs. } 31 \% \text { vs. } \\
15 \% \text { vs. } 33 \% \\
\text { Unknown: } 15 \% \text { vs. } 3 \% \text { vs. } \\
15 \% \text { vs. } 0 \%\end{array}$ & $\begin{array}{l}\text { A vs. B vs. C vs. D, mean (SD/SIQR), } \\
\text { p=between groups: } \\
\text { 6MWT: } 101 \text { (39.5) to } 121.2(47.4) \text { vs. } 70(30) \\
\text { to } 45(54.5) \text { vs. } 83.9(39.8) \text { to } 100 \text { (55) vs. } \\
83.5(44) \text { to } 90 \text { (35), p>0.05 for all group } \\
\text { comparisons } \\
\text { MSIS-29 (physical): } 50.5(9.5) \text { to } 45.9(10.5) \\
\text { vs. } 48.3(10.5) \text { to } 49.6(11.6) \text { vs. } 54(11.5) \text { to } \\
49.4(12) \text { vs. } 55.3(9.5) \text { to } 50.5(11.3), p=N R \\
\text { MSIS-29 (psychological): } 18(5.5) \text { to } 15(5.7) \\
\text { vs. } 14(2.2) \text { to } 15(4) \text { vs. } 18(5.38) \text { to } 17(4.8) \\
\text { vs. } 17(4) \text { to } 15(4.5), p>0.05 \text { for all group } \\
\text { comparisons } \\
\text { BBS: } 28.9(9.5) \text { to } 34.5(9.8) \text { vs. } 22.6(12.6) \\
\text { to } 27.9(11.5) \text { vs. } 30.4(11.6) \text { to } 34.2(9.8) \text { vs. } \\
24.9(11.6) \text { to } 21.8(11.9), p<0.05 \text { for all } \\
\text { comparisons vs. control }\end{array}$ \\
\hline $\begin{array}{l}\text { Young, } 2019^{54} \\
\text { Postural control } \\
\text { RCT } \\
\text { Fair }\end{array}$ & $\begin{array}{l}\text { A. Movement to } \\
\text { Music, } 36 \text { sessions } \\
\text { over } 12 \text { weeks } \\
(n=27) \\
\text { B. Adapted Yoga, } \\
36 \text { sessions over } \\
12 \text { weeks ( } n=26) \\
\text { C. Waitlist control } \\
(n=28)\end{array}$ & $\begin{array}{l}\text { A vs. B vs. C } \\
\text { Age: } 50 \text { vs. } 48 \text { vs. } 47 \\
\text { Female: } 81 \% \text { vs. } 77 \% \text { vs. } \\
86 \% \\
\text { White: } 44 \text { vs. } 58 \% \text { vs. } 61 \%\end{array}$ & $\begin{array}{l}\text { A vs. B vs. C, mean difference, } p=\text { between } \\
\text { groups: } \\
\text { TUG: } \\
\text { A vs. C: }-1.89,95 \% \mathrm{Cl}-3.30 \text { to }-0.48, p=0.01 \\
\text { B vs. } \mathrm{C}:-1.20,95 \% \mathrm{Cl}-2.58 \text { to } 0.18, p=0.09 \\
\text { B vs. A: } 0.69,95 \% \mathrm{Cl}-0.71 \text { to } 2.08, p=0.33 \\
\text { 6MWT: } \\
\text { A vs. C: } 40.98,95 \% \mathrm{Cl} 2.21 \text { to } 80, p=0.04 \\
\text { B vs. C: } 22.83,95 \% \mathrm{Cl}-16.67 \text { to } 6.2, p=0.25 \\
\text { B vs. A: }-18.15,95 \% \mathrm{Cl}-56.4 \text { to } 20.1, \\
\text { p=0.34 } \\
\text { 5xSit-to-Stand: } \\
\text { A vs. C: }-1.00,95 \% \mathrm{Cl}-2.58 \text { to } 0.55, p=0.20 \\
\text { B vs. C: }-0.70,95 \% \mathrm{Cl}-2.17 \text { to } 0.77, p=0.34 \\
\text { B vs. A: } 0.30,95 \%-1.21 \text { to } 1.82, p=0.69\end{array}$ \\
\hline
\end{tabular}

Abbreviations: 5x = five times; 6MWT = 6-Minute Walk Test; BBS = Berg Balance Scale; $\mathrm{CI}=$ confidence interval; EDSS = Expanded Disability Status Scale; MS = multiple sclerosis; MSIS-29 = Multiple Sclerosis Impact Scale; MSQoL = Multiple Sclerosis Quality of Life; NR = not reported; PT = physical therapy; RCT = randomized controlled trial; RRMS = relapsingremitting multiple sclerosis; $\mathrm{SD}=$ standard deviation; $\mathrm{SIQR}=$ semi-interquartile range; SF-36 QOL = Short Form Survey $(36$ Item) Quality of Life; SPMS = secondary progressive multiple sclerosis; TUG $=$ Timed Up and Go Test

${ }^{a}$ Not included in the sample size total in the Detailed Synthesis paragraph.

\section{Yoga-Cerebral Palsy}

No studies were identified.

\section{Yoga-Spinal Cord Injury}

No studies were identified.

\section{Strength Exercise Interventions}

\section{Muscle Strength Exercise}

Strength exercise focuses on muscle training interventions to increase muscle strength and includes exercises such as progressive resistance exercise, body weight resistance exercise, and Pilates. 


\section{Key Points}

- In participants with MS, muscle strength exercise was not associated with improved function, including walking, balance, or quality of life versus usual care (SOE: low).

- In participants with CP, evidence from small trials suggest muscle strength exercise was not associated with improved walking or function when compared with usual care immediately postintervention or in the short term (SOE: low).

- In participants with SCI, there was insufficient evidence from one small trial on quality of life compared with usual care (SOE: insufficient).

\section{Detailed Synthesis}

Nineteen RCTs ${ }^{52,68,83,149,198-216,218}$ and two quasiexperimental trials ${ }^{62,217}$ involving 1,070 participants evaluated muscle strengthening interventions. Muscle strength exercises included progressive resistance exercises of various kinds (e.g., resistance against external weights such as leg press and hamstring curls, as well as body weight resistance such as abdominal crunches and Pilates). Most trials compared muscle strengthening to usual care, which included continuation of usual PT or rehabilitation, ${ }^{52,200,201,204,205,213,216}$ previous activity levels, ${ }^{199,202,203}$ or an attention control of relaxation techniques at home or massage. ${ }^{198,200,201,206}$ Two trials included two comparator groups. ${ }^{68,69,206}$ Pilates was compared with aquatic exercise in one trial ${ }^{68,69}$ and progressive resistance combined with neuromuscular electrical stimulation versus neuromuscular electrical stimulation alone in another. ${ }^{210}$ Eleven trials ${ }^{52,68,69,149,198-206,215}$ and one quasiexperimental study ${ }^{62}$ enrolled participants with MS (Table 34). Seven trials ${ }^{207-216}$ and one quasiexperimental study ${ }^{217}$ were of participants with CP (Table 35), and one trial was conducted in participants with SCI (Table 36) ${ }^{218}$ Three trials ${ }^{204,205,211,212}$ met criteria for good quality, twelve for fair quality, ${ }^{52,83,149,198-203,207-210,214,215,218}$ and four trials ${ }^{68,69,206,213,216}$ and two quasiexperimental studies ${ }^{62,217}$ were rated poor quality. The poor-quality trials were judged to have high risk of bias due to unclear randomization and/or allocation concealment, lack of similarity between treatment groups at baseline, and unacceptable attrition. The quasiexperimental trials were deemed to have high risk of bias due to lack of similarity between treatment groups, the absence of controlling for potential confounding, and unacceptable attrition. The most frequently reported outcomes varied by condition. Most prioritized outcomes reported across conditions were walking-related (e.g., 2MWT, 6MWT, 10MWT) and few studies reported measures of functional capacity (e.g., TUG) or quality of life (e.g., SF-36). Metaanalyses of trials was conducted as appropriate.

\section{Muscle Strength Exercise-Multiple Sclerosis}

Eleven trials ${ }^{52,68,69,83,149,198-206}$ and one quasiexperimental study ${ }^{62}$ enrolled participants with MS ( $\mathrm{n}=584$ ) (Table 34). Weighted mean age of participants across trials was 45.7 years (range 41 to 54 years) with a weighted mean proportion female of 67.9 percent (range $29 \%$ to $100 \%$ ). Race/ethnicity generally was not reported. Ambulation status varied across studies. All studies enrolled participants who could, at minimum, ambulate using bilateral assistance (i.e., crutches, canes). The EDSS at baseline was reported in four trials with a range of means or medians of 2.9 to 5.9. The weighted mean number of sessions across trials was 22.6 over a weighted mean period of 11.6 weeks. 
Table 34. Muscle strength exercise in multiple sclerosis

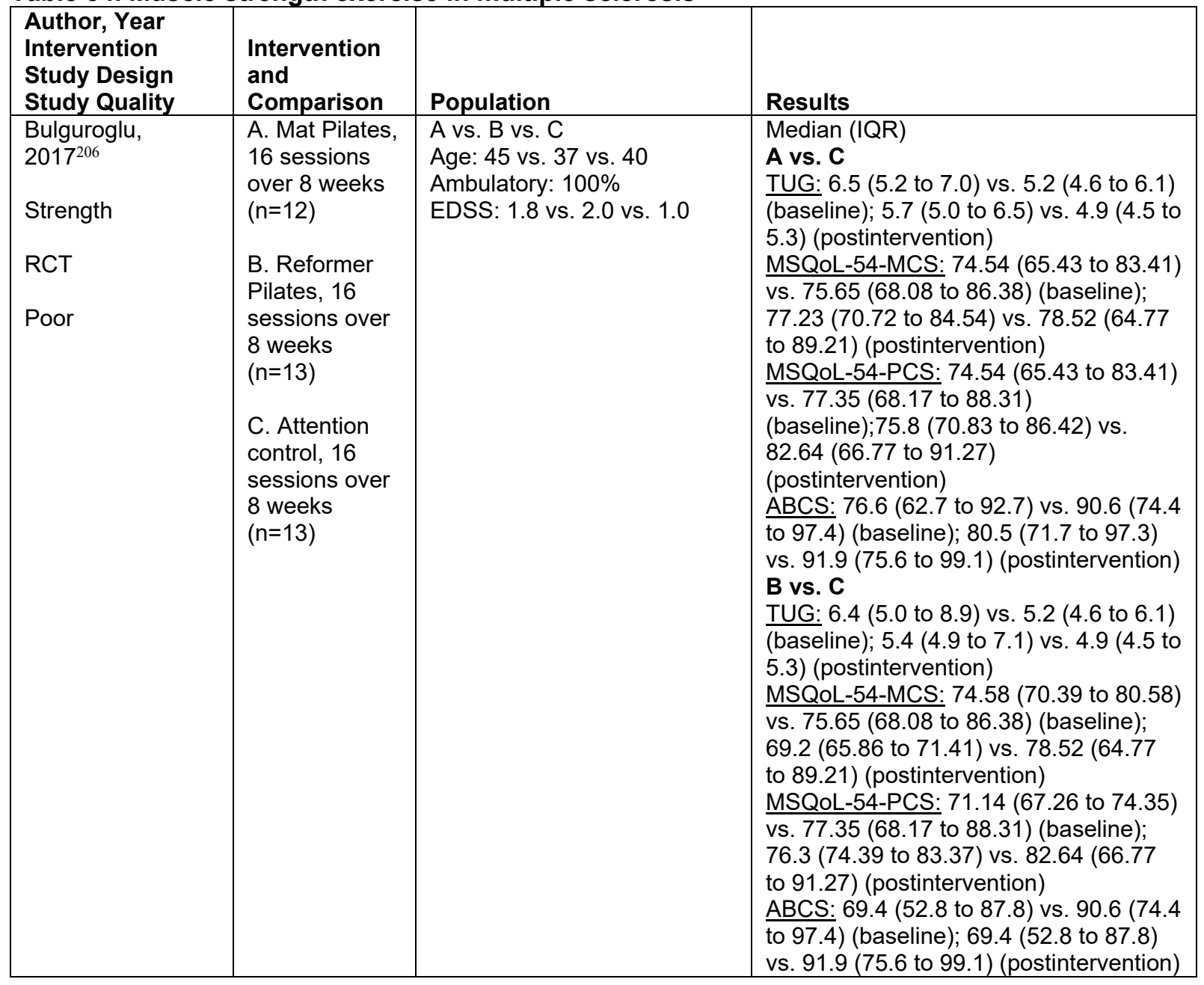




\begin{tabular}{|c|c|c|c|}
\hline $\begin{array}{l}\text { Author, Year } \\
\text { Intervention } \\
\text { Study Design } \\
\text { Study Quality }\end{array}$ & $\begin{array}{l}\text { Intervention } \\
\text { and } \\
\text { Comparison }\end{array}$ & Population & Results \\
\hline $\begin{array}{l}\text { Callesen, } 2019^{149} \\
\text { Strength } \\
\text { RCT } \\
\text { Fair }\end{array}$ & $\begin{array}{l}\text { A. Progressive } \\
\text { resistance } \\
\text { training } \\
(n=17): 20 \\
\text { sessions over } \\
10 \text { weeks } \\
\text {-median } \\
\text { number of } \\
\text { sessions } \\
\text { completed } \\
\text { (range): } 17 \text { ( } 8 \\
\text { to 19) } \\
\text { B. Balance } \\
\text { training } \\
\text { ( } n=24): 20 \\
\text { sessions over } \\
10 \text { weeks } \\
\text {-median } \\
\text { number of } \\
\text { sessions } \\
\text { completed } \\
\text { (range): } 16 \text { (6 } \\
\text { to } 20) \\
\text { C. Waitlist } \\
\text { control ( } n=18 \text { ) }\end{array}$ & $\begin{array}{l}\text { A vs. B vs. C } \\
\text { Median age: } 52 \text { vs. } 51 \text { vs. } 56 \\
\text { years } \\
\text { Female: } 70 \% \text { vs. } 82 \% \text { vs. } \\
80 \% \\
\text { Race: NR } \\
\text { Ambulatory: } 100 \% \text { vs. } 100 \% \\
\text { vs. } 100 \% \\
\text { Gait assistive devices: } 17 \% \\
\text { vs. } 11 \% \text { vs. } 10 \% \\
\text { Median duration of illness: } 15 \\
\text { vs. } 10 \text { vs. } 11 \text { years } \\
\text { MS type } \\
\text { - RRMS: } 70 \% \text { vs. } 75 \% \text { vs. } \\
65 \% \\
\text { - SPMS: } 22 \% \text { vs. } 14 \% \text { vs. } \\
15 \% \\
\text { - PPMS: } 70 \% \text { vs. } 9 \% \text { vs. } 20 \% \\
\text { Median EDSS: } 4 \text { vs. } 4 \text { vs. } 3.5\end{array}$ & 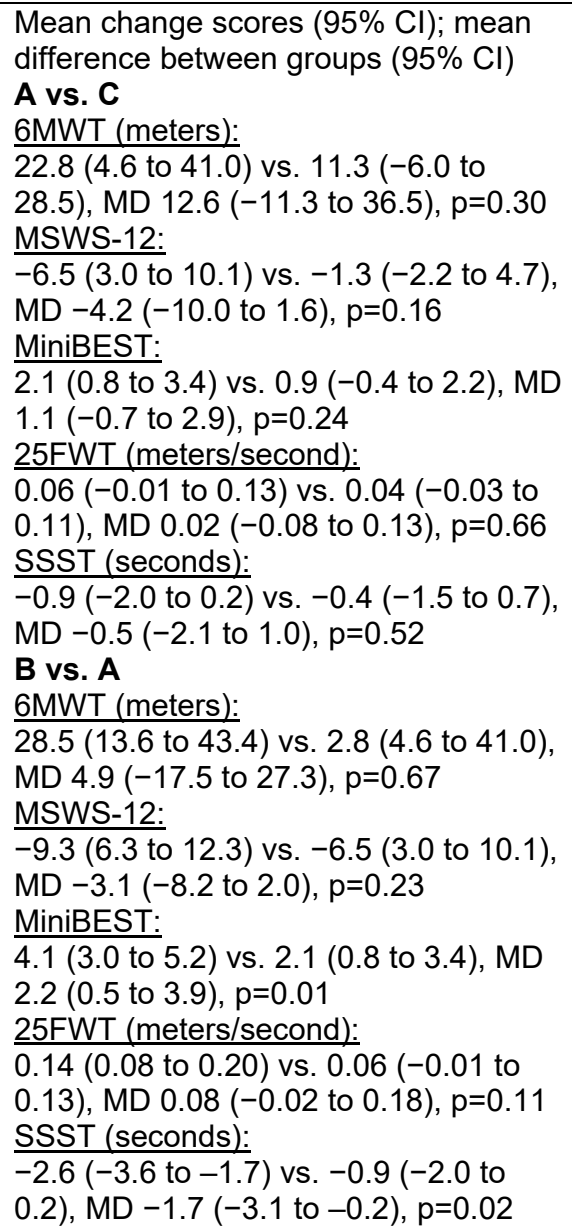 \\
\hline $\begin{array}{l}\text { Dalgas, } 2009^{202} \\
\text { Dalgas, } 2010^{203} \\
\text { Strength } \\
\text { RCT } \\
\text { Fair }\end{array}$ & $\begin{array}{l}\text { A. Progressive } \\
\text { resistance, } 24 \\
\text { sessions over } \\
12 \text { weeks } \\
(n=15) \\
\text { B. Waitlist } \\
\text { control }(n=16)\end{array}$ & $\begin{array}{l}\text { A vs. B } \\
\text { Age: } 45 \text { vs. } 48 \\
\text { Female: } 63 \% \text { vs. } 67 \% \\
\text { Ambulatory to } 100 \mathrm{~m}: 100 \% \\
\text { RRMS: } 100 \%\end{array}$ & 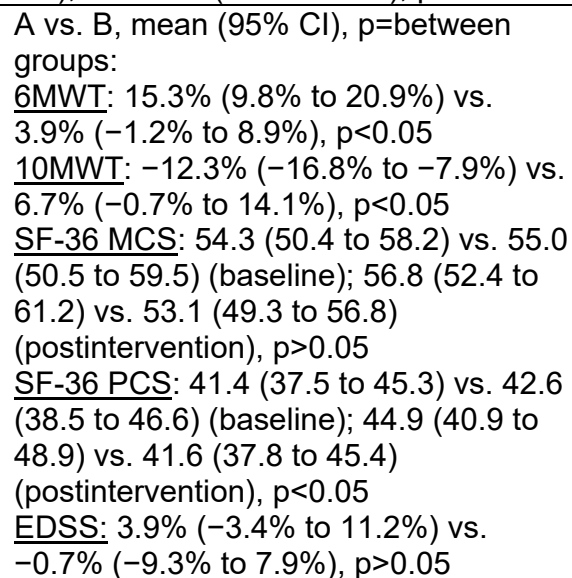 \\
\hline
\end{tabular}




\begin{tabular}{|c|c|c|c|}
\hline $\begin{array}{l}\text { Author, Year } \\
\text { Intervention } \\
\text { Study Design } \\
\text { Study Quality }\end{array}$ & $\begin{array}{l}\text { Intervention } \\
\text { and } \\
\text { Comparison }\end{array}$ & Population & Results \\
\hline $\begin{array}{l}\text { Dodd, } 2011^{204} \\
\text { Strength } \\
\text { RCT } \\
\text { Good }\end{array}$ & $\begin{array}{l}\text { A. Progressive } \\
\text { resistance, } 20 \\
\text { sessions over } \\
10 \text { weeks } \\
(n=36) \\
\\
\text { B. Attention } \\
\text { control (social } \\
\text { program), } 10 \\
\text { sessions over } \\
10 \text { weeks } \\
(n=35)\end{array}$ & $\begin{array}{l}\text { A vs. } B \\
\text { Age: } 47.7 \text { vs. } 50.4 \\
\text { Female: } 72 \% \text { vs. } 74 \% \\
\text { Ambulation index: } \\
2 \text { (mild): } 47 \% \text { vs. } 54 \% \\
3 \text { (moderate): } 39 \% \text { vs. } 26 \% \\
4 \text { (severe): } 14 \% \text { vs. } 20 \% \\
\text { Gait aid use (yes): } 33 \% \text { vs. } \\
37 \%\end{array}$ & $\begin{array}{l}\text { A vs. B, mean difference } \\
\text { 2MWT: MD 2.6, } 95 \% \mathrm{Cl}-4.0 \text { to } 9.1 \text {, } \\
\text { p>0.05 (post-pre change); } \mathrm{MD}-3.4 \\
\text { (95\% } \mathrm{Cl}-9.5 \text { to } 2.7 \text { ), p>0.05 (week } 22 \\
\text { followup) } \\
\text { WHO-QOL: } \mathrm{MD} 0.3,95 \% \mathrm{Cl}-0.1 \text { to } \\
0.6, \mathrm{p}>0.05 \text { (post-pre change); MD } \\
-0.2,95 \% \mathrm{Cl}-0.6 \text { to } 0.3, \mathrm{p}>0.05 \text { (week } \\
22 \text { followup) }\end{array}$ \\
\hline $\begin{array}{l}\text { Duff, } 2018^{198} \\
\text { Strength } \\
\text { RCT } \\
\text { Fair }\end{array}$ & $\begin{array}{l}\text { A. Pilates plus } \\
\text { massage, } 24 \\
\text { sessions of } \\
\text { Pilates and } 12 \\
\text { massages } \\
\text { over } 12 \text { weeks } \\
\text { ( } n=15) \\
\\
\text { B. Attention } \\
\text { control } \\
\text { (massage), } 12 \\
\text { massages } \\
\text { over } 12 \text { weeks } \\
(n=15)\end{array}$ & $\begin{array}{l}\text { A vs. B } \\
\text { Age: } 45.7 \text { vs. } 45.1 \\
\text { Female: } 80 \% \text { vs. } 73 \% \\
\text { Ambulatory: } 100 \% \\
\text { Wheelchair user: } 0 \% \\
\text { RRMS: } 93 \% \text { vs. } 73 \% \\
\text { SPMS: } 0 \% \text { vs. } 13 \% \\
\text { PPMS: } 7 \% \text { vs. } 13 \%\end{array}$ & 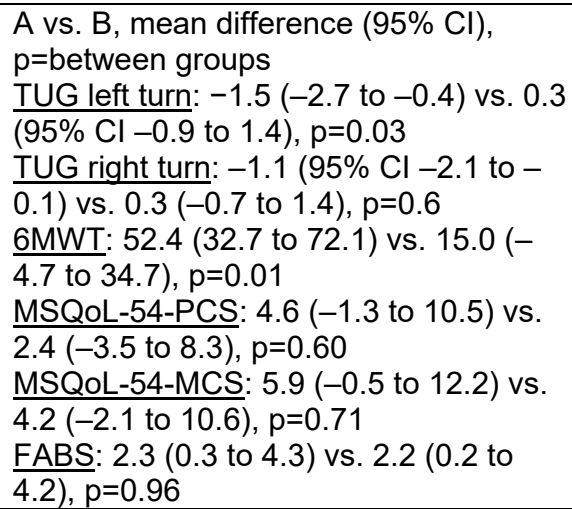 \\
\hline $\begin{array}{l}\text { Fox, 2016 } 200 \\
\text { Freeman, } 2012^{201} \\
\text { Strength } \\
\text { RCT } \\
\text { Fair }\end{array}$ & $\begin{array}{l}\text { A. Pilates, } 12 \\
\text { sessions over } \\
12 \text { weeks } \\
(n=33) \\
\text { B. Usual PT, } \\
12 \text { sessions } \\
\text { over } 12 \text { weeks } \\
(n=35) \\
\text { C. Relaxation, } \\
3 \text { sessions } \\
\text { over } 12 \text { weeks } \\
(n=32)\end{array}$ & $\begin{array}{l}\text { A vs. B vs. C } \\
\text { Age: } 53.97 \text { vs. } 54.60 \text { vs. } \\
53.78 \\
\text { Female: } 85 \% \text { vs. } 71 \% \text { vs. } \\
66 \% \\
\text { Ambulatory to } 20 \mathrm{m:}: 100 \% \\
\text { RRMS: } 39 \% \text { vs. } 37 \% \text { vs. } 38 \% \\
\text { SPMS: } 24 \% \text { vs. } 31 \% \text { vs. } 34 \% \\
\text { PPMS: } 36 \% \text { vs. } 31 \% \text { vs. } 25 \% \\
\text { Benign: } 0 \% \text { vs. } 0 \% \text { vs. } 3 \%\end{array}$ & 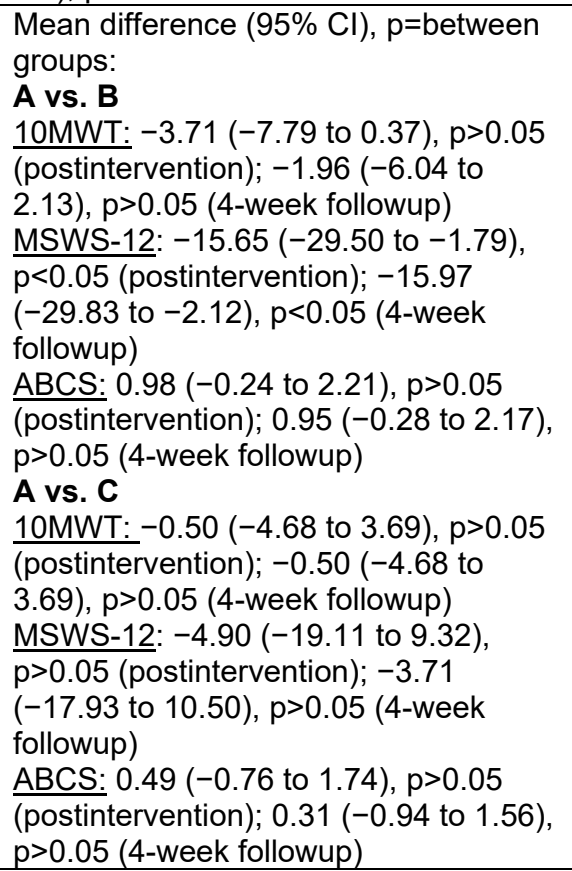 \\
\hline
\end{tabular}




\begin{tabular}{|c|c|c|c|}
\hline $\begin{array}{l}\text { Author, Year } \\
\text { Intervention } \\
\text { Study Design } \\
\text { Study Quality } \\
\end{array}$ & $\begin{array}{l}\text { Intervention } \\
\text { and } \\
\text { Comparison }\end{array}$ & Population & Results \\
\hline $\begin{array}{l}\text { Kalron, } 2017^{52} \\
\text { Strength } \\
\text { RCT } \\
\text { Fair }\end{array}$ & $\begin{array}{l}\text { A. Pilates, } 12 \\
\text { sessions over } \\
12 \text { weeks } \\
(\mathrm{n}=22) \\
\\
\text { B. Usual } \\
\text { physical } \\
\text { therapy, } 12 \\
\text { sessions over } \\
12 \text { weeks } \\
(\mathrm{n}=23)\end{array}$ & $\begin{array}{l}\text { A vs. } B \\
\text { Age: } 42.9 \text { vs. } 44.3 \\
\text { Female: } 60.9 \% \text { vs. } 68.2 \% \\
\text { Ambulatory to } 100 \mathrm{~m}: 100 \% \\
\text { EDSS: } 4.1 \text { vs. } 4.6 \\
\text { RRMS: } 100 \%\end{array}$ & $\begin{array}{l}\text { A vs. B, mean change (SD), } p=\text { between } \\
\text { group } \\
\text { TUG: }-1.8(2.1) \text { vs. }-1.7(2.1), p=0.422 \\
\text { 6MWT: } 39.1 \text { (78.3) vs. } 25.3(67.2), \\
p=0.341 \\
\text { 2MWT: } 14.5(25.8) \text { vs. } 12.7(23.0), \\
p=0.872 \\
\text { MSWS-12: } 2.8 \text { (6.3) vs. } 2.4(5.9), \\
p=0.924 \\
\text { BBS: } 1.1(4.2) \text { vs. } 1.3(5.2), M D-0.20, \\
5 \% \text { Cl }-2.888 \text { to } 2.488, p=0.561\end{array}$ \\
\hline $\begin{array}{l}\text { Kara, } 2017^{62} \\
\text { Strength } \\
\text { Quasiexperimental } \\
\text { Poor }\end{array}$ & $\begin{array}{l}\begin{array}{l}\text { A. Pilates, } 16 \\
\text { sessions over } \\
8 \text { weeks } \\
(n=27)\end{array} \\
\text { B. Multimodal } \\
\text { exercise } \\
\text { (focus on } \\
\text { aerobic), } 16 \\
\text { sessions over } \\
8 \text { weeks } \\
\text { ( } n=28)\end{array}$ & $\begin{array}{l}\text { A vs. B } \\
\text { Age: } 50 \text { vs. } 43 \\
\text { Female: } 67 \% \text { vs. } 65 \% \\
\text { EDSS: } 2.85 \text { vs. } 3.2\end{array}$ & $\begin{array}{l}\text { A vs. B, mean difference }(95 \% \mathrm{Cl}) \\
\text { p=between groups: } \\
\text { TUG right: } \\
-0.47 \text { ( }-2.98 \text { to } 2.04), p=0.71 \\
\text { TUG left: } \\
-3.07 \text { (-6.34 to } 0.20), p=0.07 \\
\left.\frac{\text { BBS: }}{-0.67} \text { ( }-10.56 \text { to } 9.22\right), p=0.89\end{array}$ \\
\hline $\begin{array}{l}\text { Kjolhede, } 2016^{199} \\
\text { Strength } \\
\text { RCT } \\
\text { Fair }\end{array}$ & $\begin{array}{l}\text { A. Progressive } \\
\text { resistance, } 48 \\
\text { sessions over } \\
24 \text { weeks } \\
(n=17) \\
\begin{array}{l}\text { B. Usual care } \\
\text { (habitual } \\
\text { lifestyle) } \\
(n=18)\end{array}\end{array}$ & $\begin{array}{l}\text { A vs. } \mathrm{B} \\
\text { Age: } 44.6 \text { vs. } 42.2 \\
\text { Female: } 75 \% \text { vs. } 75 \% \\
\text { EDSS: } 2.9 \text { vs. } 2.9 \\
\text { RRMS: } 100 \%\end{array}$ & $\begin{array}{l}\text { A vs. B, mean }(95 \% \mathrm{Cl}), \mathrm{p}=\text { between } \\
\text { group: } \\
\text { 2MWT (m/s): } 1.61 \text { (1.4 to } 1.8) \text { vs. } 1.66 \\
\text { (1.5 to } 1.8) \text { (baseline); } 1.77 \text { (1.6 to } 2.0) \\
\text { vs. } 1.69 \text { (1.5 to } 1.9) \text { (postintervention), } \\
\text { p=0.011 } \\
\text { 2MWT (meters): } 193.2(168 \text { to } 216) \text { vs. } \\
199.2 \text { (180 to } 216) \text { (baseline); } 212.2 \\
\text { (192 to } 240) \text { vs. } 202.8 \text { (180 to 228) } \\
\text { (postintervention) } \\
\text { 25FWT (m/s): } 1.66 \text { (1.5 to } 1.8) \text { vs. } 1.79 \\
\text { (1.6 to } 2.0) \text { (baseline); } 1.82(1.7 \text { to } 2.0) \\
\text { vs. } 1.80(1.6 \text { to } 2.0) \text { (postintervention), } \\
p=<0.001\end{array}$ \\
\hline $\begin{array}{l}\text { Marandi, 201368,69 } \\
\text { Strength } \\
\text { RCT } \\
\text { Poor }\end{array}$ & $\begin{array}{l}\begin{array}{l}\text { A. Pilates, } 36 \\
\text { sessions over } \\
12 \text { weeks } \\
(n=15)\end{array} \\
\text { B. Aquatics, } \\
36 \text { sessions } \\
\text { over } 12 \text { weeks } \\
(n=15) \\
\text { C. Usual care } \\
(n=15)\end{array}$ & $\begin{array}{l}\text { A vs. B vs. C } \\
\text { Age: NR } \\
\text { Female: } 100 \% \\
\text { Ambulatory: } 100 \% \\
\text { Wheelchair user: } 0 \%\end{array}$ & $\begin{array}{l}\text { Mean difference (SE), } p=\text { between } \\
\text { groups: } \\
\text { A vs. C } \\
\text { A vs. C } \\
\text { Right leg SSST: }-5.96(1.4), p=0.000 \\
\text { Left leg SSST: }-6.23(1.2), p=0.000 \\
\text { A vs. B } \\
\text { Right leg SSST: }-0.08(1.4), p=0.955 \\
\text { Left leg SSST: } 0.00(1.2), p=0.997\end{array}$ \\
\hline
\end{tabular}




\begin{tabular}{|c|c|c|c|}
\hline $\begin{array}{l}\text { Author, Year } \\
\text { Intervention } \\
\text { Study Design } \\
\text { Study Quality }\end{array}$ & $\begin{array}{l}\text { Intervention } \\
\text { and } \\
\text { Comparison }\end{array}$ & Population & Results \\
\hline $\begin{array}{l}\text { Ortiz-Rubio, } \\
2016^{205} \\
\text { Strength } \\
\text { RCT } \\
\text { Good }\end{array}$ & 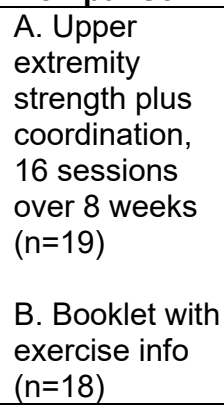 & $\begin{array}{l}\text { A vs. B } \\
\text { Age: } 42.21 \text { vs. } 44.89 \\
\text { Female: } 26 \% \text { vs. } 33 \% \\
\text { MS type: } \\
\text { RRMS: } 21 \% \text { vs. } 22 \% \\
\text { PPMS: } 16 \% \text { vs. } 11 \% \\
\text { SPMS: } 63 f \% \text { vs. } 67 \% \\
\text { EDSS: } 5.71 \text { vs. } 6.04\end{array}$ & $\begin{array}{l}\text { A vs. } B, \text { mean difference }(95 \% \mathrm{Cl}) \\
\text { p=between groups: } \\
\text { ARAT most affected upper limb: } 2.21 \\
(-2.95 \text { to }-1.46) \text { vs. } 0.16(-0.29 \text { to } \\
0.62), p=<0.001 \\
\text { ARAT least affected upper limb: } 0.68 \\
(-1.28 \text { to }-0.08) \text { vs. } 0.16(-0.08 \text { to } \\
0.42), p<0.001\end{array}$ \\
\hline $\begin{array}{l}\text { Tollar, } 2020^{83} \\
\text { Strength: } \\
\text { proprioceptive } \\
\text { neuromuscular } \\
\text { facilitation } \\
\text { RCT } \\
\text { Fair }\end{array}$ & $\begin{array}{l}\text { A. } \\
\text { Proprioceptive } \\
\text { neuromuscular } \\
\text { facilitation, } 25 \\
\text { sessions over } \\
5 \text { weeks } \\
(n=14) \\
\text { B. Usual care, } \\
25 \text { sessions } \\
\text { over } 5 \text { weeks } \\
(n=12)\end{array}$ & $\begin{array}{l}\text { Age: } 47 \text { vs. } 44 \\
\text { Female: } 93 \% \text { vs. } 92 \% \\
\text { Ambulatory: } 100 \% \text { RRMS: } \\
64 \% \text { vs. } 66 \% \\
\text { PPMS: } 36 \% \text { vs. } 34 \% \\
\text { Median EDSS score: } 5.0 \text { vs. } \\
5.0\end{array}$ & 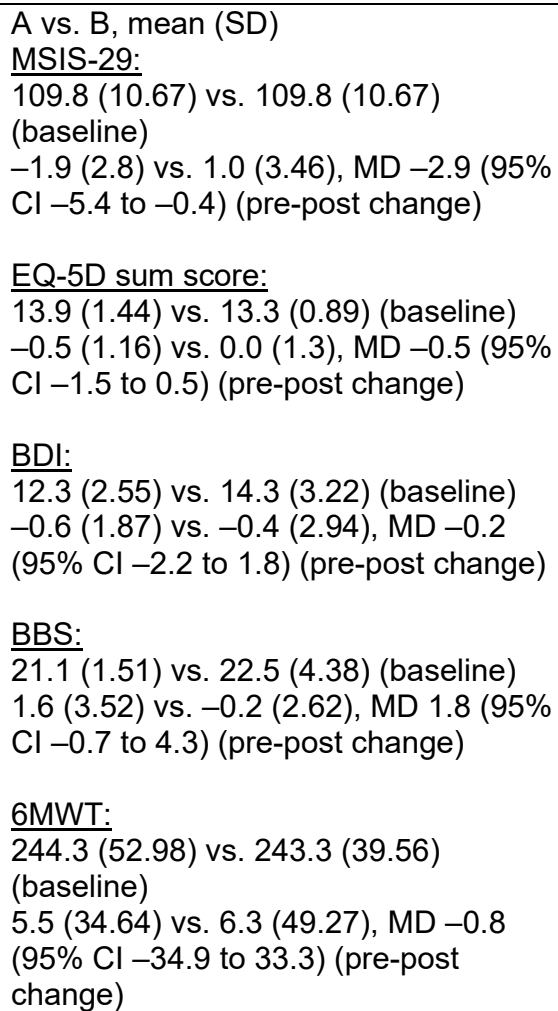 \\
\hline
\end{tabular}

Abbreviations: 2MWT = 2-Minute Walk Test; 6MWT = 6-Minute Walk Test; 10MWT = 10-Meter Walk Test; 25FWT = 25-Foot Walk Test; $\mathrm{ABCS}=$ Activities-specific Balance Confidence Scale; $\mathrm{AC}=$ attention control; $\mathrm{AE}=$ adverse event; $\mathrm{ARAT}=\mathrm{Action}$ Research Arm Tests; BBS = Berg Balance Scale; BDI = Beck Depression Index; CI = confidence interval; EDSS = Expanded Disability Status Scale; EPC = Evidence-based Practice Center; EQ-5D = EuroQOL-5 Dimension Questionnaire; FABS = Fullerton Advanced Balance Scale; IQR = interquartile range; $\mathrm{MD}=$ mean difference; MiniBEST = Mini Balance Evaluation System Test; MS = multiple sclerosis; MSIS-29 = Multiple Sclerosis Impact Scale; MSQoL-MCS = Multiple Sclerosis Quality of Life-54 instrument Mental Component Score; MSQoL-PCS = Multiple Sclerosis Quality of Life-54 instrument Physical Component Score; MSWS-12 = Multiple Sclerosis Walking Scale-12; MD = mean difference; NR = not reported; PPMS = primary progressive multiple sclerosis; $\mathrm{PT}=$ physical therapy; $\mathrm{QOL}=$ quality of life; $\mathrm{RCT}=$ randomized controlled trial; RRMS = relapsing-remitting multiple sclerosis; $\mathrm{SD}=$ standard deviation; $\mathrm{SE}=$ standard error; SF-36 MCS = Short-Form $36 \mathrm{Mental}$ Component Summary; SF-36 PCS = Short-Form 36 Physical Component Score; SPMS = secondary progressive multiple sclerosis; SSST $=$ Six Spot Step Test; TUG $=$ Timed Up and Go Test; WHOQoL = World Health Organization Quality of Life

\section{Walking Measures}

Walking-related outcome measures were most commonly reported. Strengthening exercises were generally not clearly associated with improved walking ability across measures compared with attention control (massage or social program), continuation of previous exercise, or PT 
immediately postintervention, based on pooled differences in changed scores (6MWT [Figure 9, 5 trials $^{83}$ ]; 2MWT [Figure 10, 3 trials ${ }^{52,199,204}$ ]; 10MWT [Figure 11, 2 trials with 3 comparisons $^{200-203}$ ]; and multiple sclerosis walking scale MSWS-12 [Figure 12, 0-100 scale, 3 trials with 3 comparisons $\left.{ }^{52,149,200,201}\right]$ ). Study results were generally homogeneous across trials for all walking measures despite variability in type, duration, and intensity of the strengthening exercises, and differences in the baseline activity level in the control groups. Two trials also reported no difference in walking speed in the strengthening group versus previous activity level or waitlist controls for the $25 \mathrm{FWT}^{149,199}$ ( 2 trials, MD -0.07 seconds, $95 \% \mathrm{CI}-0.19$ to 0.05 , $\mathrm{I}^{2}=47 \%$ ) (Figure 13).

There was limited evidence for a strengthening exercise effect beyond immediate posttreatment. There was no clear improvement in walking ability (10MWT) at short-term followup for strengthening exercises versus a relaxation attention control or PT ( 2 trials with 3 comparisons, ${ }^{200-203} \mathrm{MD}-1.3,95 \% \mathrm{CI}-2.75$ to 0.22 ) (Figure 11 ).

These trials provided low-strength evidence of no clear benefit on walking with muscle strength exercises compared with usual care immediately postintervention and at short-term followup.

Figure 9. Muscle strength exercise versus usual care in multiple sclerosis: 6MWT immediately following intervention

\begin{tabular}{|c|c|c|c|c|c|c|c|c|c|c|c|}
\hline Study & Cond. & Exercise & Control & $\begin{array}{c}\mathrm{N} \\
\text { Exercise }\end{array}$ & $\begin{array}{c}\mathrm{N} \\
\text { Control }\end{array}$ & Weight & $\begin{array}{c}\text { MD } \Delta \text { Scores } \\
\mathrm{PL}[95 \% \mathrm{Cl}]\end{array}$ & & & & \\
\hline Kalron 2017 & MS & Pilates & PT & 22 & 23 & $15.4 \%$ & $-13.80[-56.51,28.91]$ & & & - & \\
\hline Duff 2018 & MS & Pilates & AC Massage & 15 & 15 & $5.6 \%$ & $-37.40[-108.14,33.34]$ & & & & \\
\hline Dalgas 2010 & MS & PRE & Previous & 16 & 18 & $4.4 \%$ & $-56.10[-135.91,23.71]$ & & & 一 & \\
\hline Callesen 2019 & MS & PRE & WL & 17 & 9 & $49.2 \%$ & $-12.60[-36.50,11.30]$ & & + & - & \\
\hline Tollar 2020 & MS & Cycling & WL & 14 & 12 & $25.4 \%$ & $0.80[-32.46,34.06]$ & & & & \\
\hline \multicolumn{4}{|l|}{ Total $(95 \% \mathrm{Cl})$} & 84 & 77 & $100.0 \%$ & $-12.69[-29.45,4.07]$ & & & & \\
\hline \multicolumn{8}{|c|}{ Heterogeneity: $\mathrm{Tau}^{2}=0.00 ; \mathrm{Chi}^{2}=2.24, \mathrm{df}=4(\mathrm{P}=0.69) ; \mathrm{I}^{2}=0 \%$} & -200 & $-100 \quad 0$ & $0 \quad 100$ & 200 \\
\hline
\end{tabular}

Abbreviations: $\Delta=$ change; $6 \mathrm{MWT}=6$-Minute Walk Test; $\mathrm{AC}=$ attention control; $\mathrm{CI}=$ confidence interval; $\mathrm{MD}=$ mean difference; $\mathrm{PL}=$ profile likelihood; $\mathrm{PRE}=$ progressive resistance exercise; $\mathrm{PT}=$ physical therapy

Figure 10. Muscle strength exercise versus usual care in multiple sclerosis: 2MWT immediately following intervention

\begin{tabular}{|c|c|c|c|c|c|c|c|}
\hline Study & Exercise & Control & $\begin{array}{c}\mathrm{N} \\
\text { Exercise }\end{array}$ & $\begin{array}{c}\mathrm{N} \\
\text { Control } \\
\end{array}$ & Weight & $\begin{array}{l}\mathrm{MD} \triangle \text { Scores } \\
\mathrm{PL}[95 \% \mathrm{Cl}]\end{array}$ & \\
\hline Kjolhede 2016 & PRE & Previous & 16 & 14 & $7.5 \%$ & $-15.40[-36.32,5.52]$ & - \\
\hline Kalron 2017 & Pilates & PT & 22 & 23 & $16.0 \%$ & $-1.80[-16.10,12.50]$ & \\
\hline Dodd 2011 & PRE & AC PT + Social & 39 & 37 & $76.5 \%$ & $-2.60[-9.15,3.95]$ & \\
\hline Total $(95 \% \mathrm{Cl})$ & & & 77 & 74 & $100.0 \%$ & $-3.43[-11.92,2.81]$ & \\
\hline \multicolumn{7}{|c|}{ Heterogeneity: $\mathrm{Tau}^{2}=0.00 ; \mathrm{Chi}^{2}=1.37, \mathrm{df}=2(P=0.50) ; 1^{2}=0 \%$} & $\begin{array}{ccccc}1 & 1 & 1 & 1 \\
-50 & -25 & 0 & 25 & 50 \\
\text { Favors } & \text { Exercise } & \text { Favors Control }\end{array}$ \\
\hline
\end{tabular}

Abbreviations: $\Delta=$ change; $2 \mathrm{MWT}=2$-Minute Walk Test; $\mathrm{AC}=$ attention control; $\mathrm{CI}=$ confidence interval; $\mathrm{MD}=$ mean difference; $\mathrm{PL}=$ profile likelihood; $\mathrm{PRE}=$ progressive resistance exercise; $\mathrm{PT}=$ physical therapy 
Figure 11. Muscle strength exercise versus usual care in multiple sclerosis: 10MWT immediately following intervention and during the short-term followup

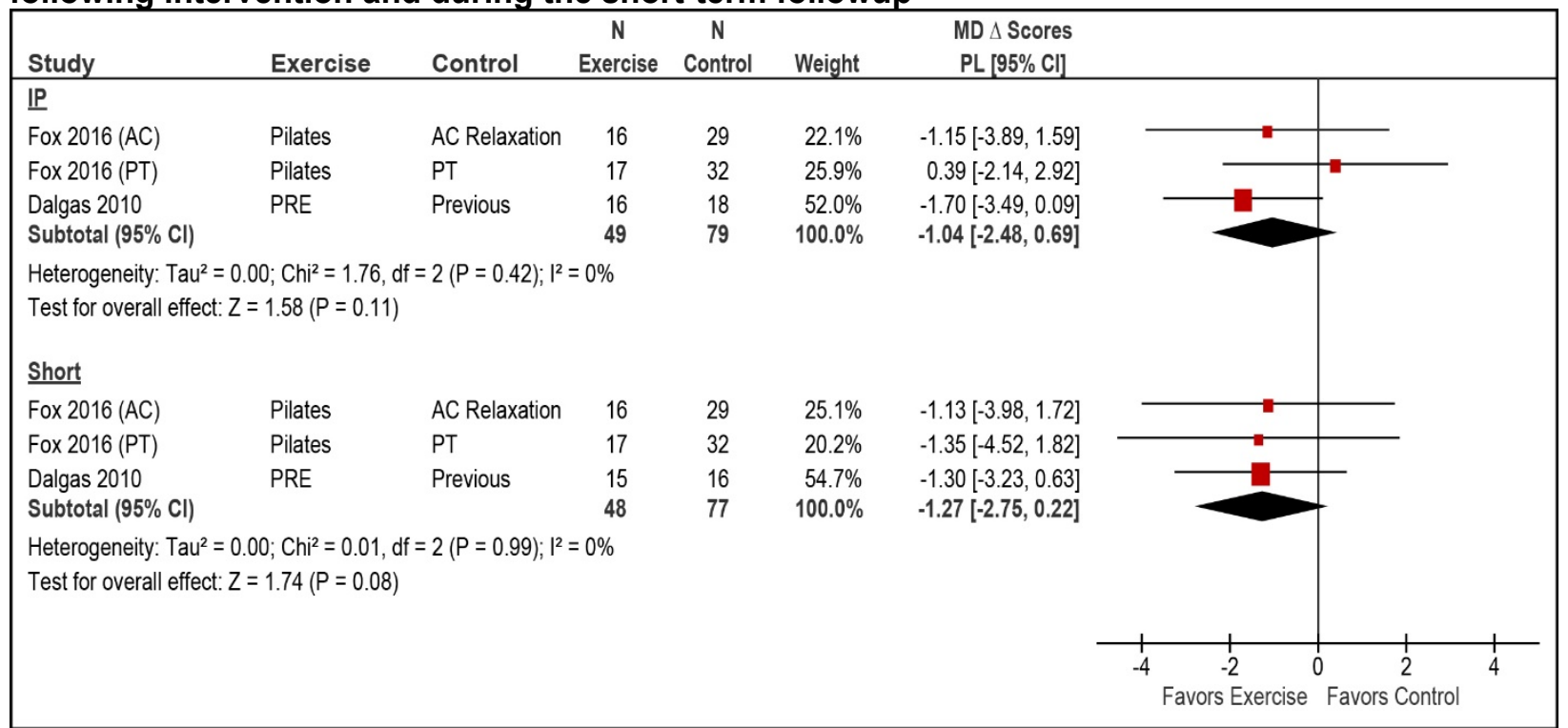

Abbreviations: $\Delta=$ change; 10MWT $=10$-Meter Walk Test; $\mathrm{AC}=$ attention control; $\mathrm{CI}=$ confidence interval; $\mathrm{MD}=$ mean difference; $\mathrm{PL}=$ profile likelihood; $\mathrm{PRE}=$ progressive resistance exercise; $\mathrm{PT}=$ physical therapy

Figure 12. Muscle strength exercise versus usual care in multiple sclerosis: MSWS-12 immediately following intervention

Figure 12 is a forest plot examining 12 minute walking test scores comparing muscle strength exercise with usual care. The pooled mean difference for the four studies is -1.36 (95\% confidence interval -4.83 to 2.10$)$, favoring exercise, with an I-squared value of $26 \%$ and a p-value of 0.44 .

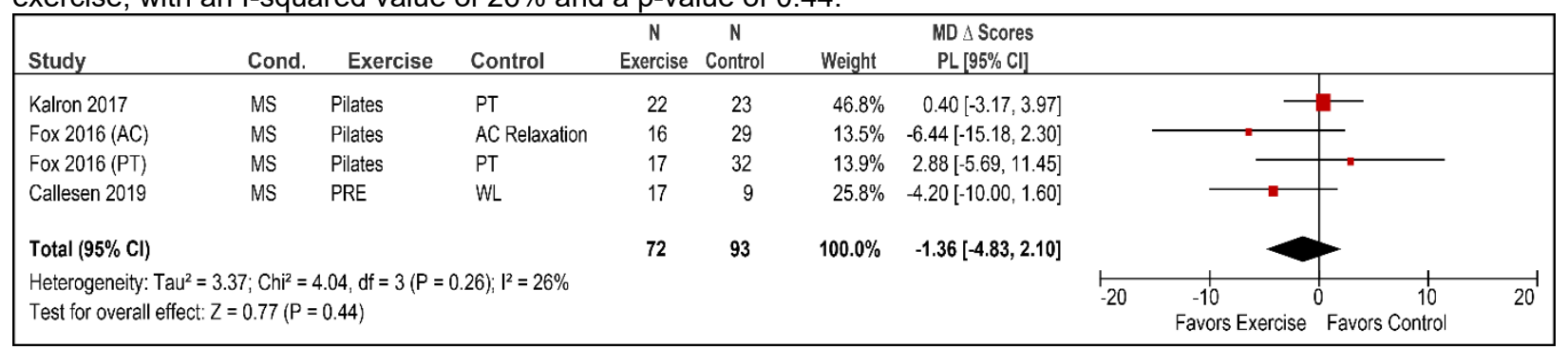

Abbreviations: $\mathrm{AC}=$ attention control; $\mathrm{CI}=$ confidence interval; $\mathrm{MD}=$ mean difference; $\mathrm{MSWS}-12=$ Multiple Sclerosis Walking Scale-12; PL = profile likelihood; PT = physical therapy

Figure 13. Muscle strength exercise versus usual care in multiple sclerosis: 25FWT immediately following intervention

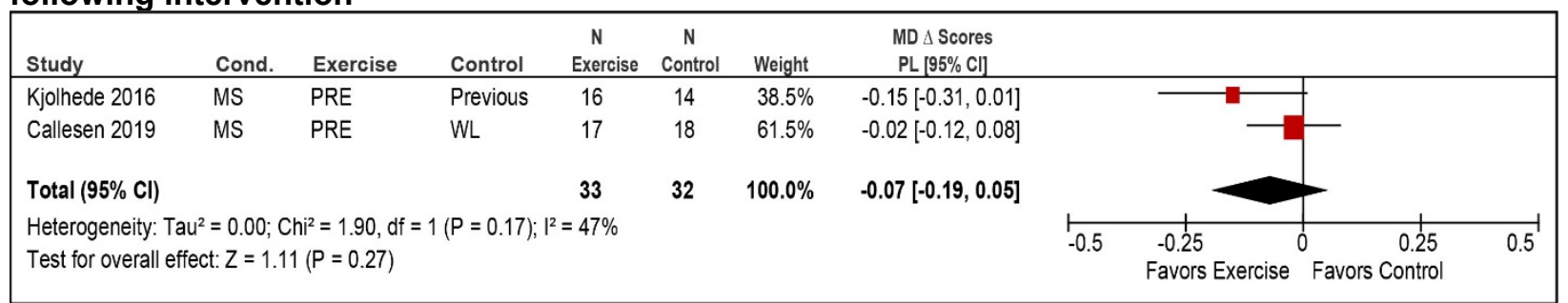

Abbreviations: $\Delta=$ change; $25 \mathrm{FWT}=25$-Foot Walk Test; $\mathrm{CI}=$ confidence interval; $\mathrm{MD}=$ mean difference; $\mathrm{MS}=$ multiple sclerosis; $\mathrm{PL}=$ profile likelihood; $\mathrm{PRE}=$ progressive resistance exercise; $\mathrm{WL}=$ waitlist 


\section{Functional Capacity Measures}

Muscle strengthening exercises resulted in no difference in improvement in functional capacity based on the TUG immediately postintervention compared with usual care or attention control ( 3 trials ${ }^{52,198,206} \mathrm{MD}-1.3$ seconds, $95 \% \mathrm{CI}-4.38$ to $1.78, \mathrm{I}^{2}=0 \%$ ) (Figure 14). Exclusion of the poor-quality trial ${ }^{206}$ resulted in a smaller but more precise effect estimate, however the difference did not reach statistical significance ( 2 trials, $\mathrm{MD}-0.61$ seconds, $95 \% \mathrm{CI}-2.00$ to $0.78, \mathrm{I}^{2}=33 \%$ ). One poor-quality study of Pilates versus aerobics found no difference between groups for the right or left TUG. ${ }^{62}$ Two trials ( 1 fair and 1 poor quality) ${ }^{68,69,149}$ found no improvement in the Six Spot Step Test (SSST), with strengthening exercises compared with usual care ( 2 trials, $\mathrm{MD}-2.88$ seconds, $95 \% \mathrm{CI}-7.51$ to $1.74, \mathrm{I}^{2}=95 \%$ ) (Figure 15 ).

Additionally, there was no difference in the SSST compared with aquatic exercise in one trial ${ }^{68,69}$ Results provided low-strength evidence of no clear benefit on function with strength exercises alone compared with usual care.

Figure 14. Muscle strength exercise versus usual care in multiple sclerosis: TUG immediately following intervention

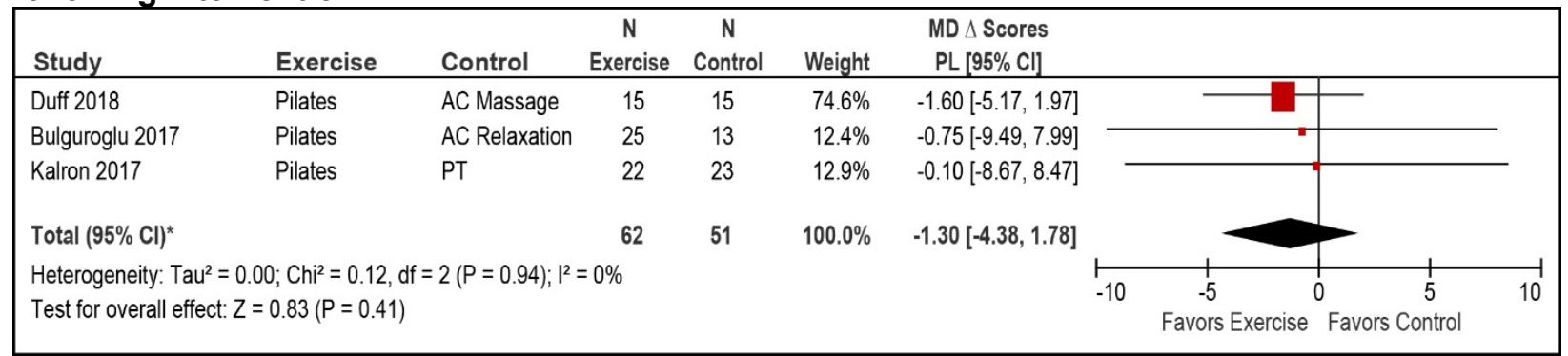

Abbreviations: $\Delta$ = change; $\mathrm{AC}=$ attention control; $\mathrm{CI}=$ confidence interval; $\mathrm{MD}=$ mean difference; $\mathrm{PL}=$ profile likelihood; $\mathrm{PT}$ $=$ physical therapy; TUG $=$ Timed Up and Go Test

Figure 15. Muscle strength exercise versus usual care in multiple sclerosis: SSST immediately following intervention

\begin{tabular}{|c|c|c|c|c|c|c|c|c|c|c|c|}
\hline Study & Cond. & Exercise & Control & $\begin{array}{c}\mathrm{N} \\
\text { Exercise }\end{array}$ & $\begin{array}{c}\mathrm{N} \\
\text { Control }\end{array}$ & Weight & $\begin{array}{l}\text { MD } \Delta \text { Scores } \\
\text { PL }[95 \% \mathrm{Cl}]\end{array}$ & & & & \\
\hline Marandi 2013 & MS & Pilates & Med-based Therapy & 15 & 15 & $50.5 \%$ & $-5.22[-6.45,-3.99]$ & & -5 & & \\
\hline Callesen 2019 & MS & PRE & WL & 17 & 18 & $49.5 \%$ & $-0.50[-2.05,1.05]$ & & - & & \\
\hline Total $(95 \% \mathrm{Cl})$ & & & & 32 & 33 & $100.0 \%$ & $-2.88[-7.51,1.74]$ & & & & \\
\hline \multicolumn{8}{|c|}{$\begin{array}{l}\text { Heterogeneity: } \text { Tau }^{2}=10.63 ; \mathrm{Chi}^{2}=21.82, \mathrm{df}=1(\mathrm{P}<0.00001) ; \mathrm{I}^{2}=95 \% \\
\text { Test for overall effect: } Z=1.22(P=0.22)\end{array}$} & -10 & $\begin{array}{c}-5 \\
\text { Favors Exercise }\end{array}$ & $\begin{array}{ll} & 1 \\
0 & 5 \\
\text { Favors Control }\end{array}$ & 10 \\
\hline
\end{tabular}

Abbreviations: $\Delta=$ change; $\mathrm{CI}=$ confidence interval; $\mathrm{MD}=$ mean difference; $\mathrm{MS}=$ multiple sclerosis; $\mathrm{PL}=$ profile likelihood; $\mathrm{PRE}=$ progressive resistance exercise $; \mathrm{SSST}=\mathrm{Six}$ Spot Step Test; $\mathrm{WL}=$ waitlist

\section{Quality of Life Measures}

Quality of life based on the Mental Component Summary (MCS) and Physical Component Summary (PCS) of either the SF-36 or MSQoL (0-100 scales) was reported in three trials. ${ }^{198,202,203,206}$ A small improvement in MCS was seen for muscle strengthening exercises versus attention control or massage ( 3 trials, ${ }^{198,202,203,206} \mathrm{MD}-3.5,95 \% \mathrm{CI}-6.61$ to -0.27 , $\mathrm{I}^{2}=0 \%$ ) (Figure 16). Exclusion of the poor-quality trial ${ }^{206}$ had a negligible impact on the effect size (MD $-3.85,95 \% \mathrm{CI}-7.33$ to $-0.37, \mathrm{I}^{2}=0 \%$ ). It is unclear whether this small difference represents a clinically meaningful improvement. There was no difference between groups for PCS across all three trials ${ }^{198,202,203,206}$ (MD -2.8, 95\% CI -6.68 to 3.12, $\mathrm{I}^{2}=34 \%$ ) (Figure 16). However, exclusion of the poor-quality trial ${ }^{206}$ resulted in a small improvement favoring muscle strengthening exercise ( 2 trials, $\mathrm{MD}-4.2,95 \% \mathrm{CI}-7.51$ to $-0.79, \mathrm{I}^{2}=0 \%$ ). Again, it is unclear 
whether this small difference represents a clinically meaningful improvement. Two additional trials found no difference in overall quality of life based on the WHOQOL immediately postintervention or 12 weeks after the intervention (difference $0.3,95 \% \mathrm{CI}-0.1$ to 0.6 , and difference $-0.2,95 \%$ CI -0.6 to 0.3 , respectively), ${ }^{83,204}$ or the EuroQOL-5 Dimension Questionnaire (EQD-5) immediately postintervention (difference $-0.5,95 \% \mathrm{CI}-1.5$ to 0.5 ) ${ }^{83}$ Together these studies found low-strength evidence of no clear benefit with strength exercises alone on quality of life compared with usual care.

Figure 16. Muscle strength exercise versus usual care in multiple sclerosis: MSQOL/SF-36 MCS and PCS immediately following intervention

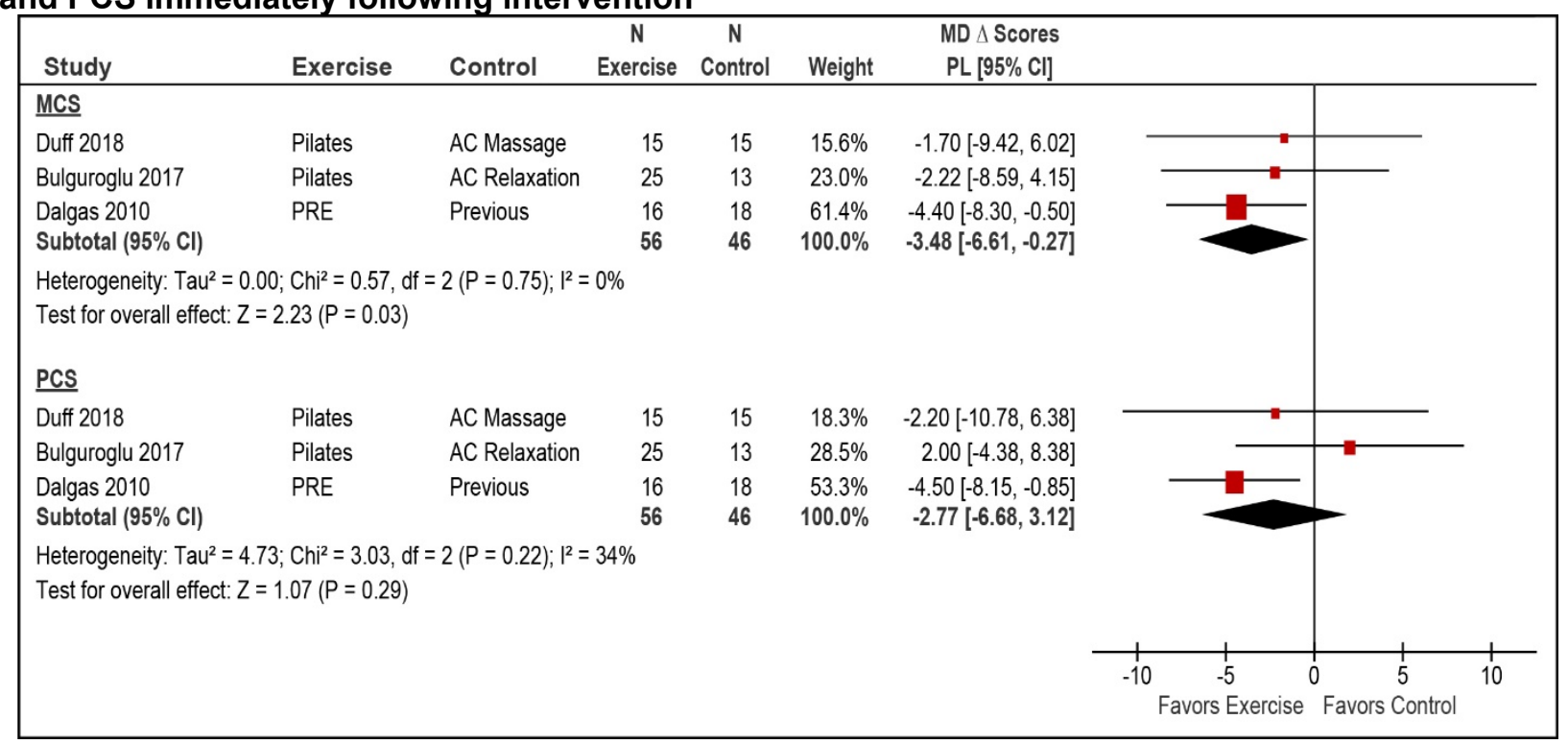

Abbreviations: $\Delta=$ change; $\mathrm{AC}=$ attention control; $\mathrm{CI}=$ confidence interval; $\mathrm{MD}=$ mean difference; $\mathrm{MSQOL}=\mathrm{Multiple}$ Sclerosis Quality of Life; PL = profile likelihood; PRE = progressive resistance exercise; SF-36 MCS/PCS = Short-Form 36 Mental Component Summary/Physical Component Summary

\section{Other Outcome Measures}

Across five trials comparing strengthening exercises with usual care, previous activity, or attention control, different measures of balance were used. Two trials with three comparisons assessed balance immediately postintervention using the Activities-specific Balance Confidence Scale (ABCS) (0-100 scale). Strengthening exercises were not associated with improved balance immediately postintervention ( $\mathrm{MD}-1.33,95 \% \mathrm{CI}-4.95$ to $2.60, \mathrm{I}^{2}=39 \%$ ) (Figure 17). ${ }^{149,200,201,206}$ There was no difference in balance improvement immediately postintervention in three other fair-quality trials, one using the Fullerton Advanced Balance Scale (scale 0-4, difference in change score MD $0.1,95 \%$ CI -5.43 to 5.63$)^{198}$ and two the BBS (scale 0-56, Figure 18, 2 trials $^{52,83}$ ). One poor-quality trial ${ }^{62}$ comparing strengthening to aerobic exercise found no difference in balance as assessed by the BBS immediately postintervention (difference in change score, MD $0.7,95 \% \mathrm{CI}-5.93$ to 7.23 ). These studies provided low-strength evidence for no benefit on balance between strength exercises and usual care, previous activity, or attention control.

Disability status was assessed in one fair-quality trial that compared strengthening exercises to previous activity level using the EDSS (0-10 scale). Strengthening exercises were not associated with an improvement in disability immediately postintervention (difference $0.1,95 \%$ CI -0.43 to 0.63$).^{202,203}$ 
Two fair-quality trials assessed depression immediately following 12 weeks of progressive resistance training (PRT) $)^{202,203}$ or 5 weeks of proprioceptive neuromuscular facilitation (PNF) ${ }^{83}$ using the Major Depression Inventory and Beck Depression Inventory, respectively. There was no difference comparing the strengthening exercise groups versus standard care (Figure 19, 2 trials $\left.^{83,202,203}\right)$. In the trial assessing PNF, the scores on the MSIS-29 improved slightly compared with usual care (MD $-2.9,95 \% \mathrm{CI}-5.4$ to -0.4$)$.

Upper extremity strengthening exercises were associated with a small functional improvement for both the more and the less affected upper extremity compared with attention control immediately postintervention based on the Action Research Arm Tests (ARAT) (scale 057 , difference in change score, $2.1,95 \%$ CI 1.54 to 2.56 and $0.5,95 \%$ CI 0.23 to 0.81 , respectively). ${ }^{205}$ However, it is not clear whether these small changes represent clinically important differences.

Figure 17. Muscle strength exercise versus usual care in multiple sclerosis: ABCS immediately following intervention

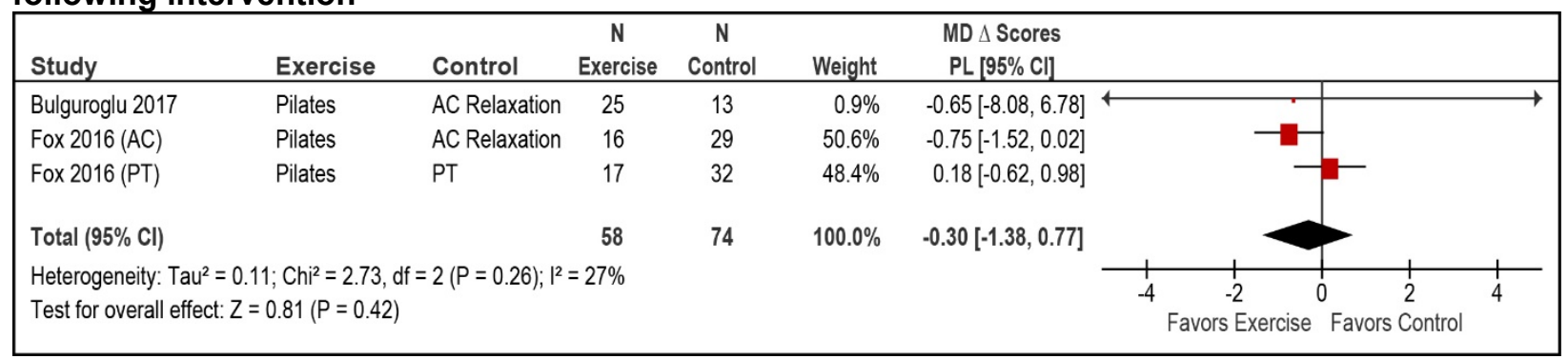

Abbreviations: $\Delta=$ change; ABCS = Activities-specific Balance Confidence Scale; $\mathrm{AC}=$ attention control; $\mathrm{CI}=$ confidence interval; $\mathrm{MD}=$ mean difference; $\mathrm{PL}=$ profile likelihood; $\mathrm{PT}=$ physical therapy

Figure 18. Muscle strength exercise versus usual care in multiple sclerosis: BBS immediately following intervention

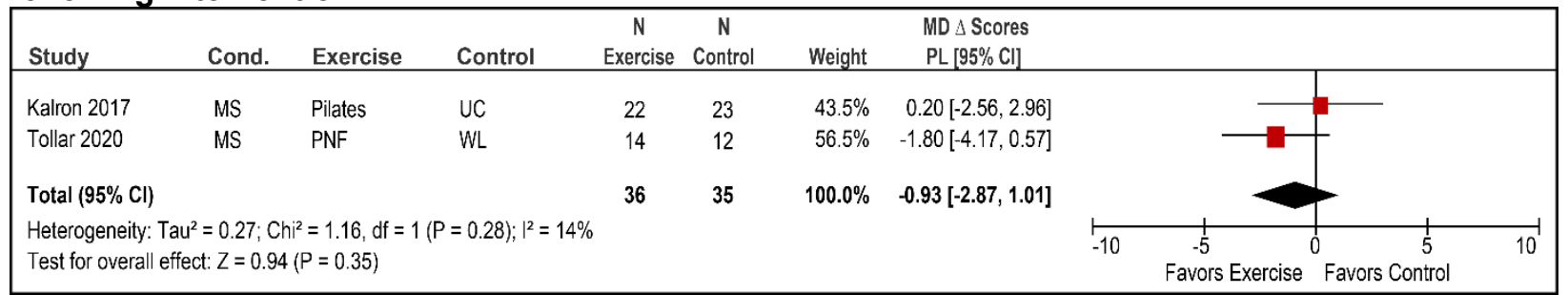

Abbreviations: $\Delta=$ change; $\mathrm{BBS}=$ Berg Balance Scale; $\mathrm{CI}=$ confidence interval; $\mathrm{MD}=$ mean difference; $\mathrm{PL}=$ profile likelihood; $\mathrm{SE}=$ standard error

Figure 19. Muscle strength exercise versus usual care in multiple sclerosis: BDI depression immediately following intervention

\begin{tabular}{|c|c|c|c|c|c|c|c|c|}
\hline Study & Cond. & Exercise & Control & $\begin{array}{c}\mathrm{N} \\
\text { Exercise } \\
\end{array}$ & $\begin{array}{c}\mathrm{N} \\
\text { Control } \\
\end{array}$ & Weight & $\begin{array}{l}\text { SMD } \triangle \text { Scores } \\
\text { PL }[95 \% \mathrm{Cl}]\end{array}$ & \\
\hline Dalgas 2010 & MS & PRE & Previous & 15 & 16 & $54.2 \%$ & $-0.32[-1.03,0.39]$ & 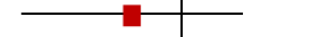 \\
\hline Tollar 2020 & MS & PNF & WL & 14 & 12 & $45.8 \%$ & $-0.04[-0.81,0.73]$ & 4 \\
\hline \multicolumn{4}{|l|}{ Total $(95 \% \mathrm{Cl})$} & 29 & 28 & $100.0 \%$ & $-0.19[-0.71,0.33]$ & \\
\hline \multicolumn{8}{|c|}{ Test for overall effect: $Z=0.72(P=0.47)$} & 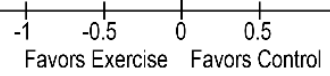 \\
\hline
\end{tabular}

Abbreviations: $\Delta=$ change; $\mathrm{BDI}=$ Beck Depression Inventory; $\mathrm{CI}=$ confidence interval; $\mathrm{MD}=$ mean difference; $\mathrm{MDI}=\mathrm{Major}$ Depression Inventory; $\mathrm{PL}=$ profile likelihood; $\mathrm{SE}=$ standard error 
Six trials did not address harms or adverse events. Three trials reported no adverse events; ${ }^{52,198,205}$ one trial reported no intervention-related adverse events but four events unrelated to the intervention ( 1 fractured ankle from falling in the snow in the Pilates group, 1 fractured humerus from falling in the snow in the PT group, and 1 pneumonia and 1 pancreatitis in the relaxation group); ${ }^{200,201}$ and one trial reported short-term muscle soreness in $25(69 \%)$ participants in the strengthening exercise group, ${ }^{204}$ however, no training sessions were missed due to any injury.

\section{Muscle Strength Exercise-Cerebral Palsy}

Seven trials ${ }^{207-216}$ and one quasiexperimental study ${ }^{217}$ enrolled participants with CP $(n=388)$ (Table 35). Weighted mean age of participants across trials was 9.9 years (range, 5.9 to 18.4 years) with weighted mean proportion female of 45 percent (range, 38\% to 53\%). No study provided data on race. Six studies enrolled participants who could, at minimum, ambulate using bilateral assistance (i.e., crutches, canes), ${ }^{207-212,215-217}$ while one enrolled children who could sit for 10 seconds with back unsupported and feet supported, ${ }^{213}$ and one did not report data on ambulatory status. ${ }^{214}$ The weighted mean baseline GMFM was 61.2 (range, 44.3 to 80.2). Seventeen percent of patients in the quasiexperimental study were wheelchair users; no other study provided data on wheelchair use. ${ }^{217}$ Six trials compared strengthening exercises with usual $\mathrm{PT},{ }^{207-209,211-216}$ and the remaining trial compared strengthening combined with neuroelectrical stimulation versus neuroelectrical stimulation alone. ${ }^{210}$ One quasiexperimental study compared strengthening exercise of the lower extremity and trunk with a nontraining group. ${ }^{217}$

Table 35. Muscle strength exercise in cerebral palsy

\begin{tabular}{|c|c|c|c|}
\hline $\begin{array}{l}\text { Author, Year } \\
\text { Intervention } \\
\text { Study Design } \\
\text { Study Quality }\end{array}$ & $\begin{array}{l}\text { Intervention } \\
\text { and Comparison }\end{array}$ & Population & Results \\
\hline $\begin{array}{l}\text { Cho, } 2020^{216} \\
\text { Strength } \\
\text { RCT } \\
\text { Poor }\end{array}$ & $\begin{array}{l}\text { A. FPRE, } 12 \\
\text { sessions over } 6 \\
\text { weeks }(n=13) \\
\text { B. Conventional } \\
\text { therapy, } 18 \\
\text { sessions over } 6 \\
\text { weeks }(n=12)\end{array}$ & $\begin{array}{l}\text { A vs. B } \\
\text { Age (mean years): } 5.54 \\
\text { vs. } 7.17 \\
\text { Female: } 9(69 \%) \text { vs. } 4 \\
(33 \%) \\
\text { Ambulatory: } 100 \% \\
\text { GMFCS: } 2.08 \text { vs. } 2.33\end{array}$ & $\begin{array}{l}\text { A vs. B, mean (SD) } \\
\text { GMFM-88 score } \\
69.98(21.55) \text { vs. } 68.15(27.15) \text { (baseline) } \\
71.78(21.05) \text { vs. } 63.48(27.48) \\
\text { (postintervention), } p=0.019 \text { for group } A \text { and } \\
0.375 \text { for group B for change from baseline } \\
\text { Increase pre-post for FPRE group } p=0.019 ; \\
\text { control group showed no significant difference, } \\
p=0.375 \text {. }\end{array}$ \\
\hline $\begin{array}{l}\text { Elnaggar } 2019^{215} \\
\text { Strength } \\
\text { RCT } \\
\text { Fair }\end{array}$ & $\begin{array}{l}\text { A. Plyometric } \\
\text { training, } 16 \\
\text { sessions over } 8 \\
\text { weeks }(n=19) \\
\begin{array}{l}\text { B. Usual care } \\
(n=20)\end{array}\end{array}$ & $\begin{array}{l}\text { Age: } 9.5 \text { vs. } 10.3 \\
\text { Female: } 32 \% \text { vs. } 45 \% \\
\text { Ambulatory: } 100 \% \text { All } \\
\text { patients were } \\
\text { considered to have mild } \\
\text { spastic CP }\end{array}$ & $\begin{array}{l}\text { A vs. B, mean (SD) } \\
\frac{10 \mathrm{MWT}(\mathrm{m} / \mathrm{s}):}{1.18(0.08) \text { vs. }} 1.21(0.09) \text { (baseline) } \\
1.29(0.06) \text { vs. } 1.25(0.05) \text { (postintervention) } \\
0.11(0.05) \text { vs. } 0.04(0.06), \text { MD } 0.07(95 \% \mathrm{Cl} \\
0.04 \text { to } 0.10) \text { (pre-post change score) }\end{array}$ \\
\hline $\begin{array}{l}\text { Kara, } 2020^{214} \\
\text { Strength } \\
\text { RCT } \\
\text { Fair }\end{array}$ & $\begin{array}{l}\text { A. Strength and } \\
\text { power training, } 36 \\
\text { sessions over } 12 \\
\text { weeks ( } n=15) \\
\text { B. Usual care } \\
\text { occupational } \\
\text { therapy, } 36 \\
\text { sessions over } 12 \\
\text { weeks }(n=15)\end{array}$ & $\begin{array}{l}\text { A vs. B } \\
\text { Age: } 12.3 \text { vs. } 11.8 \\
\text { Female: } 53 \% \text { vs. } 53 \% \\
\text { MACS Level } \\
\text { I: } 47 \% \text { vs. } 40 \% \\
\text { II: } 27 \% \text { vs. } 33 \% \\
\text { III: } 27 \% \text { vs. } 27 \% \\
\text { GMFCS Level } \\
\text { I: } 87 \% \text { vs. } 87 \% \\
\text { II: } 13 \% \text { vs. } 13 \% \\
\end{array}$ & 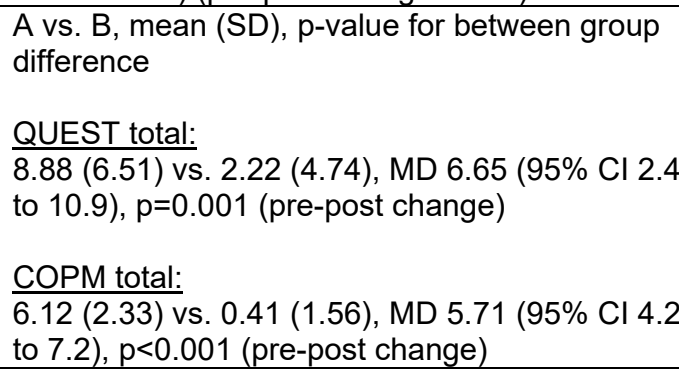 \\
\hline
\end{tabular}




\begin{tabular}{|c|c|c|c|}
\hline $\begin{array}{l}\text { Author, Year } \\
\text { Intervention } \\
\text { Study Design } \\
\text { Study Quality }\end{array}$ & $\begin{array}{l}\text { Intervention } \\
\text { and Comparison }\end{array}$ & Population & Results \\
\hline $\begin{array}{l}\text { Scholtes, 2010 } \\
\text { Scholtes, } 2012^{207} \\
\text { Scholtes, 2008 } \\
\text { Strength } \\
\text { RCT } \\
\text { Fair }\end{array}$ & $\begin{array}{l}\text { A. Progressive } \\
\text { resistance, } 36 \\
\text { sessions over } 12 \\
\text { weeks }(n=24) \\
\begin{array}{l}\text { B. Usual care } \\
(n=25)\end{array}\end{array}$ & $\begin{array}{l}\text { A vs. B } \\
\text { Age: } 10.33 \text { vs. } 10.25 \\
\text { Female: } 33 \% \text { vs. } 50 \% \\
\text { Ambulatory: } 100 \% \\
\text { Bilateral: } 71 \% \text { vs. } 60 \% \\
\text { GMFM I: } 54 \% \text { vs. } 48 \% \\
\text { GMFM II: } 33 \% \text { vs. } 36 \% \\
\text { GMFM III: } 13 \% \text { vs. } 16 \%\end{array}$ & $\begin{array}{l}\text { A vs. B, Regression effect size }(95 \% \mathrm{Cl}), \\
p=\text { between groups: } \\
\text { GMFM-66: }-0.56 \text { ( }-2.11 \text { to } 0.99), p=0.48 \\
\text { (postintervention); } 0.26(-1.23 \text { to } 1.76), p=0.73 \\
\text { (6 weeks postintervention) } \\
\text { 10MWT: }-0.04(-0.18 \text { to } 0.10), p=0.56 \\
\text { (postintervention); }-0.06(-0.17 \text { to } 0.04), p=0.25 \\
\text { (6 weeks postintervention) } \\
\text { Sit-to-Stand (reps): }-0.47(-2.28 \text { to } 1.33), \\
p=0.61 \text { (postintervention); }-0.75(-2.21 \text { to } 0.72) \text {, } \\
p=0.32 \text { (6-weeks postintervention) } \\
\text { Lateral step-up test (reps): } 0.48(-1.45 \text { to } 2.40) \text {, } \\
p=0.63 \text { (postintervention); } 0.13(-1.84 \text { to } 2.10), \\
p=0.9 \text { ( } 6 \text { weeks postintervention) } \\
\text { 1-minute fast walking test (m/s): } 0.04(-0.04 \text { to } \\
0.12), p=0.30 \text { (postintervention); }-0.01(-0.08 \text { to } \\
0.06), p=0.78 \text { (6 weeks postintervention) } \\
\text { Timed Stair Test (s): } 0.83(-2.64 \text { to } 4.30), \\
p=0.64 \text { (postintervention); } 2.87(-2.41 \text { to } 8.16), \\
p=0.29 \text { (6 weeks postintervention) }\end{array}$ \\
\hline $\begin{array}{l}\text { Taylor, } 2013^{211} \\
\text { Bania, } 2016^{212} \\
\text { Strength } \\
\text { RCT } \\
\text { Good }\end{array}$ & $\begin{array}{l}\text { A. Progressive } \\
\text { resistance, } 24 \\
\text { sessions over } 12 \\
\text { weeks }(n=23) \\
\begin{array}{l}\text { B. Usual care } \\
(n=25)\end{array}\end{array}$ & $\begin{array}{l}\text { A vs. B } \\
\text { Age: } 18.17 \text { vs. } 18.58 \\
\text { Female: } 44 \% \text { vs. } 48 \% \\
\text { No gait aid } 57 \% \text { vs. } \\
60 \% \\
\text { GMFM II: } 57 \% \text { vs. } 64 \% \\
\text { GMFM III: } 43 \% \text { vs. } 36 \%\end{array}$ & $\begin{array}{l}\text { A vs. B, mean difference }(95 \% \mathrm{Cl}) \text { between } \\
\text { groups: } \\
\text { GMFM-66-D: }-1.3(-4.9 \text { to } 2.4), p>0.05 \\
\text { (postintervention); } 2.5 \text { ( }-1.8 \text { to } 6.9), p>0.05 \text { (12 } \\
\text { weeks postintervention) } \\
\text { GMFM-66-E: } 0.9 \text { (-3.0 to } 4.7), p>0.05 \\
\text { (postintervention); } 1.0(-2.6 \text { to } 4.5), p>0.05 \text { (12 } \\
\text { weeks postintervention) } \\
\text { 6MWT: } 0.1 \text { ( }-20.6 \text { to } 20.9), p>0.05 \\
\text { (postintervention); }-12.3(-34.8 \text { to } 10.2), p>0.05 \\
\text { (12 weeks postintervention) } \\
\text { Timed Stair Test (s): }-0.9(-4.7 \text { to } 2.9) \\
\text { (postintervention); }-0.6(-4.2 \text { to } 3.0)(12 \text { weeks } \\
\text { postintervention) } \\
\text { Gait Profile Score }\left(^{\circ}\right): 0.2(-0.6 \text { to } 0.9), p>0.05 \\
\text { (postintervention); } 0.2(-0.8 \text { to } 1.2), p>0.05 \text { (12 } \\
\text { weeks postintervention) }\end{array}$ \\
\hline $\begin{array}{l}\text { Kirk, } 2016^{217} \\
\text { Strength } \\
\text { Quasiexperimental } \\
\text { Poor }\end{array}$ & $\begin{array}{l}\text { A. Progressive } \\
\text { resistance, } 36 \\
\text { sessions over } 12 \\
\text { weeks }(n=12) \\
\begin{array}{l}\text { B. Usual care } \\
(n=23)\end{array}\end{array}$ & $\begin{array}{l}\text { A+B } \\
\text { Age: } 36.5 \\
\text { Female: } 43 \% \\
\text { Wheelchair user: } 17 \%\end{array}$ & $\begin{array}{l}\text { A vs. B, mean (SD), } p=\text { between groups: } \\
\text { 10MWT: } 7.76(1.23) \text { to } 7.49(1.10) \text { vs. } 8.83 \\
\text { (0.78) to } 8.47(0.86), p>0.05 \\
\text { 6MWT: } 481(30) \text { to } 510(33) \text { vs. } 400(32) \text { to } 416 \\
\text { (33) p>0.05 } \\
\text { Timed Stair Test (s): } 30.69(4.92) \text { to } 29.15 \\
(4.62) \text { vs. } 49.82(7.27) \text { to } 45.01(6.57), p>0.05\end{array}$ \\
\hline $\begin{array}{l}\text { Qi, 2018a } 210 \\
\text { Strength } \\
\text { RCT } \\
\text { Fair }\end{array}$ & $\begin{array}{l}\text { A. Strength } \\
\text { exercises }+ \\
\text { neuromuscular } \\
\text { electrical } \\
\text { stimulation, } 30 \\
\text { sessions over } 6 \\
\text { weeks ( } n=50) \\
\text { B. Neuromuscular } \\
\text { electrical } \\
\text { stimulation, } 30 \\
\text { sessions over } 6 \\
\text { weeks }(n=50)\end{array}$ & $\begin{array}{l}\text { A vs. B } \\
\text { Age: } 5.8 \text { vs. } 6.0 \\
\text { Female: } 48 \% \text { vs. } 46 \% \\
\text { Spastic CP: } 100 \%\end{array}$ & $\begin{array}{l}\text { A vs. B, mean (SD) } \\
\text { GMFM-D/E: } \\
44.5(13.2) \text { vs. } 44 \text { (12.6), } p>0.05 \text { (baseline) } \\
70.6(15.2) \text { vs. } 56.7 \text { (14.3), } p<0.05 \\
\text { (postintervention) } \\
\text { MD } 13.4,95 \% \mathrm{Cl} 7.94 \text { to } 18.86, p<0.001 \\
71.0 \text { (16.4) vs. } 58.0 \text { (15.6), } p<0.05 \text { (6 weeks } \\
\text { postintervention) } \\
\text { MD } 12.5,95 \% \mathrm{Cl} 6.74 \text { to } 18.26, p<0.001\end{array}$ \\
\hline
\end{tabular}




\begin{tabular}{|c|c|c|c|}
\hline $\begin{array}{l}\text { Author, Year } \\
\text { Intervention } \\
\text { Study Design } \\
\text { Study Quality }\end{array}$ & $\begin{array}{l}\text { Intervention } \\
\text { and Comparison }\end{array}$ & Population & Results \\
\hline $\begin{array}{l}\text { Tedla, } 2014^{213} \\
\text { Strength } \\
\text { RCT } \\
\text { Poor }\end{array}$ & $\begin{array}{l}\text { A. Strength training } \\
18 \text { sessions over } 6 \\
\text { weeks }+ \\
\text { conventional PT } \\
(n=31) \\
\text { B. Conventional } \\
\text { PT } 3-5 \text { sessions } \\
\text { per week for } 6 \\
\text { weeks }(n=31)\end{array}$ & $\begin{array}{l}\text { A vs. B (data are for } \\
\text { completers only; n=30 } \\
\text { vs. } 30 \text { ) } \\
\text { Age: } 9.1 \text { vs. } 8.9 \text { years } \\
\text { Female: } 33 \% \text { vs. } 33 \% \\
\text { Gross motor function } \\
\text { classification system: } \\
\text { I: } 7 \% \text { vs. } 3 \% \\
\text { II: } 20 \% \text { vs. } 27 \% \\
\text { III: } 37 \% \text { vs. } 27 \% \\
\text { IV: } 37 \% \text { vs. } 43 \% \\
\end{array}$ & $\begin{array}{l}\text { A vs. B, mean change from baseline (SD): } \\
\frac{\text { PBS total score }}{7.23(3.350) \text { vs. } 1.87(1.074), p<0.001} \\
\frac{\text { GMFM-total score }}{9.9(N R) \text { vs. } 2.2(N R), p=N R}\end{array}$ \\
\hline
\end{tabular}

Abbreviations: 6MWT = 6-Minute Walk Test; 10MWT = 10-Meter Walk Test; $\mathrm{AE}=$ adverse event; COPM = Canadian Occupational Performance Measure; $\mathrm{CI}=$ confidence interval; $\mathrm{COPM}=$ Canadian Occupational Performance $\mathrm{Measure} ; \mathrm{CP}=$ cerebral palsy; $\mathrm{EPC}=$ Evidence-based Practice Center; FPRE $=$ functional progressive resistance exercise; GMFM $=$ Gross Motor Function Measure; GMFM-66 = Gross Motor Function Measure 66; GMFM-66-D = Gross Motor Function Measure 66 (standing); GMFM-66-E = Gross Motor Function Measure 66 (walking, running, jumping); MACS = manual ability classification system; MD = mean difference; $\mathrm{NR}=$ not reported; PBS = Pediatric Balance Scale; PT = physical therapy; QUEST $=$ Quality of Upper Extremity Skill Test; RCT = randomized controlled trial; SD = standard deviation

\section{Walking Measures}

There were no differences between strengthening exercise compared with usual PT care in

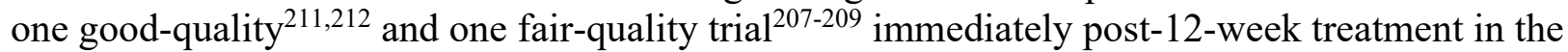
1MWT, ${ }^{207-209}$ the 6MWT, ${ }^{211,212}$ and the 10MWT (Figure 20, 2 trials ${ }^{207-209,215}$ ). In one trial, ${ }^{211,212}$ there was no improvement in gait profile scores following strength exercises versus PT in immediate- or short-term followup. One quasiexperimental study also reported no difference on 6MWT and 10MWT comparing strengthening with active training. ${ }^{217}$ At short-term followup, no difference was reported in walking ability between groups in the two trials (1 minute fast walking test), ${ }^{207-209}$ the $6 \mathrm{MWT},{ }^{211,212}$ and the 10MWT. ${ }^{207-209}$ These studies provided lowstrength evidence of no clear benefit on walking with strength exercises alone compared with usual care immediately postintervention and at short-term followup.

\section{Functional Capacity Measures}

Evidence on functional outcomes with strengthening exercise in children with CP was based on one good-quality, ${ }^{211,212}$ one fair-quality, ${ }^{207-209}$ and two poor-quality studies ${ }^{213,216}$ (Table 35) and provided low-strength evidence of no clear benefit of strengthening exercise on function versus control groups using the GMFM immediately, 6 weeks, or 12 weeks following treatment. One fair-quality trial ${ }^{207-209}$ also reported no difference between groups in the 30-second lateral step-up test in immediate and short-term followup.

In one fair-quality trial, ${ }^{210}$ strength exercises combined with neuroelectrical stimulation versus neuroelectrical stimulation alone resulted in improved functional capacity based on the GMFM in immediate- and short-term followup (difference in change scores $-13.4,95 \% \mathrm{CI}$ -16.90 to -9.90 ; and $-12.5,95 \% \mathrm{CI}-16.26$ to -8.74 , respectively). Due to study limitations, lack of corroborating evidence, and imprecision in the estimates, this evidence was insufficient to draw conclusions.

\section{Other Outcome Measures}

Across two trials ( 1 good and 1 fair quality), there was no improvement in the timed stair test with strengthening versus usual PT immediately postintervention and at short-term followup. ${ }^{207-}$ 
209,211,212 The one poor-quality quasiexperimental study reported no difference immediately posttreatment between strength exercises and usual care in the timed stair test. ${ }^{217}$ Strengthening compared with usual PT did not improve 30-second sit-to-stand in one trial in the immediate- or short-term followup. ${ }^{207-209}$

Strengthening compared with usual PT improved balance immediately after the intervention as measured by the PBS in one poor-quality trial ${ }^{213}$ (differences in change scores 7.23 , standard deviation (SD) 3.35 and 1.87, SD 1.07, p<0.001) and in the Forward and Side Functional Reach Test (before-after change, $\mathrm{p}<0.005$ ) in another poor-quality trial. ${ }^{216}$

One fair-quality trial $(n=30)$ found the quality of upper extremity movement, and both activity performance and satisfaction improved, with strength and power training versus usual occupational therapy. ${ }^{214}$ Due to the risk of bias and small sample size, the evidence for improved upper extremity movements is too limited to draw conclusion. None of the trials reported on quality of life measures.

Adverse events were reported in one RCT and in one quasiexperimental study. In the RCT, ${ }^{211,212}$ short-term muscle soreness was reported by most participants in the strength exercise group. Additionally, one minor calf strain and one minor discomfort due to plantar fasciitis occurred in the same group. In the quasiexperimental study, most subjects in the exercise group reported muscle soreness and three subjects reported irritation in tendon tissue surrounding the knee. ${ }^{217}$ One trial reported no adverse events in either treatment $\operatorname{arm}^{214}$ and the remaining four RCTs did not address harms or adverse events.

Figure 20. Muscle strength exercise versus usual care in cerebral palsy: 10MWT immediately following intervention

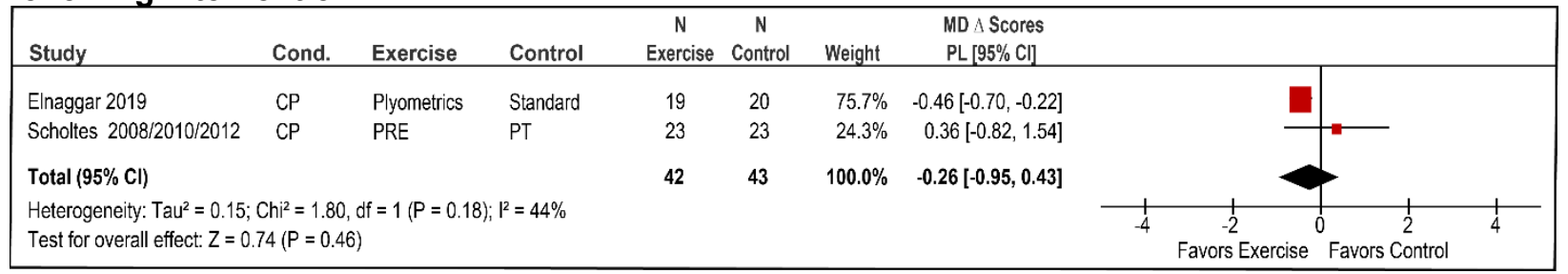

Abbreviations: $\Delta=$ change; $10 \mathrm{MWT}=10$-meter Walk Test; $\mathrm{CI}=$ confidence interval; $\mathrm{MD}=$ mean difference; $\mathrm{PL}=$ profile likelihood.

\section{Muscle Strength Exercise-Spinal Cord Injury}

One fair-quality trial ${ }^{218}$ enrolled adult males $(\mathrm{n}=98)$ with SCI (Table 36). The mean age of participants was 63 years; all participants were paraplegic. Limited evidence suggested 12 months of breathing and upper limb strength exercises improved quality of life as measured on four of five SF-36 subscales (0-100 scale) immediately after the intervention: physical function (26.7, 95\% CI 24.61 to 28.79 ); social function (28.9, $95 \%$ CI 26.06 to 31.74 ); role emotional (22.0, 95\% CI 20.11 to 23.89 ); and mental health (21.0, 95\% CI 19.10 to 22.90$)$. There were no differences between groups in any of the SF-36 subscales after 4 weeks of intervention. However, the evidence was considered too limited to draw firm conclusions on the effectiveness of strength training on quality of life (SOE: insufficient). ${ }^{218}$

This trial did not address harms or adverse events. 
Table 36. Muscle strength exercise in spinal cord injury

\begin{tabular}{|c|c|c|c|}
\hline $\begin{array}{l}\text { Author, Year } \\
\text { Intervention } \\
\text { Study Design } \\
\text { Study Quality }\end{array}$ & $\begin{array}{l}\text { Intervention } \\
\text { and Comparison }\end{array}$ & Population & Prioritized Outcomes \\
\hline $\begin{array}{l}\text { Chen, } 2016^{218} \\
\text { Strength } \\
\text { RCT } \\
\text { Fair }\end{array}$ & $\begin{array}{l}\text { A. Pulmonary } \\
\text { rehabilitation, } 365 \\
\text { sessions over } 52 \\
\text { weeks ( } n=49) \\
\begin{array}{l}\text { B. Usual care } \\
(n=49)\end{array}\end{array}$ & $\begin{array}{l}\text { A vs. B } \\
\text { Age: } 62.3 \text { vs. } 63.1 \\
\text { Female: } 0 \% \\
\text { T1-2: } 35 \% \text { vs. } 35 \% \\
\text { T3-4: } 33 \% \text { vs. } 33 \% \\
\text { T5-6: } 33 \% \text { vs. } 33 \%\end{array}$ & 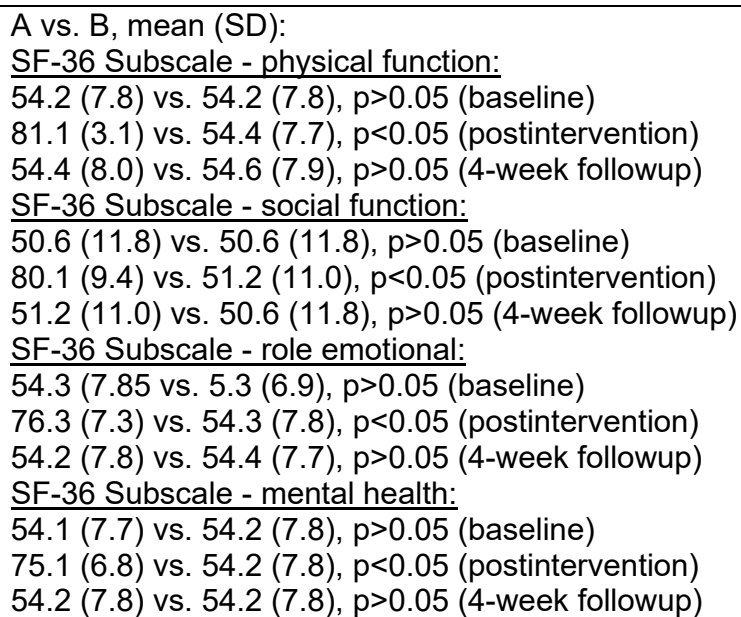 \\
\hline
\end{tabular}

Abbreviations: $\mathrm{RCT}=$ randomized controlled trial; $\mathrm{SD}=$ standard deviation; SF-36=Short-Form 36

\section{Multimodal Interventions}

\section{Progressive Resistance or Strengthening Combination Exercises}

Multimodal exercises provide information on the benefit of combining different types of interventions. Unlike single exercise interventions, multimodal exercise blends various types of exercises by linking progressive resistance exercise and/or strengthening exercises with at least one component of aerobic exercises, balance exercises, or other interventions.

\section{Key Points}

- In participants with MS, across measures of walking ability (6MWT and 10MWT), multimodal exercise was associated with improved walking ability and balance compared with usual care at the end of treatment (SOE: low). Evidence was insufficient to draw conclusions from one small trial of group versus home-based exercise with regard to walking ability or balance.

- Evidence was limited in MS on functional capacity and quality of life (SOE: insufficient).

- In participants with CP, there was low-strength evidence of no clear benefit in functional capacity or quality of life with multimodal exercises. Evidence was insufficient to draw conclusions regarding the impact of multimodal exercise on walking.

- In participants with SCI, evidence from small trials was insufficient to draw conclusions regarding the impact of exercise on walking or functional capacity (SOE: insufficient).

\section{Detailed Synthesis}

Seventeen RCTs, ${ }^{220-223,225,226,228-230,232,234-242,244-248}$ one quasiexperimental study, ${ }^{233}$ and one cohort study ${ }^{250}$ in 911 participants evaluated multimodal exercise. Multimodal exercises included progressive resistance exercises/strengthening exercises in combination with aerobic exercise. Some also included balance exercises. Most trials compared multimodal exercise to usual care. Usual care included maintaining previous activity levels (5 trials, 1 quasiexperimental 
study), ${ }^{222,223,225,226,228,233}$ inclusion of an attention control (2 trials), ${ }^{221,232} \mathrm{PT}$ (4 trials), ${ }^{234-242}$ selfregulated exercise ( 1 study), ${ }^{250}$ or waitlist ( 2 trials). ${ }^{230,231,246,247}$ Additional trials compared multimodal exercise with aerobic exercise ${ }^{220}$ or a different combination of multimodal exercises. $^{244,245,248}$ Ten trials ${ }^{220-223,225,226,228,230-232}$ and one quasiexperimental study ${ }^{233}$ enrolled participants with MS (Table 37), four trials ${ }^{234-242}$ were in participants with CP (Table 38), and four studies ( 3 RCTs, 1 cohort study) $)^{244-248,250}$ were conducted in participants with SCI (Table 39). Twelve 220-223,230-232,234-242,244,245,248 trials met criteria for fair quality and four $225,226,228,246,247^{2}$ were rated poor quality and deemed to have high risk of bias due to unclear randomization and/or allocation concealment, lack of similarity between treatment groups at baseline, and unacceptable attrition. The quasiexperimental and cohort studies were considered to be fair quality. ${ }^{233,250}$ The most frequently prioritized outcomes reported were walking-related measures (e.g., 6MWT, 10MWT) and quality of life (e.g., SF-36); few studies reported on functional capacity measures (e.g., TUG). Measures reported varied by condition. Differences in change scores (mean difference) between treatment groups were reported unless otherwise noted.

\section{Multimodal Exercises-Multiple Sclerosis}

Ten trials ${ }^{220-223,225,226,228,230-232}$ and one quasiexperimental study ${ }^{233}$ enrolled participants $(n=540)$ with MS (Table 37). Weighted mean age of participants across trials was 39.76 years (range 32.7 to 52.0 years) with weighted mean proportion female of 73.9 percent (range 55.9\% to $100 \%)$. No study provided data on race. All studies enrolled participants who could, at minimum, ambulate using bilateral assistance (i.e., crutches or canes), and the weighted mean baseline EDSS (across 4 studies) $222,223,228,232$ was 4.9 (range 1.61 to 8.7).

Table 37. Multimodal exercise in multiple sclerosis

\begin{tabular}{|c|c|c|c|}
\hline \begin{tabular}{|l} 
Author, Year \\
Intervention \\
Study Design \\
Study Quality
\end{tabular} & $\begin{array}{l}\text { Intervention } \\
\text { and Comparison }\end{array}$ & Population & Results \\
\hline $\begin{array}{l}\text { Cakit, } 2010^{225} \\
\text { Multimodal } \\
\text { exercise } \\
\text { RCT } \\
\text { Poor }\end{array}$ & $\begin{array}{l}\text { A. Progressive } \\
\text { resistance cycling } \\
\text { plus balance } \\
\text { exercises (lower } \\
\text { extremity } \\
\text { strengthening), } 16 \\
\text { sessions over } 8 \\
\text { weeks ( } n=14) \\
\text { B. Usual care } \\
(n=9)\end{array}$ & $\begin{array}{l}\text { A vs. B } \\
\text { Age: } 36.4 \text { vs. } 35.5 \\
\text { Female: } 64 \% \text { vs. } 67 \% \\
\text { RRMS or SPMS: } \\
100 \% \\
\text { Assistive device: } \\
28.5 \% \text { vs. } 37.5 \%\end{array}$ & $\begin{array}{l}\text { A vs. B, mean (SD) change, } p=\text { between groups: } \\
\text { TUG: }-1.3(1.2) \text { vs. }-0.2(0.8), p<0.05 \\
\text { 10MWT: }-1.9(1.2) \text { vs. } 0.1(0.8), p<0.05 \\
\text { DGI: } 2.7(0.5) \text { vs. } 0.4(0.4), p<0.01 \\
\text { Falls Efficiency Scale: }-11.3(7.8) \text { vs. }-2.6(3.1), \\
\text { p<0.01 } \\
\text { SF-36 Physical Function: } 21.2(14.4) \text { vs. } 7.7(7.4), \\
\text { p>0.05 } \\
\text { SF-36 Role-Physical Function: } 34.0(30.1) \text { vs. } 5.0 \\
\text { (44.7), p>0.05 } \\
\text { SF-36 General Health: } 4.3(8.4) \text { vs. } 3.2(11.7), \\
\text { p }>0.05 \\
\text { SF-36 Vitality: } 9.0(19.3) \text { vs. } 11.0(20.4), p>0.05 \\
\text { p }>0.05 \\
\text { SF-36 Rocial Functioning: } 3.4(23.1) \text { vs. } 5.0(16.7), \\
\text { (50.5), p>0.05 } \\
\text { SF-36 Mental Health: } 7.2(13.4) \text { vs. } 7.0(6.7), p>0.05\end{array}$ \\
\hline
\end{tabular}




\begin{tabular}{|c|c|c|c|}
\hline $\begin{array}{l}\text { Author, Year } \\
\text { Intervention } \\
\text { Study Design } \\
\text { Study Quality }\end{array}$ & $\begin{array}{l}\text { Intervention } \\
\text { and Comparison }\end{array}$ & Population & Results \\
\hline $\begin{array}{l}\text { Ebrahimi, } 2015^{228} \\
\text { Multimodal } \\
\text { exercise } \\
\text { RCT } \\
\text { Poor }\end{array}$ & $\begin{array}{l}\text { A. Whole body } \\
\text { vibration + low- } \\
\text { intensity exercise, } \\
30 \text { sessions over } \\
10 \text { weeks }(n=17) \\
\text { B. Usual care } \\
(n=17)\end{array}$ & $\begin{array}{l}\text { A vs. B } \\
\text { Age: } 37.06 \text { vs. } 40.75 \\
\text { Female: } 69 \% \text { vs. } 86 \% \\
\text { Ambulatory: } 100 \% \\
\text { EDSS: } 3.12 \text { vs. } 3.10\end{array}$ & $\begin{array}{l}\text { A vs. } B, \text { mean (SD), } p=\text { between groups: } \\
\text { TUG: } 11.32(5.21) \text { to } 11.16(8.82) \text { vs. } 14.43(3.20) \text { to } \\
\text { 14.57 }(4.02), p=0.05 \\
\text { 10MWT: } 17.67(8.92) \text { to } 13.37(4.59) \text { vs. } 21.16 \\
\text { (6.36) to } 19.39(6.52), p=0.56 \\
\text { 6MWT: } 184.01(101.04) \text { to } 272.32(105.60) \text { vs. } \\
\text { 150.37 }(65.18) \text { to } 162.80(60.57), p=0.01 \\
\text { MSQoL-54 PCS: } 45.80(9.70) \text { to } 53.36(11.9) \text { vs. } \\
43.38(15.43) \text { to } 45.53(7.30), p=0.40 \\
\text { MSQoL-54 MCS: } 50.87(15.46) \text { to } 58.34(14.89) \text { vs. } \\
41.66(17.07) \text { to } 50.10(14.72), p=0.42 \\
\text { EDSS: } 3.12(1.19) \text { to } 2.65(1.20) \text { vs. } 3.10(0.76) \text { to } \\
\text { 3.03 (0.69), } p=0.01 \\
\text { BBS: } 40.37(9.97) \text { to } 46.43(8.34) \text { vs. } 34.00(9.13) \text { to } \\
35.85(7.22), p=0.01\end{array}$ \\
\hline $\begin{array}{l}\text { Faramarzi, } \\
2020^{230} \\
\text { Has companion: } \\
\text { Banitalebi, } \\
2020^{231} \\
\text { Multimodal } \\
\text { Exercise } \\
\text { Immediately } \\
\text { Postintervention, } \\
12 \text { weeks } \\
\text { RCT } \\
\text { Fair }\end{array}$ & $\begin{array}{l}\text { A. Resistance + } \\
\text { endurance }+ \\
\text { Pilates }+ \text { balance } \\
+ \text { stretch), } \\
36 \text { sessions over } \\
12 \text { weeks ( } n=23 \text { ) } \\
\text { B. Combined } \\
\text { exercise - } \\
\text { Moderate } \\
\text { disability group } \\
\text { (4.5 EDSS } \leq 6) \\
36 \text { sessions (3 } \\
\text { per week) over } \\
12 \text { weeks ( } n=13) \\
\text { C. Combined } \\
\text { exercise }- \text { High } \\
\text { disability group } \\
\text { (EDSS } \geq 6.5) \\
36 \text { sessions (3 } \\
\text { per week) over } \\
12 \text { weeks ( } n=11 \text { ) } \\
\text { D. Waitlist } \\
\text { control } \\
\text { Low ( } n=23 \text { ) } \\
\text { E. Waitlist } \\
\text { control Moderate } \\
\text { ( } n=13) \\
\text { F. Waitlist } \\
\text { control } \\
\text { High ( } n=11 \text { ) }\end{array}$ & $\begin{array}{l}\text { A vs. B vs. C vs. D } \\
\text { Age: NR (between } \\
18 \text { and } \\
50 \text { years) } \\
\text { Female: } 100 \% \\
\text { Ambulatory: } 100 \% \\
\\
\text { EDSS score: } \\
\text { EDSS < } 4.5: \\
\text { A. } 23(24 \%) \text { vs. D. } \\
23(24 \%) \\
\text { EDSS } \leq 4.5 \text { to } \leq 6: \\
\text { B. } 13(14 \%) \text { vs. D. } 13 \\
(14 \%) \\
\text { EDSS } \geq 6.5: \\
\text { C. } 11(12 \%) \text { vs. D. } 11 \\
(12 \%)\end{array}$ & 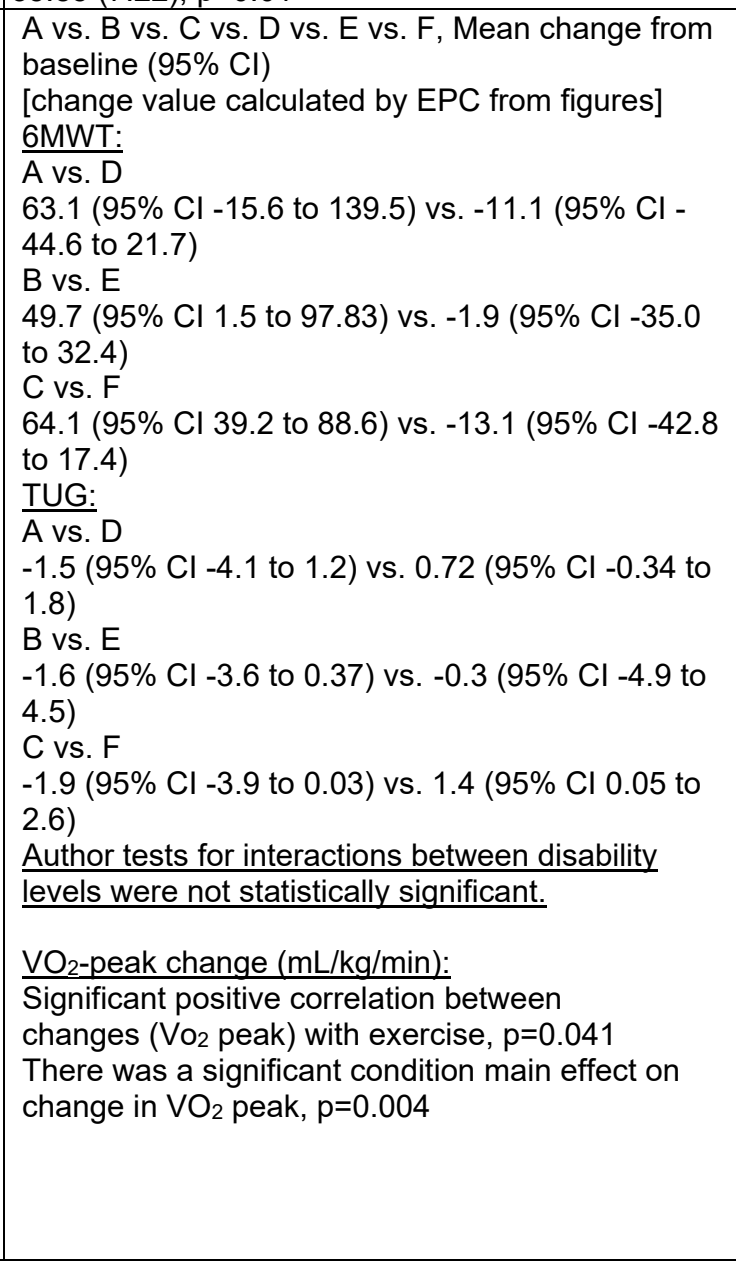 \\
\hline
\end{tabular}




\begin{tabular}{|c|c|c|c|}
\hline $\begin{array}{l}\text { Author, Year } \\
\text { Intervention } \\
\text { Study Design } \\
\text { Study Quality } \\
\end{array}$ & $\begin{array}{l}\text { Intervention } \\
\text { and Comparison }\end{array}$ & Population & Results \\
\hline $\begin{array}{l}\text { Kerling, } 2015^{220} \\
\text { Multimodal } \\
\text { exercise } \\
\text { RCT } \\
\text { Fair }\end{array}$ & $\begin{array}{l}\text { A. Full body } \\
\text { progressive } \\
\text { resistance }+ \\
\text { aerobic training, } \\
36 \text { sessions over } \\
12 \text { weeks }(n=30) \\
\\
\text { B. Aerobic } \\
\text { training, } 36 \\
\text { sessions over } 12 \\
\text { weeks }(n=30)\end{array}$ & $\begin{array}{l}\text { A vs. B } \\
\text { Age: } 42.3 \text { vs. } 45.6 \\
\text { Female: } 80 \% \text { vs. } 67 \% \\
\text { EDSS: } 2.6 \text { vs. } 3.1\end{array}$ & $\begin{array}{l}\text { A vs. B, mean (SD), p=between groups: } \\
\text { SF-36 PCS: } 44.9(9.1) \text { to } 46.2(9.1) \text { vs. } 39.0(10.8) \\
\text { to } 39.6(11.3), p=0.56 \\
\text { SF=36 MCS: } 44.9(13.6) \text { to } 45.4(13.4) \text { vs. } 46.7 \\
\text { (11.7) to } 51.4(8.6), p=0.01\end{array}$ \\
\hline $\begin{array}{l}\text { Ozkul, 2020b } 232 \\
\text { Multimodal } \\
\text { Exercise } \\
\text { RCT } \\
\text { Fair }\end{array}$ & $\begin{array}{l}\text { A. Aerobics + } \\
\text { Pilates, } 24 \\
\text { sessions over } 8 \\
\text { weeks }(n=17) \\
\\
\text { B. Control group, } \\
\text { relaxation exercise } \\
\text { at home, } 24 \\
\text { sessions over } 8 \\
\text { weeks }(n=17)\end{array}$ & $\begin{array}{l}\text { A vs. } B \\
\text { Age: } 35.8 \text { vs. } 36.7 \\
\text { Female: } 76 \% \text { vs. } 76 \% \\
\text { Ambulatory: } 100 \% \\
\text { EDSS: } 1.5 \text { vs. } 1.71\end{array}$ & $\begin{array}{l}\text { A vs. B, Mean (SD), change mean (SD), } p=\text { within } \\
\text { groups } \\
\text { 6MWT (meters): } \\
539.94(50.21) \text { vs. } 513.82(50.96) \text { (baseline) } \\
587.92(51.44) \text { vs. } 502.75(53.54) \text { (postintervention); } \\
\text { change mean (SD) } 47.98 \text { (23.34) vs. }-11.07 \\
\text { (36.40), p<0.001 } \\
\text { MSQOL-54-MCS: } \\
62.74(19.37) \text { vs. } 56.29(16.47) \text { (baseline) } \\
74.24(14.83) \text { vs. } 50.91(20.42) \text { (postintervention) } \\
\text { change mean (SD) } 11.50(15.94) \text { vs. }-5.38 \text { (17.37), } \\
\text { p=0.006 } \\
\text { MSQOL-54-PCS: } \\
120.54(29.32) \text { vs. } 109.67(27.89) \text { (baseline) } \\
140.08(18.42) \text { vs. } 97.83(35.58) \text { (postintervention) } \\
\text { change mean (SD) } 19.54 \text { (14.42) vs. }-11.84 \\
(28.36), p<0.001 \\
52\end{array}$ \\
\hline $\begin{array}{l}\text { Roppolo, } 2013^{233} \\
\text { Multimodal } \\
\text { exercise } \\
\text { Quasiexperimental } \\
\text { Fair }\end{array}$ & $\begin{array}{l}\text { A. Combination } \\
\text { therapy (aerobic }+ \\
\text { strength training), } \\
24 \text { sessions over } \\
12 \text { weeks }(n=17) \\
\text { B. Usual care } \\
(n=18)\end{array}$ & $\begin{array}{l}\text { A vs. } B \\
\text { Age: } 40 \text { vs. } 40 \text { years } \\
\text { Female: } 100 \% \text { vs. } \\
100 \% \\
\text { EDSS: } 1.5 \text { vs. } 2.0\end{array}$ & $\begin{array}{l}\text { A vs. B, mean (SD) } \\
\text { MSQOL-54 } \\
202.7(7.9) \text { vs. } 139.3 \text { (32.4), MD } 63.4(7.86)(95 \% \mathrm{Cl} \\
47.43 \text { to } 79.4), \text { p<0.001 (postintervention); } \\
29.5(36.17) \text { vs. }-22.5 \text { (55.57), MD } 52.0,95 \% \mathrm{Cl} \\
20.8 \text { to } 83.2, \text { p=NR (pre-post change) }\end{array}$ \\
\hline $\begin{array}{l}\text { Sandroff, } 2017^{221} \\
\text { Multimodal } \\
\text { exercise } \\
\text { RCT } \\
\text { Fair }\end{array}$ & $\begin{array}{l}\text { A. Resistance + } \\
\text { aerobics + } \\
\text { balance, } 72 \\
\text { sessions over } 24 \\
\text { weeks. ( }=43) \\
\\
\begin{array}{l}\text { B. Usual care- } \\
\text { stretching and } \\
\text { toning, } 72 \\
\text { sessions over } 24 \\
\text { weeks }(n=40)\end{array}\end{array}$ & $\begin{array}{l}\text { A vs. B } \\
\text { Age: } 49.8 \text { vs. } 51.2 \\
\text { Female: } 83.7 \% \text { vs. } \\
87.5 \% \\
\text { EDSS } 4-6: 100 \% \\
\text { Walking difficulties: } \\
100 \%\end{array}$ & $\begin{array}{l}\text { A vs. B mean (SD), p=between groups: } \\
\text { 6MWT: } 1073.1(529.0) \text { vs. } 1097.5(493.3) \\
\text { (baseline); } 1185.5(600.5) \text { vs. } 1115.1(512.7) \\
\text { (postintervention), p=0.05 } \\
\text { 25 foot WT: } 3.7(1.8) \text { vs. } 4.0(1.4) \text { (baseline); } 4.0 \\
\text { (1.9) vs. } 4.0(1.5) \text { (postintervention), p>0.11 } \\
\text { MSWS-12: } 64.8(24.7) \text { vs. } 51.8(24.7) \text { (baseline); } \\
59.0(23.4) \text { vs. } 49.3 \text { (27.1) (postintervention), } p=0.98\end{array}$ \\
\hline
\end{tabular}




\begin{tabular}{|c|c|c|c|}
\hline $\begin{array}{l}\text { Author, Year } \\
\text { Intervention } \\
\text { Study Design } \\
\text { Study Quality }\end{array}$ & $\begin{array}{l}\text { Intervention } \\
\text { and Comparison }\end{array}$ & Population & Results \\
\hline $\begin{array}{l}\text { Sangelaji, } 2014^{226} \\
\text { Multimodal } \\
\text { exercise } \\
\text { RCT } \\
\text { Poor }\end{array}$ & $\begin{array}{l}\text { A. Strength + } \\
\text { aerobics + } \\
\text { balance, } 30 \\
\text { sessions over } 10 \\
\text { weeks }(n=29) \\
\\
\text { B. Usual care } \\
\text { (previous activity } \\
\text { level) }(n=22)\end{array}$ & $\begin{array}{l}\text { A vs. B } \\
\text { Age: } 33.05 \text { vs. } 7.68 \\
\text { Female: } 61.5 \% \text { vs. } \\
68.2 \% \\
\text { EDSS } 0-4: 100 \%\end{array}$ & $\begin{array}{l}\text { A vs. B, mean difference (SD), } p=\text { between groups: } \\
\text { 6MWT: } 137.2 \text { (24.54), } p<0.0001 ; 184.3(51.1), \\
p=0.001 \text { ( } 1 \text {-year followup) } \\
\text { MSQoL-PCS: } 12.17(3.62), p=0.001 ; 10.90 \text { (4.55), } \\
p=0.02 \text { (1-year followup) } \\
\text { MSQoL-MCS: MD 16.36 (4.46), } p=0.001 ; 13.54 \\
\text { (5.37), } p=0.02 \text { (1-year followup) } \\
\text { EDSS: }-0.13(0.23), p=0.60 ;-0.28(0.29), p=0.35(1 \\
\text { year followup) } \\
\text { BBS: } 3.34 \text { (0.87), } p<0.0001 ; 3.21(1.44), p=0.03(1- \\
\text { year followup) }\end{array}$ \\
\hline $\begin{array}{l}\text { Sangelaji, } 2016^{222} \\
\text { Multimodal } \\
\text { exercise } \\
\text { RCT } \\
\text { Fair }\end{array}$ & $\begin{array}{l}\text { A. } 1 \text { aerobic + } 3 \\
\text { resistance } \\
\text { training, } 32 \\
\text { sessions over } 8 \\
\text { weeks ( } n=10) \\
\text { B. } 2 \text { aerobic + } 2 \\
\text { resistance } \\
\text { training, } 32 \\
\text { sessions over } 8 \\
\text { weeks ( }=10) \\
\text { C. } 3 \text { aerobic + } 1 \\
\text { resistance } \\
\text { training, } 32 \\
\text { sessions over } 8 \\
\text { weeks }(n=10) \\
\text { D. No intervention } \\
\text { control }(n=10)\end{array}$ & $\begin{array}{l}\text { A vs. B vs. C vs. D } \\
\text { Age: } 36 \text { vs. } 31 \text { vs. } 34 \\
\text { vs. } 34 \\
\text { Female: } 60 \% \text { vs. } 60 \% \\
\text { vs. } 60 \text { vs. } 60 \% \\
\text { Baseline EDSS: } 1.33 \\
\text { vs. } 2.06 \text { vs. } 1.95 \text { vs. } \\
1.81\end{array}$ & $\begin{array}{l}\text { Mean difference (SE), } p=v s \text {. control group: } \\
\text { A vs. D } \\
\text { 10MWT: } 2.31(1.04), p=0.030 \\
\text { 6MWT: }-75.22(28.21), p=0.010 \\
\text { BBS: }-5.88(1.80), p<0.001 \\
\text { B vs. D } \\
\text { 10MWT: } 1.45(1.07), p=0.190 \\
\text { 6MWT: }-63.00(29.03), p=0.040 \\
\text { BBS: }-1.25(1.85), p=0.500 \\
\text { C vs. D } \\
\frac{10 M W T:}{6 \text { GMWT: }}-27.83(1.01), p=0.080 \\
\text { BBS: }-3.10(1.75), p=0.090\end{array}$ \\
\hline $\begin{array}{l}\text { Tarakci, } 2013^{223} \\
\text { Multimodal } \\
\text { exercise } \\
\text { RCT } \\
\text { Fair }\end{array}$ & $\begin{array}{l}\text { A. Exercise (e.g., } \\
\text { ROM, strength, } \\
\text { flexibility, balance, } \\
\text { core stability), } 36 \\
\text { sessions over } 12 \\
\text { weeks ( }=51) \\
\\
\text { B. Waitlist control } \\
(n=48)\end{array}$ & $\begin{array}{l}\text { A vs. } B \\
\text { Age: } 41.5 \text { vs. } 39.7 \\
\text { Female: } 67 \% \text { vs. } 63 \% \\
\text { EDSS: } 9.0 \text { vs. } 8.4 \\
\text { RRMS: } 63 \% \text { vs. } 69 \% \\
\text { PPMS: } 20 \% \text { vs. } 17 \% \\
\text { SPMS: } 18 \% \text { vs. } 15 \%\end{array}$ & $\begin{array}{l}\text { A vs. B, mean (SD), } p=\text { between groups: } \\
\text { 10MWT: } 17.97(2.89) \text { vs. } 17.17 \text { (3.89) (baseline) } \\
15.24(2.51) \text { vs. } 18.62(4.21), \text { MD } 0.98 \\
\text { (postintervention), } p<0.001 \\
\text { MusiQoL: } 74.41(9.20) \text { vs. } 73.42 \text { (9.73) (baseline) } \\
76.39 \text { (9.53) vs. } 73.02(10.30), \text { MD } 0.34 \\
\text { (postintervention), } p=0.02 \\
\text { BBS: } 37.68 \text { (9.91) vs. } 36.94(12.55) \text { (baseline) } \\
42.01 \text { (9.32) vs. } 34.81(12.85), \text { MD } 0.64 \\
\text { (postintervention), } p=0.003 \\
\text { Stair Climbing Test: } 12.00(3.57) \text { vs. } 13.92 \text { (4.54) } \\
9.53 \text { (3.49) vs. } 18.46(16.34), \text { MD } 0.290 \\
\text { (postintervention), } p<0.001\end{array}$ \\
\hline
\end{tabular}




\begin{tabular}{|c|c|c|c|}
\hline $\begin{array}{l}\text { Author, Year } \\
\text { Intervention } \\
\text { Study Design } \\
\text { Study Quality }\end{array}$ & $\begin{array}{l}\text { Intervention } \\
\text { and Comparison }\end{array}$ & Population & Results \\
\hline $\begin{array}{l}\text { Williams, } 2020^{229} \\
\text { Multimodal } \\
\text { exercise } \\
\text { RCT } \\
\text { Fair }\end{array}$ & \begin{tabular}{|l|} 
A. Center-based \\
group strength + \\
endurance + \\
balance, 16 \\
sessions over 8 \\
weeks $(n=26)$ \\
\\
B. Home-based \\
exercise strength \\
+ endurance + \\
balance exercises, \\
16 sessions over 8 \\
weeks $(n=24)$
\end{tabular} & $\begin{array}{l}\text { Age: } 53 \text { vs. } 51 \\
\text { Female: } 65 \% \text { vs. } 88 \% \\
\text { Ambulatory: } 100 \% \\
\text { Aid use } \\
\text { None: } 27 \% \text { vs. } 58 \% \\
\text { Unilateral: } 42 \% \text { vs. } \\
29 \% \\
\text { Bilateral: } 31 \% \text { vs. } 13 \% \\
\\
\text { Type of MS } \\
\text { RRMS: } 58 \% \text { vs. } 67 \% \\
\text { PPMS: } 19 \% \text { vs. } 8 \% \\
\text { SPMS: } 15 \% \text { vs. } 8 \% \\
\text { Benign: } 4 \% \text { vs. } 8 \% \\
\text { Unknown/NR: } 4 \% \text { vs. } \\
8 \%\end{array}$ & 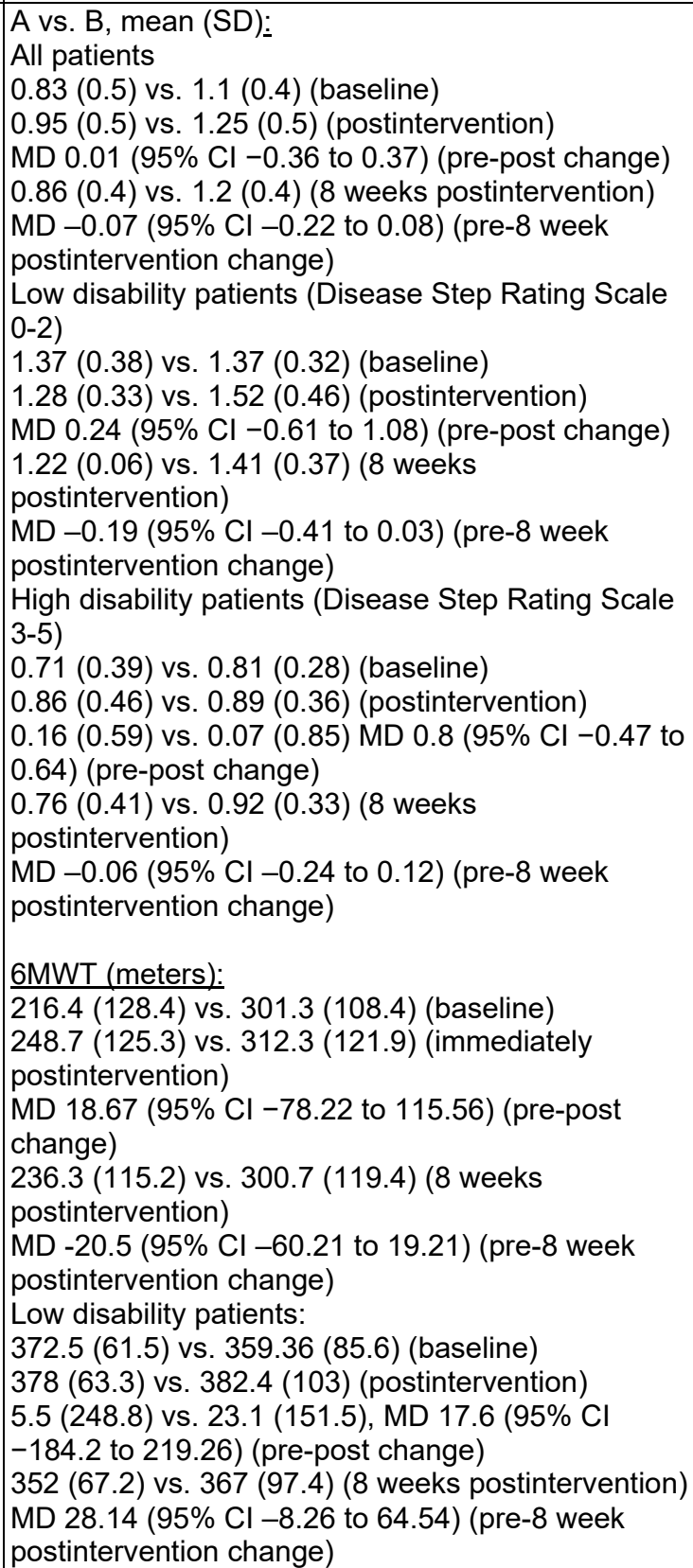 \\
\hline
\end{tabular}




\begin{tabular}{|c|c|c|c|}
\hline $\begin{array}{l}\text { Author, Year } \\
\text { Intervention } \\
\text { Study Design } \\
\text { Study Quality }\end{array}$ & $\begin{array}{l}\text { Intervention } \\
\text { and Comparison }\end{array}$ & Population & Results \\
\hline $\begin{array}{l}\text { Williams, } 2020 \\
\text { (Continued) }\end{array}$ & & & 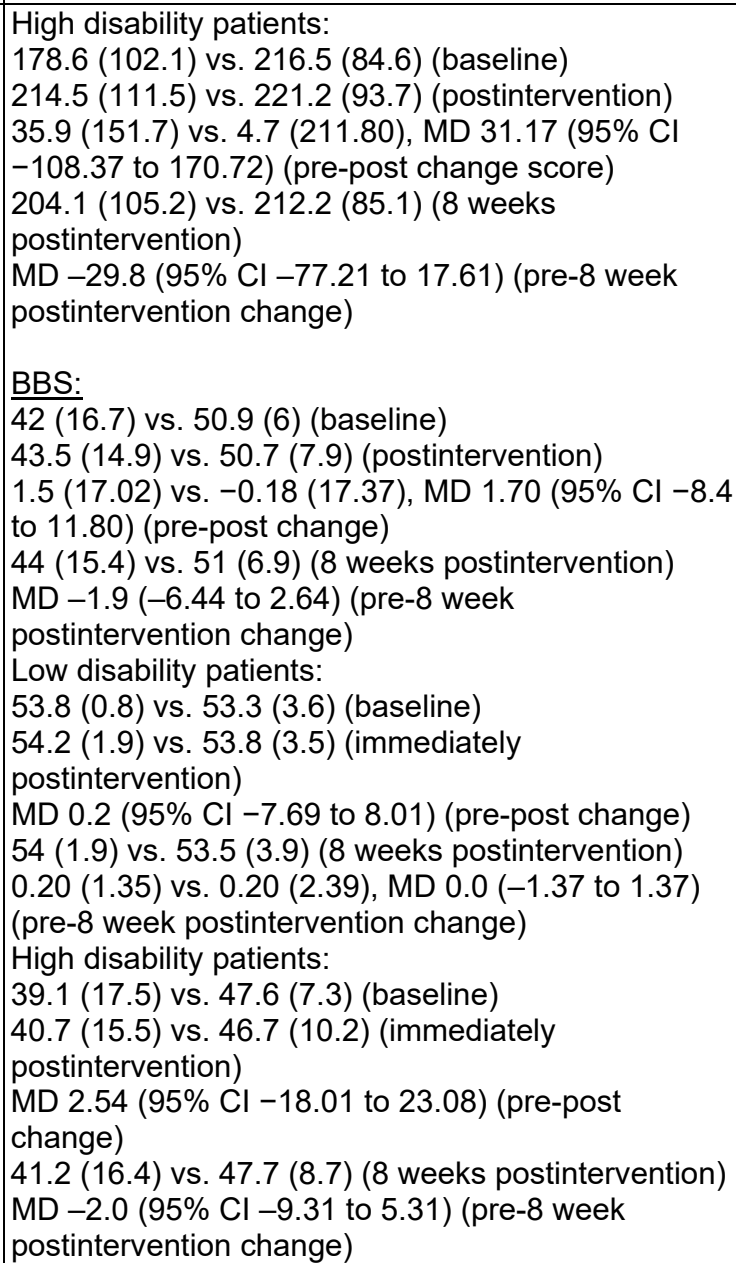 \\
\hline
\end{tabular}

Abbreviations: 6MWT = 6-Minute Walk Test; 10MWT = 10-Meter Walk Test; AE = adverse event; BBS = Berg Balance Scale; BDI = Beck Depression Inventory; CI = confidence interval; DGI = Dynamic Gait Index; EDSS = Expanded Disability Status Scale; EPC = Evidence-based Practice Center; MD = mean difference; MS = multiple sclerosis; MSQoL-MCS = Multiple Sclerosis Quality of Life-54 instrument Mental Component Score; MSQoL-PCS = Multiple Sclerosis Quality of Life-54 instrument Physical Component Score; MSWS-12 = Multiple Sclerosis Walking Scale-12; MusiQoL= Multiple Sclerosis International Quality of Life questionnaire; NR = not reported; PPMS = primary progressive multiple sclerosis; QOL $=$ quality of life; $\mathrm{RCT}=$ randomized controlled trial; $\mathrm{RRMS}=$ relapsing-remitting multiple sclerosis; $\mathrm{SD}=$ standard deviation; $\mathrm{SE}=$ standard error; SF-36 MCS = Short-Form 36 Mental Component Summary; SF-36 PCS = Short-Form 36 Physical Component Score; SF36 = Short-Form 36 Quality of Life; SPMS = secondary progressive multiple sclerosis; TUG = Timed Up and Go Test; VO 2 peak $=$ highest value of $\mathrm{VO}_{2}$ attained

\section{Walking Measures}

There was low strength of evidence that multimodal exercise was generally associated with improved walking ability across measures, compared with attention control (stretching) or continuation of previous activity based on pooled difference in change scores (6MWT, 6 trials, MD -67.7 meters, 95\% CI -85.6 to $-49.9, \mathrm{I}^{2}=58 \%,{ }^{221,222,226,228,230,232}$ 10MWT, 4 trials, MD -2.7 seconds, $95 \% \mathrm{CI}-4.2$ to $-1.2, \mathrm{I}^{2}=80 \%^{222,223,225,228}$ ) immediately posttreatment (Figures 21 and 22). Substantial heterogeneity in pooled estimates for both measures was noted; all but one trial favored multimodal exercise and all had different magnitudes of effect and variability. This may in part be due to differences in baseline measure values across studies and may also be related to 
differences in intervention. Isolated studies reported ambulatory ability or use of assistive devices, duration of MS, or status (e.g., progressive, remitting), precluding evaluation of these as sources of heterogeneity. For the 6MWT, exclusion of two poor-quality trials only slightly attenuated the effect size and but did substantially reduce heterogeneity ( 4 trials, MD -66.3 meters, $95 \%$ CI -75.1 to $\left.-38.5, \mathrm{I}^{2}=0 \%\right)^{221,222,230,232}$ and exclusion of an outlier trial resulted in a decrease in effect size and substantially reduced heterogeneity ( 4 trials, MD -64.9 meters, 95\% $\mathrm{CI}-73.5$ to $\left.-56.2, \mathrm{I}^{2}=0 \%\right),{ }^{221,222,228}$ maintaining an effect size that may be clinically meaningful. These trials provided low-strength evidence for improved walking with multimodal exercises compared with usual care, previous activity, or attention control. Authors of one trial reported that results did not statistically differ based on disability status based on EDSS scores considered low $(<4.5)$, moderate $(4.5$ to $\leq 6)$, or high $(\geq 6.5) .{ }^{230}$ Interestingly, the trial with the longest intervention length (72 sessions over 24 months) showed no difference between treatment groups and had smallest effect size, ${ }^{221}$ the other trials involved 30 to 36 sessions over 8 to 12 weeks. For the 10MWT, exclusion of poor-quality trials had negligible impact on either effect size and increased heterogeneity ( 2 trials, MD 3.1 seconds, 95\% CI -5.4 to $-0.8, \mathrm{I}^{2}=87 \%$ ). ${ }^{222,223}$ However, exclusion of an outlier trial resulted in a slightly smaller but more precise effect size and substantially reduced heterogeneity ( 3 trials $\mathrm{MD}-1.99$ seconds, $95 \% \mathrm{CI}-2.8$ to -1.2 , $\left.\mathrm{I}^{2}=0 \%\right) .{ }^{22,225,228}$ The differences between groups may not be not clinically meaningful for this outcome. The mean baseline EDSS of the excluded trial was $\sim 4.3$ compared with a mean of $\sim 2$ in the only other trial reporting this measure.

In one trial ${ }^{221}$ there was no difference in walking ability on the Multiple Sclerosis Walking Scale-12 (difference $-3.3,95 \%$ CI -10.2 to 3.6, 0-100 scale) or on a timed 25FWT (difference $0.30,95 \% \mathrm{CI}-0.2$ to 0.8 feet/second) immediately posttreatment.

One poor-quality trial ${ }^{226}$ reported that improvement in the $6 \mathrm{MWT}$ persisted long- term (42 weeks) posttreatment (MD 184.3 \pm 51.1 meters, $\mathrm{p}=0.03$ ) following multimodal exercise (strength, aerobics and balance exercises) versus usual care.

One small fair-quality trial ${ }^{229}$ found no difference in walking ability between multimodal exercise performed in a group setting or home setting based on 6MWT immediately after the 8week intervention or at the subsequent 8-week followup, or for 10MWT at either time. Authors reported higher percentage of completed sessions in the group exercise arm (83\% versus $45 \%)$. No differences between groups in either walking measure were observed based on disability level measured via the Disease Step Rating Scale (0-6 overall score with scores 0-2 for low and 3-6 for high disability) at either time frame (Table 37).

Figure 21. Multimodal exercise versus usual care in multiple sclerosis: 6MWT

\begin{tabular}{|c|c|c|c|c|c|c|c|c|c|c|}
\hline Study & Exercise & Control & $\begin{array}{c}\mathrm{N} \\
\text { Exercise } \\
\end{array}$ & $\begin{array}{c}\mathrm{N} \\
\text { Control } \\
\end{array}$ & Weight & $\begin{array}{l}\text { MD } \Delta \text { Scores } \\
\mathrm{PL}[95 \% \mathrm{Cl}]\end{array}$ & & & & \\
\hline Sandroff 2017 & PRE+Aerob+Bal & AC Stretch & 43 & 40 & $10.8 \%$ & $-28.70[-73.45,16.05]$ & & - & - & \\
\hline Sangelaji 2014 & PRE+Aerob+Bal & Previous & 35 & 20 & $9.8 \%$ & $-135.70[-183.80,-87.60]$ & & & & \\
\hline Sangelaji 2016 & PRE+Aerob & Previous & 30 & 10 & $13.0 \%$ & $-55.20[-93.95,-16.45]$ & & & & \\
\hline Ebrahimi 2015 & PRE+balance (WBV) & Previous & 16 & 14 & $13.2 \%$ & $-75.90[-114.22,-37.58]$ & & & & \\
\hline Faramarzi 2020 & PRE+Aerob+Bal+Pilates+Stretch & WL & 46 & 43 & $30.1 \%$ & $-68.22[-78.67,-57.77]$ & & - & & \\
\hline Ozkul 2020 & Aerob+Pilates & $A C$ & 17 & 17 & $23.2 \%$ & $-59.05[-79.61,-38.49]$ & & - & & \\
\hline Total $(95 \% \mathrm{Cl})$ & & & 187 & 144 & $100.0 \%$ & $-67.73[-85.56,-49.90]$ & & & & \\
\hline \multicolumn{3}{|c|}{$\begin{array}{l}\text { Heterogeneity: } \text { Tau }^{2}=246.53 ; \mathrm{Chi}^{2}=11.84, \mathrm{df}=5(\mathrm{P}=0.04) ; \mathrm{l}^{2}=58 \% \\
\text { Test for overall effect: } Z=7.44(P<0.00001)\end{array}$} & & & & & -200 & $\begin{array}{l}-100 \\
\text { Favors Exercise }\end{array}$ & $\begin{array}{cc}0 & 100 \\
\text { Favors Control }\end{array}$ & 200 \\
\hline
\end{tabular}

Abbreviations: $\Delta=$ change; $6 \mathrm{MWT}=6$-Minute Walk Test; $\mathrm{AC}=$ attention control; $\mathrm{CI}=$ confidence interval; $\mathrm{MD}=$ mean difference; $\mathrm{PA}=$ previous activity; $\mathrm{PL}=$ profile likelihood; $\mathrm{PRE}=$ progressive resistance exercise 
Figure 22. Multimodal exercise versus usual care in multiple sclerosis: 10MWT

\begin{tabular}{|c|c|c|c|c|c|c|c|}
\hline Study & Exercise & Control & $\begin{array}{c}\mathrm{N} \\
\text { Exercise }\end{array}$ & $\begin{array}{c}\mathrm{N} \\
\text { Control }\end{array}$ & Weight & $\begin{array}{l}\text { MD } \Delta \text { Scores } \\
\mathrm{PL}[95 \% \mathrm{Cl}]\end{array}$ & \\
\hline Sangelaji 2016 & PRE+Aerob & PA & 30 & 10 & $26.3 \%$ & $-1.86[-3.27,-0.45]$ & $\because$ \\
\hline Cakit 2010 & PRE+Aerob+Bal & $\mathrm{PA}$ & 14 & 9 & $31.8 \%$ & $-2.00[-2.82,-1.18]$ & - \\
\hline Ebrahimi 2015 & PRE+Bal & $\mathrm{PA}$ & 16 & 14 & $10.7 \%$ & $-2.53[-6.13,1.07]$ & \\
\hline Tarakci 2013 & PRE+Bal & PA & 55 & 55 & $31.3 \%$ & $-4.18[-5.05,-3.31]$ & $\rightarrow-$ \\
\hline Total $(95 \% \mathrm{Cl})$ & & & 115 & 88 & $100.0 \%$ & $-2.73[-4.15,-1.22]$ & \\
\hline \multicolumn{7}{|c|}{ Heterogeneity: $\mathrm{Tau}^{2}=1.46 ; \mathrm{Chi}^{2}=15.02, \mathrm{df}=3(P=0.002) ; 1^{2}=80 \%$} & $\begin{array}{ccccc}-4 & -2 & 0 & 2 & 4 \\
\text { Favors } & \text { Exercise } & \text { Favors Control }\end{array}$ \\
\hline
\end{tabular}

Abbreviations: $\Delta=$ change; $10 \mathrm{MWT}=10$-Meter Walk Test; $\mathrm{AC}=$ attention control; $\mathrm{CI}=$ confidence interval; $\mathrm{MD}=$ mean difference; $\mathrm{PA}=$ previous activity; $\mathrm{PL}=$ profile likelihood; $\mathrm{PRE}=$ progressive resistance exercise $=$ profile likelihood; $\mathrm{PRE}=$ progressive resistance exercise; $\mathrm{TUG}=$ Timed $\mathrm{Up}$ and Go Test

\section{Functional Capacity Measures}

Improvement in functional capacity based on TUG was seen immediately postmultimodal exercise intervention across two small poor-quality trials and one larger fair-quality trial (3 trials, $\mathrm{MD}-1.65$ seconds, $95 \% \mathrm{CI}-2.6$ to $\left.-0.4 ; \mathrm{I}^{2}=62 \%\right)^{225,228}$ compared with previous activity but may not be clinically meaningful. Evidence was considered insufficient (Figure 23).

Figure 23. Multimodal exercise versus usual care in multiple sclerosis: TUG

\begin{tabular}{|c|c|c|c|c|c|c|c|c|c|c|}
\hline & Exercise & Control & $\begin{array}{c}\mathbf{N} \\
\text { Exercise } \\
\end{array}$ & $\begin{array}{c}\mathrm{N} \\
\text { Control } \\
\end{array}$ & Weight & $\begin{array}{l}\mathrm{MD} \Delta \text { Scores } \\
\mathrm{PL}[95 \% \mathrm{Cl}]\end{array}$ & & & & \\
\hline & PRE+Aero+Bal & Previous & 14 & 9 & $41.8 \%$ & $-1.10[-1.92,-0.28]$ & & - & & \\
\hline$) 15$ & PRE+Balance(WBV) & Previous & 16 & 14 & $8.4 \%$ & $-0.30[-3.32,2.72]$ & & & & \\
\hline 2020 & PRE+Aerob+Bal+Pilates+Stretch & WL & 46 & 43 & $49.8 \%$ & $-2.15[-2.72,-1.58]$ & & -1 & & \\
\hline Cl) & & & 76 & 66 & $100.0 \%$ & $-1.65[-2.59,-0.36]$ & & & & \\
\hline \multicolumn{3}{|c|}{$\begin{array}{l}\text { ity: } \mathrm{Tau}^{2}=0.38 ; \mathrm{Chi}^{2}=5.21, \mathrm{df}=2(\mathrm{P}=0.07) ; \mathrm{I}^{2}=62 \% \\
\text { sall effect: } \mathrm{Z}=3.23(\mathrm{P}=0.001)\end{array}$} & & & & & -4 & $\begin{array}{c}-2 \\
\text { Favors Exercise }\end{array}$ & $\begin{array}{lc} & 1 \\
& 2 \\
\text { Favors Control }\end{array}$ & 4 \\
\hline
\end{tabular}

Abbreviations: $\Delta=$ change; $\mathrm{AC}=$ attention control; $\mathrm{CI}=$ confidence interval; $\mathrm{MD}=$ mean difference; $\mathrm{PA}=$ previous activity; $\mathrm{PRE}=$ progressive resistance exercise $\mathrm{PL}=$ profile likelihood; $\mathrm{TUG}=$ Timed $\mathrm{Up}$ and Go Test

\section{Quality of Life Measures}

Quality of life evidence, based on MSQOL-54 MCS and PCS (0 to 100 scales) across two poor-quality trials and one fair-quality trial postintervention was considered insufficient to draw firm conclusions (Figure 24). ${ }^{226,228}$ For MCS, there was substantial heterogeneity for the pooled difference ( 3 trials, MD $-10.7,95 \% \mathrm{CI}-22.6$ to $1.24, \mathrm{I}^{2}=91 \%$ ). Two trials favored exercise ${ }^{226}$ versus maintenance of usual activity but a third showed no difference between treatment groups $(0.97,95 \% \mathrm{CI}-6.2$ to 8.1$){ }^{228}$ All trials reported improvement on the PCS (3 trials, MD -13.7, $95 \% \mathrm{CI}-21.64$ to $-4.9 \mathrm{I}^{2}=81 \%$ ) but only one reached statistical significance; substantial heterogeneity was noted although estimates tended to favor exercise. Author-reported data for one trial appeared to be out of the expected range for this measure; exclusion of it slightly reduced the effect size and heterogeneity for PCS (2 poor -quality trials, MD -12.0, 95\% CI 13.8 to $-5.0, \mathrm{I}^{2}=75 \%$ ). It is unclear if this was a clinically meaningful difference.

Improved quality of life based on the Multiple Sclerosis International Quality of Life questionnaire (MusiQoL) (0-100 scale) was seen in an additional fair-quality trial (difference $2.4,95 \% \mathrm{CI}-4.7$ to -0.1$){ }^{223}$ In one fair-quality prospective quasiexperimental study, ${ }^{233}$ exercise significantly improved MSQOL-54 total scores postintervention compared with usual care (difference $52.0,95 \%$ CI 20.8 to 83.2 ). 
In one poor-quality trial, individual SF-36 domain scores that improved with exercise were physical functioning and bodily pain; no differences between groups were seen for the other domains (Table 38). ${ }^{225}$

In the only trial (poor-quality) that reported long-term outcomes (42 weeks) posttreatment, improvement on both the PCS and MCS of the MSQOL persisted long term (PCS difference $10.9 \pm 4.55, \mathrm{p}=0.02$, MCS difference $13.5 \pm 5.4, \mathrm{p}=0.02,0-100$ scale) for multimodal exercise versus usual care. ${ }^{226}$

In one trial, the control condition of aerobic exercise was associated with improvement in the SF-36 MCS compared with multimodal exercise (difference 4.2, 95\% CI 0.2 to $8.2,0$-100 scale) but there was no difference between groups on SF-36 PCS (difference -0.7, 95\% CI -3.9 to 2.2, $0-100$ scale) in one trial. ${ }^{220}$

Figure 24. Multimodal exercise versus usual care in multiple sclerosis: MSQOL MCS and MSQOL PCS

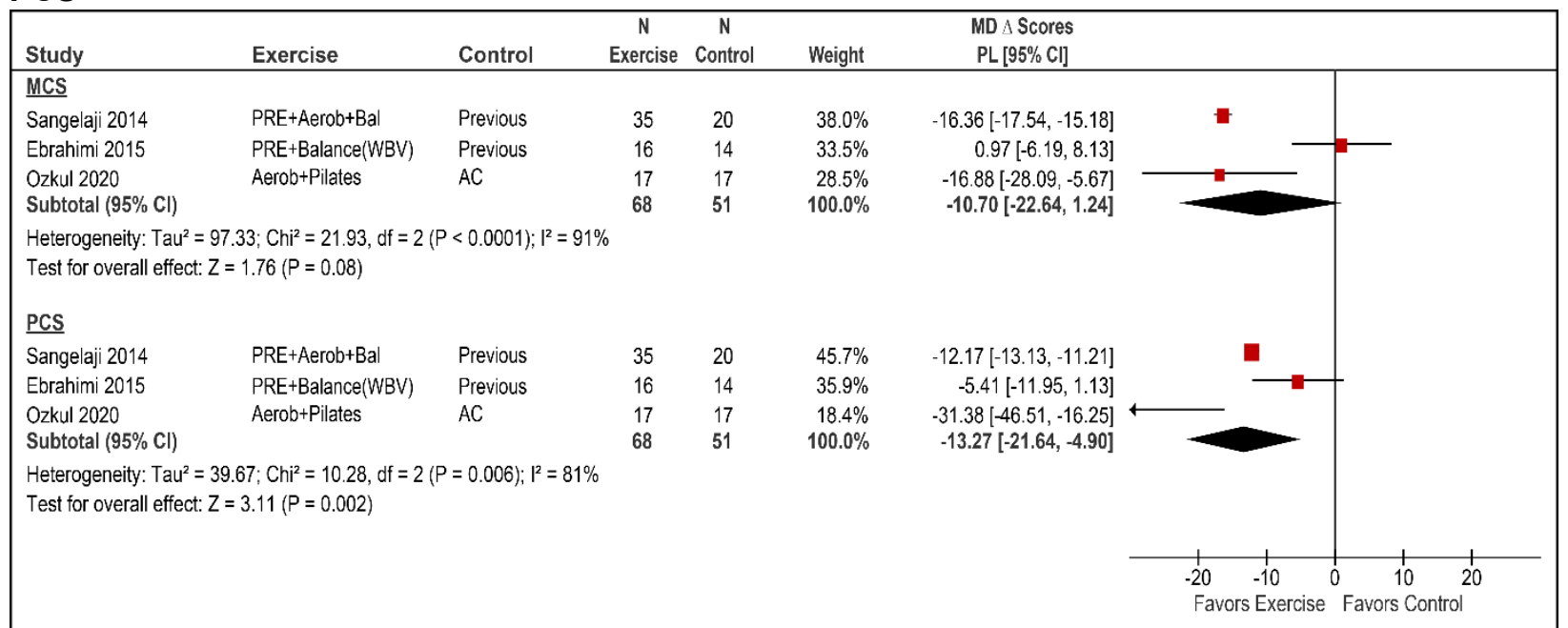

Abbreviations: $\Delta=$ change; $\mathrm{AC}=$ attention control; $\mathrm{CI}=$ confidence interval; $\mathrm{MD}=$ mean difference; $\mathrm{MCS}=$ mental component score; MSQOL = Multiple Sclerosis Quality of Life; PA = previous activity; $\mathrm{PCS}=$ physical component $\mathrm{score} ; \mathrm{PL}=$ profile likelihood; PRE = progressive resistance exercise

Functional capacity was improved with multimodal exercise based on a timed climbing test in one fair-quality trial (difference -7.0 seconds, $95 \% \mathrm{CI}-10.5$ to -3.5 ) 223 and based on the Dynamic Gait Index (DGI) (difference 0.20, 95\% CI 1.9 to 2.7) and Falls Efficacy Scale (difference $-8.7,95 \% \mathrm{CI}-14.4$ to -3.0 ) in another poor-quality trial. ${ }^{225}$

Balance improved with multimodal exercise compared with continuation of previous activity (4 trials, MD $-3.4,95 \% \mathrm{CI}-3.8$ to $-3.1, \mathrm{I}^{2}=38 \%$, BBS, 0-56 scale), ${ }^{222,223,226,228}$ individually all trials favored exercise but the magnitude of effect size varied (range -3.3 to -6.5 ) (Figure 25). Analysis excluding one outlier had no impact on effect size but substantially reduced heterogeneity ( 3 trials, MD $-3.4,95 \% \mathrm{CI}-3.6$ to $-3.1, \mathrm{I}^{2}=0 \%$ ). ${ }^{222,226,228}$ These trials provided low-strength evidence of improved balance scores with multimodal exercises. However, no difference in balance (BBS 0-56 scale) between group and home-based multimodal exercise in one trial ${ }^{229}$ was observed immediately after the intervention or at 8-week followup. Similarly, there were no differences between groups observed based on disability level (low or high) at either timeframe in this trial (Table 37).

No clear difference in disability immediately posttreatment was reported across two poorquality trials based on the EDSS $\left(0-10\right.$ scale, 2 trials, $\mathrm{MD}-0.1,95 \% \mathrm{CI}-0.3$ to $\left.0.1, \mathrm{I}^{2}=42 \%\right)$ 
(Figure 26). ${ }^{226,228}$ One of the trials reported no difference in EDSS at long term (42 weeks) between multimodal exercises and usual care. ${ }^{226}$

Figure 25. Multimodal exercise versus usual care in multiple sclerosis: BBS

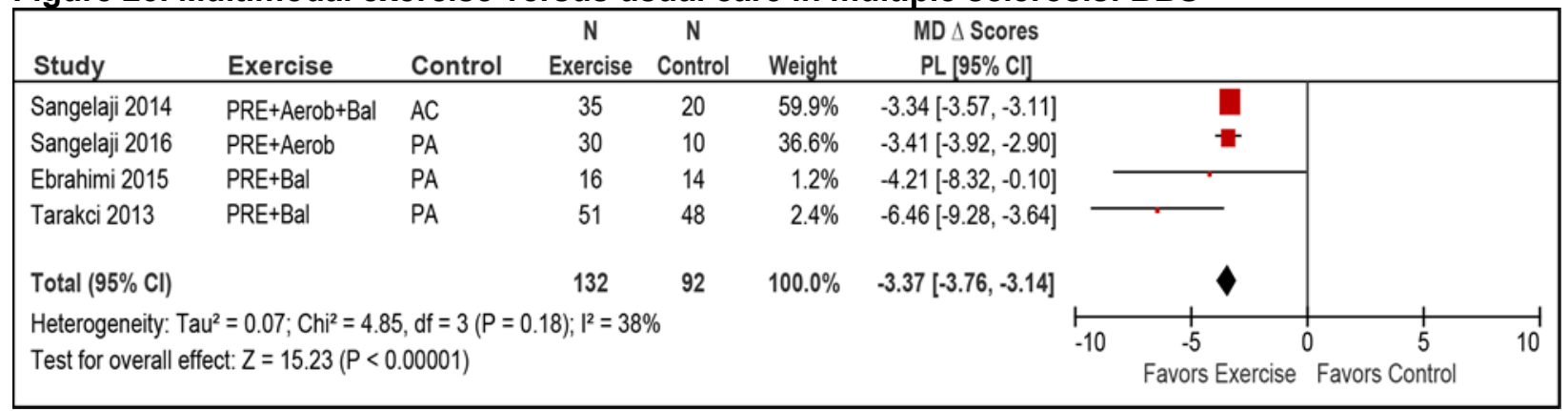

Abbreviations: $\Delta=$ change; $\mathrm{AC}=$ attention control; $\mathrm{BBS}=$ Berg Balance Scale; $\mathrm{CI}=$ confidence interval; $\mathrm{MD}=$ mean difference; $\mathrm{PA}=$ previous activity $\mathrm{PL}=$ profile likelihood; $\mathrm{PRE}=$ progressive resistance exercise

Figure 26. Multimodal exercise versus usual care in multiple sclerosis: EDSS

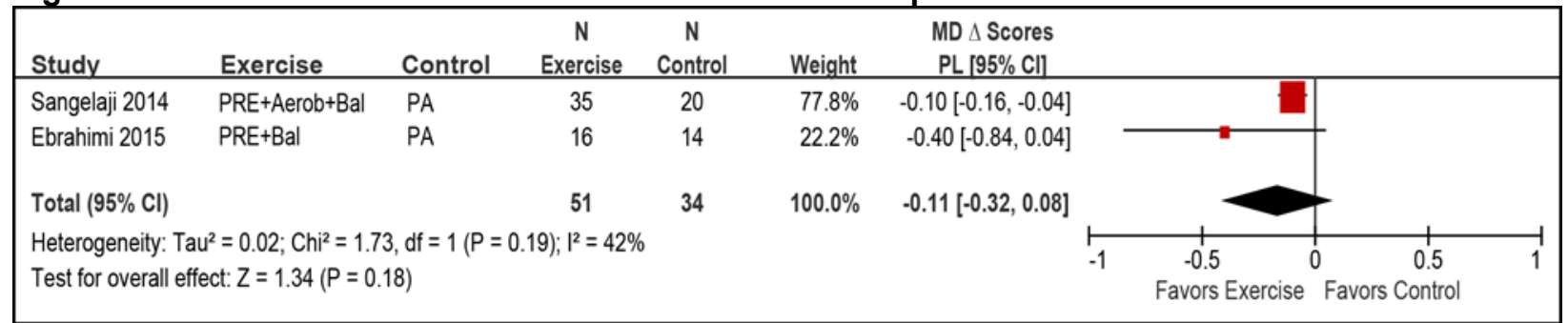

Abbreviations: $\Delta=$ change; $\mathrm{CI}=$ confidence interval; EDSS = Expanded Disability Status Scale; $\mathrm{MD}=$ mean difference; $\mathrm{PA}=$ previous activity; $\mathrm{PL}=$ profile likelihood; $\mathrm{PRE}=$ progressive resistance exercise

Six RCTs did not address harms or adverse events. Two RCTs reported that no adverse events occurred. ${ }^{223,229}$

\section{Multimodal Exercises-Cerebral Palsy}

Four trials enrolled participants $(n=177)$ with CP (Table 38). ${ }^{234-240}$ Weighted mean age of participants across trials was 13.5 years (range 9.75 to 20 years) with weighted mean proportion female of 48.5 percent (range $42 \%$ to $52.6 \%$ ). No study provided data on race. Weighted mean proportion of participants who could ambulate was 96.3 percent (range $92.3 \%$ to $100 \%$ ) and level I, II, III, and IV GMFCS levels were 56, 31, 13, and 3 percent, respectively. 
Table 38. Multimodal exercise in cerebral palsy

\begin{tabular}{|c|c|c|c|}
\hline $\begin{array}{l}\text { Author, Year } \\
\text { Intervention } \\
\text { Study Design } \\
\text { Study Quality }\end{array}$ & $\begin{array}{l}\text { Intervention } \\
\text { and Comparison }\end{array}$ & Population & Results \\
\hline $\begin{array}{l}\text { Fosdahl, } \\
2019 b^{241} \\
\text { Multimodal } \\
\text { exercise } \\
\text { RCT } \\
\text { Fair }\end{array}$ & $\begin{array}{l}\text { A. Strength training } \\
\text { (progressive } \\
\text { resistance exercise) } \\
+ \text { stretching, } 48 \\
\text { sessions over } 16 \\
\text { weeks }(n=17) \\
\text { B. Usual care }(n=20)\end{array}$ & $\begin{array}{l}\text { A vs. B } \\
\text { Age: } 10.4 \text { vs. } 10.0 \\
\text { Female: } 59 \% \text { vs. } 30 \% \\
\text { Ambulatory: } 100 \% \\
\text { GMFM: } \\
\text { I: } 59 \% \text { vs. } 60 \% \\
\text { II: } 41 \% \text { vs. } 35 \% \\
\text { III: } 0 \% \text { vs. } 5 \%\end{array}$ & $\begin{array}{l}\text { A vs. B, mean change score (SD) } \\
\text { 6MWT (meters): } \\
-45.7(55.4) \text { vs. }-55.4 \text { (55.5), adj. MD } 10.6(95 \% \\
\mathrm{Cl}-29.3 \text { to } 50.6), p=0.590 \text { (pre-post change) } \\
-51.1(72.8) \text { vs. }-56.6(59.6) \text {, adj. MD } 7.2(95 \% \mathrm{Cl} \\
-43.3 \text { to } 57.7), p=0.772 \text { (16-week change) } \\
\text { GDI: } \\
-0.4(4.4) \text { vs. }-0.8(7.14) \text {, adj. MD }-1.0(95 \% \mathrm{Cl} \\
-5.3 \text { to } 3.3), p=0.650 \text { (pre-post change) } \\
-0.7(6.0) \text { vs. } 1.01(5.9) \text {, adj. MD }-1.4(95 \% \mathrm{Cl} \\
-5.6 \text { to } 2.8), p=0.504(16 \text {-week change) }\end{array}$ \\
\hline $\begin{array}{l}\text { Kaya Kara, } \\
2019^{242} \\
\text { Multimodal } \\
\text { exercise } \\
\text { RCT } \\
\text { Fair }\end{array}$ & $\begin{array}{l}\text { A. Strength training } \\
\text { (progressive } \\
\text { resistance exercise) } \\
+ \text { balance, } 36 \\
\text { sessions over } 12 \\
\text { weeks ( } n=17) \\
\text { B. Usual care, } \\
36 \text { sessions over } 12 \\
\text { weeks }(n=16)\end{array}$ & $\begin{array}{l}\text { A vs. B } \\
\text { Age: } 11.8 \text { vs. } 11.3 \\
\text { Female: } 53 \% \text { vs. } 60 \% \\
\text { Ambulatory: } 100 \% \\
\text { Manual ability } \\
\text { classification } \\
\text { system level: } \\
\text { I: } 47 \% \text { vs. } 47 \% \\
\text { II: } 33 \% \text { vs. } 27 \% \\
\text { III: } 20 \% \text { vs. } 27 \%\end{array}$ & $\begin{array}{l}\text { A vs. B, mean change from baseline (SD) } \\
\text { (data are for completers only; } n=15 \text { vs. } 15) \\
\text { GMFM-88D: } \\
0.17(0.67) \text { vs. } 0.32(1.42), M D-0.15(95 \% \mathrm{Cl} \\
-0.93 \text { to } 0.63), p=0.632 \text {; effect size } 0.13 \\
\text { GMFM-88E: } \\
2.31(2.20) \text { vs. }-0.37(2.59), \text { MD } 2.68(95 \% \mathrm{Cl} \\
0.98 \text { to } 4.38), p=0.004 ; \text { effect size } 1.11 \\
\text { 1MWT: } \\
7.76(7.03) \text { vs. } 0.53(3.37), M D 7.23(95 \% \mathrm{Cl} \mathrm{NR}) \text {, } \\
\text { p=0.001; effect size } 1.31 \\
\text { TUG: } \\
-1.02(0.45) \text { vs. } 0.08(0.45), \mathrm{MD}-1.10(95 \% \mathrm{Cl} \\
-1.42 \text { to }-0.78), p<0.001 ; \text { effect size } 2.42\end{array}$ \\
\hline $\begin{array}{l}\text { Slaman, } \\
2015^{237} \\
\text { Slaman, } \\
2015^{234} \\
\text { Slaman, } \\
2014^{235} \\
\text { Slaman, } \\
2010^{236} \\
\text { Multimodal } \\
\text { exercise } \\
\text { RCT } \\
\text { Fair }\end{array}$ & $\begin{array}{l}\text { A. Strength training }+ \\
\text { aerobic fitness, } 48 \\
\text { sessions over } 3 \\
\text { months plus } 8-10 \\
\text { counseling sessions } \\
\text { on physical activity } \\
\text { and sports } \\
\text { participation over } 3 \\
\text { months: ( } n=28) \\
\text { B. Usual care }(n=29)\end{array}$ & $\begin{array}{l}\text { A vs. B } \\
\text { Age: } 20 \text { vs. } 20 \\
\text { Female: } 48.3 \% \text { vs. } 57.1 \% \\
\text { Ambulatory: } 97 \% \text { vs. } 89 \% \\
\text { Wheelchair user: } 3.3 \% \text { vs. } \\
10.7 \% \\
\text { Unilateral CP: } 52 \% \text { vs. } \\
50 \% \\
\text { GMFM I: } 61 \% \text { vs. } 55 \% \\
\text { GMFM II: } 32 \% \text { vs. } 31 \% \\
\text { GMFM III: } 7 \% \text { vs. } 10 \% \\
\text { GMFM IV: } 0 \% \text { vs. } 3 \%\end{array}$ & 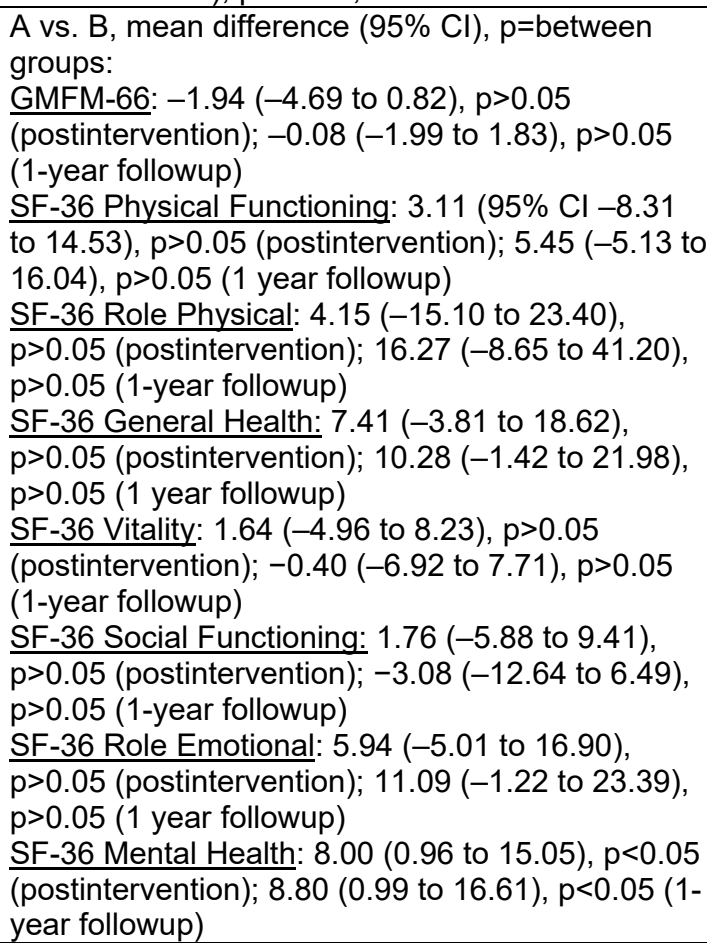 \\
\hline
\end{tabular}




\begin{tabular}{|c|c|c|c|}
\hline $\begin{array}{l}\text { Author, Year } \\
\text { Intervention } \\
\text { Study Design } \\
\text { Study Quality }\end{array}$ & $\begin{array}{l}\text { Intervention } \\
\text { and Comparison }\end{array}$ & Population & Results \\
\hline $\begin{array}{l}\text { Van Wely, } \\
2014 a^{238} \\
\text { Van Wely, } \\
2014 b^{239} \\
\text { Van Wely, } \\
2010^{240} \\
\text { Multimodal } \\
\text { exercise } \\
\text { RCT } \\
\text { Fair }\end{array}$ & $\begin{array}{l}\text { A. Strength plus } \\
\text { aerobics } 24 \text { sessions } \\
\text { over } 4 \text { months plus } \\
\text { PT and counseling } \\
\text { over } 6 \text { months plus } \\
\text { usual PT from } \\
\text { months } 4-12(n=25) \\
\text { B. Usual PT months } \\
0-12(n=25)\end{array}$ & $\begin{array}{l}\text { A vs. B } \\
\text { Age: } 9.5 \text { vs. } 10.0 \\
\text { Female: } 52 \% \text { vs. } 33 \% \\
\text { Ambulatory: } 100 \% \\
\text { Wheelchair user for long } \\
\text { distances: } 20 \%) \text { vs. }(21 \% \\
\text { GMFCS I: } 60 \% \text { vs. } 54 \% \\
\text { GMFCS II: } 24 \% \text { vs. } 25 \% \\
\text { GMFCS III: } 16 \% \text { vs. } 21 \% \\
\text { Bilateral: } 52 \% \text { vs. } 54 \%\end{array}$ & $\begin{array}{l}\text { A vs. B, mean difference }(95 \% \mathrm{Cl}), \mathrm{p}=\text { between } \\
\text { groups: } \\
\text { GMFM-66: } 2.8(0.2 \text { to } 5.4), p=0.03 \text { (month } 6) ;-0.9 \\
\text { (-3.3 to } 1.4), p>0.05 \text { (month } 12) \\
\text { 1MWT: } 5.0(0.0 \text { to } 9.0), p=0.06 \text { (month } 4) ; 2.0 \\
\text { (-4.0 to } 9.0), p>0.05 \text { (month } 6) ; 3.0(-43.0 \text { to } \\
\text { 10.0), p>0.05 (month 12) } \\
\text { CPQoL Social Well-Being \& Acceptance: } \\
\text {-3.1 (-7.9 to 1.7), } p=0.19 \text { (month 12) } \\
\text { CPQoL Functioning: }-2.5 \text { ( }-7.3 \text { to } 2.3), p=0.30 \\
\text { (month 12) } \\
\text { CPQoL Participation \& Physical Health: } \\
\text {-0.8 (-5.7 to 4.1), p=0.75 (month 12) } \\
\text { CPQoL Emotional Well-Being and Self-Esteem: } \\
\text {-0.3 (-5.3 to 4.7), p=0.90 (month 12) } \\
\text { CPQoL pain and impact on disability: } 5.0(-5.2 \text { to } \\
\text { 15.2), p=0.33 (month 12) }\end{array}$ \\
\hline
\end{tabular}

Abbreviations: 1MWT = One-Minute Walk Test; 6MWT = 6-Minute Walk Test; adj. = adjusted; $\mathrm{CI}=$ confidence interval; CPQoL = Cerebral Palsy Quality of Life questionnaire; GDI = Gait Deviation Index; GMFCS = Gross Motor Function Classification System; GMFM = Gross Motor Function Measure; GMFM-88D = Gross Motor Function Measure-88D (standing); GMFM-88E = Gross Motor Function Measure E (walking, running, jumping); $\mathrm{MD}=$ mean difference; $\mathrm{NR}=$ not reported; $\mathrm{PT}=$ physical therapy; RCT = randomized controlled trial; $\mathrm{SD}=$ standard deviation; SF-36 = Short-Form 36 Quality of Life; TUG = Timed Up and Go Test

a Van Wely was considered a fair-quality trial based on the 2014a publication which reported primary outcomes of interest; the $2014 \mathrm{~b}$ publication reported secondary outcomes (quality of life) and the 2010 publication is the trial protocol (LEARN 2 MOVE 7-12).

Walking outcomes were reported in three RCTs. ${ }^{238-242}$ Evidence was considered insufficient to draw firm conclusions on the impact of exercise on walking. There was no improvement in $6 \mathrm{MWT}$ versus usual care either immediately post-16-week intervention or at 16 weeks postintervention. ${ }^{241}$ Similarly, there was no improvement in Gait Deviation Index (GDI) at either time in the same trial. ${ }^{241}$ There was improvement on the 1MWT with multimodal exercise versus usual care immediately post-12- to 16 -week treatment across two trials (MD -5.3, 95\% CI 10.24 to $-0.33, \mathrm{I}^{2}=45$ ) (Figure 27). There was no difference between multimodal exercise and usual care in one trial at 26 weeks or 52 -week followup. ${ }^{238-240}$

There was low-strength evidence of no clear benefit to exercise on functional capacity based on GMFM-66 compared with usual care immediately posttreatment. Results across two trials reporting GMFM-66 (0-100 scale) were inconsistent with the pooled differences, showing no difference between multimodal exercise versus usual care ( 2 trials, $\mathrm{MD}-1.5,95 \% \mathrm{CI}-6.4$ to 4.7, $\mathrm{I}^{2}=71 \%$ ) (Figure 28). ${ }^{234-240}$ No difference between groups was seen in one trial (difference $1.6,95 \% \mathrm{CI}-2.7$ to 5.9$)^{234-237}$ while the other trial favored the combination of strength training, aerobics, and PT over usual care (difference $-3.1,95 \% \mathrm{CI}-5.7$ to -0.6 ). ${ }^{238-240} \mathrm{In}$ addition to variation in interventions, differences in patient populations may have contributed to the inconsistency: the first study enrolled older participants (mean age 20 years vs. 10 years) and more participants with GMFCS level II (32\% vs. $24.5 \%)$. It was unclear whether the effect sizes are clinically meaningful. In one small trial, there was no difference between exercise and usual care on the GMFM-88-D but some improvement in GMFM-88-E (difference 2.7, 95\% 1.0 to 4.4). ${ }^{242}$ In the same trial, TUG improved with exercise (difference $-1.1,95 \% \mathrm{CI}-1.4$ to -0.78 ) compared with usual care, but evidence was considered insufficient for this outcome. 
No improvement in quality of life for any CPQoL domain was reported in one trial following multimodal exercise (strength training combined with cardiopulmonary fitness exercise) versus usual care. ${ }^{238-240}$ The other trial reported no improvement for any SF-36 domain scores immediately postintervention with multimodal exercise versus usual care; only the bodily pain subscale was improved at intermediate-term (24 weeks) followup (Table 38). ${ }^{234-237}$ These trials provided low-strength evidence of no clear benefit of multimodal exercises function or quality of life.

Functional muscle strength measured as the number of lateral step-ups and sit-to-stands in 30 seconds was not different between the multimodal exercise and usual care groups immediately after the 16-week intervention or at 24 weeks followup. ${ }^{238-240}$

One RCT reported that there were no adverse events. ${ }^{234}$ Another RCT indicated that two participants were lost to followup due to medical reasons, but did not specify the medical reasons or otherwise address harms or adverse events. ${ }^{238}$ A third trial reported that no adverse events were associated with the training protocol, but that one participant in the exercise group had ankle pain following a fall while playing basketball. ${ }^{242} \mathrm{~A}$ fourth trial did not report on adverse events. $^{241}$

Figure 27. Multimodal exercise versus usual care in cerebral palsy: $1 \mathrm{MWT}^{\mathrm{a}}$

\begin{tabular}{|c|c|c|c|c|c|c|c|c|c|c|}
\hline \multirow{2}{*}{ Study or Subgroup } & Mean Difference & \multicolumn{2}{|c|}{ Exercise } & \multicolumn{2}{|c|}{ Zontrol } & $\begin{array}{l}\text { Mean Difference } \\
\text { IV, Random, } 95 \% \mathrm{Cl}\end{array}$ & \multicolumn{4}{|c|}{$\begin{array}{c}\text { Mean Difference } \\
\text { IV. Random, } 95 \% \mathrm{Cl}\end{array}$} \\
\hline & -7.23 & 2.01 & 15 & 15 & $62.8 \%$ & $-7.23[-11.17,-3.29]$ & & 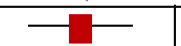 & & \\
\hline Van Wely 2014 & -2 & 3.32 & 25 & 25 & $37.2 \%$ & $-2.00[-8.51,4.51]$ & & 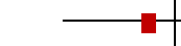 & & \\
\hline Total $(95 \% \mathrm{Cl})$ & & & 40 & 40 & $100.0 \%$ & $-5.28[-10.24,-0.33]$ & & & & \\
\hline $\begin{array}{l}\text { Heterogeneity: Tau² = } \\
\text { Test for overall effect: }\end{array}$ & $\begin{array}{l}\text { 15; } \mathrm{Chi}^{2}=1.82, \mathrm{df}= \\
=2.09(\mathrm{P}=0.04)\end{array}$ & $1(P=C$ & ); $1^{2}=4$ & & & & -20 & Favors Exercise & $\begin{array}{cc}10 \\
\text { Favors Control }\end{array}$ & 20 \\
\hline
\end{tabular}

Abbreviations: $\Delta=$ change; $1 \mathrm{MWT}=1$-Minute Walk Test; $\mathrm{CI}=$ confidence interval; $\mathrm{IV}=$ weighted mean difference; $\mathrm{SE}=$ standard error

${ }^{a}$ This figure reflects Dersimonian Laird estimates as the Profile Likelihood model did not converge.

Figure 28. Multimodal exercise versus usual care in cerebral palsy: GMFM-66

\begin{tabular}{|c|c|c|c|c|c|c|c|c|}
\hline Study & Exercise & Control & $\begin{array}{c}\mathrm{N} \\
\text { Exercise } \\
\end{array}$ & $\begin{array}{c}\mathrm{N} \\
\text { Control }\end{array}$ & Weight & $\begin{array}{l}\text { MD } \Delta \text { Scores } \\
\text { PL }[95 \% \mathrm{Cl}]\end{array}$ & & \\
\hline Slaman 2015 & PRE+Areob & PT & 28 & 27 & $43.0 \%$ & $1.59[-2.68,5.86]$ & & + \\
\hline Van Wely 2014 & $\mathrm{PRE}+$ Aerob+PT & PT & 25 & 25 & $57.0 \%$ & $-3.10[-5.65,-0.55]$ & & \\
\hline Total $(95 \% \mathrm{Cl})$ & & & 53 & 52 & $100.0 \%$ & $-1.48[-6.37,4.66]$ & & \\
\hline \multicolumn{7}{|c|}{ Heterogeneity: Tau $^{2}=7.78 ; \mathrm{Chi}^{2}=3.41, \mathrm{df}=1(P=0.06) ;\left.\right|^{2}=71 \%$} & $\begin{array}{ll}-4 & -2 \\
\text { Favors Exercise }\end{array}$ & $\begin{array}{cc}2 & 1 \\
\text { Eavors Control } & \end{array}$ \\
\hline
\end{tabular}

Abbreviations: $\Delta=$ change; $\mathrm{AC}=$ attention control; $\mathrm{CI}=$ confidence interval; GMFM-66 = Gross Motor Function Measure 66; $\mathrm{MD}=$ mean difference; $\mathrm{PA}=$ previous activity; $\mathrm{PL}=$ profile likelihood; $\mathrm{PRE}=$ progressive resistance exercise

\section{Multimodal Exercises-Spinal Cord Injury}

Three RCTs ${ }^{244-248}$ and one cohort study ${ }^{250}$ enrolled participants $(\mathrm{n}=228)$ with SCI (Table 39). Weighted mean age of participants across trials was 35.3 (range 36.8 to 41.4 years) with weighted mean proportion female of 17.6 percent (range 1\% to $15.5 \%$ ). No study provided data on race or ambulatory abilities. Evidence was considered insufficient for all outcomes based on single trials for each comparison, study quality, and imprecision of effect sizes. 
Table 39. Multimodal strength exercise in spinal cord injury

\begin{tabular}{|c|c|c|c|}
\hline $\begin{array}{l}\text { Author, Year } \\
\text { Intervention } \\
\text { Study Design } \\
\text { Study Quality }\end{array}$ & $\begin{array}{l}\text { Intervention } \\
\text { and Comparison }\end{array}$ & Population & Results \\
\hline $\begin{array}{l}\text { Galea, } 2018^{245} \\
\text { Multimodal } \\
\text { exercise } \\
\text { RCT } \\
\text { Fair }\end{array}$ & $\begin{array}{l}\text { A. Whole body } \\
\text { strength + aerobics, } \\
36 \text { sessions over } 12 \\
\text { weeks }(n=60) \\
\text { B. Upper body } \\
\text { strength + aerobics, } \\
36 \text { sessions over } 12 \\
\text { weeks }(n=56)\end{array}$ & $\begin{array}{l}\text { A vs. B } \\
\text { Age: } 40.1 \text { vs. } 42.8 \\
\text { Female: } 15 \% \text { vs. } 16 \% \\
\text { ASIA A: } 48 \% \text { vs. } 50 \% \\
\text { ASIA B: } 15 \% \text { vs. } 14 \% \\
\text { ASIA C: } 12 \% \text { vs. } 9 \% \\
\text { ASIA D: } 25 \% \text { vs. } 27 \% \\
\text { C2-C } 8: 48 \% \text { vs. } 59 \% \\
\text { T1-T6: } 30 \% \text { vs. } 23 \% \\
\text { T7-T12: } 22 \% \text { vs. } 18 \% \\
\end{array}$ & 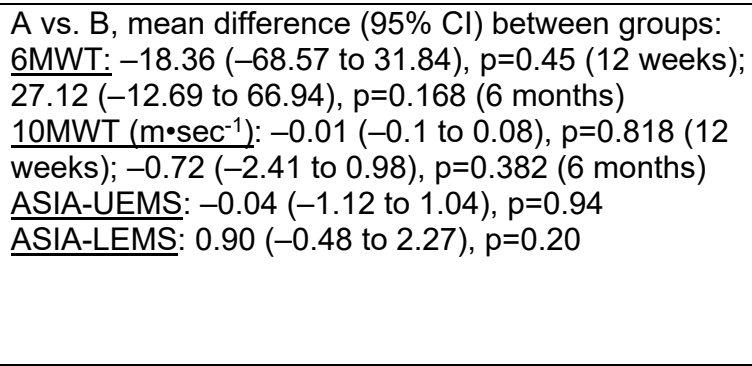 \\
\hline $\begin{array}{l}\text { Harness, } \\
2008^{250} \\
\text { Multimodal } \\
\text { exercise } \\
\text { Cohort study } \\
\text { Fair }\end{array}$ & $\begin{array}{l}\text { A. Strength + cycling } \\
+ \text { vibration, mean } 56 \\
\text { days over } 6 \text { months } \\
(n=22) \\
\text { B. Usual care (self- } \\
\text { regulated exercise), } \\
\text { mean } 98 \text { days over } \\
6 \text { months ( }=9)\end{array}$ & $\begin{array}{l}\text { A vs. B } \\
\text { Age: } 37.8 \text { vs. } 34.5 \\
\text { Female: } 13.6 \% \text { vs. } 0 \% \\
\text { ASIA-UEMS: } 31.0 \text { vs. } \\
38.0, p=0.37 \\
\text { ASIA-LEMS: } 8 \text { vs. } 4\end{array}$ & $\begin{array}{l}\text { A vs. } B \text {, mean change (SE), } p=\text { between groups: } \\
\text { EQ-5D: } 14.0(5.0) \text { vs. } 3.0(5.0), p=0.14 \\
\text { LEMS: } 3.3(0.9) \text { vs. } 0(0.2), p=0.035 \\
\text { ASIA Total Motor: } 4.8(1.0) \text { vs. }-0.1(0.5), p<0.001 \\
\text { CHART: } 12.0(15.0) \text { vs. } 0.1(18.0), p=0.60\end{array}$ \\
\hline $\begin{array}{l}\text { Jones, } \\
2014 a^{247} \\
2014 b^{246} \\
\text { Multimodal } \\
\text { exercise } \\
\text { RCT } \\
\text { Poor }\end{array}$ & $\begin{array}{l}\text { A. Activity-based } \\
\text { therapy, } 72 \text { sessions } \\
\text { over } 24 \text { weeks } \\
(n=20) \\
\text { B. Waitlist }(n=21)\end{array}$ & $\begin{array}{l}\text { A vs. B } \\
\text { Age: } 42 \text { vs. } 34 \\
\text { Female: } 5 \% \text { vs. } 48 \% \\
\text { Tetraplegia: } 75 \% \text { vs. } \\
76 \% \\
\text { AIS C: } 35 \% \text { vs. } 52 \% \\
\text { AIS D: } 65 \% \text { vs. } 48 \%\end{array}$ & 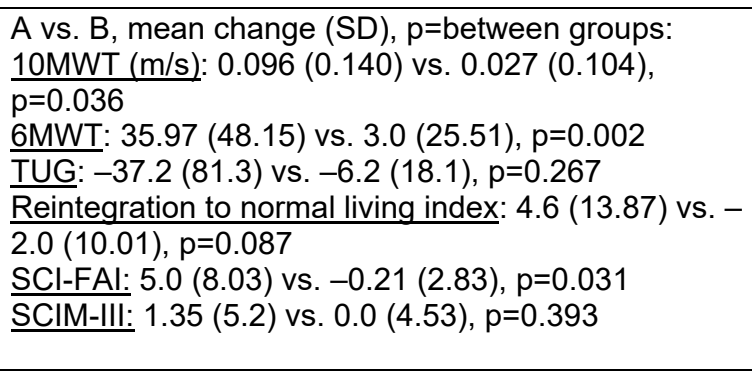 \\
\hline $\begin{array}{l}\text { Liu, } 2019^{248} \\
\text { Multimodal } \\
\text { exercise } \\
\text { RCT } \\
\text { Fair }\end{array}$ & $\begin{array}{l}\text { A. Strength exercise } \\
+ \text { treadmill + core } \\
\text { stability training on a } \\
\text { stable support } \\
\text { surface, } 60 \text { sessions } \\
\text { over } 12 \text { weeks } \\
(n=20) \\
\text { B. Strength exercise } \\
+ \text { treadmill + core } \\
\text { stability training on } \\
\text { an unstable support } \\
\text { surface, } 60 \text { sessions } \\
\text { over } 12 \text { weeks } \\
\text { ( } n=20)\end{array}$ & $\begin{array}{l}\text { A vs. } B \\
\text { (data are for completers } \\
\text { only; } n=14 \text { vs. } 15 \text { ) } \\
\text { Age: } 43 \text { vs. } 46 \\
\text { Female: } 21 \% \text { vs. } 27 \% \\
\text { Ambulatory: } 100 \% \\
\text {-paraplegia: } 36 \% \text { vs. } \\
40 \% \\
\text {-tetraplegia: } 64 \% \text { vs. } \\
60 \%\end{array}$ & $\begin{array}{l}\text { A vs. B, mean (SD), data for completers only: } \\
\text { Stride length (units NR): } \\
0.564(0.189) \text { vs. } 0.454(0.173), p=0.025 \\
\text { (postintervention) } \\
0.09(0.26) \text { vs. } 0.06(0.24), \mathrm{MD} 0.03(95 \% \mathrm{Cl}-0.16 \text { to } \\
0.22), p=N R \text { (pre-post change) } \\
\text { Walking speed (units NR): } \\
0.350 \text { (0.226) vs. } 0.209(0.171), p=0.0196 \\
\text { (postintervention) } \\
0.09(0.30) \text { vs. } 0.03(0.23), \mathrm{MD} 0.06(95 \% \mathrm{Cl}-0.14 \text { to } \\
0.26), p=N R \text { (pre-post change) }\end{array}$ \\
\hline
\end{tabular}

Abbreviations: 6MWT = 6-Minute Walk Test; 10MWT = 10-Meter Walk Test; AE = adverse event; AIS = ASIA Impairment Scale; ASIA-LEMS = American Spinal Injuries Association Impairment Scale - Lower Extremity Motor Score; ASIA-UEMS = American Spinal Injuries Association Impairment Scale - Upper Extremity Motor Score; CHART = Craig Handicap Assessment and Reporting Technique; CI = confidence interval; EQ-5D = European Quality of Life 5 dimensions; MD = mean difference; $\mathrm{NR}=$ not reported; $\mathrm{RCT}=$ randomized controlled trial; $\mathrm{SCI}=$ spinal cord injury; $\mathrm{SCIM}=$ Spinal Cord Independence Measure; $\mathrm{SD}=$ standard deviation; $\mathrm{SE}=$ standard error; $\mathrm{SEM}=$ standard error mean; TUG = Timed Up and Go Test; UEMS = Upper Extremety Motor Score

Evidence from small trials was insufficient to draw conclusions regarding the impact of multimodal exercise on functional capacity or walking in participants with SCI. 
Two studies, one $\mathrm{RCT}^{246,247}$ and one cohort study, ${ }^{250}$ compared multimodal exercise with usual care. Multimodal exercise (which consisted of progressive resistance exercise and locomotor training using manual or robot-assisted gait and aquatic exercise) was associated with improved walking ability compared with waitlist immediately postintervention in the RCT (at 24 weeks $)$, based on the 6MWT $(36.0 \pm 48.2$ vs. $3.0 \pm 25.5$ meters, $\mathrm{p}=0.002)$, the $10 \mathrm{MWT}(0.1 \pm 0.1$ vs. $0.03 \pm 0.1$ meters per second; $p=0.036$ ), and the Spinal Cord Injury Function Ambulation Index (SCI-FAI, scale not provided) $(5.0 \pm 8.0$ vs. $-0.2 \pm 2.8, p=0.031)$. It is not clear if differences are clinically meaningful. Multimodal exercise was not associated with improved functional capacity based on the TUG test in the RCT. ${ }^{247}$

The fair-quality cohort study found no difference between multimodal exercise (consisting of progressive resistance exercise, aerobic exercise, and balance training) versus self-regulated exercise in quality of life using the EQ-5D immediately posttreatment ( 26 weeks) change scores $(14.0 \pm 5.0$ vs. $3.0 \pm 5.0$, respectively, $p=0.14){ }^{250}$

Another trial compared whole-body (progressive resistance exercise for the trunk and upper and lower extremity, locomotor training, and functional electrical stimulation assisted cycling) versus upper body (circuit-based strength and aerobic training) multimodal exercise programs. ${ }^{244,245}$ Walking measures were only reported for the small subset of participants who could walk $(\mathrm{n}=26)$. There was no difference immediately following the 12 -week intervention (6MWT difference -12.30 meters, 95\% CI -68.01 to 43.41 ; $10 \mathrm{MWT}$ difference $-0.10 \mathrm{~m} \bullet \mathrm{sec}^{-1}$, $95 \%$ CI -0.30 to 0.10 ). At 12 -week followup, whole body exercise was associated with improvement in the 6MWT (difference -88.0 meters, 95\% CI -143.71 to -32.29 ) but not the 10MWT (difference $-0.80 \mathrm{~m} \cdot \mathrm{sec}^{-1}, 95 \% \mathrm{CI}-2.3$ to 0.70 ) versus upper body exercise. Evidence was considered insufficient to draw firm conclusions.

One trial compared trunk stabilization exercises done on an unstable surface to exercises performed on a stable surface. ${ }^{248}$ There were no differences between treatment groups for either walking speed or stride length immediately after the 12-week intervention. (Authors did not provide units of measure.)

One RCT comparing multimodal exercise with usual care (waitlist) found no differences in change scores between groups in disability or activities of daily life immediately posttreatment (12 weeks): Spinal Cord Independence Measure, version III (SCIM-III) (1.4 \pm 5.2 vs. $0.0 \pm 4.5$, $\mathrm{p}=0.393)$ and Reintegration to Normal Living ( $4.6 \pm 13.9$ vs. $-2.0 \pm 10.0, \mathrm{p}=0.087)$, respectively. ${ }^{244,245}$ The cohort study reported no differences in disability between multimodal exercise and usual care based on the Craig Handicap and Assessment Reporting Technique (CHART). ${ }^{250}$

Withdrawals due to injuries related to participation in intensive exercise versus usual care were reported in one trial $(7.7 \%$ vs. $0 \%) .{ }^{246,247}$ In the trial comparing whole body versus upper body multimodal exercise, withdrawal due to adverse events was similar $(3.3 \% \mathrm{vs} .1 .8 \%)$ even though the reporting of any definite or probable intervention-related event was more common with whole body strengthening and aerobics versus the upper body intervention. ${ }^{244,245}$ Skin abrasion/bruising was the most common (Table 39). The study comparing trunk stabilization exercise on an unstable support surface versus the exercises done on a stable surface reported that no adverse events occurred. ${ }^{248}$

\section{All Exercise}

The outcomes with sufficient data were analyzed using meta-analysis across all trials combined to determine a general exercise effect that was not dependent on patient population or 
intervention modality(s). The comparison group consisted of no treatment or waitlist controls, or control groups with low-intensity usual care. Usual care arms of higher intensity (e.g., 48 sessions over 12 weeks of strength, aerobic, balance, and coordination exercises) were excluded from the analysis, due to concern that they may confer some level of treatment. The analysis included the difference in outcome from before treatment (baseline) to immediately postintervention. Only RCTs were included in these meta-analyses due to the overall lower quality of nonrandomized studies in this body of evidence. Sensitivity analyses removing trials rated poor quality from the meta-analyses in this section yielded results similar to the primary analyses, with the exception of the TUG test, which was no longer statistically significant after the removal of poor quality studies.

\section{Key Points}

- Across different interventions in trials of participants with MS, there was evidence that physical exercise improves walking ability (SOE: high).

- Across different interventions in trials of participants with MS, there was evidence that physical exercise improves balance (SOE: moderate).

- There was moderate-strength evidence of no clear benefit of physical activity on function in participants with MS (SOE: moderate).

- Across different interventions in trials of participants with $\mathrm{CP}$, there was low-strength evidence that physical activity improves function (SOE: low).

- Across different interventions in trials of participants with SCI, there was low-strength evidence that physical activity improves function (SOE: low).

\section{Detailed Synthesis}

\section{Walking Ability}

In order to determine if walking ability improves with any physical activity intervention, RCTs that assessed performance on the 6MWT, the 10MWT, and/or the MSWS-12 versus no or low-intensity usual care were pooled. Twenty-seven total RCTs were included (23 RCTs for the 6MWT, 14 RCTs for 10MWT, 9 RCTs for MSWS-12). Most trials enrolled participants with MS (25 RCTs, $n=1,343)$. Seven RCTs enrolled participants with CP $(n=234)^{126,129,188,207,211,215,241}$ and two enrolled participants with SCI $(\mathrm{n}=69) .{ }^{189,247}$

\section{6-Minute Walk Test}

Twenty-five RCTs $(n=1,196)$ were included in the 6MWT analysis $52,54,66,77,80,83,126,129,149,160,177,188,192,193,197,198,203,211,221,222,226,228,230,232,241,246,247$ (Figure 29). Most trials were rated fair quality, one trial was rated good quality, ${ }^{211}$ and eight RCTs were rated poor quality. ${ }^{188,192,193,197,226,228,246,247}$ Nineteen trials were in participants with MS, ${ }^{52,54,66,77,80,83,149,160,177,192,193,197,198,203,221,222,226,228,230,232}$ five RCTs enrolled participants with $\mathrm{CP},{ }^{126,129,188,211,241}$ and one was in participants with SCI. ${ }^{247}$ Aerobic interventions included cycling, aquatics, dance, motion gaming, and treadmill training; strength interventions included Pilates and progressive resistance exercises; and multimodal interventions generally included strength training along with aerobic and/or balance training. One study included balance and motor control training as an intervention. ${ }^{149}$

In pooled analysis, participants in the intervention groups walked a mean of almost 33 more meters than those in the control groups after controlling for baseline walking distance (MD 
$-32.94,95 \% \mathrm{CI}-46.07$ to $-19.81, \mathrm{I}^{2}=78 \%, \mathrm{p}<0.001$ ) (Figure 29 ). The treatment effect was stronger and significant in trials of multimodal interventions (MD, $-51.70,95 \% \mathrm{CI}-71.92$ to $-31.48, \mathrm{p}<0.001$ ) for a significant difference based on exercise modality subgroup (i.e., aerobic, vs. strength, vs. postural control vs. multimodal exercises, $\mathrm{p}=0.02$ ).

In pooled analysis of the 19 trials that enrolled participants with MS, results also favored physical activity (MD $-42.70,95 \% \mathrm{CI}-57.05$ to $-28.35, \mathrm{I}^{2}=75 \%$ ). This was not the case in the four trials that enrolled participants with $\mathrm{CP}$ where distance walked was similar in the intervention and control groups (MD $6.85,95 \% \mathrm{CI}-13.39$ to $27.08, \mathrm{I}^{2}=0 \%$ ), or in the one SCI trial (MD $-32.97,95 \% \mathrm{CI}-68.17$ to 2.23$)$.

Figure 29. 6MWT meta-analysis of all randomized controlled trials versus no treatment/usual care

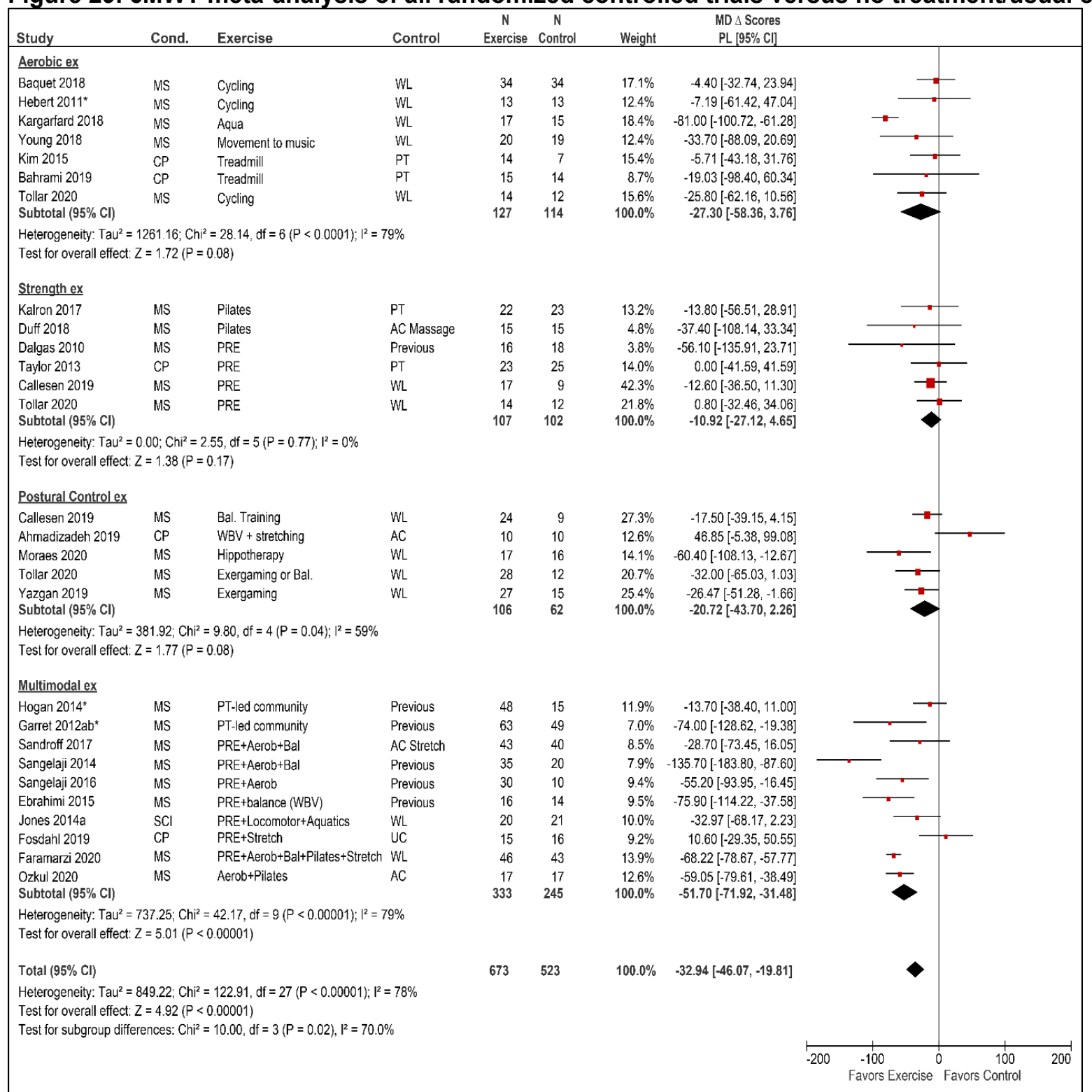

Abbreviations: $\Delta=$ change; $6 \mathrm{MWT}=6$-Minute Walk Test; $\mathrm{AC}=$ attention control; Aerob = aerobic exercise; Aqua $=$ aquatic exercise; $\mathrm{Bal}=$ balance training; $\mathrm{CI}=$ confidence interval; Cond. = condition; $\mathrm{CP}=$ cerebral palsy; $\mathrm{ex}=$ exercise; $\mathrm{MD}=$ mean difference; $\mathrm{MS}=$ multiple sclerosis; $\mathrm{PL}=$ profile likelihood; $\mathrm{PRE}=$ progressive resistance exercise; Previous $=$ continuation of previous activities; $\mathrm{PT}=$ physical therapy; $\mathrm{SCI}=$ spinal cord injury; Stretch $=$ stretching exercise; $\mathrm{UC}=$ usual care $($ not otherwise specified); WL = waitlist 


\section{0-Meter Walk Test}

Fourteen RCTs $(n=659)^{120,129,143,150,189,200,203,207,215,222,223,225,228,247}$ were included in the analysis of 10MWT. Nine trials were of participants with MS, ${ }^{120,143,150,200,203,222,223,225,228}$ two trials were of participants with CP, ${ }^{129,207,215}$ and two trials enrolled participants with SCI. ${ }^{189,247}$ Ten trials were rated fair quality and three were rated poor quality. ${ }^{225,228,247}$ Strength exercises consisted of Pilates and progressive resistance exercises, balance exercises included CoDuSe and PT along with WBV, and multimodal exercises included strength exercises plus aerobic and/or balance exercises. The physical activity intervention was associated with improved time on the 10MWT by 1.24 seconds compared with controls (MD $-1.24,95 \% \mathrm{CI}-2.04$ to $-0.44, \mathrm{I}^{2}=87 \%$ ) (Figure 30).

In pooled analysis of the nine MS RCTs, participants in the physical activity groups walked faster than participants in the control groups ( $\mathrm{MD}-1.44,95 \% \mathrm{CI}-2.74$ to $-0.13, \mathrm{I}^{2}=90 \%$ ). There were no differences between the intervention and control groups in the pooled analysis of the three CP trials (MD $-0.46,95 \% \mathrm{CI}-1.55$ to 0.63 ) or in pooled analysis of the two trials that enrolled participants with SCI (MD $-5.07,95 \% \mathrm{CI}-13.29$ to $3.15, \mathrm{I}^{2}=55 \%$ ).

Figure 30. 10MWT meta-analysis of all randomized controlled trials versus no treatment/usual care

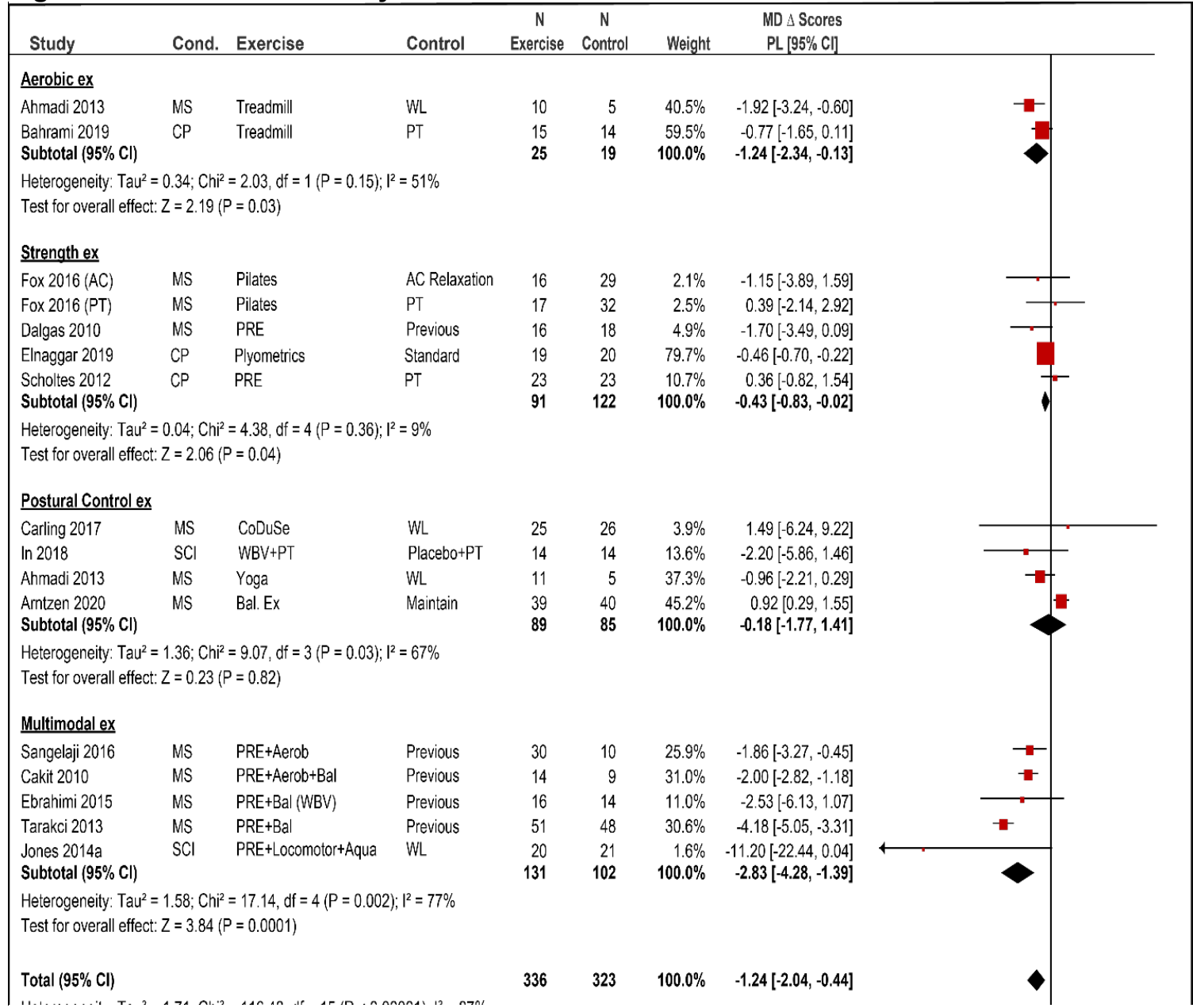

Abbreviations: $\Delta=$ change; $10 \mathrm{MWT}=10$-Meter Walk Test; $\mathrm{AC}=$ attention control; Aerob = aerobic exercise; Aqua = aquatic exercise; $\mathrm{Bal}=$ balance training; $\mathrm{CI}=$ confidence interval; $\mathrm{CoDuSe}=$ core stability, dual task and sensorimotor challenges; $\mathrm{Cond}$. = condition; $\mathrm{CP}=$ cerebral palsy; $\mathrm{ex}=$ exercise; $\mathrm{MD}=$ mean difference; $\mathrm{MS}=$ multiple sclerosis; $\mathrm{PL}=$ profile likelihood; $\mathrm{PRE}=$ 
progressive resistance exercise; Previous = continuation of previous activities; $\mathrm{PT}=$ physical therapy; $\mathrm{SCI}=$ spinal cord injury; $\mathrm{WBV}=$ whole body vibration; $\mathrm{WL}=$ waitlist

\section{Multiple Sclerosis Walking Scale-12}

Eight fair-quality RCTs ${ }^{52,77,143,144,149,150,175,200}(\mathrm{n}=632)$ assessed the effect of physical activity on walking ability in participants with MS using the MSWS-12 (Figure 31). Cycling was the intervention in the aerobic exercise RCT; strength interventions were Pilates; the balance interventions included CoDuSe, balance and motor control training, and supervised Wii training using virtual reality; and the multimodal intervention consisted of strength and aerobic and/or balance exercises. Physical activity was associated with improved scores on the MSWS-12 (MD $-2.88,95 \% \mathrm{CI}-4.80$ to $-0.96, \mathrm{I}^{2}=33 \%$ ). Although there were no differences between subgroups based on intervention category (i.e., aerobic vs. strength vs. balance vs. multimodal exercise, $\mathrm{p}=0.06$ ), pooled analysis of the five RCTs that focused on balance training did show improvement versus control with balance exercise on self-reported walking ability in participants with $\mathrm{MS}\left(\mathrm{n}=316, \mathrm{MD}-4.56,95 \% \mathrm{CI}-6.51\right.$ to $\left.-2.60, \mathrm{I}^{2}=0 \%\right)$.

\section{Figure 31. MSWS-12 meta-analysis of all RCTs versus no treatment/usual care}

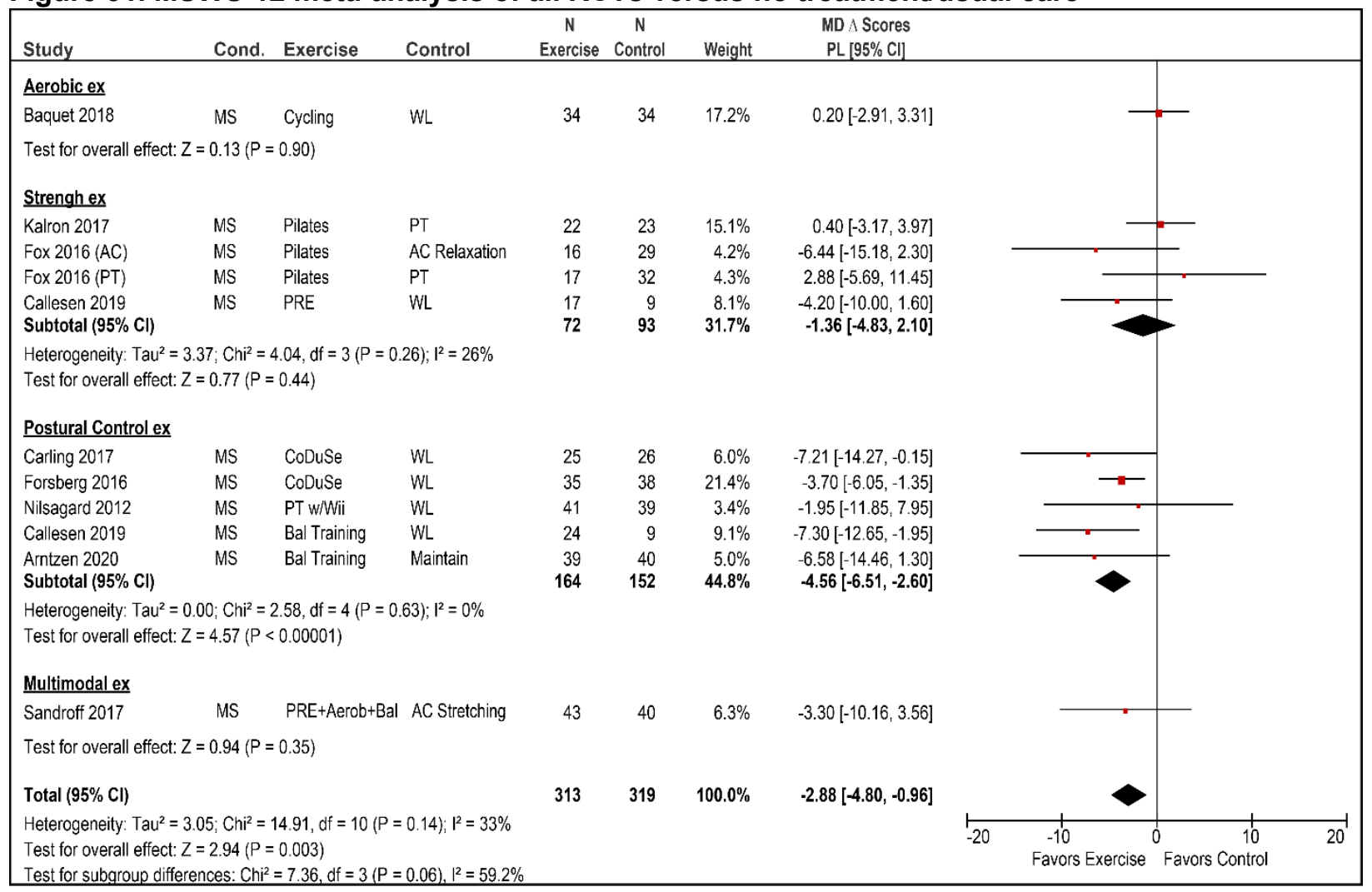

Abbreviations: $\Delta=$ change; $\mathrm{AC}=$ attention control; Aerob = aerobic exercise; $\mathrm{Bal}=$ balance training; $\mathrm{CI}=$ confidence interval; $\mathrm{CoDuSe}=$ core stability, dual task, and sensorimotor challenges; ex = exercise; $\mathrm{MD}=$ mean difference; $\mathrm{MS}=$ multiple sclerosis; MSWS-12 = Multiple Sclerosis Walking Scale-12; PL = profile likelihood; PRE = progressive resistance exercise; $\mathrm{PT}=$ physical therapy; $\mathrm{WL}=$ waitlist

\section{Other Function Outcomes}

Ten RCTs ${ }^{85-87,101,102,161,165,167,209,211,212,234,238}$ provided evidence for exercise on the GMFM or on the D (standing) and/or E (walking, running, jumping) subscales of the GMFM-66 (Figures 32-34). All trials were conducted in participants with CP. All RCTs enrolled children (8 trials 
with mean ages between 8.5 and 14) or young adults ( 2 trials with mean ages between 18 and 20). ${ }^{167,211,212,234-237}$ Eighteen trials ${ }^{50,52-54,95,143,144,151,175,177,185,189,198,206,225,228,242,246,247}$ examined the effect of physical activity on the TUG (Figure 35 ).

\section{GMFM-66}

Eight RCTs in participants with $\mathrm{CP}^{85-87,161,165,167,209,211,212,234,238}(\mathrm{n}=377)$ measured gross motor function with the GMFM-66 and provided the total scores pre- and post-exercise (Figure 32). One trial was rated poor quality ${ }^{165}$ and the remainder were judged to be fair quality. Aerobic exercises included cycling and treadmill training; strength exercises used progressive resistance; balance training used horses (hippotherapy), and multimodal exercises included strength training along with aerobic activity.

There was no change in GMFM-66 scores attributable to physical exercise (MD $-0.58,95 \%$ $\mathrm{CI}-1.62$ to $0.45, \mathrm{p}=0.27$ ) (Figure 32 ). There were subgroup differences based on the exercise category of the intervention $(\mathrm{p}=0.001)$. The results from the single trial of strength training ${ }^{209}$ significantly favored the control group (MD 1.30, 95\% CI 0.67 to $1.93, \mathrm{p}<0.001$ ). In this trial children in the control group received usual care, which consisted of one to three sessions per week of conventional PT. The intervention group underwent three sessions per week of functional progressive resistance exercise training to improve walking ability. Although strength measures were improved with strength training, GMFM-66 scores were not.

\section{GMFM-66-D}

The GMFM-66-D subscale is concerned with standing ability. Two RCTs $(n=78)$, one rated good quality ${ }^{211,212}$ and one poor quality, ${ }^{101,102}$ presented evidence for the D subscale and found no differences between RAGT or muscle strength exercises and usual care (MD $-0.89,95 \%$ CI -7.33 to 5.55, $p=0.79$ ) (Figure 33). There were no subgroup differences $(p=0.11)$. Excluding the poor-quality RCT did not alter the findings.

\section{GMFM-66-E}

Four RCTs ${ }^{101,165,167,211,212}(\mathrm{n}=175)$ reported GMFM-66-E subscale scores, which examine walking, running, and jumping ability (Figure 34). One trial was rated good quality, ${ }^{21,212}$ one fair quality, ${ }^{167}$ and the other two were considered poor quality. ${ }^{102,165}$ Included interventions were RAGT, muscle strength exercises, and hippotherapy. Pooled analysis found a significant improvement with exercises versus usual care (MD $-3.73,95 \% \mathrm{CI}-5.78$ to $-1.67, \mathrm{p}<0.001$ ). 
Figure 32. GMFM-66 meta-analysis of all randomized controlled trials versus no treatment/usual care

\begin{tabular}{|c|c|c|c|c|c|c|c|c|c|c|c|}
\hline Study & Cond. & Exercise & Control & $\begin{array}{c}\mathrm{N} \\
\text { Exercise }\end{array}$ & $\begin{array}{c}\mathrm{N} \\
\text { Control }\end{array}$ & Weight & $\begin{array}{l}\text { MD } \Delta \text { Scores } \\
\mathrm{PL}[95 \% \mathrm{Cl}]\end{array}$ & & & & \\
\hline \multicolumn{12}{|l|}{ Aerobic ex } \\
\hline Fowler 2010/Demuth 2012 & $\mathrm{CP}$ & Cycling & Previous & 29 & 29 & $19.9 \%$ & $-0.70[-1.69,0.29]$ & & & & \\
\hline $\begin{array}{l}\text { Bryant } 2012 \\
\text { Subtotal }(95 \% \mathrm{Cl})\end{array}$ & $\mathrm{CP}$ & Bike+Treadmill & PT & $\begin{array}{l}20 \\
49\end{array}$ & $\begin{array}{l}10 \\
39\end{array}$ & $\begin{array}{l}10.9 \% \\
30.8 \%\end{array}$ & $\begin{array}{l}-0.93[-3.25,1.39] \\
-0.74[-1.97,0.42]\end{array}$ & & & 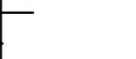 & \\
\hline \multicolumn{12}{|c|}{$\begin{array}{l}\text { Heterogeneity: } \mathrm{Tau}^{2}=0.00 ; \mathrm{Chi}^{2}=0.03, \mathrm{df}=1(\mathrm{P}=0.86) ; \mathrm{I}^{2}=0 \% \\
\text { Test for overall effect: } Z=1.58(P=0.11)\end{array}$} \\
\hline \multicolumn{12}{|l|}{ Strength ex } \\
\hline Scholtes 2010 & $\mathrm{CP}$ & PRE & PT & 24 & 25 & $22.3 \%$ & $1.30[0.67,1.93]$ & & & $\rightarrow$ & \\
\hline \multicolumn{12}{|c|}{ Test for overall effect: $Z=4.05(P<0.0001)$} \\
\hline \multicolumn{12}{|l|}{ Postural Control ex } \\
\hline Deutz 2017 & $\mathrm{CP}$ & Hippotherapy & WL & 35 & 38 & $4.8 \%$ & $-2.50[-6.71,1.71]$ & & & - & \\
\hline Herrero 2012 & $\mathrm{CP}$ & Hippotherapy & Placebo & 19 & 19 & $23.7 \%$ & $-0.27[-0.61,0.07]$ & & & & \\
\hline $\begin{array}{l}\text { Mutoh } 2019 \\
\text { Subtotal (95\% Cl) }\end{array}$ & $\mathrm{CP}$ & Hippotherapy & Outdoor Rec & $\begin{array}{l}12 \\
66\end{array}$ & $\begin{array}{l}12 \\
69\end{array}$ & $\begin{array}{r}3.8 \% \\
32.3 \%\end{array}$ & $\begin{array}{r}-5.70[-10.54,-0.86] \\
-2.12[-5.27,1.04]\end{array}$ & & & & \\
\hline \multicolumn{12}{|c|}{ Heterogeneity: $\mathrm{Tau}^{2}=5.12 ; \mathrm{Chi}^{2}=5.86, \mathrm{df}=2(\mathrm{P}=0.05) ; \mathrm{I}^{2}=66 \%$} \\
\hline \multicolumn{12}{|l|}{ Multimodal ex } \\
\hline Slaman 2015 & $\mathrm{CP}$ & PRE+Aerob & PT & 28 & 27 & $4.7 \%$ & $1.59[-2.68,5.86]$ & & & & \\
\hline $\begin{array}{l}\text { Van Wely } 2014 \\
\text { Subtotal (95\% Cl) }\end{array}$ & $\mathrm{CP}$ & $\mathrm{PRE}+$ Aerob+PT & PT & $\begin{array}{l}25 \\
53\end{array}$ & $\begin{array}{l}25 \\
52\end{array}$ & $\begin{array}{r}9.8 \% \\
14.5 \%\end{array}$ & $\begin{array}{l}-3.10[-5.65,-0.55] \\
-1.48[-6.37,4.66]\end{array}$ & & & & \\
\hline \multicolumn{12}{|c|}{ Heterogeneity: $\mathrm{Tau}^{2}=7.78 ; \mathrm{Chi}^{2}=3.41, \mathrm{df}=1(P=0.06) ; \mathrm{I}^{2}=71 \%$} \\
\hline Total $(95 \% \mathrm{Cl})$ & & & & 192 & 185 & $100.0 \%$ & $-0.58[-1.62,0.45]$ & & & & \\
\hline $\begin{array}{l}\text { Heterogeneity: } \operatorname{Tau}^{2}=1.14 \\
\text { Test for overall effect: } Z=1 \\
\text { Test for subgroup difference }\end{array}$ & $\begin{array}{l}\mathrm{Chi}^{2}=34 . \\
11(\mathrm{P}=0.2 \\
\text { s: } \text { Chi }^{2}=1\end{array}$ & $\begin{array}{l}\mathrm{df}=7(P<0.000 \\
34, \mathrm{df}=3(P=0.0\end{array}$ & $\begin{array}{l}1) ;\left.\right|^{2}=79 \% \\
010),\left.\right|^{2}=81.6 \%\end{array}$ & & & & & $!_{-10}$ & Favors Exercise $^{-5}$ & Favors Control & 10 \\
\hline
\end{tabular}

Abbreviations: $\Delta=$ change; Aerob = aerobic exercise; $\mathrm{CI}=$ confidence interval; $\mathrm{CP}=$ cerebral palsy; $\mathrm{ex}=$ exercise; $\mathrm{GMFM}=$ gross motor function measure; $\mathrm{MD}=$ mean difference; Outdoor $\mathrm{Rec}=$ outdoor recreation; $\mathrm{PL}=$ profile likelihood; $\mathrm{PRE}=$ progressive resistance exercise; Previous = continuation of previous activities; $\mathrm{PT}=$ physical therapy; $\mathrm{WL}=$ waitlist

Figure 33. GMFM-66-D meta-analysis of all randomized controlled trials versus no intervention/usual care

\begin{tabular}{|c|c|c|c|c|c|c|c|c|c|c|c|}
\hline Study & Cond. & Exercise & Control & $\begin{array}{c}\mathrm{N} \\
\text { Exercise }\end{array}$ & $\begin{array}{c}\mathrm{N} \\
\text { Control }\end{array}$ & Weight & $\begin{array}{l}\text { MD } \Delta \text { Scores } \\
\text { PL }[95 \% \text { Cl] }\end{array}$ & & & & \\
\hline \multicolumn{12}{|l|}{ Aerobic ex } \\
\hline Wallard 2018 & $\mathrm{CP}$ & RAGT & PT & 14 & 16 & $41.9 \%$ & $-4.76[-11.65,2.13]$ & & 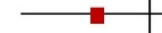 & - & \\
\hline \multicolumn{12}{|c|}{ Test for overall effect: $Z=1.35(P=0.18)$} \\
\hline \multicolumn{12}{|l|}{ Strength ex } \\
\hline Taylor 2013/Bania 2016 & $\mathrm{CP}$ & PRE & PT & 23 & 25 & $58.1 \%$ & $1.90[-2.58,6.38]$ & & & & \\
\hline \multicolumn{12}{|c|}{ Test for overall effect: $Z=0.83(P=0.41)$} \\
\hline \multicolumn{4}{|l|}{ Total $(95 \% \mathrm{Cl})$} & 37 & 41 & $100.0 \%$ & $-0.89[-7.33,5.55]$ & & & & \\
\hline \multicolumn{8}{|c|}{ Heterogeneity: $\mathrm{Tau}^{2}=13.38 ; \mathrm{Chi}^{2}=2.52, \mathrm{df}=1(P=0.11) ; \mathrm{I}^{2}=60 \%$} & -20 & -10 & 10 & 20 \\
\hline \multicolumn{8}{|c|}{ Test for overall effect: $Z=0.27(P=0.79)$} & \multicolumn{4}{|c|}{ Test for subgroup differences: $\mathrm{Chi}^{2}=2.52, \mathrm{df}=1(\mathrm{P}=0.11), \mathrm{I}^{2}=60.4 \%$} \\
\hline
\end{tabular}

Abbreviations: $\Delta=$ change; $\mathrm{CI}=$ confidence interval; Cond. = condition; $\mathrm{CP}=$ cerebral palsy; $\mathrm{ex}=$ exercise; $\mathrm{GMFM}-\mathrm{D}=$ gross motor function measure, subscale $\mathrm{D}$ (standing); $\mathrm{MD}=$ mean difference; $\mathrm{PL}=$ profile likelihood; $\mathrm{PRE}=$ progressive resistance exercise; $\mathrm{PT}=$ physical therapy; $\mathrm{RAGT}=$ robotic assisted gait training 
Figure 34. GMFM-66-E meta-analysis of all randomized controlled trials versus no intervention/usual care

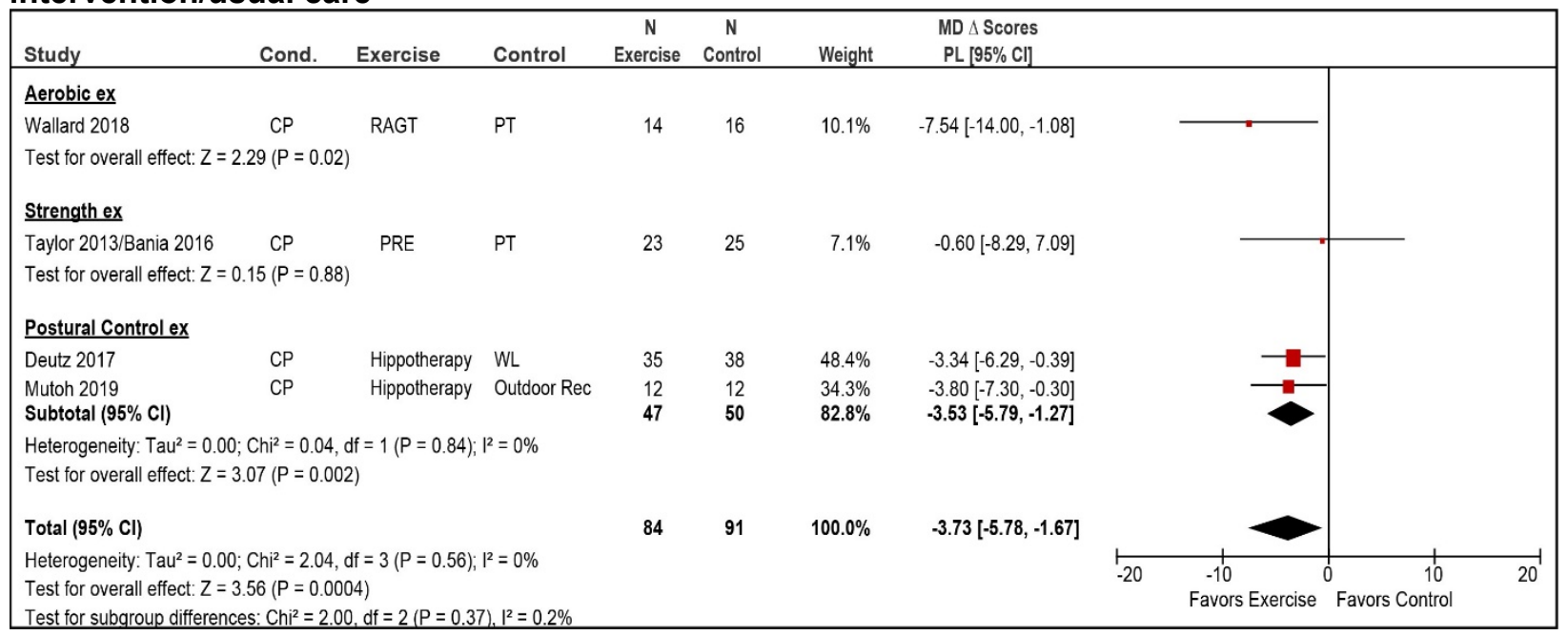

Abbreviations: $\Delta=$ change; $\mathrm{CI}=$ confidence interval; $\mathrm{CP}=$ cerebral palsy; ex = exercise; GMFM-E = Gross Motor Function Measure 66, subscale E (walking, running, jumping); $\mathrm{MD}=$ mean difference; Outdoor Rec = outdoor recreation; PL = profile likelihood; PRE = progressive resistance exercise; $\mathrm{PT}=$ physical therapy; $\mathrm{RAGT}=$ robotic assisted gait training; $\mathrm{SE}=$ standard error; WL = waitlist

\section{Timed Up and Go Test}

Nineteen trials ${ }^{50,52-54,95,143,144,151,175,177,185,189,198,206,225,228,230,242,246,247}(\mathrm{n}=793)$ examined the effect of physical activity on the TUG (Figure 35). Two studies enrolled participants with $\mathrm{CP},{ }^{50,242}$ two enrolled participants with SCI, ${ }^{189,246,247}$ and the remainder were conducted in participants with MS. Aerobic interventions included interval exercises, RAGT, and movement to music; balance exercises included motion gaming, WBV, and CoDuSe; and strength and multimodal interventions included Pilates and progressive resistance training, along with aerobic exercise or balance exercises. Physical activity was associated with improvement on the TUG ( $\mathrm{MD}-0.66,95 \% \mathrm{CI}-1.28$ to $-0.04, \mathrm{I}^{2}=85 \%$ ). However, the results were no longer statistically significant after the removal of the poor quality trials in a sensitivity analysis $(\mathrm{n}=15$ studies, MD $-0.55,95 \%$ CI -1.29 to $0.19, \mathrm{I}^{2}=89 \%$ ). Results were also not significant when the fifteen trials that enrolled participants with MS were pooled (MD $-0.30,95 \% \mathrm{CI}-1.18$ to $0.59, \mathrm{I}^{2}=89 \%$ ). There was also no difference between all exercise versus usual care when the two trials in participants with SCI were pooled (MD $-10.33,95 \% \mathrm{CI}-37.10$ to $16.45, \mathrm{I}^{2}=61 \%$ ). However, pooled analysis of the two trials in participants with CP found improved TUG with all exercise (MD $-1.05,95 \% \mathrm{CI}-1.35$ to $-0.76, \mathrm{I}^{2}=0 \%$ ). 
Figure 35. Timed Up and Go Test| versus no intervention or usual care

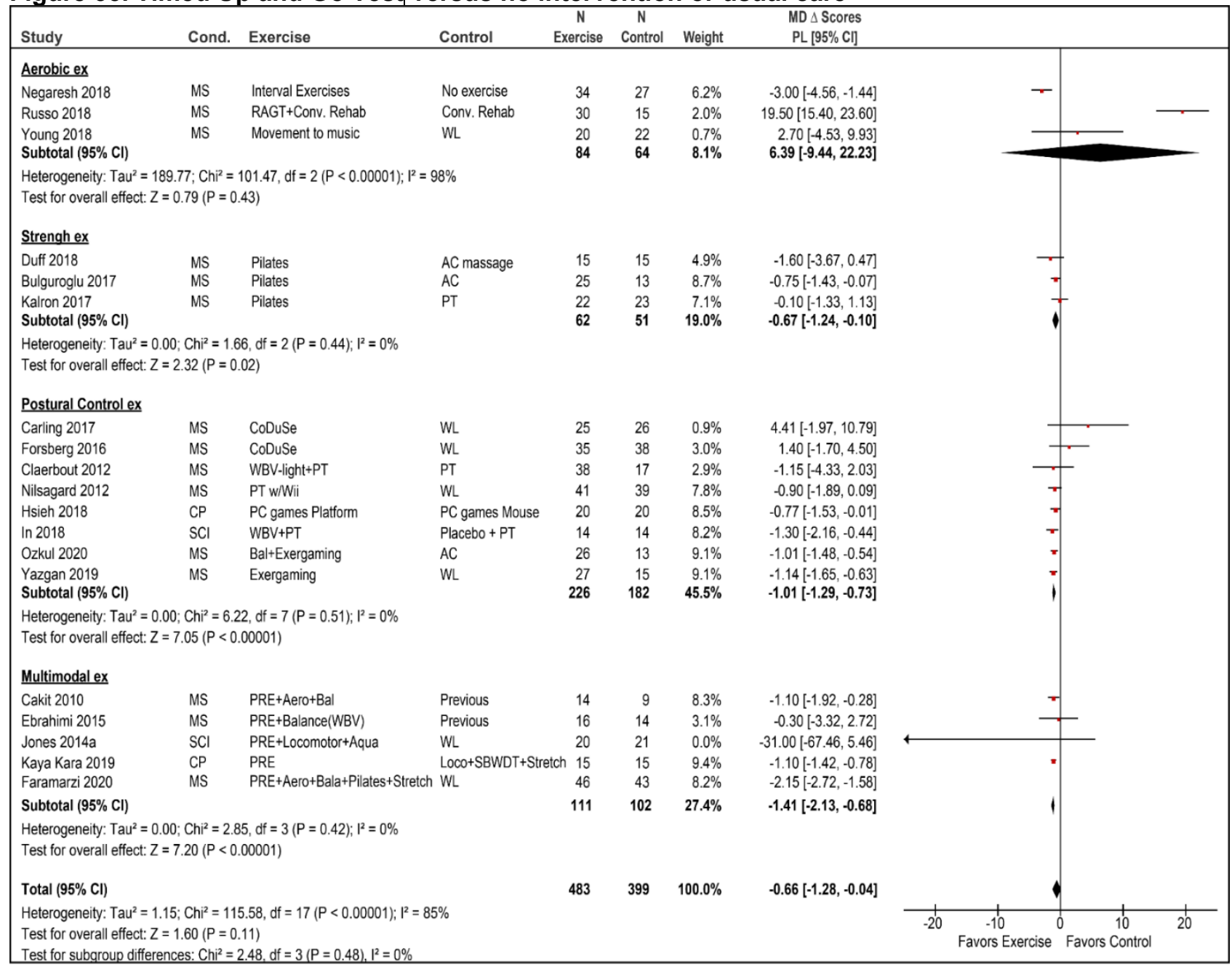

Abbreviations: $\Delta=$ change; $\mathrm{AC}=$ attention control; $\mathrm{Aerob}=$ aerobic exercise; Aqua = aquatics; $\mathrm{Bal}=$ balance; $\mathrm{Conv}$ Rehab = conventional rehabilitation; $\mathrm{CP}=$ cerebral palsy; loco = locomotor; $\mathrm{MS}=$ multiple sclerosis; $\mathrm{PRE}=$ progressive resistance exercise; PT = physical therapy; RAGT = robot-assisted gait training; SBWDT $=$ weight-bearing symmetry; $\mathrm{SCI}=$ spinal cord injury; $\mathrm{WBV}=$ whole body vibration; $\mathrm{WL}=$ waitlist

\section{Balance}

Twenty RCTs ${ }^{50,52,66,83,117,120,141,143-146,151,156-158,177,222,223,226,228}(\mathrm{n}=1,011)$ that compared a physical exercise intervention with no activity or low-intensity usual care included the BBS to assess balance prior to the intervention and immediately after treatment (Figure 36). All RCTs enrolled participants with MS except for three trials, one conducted in participants with CP $(n=40)^{50}$ and two conducted in SCI $(n=60) .{ }^{156,157}$ One trial was considered good quality, ${ }^{145}$ two trials were rated poor quality, ${ }^{226,228}$ and the remainder were rated fair quality. Aerobic interventions included treadmill training and aquatic exercise; balance interventions included CoDuSe, Cawthorne/Cooksey exercises, balance training with sensory integration, computer games using a platform, and hippotherapy; and multimodal interventions included strength plus balance and/or aerobics.

Physical activity was associated with significantly improved scores on the BBS by over 3 points (MD $-3.64,95 \% \mathrm{CI}-4.23$ to $-3.04, \mathrm{I}^{2}=68 \%$ ). Trials of aerobic exercise, balance (postural control) exercises, and trials of multimodal exercises reported significant effects (MD -3.48 , 
$95 \% \mathrm{CI}-5.68$ to $-1.28, \mathrm{p}=0.002 ; \mathrm{MD}-3.97,95 \% \mathrm{CI}-5.00$ to $-2.94, \mathrm{p}<0.001 ; \mathrm{MD}-3.45,95 \%$ $\mathrm{CI}-3.89$ to $-3.01, \mathrm{p}<0.001$, respectively) although there was no subgroup effect based on intervention category (i.e., aerobic vs. strength vs. balance vs. multimodal exercise, $p=0.06$ ). The overall results were similar when only the MS trials were analyzed (MD $-3.56,95 \% \mathrm{CI}-4.58$ to $\left.-2.54, \mathrm{I}^{2}=77 \%\right)$.

Figure 36. BBS meta-analysis of all randomized controlled trials versus no intervention/usual care

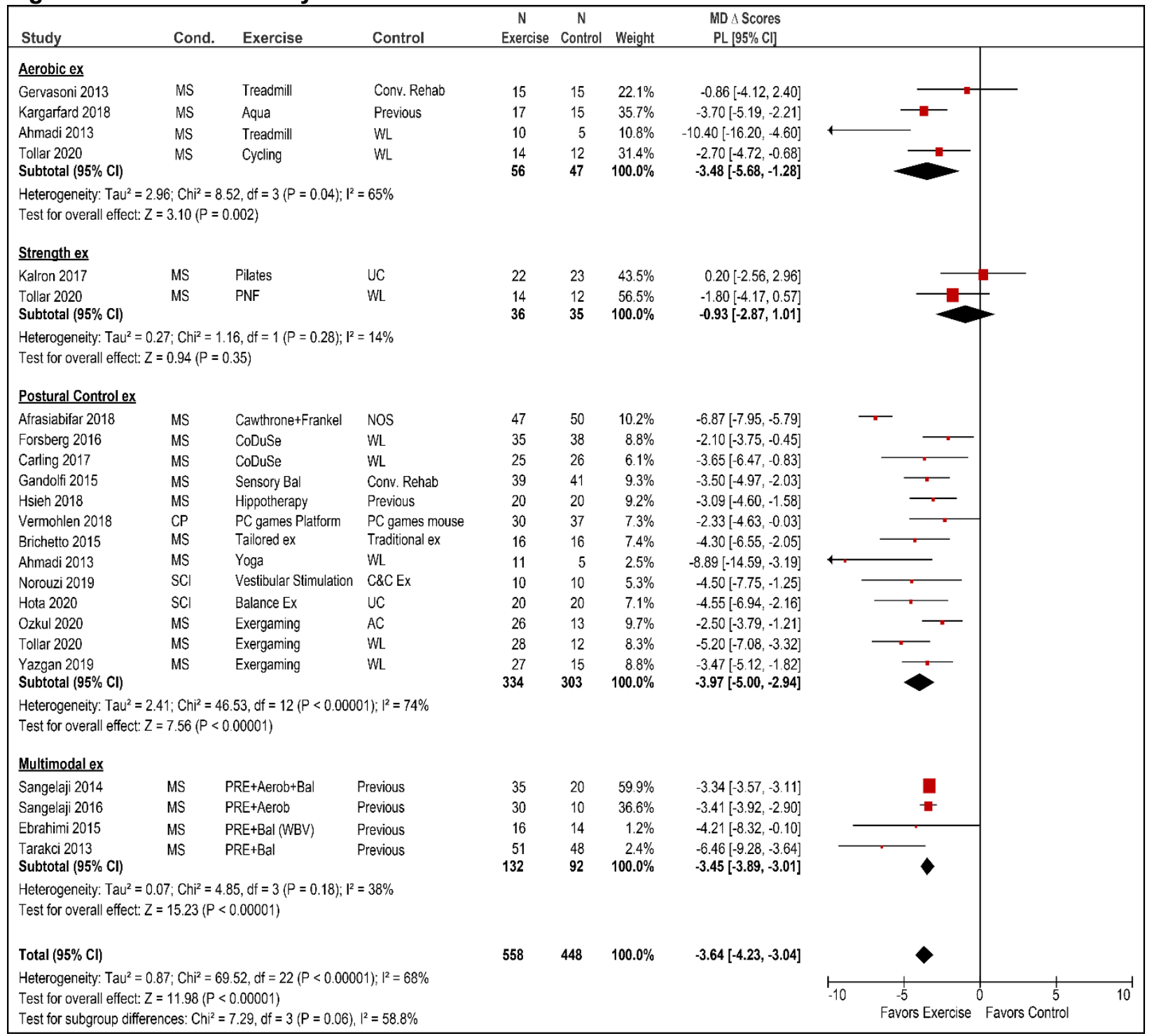

Abbreviations: $\Delta=$ change; Aerob = aerobic exercise; Aqua = aquatic exercise; $\mathrm{BBB}=$ Berg Balance Scale; Bal = balance training; $\mathrm{C} \& \mathrm{C}=$ Cawthorne and Cooksey exercises; $\mathrm{CI}=$ confidence interval; CoDuSe $=$ core stability, dual task and sensorimotor challenges; Cond. = condition; Conv. = conventional; $\mathrm{CP}=$ cerebral palsy; ex = exercise; $\mathrm{MD}=$ mean difference; $\mathrm{MS}=$ multiple sclerosis; NOS = not otherwise specified; PC = personal computer; $\mathrm{PL}=$ profile likelihood; $\mathrm{PNF}=$ proprioceptive neuromuscular facilitation; PRE = progressive resistance exercise; Previous = continuation of previous activities; Rehab = rehabilitation; $\mathrm{SCI}=$ spinal cord injury; $\mathrm{WL}=$ waitlist 


\section{KQ2a: Clinical Outcomes}

\section{Cardiometabolic Disease}

No studies were identified that assessed the long-term (or short-term) benefits of physical exercise on the primary, secondary, or tertiary prevention of cardiovascular disease and diabetes. Although some studies did examine the effect of exercise on intermediate outcomes such as resting heart rate, lipid levels, and serum glucose (discussed in KQ2b), there were no included studies that reported prevention of myocardial infarction, stroke, or the development of diabetes. A history of cardiovascular or metabolic disorders was often used as exclusion criteria in trials enrolling adults with MS and SCI. This prevented examination of the effects of exercise on improvement in cardiovascular disease or diabetes symptoms. (CP trials were mostly in children and adolescents and did not typically address cardiometabolic disease.)

\section{Obesity}

No included studies provided evidence for the development of obesity or overweight or the proportion of patients who were no longer overweight or obese following an intervention.

\section{Depression and Anxiety}

While there was a lack of evidence on cardiometabolic disease and exercise, multiple trials addressed mental health and administered validated self-report mental health instruments (e.g., Beck Depression Inventory [BDI], Hospital Anxiety and Depression Scale [HADS]) before and after physical exercise. Most trials were in participants with MS (17 RCTs, 4 quasiexperimental trials, total $n=973$ ) (Table 40). Three RCTs enrolled participants with SCI ( $n=171)$ (Table 41).

\section{Multiple Sclerosis}

The best quality evidence in MS patients was in RAGT ( 3 good-quality RCTs, ${ }^{96-98} 1$ fairquality RCT ${ }^{95} 209$ studies), followed by cycling studies ( 3 fair-quality RCTs, ${ }^{53,77,80} \mathrm{n}=169$ ) and aqua therapy ( 2 good-quality RCTs, ${ }^{70,71} \mathrm{n}=109$ ). Other interventions in trials with evidence for mental health in participants with MS included treadmill training, calisthenics, Tai Chi, Pilates, aquatics, yoga, and progressive resistance training, and multimodal exercises (Table 40). All but one trial ${ }^{117}$ enrolled more females than males, with most adults in their 30 s and 40s.

In the four RAGT RCTs (one with virtual reality) in participants with MS, two studies used the Hamilton Rating Scale for Depression ${ }^{95,96}$ (HRSD, also called the Hamilton Depression Rating Scale, abbreviated HDRS or HAM-D) and two trials used the Patient Health Questionnaire-9 (PHQ-9). ${ }^{97,98}$ Comparison groups were usual rehabilitation exercises, walking therapy without RAGT, and RAGT without virtual reality. Comparisons between RAGT and control groups indicated improved depression scores after exercise, with no differences between exercise groups.

The instruments used in the three MS cycling RCTs were the Inventory of Depressive Symptomatology Self Report 16 item (IDS-16-SR), the BDI, and the BDI-II. All trials had a usual care or waitlist control arm and one trial also had a vestibular rehabilitation comparison group. In two RCTs ${ }^{53,77}$ exercise was associated with improved baseline to postintervention depression scores that were statistically significant, whereas the usual care arms did not show significant improvement, but between group differences failed to reach significance. The third RCT found no differences between either cycling or vestibular rehabilitation and control. ${ }^{80}$ 
Table 40. Effects of exercise on depression in participants with multiple sclerosis

\begin{tabular}{|c|c|c|c|}
\hline $\begin{array}{l}\text { Author, Year } \\
\text { Intervention } \\
\text { Study Design } \\
\text { Study Quality }\end{array}$ & $\begin{array}{l}\text { Intervention } \\
\text { and Comparison }\end{array}$ & Population & Results \\
\hline $\begin{array}{l}\text { Ahmadi, } \\
2013^{120} \\
\text { Aerobic/ } \\
\text { Postural } \\
\text { control } \\
\text { RCT } \\
\text { Fair }\end{array}$ & 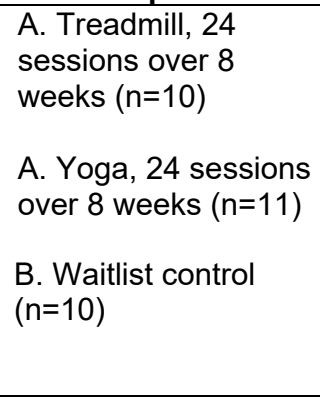 & $\begin{array}{l}\text { A vs. B vs. C } \\
\text { Age: } 37 \text { vs. } 32 \text { vs. } 37 \\
\text { Female: } 100 \% \\
\text { EDSS: } 2.40 \text { vs. } 2.00 \\
\text { vs. } 2.25\end{array}$ & $\begin{array}{l}\text { A vs. B vs. C, mean (SD), } p=\text { between groups: } \\
\text { BDI: } 8.50(3.06 \text { to } 5.60(3.40) \text { vs. } 17.36(12.42) \\
\text { to } 11.09(12.46) \text { vs. } 11.90(9.39) \text { to } 12.50(8.1) \\
\text { A vs. } B, p=0.11 \\
\text { A vs. } C, p=0.11 \\
\text { B. vs. } C, p=p=0.001 \\
\text { BAl: } 7.90(5.91) \text { to } 6.10(4.95) \text { vs. } 12.45(4.54) \\
\text { to } 6.45(3.61) \text { vs. } 7.50(6.77) \text { to } 8.20(7.39) \\
\text { A vs. } B, p=0.01 \\
\text { A vs. } C, p=0.22 \\
\text { B vs. } C, p=0.001\end{array}$ \\
\hline $\begin{array}{l}\text { Aydin, } 2014^{59} \\
\text { Aerobics } \\
\text { RCT } \\
\text { Fair }\end{array}$ & $\begin{array}{l}\text { A. Callisthenic } \\
\text { exercises (in clinic): } \\
60 \text { sessions, over } 12 \\
\text { weeks, }(n=16) \\
\\
\text { B. Callisthenic } \\
\text { exercises (home- } \\
\text { based): } 60 \text { sessions, } \\
\text { over } 12 \text { weeks, } \\
(n=20)\end{array}$ & $\begin{array}{l}\text { A vs. B } \\
\text { Age: } 32.6 \text { vs. } 33 \\
\text { Female: } 56 \% \text { vs. } \\
55 \% \\
\text { EDSS: } 3.6 \text { vs. } 3.4\end{array}$ & $\begin{array}{l}\text { A vs. B, mean (SD) } \\
\text { HADS-A: } \\
10.63(7.33) \text { vs. } 11.05(5.73), p=0.762 \\
\text { (baseline) } \\
8.69(6.11) \text { vs. } 10.00(5.36), p=0.482 \\
\text { (postintervention) } \\
\text { Pre-post exercise intra-group comparison: } \\
\text { Difference }-1.94(2.35)-1.05(1.32), p=0.412 \\
\text { HADS-D: } \\
8.50(3.74) \text { vs. } 6.75(3.23), p=0.212 \text { (baseline) } \\
6.13(3.26) \text { vs. } 8.60(2.41), p=0.011 \\
\text { (postintervention) } \\
\text { Pre-post exercise intra-group comparison: } \\
\text { Difference }-1.94(2.35) \text { vs. } 1.85(1.60), \\
p=<0.001\end{array}$ \\
\hline $\begin{array}{l}\text { Baquet, } 2018^{77} \\
\text { Aerobic } \\
\text { exercise } \\
\text { RCT } \\
\text { Fair }\end{array}$ & $\begin{array}{l}\text { A. Bicycle ergometry, } \\
24-36 \text { sessions over } \\
12 \text { weeks }(n=34) \\
\text { B. Waitlist control } \\
\text { group }(n=34)\end{array}$ & $\begin{array}{l}\text { A vs. B } \\
\text { Age: } 38.2 \text { vs. } 39.6 \\
\text { Female: } 62 \% \text { vs. } \\
74 \% \\
\text { EDSS: } 1.7 \text { vs. } 1.8 \\
\text { RRMS: } 100 \%\end{array}$ & $\begin{array}{l}\text { A vs. } B \text {, mean difference between groups: } \\
\text { IDS16-SR: } 0.5,95 \% \mathrm{Cl}-0.8 \text { to } 1.9, p=0.44\end{array}$ \\
\hline $\begin{array}{l}\text { Burschka, } \\
2014^{173} \\
\text { Postural } \\
\text { control } \\
\text { Quasiexperime } \\
\text { ntal }\end{array}$ & $\begin{array}{l}\text { A. Tai Chi, } 48 \\
\text { sessions } 6 \text { months } \\
(n=15) \\
\text { B. Usual care }(n=17)\end{array}$ & $\begin{array}{l}\text { A vs. B } \\
\text { Age: } 42 \text { vs. } 43 \\
\text { Female: } 66 \% \text { vs. } \\
71 \% \\
\text { Ambulatory: } 100 \% \\
\text { RRMS: } 93 \% \text { vs. } 76 \% \\
\text { SPMS: } 0 \% \text { vs. } 24 \% \\
\text { CIS: } 7 \% \text { vs. } 0 \%\end{array}$ & 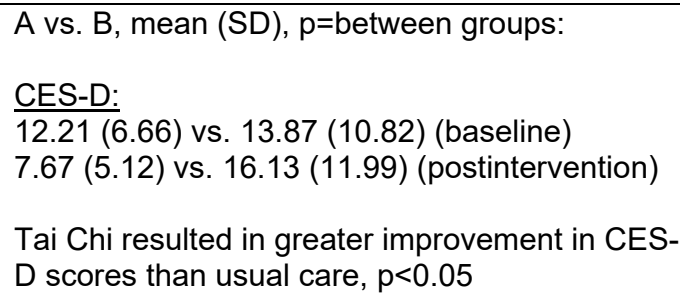 \\
\hline Poor & & & \\
\hline $\begin{array}{l}\text { Cakit, } 2010^{225} \\
\text { Multimodal } \\
\text { exercise } \\
\text { RCT } \\
\text { Poor }\end{array}$ & $\begin{array}{l}\text { A. Progressive } \\
\text { resistance cycling } \\
\text { plus balance } \\
\text { exercises (lower } \\
\text { extremity } \\
\text { strengthening), } 16 \\
\text { sessions over } 8 \\
\text { weeks ( } n=14 \text { ) } \\
\text { B. Usual care }(n=9)\end{array}$ & $\begin{array}{l}\text { A vs. B } \\
\text { Age: } 36.4 \text { vs. } 35.5 \\
\text { Female: } 64 \% \text { vs. } \\
67 \% \\
\text { RRMS or SPMS: } \\
100 \% \\
\text { Assistive device: } \\
28.5 \% \text { vs. } 37.5 \% \\
\text { Fall frequency last } \\
\text { year: } 2.0 \text { vs. } 2.4 \\
\end{array}$ & $\begin{array}{l}\text { A vs. B, mean (SD), } p=\text { between groups: } \\
\text { BDI: } \\
22.8(12.7) \text { vs. } 27.0(17.6) \text { (baseline) } \\
17.2(12.3) \text { vs. } 25.4(22.8) \text { (postintervention) } \\
-5.5(5.3) \text { vs. }-1.6(6.0), p=<0.05 \text { (pre-post } \\
\text { change) }\end{array}$ \\
\hline
\end{tabular}




\begin{tabular}{|c|c|c|c|}
\hline $\begin{array}{l}\text { Author, Year } \\
\text { Intervention } \\
\text { Study Design } \\
\text { Study Quality }\end{array}$ & $\begin{array}{l}\text { Intervention } \\
\text { and Comparison }\end{array}$ & Population & Results \\
\hline $\begin{array}{l}\text { Calabro, } \\
2017^{96} \\
\text { Aerobic } \\
\text { exercise } \\
\text { RCT } \\
\text { Good }\end{array}$ & $\begin{array}{l}\text { A. Lokomat-Pros } \\
\text { (RAGT + VR), } 40 \\
\text { sessions over } 8 \\
\text { weeks }(n=20) \\
\text { B. Lokomat-Nanos } \\
\text { (RAGT), } 40 \text { sessions } \\
\text { over } 8 \text { weeks }(n=20)\end{array}$ & $\begin{array}{l}\text { A vs. B } \\
\text { Age: } 44 \text { vs. } 41 \\
\text { Female: } 65 \% \text { vs. } \\
60 \% \\
\text { EDSS: } 4.40 \text { vs. } 4.75\end{array}$ & $\begin{array}{l}\text { HRSD median change score, } \mathrm{p}=\text { between } \\
\text { groups: } \\
-0.062,95 \% \mathrm{Cl}-4.932 \text { to } 4.808, p=0.90\end{array}$ \\
\hline $\begin{array}{l}\text { Castro- } \\
\text { Sanchez, } \\
2012^{71} \\
\text { Aerobic } \\
\text { Exercise } \\
\text { RCT } \\
\text { Good }\end{array}$ & $\begin{array}{l}\text { A. Ai-Chi aqua } \\
\text { therapy with Tai-Chi } \\
\text { music, } 40 \text { sessions } \\
\text { over } 20 \text { weeks } \\
(n=36) \\
\text { B. Relaxation } \\
\text { exercises on exercise } \\
\text { mat without music, } \\
40 \text { sessions over } 20 \\
\text { weeks ( } n=37 \text { ) }\end{array}$ & $\begin{array}{l}\text { A vs. B } \\
\text { Age: } 46 \text { vs. } 50 \\
\text { Female: } 72 \% \text { vs. } \\
65 \% \\
\text { EDSS: } 6.3 \text { vs. } 5.9 \\
\text { PPMS: } 17 \% \text { vs. } 24 \% \\
\text { SPMS: } 25 \% \text { vs. } 32 \%\end{array}$ & $\begin{array}{l}\text { A vs. B, median (SD), p-value=between groups: } \\
\text { BDI: } 14(7.72) \text { to } 5(3.2) \text { vs. } 15 \text { (8.68) to } 13 \\
\text { (5.91), p<0.05 } \\
\begin{array}{l}\text { Differences in depression scores were } \\
\text { maintained at } 24 \text { weeks ( } 4 \text { weeks } \\
\text { postintervention) but there was no difference } \\
\text { between groups at } 30 \text { weeks }\end{array}\end{array}$ \\
\hline $\begin{array}{l}\text { Dalgas, } 2009^{202} \\
\text { Dalgas, } 2010^{203} \\
\text { Strength } \\
\text { RCT } \\
\text { Fair }\end{array}$ & $\begin{array}{l}\text { A. Progressive } \\
\text { resistance, } 24 \\
\text { sessions over } 12 \\
\text { weeks ( } n=15) \\
\begin{array}{l}\text { B. Waitlist control } \\
(n=16)\end{array}\end{array}$ & $\begin{array}{l}\text { A vs. B } \\
\text { Age: } 45 \text { vs. } 48 \\
\text { Female: } 63 \% \text { vs. } \\
67 \% \\
\text { Ambulatory to } 100 \mathrm{~m}: \\
100 \% \\
\text { RRMS: } 100 \%\end{array}$ & $\begin{array}{l}\text { A vs. B, mean (SD), p=between groups: } \\
\text { MDI ( } 20 \text { to } 24, \text { mild depression; } 25 \text { to } 29, \\
\text { moderate depression; > } 29, \text { major depression): } \\
10.3,95 \% \mathrm{Cl} 7.0 \text { to } 13.5 \text { vs. } 8.8,95 \% \mathrm{Cl} 6.4 \text { to } \\
11.3 \text { (baseline) } \\
7.9,95 \% \mathrm{Cl} 5.2 \text { to } 10.6 \text { vs. } 9.9,95 \% \mathrm{Cl} 7.4 \text { to } \\
12.5 \text { (postintervention) } \\
\text { Mean change between group NR, } \mathrm{p}=0.01\end{array}$ \\
\hline $\begin{array}{l}\text { Gervasoni, } \\
2014^{117} \\
\text { Aerobic } \\
\text { Exercise } \\
\text { RCT } \\
\text { Fair }\end{array}$ & $\begin{array}{l}\text { A. } 30 \text { minutes } \\
\text { conventional therapy } \\
+15 \text { minutes } \\
\text { treadmill training, } 12 \\
\text { sessions over } 2 \\
\text { weeks ( } n=15) \\
\text { B. } 45 \text { minutes } \\
\text { conventional therapy, } \\
12 \text { sessions over } 2 \\
\text { weeks }(n=15)\end{array}$ & $\begin{array}{l}\text { A vs. B } \\
\text { Age: } 49.6 \text { vs. } 45.7 \\
\text { Female: } 40 \% \\
\text { Able to walk } 6 \text { meters } \\
\text { with or without assist } \\
\text { device } \\
\text { EDSS: } 5.25 \text { ( } 3.0 \text { to } \\
\text { 6.5) } \\
\text { RRMS: } 47.6 \% \\
\text { PPMS: } 19.0 \% \\
\text { SPMS: } 33.3 \%\end{array}$ & $\begin{array}{l}\text { Median change scores, } p=\text { between groups: } \\
\text { PANAS positive: } \\
1.0 \text { vs. } 5.0, p=0.86 \\
\frac{\text { PANAS negative }}{-5.0 \text { vs. }-2.0, p=0.48}\end{array}$ \\
\hline $\begin{array}{l}\text { Hebert, } 2011^{80} \\
\text { Aerobic } \\
\text { Exercise } \\
\text { RCT } \\
\text { Fair }\end{array}$ & $\begin{array}{l}\text { A. Bicycle } \\
\text { Ergometry, } 12 \\
\text { sessions for } 6 \text { weeks } \\
(n=12) \\
\text { B. Vestibular rehab } \\
(n=13) \\
\begin{array}{l}\text { C. Waitlist control } \\
(n=13)\end{array}\end{array}$ & $\begin{array}{l}\text { A vs. B vs. C } \\
\text { Age: } 46.8 \text { vs. } 42.6 \text { vs. } \\
50.2 \\
\text { Female: } 75 \% \text { vs. } \\
85 \% \text { vs. } 85 \% \\
\text { Ambulatory: } 100 \%\end{array}$ & $\begin{array}{l}\text { Mean difference between groups: } \\
\text { BDI-II: } \\
\text { A vs. B: } 4.4,95 \% \mathrm{Cl}-3.0 \text { to } 11.9, p=0.431 \\
\text { A vs. C: } 5.0,95 \% \mathrm{Cl}-2.5 \text { to } 12.4, p=0.307 \\
\text { B vs. C: } 0.6,95 \% \mathrm{Cl}-6.8 \text { to } 7.8, p=1.00\end{array}$ \\
\hline
\end{tabular}




\begin{tabular}{|c|c|c|c|}
\hline $\begin{array}{l}\text { Author, Year } \\
\text { Intervention } \\
\text { Study Design } \\
\text { Study Quality } \\
\end{array}$ & $\begin{array}{l}\text { Intervention } \\
\text { and Comparison }\end{array}$ & Population & Results \\
\hline $\begin{array}{l}\text { Kara, } 2017^{62} \\
\text { Strength } \\
\text { Quasiexperime } \\
\text { ntal } \\
\text { Poor }\end{array}$ & $\begin{array}{l}\text { A. Pilates, } 16 \\
\text { sessions over } 8 \\
\text { weeks }(n=27) \\
\\
\text { B. Multimodal } \\
\text { exercise (focus on } \\
\text { aerobic), } 16 \text { sessions } \\
\text { over } 8 \text { weeks }(n=28)\end{array}$ & $\begin{array}{l}\text { A vs. } B \\
\text { Age: } 50 \text { vs. } 43 \\
\text { Female: } 67 \% \text { vs. } \\
65 \% \\
\text { EDSS: } 2.85 \text { vs. } 3.2\end{array}$ & $\begin{array}{l}\text { A vs. B, mean (SD), } p=\text { between groups: } \\
\text { BDI: } \\
11.44(6.52) \text { vs. } 8.92(6.49), p=0.001 \text { (baseline) } \\
9.77(5.26) \text { vs. } 7.15(6.35), p=0.156 \\
\text { (postintervention) } \\
\text { Mean change between groups: } \\
0.1,95 \% \mathrm{Cl}-0.4 .53 \text { to } 4.73, p=0.97\end{array}$ \\
\hline $\begin{array}{l}\text { Keser, } 2011^{63} \\
\text { Aerobic } \\
\text { exercise } \\
\text { Quasiexperime } \\
\text { ntal } \\
\text { Poor }\end{array}$ & $\begin{array}{l}\text { A. Calisthenics, } 18 \\
\text { sessions over } 6 \\
\text { weeks ( } n=15) \\
\text { B. Routine neuro- } \\
\text { rehab (strength, } \\
\text { balance, } \\
\text { coordination, } \\
\text { spasticity exercises), } \\
18 \text { sessions over } 6 \\
\text { weeks ( } n=15)\end{array}$ & $\begin{array}{l}\text { A vs. B } \\
\text { Age: } 36 \text { vs. } 35 \\
\text { Female: } 53 \% \text { vs. } \\
47 \% \\
\text { EDSS: } 2.9 \text { vs. } 2.8\end{array}$ & $\begin{array}{l}\text { A vs. B, } p=\text { between groups: } \\
\text { HADS Anxiety: scores not provided } \\
\text { HADS Depression: scores not provided } \\
\text { Differences between groups were not } \\
\text { significant, } p>0.05\end{array}$ \\
\hline $\begin{array}{l}\text { Negaresh, } \\
2019^{53} \\
\text { Aerobic } \\
\text { exercise } \\
\text { RCT } \\
\text { Fair }\end{array}$ & $\begin{array}{l}\text { A. Normal BMI } \\
\text { cycling UE/LE, } 24 \\
\text { sessions over } 8 \\
\text { weeks ( } n=18) \\
\begin{array}{l}\text { B. Normal BMI } \\
\text { control ( } n=15) \\
\text { C. Overweight } \\
\text { cycling UE/LE, } 24 \\
\text { sessions over } 8 \\
\text { weeks ( } n=17) \\
\text { D. Overweight control } \\
(n=13)\end{array}\end{array}$ & $\begin{array}{l}\text { A vs. B vs. C vs. D } \\
\text { Age: } 31.2 \text { vs. } 29.1 \text { vs. } \\
32.1 \text { vs. } 2.1 \\
\text { Female: } 64 \% \text { vs. } \\
64 \% \text { vs. } 64 \% \text { vs. } \\
69 \% \\
\text { RRMS: } 100 \%\end{array}$ & $\begin{array}{l}\text { A vs. B vs. C vs. D } \\
\text { (scores are estimates from graph) } \\
\text { BDI change score from baseline: } \\
-4.8 \text { vs. } 0.1 \text { vs. }-2.5 \text { vs. }-0.1, p=0.005 \\
\text { Interaction between Weight and Exercise } \\
p=0.14\end{array}$ \\
\hline $\begin{array}{l}\text { Ozkul, } 2020 b^{232} \\
\text { Multimodal } \\
\text { exercise } \\
\text { RCT } \\
\text { Fair }\end{array}$ & $\begin{array}{l}\text { A. Aerobic exercise + } \\
\text { Pilates, } 24 \text { sessions } \\
\text { over } 8 \text { weeks }(n=17) \\
\text { B. Relaxation } \\
\text { exercises, } 24 \\
\text { sessions over } 8 \\
\text { weeks }(n=17)\end{array}$ & $\begin{array}{l}\text { A vs. B } \\
\text { Age: } 35.88 \text { vs. } 36.76 \\
\text { Female: } 76 \% \text { vs. } \\
76 \% \\
\text { RRMS: } 100 \% \\
\text { EDSS: } 1.50 \text { vs. } 1.71\end{array}$ & $\begin{array}{l}\text { A vs. } B \text {, mean change (SD), } p=\text { between groups } \\
\text { BDI: Mean change }-1.88(5.35) \text { vs. } 3.24 \text { (8.86), } \\
p=0.05\end{array}$ \\
\hline $\begin{array}{l}\text { Razazian, } \\
2016^{70} \\
\text { Aerobic/Postur } \\
\text { al control } \\
\text { RCT } \\
\text { Poor }\end{array}$ & $\begin{array}{l}\text { A. Aquatic Exercise, } \\
24 \text { sessions over } 8 \\
\text { weeks }(n=18) \\
\text { B. Yoga, } 24 \text { sessions } \\
\text { over } 8 \text { weeks }(n=18) \\
\text { C. Attention control } \\
(18)\end{array}$ & $\begin{array}{l}\text { A vs. B vs. C } \\
\text { Age: } 35.4 \text { vs. } 33.3 \text { vs. } \\
33.1 \\
\text { Female: } 100 \% \\
\text { EDSS: } 3.44 \text { vs. } 3.89 \\
\text { vs. } 3.25 \\
\text { RRMS: } 61 \% \text { vs. } 72 \% \\
\text { vs. } 67 \% \\
\text { SPMS: } 11 \% \text { vs. } 6 \% \\
\text { vs. } 11 \% \\
\text { Progressive- } \\
\text { relapsing MS: } 28 \% \\
\text { vs. } 22 \% \text { vs. } 22 \%\end{array}$ & $\begin{array}{l}\text { A vs. B vs. C, mean (SD), } p=\text { between groups: } \\
\text { BDI: } 19.17(7.83) \text { vs. } 19.72 \text { ( } 7.04) \text { vs. } 20.78 \\
\text { (6.22) (baseline) } \\
4.78(3.42) \text { vs. } 5.06(2.92) \text { vs. } 21.33(6.88) \\
\text { (postintervention) } \\
\text { Mean change in BDI between groups: } \\
\text { A vs. C } \\
\text { MD } 14.94,95 \% \mathrm{Cl} 10.57 \text { to } 19.31, p<0.001 \\
\text { B vs. C } \\
\text { MD } 15.21,95 \% \mathrm{Cl} 11.06 \text { to } 19.36, p<0.001 \\
\text { A vs. } B \\
\text { MD }-0.27,95 \% \mathrm{Cl}-4.50 \text { to } 3.96, p=0.90\end{array}$ \\
\hline
\end{tabular}




\begin{tabular}{|c|c|c|c|}
\hline $\begin{array}{l}\text { Author, Year } \\
\text { Intervention } \\
\text { Study Design } \\
\text { Study Quality }\end{array}$ & $\begin{array}{l}\text { Intervention } \\
\text { and Comparison }\end{array}$ & Population & Results \\
\hline $\begin{array}{l}\text { Roppolo, } \\
2013^{233} \\
\text { Multimodal } \\
\text { Quasiexperime } \\
\text { ntal } \\
\text { Fair }\end{array}$ & $\begin{array}{l}\text { A. Aerobic }+ \text { Strength } \\
\text { training, } 24 \text { sessions } \\
\text { over } 12 \text { weeks } \\
(n=17) \\
\text { B. Control group } \\
\text { (activity not } \\
\text { specified) }(n=18)\end{array}$ & $\begin{array}{l}\text { A vs. } \mathrm{B} \\
\text { Age: } 40 \text { vs. } 40 \\
\text { Female: } 100 \% \\
\text { EDSS: } 1.5 \text { vs. } 2.0\end{array}$ & $\begin{array}{l}\text { A vs. B, mean (SD), p=between groups } \\
\frac{\text { BDI: } 8.8(5.8) \text { to } 3.4(2.9) \text { vs. } 9.2(3.7) \text { to } 17.0}{(7.0), M D 13.2,95 \% \text { Cl } 9.86 \text { to } 16.55, p<0.001}\end{array}$ \\
\hline $\begin{array}{l}\text { Russo, } 2018^{95} \\
\text { Aerobic } \\
\text { exercise } \\
\text { RCT } \\
\text { Fair }\end{array}$ & $\begin{array}{l}\text { A. RAGT, } 18 \\
\text { sessions over } 6 \\
\text { weeks then } 36 \\
\text { sessions of rehab } \\
\text { exercises over } 12 \\
\text { weeks }(n=30) \\
\\
\text { B. Rehab exercises, } \\
54 \text { sessions over } 18 \\
\text { weeks }(n=15)\end{array}$ & $\begin{array}{l}\text { A vs. } B \\
\text { Age: } 42 \text { vs. } 41 \\
\text { Female: } 53 \% \text { vs. } \\
67 \%\end{array}$ & 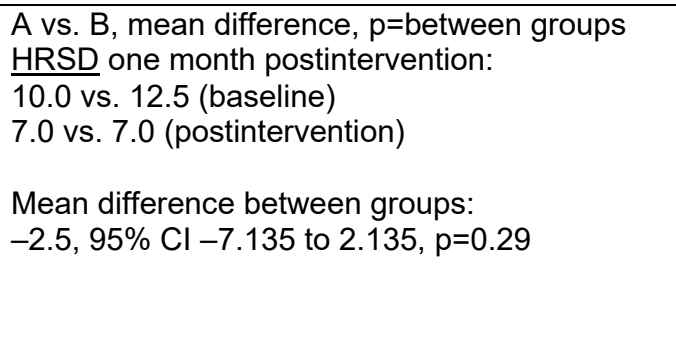 \\
\hline $\begin{array}{l}\text { Sadeghi } \\
\text { Bahmani, } \\
2019^{61} \\
\text { Aerobics/ } \\
\text { Postural } \\
\text { control } \\
\text { RCT } \\
\text { Fair }\end{array}$ & $\begin{array}{l}\text { A. Endurance } \\
\text { training (treadmill, } \\
\text { cycling, walking, } \\
\text { jogging), } 24 \text { sessions } \\
\text { over } 8 \text { weeks ( } n=26 \text { ) } \\
\text { B. Balance and } \\
\text { coordination } \\
\text { exercises, } 24 \\
\text { sessions over } 8 \\
\text { weeks ( } n=24) \\
\text { C. Attention control, } \\
24 \text { sessions over } 8 \\
\text { weeks }(n=21)\end{array}$ & $\begin{array}{l}\text { A vs. B vs. C } \\
\text { Age: } 38 \text { vs. } 39 \text { vs. } 38 \\
\text { Female: } 100 \% \\
\text { EDSS: } 2.46 \text { vs. } 3.38 \\
\text { vs. } 2.02\end{array}$ & 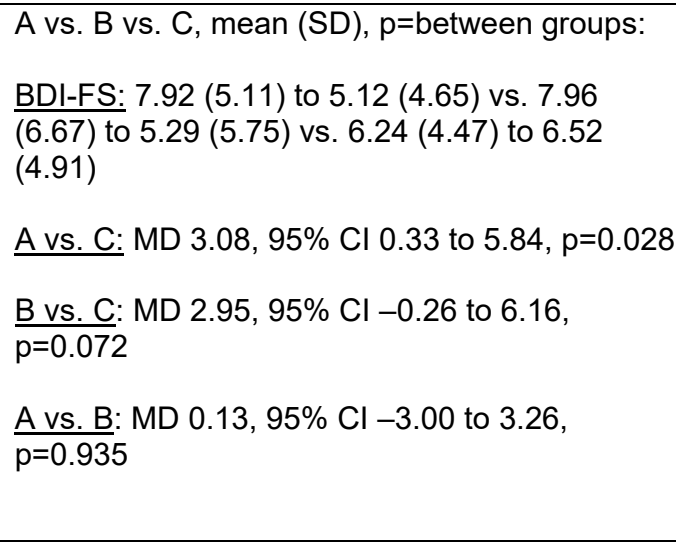 \\
\hline $\begin{array}{l}\text { Sadeghi } \\
\text { Bahmani, } 2020 \\
\text { Aerobic } \\
\text { exercise } \\
\text { RCT } \\
\text { Fair }\end{array}$ & $\begin{array}{l}\text { A. Aquatic exercise, } \\
16 \text { sessions over } 8 \\
\text { weeks }(n=20) \\
\\
\text { B. Aquatic exercise, } \\
24 \text { sessions over } 8 \\
\text { weeks }(n=18) \\
\text { C. Active control, } \\
\text { (social program) } 16 \\
\text { to } 24 \text { sessions over } 8 \\
\text { weeks }(n=22)\end{array}$ & $\begin{array}{l}\text { A vs. B vs. C } \\
\text { Age: } 39.35 \text { vs. } 40.61 \\
\text { vs. } 33.77 \\
\text { Female: } 100 \% \\
\text { EDSS (median): } 3.0 \\
\text { vs. } 1.5 \text { vs. } 1.5\end{array}$ & $\begin{array}{l}\text { Mean (SE) of change scores, } p=\text { between all } 3 \\
\text { groups } \\
\text { BDI-Fast Screen: } 4.80 \text { (5.90) vs. } 7.83 \text { (4.91) vs. } \\
6.05 \text { (4.49), } p<0.001\end{array}$ \\
\hline $\begin{array}{l}\text { Straudi, } 2016^{97} \\
\text { Aerobic } \\
\text { exercise } \\
\text { RCT } \\
\text { Good }\end{array}$ & $\begin{array}{l}\text { A. RAGT, } 12 \\
\text { sessions o)ver } 6 \\
\text { weeks }(n=27) \\
\text { B. Walking therapy } \\
\text { without RAGT, } 12 \\
\text { sessions over } 6 \\
\text { weeks }(n=25)\end{array}$ & $\begin{array}{l}\text { A vs. B } \\
\text { Age: } 52 \text { vs. } 54 \\
\text { Female: } 63 \% \text { vs. } \\
68 \% \\
\text { EDSS: } 6.43 \text { vs. } 6.46 \\
\text { PPMS: } 33 \% \text { vs. } 28 \% \\
\text { SPMS: } 67 \% \text { vs. } 72 \%\end{array}$ & $\begin{array}{l}\text { PHQ-9: } \\
\text { T2-T0: }-1.7(3.24) \text { vs. }-3.04 \text { (4.66), } p=0.213\end{array}$ \\
\hline
\end{tabular}




\begin{tabular}{|c|c|c|c|}
\hline $\begin{array}{l}\text { Author, Year } \\
\text { Intervention } \\
\text { Study Design } \\
\text { Study Quality }\end{array}$ & $\begin{array}{l}\text { Intervention } \\
\text { and Comparison }\end{array}$ & Population & Results \\
\hline $\begin{array}{l}\text { Straudi, } 2019^{98} \\
\text { Aerobic } \\
\text { exercise } \\
\text { RCT } \\
\text { Good }\end{array}$ & $\begin{array}{l}\text { A. RAGT, } 12 \\
\text { sessions over } 4 \\
\text { weeks }(n=36) \\
\text { B. Overground } \\
\text { walking, } 12 \text { sessions } \\
\text { over } 4 \text { weeks }(n=36)\end{array}$ & $\begin{array}{l}\text { A vs. B } \\
\text { Age: } 56 \text { vs. } 55 \\
\text { Female: } 67 \% \text { vs. } \\
69 \% \\
\text { EDSS: } 6.5 \text { vs. } 6.5 \\
\text { PPMS: } 50 \% \text { vs. } 45 \% \\
\text { SPMS: } 50 \% \text { vs. } 55 \%\end{array}$ & 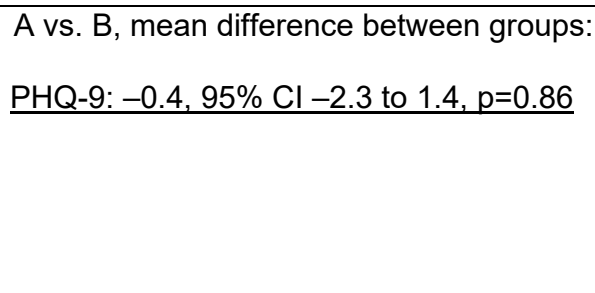 \\
\hline
\end{tabular}

Abbreviations: BDI = Beck Depression Inventory; BDI-FS = Beck Depression Inventory-Fast Screen; BMI = body mass index; CES-D = Center for Epidemiologic Studies Depression Scale; CI = confidence interval; CIS = Clinically Isolated Syndrome;

EDSS = Expanded Disability Status Scale; HADS = Hospital Anxiety and Depression Scale; HRSD = Hamilton Rating Scale for Depression; IDS16-SR = 16-item version of Inventory of Depressive Symptomatology Self-Rated; MD = mean difference; MDI = Major Depression Inventory; NR = not reported; PANAS = Positive and Negative Affect Schedule; PHQ-9=Patient Health Questionnaire-9; PPMS = primary progressive multiple sclerosis; RAGT = robot-assisted gait training; RCT = randomized controlled trial; RRMS = relapsing-remitting multiple sclerosis; $\mathrm{SD}=$ standard deviation; SE- standard error; SPMS = secondary progressive multiple sclerosis; $\mathrm{VR}=$ virtual reality

Results from head-to-head exercise interventions in MS RCTs not already discussed include cycling versus vestibular rehabilitation, ${ }^{79,80}$ treadmill versus yoga, ${ }^{120}$ and aquatic exercise versus yoga ${ }^{70}$ or versus relaxation exercises on a mat. ${ }^{70,71}$ In two trials all exercise arms demonstrated statistically significantly improved depression scores at end of treatment compared with baseline. One good-quality aquatics trial found improved depression scores at end of treatment versus the control group with the differences maintained at 10 weeks postintervention. ${ }^{70,71}$ However, there were no differences between cycling and vestibular rehabilitation or between aquatic exercise and yoga in change in depression scores with exercise. In one RCT, yoga was associated with improved depression and anxiety scores compared with control, but treadmill training was associated with greater improvement than yoga on anxiety scores. ${ }^{120}$

Two poor-quality quasiexperimental studies compared calisthenics with neuro-rehabilitation exercises $^{63}$ and Pilates with multimodal exercises. ${ }^{62}$ Scores on the HADS anxiety scale were improved with calisthenics and with neuro-rehabilitation exercises, but there were no differences between groups. There were also no differences in pre-post exercise changes on the BDI between Pilates and multimodal exercises (aerobic, strengthening, balance, and walking exercises).

\section{Spinal Cord Injury}

Three fair-quality RCTs $(n=171)$ examined the effects of physical exercise on mental health in primarily male participants with SCI, most in their 30 s and $40 \mathrm{~s}^{89,138,139,244,245}$ (Table 41). All three RCTs administered instruments to measure depression and two of the three trials administered instruments to assess anxiety. ${ }^{89,244,245}$ Exercise interventions included precision track walking, treadmill walking with body weight support as needed, arm cycling, general exercises, whole body strength plus aerobics, and upper body strength plus aerobics. No RCT utilized a waitlist or no exercise control. Exercise did not improve anxiety or depression scores from baseline in any of the trials, and there were also no differences on mental health measures between exercise groups within trials $(\mathrm{p}>0.05)$. 
Table 41. Effects of exercise on depression and anxiety in spinal cord injury

\begin{tabular}{|c|c|c|c|}
\hline $\begin{array}{l}\text { Author, Year } \\
\text { Intervention } \\
\text { Study Design } \\
\text { Study Quality }\end{array}$ & $\begin{array}{l}\text { Intervention } \\
\text { and Comparison }\end{array}$ & Population & Results \\
\hline $\begin{array}{l}\text { Akkurt, } 2017^{89} \\
\text { Aerobic exercise } \\
\text { RCT } \\
\text { Fair }\end{array}$ & $\begin{array}{l}\text { A. Arm ergometer, } \\
36 \text { sessions over } 12 \\
\text { weeks plus } 120 \\
\text { sessions general } \\
\text { exercises over } 12 \\
\text { weeks ( } n=17) \\
\text { B. General } \\
\text { exercises, } 120 \\
\text { sessions over } 12 \\
\text { weeks }(n=16)\end{array}$ & $\begin{array}{l}\text { A vs. B } \\
\text { Age: } 33 \text { vs. } 37 \\
\text { Female: } 5 \% \text { vs. } 19 \% \\
\text { Ambulatory: } 41 \% \text { vs. } \\
50 \% \\
\text { Wheelchair user: } \\
59 \% \text { vs. } 50 \% \\
\text { Paraplegia: } 100 \% \text { vs. } \\
94 \%\end{array}$ & $\begin{array}{l}\text { A vs. } B, \text { median: } \\
\text { HADS change scores: } \\
0 \text { vs. } 0.5, p>0.05 \\
\text { CES-D change scores: }-3 \text { vs. } 3, p>0.05\end{array}$ \\
\hline $\begin{array}{l}\text { Galea, } 2018^{245} \\
\text { Multimodal } \\
\text { exercise } \\
\text { RCT } \\
\text { Fair }\end{array}$ & $\begin{array}{l}\text { A. Whole body } \\
\text { strength }+ \text { aerobics, } \\
36 \text { sessions over } 12 \\
\text { weeks }(n=60) \\
\text { B. Upper body } \\
\text { strength + aerobics, } \\
36 \text { sessions over } 12 \\
\text { weeks }(n=56)\end{array}$ & $\begin{array}{l}\text { A vs. B } \\
\text { Age: } 40.1 \text { vs. } 42.8 \\
\text { Female: } 15 \% \\
\text { vs. } 16 \% \\
\text { ASIA A: } 48 \% \text { vs. } \\
50 \% \\
\text { ASIA B: } 15 \% \text { vs. } \\
\text { 14\% } \\
\text { ASIA C: } 12 \% \text { vs. } 9 \% \\
\text { ASIA D: } 25 \% \text { vs. } \\
27 \% \\
\text { C2-C8: } 48 \% \text { vs. } 59 \% \\
\text { T1-T6: } 30 \% \text { vs. } 23 \% \\
\text { T7-T12: } 22 \% \text { vs. } \\
18 \%\end{array}$ & $\begin{array}{l}\text { A vs. B, mean (SD), } p=\text { between groups: } \\
\text { HADS-Anxiety } \\
10.3(1.8) \text { vs. } 10.5(1.8) \text { (baseline) } \\
10.4(1.6) \text { vs. } 10.1(1.6) \text { (postintervention) } \\
\text { MD } 0.29,95 \% \mathrm{Cl}-0.25 \text { to } 0.83, p=0.291 \\
\text { (postintervention) } \\
\text { MD }-0.14,95 \% \mathrm{Cl}-0.89 \text { to } 0.60, p=0.701 \text { (24 } \\
\text { weeks-12 weeks postintervention) } \\
\text { HADS-Depression } \\
10.5(2) \text { vs. } 10.4(2.1) \text { (baseline) } \\
10(1.6) \text { vs. } 10.2(1.3) \text { (postintervention) } \\
\text { MD -0.28, } 95 \% \mathrm{Cl}-0.83 \text { to } 0.27, p=0.309 \\
\text { (postintervention) } \\
10.1(1.5) \text { vs. } 10.2(1.4) \\
\text { MD }-0.23(95 \% \mathrm{Cl}-0.81 \text { to } 0.35), p=0.428 \\
(24 \text { weeks-12 weeks postintervention) }\end{array}$ \\
\hline $\begin{array}{l}\text { Yang, 2014 } \\
\text { Aerobic Exercise } \\
\text { RCT (Crossover) } \\
\text { Fair }\end{array}$ & $\begin{array}{l}\text { A. Precision track } \\
\text { walking training, } 40 \\
\text { sessions over } 8 \\
\text { weeks ( } n=11) \\
\text { B. BWS (if needed) } \\
\text { treadmill walking, } 40 \\
\text { sessions over } 8 \\
\text { weeks ( } n=11)\end{array}$ & $\begin{array}{l}\text { A vs. B } \\
\text { Age: } 44 \text { vs. } 48 \\
\text { Female: } 30 \% \text { vs. } \\
30 \% \\
\text { Able to walk } \geq 5 \\
\text { meters with walking } \\
\text { aid or braces }\end{array}$ & $\begin{array}{l}\text { A vs. B } \\
\text { Pre-post change scores (estimated from } \\
\text { graph) } \\
\text { CES-D: }-2.7 \text { vs. }-2.4, p>0.05 \\
\text { Both groups achieved significant improvement } \\
\text { from baseline, } p<0.05\end{array}$ \\
\hline
\end{tabular}

Abbreviations: ASIA = American Spinal Injury Association Impairment Scale; BWS = body weight supported; CI = confidence interval; CES-D = Center for Epidemiologic Studies Depression Scale; HADS = Hospital Anxiety and Depression Scale; MD = mean difference; $\mathrm{RCT}=$ randomized controlled trial; $\mathrm{SD}=$ standard deviation

\section{Depression Across Interventions and Populations}

In order to determine if general physical exercise (e.g., RAGT, cycling, and strength training) has an effect on depression scores, we looked for RCTs that included a low-intensity usual care, waitlist control, or no intervention comparison arm in any included population. Eleven RCTs (9 fair quality ${ }^{53,61,72,77,80,83,95,120,203,232}$ and 2 poor quality ${ }^{70,225}$ ) in participants with MS reported data that could be combined in meta-analysis. Interventions included treadmill, cycling, motion gaming, interval exercises, RAGT, and progressive resistance exercises with and without aerobic plus balance exercises. Usual care consisted of waitlist controls, conventional rehabilitation, and continuation of previous activity level. One trial assessed postintervention depression at a 4week posttreatment followup, rather than immediately postintervention. ${ }^{95}$ Pooled analysis of these RCTs $(n=522)$ found physical exercise associated with greater improvement in depression 
scores compared with usual care (SMD $-0.29,95 \% \mathrm{CI}-0.50$ to $-0.03, \mathrm{I}^{2}=8 \%, \mathrm{p}=0.005$ ) (Figure 37). There were no differences between groups based on type of exercise intervention $(\mathrm{p}=0.94)$.

Figure 37. Effect of exercise versus usual care on depression scores in multiple sclerosis

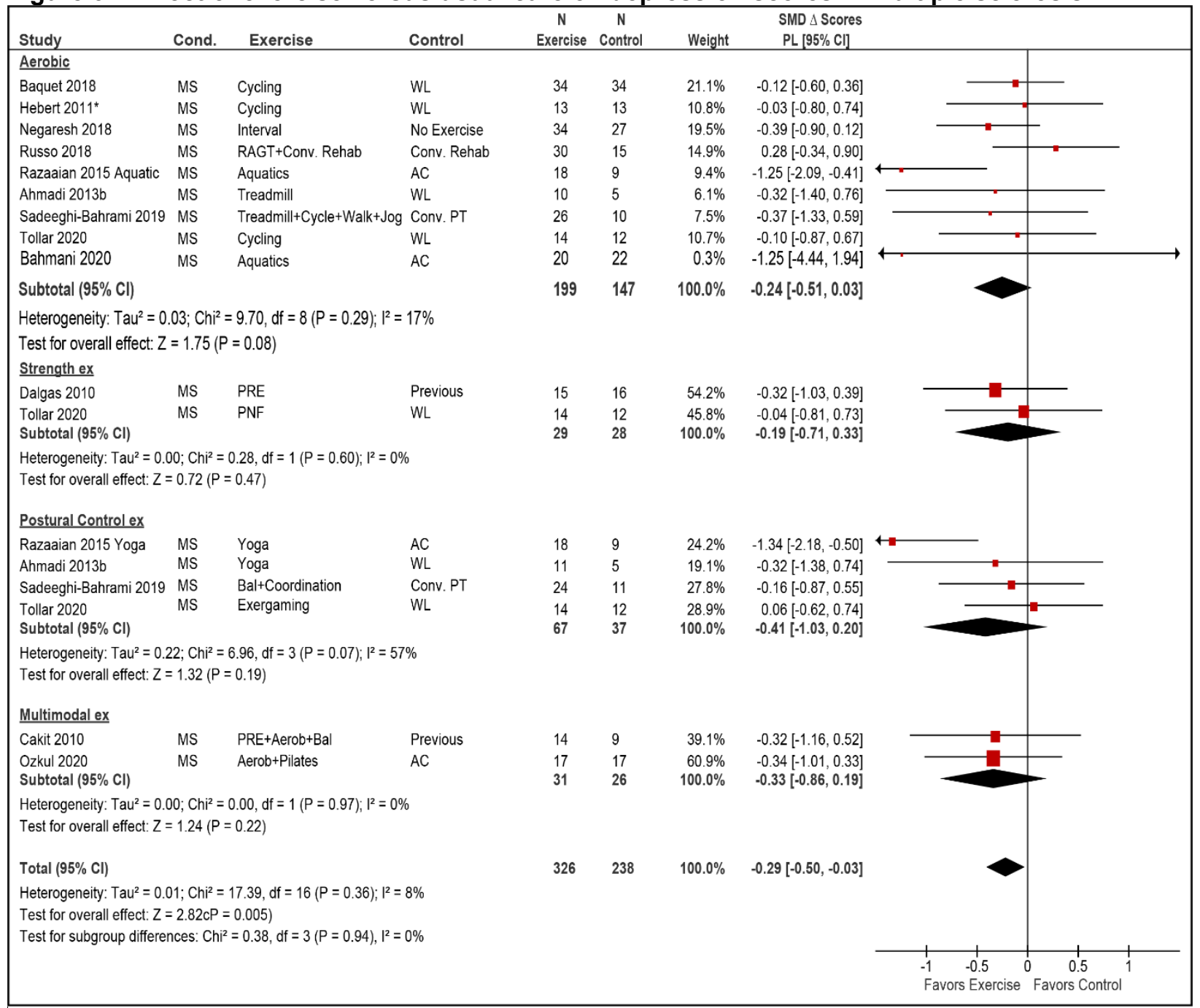

Abbreviations: $\Delta$ = change; $\mathrm{AC}=$ attention control; $\mathrm{Aerob}=$ aerobic exercise; $\mathrm{Bal}=$ balance training; $\mathrm{CI}=$ confidence interval; Cond. = condition; Conv. = conventional; ex = exercise; $\mathrm{MD}=$ mean difference; $\mathrm{MS}=$ multiple sclerosis; $\mathrm{PL}=$ profile likelihood; $\mathrm{PNF}=$ proprioceptive neuromuscular facilitation; $\mathrm{PRE}=$ progressive resistance exercise; Previous $=$ continuation of previous activities; $\mathrm{PT}=$ physical therapy; RAGT = robotic assisted gait training; $\mathrm{SMD}=$ standardized mean difference; $\mathrm{WL}=$ waitlist

These findings in trials comparing exercise with no exercise or usual care provided moderate-strength evidence of a benefit of exercise versus no exercise or versus low-intensity usual care on depression scores in adult participants with MS. There was much less evidence in SCI (3 trials, $\mathrm{n}=171)^{89,138,245}$ where there was no difference in depression with exercise, although data could not be pooled (SOE: low). No studies with depression outcomes that met inclusion criteria were identified in participants with $\mathrm{CP}$ (SOE: insufficient).

Only two studies, one in $\mathrm{MS}^{63}$ and one in $\mathrm{SCI},{ }^{244,245}$ reported results from instruments measuring anxiety, resulting in insufficient evidence from which to draw conclusions regarding the benefit of exercise on anxiety. 


\section{Sexual Function}

One fair-quality study randomized 62 women with MS to aquatic exercise two times weekly, three times weekly, or to an active control (social encounters at hospital) for 8 weeks. ${ }^{72}$ The Female Sexual Function Index contains 19 questions covering sexual desire, sexual arousal, lubrication, orgasm, sexual satisfaction, and pain. Aquatic exercises included warm up, walking, stretching, gymnastics, relay races, strength training, team competitions, and crossing the pool alone. Higher scores indicated greater sexual function. After controlling for baseline values, the highest scores were achieved in the group that exercised two times weekly (52.14) followed by three times weekly $(48.80)$, and active control $(42.80)(\mathrm{p}<0.001)$.

\section{KQ2b: Intermediate Outcomes}

Forty-two studies (36 RCTs, 5 quasiexperimental studies, and 1 cohort study) evaluated the effect of physical activity on intermediate outcomes. $53,63,75-78,82,88,89,91-93,117,125,132-$ 137,140,147,186,198,204,206-209,211-213,216-222,224,227,228,230,231,234-237,242,243,246,247,249 Eighteen studies enrolled participants with MS ( $\mathrm{n}=984),{ }^{53,63,76-78,117,147,186,198,204,206,220-222,224,227,228,230,231}$ (Table 42), 11 studies enrolled children with CP $(\mathrm{n}=401),{ }^{88,125,132,207-209,211-213,216,217,234-237,242,243}$ (Table 43), and 13 enrolled participants with SCI $(n=519)^{75,82,89,91-93,133-137,140,213,249}$ (Table 44).

Table 42. Intermediate outcomes of physical activity in participants with multiple sclerosis

\begin{tabular}{|c|c|c|c|}
\hline $\begin{array}{l}\text { Author, Year } \\
\text { Intervention } \\
\text { Study Design } \\
\text { Study Quality }\end{array}$ & $\begin{array}{l}\text { Intervention } \\
\text { and Comparison }\end{array}$ & $\begin{array}{l}\text { Population } \\
\text { (Multiple Sclerosis) }\end{array}$ & Results \\
\hline $\begin{array}{l}\text { Abbasi, } 2019^{186} \\
\text { Postural control } \\
\text { RCT } \\
\text { Fair }\end{array}$ & $\begin{array}{l}\text { A. Whole body } \\
\text { vibration, } 18 \\
\text { sessions over } 6 \\
\text { weeks }(n=22) \\
\text { B. No treatment, no } \\
\text { exercise }(n=24)\end{array}$ & $\begin{array}{l}\text { A vs. B } \\
\text { Age: } 37 \text { vs. } 39 \\
\text { Female: } 4.5 \% \text { vs. } 16.7 \% \\
\text { EDSS: } 1.54 \text { vs. } 1.55\end{array}$ & $\begin{array}{l}\text { A vs. } B, \text { median difference (interquartile } \\
\text { range), } p \text {-value is between groups } \\
\text { Strength: } \\
\text { Trunk Flexor: } 25.83 \text { ( } 8.83 \text { to } 46.41) \text { vs. }-0.33 \\
\text { (-5.67 to } 6.75), p<0.001 \\
\text { Trunk Extensor: } 38.17(20.75 \text { to } 70) \text { vs. }-1.49 \\
\text { (-11.83 to } 3.49), p<0.001\end{array}$ \\
\hline $\begin{array}{l}\text { Amiri, } 2019^{147} \\
\text { Postural control } \\
\text { RCT } \\
\text { Fair }\end{array}$ & $\begin{array}{l}\text { A. Core stability } \\
\text { training, } 30 \\
\text { sessions over } 10 \\
\text { weeks }(n=35) \\
\\
\text { B. Conventional } \\
\text { care including } \\
\text { stretching and } \\
\text { range of motion } \\
\text { exercises }(n=34)\end{array}$ & $\begin{array}{l}\text { A vs. B } \\
\text { Age: } 32 \text { vs. } 31 \\
\text { Female: } 100 \% \\
\text { EDSS: } 3.56 \text { vs. } 3.74\end{array}$ & $\begin{array}{l}\text { Core strength tests (R/L hip abduction, R/L } \\
\text { external rotation) demonstrate significant } \\
\text { differences in strength based on baseline } \\
\text { EDSS score (2.5-3.5; } 3.5-4.5 ; 4.5-5.5) \text {, } \\
p<0.001 \\
\text { Plank test: significant differences between } \\
\text { groups based on EDSS score, } p<0.001 \\
\text { Overall static balance tests demonstrate } \\
\text { significant differences in strength based on } \\
\text { baseline EDSS score and significant } \\
\text { differences compared with the control group, } \\
\text { p<0.001 } \\
\text { Greatest improvements seen in those with } \\
\text { greatest disability (least strong) }\end{array}$ \\
\hline
\end{tabular}




\begin{tabular}{|c|c|c|c|}
\hline $\begin{array}{l}\text { Author, Year } \\
\text { Intervention } \\
\text { Study Design } \\
\text { Study Quality }\end{array}$ & $\begin{array}{l}\text { Intervention } \\
\text { and Comparison }\end{array}$ & $\begin{array}{l}\text { Population } \\
\text { (Multiple Sclerosis) }\end{array}$ & Results \\
\hline $\begin{array}{l}\text { Baquet, } 2018^{77} \\
\text { Aerobic exercise } \\
\text { RCT } \\
\text { Fair }\end{array}$ & $\begin{array}{l}\text { A. Bicycle } \\
\text { ergometry, 24-36 } \\
\text { sessions over } 12 \\
\text { weeks ( } n=34) \\
\text { B. Waitlist control } \\
\text { group }(n=34)\end{array}$ & $\begin{array}{l}\text { A vs. B } \\
\text { Age: } 38.2 \text { vs. } 39.6 \\
\text { Female: } 62 \% \text { vs. } 74 \% \\
\text { EDSS: } 1.7 \text { vs. } 1.8 \\
\text { RRMS: } 100 \%\end{array}$ & $\begin{array}{l}\text { A vs. } B \text {, mean difference between groups: } \\
\frac{\mathrm{VO}_{2} \text { peak }(\mathrm{ml} / \mathrm{min})}{62.5, p=0.37}-51.4,95 \% \mathrm{Cl}-165.2 \text { to } \\
\frac{\mathrm{VO}_{2} \text { peak }(\mathrm{ml} / \mathrm{min} / \mathrm{kg})}{0.6, p=0.24}-0.9,95 \% \mathrm{Cl}-2.5 \text { to }\end{array}$ \\
\hline $\begin{array}{l}\text { Bulguroglu, } 2017^{206} \\
\text { Strength } \\
\text { RCT } \\
\text { Poor }\end{array}$ & \begin{tabular}{|l|} 
A. Mat Pilates, 16 \\
sessions over 8 \\
weeks $(n=12)$ \\
\\
B. Reformer Pilates, \\
16 sessions over 8 \\
weeks $(n=13)$ \\
\\
C. Attention control, \\
16 sessions over 8 \\
weeks $(n=13)$
\end{tabular} & $\begin{array}{l}\text { A vs. B vs. C } \\
\text { Age: } 45 \text { vs. } 37 \text { vs. } 40 \\
\text { Ambulatory: } 100 \% \\
\text { EDSS: } 1.8 \text { vs. } 2.0 \text { vs. } \\
1.0\end{array}$ & $\begin{array}{l}\text { A vs. B. vs. C, median: } \\
\text { Modified pushup (repetitions/ } 30 \text { seconds): } \\
6.5 \text { to } 10 \text { vs. } 3 \text { to } 10 \text { vs. } 7 \text { to } 7 \\
\text { Modified sit-up (repetitions/ } 30 \text { seconds): } \\
6 \text { to } 7.5 \text { vs. } 10 \text { to } 15 \text { vs. } 4 \text { to } 8 \\
\text { Trunk flexor test (seconds): } \\
2.32 \text { to } 6 \text { vs. } 4.91 \text { to } 13.3 \text { vs. } 6.46 \text { to } 6.4 \\
\text { Prone bridge (seconds): } \\
18.29 \text { to } 25.23 \text { vs. } 22.31 \text { to } 37.53 \text { vs. } 20.68 \text { to } \\
21.21 \\
\text { Pilates groups improved significantly over } \\
\text { baseline while the control group did not. }\end{array}$ \\
\hline $\begin{array}{l}\text { Collett, } 2011^{82} \\
\text { Aerobic exercise } \\
\text { RCT } \\
\text { Poor }\end{array}$ & \begin{tabular}{|l|} 
A. Combined \\
intermittent and \\
continuous static \\
cycling, 24 sessions \\
over 12 weeks \\
(n=20) \\
B. Intermittent static \\
cycling, 24 sessions \\
over 12 weeks \\
(n=21) \\
C. Continuous static \\
cycling, 24 sessions \\
over 12 weeks \\
(n=20)
\end{tabular} & $\begin{array}{l}\text { A vs. B vs. C } \\
\text { Age: } 55 \text { vs. } 50 \text { vs. } 52 \\
\text { Female: } 53 \% \text { vs. } 78 \% \\
\text { vs. } 80 \% \\
\text { Ambulatory: } 100 \%\end{array}$ & $\begin{array}{l}\text { Change postintervention: no data provided } \\
\text { Leg Power: NS }\end{array}$ \\
\hline $\begin{array}{l}\text { Dodd, } 2011^{204} \\
\text { Strength } \\
\text { RCT } \\
\text { Good }\end{array}$ & $\begin{array}{l}\text { A. Progressive } \\
\text { resistance: } 20 \\
\text { sessions over } 10 \\
\text { weeks }(n=39) \\
\text { B. Social program } \\
\text { (attention control), } \\
10 \text { sessions } x 10 \\
\text { weeks plus usual } \\
\text { care }(n=37)\end{array}$ & $\begin{array}{l}\text { A vs. B } \\
\text { Age: } 47.7 \text { vs. } 50.4 \\
\text { Female: } 72 \% \text { vs. } 74 \% \\
\text { Ambulation index } \\
2 \text { (mild): } 47 \% \text { vs. } 54 \% \\
3 \text { (moderate): } 39 \% \text { vs. } \\
26 \% \\
4 \text { (severe): } 14 \% \text { vs. } 20 \% \\
\text { Gait aid (yes): } 33 \% \text { vs. } \\
37 \%\end{array}$ & $\begin{array}{l}\text { A vs. B, mean difference between groups: } \\
\text { Max leg press (kg): MD } 10.8,95 \% \mathrm{Cl} 4.9 \text { to } \\
16.7, p<0.05 \\
\frac{\text { Reverse leg press }(\mathrm{kg}):}{9.5, p<0.05}\end{array}$ \\
\hline $\begin{array}{l}\text { Duff, } 2018^{198} \\
\text { Strength } \\
\text { RCT } \\
\text { Fair }\end{array}$ & $\begin{array}{l}\text { A. Pilates }+ \\
\text { massage, } 24 \\
\text { sessions of Pilates } \\
\text { and } 12 \text { massages } \\
\text { over } 12 \text { weeks } \\
(n=15) \\
\\
\text { B. Massage, } 12 \\
\text { massages over } 12 \\
\text { weeks }(n=15)\end{array}$ & \begin{tabular}{|l|} 
A vs. B \\
Age: 45.7 vs. 45.1 \\
Female: $80 \%$ vs. $73 \%$ \\
Ambulatory: $100 \%$ \\
Wheelchair user: $0 \%$ \\
RRMS: $93 \%$ vs. $73 \%$ \\
SPMS: $0 \%$ vs. $13 \%$ \\
PPMS: $7 \%$ vs. $13 \%$
\end{tabular} & $\begin{array}{l}\text { A vs. } \mathrm{B}, \text { mean change: } \\
\% \text { body fat: }-0.2,95 \% \mathrm{Cl}-1.4 \text { to } 1.0 \text { vs. }-0.8 \text {, } \\
95 \% \mathrm{Cl}-2.0 \text { to } 0.4, p=0.51\end{array}$ \\
\hline
\end{tabular}




\begin{tabular}{|c|c|c|c|}
\hline $\begin{array}{l}\text { Author, Year } \\
\text { Intervention } \\
\text { Study Design } \\
\text { Study Quality }\end{array}$ & $\begin{array}{l}\text { Intervention } \\
\text { and Comparison }\end{array}$ & $\begin{array}{l}\text { Population } \\
\text { (Multiple Sclerosis) }\end{array}$ & Results \\
\hline $\begin{array}{l}\text { Ebrahimi, } 2015^{228} \\
\text { Multimodal exercise } \\
\text { RCT } \\
\text { Poor }\end{array}$ & $\begin{array}{l}\text { A. Whole body } \\
\text { vibration + low- } \\
\text { intensity exercise, } \\
30 \text { sessions over } 10 \\
\text { weeks }(n=17) \\
\begin{array}{l}\text { B. Usual care } \\
(n=17)\end{array}\end{array}$ & $\begin{array}{l}\text { A vs. B } \\
\text { Age: } 37.06 \text { vs. } 40.75 \\
\text { Female: } 69 \% \text { vs. } 86 \% \\
\text { Ambulatory: } 100 \% \\
\text { EDSS: } 3.12 \text { vs. } 3.10\end{array}$ & $\begin{array}{l}\text { A vs. B, mean (SD) } \\
\text { Modified pushup: } \\
5.31(4.75) \text { vs. } 2.42(3.99) \text { (baseline) } \\
12.12(6.54) \text { vs. } 2.92(3.83) \text { (postintervention) } \\
\text { Time X Group } p=0.07\end{array}$ \\
\hline $\begin{array}{l}\text { Faramarzi, } 2020^{230} \\
\text { Banitalebi, 2020231 } \\
\text { Multimodal exercise } \\
\text { RCT } \\
\text { Fair }\end{array}$ & $\begin{array}{l}\text { A. Resistance + } \\
\text { cycling or running }+ \\
\text { balance exercises }+ \\
\text { Pilates + stretching, } \\
36 \text { sessions over } 12 \\
\text { weeks }(n=46) \\
\text { B. Waitlist control } \\
(n=43)\end{array}$ & $\begin{array}{l}\text { A vs. B } \\
\text { Age criteria: (18 to } 50) \\
\text { Female: } 100 \% \\
\text { EDSS } 0 \text { to } 4: 48 \% \text { to } \\
48 \% \\
\text { EDSS } 4.5 \text { to } 6: 27 \% \text { vs. } \\
27 \% \\
\text { EDSS } 6.5 \text { to } 8: 23 \% \text { vs. } \\
23 \%\end{array}$ & 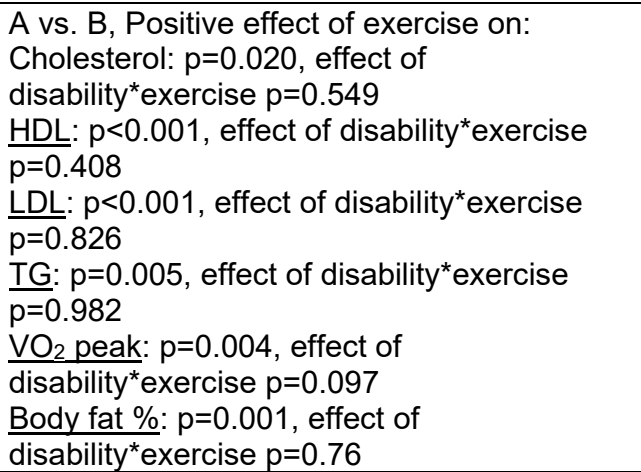 \\
\hline $\begin{array}{l}\text { Gervasoni, } 2014^{117} \\
\text { Aerobic } \\
\text { Exercise } \\
\text { RCT } \\
\text { Fair }\end{array}$ & $\begin{array}{l}\text { A. } 30 \text { minutes } \\
\text { conventional } \\
\text { therapy }+15 \\
\text { minutes treadmill } \\
\text { training, } 12 \\
\text { sessions over } 2 \\
\text { weeks ( } n=15) \\
\text { B. } 45 \text { minutes } \\
\text { conventional } \\
\text { therapy, } 12 \\
\text { sessions over } 2 \\
\text { weeks }(n=15)\end{array}$ & $\begin{array}{l}\text { A vs. B } \\
\text { Age: } 49.6 \text { vs. } 45.7 \\
\text { Female: } 40 \% \\
\text { Able to walk } 6 \text { meters } \\
\text { with or without assist } \\
\text { device } \\
\text { RRMS: } 47.6 \% \\
\text { PPMS: } 19.0 \% \\
\text { SPMS: } 33.3 \%\end{array}$ & $\begin{array}{l}\text { A vs. } B \text {, mean difference between groups: } \\
\frac{\text { Resting HR: }}{p=0.40} 3.76,95 \% \mathrm{Cl}-4.92 \text { to } 12.44 \text {, }\end{array}$ \\
\hline $\begin{array}{l}\text { Heine, } 2017^{78} \\
\text { Aerobic exercise } \\
\text { RCT } \\
\text { Fair }\end{array}$ & $\begin{array}{l}\text { A. Leg cycling, } 48 \\
\text { sessions over } 16 \\
\text { weeks ( } n=43) \\
\\
\text { B. MS nurse } \\
\text { consultation, } 3 \\
\text { consultations over } \\
16 \text { weeks }(n=46)\end{array}$ & $\begin{array}{l}\text { A vs. B } \\
\text { Age: } 43.1 \text { vs. } 48.2 \\
\text { Female: } 74 \% \text { vs. } 72 \% \\
\text { Ambulatory: } 100 \% \\
\text { EDSS: } 2.5 \text { vs. } 3.0 \\
\text { RRMS: } 72 \% \text { vs. } 74 \% \\
\text { SPMS: } 7 \% \text { vs. } 11 \% \\
\text { PPMS: } 21 \% \text { vs. } 15 \%\end{array}$ & $\begin{array}{l}\text { A vs. } B, \text { mean }(\mathrm{SD}), \mathrm{p}=\text { between groups } \\
\mathrm{VO}_{2} \text { peak }(\mathrm{L} / \mathrm{min}): \mathrm{MD} 0.048(0.082), \mathrm{p}=0.561 \\
\mathrm{VO}_{2} \text { peak }(\mathrm{mL} / \mathrm{kg} / \mathrm{min}): \mathrm{MD} 0.979(1.075),\end{array}$ \\
\hline
\end{tabular}




\begin{tabular}{|c|c|c|c|}
\hline $\begin{array}{l}\text { Author, Year } \\
\text { Intervention } \\
\text { Study Design } \\
\text { Study Quality }\end{array}$ & $\begin{array}{l}\text { Intervention } \\
\text { and Comparison }\end{array}$ & $\begin{array}{l}\text { Population } \\
\text { (Multiple Sclerosis) }\end{array}$ & Results \\
\hline $\begin{array}{l}\text { Kerling, } 2015^{220} \\
\text { Multimodal exercise } \\
\text { RCT } \\
\text { Fair }\end{array}$ & $\begin{array}{l}\text { A. Full body } \\
\text { progressive } \\
\text { resistance }+ \\
\text { aerobic training, } 36 \\
\text { sessions over } 12 \\
\text { weeks }(n=30) \\
\text { B. Aerobic training, } \\
36 \text { sessions over } 12 \\
\text { weeks }(n=30)\end{array}$ & $\begin{array}{l}\text { A vs. B } \\
\text { Age: } 42.3 \text { vs. } 45.6 \\
\text { Female: } 80 \% \text { vs. } 67 \% \\
\text { EDSS: } 2.6 \text { vs. } 3.1\end{array}$ & 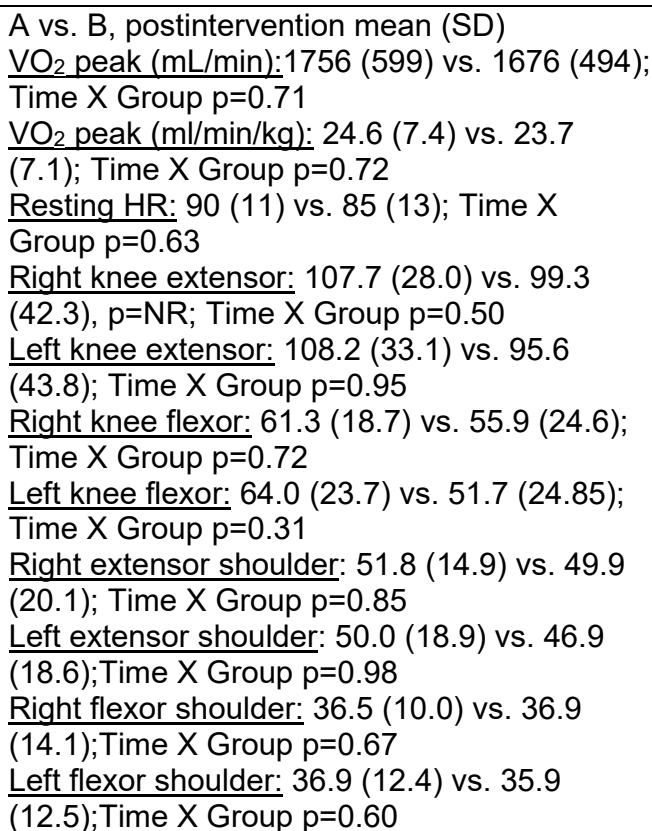 \\
\hline $\begin{array}{l}\text { Keser, } 2011^{63} \\
\text { Aerobic exercise } \\
\text { Quasiexperimental } \\
\text { Poor }\end{array}$ & $\begin{array}{l}\text { A. Calisthenics, } 18 \\
\text { sessions over } 6 \\
\text { weeks (15) } \\
\text { B. Neuro- } \\
\text { rehabilitation } 18 \\
\text { sessions over } 6 \\
\text { weeks (15) }\end{array}$ & $\begin{array}{l}\text { A vs. B } \\
\text { Age: } 36 \text { vs. } 35 \\
\text { Female: } 53 \% \text { vs. } 47 \% \\
\text { EDSS: } 2.9 \text { vs. } 2.8\end{array}$ & $\begin{array}{l}\text { A vs. } B, \text { mean change, } p=\text { between groups: } \\
\text { UE Right Strength: } 8.67 \text { (10.17) vs. } 15.19 \\
\text { (7.77), } p<0.05 \\
\text { UE Left Strength: } 7.86 \text { (11.97) vs. } 16.25 \\
\text { (10.95), p<0.05 } \\
\frac{\text { LE Right Strength: } 15.76(11.17) \text { vs. } 20.66}{(6.18), p>0.05} \\
\frac{\text { LE Left Strength: }}{(16.69), p>0.05}\end{array}$ \\
\hline $\begin{array}{l}\text { Negaresh, } 2019^{53} \\
\text { Aerobic exercise } \\
\text { RCT } \\
\text { Fair }\end{array}$ & $\begin{array}{l}\text { A. Normal BMI } \\
\text { cycling UE/LE, } 24 \\
\text { sessions over } 8 \\
\text { weeks ( } n=18 \text { ) } \\
\text { B. Normal BMI } \\
\text { control ( } n=15) \\
\text { C. Overweight } \\
\text { cycling UE/LE, } 24 \\
\text { sessions over } 8 \\
\text { weeks ( } n=17 \text { ) } \\
\text { D. Overweight } \\
\text { control ( } n=13 \text { ) }\end{array}$ & $\begin{array}{l}\text { A vs. B vs. C vs. D } \\
\text { Age: } 31.2 \text { vs. } 29.1 \text { vs. } \\
32.1 \text { vs. } 2.1 \\
\text { Female: } 64 \% \text { vs. } 64 \% \\
\text { vs. } 64 \% \text { vs. } 69 \% \\
\text { RRMS: } 100 \%\end{array}$ & $\begin{array}{l}\text { A vs. } B \text { vs. } C \text { vs. } D \text {, mean difference between } \\
\text { groups (scores are estimates from graph) } \\
\frac{\mathrm{VO}_{2} \text { peak: } 2.7 \text { vs. } 0 \text { vs. } 1.9 \text { vs. } 0.6, p=0.001}{\text { Interaction, } p=0.17} \\
\frac{\text { BMl: }}{-0.10} \text { vs. }-0.15 \text { vs. }-0.45 \text { vs. }-0.20, p=0.53 \\
\text { Interaction } p=0.38\end{array}$ \\
\hline $\begin{array}{l}\text { Sandroff, } 2017^{221} \\
\text { Multimodal exercise } \\
\text { RCT } \\
\text { Fair }\end{array}$ & $\begin{array}{l}\text { A. Resistance }+ \\
\text { aerobics }+ \text { balance: } \\
72 \text { sessions over } 24 \\
\text { weeks. ( } n=43) \\
\\
\text { B. Stretching and } \\
\text { toning, } 72 \text { sessions } \\
\text { over } 24 \text { weeks } \\
(n=40)\end{array}$ & $\begin{array}{l}\text { A vs. B } \\
\text { Age: } 49.8 \text { vs. } 51.2 \\
\text { Female: } 83.7 \% \text { vs. } \\
87.5 \% \\
\text { EDSS } 4-6: 100 \% \\
\text { Walking difficulties: } \\
100 \%\end{array}$ & $\begin{array}{l}\text { A vs. } \mathrm{B}, \text { mean (SD) } \\
\mathrm{VO}_{2} \text { Peak (ml/kg/min): } \\
16.5(6.5) \text { vs. } 15.4(6.2), p=N R \text { (baseline) } \\
17.1(5.9) \text { vs. } 15.9(5.5), p=N R \\
\text { (postintervention) } \\
\text { Time } X \text { Group interaction } p>0.20\end{array}$ \\
\hline
\end{tabular}




\begin{tabular}{|c|c|c|c|}
\hline $\begin{array}{l}\text { Author, Year } \\
\text { Intervention } \\
\text { Study Design } \\
\text { Study Quality }\end{array}$ & $\begin{array}{l}\text { Intervention } \\
\text { and Comparison }\end{array}$ & $\begin{array}{l}\text { Population } \\
\text { (Multiple Sclerosis) }\end{array}$ & Results \\
\hline $\begin{array}{l}\text { Sangelaji, } 2016^{222} \\
\text { Multimodal exercise } \\
\text { RCT } \\
\text { Fair }\end{array}$ & $\begin{array}{l}\text { A. } 1 \text { aerobic }+3 \\
\text { resistance training, } \\
32 \text { sessions over } 8 \\
\text { weeks }(n=10) \\
\text { B. } 2 \text { aerobic }+2 \\
\text { resistance training, } \\
32 \text { sessions over } 8 \\
\text { weeks ( } n=10) \\
\text { C. } 3 \text { aerobic }+1 \\
\text { resistance training, } \\
32 \text { sessions over } 8 \\
\text { weeks ( } n=10) \\
\text { D. No intervention } \\
\text { control }(n=10)\end{array}$ & $\begin{array}{l}\text { A vs. B vs. C vs. D } \\
\text { Age: } 36 \text { vs. } 31 \text { vs. } 34 \text { vs. } \\
34 \\
\text { Female: } 60 \% \text { vs. } 60 \% \\
\text { vs. } 60 \text { vs. } 60 \% \\
\text { Baseline EDSS: } 1.33 \text { vs. } \\
2.06 \text { vs. } 1.95 \text { vs. } 1.81\end{array}$ & $\begin{array}{l}\text { Mean difference (SE) vs. control group }(\mathrm{Kg}) \text { : } \\
\text { Left Knee flexion: } \\
\text { A. }-5.57(2.09), p=0.01 \\
\text { B. }-3.17(2.14), p=0.15 \\
\text { C. }-5.54(2.04), p=0.01 \\
\text { Right Knee flexion: } \\
\text { A. }-4.61(1.89), p=0.02 \\
\text { B. }-5.08(1.94), p=0.04 \\
\text { C. }-4.05,1.85, p=0.01 \\
\text { Left Knee Extension: } \\
\text { A. }-7.77(2.73, p=0.01 \\
\text { B. }-5.08 \text { (2.80), } p=0.08 \\
\text { C. }-7.95(2.68, p=0.01 \\
\text { Right Knee Extension: } \\
\text { A. }-4.88 \text { (3.48), } p=0.17 \\
\text { B. }-1.62(3.56), p=0.65 \\
\text { C. }-6.30 \text { (3.41), } p=0.07\end{array}$ \\
\hline $\begin{array}{l}\text { Wens, 2015b } \\
\text { (high intensity) } \\
\text { Multimodal exercise } \\
\text { RCT } \\
\text { Fair }\end{array}$ & $\begin{array}{l}\text { A. Resistance } \\
\text { training + high- } \\
\text { intensity interval } \\
\text { training, } 30 \\
\text { sessions over } 12 \\
\text { weeks ( } n=12 \text { ) } \\
\text { B. Resistance } \\
\text { training + high- } \\
\text { intensity continuous } \\
\text { cardiovascular } \\
\text { training, } 30 \\
\text { sessions over } 12 \\
\text { weeks (n=11) } \\
\text { C. No intervention - } \\
\text { "sedentary control" } \\
\text { ( } n=11 \text { ) }\end{array}$ & $\begin{array}{l}\text { A vs. B vs. C } \\
\text { Age: } 43 \text { vs. } 47 \text { vs. } 47 \\
\text { Female: } 42 \% \text { vs. } 45 \% \\
\text { vs. } 82 \% \\
\text { EDSS: } 2.3 \text { vs. } 2.7 \text { vs. } \\
2.5 \\
\text { RRMS: } 83 \% \text { vs. } 73 \% \text { vs. } \\
73 \% \\
\text { CPMS: } 17 \% \text { vs. } 27 \% \text { vs. } \\
27 \%\end{array}$ & 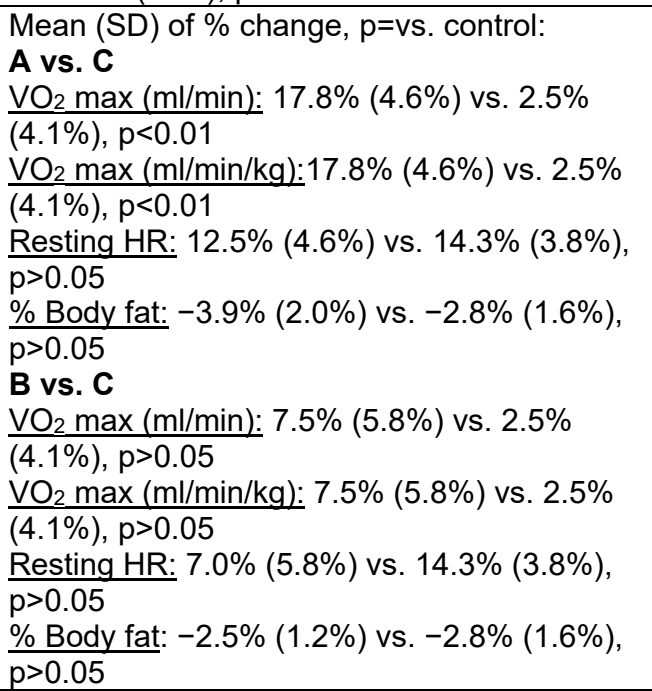 \\
\hline $\begin{array}{l}\text { Wens, 2015a } 227 \\
\text { (impact of 24) } \\
\text { Multimodal exercise } \\
\text { RCT } \\
\text { Poor }\end{array}$ & $\begin{array}{l}\text { A. Progressive } \\
\text { resistance }+ \\
\text { aerobics, } 60 \\
\text { sessions over } 24 \\
\text { weeks }(n=29) \\
\text { B. Nonexercise } \\
\text { control }(n=15)\end{array}$ & $\begin{array}{l}\text { A vs. } B \\
\text { Age: } 48 \text { vs. } 49 \\
\text { Female: } 59 \% \text { vs. } 53 \% \\
\text { EDSS: } 3.25 \text { vs. } 3.36\end{array}$ & $\begin{array}{l}\text { A vs. B, mean difference between groups: } \\
\text { Resting HR: } 9.0,95 \% \mathrm{Cl} 6.57 \text { to } 11.43, \\
p<0.001 \\
\text { Body weight }(\mathrm{kg}): 1.9,95 \% \mathrm{Cl}-0.124 \text { to } 0.07 \\
\text { Body fat \%: } 2.0,95 \% \mathrm{Cl} 0.67 \text { to } 3.33, \mathrm{p}=0.003 \\
\text { No differences in glucose and insulin } \\
\text { Knee extension and flexion improved with } \\
\text { exercise. Group X Time interaction } \mathrm{p}<0.05\end{array}$ \\
\hline
\end{tabular}

Abbreviations: $\mathrm{BMI}=$ body mass index; $\mathrm{CI}=$ confidence interval; CPMS = chronic progressive multiple sclerosis; EDSS = Expanded Disability Status Scale; HR = heart rate; LE = lower extremity; MD = mean difference; NR = not reported; NS = not significant; PPMS = primary progressive multiple sclerosis; RCT = randomized controlled trial; RRMS = relapsing-remitting multiple sclerosis; $\mathrm{SD}=$ standard deviation; $\mathrm{SPMS}=$ secondary progressive multiple sclerosis; $\mathrm{UE}=$ upper extremity; $\mathrm{VO}_{2} \mathrm{max}=$ maximal oxygen uptake 
Table 43. Intermediate outcomes of physical activity in participants with cerebral palsy

\begin{tabular}{|c|c|c|c|}
\hline $\begin{array}{l}\text { Author, Year } \\
\text { Intervention } \\
\text { Study Design } \\
\text { Study Quality }\end{array}$ & $\begin{array}{l}\text { Intervention } \\
\text { and } \\
\text { Comparison } \\
\end{array}$ & $\begin{array}{l}\text { Population } \\
\text { (Cerebral Palsy) }\end{array}$ & Results \\
\hline $\begin{array}{l}\text { Cho, } 2020^{216} \\
\text { Strength } \\
\text { RCT } \\
\text { Poor }\end{array}$ & $\begin{array}{l}\text { A. Progressive } \\
\text { resistance, } 18 \\
\text { sessions over } \\
6 \text { weeks }(n=13) \\
\text { B. } \\
\text { Conventional } \\
\text { therapy, } 18 \\
\text { sessions over } \\
6 \text { weeks }(n=12)\end{array}$ & $\begin{array}{l}\text { A vs. B } \\
\text { Age: } 5.54 \text { vs. } 7.17 \\
\text { Female: } 69 \% \text { vs. } 33 \% \\
\text { GMFCS: } 2.08 \text { vs. } 2.33 \\
\text { GMFM: } 69.98 \text { vs. } 68.15\end{array}$ & $\begin{array}{l}\text { A vs. } B, \text { mean }(S D) \text { of change, } p=\text { between } \\
\text { groups } \\
\text { Knee extensor strength: } \\
\frac{\text { Nondominant: }-2.196(0.048) \text { vs. }-2.078}{(0.062), p=0.436} \\
\frac{\text { Dominant: }-3.065(0.010) \text { vs. }-590(0.567),}{p=0.029}\end{array}$ \\
\hline $\begin{array}{l}\text { Johnston, } 2011^{125} \\
\text { Aerobic exercise } \\
\text { RCT } \\
\text { Fair }\end{array}$ & $\begin{array}{l}\text { A. Partial BWS } \\
\text { treadmill } \\
\text { training } x 20 \\
\text { sessions over } \\
2 \text { weeks, then } \\
50 \text { sessions at } \\
\text { home over } 10 \\
\text { weeks ( } n=14 \text { ) } \\
\text { B. } \\
\text { Individualized } \\
\text { strength-based } \\
\text { PT, } 20 \\
\text { sessions over } \\
2 \text { weeks, then } \\
50 \text { session at } \\
\text { home over } 10 \\
\text { weeks ( } n=12 \text { ) }\end{array}$ & $\begin{array}{l}\text { A vs. B } \\
\text { Age: } 9.6 \text { vs. } 9.5 \\
\text { Female: } 50 \% \text { vs. } 42 \% \\
\text { GMFCS II: } 7 \% \text { vs. } 8 \% \\
\text { GMFCS III: } 64 \% \text { vs. } 50 \% \\
\text { GMFCS IV: } 29 \% \text { vs. } \\
42 \% \\
\text { Diplegic CP: } 57 \% \text { vs. } \\
33 \% \\
\text { Triplegic CP: } 0 \% \text { vs. } \\
17 \% \\
\text { Quadriplegic CP: } 43 \% \\
\text { vs. } 50 \%\end{array}$ & $\begin{array}{l}\text { A vs. B, mean scores (SD) } \\
\text { Knee extension strength: } 3.90(3.09) \text { to } 3.58 \\
(2.82) \text { vs. } 3.09(3.15) \text { to } 3.80(4.22), p>0.05 \\
\text { Knee flexion strength: } 2.47(1.45) \text { to } 2.43 \\
(1.54) \text { vs. } 2.35(2.04) \text { to } 2.98(3.26), p>0.05 \\
\text { Dorsiflexion strength: } 0.86(1.21) \text { to } 0.69 \\
(0.78) \text { vs. } 0.62(0.75) \text { to } 0.77(0.66), p>0.05 \\
\text { Plantarflexion strength: } 3.44(1.91) \text { to } 3.23 \\
(1.45) \text { vs. } 3.06(3.62) \text { to } 3.14(3.32), p>0.05\end{array}$ \\
\hline $\begin{array}{l}\text { Kaya Kara, } 2019^{242} \\
\text { Multimodal } \\
\text { RCT } \\
\text { Fair }\end{array}$ & $\begin{array}{l}\text { A. Strength }+ \\
\text { balance } \\
\text { training, } 36 \\
\text { sessions over } \\
12 \text { weeks } \\
(n=15) \\
\begin{array}{l}\text { B. Usual care } \\
(n=15)\end{array} \\
\end{array}$ & $\begin{array}{l}\text { A vs. B } \\
\text { Age: } 11.8 \text { vs. } 11.3 \\
\text { Female: } 53 \% \text { vs. } 53 \% \\
\text { MAC I: } 47 \% \text { vs. } 47 \% \\
\text { MAC II: } 33 \% \text { vs. } 27 \% \\
\text { MAC III: } 20 \% \text { vs. } 28 \%\end{array}$ & $\begin{array}{l}\text { A vs. B, mean difference, Effect size, } p \text { - } \\
\text { value is between groups } \\
\text { Affected lower leg } 1 \mathrm{RM}(\mathrm{kg}): 54.33, \mathrm{ES} \\
3.23, \mathrm{p}<0.001 \\
\frac{\text { Unaffected lower leg } 1 \mathrm{RM}(\mathrm{kg})}{2.74, \mathrm{p}<0.001} 44.33, \text { ES }\end{array}$ \\
\hline $\begin{array}{l}\text { Kirk, } 2016^{217} \\
\text { Strength } \\
\text { Quasiexperimental } \\
\text { Poor }\end{array}$ & $\begin{array}{l}\text { A. Progressive } \\
\text { resistance, } 36 \\
\text { sessions over } \\
12 \text { weeks } \\
(n=12) \\
\text { B. Usual care } \\
(n=23)\end{array}$ & $\begin{array}{l}\text { A vs. B } \\
\text { Age: } 36.5 \\
\text { Female: } 43 \% \\
\text { Wheelchair user: } 17 \%\end{array}$ & $\begin{array}{l}\text { A, mean (SD): } \\
\text { Statistically significant Groups X Time } \\
\text { interaction for all exercises below for the } \\
\text { most affected leg }(\mathrm{kg}): \\
\frac{\text { Ankle dorsiflexion 1RM: }}{\text { (1.1) }} 5.7(0.6) \text { to } 10.4 \\
\frac{\text { Ankle plantarflexion 1RM: }}{71.8(6.7)} \\
\frac{\text { Knee flexion 1RM: } 16.3(4.9) \text { to }}{\text { Knee extension 1RM: }} 72.3(5.8) \text { to } 29.5(3.1) \\
(6.7)\end{array}$ \\
\hline
\end{tabular}




\begin{tabular}{|c|c|c|c|}
\hline $\begin{array}{l}\text { Author, Year } \\
\text { Intervention } \\
\text { Study Design } \\
\text { Study Quality }\end{array}$ & $\begin{array}{l}\text { Intervention } \\
\text { and } \\
\text { Comparison }\end{array}$ & $\begin{array}{l}\text { Population } \\
\text { (Cerebral Palsy) }\end{array}$ & Results \\
\hline $\begin{array}{l}\text { Makhov, } 2018^{243} \\
\text { Multimodal } \\
\text { RCT } \\
\text { Poor }\end{array}$ & \begin{tabular}{|l} 
A. Therapeutic \\
gymnastics + \\
strength 94 \\
sessions over \\
15 weeks \\
$(n=18)$ \\
B. Therapeutic \\
gymnastics \\
(passive \\
exercises only) \\
$(n=17)$
\end{tabular} & $\begin{array}{l}\text { A vs. B } \\
\text { Age: } 7-9 \text { years } \\
\text { Female: } \\
\text { Spastic diplegia or } \\
\text { spastic tetra paresis: } \\
100 \%\end{array}$ & $\begin{array}{l}\text { A vs. B, mean (SD), p-value is between } \\
\text { groups } \\
\text { Strength quadriceps femoris: } 1.29(0.49) \text { to } \\
\begin{array}{l}1.92(0.38) \text { vs. } 1.36(0.56) \text { to } 1.61(0.61) \\
p<0.05\end{array}\end{array}$ \\
\hline $\begin{array}{l}\text { Nsenga, } 2013^{88} \\
\text { Aerobic exercise } \\
\text { Quasiexperimental } \\
\text { Fair }\end{array}$ & $\begin{array}{l}\text { A. Cycle } \\
\text { ergometry, } 24 \\
\text { sessions over } \\
8 \text { weeks }(n=10) \\
\text { B. No training } \\
\text { control }(n=10)\end{array}$ & $\begin{array}{l}\text { A vs. B } \\
\text { Age: } 14.2 \text { vs. } 14.2 \\
\text { Female: } 40 \% \text { vs. } 40 \% \\
\text { Ambulatory: } 100 \% \\
\text { Hemiplegia: } 80 \% \text { vs. } \\
80 \%\end{array}$ & 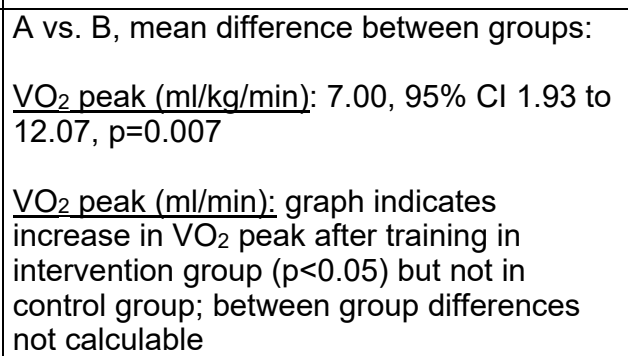 \\
\hline $\begin{array}{l}\text { Nsenga Leunkeu, } 2012^{132} \\
\text { Aerobic exercise } \\
\text { Quasiexperimental } \\
\text { Fair }\end{array}$ & $\begin{array}{l}\text { A. Treadmill } \\
\text { walking, } 24 \\
\text { sessions over } \\
8 \text { weeks, } \\
(n=12) \\
\text { B. No training, } \\
(n=12)\end{array}$ & $\begin{array}{l}\text { A vs. B } \\
\text { Age: } 14.2 \text { vs. } 14.2 \\
\text { Female: } 50 \% \text { vs. } 50 \% \\
\text { Hemiplegic CP: } 83 \% \text { vs. } \\
83 \% \\
\text { GMFCS I: } 67 \% \text { vs. } 67 \% \\
\text { GMFCS II: } 33 \% \text { vs. } 33 \%\end{array}$ & $\begin{array}{l}\text { A vs. B, mean (estimates from bar graph): } \\
\text { } \mathrm{VO}_{2} \text { peak: } 32.5 \text { to } 39.0 \text { vs. } 32.5 \text { to } 32.5 \text {, no } \\
\text { difference in baseline values, significant } \\
\text { difference in postintervention values } \\
\text { favoring treatment, statistical significance } \\
\text { between groups not clear }\end{array}$ \\
\hline $\begin{array}{l}\text { Scholtes, } 2010^{209} \\
\text { Scholtes, } 2012^{207} \\
\text { Scholtes, } 2008^{208} \\
\text { Strength } \\
\text { RCT } \\
\text { Fair }\end{array}$ & $\begin{array}{l}\text { A. Progressive } \\
\text { resistance, } 36 \\
\text { sessions over } \\
12 \text { weeks } \\
(n=26) \\
\begin{array}{l}\text { B. Usual care } \\
(n=25)\end{array}\end{array}$ & $\begin{array}{l}\text { A vs. B } \\
\text { Age: } 10.33 \text { vs. } 10.25 \\
\text { Female: } 33 \% \text { vs. } 50 \% \\
\text { Ambulatory: } 100 \% \\
\text { Bilateral: } 71 \% \text { vs. } 60 \% \\
\text { GMFM I: } 54 \% \text { vs. } 48 \% \\
\text { GMFM II: } 33 \% \text { vs. } 36 \% \\
\text { GMFM III: } 13 \% \text { vs. } 16 \%\end{array}$ & $\begin{array}{l}\text { A vs. B, Regression effect size } \\
\text { Knee extensors (N/kg): } 0.56,95 \% \mathrm{Cl} 0.13 \\
\text { to } 0.99, p=0.01 \\
\text { Knee flexors (N/kg): } 0.05,95 \% \mathrm{Cl}-0.25 \text { to } \\
0.36, p=0.71 \\
\text { Hip flexor }(\mathrm{N} / \mathrm{kg}): 0.16,95 \% \mathrm{Cl}-0.22 \text { to } \\
0.55, p=0.41 \\
\text { Hip abductor (N/kg): } 0.27,95 \% \mathrm{Cl} 0.00 \text { to } \\
0.54, p=0.05\end{array}$ \\
\hline $\begin{array}{l}\text { Slaman, 2014 } 235 \\
\text { Slaman, 2015a } a^{237} \\
\text { Slaman, 2015b } \\
\text { Multimodal exercise } \\
\text { RCT } \\
\text { Fair }\end{array}$ & $\begin{array}{l}\text { A. Strength } \\
\text { training + } \\
\text { aerobic fitness, } \\
48 \text { sessions } \\
\text { over } 3 \text { months } \\
\text { plus } 8-10 \\
\text { counseling } \\
\text { sessions on } \\
\text { physical } \\
\text { activity and } \\
\text { sports } \\
\text { participation } \\
\text { over } 3 \text { months: } \\
(n=28) \\
\\
\text { B. Usual care } \\
(n=29)\end{array}$ & $\begin{array}{l}\text { A vs. B } \\
\text { Age: } 20 \text { vs. } 20 \\
\text { Female: } 48.3 \% \text { vs. } \\
57.1 \% \\
\text { Ambulatory: } 97 \% \text { vs. } \\
89 \% \\
\text { Wheelchair user: } 3.3 \% \\
\text { vs. } 10.7 \% \\
\text { Unilateral CP: } 52 \% \text { vs. } \\
50 \% \\
\text { GMFM I: } 61 \% \text { vs. } 55 \% \\
\text { GMFM II: } 32 \% \text { vs. } 31 \% \\
\text { GMFM III: } 7 \% \text { vs. } 10 \% \\
\text { GMFM IV: } 0 \% \text { vs. } 3 \%\end{array}$ & 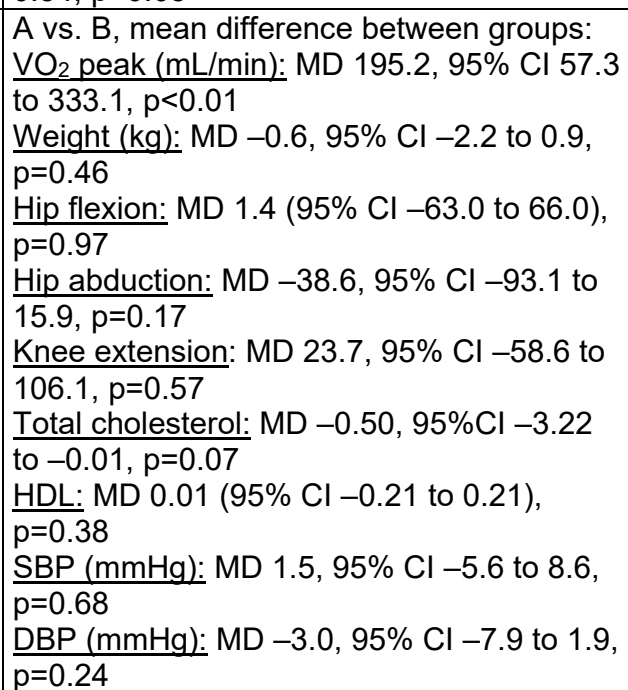 \\
\hline
\end{tabular}




\begin{tabular}{|c|c|c|c|}
\hline $\begin{array}{l}\text { Author, Year } \\
\text { Intervention } \\
\text { Study Design } \\
\text { Study Quality }\end{array}$ & $\begin{array}{l}\text { Intervention } \\
\text { and } \\
\text { Comparison }\end{array}$ & $\begin{array}{l}\text { Population } \\
\text { (Cerebral Palsy) }\end{array}$ & Results \\
\hline $\begin{array}{l}\text { Taylor, } 2013^{211} \\
\text { Strength } \\
\text { RCT } \\
\text { Good }\end{array}$ & $\begin{array}{l}\text { A. Progressive } \\
\text { resistance, } 24 \\
\text { sessions over } \\
12 \text { weeks } \\
(n=23) \\
\text { B. Usual care } \\
(n=25)\end{array}$ & $\begin{array}{l}\text { A vs. B } \\
\text { Age: } 18.17 \text { vs. } 18.58 \\
\text { Female: } 44 \% \text { vs. } 48 \% \\
\text { No gait aid } 57 \% \text { vs. } 60 \% \\
\text { GMFM II: } 57 \% \text { vs. } 64 \% \\
\text { GMFM III: } 43 \% \text { vs. } 36 \%\end{array}$ & $\begin{array}{l}\text { A vs. B, mean difference between groups: } \\
\text { Max leg press (1Rep Max; } k g): 14.8,95 \% \\
\mathrm{Cl} 4.3 \text { to } 25.3, p<0.05 \\
\text { Reverse leg press (1RepMax; } \mathrm{kg}):-0.7 \\
95 \% \mathrm{Cl}-4.3 \text { to } 2.8), \mathrm{p}>0.05\end{array}$ \\
\hline $\begin{array}{l}\text { Tedla, } 2014^{213} \\
\text { Strength } \\
\text { RCT } \\
\text { Poor }\end{array}$ & $\begin{array}{l}\text { A. Strength } \\
\text { training } 18 \\
\text { sessions over } \\
6 \text { weeks + } \\
\text { conventional } \\
\text { PT 1-2 } \\
\text { days/week } \\
(n=31) \\
\text { B. } \\
\text { Conventional } \\
\text { PT 3-5 } \\
\text { sessions/per } \\
\text { for 6 weeks } \\
(n=31)\end{array}$ & $\begin{array}{l}\text { A vs. } B \text { (data are for } \\
\text { completers only; } n=30 \\
\text { vs. } 30 \text { ) } \\
\text { Age: } 9.1 \text { vs. } 8.9 \text { years } \\
\text { Female: } 33 \% \text { vs. } 33 \% \\
\text { Gross motor function } \\
\text { classification system: } \\
\text { I: } 7 \% \text { vs. } 3 \% \\
\text { II: } 20 \% \text { vs. } 27 \% \\
\text { III: } 37 \% \text { vs. } 27 \% \\
\text { IV: } 37 \% \text { vs. } 43 \%\end{array}$ & $\begin{array}{l}\text { A vs. } B \text {, change in scores, } p \text {-value is } \\
\text { between groups } \\
\text { Change in Strength of Trunk, Hip, Knee, } \\
\frac{\text { Ankle: significantly better in group A than } B}{p<0.05}\end{array}$ \\
\hline
\end{tabular}

Abbreviations: BWS = body weight supported; $\mathrm{CI}=$ confidence interval; $\mathrm{CP}=$ cerebral palsy; $\mathrm{DBP}=$ diastolic blood pressure; EDSS $=$ Expanded Disability Status Scale; GMFCS = Gross Motor Function Classification System; GMFM = Gross Motor Function Measure; HDL = high-density lipoprotein cholesterol; $\mathrm{HR}=$ heart rate; $\mathrm{MD}=$ mean difference; $\mathrm{NR}=$ not reported; $\mathrm{PT}=$ physical therapy; $\mathrm{RM}=$ one-repetition maximum; $\mathrm{SBP}=$ systolic blood pressure; $\mathrm{SD}=$ standard deviation; $\mathrm{VO}_{2}$ peak $=$ highest value of $\mathrm{VO}_{2}$ attained

Table 44. Intermediate outcomes of physical activity in participants with spinal cord injury

\begin{tabular}{|c|c|c|c|}
\hline $\begin{array}{l}\text { Author, Year } \\
\text { Intervention } \\
\text { Study Design } \\
\text { Study Quality }\end{array}$ & $\begin{array}{l}\text { Intervention } \\
\text { and Comparison }\end{array}$ & $\begin{array}{l}\text { Population } \\
\text { (Spinal Cord Injury) }\end{array}$ & esults \\
\hline $\begin{array}{l}\text { Akkurt, } 2017^{89} \\
\text { Aerobic exercise } \\
\text { RCT } \\
\text { Fair }\end{array}$ & $\begin{array}{l}\text { A. Arm ergometer, } \\
36 \text { sessions over } 12 \\
\text { weeks plus general } \\
\text { exercises, } 120 \\
\text { sessions over } 12 \\
\text { weeks ( } n=17) \\
\\
\text { B. General } \\
\text { exercises, } 120 \\
\text { sessions over } 12 \\
\text { weeks }(n=16)\end{array}$ & $\begin{array}{l}\text { A vs. B } \\
\text { Age: } 33 \text { vs. } 37 \\
\text { Female: } 5 \% \text { vs. } 19 \% \\
\text { Ambulatory: } 41 \% \text { vs. } \\
50 \% \\
\text { Wheelchair user: } 59 \% \\
\text { vs. } 50 \% \\
\text { Paraplegia: } 100 \% \text { vs. } \\
94 \%\end{array}$ & $\begin{array}{l}\text { A vs. B, median change, } p=\text { between } \\
\text { groups: } \\
\text { VO } 2 \text { peak }(\mathrm{ml} / \mathrm{kg} / \mathrm{min}): 4.30 \text { vs. } 1.35, p=0.02 \\
\text { FEV1 }(\mathrm{ml}):-0.14 \text { vs. } 0.17, p>0.05 \\
\text { FVC }(\mathrm{ml}):-0.31 \text { vs. }-0.20, p>0.05 \\
\text { FEV1/FVC, } 3.51 \text { vs. }-0.50, p>0.05 \\
\text { SBP }(\mathrm{mmH}): 0 \text { vs. } 0, p>0.05 \\
\text { DBP }(\mathrm{mmHg}): 0 \text { vs. } 0, p>0.05 \\
\text { T-chol: } 10 \text { vs. } 2, p>0.05 \\
\text { TG: } 5.5 \text { vs. } 26, p>0.05 \\
\text { LDL: } 0 \text { vs. }-3.5, p>0.05 \\
\text { HDL: } 0 \text { vs. } 5.5, p>0.05\end{array}$ \\
\hline
\end{tabular}




\begin{tabular}{|c|c|c|c|}
\hline $\begin{array}{l}\text { Author, Year } \\
\text { Intervention } \\
\text { Study Design } \\
\text { Study Quality }\end{array}$ & $\begin{array}{l}\text { Intervention } \\
\text { and Comparison }\end{array}$ & $\begin{array}{l}\text { Population } \\
\text { (Spinal Cord Injury) }\end{array}$ & Results \\
\hline $\begin{array}{l}\text { Alexeeva, } 2011^{133} \\
\text { Aerobic exercise } \\
\text { RCT } \\
\text { Fair }\end{array}$ & \begin{tabular}{|l|} 
A. BWS treadmill \\
training, 39 \\
sessions over 13 \\
weeks $(n=9)$ \\
B. BWS track \\
training, 39 \\
sessions over 13 \\
weeks $(n=14)$ \\
C. Structured PT, \\
39 sessions over 13 \\
weeks $(n=12)$
\end{tabular} & $\begin{array}{l}\text { A vs. B vs. C } \\
\text { Age: } 43 \text { vs. } 36 \text { vs. } 35 \\
\text { Female: } 11 \% \text { vs. } 14 \% \\
\text { vs. } 17 \% \\
\text { ASIA D: } 100 \% \\
\text { Cervical: } 89 \% \text { vs. } 57 \% \\
\text { vs. } 58 \%\end{array}$ & $\begin{array}{l}\text { A vs. B vs. C, mean (SD) } \\
\text { VO2 peak (ml/km/min): } 12 \% \text { nonsignificant } \\
\text { increase within groups, but no differences } \\
\text { between groups, } p>0.05 \\
\\
\frac{\mathrm{MMT} \text { (combined upper and lower limb }}{\text { strength): } 71.5(15.1) \text { to } 78.1(15.3) \text { vs. } 69.5} \\
\frac{\text { (12.1) to } 73.3(11.5) \text { vs. } 76.3(11.6) \text { to } 81.8}{(11.0)(6-9 \% \text { significant increase within }} \\
\text { groups; no difference among groups, } \\
\text { p>0.05) }\end{array}$ \\
\hline $\begin{array}{l}\text { Chen, } 2016^{218} \\
\text { Strength } \\
\text { RCT } \\
\text { Fair }\end{array}$ & $\begin{array}{l}\text { A. Pulmonary } \\
\text { rehabilitation: } 365 \\
\text { sessions over } 52 \\
\text { weeks }(n=49) \\
\begin{array}{l}\text { B. Usual care } \\
(\mathrm{n}=49)\end{array}\end{array}$ & $\begin{array}{l}\text { A vs. B } \\
\text { Age: } 62.3 \text { vs. } 63.1 \\
\text { Female: } 0 \% \\
\text { T1-2: } 35 \% \text { vs. } 35 \% \\
\text { T3-4: } 33 \% \text { vs. } 33 \% \\
\text { T5-6: } 33 \% \text { vs. } 33 \%\end{array}$ & $\begin{array}{l}\text { A vs. B, mean (SD), } p=\text { postintervention: } \\
\text { FEV1: } 1.17(0.25) \text { to } 2.20(0.45) \text { vs. } 1.17 \\
\text { (0.45) to } 1.14(0.44), p<0.05 \\
\text { FVC: } 2.16(0.36) \text { to } 2.98(0.54) \text { vs. } 2.16 \\
\text { (0.42) to } 2.17(0.42), p<0.05 \\
\text { FEV1/FVC: } 0.53(0.17 \text { to } 0.75(0.08) \text { vs. } \\
0.53(0.17) \text { to } 0.52(0.15), p<0.05\end{array}$ \\
\hline $\begin{array}{l}\text { Giangregorio, } 2012^{134} \\
\text { Craven, } 2017^{137} \\
\text { Aerobic exercise } \\
\text { RCT } \\
\text { Fair }\end{array}$ & $\begin{array}{l}\text { A. BWS treadmill } \\
\text { walking with FES, } \\
48 \text { sessions over } 16 \\
\text { weeks ( } n=17) \\
\\
\text { B. Aerobic and } \\
\text { resistance training, } \\
48 \text { sessions over } 16 \\
\text { weeks }(n=17)\end{array}$ & $\begin{array}{l}\text { A vs. B } \\
\text { Age: } 56.6 \text { vs. } 54.1 \\
\text { Female: } 18 \% \text { vs. } 29 \% \\
\text { Tetraplegia: } 82 \% \text { vs. } \\
71 \% \\
\text { UEMS: } 38.3 \text { vs. } 37.5 \\
\text { LEMS: } 30.4 \text { vs. } 27.9\end{array}$ & $\begin{array}{l}\text { A vs. B, mean (SD), } p=\text { between groups } \\
\text { BMD Total Hip: } 0.89(0.20) \text { to } 0.88(0.20) \\
\text { vs. } 0.86(0.24) \text { to } 0.87(0.23), p>0.05 \\
\text { BMD Distal Femur: } 0.89(0.16) \text { to } 0.87 \text { to } \\
0.15) \text { vs. } 0.81(0.18) \text { to } 0.80(0.18), p>0.05 \\
\text { BMD Proximal Tibia: } 0.71(0.18) \text { to } 0.71 \\
\text { (0.15) vs. } 0.68(0.19) \text { to } 0.66(0.19), p>0.05 \\
\text { Fat mass (kg): } 25.4(9.5) \text { to } 24.3(9.5) \text { vs. } \\
\text { 23.2 (10.8) to } 23.0(10.7), p>0.05\end{array}$ \\
\hline $\begin{array}{l}\text { Gorman, } 2019^{75} \\
\text { Aerobic exercise } \\
\text { RCT }\end{array}$ & $\begin{array}{l}\text { A. RAGT, } 36 \\
\text { sessions over } 3 \\
\text { months }(n=17) \\
\\
\text { B. Aquatic therapy, } \\
36 \text { sessions over } 3 \\
\text { months }(n=15)\end{array}$ & $\begin{array}{l}\text { A vs. B } \\
\text { Age: } 45.4 \text { vs. } 46.9 \\
\text { Community Ambulation: } \\
83 \% \text { vs. } 67 \% \\
\text { Tetraplegic: } 67 \% \text { vs. } \\
73 \%\end{array}$ & $\begin{array}{l}\text { A vs. } B, \text { mean }(\mathrm{SD}), \mathrm{p}=\text { between groups } \\
\mathrm{VO} \mathrm{V}_{2} \text { peak }(\mathrm{ml} / \mathrm{kg} / \mathrm{min}): 16.48(5.39) \text { to } 16.18 \\
(5.11) \text { vs. } 13.33(3.06) \text { to } 14.31(3.88), \\
\mathrm{p}=0.063\end{array}$ \\
\hline $\begin{array}{l}\text { Jones, 2014a } \\
\text { Jones, 2014b } \\
\text { Multimodal exercise } \\
\text { RCT }\end{array}$ & $\begin{array}{l}\text { A. Activity-based } \\
\text { therapy, } 72 \\
\text { sessions over } 24 \\
\text { weeks }(n=20) \\
\text { B. Waitlist }(n=21)\end{array}$ & $\begin{array}{l}\text { A vs. B } \\
\text { Age: } 42 \text { vs. } 34 \\
\text { Female: } 5 \% \text { vs. } 48 \% \\
\text { Tetraplegia: } 75 \% \text { vs. } \\
76 \% \\
\text { AIS C: } 35 \% \text { vs. } 52 \% \\
\text { AIS D: } 65 \% \text { vs. } 48 \%\end{array}$ & $\begin{array}{l}\text { A vs. B, mean change (SD), } p=\text { between } \\
\text { groups: } \\
\text { BMI: } 0.005(1.15) \text { vs. } 0.723(2.22), p=0.288 \\
\frac{\text { Weight (lbs): }-2.0(8.29) \text { vs. } 5.03(14.05)}{p=0.314}\end{array}$ \\
\hline $\begin{array}{l}\text { Jung, } 2014^{76} \\
\text { Aerobic exercise } \\
\text { RCT } \\
\text { Fair }\end{array}$ & $\begin{array}{l}\text { A. Aquatic exercise, } \\
24 \text { sessions over } 8 \\
\text { weeks }(n=10) \\
\\
\text { B. Land exercise, } \\
24 \text { sessions over } 8 \\
\text { weeks }(n=10)\end{array}$ & $\begin{array}{l}\text { A vs. } B \\
\text { Age: } 42.1 \text { vs. } 51.1 \\
\text { Female: } 30 \% \text { vs. } 50 \%\end{array}$ & $\begin{array}{l}\text { A vs. B, mean change scores, } p=\text { between } \\
\text { groups: } \\
\text { FVC(L): } 1.8(1.3) \text { vs. } 0.31(1.6), p=0.031 \\
\text { FEV1(L): } 1.1(1.2) \text { vs. } 0.21(0.3) ; p=0.038 \\
\text { FER(L/sec): } 10.0(9.7) \text { vs. } 5.4(7.0), \\
p=0.238 \\
\text { FEV1/FVC: } 3.7(2.3) \text { vs. } 2.1(3.4), p=0.243\end{array}$ \\
\hline
\end{tabular}




\begin{tabular}{|c|c|c|c|}
\hline $\begin{array}{l}\text { Author, Year } \\
\text { Intervention } \\
\text { Study Design } \\
\text { Study Quality }\end{array}$ & $\begin{array}{l}\text { Intervention } \\
\text { and Comparison }\end{array}$ & $\begin{array}{l}\text { Population } \\
\text { (Spinal Cord Injury) }\end{array}$ & Results \\
\hline $\begin{array}{l}\text { Lavado, } 2013^{92} \\
\text { Aerobic exercise } \\
\text { RCT } \\
\text { Fair }\end{array}$ & $\begin{array}{l}\text { A. Hand cycling }+ \\
\text { distance with } \\
\text { wheelchair }+ \\
\text { general exercises, } \\
32-48 \text { sessions over } \\
16 \text { weeks }(n=21) \\
\begin{array}{l}\text { B. Usual care } \\
(n=21)\end{array}\end{array}$ & $\begin{array}{l}\text { A vs. B } \\
\text { Age: } 34.1 \text { vs. } 38.5 \\
\text { Female: } 14 \% \text { vs. } 19 \% \\
\text { Community ambulators: } \\
\text { 0\% } \\
\text { C5-L2: } 100 \%\end{array}$ & $\begin{array}{l}\text { A vs. B, median: } \\
\text { VO2 peak ( } \mathrm{mL} / \mathrm{min}): 939 \text { to } 1154(\mathrm{p}=0.009) \\
\text { vs. } 896 \text { to } 834, \mathrm{p}=0.906 ; \text { Postintervention } \\
\text { comparison (no control for baseline values) } \\
p<0.001\end{array}$ \\
\hline $\begin{array}{l}\text { Lai, } 2010^{91} \\
\text { Aerobic Exercise } \\
\text { Quasiexperimental } \\
\text { Fair }\end{array}$ & $\begin{array}{l}\text { A. Functional } \\
\text { electrical } \\
\text { stimulation cycling } \\
\text { exercises, } 36 \\
\begin{array}{l}\text { sessions over } 12 \\
\text { weeks }(n=12)\end{array} \\
\begin{array}{l}\text { B. Control group } \\
(n=12)\end{array} \\
\end{array}$ & $\begin{array}{l}\text { A vs. B } \\
\text { Age: } 28.9 \text { vs. } 28.2 \\
\text { Female: } 17 \% \text { vs. } 17 \% \\
\text { Ambulatory: } 0 \% \\
\text { Paraplegia: } \\
10(67 \%) \text { vs. } 10(67 \%)\end{array}$ & $\begin{array}{l}\text { A vs. B, mean difference between groups: } \\
\text { BMD Femoral Neck: }-0.003,95 \% \mathrm{Cl}-0.12 \\
\text { to } 0.11, p=0.96 \\
\text { BMD Distal Femur: }-0.05,95 \% \mathrm{Cl}-0.12 \text { to } \\
0.03, p=0.21\end{array}$ \\
\hline $\begin{array}{l}\text { Mogharnasi, } 2019^{219} \\
\text { Strength } \\
\text { RCT } \\
\text { Poor }\end{array}$ & $\begin{array}{l}\text { A. Upper body } \\
\text { resistance training: } \\
24 \text { sessions over } 8 \\
\text { weeks }(n=10) \\
\begin{array}{l}\text { B. Usual care } \\
(n=10)\end{array}\end{array}$ & $\begin{array}{l}\text { A vs. B } \\
\text { Age: } 25.33 \text { vs. } 25.50 \\
\text { Female: } 0 \% \\
\text { Ambulatory: } 0 \% \\
\text { Wheelchair user: } 100 \% \\
\text { T9: } 10 \% \text { vs. } 20 \% \\
\text { T10: } 20 \% \text { vs. } 20 \% \\
\text { T11: } 20 \% \text { vs. } 0 \% \\
\text { T12: } 50 \% \text { vs. } 60 \%\end{array}$ & $\begin{array}{l}\text { A vs. B, mean difference between groups: } \\
\text { BMl: }-0.83,95 \% \mathrm{Cl}-1.85 \text { to } 0.19, p=0.11 \\
\text { \% Body fat: }-1.2,95 \% \mathrm{Cl}-3.11 \text { to } \\
0.71, p=0.22 \\
\text { T-chol: }-16.00,95 \% \mathrm{Cl}-11.21 \text { to }-20.78 \\
p<0.001 \\
\text { HDL: } 4.2,95 \% \mathrm{Cl} 0.84 \text { to } 7.56, p=0.01 \\
\text { LDL: }-6.5,95 \% \mathrm{Cl}-9.81 \text { to }-3.20, p<0.001 \\
\text { TG: }-25.3,95 \% \mathrm{Cl}-32.74 \text { to }-17.86 \\
p<0.001\end{array}$ \\
\hline $\begin{array}{l}\text { Totosy de Zepetnek, } \\
2015^{249} \\
\text { Multimodal } \\
\text { RCT } \\
\text { Fair }\end{array}$ & $\begin{array}{l}\text { A. Progressive } \\
\text { resistance }+ \\
\text { aerobic training, } 32 \\
\text { sessions over } 16 \\
\text { weeks }(n=12) \\
\text { B. Maintain existing } \\
\text { physical activity } \\
\text { levels }(n=11)\end{array}$ & $\begin{array}{l}\text { A vs. B } \\
\text { Age: } 39 \text { vs. } 42 \\
\text { Female: } 0 \% \text { vs. } 18 \% \\
\text { AIS A-B: } 25 \% \text { vs. } 45 \% \\
\text { AIS C-D: } 75 \% \text { vs. } 55 \%\end{array}$ & 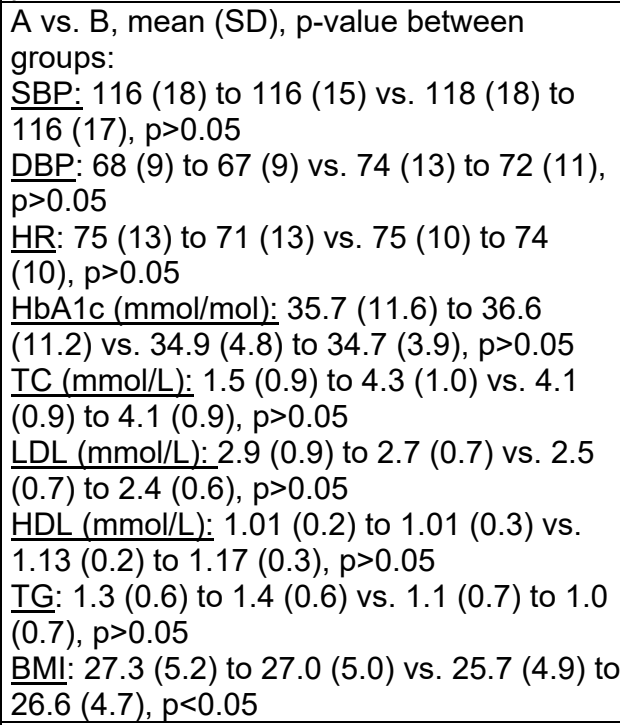 \\
\hline $\begin{array}{l}\text { Valent, } 2010^{93} \\
\text { Aerobic exercise } \\
\text { Cohort study } \\
\text { Fair }\end{array}$ & $\begin{array}{l}\text { A. Hand cycle } \\
\text { ergometry, 15-72 } \\
\text { sessions over 9-33 } \\
\text { weeks }(n=20) \\
\text { B. Unclear } \\
\text { (matched control) } \\
(n=17)\end{array}$ & $\begin{array}{l}\text { A vs. B } \\
\text { Age: } 46 \text { vs. } 40 \\
\text { Female: } 24 \% \text { vs. } 24 \% \\
\text { Paraplegia: } 10(59 \%) \text { vs. } \\
11(65 \%)\end{array}$ & $\begin{array}{l}\text { A vs. B, mean change scores } \\
\text { FVC\%: }-9.4 \text { vs. }-7.8, p=0.619 \\
\text { PEF\%: }-12.6 \text { vs. }-10.0, p=0.722 \\
\frac{V O_{2} \text { peak }(\mathrm{ml} / \mathrm{min}):}{} 0.21 \text { vs. } 0.13, p=0.356 \\
\mathrm{VO}_{2} \text { peak }(\mathrm{ml} / \mathrm{kg} / \mathrm{min}):\end{array}$ \\
\hline
\end{tabular}




\begin{tabular}{|c|c|c|c|}
\hline $\begin{array}{l}\text { Author, Year } \\
\text { Intervention } \\
\text { Study Design } \\
\text { Study Quality }\end{array}$ & $\begin{array}{l}\text { Intervention } \\
\text { and Comparison }\end{array}$ & $\begin{array}{l}\text { Population } \\
\text { (Spinal Cord Injury) }\end{array}$ & Results \\
\hline $\begin{array}{l}\text { Van der Scheer, } \\
2016^{140} \\
\text { Aerobic exercise } \\
\text { RCT } \\
\text { Fair }\end{array}$ & $\begin{array}{l}\text { A. Low-intensity } \\
\text { wheelchair treadmill } \\
\text { training, } 32 \\
\text { sessions over } 16 \\
\text { weeks }(n=14) \\
\text { B. Usual care } \\
(n=15)\end{array}$ & $\begin{array}{l}\text { A vs. B } \\
\text { Age: } 55 \text { vs. } 57 \\
\text { Female: } 14 \% \text { vs. } 33 \% \\
\text { Paraplegia: } 64 \% \text { vs. } 73 \%\end{array}$ & $\begin{array}{l}\text { A vs. } \mathrm{B}, \text { median change, } p=\text { between } \\
\text { groups: } \\
\mathrm{VO}_{2} \text { peak (L/min): } 0.05 \text { to }-0.07, p=0.01\end{array}$ \\
\hline
\end{tabular}

Abbreviations: ASIA = American Spinal Injuries Association Impairment Scale; BMD = bone mineral density; BMI = body mass index; $\mathrm{CI}=$ confidence interval; $\mathrm{DBP}=$ diastolic blood pressure; FER $=$ forced expiratory ratio; FEV1 = forced expiratory volume; $\mathrm{FVC}=$ forced vital capacity; HbAlc = hemoglobin A1c; HR = heart rate; $\mathrm{LDL}=$ low-density lipoprotein cholesterol; MMT = Maximal Muscle Testing combined upper and lower limb strength; NS = not significant; PEF = peak expiratory flow; PT $=$ physical therapy; RAGT = robot-assisted gait training; $\mathrm{SBP}=$ systolic blood pressure; $\mathrm{SD}=$ standard deviation; $\mathrm{TG}=$ triglyceride; UEMS = Upper Extremity Motor Score

The range of mean ages enrolled in studies of participants with CP was 6.3 years to 38 years, in studies of participants with MS was 29 years to 52 years, and for participants with SCI was 28 years to 63 years. The proportion of females enrolled ranged from a low of none in an SCI study, to a high of 100 percent two studies of participants with MS. Race was not reported in any of these studies. Reporting of characteristics at baseline related to spasticity or overall mobility varied according to condition. In studies of participants with CP, most were in GMFCS level I or II. In the studies of participants with MS, baseline disability ranged from 1.7 to 3.7 on the EDSS (range of scale $0-10$ with higher values representing more problems walking), except for one study that enrolled only participants with EDSS scores of 4-6 ${ }^{221}$ and one with 23 percent of enrolled subjects in the range of 6.5 to 8 points on the EDSS/Wheelchair use was not reported in studies of participants with CP or MS. The studies of participants with SCI varied in the way disability was reported and the level of disability; 59 to 100 percent had paraplegia and wheelchair use ranged from 50 to 100 percent.

Interventions studied and comparisons made also varied widely. Eighteen studies evaluated aerobic interventions (10 vs. usual care), nine evaluated strength interventions ( 8 vs. usual care, 1 vs. attention control), and fourteen evaluated multimodal interventions (12 vs. usual care). In the 17 of 20 studies categorized as comparing interventions with usual care, the control groups consisted of standard PT regimens, waitlist, "attention control" (nonphysical activity social interactions), and unspecified "usual care" interventions. The two prioritized intermediate outcomes were $\mathrm{VO}_{2}$ peak, reported in 15 studies, and pulmonary function tests, reported in 4 studies (see Table 42 for more details). Other intermediate outcomes reported included weightbased outcomes, lipids, heart rate, glucose, strength changes, percent body fat, and bone mineral density.

Evidence on the impact of physical activity interventions on intermediate outcomes is mostly insufficient to draw conclusions. There was low-strength evidence that aerobic interventions improve $\mathrm{VO}_{2}$ peak in participants with $\mathrm{CP}$ and $\mathrm{SCI}$, but evidence was inconsistent in patients with MS. For other comparisons and outcomes there were several instances where a single study found a significant benefit of an intervention on one or more outcomes or that there was no difference between groups, but interpretation is limited due to small sample size, methodological limitations, and lack of corroborating evidence. 


\section{Comparisons With Usual Care}

\section{Aerobic Exercises Versus Usual Care}

$\mathrm{VO}_{2}$ peak was the most commonly reported intermediate outcome in studies of aerobic exercise. Evidence in participants with $\mathrm{CP}$ and $\mathrm{SCI}$ indicated improved $\mathrm{VO}_{2}$ peak with exercise (SOE: low), while evidence in participants with MS was inconsistent (SOE: insufficient). In three fair-quality RCTs of participants with MS (Table 42), only one found a significant improvement in $\mathrm{VO}_{2}$ peak, a study of weight-based upper and lower body cycling training, ${ }^{53}$ while regular cycling programs versus usual care did not impact $\mathrm{VO}_{2}$ peak (Table 42). ${ }^{77,78}$ Two fair-quality quasiexperimental studies compared an aerobic exercise with usual care in adolescents with CP over 8 weeks ( $\mathrm{n}=44$ total) ${ }^{88,132} \mathrm{VO}_{2}$ peak measured in $\mathrm{ml} / \mathrm{kg} / \mathrm{min}$ was increased significantly more with cycle-ergometry in one study, and in both studies $\mathrm{VO}_{2}$ peak measured in $\mathrm{ml} / \mathrm{min}$ was increased significantly with aerobic exercise (Table 43) (SOE: low). In participants with SCI, three studies reported $\mathrm{VO}_{2}$ peak, with two fair-quality RCTs $(\mathrm{n}=71)$ finding a significant increase with aerobic exercise training, ${ }^{92,140}$ and a small $(\mathrm{n}=17)$, fair-quality cohort study not finding a difference, although the endpoint values were higher in the aerobic exercise group (Table 44) (SOE: low). ${ }^{93}$

Pulmonary function was not improved with aerobic training in a cohort study of participants with SCI (Table 44). ${ }^{93}$ This is insufficient-strength evidence to draw conclusions due to study limitations, lack of corroborating evidence, and imprecision. Other intermediate outcomes reported in these studies were not found to have significant improvement with aerobic exercise (e.g., pulmonary function tests, bone density, weight, BMI). ${ }^{53,91,93,117}$

\section{Strength Exercises Versus Usual Care}

Nine studies evaluated strength exercise programs and reported intermediate outcomes. Three studies were in participants with MS, ${ }^{198,204,206}$ four in participants with $\mathrm{CP},{ }^{207,211,213,217}$ and two in participants with SCI. ${ }^{213,218,219}$ One of these was a poor-quality quasiexperimental study (Table 43) ${ }^{217}$ None of these RCTs reported on $\mathrm{VO}_{2}$ peak/max. A fair-quality RCT $(\mathrm{n}=98)$ of a pulmonary rehabilitation program in participants with SCI over 52 weeks found all pulmonary function tests measured to be significantly improved with the intervention (Table 44). ${ }^{218}$ For example, forced expiratory volume (FEV1) increased from 1.17 to 2.20 with pulmonary rehabilitation compared with a small decrease $(1.17$ to 1.14$)$ in the control group $(\mathrm{p}<0.05)$.

In participants with MS, three RCTs measured improvement in strength in various ways, comparing strength training compared with usual care or social programs (attention control) (Table 42). ${ }^{147,204,206}$ Each study found one or more measures of strength were significantly improved with strength training over 8 to 12 weeks (Table 42 ). A single fair-quality RCT compared Pilates plus massage to massage alone and found no impact on percent body fat over 12 weeks. ${ }^{198}$

In studies that enrolled participants with $\mathrm{CP}$, results were mixed with improved strength demonstrated on one or more measures in each study ${ }^{207,211,213,217}$ but not on all measures. ${ }^{207,211,216}$ Strength training lasted for 12 weeks in three of these studies ${ }^{207,211,217}$ and 6 weeks in two studies. ${ }^{213,216}$

\section{Multimodal Exercises Versus Usual Care}

Ten RCTs (in 11 publications) evaluated multimodal exercise programs with usual care and reported an intermediate outcome. ${ }^{221,224,227,228,230,231,234-237,242,243,246,247,249}$ Four were poor 
quality, ${ }^{227,228,243,246,247}$ and the rest were fair. In participants with MS, three fair-quality RCTs evaluated multimodal exercise programs and reported $\mathrm{VO}_{2}$ peak ${ }^{221}$ or $\mathrm{VO}_{2}$ max. ${ }^{24,231}$ In a study of mostly women with baseline EDSS scores all 4-6 (some walking impairment), resistance training, aerobics, and balance training over 24 weeks did not improve $\mathrm{VO}_{2}$ peak compared with stretching and toning. ${ }^{221}$ The second study enrolled participants with mean baseline EDSS of 2.5 (little or no walking impairment), and the intervention groups had less than half women, while the control group had 82 percent women. ${ }^{224}$ This study compared resistance training plus either high-intensity interval training $(\mathrm{n}=12)$ or plus high-intensity continuous cardio training $(\mathrm{n}=11)$ with usual care $(n=11)$ over 12 weeks. The addition of interval training resulted in greater improvement in $\mathrm{VO}_{2}$ peak than usual care, while the addition of continuous cardio training did not improve $\mathrm{VO}_{2}$ max or $\mathrm{VO}_{2}$ peak significantly compared with usual care (Table 42). ${ }^{224}$ The disparity in the proportion of women in the intervention versus control arms added to the study limitations. The third study enrolled women only, with 50 percent having EDSS scores of 4 or higher (23\% with 6.5 to 8 , more significant impairment). A multimodal intervention of resistance training, aerobic training, balance, Pilates, and stretching for 12 weeks resulted significantly improved $\mathrm{VO}_{2}$ peak in the overall analysis, however when differences between groups in disability at baseline were taken into account the difference was no longer significant. ${ }^{231}$ In participants with $\mathrm{CP}$, a single fair-quality $\mathrm{RCT}(\mathrm{n}=57)$ found strength training plus aerobic training and counseling over 12 weeks improved $\mathrm{VO}_{2}$ peak significantly in young adults (mean age 20 years), most of whom were in GMFM categories I and II at enrollment. ${ }^{234-237}$

Changes in strength were measured in four RCTs of multimodal interventions. In three studies of multimodal exercise programs in participants with MS (2 with progressive resistance training and aerobics, 2 with WBV, and 1 with low-intensity exercise), results varied in terms of significance depending on the specific measure reported, but overall there was some benefit seen across the studies (Table 42). ${ }^{222,224,227,228}$ In participants with MS, effect on percent body fat was positive in two studies of progressive resistance training plus aerobic exercise, ${ }^{224,227}$ but weight or BMI was not significantly different between groups in three RCTs. ${ }^{224,227,234-237}$ In young adults with CP, no improvement was seen (Table 43). ${ }^{234-237}$ Resting heart rate, lipids, and glucose were reported in few studies and were not different compared with control groups. In one study of participants with SCI, multimodal exercise maintained BMI, while the control group BMI increased. ${ }^{249}$ Other intermediate outcomes (blood pressure, heart rate, A1c, and lipids) were not significantly different between groups.

\section{Head-to-Head Comparisons}

\section{Aerobic Exercises}

Seven fair-quality RCTs ${ }^{75,76,82,89,125,133-137}$ evaluated comparisons of different multimodal interventions. In participants with MS ( $n=20), 8$ weeks of aquatic exercise improved FEV1 and forced vital capacity (FVC) significantly more than land exercises (Table 42); other pulmonary function test measures were not significantly different. ${ }^{76} \mathrm{VO}_{2}$ peak was reported in three RCTs in participants with SCI, with two studies $(n=67)$ not finding a significant difference between groups (RAGT vs. aquatic therapy and body-weight supported treadmill vs. track training) ${ }^{75,133}$ A third study $(n=33)$ found significantly greater improvement with an arm ergometry training over general exercises over 12 weeks. ${ }^{89}$ Pulmonary function tests were reported in two RCTs (Table 44). In participants with SCI $(n=33)$ pulmonary function tests showed small changes with 
no significant differences between an arm ergometer program compared with a general exercise program over 12 weeks. ${ }^{89}$

Change in strength was measured in three RCTs of participants with SCI, ${ }^{89,133-137}$ and one each of participants with $\mathrm{CP}^{125}$ and with $\mathrm{MS},{ }^{82}$ with none finding differences between interventions. Other intermediate outcomes reported in head-to-head comparisons of aerobic exercise programs included resting heart rate, waist circumference, fat mass, blood pressure, and lipids. These were reported in very few studies and no differences were found between interventions.

\section{Multimodal Exercises}

Three fair-quality RCTs of participants with MS compared a multimodal exercise program with either another multimodal program (2 studies), ${ }^{222,224}$ or an aerobic exercise program (1 study). ${ }^{220}$ One study reported $\mathrm{VO}_{2}$ peak, ${ }^{220,224}$ and one study reported $\mathrm{VO}_{2}$ max. ${ }^{224}$ In a study comparing resistance training plus high-intensity interval training or plus high-intensity continuous cardio training over 12 weeks, the interval training group had a greater improvement in $\mathrm{VO}_{2}$ peak (17.8\% increase vs. $7.5 \%$ increase), but a formal statistical analysis was not undertaken. ${ }^{224}$ Resting heart rate increased more with interval training (12.5\% vs. $\left.7.0 \%\right)$, and percent body fat $(-3.9 \%$ vs. $-2.5 \%)$ was reduced in both groups, slightly more in the interval training group. In the other study resistance training plus aerobic training did not improve $\mathrm{VO}_{2}$ peak more than aerobic training alone over 12 weeks. $^{220}$ This study also reported that resting heart rate and strength changes did not differ between groups. The third study compared three multimodal groups, with varying levels of aerobic and resistance training with control, but did not make statistical comparisons across the interventions groups directly. Strength outcomes were greater in the groups with more resistance or more aerobic training, compared with equal amounts of each, although the differences were small. ${ }^{222}$

A single study of children with CP compared multimodal training with gymnastics and strength training with passive gymnastics only, finding that quadriceps strength was improved more with multimodal training after 15 weeks (Table 43). ${ }^{243}$

\section{KQ2c: Harms of Immobility}

Reduction of harms due to immobility was rarely studied in trials of physical activity in MS, $\mathrm{CP}$, and SCI. Two RCTs in participants with SCI provided evidence for this subquestion.

\section{Decubitus Ulcer}

There were no trials identified that assessed the prevention, formation, or improvement of decubitus ulcer as a function of physical activity.

\section{Urinary Tract Infection}

One fair-quality RCT $(n=42)$ in participants with SCI examined the effectiveness of aerobic exercise as treatment for chronic asymptomatic bacteriuria. ${ }^{92}$ All spinal cord lesions were between $\mathrm{C} 8$ and $\mathrm{T} 12$ segments. The mean age of participants was 36 years and 17 percent were female with a mean time since injury of 4.8 years. The intervention group received 16 weeks of arm cycling, performed distance with a wheelchair, strength exercises, and muscle stretching two to three times a week in addition to usual PT sessions. The control group received only the PT sessions. Urine was collected by catheter or urine jet. The outcome was eradication of bacteriuria or continued negative urine culture versus the need for antibiotics regardless of bacteriuria. 
Chronic asymptomatic bacteriuria was identified in 24 patients (57\%) before treatment $(52 \%$ intervention vs. $62 \%$ control) and in 18 patients ( $43 \%$ ) after treatment (14\% intervention vs. $71 \%$ control), which was a statistically significant difference between groups (relative risk $0.20,95 \%$ CI 0.07 to $0.54, p<0.001$ ). The authors pointed out that there was no adjustment made for individual fluid intake, which may have impacted the findings. In patients who required antibiotics, the locus of infection was not specified and urinary culture not conducted prior to the initiation of antibiotics. No other trials of urinary tract infection were identified.

\section{Bowel Dysfunction}

Twenty-four participants with incomplete T8 to L2 SCI were randomized to RAGT or body weight-supported treadmill training in one fair-quality RCT. ${ }^{113}$ Participant mean age was 40 years, 33 percent were female, and all participants had a duration of injury of less than 6 months. Both groups underwent defecation management training before beginning walking training four times weekly for 1 month. Outcomes were enema dose needed and defecation time. After 16 training sessions, the RAGT group required a lower enema dose after training than the treadmill group when compared with baseline dose requirements $(-29 \mathrm{~mL}$ vs. $-11 \mathrm{~mL}, \mathrm{p}<0.05)$. The RAGT group also had a reduced defecation time compared with defecation times before training ( -29 min vs. $-15 \mathrm{~min}, \mathrm{p}<0.05$ ), indicating improved bowl function with RAGT. No other trials of bowel dysfunction were identified.

\section{Autonomic Dysreflexia}

No study meeting inclusion criteria for this review reported incidence of autonomic dysreflexia as a function of harm reduction with physical activity. Autonomic dysreflexia as potential harm of physical activity is discussed in KQ2e.

\section{KQ2d: Risk of Adverse Outcomes Due to MS, CP, SCI}

Nineteen studies (18 RCTs and 1 cohort study) representing 945 participants evaluated the effect of physical activity on spasticity in participants using or at risk for requiring wheelchairs (Tables 45, 46, 47). ${ }^{71,73,74,94,96,99,100,111,121,125,134,136,158,159,163,174,204,207-210,223,244,245,267}$ We did not find eligible studies that reported other relevant outcomes.

Table 45. Impact of physical activity interventions on spasticity in participants with multiple sclerosis

\begin{tabular}{|c|c|c|c|}
\hline $\begin{array}{l}\text { Author, Year } \\
\text { Intervention } \\
\text { Study Design } \\
\text { Study Quality }\end{array}$ & $\begin{array}{l}\text { Intervention } \\
\text { and Comparison }\end{array}$ & $\begin{array}{l}\text { Population } \\
\text { (Multiple Sclerosis) }\end{array}$ & Results \\
\hline $\begin{array}{l}\text { Calabro, } 2017^{96} \\
\text { Aerobic exercise } \\
\text { RCT } \\
\text { Good }\end{array}$ & $\begin{array}{l}\text { A. Lokomat-Pros (RAGT + } \\
\text { VR), } 40 \text { sessions over } 8 \text { weeks } \\
(n=20) \\
\text { B. Lokomat-Nanos (RAGT), } 40 \\
\text { sessions over } 8 \text { weeks }(n=20)\end{array}$ & $\begin{array}{l}\text { A vs. } \mathrm{B} \\
\text { Age: } 44 \text { vs. } 41 \\
\text { Female: } 65 \% \text { vs. } 60 \% \\
\text { EDSS: } 4.40 \text { vs. } 4.75\end{array}$ & $\begin{array}{l}\text { Effect size, } p \text {-value is between } \\
\text { groups: } \\
\text { MAS: }-0.01,95 \% \mathrm{Cl}-0.539 \text { to } \\
0.539, p=0.40\end{array}$ \\
\hline
\end{tabular}




\begin{tabular}{|c|c|c|c|}
\hline $\begin{array}{l}\text { Author, Year } \\
\text { Intervention } \\
\text { Study Design } \\
\text { Study Quality }\end{array}$ & $\begin{array}{l}\text { Intervention } \\
\text { and Comparison }\end{array}$ & $\begin{array}{l}\text { Population } \\
\text { (Multiple Sclerosis) }\end{array}$ & Results \\
\hline $\begin{array}{l}\text { Castro-Sanchez, } \\
2012^{71} \\
\text { Aerobic exercise } \\
\text { RCT } \\
\text { Good }\end{array}$ & $\begin{array}{l}\text { A. Ai-Chi aqua therapy with } \\
\text { Tai-Chi music, } 60 \text { sessions } \\
\text { over } 20 \text { weeks }(n=36) \\
\text { B. Relaxation exercises on } \\
\text { exercise mat without music, } 60 \\
\text { sessions over } 20 \text { weeks } \\
(n=37)\end{array}$ & $\begin{array}{l}\text { A vs. B } \\
\text { Age: } 46 \text { vs. } 50 \\
\text { Female: } 72 \% \text { vs. } 65 \% \\
\text { EDSS: } 6.3 \text { vs. } 5.9 \\
\text { PPMS: } 17 \% \text { vs. } 24 \% \\
\text { SPMS: } 25 \% \text { vs. } 32 \%\end{array}$ & $\begin{array}{l}\text { Mean (SD) baseline to } \\
\text { postintervention, p-value between } \\
\text { groups: } \\
\text { Spasm VAS: } 5(2.8) \text { to } 2(4.3) \text { vs. } \\
6(3.1) \text { to } 4(4.5), 91 \% \text { improvement } \\
\text { vs. } 10 \% \text { improvement, } p<0.05 \\
\text { The difference on the spasm VAS } \\
\text { was maintained at } 24 \text { weeks (4 } \\
\text { weeks postintervention) but there } \\
\text { was no difference between groups } \\
\text { at } 30 \text { weeks. }\end{array}$ \\
\hline $\begin{array}{l}\text { Dodd, } 2011^{204} \\
\text { Strength } \\
\text { RCT } \\
\text { Good }\end{array}$ & $\begin{array}{l}\text { A. Progressive resistance, } 20 \\
\text { sessions over } 10 \text { weeks } \\
(n=36) \\
\text { B. Social program (attention } \\
\text { control), } 10 \text { sessions over } 10 \\
\text { weeks }(n=35)\end{array}$ & $\begin{array}{l}\text { A vs. B } \\
\text { Age: } 47.7 \text { vs. } 50.4 \\
\text { Female: } 72 \% \text { vs. } 74 \% \\
\text { Ambulation index } \\
2 \text { (mild): } 47 \% \text { vs. } 54 \% \\
3 \text { (moderate): } 39 \% \text { vs. } \\
26 \% \\
4 \text { (severe): } 14 \% \text { vs. } 20 \%\end{array}$ & $\begin{array}{l}\text { Mean difference between groups: } \\
\text { MSIS-88 stiffness: }-2.4,95 \% \mathrm{Cl}- \\
0.52 \text { to } 0.5 \\
\frac{\text { MSIS-88 muscle spasms: }-2.8,95 \%}{\mathrm{CI}-5.6 \text { to } 0.03)}\end{array}$ \\
\hline $\begin{array}{l}\text { Pompa, } 2017^{94} \\
\text { Aerobic exercise } \\
\text { RCT } \\
\text { Fair }\end{array}$ & $\begin{array}{l}\text { A. RAGT, } 12 \text { sessions over } 4 \\
\text { weeks }(n=21) \\
\text { B. Conventional walking } \\
\text { training, } 12 \text { sessions over } 4 \\
\text { weeks }(n=22)\end{array}$ & $\begin{array}{l}\text { A vs. } B \\
\text { Age: } 47 \text { vs. } 50 \\
\text { Female: } 48 \% \text { vs. } 55 \% \\
\text { PPMS: } 0 \% \text { vs. } 13.6 \% \\
\text { EDSS } \\
6.62 \text { vs. } 6.50\end{array}$ & $\begin{array}{l}\text { A vs. B, mean SD, } p=\text { between } \\
\text { groups: } \\
\text { Spasticity VAS } 100 \mathrm{~mm} \text { ranged from } \\
\text { "no problem" to "very bad": } \\
5.05 \text { to } 3.40 \text { vs. } 5.31 \text { to } 5.23, \\
p=0.048 .\end{array}$ \\
\hline $\begin{array}{l}\text { Tarakci, } 2013^{223} \\
\text { Multimodal } \\
\text { exercise } \\
\text { RCT } \\
\text { Fair }\end{array}$ & $\begin{array}{l}\text { A. Exercise (e.g., range of } \\
\text { motion, strength, flexibility, } \\
\text { balance, core stability), } 36 \\
\text { sessions over } 12 \text { weeks } \\
(n=51) \\
\text { B. Waitlist control }(n=48)\end{array}$ & $\begin{array}{l}\text { A vs. } B \\
\text { Age: } 41.5 \text { vs. } 39.7 \\
\text { Female: } 67 \% \text { vs. } 63 \% \\
\text { EDSS: } 9.0 \text { vs. } 8.4 \\
\text { RRMS: } 63 \% \text { vs. } 69 \%\end{array}$ & $\begin{array}{l}\text { A vs. B, mean, } p \text {-values are } \\
\text { between groups, MAS: } \\
\text { RHipFlexors: } 1.35 \text { to } 0.68 \text { vs. } 1.52 \text { to } \\
1.65, p<0.001 \\
\text { LHipFlexors: } 1.29 \text { to } 1.00 \text { vs. } 1.52 \text { to } \\
1.65, p=0.015 \\
\frac{\text { RHamstring: } 1.35 \text { to } 0.70 \text { vs. } 1.28 \text { to }}{1.47, p<0.001} \\
\text { LHamstring: } 1.01 \text { to } 0.54 \text { vs. } 1.02 \text { to } \\
1.26, p<0.001 \\
\text { RAchilles: } 0.86 \text { to } 0.68 \text { vs. } 0.94 \text { to } \\
1.10, p=0.014 \\
\text { LAchilles: } 0.58 \text { to } .27 \text { vs. } 0.81 \text { to } \\
0.89, p<0.001\end{array}$ \\
\hline $\begin{array}{l}\text { Vermohlen, } \\
2018^{158} \\
\text { Postural control } \\
\text { RCT } \\
\text { Fair }\end{array}$ & $\begin{array}{l}\text { A. Hippotherapy, } 12 \text { sessions } \\
\text { over } 12 \text { weeks plus standard } \\
\text { care }(n=32) \\
\text { B. Standard care }(n=38)\end{array}$ & $\begin{array}{l}\text { A vs. B } \\
\text { Age (median years): } 50 \\
\text { vs. } 51 \\
\text { Female: } 90 \% \text { vs. } 73 \% \\
\text { EDSS: } 5.4 \text { vs. } 5.3\end{array}$ & $\begin{array}{l}\text { A vs. B, mean difference between } \\
\text { groups: } \\
\text { Spasticity NRS: }-0.9(95 \% \mathrm{Cl}:-1.9 \\
\text { to }-0.1), p=0.031\end{array}$ \\
\hline
\end{tabular}

Abbreviations: CI = confidence interval; EDSS = Expanded Disability Status Scale; MAS = Modified Ashworth Scale; MSIS-88 = Multiple Sclerosis Impact Scale; NRS = numeric rating scale; PPMS = primary progressive multiple sclerosis; RAGT = robotassisted gait training; RCT = randomized controlled trial; RRMS = relapsing-remitting multiple sclerosis; $\mathrm{SD}=$ standard deviation; VAS = Visual Analog Scale; VR = virtual reality 
Table 46. Impact of physical activity interventions on spasticity in children with cerebral palsy

\begin{tabular}{|c|c|c|c|}
\hline $\begin{array}{l}\text { Author, Year } \\
\text { Intervention } \\
\text { Study Design } \\
\text { Study Quality }\end{array}$ & $\begin{array}{l}\text { Intervention } \\
\text { and Comparison }\end{array}$ & $\begin{array}{l}\text { Population } \\
\text { (Cerebral Palsy) }\end{array}$ & Results \\
\hline $\begin{array}{l}\text { Adar, } 2017^{73} \\
\text { Aerobic exercise } \\
\text { RCT } \\
\text { Fair }\end{array}$ & $\begin{array}{l}\text { A. Aquatic exercise, } 30 \\
\text { sessions over } 6 \text { weeks } \\
(n=17) \\
\text { B. Land-based exercise, } 30 \\
\text { sessions over } 6 \text { weeks } \\
(n=15)\end{array}$ & $\begin{array}{l}\text { A vs. B } \\
\text { Age: } 10.1 \text { vs. } 9.3 \\
\text { Female: } 53 \% \text { vs. } 40 \% \\
\text { GMFCS median } \\
\text { (range): } 2 \text { (1-4) vs. } 2 \text { (1- } \\
\text { 4) }\end{array}$ & $\begin{array}{l}\text { Median pre-post } p \text {-values on MAS } \\
\text { for each treatment arm } \\
\text { Median pre-post } p \text {-values on MAS } \\
\text { for each treatment arm } \\
\text { RKneeFlexors } \\
\text { Location: Aquatics } 0.039 \text {, Land } \\
0.008 \\
\text { LKneeFlexors } \\
\text { Location: Aquatics } 0.003 \text {, Land } \\
0.003 \\
\text { RAnkleFlexors } \\
\text { Location: Aquatics } 0.005 \text {, Land } \\
0.001 \\
\text { LAnkleFlexors } \\
\text { Location: Aquatics } 0.046 \text {, Land } \\
0.046 \\
\text { RHipAdductors } \\
\text { Location: Aquatics } 0.025 \text {, Land } \\
\text { LHipAdductors } \\
\text { Location: Aquatics } 0.003 \text {, Land } \\
0.013\end{array}$ \\
\hline $\begin{array}{l}\text { Chrysagis, } \\
2012^{121} \\
\text { Aerobic exercise } \\
\text { RCT } \\
\text { Fair }\end{array}$ & $\begin{array}{l}\text { A. Treadmill training, } 36 \\
\text { sessions over } 12 \text { weeks } \\
(n=11) \\
\text { B. Conventional PT, } 36 \\
\text { sessions over } 12 \text { weeks } \\
(n=11)\end{array}$ & $\begin{array}{l}\text { A vs. } B \\
\text { Age: } 15.90 \text { vs. } 16.09 \\
\text { Female: } 45 \% \text { vs. } 36 \% \\
\text { Ambulatory: } 100 \%\end{array}$ & 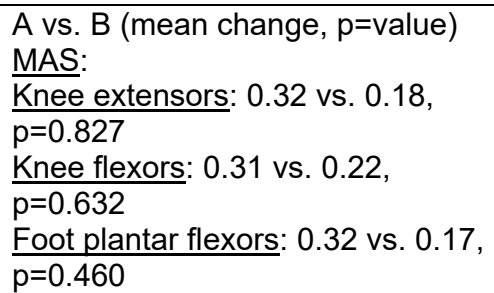 \\
\hline $\begin{array}{l}\text { El-Shamy, } \\
2018^{181} \\
\text { Postural control } \\
\text { RCT } \\
\text { Fair }\end{array}$ & $\begin{array}{l}\text { A. Robotic upper-limb } \\
\text { therapy, } 36 \text { sessions over } 12 \\
\text { weeks }(n=15) \\
\text { B. Conventional therapy of } \\
\text { stretching and strength } \\
\text { exercises, } 36 \text { sessions over } \\
12 \text { weeks }(n=15)\end{array}$ & $\begin{array}{l}\text { A vs. B } \\
\text { Age: } 6.9 \text { vs. } 6.8 \\
\text { Female: } 40 \% \text { vs. } 27 \% \\
\text { MACS I: } 33 \% \text { vs. } 40 \% \\
\text { MACS II: } 53 \% \text { vs. } 40 \% \\
\text { MACS III: } 13 \% \text { vs. } 20 \%\end{array}$ & $\begin{array}{l}\text { Mean difference between groups: } \\
\text { Spasticity MAS: }-0.4,95 \% \mathrm{Cl}-0.8 \\
\text { to }-0.1, p<0.05\end{array}$ \\
\hline $\begin{array}{l}\text { Johnston, } \\
2011^{125} \\
\text { Aerobic exercise } \\
\text { RCT } \\
\text { Fair }\end{array}$ & $\begin{array}{l}\text { A. Partial BWS treadmill } \\
\text { training with } 20 \text { sessions over } \\
2 \text { weeks, then } 50 \text { sessions at } \\
\text { home over } 10 \text { weeks ( } n=14) \\
\text { B. Individualized strength- } \\
\text { based PT, } 20 \text { sessions over } \\
2 \text { weeks, then } 50 \text { session at } \\
\text { home over } 10 \text { weeks }(n=12)\end{array}$ & $\begin{array}{l}\text { A vs. B } \\
\text { Age: } 9.6 \text { vs. } 9.5 \\
\text { Female: } 50 \% \text { vs. } 42 \% \\
\text { GMFCS II: } 7 \% \text { vs. } 8 \% \\
\text { GMFCS III: } 64 \% \text { vs. } \\
50 \% \\
\text { GMFCS IV: } 29 \% \text { vs. } \\
42 \%\end{array}$ & $\begin{array}{l}\text { Mean difference between groups, } \\
\mathrm{p}=\text { between groups } \\
\text { KinCom computerized } \\
\frac{\text { dynamometer: }}{\text { Plantar Flexor Spasticity }(\mathrm{J} / \mathrm{O} / \mathrm{s}):} \\
-0.0003, \mathrm{p}=0.75 \\
\frac{\text { Knee flexor spasticity }(\mathrm{J} / \mathrm{O} / \mathrm{s}):}{-0.0026, \mathrm{p}=0.59}\end{array}$ \\
\hline $\begin{array}{l}\text { Lai, } 2015^{74} \\
\text { Aerobic exercise } \\
\text { Cohort study } \\
\text { Fair }\end{array}$ & $\begin{array}{l}\text { A. Aquatic therapy, } 24 \\
\text { sessions over } 12 \text { weeks, } \\
\text { rehab exercises, } 24-36 \\
\text { sessions over } 12 \text { weeks } \\
(n=11) \\
\text { B. Rehab exercises, } 24-36 \\
\text { sessions over } 12 \text { weeks } \\
(n=13)\end{array}$ & $\begin{array}{l}\text { A vs. B } \\
\text { Age: } 7.6 \text { vs. } 6.6 \\
\text { Female: } 64 \% \text { vs. } 31 \% \\
\text { GMFCS I: } 9 \% \text { vs. } 8 \% \\
\text { GMFCS II: } 36 \% \text { vs. } 46 \% \\
\text { GMFCS III: } 27 \% \text { vs. } \\
23 \% \\
\text { GMFCS IV: } 27 \% \text { vs. } \\
23 \%\end{array}$ & $\begin{array}{l}\text { A vs. B (ANCOVA p-values) } \\
\text { MAS: } \\
\text { Ankle: } 0.614 \\
\text { Knee: } 1.000 \\
\text { Wrist: } 1.000 \\
\text { Elbow: } 1.000\end{array}$ \\
\hline
\end{tabular}




\begin{tabular}{|c|c|c|c|}
\hline $\begin{array}{l}\text { Author, Year } \\
\text { Intervention } \\
\text { Study Design } \\
\text { Study Quality }\end{array}$ & $\begin{array}{l}\text { Intervention } \\
\text { and Comparison }\end{array}$ & $\begin{array}{l}\text { Population } \\
\text { (Cerebral Palsy) }\end{array}$ & Results \\
\hline $\begin{array}{l}\text { Lucena-Anton, } \\
2018^{163} \\
\text { Postural control } \\
\text { RCT } \\
\text { Fair }\end{array}$ & $\begin{array}{l}\text { A. Hippotherapy, } 12 \text { sessions } \\
\text { over } 12 \text { weeks plus } \\
\text { physiotherapy, } 24 \text { sessions } \\
\text { over } 12 \text { weeks }(n=22) \\
\text { B. PT, } 24 \text { sessions over } 12 \\
\text { weeks }(n=22)\end{array}$ & $\begin{array}{l}\text { A vs. B } \\
\text { Age: } 9.5 \text { vs. } 8.2 \\
\text { Female: } 41 \% \text { vs. } 32 \% \\
\text { Nonambulatory: } 100 \%\end{array}$ & $\begin{array}{l}\text { A vs. } B, \text { mean (baseline to } \\
\text { posttreatment), } p=\text { between groups } \\
\text { MAS: } \\
\text { Left Abductors: } 2.77 \text { to } 2.50 \text { vs. } \\
2.59 \text { to } 2.54, p=0.040 \\
\frac{\text { Right Abductors: } 2.22 \text { to } 1.77 \text { vs. }}{2.40 \text { to } 2.31, p=0.047}\end{array}$ \\
\hline $\begin{array}{l}\text { Qi, 2018a } 210 \\
\text { Strength } \\
\text { RCT } \\
\text { Fair }\end{array}$ & $\begin{array}{l}\text { A. Strength exercises }+ \\
\text { neuromuscular electrical } \\
\text { stimulation, } 30 \text { sessions over } \\
6 \text { weeks }(n=50) \\
\text { B. Neuromuscular electrical } \\
\text { stimulation, } 30 \text { sessions over } \\
6 \text { weeks }(n=50)\end{array}$ & $\begin{array}{l}\text { A vs. B } \\
\text { Age: } 5.8 \text { vs. } 6.0 \\
\text { Female: } 48 \% \text { vs. } 46 \% \\
\text { Comprehensive } \\
\text { Spasticity Scale: } \\
12.0 \text { vs. } 12.3\end{array}$ & $\begin{array}{l}\text { Mean difference between groups: } \\
\text { CSS: } 1.6,95 \% \mathrm{Cl} 0.33 \text { to } 2.87 \text {, } \\
\mathrm{p}=0.01\end{array}$ \\
\hline $\begin{array}{l}\text { Scholtes, } \\
2010^{209} \\
\text { Scholtes, } \\
2012^{207} \\
\text { Scholtes, } \\
2008^{208} \\
\text { Strength } \\
\text { RCT } \\
\text { Fair }\end{array}$ & $\begin{array}{l}\text { A. Progressive resistance, } 36 \\
\text { sessions over } 12 \text { weeks } \\
(n=24) \text { : } \\
\text { B. Usual care }(n=25)\end{array}$ & $\begin{array}{l}\text { A vs. B } \\
\text { Age: } 10.33 \text { vs. } 10.25 \\
\text { Female: } 33 \% \text { vs. } 50 \% \\
\text { Ambulatory: } 100 \\
\text { GMFCS I: } 54 \% \text { vs. } 48 \% \\
\text { GMFCS II: } 33 \% \text { vs. } 36 \% \\
\text { GMFCS III: } 13 \% \text { vs. } \\
16 \%\end{array}$ & $\begin{array}{l}\text { A vs. B, mean (SD), } p=\text { between } \\
\text { groups } \\
\text { Spasticity }(0-5, \text { higher=greater } \\
\text { spasticity): } 1.00(1.32) \text { vs. } 2.00 \\
\text { (1.32) (baseline) } \\
2.00 \text { (1.11) vs. } 1.50(1.10) \\
\text { (postintervention) } \\
\text { Effect Size: } 0.46,95 \% \mathrm{Cl}-0.34 \text { to } \\
1.26, p=0.26\end{array}$ \\
\hline $\begin{array}{l}\text { Wu, } 2017 b^{99} \\
\text { Aerobic exercise } \\
\text { RCT } \\
\text { Fair }\end{array}$ & $\begin{array}{l}\text { A. RAGT (resistive force), } 18 \\
\text { sessions over } 6 \text { weeks } \\
(n=11) \\
\text { B. Treadmill only training, } 18 \\
\text { sessions over } 6 \text { weeks } \\
(n=10)\end{array}$ & $\begin{array}{l}\text { A vs. B } \\
\text { Age: } \\
11.3 \text { vs. } 10.5 \\
\text { Female: } 45 \% \text { vs. } 40 \% \\
\text { Nonwhite: } 54.5 \% \text { vs. } \\
50 \% \\
\text { GMFCS I: } 9 \% \text { vs. } 17 \% \\
\text { GMFCS II: } 55 \% \text { vs. } 25 \% \\
\text { GMFCS III: } 27 . \text { vs. } 42 \% \\
\text { GMFCS IV: } 9 \% \text { vs. } 17 \% \\
\end{array}$ & $\begin{array}{l}\text { A vs. B, mean (SD) } \\
\text { MAS } \\
\text { (Baseline vs. } 6 \text { weeks vs. } 8 \text { weeks } \\
\text { followup) } \\
0.62(0.46) \text { to } 0.67(0.60) \text { to } 0.41 \\
(0.38), p=0.18, \text { vs. } \\
0.65(0.36) \text { to } 0.48(0.47) \text { to } 0.58 \\
(0.44), p=0.19\end{array}$ \\
\hline $\begin{array}{l}\text { Wu, } 2017 a^{100} \\
\text { Aerobic exercise } \\
\text { RCT } \\
\text { Fair }\end{array}$ & $\begin{array}{l}\text { A. RAGT with resistance, } 18 \\
\text { sessions over } 6 \text { weeks } \\
(n=10) \\
\text { B. RAGT with assistance, } 18 \\
\text { sessions over } 6 \text { weeks } \\
(n=10)\end{array}$ & $\begin{array}{l}\text { A vs. B } \\
\text { Age: } 10.6 \text { vs. } 10.8 \\
\text { Female: } 50 \% \text { vs. } 45 \% \\
\text { GMFCS I: } 8 \% \text { vs. } 0 \% \\
\text { GMFCS II: } 42 \% \text { vs. } 45 \% \\
\text { GMFCS III: } 42 \% \text { vs. } \\
36 \% \\
\text { GMFCS IV: } 8 \% \text { vs. } 18 \%\end{array}$ & $\begin{array}{l}\text { Mean (SD), } p=\text { between groups } \\
\text { postintervention: } \\
\frac{\mathrm{MAS}}{0.65}(0.57) \text { to } 0.83(0.66) \text { to } 0.63 \\
(0.39) \text { followup vs. } 0.85(0.67) \text { to } \\
0.68(0.56) \text { to } 0.87(0.55) \text { followup } \\
\text { MD } 0.35,95 \% \mathrm{Cl}-0.19 \text { to } 0.89 \text {, } \\
p=0.21\end{array}$ \\
\hline
\end{tabular}

Abbreviations: ANCOVA = analysis of covariance; $\mathrm{BWS}=$ body weight supported; $\mathrm{CSS}=$ Comprehensive Spasticity Scale; $\mathrm{CI}=$ confidence interval; GMFCS = Gross Motor Function Classification System; IQR = interquartile range; MAS=Modified Ashworth Scale; MACS = manual ability classification system; $\mathrm{MD}=$ mean difference; $\mathrm{PT}=$ physical therapy; RAGT $=$ robotassisted gait training; $\mathrm{RCT}=$ randomized controlled trial; $\mathrm{SD}=$ standard deviation; $\mathrm{VR}=$ virtual reality 
Table 47. Impact of physical activity interventions on spasticity in participants with spinal cord injury

\begin{tabular}{|c|c|c|c|}
\hline $\begin{array}{l}\text { Author, Year } \\
\text { Intervention } \\
\text { Study Design } \\
\text { Study Quality }\end{array}$ & $\begin{array}{l}\text { Intervention } \\
\text { and Comparison }\end{array}$ & $\begin{array}{l}\text { Population } \\
\text { (Spinal Cord Injury) }\end{array}$ & Results \\
\hline $\begin{array}{l}\text { Galea, } 2018^{245} \\
\text { Multimodal } \\
\text { exercise } \\
\text { RCT } \\
\text { Fair }\end{array}$ & $\begin{array}{l}\text { A. Whole body strength }+ \\
\text { aerobics, } 36 \text { sessions over } 12 \\
\text { weeks }(n=60) \\
\text { B. Upper body strength }+ \\
\text { aerobics, } 36 \text { sessions over } 12 \\
\text { weeks }(n=56)\end{array}$ & $\begin{array}{l}\text { A vs. B } \\
\text { Age: } 40.1 \text { vs. } 42.8 \\
\text { Female: } 15 \% \text { vs. } 16 \% \\
\text { ASIA A: } 48 \% \text { vs. } 50 \% \\
\text { ASIA B: } 15 \% \text { vs. } 14 \% \\
\text { ASIA C: } 12 \% \text { vs. } 9 \% \\
\text { ASIA D: } 25 \% \text { vs. } 27 \% \\
\\
\text { C2-C8: } 48 \% \text { vs. } 59 \% \\
\text { T1-T6: } 30 \% \text { vs. } 23 \% \\
\text { T7-T12: } 22 \% \text { vs. } 18 \%\end{array}$ & $\begin{array}{l}\text { A vs. } B \\
1.8(1.1) \text { vs. } 1.5(1) \text { (baseline) } \\
1.6(1.1) \text { vs. } 1.8(1.1), M D-0.25 \\
(95 \% \mathrm{Cl}-0.61 \text { to } 0.1), p=0.163\end{array}$ \\
\hline $\begin{array}{l}\text { Kapadia, } 2014^{136} \\
\text { Aerobic exercise } \\
\text { RCT } \\
\text { Fair }\end{array}$ & $\begin{array}{l}\text { A. BWS treadmill walking with } \\
\text { FES, } 48 \text { sessions over } 16 \\
\text { weeks }(n=17) \\
\text { B. Aerobic and resistance } \\
\text { training, } 48 \text { sessions over } 16 \\
\text { weeks ( } n=17 \text { ) }\end{array}$ & $\begin{array}{l}\text { A vs. B } \\
\text { Age: } 56.6 \text { vs. } 54.1 \\
\text { Female: } 18 \% \text { vs. } 29 \% \\
\text { Tetraplegia: } 82 \% \text { vs. } 71 \% \\
\text { UEMS: } 38.3 \text { vs. } 37.5 \\
\text { LEMS: } 30.4 \text { vs. } 27.9\end{array}$ & $\begin{array}{l}\text { MAS: No between group differences } \\
\text { in MAS involving the hip, knee, and } \\
\text { ankle joints. }\end{array}$ \\
\hline $\begin{array}{l}\text { Kumru, } 2016^{111} \\
\text { Aerobic exercise } \\
\text { RCT } \\
\text { Fair }\end{array}$ & $\begin{array}{l}\text { A. RAGT with rTMS, } 20 \\
\text { sessions over } 4 \text { weeks }(n=15) \\
\text { B. RAGT with Sham rTMS, } 20 \\
\text { sessions over } 4 \text { weeks }(n=16)\end{array}$ & $\begin{array}{l}\text { A vs. B } \\
\text { Age: } 51 \text { vs. } 49 \\
\text { Female: } 33 \% \text { vs. } 13 \% \\
\text { ASIA A; } 0 \% \\
\text { ASIA B: } 0 \% \\
\text { ASIA C: } 80 \% \text { vs. } 88 \% \\
\text { ASIA D: } 20 \% \text { vs. } 22 \% \\
\text { Cervical or Thoracic: } \\
100 \% \\
\text { Cervical: } 53 \% \text { vs. } 38 \%\end{array}$ & $\begin{array}{l}\text { A vs. B } \\
\text { Mean difference between groups: } \\
\text { MAS: }-0.20,95 \% \mathrm{Cl}-0.94 \text { to } 0.54 \text {, } \\
p=0.59\end{array}$ \\
\hline
\end{tabular}

Abbreviations: ASIA = American Spinal Injury Association Impairment Scale; BWS = bodyweight supported; CI = confidence interval; FES = functional electrical stimulation; LEMS = Lower Extremity Motor Score; MAS = Modified Ashworth Scale; RAGT = robot-assisted gait training; rTMS = transcranial magnetic stimulation; UEMS = Upper Extremety Motor Score

Six studies enrolled participants with MS ( $\mathrm{n}=396)$ (Table 45), ten enrolled children or adolescents with CP ( $\mathrm{n}=457)$ (Table 46), and three enrolled participants with SCI ( $\mathrm{n}=181)$ (Table 47). The range of mean ages enrolled in studies of participants with $\mathrm{CP}$ was 4.7 to 16 years, in studies of participants with MS was 39.7 to 51 years, and in participants with SCI was 40.1 years to 56.6 years. The proportion of females enrolled ranged from a low of 15 percent in a SCI study, to a high of 90 percent in a study of participants with MS. Race was reported in only two studies, both of children with CP (nonwhite race 52\% and $48 \%$ ). ${ }^{99,100}$ Reporting of characteristics at baseline related to spasticity or overall mobility varied according to condition. In studies of participants with CP, most were in GMFCS level II or III, with fewer participants in levels I and IV, except for the study of the youngest participants (mean age 4.8 years) where over 73 percent were in GMFCS level V. In the studies of participants with MS, baseline disability ranged from 4.4 to 9 on the EDSS (range of scale 0 to 10 with higher values representing more problems walking). The studies of participants with spinal cord injuries varied in the way disability was reported and the level of disability. Two of the RCTs enrolled mostly participants ( $100 \%$ and 77\%) at the ASIA scale C and D level (motor incomplete, motor function preserved at some level), ${ }^{11,134-136}$ while the third enrolled participants at each ASIA score level with more than 60 percent at level A (complete impairment, no sensory or motor function) or B (sensory 
incomplete, but motor function complete). ${ }^{244,245}$ The proportion of participants using wheelchairs, full or part-time, was not explicitly reported.

Interventions studied and comparisons made varied widely. Eleven studies evaluated aerobic interventions s $^{71,73,74,94,96,99,100,111,121,125,136}$ (2 vs. usual care, including standard PT), four evaluated strength interventions ${ }^{181,204,207-210}$ (1 vs. usual care, 1 vs. attention control), two evaluated balance interventions $^{158,163,267}$ (all vs. usual care), and two evaluated multimodal interventions (1 vs. usual care). In the 8 of 18 studies categorized as comparing interventions with usual care, the control groups consisted of standard PT regimens, waitlist, "attention control" (nonphysical activity social interactions) and unspecified "usual care" interventions. The most commonly reported outcome of spasticity was the Modified Ashworth Scale (MAS) (range 0-4, measure of resistance on passive soft tissue stretching), used in 10 studies. $^{73,74,96,99,100,111,121,136,163,181}$

Seven of 19 studies found a significant difference between groups. In comparisons to usual care control groups, balance interventions in four RCTs (1 of hippotherapy in participants with $\mathrm{CP},{ }^{158} 1$ of hippotherapy in participants in $\mathrm{MS},{ }^{163} 1$ of robotic upper-limb therapy in children with $\mathrm{CP},{ }^{181}$ and 1 of a multimodal therapy in participants with $\mathrm{MS}^{223}$ ) significantly improved spasticity. In head-to-head comparisons, robot-assisted treadmill training improved spasticity more than nonassisted treadmill training, ${ }^{99}$ aquatic Tai Chi with music improved spasticity more than relaxation exercises on land without music, ${ }^{71}$ and neuromuscular electrical stimulation with strength training improved spasticity more than neuromuscular electrical stimulation alone. ${ }^{210}$ All other comparisons did not find differences in the effect on spasticity. The details of these studies are summarized below.

Because the studies were small ( $\mathrm{n}=11$ to 116$)$, most were fair quality, and for each population-intervention-comparison there was only a single study, the majority of this evidence is insufficient to draw conclusions. The two exceptions were a good-quality RCT of progressive resistance training compared with a social program (attention control) that found no difference in spasticity between groups in participants with MS, providing low-strength evidence of no clear benefit of strength training on spasticity, ${ }^{204}$ and a good-quality RCT, also in participants with MS, of aquatic-based Ai-Chi versus relaxation exercises on land that provided low-strength evidence of benefit with aquatics on a spasm visual analogue

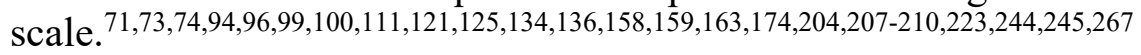

\section{Comparisons With Usual Care}

\section{Aerobic Exercises Versus Usual Care}

Two fair-quality studies compared an aerobic exercise with usual care in participants with $\mathrm{CP}$ over 12 weeks, with neither finding a significant benefit on measures of spasticity. ${ }^{74,121}$ Both were small studies ( $\mathrm{n}=22$ total), with one being an RCT of treadmill training in adolescents with CP. ${ }^{121}$ Evaluation of knee extensors and flexors and foot flexors showed small differences $(<0.5$ difference on a 5-point scale) favoring the intervention numerically, which did not reach statistical significance. The other study was a cohort study that evaluated aquatic therapy compared with standard rehabilitation exercises, with adjusted analysis not finding differences at the ankle, knee, wrist, or elbow. ${ }^{74}$

\section{Strength Exercises Versus Usual Care}

Two RCTs evaluated the effect of progressive resistance training on spasticity, with neither finding a significant benefit. ${ }^{204,207-209}$ In a small good-quality RCT of participants with MS 
$(\mathrm{n}=71)$, an attention control social program was used as the comparison group intervention. Using the Multiple Sclerosis Spasticity Scale-88, muscle stiffness and muscle spasms were not significantly improved with strength training (Table 45). ${ }^{204}$ A small fair-quality trial $(n=49)$ in children with CP reported small improvement in the intervention group compared with the control group, but the difference between groups was not significant (Table 46). Spasticity was measured using goniometry to identify the joint angle at which a sudden increase in muscle tone occurred during a fast passive stretch ( 0 -5-point scale, 5 being the worst). Another small $(n=30)$ fair-quality RCT in children with CP assessed robotic upper limb therapy over 12 weeks, compared with conventional therapy and found that spasticity was improved with the nintervention, ${ }^{181}$ based on the MAS (Table 46).

\section{Balance Exercises Versus Usual Care}

Two fair-quality RCTs evaluated hippotherapy over 12 weeks. One was of participants with $\mathrm{CP}(\mathrm{n}=44),{ }^{163}$ and the other in participants with MS $(\mathrm{n}=70) .{ }^{158}$ In the study of children with $\mathrm{CP}$, none of whom were ambulatory, there was a significant difference in spasticity between groups on the MAS on both the left and right adductors. ${ }^{163}$ The magnitude of difference was small $(0.22$ and 0.36 on a 5-point scale), but the effect size was considered medium to large (Cohen's $\mathrm{d}=$ 0.638 and 0.646 , respectively). In the other RCT of mostly women (82\%) with MS with mean baseline EDSS of 5.4, 12 weeks of hippotherapy also had significantly lower spasticity based on a $0-10$ numeric rating scale (numeric rating scale $-0.9,95 \% \mathrm{CI}-1.9$ to $-0.1, \mathrm{p}=0.031$ ). ${ }^{158}$ The magnitude of effect is small, but larger than seen in other studies.

\section{Multimodal Exercises Versus Usual Care}

A single small fair-quality RCT of participants with MS, whose mean baseline EDSS score was 8.7, evaluated an exercise program that included range of motion, strength, flexibility, balance, and core stability exercises over 12 weeks compared with a waitlist control group. ${ }^{223}$ Based on the MAS, significant improvement was seen at all testing points compared with control (Table 45). The magnitude of difference varied based on the location of testing (e.g. difference of 0.7 at right hip flexor vs. 0.39 at left Achilles-tendon on a 5-point scale), but the control group value deteriorated slightly over the 12 -week period.

\section{Head-to-Head Comparisons}

\section{Aerobic Exercises}

In participants with MS (Table 45), one RCT compared RAGT with a conventional walking program. ${ }^{94}$ In this RCT of participants with baseline EDSS scores of 6.6, using a Visual Analog Scale (VAS) (0-100), RAGT improved spasticity more than conventional walking exercises $(-1.65$ vs. $0.08, p=0.048)$, but the difference is small and the spasticity level was low in both groups at baseline (5.05 and 5.31). In another RCT (good quality), the Lokomat ${ }^{\circledR}$ RAGT was compared with and without a virtual reality program $(n=40) .{ }^{96}$ At baseline, participants' disability was less than the previous study (EDSS 4.6). At 8 weeks, there was not a significant improvement from baseline in either group, nor was the difference between groups significant based on the MAS. In a third study (good quality, $n=73$ ), participants with mean EDSS at baseline of 6.1 were assigned to aquatic Ai Chi with music or to land-based relaxation exercises without music. Based on a 0 to 100 VAS scale, spasticity was improved more in the aquatic 
group after 20 weeks (Table 46). ${ }^{71}$ The difference between groups remained significant at week 24 (4 weeks postintervention) but not at week 30 .

In children with CP (Table 46), four fair-quality RCTs compared one form of aerobic exercise with another and reported on spasticity. ${ }^{73,99,100,125}$ Three studies found no benefit of either intervention on measures of spasticity, one comparing partial body weight supporttreadmill training with individualized strength training for 12 weeks $(n=26),{ }^{125}$ one comparing RAGT with nonassisted treadmill training over 6 weeks with an 8-week followup $(n=21),{ }^{99}$ and one comparing RAGT with resistance or assistance (Table 46). ${ }^{100}$ These studies were very small, such that differences may not have been found due to inadequate statistical power. The fourth RCT compared an aquatic exercise program with a land-based program for 6 weeks $(n=32)$, finding statistically significant improvement in both groups. ${ }^{73}$ Statistical comparisons were not made between groups, and data were not provided to conduct such calculations.

In participants with incomplete SCI, two fair-quality RCTs compared aerobic interventions with each other, but neither found a difference in the effect on spasticity (Table 47). ${ }^{111,134-136}$ The first compared functional electric stimulation in body weight supported treadmill training with an aerobic and resistance training program over 16 weeks $(n=34) .{ }^{136}$ At baseline 78 percent were ambulatory. There was no difference in spasticity at the end of the study in either group, or between groups. In the second RCT $(n=31)$, RAGT with and without rTMS was evaluated over 4 weeks, using a sham rTMS for the control group. ${ }^{111}$ At baseline, 84 percent were ASIA level C for impairment (motor incomplete). Neither group had significant improvements in spasticity at 4 weeks, nor was there a significant difference between groups.

\section{Strength Exercises}

A fair-quality RCT in children with CP compared neuromuscular electrical stimulation with and without strength training exercises over 6 weeks $(n=100) .{ }^{210}$ Mean age was 6 years, and the Comprehensive Spasticity Scale score was 12.1 (mean) at baseline (scores of 10-12 defined as moderate spasm). After 6 weeks, while the score was reduced to the level of "mild spasm" in both groups, the combined neuromuscular electrical stimulation and strength training group had a significantly greater reduction, resulting in a mean score of 7.6 compared with a mean score of 9.5 in the control group $(\mathrm{p}<0.05)$.

\section{Multimodal Exercises}

A fair-quality RCT of participants with incomplete SCI $(n=116)$ compared whole body strength and aerobic training (locomotor training, functional electrical stimulation-assisted leg cycling, and trunk and lower extremity exercises) with upper body strength and aerobic training

only. ${ }^{244,245}$ In this study, 49 percent of participants had ASIA scale level A impairment at baseline (complete SCI). Spasticity was measured using the self-reported Penn Spasm Frequency Score, rated 0 (no spasms) to 4 ( 4 being spontaneous spasms occurring $>10 /$ hour) over the past week. The baseline mean score for whole body strength and aerobic training was 1.8, and for upper body strength and aerobic training was 1.5 . There was no improvement and no difference between groups after 12 weeks $(p=0.163)$. 


\section{KQ2e: Physical Activity Harms}

Most included studies of physical activity in participants with MS, CP, and SCI did not assess or did not report adverse events or harms experienced by study participants. This included greater than 60 percent of studies in participants with SCI and CP and greater than 40 percent of studies in participants with MS. A small proportion of trials (11\%) reported that there were no harms, adverse events, serious adverse events, and/or study withdrawals due to adverse events.

In studies that reported adverse events, sometimes the events were not broken down into intervention versus control groups. That is, some studies reported that many or most study participants experienced "sore muscles" or "aches and pains," contributing to the challenge of determining which interventions are associated with which harms. Overuse injuries were rarely described as an "overuse" injury but musculoskeletal issues (i.e., joint pain, muscle soreness, sprains, muscle cramps) were frequently cited without being associated with a particular intervention.

Other potential harms that may be associated with physical exercise and that are especially concerning include autonomic dysreflexia (that could be fatal), fractures, and falls.

One fair-quality RCT $(n=116)$ in participants with $\mathrm{C} 2$ to T12 SCI (49\% ASIA Impairment Scale [AIS]-A, 26\% AIS-D) randomized participants to intensive whole-body exercises versus intensive upper-body exercise for 12 weeks (36 sessions). ${ }^{244,245}$ Whole-body exercises included locomotor training, FES-assisted leg cycling, and assisted and resisted exercises to strengthen the trunk, upper limbs, and lower limbs. Upper-body exercises involved arm cycling and upper-body strength exercises, such as chest press and biceps/triceps curls.

This trial ${ }^{245}$ systematically monitored participants for adverse events and recorded 719 total such events (404 with full-body exercise and 309 with upper-body exercise). In the full-body exercise group $(n=60)$, there were 26 instances of autonomic dysreflexia ( 3 were considered serious) and 5 episodes of dizziness/nausea (possibly related to autonomic dysreflexia). In the upper-body exercise group $(n=56)$, there were 7 episodes of autonomic dysreflexia and 15 episodes of headache (possibly related to autonomic dysreflexia). Data on the number of participants who experienced each adverse event were not provided. Although only three episodes of autonomic dysreflexia were rated as serious by study personnel, this trial demonstrates the need for cardiovascular monitoring of exercise participants, especially participants with SCI and those experiencing intense interventions to minimize cardiovascular risk. This trial provides low-strength evidence of increased episodes of dysreflexia with more intense exercise versus less intense exercise, in this case whole body exercise versus upper body exercises.

In addition to episodes of autonomic dysreflexia, one participant in the full-body intervention group above experienced bilateral insufficiency fractures of the medial femoral condyle and tibial plateau. ${ }^{245}$ Across all included studies reporting adverse events, fracture was one of the most commonly cited specific harms and occurred in at least eight trials. However, not all fractures were study related; fractures also occurred in participants assigned to various exercise groups (e.g., aerobics, aquatics, cycling, hippotherapy) in addition to control groups. There was no indication of increased fracture risk with any particular exercise intervention versus another intervention or versus a control intervention, but evidence was limited.

Six trials reported the occurrence of one or more falls. Falls were reported in hippotherapy, ${ }^{158,164,165}$ Pilates, ${ }^{200,201}$ balance training, ${ }^{141,143}$ and usual physiotherapy. ${ }^{200,201}$ Although one fall from a horse resulted in a fractured humerus, ${ }^{165}$ data were too sparse to determine if falls were more strongly associated or if the consequences of falls were more severe 
with one intervention versus a no exercise or usual care control (RR 3.74, 95\% CI 0.80 to 17.45 , $\mathrm{p}=0.093$ ) (SOE: Insufficient).

\section{KQ2f: Physical Activity Characteristics}

Three RCTs provided evidence for this KQ and all studies enrolled participants with MS $(\mathrm{n}=397) .{ }^{59,192,193,197}$ Details for each RCT are provided below.

\section{Hospital-Based Versus Home-Based Calisthenics}

Two fair-quality RCTs ( $n=83)$ enrolled participants with MS and compared hospital or center-based exercise with home-based exercise. ${ }^{59,229}$ In one trial, participants were, on average, 33 years old (range 18 to 50 years), were 56 percent female, and had a mean EDSS score of 3.5. ${ }^{59}$ All participants received a 12-week, 36-session exercise program that consisted of calisthenics 3 days per week and relaxation 2 days per week. Sessions included 15 minutes of warmup, 20 minutes of intensive calisthenics, and 15 minutes of cool down and relaxation. In the hospital group, exercises were conducted by a physiatrist. Participants in the control group were to conduct the same exercises at home with daily telephone followup. Both groups significantly improved on the 10MWT from baseline with no differences between groups $(p=0.442)$. Quality of life was also significantly improved in both groups but was not different between groups $(p=0.146)$. Both the hospital-based and the home-based group improved significantly on the BBS compared with baseline, but the hospital-based group saw a greater improvement $(\mathrm{p}=0.031)$. At baseline, 62.5 percent of participants had depressive symptoms and 52.7 percent had symptoms of anxiety. At the conclusion of the 12-week exercise program, both groups saw statistically significant improvement on both the HADS-D (depression) and the HADS-A (anxiety) scales, but the hospital-based exercise group improved to a greater degree than the home-based exercise group $(\mathrm{p}<0.001)$.

In the second trial, participants were 51 years of age on average, 76 percent were female, and most required unilateral $(36 \%)$ or bilateral $(22 \%)$ ambulation aids. ${ }^{22}$ The intervention group received sixteen 60-minute group exercise sessions aimed at improving gait and balance. Sessions were led by physiotherapists and exercises were performed at moderate to high intensity. The control group performed similar exercises at home. Gait speed, walking endurance, and balance did not improve over time and there were no statistically significant differences between the groups on the 10MWT, 6MWT, or the BBS ( $p>0.05$ for all comparisons).

Findings from these trials are mixed and suggest that for some outcomes, home exercise with close followup may yield similar improvements as hospital-based interventions. In one trial, ${ }^{59}$ depression scores improved to a greater degree with hospital-based exercises, but confirmation of these findings are needed to determine when home-based, unobserved activity provides similar benefits to clinic or hospital-based physical activity interventions.

\section{Physiotherapist-Led Versus Fitness-Instructor-Led Exercise}

One poor-quality RCT in participants with MS randomized individuals with minimal gait impairment (Guy's Neurological Disability Scale [GNDS] mobility section score of 0 to 2) to group exercise led by a physiotherapist $(n=63)$ or by a fitness instructor $(n=67) .{ }^{192,193}$ The mean age of participants was 51 years, 73 percent were female, and 52 percent had RRMS. Only the physiotherapist-led exercise program was predefined and consisted of aerobic and strength exercises weekly for 10 weeks. Participants were also advised to continue walking, cycling, 
swimming, or running at home for 30 minutes twice a week, and from week 6 on, an additional self-directed strength and aerobic session was added.

The fitness instructor-led program was not predefined and was conducted at 11 different sites across Ireland to reflect typical community programs available to MS patients. Most weekly sessions consisted of a combination of aerobic and strength training with no additional, selfdirected training at home specified.

Both groups saw statistically significant improvement on the MSIS-29 physical and psychological components and on the 6MWT from baseline measurements. However, the groups were not compared with each other but were each compared with a control group $(n=49)$ that was instructed not to change exercise habits. Both the physiotherapist-led and the fitness instructorled groups saw greater improvement over the control group on all measures. Due to high attrition, this trial provides limited support for the effectiveness of aerobic and strength focused community-based programs in MS patients with no or low levels of gait impairment.

\section{Group Versus Individual Physiotherapy}

One poor-quality RCT randomized participants with MS who needed bilateral support for gait and possibility a wheelchair for longer distances (GNDS mobility section 3 or 4) to group versus individual physiotherapy. ${ }^{197}$ The mean age of participants was 55 years, 60 percent were female, and 43 percent were diagnosed with secondary-progressive MS. Both groups received 10 weekly sessions of group or individual physiotherapy.

The group physiotherapy program was self-paced and consisted of strength and balance exercises to reduce falls and improve balance and mobility. Progression was based on individual ability. Participants in the individual physiotherapy group received treatment based on the individual's problem list and goals.

Both group physiotherapy and individual physiotherapy were associated with improved scores from baseline on the MSIS-29 physical component and on the BBS. Group physiotherapy was also associated with improved scores from baseline on the MSIS-29 psychological component, while individual physiotherapy was associated with improved walking distance on the 6MWT. Due to breaking of randomization and attrition greater than 20 percent, this study provided limited evidence for similar benefits of group versus individual physiotherapy in MS patients with reduced mobility.

\section{Other Comparisons}

No included studies utilized telehealth or varied the level of training provided to study participants. Physical activity in trials of home-based exercise were typically not observed and therefore did not meet inclusion criteria for this review. Analysis across trials to further examine the effects of intervention location, amount of instruction, or level of supervision was not feasible due to significant heterogeneity in study populations, interventions, and comparators.

\section{KQ3: Patient Factors and Physical Activity}

This KQ evaluates the benefits and harms of the interventions according to patient characteristics, subgroups, demographics, condition, and intervention variations reported in the included studies. 


\section{Key Points}

- In participants with incomplete SCI, having better function and more recent injury at baseline was associated with better response to aerobic interventions than those with worse function and longer time since injury (2 RCTs). A study of women with MS found more improvement in strength and balance with core stability training in those whose baseline disability was worse. Other subgroup analyses (3 RCTs) did not find evidence of variation in effects based on baseline function or spasticity in children with CP (total body vibration), or based on weight category in participants with MS (cycling).

- Comparisons of findings across studies of participants with CP did not suggest differences in results on walking outcomes by age group (children, adolescents, adults). This finding required confirmation from direct evidence based on subgroup analyses of age within studies. Data were too homogeneous to compare outcomes by age in MS or SCI. Evaluations of differences by sex or race/ethnicity were not possible.

- Comparisons of findings according to condition (CP, MS, SCI) across studies was limited by small numbers of studies in each comparison. With aerobic interventions, $\mathrm{VO}_{2}$ peak was significantly improved in participants with SCI (2 RCTs) and CP (2 RCTs), but not in participants with MS (2 RCTs). No other differences were identified. These findings required confirmation from direct evidence based on subgroup analyses of participant diagnosis within studies.

\section{Detailed Synthesis}

Few studies evaluated the effect of the interventions according to patient characteristics or other subgroups. Only one study ${ }^{168}$ undertook analysis of the effects of the exercise intervention according to demographic characteristics (KQs $3 a$ ), and no study evaluated harms according to baseline patient characteristics. Six studies ( 2 in SCI, ${ }^{138,247} 3$ in $\mathrm{MS}^{53,147,231}$ and 1 in $\mathrm{CP}^{168}$ ) evaluated the effects of interventions according to patient characteristics or factors such as baseline functional ability, recency of onset of condition, and weight (Table 48).

In participants with incomplete SCI, two small studies found that those with better function or more recent injury had better response to physical activity interventions. In a small $(n=22)$ crossover RCT in participants with incomplete SCI ( $>7$ months since injury), two methods of walking retraining were compared (endurance and precision training) over 2 months ${ }^{138}$

Improvement on the 6MWT was significant with endurance training among participants with better walking speed at enrollment ( $>0.5$ meters per second; $\mathrm{p}=0.03$ ), while for those with lower walking function at baseline, no test was significantly improved with either training method. A secondary analysis of data from a small RCT $(\mathrm{n}=38)$ of participants with chronic ( $>12$ months) incomplete SCI who were assigned to activity-based therapy evaluated predictors of response to intervention according to baseline characteristics ${ }^{246,247}$ Response was defined as improvement of at least 45.1 meters on the $6 \mathrm{MWT}, 0.13 \mathrm{~m} / \mathrm{s}$ on the 10MWT, and reduction of at least 25.7 seconds for the TUG test, representing what the authors considered conservative estimates of minimally important differences. Participants having a response on the 6MWT were greatest (statistically significant) among those with AIS grade D (vs. grade C), and in those whose injury occurred less than 3 years before treatment (vs. $>3$ years). Changes on the 10MWT and the TUG test were not significantly different based on these patient factors. Other patient factors evaluated, including injury level, lower-extremity motor score, and use of a walker prior to study, were not found to impact the likelihood of improvement on any measure. A trial of 
women with MS ( $n=69)$ compared core stability training with conventional care (including stretching) over 10 weeks and found that improvement in strength and balance outcomes was greater in participants with greater disability at baseline. ${ }^{147}$ Specifically, women with worse baseline EDSS (scores ranging from 3.5 to 4.5 and 4.5 to 5.5) improved significantly more than those with better baseline scores (range 2.5 to 3.5). One study of children with CP found hippotherapy associated with improved sitting assessment scores compared with no hippotherapy in children with less disability (GMFCS I), whereas those with GMFCS II did not show improvement. ${ }^{168}$

In contrast, analyses in three other studies of physical activity interventions did not find evidence of variation in effects based on baseline function or spasticity in children with $\mathrm{CP}$, baseline function in women with MS, or based on weight category in participants with MS.

A study of participants with RRMS evaluated interval cycling training (upper and lower extremity) for 2 months, stratifying analysis by weight categories (normal BMI $<25$, overweight $>25) .{ }^{53}$ Although fatigue and depression scores improved in the exercise groups, no interactions were found between weight subgroups on weight status (BMI category), fatigue, or depression. A trial of a multimodal exercise program over 12 weeks adjusted analyses based on disability at baseline (grouped by low, moderate and severe based on EDSS). ${ }^{230,231}$ While improvements were seen on the $6 \mathrm{MWT}$ and the TUG and in serum lipids, $\mathrm{VO}_{2}$, and percent body fat prior to adjustment, none were found to show significant differences between the intervention and control after adjustment.

Table 48. Within-study subgroup analyses of effects of exercise in participants with MS, SCl, or CP

\begin{tabular}{|c|c|c|c|}
\hline $\begin{array}{l}\text { Author, Year } \\
\text { Intervention } \\
\text { Study Design } \\
\text { Study Quality }\end{array}$ & Intervention & Population & Results \\
\hline $\begin{array}{l}\text { Amiri, 2019 } \\
\text { Postural control } \\
\text { RCT } \\
\text { Fair }\end{array}$ & $\begin{array}{l}\text { A. Core stability } \\
\text { training, } 30 \text { sessions } \\
\text { over } 10 \text { weeks }(n=35) \\
\text { B. Conventional care } \\
\text { including stretching } \\
\text { and range of motion } \\
\text { exercises }(n=34)\end{array}$ & $\begin{array}{l}\text { A vs. B } \\
\text { Age: } 32 \text { vs. } 31 \\
\text { Female: } 100 \% \\
\text { EDSS: } 3.56 \text { vs. } 3.74 \\
\text { MS }\end{array}$ & $\begin{array}{l}\text { Core strength tests (R/L hip abduction, R/L } \\
\text { external rotation) demonstrated significant } \\
\text { differences in strength based on baseline } \\
\text { EDSS score (2.5-3.5; } 3.5-4.5 ; 4.5-5.5) \text {, } \\
p<0.001 \\
\text { Plank test: significant differences between } \\
\text { groups based on EDSS score, } p<0.001 \\
\text { Overall static balance tests demonstrated } \\
\text { significant differences in strength based on } \\
\text { baseline EDSS score and significant } \\
\text { differences compared with the control group, } \\
\text { p<0.001 } \\
\text { Greatest improvements seen in those with } \\
\text { greatest disability (least strong) }\end{array}$ \\
\hline
\end{tabular}




\begin{tabular}{|c|c|c|c|}
\hline $\begin{array}{l}\text { Author, Year } \\
\text { Intervention } \\
\text { Study Design } \\
\text { Study Quality }\end{array}$ & Intervention & Population & Results \\
\hline $\begin{array}{l}\text { Faramarzi, } \\
2020^{230} \\
\text { Banitalebi, } \\
2020^{231} \\
\text { Multimodal } \\
\text { exercise } \\
\text { RCT } \\
\text { Fair }\end{array}$ & $\begin{array}{l}\text { A. Resistance }+ \\
\text { cycling or running + } \\
\text { balance exercises }+ \\
\text { Pilates }+ \text { stretching, } \\
36 \text { sessions over } 12 \\
\text { weeks }(n=46) \\
\text { B. Waitlist control } \\
(n=43)\end{array}$ & $\begin{array}{l}\text { A vs. B } \\
\text { Age criteria: (18 to } 50) \\
\text { Female: } 100 \% \\
\text { EDSS } 0 \text { to } 4: 48 \% \text { to } \\
48 \% \\
\text { EDSS } 4.5 \text { to } 6: 27 \% \text { vs. } \\
27 \% \\
\text { EDSS } 6.5 \text { to } 8: 23 \% \text { vs. } \\
23 \%\end{array}$ & $\begin{array}{l}\text { A vs. B, Positive effect of exercise on: } \\
\text { Cholesterol: } p=0.020 \text {, effect of } \\
\text { disability*exercise } p=0.549 \\
\text { HDL: } p<0.001 \text {, effect of disability*exercise } \\
p=0.408 \\
\text { LDL: } p<0.001 \text {, effect of disability*exercise } \\
p=0.826 \\
\text { TG: } p=0.005 \text {, effect of disability }{ }^{*} \text { exercise } \\
p=0.982 \\
\text { VO } 2 \text { peak: } p=0.004 \text {, effect of disability*exercise } \\
p=0.097 \\
\text { Body fat } \%: p=0.001 \text {, effect of } \\
\text { disability*exercise } p=0.76 \\
\text { TUG: } p<0.001 \text {, effect of disability*exercise } \\
p=0.396 \\
\text { 6MWT: } p<0.001 \text {, effect of disability*exercise } \\
p=0.587\end{array}$ \\
\hline $\begin{array}{l}\text { Jones, } 2014^{246} \\
\text { Multimodal } \\
\text { exercise } \\
\text { Secondary } \\
\text { analysis of } \\
\text { responders in } \\
\text { an RCT } \\
\text { Poor }\end{array}$ & $\begin{array}{l}\text { A. Activity-based } \\
\text { therapy } \\
\text { (developmental } \\
\text { sequence activities, } \\
\text { resistance training, } \\
\text { and locomotor } \\
\text { training) ( } \mathrm{n}=38 \text { ) } \\
\text { No control for this } \\
\text { analysis }\end{array}$ & $\begin{array}{l}\text { Age: } 38 \text { years } \\
\text { Female: } 29 \% \\
\text { Motor Incomplete SCI } \\
\text { ASIA C or D } \\
\text { SCI }\end{array}$ & $\begin{array}{l}\text { 6MWT response (>45.1 meters improvement): } \\
\text { AIS Grade C vs. D: } \\
\text { OR } 11.00 \text { ( } 95 \% \mathrm{CI} 1.24 \text { to } 7.97) \\
</>3 \text { years since injury: } \\
\text { OR } 4.80 \text { ( } 95 \% \mathrm{Cl} 1.04 \text { to } 22.10) \\
\text { Other outcomes (10MWT and TUG): not } \\
\text { significantly different based on AIS grade or } \\
\text { time since injury } \\
\text { No outcome found significantly different in } \\
\text { other subgroups (injury level, lower extremity } \\
\text { function, use of a walker) }\end{array}$ \\
\hline $\begin{array}{l}\text { Matusiak- } \\
\text { Wieczorek, } \\
\text { 2020Matusiak- } \\
\text { Wieczorek, } \\
2020 \# 19901 \\
\text { Postural control } \\
\text { RCT } \\
\text { Fair }\end{array}$ & $\begin{array}{l}\text { A. Hippotherapy, } 24 \\
\text { sessions over } 12 \\
\text { weeks }(n=15) \\
\text { B. Hippotherapy, } 12 \\
\text { sessions over } 12 \\
\text { weeks ( } n=15) \\
\text { C. No hippotherapy } \\
(n=15)\end{array}$ & $\begin{array}{l}\text { A vs. B vs. C } \\
\text { Age: } 7.93 \text { vs. } 7.60 \text { vs. } \\
8.13 \\
\text { Female: } 40 \% \text { vs. } 47 \% \\
\text { vs. } 47 \% \\
\text { GMFCS I: } 67 \% \text { vs. } 80 \% \\
\text { vs. } 47 \% \\
\text { GMFCS II: } 33 \% \text { vs. } 20 \% \\
\text { vs. } 53 \%\end{array}$ & $\begin{array}{l}\text { A vs. B vs. C, mean (SD), } p=\text { between groups } \\
\text { SAS improvement vs. no improvement: } \\
\text { A vs. C (6-7 year olds): } p<0.001 \\
\text { B vs. C (6-7 year olds): } p=0.022 \\
\text { A vs. B (6-7 year olds): } p=0.105 \\
\text { A vs. C (8-12 year olds): } p=0.379 \\
\text { B vs. C (8-12 year olds): } p=0.442 \\
\text { A vs. C (8-12 year olds): } p=0.397 \\
\text { A vs. C (GMFCS I): } p=0.001 \\
\text { B vs. C (GMFCS I): } p=0.073 \\
\text { A vs. B (GMFCS I): } p=0.030 \\
\text { A vs. C (GMFCS II): } p=0.326 \\
\text { B vs. C (GMFCS II): } p=0.509 \\
\text { A vs. B (GMFCS II): } p=0.429\end{array}$ \\
\hline $\begin{array}{l}\text { Negaresh, } \\
2019^{53} \\
\text { Aerobic } \\
\text { Exercise } \\
\text { RCT } \\
\text { Fair }\end{array}$ & $\begin{array}{l}\text { A. Interval cycling } \\
\text { training of upper and } \\
\text { lower extremity } \\
24 \text { sessions over } 8 \\
\text { weeks } \\
\text { Normal BMI }(n=18) \\
\text { Overweight }(n=17) \\
\text { B. Control } \\
\text { Normal BMI }(n=15) \\
\text { Overweight: }(n=13)\end{array}$ & $\begin{array}{l}\text { A vs. } B \\
\text { Age: } 31 \text { vs. } 31 \\
\text { Female: } 65 \% \text { vs. } 67 \% \\
\text { EDSS: } 1.65 \text { vs. } 1.54 \\
\text { MS }\end{array}$ & $\begin{array}{l}\text { No significant interactions between weight } \\
\text { status and fatigue or depression outcomes } \\
(p>0.05)\end{array}$ \\
\hline
\end{tabular}




\begin{tabular}{|c|c|c|c|}
\hline $\begin{array}{l}\text { Author, Year } \\
\text { Intervention } \\
\text { Study Design } \\
\text { Study Quality }\end{array}$ & Intervention & Population & Results \\
\hline $\begin{array}{l}\text { Yang, 2014 } \\
\text { Aerobic } \\
\text { Exercise } \\
\text { RCT } \\
\text { (Crossover) } \\
\text { Fair }\end{array}$ & $\begin{array}{l}\text { A. BWS (if needed) } \\
\text { treadmill walking, } 40 \\
\text { sessions over } 8 \\
\text { weeks ( } n=10) \\
\text { B. Precision track } \\
\text { walking training, } 40 \\
\text { sessions over } 8 \\
\text { weeks ( } n=10)\end{array}$ & $\begin{array}{l}\text { A vs. B } \\
\text { Age: } 48 \text { vs. } 44 \\
\text { Female: } 30 \% \text { vs. } 30 \% \\
\text { Able to walk } \geq 5 \text { meters } \\
\text { with walking aid or } \\
\text { braces: } 100 \% \\
\text { SCl }\end{array}$ & $\begin{array}{l}\text { A. Precision training: No significant } \\
\text { improvements across groups } \\
\text { B. Endurance training: } 6 \mathrm{MWT} \text { improved } \\
\text { significantly only in those with baseline walking } \\
\text { speed }>0.5 \mathrm{~m} / \mathrm{s} \\
\text { No changes in } 10 \mathrm{MWT} \text { (patient selected speed } \\
\text { or fast speed) or Spinal Cord Injury-Functional } \\
\text { Ambulation Profile }\end{array}$ \\
\hline
\end{tabular}

Abbreviations: 6MWT = 6-Minute Walk Test; 10MWT = 10-Meter Walk Test; AIS = ASIA Impairment Scale; ASIA = American Spinal Injury Association Impairment Scale; BMI = body mass index; BWS = body weight supported; CI = confidence interval; $\mathrm{CP}=$ cerebral palsy; EDSS = Expanded Disability Status Scale; GMFCS = Gross Motor Function Classification System; GMFM-88 = Gross Motor Function Measure 88; MMAS = Modified Modified Ashworth Scale; MS = multiple sclerosis; OR = odds ratio; $\mathrm{RCT}=$ randomized controlled trial; RRMS = relapsing-remitting multiple sclerosis; $\mathrm{SCI}=$ spinal cord injury; $\mathrm{TUG}=$ Timed Up and Go Test; VO2 max = maximal oxygen uptake

\section{KQ3a: Patient Demographics}

One included study conducted subgroup analyses on patient demographics (i.e., age). ${ }^{168}$ Qualitative comparison of effects seen in these subgroups (age, sex, or race/ethnicity) may provide some insight into potential variation.

\section{Age}

One CP study ${ }^{168}(\mathrm{n}=45)$ compared the results of 12 weeks of hippotherapy on the Sitting Assessment Scale based on participant age and found that compared to a no hippotherapy control group, younger children aged 6 and 7 years had improved sitting scores, whereas there was no significant improvement on the Sitting Assessment Scale with hippotherapy among children age 8 through 12 years versus no hippotherapy.

Within populations, comparing similar interventions, there was either inadequate variation in age or heterogeneity in outcomes assessed to evaluate impact of age. For example, across seven studies of cycling exercises in participants with MS, mean age varied from 31 to 59 years (with two studies not clearly reporting age of participants), with the study of the youngest cohort evaluating TUG, fatigue, depression, and aerobic capacity, ${ }^{53}$ and the study of the oldest cohort evaluating disability (using EDSS) and quality of life. Across 15 studies of mainly cycling or treadmill interventions in participants with SCI (that reported age and a prioritized outcome), there was not a wide variation in the age of enrolled participants, with a median of 43 years (range 33 to 56 years). ${ }^{75,76,89,90,92,93,107-113,133-136,138-140}$ Studies of participants in their 30s predominately evaluated oxygen consumption outcomes, where studies of participants in their 50s more commonly evaluated walking tests. The 10MWT was the most commonly reported across these studies, with no apparent differences in findings for younger and older participants, in that improvements were not found. ${ }^{111,133-136}$ Other intervention types had fewer studies, with no ability to evaluate the effect of age.

Across 22 studies of aerobic-type exercise in participants with CP, 13 included children (median age 9 years), 8 included adolescents (median age 16 years), and 1 included adults (median age 27 years). ${ }^{73,74,85-88,99-102,105,121-128,131,132,218}$ Looking at studies of children versus teens, in very global terms, the findings are similar; three of six and four of nine studies reported 
positive findings on walking tests, while one of three and one of four reported positive findings in gross motor function in children and adolescents (respectively). The measures reported are too varied to be compared with evaluate any potential differences in magnitude of effects. The single study of adults found improved walking speed on the 6MWT. ${ }^{126}$ Other intervention types had fewer studies, with no ability to evaluate the effect of age.

\section{Sex}

As noted above, there were no studies that evaluated males and females as subgroups. In order to evaluate this factor across studies, it would be necessary to compare studies that enrolled largely males (e.g. $>75 \%$ ) with those that enrolled largely females where the other patient characteristics were similar and that studied similar interventions. While there is some variation across conditions in the proportions of females enrolled, we found no instance of studies that would be comparable based on sex alone.

\section{Race/Ethnicity}

Race and ethnicity were poorly reported in the included studies. For example, in the 22 studies of aerobic interventions in participants with $\mathrm{CP}$, half did not report race or ethnicity, while in 17 studies of cycling or treadmill exercise interventions in participants with SCI, only one reported on the race of participants. While several studies were conducted in countries outside of the United States (e.g., Iran or China), they were also mostly unclear on the race or ethnicity of the participants enrolled. Because of this poor reporting, and lack of heterogeneous groups to compare, we were unable to evaluate any impact of race/ethnicity on the outcomes of the interventions.

\section{KQ3b: Variations by Condition and Intervention}

In evaluating the potential variation of effects of physical activity interventions across the three populations, we compared results where we had adequate data to conduct meta-analysis, where the comparison was some form of usual care, where there was more than one condition with the same outcome measure reported, and measures of balance (e.g., BBS) according to intervention category (aerobic, balance, strength).

\section{Aerobic Interventions}

One RCT in adults with $\mathrm{CP}^{126}$ and four RCTs in participants with MS reported on the 6MWT for aerobic interventions. ${ }^{54,66,77,79,80}$ None found a significant benefit and there was no clear difference according to population. Two studies each in participants with MS, SCI, and CP reported $\mathrm{VO}_{2}$ peak. The results appeared to vary by population in these studies. Studies in patients with SCI ( 2 RCTs, MD $-206 \mathrm{~mL} / \mathrm{min}, 95 \% \mathrm{CI}-359$ to $\left.-53, \mathrm{I}^{2} 51 \%\right),{ }^{92,140}$ and with $\mathrm{CP}(2$ studies, MD of 6.5 to $7.0 \mathrm{ml} / \mathrm{kg} / \mathrm{min}$, both statistically significant but not combinable $)^{88,132}$ found a benefit with aerobic exercise. In contrast, participants with MS did not show a benefit (2 RCTs, MD $0.20 \mathrm{~mL} / \mathrm{min}, 95 \% \mathrm{CI}-127$ to $\left.127, \mathrm{I}^{2} 0 \%\right) .{ }^{77,78}$ No other outcomes were reported across the different populations.

\section{Strength Interventions}

The 6MWT was reported in one RCT of children with $\mathrm{CP},{ }^{211,212}$ and in three RCTs of participants with MS..$^{52,198,202,203}$ None found a significant benefit and there were no clear 
difference according to population. No other outcomes were reported across the different populations.

\section{Balance Interventions}

One RCT of participants with $\mathrm{MS}^{143}$ and one in participants with $\mathrm{SCI}^{189}$ reported no effect of the intervention on the 10MWT, and no clear difference according to population. The BBS score was reported in one RCT of participants with $\mathrm{CP}^{50}$ and five RCTs in participants with MS. ${ }^{141,143,144,158,159}$ All studies showed improved balance, with variation in the magnitude of benefit, and no clear difference according to population. Results for the TUG test varied by population; one RCT in participants with CP found no benefit, ${ }^{50}$ four RCTs of participants with MS found no benefit but variation in the direction of the nonsignificant effects across studies, ${ }^{143,144,175,185}$ and one RCT in participants with SCI found a small benefit $(-1.30$ seconds, $95 \% \mathrm{CI}-2.16$ to -0.44$).{ }^{189}$ No other outcomes were reported across the different populations.

\section{Multimodal Interventions}

On the 6MWT, six of seven RCTs of participants with MS $192,193,197,221,222,226,228$ and one of participants with $\mathrm{SCI}^{247}$ showed improvements and no clear difference according to population. The seventh study of patients with MS did not find a significant improvement, after adjusting for baseline disability scores. ${ }^{231}$ In contrast, on the 10MWT, four RCTs of participants with $\mathrm{MS}^{222,223,225,228}$ did not find a benefit, while one RCT in participants with SCI found a benefit $(-11.20$ seconds, $95 \% \mathrm{CI}-22.44$ to -0.04$){ }^{247}$ The TUG test was not improved in either MS patients ( $3 \mathrm{RCTs})^{225,228,231}$ or in SCI patients (1 RCT), ${ }^{247}$ and there were no clear difference according to population. No other outcomes were reported across the different populations.

\section{KQ4: Methodological Gaps}

\section{Key Points}

- Conclusions that can be drawn from research on physical activity in patients with MS, $\mathrm{CP}$, and SCI were limited by small sample sizes, inadequate descriptions of population characteristics and control group activities, incomplete data analysis, inadequate reporting of adverse events, and few RCTs rated good quality (low risk of bias). There were few studies in MS and CP that enrolled a more disabled population.

- A few large, well-conducted RCTs of longer duration would greatly strengthen the evidence base. Large, cohort studies could provided data on long-term health outcomes, as well as potential harms from the intervention

\section{Detailed Synthesis}

Methodological weakness not discussed in subquestions below included inadequate description of control groups, inadequate reporting of baseline data, inadequate reporting of harms or adverse events, and inadequate between group analysis. Gaps in the evidence included fewer studies in CP and SCI than in MS with less evidence available for MS in males, SCI in females, and CP in adults. The lack of harms data is also a research gap, as is the relative lack of studies in MS and CP that enrolled a more disabled population. 
Although interventions received in the intervention groups were generally well described, in many cases participants in the control groups were described as maintaining their usual level of activity without comment on what that usual level of activity was (e.g., no physical activity at all, daily walk to the mailbox, balance exercises). The control group was also described as continuing their usual physiotherapy without comment on what that physiotherapy entailed (e.g., 2 hourly sessions of free weights, 3 sets of 12 reps biceps curls and triceps extensions plus walking on a treadmill at 1.5 miles per hour for 15 minutes plus leg lifts and abdominal crunches on mat). In order to minimize across-study heterogeneity, it is important to pool trials with not just similar interventions, but also similar control groups, which was challenging at times when control group participation was not well described.

Participant baseline data were also not always well presented. Most studies provided mean age and the proportion of males and females per study arms, but often data were lacking in characteristics that may predict a better or worse outcome. For example, studies often gave no indication of the level of impairment of participants per treatment arms. The EDSS was the characteristic most often provided to indicate degree of impairment. But many studies did not provide that information or the type of MS with which MS participants were diagnosed (e.g., RRMS, PPMS), the specific level of injury in patients with SCI, or the GMFCS and degree of spasticity in patients with CP. Baseline participant data was also often provided for only the participants who were analyzed rather than all patients randomized. Many studies also did not provide disease severity or use of assistive technology (including use of wheelchairs) and did not control for these factors, although some trials did limit eligibility to patients with a range of disease severity.

Another methodological weakness was how data were analyzed in trials. Many studies did not fully take baseline data into account when comparing the performance between intervention and control groups. In some cases, the intervention group improved significantly from baseline whereas the control group had not. This was given as evidence of the superiority of the intervention. Another data analysis method that can yield misleading results occurred when studies compared baseline data and found no difference between groups and then measured postintervention data and found a statistically significant difference between groups favoring the intervention. Neither of these methods considered the difference between the changes in outcome measure before and after the intervention which can lead to faulty conclusions.

Another weakness, which is also a gap, is the lack of information on harms of the intervention. Many trials did not report any harms or adverse events and did not report that there were no harms or adverse events. In this case, it is impossible to determine whether adverse events occurred but were not reported in the publication, whether adverse events occurred but were not captured by the researchers, or whether no adverse events occurred. In trials designed to demonstrate that a treatment is effective, harms are often not adequately addressed but all trials should have an adequate means to document harms and adverse events experienced by study participants and report all of them in publications.

Another research gap is the limited information in certain populations based on the lower prevalence of disease (i.e., MS in males, SCI in females). Evidence in CP is largely limited to trials of children. Expanding the sample size would assist in capturing a broader range of individuals and provide information to fill in research gaps. 


\section{KQ4a: Types of Studies}

Out of the 168 included studies in this review, 44 percent enrolled participants with MS, 38 percent were conducted in participants with $\mathrm{CP}$, and 18 percent were in participants with SCI. Most of these studies were RCTs $(n=146,87 \%)$, a few were quasiexperimental trials where participants were not randomized into groups $(\mathrm{n}=15,9 \%)$, and the remainder were cohort studies with at least two groups of participants $(n=7,4 \%)$. Most studies were rated fair quality, however most of the nonrandomized studies (quasiexperimental and cohort studies) were rated poor quality $(n=12,55 \%)$ and were primarily conducted in participants with $C P(n=11,50 \%)$.

\section{KQ4b: Weaknesses in Study Design}

Within the included studies, multiple weakness in study design were identified. These involved sample size, study duration, and inclusion/exclusion criteria.

One weakness in study design concerned small sample sizes. Sample size cutoffs for eligibility in this review were at least $n=20$ in CP and SCI and $n=30$ in MS. These sample sizes

are actually rather small and reflect the difficulty in recruiting large numbers of participants. This could be due to the prevalence of the diseases included (i.e., MS, CP, and SCI), potentially reduced mobility of the patient sample, other patient comorbidities, and/or logistical difficulties that may make participation in research less likely. Small sample sizes (vs. larger sample sizes) increase the difficulty in demonstrating a treatment effect as it is harder to achieve statistical significance with fewer numbers. Small sample sizes also increase the likelihood that even a RCT will have differences in prognostic factors between treatment and control groups that may render findings unreliable. In this review, only a few studies enrolled more than 100 participants.

An additional study design weakness regarded study duration. Most studies were terminated immediately postintervention, which ran typically 12 or 16 weeks. Without longitudinal followup, it is impossible to determine if the intervention is associated with prevention of detrimental clinical health outcomes (e.g., stroke or development of diabetes). To determine a treatment effect, some studies included intermediate health outcomes such as heart rate, blood pressure, and blood glucose. Extrapolation from these intermediate outcomes to long-term health benefit is not ideal. Also, since most studies excluded individuals with known cardiovascular or metabolic disease, it is impossible to comment on the benefit of the intervention regarding secondary prevention (e.g., preventing a second heart attack) or tertiary prevention (e.g., reducing angina or heart failure symptoms).

\section{KQ4c: Future Research}

An ideal study would be a RCT that includes a no treatment arm, such as waitlist control or attention control group. This would provide the information needed to determine if the intervention worked or not in the included patient population. Including a usual care arm as a comparator would provide additional information but only if what usual care entails is adequately described. The intervention(s) should use standard methods, when possible, that also need to be well described, either in the publication or in a cited or included protocol. In order to 
maintain the statistical power needed to demonstrate a difference between groups, the number of intervention groups would be limited to that supported by the sample size.

An ideal study would also be large enough to permit subgroup analyses. For example, a study that enrolled sufficient males and females would be able to demonstrate if there is a difference in treatment effect that could be attributed to gender. A trial that enrolled individuals with varying degrees of disability could suggest whether the intervention has a greater effect in those with greater versus lesser impairment. A trial that enrolled participants across a spectrum of ages could comment on the impact of age on the treatment effect. A large study (RCT or cohort study) would also be more likely to retain sufficient numbers of participants to facilitate a longitudinal analysis. This would enable the investigation of clinical outcomes that take time to develop (e.g., coronary artery disease) as well as potential harms of the intervention.

An ideal study would have a prespecified and consistent method for identifying harms and adverse events experienced during the study. Any data collection forms should be available for review. Assessors should be blinded. The number of study participants who experienced a specific adverse event should be provided for each study group, not just the number of total adverse events, as any participant may have multiple or repeated adverse events. All adverse events should be specified, not just those experienced by more than 5 or 10 percent - the death of only one participant could be due to the intervention and is important to report, even if fewer than 5 percent died.

An ideal study would also receive a good-quality rating or demonstrate low risk of bias. Studies with low risk of bias tend to generate conservative estimates of effect compared with studies rated medium or high risk of bias (fair or poor quality). Requirements for low risk of bias include appropriate methods of randomization (e.g., computer generated random numbers) and concealment of the allocation (e.g., centrally managed), and successful randomization, that is, baseline characteristics of participants, especially those known to be prognostic factors (e.g., participant BMI on the development of diabetes). Other criteria for low risk of bias (high quality) include blinding of all involved when possible, especially blinding of outcome assessors, analyzing all participants in the groups to which they were randomized with minimum attrition, and no or minimal difference in attrition between groups. Two or more larger, well-conducted RCTs typically generate more reliable and stable estimates of effect than would a greater number of smaller studies rated fair or poor quality. 


\section{Discussion}

\section{Key Findings and Strength of Evidence}

We included 168 studies ( $n=7,511)$, of which 146 were randomized controlled trials (RCTs). Key findings and strength of evidence are summarized in Table 49. Overall strength of evidence grades and detailed domain assessments appear in Appendix $\mathrm{H}$.

The average sample size was 45 (range 20 to 242), with only 3 studies with samples sizes of 100 or more. Most studies were rated moderate risk of bias. The bulk of the evidence was in participants with multiple sclerosis (MS). In participants with MS, walking ability may be improved with treadmill training and multimodal exercise regimens that include strength training; function may be improved with treadmill training, balance exercises, and motion gaming; balance is likely improved with postural control exercises (that may also reduce risk of falls) and may be improved with aquatic exercises, robot-assisted gait training (RAGT), treadmill training, motion gaming, and multimodal exercises; activities of daily living (ADL) may be improved with aquatic therapy; sleep may be improved with aerobic exercises; female sexual function may be improved with aquatic exercise; and cardiovascular fitness $\left(\mathrm{VO}_{2}\right.$ peak) may be improved with multimodal exercises. In participants with cerebral palsy (CP), balance may be improved with hippotherapy and motion gaming and function may be improved with cycling, hippotherapy, and treadmill training. In participants with spinal cord injury (SCI), evidence suggests that ADL may be improved with RAGT. When RCTs were pooled across types of exercise, physical activity interventions were found to improve walking in MS, to likely improve balance and depression in MS, and may improve aerobic fitness and function in participants with CP or with SCI. When populations were combined, dance may improve function in participants with MS and CP. The majority of this evidence is low strength. Evidence on long-term health outcomes was not found. For intermediate outcomes such as blood pressure, lipid profile, and blood glucose, there was insufficient evidence from which to draw conclusions. There was inadequate reporting of adverse events in many trials. However, physical activity was associated with low-strength evidence of increased autonomic dysreflexia episodes in SCI.

Table 49. Effects of physical activity interventions compared with usual care ${ }^{a}$

\begin{tabular}{|c|c|c|c|}
\hline $\begin{array}{l}\text { Intervention } \\
\text { Category } \\
\text { Intervention }\end{array}$ & $\begin{array}{l}\text { Multiple Sclerosis } \\
\text { Studies } \\
\text { Strength of Evidence } \\
\text { (Direction of Finding) }\end{array}$ & $\begin{array}{l}\text { Cerebral Palsy } \\
\text { Studies } \\
\text { Strength of Evidence } \\
\text { (Direction of Finding) }^{\text {b }}\end{array}$ & $\begin{array}{l}\text { Spinal Cord Injury } \\
\text { Studies } \\
\text { Strength of Evidence } \\
\text { (Direction of Finding) }\end{array}$ \\
\hline $\begin{array}{l}\text { Aerobic Exercise } \\
\text { Dance }(1 \mathrm{RCT} \text { in } \mathrm{MS} \text { and } \\
1 \mathrm{RCT} \text { in } \mathrm{CP})^{\mathrm{a}}\end{array}$ & $\begin{array}{c}\text { Low } \\
\text { (function improvement) }\end{array}$ & $\begin{array}{c}\text { Low } \\
\text { (function improvement) }\end{array}$ & Insufficient \\
\hline $\begin{array}{l}\text { Aerobic Exercise } \\
\text { Aerobics }\end{array}$ & $\begin{array}{c}\text { Low } \\
\text { (sleep improvement) }\end{array}$ & Insufficient & Insufficient \\
\hline $\begin{array}{l}\text { Aerobic Exercise } \\
\text { Aquatics }\end{array}$ & $\begin{array}{l}\text { Low } \\
\text { (balance, ADL } \\
\text { improvement, female } \\
\text { sexual function) }\end{array}$ & Insufficient & Insufficient \\
\hline $\begin{array}{l}\text { Aerobic Exercise } \\
\text { Cycling }\end{array}$ & $\begin{array}{l}\text { Low } \\
\text { (no clear benefit on } \\
\text { walking) }\end{array}$ & $\begin{array}{c}\text { Low } \\
\text { (function improvement) }\end{array}$ & Insufficient \\
\hline
\end{tabular}




\begin{tabular}{|c|c|c|c|}
\hline $\begin{array}{l}\text { Intervention } \\
\text { Category } \\
\text { Intervention }\end{array}$ & $\begin{array}{l}\text { Multiple Sclerosis } \\
\text { Studies } \\
\text { Strength of Evidence } \\
\text { (Direction of Finding) }\end{array}$ & $\begin{array}{c}\text { Cerebral Palsy } \\
\text { Studies } \\
\text { Strength of Evidence } \\
\text { (Direction of Finding) }\end{array}$ & $\begin{array}{l}\text { Spinal Cord Injury } \\
\text { Studies } \\
\text { Strength of Evidence } \\
\text { (Direction of Finding) }\end{array}$ \\
\hline $\begin{array}{l}\text { Aerobic Exercise } \\
\text { Robot-Assisted Gait } \\
\text { Training }\end{array}$ & $\begin{array}{c}\text { Low } \\
\text { (balance improvement) } \\
\text { Low } \\
\text { (no clear benefit in } \\
\text { function) }\end{array}$ & Insufficient & $\begin{array}{c}\text { Low } \\
\text { (ADL improvement) } \\
\text { Low } \\
\text { (no clear benefit on walking, } \\
\text { function) }\end{array}$ \\
\hline $\begin{array}{l}\text { Aerobic Exercise } \\
\text { Treadmill }\end{array}$ & $\begin{array}{l}\text { Low } \\
\text { (walking, function, and } \\
\text { balance improvement) }\end{array}$ & $\begin{array}{c}\text { Low } \\
\text { (function improvement) }\end{array}$ & Insufficient \\
\hline $\begin{array}{l}\text { Postural Control } \\
\text { Balance Exercises }\end{array}$ & $\begin{array}{c}\text { Moderate } \\
\text { (balance improvement) }\end{array}$ & Insufficient & Insufficient \\
\hline $\begin{array}{l}\text { Postural Control } \\
\text { Balance Exercises }\end{array}$ & $\begin{array}{c}\text { Low } \\
\text { (fall risk improvement) }\end{array}$ & Insufficient & Insufficient \\
\hline $\begin{array}{l}\text { Postural Control } \\
\text { Balance Exercises }\end{array}$ & $\begin{array}{c}\text { Low } \\
\text { (function improvement) }\end{array}$ & Insufficient & Insufficient \\
\hline $\begin{array}{l}\text { Postural Control } \\
\text { Hippotherapy }\end{array}$ & Insufficient & $\begin{array}{l}\text { Low } \\
\text { (balance and function } \\
\text { improvement) }\end{array}$ & Insufficient \\
\hline $\begin{array}{l}\text { Postural Control } \\
\text { Tai Chi }\end{array}$ & Insufficient & Insufficient & Insufficient \\
\hline $\begin{array}{l}\text { Postural Control } \\
\text { Motion Gaming }\end{array}$ & $\begin{array}{l}\text { Low } \\
\text { (function, balance } \\
\text { improvement) }\end{array}$ & $\begin{array}{c}\text { Low } \\
\text { (balance improvement) }\end{array}$ & Insufficient \\
\hline $\begin{array}{l}\text { Postural Control } \\
\text { Whole Body Vibration }\end{array}$ & Insufficient & Insufficient & Insufficient \\
\hline $\begin{array}{l}\text { Postural Control } \\
\text { Yoga }\end{array}$ & $\begin{array}{c}\text { Low } \\
\text { (no clear benefit on } \\
\text { function) }\end{array}$ & Insufficient & Insufficient \\
\hline $\begin{array}{l}\text { Strength Interventions } \\
\text { Muscle Strength } \\
\text { Exercise }\end{array}$ & $\begin{array}{c}\text { Low } \\
\text { (no clear benefit in } \\
\text { walking, function, } \\
\text { balance, quality of life, } \\
\text { spasticity) }\end{array}$ & $\begin{array}{l}\text { Low } \\
\text { (no clear benefit in } \\
\text { walking and function) }\end{array}$ & Insufficient \\
\hline $\begin{array}{l}\text { Multimodal Exercise } \\
\text { Progressive Resistance } \\
\text { or Strength Exercise } \\
\text { Plus Aerobic or Balance }\end{array}$ & $\begin{array}{l}\text { Low } \\
\text { (walking, balance, } \mathrm{VO}_{2} \\
\text { improvement) }\end{array}$ & $\begin{array}{l}\text { Low } \\
\text { (no clear benefit in } \\
\text { function, quality of life) }\end{array}$ & Insufficient \\
\hline
\end{tabular}




\begin{tabular}{|c|c|c|c|}
\hline $\begin{array}{l}\text { Intervention } \\
\text { Category } \\
\text { Intervention }\end{array}$ & $\begin{array}{l}\text { Multiple Sclerosis } \\
\text { Studies } \\
\text { Strength of Evidence } \\
\text { (Direction of Finding) }\end{array}$ & $\begin{array}{c}\text { Cerebral Palsy } \\
\text { Studies } \\
\text { Strength of Evidence } \\
\text { (Direction of Finding) }\end{array}$ & $\begin{array}{l}\text { Spinal Cord Injury } \\
\text { Studies } \\
\text { Strength of Evidence } \\
\text { (Direction of Finding) }\end{array}$ \\
\hline & $\begin{array}{c}\text { High } \\
\text { (walking improvement) }\end{array}$ & $\begin{array}{c}\text { Low } \\
\text { (function) }\end{array}$ & $\begin{array}{c}\text { Low } \\
\text { (function) }\end{array}$ \\
\hline All Types of Exercise & $\begin{array}{c}\text { Moderate } \\
\text { (balance, depression } \\
\text { improvement, no clear } \\
\text { benefit on function) }\end{array}$ & $\begin{array}{c}\text { Low } \\
\left(\mathrm{VO}_{2} \text { improvement }\right)\end{array}$ & $\begin{array}{c}\text { Low } \\
\text { (VO }_{2} \text { improvement, } \\
\text { increased episodes of } \\
\text { autonomic dysreflexiac, no } \\
\text { clear benefit on depression) }\end{array}$ \\
\hline
\end{tabular}

Abbreviations: $\mathrm{ADL}=$ activities of daily living; $\mathrm{CP}=$ cerebral palsy; $\mathrm{MS}=$ multiple sclerosis; $\mathrm{RCT}=$ randomized controlled trial ${ }^{a}$ Strength of evidence color shading: blue $=$ high strength of evidence, green $=$ moderate, yellow $=$ low, white $=$ insufficient

${ }^{b}$ Strength of evidence based on combining the two populations, multiple sclerosis and cerebral palsy

${ }^{\mathrm{c}}$ Whole-body exercise versus exercise limited to upper body

\section{Findings in Relationship to What Is Already Known}

The 2018 Physical Activity Guideline Advisory Committee Scientific Report ${ }^{268}$ found strong evidence that within the general population, sedentariness is linked to increased risk of all-cause mortality and cardiovascular mortality, in a dose-response fashion. Additionally, there was strong evidence of an association between sedentary behavior and increased risk of developing type 2 diabetes and cardiovascular disease. The committee also found moderate evidence that moderate to vigorous exercise of any duration was associated with health benefits, such as improved blood pressure and lipid profile.

Unfortunately, we identified no evidence in people with MS, CP, and SCI concerning risk of mortality, the development of diabetes, or the development of cardiovascular disease in relation to physical exercise as defined in this review. The evidence for improvement in intermediate outcomes was limited to low-strength evidence for improvement in $\mathrm{VO}_{2}$ peak with exercise in participants with CP and SCI. Evidence for other intermediate health outcomes such as blood pressure and lipid profile was too sparse to draw conclusions.

We were also not able to draw general conclusions regarding potential harms of physical exercise; all trials were designed to assess benefits and only one trial in participants with SCI appeared to systematically monitor participants for adverse events, recording over 700 adverse events. ${ }^{245}$ This trial demonstrated the need for cardiovascular monitoring during aerobic exercise, especially in people with SCI, as out of 33 episodes of autonomic dysreflexia, three were considered serious. While a 2014 systematic review ${ }^{269}$ of adverse events in cardiovascularrelated training programs in SCI ( $\mathrm{n}=38$ studies) reported no serious episodes of autonomic dysreflexia, this review found functional electrical stimulation ambulation associated with a 4 percent fracture rate, although there were few adverse events reported in studies of volitional exercise in SCI.

A 2014 systematic review ${ }^{270}$ examined the safety of exercise training in MS ( $n=26$ studies) and found no increased risk for relapse between exercise and control groups (4.6\% vs. 6.3\%) or the risk of experiencing any adverse event $(2.0 \%$ vs. $1.2 \%)$.

We identified no systematic reviews of safety in people with $\mathrm{CP}$. 


\section{Multiple Sclerosis}

A 2013 systematic review was conducted to inform guideline development on the effects of exercise on fitness, mobility, fatigue, and health-related quality of life in adults with MS. ${ }^{271}$ This review included 54 studies published before December 2011 and found that in people with mild to moderate MS, physical exercise improved aerobic capacity and muscle strength. The authors also concluded that exercise may improve mobility and health-related quality of life. These findings are largely consistent with our review, which determined that physical exercise improved walking ability, balance, and depression in participants with MS, although support for improvement in health-related quality of life and aerobic fitness was limited. The evidence for strength was mixed but the sole good-quality trial found a strength benefit in participants with MS.

A 2017 systematic review of 18 studies $(\mathrm{n}=290)$ that enrolled participants with MS and severe mobility disability (e.g., Expanded Disability Status EDSS score $\geq 6$ ) concluded that limited evidence suggests conventional resistance exercise and adapted exercise training may improve physical fitness and function in this population. ${ }^{272}$ The authors also note that adapted exercise may not be feasible due to cost and accessibility.

\section{Cerebral Palsy}

2016 guidelines from The Netherlands were based on a systematic review that included five RCTs to determine physical activity recommendations for people with $\mathrm{CP} .{ }^{46}$ The included interventions consisted of cardiorespiratory endurance training, which was compared with no intervention. Some of the included trials indicated improved aerobic capacity after training and some showed improved strength. These results are similar to this review, which found evidence for improved aerobic fitness with exercise, although the evidence for strength outcomes was mixed. The current review also found evidence for improved balance and function with physical activity that was not identified in the Dutch review.

\section{Spinal Cord Injury}

A 2017 systematic review ${ }^{273}$ conducted as the foundation for exercise guidelines for people with SCI included 211 studies, 189 studies in chronic SCI. Search dates were between 1980 and 2016 and included RCTs, non-RCTs, pre-post series, case series, and cross-sectional cohort studies (in chronic SCI most were pre-post studies, 16 RCTs). The review concluded that upper body aerobic exercise at moderate to vigorous intensity plus upper body strength exercises can improve cardiorespiratory fitness, power, strength, and body composition in participants with SCI. The current review has similar conclusions for improved aerobic fitness with physical exercise and improved walking and function with RAGT, but there was insufficient evidence from trials meeting inclusion criteria for improved body composition with physical exercises in participants with SCI.

This current systematic review has stricter criteria for study inclusion than other systematic reviews. For example, case series and single-arm pre-post studies were not included.

Additionally, the physical exercise intervention had to include at least 10 sessions on 10 different days and the activity had to be observed by a researcher or healthcare provider. Sample sizes also had to be met ( $\mathrm{n}=20$ in $\mathrm{CP}$ and SCI, $\mathrm{n}=30$ in MS). These stricter criteria alone may explain any differences between previous systematic reviews and this review. The current review was also limited to studies published in 2008 and beyond; the other reviews included studies published in 
the 1980s and 1990s, which may not have the methodological rigor as trials conducted more recently.

\section{Applicability and Generalizability}

Due to the strict criteria for trial inclusion in this review and because participants in trials received extra attention, training, and supervision and may have been healthier and more mobile than individuals not participating in trials, applicability to individual patients with $\mathrm{MS}, \mathrm{CP}$, and SCI and generalizability to other populations may be reduced.

Factors that could impact the applicability of our findings include the trial setting. Some trials were conducted in a rehabilitation facility, a special school, university, hospital or other location, but often the setting was not specified. Additionally, the cost of equipment may limit the ability of patients to participate in some types of exercise evaluated in clinical trials. For example, a treadmill with body weight support or the robotic equipment needed to engage in RAGT may be cost prohibitive for many patients, making these interventions less applicable to patients seen in primary care.

Another factor concerns the selection of participants. Patients were often excluded from trials if they had known cardiovascular disease, metabolic disease, or mental illness. Children with CP were typically excluded for recent surgery, an uncontrolled seizure disorder, contractures or significant spasticity in addition to a lack of other major medical or cognitive problems. This could reduce applicability to primary care patients who may have a medical, psychological, or cognitive issue not represented in clinical trials. Most studies enrolled participants with less disability, rather than the full spectrum of ability, although across studies the distribution of ability was wide. Additionally, this review included patients with MS, CP, or SCI and the findings may not be as applicable to primary care patients with a different disease or condition, although disabilities may be similar across conditions (e.g., Parkinson's disease with MS, severe arthritis with SCI) that would increase applicability.

Exercise dose may also influence applicability. We required a minimum of 10 exercise sessions on 10 different days of any intensity, for any duration, and over any period of time for a trial to be eligible for inclusion. No trials included identical training arms where only the duration of the exercise session or the period of time over which the exercise occurred varied. Four trials in this review varied intensity of exercise (two in MS, two in CP). In MS, downhill treadmill training was associated with significantly better results on mobility and function than uphill treadmill training, ${ }^{119}$ but there was no difference on mobility or balance with whole body vibration (WBV) versus whole body light vibration. ${ }^{185} \mathrm{In} \mathrm{CP}$, there was no difference on function between RAGT with resistance compared with RAGT with assistance ${ }^{100}$ but improved sitting scores with 24 hippotherapy sessions over 12 weeks compared with 12 sessions. ${ }^{168} \mathrm{~A}$ 2019 systematic review ${ }^{274}$ of trials that enrolled participants with CP found that improvement in Gross Motor Function Measure scores was positively related to the number of hours trained daily. Additionally, this report focused on supervised exercise training and excluded all leisuretime and lifestyle physical activity interventions, which may have greater and more sustained short- as well as long-term health effects.

\section{Limitations of the Evidence Base}

Interventions tended to vary by population. For example, most hippotherapy trials were conducted in participants with $\mathrm{CP}$, whereas most trials with a strength component were in participants with MS, while RAGT trials were well dispersed across the three included 
populations. However, there were few trials or no trials of several interventions conducted in participants with SCI, limiting the ability to draw firm conclusions on benefits of these particular training modalities. Even when trials of various interventions were pooled in meta-analyses, few trials were conducted in an SCI population, resulting in insufficient evidence for several outcomes. For some of the interventions, there was also little evidence in participants with CP.

Another limitation is the rather large proportion of included studies that were rated poor quality (25\%). This rating was given because of serious methodological limitations in trials such as high attrition or lack of similarity of patient characteristics between groups at baseline, which could jeopardize the reliability of the findings. Additionally, it is often impossible to blind participants to exercise category, particularly if they are in the no exercise, attention control, or a waitlist control group. We conducted sensitivity analyses excluding poor-quality trials to determine if pooled results depended on the inclusion of poor-quality studies and reported both results. Additionally, studies were usually less than 6 months in duration, which did not permit assessment of clinical health outcomes that take time to develop, such as coronary artery disease. Few studies conducted subgroup analysis, which was often not possible because sample sizes of trials were so small, often less than 40 participants. Although some studies reported the physical activity to be low, moderate, or high intensity, most studies did not include a description of involved effort and studies often did not include a measure of intensity of the intervention (e.g., perceived effort or degree of energy expenditure) making it impossible to compare studies based on intensity, or describe the activities the control group experienced, making it difficult to determine which studies could be pooled in a meta-analysis. Many studies did not report harms or did not report that there were no harms or adverse events. Without adequate assessment and reporting of adverse events, the potential harms of a particular physical exercise regime are unknown. See Key Question 4 results for additional information on weakness and gaps in the evidence base. Additionally, many studies did not include a usual care or no treatment arm. Without a usual care comparator, it is difficult to be certain if a particular intervention is effective, even if postintervention assessment values are statistically improved from baseline values. It could be that just being in a study results in improvement unrelated to the intervention. Most of the RAGT studies in CP and SCI included in this review did not have a usual care arm, limiting the ability to draw conclusions regarding RAGT effectiveness in these populations. Below is a discussion of the limitations stratified by the effort needed to overcome major limitations of the evidence base.

\section{Addressing Limitations: Minimal Effort}

Studies often did not describe the activities the control group experienced, making it difficult to determine which studies could be pooled in a meta-analysis. In studies that did not use a waitlist control or a no-treatment control (including no usual care physical activities), it is important to specify the nature of the control intervention. This includes number of sessions, length of sessions, and specifics of physical activities involved, rather than just stating "usual care," "routine physiotherapy," or "conventional rehabilitation," since what is usual care in one medical center, geographic area, or country may be very different from another.

Studies often did not report harms or did not report that there were no harms or adverse events. Without adequate assessment and reporting of adverse events, the potential harms of a particular physical exercise regime are unknown. Studies should report that "adverse events were not assessed" or indicate how adverse events and harms were systematically identified (e.g., by 
questionnaire, by standardized interview) and provide documentation of any questionnaire or list of interview questions used.

Another limitation is the rather large proportion of included studies that were rated poor quality. Straightforward ways to improve study quality ratings are to report the specifics of randomization (e.g., random numbers table, cite randomization website used) and to report how the allocation was concealed (e.g., opaque, sealed, sequentially-numbered envelopes; central, Web-based reporting of allocation). Reporting who was blinded in the study is also an important aspect of trial design that should be mentioned (and blinding those who can be blinded, especially the outcome assessor, improves the reliability of the results).

These are simple additions to reporting how a given trial was actually conducted and require little or no additional work.

\section{Addressing Limitations: Moderate to Large Effort}

Not all the elements assessed in quality rating a trial are as easy to improve upon. Studies rated poor quality usually have other flaws in addition to inadequate reporting of study methodology. Serious methodological limitations in trials such as high attrition and/or lack of similarity of patient characteristics between groups at baseline could also jeopardize the reliability of the findings.

To reduce baseline differences in patient characteristics between treatment groups in trials with small sample sizes, the technique of minimization can decrease the risk of bias that happens when the two groups being compared are dissimilar on prognostic participant characteristics such as age, gender, or comorbidities. Low quality ratings due to large or unequal attrition between groups is more difficult to remedy without anticipating why participants are likely to leave the study.

\section{Addressing Limitations: Large Effort}

Small sample size is perhaps the most difficult limitation to overcome and this has no easy remedy given the populations we have included in this review. The cost of conducting studies with larger sample sizes are generally higher and larger studies may be more time-consuming to complete, especially if study enrollment is slow. Crossover studies reduce the required sample size needed to demonstrate a treatment effect, but are associated with their own potential bias due to potentially inadequate washout from the previous treatment(s). Several smaller studies can be pooled to demonstrate a treatment effect, but require standardized methodology across trials.

Another technique to increase sample size would be to broaden the definition of the study population. For example, the population could be wheelchair users without mobility due to lower limb dysfunction. This could include participants with limb paralysis, weakness, or absence, broadening the population to individuals with MS, CP, SCI, as well as stroke, amputation, amyotrophic lateral sclerosis, and others. In addition to reporting overall findings, results could be stratified by condition or by category of condition (e.g., MS or neurological disease) or some other method of grouping populations that would be meaningful.

\section{Implications for Clinical and Policy Decision Making}

This review has implications for clinical and policy decision making for patients using a wheelchair or patients who may potentially benefit from using a wheelchair in the future. This review provides evidence for the necessity of implementing physical activity programs for 
people with disability and/or chronic conditions. Not only is physical activity in general associated with improved physical function, but it is also associated with improved mental health as well. This review also provides limited evidence that physical activity may help prevent negative consequences of sustained sedentariness, such as increased spasticity. Physical activity should be a prescribed element in overall healthcare for those with disabilities and not just an afterthought. Findings of this review are consistent with previous reviews and support current guidelines that advise regular exercise in people with MS, CP, and SCI. Exercise interventions that are strength focused should include aerobic elements (and balance exercises as needed). Exercise interventions that consist primarily of cycling should include strength and/or balance exercises as well for optimum improvement in function. In general, evidence supports physical exercise to improve walking ability, function, balance, depression, strength, and aerobic fitness.

\section{Implications for All Providers}

It is important for providers to understand the barriers to physical exercise for their patients. This may include lack of accessibility, ${ }^{275,276}$ lack of time, ${ }^{275,277-279}$ lack of enjoyment with prescribed exercise, ${ }^{275,279}$ and lack of adequate social support. ${ }^{275,278,280,281}$ Other potential barriers to exercise include lack of transportation, ${ }^{276,279,281}$ lack of awareness of the relationship between exercise and health, ${ }^{277,279}$ and high cost. ${ }^{276,277,281}$ All providers should address these and any other potential barriers that may exist with their patients when prescribing physical activity.

Motivational interviewing may be helpful. ${ }^{282-284}$ Providers need to take the individual patient into account. The exercise modality with the greatest evidence for benefit in MS may not be the best choice for their particular patient with MS.

\section{Implications for Primary Care Providers with MS, CP, and SCI Patients}

Broadly speaking, in patients with MS, CP, and SCI, moving the body in an effort to improve cardiovascular fitness is desired. In patients with SCI, consideration should be given to monitoring the patient's cardiovascular and thermodynamic response to ensure a particular cardiovascular activity at a specific intensity is safe for the patient, so as to avoid serious episodes of autonomic dysreflexia, which may be life threatening. We found benefits in all three included populations with aerobic exercise.

Strength exercises should also be an included part of any exercise routine for patients with MS, CP, and SCI. Although this review found support for improved walking with combined strength and aerobic exercises in study participants with MS but insufficient evidence for benefit in CP and SCI, a 2019 systematic review ${ }^{285}$ found improved function (Gross Motor Function Measure [GMFM] scores) in children with CP. Cardiovascular fitness and muscle strength may be improved with aerobic and resistance training, based on a 2019 systematic review of systematic reviews in people with SCI.

Balance exercises may also prove beneficial additions to a physical exercise program for people with MS, CP, and SCI. This review found that balance training may improve balance, function, and/or quality of life in MS and CP. While the evidence was too sparse to draw a conclusion regarding balance training in SCI, a $2019 \mathrm{RCT}^{248}$ that enrolled people with chronic SCI reported improved balance with a combination of aerobic, strength, and core stability training. 


\section{Implications for Primary Care Providers With Patients With Disabilities Other than MS, CP, or SCI}

Although we limited this review to evidence in MS, CP, and SCI, other medical illnesses and injuries may respond similarly to physical activity as our included populations. For instance, patients with Parkinson's disease or Lyme disease may have similar issues and challenges as patients with MS. Patients with intellectual disability and motor impairment due to other neurological disease or inborn errors of metabolism may face similar challenges as patients with $\mathrm{CP}$. And patients with stroke, arthritis, or the wheelchair-using elderly may have issues and challenges similar to those with SCI. As long as physical exercise can be performed safely, aerobic, strength, and balance training may benefit these populations as well.

Several systematic reviews of the effects of physical exercise on the health of people with other conditions have found benefits to exercise. For example, a 2016 review $^{286}$ found gait performance improved with gait and strength training in people with lower limb amputation using a prosthesis. A 2019 systematic review ${ }^{287}$ found that home-based exercise improved balance and gait speed in people with Parkinson's disease and that the improvement was similar to that seen in center-based exercise. A 2019 systematic review ${ }^{288}$ in stroke patients reported improved walking speed and endurance with a combination of aerobic and strength exercises. A 2015 systematic $^{289}$ review of elderly patients reported a large effect of Pilates in improving muscle strength, walking, ADL, and quality of life. A 2015 systematic review ${ }^{290}$ found improved depression scores with exercise in adult patients with arthritis.

Similar to able-bodied people, physical exercise has the potential to benefit those with various disabilities.

\section{Implications for Physical Activity During a Pandemic}

Life during a pandemic may present unique challenges to those with mobility constraints. Quarantined individuals may be less likely to exercise and frailty may increase without regular physical activity. A rapid review concerning those who are now housebound due to COVID-19 concluded that people should continue to engage in strength, resistance, and balance training, that adding a social element may help with motivation and decrease mental distress, and that technology that supports physical activity such as use of the internet or video games may be helpful. ${ }^{291}$ The Multiple Sclerosis Association of America has a Webinar on dealing with the COVID-19 pandemic and recommends continuing to keep physically active. ${ }^{292}$ An article in Frontiers in Neurology recommends accelerating the use of telemedicine to care for patients with $\mathrm{CP}$ during a pandemic indicating that telemedicine can enable healthcare personnel to manage medication and provide exercises for the patient in a home environment. ${ }^{293}$ A Department of Veterans Affairs tip sheet for veterans with SCI recommends continuing to care for oneself and move the body during the pandemic. ${ }^{294}$ There are several online resources that patients with MS, $\mathrm{CP}, \mathrm{SCI}$, and other conditions may find useful to modify or jumpstart an exercise routine. One such website provides a chair-based 10-minute workout for those whose exercise routines have been upended by COVID-19. ${ }^{295}$ Results from this report can also inform efforts to maintain physical activity during the COVID-19 pandemic. Exercise activities that can be done at home and were found to have benefits include dance, stationary cycling, treadmill, motion gaming and multimodal progressive training activities for children with CP and adults with MS. Balance exercises can be done at home, and were found beneficial for patients with MS. Combining the evidence on all aerobic activities, many of which can be done at home, showed beneficial results 
for patients with $\mathrm{CP}, \mathrm{MS}$, or SCI. Even though keeping physically active may be more of a challenge during a pandemic, it is important to continue to do so.

\section{Limitations of the Systematic Review Process}

We excluded non-English language articles and studies published only as abstracts. Additionally we did not check for publication bias due to insufficient number of trials available for most meta-analyses and the heterogeneity in physical exercise interventions, comparisons groups, and patient populations evaluated in trials. Statistical heterogeneity was present in a number of meta-analyses. We used a random effects model and conducted stratified analysis based on the intervention. Due to scope limitations, we did not include leisure-time physical activity or physical activity conducted outside of the research study, which is the bulk of physical activity in all populations; it is also difficult to compare the results between different leisure-time physical activities without well-defined physical activity parameters that exists in trials.

\section{Research Recommendations}

Larger, well-conducted RCTs are needed in patients with MS, CP, and SCI to address evidence gaps and to confirm current findings. Large, controlled cohort studies could also provide data on long-term outcomes and harms of the intervention. Larger sample sizes would enable subgroup analyses based on patient characteristics and comorbidities. Longer duration studies would enable identification of interventions that demonstrate reduced cardiovascular and metabolic adverse events and improved mortality, fitness, function, and quality of life over the long term. Studies providing data on the intensity of physical activity are needed. Studies that enroll participants with high degrees of disability are also needed. Studies, if possible, should have a control arm that receives no treatment, such as a waitlist control, to demonstrate that a particular intervention is effective when compared with no treatment. 


\section{Conclusion}

Physical activity was associated with improvements in walking ability, general function, balance (including fall risk), depression, sleep, activities of daily living, female sexual function, and aerobic capacity, depending on population enrolled and type of exercise utilized. No studies reported long-term cardiovascular or metabolic disease health outcomes. Future trials could alter these findings, and further research is needed to examine health outcomes and to understand the magnitude and clinical importance of benefits seen in intermediate outcomes. 


\section{References}

1. Physical Activity Guidelines Advisory Committee. 2008 Physical Activity Guidelines for Americans [Archived]. Washington, DC: U.S. Department of Health and Human Services--National Institutes of Health--Office of Disease Prevention and Health Promotion; 2008. Available at: https:/health.gov/paguidelines/2008/.

2. U.S. Department of Health and Human Services. Physical Activity Guidelines for Americans, 2nd edition. Washington, DC: U.S. Department of Health and Human Services; 2018. Available at: https://health.gov/paguidelines/secondedition/pdf/Physical_Activity_Guidelines 2 nd edition.pdf.

3. Confavreux C, Vukusic S. Natural history of multiple sclerosis: a unifying concept. Brain. 2006 Mar;129(Pt 3):606-16. doi: 10.1093/brain/awl007. PMID: 16415308.

4. Okoro CA, Hollis ND, Cyrus AC, et al. Prevalence of Disabilities and Health Care Access by Disability Status and Type Among Adults - United States, 2016. MMWR. Morbidity and mortality weekly report. 2018 Aug 17;67(32):882-7. doi: 10.15585/mmwr.mm6732a3. PMID: 30114005 .

5. UK Chief Medical Officers' Physical Activity Guidelines. London, England: U.K. Department of Health \& Social Care; September 7, 2019. Available at: https://assets.publishing.service.gov.uk/gove rnment/uploads/system/uploads/attachment data/file/829841/uk-chief-medical-officersphysical-activity-guidelines.pdf.

6. Centers for Disease Control and Prevention. Data and Statistics for Cerebral Palsy. National Center on Birth Defects and Developmental Disabilities. 2018. https://www.cdc.gov/ncbddd/cp/data.html. Accessed July 30, 2019.

7. Wallin MT, Culpepper WJ, Campbell JD, et al. The prevalence of MS in the United States: A population-based estimate using health claims data. Neurology. 2019 Mar 5;92(10):e1029-e40. doi: 10.1212/wnl.0000000000007035. PMID: 30770430 .
8. National Spinal Cord Injury Statistical Center. Facts and Figures at a Glance. Birmingham, AL: University of Alabama at Birmingham; 2016.

https://www.nscisc.uab.edu/Public/Facts $\% 2$ 02016.pdf. Accessed September 12, 2019.

9. SCI-Info-Pages. Spinal Cord Injury Facts \& Statistics. https://www.sci-infopages.com/spinal-cord-injury-facts-andstatistics. Accessed October 22, 2019.

10. Kister I, Chamot E, Salter AR, et al. Disability in multiple sclerosis: a reference for patients and clinicians. Neurology. 2013 Mar 12;80(11):1018-24. doi: 10.1212/WNL.0b013e3182872855. PMID: 23427319 .

11. Hernandez Clares R, Carreon Guarnizo E, Sanchez Galvez RM, et al. A change in multiple sclerosis morbidity spectrum [abstract]. In: Thompson, AJ, ed. Poster Session 1. MSParis2017. 7th Joint ECTRIMS-ACTRIMS Meeting; 2017 Oct 25-28; Paris, France. Newbury Park, California: SAGE Publishing; 2017. Mult Scler. 2017;23(S3):85-426. doi: 10.1177/1352458517731404. PMID: 29065758.

12. Sweis R, Biller J. Systemic Complications of Spinal Cord Injury. Curr Neurol Neurosci Rep. 2017 Feb;17(2):8. doi: 10.1007/s11910-017-0715-4. PMID: 28188542.

13. Froehlich-Grobe K, Washburn RA. Exploring obesity among wheelchair users: BMI vs body compostion. International Journal of Excercise Science: Conference Proceedings; 2010 Nov 18-19; Houston. 2010;6(1):Article 28.

14. Gupta N, White KT, Sandford PR. Body mass index in spinal cord injury -- a retrospective study. Spinal Cord. 2006 Feb;44(2):92-4. doi: 10.1038/sj.sc.3101790. PMID: 16030513.

15. Khurana SR, Bamer AM, Turner AP, et al. The prevalence of overweight and obesity in veterans with multiple sclerosis. Am J Phys Med Rehabil. 2009 Feb;88(2):83-91. doi: 10.1097/PHM.0b013e318194f8b5. PMID: 19169174. 
16. McPhee PG, Gorter JW, Cotie LM, et al. Descriptive data on cardiovascular and metabolic risk factors in ambulatory and non-ambulatory adults with cerebral palsy. Data in brief. 2015 Dec;5:967-70. doi: 10.1016/j.dib.2015.10.045. PMID: 26759816.

17. Whitney DG, Hurvitz EA, Ryan JM, et al. Noncommunicable disease and multimorbidity in young adults with cerebral palsy. Clinical epidemiology. 2018;10:5119. doi: 10.2147/clep.s159405. PMID: 29750055.

18. Mincu RI, Magda LS, Florescu M, et al. Cardiovascular dysfunction in multiple sclerosis. Maedica. 2015 Sep;10(4):364-70. PMID: 28465741.

19. Peterson MD, Ryan JM, Hurvitz EA, et al. Chronic conditions in adults with cerebral palsy. Jama. 2015 Dec 1;314(21):2303-5. doi: 10.1001/jama.2015.11025. PMID: 26624831.

20. Wu CW, Huang SW, Lin JW, et al. Risk of stroke among patients with cerebral palsy: a population-based cohort study. Dev Med Child Neurol. 2017 Jan;59(1):52-6. doi: 10.1111/dmcn.13180. PMID: 27346658.

21. National Academies of Sciences E, Medicine, Health, et al. The Promise of Assistive Technology to Enhance Activity and Work Participation. Washington (DC): National Academies Press (US) Copyright 2017 by the National Academy of Sciences. All rights reserved.; 2017.

22. Dionne CD, Gainforth HL, Malley DA, et al. Examining implicit attitudes towards exercisers with a physical disability. ScientificWorldJournal. 2013;621596:1-8. doi: 10.1155/2013/621596. PMID: 23710142 .

23. Backus D. Increasing physical activity and participation in people with multiple sclerosis: a review. Arch Phys Med Rehabil. 2016 Sep;97(9 Suppl):S210-7. doi: 10.1016/j.apmr.2015.09.027. PMID: 27211007.
24. Casey B, Coote S, Shirazipour C, et al. Modifiable psychosocial constructs associated with physical activity participation in people with multiple sclerosis: a systematic review and metaanalysis. Arch Phys Med Rehabil. 2017 07;98(7):1453-75. doi: 10.1016/j.apmr.2017.01.027. PMID: 28254635.

25. de Groot S, van der Scheer JW, Bakkum AJ, et al. Wheelchair-specific fitness of persons with a long-term spinal cord injury: crosssectional study on effects of time since injury and physical activity level. Disabil Rehabil. 2016;38(12):1180-6. doi: 10.3109/09638288.2015.1076072. PMID: 26308969.

26. Phang SH, Martin Ginis KA, Routhier F, et al. The role of self-efficacy in the wheelchair skills-physical activity relationship among manual wheelchair users with spinal cord injury. Disabil Rehabil. 2012;34(8):625-32. doi: 10.3109/09638288.2011.613516. PMID: 21981243.

27. Mezini S, Soundy A. A thematic synthesis considering the factors which influence multiple sclerosis related fatigue during physical activity. Behav Sci (Basel). 2019 Jul 1;9(7):70. doi: 10.3390/bs9070070. PMID: 31266231.

28. Usuba K, Oddson B, Gauthier A, et al. Leisure-time physical activity in adults with Cerebral Palsy. Disabil Health J. 2015 Oct;8(4):611-8. doi: 10.1016/j.dhjo.2015.05.006. PMID: 26141088.

29. Claydon VE, Hol AT, Eng JJ, et al. Cardiovascular responses and postexercise hypotension after arm cycling exercise in subjects with spinal cord injury. Arch Phys Med Rehabil. 2006 Aug;87(8):1106-14. doi: 10.1016/j.apmr.2006.05.011. PMID: 16876557.

30. Myers JN, Hsu L, Hadley D, et al. Postexercise heart rate recovery in individuals with spinal cord injury. Spinal Cord. 2010 Aug;48(8):639-44. doi: 10.1038/sc.2009.196. PMID: 20125108. 
31. Get the Facts! Birmingham, AL: National Center on Health, Physical Activity and Disability. https:/www.nchpad.org/fppics/GettheFactsF INAL.pdf. Accessed September 21, 2019.

32. Best KL, Arbour-Nicitopoulos KP, Sweet SN. Community-based physical activity and wheelchair mobility programs for individuals with spinal cord injury in Canada: current reflections and future directions. J Spinal Cord Med. 2017 Nov;40(6):777-82. doi: 10.1080/10790268.2017.1367363. PMID: 28872428 .

33. North Carolina Office on Disability and Health. Removing Barriers to Health Clubs and Fitness Facilities: A Guide for Accommodating All Members, Including People With Disabilities and Older Adults. Chapel Hill: The University of North Carolina, Frank Porter Graham Child Development Institute; 2008. https://fpg.unc.edu/node/4110. Accessed September, 21, 2019.

34. Martin Ginis KA, Papathomas A, Perrier MJ, et al. Psychosocial factors associated with physical activity in ambulatory and manual wheelchair users with spinal cord injury: a mixed-methods study. Disabil Rehabil. 2017 01;39(2):187-92. doi: 10.3109/09638288.2015.1045991. PMID: 26104107.

35. de Groot S, van der Scheer JW, Bakkum AJ, et al. Wheelchair-specific fitness of persons with a long-term spinal cord injury: crosssectional study on effects of time since injury and physical activity level. Disabil Rehabil. 2016;38(12):1180-6. doi: 10.3109/09638288.2015.1076072. PMID: 26308969 .

36. Requejo PS, Furumasu J, Mulroy SJ. Evidence-based strategies for preserving mobility for elderly and aging manual wheelchair users. Top Geriatr Rehabil. 2015 Jan-Mar;31(1):26-41. doi: 10.1097/TGR.0000000000000042. PMID: 26366040 .
37. Bradbury KE, Guo W, Cairns BJ, et al. Association between physical activity and body fat percentage, with adjustment for BMI: a large cross-sectional analysis of UK Biobank. BMJ Open. 2017 Mar 24;7(3):e011843. doi: 10.1136/bmjopen2016-011843. PMID: 28341684.

38. $\mathrm{Hu}$ G, Jousilahti P, Barengo NC, et al. Physical activity, cardiovascular risk factors, and mortality among Finnish adults with diabetes. Diabetes Care. 2005 Apr;28(4):799-805. PMID: 15793176.

39. Salonen MK, Wasenius N, Kajantie E, et al. Physical activity, body composition and metabolic syndrome in young adults. PLoS One. 2015;10(5):e0126737. doi: 10.1371/journal.pone.0126737. PMID: 25992848.

40. Angevaren M, Aufdemkampe G, Verhaar HJJ, et al. Physical activity and enhanced fitness to improve cognitive function in older people without known cognitive impairment. The Cochrane database of systematic reviews. 2008(3):CD005381-CD. doi: 10.1002/14651858.CD005381.pub3. PMID: 18646126.

41. Helmrich SP, Ragland DR, Leung RW, et al. Physical activity and reduced occurrence of non-insulin-dependent diabetes mellitus. N Engl J Med. 1991 Jul 18;325(3):147-52. doi: 10.1056/nejm199107183250302. PMID: 2052059.

42. Jeon CY, Lokken RP, Hu FB, et al. Physical activity of moderate intensity and risk of type 2 diabetes: a systematic review. Diabetes Care. 2007 Mar;30(3):744-52. doi: 10.2337/dc06-1842. PMID: 17327354.

43. Mann S, Beedie C, Jimenez A. Differential effects of aerobic exercise, resistance training and combined exercise modalities on cholesterol and the lipid profile: review, synthesis and recommendations. Sports Med. 2014 Feb;44(2):211-21. doi: 10.1007/s40279-013-0110-5. PMID: 24174305.

44. Wen CP, Wai JP, Tsai MK, et al. Minimum amount of physical activity for reduced mortality and extended life expectancy: a prospective cohort study. Lancet. 2011 Oct 1;378(9798):1244-53. doi: 10.1016/s01406736(11)60749-6. PMID: 21846575. 
45. Herring MP, Fleming KM, Hayes SP, et al. Moderators of exercise effects on depressive symptoms in multiple sclerosis: a metaregression. Am J Prev Med. 2017 Oct;53(4):508-18. doi:

10.1016/j.amepre.2017.04.011. PMID: 28602542.

46. Verschuren O, Peterson MD, Balemans AC, et al. Exercise and physical activity recommendations for people with cerebral palsy. Dev Med Child Neurol. 2016 08;58(8):798-808. doi: 10.1111/dmcn.13053. PMID: 26853808.

47. Halabchi F, Alizadeh Z, Sahraian MA, et al. Exercise prescription for patients with multiple sclerosis; potential benefits and practical recommendations. BMC Neurol. 2017 Sep 16;17(1):185. doi: 10.1186/s12883-017-0960-9. PMID: 28915856.

48. Dalgas U. Rehabilitation and multiple sclerosis: hot topics in the preservation of physical functioning. J Neurol Sci. 2011 Dec;311 Suppl 1:S43-7. doi: 10.1016/s0022510x(11)70008-9. PMID: 22206766.

49. Methods Guide for Effectiveness and Comparative Effectiveness Reviews. Rockville, MD: Agency for Healthcare Research and Quality. https:/effectivehealthcare.ahrq.gov/topics/ce r-methods-guide/overview. Accessed September 12, 2019.

50. Hsieh HC. Effects of a gaming platform on balance training for children with cerebral palsy. Pediatr Phys Ther. 2018 Oct;30(4):303-8. doi: 10.1097/PEP.0000000000000521. PMID: 30199515.

51. Kalron A, Fonkatz I, Frid L, et al. The effect of balance training on postural control in people with multiple sclerosis using the CAREN virtual reality system: a pilot randomized controlled trial. J Neuroengineering Rehabil. 2016 Mar 1;13:13. doi: 10.1186/s12984-016-0124-y. PMID: 26925955.
52. Kalron A, Rosenblum U, Frid L, et al. Pilates exercise training vs. physical therapy for improving walking and balance in people with multiple sclerosis: a randomized controlled trial. Clin Rehabil. 2017 Mar;31(3):319-28. doi: 10.1177/0269215516637202. PMID: 26951348.

53. Negaresh R, Motl R, Mokhtarzade M, et al. Effect of short-term interval excercise training on fatigue, depression, and fitness in normal weight vs. overweight person with multiple sclerosis. Explore (NY). 2019 MarApr;15(2):134-41. doi: 10.1016/j.explore.2018.07.007. PMID: 30122328.

54. Young HJ, Mehta TS, Herman C, et al. The effects of M2M and adapted yoga on physical and psychosocial outcomes in people wth multiple sclerosis. Arch Phys Med Rehabil. 2019 Aug 6;100(3):391-400. doi: 10.1016/j.apmr.2018.06.032. PMID: 30092206.

55. Nelson HD. Systematic Reviews to Answer Health Care Questions. In: Gaertner R, editor. Philadelphia, PA: Wolters Kluwer; 2014.

56. Methods Guide for Effectiveness and Comparative Effectiveness Reviews. AHRQ Publication No. 10(14)-EHC063-EF. Rockville, MD: Agency for Healthcare Research and Quality; January 2014. Current chapters available at https://effectivehealthcare.ahrq.gov/topics/ce r-methods-guide/overview/.

57. Force USPST. U.S. Preventative Services Task Force Procedure Manual. Rockville, MD: 2017. https://www.uspreventiveservicestaskforce.o $\mathrm{rg} /$ Page/Name/procedure-manual.

58. Higgins JPT, Green S, editors. Cochrane Handbook for Systematic Reviews of Interventions Version 5.1.0 [updated March 2011]. The Cochrane Collaboration; 2011. Available at www.cochrane-handbook.org.

59. Aydin T, Akif Sariyildiz M, Guler M, et al. Evaluation of the effectiveness of home based or hospital based calisthenic exercises in patients with multiple sclerosis. Eur Rev Med Pharmacol Sci. 2014;18(8):1189-98. PMID: 24817294. 
60. Al-Sharman A, Khalil H, El-Salem K, et al. The effects of aerobic exercise on sleep quality measures and sleep-related biomarkers in individuals with Multiple Sclerosis: a pilot randomised controlled trial. NeuroRehabilitation. 2019;45(1):107-15. doi: 10.3233/NRE-192748. PMID: 31403958 .

61. Sadeghi Bahmani D, Razazian N, Farnia V, et al. Compared to an active control condition, in persons with multiple sclerosis two different types of exercise training improved sleep and depression, but not fatigue, paresthesia, and intolerance of uncertainty. Mult Scler Relat Disord. 2019 Nov;36:101356. doi: 10.1016/j.msard.2019.07.032. PMID: 31521917.

62. Kara B, Kucuk F, Poyraz EC, et al. Different types of exercise in multiple sclerosis: aerobic exercise or pilates, a single-blind clinical study. J Back Musculoskeletal Rehabil. 2017;30(3):565-73. doi: 10.3233/BMR-150515. PMID: 27911284.

63. Keser I, Meric A, Kirdi N, et al. Comparing routine neurorehabilitation programme with callisthenic exercises in multiple sclerosis. NeuroRehabilitation. 2011;29(1):91-8. doi: 10.3233/NRE-2011-0682. PMID: 21876301.

64. Teixeira-Machado L, Azevedo-Santos I, Desantana JM. Dance improves functionality and psychosocial adjustment in cerebral palsy: a randomized controlled clinical trial. Am J Phys Med Rehabil. 2017 Jun;96(6):424-9. doi: 10.1097/PHM.0000000000000646. PMID: 27820729.

65. Gibson N, Chappell A, Blackmore AM, et al. The effect of a running intervention on running ability and participation in children with cerebral palsy: a randomized controlled trial. Disabil Rehabil. 2018

Dec;40(25):3041-49. doi: 10.1080/09638288.2017.1367426. PMID: 28826274.
66. Kargarfard M, Shariat A, Ingle L, et al. Randomized controlled trial to examine the impact of aquatic exercise training on functional capacity, balance, and perceptions of fatigue in female patients with multiple sclerosis. Arch Phys Med Rehabil. 2018 Feb;99(2):234-41. doi: 10.1016/j.apmr.2017.06.015. PMID: 28735720.

67. Kooshiar H, Moshtagh M, Sardar MA, et al. Fatigue and quality of life of women with multiple sclerosis: a randomized controlled clinical trial. J Sports Med Phys Fitness. 2015 Jun;55(6):668-74. PMID: 25303070.

68. Marandi SM, Nejad VS, Shanazari Z, et al. A comparison of 12 weeks of pilates and aquatic training on the dynamic balance of women with mulitple sclerosis. Int J Prev Med. 2013 Apr;4(Suppl 1):S110-7. PMID: 23717760.

69. Marandi SM, Shahnazari Z, Minacian V, et al. A comparison between pilates exercise and aquatic training effects on mascular strength in women with mulitple sclerosis. Pak J Med Sci. 2013;29(1 Suppl):285-9. doi: 10.12669/pjms.291(Suppl).3518.

70. Razazian N, Yavari Z, Farnia V, et al. Exercising impacts on fatigue, depression, and paresthesia in female patients with multiple sclerosis. Med Sci Sports Exerc. 2016 May;48(5):796-803. doi: 10.1249/MSS.0000000000000834. PMID: 26656775.

71. Castro-Sanchez AM, Mataran-Penarrocha GA, Lara-Palomo I, et al. Hydrotherapy for the treatment of pain in people with multiple sclerosis: a randomized controlled trial. Evid Based Complement Alternat Med. 2012;473963:1-8. doi: 10.1155/2012/473963. PMID: 21785645.

72. Sadeghi Bahmani D, Motl RW, Razazian N, et al. Aquatic exercising may improve sexual function in females with multiple sclerosis - an exploratory study. Mult Scler Relat Disord. 2020 Apr 26;43:102106. doi: 10.1016/j.msard.2020.102106. PMID: 32428843 . 
73. Adar S, Dundar U, Demirdal ÜS, et al. The effect of aquatic exercise on spasticity, quality of life, and motor function in cerebral palsy. Turk J Phys Med Rehab. 2017 Aug 14;63(3):239-48. doi: 10.5606/tftrd.2017.280. PMID: 31453460.

74. Lai CJ, Liu WY, Yang TF, et al. Pediatric aquatic therapy on motor function and enjoyment in children diagnosed with cerebral palsy of various motor severities. J Child Neurol. 2015 Feb;30(2):200-8. doi: 10.1177/0883073814535491. PMID: 24907137.

75. Gorman PH, Scott W, VanHiel L, et al. Comparison of peak oxygen consumption response to aquatic and robotic therapy in individuals with chronic motor incomplete spinal cord injury: a randomized controlled trial. Spinal Cord. 2019 Jun;57(6):471-81. doi: 10.1038/s41393-019-0239-7. PMID: 30659286.

76. Jung J, Chung E, Kim K, et al. The effects of aquatic exercise on pulmonary function in patients with spinal cord injury. J Phys Ther Sci. 2014 May;26(5):707-9. doi: 10.1589/jpts.26.707. PMID: 24926136.

77. Baquet L, Hasselmann H, Patra S, et al. Short-term interval aerobic exercise training does not improve memory functioning in relapsing-remitting multiple sclerosis-a randomized controlled trial. PeerJ. 2018 Dec 12;6:e6037. doi: 10.7717/peerj.6037. PMID: 30581662 .

78. Heine M, Verschuren O, Hoogervorst EL, et al. Does aerobic training alleviate fatigue and improve societal participation in patients with multiple sclerosis? A randomized controlled trial. Mult Scler. 2017 Oct;23(11):1517-26. doi: $10.1177 / 1352458517696596$. PMID: 28528566 .

79. Hebert JR. Effects of vestibular rehabilitation on MS-related fatigue: Randomized control trial. Dissertation Abstracts International: Section B: The Sciences and Engineering. 2009;70(6B):3470.
80. Hebert JR, Corboy JR, Manago MM, et al. Effects of vestibular rehabilitation on multiple sclerosis-related fatigue and upright postural control: a randomized controlled trial. Phys Ther. 2011 Aug;91(8):1166-83. doi: 10.2522/ptj.20100399. PMID: 21680771.

81. Hochsprung A, Granja Dominguez A, Magni E, et al. Effect of visual biofeedback cycling training on gait in patients with multiple sclerosis. Neurologia. 2017 Sep 6 doi: 10.1016/j.nrl.2017.07.008. PMID: 28888468.

82. Collett J, Dawes H, Meaney A, et al. Exercise for multiple sclerosis: a singleblind randomized trial comparing three exercise intensities. Mult Scler. 2011 May;17(5):594-603. doi: 10.1177/1352458510391836. PMID: 21247971.

83. Tollar J, Nagy F, Toth BE, et al. Exercise effects on multiple sclerosis quality of life and clinical-motor symptoms. Med Sci Sports Exerc. 2020 May;52(5):1007-14. doi: 10.1249/MSS.0000000000002228. PMID: 31876670 .

84. Niwald M, Redlicka J, Miller E. The effects of aerobic training on the functional status, quality of life, the level of fatigue and disability in patients with multiple sclerosisa preliminary report. Aktualnosci Neurologiczne. 2017;17(1):15-22. doi: 10.15557/AN.2017.0002.

85. Fowler EG, Knutson LM, Demuth SK, et al. Pediatric endurance and limb strengthening (PEDALS) for children with cerebral palsy using stationary cycling: a randomized controlled trial. Phys Ther. 2010 Mar;90(3):367-81. doi: 10.2522/ptj.20080364. PMID: 20093327.

86. Demuth SK, Knutson LM, Fowler EG. The PEDALS stationary cycling intervention and health-related quality of life in children with cerebral palsy: a randomized controlled trial. Dev Med Child Neurol. 2012 Jul;54(7):65461. doi: $10.1111 /$ j.1469-8749.2012.04321.x. PMID: 22582760. 
87. Bryant E, Pountney T, Williams H, et al. Can a six-week exercise intervention improve gross motor function for nonambulant children with cerebral palsy? A pilot randomized controlled trial. Clin Rehabil. 2013 Feb;27(2):150-9. doi: 10.1177/0269215512453061. PMID: 22850757.

88. Nsenga AL, Shephard RJ, Ahmaidi S. Aerobic training in children with cerebral palsy. Int J Sports Med. 2013 Jun;34(6):5337. doi: 10.1055/s-0032-1321803. PMID: 23184482 .

89. Akkurt H, Karapolat HU, Kirazli Y, et al. The effects of upper extremity aerobic exercise in patients with spinal cord injury: a randomized controlled study. Eur J Phys Rehabil Med. 2017 Apr;53(2):219-27. doi: 10.23736/S1973-9087.16.03804-1. PMID: 27824234.

90. Sadowsky CL, Hammond ER, Strohl AB, et al. Lower extremity functional electrical stimulation cycling promotes physical and functional recovery in chronic spinal cord injury. J Spinal Cord Med. 2013

Nov;36(6):623-31. doi: 10.1179/2045772313Y.0000000101. PMID: 24094120.

91. Lai CH, Chang WH, Chan WP, et al. Effects of functional electrical stimulation cycling exercise on bone mineral density loss in the early stages of spinal cord injury. J Rehabil Med. 2010 Feb;42(2):150-4. doi: 10.2340/16501977-0499. PMID: 20140411.

92. Lavado EL, Cardoso JR, Silva LG, et al. Effectiveness of aerobic physical training for treatment of chronic asymptomatic bacteriuria in subjects with spinal cord injury: a randomized controlled trial. Clin Rehabil. 2013 Feb;27(2):142-9. doi: 10.1177/0269215512450522. PMID: 22837545.

93. Valent L, Dallmeijer A, Houdijk H, et al. Effects of hand cycle training on wheelchair capacity during clinical rehabilitation in persons with a spinal cord injury. Disabil Rehabil. 2010;32(26):2191-200. doi: 10.3109/09638288.2010.509461. PMID: 20726737.
94. Pompa A, Morone G, Iosa M, et al. Does robot-assisted gait training improve ambulation in highly disabled multiple sclerosis people? A pilot randomized control trial. Mult Scler. 2017 Apr;23(5):696-703. doi: $10.1177 / 1352458516663033$. PMID: 27486219.

95. Russo M, Dattola V, De Cola MC, et al. The role of robotic gait training coupled with virtual reality in boosting the rehabilitative outcomes in patients with multiple sclerosis. Int J Rehabil Res. 2018 Jun;41(2): 166-72. doi: 10.1097/MRR.0000000000000270. PMID: 29384762.

96. Calabro RS, Russo M, Naro A, et al. Robotic gait training in multiple sclerosis rehabilitation: can virtual reality make the difference? Findings from a randomized controlled trial. J Neurol Sci. 2017 Jun 15;377:25-30. doi: 10.1016/j.jns.2017.03.047. PMID: 28477702.

97. Straudi S, Fanciullacci C, Martinuzzi C, et al. The effects of robot-assisted gait training in progressive multiple sclerosis: a randomized controlled trial. Mult Scler. 2016 Mar;22(3):373-84. doi: 10.1177/1352458515620933. PMID: 26658817.

98. Straudi S, Manfredini F, Lamberti N, et al. Robot-assisted gait training is not superior to intensive overground walking in multiple sclerosis with severe disability (the RAGTIME study): a randomized controlled trial. Mult Scler. 2019 Mar 4 doi: 10.1177/1352458519833901. PMID: 30829117.

99. Wu M, Kim J, Arora P, et al. Effects of the integration of dynamic weight shifting training into treadmill training on walking function of children with cerebral palsy: a randomized controlled study. Am J Phys Med Rehabil. 2017 Nov;96(11):765-72. doi: 10.1097/PHM.0000000000000776. PMID: 28644244.

100. Wu M, Kim J, Gaebler-Spira DJ, et al. Robotic resistance treadmill training improves locomotor function in children with cerebral palsy: a randomized controlled pilot study. Arch Phys Med Rehabil. 2017 Nov;98(11):2126-33. doi: 10.1016/j.apmr.2017.04.022. PMID: 28576629. 
101. Wallard L, Dietrich G, Kerlirzin Y, et al. Robotic-assisted gait training improves walking abilities in diplegic children with cerebral palsy. Europ J Paediatr Neurol. 2017 May;21(3):557-64. doi: 10.1016/j.gaitpost.2017.11.007. PMID: 28188024.

102. Wallard L, Dietrich G, Kerlirzin Y, et al. Effect of robotic-assisted gait rehabilitation on dynamic equilibrium control in the gait of children with cerebral palsy. Gait Posture. 2018 Feb;60:55-60. doi: 10.1016/j.gaitpost.2017.11.007. PMID: 29156378.

103. Aras B, Yasar E, Kesikburun S, et al. Comparison of the effectiveness of partial body weight-supported treadmill exercises, robotic-assisted treadmill exercises, and anti-gravity treadmill exercises in spastic cerebral palsy. Turk J Phys Med Rehabil. 2019 Jun;65(4):361-70. doi: 10.5606/tftrd.2019.3078. PMID: 31893273.

104. Klobucka S, Klobucky R, Kollar B. Effect of robot-assisted gait training on motor functions in adolescent and young adult patients with bilateral spastic cerebral palsy: A randomized controlled trial.

NeuroRehabilitation. 2020 Oct 29;29:29. doi: 10.3233/NRE-203102. PMID: 33136072 .

105. Peri E, Turconi AC, Biffi E, et al. Effects of dose and duration of Robot-Assisted Gait Training on walking ability of children affected by cerebral palsy. Technol Health Care. 2017 Aug 9;25(4):671-81. doi: 10.3233/THC-160668. PMID: 28436398.

106. Yazici M, Livanelioglu A, Gucuyener K, et al. Effects of robotic rehabilitation on walking and balance in pediatric patients with hemiparetic cerebral palsy. Gait Posture. 2019 May;70:397-402. doi: 10.1016/j.gaitpost.2019.03.017. PMID: 30974395.

107. Esclarin-Ruz A, Alcobendas-Maestro M, Casado-Lopez R, et al. A comparison of robotic walking therapy and conventional walking therapy in individuals with upper versus lower motor neuron lesions: a randomized controlled trial. Arch Phys Med Rehabil. 2014 Jun;95(6):1023-31. doi: 10.1016/j.apmr.2013.12.017. PMID: 24393781.
108. Field-Fote EC, Roach KE. Influence of a locomotor training approach on walking speed and distance in people with chronic spinal cord injury: a randomized clinical trial. Phys Ther. 2011 Jan;91(1):48-60. doi: 10.2522/ptj.20090359. PMID: 21051593.

109. Sandler EB, Roach KE, Field-Fote EC. Dose-response outcomes associated with different forms of locomotor training in persons with chronic motor-incomplete spinal cord injury. J Neurotrauma. 2017 May 15;34(10):1903-8. doi: 10.1089/neu.2016.4555. PMID: 27901413.

110. Kressler J, Nash MS, Burns PA, et al. Metabolic responses to 4 different body weight-supported locomotor training approaches in persons with incomplete spinal cord injury. Arch Phys Med Rehabil. 2013 Aug;94(8):1436-42. doi: 10.1016/j.apmr.2013.02.018. PMID: 23473703.

111. Kumru H, Benito-Penalva J, Valls-Sole J, et al. Placebo-controlled study of rTMS combined with Lokomat ${ }^{\circledR}$ gait training for treatment in subjects with motor incomplete spinal cord injury. Exp Brain Res. 2016 Dec;234(12):3447-55. doi: 10.1007/s00221016-4739-9. PMID: 27469242.

112. Shin JC, Kim JY, Park HK, et al. Effect of robotic-assisted gait training in patients with incomplete spinal cord injury. Ann Rehabil Med. 2014 Dec;38(6):719-25. doi: 10.5535/arm.2014.38.6.719. PMID: 25566469 .

113. Huang Q, Yu L, Gu R, et al. Effects of robot training on bowel function in patients with spinal cord injury. J Phys Ther Sci. 2015 May;27(5):1377-8. doi: 10.1589/jpts.27.1377. PMID: 26157223.

114. Duffell LD, Niu X, Brown G, et al. Variability in responsiveness to interventions in people with spinal cord injury: do some respond better than others? Conf Proc IEEE Eng Med Biol Soc. 2014;2014:5872-5. doi: 10.1109/EMBC.2014.6944964. PMID: 25571332 . 
115. Yildirim MA, Ones K, Goksenoglu G. Early term effects of robotic assisted gait training on ambulation and functional capacity in patients with spinal cord injury. Turk J Med Sci. 2019 Jun 18;49(3):838-43. doi: 10.3906/sag-1809-7. PMID: 31134784.

116. Midik M, Paker N, Bugdayci D, et al. Effects of robot-assisted gait training on lower extremity strength, functional independence, and walking function in men with incomplete traumatic spinal cord injury. Turk J Phys Med Rehabil. 2020 Mar;66(1):54-9. doi: 10.5606/tftrd.2020.3316. PMID: 32318675.

117. Gervasoni E, Cattaneo D, Jonsdottir J. Effect of treadmill training on fatigue in multiple sclerosis: a pilot study. Int J Rehabil Res. 2014 Mar;37(1):54-60. doi: 10.1097/MRR.0000000000000034. PMID: 24220224.

118. Jonsdottir J, Gervasoni E, Bowman T, et al. Intensive multimodal training to improve gait resistance, mobility, balance and cognitive function in persons with multiple sclerosis: a pilot randomized controlled trial. Front Neurol. 2018 Sep 28;9:800. doi: 10.3389/fneur.2018.00800. PMID: 30323787 .

119. Samaei A, Bakhtiary AH, Hajihasani A, et al. Uphill and downhill walking in multiple sclerosis: a randomized controlled trial. Int J MS Care. 2016 Jan-Feb;18(1):34-41. doi: 10.7224/1537-2073.2014-072. PMID: 26917996.

120. Ahmadi A, Arastoo AA, Nikbakht M, et al. Comparison of the effect of 8 weeks aerobic and yoga training on ambulatory function, fatigue and mood status in MS patients. Iran Red Crescent Med J. 2013 Jun;15(6):44954. doi: 10.5812/ircmj.3597. PMID: 24349740 .

121. Chrysagis N, Skordilis EK, Stavrou N, et al. The effect of treadmill training on gross motor function and walking speed in ambulatory adolescents with cerebral palsy: a randomized controlled trial. Am J Phys Med Rehabil. 2012 Sep;91(9):747-60. doi: 10.1097/PHM.0b013e3182643eba. PMID: 22902937.
122. Emara HA, El-Gohary TM, Al-Johany AA. Effect of body-weight suspension training versus treadmill training on gross motor abilities of children with spastic diplegic cerebral palsy. Eur J Phys Rehabil Med. 2016 Jun;52(3):356-63. PMID: 26845668.

123. Grecco LA, de Almeida Carvalho Duarte N, Mendonca ME, et al. Transcranial direct current stimulation during treadmill training in children with cerebral palsy: a randomized controlled double-blind clinical trial. Res Dev Disabil. 2014

Nov;35(11):2840-8. doi: 10.1016/j.ridd.2014.07.030. PMID: 25105567.

124. Grecco LA, Zanon N, Sampaio LM, et al. A comparison of treadmill training and overground walking in ambulant children with cerebral palsy: randomized controlled clinical trial. Clin Rehabil. 2013

Aug;27(8):686-96. doi: 10.1177/0269215513476721. PMID: 23503736 .

125. Johnston TE, Watson KE, Ross SA, et al. Effects of a supported speed treadmill training exercise program on impairment and function for children with cerebral palsy. Dev Med Child Neurol. 2011 Aug;53(8):742-50. doi: 10.1111/j.14698749.2011.03990.x. PMID: 21679357.

126. Kim OY, Shin YK, Yoon YK, et al. The effect of treadmill exercise on gait efficiency during overground walking in adults with cerebral palsy. Ann Rehabil Med. 2015 Feb;39(1):25-31. doi: 10.5535/arm.2015.39.1.25. PMID: 25750868 .

127. Willoughby KL, Dodd KJ, Shields N, et al. Efficacy of partial body weight-supported treadmill training compared with overground walking practice for children with cerebral palsy: a randomized controlled trial. Arch Phys Med Rehabil. 2010 Mar;91(3):333-9. doi: 10.1016/j.apmr.2009.10.029. PMID: 20298820. 
128. Swe NN, Sendhilnnathan S, van Den Berg $\mathrm{M}$, et al. Over ground walking and body weight supported walking improve mobility equally in cerebral palsy: a randomised controlled trial. Clin Rehabil. 2015 Nov;29(11):1108-16. doi: 10.1177/0269215514566249. PMID: 25636992.

129. Bahrami F, Noorizadeh Dehkordi S, Dadgoo $M$. The efficacy of treadmill training on walking and quality of life of adults with spastic cerebral palsy: a randomized controlled trial. Iran J Child Neurol. 2019 Fall;13(4):121-33. PMID: 31645872.

130. Duarte Nde A, Grecco LA, Galli M, et al. Effect of transcranial direct-current stimulation combined with treadmill training on balance and functional performance in children with cerebral palsy: a double-blind randomized controlled trial. PLoS One. 2014 Aug 29;9(8):e105777. doi: 10.1371/journal.pone.0105777. PMID: 25171216.

131. Aviram R, Harries N, Namourah I, et al. Effects of a group circuit progressive resistance training program compared with a treadmill training program for adolescents with cerebral palsy. Dev Neurorehabil. 2017 Aug;20(6):347-54. doi: 10.1080/17518423.2016.1212946. PMID: 27536805.

132. Nsenga Leunkeu A, Shephard RJ, Ahmaidi S. Six-minute walk test in children with cerebral palsy gross motor function classification system levels I and II: reproducibility, validity, and training effects. Arch Phys Med Rehabil. 2012

Dec;93(12):2333-9. doi: 10.1016/j.apmr.2012.06.005. PMID: 22721868.

133. Alexeeva N, Sames C, Jacobs PL, et al. Comparison of training methods to improve walking in persons with chronic spinal cord injury: a randomized clinical trial. J Spinal Cord Med. 2011;34(4):362-79. doi: 10.1179/2045772311Y.0000000018. PMID: 21903010 .
134. Giangregorio L, Craven C, Richards K, et al. A randomized trial of functional electrical stimulation for walking in incomplete spinal cord injury: effects on body composition. J Spinal Cord Med. 2012 Sep;35(5):351-60. doi: 10.1179/2045772312Y.0000000041. PMID: 23031172.

135. Hitzig SL, Craven BC, Panjwani A, et al. Randomized trial of functional electrical stimulation therapy for walking in incomplete spinal cord injury: effects on quality of life and community participation. Top Spinal Cord Inj Rehabil. 2013 Fall;19(4):245-58. doi: 10.1310/sci1904245. PMID: 24244090.

136. Kapadia N, Masani K, Catharine Craven B, et al. A randomized trial of functional electrical stimulation for walking in incomplete spinal cord injury: effects on walking competency. J Spinal Cord Med. 2014 Sep;37(5):511-24. doi: 10.1179/2045772314Y.0000000263. PMID: 25229735 .

137. Craven BC, Giangregorio LM, Alavinia SM, et al. Evaluating the efficacy of functional electrical stimulation therapy assisted walking after chronic motor incomplete spinal cord injury: effects on bone biomarkers and bone strength. J Spinal Cord Med. 2017 Nov;40(6):748-58. doi: 10.1080/10790268.2017.1368961. PMID: 28929919.

138. Yang JF, Musselman KE, Livingstone D, et al. Repetitive mass practice or focused precise practice for retraining walking after incomplete spinal cord injury? A pilot randomized clinical trial. Neurorehabil Neural Repair. 2014 May;28(4):314-24. doi: 10.1177/1545968313508473. PMID: 24213960 .

139. Musselman KE, Yang JF. Spinal cord injury functional ambulation profile: a preliminary look at responsiveness. Phys Ther. 2014 Feb;94(2):240-50. doi: 10.2522/ptj.20130071. PMID: 24114437.

140. van der Scheer JW, de Groot S, Tepper M, et al. Low-intensity wheelchair training in inactive people with long-term spinal cord injury: a randomized controlled trial on fitness, wheelchair skill performance and physical activity levels. J Rehabil Med. 2016 Jan;48(1):33-42. doi: 10.2340/165019772037. PMID: 26660337. 
141. Gandolfi M, Munari D, Geroin C, et al. Sensory integration balance training in patients with multiple sclerosis: a randomized, controlled trial. Mult Scler. 2015 Oct;21(11):1453-62. doi: 10.1177/1352458514562438. PMID: 25583852 .

142. Salci Y, Fil A, Armutlu K, et al. Effects of different exercise modalities on ataxia in multiple sclerosis patients: a randomized controlled study. Disabil Rehabil. 2017 Dec;39(26):2626-32. doi: 10.1080/09638288.2016.1236411. PMID: 27794631.

143. Carling A, Forsberg A, Gunnarsson M, et al. CoDuSe group exercise programme improves balance and reduces falls in people with multiple sclerosis: a multi-centre, randomized, controlled pilot study. Mult Scler. 2017 Sep;23(10):1394-404. doi: 10.1177/1352458516677591. PMID: 27834736.

144. Forsberg A, von Koch L, Nilsagard Y. Effects on balance and walking with the CoDuSe balance exercise program in people with multiple sclerosis: a multicenter randomized controlled trial. Mult Scler Int. 2016;2016 doi: 10.1155/2016/7076265. PMID: 28042485.

145. Afrasiabifar A, Karami F, Najafi Doulatabad S. Comparing the effect of CawthorneCooksey and Frenkel exercises on balance in patients with multiple sclerosis: a randomized controlled trial. Clin Rehabil. 2018 Jan;32(1):57-65. doi: 10.1177/0269215517714592. PMID: 28629268.

146. Brichetto G, Piccardo E, Pedulla L, et al. Tailored balance exercises on people with multiple sclerosis: a pilot randomized, controlled study. Mult Scler. 2015 Jul;21(8):1055-63. doi: 10.1177/1352458514557985. PMID: 25392337.

147. Amiri B, Sahebozamani M, Sedighi B. The effects of 10-week core stability training on balance in women with multiple sclerosis according to Expanded Disability Status Scale: a single-blinded randomized controlled trial. Eur J Phys Rehabil Med. 2019 Apr;55(2):199-208. doi: 10.23736/S1973-9087.18.04778-0. PMID: 29307152 .
148. Arntzen EC, Straume BK, Odeh F, et al. Group-based individualized comprehensive core stability intervention improves balance in persons with multiple sclerosis: a randomized controlled trial. Phys Ther. 2019 Aug 1;99(8):1027-38. doi: 10.1093/ptj/pzz017. PMID: 30722036.

149. Callesen J, Cattaneo D, Brincks J, et al. How do resistance training and balance and motor control training affect gait performance and fatigue impact in people with multiple sclerosis? A randomized controlled multicenter study. Mult Scler. 2019 Jul 24 doi: 10.1177/1352458519865740. PMID: 31339460 .

150. Arntzen EC, Straume B, Odeh F, et al. Group-based, individualized, comprehensive core stability and balance intervention provides immediate and long-term improvements in walking in individuals with multiple sclerosis: A randomized controlled trial. Physiother Res Int. 2020

Jan;25(1):e1798. doi: 10.1002/pri.1798. PMID: 31268223.

151. Ozkul C, Guclu-Gunduz A, Yazici G, et al. Effect of immersive virtual reality on balance, mobility, and fatigue in patients with multiple sclerosis: A single-blinded randomized controlled trial. European Journal of Integrative Medicine. 2020;35 doi: 10.1016/j.eujim.2020.101092.

152. Curtis DJ, Woollacott M, Bencke J, et al. The functional effect of segmental trunk and head control training in moderate-to-severe cerebral palsy: a randomized controlled trial. Dev Neurorehabil. 2018 Feb;21(2):91-100. doi: $10.1080 / 17518423.2016 .1265603$. PMID: 28045553.

153. Bleyenheuft Y, Ebner-Karestinos D, Surana $\mathrm{B}$, et al. Intensive upper- and lowerextremity training for children with bilateral cerebral palsy: a quasi-randomized trial. Dev Med Child Neurol. 2017 Jun;59(6):625-33. doi: 10.1111/dmcn.13379. PMID: 28133725 .

154. Lorentzen J, Greve LZ, Kliim-Due M, et al. Twenty weeks of home-based interactive training of children with cerebral palsy improves functional abilities. BMC Neurol. 2015 May 10;15:75. doi: 10.1186/s12883015-0334-0. PMID: 25956055. 
155. Kim BJ, Kim SM, Kwon HY. The effect of group exercise program on the self-efficacy and activities of daily living in adults with cerebral palsy. J Phys Ther Sci. 2017

Dec;29(12):2184-9. doi: 10.1589/jpts.29.2184. PMID: 29643601.

156. Norouzi E, Vaezmousavi M. Neurofeedback training and physical training differentially impacted on reaction time and balance skills among Iranian veterans with spinal cord injury. J Mil Veterans Health. 2019 Jul;27(3):11-8.

157. Hota D, Das S, Joseph NM. Effect of dual task exercise to develop body balance, movement co-ordination and walking speed among post cervical injury clients. International Journal of Research in Pharmaceutical Sciences. 2020;11(1):111722. doi: 10.26452/ijrps.v11i1.1944.

158. Vermohlen V, Schiller P, Schickendantz S, et al. Hippotherapy for patients with multiple sclerosis: a multicenter randomized controlled trial (MS-HIPPO). Mult Scler. 2018 Sep;24(10):1375-82. doi: 10.1177/1352458517721354. PMID: 28770664.

159. Wollenweber V, Drache M, Schickendantz S, et al. Study of the effectiveness of hippotherapy on the symptoms of multiple sclerosis - outline of a randomised controlled multicentre study (MS-HIPPO). Contemp Clin Trials Commun. 2016 Aug 15;3:6-11. doi:

10.1016/j.conctc.2016.02.001. PMID: 29736452.

160. Moraes AG, Neri SGR, Motl RW, et al. Effect of hippotherapy on walking performance and gait parameters in people with multiple sclerosis. Mult Scler Relat Disord. 2020 May 26;43:102203. doi: 10.1016/j.msard.2020.102203. PMID: 32485633 .

161. Herrero P, Gomez-Trullen EM, Asensio A, et al. Study of the therapeutic effects of a hippotherapy simulator in children with cerebral palsy: a stratified single-blind randomized controlled trial. Clin Rehabil. 2012 Dec;26(12):1105-13. doi: $10.1177 / 0269215512444633$. PMID: 22610128 .
162. Silva e Borges MB, Werneck MJ, da Silva Mde L, et al. Therapeutic effects of a horse riding simulator in children with cerebral palsy. Arq Neuropsiquiatr. 2011 Oct;69(5):799-804. doi: 10.1590/s0004282x2011000600014. PMID: 22042184.

163. Lucena-Anton D, Rosety-Rodriguez I, Moral-Munoz JA. Effects of a hippotherapy intervention on muscle spasticity in children with cerebral palsy: a randomized controlled trial. Complement Ther Clin Pract. 2018 May;31:188-92. doi: 10.1016/j.ctcp.2018.02.013. PMID: 29705454.

164. Kwon JY, Chang HJ, Yi SH, et al. Effect of hippotherapy on gross motor function in children with cerebral palsy: a randomized controlled trial. J Altern Complement Med. 2015 Jan;21(1):15-21. doi: 10.1089/acm.2014.0021. PMID: 25551626.

165. Deutz U, Heussen N, Weigt-Usinger K, et al. Impact of hippotherapy on gross motor function and quality of life in children with bilateral cerebral palsy: a randomized openlabel crossover study. Neuropediatrics. 2018 Jun;49(3):185-92. doi: 10.1055/s-00381635121. PMID: 29486504.

166. Lee CW, Kim SG, Na SS. The effects of hippotherapy and a horse riding simulator on the balance of children with cerebral palsy. J Phys Ther Sci. 2014 Mar;26(3):423-5. doi: 10.1589/jpts.26.423. PMID: 24707098.

167. Mutoh T, Mutoh T, Tsubone H, et al. Impact of long-term hippotherapy on the walking ability of children with cerebral palsy and quality of life of their caregivers. Frontiers in neurology 2019 Aug 13;10:834. doi: 10.3389/fneur.2019.00834. PMID: 31456733 .

168. Matusiak-Wieczorek E, DziankowskaZaborszczyk E, Synder M, et al. The Influence of Hippotherapy on the Body Posture in a Sitting Position among Children with Cerebral Palsy. Int J Environ Res Public Health. 2020 Sep 19;17(18):19. doi: 10.3390/ijerph17186846. PMID: 32961681. 
169. Kwon JY, Chang HJ, Lee JY, et al. Effects of hippotherapy on gait parameters in children with bilateral spastic cerebral palsy. Arch Phys Med Rehabil. 2011

May;92(5):774-9. doi:

10.1016/j.apmr.2010.11.031. PMID:

21530725.

170. Matusiak-Wieczorek E, MalachowskaSobieska M, Synder M. Influence of hippotherapy on body balance in the sitting position among children with cerebral palsy. Ortop Traumatol Rehabil. 2016 Mar 23;18(2):165-75. doi: 10.5604/15093492.1205024. PMID: 28155825 .

171. Park ES, Rha DW, Shin JS, et al. Effects of hippotherapy on gross motor function and functional performance of children with cerebral palsy. Yonsei Med J. 2014 Nov;55(6):1736-42. doi: 10.3349/ymj.2014.55.6.1736. PMID: 25323914.

172. Azimzadeh E, Hosseini MA, Nourozi K, et al. Effect of Tai Chi Chuan on balance in women with multiple sclerosis. Complement Ther Clin Pract. 2015 Feb;21(1):57-60. doi: 10.1016/j.ctcp.2014.09.002. PMID: 25534298 .

173. Burschka JM, Keune PM, Oy UH, et al. Mindfulness-based interventions in multiple sclerosis: beneficial effects of Tai Chi on balance, coordination, fatigue and depression. BMC Neurol. 2014 Aug 23;14:165. doi: 10.1186/s12883-014-01654. PMID: 25145392.

174. Qi Y, Zhang X, Zhao Y, et al. The effect of wheelchair Tai Chi on balance control and quality of life among survivors of spinal cord injuries: a randomized controlled trial. Complement Ther Clin Pract. 2018

Nov;33:7-11. doi:

10.1016/j.ctcp.2018.07.004. PMID: 30396629 .

175. Nilsagard YE, Forsberg AS, von Koch L. Balance exercise for persons with multiple sclerosis using Wii games: a randomised, controlled multi-centre study. Mult Scler. 2013 Feb;19(2):209-16. doi: 10.1177/1352458512450088. PMID: 22674972 .
176. Khalil H, Al-Sharman A, El-Salem K, et al. The development and pilot evaluation of virtual reality balance scenarios in people with multiple sclerosis (MS): a feasibility study. NeuroRehabilitation. 2018;43(4):47382. doi: 10.3233/NRE-182471. PMID: 30400117.

177. Yazgan YZ, Tarakci E, Tarakci D, et al. Comparison of the effects of two different exergaming systems on balance, functionality, fatigue, and quality of life in people with multiple sclerosis: A randomized controlled trial. Mult Scler Relat Disord. 2019 Dec 21;39:101902. doi: 10.1016/j.msard.2019.101902. PMID: 31924591 .

178. Tarakci D, Ersoz Huseyinsinoglu B, Tarakci E, et al. Effects of Nintendo Wii-Fit ${ }^{\circledR}$ video games on balance in children with mild cerebral palsy. Pediatr Int. 2016

Oct;58(10):1042-50. doi: 10.1111/ped.12942. PMID: 26858013.

179. Acar G, Altun GP, Yurdalan S, et al. Efficacy of neurodevelopmental treatment combined with the Nintendo ${ }^{\circledR}$ Wii in patients with cerebral palsy. J Phys Ther Sci. 2016 Mar;28(3):774-80. doi: 10.1589/jpts.28.774. PMID: 27134357.

180. Zoccolillo L, Morelli D, Cincotti F, et al. Video-game based therapy performed by children with cerebral palsy: a cross-over randomized controlled trial and a crosssectional quantitative measure of physical activity. Eur J Phys Rehabil Med. 2015 Dec;51(6):669-76. PMID: 25653079.

181. El-Shamy SM. Efficacy of Armeo ${ }^{\circledR}$ Robotic Therapy versus conventional therapy on upper limb function in children with hemiplegic cerebral palsy. Am J Phys Med Rehabil. 2018 Mar;97(3):164-69. doi: 10.1097/PHM.0000000000000852. PMID: 29059068.

182. Hsieh H-C. Preliminary study of the effect of training with a gaming balance board on balance control in children with cerebral palsy: a randomized controlled trial. Am J Phys Med Rehabil. 2020;99(2):142-8. doi: 10.1097/PHM.0000000000001300. PMID: 31464757. 
183. Pourazar M, Bagherzadeh F, Mirakhori F. Virtual reality training improves dynamic balance in children with cerebral palsy. International Journal of Developmental Disabilities. 2019 doi: 10.1080/20473869.2019.1679471.

184. Tak S, Choi W, Lee S. Game-based virtual reality training improves sitting balance after spinal cord injury: a single-blinded, randomized controlled trial. Med Sci Technol. 2015;56:53-9.

185. Claerbout M, Gebara B, Ilsbroukx S, et al. Effects of 3 weeks' whole body vibration training on muscle strength and functional mobility in hospitalized persons with multiple sclerosis. Mult Scler. 2012 Apr;18(4):498-505. doi: 10.1177/1352458511423267. PMID: 22084490 .

186. Abbasi M, Kordi Yoosefinejad A, Poursadeghfard M, et al. Whole body vibration improves core muscle strength and endurance in ambulant individuals with multiple sclerosis: a randomized clinical trial. Mult Scler Relat Disord. 2019 Jul;32:88-93. doi:

10.1016/j.msard.2019.04.028. PMID: 31071658 .

187. Lee BK, Chon SC. Effect of whole body vibration training on mobility in children with cerebral palsy: a randomized controlled experimenter-blinded study. Clin Rehabil. 2013 Jul;27(7):599-607. doi: 10.1177/0269215512470673. PMID: 23411791 .

188. Ahmadizadeh Z, Khalili MA, Ghalam MS, et al. Effect of whole body vibration with stretching exercise on active and passive range of motion in lower extremities in children with cerebral palsy: A randomized clinical trial. Iranian Journal of Pediatrics. 2019;29(5) doi: 10.5812/ijp.84436.

189. In T, Jung K, Lee MG, et al. Whole-body vibration improves ankle spasticity, balance, and walking ability in individuals with incomplete cervical spinal cord injury. NeuroRehabilitation. 2018;42(4):491-7. doi: 10.3233/NRE-172333. PMID: 29660953.
190. Doulatabad SN, Nooreyan K, Doulatabad AN, et al. The effects of pranayama, hatha and raja yoga on physical pain and the quality of life of women with multiple sclerosis. Afr J Tradit Complement Altern Med. 2012 Oct 1;10(1):49-52. PMID: 24082325.

191. Najafidoulatabad S, Mohebbi Z, Nooryan K. Yoga effects on physical activity and sexual satisfaction among the Iranian women with multiple sclerosis: a randomized controlled trial. Afr J Tradit Complement Altern Med. 2014 Aug 23;11(5):78-82. doi: 10.4314/ajtcam.v11i5.13. PMID: 25395709.

192. Garrett M, Hogan N, Larkin A, et al. Exercise in the community for people with multiple sclerosis--a follow-up of people with minimal gait impairment. Mult Scler. 2013 May;19(6):790-8. doi: 10.1177/1352458512461390. PMID: 23132904.

193. Garrett M, Hogan N, Larkin A, et al. Exercise in the community for people with minimal gait impairment due to MS: an assessor-blind randomized controlled trial. Mult Scler. 2013 May;19(6):782-9. doi: 10.1177/1352458512461966. PMID: 23128667.

194. Hasanpour-Dehkordi A. Influence of yoga and aerobics exercise on fatigue, pain and psychosocial status in patients with multiple sclerosis: a randomized trial. J Sports Med Phys Fitness. 2016 Nov;56(11):1417-22. PMID: 26223004.

195. Hasanpour-Dehkordi A, Jivad N, Solati K. Effects of yoga on physiological indices, anxiety and social functioning in multiple sclerosis patients: a randomized trial. J Clin Diagn Res. 2016 Jun;10(6):VC01-VC5. doi: 10.7860/JCDR/2016/18204.7916. PMID: 27504387.

196. Hasanpour-Dehkordi A, Jivad N. Comparison of regular aerobic and yoga on the quality of life in patients with multiple sclerosis. Med J Islam Repub Iran. 2014 Dec 6;28:141. PMID: 25694999.

197. Hogan N, Kehoe M, Larkin A, et al. The effect of community exercise interventions for people with MS who use bilateral support for gait. Mult Scler Int. 2014 Jan;2014 doi: 10.1155/2014/109142. PMID: 24575302 . 
198. Duff WRD, Andrushko JW, Renshaw DW, et al. Impact of pilates exercise in multiple sclerosis: a randomized controlled trial. Int $\mathrm{J}$ MS Care. 2018 Mar-Apr;20(2):92-100. doi: 10.7224/1537-2073.2017-066. PMID: 29670495.

199. Kjolhede T, Dalgas U, Gade AB, et al. Acute and chronic cytokine responses to resistance exercise and training in people with multiple sclerosis. Scand J Med Sci Sports. 2016 Jul;26(7):824-34. doi: 10.1111/sms.12504. PMID: 26105554.

200. Fox EE, Hough AD, Creanor S, et al. Effects of pilates-based core stability training in ambulant people with multiple sclerosis: multicenter, assessor-blinded, randomized controlled trial. Phys Ther. 2016 Aug;96(8):1170-8. doi: 10.2522/ptj.20150166. PMID: 26893507.

201. Freeman J, Fox E, Gear M, et al. Pilates based core stability training in ambulant individuals with multiple sclerosis: protocol for a multi-centre randomised controlled trial. BMC Neurol. 2012 Apr 5;12:19. doi: 10.1186/1471-2377-12-19. PMID: 22480437.

202. Dalgas U, Stenager E, Jakobsen J, et al. Resistance training improves muscle strength and functional capacity in multiple sclerosis. Neurology. 2009 Nov 3;73(18):1478-84. doi: 10.1212/WNL.0b013e3181bf98b4. PMID: 19884575.

203. Dalgas U, Stenager E, Jakobsen J, et al. Fatigue, mood and quality of life improve in MS patients after progressive resistance training. Mult Scler. 2010 Apr;16(4):480-90. doi: $10.1177 / 1352458509360040$. PMID: 20194584.

204. Dodd KJ, Taylor NF, Shields N, et al. Progressive resistance training did not improve walking but can improve muscle performance, quality of life and fatigue in adults with multiple sclerosis: a randomized controlled trial. Mult Scler. 2011

Nov;17(11):1362-74. doi: 10.1177/1352458511409084. PMID: 21677021.
205. Ortiz-Rubio A, Cabrera-Martos I, Rodriguez-Torres J, et al. Effects of a homebased upper limb training program in patients with multiple sclerosis: a randomized controlled trial. Arch Phys Med Rehabil. 2016 Dec;97(12):2027-33. doi: 10.1016/j.apmr.2016.05.018. PMID: 27343343.

206. Bulguroglu I, Guclu-Gunduz A, Yazici G, et al. The effects of mat pilates and reformer pilates in patients with multiple sclerosis: a randomized controlled study. NeuroRehabilitation. 2017;41(2):413-22. doi: 10.3233/NRE-162121. PMID: 28946576.

207. Scholtes VA, Becher JG, Janssen-Potten YJ, et al. Effectiveness of functional progressive resistance exercise training on walking ability in children with cerebral palsy: a randomized controlled trial. Res Dev Disabil. 2012 Jan-Feb;33(1):181-8. doi: 10.1016/j.ridd.2011.08.026. PMID: 22093663.

208. Scholtes VA, Dallmeijer AJ, Rameckers EA, et al. Lower limb strength training in children with cerebral palsy--a randomized controlled trial protocol for functional strength training based on progressive resistance exercise principles. BMC Pediatr. 2008 Oct 8;8:41. doi: 10.1186/1471-2431-841. PMID: 18842125.

209. Scholtes VA, Becher JG, Comuth A, et al. Effectiveness of functional progressive resistance exercise strength training on muscle strength and mobility in children with cerebral palsy: a randomized controlled trial. Dev Med Child Neurol. 2010 Jun;52(6):e107-13. doi: 10.1111/j.14698749.2009.03604.x. PMID: 20132136.

210. Qi YC, Niu XL, Gao YR, et al. Therapeutic effect evaluation of neuromuscular electrical stimulation with or without strengthening exercise on spastic cerebral palsy. Clin Pediatr (Phila). 2018 May;57(5):580-3. doi: 10.1177/0009922817732619. PMID: 28990434.

211. Taylor NF, Dodd KJ, Baker RJ, et al. Progressive resistance training and mobilityrelated function in young people with cerebral palsy: a randomized controlled trial. Dev Med Child Neurol. 2013 Sep;55(9):806-12. doi: 10.1111/dmcn.12190. PMID: 23789741. 
212. Bania TA, Dodd KJ, Baker RJ, et al. The effects of progressive resistance training on daily physical activity in young people with cerebral palsy: a randomised controlled trial. Disabil Rehabil. 2016;38(7):620-6. doi: 10.3109/09638288.2015.1055376. PMID: 26056856.

213. Tedla JS. Strength training effects on balance in spastic diplegia subjects: a randomized controlled trial. J Pediatr Neurol. 2014;12(1):15-28. doi: $10.3233 / \mathrm{JPN}-140634$.

214. Kara OK, Yardimci BN, Sahin S, et al. Combined effects of mirror therapy and exercises on the upper extremities in children with unilateral cerebral palsy: A randomized controlled trial. Dev Neurorehabil. 2020 May;23(4):253-64. doi: 10.1080/17518423.2019.1662853. PMID: 31514564 .

215. Elnaggar RK, Elbanna MF, Mahmoud WS, et al. Plyometric exercises: subsequent changes of weight-bearing symmetry, muscle strength and walking performance in children with unilateral cerebral palsy. J Musculoskelet Neuronal Interact. 201912 01;19(4):507-15. PMID: 31789302.

216. Cho HJ, Lee BH. Effect of Functional Progressive Resistance Exercise on Lower Extremity Structure, Muscle Tone, Dynamic Balance and Functional Ability in Children with Spastic Cerebral Palsy. Children (Basel). 2020 Jul 31;7(8):31. doi: 10.3390/children7080085. PMID: 32751813 .

217. Kirk H, Geertsen SS, Lorentzen J, et al. Explosive resistance training increases rate of force development in ankle dorsiflexors and gait function in adults with cerebral palsy. J Strength Cond Res. 2016 Oct;30(10):2749-60. doi: 10.1519/JSC.0000000000001376. PMID: 26890969.

218. Chen X, Wei K, Miao F, et al. Improvement of pulmonary function and life quality on high paraplegia patients through pulmonary rehabilitation. Int J Clin Exp Med. 2016;9(11):22275-81.
219. Mogharnasi M, TaheriChadorneshin H, Papoli-Baravati SA, et al. Effects of upperbody resistance exercise training on serum nesfatin-1 level, insulin resistance, and body composition in obese paraplegic men. Disabil Health J. 2019 Jan;12(1):29-34. doi: 10.1016/j.dhjo.2018.07.003. PMID: 30061073 .

220. Kerling A, Keweloh K, Tegtbur U, et al. Effects of a short physical excercise intervention on patients with multiple sclerosis (MS). Int J Mol Sci. $2015 \mathrm{Jul}$ 10;16(7):15761-75. doi: 10.3390/ijms160715761. PMID: 26184173.

221. Sandroff BM, Bollaert RE, Pilutti LA, et al. Multimodal exercise training in multiple sclerosis: a randomized controlled trial in persons with substantial mobility disability. Contemp Clin Trials. 2017 Oct;61:39-47. doi: 10.1016/j.cct.2017.07.016. PMID: 28732757.

222. Sangelaji B, Kordi M, Banihashemi F, et al. A combined exercise model for improving muscle strength, balance, walking distance, and motor agility in multiple sclerosis patients: a randomized clinical trial. Iran $\mathrm{J}$ Neurol. 2016 Jul 6;15(3):111-20. PMID: 27648171.

223. Tarakci E, Yeldan I, Huseyinsinoglu BE, et al. Group exercise training for balance, functional status, spasticity, fatigue and quality of life in multiple sclerosis: a randomized controlled trial. Clin Rehabil. 2013 Sep;27(9):813-22. doi: 10.1177/0269215513481047. PMID: 23543341.

224. Wens I, Dalgas U, Vandenabeele F, et al. High intensity excercise in multiple sclerosis: effects on muscle contractile characteristics and excercise capacity, a randomised controlled trial. PLoS One. 2015 Oct;10(9) doi: 10.1371/journal.pone.0133697. PMID: 26418222.

225. Cakit BD, Nacir B, Genc H, et al. Cycling progressive resistance training for people with multiple sclerosis: a randomized controlled study. Am J Phys Med Rehabil. 2010 Jun;89(6):446-57. doi: 10.1097/PHM.0b013e3181d3e71f. PMID: 20216060 . 
226. Sangelaji B, Nabavi SM, Estebsari F, et al. Effect of combination exercise therapy on walking distance, postural balance, fatigue and quality of life in multiple sclerosis patients: a clinical trial study. Iran Red Crescent Med J. 2014 Jun;16(6):e17173. doi: 10.5812/ircmj.17173. PMID: 25068045.

227. Wens I, Hansen D, Verboven K, et al. Impact of 24 weeks of resistance and endurance excercise on glucose tolerance in persons with multiple sclerosis. Am J Phys Med Rehabil. 2015 Oct;94(10 Suppl 1):83847. doi: 10.1097/PHM.0000000000000257. PMID: 25768064.

228. Ebrahimi A, Eftekhari E, Etemadifar M. Effects of whole body vibration on hormonal \& functional indices in patients with multiple sclerosis. Indian J Med Res. 2015 Oct; 142(4):450-8. doi: 10.4103/09715916.169210. PMID: 26609037.

229. Williams KL, Low Choy NL, Brauer SG. Center-Based Group and Home-Based Individual Exercise Programs Have Similar Impacts on Gait and Balance in People With Multiple Sclerosis: A Randomized Trial. Pm R. 2020 Apr 06;06:06. doi: 10.1002/pmrj.12377. PMID: 32249509.

230. Faramarzi M, Banitalebi E, Raisi Z, et al. Effect of combined exercise training on pentraxins and pro- inflammatory cytokines in people with multiple sclerosis as a function of disability status. Cytokine. 2020 Jul 16;134:155196. doi: 10.1016/j.cyto.2020.155196. PMID: 32683106.

231. Banitalebi E, Ghahfarrokhi MM, Negaresh $\mathrm{R}$, et al. Exercise improves neurotrophins in multiple sclerosis independent of disability status. Mult Scler Relat Disord. 2020 May 13;43:102143. doi:

10.1016/j.msard.2020.102143. PMID: 32473563 .

232. Ozkul C, Guclu-Gunduz A, Eldemir K, et al. Combined exercise training improves cognitive functions in multiple sclerosis patients with cognitive impairment: A single-blinded randomized controlled trial. Mult Scler Relat Disord. 2020 Jul $23 ; 45: 102419$. doi: 10.1016/j.msard.2020.102419. PMID: 32736216.
233. Roppolo M, Mulasso A, Gollin M, et al. The role of fatigue in the associations between exercise and psychological health in multiple sclerosis: direct and indirect effects. Ment Health Phys Act. 2013 Jun;6(2):87-94. doi: 10.1016/j.mhpa.2013.05.002.

234. Slaman J, Roebroeck M, Dallmijer A, et al. Can a lifestyle intervention programme improve physical behaviour among adolescents and young adults with spastic cerebral palsy? A randomized controlled trial. Dev Med Child Neurol. 2015 Feb;57(2):159-66. doi: 10.1111/dmcn.12602. PMID: 25303096.

235. Slaman J, Roebroeck M, van der Slot W, et al. Can a lifestyle intervention improve physical fitness in adolescents and young adults with spastic cerebral palsy? A randomized controlled trial. Arch Phys Med Rehabil. 2014 Sep;95(9):1646-55. doi: 10.1016/j.apmr.2014.05.011. PMID: 25067790 .

236. Slaman J, Roebroeck ME, van Meeteren J, et al. Learn 2 Move 16-24: effectiveness of an intervention to stimulate physical activity and improve physical fitness of adolescents and young adults with spastic cerebral palsy; a randomized controlled trial. BMC Pediatr. 2010 Nov 5;10:79. doi: 10.1186/1471-243110-79. PMID: 21054829.

237. Slaman J, van den Berg-Emons HJ, van Meeteren J, et al. A lifestyle intervention improves fatigue, mental health and social support among adolescents and young adults with cerebral palsy: focus on mediating effects. Clin Rehabil. 2015 Jul;29(7):717-27. doi: 10.1177/0269215514555136. PMID: 25352613.

238. Van Wely L, Balemans AC, Becher JG, et al. Physical activity stimulation program for children with cerebral palsy did not improve physical activity: a randomised trial. J Physiother. 2014 Mar;60(1):40-9. doi: 10.1016/j.jphys.2013.12.007. PMID: 24856939. 
239. Van Wely L, Balemans AC, Becher JG, et al. The effectiveness of a physical activity stimulation programme for children with cerebral palsy on social participation, selfperception and quality of life: a randomized controlled trial. Clin Rehabil. 2014 Oct;28(10):972-82. doi: 10.1177/0269215513500971. PMID: 24047644.

240. Van Wely L, Becher JG, ReindersMesselink HA, et al. LEARN 2 MOVE 7-12 years: a randomized controlled trial on the effects of a physical activity stimulation program in children with cerebral palsy. BMC Pediatr. 2010 Nov 2;10:77. doi: 10.1186/1471-2431-10-77. PMID: 21044314.

241. Fosdahl MA, Jahnsen R, Kvalheim K, et al. Effect of a combined stretching and strength training program on gait function in children with cerebral palsy, GMFCS level I \& II: a randomized controlled trial. Medicina. 2019 Jun 6;55(6) doi: 10.3390/medicina55060250. PMID: 31174397.

242. Kaya Kara O, Livanelioglu A, Yardimci $\mathrm{BN}$, et al. The effects of functional progressive strength and power training in children with unilateral cerebral palsy. Pediatr Phys Ther. 2019 Jul;31(3):286-95. doi: 10.1097/PEP.0000000000000628. PMID: 31220015.

243. Makhov AS, Medvedev IN. The ability to reduce the severity of motor disorders in children with cerebral palsy. Res J Pharm Biol Chem Sci. 2018;9(2):991-6.

244. Galea MP, Dunlop SA, Davis GM, et al. Intensive exercise program after spinal cord injury ("Full-On"): study protocol for a randomized controlled trial. Trials. 2013 Sep 11;14:291. doi: 10.1186/1745-6215-14-291. PMID: 24025260.

245. Galea MP, Dunlop SA, Geraghty T, et al. SCIPA Full-On: a randomized controlled trial comparing intensive whole-body exercise and upper body exercise after spinal cord injury. Neurorehabil Neural Repair. 2018 Jun;32(6-7):557-67. doi: $10.1177 / 1545968318771213$.
246. Jones ML, Evans N, Tefertiller C, et al. Activity-based therapy for recovery of walking in chronic spinal cord injury: results from a secondary analysis to determine responsiveness to therapy. Arch Phys Med Rehabil. 2014 Dec;95(12):2247-52. doi: 10.1016/j.apmr.2014.07.401. PMID: 25102385 .

247. Jones ML, Evans N, Tefertiller C, et al. Activity-based therapy for recovery of walking in individuals with chronic spinal cord injury: results from a randomized clinical trial. Arch Phys Med Rehabil. 2014 Dec;95(12):2239-46.e2. doi: 10.1016/j.apmr.2014.07.400. PMID: 25102384.

248. Liu H, Li J, Du L, et al. Short-term effects of core stability training on the balance and ambulation function of individuals with chronic spinal cord injury: a pilot randomized controlled trial. Minerva Med. 2019 Jun;110(3):216-23. doi: 10.23736/S0026-4806.19.05952-4. PMID: 30809995 .

249. Totosy de Zepetnek JO, Pelletier CA, Hicks $\mathrm{AL}$, et al. Following the physical activity guidelines for adults with spinal cord injury for 16 weeks does not improve vascular health: a randomized controlled trial. Arch Phys Med Rehabil. 2015 Sep;96(9):1566-75. doi: 10.1016/j.apmr.2015.05.019. PMID: 26070976.

250. Harness ET, Yozbatiran N, Cramer SC. Effects of intense exercise in chronic spinal cord injury. Spinal Cord. 2008

Nov;46(11):733-7. doi: 10.1038/sc.2008.56. PMID: 18521094.

251. Brochetti AM, Brose SW, Kuemmel AM, et al. Interdisciplinary bodyweight management program for persons with SCI. J Spinal Cord Med. 2018 Dec 1-7. doi: 10.1080/10790268.2018.1547860. PMID: 30517834 .

252. de Groot S, Kouwijzer I, Baauw M, et al. Effect of self-guided training for the HandbikeBattle on body composition in people with spinal cord injury. Spinal Cord Ser Cases. 2018 Aug 24;4(79) doi: 10.1038/s41394-018-0103-6. PMID: 30155275 . 
253. de Rossi G, Matos-Souza JR, Costa ESAD, et al. Physical activity and improved diastolic function in spinal cord-injured subjects. Med Sci Sports Exerc.

2014;46(5):887-92. doi:

10.1249/MSS.0000000000000187. PMID: 24126969.

254. Gibbons RS, Shave RE, Gall A, et al. FESrowing in tetraplegia: a preliminary report. Spinal Cord. 2014 Dec;52(12):880-6. doi: 10.1038/sc.2014.159. PMID: 25266698.

255. Gibbons RS, Stock CG, Andrews BJ, et al. The effect of FES-rowing training on cardiac structure and function: pilot studies in people with spinal cord injury. Spinal Cord. 2016 Oct;54(10):822-9. doi: 10.1038/sc.2015.228. PMID: 26754476.

256. Hubli M, Currie KD, West CR, et al. Physical exercise improves arterial stiffness after spinal cord injury. J Spinal Cord Med. 2014 Nov;37(6):782-5. doi: 10.1179/2045772314Y.0000000232. PMID: 24976366 .

257. Jorissen W, Vanmierlo T, Wens I, et al. Twelve weeks of medium-intensity exercise therapy affects the lipoprotein profile of multiple sclerosis patients. Int J Mol Sci. 2018 Jan 8;19(1) doi: 10.3390/ijms19010193. PMID: 29316715.

258. Keytsman C, Hansen D, Wens I, et al. Impact of high-intensity concurrent training on cardiovascular risk factors in persons with multiple sclerosis - pilot study. Disabil Rehabil. 2017 Oct 27;41(4):430-5. doi: 10.1080/09638288.2017.1395086. PMID: 29076386.

259. Lauglo R, Vik T, Lamvik T, et al. Highintensity interval training to improve fitness in children with cerebral palsy. BMJ Open Sport Exerc Med. 2016;2(1) doi: 10.1136/bmjsem-2016-000111. PMID: 27900177.

260. Magnani S, Olla S, Pau M, et al. Effects of six months training on physical capacity and metaboreflex activity in patients with multiple sclerosis. Front Physiol. 2016;7:531. doi: 10.3389/fphys.2016.00531. PMID: 27895592.
261. Myers J, Gopalan R, Shahoumian T, et al. Effects of customized risk reduction program on cardiovascular risk in males with spinal cord injury. J Rehabil Res Dev. 2012;49(9):1355-64. PMID: 23408217.

262. Zabay Neiro C, Martínez Lerín N. How to decrease cardiovascular risk factors in people with multiple sclerosis. Revista Cientifica de la Sociedad Espanola de Enfermeria Neurologica. 2018;47:11-7. doi: 10.1016/j.sedene.2017.10.003. PMID: 8112704

263. Orban A, Garg B, Sammi MK, et al. Effect of high-intensity exercise on multiple sclerosis function and 31P MRS outcomes. Med Sci Sports Exerc. 2019 Jan 30;30:30. doi: 10.1249/MSS.0000000000001914. PMID: 30707115.

264. Lokomat ${ }^{\circledR}$. Hocoma. https://www.hocoma.com/us/solutions/loko mat/. Accessed September 17, 2019.

265. Stergiou A, Tzoufi M, Ntzani E, et al. Therapeutic effects of horseback riding interventions: a systematic review and metaanalysis. Am J Phys Med Rehabil. 2017 Oct;96(10):717-25. doi: 10.1097/PHM.0000000000000726. PMID: 28252520 .

266. What is Exergaming? South Dakota Department of Health,. https://healthysd.gov/what-is-exergaming-5/. Accessed Sept. 15, 2019.

267. Katusic A, Alimovic S, Mejaski-Bosnjak V. The effect of vibration therapy on spasticity and motor function in children with cerebral palsy: a randomized controlled trial. NeuroRehabilitation. 2013;32(1):1-8. doi: 10.3233/NRE-130817. PMID: 23422453.

268. 2018 Physical Activity Guidelines Advisory Committee. 2018 Physical Activity Guidelines Advisory Committee Scientific Report. Washington, DC: U.S. Department of Health and Human Services; February 2018. Available at: https://health.gov/paguidelines/secondedition/report/pdf/PAG_Advisory_Committ ee_Report.pdf. 
269. Warms CA, Backus D, Rajan S, et al. Adverse events in cardiovascular-related training programs in people with spinal cord injury: a systematic review. J Spinal Cord Med. 2014 Nov;37(6):672-92. doi: 10.1179/2045772313Y.0000000115. PMID: 24090603.

270. Pilutti LA, Platta ME, Motl RW, et al. The safety of exercise training in multiple sclerosis: a systematic review. J Neurol Sci. 2014 Aug 15;343(1-2):3-7. doi: 10.1016/j.jns.2014.05.016. PMID: 24880538.

271. Latimer-Cheung AE, Pilutti LA, Hicks AL, et al. Effects of exercise training on fitness, mobility, fatigue, and health-related quality of life among adults with multiple sclerosis: a systematic review to inform guideline development. Arch Phys Med Rehabil. 2013 Sep;94(9):1800-28.e3. doi:

10.1016/j.apmr.2013.04.020. PMID: 23669008 .

272. Edwards T, Pilutti LA. The effect of exercise training in adults with multiple sclerosis with severe mobility disability: A systematic review and future research directions. Mult Scler Relat Disord. 2017 Aug;16:31-9. doi:

10.1016/j.msard.2017.06.003. PMID: 28755682.

273. van der Scheer JW, Martin Ginis KA, Ditor DS, et al. Effects of exercise on fitness and health of adults with spinal cord injury: a systematic review. Neurology. 2017 Aug 15;89(7):736-45. doi: 10.1212/WNL.0000000000004224. PMID: 28733344.

274. Hsu CW, Kang YN, Tseng SH. Effects of therapeutic exercise intensity on cerebral palsy outcomes: a systematic review with meta-regression of randomized clinical trials. Front Neurol. 2019;10:657. doi: 10.3389/fneur.2019.00657. PMID: 31293501 .

275. Wright A, Roberts R, Bowman G, et al. Barriers and facilitators to physical activity participation for children with physical disability: comparing and contrasting the views of children, young people, and their clinicians. Disabil Rehabil. 2019 Jun;41(13):1499-507. doi: 10.1080/09638288.2018.1432702. PMID: 29382235 .
276. Vanner EA, Block P, Christodoulou CC, et al. Pilot study exploring quality of life and barriers to leisure-time physical activity in persons with moderate to severe multiple sclerosis. Disabil Health J. 2008 Jan;1(1):58-65. doi: 10.1016/j.dhjo.2007.11.001. PMID: 21122712.

277. Ige-Elegbede J, Pilkington P, Gray S, et al. Barriers and facilitators of physical activity among adults and older adults from Black and Minority Ethnic groups in the UK: A systematic review of qualitative studies. Prev Med Rep. 2019 Sep;15:100952. doi: 10.1016/j.pmedr.2019.100952. PMID: 31367514.

278. Cadmus-Bertram LA, Gorzelitz JS, Dorn DC, et al. Understanding the physical activity needs and interests of inactive and active rural women: a cross-sectional study of barriers, opportunities, and intervention preferences. J Behav Med. 2019 Jun 13 doi: 10.1007/s10865-019-00070-z. PMID: 31197537.

279. Verschuren O, Wiart L, Hermans D, et al. Identification of facilitators and barriers to physical activity in children and adolescents with cerebral palsy. J Pediatr. 2012

Sep;161(3):488-94. doi:

10.1016/j.jpeds.2012.02.042. PMID: 22494875.

280. Vader K, Doulas T, Patel R, et al. Experiences, barriers, and facilitators to participating in physical activity and exercise in adults living with chronic pain: a qualitative study. Disabil Rehabil. 2019 Oct 15:1-9. doi: 10.1080/09638288.2019.1676834. PMID: 31613655 .

281. Jackson S, Mercer C, Singer BJ. An exploration of factors influencing physical activity levels amongst a cohort of people living in the community after stroke in the south of England. Disabil Rehabil. 2018 Feb;40(4):414-24. doi: 10.1080/09638288.2016.1258437. PMID: 28029070 . 
282. Bennett JA, Lyons KS, Winters-Stone K, et al. Motivational interviewing to increase physical activity in long-term cancer survivors: a randomized controlled trial. Nurs Res. 2007 Jan-Feb;56(1):18-27. doi: 10.1097/00006199-200701000-00003. PMID: 17179870.

283. Falahee M, Benkert R, George N, et al. Motivational interviewing to increase physical activity in underserved women. Journal for Nurse Practitioners. 2016 NovDec;12(10):704-9. doi: 10.1016/j.nurpra.2016.11.002.

284. Sjoling M, Lundberg K, Englund E, et al. Effectiveness of motivational interviewing and physical activity on prescription on leisure exercise time in subjects suffering from mild to moderate hypertension. BMC Res Notes. 2011 Sep 12;4:352. doi: 10.1186/1756-0500-4-352. PMID: 21906404.

285. Collado-Garrido L, Paras-Bravo P, CalvoMartin P, et al. Impact of Resistance Therapy on Motor Function in Children with Cerebral Palsy: A Systematic Review and Meta-Analysis. Int J Environ Res Public Health. 2019 Nov 15;16(22) doi: 10.3390/ijerph16224513. PMID: 31731636.

286. Wong CK, Ehrlich JE, Ersing JC, et al. Exercise programs to improve gait performance in people with lower limb amputation: A systematic review. Prosthet Orthot Int. 2016 Feb;40(1):8-17. doi: 10.1177/0309364614546926. PMID: 25261490 .

287. Flynn A, Allen NE, Dennis S, et al. Homebased prescribed exercise improves balancerelated activities in people with Parkinson's disease and has benefits similar to centrebased exercise: a systematic review. J Physiother. 2019 Oct;65(4):189-99. doi: 10.1016/j.jphys.2019.08.003. PMID: 31521554.

288. Pogrebnoy D, Dennett A. Exercise Programs Delivered According to Guidelines Improve Mobility in People With Stroke: A Systematic Review and Meta-analysis. Arch Phys Med Rehabil. 2019 Aug 8 doi: 10.1016/j.apmr.2019.06.015. PMID: 31400308 .
289. Bullo V, Bergamin M, Gobbo S, et al. The effects of Pilates exercise training on physical fitness and wellbeing in the elderly: A systematic review for future exercise prescription. Prev Med. 2015 Jun;75:1-11. doi: 10.1016/j.ypmed.2015.03.002. PMID: 25773473.

290. Kelley GA, Kelley KS, Hootman JM. Effects of exercise on depression in adults with arthritis: a systematic review with meta-analysis of randomized controlled trials. Arthritis Res Ther. 2015 Feb 3;17:21. doi: 10.1186/s13075-015-0533-5. PMID: 25645739 .

291. Hartmann-Boyce J, Davies N, Frost R, et al. Maximising mobility in older people when isolated with COVID-19. March 2020. https://www.cebm.net/covid-19/maximisingmobility-in-the-older-people-when-isolatedwith-covid-19/. Accessed September 21, 2020.

292. What You Need to Know About COVID-19 and MS - Program 6. 2020.

https://mymsaa.org/videos/what-you-needto-know-covid-and-ms-6/. Accessed September 21, 2020.

293. Ben-Pazi H, Beni-Adani L, Lamdan R. Accelerating Telemedicine for Cerebral Palsy During the COVID-19 Pandemic and Beyond. Front Neurol. 2020 Jun 26;11:746. doi: 10.3389/fneur.2020.00746. PMID: 32670193 .

294. Department of Veterans Affairs (VA) Spinal Cord Injuries and Disorders (SCI/D) System of Care: Information for Veterans with SCI/D During the COVID-19 Public Health Emergency. https://www.sci.va.gov/docs/COVIDTipsFor VeteransWithSCID.pdf. Accessed September 21, 2020.

295. NHS - Chair based Exercises. https://www.youtube.com/watch?v=EJgZyg

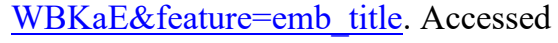
September 21, 2020. 


\section{Abbreviations and Acronyms}

\begin{tabular}{|c|c|}
\hline Abbreviation & Definition \\
\hline 1MWT & 1-Minute Walk Test \\
\hline 2MWT & 2-Minute Walk Test \\
\hline 6MWT & 6-Minute Walk Test \\
\hline 10MWT & 10-Meter Walk Test \\
\hline 25FWT & 25-Foot Walk Test \\
\hline ABCS & Activities-specific Balance Confidence Scale \\
\hline$A C$ & attention control \\
\hline ADL & Activities of Daily Living \\
\hline $\mathrm{AE}$ & adverse event \\
\hline $\mathrm{AHRQ}$ & Agency for Healthcare Research and Quality \\
\hline AIS & Asia Impairment Scale \\
\hline ANCOVA & analysis of covariance \\
\hline ARAT & Action Research Arm tests \\
\hline ASIA & American Spinal Injury Association Impairment Scale \\
\hline ASIA-LEMS & American Spinal Injuries Association Impairment Scale - Lower Extremity Motor Score \\
\hline ASIA-UEMS & American Spinal Injuries Association Impairment Scale - Upper Extremity Motor Score \\
\hline BBS & Berg Balance Scale \\
\hline $\mathrm{BDI}$ & Beck Depression Inventory \\
\hline BDI-FS & Beck Depression Inventory-Fast Screen \\
\hline BMD & bone mineral density \\
\hline BMI & body mass index \\
\hline BWS & body weight supported \\
\hline CES-D & Center for Epidemiologic Studies Depression Scale \\
\hline CHART & Craig Handicap and Assessment Reporting Technique \\
\hline $\mathrm{Cl}$ & confidence interval \\
\hline CIS & Clinically Isolated Syndrome \\
\hline CoDuse & core stability, dual tasking, sensory strategies \\
\hline $\mathrm{CP}$ & cerebral palsy \\
\hline CPMS & chronic progressive multiple sclerosis \\
\hline CPQoL & Cerebral Palsy Quality of Life scale \\
\hline CV & Cardiovascular \\
\hline CVD & cardiovascular disease \\
\hline DBP & diastolic blood pressure \\
\hline DGI & Dynamic Gait Index \\
\hline EDSS & Expanded Disability Status Scale \\
\hline EPC & Evidence-based Practice Center \\
\hline EQ-5D & EuroQOL-5 Dimension Questionnaire \\
\hline FABS & Fullerton Advanced Balance Scale \\
\hline FAC & functional ambulation category \\
\hline
\end{tabular}




\begin{tabular}{|c|c|}
\hline Abbreviation & Definition \\
\hline FAP & Functional Ambulation Profile \\
\hline FER & forced expiratory ratio \\
\hline FES & functional electrical stimulation \\
\hline FEV1 & forced expiratory volume \\
\hline FIM & Functional Independence Measure \\
\hline FPRE & functional progressive resistance exercise \\
\hline FVC & forced vital capacity \\
\hline GMFCS & Gross Motor Function Classification System \\
\hline GMFM & Gross Motor Function Measure \\
\hline GMFM-66 & Gross Motor Function Measure 66 \\
\hline GMFM-66-D & Gross Motor Function Measure 66 (standing) \\
\hline GMFM-66-E & Gross Motor Function Measure 66 (walking, running, jumping) \\
\hline GMFM-88 & Gross Motor Function Measure 88 \\
\hline GMFM-88-D & Gross Motor Function Measure 88 (standing) \\
\hline GMFM-88-E & Gross Motor Function Measure 88 (walking, running, jumping) \\
\hline GNDS & Guy's Neurological Disability Scale \\
\hline HADS & Hospital Anxiety and Depression Scale \\
\hline HAQUAMS & Hamburg Quality of Life Questionnaire in Multiple Sclerosis questionnaire \\
\hline $\mathrm{HbA1c}$ & Hemoglobin A1c \\
\hline HiMAT & High-level Mobility Assessment Tool \\
\hline HOMA & homeostatic model assessment \\
\hline $\mathrm{HR}$ & heart rate \\
\hline HRSD & Hamilton Rating Scale for Depression \\
\hline ICF & International Classification of Functioning \\
\hline IDS16-SR & 16-item version of Inventory of Depressive Symptomatology Self-Rated \\
\hline IPA & Impact on Participation and Autonomy \\
\hline IQR & interquartile range \\
\hline $\mathrm{KQ}$ & Key Question \\
\hline LEMS & Lower Extremity Motor Score \\
\hline LMN & lower motor neuron \\
\hline MACS & manual ability classification system \\
\hline MAS & Modified Ashworth Scale \\
\hline MD & mean difference \\
\hline $\mathrm{MDI}$ & Major Depression Inventory \\
\hline MiniBEST & Mini Balance Evaluation System Test \\
\hline MMAS & Modified Modified Ashworth Scale \\
\hline MMT & Maximal Muscle Testing combined upper and lower limb strength \\
\hline MQLIM & Multicultural Quality of Life Index \\
\hline MS & multiple sclerosis \\
\hline MSFC & multiple sclerosis functional composite \\
\hline MSIS-29 & Multiple Sclerosis Impact Scale-29 \\
\hline MSIS-88 & Multiple Sclerosis Impact Scale-88 \\
\hline
\end{tabular}




\begin{tabular}{|c|c|}
\hline Abbreviation & Definition \\
\hline MSQOL & Multiple Sclerosis Quality of Life \\
\hline MSWS-12 & Multiple Sclerosis Walking Scale-12 \\
\hline MusiQoL & Multiple Sclerosis International Quality of Life questionnaire \\
\hline $\mathrm{NIH}$ & National Institutes of Health \\
\hline NR & not reported \\
\hline NRS & numeric rating scale \\
\hline NS & not significant \\
\hline $\mathrm{PA}$ & previous activity \\
\hline PANAS & Positive and Negative Affect Schedule \\
\hline PBS & Pediatric Balance Scale \\
\hline PDDS & Patient Determined Disease Steps \\
\hline PEDI & Pediatric Evaluation Disability Inventory \\
\hline PEF & peak expiratory flow \\
\hline PHQ-9 & Patient Health Questionnaire-9 \\
\hline PICOTS & Population, Intervention, Comparator, Outcome, Timing, Setting \\
\hline $\mathrm{PL}$ & profile likelihood \\
\hline $\mathrm{PODCl}$ & Pediatric Outcomes Data Collection Instrument \\
\hline PPMS & primary progressive multiple sclerosis \\
\hline PRE & progressive resistance exercise \\
\hline PT & physical therapy \\
\hline QLS & Questionnaire of Life Satisfaction \\
\hline QOL & quality of life \\
\hline RAGT & robot-assisted gait training \\
\hline $\mathrm{RCT}$ & randomized controlled trial \\
\hline RRMS & relapsing-remitting multiple sclerosis \\
\hline rTMS & transcranial magnetic stimulation \\
\hline SAWS & Satisfaction with Abilities and Well-Being Scale \\
\hline SBP & systolic blood pressure \\
\hline $\mathrm{SCl}$ & spinal cord injury \\
\hline SCIM & Spinal Cord Independence Measure \\
\hline SCiM3-M & Spinal Cord Independence Measurement III mobility section \\
\hline SD & standard deviation \\
\hline SE & standard error \\
\hline SF-12 & Short Form (12) Health Survey \\
\hline SF-36 MCS & Short-Form 36 Mental Component Score \\
\hline SF-36 PCS & Short-Form 36 Physical Component Score \\
\hline SIQR & semi-interquartile range \\
\hline SOE & strength of evidence \\
\hline SPMS & secondary progressive multiple sclerosis \\
\hline SSST & Six Spot Step Test \\
\hline STATA & Software for Statistics and Data Science \\
\hline
\end{tabular}




\begin{tabular}{|l|l|}
\hline Abbreviation & Definition \\
\hline TBS & Tinetti Balance Scale \\
\hline tDCS & transcranial direct current stimulation \\
\hline TEP & Technical Expert Panel \\
\hline TG & triglyceride \\
\hline TOP & task-oriented physical therapy \\
\hline TOO & Task Order Officer \\
\hline TUG & Timed Up and Go Test \\
\hline UEMS & Upper Extremity Motor Score \\
\hline UMN & upper motor neuron \\
\hline & \\
\hline VAS & visual analog scale \\
\hline VO 2 max & maximal oxygen uptake \\
\hline VO 2 peak & highest value of VO ${ }_{2}$ attained upon an incremental or other high-intensity exercise test \\
\hline VR & virtual reality \\
\hline WBV & whole body vibration \\
\hline WeeFIM & Wee-Functional Independence Measure for children \\
\hline WHOQOL & World Health Organization Quality of Life \\
\hline WISCl & Walking Index for Spinal Cord Injury \\
\hline
\end{tabular}




\section{Appendixes}

Appendix A. Literature Search Strategies............................................................................... A-1

Appendix B. Included Studies List ................................................................................................. B-1

Appendix C. Excluded Studies List .................................................................................. C-1

Table C-1. Study exclusion reasons............................................................................... C-1

Appendix D. Included and Excluded Study Details and Systematic Reviews Evaluated .. D-1 Table D-1. Included and excluded study definitions and characteristics....................... D-1

Appendix E. Criteria for Assessing Quality and Internal Validity of Individual Studies . E-1

Quality Assessment Criteria: Randomized Controlled Trials..................................................E-1

Table E-1. Methods to assess quality of trials: assessment of internal validity................E-1

Quality Assessment Criteria: Nonrandomized Studies ........................................................E-2

Table E-2. Nonrandomized studies: guidance for quality assessment.............................E-2

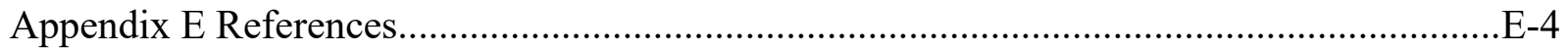

Appendix F. Evidence Tables ..................................................................................................1

Table F-1. Study description and results (continued in Table F-2) .................................F-1

Table F-2. Harms and study characteristics (continuation of Table F-1 results by

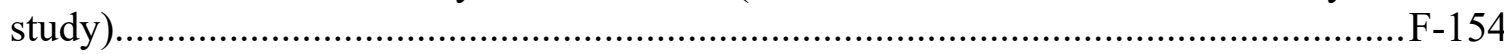

Appendix G. Quality Assessment ........................................................................................... G-1

Table G-1. Quality assessment of randomized controlled trials ..................................... G-1

Table G-2. Quality assessment of quasiexperimental studies......................................... G-7

Table G-3. Quality assessment of cohort studies ........................................................ G-9

Appendix H. Strength of Evidence ..............................................................................................H-1

Table H-1. Strength of evidence for Key Question 2: aerobic exercise for multiple

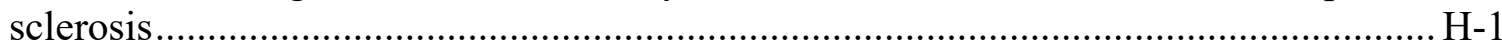

Table H-2. Strength of evidence for Key Question 2: aerobic exercise for cerebral

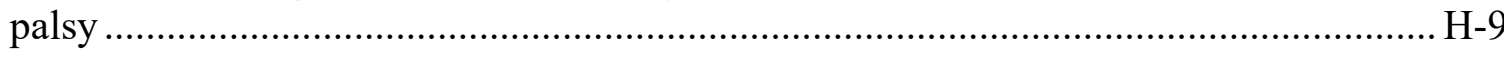

Table H-3. Strength of evidence for Key Question 2: aerobic exercise for cerebral palsy and multiple sclerosis.

Table H-4. Strength of evidence for Key Question 2: aerobic exercise for spinal cord injury

Table H-5. Strength of evidence for Key Question 2: balance exercise for multiple sclerosis

Table H-6. Strength of evidence for Key Question 2: balance exercise for cerebral palsy

Table H-7. Strength of evidence for Key Question 2: balance exercise for spinal cord injury

Table H-8. Strength of evidence for Key Question 2: muscle strength exercise for multiple sclerosis 
Table H-9. Strength of evidence for Key Question 2: muscle strength exercise for cerebral palsy

Table H-10. Strength of evidence for Key Question 2: muscle strength exercise for spinal cord injury $\mathrm{H}-45$

Table H-11. Strength of evidence for Key Question 2: Progressive resistance or strength exercise plus aerobic or balance

exercise that includes strengthening for multiple sclerosis. H-46

Table H-12. Strength of evidence for Key Question 2: multimodal exercise that includes strengthening for cerebral palsy.

Table H-13. Strength of evidence for Key Question 2: multimodal exercise that includes strengthening for spinal cord injury....

Table H-14. Strength of evidence for Key Question 2a clinical outcomes: mental health

Table H-15. Strength of evidence for Key Question 2: general exercise effect across interventions and populations

Table H-16. Strength of evidence for Key Question 2b intermediate outcomes: effect of physical activity interventions on $\mathrm{VO}_{2}$ peak and $\mathrm{VO}_{2}$ max H-62

Table H-17. Strength of evidence for Key Question 2b intermediate outcomes: effect of physical activity interventions on pulmonary function tests. H-65

Table H-18. Strength of evidence for Key Question 2c: reduction of harms of immobility..... H-66

Table H-19. Strength of evidence for Key Question 2d decreased risk of adverse outcomes of mobility devices: effect of physical activity interventions on spasticity . H-67 Table H-20. Strength of evidence for Key Question 2e: harms of physical activity .... H-70 


\section{Appendix A. Literature Search Strategies}

Database: Ovid MEDLINE(R) and Epub Ahead of Print, In-Process \& Other Non-Indexed Citations, Daily and Versions(R) 1946 to November 3, 2020

Search: RCTs and controlled observational studies

1. Spinal Cord Injuries/

2. ("spinal cord injury" or "SCI" or (spin* adj2 injur*)).ti,ab.

3. exp Multiple Sclerosis/

4. "multiple sclerosis".ti,ab.

5. Cerebral Palsy/

6. "cerebral palsy".ti,ab.

7. Disabled Persons/

8. Paraplegia/ or Quadriplegia/

9. (wheelchair or quadripleg* or parapleg* or tetrapleg*).ti,ab.

10. or/1-9

11. $\exp$ Exercise/

12. exp Exercise Therapy/

13. exp Physical Fitness/

14. Weight Lifting/

15. Yoga/

16. exp Martial Arts/

17. Equine-Assisted Therapy/

18. Bicycling/

19. Hydrotherapy/

20. exp Balneology/

21. Swimming/

22. Vibration/

23. sports/ or sports for persons with disabilities/

24. (exercise or "standing frame" or vibration or stretch* or flexibility or yoga or "martial art*" or "tai chi" or "tai ji" or hippotherapy or (equine adj2 therapy) or resistance or "weight lift*" or "weight train*" or ergometry or bicycl* or "strength train*" or treadmill or "gait train*" or swim* or aquatherapy or hydrotherapy or sport*).ti,ab.

25. ("physical fitness" or "physical activity").ti,ab.

26. or/11-25

27. 10 and 26

28. limit 27 to randomized controlled trial

29. 27 and (random* or control* or trial or cohort or group* or arm*).ti,ab.

30.28 or 29

31. limit 30 to $\mathrm{yr}=" 2008$-Current"

32. limit 31 to english language 


\section{Search: Systematic reviews}

1. Spinal Cord Injuries/

2. ("spinal cord injury" or "SCI" or (spin* adj2 injur*)).ti,ab.

3. exp Multiple Sclerosis/

4. "multiple sclerosis".ti,ab.

5. Cerebral Palsy/

6. "cerebral palsy".ti,ab.

7. Disabled Persons/

8. Paraplegia/ or Quadriplegia/

9. (wheelchair or quadripleg* or parapleg* or tetrapleg*).ti,ab.

10. or/1-9

11. exp Exercise/

12. exp Exercise Therapy/

13. exp Physical Fitness/

14. Weight Lifting/

15. Yoga/

16. exp Martial Arts/

17. Equine-Assisted Therapy/

18. Bicycling/

19. Hydrotherapy/

20. exp Balneology/

21. Swimming/

22. Vibration/

23. sports/ or sports for persons with disabilities/

24. (exercise or "standing frame" or vibration or stretch* or flexibility or yoga or "martial art*" or "tai chi" or "tai ji" or hippotherapy or (equine adj2 therapy) or resistance or "weight lift*" or "weight train*" or ergometry or bicycl* or "strength train*" or treadmill or "gait train*" or swim* or aquatherapy or hydrotherapy or sport*).ti,ab.

25. ("physical fitness" or "physical activity").ti,ab.

26. or/11-25

27. 10 and 26

28. 27 and (systematic or meta*).ti,ab.

29 . limit 27 to (meta analysis or systematic reviews)

30.28 or 29

31. limit 30 to $y r=" 2008-C u r r e n t "$ 


\section{Search: Evaluation studies}

1. Spinal Cord Injuries/

2. ("spinal cord injury" or "SCI" or (spin* adj2 injur*)).ti,ab.

3. exp Multiple Sclerosis/

4. "multiple sclerosis".ti,ab.

5. Cerebral Palsy/

6. "cerebral palsy".ti,ab.

7. Disabled Persons/

8. Paraplegia/ or Quadriplegia/

9. (wheelchair or quadripleg* or parapleg* or tetrapleg*).ti,ab.

10. or/1-9

11. exp Exercise/

12. exp Exercise Therapy/

13. exp Physical Fitness/

14. Weight Lifting/

15. Yoga/

16. exp Martial Arts/

17. Equine-Assisted Therapy/

18. Bicycling/

19. Hydrotherapy/

20. exp Balneology/

21. Swimming/

22. Vibration/

23. sports/ or sports for persons with disabilities/

24. (exercise or "standing frame" or vibration or stretch* or flexibility or yoga or "martial art*" or "tai chi" or "tai ji" or hippotherapy or (equine adj2 therapy) or resistance or "weight lift*" or "weight train*" or ergometry or bicycl* or "strength train*" or treadmill or "gait train*" or swim* or aquatherapy or hydrotherapy or sport*).ti,ab.

25. ("physical fitness" or "physical activity").ti,ab.

26. or/11-25

27. 10 and 26

28. (pre or before).ti,ab.

29. (post or after).ti,ab.

30. limit 27 to (comparative study or evaluation studies)

31.27 and (28 or 29$)$

32. Pilot Projects/

33. pilot.ti,ab.

34. 27 and (32 or 33$)$

35.30 or 31 or 34

36. limit 35 to $y r=" 2008$-Current"

37. limit 36 to english language

38. limit 37 to randomized controlled trial

39. 37 and (random* or control* or trial or cohort or group* or arm*).ti,ab.

40. 37 not (38 or 39 ) 
Database: EBM Reviews - Cochrane Central Register of Controlled Trials November 3, 2020

1. Spinal Cord Injuries/

2. ("spinal cord injury" or "SCI" or (spin* adj2 injur*)).ti,ab.

3. exp Multiple Sclerosis/

4. "multiple sclerosis".ti,ab.

5. Cerebral Palsy/

6. "cerebral palsy".ti,ab.

7. Disabled Persons/

8. Paraplegia/ or Quadriplegia/

9. (wheelchair or quadripleg* or parapleg* or tetrapleg*).ti,ab.

10. or/1-9

11. $\exp$ Exercise/

12. exp Exercise Therapy/

13. exp Physical Fitness/

14. Weight Lifting/

15. Yoga/

16. exp Martial Arts/

17. Equine-Assisted Therapy/

18. Bicycling/

19. Hydrotherapy/

20. exp Balneology/

21. Swimming/

22. Vibration/

23. sports/ or sports for persons with disabilities/

24. (exercise or "standing frame" or vibration or stretch* or flexibility or yoga or "martial art*" or "tai chi" or "tai ji" or hippotherapy or (equine adj2 therapy) or resistance or "weight lift*" or "weight train*" or ergometry or bicycl* or "strength train*" or treadmill or "gait train*" or swim* or aquatherapy or hydrotherapy or sport*).ti,ab.

25. ("physical fitness" or "physical activity").ti,ab.

26. or/11-25

27. 10 and 26

28. limit 27 to randomized controlled trial

29. 27 and (random* or control* or trial or cohort or group* or arm*).ti,ab.

30.28 or 29

31. limit 30 to $y r=" 2008$-Current"

32. limit 31 to english language

33. limit 32 to medline records

34. 32 not 33 
Database: EBM Reviews - Cochrane Database of Systematic Reviews 2005 to November 3, 2020

1. ("spinal cord injury" or "SCI" or (spin* adj2 injur*)).ti,ab.

2. "multiple sclerosis".ti,ab.

3. "cerebral palsy".ti,ab.

4. (wheelchair or quadripleg* or parapleg* or tetrapleg*).ti,ab.

5. (exercise or "standing frame" or vibration or stretch* or flexibility or yoga or "martial art*" or "tai chi" or "tai ji" or hippotherapy or (equine adj2 therapy) or resistance or "weight lift*" or

"weight train*" or ergometry or bicycl* or "strength train*" or treadmill or "gait train*" or swim* or aquatherapy or hydrotherapy or sport*).ti,ab.

6. ("physical fitness" or "physical activity").ti,ab.

7. ( 1 or 2 or 3 or 4$)$ and ( 5 or 6$)$

8 . limit 7 to full systematic reviews

Database: PsycINFO 1806 to November Week 12020

1. spinal cord injuries/

2. ("spinal cord injury" or "SCI" or (spin* adj2 injur*)).ti,ab.

3. multiple sclerosis/

4. "multiple sclerosis".ti,ab.

5. exp paralysis/

6. ("cerebral palsy" or wheelchair or quadripleg* or parapleg* or tetrapleg*).ti,ab.

7. or/1-6

8. physical activity/ or exp exercise/

9. physical fitness/

10. yoga/

11. recreation/ or athletic participation/ or martial arts/ or weightlifting/ or sports/

12. vibration/

13. (exercise or "standing frame" or vibration or stretch* or flexibility or yoga or "martial art*" or "tai chi" or "tai ji" or hippotherapy or (equine adj2 therapy) or resistance or "weight lift*" or "weight train*" or ergometry or bicycl* or "strength train*" or treadmill or "gait train*" or swim* or aquatherapy or hydrotherapy or sport*).ti,ab.

14. ("physical fitness" or "physical activity").ti,ab.

15. or/8-14

16. 7 and 15

17. limit 16 to $y r=" 2008$-Current"

18. limit 17 to english language

19. 18 and (random* or control* or trial or cohort or group* or arm*).ti,ab.

20. limit 18 to ("0300 clinical trial" or 2100 treatment outcome)

21. 19 or 20 
Database: EBSCO CINAHL Plus with Full Text to November 3, 2020

1. (MH "spinal cord injuries")

2. TI "spinal cord injur*" OR TI sci

3. (MH "Multiple Sclerosis")

4. TI multiple sclerosis

5. (MH "Cerebral Palsy")

6. TI cerebral palsy

7. (MH "Paraplegia") OR (MH "Quadriplegia")

8. TI wheelchair OR TI parapleg* OR TI quadripleg* OR TI tetrapleg*

9. S1 OR S2 OR S3 OR S4 OR S5 OR S6 OR S7 OR S8

10. (MH "Exercise+") OR (MH "Leisure Activities+") OR (MH "Physical Fitness+") OR (MH "Physical Activity") OR (MH "Sports+")

11. (MH "Weight Lifting") OR (MH "Resistance Training")

12. (MH "Yoga")

13. (MH "Vibration")

14. TI exercise OR TI "standing frame" OR TI vibration OR TI stretch* OR TI flexibility OR TI yoga OR TI "martial art*" OR TI "tai chi" OR TI "tai ji" OR TI hippotherapy OR TI "equine therapy" OR TI "resistance train*"

15. TI "weight train*" OR TI ergometry OR TI bicycl* OR TI "strength train*" OR TI treadmill OR TI "gait train*" OR TI swim* OR TI aquatherapy OR TI hydrotherapy OR TI sport*

16. TI "physical fitness" OR TI "physical activity"

17. S10 OR S11 OR S12 OR S13 OR S14 OR S15 OR S16

18. S9 AND S17

19. TI random* or TI control* or TI trial or TI cohort or TI group* or TI arm*

20. $\mathrm{AB}$ random* or $\mathrm{AB}$ control* or $\mathrm{AB}$ trial or $\mathrm{AB}$ cohort or $\mathrm{AB}$ group* or $\mathrm{AB}$ arm*

21. S19 OR S20

22. S18 AND S21

23. S18 AND S21 Limiters - Published Date: 20080101-20191231; Exclude MEDLINE records

Database: Elsevier Embase Web to February 6, 2019

('spinal cord injury'/exp OR 'spinal cord injury' OR 'multiple sclerosis'/exp OR 'multiple sclerosis' OR 'cerebral palsy' OR 'disabled person' OR 'paraplegia' OR 'quadriplegia' OR 'tetraplegia') AND ('exercise' OR 'kinesiotherapy' OR 'fitness' OR 'physical activity' OR 'sport' OR 'weight lifting' OR 'yoga' OR 'martial art' OR 'hippotherapy' OR 'cycling' OR 'swimming' OR 'hydrotherapy' OR 'vibration' OR 'resistance training') AND 'article'/it AND (2008:py OR 2009:py OR 2010:py OR 2011:py OR 2012:py OR 2013:py OR 2014:py OR 2015:py OR 2016:py OR 2017:py OR 2018:py OR 2019:py) AND [english]/lim AND [embase]/lim NOT ([embase]/lim AND [medline]/lim) 
Database: EBSCO Rehabilitation \& Sports Medicine Source to November 3, 2020

1. (MH "spinal cord injuries")

2. TI "spinal cord injur*" OR TI sci

3. (MH "Multiple Sclerosis")

4. TI multiple sclerosis

5. (MH "Cerebral Palsy")

6. TI cerebral palsy

7. (MH "Paraplegia") OR (MH "Quadriplegia")

8. TI wheelchair OR TI parapleg* OR TI quadripleg* OR TI tetrapleg*

9. S1 OR S2 OR S3 OR S4 OR S5 OR S6 OR S7 OR S8

10. (MH "Exercise+") OR (MH "Leisure Activities+") OR (MH "Physical Fitness+") OR (MH

"Physical Activity") OR (MH "Sports+")

11. (MH "Weight Lifting") OR (MH "Resistance Training")

12. (MH "Yoga")

13. (MH "Vibration")

14. TI exercise OR TI "standing frame" OR TI vibration OR TI stretch* OR TI flexibility OR TI yoga OR TI "martial art*" OR TI "tai chi" OR TI "tai ji" OR TI hippotherapy OR TI "equine therapy" OR TI "resistance train*"

15. TI "weight train*" OR TI ergometry OR TI bicycl* OR TI "strength train*" OR TI treadmill OR TI "gait train*" OR TI swim* OR TI aquatherapy OR TI hydrotherapy OR TI sport*

16. TI "physical fitness" OR TI "physical activity"

17. S10 OR S11 OR S12 OR S13 OR S14 OR S15 OR S16

18. S9 AND S17

19. TI random* or TI control* or TI trial or TI cohort or TI group* or TI arm*

20. $\mathrm{AB}$ random* or $\mathrm{AB}$ control* or $\mathrm{AB}$ trial or $\mathrm{AB}$ cohort or $\mathrm{AB}$ group* or $\mathrm{AB}$ arm*

21. S19 OR S20

22. S18 AND S21

23. S18 AND S21 Limiters - Published Date: 20080101-2019123 


\section{Appendix B. Included Studies List}

1. Abbasi M, Kordi Yoosefinejad A, Poursadeghfard M, et al. Whole body vibration improves core muscle strength and endurance in ambulant individuals with multiple sclerosis: a randomized clinical trial. Mult Scler Relat Disord. 2019 Jul;32:88-93. doi:

10.1016/j.msard.2019.04.028. PMID: 31071658 .

2. Acar G, Altun GP, Yurdalan S, et al. Efficacy of neurodevelopmental treatment

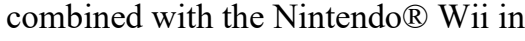
patients with cerebral palsy. J Phys Ther Sci. 2016 Mar;28(3):774-80. doi: 10.1589/jpts.28.774. PMID: 27134357.

3. Adar S, Dundar U, Demirdal ÜS, et al. The effect of aquatic exercise on spasticity, quality of life, and motor function in cerebral palsy. Turk J Phys Med Rehab. 2017 Aug 14;63(3):239-48. doi: 10.5606/tftrd.2017.280. PMID: 31453460.

4. Afrasiabifar A, Karami F, Najafi Doulatabad S. Comparing the effect of CawthorneCooksey and Frenkel exercises on balance in patients with multiple sclerosis: a randomized controlled trial. Clin Rehabil. 2018 Jan;32(1):57-65. doi: 10.1177/0269215517714592. PMID: 28629268.

5. Ahmadi A, Arastoo AA, Nikbakht M, et al. Comparison of the effect of 8 weeks aerobic and yoga training on ambulatory function, fatigue and mood status in MS patients. Iran Red Crescent Med J. 2013 Jun;15(6):44954. doi: 10.5812/ircmj.3597. PMID: 24349740 .

6. Ahmadizadeh Z, Khalili MA, Ghalam MS, et al. Effect of whole body vibration with stretching exercise on active and passive range of motion in lower extremities in children with cerebral palsy: A randomized clinical trial. Iranian Journal of Pediatrics. 2019;29(5) doi: 10.5812/ijp.84436.
7. Akkurt H, Karapolat HU, Kirazli Y, et al. The effects of upper extremity aerobic exercise in patients with spinal cord injury: a randomized controlled study. Eur J Phys Rehabil Med. 2017 Apr;53(2):219-27. doi: 10.23736/S1973-9087.16.03804-1. PMID: 27824234.

8. Al-Sharman A, Khalil H, El-Salem K, et al. The effects of aerobic exercise on sleep quality measures and sleep-related biomarkers in individuals with Multiple Sclerosis: a pilot randomised controlled trial. NeuroRehabilitation. 2019;45(1):107-15. doi: 10.3233/NRE-192748. PMID: 31403958 .

9. Alexeeva N, Sames C, Jacobs PL, et al. Comparison of training methods to improve walking in persons with chronic spinal cord injury: a randomized clinical trial. J Spinal Cord Med. 2011;34(4):362-79. doi: 10.1179/2045772311Y.0000000018. PMID: 21903010 .

10. Amiri B, Sahebozamani M, Sedighi B. The effects of 10-week core stability training on balance in women with multiple sclerosis according to Expanded Disability Status Scale: a single-blinded randomized controlled trial. Eur J Phys Rehabil Med. 2019 Apr;55(2):199-208. doi: 10.23736/S1973-9087.18.04778-0. PMID: 29307152.

11. Aras B, Yasar E, Kesikburun S, et al. Comparison of the effectiveness of partial body weight-supported treadmill exercises, robotic-assisted treadmill exercises, and anti-gravity treadmill exercises in spastic cerebral palsy. Turk J Phys Med Rehabil. 2019 Jun;65(4):361-70. doi: 10.5606/tftrd.2019.3078. PMID: 31893273.

12. Arntzen EC, Straume B, Odeh F, et al. Group-based, individualized, comprehensive core stability and balance intervention provides immediate and long-term improvements in walking in individuals with multiple sclerosis: A randomized controlled trial. Physiother Res Int. 2020 Jan;25(1):e1798. doi: 10.1002/pri.1798. PMID: 31268223. 
13. Arntzen EC, Straume BK, Odeh F, et al. Group-based individualized comprehensive core stability intervention improves balance in persons with multiple sclerosis: a randomized controlled trial. Phys Ther. 2019 Aug 1;99(8):1027-38. doi: 10.1093/ptj/pzz017. PMID: 30722036.

14. Aviram R, Harries N, Namourah I, et al. Effects of a group circuit progressive resistance training program compared with a treadmill training program for adolescents with cerebral palsy. Dev Neurorehabil. 2017 Aug;20(6):347-54. doi: 10.1080/17518423.2016.1212946. PMID: 27536805 .

15. Aydin T, Akif Sariyildiz M, Guler M, et al. Evaluation of the effectiveness of home based or hospital based calisthenic exercises in patients with multiple sclerosis. Eur Rev Med Pharmacol Sci. 2014;18(8):1189-98. PMID: 24817294.

16. Azimzadeh E, Hosseini MA, Nourozi K, et al. Effect of Tai Chi Chuan on balance in women with multiple sclerosis. Complement Ther Clin Pract. 2015 Feb;21(1):57-60. doi: 10.1016/j.ctcp.2014.09.002. PMID: 25534298.

17. Bahrami F, Noorizadeh Dehkordi S, Dadgoo $\mathrm{M}$. The efficacy of treadmill training on walking and quality of life of adults with spastic cerebral palsy: a randomized controlled trial. Iran J Child Neurol. 2019 Fall;13(4):121-33. PMID: 31645872.

18. Bania TA, Dodd KJ, Baker RJ, et al. The effects of progressive resistance training on daily physical activity in young people with cerebral palsy: a randomised controlled trial. Disabil Rehabil. 2016;38(7):620-6. doi: 10.3109/09638288.2015.1055376. PMID: 26056856.

19. Banitalebi E, Ghahfarrokhi MM, Negaresh $\mathrm{R}$, et al. Exercise improves neurotrophins in multiple sclerosis independent of disability status. Mult Scler Relat Disord. 2020 May 13;43:102143. doi: 10.1016/j.msard.2020.102143. PMID: 32473563.
20. Baquet L, Hasselmann H, Patra S, et al. Short-term interval aerobic exercise training does not improve memory functioning in relapsing-remitting multiple sclerosis-a randomized controlled trial. PeerJ. 2018 Dec 12;6:e6037. doi: 10.7717/peerj.6037. PMID: 30581662 .

21. Bleyenheuft Y, Ebner-Karestinos D, Surana $\mathrm{B}$, et al. Intensive upper- and lowerextremity training for children with bilateral cerebral palsy: a quasi-randomized trial. Dev Med Child Neurol. 2017 Jun;59(6):625-33. doi: 10.1111/dmcn.13379. PMID: 28133725.

22. Brichetto G, Piccardo E, Pedulla L, et al. Tailored balance exercises on people with multiple sclerosis: a pilot randomized, controlled study. Mult Scler. 2015 Jul;21(8):1055-63. doi: 10.1177/1352458514557985. PMID: 25392337.

23. Bryant E, Pountney T, Williams H, et al. Can a six-week exercise intervention improve gross motor function for nonambulant children with cerebral palsy? A pilot randomized controlled trial. Clin Rehabil. 2013 Feb;27(2):150-9. doi: 10.1177/0269215512453061. PMID: 22850757.

24. Bulguroglu I, Guclu-Gunduz A, Yazici G, et al. The effects of mat pilates and reformer pilates in patients with multiple sclerosis: a randomized controlled study. NeuroRehabilitation. 2017;41(2):413-22. doi: 10.3233/NRE-162121. PMID: 28946576.

25. Burschka JM, Keune PM, Oy UH, et al. Mindfulness-based interventions in multiple sclerosis: beneficial effects of Tai Chi on balance, coordination, fatigue and depression. BMC Neurol. 2014 Aug 23;14:165. doi: 10.1186/s12883-014-01654. PMID: 25145392.

26. Cakit BD, Nacir B, Genc H, et al. Cycling progressive resistance training for people with multiple sclerosis: a randomized controlled study. Am J Phys Med Rehabil. 2010 Jun;89(6):446-57. doi: 10.1097/PHM.0b013e3181d3e71f. PMID: 20216060. 
27. Calabro RS, Russo M, Naro A, et al. Robotic gait training in multiple sclerosis rehabilitation: can virtual reality make the difference? Findings from a randomized controlled trial. J Neurol Sci. 2017 Jun 15;377:25-30. doi: 10.1016/j.jns.2017.03.047. PMID: 28477702 .

28. Callesen J, Cattaneo D, Brincks J, et al. How do resistance training and balance and motor control training affect gait performance and fatigue impact in people with multiple sclerosis? A randomized controlled multicenter study. Mult Scler. 2019 Jul 24 doi: 10.1177/1352458519865740. PMID: 31339460 .

29. Carling A, Forsberg A, Gunnarsson M, et al. CoDuSe group exercise programme improves balance and reduces falls in people with multiple sclerosis: a multi-centre, randomized, controlled pilot study. Mult Scler. 2017 Sep;23(10):1394-404. doi: 10.1177/1352458516677591. PMID: 27834736.

30. Castro-Sanchez AM, Mataran-Penarrocha GA, Lara-Palomo I, et al. Hydrotherapy for the treatment of pain in people with multiple sclerosis: a randomized controlled trial. Evid Based Complement Alternat Med. 2012;473963:1-8. doi: 10.1155/2012/473963. PMID: 21785645.

31. Chen X, Wei K, Miao F, et al. Improvement of pulmonary function and life quality on high paraplegia patients through pulmonary rehabilitation. Int J Clin Exp Med. 2016;9(11):22275-81.

32. Cho HJ, Lee BH. Effect of Functional Progressive Resistance Exercise on Lower Extremity Structure, Muscle Tone, Dynamic Balance and Functional Ability in Children with Spastic Cerebral Palsy. Children (Basel). 2020 Jul 31;7(8):31. doi: 10.3390/children7080085. PMID: 32751813 .

33. Chrysagis N, Skordilis EK, Stavrou N, et al. The effect of treadmill training on gross motor function and walking speed in ambulatory adolescents with cerebral palsy: a randomized controlled trial. Am J Phys Med Rehabil. 2012 Sep;91(9):747-60. doi: 10.1097/PHM.0b013e3182643eba. PMID: 22902937.
34. Claerbout M, Gebara B, Ilsbroukx S, et al. Effects of 3 weeks' whole body vibration training on muscle strength and functional mobility in hospitalized persons with multiple sclerosis. Mult Scler. 2012 Apr;18(4):498-505. doi: 10.1177/1352458511423267. PMID: 22084490 .

35. Collett J, Dawes H, Meaney A, et al. Exercise for multiple sclerosis: a singleblind randomized trial comparing three exercise intensities. Mult Scler. 2011 May;17(5):594-603. doi: 10.1177/1352458510391836. PMID: 21247971.

36. Craven BC, Giangregorio LM, Alavinia SM, et al. Evaluating the efficacy of functional electrical stimulation therapy assisted walking after chronic motor incomplete spinal cord injury: effects on bone biomarkers and bone strength. J Spinal Cord Med. 2017 Nov;40(6):748-58. doi: 10.1080/10790268.2017.1368961. PMID: 28929919.

37. Curtis DJ, Woollacott M, Bencke J, et al. The functional effect of segmental trunk and head control training in moderate-to-severe cerebral palsy: a randomized controlled trial. Dev Neurorehabil. 2018 Feb;21(2):91-100. doi: 10.1080/17518423.2016.1265603. PMID: 28045553.

38. Dalgas U, Stenager E, Jakobsen J, et al. Resistance training improves muscle strength and functional capacity in multiple sclerosis. Neurology. 2009 Nov 3;73(18):1478-84. doi: 10.1212/WNL.0b013e3181bf98b4. PMID: 19884575.

39. Dalgas U, Stenager E, Jakobsen J, et al. Fatigue, mood and quality of life improve in MS patients after progressive resistance training. Mult Scler. 2010 Apr;16(4):480-90. doi: 10.1177/1352458509360040. PMID: 20194584.

40. Demuth SK, Knutson LM, Fowler EG. The PEDALS stationary cycling intervention and health-related quality of life in children with cerebral palsy: a randomized controlled trial. Dev Med Child Neurol. 2012 Jul;54(7):65461. doi: 10.1111/j.1469-8749.2012.04321.x. PMID: 22582760. 
41. Deutz U, Heussen N, Weigt-Usinger K, et al. Impact of hippotherapy on gross motor function and quality of life in children with bilateral cerebral palsy: a randomized openlabel crossover study. Neuropediatrics. 2018 Jun;49(3):185-92. doi: 10.1055/s-00381635121. PMID: 29486504.

42. Dodd KJ, Taylor NF, Shields N, et al. Progressive resistance training did not improve walking but can improve muscle performance, quality of life and fatigue in adults with multiple sclerosis: a randomized controlled trial. Mult Scler. 2011

Nov;17(11):1362-74. doi: 10.1177/1352458511409084. PMID: 21677021 .

43. Doulatabad SN, Nooreyan K, Doulatabad AN, et al. The effects of pranayama, hatha and raja yoga on physical pain and the quality of life of women with multiple sclerosis. Afr J Tradit Complement Altern Med. 2012 Oct 1;10(1):49-52. PMID: 24082325 .

44. Duarte Nde A, Grecco LA, Galli M, et al. Effect of transcranial direct-current stimulation combined with treadmill training on balance and functional performance in children with cerebral palsy: a double-blind randomized controlled trial. PLoS One. 2014 Aug 29;9(8):e105777. doi: 10.1371/journal.pone.0105777. PMID: 25171216 .

45. Duff WRD, Andrushko JW, Renshaw DW, et al. Impact of pilates exercise in multiple sclerosis: a randomized controlled trial. Int J MS Care. 2018 Mar-Apr;20(2):92-100. doi: 10.7224/1537-2073.2017-066. PMID: 29670495.

46. Duffell LD, Niu X, Brown G, et al. Variability in responsiveness to interventions in people with spinal cord injury: do some respond better than others? Conf Proc IEEE Eng Med Biol Soc. 2014;2014:5872-5. doi: 10.1109/EMBC.2014.6944964. PMID: 25571332 .

47. Ebrahimi A, Eftekhari E, Etemadifar M. Effects of whole body vibration on hormonal \& functional indices in patients with multiple sclerosis. Indian J Med Res. 2015 Oct;142(4):450-8. doi: 10.4103/09715916.169210. PMID: 26609037.
48. El-Shamy SM. Efficacy of Armeo ${ }^{\circledR}$ Robotic Therapy versus conventional therapy on upper limb function in children with hemiplegic cerebral palsy. Am J Phys Med Rehabil. 2018 Mar;97(3):164-69. doi: 10.1097/PHM.0000000000000852. PMID: 29059068.

49. Elnaggar RK, Elbanna MF, Mahmoud WS, et al. Plyometric exercises: subsequent changes of weight-bearing symmetry, muscle strength and walking performance in children with unilateral cerebral palsy. J Musculoskelet Neuronal Interact. 201912 01;19(4):507-15. PMID: 31789302.

50. Emara HA, El-Gohary TM, Al-Johany AA. Effect of body-weight suspension training versus treadmill training on gross motor abilities of children with spastic diplegic cerebral palsy. Eur J Phys Rehabil Med. 2016 Jun;52(3):356-63. PMID: 26845668.

51. Esclarin-Ruz A, Alcobendas-Maestro M, Casado-Lopez R, et al. A comparison of robotic walking therapy and conventional walking therapy in individuals with upper versus lower motor neuron lesions: a randomized controlled trial. Arch Phys Med Rehabil. 2014 Jun;95(6):1023-31. doi: 10.1016/j.apmr.2013.12.017. PMID: 24393781.

52. Faramarzi M, Banitalebi E, Raisi Z, et al. Effect of combined exercise training on pentraxins and pro- inflammatory cytokines in people with multiple sclerosis as a function of disability status. Cytokine. 2020 Jul 16;134:155196. doi: 10.1016/j.cyto.2020.155196. PMID: 32683106.

53. Field-Fote EC, Roach KE. Influence of a locomotor training approach on walking speed and distance in people with chronic spinal cord injury: a randomized clinical trial. Phys Ther. 2011 Jan;91(1):48-60. doi: 10.2522/ptj.20090359. PMID: 21051593.

54. Forsberg A, von Koch L, Nilsagard Y. Effects on balance and walking with the $\mathrm{CoDuSe}$ balance exercise program in people with multiple sclerosis: a multicenter randomized controlled trial. Mult Scler Int. 2016;2016 doi: 10.1155/2016/7076265. PMID: 28042485. 
55. Fosdahl MA, Jahnsen R, Kvalheim K, et al. Effect of a combined stretching and strength training program on gait function in children with cerebral palsy, GMFCS level I \& II: a randomized controlled trial. Medicina. 2019 Jun 6;55(6) doi:

10.3390/medicina55060250. PMID: 31174397.

56. Fowler EG, Knutson LM, Demuth SK, et al. Pediatric endurance and limb strengthening (PEDALS) for children with cerebral palsy using stationary cycling: a randomized controlled trial. Phys Ther. 2010

Mar;90(3):367-81. doi: 10.2522/ptj.20080364. PMID: 20093327.

57. Fox EE, Hough AD, Creanor S, et al. Effects of pilates-based core stability training in ambulant people with multiple sclerosis: multicenter, assessor-blinded, randomized controlled trial. Phys Ther. 2016 Aug;96(8):1170-8. doi: 10.2522/ptj.20150166. PMID: 26893507.

58. Freeman J, Fox E, Gear M, et al. Pilates based core stability training in ambulant individuals with multiple sclerosis: protocol for a multi-centre randomised controlled trial. BMC Neurol. 2012 Apr 5;12:19. doi: 10.1186/1471-2377-12-19. PMID: 22480437.

59. Galea MP, Dunlop SA, Davis GM, et al. Intensive exercise program after spinal cord injury ("Full-On"): study protocol for a randomized controlled trial. Trials. $2013 \mathrm{Sep}$ 11;14:291. doi: 10.1186/1745-6215-14-291. PMID: 24025260.

60. Galea MP, Dunlop SA, Geraghty T, et al. SCIPA Full-On: a randomized controlled trial comparing intensive whole-body exercise and upper body exercise after spinal cord injury. Neurorehabil Neural Repair. 2018 Jun;32(6-7):557-67. doi: $10.1177 / 1545968318771213$.

61. Gandolfi M, Munari D, Geroin C, et al. Sensory integration balance training in patients with multiple sclerosis: a randomized, controlled trial. Mult Scler. 2015 Oct;21(11):1453-62. doi: $10.1177 / 1352458514562438$. PMID: 25583852 .
62. Garrett M, Hogan N, Larkin A, et al. Exercise in the community for people with multiple sclerosis--a follow-up of people with minimal gait impairment. Mult Scler. 2013 May;19(6):790-8. doi: 10.1177/1352458512461390. PMID: 23132904 .

63. Garrett M, Hogan N, Larkin A, et al. Exercise in the community for people with minimal gait impairment due to MS: an assessor-blind randomized controlled trial. Mult Scler. 2013 May;19(6):782-9. doi: 10.1177/1352458512461966. PMID: 23128667.

64. Gervasoni E, Cattaneo D, Jonsdottir J. Effect of treadmill training on fatigue in multiple sclerosis: a pilot study. Int $\mathrm{J}$ Rehabil Res. 2014 Mar;37(1):54-60. doi: 10.1097/MRR.0000000000000034. PMID: 24220224.

65. Giangregorio L, Craven C, Richards K, et al. A randomized trial of functional electrical stimulation for walking in incomplete spinal cord injury: effects on body composition. J Spinal Cord Med. 2012 Sep;35(5):351-60. doi: 10.1179/2045772312Y.0000000041. PMID: 23031172.

66. Gibson N, Chappell A, Blackmore AM, et al. The effect of a running intervention on running ability and participation in children with cerebral palsy: a randomized controlled trial. Disabil Rehabil. 2018

Dec;40(25):3041-49. doi: 10.1080/09638288.2017.1367426. PMID: 28826274.

67. Gorman PH, Scott W, VanHiel L, et al. Comparison of peak oxygen consumption response to aquatic and robotic therapy in individuals with chronic motor incomplete spinal cord injury: a randomized controlled trial. Spinal Cord. 2019 Jun;57(6):471-81. doi: 10.1038/s41393-019-0239-7. PMID: 30659286.

68. Grecco LA, de Almeida Carvalho Duarte N, Mendonca ME, et al. Transcranial direct current stimulation during treadmill training in children with cerebral palsy: a randomized controlled double-blind clinical trial. Res Dev Disabil. 2014

Nov;35(11):2840-8. doi: 10.1016/j.ridd.2014.07.030. PMID: 25105567. 
69. Grecco LA, Zanon N, Sampaio LM, et al. A comparison of treadmill training and overground walking in ambulant children with cerebral palsy: randomized controlled clinical trial. Clin Rehabil. 2013

Aug;27(8):686-96. doi: 10.1177/0269215513476721. PMID: 23503736 .

70. Harness ET, Yozbatiran N, Cramer SC. Effects of intense exercise in chronic spinal cord injury. Spinal Cord. 2008

Nov;46(11):733-7. doi: 10.1038/sc.2008.56. PMID: 18521094.

71. Hasanpour-Dehkordi A. Influence of yoga and aerobics exercise on fatigue, pain and psychosocial status in patients with multiple sclerosis: a randomized trial. J Sports Med Phys Fitness. 2016 Nov;56(11):1417-22. PMID: 26223004.

72. Hasanpour-Dehkordi A, Jivad N. Comparison of regular aerobic and yoga on the quality of life in patients with multiple sclerosis. Med J Islam Repub Iran. 2014 Dec 6;28:141. PMID: 25694999.

73. Hasanpour-Dehkordi A, Jivad N, Solati K. Effects of yoga on physiological indices, anxiety and social functioning in multiple sclerosis patients: a randomized trial. J Clin Diagn Res. 2016 Jun;10(6):VC01-VC5. doi: 10.7860/JCDR/2016/18204.7916. PMID: 27504387.

74. Hebert JR. Effects of vestibular rehabilitation on MS-related fatigue: Randomized control trial. Dissertation Abstracts International: Section B: The Sciences and Engineering. 2009;70(6B):3470.

75. Hebert JR, Corboy JR, Manago MM, et al. Effects of vestibular rehabilitation on multiple sclerosis-related fatigue and upright postural control: a randomized controlled trial. Phys Ther. 2011 Aug;91(8):1166-83. doi: 10.2522/ptj.20100399. PMID: 21680771.

76. Heine M, Verschuren O, Hoogervorst EL, et al. Does aerobic training alleviate fatigue and improve societal participation in patients with multiple sclerosis? A randomized controlled trial. Mult Scler. 2017 Oct;23(11):1517-26. doi: 10.1177/1352458517696596. PMID: 28528566 .
77. Herrero P, Gomez-Trullen EM, Asensio A, et al. Study of the therapeutic effects of a hippotherapy simulator in children with cerebral palsy: a stratified single-blind randomized controlled trial. Clin Rehabil. 2012 Dec;26(12):1105-13. doi: 10.1177/0269215512444633. PMID: 22610128.

78. Hitzig SL, Craven BC, Panjwani A, et al. Randomized trial of functional electrical stimulation therapy for walking in incomplete spinal cord injury: effects on quality of life and community participation. Top Spinal Cord Inj Rehabil. 2013 Fall;19(4):245-58. doi: 10.1310/sci1904245. PMID: 24244090.

79. Hochsprung A, Granja Dominguez A, Magni E, et al. Effect of visual biofeedback cycling training on gait in patients with multiple sclerosis. Neurologia. 2017 Sep 6 doi: 10.1016/j.nrl.2017.07.008. PMID: 28888468.

80. Hogan N, Kehoe M, Larkin A, et al. The effect of community exercise interventions for people with MS who use bilateral support for gait. Mult Scler Int. 2014 Jan;2014 doi: 10.1155/2014/109142. PMID: 24575302.

81. Hota D, Das S, Joseph NM. Effect of dual task exercise to develop body balance, movement co-ordination and walking speed among post cervical injury clients. International Journal of Research in Pharmaceutical Sciences. 2020;11(1):111722. doi: 10.26452/ijrps.v11i1.1944.

82. Hsieh H-C. Preliminary study of the effect of training with a gaming balance board on balance control in children with cerebral palsy: a randomized controlled trial. Am J Phys Med Rehabil. 2020;99(2):142-8. doi: 10.1097/PHM.0000000000001300. PMID: 31464757.

83. Hsieh HC. Effects of a gaming platform on balance training for children with cerebral palsy. Pediatr Phys Ther. 2018

Oct;30(4):303-8. doi: 10.1097/PEP.0000000000000521. PMID: 30199515. 
84. Huang Q, Yu L, Gu R, et al. Effects of robot training on bowel function in patients with spinal cord injury. J Phys Ther Sci. 2015 May;27(5):1377-8. doi: 10.1589/jpts.27.1377. PMID: 26157223.

85. In T, Jung K, Lee MG, et al. Whole-body vibration improves ankle spasticity, balance, and walking ability in individuals with incomplete cervical spinal cord injury. NeuroRehabilitation. 2018;42(4):491-7. doi: 10.3233/NRE-172333. PMID: 29660953.

86. Johnston TE, Watson KE, Ross SA, et al. Effects of a supported speed treadmill training exercise program on impairment and function for children with cerebral palsy. Dev Med Child Neurol. 2011 Aug;53(8):742-50. doi: 10.1111/j.14698749.2011.03990.x. PMID: 21679357.

87. Jones ML, Evans N, Tefertiller C, et al. Activity-based therapy for recovery of walking in chronic spinal cord injury: results from a secondary analysis to determine responsiveness to therapy. Arch Phys Med Rehabil. 2014 Dec;95(12):2247-52. doi: 10.1016/j.apmr.2014.07.401. PMID: 25102385 .

88. Jones ML, Evans N, Tefertiller C, et al. Activity-based therapy for recovery of walking in individuals with chronic spinal cord injury: results from a randomized clinical trial. Arch Phys Med Rehabil. 2014 Dec;95(12):2239-46.e2. doi: 10.1016/j.apmr.2014.07.400. PMID: 25102384.

89. Jonsdottir J, Gervasoni E, Bowman T, et al. Intensive multimodal training to improve gait resistance, mobility, balance and cognitive function in persons with multiple sclerosis: a pilot randomized controlled trial. Front Neurol. 2018 Sep 28;9:800. doi: 10.3389/fneur.2018.00800. PMID: 30323787.

90. Jung J, Chung E, Kim K, et al. The effects of aquatic exercise on pulmonary function in patients with spinal cord injury. J Phys Ther Sci. 2014 May;26(5):707-9. doi: 10.1589/jpts.26.707. PMID: 24926136.
91. Kalron A, Fonkatz I, Frid L, et al. The effect of balance training on postural control in people with multiple sclerosis using the CAREN virtual reality system: a pilot randomized controlled trial. J Neuroengineering Rehabil. 2016 Mar 1;13:13. doi: 10.1186/s12984-016-0124-y. PMID: 26925955.

92. Kalron A, Rosenblum U, Frid L, et al. Pilates exercise training vs. physical therapy for improving walking and balance in people with multiple sclerosis: a randomized controlled trial. Clin Rehabil. 2017 Mar;31(3):319-28. doi: 10.1177/0269215516637202. PMID: 26951348.

93. Kapadia N, Masani K, Catharine Craven B, et al. A randomized trial of functional electrical stimulation for walking in incomplete spinal cord injury: effects on walking competency. J Spinal Cord Med. 2014 Sep;37(5):511-24. doi: 10.1179/2045772314Y.0000000263. PMID: 25229735 .

94. Kara B, Kucuk F, Poyraz EC, et al. Different types of exercise in multiple sclerosis: aerobic exercise or pilates, a single-blind clinical study. J Back Musculoskeletal Rehabil. 2017;30(3):565-73. doi: 10.3233/BMR-150515. PMID: 27911284.

95. Kara OK, Yardimci BN, Sahin S, et al. Combined effects of mirror therapy and exercises on the upper extremities in children with unilateral cerebral palsy: A randomized controlled trial. Dev Neurorehabil. 2020 May;23(4):253-64. doi: 10.1080/17518423.2019.1662853. PMID: 31514564 .

96. Kargarfard M, Shariat A, Ingle L, et al. Randomized controlled trial to examine the impact of aquatic exercise training on functional capacity, balance, and perceptions of fatigue in female patients with multiple sclerosis. Arch Phys Med Rehabil. 2018 Feb;99(2):234-41. doi: 10.1016/j.apmr.2017.06.015. PMID: 28735720. 
97. Kaya Kara O, Livanelioglu A, Yardimci $\mathrm{BN}$, et al. The effects of functional progressive strength and power training in children with unilateral cerebral palsy. Pediatr Phys Ther. 2019 Jul;31(3):286-95. doi: 10.1097/PEP.0000000000000628. PMID: 31220015.

98. Kerling A, Keweloh K, Tegtbur U, et al. Effects of a short physical excercise intervention on patients with multiple sclerosis (MS). Int J Mol Sci. $2015 \mathrm{Jul}$ 10;16(7):15761-75. doi: 10.3390/ijms160715761. PMID: 26184173.

99. Keser I, Meric A, Kirdi N, et al. Comparing routine neurorehabilitation programme with callisthenic exercises in multiple sclerosis. NeuroRehabilitation. 2011;29(1):91-8. doi: 10.3233/NRE-2011-0682. PMID: 21876301.

100. Khalil H, Al-Sharman A, El-Salem K, et al. The development and pilot evaluation of virtual reality balance scenarios in people with multiple sclerosis (MS): a feasibility study. NeuroRehabilitation. 2018;43(4):47382. doi: 10.3233/NRE-182471. PMID: 30400117.

101. Kim BJ, Kim SM, Kwon HY. The effect of group exercise program on the self-efficacy and activities of daily living in adults with cerebral palsy. J Phys Ther Sci. 2017 Dec;29(12):2184-9. doi: 10.1589/jpts.29.2184. PMID: 29643601.

102. Kim OY, Shin YK, Yoon YK, et al. The effect of treadmill exercise on gait efficiency during overground walking in adults with cerebral palsy. Ann Rehabil Med. 2015 Feb;39(1):25-31. doi: 10.5535/arm.2015.39.1.25. PMID: 25750868 .

103. Kirk H, Geertsen SS, Lorentzen J, et al. Explosive resistance training increases rate of force development in ankle dorsiflexors and gait function in adults with cerebral palsy. J Strength Cond Res. 2016 Oct;30(10):2749-60. doi: 10.1519/JSC.0000000000001376. PMID: 26890969 .

104. Kjolhede T, Dalgas U, Gade AB, et al. Acute and chronic cytokine responses to resistance exercise and training in people with multiple sclerosis. Scand J Med Sci Sports. 2016 Jul;26(7):824-34. doi: 10.1111/sms.12504. PMID: 26105554.
105. Klobucka S, Klobucky R, Kollar B. Effect of robot-assisted gait training on motor functions in adolescent and young adult patients with bilateral spastic cerebral palsy: A randomized controlled trial.

NeuroRehabilitation. 2020 Oct 29;29:29. doi: 10.3233/NRE-203102. PMID: 33136072 .

106. Kooshiar H, Moshtagh M, Sardar MA, et al. Fatigue and quality of life of women with multiple sclerosis: a randomized controlled clinical trial. J Sports Med Phys Fitness. 2015 Jun;55(6):668-74. PMID: 25303070.

107. Kressler J, Nash MS, Burns PA, et al. Metabolic responses to 4 different body weight-supported locomotor training approaches in persons with incomplete spinal cord injury. Arch Phys Med Rehabil. 2013 Aug;94(8):1436-42. doi: 10.1016/j.apmr.2013.02.018. PMID: 23473703.

108. Kumru H, Benito-Penalva J, Valls-Sole J, et al. Placebo-controlled study of rTMS combined with Lokomat ${ }^{\circledR}$ gait training for treatment in subjects with motor incomplete spinal cord injury. Exp Brain Res. 2016 Dec;234(12):3447-55. doi: 10.1007/s00221016-4739-9. PMID: 27469242.

109. Kwon JY, Chang HJ, Lee JY, et al. Effects of hippotherapy on gait parameters in children with bilateral spastic cerebral palsy. Arch Phys Med Rehabil. 2011 May;92(5):774-9. doi: 10.1016/j.apmr.2010.11.031. PMID: 21530725.

110. Kwon JY, Chang HJ, Yi SH, et al. Effect of hippotherapy on gross motor function in children with cerebral palsy: a randomized controlled trial. J Altern Complement Med. 2015 Jan;21(1):15-21. doi: 10.1089/acm.2014.0021. PMID: 25551626.

111. Lai CH, Chang WH, Chan WP, et al. Effects of functional electrical stimulation cycling exercise on bone mineral density loss in the early stages of spinal cord injury. J Rehabil Med. 2010 Feb;42(2):150-4. doi: 10.2340/16501977-0499. PMID: 20140411. 
112. Lai CJ, Liu WY, Yang TF, et al. Pediatric aquatic therapy on motor function and enjoyment in children diagnosed with cerebral palsy of various motor severities. J Child Neurol. 2015 Feb;30(2):200-8. doi: 10.1177/0883073814535491. PMID: 24907137.

113. Lavado EL, Cardoso JR, Silva LG, et al. Effectiveness of aerobic physical training for treatment of chronic asymptomatic bacteriuria in subjects with spinal cord injury: a randomized controlled trial. Clin Rehabil. 2013 Feb;27(2):142-9. doi: 10.1177/0269215512450522. PMID: 22837545.

114. Lee BK, Chon SC. Effect of whole body vibration training on mobility in children with cerebral palsy: a randomized controlled experimenter-blinded study. Clin Rehabil. 2013 Jul;27(7):599-607. doi: 10.1177/0269215512470673. PMID: 23411791.

115. Lee CW, Kim SG, Na SS. The effects of hippotherapy and a horse riding simulator on the balance of children with cerebral palsy. J Phys Ther Sci. 2014 Mar;26(3):423-5. doi: 10.1589/jpts.26.423. PMID: 24707098.

116. Liu H, Li J, Du L, et al. Short-term effects of core stability training on the balance and ambulation function of individuals with chronic spinal cord injury: a pilot randomized controlled trial. Minerva Med. 2019 Jun;110(3):216-23. doi: 10.23736/S0026-4806.19.05952-4. PMID: 30809995.

117. Lorentzen J, Greve LZ, Kliim-Due M, et al. Twenty weeks of home-based interactive training of children with cerebral palsy improves functional abilities. BMC Neurol. 2015 May 10;15:75. doi: 10.1186/s12883015-0334-0. PMID: 25956055.

118. Lucena-Anton D, Rosety-Rodriguez I, Moral-Munoz JA. Effects of a hippotherapy intervention on muscle spasticity in children with cerebral palsy: a randomized controlled trial. Complement Ther Clin Pract. 2018 May;31:188-92. doi: 10.1016/j.ctcp.2018.02.013. PMID: 29705454.
119. Makhov AS, Medvedev IN. The ability to reduce the severity of motor disorders in children with cerebral palsy. Res J Pharm Biol Chem Sci. 2018;9(2):991-6.

120. Marandi SM, Nejad VS, Shanazari Z, et al. A comparison of 12 weeks of pilates and aquatic training on the dynamic balance of women with mulitple sclerosis. Int J Prev Med. 2013 Apr;4(Suppl 1):S110-7. PMID: 23717760.

121. Marandi SM, Shahnazari Z, Minacian V, et al. A comparison between pilates exercise and aquatic training effects on mascular strength in women with mulitple sclerosis. Pak J Med Sci. 2013;29(1 Suppl):285-9. doi: 10.12669/pjms.291(Suppl).3518.

122. Matusiak-Wieczorek E, DziankowskaZaborszczyk E, Synder M, et al. The Influence of Hippotherapy on the Body Posture in a Sitting Position among Children with Cerebral Palsy. Int J Environ Res Public Health. 2020 Sep 19;17(18):19. doi: 10.3390/ijerph17186846. PMID: 32961681.

123. Matusiak-Wieczorek E, MalachowskaSobieska M, Synder M. Influence of hippotherapy on body balance in the sitting position among children with cerebral palsy. Ortop Traumatol Rehabil. 2016 Mar 23;18(2):165-75. doi: 10.5604/15093492.1205024. PMID: 28155825.

124. Midik M, Paker N, Bugdayci D, et al. Effects of robot-assisted gait training on lower extremity strength, functional independence, and walking function in men with incomplete traumatic spinal cord injury. Turk J Phys Med Rehabil. 2020 Mar;66(1):54-9. doi: 10.5606/tftrd.2020.3316. PMID: 32318675.

125. Mogharnasi M, TaheriChadorneshin H, Papoli-Baravati SA, et al. Effects of upperbody resistance exercise training on serum nesfatin-1 level, insulin resistance, and body composition in obese paraplegic men. Disabil Health J. 2019 Jan;12(1):29-34. doi: 10.1016/j.dhjo.2018.07.003. PMID: 30061073 . 
126. Moraes AG, Neri SGR, Motl RW, et al. Effect of hippotherapy on walking performance and gait parameters in people with multiple sclerosis. Mult Scler Relat Disord. 2020 May 26;43:102203. doi: 10.1016/j.msard.2020.102203. PMID: 32485633 .

127. Musselman KE, Yang JF. Spinal cord injury functional ambulation profile: a preliminary look at responsiveness. Phys Ther. 2014 Feb;94(2):240-50. doi: 10.2522/ptj.20130071. PMID: 24114437.

128. Mutoh T, Mutoh T, Tsubone H, et al. Impact of long-term hippotherapy on the walking ability of children with cerebral palsy and quality of life of their caregivers. Frontiers in neurology 2019 Aug 13;10:834. doi: 10.3389/fneur.2019.00834. PMID: 31456733 .

129. Najafidoulatabad S, Mohebbi Z, Nooryan K. Yoga effects on physical activity and sexual satisfaction among the Iranian women with multiple sclerosis: a randomized controlled trial. Afr J Tradit Complement Altern Med. 2014 Aug 23;11(5):78-82. doi: 10.4314/ajtcam.v11i5.13. PMID: 25395709.

130. Negaresh R, Motl R, Mokhtarzade M, et al. Effect of short-term interval excercise training on fatigue, depression, and fitness in normal weight vs. overweight person with multiple sclerosis. Explore (NY). 2019 MarApr;15(2):134-41. doi: 10.1016/j.explore.2018.07.007. PMID: 30122328.

131. Nilsagard YE, Forsberg AS, von Koch L. Balance exercise for persons with multiple sclerosis using Wii games: a randomised, controlled multi-centre study. Mult Scler. 2013 Feb;19(2):209-16. doi: $10.1177 / 1352458512450088$. PMID: 22674972.

132. Niwald M, Redlicka J, Miller E. The effects of aerobic training on the functional status, quality of life, the level of fatigue and disability in patients with multiple sclerosisa preliminary report. Aktualnosci Neurologiczne. 2017;17(1):15-22. doi: 10.15557/AN.2017.0002.
133. Norouzi E, Vaezmousavi M. Neurofeedback training and physical training differentially impacted on reaction time and balance skills among Iranian veterans with spinal cord injury. J Mil Veterans Health. 2019 Jul;27(3):11-8.

134. Nsenga AL, Shephard RJ, Ahmaidi S. Aerobic training in children with cerebral palsy. Int J Sports Med. 2013 Jun;34(6):5337. doi: 10.1055/s-0032-1321803. PMID: 23184482.

135. Nsenga Leunkeu A, Shephard RJ, Ahmaidi S. Six-minute walk test in children with cerebral palsy gross motor function classification system levels I and II: reproducibility, validity, and training effects. Arch Phys Med Rehabil. 2012

Dec;93(12):2333-9. doi: 10.1016/j.apmr.2012.06.005. PMID: 22721868.

136. Ortiz-Rubio A, Cabrera-Martos I, Rodriguez-Torres J, et al. Effects of a homebased upper limb training program in patients with multiple sclerosis: a randomized controlled trial. Arch Phys Med Rehabil. 2016 Dec;97(12):2027-33. doi: 10.1016/j.apmr.2016.05.018. PMID: 27343343.

137. Ozkul C, Guclu-Gunduz A, Eldemir K, et al. Combined exercise training improves cognitive functions in multiple sclerosis patients with cognitive impairment: A single-blinded randomized controlled trial. Mult Scler Relat Disord. 2020 Jul 23;45:102419. doi: 10.1016/j.msard.2020.102419. PMID: 32736216.

138. Ozkul C, Guclu-Gunduz A, Yazici G, et al. Effect of immersive virtual reality on balance, mobility, and fatigue in patients with multiple sclerosis: A single-blinded randomized controlled trial. European Journal of Integrative Medicine. 2020;35 doi: 10.1016/j.eujim.2020.101092.

139. Park ES, Rha DW, Shin JS, et al. Effects of hippotherapy on gross motor function and functional performance of children with cerebral palsy. Yonsei Med J. 2014 Nov;55(6):1736-42. doi: 10.3349/ymj.2014.55.6.1736. PMID: 25323914. 
140. Peri E, Turconi AC, Biffi E, et al. Effects of dose and duration of Robot-Assisted Gait Training on walking ability of children affected by cerebral palsy. Technol Health Care. 2017 Aug 9;25(4):671-81. doi: 10.3233/THC-160668. PMID: 28436398.

141. Pompa A, Morone G, Iosa M, et al. Does robot-assisted gait training improve ambulation in highly disabled multiple sclerosis people? A pilot randomized control trial. Mult Scler. 2017 Apr;23(5):696-703. doi: 10.1177/1352458516663033. PMID: 27486219.

142. Pourazar M, Bagherzadeh F, Mirakhori F. Virtual reality training improves dynamic balance in children with cerebral palsy. International Journal of Developmental Disabilities. 2019 doi: 10.1080/20473869.2019.1679471.

143. Qi Y, Zhang X, Zhao Y, et al. The effect of wheelchair Tai Chi on balance control and quality of life among survivors of spinal cord injuries: a randomized controlled trial. Complement Ther Clin Pract. 2018 Nov;33:7-11. doi: 10.1016/j.ctcp.2018.07.004. PMID: 30396629.

144. Qi YC, Niu XL, Gao YR, et al. Therapeutic effect evaluation of neuromuscular electrical stimulation with or without strengthening exercise on spastic cerebral palsy. Clin Pediatr (Phila). 2018 May;57(5):580-3. doi: 10.1177/0009922817732619. PMID: 28990434.

145. Razazian N, Yavari Z, Farnia V, et al. Exercising impacts on fatigue, depression, and paresthesia in female patients with multiple sclerosis. Med Sci Sports Exerc. 2016 May;48(5):796-803. doi: 10.1249/MSS.0000000000000834. PMID: 26656775.

146. Roppolo M, Mulasso A, Gollin M, et al. The role of fatigue in the associations between exercise and psychological health in multiple sclerosis: direct and indirect effects. Ment Health Phys Act. 2013 Jun;6(2):87-94. doi: 10.1016/j.mhpa.2013.05.002.
147. Russo M, Dattola V, De Cola MC, et al. The role of robotic gait training coupled with virtual reality in boosting the rehabilitative outcomes in patients with multiple sclerosis. Int J Rehabil Res. 2018 Jun;41(2):166-72. doi: 10.1097/MRR.0000000000000270. PMID: 29384762.

148. Sadeghi Bahmani D, Motl RW, Razazian N, et al. Aquatic exercising may improve sexual function in females with multiple sclerosis - an exploratory study. Mult Scler Relat Disord. 2020 Apr 26;43:102106. doi: 10.1016/j.msard.2020.102106. PMID: 32428843 .

149. Sadeghi Bahmani D, Razazian N, Farnia V, et al. Compared to an active control condition, in persons with multiple sclerosis two different types of exercise training improved sleep and depression, but not fatigue, paresthesia, and intolerance of uncertainty. Mult Scler Relat Disord. 2019 Nov;36:101356. doi: 10.1016/j.msard.2019.07.032. PMID: 31521917.

150. Sadowsky CL, Hammond ER, Strohl AB, et al. Lower extremity functional electrical stimulation cycling promotes physical and functional recovery in chronic spinal cord injury. J Spinal Cord Med. 2013

Nov;36(6):623-31. doi: 10.1179/2045772313Y.0000000101. PMID: 24094120.

151. Salci Y, Fil A, Armutlu K, et al. Effects of different exercise modalities on ataxia in multiple sclerosis patients: a randomized controlled study. Disabil Rehabil. 2017 Dec;39(26):2626-32. doi: 10.1080/09638288.2016.1236411. PMID: 27794631.

152. Samaei A, Bakhtiary AH, Hajihasani A, et al. Uphill and downhill walking in multiple sclerosis: a randomized controlled trial. Int $\mathrm{J}$ MS Care. 2016 Jan-Feb;18(1):34-41. doi: 10.7224/1537-2073.2014-072. PMID: 26917996.

153. Sandler EB, Roach KE, Field-Fote EC. Dose-response outcomes associated with different forms of locomotor training in persons with chronic motor-incomplete spinal cord injury. J Neurotrauma. 2017 May 15;34(10):1903-8. doi: 10.1089/neu.2016.4555. PMID: 27901413. 
154. Sandroff BM, Bollaert RE, Pilutti LA, et al. Multimodal exercise training in multiple sclerosis: a randomized controlled trial in persons with substantial mobility disability. Contemp Clin Trials. 2017 Oct;61:39-47. doi: 10.1016/j.cct.2017.07.016. PMID: 28732757.

155. Sangelaji B, Kordi M, Banihashemi F, et al. A combined exercise model for improving muscle strength, balance, walking distance, and motor agility in multiple sclerosis patients: a randomized clinical trial. Iran J Neurol. 2016 Jul 6;15(3):111-20. PMID: 27648171.

156. Sangelaji B, Nabavi SM, Estebsari F, et al. Effect of combination exercise therapy on walking distance, postural balance, fatigue and quality of life in multiple sclerosis patients: a clinical trial study. Iran Red Crescent Med J. 2014 Jun;16(6):e17173. doi: 10.5812/ircmj.17173. PMID: 25068045.

157. Scholtes VA, Becher JG, Comuth A, et al. Effectiveness of functional progressive resistance exercise strength training on muscle strength and mobility in children with cerebral palsy: a randomized controlled trial. Dev Med Child Neurol. 2010 Jun;52(6):e107-13. doi: 10.1111/j.14698749.2009.03604.x. PMID: 20132136.

158. Scholtes VA, Becher JG, Janssen-Potten YJ, et al. Effectiveness of functional progressive resistance exercise training on walking ability in children with cerebral palsy: a randomized controlled trial. Res Dev Disabil. 2012 Jan-Feb;33(1):181-8. doi: 10.1016/j.ridd.2011.08.026. PMID: 22093663.

159. Scholtes VA, Dallmeijer AJ, Rameckers EA, et al. Lower limb strength training in children with cerebral palsy--a randomized controlled trial protocol for functional strength training based on progressive resistance exercise principles. BMC Pediatr. 2008 Oct 8;8:41. doi: 10.1186/1471-2431-841. PMID: 18842125.

160. Shin JC, Kim JY, Park HK, et al. Effect of robotic-assisted gait training in patients with incomplete spinal cord injury. Ann Rehabil Med. 2014 Dec;38(6):719-25. doi: 10.5535/arm.2014.38.6.719. PMID: 25566469 .
161. Silva e Borges MB, Werneck MJ, da Silva Mde L, et al. Therapeutic effects of a horse riding simulator in children with cerebral palsy. Arq Neuropsiquiatr. 2011 Oct;69(5):799-804. doi: 10.1590/s0004282x2011000600014. PMID: 22042184.

162. Slaman J, Roebroeck M, Dallmijer A, et al. Can a lifestyle intervention programme improve physical behaviour among adolescents and young adults with spastic cerebral palsy? A randomized controlled trial. Dev Med Child Neurol. 2015 Feb;57(2):159-66. doi: 10.1111/dmcn.12602. PMID: 25303096.

163. Slaman J, Roebroeck M, van der Slot W, et al. Can a lifestyle intervention improve physical fitness in adolescents and young adults with spastic cerebral palsy? A randomized controlled trial. Arch Phys Med Rehabil. 2014 Sep;95(9):1646-55. doi: 10.1016/j.apmr.2014.05.011. PMID: 25067790.

164. Slaman J, Roebroeck ME, van Meeteren J, et al. Learn 2 Move 16-24: effectiveness of an intervention to stimulate physical activity and improve physical fitness of adolescents and young adults with spastic cerebral palsy; a randomized controlled trial. BMC Pediatr. 2010 Nov 5;10:79. doi: 10.1186/1471-243110-79. PMID: 21054829.

165. Slaman J, van den Berg-Emons HJ, van Meeteren J, et al. A lifestyle intervention improves fatigue, mental health and social support among adolescents and young adults with cerebral palsy: focus on mediating effects. Clin Rehabil. 2015 Jul;29(7):717-27. doi: 10.1177/0269215514555136. PMID: 25352613.

166. Straudi S, Fanciullacci C, Martinuzzi C, et al. The effects of robot-assisted gait training in progressive multiple sclerosis: a randomized controlled trial. Mult Scler. 2016 Mar;22(3):373-84. doi: 10.1177/1352458515620933. PMID: 26658817.

167. Straudi S, Manfredini F, Lamberti N, et al. Robot-assisted gait training is not superior to intensive overground walking in multiple sclerosis with severe disability (the RAGTIME study): a randomized controlled trial. Mult Scler. 2019 Mar 4 doi: 10.1177/1352458519833901. PMID: 30829117. 
168. Swe NN, Sendhilnnathan S, van Den Berg $\mathrm{M}$, et al. Over ground walking and body weight supported walking improve mobility equally in cerebral palsy: a randomised controlled trial. Clin Rehabil. 2015

Nov;29(11):1108-16. doi: 10.1177/0269215514566249. PMID: 25636992.

169. Tak S, Choi W, Lee S. Game-based virtual reality training improves sitting balance after spinal cord injury: a single-blinded, randomized controlled trial. Med Sci Technol. 2015;56:53-9.

170. Tarakci D, Ersoz Huseyinsinoglu B, Tarakci E, et al. Effects of Nintendo Wii-Fit ${ }^{\circledR}$ video games on balance in children with mild cerebral palsy. Pediatr Int. 2016 Oct;58(10):1042-50. doi: 10.1111/ped.12942. PMID: 26858013.

171. Tarakci E, Yeldan I, Huseyinsinoglu BE, et al. Group exercise training for balance, functional status, spasticity, fatigue and quality of life in multiple sclerosis: a randomized controlled trial. Clin Rehabil. 2013 Sep;27(9):813-22. doi: 10.1177/0269215513481047. PMID: 23543341 .

172. Taylor NF, Dodd KJ, Baker RJ, et al. Progressive resistance training and mobilityrelated function in young people with cerebral palsy: a randomized controlled trial. Dev Med Child Neurol. 2013

Sep;55(9):806-12. doi: 10.1111/dmen.12190. PMID: 23789741.

173. Tedla JS. Strength training effects on balance in spastic diplegia subjects: a randomized controlled trial. J Pediatr Neurol. 2014;12(1):15-28. doi: 10.3233/JPN-140634.

174. Teixeira-Machado L, Azevedo-Santos I, Desantana JM. Dance improves functionality and psychosocial adjustment in cerebral palsy: a randomized controlled clinical trial. Am J Phys Med Rehabil. 2017 Jun;96(6):424-9. doi: 10.1097/PHM.0000000000000646. PMID: 27820729.
175. Tollar J, Nagy F, Toth BE, et al. Exercise effects on multiple sclerosis quality of life and clinical-motor symptoms. Med Sci Sports Exerc. 2020 May;52(5):1007-14. doi: 10.1249/MSS.0000000000002228. PMID: 31876670 .

176. Totosy de Zepetnek JO, Pelletier CA, Hicks $\mathrm{AL}$, et al. Following the physical activity guidelines for adults with spinal cord injury for 16 weeks does not improve vascular health: a randomized controlled trial. Arch Phys Med Rehabil. 2015 Sep;96(9):1566-75. doi: 10.1016/j.apmr.2015.05.019. PMID: 26070976.

177. Valent L, Dallmeijer A, Houdijk H, et al. Effects of hand cycle training on wheelchair capacity during clinical rehabilitation in persons with a spinal cord injury. Disabil Rehabil. 2010;32(26):2191-200. doi: 10.3109/09638288.2010.509461. PMID: 20726737.

178. van der Scheer JW, de Groot S, Tepper M, et al. Low-intensity wheelchair training in inactive people with long-term spinal cord injury: a randomized controlled trial on fitness, wheelchair skill performance and physical activity levels. J Rehabil Med. 2016 Jan;48(1):33-42. doi: 10.2340/165019772037. PMID: 26660337.

179. Van Wely L, Balemans AC, Becher JG, et al. Physical activity stimulation program for children with cerebral palsy did not improve physical activity: a randomised trial. J Physiother. 2014 Mar;60(1):40-9. doi: 10.1016/j.jphys.2013.12.007. PMID: 24856939.

180. Van Wely L, Balemans AC, Becher JG, et al. The effectiveness of a physical activity stimulation programme for children with cerebral palsy on social participation, selfperception and quality of life: a randomized controlled trial. Clin Rehabil. 2014 Oct;28(10):972-82. doi: 10.1177/0269215513500971. PMID: 24047644.

181. Van Wely L, Becher JG, ReindersMesselink HA, et al. LEARN 2 MOVE 7-12 years: a randomized controlled trial on the effects of a physical activity stimulation program in children with cerebral palsy. BMC Pediatr. 2010 Nov 2;10:77. doi: 10.1186/1471-2431-10-77. PMID: 21044314. 
182. Vermohlen V, Schiller P, Schickendantz S, et al. Hippotherapy for patients with multiple sclerosis: a multicenter randomized controlled trial (MS-HIPPO). Mult Scler. 2018 Sep;24(10):1375-82. doi: 10.1177/1352458517721354. PMID: 28770664.

183. Wallard L, Dietrich G, Kerlirzin Y, et al. Robotic-assisted gait training improves walking abilities in diplegic children with cerebral palsy. Europ J Paediatr Neurol. 2017 May;21(3):557-64. doi: 10.1016/j.gaitpost.2017.11.007. PMID: 28188024 .

184. Wallard L, Dietrich G, Kerlirzin Y, et al. Effect of robotic-assisted gait rehabilitation on dynamic equilibrium control in the gait of children with cerebral palsy. Gait Posture. 2018 Feb;60:55-60. doi:

10.1016/j.gaitpost.2017.11.007. PMID: 29156378.

185. Wens I, Dalgas U, Vandenabeele F, et al. High intensity excercise in multiple sclerosis: effects on muscle contractile characteristics and excercise capacity, a randomised controlled trial. PLoS One. 2015 Oct;10(9) doi:

10.1371/journal.pone.0133697. PMID: 26418222.

186. Wens I, Hansen D, Verboven K, et al. Impact of 24 weeks of resistance and endurance excercise on glucose tolerance in persons with multiple sclerosis. Am J Phys Med Rehabil. 2015 Oct;94(10 Suppl 1):83847. doi: 10.1097/PHM.0000000000000257. PMID: 25768064.

187. Williams KL, Low Choy NL, Brauer SG. Center-Based Group and Home-Based Individual Exercise Programs Have Similar Impacts on Gait and Balance in People With Multiple Sclerosis: A Randomized Trial. Pm R. 2020 Apr 06;06:06. doi: 10.1002/pmrj.12377. PMID: 32249509.

188. Willoughby KL, Dodd KJ, Shields N, et al. Efficacy of partial body weight-supported treadmill training compared with overground walking practice for children with cerebral palsy: a randomized controlled trial. Arch Phys Med Rehabil. 2010

Mar;91(3):333-9. doi: 10.1016/j.apmr.2009.10.029. PMID: 20298820.
189. Wollenweber V, Drache M, Schickendantz $\mathrm{S}$, et al. Study of the effectiveness of hippotherapy on the symptoms of multiple sclerosis - outline of a randomised controlled multicentre study (MS-HIPPO). Contemp Clin Trials Commun. 2016 Aug 15;3:6-11. doi: 10.1016/j.conctc.2016.02.001. PMID: 29736452.

190. Wu M, Kim J, Arora P, et al. Effects of the integration of dynamic weight shifting training into treadmill training on walking function of children with cerebral palsy: a randomized controlled study. Am J Phys Med Rehabil. 2017 Nov;96(11):765-72. doi: 10.1097/PHM.0000000000000776. PMID: 28644244.

191. Wu M, Kim J, Gaebler-Spira DJ, et al. Robotic resistance treadmill training improves locomotor function in children with cerebral palsy: a randomized controlled pilot study. Arch Phys Med Rehabil. 2017 Nov;98(11):2126-33. doi: 10.1016/j.apmr.2017.04.022. PMID: 28576629.

192. Yang JF, Musselman KE, Livingstone D, et al. Repetitive mass practice or focused precise practice for retraining walking after incomplete spinal cord injury? A pilot randomized clinical trial. Neurorehabil Neural Repair. 2014 May;28(4):314-24. doi: 10.1177/1545968313508473. PMID: 24213960 .

193. Yazgan YZ, Tarakci E, Tarakci D, et al. Comparison of the effects of two different exergaming systems on balance, functionality, fatigue, and quality of life in people with multiple sclerosis: A randomized controlled trial. Mult Scler Relat Disord. 2019 Dec 21;39:101902. doi: 10.1016/j.msard.2019.101902. PMID: 31924591 .

194. Yazici M, Livanelioglu A, Gucuyener K, et al. Effects of robotic rehabilitation on walking and balance in pediatric patients with hemiparetic cerebral palsy. Gait Posture. 2019 May;70:397-402. doi: 10.1016/j.gaitpost.2019.03.017. PMID: 30974395 . 
195. Yildirim MA, Ones K, Goksenoglu G. Early term effects of robotic assisted gait training on ambulation and functional capacity in patients with spinal cord injury. Turk J Med Sci. 2019 Jun 18;49(3):838-43. doi: 10.3906/sag-1809-7. PMID: 31134784.

196. Young HJ, Mehta TS, Herman C, et al. The effects of M2M and adapted yoga on physical and psychosocial outcomes in people wth multiple sclerosis. Arch Phys Med Rehabil. 2019 Aug 6;100(3):391-400. doi: 10.1016/j.apmr.2018.06.032. PMID: 30092206 .

197. Zoccolillo L, Morelli D, Cincotti F, et al. Video-game based therapy performed by children with cerebral palsy: a cross-over randomized controlled trial and a crosssectional quantitative measure of physical activity. Eur J Phys Rehabil Med. 2015 Dec;51(6):669-76. PMID: 25653079. 


\section{Appendix C. Excluded Studies List}

\section{Table C-1. Study exclusion reasons}

\begin{tabular}{|l|c|}
\hline $\begin{array}{l}\text { Full Text } \\
\text { Exclusion Reason }\end{array}$ & $\begin{array}{c}\text { Exclusion } \\
\text { Code }\end{array}$ \\
\hline Ineligible population (not multiple sclerosis, cerebral palsy or spinal cord injury) & 3 \\
\hline Ineligible intervention (e.g., < than 10 sessions and or < than 10 days, only family/caregiver observed) & 4 \\
\hline Ineligible comparator & 5 \\
\hline Ineligible outcomes & 6 \\
\hline Ineligible design (i.e., case reports, case series) & 7 \\
\hline Pre-post studies & 8 \\
\hline Studies outside of search dates (before January 2008 or for systematic reviews 2014 or older) & 9 \\
\hline Not a study (letter, editorial, nonsystematic review) & 10 \\
\hline Inadequate samples size (MS and SCl n<30 and CP n<20) & 11 \\
\hline Systematic review, not used, but checked for includable studies & 12 \\
\hline Not English language & 13 \\
\hline Non-U.S. applicable study setting & 14 \\
\hline Nonhuman population (animal study) & 15 \\
\hline
\end{tabular}

Note: Codes 1-2 used for included studies and background

1. Abasiyanik Z, Ertekin O, Kahraman T, et al. The effects of clinical pilates training on walking, balance, fall risk, respiratory and cognitive functions in persons with multiple sclerosis: a randomized controlled trial. Mult Scler. 2018;Conference: 23rd annual RIMS conference. 2018. Netherlands 24(6):862. Exclusion: 10.

2. Abasiyanik Z, Ertekin O, Kahraman T, et al. The effects of Clinical Pilates training on walking, balance, fall risk, respiratory, and cognitive functions in persons with multiple sclerosis: A randomized controlled trial. Explore (NY). 2019 Jul 17;17:17. doi: 10.1016/j.explore.2019.07.010. PMID: 31377306. Exclusion: 4.

3. Abbaspoor E, Zolfaghari M, Ahmadi B, et al. The effect of combined functional training on BDNF, IGF-1, and their association with health-related fitness in the multiple sclerosis women. Growth Horm IGF Res. 2020 Jun;52:101320. doi: 10.1016/j.ghir.2020.101320. PMID: 32305012. Exclusion: 11.
4. Abd-Elmonem AM, Elhady HSA. Effect of rebound exercises on balance in children with spastic diplegia. Int J Ther Rehabil. 2018 Sep;25(9):467-74. doi: 10.12968/ijtr.2018.25.9.467. Exclusion: 14.

5. Abdel Gawad HA, Abdel Karim AE, Mohammed AH. Shock wave therapy for spastic plantar flexor muscles in hemiplegic cerebral palsy children. Egypt J Med Hum Genet. 2015;16(3):269-75. doi: 10.1016/j.ejmhg.2014.12.007. Exclusion: 14.

6. Abdel-Aziem AA, El-Basatiny HM. Effectiveness of backward walking training on walking ability in children with hemiparetic cerebral palsy: a randomized controlled trial. Clin Rehabil. 2017 Jun;31(6):790-7. doi: 10.1177/0269215516656468. PMID: 27356944. Exclusion: 14. 
7. Abouelkheir RAA, Khalil ME, Abd-Elfattah HM. Hippotherapy versus traditional physiotherapy on gait in spastic diaplegic children. Indian J Public Health Res Dev. 2019;10(12):1472-5. doi: 10.37506/v10/i12/2019/ijphrd/192416. Exclusion: 6.

8. Aburub A, Khalil H, Al-Sharman A, et al. The association between physical activity and sleep characteristics in people with multiple sclerosis. Mult Scler Relat Disord. 2017 Feb;12:29-33. doi: 10.1016/j.msard.2016.12.010. PMID: 28283102. Exclusion: 4.

9. Adamson BC, Ensari I, Motl RW. Effect of exercise on depressive symptoms in adults with neurologic disorders: a systematic review and meta-analysis. Arch Phys Med Rehabil. 2015 Jul;96(7):1329-38. doi: 10.1016/j.apmr.2015.01.005. PMID: 25596001. Exclusion: 12.

10. Adepoju F, Hamzat T, Akinyinka O. Comparative efficacy of progressive resistance exercise and biomechanical ankle platform system on functional indices of children with cerebral palsy. Ethiop. 2017 Jan;27(1):11-6. doi: 10.4314/ejhs.v27i1.3. PMID: 28458486. Exclusion: 14.

11. Afkar A, Ashouri A, Rahmani M, et al. Effect of exercise therapy on quality of life of patients with multiple sclerosis in Iran: a systematic review and meta-analysis. Neurol Sci. 2017 Nov;38(11):1901-11. doi: 10.1007/s10072-017-3047-x. PMID: 28687973. Exclusion: 12.

12. Aguirre-Guemez AV, Perez-Sanpablo AI, Quinzanos-Fresnedo J, et al. Walking speed is not the best outcome to evaluate the effect of robotic assisted gait training in people with motor incomplete spinal cord injury: a systematic review with meta-analysis. J Spinal Cord Med. 2017 Oct 25;42(2):14254. doi: 10.1080/10790268.2017.1390644. PMID: 29065788. Exclusion: 12.

13. Aidar FJ, Carneiro AL, Costa Moreira O, et al. Effects of resistance training on the physical condition of people with multiple sclerosis. J Sports Med Phys Fitness. 2018 Jul-Aug;58(7-8):1127-34. doi: 10.23736/S0022-4707.17.07621-6. PMID: 28944644. Exclusion: 11.
14. Aidar FJ, Gama de Matos D, de Souza RF, et al. Influence of aquatic exercises in physical condition in patients with multiple sclerosis. J Sports Med Phys Fitness. 2018 May;58(5):684-9. doi: 10.23736/S00224707.17.07151-1. PMID: 28462574. Exclusion: 11.

15. Akbar M, Brunner M, Ewerbeck V, et al. Do overhead sports increase risk for rotator cuff tears in wheelchair users? Arch Phys Med Rehabil. 2015 Mar;96(3):484-8. doi: 10.1016/j.apmr.2014.09.032. PMID: 25449196. Exclusion: 4.

16. Akbar N, Sandroff BM, Wylie GR, et al. Progressive resistance exercise training and changes in resting-state functional connectivity of the caudate in persons with multiple sclerosis and severe fatigue: a proof-of-concept study. Neuropsychol. 2018 Apr 2018:1-13. doi: 10.1080/09602011.2018.1449758. PMID: 29618280. Exclusion: 11.

17. Akkurt H, Kirazli Y, Karapolat H, et al. The effects of aerobic exercise on cardiopulmonary functions, quality of life, psychological state, disability and metabolic syndrome parameters in patients with spinal cord injury. Turk J Phys Med Rehabil 2013;59:409. doi: 10.4274/tftr.24.59.1. Exclusion: 10.

18. Alabdulwahab SS. Electrical stimulation improves gait in children with spastic diplegic cerebral palsy. NeuroRehabilitation. 2011;29(1):37-43. doi: 10.3233/NRE-20110675. PMID: 21876294. Exclusion: 4.

19. Alashram AR, Padua E, Annino G. Effects of whole body vibration on motor impairments in patients with neurological disorders: a systematic review. Am J Phys Med Rehabil. 2019 Jun 21;21:21. doi: 10.1097/PHM.0000000000001252. PMID: 31246611. Exclusion: 12.

20. Alemdaroglu E, Yanikoglu I, Oken O, et al. Horseback riding therapy in addition to conventional rehabilitation program decreases spasticity in children with cerebral palsy: a small sample study. Complement Ther Clin Pract. 2016 May;23:26-9. doi: 10.1016/j.ctcp.2016.02.002. PMID: 27157954. Exclusion: 11. 
21. Ali MS, Awad AS, Elassal MI. The effect of two therapeutic interventions on balance in children with spastic cerebral palsy: a comparative study. J Taibah Univ Med Sci. 2019 Aug;14(4):350-6. doi: 10.1016/j.jtumed.2019.05.005. PMID: 31488967. Exclusion: 14.

22. Ali MSM, Elazem FHA, Anwar GM. Effect of core stabilizing program on balance in spastic diplegic cerebral palsy children. Int $\mathrm{J}$ Pharmtech Res. 2016;9(5):129-36.

Exclusion: 14.

23. Alphonsus KB, Su Y, D'Arcy C. The effect of exercise, yoga and physiotherapy on the quality of life of people with multiple sclerosis: systematic review and metaanalysis. Complement Ther Med. 2019 Apr;43:188-95. doi: 10.1016/j.ctim.2019.02.010. PMID: 30935529. Exclusion: 12.

24. AlSaif AA, Alsenany S. Effects of interactive games on motor performance in children with spastic cerebral palsy. J Phys Ther Sci. 2015 Jun;27(6):2001-3. doi: 10.1589/jpts.27.2001. PMID: 26180367. Exclusion: 4.

25. Alvarenga-Filho H, Sacramento PM, Ferreira TB, et al. Combined exercise training reduces fatigue and modulates the cytokine profile of T-cells from multiple sclerosis patients in response to neuromediators. J Neuroimmunol. 2016 Apr 15;293:91-9. doi:

10.1016/j.jneuroim.2016.02.014. PMID: 27049568. Exclusion: 11.

26. Ameer MA, Fayez ES, Elkholy HH. Improving spatiotemporal gait parameters in spastic diplegic children using treadmill gait training. J Bodywork Mov Ther.

2019;23(4):937-42. doi: 10.1016/j.jbmt.2019.02.003. Exclusion: 14.

27. Ammann-Reiffer $\mathrm{C}$, Bastiaenen $\mathrm{CH}$, MeyerHeim AD, et al. Effectiveness of robotassisted gait training in children with cerebral palsy: a bicenter, pragmatic, randomized, cross-over trial (PeLoGAIT). BMC Pediatr. 2017 Mar 2;17(1):64. doi: 10.1186/s12887-017-0815-y. PMID: 28253887. Exclusion: 10.
28. Andreu L, Ramos-Campo DJ, Avila-Gandia $\mathrm{V}$, et al. Acute effects of whole-body vibration training on neuromuscular performance and mobility in hypoxia and normoxia in persons with multiple sclerosis: A crossover study. Mult Scler Relat Disord. 2020 Jan;37:101454. doi:

10.1016/j.msard.2019.101454. PMID: 31670008. Exclusion: 11.

29. Angeli CA, Boakye M, Morton RA, et al. Recovery of over-ground walking after chronic motor complete spinal cord injury. N Engl J Med. 2018 Sep 27;379(13):124450. doi: 10.1056/NEJMoa1803588. PMID: 30247091. Exclusion: 5.

30. Angsupaisal M, Visser B, Alkema A, et al. Therapist-designed adaptive riding in children with cerebral palsy: results of a feasibility study. Phys Ther. 2015 Aug;95(8):1151-62. doi: 10.2522/ptj.20140146. PMID: 25908525. Exclusion: 7.

31. Anonymous. Erratum. Mult Scler. 2016 Oct;22(12):9-11. PMID: 26041800. Exclusion: 10.

32. Antonelli CBB, Hartz CS, da Silva Santos S, et al. Effects of inspiratory muscle training with progressive loading on respiratory muscle function and sports performance in high-performance wheelchair basketball athletes: a randomized clinical trial. Int J Sports Physiol Perform. 2019 Oct 28:1-5. doi: 10.1123/ijspp.2018-0979. PMID: 31172823. Exclusion: 3.

33. Antunes FN, Pinho ASD, Kleiner AFR, et al. Different horse's paces during hippotherapy on spatio-temporal parameters of gait in children with bilateral spastic cerebral palsy: a feasibility study. Res Dev Disabil. 2016 Dec;59:65-72. doi: 10.1016/j.ridd.2016.07.015. PMID: 27518920. Exclusion: 4.

34. Aravind N, Harvey LA, Glinsky JV. Physiotherapy interventions for increasing muscle strength in people with spinal cord injuries: a systematic review. Spinal Cord. 2019 Jun;57(6):449-60. doi: 10.1038/s41393-019-0242-z. PMID: 30723256. Exclusion: 12. 
35. Arbour-Nicitopoulos K, Sweet S, Lamontagne ME, et al. A randomized controlled trial to test the effects of the SCI get fit toolkit on physical activity behavior and cognitions in adults with spinal cord injury: a preliminary analysis. J Spinal Cord Med. 2014 Sep;37(5):650. doi: 10.1179/1079026814Z.000000000315. PMID: 25229743. Exclusion: 10.

36. Armstrong EL, Spencer S, Kentish MJ, et al. Efficacy of cycling interventions to improve function in children and adolescents with cerebral palsy: a systematic review and meta-analysis. Clin Rehabil. 2019 Jul;33(7):1113-29. doi: 10.1177/0269215519837582. PMID: 30935240. Exclusion: 12.

37. Arntzen EC. Does a combination of individualised physiotherapy treatment and gait training on a treadmill influence gait in persons with multiple sclerosis?

Physiotherapy. 2011;97(Supplement 1):181415. Exclusion: 10.

38. Ashe MC, Eng JJ, Krassioukov AV, et al. Response to functional electrical stimulation cycling in women with spinal cord injuries using dual-energy X-ray absorptiometry and peripheral quantitative computed tomography: a case series. J Spinal Cord Med. 2010;33(1):68-72. doi: 10.1080/10790268.2010.11689676. PMID: 20397446. Exclusion: 7.

39. Astorino TA, Thum JS. Within-session responses to high-intensity interval training in spinal cord injury. Disabil Rehabil. 2018 Feb;40(4):444-9. doi: 10.1080/09638288.2016.1260648. PMID: 27930890. Exclusion: 5.

40. Awan WA, Masood T. Role of stretching exercises in the management of constipation in spastic cerebral palsy. J Ayub Med Coll Abbottabad. 2016 Oct-Dec;28(4):798-801. PMID: 28586619. Exclusion: 4.

41. Aye T, Thein S, Hlaing T. Effects of strength training program on hip extensors and knee extensors strength of lower limb in children with spastic diplegic cerebral palsy. J Phys Ther Sci. 2016 Jan;28(2):671-6. doi: 10.1589/jpts.28.671. PMID: 27065561. Exclusion: 14.
42. Azizi S, Marzbani H, Raminfard S, et al. The impact of an anti-gravity treadmill (AlterG) training on walking capacity and corticospinal tract structure in children with cerebral palsy. Conf Proc IEEE Eng Med Biol Soc. 2017 Jul;2017:1150-3. doi: 10.1109/EMBC.2017.8037033. PMID: 29060079. Exclusion: 4.

43. Bach Baunsgaard C, Vig Nissen U, Katrin Brust A, et al. Gait training after spinal cord injury: safety, feasibility and gait function following 8 weeks of training with the exoskeletons from Ekso Bionics. Spinal Cord. 2018 Feb;56(2):106-16. doi: 10.1038/s41393-017-0013-7. PMID: 29105657. Exclusion: 8.

44. Backus D, Berry A. Commentary on: "Calling Out MS Fatigue: Feasibility and Preliminary Effects of a Pilot Randomized Telephone-Delivered Exercise Intervention for Multiple Sclerosis Fatigue". J Neurol Phys Ther. 2020 Jan;44(1):32-3. doi: 10.1097/NPT.0000000000000304. PMID: 31834218. Exclusion: 4.

45. Backus D, Burdett B, Hawkins L, et al. Outcomes after functional electrical stimulation cycle training in individuals with multiple sclerosis who are nonambulatory. Int J MS Care. 2017 May-Jun;19(3):113-21. doi: 10.7224/1537-2073.2015-036. PMID: 28603459. Exclusion: 11.

46. Baek J-H, Kim J-W, Kim S-Y, et al. Acute effect of repeated passive motion exercise on shoulder position sense in patients with hemiplegia: a pilot study.

NeuroRehabilitation. 2009;25(2):101-6. doi: 10.3233/NRE-2009-0504. PMID: 19822940. Exclusion: 3 .

47. Bakkum AJ, de Groot S, Onderwater MQ, et al. Metabolic rate and cardiorespiratory response during hybrid cycling versus handcycling at equal subjective exercise intensity levels in people with spinal cord injury. J Spinal Cord Med. 2014 Nov;37(6):758-64. doi: 10.1179/2045772313Y.0000000164. PMID: 24621028. Exclusion: 4. 
48. Bania T, Todd K, Taylor N. Effects of strength training on the habitual physical activity and sedentary behaviour of young people with diplegic cerebral palsy. Abstracts of the European Academy of Childhood Disability 25th Annual Meeting, 10-12 October 2013, Newcastle-Gateshead, UK. Dev Med Child Neurol. 2013

Oct;55(S2):19. doi: 10.1111/dmcn.12258. Exclusion: 10.

49. Bansi J, Bloch W, Gamper U, et al. Training in MS: influence of two different endurance training protocols (aquatic versus overland) on cytokine and neurotrophin concentrations during three week randomized controlled trial. Mult Scler. 2013 Apr;19(5):613-21. doi: $10.1177 / 1352458512458605$. PMID: 22936334. Exclusion: 4.

50. Bansi J, Bloch W, Gamper U, et al. Cycling under immersion affects growth factor BDNF, cardiorespiratory fitness, fatigue and health-related quality of life in persons with multiple sclerosis. A randomized controlled study. Mult Scler J Exp Transl Clin. 2014 START: 2014 Jun 6 CONFERENCE END: 2014 Jun 7, 19th Annual Rehabilitation in Multiple Sclerosis Conference, RIMS 2014 Brighton United Kingdom;20(7):979-80. doi: $10.1177 / 1352458514533628$.

Exclusion: 4.

51. Bansi J, Bloch W, Gamper U, et al. Endurance training in MS: short-term immune responses and their relation to cardiorespiratory fitness, health-related quality of life, and fatigue. J Neurol. 2013 Dec;260(12):2993-3001. doi: 10.1007/s00415-013-7091-z. PMID: 24036849. Exclusion: 4.

52. Barati AA, Rajabi R, Shahrbanian S, et al. Investigation of the effect of sensorimotor exercises on proprioceptive perceptions among children with spastic hemiplegic cerebral palsy. J Hand Ther. 2020 Mar 10;10:10. doi: 10.1016/j.jht.2019.12.003. PMID: 32169259. Exclusion: 4.

53. Barbarulo AM, Lus G, Signoriello E, et al. Integrated cognitive and neuromotor rehabilitation in multiple sclerosis: a pragmatic study. Front Behav Neurosci. 2018 Sep 5;12(196) doi: 10.3389/fnbeh.2018.00196. PMID: 30271331. Exclusion: 5.
54. Barclay A, Paul L, MacFarlane N, et al. The effect of cycling using active-passive trainers on spasticity, cardiovascular fitness, function and quality of life in people with moderate to severe Multiple Sclerosis (MS); a feasibility study. Mult Scler Relat Disord. 2019 Sep;34:128-34. doi: 10.1016/j.msard.2019.06.019. PMID: 31260943. Exclusion: 11.

55. Barfield JP, Malone LA. Perceived exercise benefits and barriers among power wheelchair soccer players. J Rehabil Res Dev. 2013;50(2):231-8. PMID: 23761004. Exclusion: 7.

56. Barfield JP, Newsome L, Malone LA. Exercise intensity during power wheelchair soccer. Arch Phys Med Rehabil. 2016 Nov;97(11):1938-44. doi: 10.1016/j.apmr.2016.05.012. PMID: 27288710. Exclusion: 3.

57. Barry A, Cronin O, Ryan AM, et al. Impact of short-term cycle ergometer training on quality of life, cognition and depressive symptomatology in multiple sclerosis patients: a pilot study. Neurol Sci. 2018 Mar;39(3):461-9. doi: 10.1007/s10072-0173230-0. PMID: 29280019. Exclusion: 5.

58. Barthelemy A, Gagnon DH, Duclos C. Gaitlike vibration training improves gait abilities: a case report of a 62-year-old person with a chronic incomplete spinal cord injury. Spinal Cord Ser Cases. 2016 Jul 21;2:16012. doi: 10.1038/scsandc.2016.12. PMID: 28053756. Exclusion: 7.

59. Bauerfeind J, Koper M, Wieczorek J, et al. Sports injuries in wheelchair rugby - A pilot study. J Hum Kinet. 2015 Nov 22;48:12332. doi: 10.1515/hukin-2015-0098. PMID: 26834880. Exclusion: 11.

60. Baunsgaard CB, Nissen UV, Brust AK, et al. Exoskeleton gait training after spinal cord injury: an exploratory study on secondary health conditions. J Rehabil Med. 2018 Sep 28;50(9):806-13. doi: 10.2340/16501977-2372. PMID: 30183055. Exclusion: 8. 
61. Baxter $\mathrm{K}$. The effect of core strengthening versus extremity strengthening on mobility measures in patients with multiple sclerosis. Dissertation Abstracts International: Section B: The Sciences and Engineering. 2018;78(10-B(E)):No Pagination Specified. Exclusion: 12.

62. Bayon C, Martin-Lorenzo T, Moral-Saiz B, et al. A robot-based gait training therapy for pediatric population with cerebral palsy: goal setting, proposal and preliminary clinical implementation. J Neuroengineering Rehabil. 2018 Jul 27;15(1):69. doi: 10.1186/s12984-018-0412-9. PMID: 30053857. Exclusion: 11.

63. Beani E, Menici V, Ferrari A, et al. Feasibility of a home-based action observation training for children with unilateral cerebral palsy: An explorative study. Front Neurol. 2020;11 doi: 10.3389/fneur.2020.00016. Exclusion: 4.

64. Bedair R, Al-Talawy H, Shoukry K, et al. Impact of virtual reality games as an adjunct treatment tool on upper extremity function of spastic hemiplegic children. Int J Pharmtech Res. 2016;9(6):1-8. Exclusion: 14.

65. Bernhardt L, Marziniak M. Specific resistance training for patients with multiple sclerosis. Mult Scler J Exp Transl Clin. START: 2011 Oct 19 CONFERENCE END: 2011 Oct 22, 5th Joint Triennial Congress of the European and Americas Committees for Treatment and Research in Multiple Sclerosis Amsterdam

Netherlands;17(10 SUPPL. 1):S465.

Exclusion: 10.

66. Biasotto-Gonzalez DA, Grecco LC, Neto $\mathrm{HP}$, et al. Effect of treadmill gait training on static and functional balance in children with cerebral palsy: randomized controlled clinical trial. Gait Posture. 2013;38:S69S70. doi: 10.1016/j.gaitpost.2013.07.150. Exclusion: 10.

67. Bickel CS, Yarar-Fisher C, Mahoney ET, et al. Neuromuscular electrical stimulationinduced resistance training after SCI: A review of the dudley protocol. Top Spinal Cord Inj Rehabil. 2015;21(4):294-302. doi: 10.1310/sci2104-294. PMID: 26689694. Exclusion: 8.
68. Birgani PM, Ashtiyani M, Rasooli A, et al. Can an anti-gravity treadmill improve stability of children with cerebral palsy? Conf Proc IEEE Eng Med Biol Soc. 2016 08;2016:5465-8. doi: 10.1109/EMBC.2016.7591963. PMID: 28269494. Exclusion: 11.

69. Bisson EJ, Fakolade A, Petrin J, et al. Exercise interventions in multiple sclerosis rehabilitation need better reporting on comorbidities: a systematic scoping review. Clin Rehabil. 2017 Oct;31(10):1305-12. doi: 10.1177/0269215517698734. PMID: 28933610. Exclusion: 12.

70. Blackmore AM, Gibson N, Cooper MS, et al. Interventions for management of respiratory disease in young people with cerebral palsy: a systematic review. Child Care Health Dev. 2019 Sep;45(5):754-71. doi: 10.1111/cch.12703. PMID: 31276598. Exclusion: 4.

71. Bloemen M, Van Wely L, Mollema J, et al. Evidence for increasing physical activity in children with physical disabilities: a systematic review. Dev Med Child Neurol. 2017 10;59(10):1004-10. doi:

10.1111/dmcn.13422. PMID: 28374442.

Exclusion: 12.

72. Bochkezanian V, Newton RU, Trajano GS, et al. Effects of neuromuscular electrical stimulation in people with spinal cord injury. Med Sci Sports Exerc. 2018 Sep;50(9):1733-9. doi: 10.1249/MSS.0000000000001637. PMID: 29634640. Exclusion: 11.

73. Bochkezanian V, Raymond J, de Oliveira $\mathrm{CQ}$, et al. Can combined aerobic and muscle strength training improve aerobic fitness, muscle strength, function and quality of life in people with spinal cord injury? A systematic review. Spinal Cord. 2015 Jun;53(6):418-31. doi: 10.1038/sc.2015.48. PMID: 25823799. Exclusion: 12.

74. Booth ATC, Buizer AI, Meyns P, et al. The efficacy of functional gait training in children and young adults with cerebral palsy: a systematic review and metaanalysis. Dev Med Child Neurol. 2018 Sep;60(9):866-83. doi: 10.1111/dmcn.13708. PMID: 29512110. Exclusion: 12. 
75. Booth S. Aquatic exercise rehabilitation for the paediatric Cerebral Palsied client group specifically children aged 10-12 yrs, presenting with Spastic Diplegia and classified as GMFCS-E\&R Level III Aqualines: The Journal of the Hydrotherapy Association of Chartered Physiotherapists. 2014;26(2):14-22. Exclusion: 10.

76. Borel B, Lacroix J, Daviet JC, et al. Intensity level and on-court role of wheelchair rugby players during competition. J Sports Med Phys Fitness. 2018 May 29;29:29. doi: 10.23736/S00224707.18.08214-2. PMID: 29845832. Exclusion: 3.

77. Borggraefe I, Schaefer JS, Klaiber M, et al. Robotic-assisted treadmill therapy improves walking and standing performance in children and adolescents with cerebral palsy. Europ J Paediatr Neurol. 2010

Nov;14(6):496-502. doi: 10.1016/j.ejpn.2010.01.002. PMID: 20138788. Exclusion: 8.

78. Boyd RN. Functional progressive resistance training improves muscle strength but not walking ability in children with cerebral palsy. J Physiother. 2012;58(3):197. doi: 10.1016/S1836-9553(12)70111-X. PMID: 22884187. Exclusion: 10.

79. Braendvik SM, Koret T, Helbostad JL, et al. Treadmill training or progressive strength training to improve walking in people with multiple sclerosis? A randomized parallel group trial. Physiother Res Int. 2016 Dec;21(4):228-36. doi: 10.1002/pri.1636. PMID: 26110230. Exclusion: 11.

80. Brazg G, Fahey M, Holleran CL, et al. Effects of training intensity on locomotor performance in individuals with chronic spinal cord injury: a randomized crossover study. Neurorehabil Neural Repair. 2017 Oct-Nov;31(10-11):944-54. doi: 10.1177/1545968317731538. PMID: 29081250. Exclusion: 11.

81. Brochetti AM, Brose SW, Kuemmel AM, et al. Interdisciplinary bodyweight management program for persons with SCI. J Spinal Cord Med. 2018 Dec 1-7. doi: 10.1080/10790268.2018.1547860. PMID: 30517834. Exclusion: 11.
82. Broekmans T, Roelants M, Alders G, et al. Exploring the effects of a 20 -week wholebody vibration training programme on leg muscle performance and function in persons with multiple sclerosis. J Rehabil Med. 2010 Oct;42(9):866-72. doi: 10.2340/165019770609. PMID: 20878048. Exclusion: 11.

83. Broekmans T, Roelants M, Feys P, et al. Effects of long-term resistance training and simultaneous electro-stimulation on muscle strength and functional mobility in multiple sclerosis. Mult Scler. 2010 Apr;17(4):46877. doi: $10.1177 / 1352458510391339$. PMID: 21148266. Exclusion: 11.

84. Brown SH, Lewis CA, McCarthy JM, et al. The effects of internet-based home training on upper limb function in adults with cerebral palsy. Neurorehabil Neural Repair. 2010 Jul-Aug;24(6):575-83. doi: 10.1177/1545968310361956. PMID: 20581338. Exclusion: 11.

85. Brurok B. Effect from lower extremity vascular occlusion during arm cycling on Vo2peak in persons with SCI. Med Sci Sports Exerc. 2011 May;43(5):84. doi: 10.1249/01.MSS.0000402927.14509.8c. Exclusion: 11.

86. Brurok B, Helgerud J, Karlsen T, et al. Effect of aerobic high-intensity hybrid training on stroke volume and peak oxygen consumption in men with spinal cord injury. Am J Phys Med Rehabil. 2011 May;90(5):407-14. doi: 10.1097/PHM.0b013e31820f960f. PMID: 21389841. Exclusion: 11.

87. Brurok B, Torhaug T, Leivseth G, et al. Effect of leg vascular occlusion on arm cycling peak oxygen uptake in spinal cordinjured individuals. Spinal Cord. 2012 Apr;50(4):298-302. doi: 10.1038/sc.2011.129. PMID: 22124351. Exclusion: 11.

88. Buehner JJ, Forrest GF, Schmidt-Read M, et al. Relationship between ASIA examination and functional outcomes in the NeuroRecovery Network Locomotor Training Program. Arch Phys Med Rehabil. 2012 Sep;93(9):1530-40. doi: 10.1016/j.apmr.2012.02.035. PMID: 22920450. Exclusion: 8. 
89. Buhroo AM, Hussain SA. Role of IFT in rehabilitation of neurogenic bladder in patients with traumatic spinal cord injuries. JK Practitioner. 2011 Jan-Jun;16(1-2):24-6. Exclusion: 11.

90. Buker DB, Oyarce CC, Plaza RS. Effects of spinal cord injury in heart rate variability after acute and chronic exercise: a systematic review. Top Spinal Cord Inj Rehabil. 2018;24(2):167-76. doi: 10.1310/sci17-00028. PMID: 29706761. Exclusion: 12.

91. Burdea GC, Cioi D, Kale A, et al. Robotics and gaming to improve ankle strength, motor control, and function in children with cerebral palsy--a case study series. IEEE Trans Neural Syst Rehabil Eng. 2013 Mar;21(2):165-73. doi: 10.1109/TNSRE.2012.2206055. PMID: 22773059. Exclusion: 7.

92. Bures AK, Kuld S, Weiland AC, et al. Efficacy of an internet-based program (MS Intakt) to promote physical activity after inpatient rehabilitation in persons with multiple sclerosis-a randomized controlled study. Mult Scler. 2017 Oct;Conference: 7th joint ECTRIMS-ACTRIMS, MSPARIS2017. France. 23(S3):413. doi: 10.1177/1352458517731404. Exclusion: 10.

93. Burnfield JM, Buster TW, Pfeifer CM, et al. Adapted motor-assisted elliptical for rehabilitation of children with physical disabilities. J Med Device. 2019;13(1) doi: 10.1115/1.4041588. Exclusion: 11.

94. Burns AS, Marino RJ, Kalsi-Ryan S, et al. Type and timing of rehabilitation following acute and subacute spinal cord injury: a systematic review. Global Spine J. 2017 Sep;7(3 ):175S-94S. doi: 10.1177/2192568217703084. PMID: 29164023. Exclusion: 12.

95. Byrnes $\mathrm{K}, \mathrm{Wu} \mathrm{PJ}$, Whillier $\mathrm{S}$. Is pilates an effective rehabilitation tool? A systematic review. J Bodywork Mov Ther. 2018 Jan;22(1):192-202. doi: 10.1016/j.jbmt.2017.04.008. PMID: 29332746. Exclusion: 12.
96. Calabro RS, Naro A, Leo A, et al. Usefulness of robotic gait training plus neuromodulation in chronic spinal cord injury: a case report. J Spinal Cord Med. 2017 Jan;40(1):118-21. doi: 10.1080/10790268.2016.1153275. PMID: 27077568. Exclusion: 7.

97. Callesen J, Cattaneo D, Brincks J, et al. How does strength training and balance training affect gait and fatigue in patients with multiple sclerosis? A study protocol of a randomized controlled trial. NeuroRehabilitation. 2018;42(2):131-42. doi: 10.3233/NRE-172238. PMID: 29562556. Exclusion: 10.

98. Camerota F, Celletti C, Di Sipio E, et al. Focal muscle vibration, an effective rehabilitative approach in severe gait impairment due to multiple sclerosis. J Neurol Sci. 2017 Jan 15;372:33-9. doi: 10.1016/j.jns.2016.11.025. PMID: 28017240. Exclusion: 4.

99. Campbell E, Coulter EH, Mattison PG, et al. Physiotherapy rehabilitation for people with progressive multiple sclerosis: a systematic review. Arch Phys Med Rehabil. 2016 Jan;97(1):141-51. doi: 10.1016/j.apmr.2015.07.022. PMID: 26281954. Exclusion: 12.

100. Canning KL, Hicks AL. Benefits of adhering to the Canadian physical activity guidelines for adults with multiple sclerosis beyond aerobic fitness and strength. Int $\mathrm{J}$ MS Care. 2020 Jan-Feb;22(1):15-21. doi: 10.7224/1537-2073.2018-061. PMID: 32123524. Exclusion: 6 .

101. Canori A, Amiri AM, Thapa-Chhetry B, et al. Relationship between pain, fatigue, and physical activity levels during a technologybased physical activity intervention. J Spinal Cord Med. 2020 Jun 04:1-8. doi: 10.1080/10790268.2020.1766889. PMID: 32496966. Exclusion: 4.

102. Cardenas DD, Felix ER, Cowan R, et al. Effects of home exercises on shoulder pain and pathology in chronic spinal cord injury: A randomized controlled trial. Am J Phys Med Rehabil. 2020 Jun;99(6):504-13. doi: 10.1097/PHM.0000000000001362. PMID: 31851011. Exclusion: 4. 
103. Carlon SL, Taylor NF, Shields N, et al. Aerobic training for young people with cerebral palsy in specialist schools: a pilot randomised controlled trial. Dev Med Child Neurol. 2014;56(12). Exclusion: 10.

104. Carter AM. The effects of a pragmatic exercise intervention in people with multiple sclerosis. Dissertation Abstracts International Section C: Worldwide. 2018 Apr;75(4-C):No Pagination Specified. doi: 10.1177/1352458513519354. PMID: 24421303. Exclusion: 12.

105. Carter AM, Daley AJ, Kesterton SW, et al. Pragmatic exercise intervention in people with mild to moderate multiple sclerosis: a randomised controlled feasibility study. Contemp Clin Trials. 2013 Jul;35(2):40-7. doi: 10.1016/j.cct.2013.04.003. PMID: 23612222. Exclusion: 11.

106. Carty A, McCormack K, Coughlan GF, et al. Increased aerobic fitness after neuromuscular electrical stimulation training in adults with spinal cord injury. Arch Phys Med Rehabil. 2012 May;93(5):790-5. doi: 10.1016/j.apmr.2011.10.030. PMID: 22444027. Exclusion: 4.

107. Carvalho A, Santos M, Maciel P, et al. T1237C polymorphism of TLR9 gene is not associated with multiple sclerosis in the Portuguese population. Mult Scler. 2008 May;14(4):550-2. doi: 10.1177/1352458507084594. PMID: 18208876. Exclusion: 4.

108. Carvalho I, Pinto SM, Chagas DDV, et al. Robotic gait training for individuals with cerebral palsy: a systematic review and meta-analysis. Arch Phys Med Rehabil. 2017 11;98(11):2332-44. doi: 10.1016/j.apmr.2017.06.018. PMID: 28751254. Exclusion: 12.

109. Castillo-Bueno I, Ramos-Campo DJ, RubioArias JA. Effects of whole-body vibration training in patients with multiple sclerosis: a systematic review. Neurologia. 2018 Oct;33(8):534-48. doi: 10.1016/j.nrl.2016.04.007. PMID: 27448520. Exclusion: 13.
110. Castro O, $\mathrm{Ng} \mathrm{K}$, Novoradovskaya E, et al. A scoping review on interventions to promote physical activity among adults with disabilities. Disabil Health J. 2018 Apr;11(2):174-83. doi: 10.1016/j.dhjo.2017.10.013. PMID: 29132847. Exclusion: 12.

111. Cattaneo D, Gervasoni E, Pupillo E, et al. Educational and exercise intervention to prevent falls and improve participation in subjects with neurological conditions: The NEUROFALL randomized controlled trial. Front Neurol. 2019;10:865. doi: 10.3389/fneur.2019.00865. PMID: 31572282. Exclusion: 3.

112. Chaikhot D, Reed K, Petroongrad W, et al. Effects of an Upper-Body Training Program Involving Resistance Exercise and HighIntensity Arm Cranking on Peak Handcycling Performance and Wheelchair Propulsion Efficiency in Able-Bodied Men. J Strength Cond Res. 2018 Jul 17;17:17. doi: 10.1519/JSC.0000000000002738. PMID: 30024482. Exclusion: 3.

113. Champagne D, Corriveau H, Dugas C. Effect of hippotherapy on motor proficiency and function in children with cerebral palsy who walk. Phys Occup Ther Pediatr. 2017 Feb;37(1):51-63. doi: 10.3109/01942638.2015.1129386. PMID: 26930110. Exclusion: 11.

114. Chamudot R, Parush S, Rigbi A, et al. Effectiveness of modified constraintinduced movement therapy compared with bimanual therapy home programs for infants with hemiplegia: A randomized controlled trial. Am J Occup Ther. 2018;72(6):1-9. doi: 10.5014/ajot.2018.025981. PMID: 30760393. Exclusion: 4.

115. Chang HJ, Kwon J-Y, Lee J-Y, et al. The effects of hippotherapy on the motor function of children with spastic bilateral cerebral palsy. J Phys Ther Sci. 2012;24(12):1277-80. Exclusion: 8.

116. Chang SH, Afzal T, Group TSCE, et al. Exoskeleton-assisted gait training to improve gait in individuals with spinal cord injury: a pilot randomized study. Pilot feasibility stud. 2018 Mar 5;4:62. doi: 10.1186/s40814-018-0247-y. PMID: 29556414. Exclusion: 11. 
117. Chappell A, Allison G, Gibson N, et al. Functional leg stiffness in children with cerebral palsy: Can it be optimised? Dev Med Child Neurol. 2020;62:19. doi: 10.1111/dmcn.14469. Exclusion: 10.

118. Chappell A, Allison GT, Williams G, et al. The effect of a running training intervention on ankle power generation in children and adolescents with cerebral palsy: A randomized controlled trial. Clin Biomech. 2020;76:N.PAG-N.PAG. doi: 10.1016/j.clinbiomech.2020.105024. Exclusion: 6.

119. Charron S, McKay KA, Tremlett H. Physical activity and disability outcomes in multiple sclerosis: a systematic review (2011-2016). Mult Scler Relat Disord. 2018 Feb;20:169-77. doi: 10.1016/j.msard.2018.01.021. PMID: 29414293. Exclusion: 12.

120. Chen CL, Chen CY, Liaw MY, et al. Efficacy of home-based virtual cycling training on bone mineral density in ambulatory children with cerebral palsy. Osteoporos Int. 2013 Apr;24(4):1399-406. doi: 10.1007/s00198-012-2137-0. PMID: 23052930. Exclusion: 4.

121. Chen CL, Hong WH, Cheng HY, et al. Muscle strength enhancement following home-based virtual cycling training in ambulatory children with cerebral palsy. Res Dev Disabil. 2012 Jul-Aug;33(4):1087-94. doi: 10.1016/j.ridd.2012.01.017. PMID: 22502833. Exclusion: 4.

122. Cheng HY, Ju YY, Chen CL, et al. Managing lower extremity muscle tone and function in children with cerebral palsy via eight-week repetitive passive knee movement intervention. Res Dev Disabil. 2013 Jan;34(1):554-61. doi: 10.1016/j.ridd.2012.09.020. PMID: 23123868. Exclusion: 4.

123. Cheng HY, Ju YY, Chen CL, et al. Effects of whole body vibration on spasticity and lower extremity function in children with cerebral palsy. Hum Mov Sci. 2015

Feb;39:65-72. doi: 10.1016/j.humov.2014.11.003. PMID: 25461434. Exclusion: 11.
124. Cheng HY, Ju YY, Chen CL, et al. Managing spastic hypertonia in children with cerebral palsy via repetitive passive knee movements. J Rehabil Med. 2012 Mar;44(3):235-40. doi: 10.2340/165019770937. PMID: 22366894. Exclusion: 4.

125. Cheng HY, Yu YC, Wong AM, et al. Effects of an eight-week whole body vibration on lower extremity muscle tone and function in children with cerebral palsy. Res Dev Disabil. 2015 Mar;38:256-61. doi: 10.1016/j.ridd.2014.12.017. PMID: 25575288. Exclusion: 11.

126. Cherni Y, Ballaz L, Lemaire J, et al. Effect of low dose robotic-gait training on walking capacity in children and adolescents with cerebral palsy. Neurophysiol Clin. 2020 Sep 30;30:30. doi: 10.1016/j.neucli.2020.09.005. PMID: 33011059. Exclusion: 8.

127. Chinniah H, Natarajan M, Ramanathan R, et al. Effects of horse riding simulator on sitting motor function in children with spastic cerebral palsy. Physiother Res Int. e1870 p. 2020 Oct;25(4):e1870. doi: 10.1002/pri.1870. PMID: 32808394. Exclusion: 14.

128. Chiu HC, Ada L, Lee HM. Upper limb training using Wii Sports Resort for children with hemiplegic cerebral palsy: a randomized, single-blind trial. Clin Rehabil. 2014 Oct;28(10):1015-24. doi: 10.1177/0269215514533709. PMID: 24849793. Exclusion: 4.

129. Cho C, Hwang W, Hwang S, et al. Treadmill training with virtual reality improves gait, balance, and muscle strength in children with cerebral palsy. Tohoku J Exp Med. 2016 Mar;238(3):213-8. doi: 10.1620/tjem.238.213. PMID: 26947315. Exclusion: 11.

130. Chochowska M, Zgorzalewicz-Stachowiak $\mathrm{M}$, Sereda-Wiszowaty E. Influence of chosen factors on efficiency of NDB-Bobath method in rehabilitation of children with cerebral palsy. Fizjoterapia. 2008;16(3):824. doi: 10.2478/v10109-009-0024-9. Exclusion: 8 . 
131. Chong HJ, Cho SR, Jeong E, et al. Finger exercise with keyboard playing in adults with cerebral palsy: a preliminary study. J Exerc Rehabil. 2013;9(4):420-5. doi: 10.12965/jer.130050. PMID: 24278895. Exclusion: 4.

132. Chou RC, Taylor JA, Solinsky R. Effects of hybrid-functional electrical stimulation (FES) rowing whole-body exercise on neurologic improvement in subacute spinal cord injury: secondary outcomes analysis of a randomized controlled trial. Spinal Cord. 2020 Aug;58(8):914-20. doi: 10.1038/s41393-020-0445-3. PMID: 32094516. Exclusion: 4.

133. Choudhary A, Gulati S, Kabra M, et al. Efficacy of modified constraint induced movement therapy in improving upper limb function in children with hemiplegic cerebral palsy: a randomized controlled trial. Brain Dev. 2013 Oct;35(9):870-6. doi: 10.1016/j.braindev.2012.11.001. PMID: 23238223. Exclusion: 4.

134. Christy JB, Chapman CG, Murphy P. The effect of intense physical therapy for children with cerebral palsy. J Pediatr Rehabil Med. 2012;5(3):159-70. doi: 10.3233/PRM-2012-0208. PMID: 23023248. Exclusion: 11.

135. Cichon R, Maszczyk A, Stastny P, et al. Effects of krankcycle training on performance and body composition in wheelchair users. J Hum Kinet. 2015 Nov 22;48:71-8. doi: 10.1515/hukin-2015-0093. PMID: 26834875. Exclusion: 11.

136. Cimino V, Chisari CG, Raciti G, et al. Objective evaluation of Nintendo Wii Fit Plus balance program training on postural stability in Multiple Sclerosis patients: a pilot study. Int J Rehabil Res. 2020 May 02;02:02. doi: 10.1097/MRR.0000000000000408. PMID: 32371848. Exclusion: 11.

137. Clapham ED, Lamont LS, Shim M, et al. Effectiveness of surf therapy for children with disabilities. Disabil Health J. 2019 Aug 2:100828. doi: 10.1016/j.dhjo.2019.100828. PMID: 31422168. Exclusion: 3.
138. Cleary SL, Taylor NF, Dodd KJ, et al. An aerobic exercise program for young people with cerebral palsy in specialist schools: a phase I randomized controlled trial. Dev Neurorehabil. 2017 Aug;20(6):331-8. doi: 10.1080/17518423.2016.1265602. PMID: 28045554. Exclusion: 11.

139. Clutterbuck G, Auld M, Johnston L. Active exercise interventions improve gross motor function of ambulant/semi-ambulant children with cerebral palsy: a systematic review. Disabil Rehabil. 2019 Jan 05;41(10):1131-51. doi: 10.1080/09638288.2017.1422035. PMID: 29303007. Exclusion: 12.

140. Clutterbuck GL, Auld ML, Johnston LM. SPORTS STARS: a practitioner-led, peergroup sports intervention for ambulant children with cerebral palsy. Activity and participation outcomes of a randomised controlled trial. Disabil Rehabil. 2020 Jun 30;30:1-9. doi: 10.1080/09638288.2020.1783376 PMID: 32603238. Exclusion: 4.

141. Clutterbuck GL, Auld ML, Johnston LM. SPORTS STARS: a practitioner-led, peergroup sports intervention for ambulant, school-aged children with cerebral palsy. Parent and physiotherapist perspectives. Disabil Rehabil. 2020 Jul 7;7:1-10. doi: 10.1080/09638288.2020.1785558. PMID: 32633156. Exclusion: 4.

142. Cohen ET, Kietrys D, Fogerite SG, et al. Feasibility and impact of an 8-week integrative yoga program in people with moderate multiple sclerosis-related disability: a pilot study. Int J MS Care. 2017 Jan-Feb;19(1):30-9. doi: 10.7224/15372073.2015-046. PMID: 28243184. Exclusion: 11.

143. Cohen ET, Kietrys D, Gould Fogerite S, et al. Feasibility and impact of an 8-week integrative yoga program in people with moderate multiple sclerosis- related disability. Int J MS Care. 2017;19(1):30-9. doi: 10.7224/1537-2073.2015-046. Exclusion: 11.

144. Cohen L, Delafontaine A. Impact of pectoralis major stretching on vital capacity in patients with low tetraplegia: randomized controlled study. Kinesitherapie. 2018;18(195):3-9. doi: 10.1016/j.kine.2017.11.008. Exclusion: 13. 
145. Cohen M, Wetzel J, Pohle-Krauza RJ, et al. Impact of locomotor training and level of injury on ventilatory function in persons' with motor-incomplete spinal cord injury. Physiotherapy. 2011 Jun;97:eS1338. doi: 10.1016/j.physio.2011.04.002. PMID: 29339898. Exclusion: 10.

146. Cohen-Holzer M, Katz-Leurer M, Meyer S, et al. The effect of bimanual training with or without constraint on hand functions in children with unilateral cerebral palsy: a non-randomized clinical trial. Phys Occup Ther Pediatr. 2017 Oct 20;37(5):516-27. doi: 10.1080/01942638.2017.1280871. PMID: 28266881. Exclusion: 11.

147. Cohen-Holzer M, Katz-Leurer M, Reinstein $\mathrm{R}$, et al. The effect of combining daily restraint with bimanual intensive therapy in children with hemiparetic cerebral palsy: a self-control study. NeuroRehabilitation. 2011;29(1):29-36. doi: 10.3233/NRE-20110674. PMID: 21876293. Exclusion: 4.

148. Cohen-Holzer M, Sorek G, Kerem J, et al. The impact of combined constraint-induced and bimanual arm training program on the perceived hand-use experience of children with unilateral cerebral palsy. Dev Neurorehabil. 2017 Aug;20(6):355-60. doi: 10.1080/17518423.2016.1238017. PMID: 27739919. Exclusion: 4.

149. Cohen-Holzer M, Sorek G, Schweizer M, et al. The influence of a constraint and bimanual training program using a variety of modalities on endurance and on the cardiac autonomic regulation system of children with unilateral cerebral palsy: a self-control clinical trial. NeuroRehabilitation. 2017;41(1):119-26. doi: 10.3233/NRE171463. PMID: 28505992. Exclusion: 4.

150. Collett J, Meaney A, Howells K, et al. Acute recovery from exercise in people with multiple sclerosis: an exploratory study on the effect of exercise intensities. Disabil Rehabil. 2017 Mar;39(6):551-8. doi: 10.3109/09638288.2016.1152604. PMID: 26972274. Exclusion: 4.
151. Conklyn D, Stough D, Novak E, et al. A home-based walking program using rhythmic auditory stimulation improves gait performance in patients with multiple sclerosis: a pilot study. Neurorehabil Neural Repair. 2010 Nov-Dec;24(9):835-42. doi: 10.1177/1545968310372139. PMID: 20643882. Exclusion: 4.

152. Cook O, Frost G, Twose D, et al. CAN-flip: A pilot gymnastics program for children with cerebral palsy. Adapt Phys Act Q. 2015 Oct;32(4):349-70. doi: 10.1123/APAQ.2015-0026. PMID: 26485738. Exclusion: 11.

153. Coote S, Hayes S, Uszynski M, et al. Group aerobic and strength training combined with social cognitive theory based education, results of the step it up RCT. Arch Phys Med Rehabil. 2016;Conference:. 2016 american congress of rehabilitation medicine annual conference, ACRM 2016. United states. Conference start: 20161030. Conference end: 20161104 97(12):e4. Exclusion: 10.

154. Coote S, Hayes S, Uszynski M, et al. Group Aerobic and Strength Training Combined With Social Cognitive Theory Based Education, Results of The Step it up RCT...2016 ACRM / American Congress of Rehabilitation Medicine Annual Conference 30 October - 4 November 2016, Chicago, IL. Arch Phys Med Rehabil. 2016;97(12):e4-e. doi: 10.1016/j.apmr.2016.09.009. Exclusion: 10.

155. Coote S, Hughes L, Rainsford G, et al. Pilot randomized trial of progressive resistance exercise augmented by neuromuscular electrical stimulation for people with multiple sclerosis who use walking aids. Arch Phys Med Rehabil. 2015 Feb;96(2):197-204. doi: 10.1016/j.apmr.2014.09.021. PMID: 25308884. Exclusion: 4.

156. Coote S, Uszynski M, Herring MP, et al. Effect of exercising at minimum recommendations of the multiple sclerosis exercise guideline combined with structured education or attention control education secondary results of the step it up randomised controlled trial. BMC Neurol. 2017 Jun 24;17(1):119. doi: 10.1186/s12883-017-0898-y. PMID: 28646860. Exclusion: 4. 
157. Corsi C, Santos MM, Moreira RFC, et al. Effect of physical therapy interventions on spatiotemporal gait parameters in children with cerebral palsy: a systematic review. Disabil Rehabil. 2019 Oct 7:1-10. doi: 10.1080/09638288.2019.1671500. PMID: 31588810. Exclusion: 12.

158. Corvillo I, Varela E, Armijo F, et al. Efficacy of aquatic therapy for multiple sclerosis: a systematic review. Eur J Phys Rehabil Med. 2017 Dec;53(6):944-52. doi: 10.23736/S1973-9087.17.04570-1. PMID: 28215060. Exclusion: 12.

159. Costantino C, Pedrini MF, Licari O. Neuromuscular taping versus sham therapy on muscular strength and motor performance in multiple sclerosis patients. Disabil Rehabil. 2016;38(3):277-81. doi: 10.3109/09638288.2015.1038365. PMID: 25893397 Exclusion: 4.

160. Coulter EH, McLean AN, Hasler JP, et al. The effectiveness and satisfaction of webbased physiotherapy in people with spinal cord injury: a pilot randomised controlled trial. Spinal Cord. 2017 Apr;55(4):383-9. doi: 10.1038/sc.2016.125. PMID: 27596027. Exclusion: 4.

161. Covarrubias-Escudero F, Rivera-Lillo G, Torres-Castro R, et al. Effects of body weight-support treadmill training on postural sway and gait independence in patients with chronic spinal cord injury. J Spinal Cord Med. 2019;42(1):57-64. doi: 10.1080/10790268.2017.1389676. PMID: 29058553. Exclusion: 11.

162. Crane DA, Hoffman JM, Reyes MR. Benefits of an exercise wellness program after spinal cord injury. J Spinal Cord Med. 2017 03;40(2):154-8. doi: 10.1179/2045772315Y.0000000038. PMID: 26108561. Exclusion: 8.

163. Cratsenberg KA, Deitrick CE, Harrington TK, et al. Effectiveness of exercise programs for management of shoulder pain in manual wheelchair users with spinal cord injury. J Neurol Phys Ther. 2015

Oct;39(4):197-203. doi: 10.1097/NPT.0000000000000103. PMID: 26308939. Exclusion: 3.
164. Credeur DP, Stoner L, Dolbow DR, et al. Increasing physical activity in spinal cord injury: upper-body exercise alone not enough? Arch Phys Med Rehabil. 2016;97(1):171-3. doi: 10.1016/j.apmr.2015.09.009. PMID: 26710856. Exclusion: 10.

165. Cruickshank TM, Reyes AR, Ziman MR. A systematic review and meta-analysis of strength training in individuals with multiple sclerosis or Parkinson disease. Medicine (Baltimore). 2015 Jan;94(4):e411. doi: 10.1097/MD.0000000000000411. PMID: 25634170. Exclusion: 12.

166. D'Ancona CA, Clilclet A, Ikari LY, et al. Impact of treadmill gait training with neuromuscular electrical stimulation on the urodynamic profile of patients with high cervical spinal cord injury. Einstein. 2010 Sep;8(3):325-8. doi: 10.1590/S167945082010AO1691. PMID: 26760148. Exclusion: 11.

167. D'Hooghe M, Van Gassen G, Kos D, et al. Improving fatigue in multiple sclerosis by smartphone-supported energy management: the MS TeleCoach feasibility study. Mult Scler Relat Disord. 2018 May;22:90-6. doi: 10.1016/j.msard.2018.03.020. PMID: 29649789. Exclusion: 4.

168. D'Hooghe M B, Feys P, Deltour S, et al. Impact of a 5-day expedition to machu picchu on persons with multiple sclerosis. Mult Scler Int. 2014;2014:761210. doi: 10.1155/2014/761210. PMID: 24967103. Exclusion: 4.

169. D'Oliveira GL, Figueiredo FA, Passos MC, et al. Physical exercise is associated with better fat mass distribution and lower insulin resistance in spinal cord injured individuals. J Spinal Cord Med. 2014 Jan;37(1):79-84. doi: 10.1179/2045772313Y.0000000147. PMID: 24090139. Exclusion: 11.

170. D'Orio VL, Foley FW, Armentano F, et al. Cognitive and motor functioning in patients with multiple sclerosis: neuropsychological predictors of walking speed and falls. $\mathbf{J}$ Neurol Sci. 2012 May 15;316(1-2):42-6. doi: 10.1016/j.jns.2012.02.003. PMID: 22353853. Exclusion: 4. 
171. Dalgas U, Stenager E, Jakobsen J, et al. Effects of resistance training in relapsingremitting multiple sclerosis-a randomised controlled trial. Mult Scler. 2009

Sep;15(9):S13. doi: 10.1177/1352458509106961. Exclusion: 8.

172. Dalgas U, Stenager E, Lund C, et al. Maximal EMG activity increases following resistance training in patients with multiple sclerosis (\#140). Mult Scler J Exp Transl Clin. START: 2012 May 31 CONFERENCE END: 2012 Jun 2, 17th Annual Conference of Rehabilitation in Multiple Sclerosis, RIMS 2012 Hamburg Germany;18(5):S24. Exclusion: 10.

173. Dalgas U, Stenager E, Sloth M, et al. The effect of exercise on depressive symptoms in multiple sclerosis based on a meta-analysis and critical review of the literature. Eur J Neurol. 2015 Mar;22(3):443-e34. doi: 10.1111/ene.12576. PMID: 25327395. Exclusion: 12.

174. Darrah J, Law M. Focus on function: a clinical trial of two intervention approaches for children with cerebral palsy. Physiotherapy. 2011 Jun;97 doi: 10.1016/j.physio.2011.04.002. PMID: 17900362 Exclusion: 10.

175. Das SP, Ganesh GS. Evidence-based approach to physical therapy in cerebral palsy. Indian J Orthop. 2019 JanFeb;53(1):20-34. doi: 10.4103/ortho.IJOrtho_241_17. PMID: 30905979. Exclusion: $\overline{7}$.

176. Dauwan M, Begemann MJH, Slot MIE, et al. Physical exercise improves quality of life, depressive symptoms, and cognition across chronic brain disorders: a transdiagnostic systematic review and metaanalysis of randomized controlled trials. $\mathrm{J}$ Neurol. 2019 Aug 14;14:14. doi: 10.1007/s00415-019-09493-9. PMID: 31414194. Exclusion: 12.

177. Davies BL, Arpin DJ, Liu M, et al. Two different types of high-frequency physical therapy promote improvements in the balance and mobility of persons with multiple sclerosis. Arch Phys Med Rehabil. 2016 Dec;97(12):2095-101. doi: 10.1016/j.apmr.2016.05.024. PMID: 27373745. Exclusion: 11.
178. de Freitas GR, Szpoganicz C, Ilha J. Does neuromuscular electrical stimulation therapy increase voluntary muscle strength after spinal cord injury? A systematic review. Top Spinal Cord Inj Rehabil. 2018;24(1):617. doi: 10.1310/sci16-00048. PMID: 29434456. Exclusion: 12.

179. de Groot S, de Bruin M, Noomen SP, et al. Mechanical efficiency and propulsion technique after 7 weeks of low-intensity wheelchair training. Clin Biomech. 2008 May;23(4):434-41. doi: 10.1016/j.clinbiomech.2007.11.001. PMID: 18077065. Exclusion: 11.

180. de Groot S, Kouwijzer I, Baauw M, et al. Effect of self-guided training for the HandbikeBattle on body composition in people with spinal cord injury. Spinal Cord Ser Cases. 2018 Aug 24;4(79) doi: 10.1038/s41394-018-0103-6. PMID: 30155275. Exclusion: 8 .

181. De Keersmaecker E, Lefeber N, Geys M, et al. Virtual reality during gait training: does it improve gait function in persons with central nervous system movement disorders? A systematic review and meta-analysis. NeuroRehabilitation. 2019;44(1):43-66. doi: 10.3233/NRE-182551. PMID: 30814368. Exclusion: 12.

182. de Oliveira BI, Howie EK, Dunlop SA, et al. SCIPA Com: outcomes from the spinal cord injury and physical activity in the community intervention. Spinal Cord. 2016 Oct;54(10):855-60. doi: 10.1038/sc.2015.235. PMID: 26782840. Exclusion: 8 .

183. de Oliveira G, Tavares Mda C, de Faria Oliveira JD, et al. Yoga training has positive effects on postural balance and its influence on activities of daily living in people with multiple sclerosis: a pilot study. Explore (NY). 2016 Sep-Oct;12(5):325-32. doi: 10.1016/j.explore.2016.06.005. PMID: 27426024. Exclusion: 11.

184. de Paz RH, Serrano-Munoz D, PerezNombela $S$, et al. Combining transcranial direct-current stimulation with gait training in patients with neurological disorders: a systematic review. J Neuroengineering Rehabil. 2019 Sep 14;16(1):114. doi: 10.1186/s12984-019-0591-z. PMID: 31521179. Exclusion: 12. 
185. de Rossi G, Matos-Souza JR, Costa ESAD, et al. Physical activity and improved diastolic function in spinal cord-injured subjects. Med Sci Sports Exerc. 2014;46(5):887-92. doi: 10.1249/MSS.0000000000000187. PMID: 24126969. Exclusion: 8.

186. de Souza JA, Corrêa JCF, Agnol LD, et al. Effects of transcranial direct current stimulation on the rehabilitation of painful shoulder following a stroke: protocol for a randomized, controlled, double-blind, clinical trial. Trials. 2019 Mar 15;20(1):165. doi: 10.1186/s13063-019-3266-y. PMID: 30876431. Exclusion: 3.

187. de Souza-Teixeira F, Costilla S, Ayan C, et al. Effects of resistance training in multiple sclerosis. Int J Sports Med. 2009 Apr;30(4):245-50. doi: 10.1055/s-00281105944. PMID: 19199197. Exclusion: 11.

188. Declerck M, Verheul M, Daly D, et al. Benefits and enjoyment of a swimming intervention for youth with cerebral palsy: an RCT study. Pediatr Phys Ther. 2016;28(2):162-9. doi: 10.1097/PEP.0000000000000235. PMID: 26871379. Exclusion: 11.

189. Dehghansai N, Lemez S, Wattie N, et al. A systematic review of influences on development of athletes with disabilities. Adapt Phys Act Q. 2017 Jan;34(1):72-90. doi: 10.1123/APAQ.2016-0030. PMID: 28218871. Exclusion: 12.

190. Dehghansai N, Lemez S, Wattie N, et al. Training and development of Canadian wheelchair basketball players. EJSS (Champaign). 2017 Jun;17(5):511-8. doi: 10.1080/17461391.2016.1276636. PMID: 28099092. Exclusion: 4.

191. Del Rosario-Montejo O, Molina-Rueda F, Munoz-Lasa S, et al. Effectiveness of equine therapy in children with psychomotor impairment. Neurologia. 2015 Sep;30(7):425-32. doi: 10.1016/j.nrl.2013.12.023. PMID: 24656851. Exclusion: 13.
192. Deley G, Denuziller J, Casillas JM, et al. One year of training with FES has impressive beneficial effects in a 36-yearold woman with spinal cord injury. J Spinal Cord Med. 2017 Jan;40(1):107-12. doi: 10.1080/10790268.2015.1117192. PMID: 26832125. Exclusion: 7.

193. Demaneuf T, Aitken Z, Karahalios A, et al. Effectiveness of exercise interventions for pain reduction in people with multiple sclerosis: a systematic review and metaanalysis of randomized controlled trials. Arch Phys Med Rehabil. 2019 Jan;100(1):128-39. doi: 10.1016/j.apmr.2018.08.178. PMID: 30240593. Exclusion: 6.

194. Dennett R, Hendrie W, Jarrett L, et al. "I'm in a very good frame of mind": a qualitative exploration of the experience of standing frame use in people with progressive multiple sclerosis. BMJ Open. 2020 Oct 28;10(10):e037680. doi: 10.1136/bmjopen2020-037680. PMID: 33115893. Exclusion: 4.

195. Depiazzi J, Smith N, Gibson N, et al. Aquatic high intensity interval training to improve aerobic capacity is feasible in adolescents with cerebral palsy: pilot randomised controlled trial. Clin Rehabil. 2020 Sep 09:269215520956499. doi: 10.1177/0269215520956499. PMID: 32907375. Exclusion: 11.

196. Dettmers C, Sulzmann M, Ruchay-Plossl A, et al. Endurance exercise improves walking distance in MS patients with fatigue. Acta Neurol Scand. 2009 Oct;120(4):251-7. doi: 10.1111/j.1600-0404.2008.01152.x. PMID: 19178385. Exclusion: 4.

197. Deutsch JE, Guarrera-Bowlby P, Myslinski $\mathrm{MJ}$, et al. Is there evidence that active videogames increase energy expenditure and exercise intensity for people poststroke and with cerebral palsy? Games Health J. 2015 Feb;4(1):31-7. doi: 10.1089/g4h.2014.0082. PMID: 26181678. Exclusion: 12.

198. Devasahayam AJ, Downer MB, Ploughman $M$. The effects of aerobic exercise on the recovery of walking ability and neuroplasticity in people with multiple sclerosis: a systematic review of animal and clinical studies. Mult Scler Int. 2017;2017 doi: 10.1155/2017/4815958. PMID: 29181199. Exclusion: 12. 
199. Dewar R, Love S, Johnston LM. Exercise interventions improve postural control in children with cerebral palsy: a systematic review. Dev Med Child Neurol. 2015 Jun;57(6):504-20. doi: 10.1111/dmcn.12660. PMID: 25523410. Exclusion: 12.

200. Dingley AA, Pyne DB, Youngson J, et al. Effectiveness of a dry-land resistance training program on strength, power, and swimming performance in paralympic swimmers. J Strength Cond Res. 2015 Mar;29(3):619-26. doi: 10.1519/JSC.0000000000000684. PMID: 25226306. Exclusion: 11.

201. Diong J, Boswell-Ruys C. Exercise training programmes to improve hand-rim wheelchair propulsion capacity: PEDro systematic review update. Br J Sports Med. 2015 Oct;49(19):1284-5. doi: 10.1136/bjsports-2015-094583. PMID: 25645117. Exclusion: 10.

202. DiPiro ND, Embry AE, Fritz SL, et al. Effects of aerobic exercise training on fitness and walking-related outcomes in ambulatory individuals with chronic incomplete spinal cord injury. Spinal Cord. 2016 Sep;54(9):675-81. doi: 10.1038/sc.2015.212. PMID: 26666508. Exclusion: 11.

203. DiPiro ND, Holthaus KD, Morgan PJ, et al. Lower extremity strength is correlated with walking function after incomplete SCI. Top Spinal Cord Inj Rehabil. 2015;21(2):133-9. doi: 10.1310/sci2102-133. PMID: 26364282. Exclusion: 11.

204. Ditunno JF, Scivoletto G, Patrick M, et al. Validation of the walking index for spinal cord injury in a US and European clinical population. Spinal Cord. 2008 Mar;46(3):181-8. PMID: 17502878. Exclusion: 4.

205. Divanoglou A, Georgiou M. Perceived effectiveness and mechanisms of community peer-based programmes for spinal cord injuries-a systematic review of qualitative findings. Spinal Cord. 2017 Mar;55(3):22534. doi: 10.1038/sc.2016.147. PMID: 27845356. Exclusion: 7.
206. Dlugonski D, Motl RW, Mohr DC, et al. Internet-delivered behavioral intervention to increase physical activity in persons with multiple sclerosis: sustainability and secondary outcomes. Psychol Health Med. 2012;17(6):636-51. doi: 10.1080/13548506.2011.652640. PMID: 22313192. Exclusion: 4.

207. Do J-H, Yoo E-Y, Jung M-Y, et al. The effects of virtual reality-based bilateral arm training on hemiplegic children's upper limb motor skills. NeuroRehabilitation. 2016;38(2):115-27. doi: 10.3233/NRE161302. PMID: 26923353. Exclusion: 11.

208. Dodd K, Taylor NF, Shields N, et al. Strength training can improve muscle performance, quality of life and fatigue in adults with multiple sclerosis: a randomised controlled trial. Physiotherapy. 2011 Jun;97:eS293-4. doi: 10.1016/j.physio.2011.04.002. Exclusion: 10.

209. Dolbow DR, Gorgey AS, Khalil RK, et al. Effects of a fifty-six month electrical stimulation cycling program after tetraplegia: case report. J Spinal Cord Med. 2017 Jul;40(4):485-8. doi: 10.1080/10790268.2016.1234750. PMID: 27808003. Exclusion: 7.

210. Dolbow DR, Gorgey AS, Recio AC, et al. Activity-based restorative therapies after spinal cord injury: inter-institutional conceptions and perceptions. Aging Dis. 2015 Aug;6(4):254-61. doi: 10.14336/AD.2014.1105. PMID: 26236547. Exclusion: 10.

211. Dolbow JD, Dolbow DR, Stevens SL, et al. Restorative effects of aquatic exercise therapies on motor, gait, and cardiovascular function in children with cerebral palsy: a review of literature. Journal of Aquatic Physical Therapy. 2017 Summer;25(1):22-9. Exclusion: 12.

212. Domagalska-Szopa M, Szopa A, Czamara A. Dependence of gait deviation on weightbearing asymmetry and postural instability in children with unilateral cerebral palsy. PLoS One. 2016;11(10):e0165583. doi: 10.1371/journal.pone.0165583. PMID: 27788247. Exclusion: 4. 
213. Donati AR, Shokur S, Morya E, et al. Longterm training with a brain-machine interface-based gait protocol induces partial neurological recovery in paraplegic patients. Sci Rep. 2016 Aug 11;6 doi: 10.1038/srep30383. PMID: 27513629. Exclusion: 11.

214. Donenberg JG, Fetters L, Johnson R. The effects of locomotor training in children with spinal cord injury: a systematic review. Dev Neurorehabil. 2019 Jun 19;22(4):27287. doi: $10.1080 / 17518423.2018 .1487474$. PMID: 29920126. Exclusion: 12.

215. Dong VA, Fong KN, Chen YF, et al. 'Remind-to-move' treatment versus constraint-induced movement therapy for children with hemiplegic cerebral palsy: a randomized controlled trial. Dev Med Child Neurol. 2017 Feb;59(2):160-7. doi: 10.1111/dmen.13216. PMID: 27503605. Exclusion: 4.

216. Dong VA, Tung IH, Siu HW, et al. Studies comparing the efficacy of constraint-induced movement therapy and bimanual training in children with unilateral cerebral palsy: a systematic review. Dev Neurorehabil. 2013;16(2):133-43. doi: 10.3109/17518423.2012.702136. PMID: 22946588. Exclusion: 9.

217. Dost G, Dulgeroglu D, Yildirim A, et al. The effects of upper extremity progressive resistance and endurance exercises in patients with spinal cord injury. J Back Musculoskeletal Rehabil. 2014;27(4):41926. doi: 10.3233/BMR-140462. PMID: 24614829. Exclusion: 11.

218. Dowling AV, Eberly V, Maneekobkunwong $\mathrm{S}$, et al. Telehealth monitor to measure physical activity and pressure relief maneuver performance in wheelchair users. Assist Technol. 2017;29(4):202-9. doi: 10.1080/10400435.2016.1220993. PMID: 27687753. Exclusion: 4.

219. Druzbicki M, Rusek W, Snela S, et al. Functional effects of robotic-assisted locomotor treadmill thearapy in children with cerebral palsy. J Rehabil Med. 2013 Apr;45(4):358-63. doi: 10.2340/165019771114. PMID: 23450428. Exclusion: 6.
220. Druzbicki M, Rusek W, Szczepanik M, et al. Assessment of the impact of orthotic gait training on balance in children with cerebral palsy. Acta Bioeng. 2010;12(3):53-8. PMID: 21243970. Exclusion: 11.

221. Duffell LD, Brown GL, Mirbagheri MM. Facilitatory effects of anti-spastic medication on robotic locomotor training in people with chronic incomplete spinal cord injury. J Neuroengineering Rehabil. 2015 Mar 20;12:29. doi: 10.1186/s12984-0150018-4. PMID: 25881322. Exclusion: 4.

222. Duffell LD, Brown GL, Mirbagheri MM. Interventions to reduce spasticity and improve function in people with chronic incomplete spinal cord injury: distinctions revealed by different analytical methods. Neurorehabil Neural Repair. 2015 Jul;29(6):566-76. doi: 10.1177/1545968314558601. PMID: 25398727. Exclusion: 6.

223. Duffell LD, Rowlerson AM, Donaldson Nde $\mathrm{N}$, et al. Effects of endurance and strengthdirected electrical stimulation training on the performance and histological properties of paralyzed human muscle: a pilot study.[Erratum appears in Muscle Nerve. 2011 Jan;43(1):154]. Muscle Nerve. 2010 Nov;42(5):756-63. doi: 10.1002/mus.21746. PMID: 20976779. Exclusion: 11.

224. Duncan B, Shen K, Zou LP, et al. Evaluating intense rehabilitative therapies with and without acupuncture for children with cerebral palsy: a randomized controlled trial. Arch Phys Med Rehabil. 2012 May;93(5):808-15. doi: 10.1016/j.apmr.2011.12.009. PMID: 22541308. Exclusion: 4.

225. Dunne AC, Allan DB, Hunt KJ. Characterisation of oxygen uptake response to linearly increasing work rate during robotics-assisted treadmill exercise in incomplete spinal cord injury. Biomed Signal Process Control. 2010;5(1):70-5. doi: 10.1016/j.bspc.2009.09.003. Exclusion: 4.

226. Duquette SA, Guiliano AM, Starmer DJ. Whole body vibration and cerebral palsy: a systematic review. J Can Chiropractic Assoc. 2015 Sep;59(3):245-52. PMID: 26500358. Exclusion: 12. 
227. Dybesland AR, Normann B. Group physiotherapy targeting core stability and balance in individuals with multiple sclerosis. 'Movement analyses and individualisations: potent change-making tools'. Eur J Physiother. 2019;21(2):98-106. doi: 10.1080/21679169.2018.1496473. Exclusion: 7.

228. Edwards KA, Molton IR, Smith AE, et al. Relative importance of baseline pain, fatigue, sleep, and physical activity: predicting change in depression in adults with multiple sclerosis. Arch Phys Med Rehabil. 2016 Aug;97(8):1309-15. doi: 10.1016/j.apmr.2016.02.025. PMID: 27016262. Exclusion: 4.

229. Edwards T, Barfield JP, Niemiro GM, et al. Physiological responses during a $25-\mathrm{km}$ time trial in elite wheelchair racing athletes. Spinal Cord Ser Cases. 2018 Aug 14;4(77) doi: 10.1038/s41394-018-0114-3. PMID: 30131876. Exclusion: 11.

230. Edwards T, Motl RW, Pilutti LA. Cardiorespiratory demand of acute voluntary cycling with functional electrical stimulation in individuals with multiple sclerosis with severe mobility impairment. Appl Physiol Nutr Metab. 2018 Jan;43(1):71-6. doi: 10.1139/apnm-2017-0397. PMID: 28881147. Exclusion: 11.

231. Edwards T, Motl RW, Sebastiao E, et al. Pilot randomized controlled trial of functional electrical stimulation cycling exercise in people with multiple sclerosis with mobility disability. Mult Scler Relat Disord. 2018 Nov 8;26:103-11. doi: 10.1016/j.msard.2018.08.020. PMID: 30243234. Exclusion: 11.

232. Edwards T, Pilutti LA. The effect of exercise training in adults with multiple sclerosis with severe mobility disability: a systematic review and future research directions. Mult Scler Relat Disord. 2017 Aug;16:31-9. doi: 10.1016/j.msard.2017.06.003. PMID: 28755682. Exclusion: 12.
233. Eerden S, Dekker R, Hettinga FJ. Maximal and submaximal aerobic tests for wheelchair-dependent persons with spinal cord injury: a systematic review to summarize and identify useful applications for clinical rehabilitation. Disabil Rehabil. 2018 Mar;40(5):497-521. doi: 10.1080/09638288.2017.1287623. PMID: 28637157. Exclusion: 12.

234. Eftekhari E, Etemadifar M. Impact of clinical mat pilates on body composition and functional indices in female patients with multiple sclerosis. Crescent Journal of Medical and Biological Sciences. 2018 Oct;5(4):297-305. Exclusion: 11.

235. Eftekhari E, Etemadifar M. Interleukin-10 and brain-derived neurotrophic factor responses to the mat pilates training in women with multiple sclerosis. Scientia Medica. 2018;28(4) doi: 10.15448/19806108.2018.4.31668. Exclusion: 11.

236. Eftekhari E, Mostahfezian M, Etemadifar M, et al. Resistance training and vibration improve muscle strength and functional capacity in female patients with multiple sclerosis. Asian J Sports Med. 2012 Dec;3(4):279-84. PMID: 23342227. Exclusion: 11.

237. Ehling R, Edlinger M, Hermann K, et al. Successful long-term management of spasticity in patients with multiple sclerosis using a software application (APP): a pilot study. Mult Scler Relat Disord. 2017 Oct;17:15-21. doi: 10.1016/j.msard.2017.06.013. PMID: 29055449. Exclusion: 11.

238. El Shemy SA. Trunk endurance and gait changes after core stability training in children with hemiplegic cerebral palsy: a randomized controlled trial. J Back Musculoskeletal Rehabil. 2018 Nov 28;31(6):1159-67. doi: 10.3233/BMR181123. PMID: 30056415. Exclusion: 14.

239. El-Basatiny HM, Abdel-Aziem AA. Effect of backward walking training on postural balance in children with hemiparetic cerebral palsy: a randomized controlled study. Clin Rehabil. 2015 May;29(5):45767. doi: $10.1177 / 0269215514547654$. PMID: 25258425. Exclusion: 14. 
240. El-Kader SMA. Impact of respiratory muscle training on blood gases and pulmonary function among patients with cervical spinal cord injury. Electron J Gen Med. 2018;15(3) doi: 10.29333/ejgm/85190. Exclusion: 14.

241. El-Meniawy GH, Thabet NS. Modulation of back geometry in children with spastic diplegic cerebral palsy via hippotherapy training. Egypt J Med Hum Genet. 2012;13(1):63-71. doi: 10.1016/j.ejmhg.2011.10.004. Exclusion: 14.

242. El-Shamy SM. Effect of whole-body vibration on muscle strength and balance in diplegic cerebral palsy: a randomized controlled trial. Am J Phys Med Rehabil. 2014 Feb;93(2):114-21. doi: 10.1097/PHM.0b013e3182a541a4. PMID: 24434887. Exclusion: 14.

243. El-Shamy SM. Effects of antigravity treadmill training on gait, balance, and fall risk in children with diplegic cerebral palsy. Am J Phys Med Rehabil. 2017

Nov;96(11):809-15. doi: 10.1097/PHM.000000000000752. PMID: 28410250. Exclusion: 14.

244. El-Shamy SM, Abd El Kafy EM. Effect of balance training on postural balance control and risk of fall in children with diplegic cerebral palsy. Disabil Rehabil. 2014;36(14):1176-83. doi: 10.3109/09638288.2013.833312. PMID: 24032716. Exclusion: 14.

245. El-Shamy SM, Abdelaal AA. WalkAide efficacy on gait and energy expenditure in children with hemiplegic cerebral palsy: a randomized controlled trial. Am J Phys Med Rehabil. 2016 Sep;95(9):629-38. doi: 10.1097/PHM.000000000000514. PMID: 27149586. Exclusion: 14.

246. El-Shamy SM, Eid MA, El-Banna MF. Effect of extracorporeal shock wave therapy on gait pattern in hemiplegic cerebral palsy: a randomized controlled trial. Am J Phys Med Rehabil. 2014 Dec;93(12):1065-72. doi: 10.1097/PHM.0000000000000133. PMID: 24879552. Exclusion: 4.
247. El-Shamy SM, El-Banna MF. Effect of Wii training on hand function in children with hemiplegic cerebral palsy. Physiother Theory Pract. 2020;36(1):38-44. doi: 10.1080/09593985.2018.1479810. PMID: 29792556. Exclusion: 14.

248. Ellapen TJ, Hammill HV, Swanepoel M, et al. The health benefits and constraints of exercise therapy for wheelchair users: a clinical commentary. Afr J Disabil. 2017;6:337. doi: 10.4102/ajod.v6i0.337. PMID: 28936414. Exclusion: 12.

249. Ellapen TJ, Hammill HV, Swanepoel M, et al. The benefits of hydrotherapy to patients with spinal cord injuries. Afr J Disabil. 2018 May 16;7(0):450. doi: 10.4102/ajod.v7i0.450. PMID: 29850439. Exclusion: 12.

250. Elnaggar RK, Elbanna MF. Evaluation of independent versus integrated effects of reciprocal electrical stimulation and botulinum toxin-A on dynamic limits of postural stability and ankle kinematics in spastic diplegia: a single-blinded randomized trial. Eur J Phys Rehabil Med. 2019 Apr;55(2):241-9. doi: 10.23736/S1973-9087.18.05196-1. PMID: 29904047. Exclusion: 4.

251. Elnahhas AM, Elshennawy S, Aly MG. Effects of backward gait training on balance, gross motor function, and gait in children with cerebral palsy: a systematic review. Clin Rehabil. 2018 Jul 25;33(1):3-12. doi: 10.1177/0269215518790053. PMID: 30043634. Exclusion: 12.

252. Elshafey MA, Abd-Elaziem A, Gouda RE. Corrigendum to "functional stretching exercise submitted for spastic diplegic children: a randomized control study". Rehabil Res Pract. 2016;2016 doi: 10.1155/2016/1615024. PMID: 27721993 Exclusion: 10.

253. Elsworth C, Winward C, Sackley C, et al. Supported community exercise in people with long-term neurological conditions: a phase II randomized controlled trial. Clin Rehabil. 2011 Jul;25(7):588-98. doi: 10.1177/0269215510392076. PMID: 21382866. Exclusion: 3. 
254. Elvrum AK, Braendvik SM, Saether R, et al. Effectiveness of resistance training in combination with botulinum toxin-A on hand and arm use in children with cerebral palsy: a pre-post intervention study. BMC

Pediatr. 2012 Jul 2;12:91. doi: 10.1186/1471-2431-12-91. PMID: 22747635. Exclusion: 11.

255. Emara H. Effect of a new physical therapy concept on dynamic balance in children with spastic diplegic cerebral palsy. Egypt J Med Hum Genet. 2015 Jan;16(1):77-83. doi: 10.1016/j.ejmhg.2014.09.001. Exclusion: 14.

256. English C. Patients spend an alarmingly high proportion of the day sedentary. Int $\mathrm{J}$ Ther Rehabil. 2011 Feb;18(2):117. doi: 10.12968/ijtr.2011.18.2.117. Exclusion: 10.

257. Ensari I, Motl RW, Pilutti LA. Exercise training improves depressive symptoms in people with multiple sclerosis: results of a meta-analysis. J Psychosom Res. 2014 Jun;76(6):465-71. doi: 10.1016/j.jpsychores.2014.03.014. PMID: 24840141. Exclusion: 9.

258. Ensari I, Sandroff BM, Motl RW. Effects of single bouts of walking exercise and yoga on acute mood symptoms in people with multiple sclerosis. Int J MS Care. 2016 JanFeb;18(1):1-8. doi: 10.7224/15372073.2014-104. PMID: 26917992. Exclusion: 11.

259. Ensari I, Sandroff BM, Motl RW. Intensity of treadmill walking exercise on acute mood symptoms in persons with multiple sclerosis. Anxiety Stress Coping. 2017 Jan;30(1):1525. doi: $10.1080 / 10615806.2016 .1146710$. PMID: 26828264. Exclusion: 11.

260. Erickson ML, Ryan TE, Backus D, et al. Endurance neuromuscular electrical stimulation training improves skeletal muscle oxidative capacity in individuals with motor-complete spinal cord injury. Muscle Nerve. 2017 May;55(5):669-75. doi: 10.1002/mus.25393. PMID: 27576602. Exclusion: 11.
261. Eriks-Hoogland I, de Groot S, Snoek G, et al. Association of shoulder problems in persons with spinal cord injury at discharge from inpatient rehabilitation with activities and participation 5 years later. Arch Phys Med Rehabil. 2016 Jan;97(1):84-91. doi: 10.1016/j.apmr.2015.08.432. PMID: 26376446. Exclusion: 8.

262. Ertekin O, Özakbaş S, Idiman E, et al. The effects of low and severe disability on walking abilities and quality of life in multiple sclerosis patients: 6-month followup study. Noropsikiyatri Arsivi. 2013;50(1):23-9. doi: 10.4274/npa.y6213. Exclusion: 11.

263. ErtekIn O, Özakbaş S, Idiman E, et al. Quality of life, fatigue and balance improvements after home-based exercise program in multiple sclerosis patients. Noropsikiyatri Arsivi. 2012;49(1):33-8. doi: 10.4274/npa.y6091. Exclusion: 4.

264. Esclarin-De Ruz A, Alcobendas-Maestro M, Casado-Lopez R, et al. [A robotic system for gait re-education in patients with an incomplete spinal cord injury]. Rev Neurol. 2009 Dec 16-31;49(12):617-22. PMID: 20013712. Exclusion: 13.

265. Esnouf JE, Taylor PN, Mann GE, et al. Impact on activities of daily living using a functional electrical stimulation device to improve dropped foot in people with multiple sclerosis, measured by the Canadian Occupational Performance Measure. Mult Scler. 2010 Sep;16(9):11417. doi: $10.1177 / 1352458510366013$. PMID: 20601398. Exclusion: 4.

266. Espinosa IB, Platas MG, Chavez JJR, et al. Randomized and blinded study to assess the complementary therapies contribution to treat MS. Mult Scler. 2015;Conference:. 2015 progress in MS research conference. Melbourne, VIC australia. Conference start: 20151029. Conference end: 20151030. Conference publication:(var.pagings) 21(14):21. Exclusion: 10.

267. Estes S, Iddings JA, Ray S, et al. Comparison of single-session dose response effects of whole body vibration on spasticity and walking speed in persons with spinal cord injury. Neurother. 2018 Jul;15(3):68496. doi: 10.1007/s13311-018-0644-1. PMID: 29959653. Exclusion: 4. 
268. Estes SP, Iddings JA, Field-Fote EC. Priming neural circuits to modulate spinal reflex excitability. Front Neurol. 2017 Feb 3;8:17. doi: 10.3389/fneur.2017.00017. PMID: 28217104. Exclusion: 4.

269. Etoom M, Khraiwesh Y, Lena F, et al. Effectiveness of physiotherapy interventions on spasticity in people with multiple sclerosis: a systematic review and metaanalysis. Am J Phys Med Rehabil. 2018 Nov;97(11):793-807. doi: 10.1097/PHM.000000000000970. PMID: 29794531. Exclusion: 12.

270. Eugster-Buesch F, de Bruin ED, Boltshauser E, et al. Forced-use therapy for children with cerebral palsy in the community setting: a single-blinded randomized controlled pilot trial. J Pediatr Rehabil Med. 2012;5(2):6574. doi: 10.3233/PRM-2012-0198. PMID: 22699097. Exclusion: 4.

271. Evans N, Hartigan C, Kandilakis C, et al. Acute cardiorespiratory and metabolic responses during exoskeleton-assisted walking overground among persons with chronic spinal cord injury. Top Spinal Cord Inj Rehabil. 2015 Spring;21(2):122-32. doi: 10.1310/sci2102-122. PMID: 26364281. Exclusion: 11.

272. Faes Y, Banz N, Buscher N, et al. Acute effects of partial-body vibration in sitting position. World J Orthop. 2018 Sep 18;9(9):156-64. doi: 10.5312/wjo.v9.i9.156. PMID: 30254972. Exclusion: 3.

273. Fakolade A, Bisson EJ, Petrin J, et al. Effect of comorbidities on outcomes of neurorehabilitation interventions in multiple sclerosis: A scoping review. Int J MS Care. 2016 Nov-Dec;18(6):282-90. doi: 10.7224/1537-2073.2016-015. PMID: 27999522. Exclusion: 12.

274. Fakolade A, Finlayson M, Plow M. Using telerehabilitation to support people with multiple sclerosis: a qualitative analysis of interactions, processes, and issues across three interventions. Br J Occup Ther. 2017;80(4):259-68. doi: 10.1177/0308022617690405. Exclusion: 4.
275. Fang Y, Morse LR, Nguyen N, et al. Functional electrical stimulation (FES)assisted rowing combined with zoledronic acid, but not alone, preserves distal femur strength and stiffness in people with chronic spinal cord injury. Osteoporos Int. 2020 Sep 04;04:04. doi: 10.1007/s00198-020-05610x. PMID: 32888047. Exclusion: 4.

276. Fasoli SE, Fragala-Pinkham M, Hughes R, et al. Upper limb robotic therapy for children with hemiplegia. Am J Phys Med Rehabil. 2008 Nov;87(11):929-36. doi: 10.1097/PHM.0b013e31818a6aa4. PMID: 18936558. Exclusion: 11.

277. Fasoli SE, Fragala-Pinkham M, Hughes R, et al. Robotic therapy and botulinum toxin type A: a novel intervention approach for cerebral palsy. Am J Phys Med Rehabil. 2008 Dec;87(12):1022-5. doi: 10.1097/PHM.0b013e31817fb346. PMID: 18617860. Exclusion: 7.

278. Federici S, Meloni F, Bracalenti M, et al. The effectiveness of powered, active lower limb exoskeletons in neurorehabilitation: a systematic review. NeuroRehabilitation. 2015;37(3):321-40. doi: 10.3233/NRE151265. PMID: 26529583. Exclusion: 12.

279. Fehlings D, Switzer L, Findlay B, et al. Interactive computer play as "motor therapy" for individuals with cerebral palsy. Semin Pediatr Neurol. 2013 Jun;20(2):12738. doi: 10.1016/j.spen.2013.06.003. PMID: 23948687. Exclusion: 9.

280. Feitosa LC, Muzzolon SRB, Rodrigues DCB, et al. The effect of adapted sports in quality of life and biopsychosocial profile of children and adolescents with cerebral palsy. Rev Paul Pediatr. 2017 Oct-Dec;35(4):42935. doi: 10.1590/19840462/;2017;35;4;00001. PMID: 29185624. Exclusion: 11.

281. Felippe LA, Salgado PR, de Souza Silvestre $\mathrm{D}$, et al. A controlled clinical trial on the effects of exercise on cognition and mobility in adults with multiple sclerosis. Am J Phys Med Rehabil. 2019 Feb;98(2):97-102. doi: 10.1097/PHM.0000000000000987. PMID: 29927751. Exclusion: 11. 
282. Felippe LA, Salgado PR, Silvestre DS, et al. A controlled clinical trial on the effects of exercise on cognition and mobility in adults with multiple sclerosis. Am J Phys Med Rehabil. 2019 Feb;98(2):97-102. doi: 10.1097/PHM.000000000000987. PMID: 29927751. Exclusion: 11.

283. Feltham MG, Collett J, Izadi H, et al. Cardiovascular adaptation in people with multiple sclerosis following a twelve week exercise programme suggest deconditioning rather than autonomic dysfunction caused by the disease. Results from a randomized controlled trial. Eur J Phys Rehabil Med. 2013 Dec;49(6):765-74. PMID: 23877228. Exclusion: 11.

284. Fenuta AM, Hicks AL. Metabolic demand and muscle activation during different forms of bodyweight supported locomotion in men with incomplete SCI. Biomed Res Int. 2014;2014 doi: 10.1155/2014/632765. PMID: 24971340. Exclusion: 11.

285. Fernandes LC, Chitra J, Metgud D, et al. Effectiveness of artifical [sic] horse riding on postural control in spastic diplegics RCT. Indian J Physiother Occup Ther. 2008;2(4):36-40. Exclusion: 3.

286. Ferre CL, Brandao M, Surana B, et al. Caregiver-directed home-based intensive bimanual training in young children with unilateral spastic cerebral palsy: a randomized trial. Dev Med Child Neurol. 2017 May;59(5):497-504. doi: 10.1111/dmen.13330. PMID: 27864822. Exclusion: 4.

287. Ferreira AP, Pegorare AB, Salgado PR, et al. Impact of a pelvic floor training program among women with multiple sclerosis: a controlled clinical trial. Am J Phys Med Rehabil. 2016 Jan;95(1):1-8. doi: 10.1097/PHM.000000000000302. PMID: 25888662. Exclusion: 11.

288. Feys P, Coninx K, Kerkhofs L, et al. Robotsupported upper limb training in a virtual learning environment : a pilot randomized controlled trial in persons with MS. J Neuroengineering Rehabil. 2015 Jul 23;12:60. doi: 10.1186/s12984-015-0043-3. PMID: 26202325. Exclusion: 11.
289. Feys P, Moumdjian L, Van Halewyck F, et al. Effects of an individual 12-week community-located "start-to-run" program on physical capacity, walking, fatigue, cognitive function, brain volumes, and structures in persons with multiple sclerosis. Mult Scler. 2019 Nov 1;25(1):92-103. doi: 10.1177/1352458517740211. PMID: 29113572. Exclusion: 4.

290. Field T. Yoga research review. Complement Ther Clin Pract. 2016 Aug;24:145-61. doi: 10.1016/j.ctcp.2016.06.005. PMID: 27502816. Exclusion: 3.

291. Filipi ML, Kucera DL, Filipi EO, et al. Improvement in strength following resistance training in MS patients despite varied disability levels. NeuroRehabilitation. 2011;28(4):373-82. doi: 10.3233/NRE2011-0666. PMID: 21725171. Exclusion: 8.

292. Filipi ML, Leuschen MP, Huisinga J, et al. Impact of resistance training on balance and gait in multiple sclerosis. Int J MS Care. 2010;12(1):6-12. doi: 10.7224/1537-207312.1.6. Exclusion: 8.

293. Fischer M, Vialleron T, Laffaye G, et al. Long-term effects of whole-body vibration on human gait: a systematic review and meta-analysis. Front Neurol. 2019;10:627. doi: 10.3389/fneur.2019.00627. PMID: 31316447. Exclusion: 12.

294. Fisher-Pipher SPD, Kenyon LKPDPP, Westman MPD. Improving balance, mobility, and dual-task performance in an adolescent with cerebral palsy: a case report. Physiother Theory Pract. 2017

Jul;33(7):586-95. doi: 10.1080/09593985.2017.1323359. PMID: 28509631. Exclusion: 7.

295. Flachenecker P, Bures AK, Gawlik A, et al. Efficacy of an Internet-Based Program to Promote Physical Activity and Exercise after Inpatient Rehabilitation in Persons with Multiple Sclerosis: A Randomized, SingleBlind, Controlled Study. Int J Environ Res Public Health. 2020 Jun 24;17(12):24. doi: 10.3390/ijerph17124544. PMID: 32599767. Exclusion: 4. 
296. Fleerkotte BM, Koopman B, Buurke JH, et al. The effect of impedance-controlled robotic gait training on walking ability and quality in individuals with chronic incomplete spinal cord injury: an explorative study. J Neuroengineering Rehabil. 2014 Mar 4;11:26. doi: 10.1186/1743-0003-1126. PMID: 24594284. Exclusion: 11.

297. Fluet GG, Qiu Q, Kelly D, et al. Interfacing a haptic robotic system with complex virtual environments to treat impaired upper extremity motor function in children with cerebral palsy. Dev Neurorehabil. 2010;13(5):335-45. doi: 10.3109/17518423.2010.501362. PMID: 20828330. Exclusion: 11.

298. Fornusek C, Davis GM, Russold MF. Pilot study of the effect of low-cadence functional electrical stimulation cycling after spinal cord injury on thigh girth and strength. Arch Phys Med Rehabil. 2013 May;94(5):990-3. doi: 10.1016/j.apmr.2012.10.010. PMID: 23123504. Exclusion: 11.

299. Fornusek C, Hoang P. Neuromuscular electrical stimulation cycling exercise for persons with advanced multiple sclerosis. J Rehabil Med. 2014 Jul;46(7):698-702. doi: 10.2340/16501977-1792. PMID: 24763902. Exclusion: 11.

300. Forrest GF, Lorenz DJ, Hutchinson K, et al. Ambulation and balance outcomes measure different aspects of recovery in individuals with chronic, incomplete spinal cord injury. Arch Phys Med Rehabil. 2012

Sep;93(9):1553-64. doi:

10.1016/j.apmr.2011.08.051. PMID:

22920452. Exclusion: 7.

301. Forsberg AS, Von Koch L, Nilsagard YE. Effects of a balance programme focusing on core stability exercises and dual-task activities in persons with multiple sclerosis: a randomized controlled trial. Mult Scler J Exp Transl Clin. 2013 Oct;19(11 ):62-3. doi: 10.1177/1352458513502433. Exclusion: 10.

302. Fosdahl M, Holm I, Jahnsen R. The effect of progressive resistance exercise training and stretching of the hamstrings muscle in ambulant children with cerebral palsy - a randomized controlled trial. Dev Med Child Neurol. 2017;59:48. doi:

10.1111/dmcn.71_13511. Exclusion: 10.
303. Fosdahl MA, Jahnsen R, Kvalheim K, et al. Stretching and progressive resistance exercise in children with cerebal palsy: a randomised controlled trial. Pediatr Phys Ther. 2019 Jul;31(3):264-71. doi: 10.1097/PEP.0000000000000616. PMID: 31220010. Exclusion: 6.

304. Foster H, DeMark L, Spigel PM, et al. The effects of backward walking training on balance and mobility in an individual with chronic incomplete spinal cord injury: a case report. Physiother Theory Pract. 2016 Oct;32(7):536-45. doi: 10.1080/09593985.2016.1206155. PMID: 27482619. Exclusion: 7.

305. Fox EE, Hough AD, Creanor S, et al. The effects of pilates-based core stability training in ambulant people with multiple sclerosis: a multicentre, block randomised, double blinded placebo controlled trial. Mult Scler J Exp Transl Clin. 2013 Oct;19(11 ):40. doi: 10.1177/1352458513502433 . Exclusion: 10.

306. Fox EJ, Tester NJ, Butera KA, et al. Retraining walking adaptability following incomplete spinal cord injury. Spinal Cord Ser Cases. 2017;3 doi: 10.1038/s41394-0170003-10. PMID: 29449967. Exclusion: 7.

307. Foy T, Perritt G, Thimmaiah D, et al. The SCIRehab project: treatment time spent in SCI rehabilitation. Occupational therapy treatment time during inpatient spinal cord injury rehabilitation. J Spinal Cord Med. 2011;34(2):162-75. doi: 10.1179/107902611X12971826988093. PMID: 21675355. Exclusion: 3.

308. Fragala-Pinkham MA, Smith HJ, Lombard $\mathrm{KA}$, et al. Aquatic aerobic exercise for children with cerebral palsy: a pilot intervention study. Physiother Theory Pract. 2014 Feb;30(2):69-78. doi: 10.3109/09593985.2013.825825. PMID: 24328930. Exclusion: 11.

309. Fragoso YD, Santana DL, Pinto RC. The positive effects of a physical activity program for multiple sclerosis patients with fatigue. NeuroRehabilitation. 2008;23(2):153-7. PMID: 18525136. Exclusion: 11. 
310. Francisco GE, Yozbatiran N, Berliner J, et al. Robot-assisted training of arm and hand movement shows functional improvements for incomplete cervical spinal cord injury. Am J Phys Med Rehabil. 2017 Oct;96(10 Suppl 1):S171-S7. doi: 10.1097/PHM.000000000000815. PMID: 28857769. Exclusion: 11.

311. Frank R, Larimore J. Yoga as a method of symptom management in multiple sclerosis. Front Neurosci. 2015;9:133. doi: 10.3389/fnins.2015.00133. PMID: 25983675. Exclusion: 12.

312. Freeman J, Hendrie W, Jarrett L, et al. Assessment of a home-based standing frame programme in people with progressive multiple sclerosis (SUMS): a pragmatic, multi-centre, randomised, controlled trial and cost-effectiveness analysis. Lancet neurol. 2019 Aug;18(8):736-47. doi: 10.1016/S1474-4422(19)30190-5. PMID: 31301748. Exclusion: 4.

313. Freeman JA, Gear M, Pauli A, et al. The effect of core stability training on balance and mobility in ambulant individuals with multiple sclerosis: a multi-centre series of single case studies. Mult Scler. 2010 Nov;16(11):1377-84. doi: 10.1177/1352458510378126. PMID: 20699285. Exclusion: 7.

314. Freeman JA, Hendrie W, Creanor S, et al. Standing up in multiple sclerosis (SUMS): protocol for a multi-centre randomised controlled trial evaluating the clinical and cost effectiveness of a home-based selfmanagement standing frame programme in people with progressive multiple sclerosis. BMC Neurol. 2016 May 5;16:62. doi: 10.1186/s12883-016-0581-8. PMID: 27149954. Exclusion: 10.

315. Frevel D, Maurer M. Internet-based home training is capable to improve balance in multiple sclerosis: a randomized controlled trial. Eur J Phys Rehabil Med. 2015 Feb;51(1):23-30. PMID: 24755773. Exclusion: 11.

316. Frisk RF, Jensen P, Kirk H, et al. Contribution of sensory feedback to plantar flexor muscle activation during push-off in adults with cerebral palsy. J Neurophysiol. 2017 Dec 1;118(6):3165-74. doi: 10.1152/jn.00508.2017. PMID: 28904105. Exclusion: 4.
317. Froehlich-Grobe K, Aaronson LS, Washburn RA, et al. An exercise trial for wheelchair users: project workout on wheels. Contemp Clin Trials. 2012 Mar;33(2):351-63. doi: 10.1016/j.cct.2011.10.014. PMID: 22101206. Exclusion: 4.

318. Froehlich-Grobe K, Lee J, Aaronson L, et al. Exercise for everyone: a randomized controlled trial of project workout on wheels in promoting exercise among wheelchair users. Arch Phys Med Rehabil. 2014 Jan;95(1):20-8. doi: 10.1016/j.apmr.2013.07.006. PMID: 23872080. Exclusion: 4.

319. Fundaro C, Giardini A, Maestri R, et al. Motor and psychosocial impact of robotassisted gait training in a real-world rehabilitation setting: a pilot study. PLoS One. 2018;13(2):e0191894. doi: 10.1371/journal.pone.0191894. PMID: 29444172. Exclusion: 5.

320. Gagliardi C, Turconi AC, Biffi E, et al. Immersive virtual reality to improve walking abilities in cerebral palsy: a pilot study. Ann Biomed Eng. 2018 Sep;46(9):1376-84. doi: 10.1007/s10439018-2039-1. PMID: 29704186. Exclusion: 11.

321. Galea M, Dunlop S, Geraghty T, et al. Intensive exercise program after spinal cord injury (SCIPA full-on): a randomized controlled trial. Ann Phys Rehabil Med. 2018 Jul;61:e42. doi: 10.1016/j.rehab.2018.05.094. Exclusion: 10.

322. Galea MP, Panisset MG, El-Ansary D, et al. SCIPA Switch-On: a randomized controlled trial investigating the efficacy and safety of functional electrical stimulation-assisted cycling and passive cycling initiated early after traumatic spinal cord injury. Neurorehabil Neural Repair. 2017;31(6):540-51. doi: 10.1177/1545968317697035. Exclusion: 3.

323. Gandhi P, Chan K, Verrier MC, et al. Training to improve walking after pediatric spinal cord injury: a systematic review of parameters and walking outcomes. $\mathrm{J}$ Neurotrauma. 2017 May 1;34(9):1713-25. doi: 10.1089/neu.2016.4501. PMID: 27869534. Exclusion: 12. 
324. Gandolfi M, Geroin C, Picelli A, et al. Robot-assisted vs. sensory integration training in treating gait and balance dysfunctions in patients with multiple sclerosis: a randomized controlled trial. Front Hum Neurosci. 2014;8:318. doi: 10.3389/fnhum.2014.00318. PMID: 24904361. Exclusion: 11.

325. Gant KL, Nagle KG, Cowan RE, et al. Body system effects of a multi-modal training program targeting chronic, motor complete thoracic spinal cord injury. J Neurotrauma. 2018 Feb 1;35(3):411-23. doi: 10.1089/neu.2017.5105. PMID: 28795657. Exclusion: 11.

326. Gao J, He L, Yu X, et al. Rehabilitation with a combination of scalp acupuncture and exercise therapy in spastic cerebral palsy. Complement Ther Clin Pract. 2019 May;35:296-300. doi: 10.1016/j.ctcp.2019.03.002. PMID: 31003673. Exclusion: 4.

327. Garcia-Gomez S, Perez-Tejero J, Hoozemans M, et al. Effect of a home-based exercise program on shoulder pain and range of motion in elite wheelchair basketball players: a non-randomized controlled trial. Sports (Basel). 2019 Jul 24;7(8):24. doi: 10.3390/sports7080180. PMID: 31344871. Exclusion: 4.

328. Garcia-Mendez Y, Pearlman JL, Boninger ML, et al. Health risks of vibration exposure to wheelchair users in the community. J Spinal Cord Med. 2013 Jul;36(4):365-75. doi: 10.1179/2045772313Y.0000000124. PMID: 23820152. Exclusion: 7.

329. Garshick E, Mulroy S, Graves DE, et al. Active lifestyle is associated with reduced dyspnea and greater life satisfaction in spinal cord injury. Arch Phys Med Rehabil. 2016 10;97(10):1721-7. doi: 10.1016/j.apmr.2016.02.010. PMID: 26951870. Exclusion: 4.

330. Gaspard L, Tombal B, Castille Y, et al. Pelvic floor muscles training, electrical stimulation, bladder training and lifestyle interventions to manage lower urinary tract dysfunction in multiple sclerosis: a systematic review. Prog Urol. 2014 Mar;24(4):222-8. doi: 10.1016/j.purol.2013.11.004. PMID: 24560290. Exclusion: 13.
331. Gaspard L, Tombal B, Opsomer RJ, et al. [Physiotherapy and neurogenic lower urinary tract dysfunction in multiple sclerosis patients: a randomized controlled trial]. Prog Urol. 2014 Sep;24(11):697-707. doi: 10.1016/j.purol.2014.05.003. PMID: 25214451. Exclusion: 13.

332. Gates PE, Banks D, Johnston TE, et al. Randomized controlled trial assessing participation and quality of life in a supported speed treadmill training exercise program vs. a strengthening program for children with cerebral palsy. J Pediatr Rehabil Med. 2012;5(2):75-88. doi: 10.3233/PRM-2012-0199. PMID: 22699098. Exclusion: 4.

333. Gatica-Rojas V, Cartes-Velasquez R, Guzman-Munoz E, et al. Effectiveness of a Nintendo Wii balance board exercise programme on standing balance of children with cerebral palsy: a randomised clinical trial protocol. Contemp Clin Trials Commun. 2017 Jun;6:17-21. doi: 10.1016/j.conctc.2017.02.008. PMID: 29740634. Exclusion: 10.

334. Gatica-Rojas V, Cartes-Velasquez R, Mendez-Rebolledo G, et al. Effects of a Nintendo Wii exercise program on spasticity and static standing balance in spastic cerebral palsy. Dev Neurorehabil. 2017 Aug;20(6):388-91. doi: 10.1080/17518423.2016.1211770. PMID: 27538127. Exclusion: 11.

335. Gatica-Rojas V, Cartes-Velasquez R, Mendez-Rebolledo G, et al. Change in functional balance after an exercise program with Nintendo Wii in Latino patients with cerebral palsy: a case series. J Phys Ther Sci. 2016 Aug;28(8):2414-7. doi: 10.1589/jpts.28.2414. PMID: 27630446. Exclusion: 7.

336. Gatica-Rojas V, Mendez-Rebolledo G, Guzman-Munoz E, et al. Does Nintendo Wii balance board improve standing balance? A randomized controlled trial in children with cerebral palsy. Eur J Phys Rehabil Med. 2017 Aug;53(4):535-44. doi: 10.23736/S1973-9087.16.04447-6. PMID: 27882910. Exclusion: 11. 
337. Gauthier C, Brosseau R, Hicks AL, et al. Feasibility, safety, and preliminary effectiveness of a home-based self-managed high-intensity interval training program offered to long-term manual wheelchair users. Rehabil Res Pract.

2018;2018:8209360. doi:

10.1155/2018/8209360. PMID: 29888007.

Exclusion: 4.

338. Geddes EL, Costello E, Raivel K, et al. The effects of a twelve-week home walking program on cardiovascular parameters and fatigue perception of individuals with multiple sclerosis: a pilot study. Cardiopulm Phys Ther J. 2009 Mar;20(1):5-12. PMID: 20467528 Exclusion: 11.

339. Ghai S, Ghai I, Effenberg AO. Effects of dual tasks and dual-task training on postural stability: a systematic review and metaanalysis. Clin Interv Aging. 2017;12:557-77. doi: 10.2147/CIA.S125201. PMID: 28356727. Exclusion: 12.

340. Gharib NM, El-Maksoud GM, Rezk-Allah SS. Efficacy of gait trainer as an adjunct to traditional physical therapy on walking performance in hemiparetic cerebral palsied children: a randomized controlled trial. Clin Rehabil. 2011 Oct;25(10):924-34. doi: 10.1177/0269215511400768. PMID: 21427153. Exclusion: 14.

341. Giacobbi PR, Jr., Dietrich F, Larson R, et al. Exercise and quality of life in women with multiple sclerosis. Adapt Phys Act Q. 2012 Jul;29(3):224-42. PMID: 22811564.

Exclusion: 11.

342. Gibbons RS, Stock CG, Andrews BJ, et al. The effect of FES-rowing training on cardiac structure and function: pilot studies in people with spinal cord injury. Spinal Cord. 2016 Oct;54(10):822-9. doi: 10.1038/sc.2015.228. PMID: 26754476. Exclusion: 11.

343. Gibbs JC, Gagnon DH, Bergquist AJ, et al. Rehabilitation interventions to modify endocrine-metabolic disease risk in individuals with chronic spinal cord injury living in the community (RIISC): a systematic review and scoping perspective. $\mathrm{J}$ Spinal Cord Med. 2017 Nov;40(6):733-47. doi: 10.1080/10790268.2017.1350341. PMID: 28703038. Exclusion: 12.
344. Giesser B, Herlihy E, Plummer D'Amato P, et al. Randomised, controlled trial of robotic locomotor training in persons with multiple sclerosis. Mult Scler J Exp Transl Clin. 2011 Oct;17(10 SUPPL. 1):S480-S1. doi: 10.1177/1352458511422301. Exclusion: 10.

345. Gilbertson RM, Klatt MD. Mindfulness in motion for people with multiple sclerosis: a feasibility study. Int J MS Care. 2017 SepOct;19(5):225-31. doi: 10.7224/15372073.2015-095. PMID: 29070962.

Exclusion: 4.

346. Gillett JG, Boyd RN, Carty CP, et al. The impact of strength training on skeletal muscle morphology and architecture in children and adolescents with spastic cerebral palsy: a systematic review. Res Dev Disabil. 2016 Sep;56:183-96. doi: 10.1016/j.ridd.2016.06.003. PMID: 27337690. Exclusion: 12.

347. Gillett JG, Lichtwark GA, Boyd RN, et al. Functional anaerobic and strength training in young adults with cerebral palsy. Med Sci Sports Exerc. 2018 Aug;50(8):1549-57. doi: 10.1249/MSS.0000000000001614. PMID: 29557839. Exclusion: 11.

348. Giray E, Karadag-Saygi E, Ozsoy T, et al. The effects of vest type dynamic elastomeric fabric orthosis on sitting balance and gross manual dexterity in children with cerebral palsy: a single-blinded randomised controlled study. Disabil Rehabil. 2020 Oct 7;42(3):410-8. doi: 10.1080/09638288.2018.1501098. PMID: 30293457. Exclusion: 4.

349. Glinsky J, Harvey L, Korten M, et al. Shortterm progressive resistance exercise may not be effective at increasing wrist strength in people with tetraplegia: a randomised controlled trial. Aust J Physiother. 2008;54(2):103-8. PMID: 18492001. Exclusion: 4.

350. Glinsky J, Harvey L, van Es P, et al. The addition of electrical stimulation to progressive resistance training does not enhance the wrist strength of people with tetraplegia: a randomized controlled trial. Clin Rehabil. 2009 Aug;23(8):696-704. doi: 10.1177/0269215509104171. PMID: 19470552. Exclusion: 4. 
351. Gollie JM, Guccione AA, Panza GS, et al. Effects of overground locomotor training on walking performance in chronic cervical motor incomplete spinal cord injury: a pilot study. Arch Phys Med Rehabil. 2017 Jun;98(6):1119-25. doi: 10.1016/j.apmr.2016.10.022. PMID: 27965006. Exclusion: 11.

352. Golzari Z, Shabkhiz F, Soudi S, et al. Combined exercise training reduces IFNgamma and IL-17 levels in the plasma and the supernatant of peripheral blood mononuclear cells in women with multiple sclerosis. Int Immunopharmacol. 2010 Nov;10(11):1415-9. doi: 10.1016/j.intimp.2010.08.008. PMID: 20797460. Exclusion: 11.

353. Gomes-Osman J, Tibbett JA, Poe BP, et al. Priming for improved hand strength in persons with chronic tetraplegia: a comparison of priming-augmented functional task practice, priming alone, and conventional exercise training. Front Neurol. 2017;7(JAN) doi: 10.3389/fneur.2016.00242. Exclusion: 4.

354. Gonzales B, Chopard G, Charry B, et al. Effects of a training program involving body cooling on physical and cognitive capacities and quality of life in multiple sclerosis patients: a pilot study. Eur Neurol. 2017;78(1-2):71-7. doi: 10.1159/000477580. PMID: 28697504. Exclusion: 11.

355. Goosey-Tolfrey V, Foden E, Perret C, et al. Effects of inspiratory muscle training on respiratory function and repetitive sprint performance in wheelchair basketball players. Br J Sports Med. 2010 Jul;44(9):665-8. doi: 10.1136/bjsm.2008.049486. PMID: 18603575. Exclusion: 11.

356. Gorgey AS, Dolbow DR, Cifu DX, et al. Neuromuscular electrical stimulation attenuates thigh skeletal muscles atrophy but not trunk muscles after spinal cord injury. $\mathrm{J}$ Electromyogr Kinesiol. 2013

Aug;23(4):977-84. doi: 10.1016/j.jelekin.2013.04.007. PMID: 23683374. Exclusion: 11.
357. Gorgey AS, Khalil RE, Gill R, et al. Effects of testosterone and evoked resistance exercise after spinal cord injury (TEREXSCI): study protocol for a randomised controlled trial. BMJ Open. 2017 Apr 4;7(4) doi: 10.1136/bmjopen-2016-014125. PMID: 28377392. Exclusion: 11.

358. Gorgey AS, Martin H, Metz A, et al. Longitudinal changes in body composition and metabolic profile between exercise clinical trials in men with chronic spinal cord injury. J Spinal Cord Med. 2016 Nov;39(6):699-712. doi: 10.1080/10790268.2016.1157970. PMID: 27077574. Exclusion: 11.

359. Gorgey AS, Poarch HJ, Dolbow DD, et al. Effect of adjusting pulse durations of functional electrical stimulation cycling on energy expenditure and fatigue after spinal cord injury. J Rehabil Res Dev.

2014;51(9):1455-68. doi: 10.1682/JRRD.2014.02.0054. PMID: 25803753. Exclusion: 4.

360. Gorgey AS, Timmons MK, Dolbow DR, et al. Electrical stimulation and blood flow restriction increase wrist extensor crosssectional area and flow meditated dilatation following spinal cord injury. Eur J Appl Physiol. 2016 Jun;116(6):1231-44. doi: 10.1007/s00421-016-3385-z. PMID: 27155846. Exclusion: 11.

361. Gorgey AS, Wade R, Sumrell R, et al. Exoskeleton training may improve level of physical activity after spinal cord injury: a case series. Top Spinal Cord Inj Rehabil. 2017;23(3):245-55. doi: 10.1310/sci1600025. PMID: 29339900. Exclusion: 7.

362. Gorla JI, Costa e Silva Ade A, Borges M, et al. Impact of wheelchair rugby on body composition of subjects with tetraplegia: a pilot study. Arch Phys Med Rehabil. 2016 Jan;97(1):92-6. doi: 10.1016/j.apmr.2015.09.007. PMID: 26433046. Exclusion: 11.

363. Gorman P, Scott W, York H, et al. Robotic treadmill training does not improve timed measures of ambulatory function in chronic motor incomplete spinal cord injury: a pilot controlled clinical trial. Top Spinal Cord Inj Rehabil. 2011 Jun;16:63. doi: 10.1310/sci16S1-53. Exclusion: 10. 
364. Gorman PH, Geigle PR, Chen K, et al. Reliability and relatedness of peak VO2 assessments during body weight supported treadmill training and arm cycle ergometry in individuals with chronic motor incomplete spinal cord injury. Spinal Cord. 2014 Apr;52(4):287-91. doi:

10.1038/sc.2014.6. PMID: 24534779.

Exclusion: 4.

365. Gorman PH, Scott W, York H, et al. Robotically assisted treadmill exercise training for improving peak fitness in chronic motor incomplete spinal cord injury: a randomized controlled trial. J Spinal Cord Med. 2016;39(1):32-44. doi:

10.1179/2045772314Y.0000000281. PMID: 25520035. Exclusion: 11.

366. Govil K, Noohu MM. Effect of EMG biofeedback training of gluteus maximus muscle on gait parameters in incomplete spinal cord injury. NeuroRehabilitation. 2013;33(1):147-52. doi: 10.3233/NRE130939. PMID: 23949032. Exclusion: 14.

367. Granja Dominguez A, Magni E, Hochsprung A. Gait and physiotherapy: effectiveness of a physiotherapy circuit training on the ability to walk and quality of live in a multiple sclerosis's patients. Mult Scler. 2017;Conference: 7th joint ECTRIMSACTRIMS, MSPARIS2017. France. 23(3 Supplement 1):970. Exclusion: 10.

368. Grasmucke D, Zieriacks A, Jansen O, et al. Against the odds: what to expect in rehabilitation of chronic spinal cord injury with a neurologically controlled Hybrid Assistive Limb exoskeleton. A subgroup analysis of 55 patients according to age and lesion level. Neurosurg Focus. 2017 May;42(5):15. doi: 10.3171/2017.2.FOCUS171. PMID: 28463613. Exclusion: 8.

369. Grazioli E, Tranchita E, Borriello G, et al. The effects of concurrent resistance and aerobic exercise training on functional dtatus in patients with multiple sclerosis. Curr Sports Med Rep. 2019 Dec;18(12):4527. doi: $10.1249 /$ JSR.0000000000000661. PMID: 31834177. Exclusion: 11.
370. Grecco LA, de Freitas TB, Satie J, et al. Treadmill training following orthopedic surgery in lower limbs of children with cerebral palsy. Pediatr Phys Ther. 2013;25(2):187-92; discussion 93. doi: 10.1097/PEP.0b013e3182888495. PMID: 23542199. Exclusion: 11.

371. Grecco LA, Oliveira CS, Duarte NA, et al. Cerebellar transcranial direct current stimulation in children with ataxic cerebral palsy: a sham-controlled, crossover, pilot study. Dev Neurorehabil. 2017 Apr;20(3):142-8. doi: 10.3109/17518423.2016.1139639. PMID: 27003795. Exclusion: 11.

372. Grecco LA, Tomita SM, Christovao TC, et al. Effect of treadmill gait training on static and functional balance in children with cerebral palsy: a randomized controlled trial. Braz J Phys Ther. 2013 Jan-Feb;17(1):1723. PMID: 23538455. Exclusion: 11.

373. Griffin L, Decker MJ, Hwang JY, et al. Functional electrical stimulation cycling improves body composition, metabolic and neural factors in persons with spinal cord injury. J Electromyogr Kinesiol. 2009 Aug;19(4):614-22. doi: 10.1016/j.jelekin.2008.03.002. PMID: 18440241. Exclusion: 11.

374. Guclu-Gunduz A, Citaker S, Irkec C, et al. The effects of pilates on balance, mobility and strength in patients with multiple sclerosis. NeuroRehabilitation. 2014;34(2):337-42. doi: 10.3233/NRE130957. PMID: 23949064. Exclusion: 11.

375. Guillamo E, Cobo-Calvo A, Oviedo GR, et al. Feasibility and effects of structured physical exercise interventions in adults with relapsing-remitting multiple sclerosis: a pilot study. J Sports Sci Med. 2018 Sep;17(3):426-36. PMID: 30116116. Exclusion: 11.

376. Gültekin Ö, Öneş K, Harman H, et al. The effects of respiratory exercises on respiratory functions and the quality of life in patients with thoracal spinal cord injury. Nobel Medicus. 2013;9(3):82-7. Exclusion: 8. 
377. Gunn H, Andrade J, Paul L, et al. A selfmanagement programme to reduce falls and improve safe mobility in people with secondary progressive MS: the BRiMS feasibility RCT. Health Technol Assess. 2019 Jun;23(27):1-166. doi: 10.3310/hta23270. PMID: 31217069. Exclusion: 4.

378. Gunn H, Markevics S, Haas B, et al. Systematic review: The effectiveness of interventions to reduce falls and improve balance in adults with multiple sclerosis. Arch Phys Med Rehabil. 2015 Oct;96(10):1898-912. doi: 10.1016/j.apmr.2015.05.018. PMID: 26070975. Exclusion: 2.

379. Gurpinar B, Kara B, Idiman E. Effects of aquatic exercises on postural control and hand function in Multiple Sclerosis:

Halliwick versus Aquatic Plyometric Exercises: a randomised trial. $\mathrm{J}$ Musculoskelet Neuronal Interact. 2020 Jun 01;20(2):249-55. PMID: 32481240.

Exclusion: 11.

380. Hakansson NA, Hull ML. The effects of stimulating lower leg muscles on the mechanical work and metabolic response in functional electrically stimulated pedaling. IEEE Trans Neural Syst Rehabil Eng. 2010 Oct;18(5):498-504. doi:

10.1109/TNSRE.2010.2052132. PMID: 20529755. Exclusion: 4.

381. Hale LA, Mulligan HF, Treharne GJ, et al. The feasibility and short-term benefits of Blue Prescription: a novel intervention to enable physical activity for people with multiple sclerosis. Disabil Rehabil. 2013 Jul;35(14):1213-20. doi: 10.3109/09638288.2012.723787. PMID: 23025293. Exclusion: 4.

382. Hameau S, Bensmail D, Roche N, et al. Adaptations of fatigue and fatigability after a short intensive, combined rehabilitation program in patients with multiple sclerosis. $\mathrm{J}$ Rehabil Med. 2018 Jan 10;50(1):59-66. doi: 10.2340/16501977-2277. PMID: 28980009. Exclusion: 11.
383. Hamed NS, Abd-elwahab MS. Pedometerbased gait training in children with spastic hemiparetic cerebral palsy: a randomized controlled study. Clin Rehabil. 2011 Feb;25(2):157-65. doi: 10.1177/0269215510382147. PMID: 20943714. Exclusion: 14.

384. Hamed SA, El-Negamy TE, Waked NM. Effect of functional electrical stimulation on trunk curvature in spastic quadriplegic cerebral palsied children. Int J Pharmtech Res. 2016;9(5):31-6. Exclusion: 6.

385. Hammond ER, Recio AC, Sadowsky CL, et al. Functional electrical stimulation as a component of activity-based restorative therapy may preserve function in persons with multiple sclerosis. J Spinal Cord Med. 2015 Jan;38(1):68-75. doi: 10.1179/2045772314Y.0000000238. PMID: 24976037. Exclusion: 8.

386. Han DS, Hsiao MY, Wang TG, et al. Association of serum myokines and aerobic exercise training in patients with spinal cord injury: an observational study. BMC Neurol. 2016 Aug 17;16(1):142. doi: 10.1186/s12883-016-0661-9. PMID: 27534935. Exclusion: 5.

387. Han EY, Choi JH, Kim SH, et al. The effect of weight bearing on bone mineral density and bone growth in children with cerebral palsy: a randomized controlled preliminary trial. Medicine (Baltimore). 2017 Mar;96(10) doi: 10.1097/MD.0000000000005896. PMID: 28272197. Exclusion: 11.

388. Hansen D, Wens I, Keytsman C, et al. Is long-term exercise intervention effective to improve cardiac autonomic control during exercise in subjects with multiple sclerosis? A randomized controlled trial. Eur J Phys Rehabil Med. 2015 Apr;51(2):223-31. PMID: 24603938. Exclusion: 11.

389. Hansen D, Wens I, Keytsman C, et al. Ventilatory function during exercise in multiple sclerosis and impact of training intervention: cross-sectional and randomized controlled trial. Eur J Phys Rehabil Med. 2015 Oct;51(5):557-68. PMID: 25366519. Exclusion: 11. 
390. Harkema SJ, Schmidt-Read M, Lorenz DJ, et al. Balance and ambulation improvements in individuals with chronic incomplete spinal cord injury using locomotor trainingbased rehabilitation. Arch Phys Med Rehabil. 2012 Sep;93(9):1508-17. doi: 10.1016/j.apmr.2011.01.024. PMID: 21777905. Exclusion: 11.

391. Hartley S. Developing a self-management and exercise model for people with multiple sclerosis...including commentary by Plummer-D'Amato P and Giesser B. Int J Ther Rehabil. 2009;16(1):34-42. doi: 10.12968/ijtr.2009.16.1.37938. Exclusion: 8.

392. Harvey L, Fornusek C, Bowden J, et al. Electrical stimulation combined with progressive resistance training increases strength in people with spinal cord injury. Physiotherapy. 2011;97. Exclusion: 10.

393. Harvey LA, Dunlop SA, Churilov L, et al. Early intensive hand rehabilitation is not more effective than usual care plus one-toone hand therapy in people with sub-acute spinal cord injury ('Hands On'): a randomised trial. J Physiother. 2016 Apr;62(2):88-95. doi: 10.1016/j.jphys.2016.02.013. PMID: 27008910. Exclusion: 4.

394. Harvey LA, Dunlop SA, Churilov L, et al. Early intensive hand rehabilitation is not more effective than usual care plus one-toone hand therapy in people with sub-acute spinal cord injury ('Hands On'): a randomised trial. J Physiother. 2017 Oct;63(4):197-204. doi: 10.1016/j.jphys.2017.08.005. PMID: 28970100. Exclusion: 4.

395. Harvey LA, Fornusek C, Bowden JL, et al. Electrical stimulation plus progressive resistance training for leg strength in spinal cord injury: a randomized controlled trial. Spinal Cord. 2010 Jul;48(7):570-5. doi: 10.1038/sc.2009.191. PMID: 20065991. Exclusion: 4.

396. Harvey LA, Glinsky JV, Bowden JL. The effectiveness of 22 commonly administered physiotherapy interventions for people with spinal cord injury: a systematic review. Spinal Cord. 2016 Nov;54(11):914-23. doi: 10.1038/sc.2016.95. PMID: 27349607. Exclusion: 2.
397. Harvey LA, Herbert RD, Glinsky J, et al. Effects of 6 months of regular passive movements on ankle joint mobility in people with spinal cord injury: a randomized controlled trial. Spinal Cord. 2009 Jan;47(1):62-6. doi: 10.1038/sc.2008.71. PMID: 18574489. Exclusion: 4.

398. Harvey LA, Katalinic OM, Herbert RD, et al. Stretch for the treatment and prevention of contractures. Cochrane Database Syst Rev. 2017 Jan 9;1 doi: 10.1002/14651858.CD007455.pub3. PMID: 28146605. Exclusion: 4.

399. Harvey LA, Lin CW, Glinsky JV, et al. The effectiveness of physical interventions for people with spinal cord injuries: a systematic review. Spinal Cord. 2009 Mar;47(3):184-95. doi: 10.1038/sc.2008.100. PMID: 18725889. Exclusion: 2.

400. Hasnan N, Ektas N, Tanhoffer AI, et al. Exercise responses during functional electrical stimulation cycling in individuals with spinal cord injury. Med Sci Sports Exerc. 2013 Jun;45(6):1131-8. doi: 10.1249/MSS.0b013e3182805d5a. PMID: 23685444. Exclusion: 11.

401. Hassani S, Joseph K, Ann F, et al. Assessment of strength and function in ambulatory children with cerebral palsy by GMFCS level and age: a cross-sectional study. Crit Rev Phys Rehabil Med. 2011;23(1-4):1-14. doi: 10.1615/CritRevPhysRehabilMed.v23.i14.10. Exclusion: 4.

402. Hayes HA, Gappmaier E, LaStayo PC. Effects of high-intensity resistance training on strength, mobility, balance, and fatigue in individuals with multiple sclerosis: a randomized controlled trial. J Neurol Phys Ther. 2011 Mar;35(1):2-10. doi: 10.1097/NPT.0b013e31820b5a9d. PMID: 21475078. Exclusion: 11.

403. Hayes HB, Jayaraman A, Herrmann M, et al. Daily intermittent hypoxia enhances walking after chronic spinal cord injury: a randomized trial. Neurology. 2014 Jan 14;82(2):104-13. doi: 10.1212/01.WNL.0000437416.34298.43. PMID: 24285617. Exclusion: 11. 
404. Hayes SC, James Wilcox CR, Forbes White HS, et al. The effects of robot assisted gait training on temporal-spatial characteristics of people with spinal cord injuries: a systematic review. J Spinal Cord Med. 2018 Sep;41(5):529-43. doi: 10.1080/10790268.2018.1426236. PMID: 29400988. Exclusion: 2.

405. Hebert JR, Corboy JR, Manago MM, et al. Effects of vestibular rehabilitation on multiple sclerosis-related fatigue and upright postural control: a randomized controlled trial. Ann Neurol. 2012 Dec;72(S16):S34-5. doi: 10.1002/ana.23769. Exclusion: 10.

406. Hebert JR, Corboy JR, Vollmer T, et al. Efficacy of balance and eye-movement exercises for persons with multiple sclerosis (BEEMS). Neurology. 2018 Feb 27;90(9):797-807. doi: 10.1212/wnl.0000000000005013. PMID: 29386274. Exclusion: 4.

407. Hegarty AK, Kurz MJ, Stuberg W, et al. Changes in mobility and muscle function of children with cerebral palsy after gait training: a pilot study. J Appl Biomech. 2016 Oct;32(5):469-86. doi: 10.1123/jab.2015-0311. PMID: 27348240. Exclusion: 7.

408. Heine M, van de Port I, Rietberg MB, et al. Exercise therapy for fatigue in multiple sclerosis. Cochrane Database Syst Rev. 2015 Sep 11(9) doi: 10.1002/14651858.CD009956.pub2. PMID: 26358158. Exclusion: 6.

409. Hemachithra C, Meena N, Ramanathan R, et al. Immediate effect of horse riding simulator on adductor spasticity in children with cerebral palsy: A randomized controlled trial. Physiother Res Int. 2020 Jan;25(1) doi: 10.1002/pri.1809. PMID: 31502387. Exclusion: 4.

410. Heneidy WE, Eltalawy HA, Kassem HI, et al. Impact of task-oriented training on balance in spastic hemiplegic cerebral palsied children. Physiotherapy quarterly. 2020;28(2):52-6. Exclusion: 14.
411. Hernandez ME, Holtzer R, Chaparro G, et al. Brain activation changes during locomotion in middle-aged to older adults with multiple sclerosis. J Neurol Sci. 2016 Nov 15;370:277-83. doi: 10.1016/j.jns.2016.10.002. PMID: 27772776. Exclusion: 11.

412. Herring MP, Fleming KM, Hayes SP, et al. Moderators of exercise effects on depressive symptoms in multiple sclerosis: a metaregression. Am J Prev Med. 2017 Oct;53(4):508-18. doi: 10.1016/j.amepre.2017.04.011. PMID: 28602542. Exclusion: 12.

413. Hilderley AJ, Fehlings D, Lee GW, et al. Comparison of a robotic-assisted gait training program with a program of functional gait training for children with cerebral palsy: design and methods of a two group randomized controlled cross-over trial. Springerplus. 2016;5(1):1886. doi: 10.1186/s40064-016-3535-0. PMID: 27843743. Exclusion: 10.

414. Hilderley AJ, Fehlings D, Taylor M, et al. BeFAST or BeSTRONG: a randomized control trial comparing sports skill training to lower extremity strength training for children who have cerebral palsy. Dev Med Child Neurol. 2018 Oct;60:30-. doi: 10.1111/dmcn.44_14017. Exclusion: 10.

415. Hilgers C, Mundermann A, Riehle H, et al. Effects of whole-body vibration training on physical function in patients with multiple sclerosis. NeuroRehabilitation. 2013;32(3):655-63. doi: 10.3233/NRE130888. PMID: 23648620. Exclusion: 4.

416. Hiremath SV, Amiri AM, Thapa-Chhetry B, et al. Mobile health-based physical activity intervention for individuals with spinal cord injury in the community: A pilot study. PLoS One. 2019;14(10):e0223762. doi: 10.1371/journal.pone.0223762. PMID: 31613909. Exclusion: 7.

417. Hiremath SV, Amiri AM, Thapa-Chhetry B, et al. Correction: Mobile health-based physical activity intervention for individuals with spinal cord injury in the community: A pilot study. PLoS One. 2019;14(11) doi: 10.1371/journal.pone.0225490. PMID: 31725792. Exclusion: 10. 
418. Hoang P, Lord S, Gandevia S, et al. Effects of a home-based step training program on balance, stepping, cognition and functional performance in people with multiple sclerosis-a randomized controlled trial. Mult Scler J Exp Transl Clin. 2015 Oct 21(14):11. doi: $10.1177 / 1352458515616527$. Exclusion: 4.

419. Hoang P, Schoene D, Gandevia S, et al. Effects of a home-based step training programme on balance, stepping, cognition and functional performance in people with multiple sclerosis--a randomized controlled trial. Mult Scler. 2016 Jan;22(1):94-103. doi: $10.1177 / 1352458515579442$. PMID: 25921035. Exclusion: 4.

420. Hoare B, Imms C, Villanueva E, et al. Intensive therapy following upper limb botulinum toxin A injection in young children with unilateral cerebral palsy: a randomized trial. Dev Med Child Neurol. 2013 Mar;55(3):238-47. doi:

10.1111/dmcn.12054. PMID: 23236956. Exclusion: 4.

421. Hobbs D. Can a novel 'serious gaming' technology improve upper limb sensation and function in children with cerebral palsy? A population-based cohort study and pilot randomised controlled trial. Dev Med Child Neurol. 2020 or 2018?;62:74. doi: 10.1111/dmcn.14469. Exclusion: 11.

422. Hoekstra F, van Nunen MP, Gerrits KH, et al. Effect of robotic gait training on cardiorespiratory system in incomplete spinal cord injury. J Rehabil Res Dev. 2013;50(10):1411-22. doi: 10.1682/JRRD.2012.10.0186. PMID: 24699976. Exclusion: 11.

423. Hoekstra S, Valent L, Gobets D, et al. Effects of four-month handbike training under free-living conditions on physical fitness and health in wheelchair users. Disabil Rehabil. 2017 Aug;39(16):1581-8. doi: $10.1080 / 09638288.2016 .1200677$. PMID: 27385560. Exclusion: 8.

424. Holleran CL, Hennessey PW, Leddy AL, et al. High-intensity variable stepping training in patients with motor incomplete spinal cord Injury: a case series. J Neurol Phys Ther. 2018 Apr;42(2):94-101. doi: 10.1097/NPT.0000000000000217. PMID: 29547484. Exclusion: 7.
425. Horner-Johnson W, Drum CE, Abdullah N. A randomized trial of a health promotion intervention for adults with disabilities. Disabil Health J. 2011 Oct;4(4):254-61. doi: 10.1016/j.dhjo.2011.06.003. PMID: 22014673. Exclusion: 3.

426. Hosl M, Bohm H, Eck J, et al. Effects of backward-downhill treadmill training versus manual static plantarflexor stretching on muscle-joint pathology and function in children with spastic cerebral palsy. Gait Posture. 2018 Sep;65:121-8. doi: 10.1016/j.gaitpost.2018.07.171. PMID: 30055388. Exclusion: 11.

427. Hosseini SS, Rajabi H, Sahraian MA, et al. Effects of 8-week home-based yoga and resistance training on muscle strength, functional capacity and balance in patients with multiple sclerosis: a randomized controlled study. Asian J Sports Med. 2018;9(3):1-7. doi: 10.5812/asjsm.68807. Exclusion: 11.

428. Hou J, Nelson R, Mohammad N, et al. Effect of Simultaneous Combined Treadmill Training and Magnetic Stimulation on Spasticity and Gait Impairments after Cervical Spinal Cord Injury. J Neurotrauma. 2020 Jul 20;20:20. doi: 10.1089/neu.2019.6961. PMID: 32340533. Exclusion: 15.

429. Hoyme D. Body weight supported treadmill training versus gait trainer in young children with cerebral palsy. Dissertation Abstracts International: Section B: The Sciences and Engineering. 2017;78(4-B(E)):No Pagination Specified. Exclusion: 12.

430. Hsieh YL, Yang CC, Sun SH, et al. Effects of hippotherapy on body functions, activities and participation in children with cerebral palsy based on ICF-CY assessments. Disabil Rehabil. 2017 Aug;39(17):1703-13. doi: 10.1080/09638288.2016.1207108. PMID: 27440177. Exclusion: 11.

431. Hsu CW, Kang YN, Tseng SH. Effects of therapeutic exercise intensity on cerebral palsy outcomes: a systematic review with meta-regression of randomized clinical trials. Front Neurol. 2019;10:657. doi: 10.3389/fneur.2019.00657. PMID: 31293501. Exclusion: 12. 
432. Huang M, Liao LR, Pang MY. Effects of whole body vibration on muscle spasticity for people with central nervous system disorders: a systematic review. Clin Rehabil. 2017 Jan;31(1):23-33. doi: 10.1177/0269215515621117. PMID: 26658333. Exclusion: 12.

433. Hubbard EA. The acute effects of highintensity interval and continuous aerobic exercise on physiological and functional outcomes in persons with multiple sclerosis. Dissertation abstracts International: Section B: The Sciences and Engineering. 2019;80(5-B(E)):No Pagination Specified. Exclusion: 10.

434. Hubli M, Currie KD, West CR, et al. Physical exercise improves arterial stiffness after spinal cord injury. J Spinal Cord Med. 2014 Nov;37(6):782-5. doi: 10.1179/2045772314Y.0000000232. PMID: 24976366. Exclusion: 6.

435. Hughes L, Donnelly A, Rainsford G, et al. The clinical outcomes of augmenting an exercise programme with neuromuscular electrical stimulation in people with multiple sclerosis. Physiotherapy. 2011;97.

Exclusion: 10.

436. Hughes L, Donnelly A, Rainsford G, et al. Augmenting an exercise programme with neuromuscular electrical stimulation for people with multiple sclerosis-the effect on strength and fatigue. Physiotherapy. 2011;97. Exclusion: 10.

437. Hugos CL, Bourdette D, Chen Y, et al. A group-delivered self-management program reduces spasticity in people with multiple sclerosis: a randomized, controlled pilot trial. Mult Scler J Exp Transl Clin. 2017 Mar 23;3(1) doi: 10.1177/2055217317699993. PMID: 28607753. Exclusion: 4.

438. Hugos CL, Cameron MH. MS Spasticity: Take Control (STC) for ambulatory adults: protocol for a randomized controlled trial. BMC Neurol. 2020 Oct 07;20(1):368. doi: 10.1186/s12883-020-01902-1. PMID: 33028236. Exclusion: 4.
439. Hugos CL, Cameron MH, Chen Y, et al. Effects of a group spasticity management program versus usual care in people with multiple sclerosis: a randomized controlled pilot trial. Multiple sclerosis. Conference. 2017 Feb;23(1):88-9. doi: 10.1177/1352458517693959. Exclusion: 10.

440. Hugos CL, Chen Z, Chen Y, et al. A multicenter randomized controlled trial of two group education programs for fatigue in multiple sclerosis: Short- and medium-term benefits. Mult Scler. 2019 02;25(2):275-85. doi: $10.1177 / 1352458517745723$. PMID: 29226778. Exclusion: 4.

441. Hugos CL, Frankel D, Tompkins SA, et al. Community delivery of a comprehensive fall-prevention program in people with multiple sclerosis: A retrospective observational study. Int J MS Care. 2016 Jan-Feb;18(1):42-8. doi: 10.7224/15372073.2014-086. PMID: 26917997. Exclusion: 4.

442. Huisinga JM, Filipi ML, Stergiou N. Elliptical exercise improves fatigue ratings and quality of life in patients with multiple sclerosis. J Rehabil Res Dev.

2011;48(7):881-90. PMID: 21938671. Exclusion: 11.

443. Huisinga JM, Filipi ML, Stergiou N. Supervised resistance training results in changes in postural control in patients with multiple sclerosis. Motor Control. 2012 Jan;16(1):50-63. PMID: 22402220. Exclusion: 11.

444. Huisinga JM, Schmid KK, Filipi ML, et al. Persons with multiple sclerosis show altered joint kinetics during walking after participating in elliptical exercise. J Appl Biomech. 2012 Jul;28(3):249-57. PMID: 21975419. Exclusion: 11.

445. Hung Y-C, Spingarn A, Friel KM, et al. Intensive Unimanual Training Leads to Better Reaching and Head Control than Bimanual Training in Children with Unilateral Cerebral Palsy. Phys Occup Ther Pediatr. 2020;40(5):491-505. doi: 10.1080/01942638.2020.1712513. Exclusion: 4. 
446. Hussein ZA, Abd-Elwahab MS, ElShennawy SAW. Effect of arm cycling on gait of children with hemiplegic cerebral palsy. Egypt J Med Hum Genet. 2014 Jul;15(3):273-9. doi: 10.1016/j.ejmhg.2014.02.008. Exclusion: 14.

447. Hussein ZA, Salem IA, Ali MS. Effect of simultaneous proprioceptive-visual feedback on gait of children with spastic diplegic cerebral palsy. J Musculoskelet Neuronal Interact. 201912 01;19(4):500-6. PMID: 31789301. Exclusion: 14.

448. Hutchinson MJ, MacDonald MJ, Eston R, et al. Peak oxygen uptake measured during a perceptually-regulated exercise test is reliable in community-based manual wheelchair users. J Sports Sci. 2019 Mar;37(6):701-7. doi: 10.1080/02640414.2018.1522941. PMID: 30547732. Exclusion: 4.

449. Hutchinson MJ, Valentino SE, Totosy de Zepetnek JO, et al. Perceptually regulated training does not influence the differentiated RPE response following 16-weeks of aerobic exercise in adults with spinal cord injury. Appl Physiol Nutr Metab. 2019 Jun 28;28:28. doi: 10.1139/apnm-2019-0062. PMID: 31251892. Exclusion: 6.

450. Hutzler Y, Lamela Rodriguez B, Mendoza Laiz N, et al. The effects of an exercise training program on hand and wrist strength, and function, and activities of daily living, in adults with severe cerebral palsy. Res Dev Disabil. 2013 Dec;34(12):4343-54. doi: 10.1016/j.ridd.2013.09.015. PMID: 24145046. Exclusion: 11.

451. Hwang J, Shin Y, Park JH, et al. Effects of Walkbot gait training on kinematics, kinetics, and clinical gait function in paraplegia and quadriplegia.

NeuroRehabilitation. 2018;42(4):481-9. doi: 10.3233/NRE-172226. PMID: 29660947. Exclusion: 3.

452. Hwang S, Kim HR, Han ZA, et al. Improved gait speed after robot-assisted gait training in patients with motor incomplete spinal cord injury: a preliminary study. Ann Rehabil Med. 2017 Feb;41(1):34-41. doi: 10.5535/arm.2017.41.1.34. PMID: 28289633. Exclusion: 11.
453. Hwang YS, Kwon JY. Effects of modified constraint-induced movement therapy in real-world arm use in young children with unilateral cerebral palsy: A single-blind randomized trial. Neuropediatrics. $2020 \mathrm{Mar}$ 06;06:06. doi: 10.1055/s-0040-1702220. PMID: 32143221. Exclusion: 4.

454. Hyungkyu K, Jinhwa J, Jaeho Y. Effects of hippotherapy on the sitting balance of children with cerebral palsy: a randomized control trial. J Phys Ther Sci.

2012;24(9):833-6. doi: 10.1589/jpts.24.833. Exclusion: 6 .

455. Ibitoye MO, Hamzaid NA, Hasnan N, et al. Strategies for rapid muscle fatigue reduction during FES exercise in individuals with spinal cord injury: a systematic review. PLoS One. 2016;11(2) doi: 10.1371/journal.pone.0149024. PMID: 26859296. Exclusion: 6.

456. Ibrahim MM, Eid MA, Moawd SA. Effect of whole-body vibration on muscle strength, spasticity, and motor performance in spastic diplegic cerebral palsy children. Egypt J Med Hum Genet. 2014 Apr;15(2):173-9. doi: 10.1016/j.ejmhg.2014.02.007. Exclusion: 14.

457. Ishihara M, Higuchi $\mathrm{Y}$, Yonetsu R, et al. Plantarflexor training affects propulsive force generation during gait in children with spastic hemiplegic cerebral palsy: a pilot study. J Phys Ther Sci. 2015 May;27(5):1283-6. doi: 10.1589/jpts.27.1283. PMID: 26157201. Exclusion: 7.

458. Iturricastillo A, Yanci J, Granados C. Neuromuscular responses and physiological changes during small-sided games in wheelchair basketball. Adapt Phys Act Q. 2018 Jan 1;35(1):20-35. doi: 10.1123/apaq.2016-0139. PMID: 29256634. Exclusion: 11.

459. Jackel N, Tallner A, Virsevci O, et al. Effects of internet-based exercise (etraining) on fatigue and other patient reported behavioural outcomes (PRO) in patients with relapsing-remitting multiple sclerosis (RRMS). Mult Scler J Exp Transl Clin. 2015 Oct;21(S11):616. doi: 10.1177/1352458515602642. Exclusion: 7. 
460. Jackson K, Bigelow KE, Cooper C, et al. Effectiveness of group kickboxing as a means to improve gait and balance in individuals with multiple sclerosis. Int J MS Care. 2011 Winter;13(4):201. Exclusion: 7.

461. Jackson K, Edginton-Bigelow K, Bowsheir $\mathrm{C}$, et al. Feasibility and effects of a group kickboxing program for individuals with multiple sclerosis: a pilot report. J

Bodywork Mov Ther. 2012 Jan;16(1):7-13. doi: 10.1016/j.jbmt.2010.09.002. PMID: 22196421. Exclusion: 11.

462. Jackson KJ, Merriman HL, Vanderburgh PM, et al. Acute effects of whole-body vibration on lower extremity muscle performance in persons with multiple sclerosis. J Neurol Phys Ther. 2008 Dec;32(4):171-6. doi: 10.1097/NPT.0b013e31818ee760. PMID: 19265758. Exclusion: 11.

463. Jacobs PL. Effects of resistance and endurance training in persons with paraplegia. Med Sci Sports Exerc. 2009 May;41(5):992-7. doi: 10.1249/MSS.0b013e318191757f. PMID: 19346989. Exclusion: 11.

464. James S, Ziviani J, Ware RS, et al. Randomized controlled trial of web-based multimodal therapy for unilateral cerebral palsy to improve occupational performance. Dev Med Child Neurol. 2015 Jun;57(6):5308. doi: 10.1111/dmen.12705. PMID: 25955443. Exclusion: 4.

465. Jang CH, Joo MC, Noh SE, et al. Effects of hippotherapy on psychosocial aspects in children with cerebral palsy and their caregivers: a pilot study. Ann Rehabil Med. 2016 Apr;40(2):230-6. doi: 10.5535/arm.2016.40.2.230. PMID: 27152272. Exclusion: 11.

466. Jansen O, Grasmuecke D, Meindl RC, et al. Hybrid assistive limb exoskeleton HAL in the rehabilitation of chronic spinal cord injury: proof of concept; the results in 21 patients. World Neurosurg. 2018 Feb;110:73-8. doi: 10.1016/j.wneu.2017.10.080. PMID: 29081392. Exclusion: 8.
467. Jansen O, Schildhauer TA, Meindl RC, et al. Functional outcome of neurologic-controlled HAL-exoskeletal neurorehabilitation in chronic spinal cord injury: a pilot with one year treatment and variable treatment frequency. Global Spine J. 2017 Dec;7(8):735-43. doi: 10.1177/2192568217713754. PMID: 29238636. Exclusion: 11.

468. Jaume-i-Capo A, Martinez-Bueso P, MoyaAlcover B, et al. Interactive rehabilitation system for improvement of balance therapies in people with cerebral palsy. IEEE Trans Neural Syst Rehabil Eng. 2014 Mar;22(2):419-27. doi: 10.1109/TNSRE.2013.2279155. PMID: 24122561. Exclusion: 11.

469. Jayaraman A, Thompson CK, Rymer WZ, et al. Short-term maximal-intensity resistance training increases volitional function and strength in chronic incomplete spinal cord injury: a pilot study. J Neurol Phys Ther. 2013 Sep;37(3):112-7. doi: 10.1097/NPT.0b013e31828390a1. PMID: 23673372. Exclusion: 11.

470. Jayaraman P, Puckree T. The effects of a 12week program of static upper extremity weight bearing exercises on weight bearing in children with hemiplegic type of cerebral palsy. S Afr J Physiother. 2010;66(2):22-9. Exclusion: 11.

471. Jayaraman P, Puckree T. The effect of upper extremity weight bearing on upper extremity function in children with hemiplegic type of cerebral palsy. S Afr J Physiother. 2010;66(1):15-20. doi: 10.4102/sajp.v66i1.58. Exclusion: 11.

472. Jelsma J, Pronk M, Ferguson G, et al. The effect of the Nintendo Wii Fit on balance control and gross motor function of children with spastic hemiplegic cerebral palsy. Dev Neurorehabil. 2013;16(1):27-37. doi: 10.3109/17518423.2012.711781. PMID: 23030836. Exclusion: 11.

473. Jeng SC, Yeh KK, Liu WY, et al. A physical fitness follow-up in children with cerebral palsy receiving 12-week individualized exercise training. Res Dev Disabil. 2013 Nov;34(11):4017-24. doi: 10.1016/j.ridd.2013.08.032. PMID: 24036390. Exclusion: 11. 
474. Jeong JH, Yoo WG. Effects of air stacking on pulmonary function and peak cough flow in patients with cervical spinal cord injury. $\mathrm{J}$ Phys Ther Sci. 2015 Jun;27(6):1951-2. doi: 10.1589/jpts.27.1951. PMID: 26180355. Exclusion: 11.

475. Ji YH, Sun BD, Zhang J, et al. Therapeutic effect of scalp-acupuncture combined with exercise therapy on spastic cerebral palsy of the child. Zhongguo zhenjiu. 2008 Oct;28(10):723-6. PMID: 18972727. Exclusion: 13.

476. Jimenez-Morales RM, Herrera-Jimenez LF, Macias-Delgado Y, et al. Cognitive training combined with aerobic exercises in multiple sclerosis patients: a pilot study. Rev Neurol. 2017 Jun 1;64(11):489-95. PMID: 28555454. Exclusion: 13.

477. Jo HJ, Perez MA. Corticospinal-motor neuronal plasticity promotes exercisemediated recovery in humans with spinal cord injury. Brain. 2020 May

01;143(5):1368-82. doi: 10.1093/brain/awaa052. PMID: 32355959. Exclusion: 6.

478. Johnston TE, Betz RR, Lauer RT. Impact of cycling on hip subluxation in children with spinal cord injury. J Pediatr Orthop. 2009 Jun;29(4):402-5. doi: 10.1097/BPO.0b013e3181a5ec3b. PMID: 19461385. Exclusion: 4.

479. Johnston TE, Marino RJ, Oleson CV, et al. Musculoskeletal effects of 2 functional electrical stimulation cycling paradigms conducted at different cadences for people with spinal cord Injury: a pilot study. Arch Phys Med Rehabil. 2016 Sep;97(9):1413-22. doi: 10.1016/j.apmr.2015.11.014. PMID: 26705884. Exclusion: 11.

480. Johnston TE, Modlesky CM, Betz RR, et al. Muscle changes following cycling and/or electrical stimulation in pediatric spinal cord injury. Arch Phys Med Rehabil. 2011 Dec;92(12):1937-43. doi: 10.1016/j.apmr.2011.06.031. PMID: 22133240. Exclusion: 4.
481. Johnston TE, Smith BT, Mulcahey MJ, et al. A randomized controlled trial on the effects of cycling with and without electrical stimulation on cardiorespiratory and vascular health in children with spinal cord injury. Arch Phys Med Rehabil. 2009 Aug;90(8):1379-88. doi: 10.1016/j.apmr.2009.02.018. PMID: 19651272. Exclusion: 4.

482. Jolk C, Alcantara R, Bernhardt L, et al. Effects of different physical activities on the walking capacity for people with MS. Mult Scler J Exp Transl Clin. 2012

Oct;18(S4):482-3. doi: 10.1177/1352458512459021. Exclusion: 7.

483. Jolk C, Alcantara R, Bernhardt L, et al. Effects of 24 weeks progressive resistance training in comparison to core and stability training performed in groups on muscle performance, balance and spasticity in people with multiple sclerosis. Mult Scler J Exp Transl Clin. 2012 Oct;18(4 SUPPL. 1):250-1. doi: 10.1177/1352458512459019. Exclusion: 7.

484. Jonsdottir J, Bertoni R, Lawo M, et al. Serious games for arm rehabilitation of persons with multiple sclerosis. A randomized controlled pilot study. Mult Scler Relat Disord. 2018 Jan;19:25-9. doi: 10.1016/j.msard.2017.10.010. PMID: 29112939. Exclusion: 11.

485. Jorgensen ML, Dalgas U, Kjolhede T, et al. Serum brain-derived neurotrophic factor is not a mediator of neuroplasticity induced by resistance training in persons with multiple sclerosis. Mult Scler. 2018;Conference: 23rd annual RIMS conference. 2018. Netherlands 24(6):874. doi:

10.1016/j.exger.2017.03.019. PMID: 28392271. Exclusion: 10.

486. Jorissen W, Vanmierlo T, Wens I, et al. Twelve weeks of medium-intensity exercise therapy affects the lipoprotein profile of multiple sclerosis patients. Int J Mol Sci. 2018 Jan 8;19(1) doi: 10.3390/ijms19010193. PMID: 29316715. Exclusion: 8. 
487. Juckes FM, Marceniuk G, Seary C, et al. A cohort study of functional electrical stimulation in people with multiple sclerosis demonstrating improvements in quality of life and cost-effectiveness. Clin Rehabil. 2019 Jul;33(7):1163-70. doi: 10.1177/0269215519837326. PMID: 30971113. Exclusion: 4.

488. Jung JW, Her JG, Ko J. Effect of strength training of ankle plantarflexors on selective voluntary motor control, gait parameters, and gross motor function of children with cerebral palsy. J Phys Ther Sci. 2013 Oct;25(10):1259-63. doi: 10.1589/jpts.25.1259. PMID: 24259771. Exclusion: 11.

489. Kahraman T, Ozdogar AT, Yigit P, et al. Feasibility of a 6-Month yoga program to improve the physical and psychosocial status of persons with multiple sclerosis and their family members. Explore (NY). 2018 Jan - Feb;14(1):36-43. doi: 10.1016/j.explore.2017.07.006. PMID: 29174060. Exclusion: 11.

490. Kahraman T, Savci S, Coskuner-Poyraz E, et al. Determinants of physical activity in minimally impaired people with multiple sclerosis. Clin Neurol Neurosurg.

2015;138:20-4. doi: 10.1016/j.clineuro.2015.07.018. PMID: 26264722. Exclusion: 4.

491. Kakade NY, Kanase SB. Effect of core stability and functional mobility exercises on muscle strength after lumbar spinal cord injury. Indian J Public Health Res Dev. 2020;11(3):996-1001. Exclusion: 8.

492. Kalron A, Frid L, Berkowitz S, et al. Changes in gait and balance in people with multiple sclerosis attending a 12-week pilates exercise program. Multiple sclerosis. Conference: 32 nd congress of the european committee for treatment and research in multiple sclerosis, ECTRIMS. 2016 Sep;22(S3):396. Exclusion: 10.

493. Kannan M, Hildebrand A, Hugos CL, et al. Evaluation of a web-based fall prevention program among people with multiple sclerosis. Mult Scler Relat Disord. 2019 Jun;31:151-6. doi: 10.1016/j.msard.2019.04.015. PMID: 31004969. Exclusion: 4.
494. Karami F, Afrasiabifar A, Doulatabad SN. Comparing the effectiveness of vestibular rehabilitation and frenkel exercise on fatigue reduction in patients with multiple sclerosis: a randomized controlled trial. Iran Red Crescent Med J. 2018;20(12) doi: 10.5812/ircmj.68913. Exclusion: 6.

495. Karbandi S, Gorji MA, Mazloum SR, et al. Effectiveness of group versus individual yoga exercises on fatigue of patients with multiple sclerosis. N Am J Med Sci. 2015 Jun;7(6):266-70. doi: 10.4103/19472714.159332. PMID: 26199923. Exclusion: 6.

496. Karelis AD, Carvalho LP, Castillo MJ, et al. Effect on body composition and bone mineral density of walking with a robotic exoskeleton in adults with chronic spinal cord injury. J Rehabil Med. 2017 Jan 19;49(1):84-7. doi: 10.2340/16501977-2173. PMID: 27973679. Exclusion: 11.

497. Kargarfard M, Etemadifar M, Baker P, et al. Effect of aquatic exercise training on fatigue and health-related quality of life in patients with multiple sclerosis. Arch Phys Med Rehabil. 2012 Oct;93(10):1701-8. doi: 10.1016/j.apmr.2012.05.006. PMID: 22609300. Exclusion: 11.

498. Karpatkin H, Cohen ET, Rzetelny A, et al. Effects of intermittent versus continuous walking on distance walked and fatigue in persons with multiple sclerosis: a randomized crossover trial. J Neurol Phys Ther. 2015 Jul;39(3):172-8. doi: 10.1097/NPT.0000000000000091. PMID: 26050076. Exclusion: 11.

499. Karpatkin HI, Cohen ET, Klein S, et al. The effect of maximal strength training on strength, walking, and balance in people with multiple sclerosis: a pilot study. Mult Scler Int. 2016;2016:5235971. doi: 10.1155/2016/5235971. PMID: 28116161. Exclusion: 11.

500. Kassee C, Hunt C, Holmes MWR, et al. Home-based Nintendo Wii training to improve upper-limb function in children ages 7 to 12 with spastic hemiplegic cerebral palsy. J Pediatr Rehabil Med. 2017 May 17;10(2):145-54. doi: 10.3233/PRM170439. PMID: 28582885. Exclusion: 4. 
501. Kasser SL, Jacobs JV, Ford M, et al. Effects of balance-specific exercises on balance, physical activity and quality of life in adults with multiple sclerosis: a pilot investigation. Disabil Rehabil. 2015;37(24):2238-49. doi: 10.3109/09638288.2015.1019008. PMID: 25738911. Exclusion: 11.

502. Katusic A, Alimovic S, Mejaski-Bosnjak V. The effect of vibration therapy on spasticity and motor function in children with cerebral palsy: a randomized controlled trial. NeuroRehabilitation. 2013;32(1):1-8. doi: 10.3233/NRE-130817. PMID: 23422453. Exclusion: 4.

503. Katz-Leurer M, Rotem H, Keren O, et al. The effects of a 'home-based' task-oriented exercise programme on motor and balance performance in children with spastic cerebral palsy and severe traumatic brain injury. Clin Rehabil. 2009 Aug;23(8):71424. doi: $10.1177 / 0269215509335293$. PMID: 19506005. Exclusion: 3.

504. Keles MN, Elbasan B, Apaydin U, et al. Effects of inspiratory muscle training in children with cerebral palsy: a randomized controlled trial. Braz J Phys Ther. 2018 Nov;22(6):493-501. doi: 10.1016/j.bjpt.2018.03.010. PMID: 29636305. Exclusion: 4.

505. Kemp BJ, Bateham AL, Mulroy SJ, et al. Effects of reduction in shoulder pain on quality of life and community activities among people living long-term with SCI paraplegia: a randomized control trial. J Spinal Cord Med. 2011;34(3):278-84. doi: 10.1179/107902611X12972448729486. PMID: 21756566. Exclusion: 4.

506. Kenyon LKPDPP, Westman MPD, Hefferan APD, et al. A home-based body weight supported treadmill training program for children with cerebral palsy: a case series. Physiother Theory Pract. 2017 Jul;33(7):576-85. doi: 10.1080/09593985.2017.1325956. PMID: 28557625. Exclusion: 11.

507. Kersten S, Mahli M, Drosselmeyer J, et al. A pilot study of an exercise-based patient education program in people with multiple sclerosis. Mult Scler Int. 2014;2014:306878. doi: 10.1155/2014/306878. PMID: 25587449. Exclusion: 11.
508. Keser I, Kirdi N, Meric A, et al. Comparing routine neurorehabilitation program with trunk exercises based on Bobath concept in multiple sclerosis: pilot study. J Rehabil Res Dev. 2013;50(1):133-40. PMID: 23516089. Exclusion: 11.

509. Keytsman C, Hansen D, Wens I, et al. Impact of high-intensity concurrent training on cardiovascular risk factors in persons with multiple sclerosis - pilot study. Disabil Rehabil. 2017 Oct 27;41(4):430-5. doi: 10.1080/09638288.2017.1395086. PMID: 29076386. Exclusion: 11.

510. Keytsman C, Van Noten P, Spaas J, et al. Periodized home-based training: a new strategy to improve high intensity exercise therapy adherence in mildly affected patients with multiple sclerosis. Mult Scler Relat Disord. 2019;28:91-7. doi: 10.1016/j.msard.2018.12.018. PMID: 30576848. Exclusion: 7.

511. Khan AS, Patrick SK, Roy FD, et al. Training-specific neural plasticity in spinal reflexes after incomplete spinal cord injury. Neural Plast. 2016;2016:6718763. doi: 10.1155/2016/6718763. PMID: 27725887. Exclusion: 11.

512. Khanal D. Effectiveness of pelvic proprioceptive neuromuscular facilitation technique on facilitation of trunk movement in hemiparetic stroke patients. 4th EuroGlobal Physiotherapy Congress 2017. December 07-08, 2017. Rome, Italy. J Phys Ther Sports Med. 2017. Exclusion: 10.

513. Khichadiya PM, Kanase SB. Effect of specific transverse abdominal muscle strengthening and conventional therapy for trunk control in paraplegic subjects. Indian J Physiother Occup Ther. 2017;11(2):184-9. doi: 10.5958/0973-5674.2017.00058.2. Exclusion: 6.

514. Khurana M, Walia S, Noohu MM. Study on the effectiveness of virtual reality gamebased training on balance and functional performance in individuals with paraplegia. Top Spinal Cord Inj Rehabil. 2017;23(3):263-70. doi: 10.1310/sci1600003. PMID: 29339902. Exclusion: 14. 
515. Khurana S, Singh P, Razdan S. Effect of diaphragmatic breathing techniques on perceived exertion and cardiovascular variables during resistance exercises performed by tetraplegic rugby athletes. Arch Phys Med Rehabil. 2016; Conference:. 2016 american congress of rehabilitation medicine annual conference, ACRM 2016. United states. Conference start: 20161030. Conference end: 20161104 97(12):e34. Exclusion: 10.

516. Kierkegaard M, Lundberg IE, Olsson T, et al. High-intensity resistance training in multiple sclerosis - An exploratory study of effects on immune markers in blood and cerebrospinal fluid, and on mood, fatigue, health-related quality of life, muscle strength, walking and cognition. J Neurol Sci. 2016 Mar 15;362:251-7. doi: 10.1016/j.jns.2016.01.063. PMID: 26944158. Exclusion: 11.

517. Kim CY, Lee JS, Kim HD, et al. Short-term effects of respiratory muscle training combined with the abdominal drawing-in maneuver on the decreased pulmonary function of individuals with chronic spinal cord injury: a pilot randomized controlled trial. J Spinal Cord Med. 2017 Jan;40(1):1725. doi: $10.1080 / 10790268.2016 .1198576$. PMID: 27463071. Exclusion: 4.

518. Kim DA, Lee JA, Hwang PW, et al. The effect of comprehensive hand repetitive intensive strength training (CHRIST) using motion analysis in children with cerebral palsy. Ann Rehabil Med. 2012 Feb;36(1):39-46. doi: 10.5535/arm.2012.36.1.39. PMID: 22506234. Exclusion: 11.

519. Kim DI, Lee H, Lee BS, et al. Effects of a 6Week indoor hand-bike exercise program on health and fitness levels in people with spinal cord injury: a randomized controlled trial study. Arch Phys Med Rehabil. 2015

Nov;96(11):2033-40.e1. doi: 10.1016/j.apmr.2015.07.010. PMID: 26254953. Exclusion: 11.
520. Kim MR, Lee BH, Park DS. Effects of combined Adeli suit and neurodevelopmental treatment in children with spastic cerebral palsy with gross motor function classification system levels I and II. Hong Kong Physiother J. 2016 Jun;34:10-8. doi: 10.1016/j.hkpj.2015.09.036. PMID: 30931022. Exclusion: 11.

521. Kimoto M, Yonetsu R, Okada K, et al. Effect of home-based training focused on increasing maximum step length in walking function of children with cerebral palsy. Physical Therapy Reviews. 2019;24(6):35865. doi: 10.1080/10833196.2019.1664083. Exclusion: 4.

522. Kinnett-Hopkins D, Motl R. Results of a feasibility study of a patient informed, racially tailored home-based exercise program for black persons with multiple sclerosis. Contemp Clin Trials. 2018 Oct 17;17:17. doi: 10.1016/j.cct.2018.10.009. PMID: 30342254. Exclusion: 11.

523. Kjolhede T, Dalgas U, Gade AB, et al. Progressive resistance training improves muscle strength and function, but does not affect circulating cytokines for people with multiple sclerosis treated with interferonbeta. Mult Scler J Exp Transl Clin. Apr 10 21(4):526. Exclusion: 10.

524. Kjolhede T, Siemonsen S, Wenzel D, et al. Can resistance training impact MRI outcomes in relapsing-remitting multiple sclerosis? Mult Scler. 2018 Sep;24(10):1356-65. doi: 10.1177/1352458517722645. PMID: 28752800. Exclusion: 11.

525. Kjolhede T, Vissing K, de Place L, et al. Neuromuscular adaptations to long-term progressive resistance training translates to improved functional capacity for people with multiple sclerosis and is maintained at follow-up. Mult Scler. 2015 Apr;21(5):599611. doi: $10.1177 / 1352458514549402$. PMID: 25257612. Exclusion: 11.

526. Kjolhede T, Vissing K, Stenager E, et al. Long-term progressive resistance training improves functional capacity for people with mild-moderate multiple sclerosis: preliminary data. Mult Scler J Exp Transl Clin. 2013 Oct;19(11 SUPPL. 1):556-7. doi: 10.1177/1352458513502429. Exclusion: 7. 
527. Klaren RE, Hubbard EA, Motl RW. Efficacy of a behavioral intervention for reducing sedentary behavior in persons with multiple sclerosis: a pilot examination. Am J Prev Med. 2014 Nov;47(5):613-6. doi: 10.1016/j.amepre.2014.05.036. PMID: 25070271. Exclusion: 4.

528. Klingels K, Feys H, Molenaers G, et al. Randomized trial of modified constraintinduced movement therapy with and without an intensive therapy program in children with unilateral cerebral palsy. Neurorehabil Neural Repair. 2013 Nov-Dec;27(9):799807. doi: $10.1177 / 1545968313496322$. PMID: 23901061. Exclusion: 4.

529. Knight S, Fetters L. Clinical bottom line. Intensive motor skills training program combining group and individual sessions for children with cerebral palsy. Pediatr Phys Ther. 2010;22(2):160. doi: 10.1097/PEP.0b013e3181dced27. PMID: 20473098. Exclusion: 11.

530. Knights S, Graham N, Switzer L, et al. An innovative cycling exergame to promote cardiovascular fitness in youth with cerebral palsy. Dev Neurorehabil. 2016;19(2):13540. doi: 10.3109/17518423.2014.923056. PMID: 24950349. Exclusion: 11.

531. Knikou M, Mummidisetty CK. Locomotor training improves premotoneuronal control after chronic spinal cord injury. J Neurophysiol. 2014 Jun 1;111(11):2264-75. doi: $10.1152 /$ jn.00871.2013. PMID: 24598526. Exclusion: 11.

532. Knikou M, Smith AC, Mummidisetty CK. Locomotor training improves reciprocal and nonreciprocal inhibitory control of soleus motoneurons in human spinal cord injury. J Neurophysiol. 2015 Apr 1;113(7):2447-60. doi: $10.1152 /$ jn.00872.2014. PMID: 25609110. Exclusion: 11.

533. Ko M-S, Doo J-H, Kim J-S, et al. Effect of whole body vibration training on gait function and activities of daily living in children with cerebral palsy. Int J Ther Rehabil. 2015;22(7):321-8. doi: 10.12968/ijtr.2015.22.7.321. Exclusion: 4.

534. Ko Y, Kim H, Chio E, et al. Do virtual reality sports games improve sports specific skills in unilateral cerebral palsy patients? Dev Med Child Neurol. 2013 Oct;55:12-3. doi: 10.1111/dmen.12258. Exclusion: 7.
535. Kobetic R, To CS, Schnellenberger JR, et al. Development of hybrid orthosis for standing, walking, and stair climbing after spinal cord injury. J Rehabil Res Dev. 2009;46(3):447-62. PMID: 19675995. Exclusion: 10.

536. Kooijmans H, Post MWM, Stam HJ, et al. Effectiveness of a self-management intervention to promote an active lifestyle in persons with long-term spinal cord injury: the HABITS randomized clinical trial. Neurorehabil Neural Repair. 2017 Dec;31(12):991-1004. doi: 10.1177/1545968317736819. PMID: 29256337. Exclusion: 4.

537. Koontz AM, Garfunkel CE, Crytzer TM, et al. Feasibility, acceptability, and preliminary efficacy of a handcycling high-intensity interval training program for individuals with spinal cord injury. Spinal Cord. 2020 Sep 09;09:09. doi: 10.1038/s41393-02000548-7. PMID: 32908194. Exclusion: 4.

538. Koopman AD, Eken MM, van Bezeij T, et al. Does clinical rehabilitation impose sufficient cardiorespiratory strain to improve aerobic fitness? J Rehabil Med. 2013 Jan;45(1):92-8. doi: 10.2340/165019771072. PMID: 23096222. Exclusion: 4.

539. Kordi MR, Anooshe L, Khodadade S, et al. Comparing the effect of three methods of combined training on serum levels of Ghrelin, pro and anti-inflammatory cytokines in multiple sclerosis (MS) patients. Journal of Zanjan University of Medical Sciences and Health Services. 2013;22(91):39-51. Exclusion: 13.

540. Kott K, Lesher K, DeLeo G. Combining a virtual reality system with treadmill training for children with cerebral palsy. J Cyber Ther Rehabil. 2009;2(1):35-42. doi: 10.1186/1471-2431-13-168. PMID: 24112817 Exclusion: 11.

541. Kowalczewski J, Chong SL, Galea M, et al. In-home tele-rehabilitation improves tetraplegic hand function. Neurorehabil Neural Repair. 2011 Jun;25(5):412-22. doi: 10.1177/1545968310394869. PMID: 21372246. Exclusion: 11. 
542. Kramer A, Dettmers C, Gruber M. Exergaming with additional postural demands improves balance and gait in patients with multiple sclerosis as much as conventional balance training and leads to high adherence to home-based balance training. Arch Phys Med Rehabil. 2014 Oct;95(10):1803-9. doi: 10.1016/j.apmr.2014.04.020. PMID: 24823959. Exclusion: 4.

543. Kratz AL, Atalla M, Whibley D, et al. Calling Out MS Fatigue: Feasibility and Preliminary Effects of a Pilot Randomized Telephone-Delivered Exercise Intervention for Multiple Sclerosis Fatigue. J Neurol Phys Ther. 2020 Jan;44(1):23-31. doi: 10.1097/NPT.0000000000000296. PMID: 31738192. Exclusion: 11.

544. Kratz AL, Ehde DM, Bombardier CH. Affective mediators of a physical activity intervention for depression in multiple sclerosis. Rehabil Psychol. 2014 Feb;59(1):57-67. doi: 10.1037/a0035287. PMID: 24611925. Exclusion: 4.

545. Kratz AL, Molton IR, Jensen MP, et al. Further evaluation of the motivational model of pain self-management: coping with chronic pain in multiple sclerosis. Ann Behav Med. 2011 Jun;41(3):391-400. doi: 10.1007/s12160-010-9249-6. PMID: 21213092. Exclusion: 4.

546. Krause A, Schonau E, Duran I, et al. High frequency whole body vibration improves motor control in subjects with spastic cerebral palsy. Dev Med Child Neurol. 2016 Oct;58:44. doi: 10.1111/dmcn.94_13241. Exclusion: 6.

547. Krebs HI, Fasoli SE, Dipietro L, et al. Motor learning characterizes habilitation of children with hemiplegic cerebral palsy. Neurorehabil Neural Repair. 2012 Sep;26(7):855-60. doi: 10.1177/1545968311433427. PMID: 22331211. Exclusion: 11.

548. Kressler J, Burns PA, Betancourt L, et al. Circuit training and protein supplementation in persons with chronic tetraplegia. Med Sci Sports Exerc. 2014 Jul;46(7):1277-84. doi: 10.1249/MSS.0000000000000250. PMID: 24389521. Exclusion: 11.
549. Kressler J, Jacobs K, Burns P, et al. Effects of circuit resistance training and timely protein supplementation on exercise-induced fat oxidation in tetraplegic adults. Top Spinal Cord Inj Rehabil. 2014;20(2):113-22. doi: 10.1310/sci2002-113. PMID: 25477733. Exclusion: 11.

550. Kruse A, Schranz C, Svehlik M, et al. The effect of functional home-based strength training programs on the mechanomorphological properties of the plantar flexor muscle-tendon unit in children with spastic cerebral palsy. Pediatr Exerc Sci. 2019 Feb 1;31(1):67-76. doi: 10.1123/pes.2018-0106. PMID: 30424684. Exclusion: 4.

551. Kruse A, Schranz C, Tilp M, et al. Effects of progressive resistance or high-intensity strength training on muscle function and achilles tendon mechanical properties in children with cerebral palsy. Gait Posture. 2017; Conference: 26th annual meeting of the european society for movement analysis in adults and children, ESMAC. 2017. Norway 57(Supplement 1):6. Exclusion: 10.

552. Kubsik A, Klimkiewicz P, Klimkiewicz R, et al. The influence of high-tone power therapy on the functional status of patients with multiple sclerosis. Pol Merkuriusz Lek. 2014 Jul;37(217):24-9. PMID: 25154195. Exclusion: 13.

553. Kucuk F, Kara B, Poyraz EC, et al. Improvements in cognition, quality of life, and physical performance with clinical pilates in multiple sclerosis: a randomized controlled trial. J Phys Ther Sci. 2016 Mar;28(3):761-8. doi: 10.1589/jpts.28.761. PMID: 27134355. Exclusion: 11.

554. Kuhn D, Leichtfried V, Schobersberger W. Four weeks of functional electrical stimulated cycling after spinal cord injury: a clinical cohort study. Int J Rehabil Res.

2014 Sep;37(3):243-50. doi: 10.1097/MRR.0000000000000062. PMID: 24802976. Exclusion: 3.

555. Kumaresan A, Rabha D. Effects of quadriceps femoris muscle strengthening exercises on gait parameters in spastic diplegia with children with cerebral palsy. Biomedicine (India). 2017;37(4):501-5. Exclusion: 14. 
556. Kumari R, Verma M, Gupta A. To determine effectiveness of closed-kinetic chain exercise on motor control and function as compared to open-kinetic chain exercise in children with spastic diplegic CP. Indian J Physiother Occup Ther. 2014;8(2):33-7. Exclusion: 6.

557. Kurz MJ, Stuberg W, DeJong SL. Body weight supported treadmill training improves the regularity of the stepping kinematics in children with cerebral palsy. Dev Neurorehabil. 2011;14(2):87-93. doi: 10.3109/17518423.2011.552459. PMID: 21410400. Exclusion: 11.

558. Kusumoto Y, Nitta O, Takaki K. Impact of loaded sit-to-stand exercises at different speeds on the physiological cost of walking in children with spastic diplegia: a singleblind randomized clinical trial. Res Dev Disabil. 2016 Oct; 57:85-91. doi: 10.1016/j.ridd.2016.06.006. PMID: 27394691. Exclusion: 11.

559. Kwon HY, Kim BJ. Effects of task-specific movement patterns during resistance exercise on the respiratory functions and thickness of abdominal muscles of children with cerebral palsy: randomized placebocontrolled double-blinded clinical trial. J Phys Ther Sci. 2018 Aug;30(8):1073-80. doi: 10.1589/jpts.30.1073. PMID: 30154603. Exclusion: 11.

560. Kwon JY, Chang WH, Chang HJ, et al. Changes in diffusion tensor tractographic findings associated with constraint-induced movement therapy in young children with cerebral palsy. Clin Neurophysiol. 2014 Dec;125(12):2397-403. doi: 10.1016/j.clinph.2014.02.025. PMID: 24746686. Exclusion: 4.

561. Labaf S, Shamsoddini A, Taghi Hollisaz M, et al. Effects of neurodevelopmental therapy on gross motor function in children with cerebral palsy. Iran J Child Neurol. 2015;9(2):36-41. PMID: 26221161. Exclusion: 4.

562. Labruyere R, van Hedel HJ. Instrument validity and reliability of a choice response time test for subjects with incomplete spinal cord injury: relationship with function. Arch Phys Med Rehabil. 2011 Sep;92(9):1443-9. doi: 10.1016/j.apmr.2011.04.006. PMID: 21878215. Exclusion: 4.
563. Labruyere R, van Hedel HJ. Curve walking is not better than straight walking in estimating ambulation-related domains after incomplete spinal cord injury. Arch Phys Med Rehabil. 2012 May;93(5):796-801. doi: 10.1016/j.apmr.2011.11.009. PMID: 22386212. Exclusion: 4.

564. Labruyere R, van Hedel HJ. Strength training versus robot-assisted gait training after incomplete spinal cord injury: a randomized pilot study in patients depending on walking assistance. J Neuroengineering Rehabil. 2014 Jan 9;11:4. doi: 10.1186/1743-0003-11-4. PMID: 24401143. Exclusion: 11.

565. Lahelle AF, Oberg GK, Normann B. A group-based, individualized physiotherapy intervention for people with multiple sclerosis-a qualitative study. Physiother Res Int. 2018 Jul 23;23 doi: 10.1002/pri.1734. PMID: 30039598. Exclusion: 3.

566. Lahelle Af Pt MP, Oberg Gk Pt P, Normann B Pt P. Physiotherapy assessment of individuals with multiple sclerosis prior to a group intervention - a qualitative observational and interview study. Physiother Theory Pract. 2018 Jul 9:1-11. doi: 10.1080/09593985.2018.1488022. PMID: 29985730. Exclusion: 4.

567. Lai B, Cederberg K, Vanderbom KA, et al. Characteristics of adults with neurologic disability recruited for exercise trials: a secondary analysis. Adapt Phys Act Q. 2018;35(4):476-97. doi: 10.1123/apaq.20170109. Exclusion: 12.

568. Lai B, Rimmer J, Barstow B, et al. Teleexercise for persons with spinal cord injury: a mixed-methods feasibility case series. JMIR Rehabil Assist Technol. 2016 Jul 14;3(2) doi: 10.2196/rehab.5524. PMID: 28582252. Exclusion: 11.

569. Lai B, Young HJ, Bickel CS, et al. Current trends in exercise intervention research, technology, and behavioral change strategies for people with disabilities: a scoping review. Am J Phys Med Rehabil. 2017 Oct;96(10):748-61. doi: 10.1097/PHM.0000000000000743. PMID: 28398967. Exclusion: 12. 
570. Lai BH, Wu JB, Gao ZW, et al. Strategy by stages for preventing respiratory complications of acute cervical spinal cord injury. Zhongguo Gu Shang. 2015 Aug;28(8):690-4. PMID: 26502516. Exclusion: 13.

571. Lajeunesse V, Vincent C, Routhier F, et al. Exoskeletons' design and usefulness evidence according to a systematic review of lower limb exoskeletons used for functional mobility by people with spinal cord injury.

Disabil Rehabil Assist Technol. 2016

Oct;11(7):535-47. doi: 10.3109/17483107.2015.1080766. PMID: 26340538. Exclusion: 12.

572. Lakomy-Gawryszewska AA, Józefowicz K, Raniszewska A, et al. The impact of hippotherapy on the quality of trunk stabilisation, evaluated by EMG biofeedback, in children with infantile cerebral palsy. Polish Annals of Medicine. 2017;24(1):9-12. doi: 10.1016/j.poamed.2016.06.001. Exclusion: 8.

573. Lam T, Pauhl K, Ferguson A, et al. A new training paradigm using robot-applied resistance to enhance skilled walking in people with spinal cord injury.

Physiotherapy. 2015;101(1). Exclusion: 10.

574. Lam T, Pauhl K, Ferguson A, et al. Training with robot-applied resistance in people with motor-incomplete spinal cord injury: pilot study. J Rehabil Res Dev. 2015;52(1):11329. doi: 10.1682/JRRD.2014.03.0090. PMID: 26230667. Exclusion: 11.

575. Lamberti N, Straudi S, Donadi M, et al. Effectiveness of blood flow-restricted slow walking on mobility in severe multiple sclerosis: A pilot randomized trial. Scand J Med Sci Sports. 2020 Jul 09;09:09. doi: 10.1111/sms.13764. PMID: 32645227. Exclusion: 11.

576. Lan C, Chen SY, Lai JS, et al. Tai Chi Chuan in medicine and health promotion. Evid Based Complement Alternat Med. 2013;2013 doi: 10.1155/2013/502131. Exclusion: 10.
577. Langeskov-Christensen M, Heine M, Kwakkel G, et al. Aerobic capacity in persons with multiple sclerosis: a systematic review and meta-analysis. Sports Med. 2015 Jun;45(6):905-23. doi: 10.1007/s40279-0150307-x. PMID: 25739555. Exclusion: 12.

578. Larson RD, McCully KK, Larson DJ, et al. Bilateral differences in lower-limb performance in individuals with multiple sclerosis. J Rehabil Res Dev. 2013;50(2):215-22. PMID: 23761002. Exclusion: 5.

579. Larson RD, McCully KK, Larson DJ, et al. Lower-limb performance disparities: implications for exercise prescription in multiple sclerosis. J Rehabil Res Dev. 2014;51(10):1537-44. doi: 10.1682/JRRD.2013.09.0191. PMID: 25855905. Exclusion: 4.

580. Latimer-Cheung AE, Arbour-Nicitopoulos KP, Brawley LR, et al. Developing physical activity interventions for adults with spinal cord injury. Part 2: motivational counseling and peer-mediated interventions for people intending to be active. Rehabil Psychol. 2013 Aug;58(3):307-15. doi: 10.1037/a0032816. PMID: 23978086. Exclusion: 4.

581. Lau SCL, Fong M, Lenze E, et al. Psychometric evaluation of the center for epidemiologic studies depression scale (CES-D) for patients after stroke. Arch Phys Med Rehabil. 2019 Oct;100(10):26. doi: 10.1016/j.apmr.2019.08.060. Exclusion: 9.

582. Lauer RT, Johnston TE, Smith BT, et al. Lower extremity muscle activity during cycling in adolescents with and without cerebral palsy. Clin Biomech. 2008 May;23(4):442-9. doi: 10.1016/j.clinbiomech.2007.11.004. PMID: 18082920. Exclusion: 4.

583. Lauer RT, Smith BT, Mulcahey MJ, et al. Effects of cycling and/or electrical stimulation on bone mineral density in children with spinal cord injury. Spinal Cord. 2011 Aug;49(8):917-23. doi: 10.1038/sc.2011.19. PMID: 21423253. Exclusion: 4. 
584. Lauglo R, Vik T, Lamvik T, et al. Highintensity interval training to improve fitness in children with cerebral palsy. BMJ Open Sport Exerc Med. 2016;2(1) doi: 10.1136/bmjsem-2016-000111. PMID: 27900177. Exclusion: 11.

585. Lauruschkus K, Hallstrom I, Westbom L, et al. Participation in physical activities for children with cerebral palsy: feasibility and effectiveness of physical activity on prescription. Arch Physiother. 2017;7:13. doi: 10.1186/s40945-017-0041-9. PMID: 29340207. Exclusion: 4.

586. Lawrence H, Hills S, Kline N, et al. Effectiveness of exercise on functional mobility in adults with cerebral palsy: a systematic review. Physiother Can. 2016;68(4):398-407. doi: 10.3138/ptc.201538LHC. PMID: 27904240. Exclusion: 12.

587. Lazzari RD, Politti F, Belina SF, et al. Effect of transcranial direct current stimulation combined with virtual reality training on balance in children with cerebral palsy: a randomized, controlled, double-blind, clinical trial. J Mot Behav. 2017 MayJun;49(3):329-36. doi: 10.1080/00222895.2016.1204266. PMID: 27644454. Exclusion: 4.

588. Learmonth Y, Miller LJ, Paul L, et al. Excercise therapy for those moderately affected by multiple sclerosis: results of a 12-week community-based excercise programme. Mult Scler J Exp Transl Clin. 2011 Oct;17(10 SUPPL. 1):S477-S8. doi: 10.1177/1352458511422301. PMID: 21984532 Exclusion: 7.

589. Learmonth YC, Marshall-McKenna R, Paul $\mathrm{L}$, et al. A qualitative exploration of the impact of a 12-week group exercise class for those moderately affected with multiple sclerosis. Disabil Rehabil. 2013

Jan;35(1):81-8. doi: 10.3109/09638288.2012.688922. PMID: 22656959. Exclusion: 6.

590. Learmonth YC, Paul L, Miller L, et al. The effects of a 12-week leisure centre-based, group exercise intervention for people moderately affected with multiple sclerosis: a randomized controlled pilot study. Clin Rehabil. 2012 Jul;26(7):579-93. doi: 10.1177/0269215511423946. PMID: 21984532. Exclusion: 11.
591. Leavitt VM, Cirnigliaro C, Cohen A, et al. Aerobic exercise increases hippocampal volume and improves memory in multiple sclerosis: preliminary findings. Neurocase. 2014;20(6):695-7. doi: 10.1080/13554794.2013.841951. PMID: 24090098. Exclusion: 6.

592. Lee BH. Clinical usefulness of augmented reality using infrared camera based real-time feedback on gait function in cerebral palsy: a case study. J Phys Ther Sci. 2016 Apr;28(4):1387-91. doi: 10.1589/jpts.28.1387. PMID: 27190489. Exclusion: 7.

593. Lee DR, Kim YH, Kim DA, et al. Innovative strength training-induced neuroplasticity and increased muscle size and strength in children with spastic cerebral palsy: an experimenter-blind case study-three-month follow-up. NeuroRehabilitation. 2014;35(1):131-6. doi: 10.3233/NRE131036. PMID: 24419014. Exclusion: 7.

594. Lee JH, Sung IY, Yoo JY. Therapeutic effects of strengthening exercise on gait function of cerebral palsy. Disabil Rehabil. 2008;30(19):1439-44. PMID: 19230216. Exclusion: 11.

595. Lee Y, Chen K, Ren Y, et al. Robot-guided ankle sensorimotor rehabilitation of patients with multiple sclerosis. Mult Scler Relat Disord. 2017 Jan;11:65-70. doi: 10.1016/j.msard.2016.12.006. PMID: 28104260. Exclusion: 11.

596. Lee YH, Lee JH, Kim SH, et al. Hemodynamic adaptations to regular exercise in people with spinal cord injury. Ann Rehabil Med. 2017 Feb;41(1):25-33. doi: 10.5535/arm.2017.41.1.25. PMID: 28289632. Exclusion: 4.

597. Lee YH, Oh KJ, Kong ID, et al. Effect of regular exercise on cardiopulmonary fitness in males with spinal cord injury. Ann Rehabil Med. 2015 Feb;39(1):91-9. doi: 10.5535/arm.2015.39.1.91. PMID: 25750877. Exclusion: 4.

598. Lee YS, Kim WB, Park JW. The effect of exercise using a sliding rehabilitation machine on the gait function of children with cerebral palsy. J Phys Ther Sci. 2014 Nov;26(11):1667-9. doi: 10.1589/jpts.26.1667. PMID: 25435673. Exclusion: 11. 
599. Leech KA, Hornby TG. High-intensity locomotor exercise increases brain-derived neurotrophic factor in individuals with incomplete spinal cord injury. $\mathrm{J}$

Neurotrauma. 2017 Mar 15;34(6):1240-8. doi: 10.1089/neu.2016.4532. PMID:

27526567. Exclusion: 4.

600. Leech KA, Kinnaird CR, Holleran CL, et al. Effects of locomotor exercise intensity on gait performance in individuals with incomplete spinal cord injury. Phys Ther. 2016 Dec;96(12):1919-29. doi: 10.2522/ptj.20150646. PMID: 27313241. Exclusion: 11.

601. Lefmann S, Russo R, Hillier S. The effectiveness of robotic-assisted gait training for paediatric gait disorders: systematic review. J Neuroengineering Rehabil. 2017 Jan 5;14(1):1. doi: 10.1186/s12984-0160214-x. PMID: 28057016. Exclusion: 12.

602. Legg Ditterline BE, Aslan SC, Randall DC, et al. Effects of respiratory training on heart rate variability and baroreflex sensitivity in individuals with chronic spinal cord injury. Arch Phys Med Rehabil. 2018;99(3):423-32. doi: 10.1016/j.apmr.2017.06.033. Exclusion: 4.

603. Lenze EJ, Host HH, Hildebrand MW, et al. Enhanced medical rehabilitation increases therapy intensity and engagement and improves functional outcomes in postacute rehabilitation of older adults: a randomizedcontrolled trial. J Am Med Dir Assoc. 2012 Oct;13(8):708-12. doi:

10.1016/j.jamda.2012.06.014. PMID: 22863663. Exclusion: 3.

604. Lerma-Castaño PR, Rodríguez-Laiseca YA, Falla JD, et al. Effects of hippotherapy on gross motor function in children with spastic cerebral palsy: quasi-experimental study. Revista Mexicana de Pediatria. 2017 JulAug;84(4):143-8. doi:

10.3109/01942638.2015.1129386.

Exclusion: 13.

605. Leving MT, Vegter RJ, Hartog J, et al. Effects of visual feedback-induced variability on motor learning of handrim wheelchair propulsion. PLoS One. 2015;10(5) doi: 10.1371/journal.pone.0127311. PMID: 25992626. Exclusion: 3.
606. Lewis J. A progressive running program for an adolescent with cerebral palsy. Pediatr Phys Ther. 2017 07;29(3):E12-E6. doi: 10.1097/PEP.0000000000000429. PMID: 28654506. Exclusion: 7.

607. Li C, Khoo S, Adnan A. Effects of aquatic exercise on physical function and fitness among people with spinal cord injury: a systematic review. Medicine (Baltimore). 2017 Mar;96(11) doi: 10.1097/MD.0000000000006328. PMID: 28296754. Exclusion: 12.

608. Li J, Polston KFL, Eraslan M, et al. A highprotein diet or combination exercise training to improve metabolic health in individuals with long-standing spinal cord injury: a pilot randomized study. Physiol Rep. 2018 Aug 28;6(16) doi: 10.14814/phy2.13813. PMID: 30156033. Exclusion: 11.

609. Li W, Lam-Damji S, Chau T, et al. The development of a home-based virtual reality therapy system to promote upper extremity movement for children with hemiplegic cerebral palsy. Technol Disabil. 2009;21(3):107-13. doi: 10.3233/TAD2009-0277. Exclusion: 11.

610. Lin C-Y, Chang Y-M. Increase in physical activities in kindergarten children with cerebral palsy by employing MaKeyMaKey-based task systems. Res Dev Disabil. 2014 Sep;35(9):1963-9. doi: 10.1016/j.ridd.2014.04.028. Exclusion: 4.

611. Lin KC, Wang TN, Wu CY, et al. Effects of home-based constraint-induced therapy versus dose-matched control intervention on functional outcomes and caregiver wellbeing in children with cerebral palsy. Res Dev Disabil. 2011 Sep-Oct;32(5):1483-91. doi: 10.1016/j.ridd.2011.01.023. PMID: 21429706. Exclusion: 4.

612. Lins C, Castro A, Medina GIS, et al. Alternative scapular stabilization exercises to target strength, endurance and function of shoulders in tetraplegia: a prospective noncontrolled intervention study. J Spinal Cord Med. 2019 Nov 16;42(1):65-76. doi: 10.1080/10790268.2017.1398943. PMID: 29141513. Exclusion: 4. 
613. Litchke L, Lloyd L, Schmidt E, et al.

Comparison of two concurrent respiratory resistance devices on pulmonary function and time trial performance of wheel chair athletes. Ther Recreation J. 2010;44(1):5162. Exclusion: 4.

614. Litchke LG, Lloyd LK, Schmidt EA, et al. Effects of concurrent respiratory resistance training on health-related quality of life in wheelchair rugby athletes: a pilot study. Top Spinal Cord Inj Rehabil. 2012;18(3):264-72. doi: 10.1310/sci1803-264. PMID: 23459144. Exclusion: 4.

615. Litchke LG, Russian CJ, Lloyd LK, et al. Effects of respiratory resistance training with a concurrent flow device on wheelchair athletes. J Spinal Cord Med. 2008;31(1):6571. doi: $10.1080 / 10790268.2008 .11753983$. PMID: 18533414. Exclusion: 9.

616. Liu L, Chen K, Yang C, et al. Home- and lab-based robotic passive and active movement training of ankle impairments in cerebral palsy. Dev Med Child Neurol. 2013;55(25). Exclusion: 10.

617. Lopes S, Magalhaes P, Pereira A, et al. Games used with serious purposes: a systematic review of interventions in patients with cerebral palsy. Front Psychol. 2018 Sep 19;9(1712) doi: 10.3389/fpsyg.2018.01712. PMID: 30283377. Exclusion: 12.

618. Lorentzen J, Kirk H, Fernandez-Lago H, et al. Randomized controlled clinical trial of the effect of gait training on muscle function and gait kinematics in adults with cerebral palsy. Dev Med Child Neurol. 2016;58:44. doi: 10.1111/dmcn.93_13241. Exclusion: 10.

619. Lorentzen J, Kirk H, Fernandez-Lago H, et al. Treadmill training with an incline reduces ankle joint stiffness and improves active range of movement during gait in adults with cerebral palsy. Disabil Rehabil. 2017 May;39(10):987-93. doi:

10.1080/09638288.2016.1174745. PMID: 27237772. Exclusion: 4.
620. Lorentzen J, Kirk H, Lago Fernandez H, et al. Randomized controlled clinical trial of the effect of gait training in adults with cerebral palsy on ankle joint stiffness and kinematics. Dev Med Child Neurol. 2015;57:98. doi: 10.1111/dmcn.39_12886. Exclusion: 10.

621. Lorenz DJ, Datta S, Harkema SJ. Longitudinal patterns of functional recovery in patients with incomplete spinal cord injury receiving activity-based rehabilitation. Arch Phys Med Rehabil. 2012 Sep;93(9):1541-52. doi: 10.1016/j.apmr.2012.01.027. PMID: 22920451. Exclusion: 7.

622. Lotfian M, Kharazi MR, Mirbagheri A, et al. Therapeutic effects of an anti-gravity treadmill (AlterG) training on gait and lower limbs kinematics and kinetics in children with cerebral palsy. IEEE Int. 2017 Jul;2017:170-4. doi: 10.1109/ICORR.2017.8009241. PMID: 28813813. Exclusion: 11.

623. Lotter JK, Henderson CE, Plawecki A, et al. Task-specific versus impairment-based training on locomotor performance in Individuals with chronic spinal cord injury: A randomized crossover study. Neurorehabil Neural Repair. 2020 Jun 01 doi: 10.1177/1545968320927384. PMID: 32476619. Exclusion: 11.

624. Louie J, Baquie K, Offerman J, et al. Selfmanagement and multiple sclerosis: the impact of a community-based exercise and education program. Mult Scler J Exp Transl Clin. Oct 30;21(14):11-2. Exclusion: 10.

625. Louie J, Baquie KA, Offerman J, et al. Maximising abilities, negotiating and generating exercise options (manage) program: a pilot randomised controlled trial in persons with multiple sclerosis. Physiotherapy. 2015;101(1). Exclusion: 10.

626. Low DA, da Nobrega AC, Mathias CJ. Exercise-induced hypotension in autonomic disorders. Auton Neurosci. $2012 \mathrm{Nov}$ 2;171(1-2):66-78. doi: 10.1016/j.autneu.2012.07.008. PMID: 23040841. Exclusion: 10. 
627. Lu AL, Zhang XJ, Xu MF. Cohort study of hyperbaric oxygention (HBO) in controlling hypermyotonia caused by spinal cord injury. Zhongguo Gu Shang. 2012 Sep;25(9):743-6. PMID: 23256362. Exclusion: 13.

628. Lu X, Battistuzzo CR, Zoghi M, et al. Effects of training on upper limb function after cervical spinal cord injury: a systematic review. Clin Rehabil. 2015 Jan;29(1):3-13. doi: 10.1177/0269215514536411. PMID: 25575932. Exclusion: 12.

629. Lucio A, D'Ancona C A, Perissinotto MC, et al. Pelvic floor muscle training with and without electrical stimulation in the treatment of lower urinary tract symptoms in women with multiple sclerosis. J Wound Ostomy Continence Nurs. 2016 JulAug;43(4):414-9. doi: 10.1097/WON.0000000000000223. PMID: 27014935. Exclusion: 4.

630. Lucio AC, Campos RM, Perissinotto MC, et al. Pelvic floor muscle training in the treatment of lower urinary tract dysfunction in women with multiple sclerosis. Neurourol Urodyn. 2010 Nov;29(8):1410-3. doi: 10.1002/nau.20941. PMID: 20976816. Exclusion: 4.

631. Lucio AC, D'Ancona CA, Lopes MH, et al. The effect of pelvic floor muscle training alone or in combination with electrostimulation in the treatment of sexual dysfunction in women with multiple sclerosis. Mult Scler. 2014

Nov;20(13):1761-8. doi:

10.1177/1352458514531520. PMID: 24876156. Exclusion: 4.

632. Lucio AC, Perissinoto MC, Natalin RA, et al. A comparative study of pelvic floor muscle training in women with multiple sclerosis: its impact on lower urinary tract symptoms and quality of life. Clinics. 2011;66(9):1563-8. doi: 10.1590/s180759322011000900010. PMID: 22179160. Exclusion: 4.

633. Luu K, Hall PA. Hatha yoga and executive function: a systematic review. J Altern Complement Med. 2016 Feb;22(2):125-33. doi: 10.1089/acm.2014.0091. PMID: 26398441. Exclusion: 6.
634. Lyp M, Stanislawska I, Witek B, et al. Robot-assisted body-weight-supported treadmill training in gait impairment in multiple sclerosis patients: a pilot study. Adv Exp Med Biol. 2018 Feb 13;1070:1115. doi: 10.1007/5584_2018_158. PMID: 29435956. Exclusion: 11.

635. M Piovesana A, Ross S, Lloyd O, et al. Randomized controlled trial of a web-based multi-modal therapy program for executive functioning in children and adolescents with unilateral cerebral palsy. Disabil Rehabil. 2017 Oct;39(20):2021-8. doi: 10.1080/09638288.2016.1213899. PMID: 27665941. Exclusion: 6.

636. Ma DN, Zhang XQ, Ying J, et al. Efficacy and safety of 9 nonoperative regimens for the treatment of spinal cord injury: a network meta-analysis. Medicine (Baltimore). 2017 Nov;96(47):e8679. doi: 10.1097/MD.0000000000008679. PMID: 29381946. Exclusion: 12.

637. Ma JK, West CR, Martin Ginis KA. The effects of a patient and provider codeveloped, behavioral physical activity intervention on physical activity, psychosocial predictors, and fitness in individuals with spinal cord injury: a randomized controlled trial. Sports Med. 2019 Jul;49(7):1117-31. doi: 10.1007/s40279-019-01118-5. PMID: 31119717. Exclusion: 4.

638. Maas JC, Dallmeijer AJ, Huijing PA, et al. Splint: the efficacy of orthotic management in rest to prevent equinus in children with cerebral palsy, a randomised controlled trial. BMC Pediatr. 2012 Mar 26;12:38. doi: 10.1186/1471-2431-12-38. PMID: 22448907. Exclusion: 4.

639. Macias-Merlo L, Bagur-Calafat C, GirabentFarres M, et al. Effects of the standing program with hip abduction on hip acetabular development in children with spastic diplegia cerebral palsy. Disabil Rehabil. 2016;38(11):1075-81. doi: 10.3109/09638288.2015.1100221. PMID: 26517269. Exclusion: 6. 
640. MacIntosh A, Switzer L, Hwang S, et al. Ability-based balancing using the gross motor function measure in exergaming for youth with cerebral palsy. Games Health J. 2017 Dec;6(6):379-85. doi:

10.1089/g4h.2017.0053. PMID: 29016199. Exclusion: 11.

641. Mackow A, Malachowska-Sobieska M, Demczuk-Wlodarczyk E, et al. Influence of neurophysiological hippotherapy on the transference of the centre of gravity among children with cerebral palsy. Ortop

Traumatol Rehabil. 2014 Nov-

Dec;16(6):581-93. doi:

10.5604/15093492.1135048. PMID: 25694373. Exclusion: 4.

642. Madhusmita M, Ebnezar J, Srinivasan TM, et al. Efficacy of yoga as an add-on to physiotherapy in the management of patients with paraplegia: randomised controlled trial. J Clin Diagn Res. 2019;13(3):KC01-KC6. Exclusion: 14.

643. Madhusmita M, Srinivasan TM, Ebnezar J, et al. Effect of integrated yoga as an add-on to physiotherapy on walking index, esr, pain, and spasticity among subjects with traumatic spinal cord injury: a randomized control study. J Stem Cells. 2018;13(1):5866. Exclusion: 14.

644. Magalhães R, Martins EW, Marocolo M, et al. Profile of the cardiac repolarization in cervical spinal cord injury subjects performing physical exercise. Acta Scientiarum - Health Sciences.

2017;39(2):141-8. doi:

10.4025/actascihealthsci.v39i2.33468.

Exclusion: 4.

645. Magia F, Bhise A, Prabhakar M, et al. Effect of pranayama (yogic breathing) on lung function in traumatic thoracic spinal cord injury patients: an interventional study. Physiotherapy. 2015;101. Exclusion: 10.

646. Magnani S, Olla S, Pau M, et al. Effects of six months training on physical capacity and metaboreflex activity in patients with multiple sclerosis. Front Physiol. 2016;7:531. doi: 10.3389/fphys.2016.00531. PMID: 27895592. Exclusion: 11.
647. Maher CA, Williams MT, Olds T, et al. An internet-based physical activity intervention for adolescents with cerebral palsy: a randomized controlled trial. Dev Med Child Neurol. 2010 May;52(5):448-55. doi: 10.1111/j.1469-8749.2009.03609.x. PMID: 20132138. Exclusion: 4.

648. Maira B, Sholpan B. Effectiveness of the combined use of robotic kinesiotherapy and botulinum therapy in the complex rehabilitation of children with cerebral palsy. Asian J Pharm Clin Res. 2018;11(9):360-4. doi: 10.22159/ajpcr.2018.v11i9.26541. Exclusion: 4.

649. Mak C, Whittingham K, Boyd R, et al. Miyoga-a randomized controlled trial of a novel mindfulness yoga program to enhance attention for child-parent dyads with unilateral and bilateral cerebral palsy. Developmental medicine and child neurology. Conference: 71 st annual meeting of the american academy for cerebral palsy and developmental medicine, AACPDM. 2017;59:20-1. Exclusion: 10.

650. Mak C, Whittingham K, Cunnington R, et al. MiYoga: cognitive effects of a mindfulness yoga program targeting attention for children with cerebral palsy: a randomised controlled trial. Conference: 9th biennial conference of the australasian academy of cerebral palsy and developmental medicine, ausacpdm. 2018. New zealand 60. Dev Med Child Neurol. 2018 Mar;60(Supplement 1):14-5. doi: 10.1111/dmcn.13665. Exclusion: 10.

651. Mak C, Whittingham K, Cunnington R, et al. MiYoga: a randomised controlled trial of a mindfulness movement programme based on hatha yoga principles for children with cerebral palsy: a study protocol. BMJ Open. 2017 Jul 10;7(7):e015191. doi: 10.1136/bmjopen-2016-015191. PMID: 28698326. Exclusion: 4.

652. Mak C, Whittingham K, Cunnington R, et al. Effect of mindfulness yoga programme MiYoga on attention, behaviour, and physical outcomes in cerebral palsy: a randomized controlled trial. Dev Med Child Neurol. 2018 Sep;60(9):922-32. doi: 10.1111/dmcn.13923. PMID: 29869333. Exclusion: 4. 
653. Mak C, Whittingham K, Cunnington R, et al. Six-month follow-up of a mindfulness yoga program, MiYoga, on attention, executive function, behaviour and physical outcomes in cerebral palsy. Disabil Rehabil. 2020 Jun 26:1-7. doi: 10.1080/09638288.2020.1783582. PMID: 32589851. Exclusion: 4.

654. Mak CK, Whittingham K, Boyd RN. Experiences of children and parents in MiYoga, an embodied mindfulness yoga program for cerebral palsy: a mixed method study. Complement Ther Clin Pract. 2019 Feb;34:208-16. doi:

10.1016/j.ctcp.2018.12.006. PMID: 30712729. Exclusion: 4.

655. Mak CK, Whittingham K, Cunnington R, et al. MiYoga: a randomized controlled trial of an embodied mindfulness yoga program to enhance attention for child-parent dyads with unilateral and bilateral cerebral palsy. Developmental medicine and child neurology. Conference: 29th annual meeting of the european academy of childhood disability, EACD. 2017;59:26-7. Exclusion: 10.

656. Makhov AS, Medvedev IN. The effectiveness of static exercises in the rehabilitation of cerebral palsy. Res J Pharm Biol Chem Sci. 2018;9(6):603-8. doi: 10.1097/PEP.0b013e3180302111. PMID: 17304099 Exclusion: 6.

657. Malin SK, Cotugna N, Fang CS. Effect of creatine supplementation on muscle capacity in individuals with multiple sclerosis. J Diet Suppl. 2008;5(1):20-32. doi: 10.1080/19390210802328974. PMID: 22433042. Exclusion: 4.

658. Manago MM, Glick S, Hebert JR, et al. Strength training to improve gait in people with multiple sclerosis: a critical review of exercise parameters and intervention approaches. Int J MS Care. 2019 MarApr;21(2):47-56. doi: 10.7224/15372073.2017-079. PMID: 31049034.

Exclusion: 12.
659. Manca A, Cabboi MP, Dragone D, et al. Resistance training for muscle weakness in multiple sclerosis: direct versus contralateral approach in individuals with ankle dorsiflexors' disparity in strength. Arch Phys Med Rehabil. 2017 Jul;98(7):1348-56.e1. doi: 10.1016/j.apmr.2017.02.019. PMID: 28342828. Exclusion: 4.

660. Manca A, Dvir Z, Deriu F. Meta-analytic and scoping study on strength training in people with multiple sclerosis. J Strength Cond Res. 2019 Mar;33(3):874-89. doi: 10.1519/JSC.0000000000002381. PMID: 30192317. Exclusion: 12.

661. Manca A, Dvir Z, Dragone D, et al. Time course of strength adaptations following high-intensity resistance training in individuals with multiple sclerosis. Eur J Appl Physiol. 2017 Apr;117(4):731-43. doi: 10.1007/s00421-017-3534-z. PMID: 28251398. Exclusion: 4.

662. Manella KJ, Roach KE, Field-Fote EC. Operant conditioning to increase ankle control or decrease reflex excitability improves reflex modulation and walking function in chronic spinal cord injury. $\mathrm{J}$ Neurophysiol. 2013 Jun;109(11):2666-79. doi: 10.1152/jn.01039.2011. PMID: 23468393. Exclusion: 11.

663. Manfredini F, Straudi S, Lamberti N, et al. Rehabilitation Improves Mitochondrial Energetics in Progressive Multiple Sclerosis: The Significant Role of Robot-Assisted Gait Training and of the Personalized Intensity. Diagnostics. 2020 Oct 17;10(10):17. doi: 10.3390/diagnostics10100834. PMID: 33080806. Exclusion: 6.

664. Manning KY, Menon RS, Gorter JW, et al. Neuroplastic sensorimotor resting state network reorganization in children with hemiplegic cerebral palsy treated with constraint-induced movement therapy. J Child Neurol. 2016 Feb;31(2):220-6. doi: 10.1177/0883073815588995. PMID: 26078420. Exclusion: 11.

665. Mansour WT, Atya AM, Aboumousa AM. Improving gait and balance in multiple sclerosis using partial body weight supported treadmill training. Egypt J Neurol Psychiatr Neurosurg. 2013;50(3):271-6. Exclusion: 14. 
666. Marck CH, De Livera AM, Brown CR, et al. Health outcomes and adherence to a healthy lifestyle after a multimodal intervention in people with multiple sclerosis: three year follow-up. PLoS One. 2018;13(5):e0197759. doi: 10.1371/journal.pone.0197759. PMID: 29791509. Exclusion: 4.

667. Marcucci A, Edouard P, Loustalet E, et al. Efficiency of flexible derotator in walking cerebral palsy children. Ann Phys Rehabil Med. 2011 Sep;54(6):337-47. doi: 10.1016/j.rehab.2011.07.960. PMID: 21868301. Exclusion: 4.

668. Marinho-Buzelli AR, Bonnyman AM, Verrier MC. The effects of aquatic therapy on mobility of individuals with neurological diseases: a systematic review. Clin Rehabil. 2015 Aug;29(8):741-51. doi: 10.1177/0269215514556297. PMID: 25394397. Exclusion: 12.

669. Marques Catelli A, Ferreira Gattino LA, da Campo LA, et al. Cycle ergometer in the improvement of gross motor function of children with cerebral palsy: a systematic review with meta-analysis. Fisioterapia e Pesquisa. 2019;26(1):101-9. doi: 10.1590/1809-2950/18011026012019. Exclusion: 12.

670. Marryam M, Umar M, ul Ain SQ. Effectiveness of task specific training in improving functional independence after spinal cord injury. Rawal Medical Journal. 2018;43(2):245-7. Exclusion: 14.

671. Marsh AP, Applegate WB, Guralnik JM, et al. Hospitalizations during a physical activity intervention in older adults at risk of mobility disability: analyses from the lifestyle interventions and independence for elders randomized clinical trial. J Am Geriatr Soc. 2016 May;64(5):933-43. doi: 10.1111/jgs.14114. PMID: 27225353. Exclusion: 3.

672. Martakis K, Stark C, Rehberg M, et al. Oneminute walk test in children with cerebral palsy GMFCS Level 1 and 2: reference values to identify therapeutic effects after rehabilitation. Dev Neurorehabil. 2019 Jun 10:1-9. doi: 10.1080/17518423.2019.1625981. PMID: 31177878. Exclusion: 4.
673. Martin Ginis KA, van der Scheer JW, Latimer-Cheung AE, et al. Evidence-based scientific exercise guidelines for adults with spinal cord injury: an update and a new guideline. Spinal Cord. 2018 Apr;56(4):30821. doi: 10.1038/s41393-017-0017-3. PMID: 29070812. Exclusion: 12.

674. Martinez SA, Nguyen ND, Bailey E, et al. Multimodal cortical and subcortical exercise compared with treadmill training for spinal cord injury. PLoS One. 2018 Aug 9;13(8):e0202130. doi: 10.1371/journal.pone.0202130. PMID: 30092092. Exclusion: 11.

675. Martini DN, Zeeboer E, Hildebrand A, et al. ADSTEP: preliminary investigation of a multicomponent walking aid program in people with multiple sclerosis. Arch Phys Med Rehabil. 2018 Oct;99(10):2050-8. doi: 10.1016/j.apmr.2018.05.023. PMID: 29958906. Exclusion: 4.

676. Martins E, Cordovil R, Oliveira R, et al. Efficacy of suit therapy on functioning in children and adolescents with cerebral palsy: a systematic review and meta-analysis. Dev Med Child Neurol. 2016 Apr;58(4):348-60. doi: 10.1111/dmcn.12988. PMID: 26613800. Exclusion: 12.

677. Marvi-Esfahani M, Karimi MT, Etemadifar $\mathrm{M}$, et al. Comparison of energy consumption in different clinical forms multiple sclerosis with normal subjects (cohort study). Mult Scler Relat Disord. 2016 Mar;6:97-101. doi: 10.1016/j.msard.2016.02.007. PMID: 27063632. Exclusion: 4.

678. Mat Rosly M, Mat Rosly H, Davis Oam $\mathrm{GM}$, et al. Exergaming for individuals with neurological disability: a systematic review. Disabil Rehabil. 2017 04;39(8):727-35. doi: 10.3109/09638288.2016.1161086. PMID: 27108475. Exclusion: 12.

679. Mathes S, Wahl P. Reply to: Could superimposed electromyostimulation be an effective training to improve aerobic and anaerobic capacity? Methodological considerations for its development. Eur J Appl Physiol. 2017 Jul;117(7):1517-8. doi: 10.1007/s00421-017-3626-9. PMID: 28477075. Exclusion: 10. 
680. Mathevon L, Bonan I, Barnais JL, et al. Adjunct therapies to improve outcomes after botulinum toxin injection in children: a systematic review. Ann Phys Rehabil Med. 2019 Jul 29;62(4):283-90. doi: 10.1016/j.rehab.2018.06.010. PMID: 30063979. Exclusion: 12.

681. Matos-Souza JR, de Rossi G, Costa ESAA, et al. Impact of adapted sports activities on the progression of carotid atherosclerosis in subjects with spinal cord injury. Arch Phys Med Rehabil. 2016 Jun;97(6):1034-7. doi: 10.1016/j.apmr.2015.11.002. PMID: 26625710. Exclusion: 4.

682. Mattern-Baxter K. Effects of a group-based treadmill program on children with neurodevelopmental impairment who are not yet ambulating. Pediatr Phys Ther.

2016;28(3):312-9. doi: 10.1097/PEP.0000000000000250. PMID: 27008580. Exclusion: 11.

683. Mattern-Baxter K, McNeil S, Mansoor JK. Effects of home-based locomotor treadmill training on gross motor function in young children with cerebral palsy: a quasirandomized controlled trial. Arch Phys Med Rehabil. 2013 Nov;94(11):2061-7. doi: 10.1016/j.apmr.2013.05.012. PMID: 23747646. Exclusion: 4.

684. Mattioli F, Bellomi F, Stampatori C, et al. Neuroenhancement through cognitive training and anodal tDCS in multiple sclerosis. Mult Scler. 2016 Feb;22(2):22230. doi: $10.1177 / 1352458515587597$. PMID: 26014600. Exclusion: 4.

685. Maurer M. Telerehabilitation in multiple sclerosis. Mult Scler J Exp Transl Clin. START: 2015 Oct 7 CONFERENCE END: 2015 Oct 10, 31st Congress of the European Committee for Treatment and Research in Multiple Sclerosis, ECTRIMS 2015 Barcelona Spain;23(11 SUPPL. 1):41. Exclusion: 10.

686. Maurer M, Kallmann B, Hofmann WE, et al. A 6-month, multicenter, randomized, controlled study to evaluate the effect of physical training on fatigue in patients with relapsing-remitting multiple sclerosis treated with Fingolimod (Gilenya)-first results of the PACE study. Mult Scler J Exp Transl Clin. 2011 Oct;21(11 SUPPL. 1):405. doi: 10.1177/1352458515602642. PMID: 29479457. Exclusion: 10.
687. Maurer M, Schuh K, Seibert S, et al. A randomized study to evaluate the effect of exercise on fatigue in people with relapsingremitting multiple sclerosis treated with fingolimod. Mult Scler J Exp Transl Clin. 2018 Jan-Mar;4(1) doi: 10.1177/2055217318756688. PMID: 29479457. Exclusion: 4.

688. Mayo NE, Mate KK, Reid R, et al. Participation in and outcomes from a 12month tailored exercise programme for people with multiple sclerosis (MSTEP(): a randomized trial. Clin Rehabil. 2020 May 21 doi: 10.1177/0269215520923089. PMID: 32438828. Exclusion: 4.

689. Mayson TA, Harris SR. Functional electrical stimulation cycling in youth with spinal cord injury: a review of intervention studies. $\mathrm{J}$ Spinal Cord Med. 2014 May;37(3):266-77. doi: 10.1179/2045772313Y.0000000183. PMID: 24621033. Exclusion: 9.

690. Mazzoleni S, Battini E, Rustici A, et al. An integrated gait rehabilitation training based on functional electrical stimulation cycling and overground robotic exoskeleton in complete spinal cord injury patients: preliminary results. IEEE Int. 2017 Jul;2017:289-93. doi: 10.1109/ICORR.2017.8009261. PMID: 28813833. Exclusion: 11.

691. Mazzoleni S, Boldrini E, Laschi C, et al. Changes on EMG activation in healthy subjects and incomplete SCI patients following a robot-assisted locomotor training. IEEE Int. 2011;2011:5975467. doi: 10.1109/ICORR.2011.5975467. PMID: 22275665. Exclusion: 5.

692. McAuley E, Wojcicki TR, Learmonth YC, et al. Effects of a DVD-delivered exercise intervention on physical function in older adults with multiple sclerosis: a pilot randomized controlled trial. Mult Scler J Exp Transl Clin. 2015 JanDec;1:2055217315584838. doi: 10.1177/2055217315584838. PMID: 28607692. Exclusion: 4.

693. McClurg D, Lowe-Strong A, Ashe RG. The benefits of pelvic floor muscle training in people with multiple sclerosis and lower urinary tract dysfunction. Journal of the Association of Chartered Physiotherapists in Women's Health. 2008(103):21-8. Exclusion: 4. 
694. McCullagh R, Fitzgerald AP, Murphy RP, et al. Long-term benefits of exercising on quality of life and fatigue in multiple sclerosis patients with mild disability: a pilot study. Clin Rehabil. 2008 Mar;22(3):206-14. doi: 10.1177/0269215507082283. PMID:

18285430. Exclusion: 11.

695. McDaniel J, Lombardo LM, Foglyano KM, et al. Setting the pace: insights and advancements gained while preparing for an FES bike race. J Neuroengineering Rehabil. 2017 Nov 17;14(1):118. doi:

10.1186/s12984-017-0326-y. PMID: 29149885. Exclusion: 10.

696. McGibbon CA, Sexton A, Jayaraman A, et al. Evaluation of the Keeogo exoskeleton for assisting ambulatory activities in people with multiple sclerosis: an open-label, randomized, cross-over trial. $\mathrm{J}$

Neuroengineering Rehabil. 2018 Dec 12;15(1):117. doi: 10.1186/s12984-0180468-6. PMID: 30541585. Exclusion: 11.

697. McGibbon NH, Benda W, Duncan BR, et al. Immediate and long-term effects of hippotherapy on symmetry of adductor muscle activity and functional ability in children with spastic cerebral palsy. Arch Phys Med Rehabil. 2009 Jun;90(6):966-74. doi: 10.1016/j.apmr.2009.01.011. PMID: 19480872. Exclusion: 8.

698. McLeod JC, Diana H, Hicks AL. Sprint interval training versus moderate-intensity continuous training during inpatient rehabilitation after spinal cord injury: a randomized trial. Spinal Cord. 2019 Aug 28;28:28. doi: 10.1038/s41393-019-0345-6. PMID: 31462757. Exclusion: 3.

699. McLoughlin JV, Lord SR, Barr CJ, et al. Dorsiflexion assist orthosis reduces the physiological cost and mitigates deterioration in strength and balance associated with walking in people with multiple sclerosis. Arch Phys Med Rehabil. 2015 Feb;96(2):226-32.e1. doi: 10.1016/j.apmr.2014.09.005. PMID: 25264109. Exclusion: 4.

700. McNee AE, Gough M, Morrissey MC, et al. Increases in muscle volume after plantarflexor strength training in children with spastic cerebral palsy. Dev Med Child Neurol. 2009 Jun;51(6):429-35. doi: 10.1111/j.1469-8749.2008.03230.x. PMID: 19170722. Exclusion: 11.
701. McPhee PG, Wong-Pack M, Obeid J, et al. Differences in cardiovascular health in ambulatory persons with cerebral palsy. $\mathrm{J}$ Rehabil Med. 2018 Oct 9;09:09. doi: 10.2340/16501977-2491. PMID: 30299522. Exclusion: 6.

702. Medina-Perez C, de Souza-Teixeira F, Fernandez-Gonzalo R, et al. Effects of a resistance training program and subsequent detraining on muscle strength and muscle power in multiple sclerosis patients. NeuroRehabilitation. 2014;34(3):523-30. doi: 10.3233/NRE-141062. PMID: 24463236. Exclusion: 4.

703. Medina-Perez C, de Souza-Teixeira F, Fernandez-Gonzalo R, et al. Effects of highspeed power training on muscle strength and power in patients with multiple sclerosis. J Rehabil Res Dev. 2016;53(3):359-68. doi: 10.1682/JRRD.2014.08.0186. PMID: 27270470. Exclusion: 11.

704. Mehrholz J, Harvey LA, Thomas S, et al. Is body-weight-supported treadmill training or robotic-assisted gait training superior to overground gait training and other forms of physiotherapy in people with spinal cord injury? A systematic review. Spinal Cord. 2017 Aug;55(8):722-9. doi: 10.1038/sc.2017.31. PMID: 28398300. Exclusion: 12.

705. Mehrvar K, Ghorbanian B, Mahmoudpoor A. The effect of pilates training and massage therapy on plasma serum levels of IL-17 and IFNbeta as pro- inflammatory cytokines in patients with multiple sclerosis (MS). Mult Scler. 2017;Conference: 7th joint ECTRIMS-ACTRIMS, MSPARIS2017. France. 23(3 Supplement 1):951-2. Exclusion: 10.

706. Mei-Ying Poon D. Effects of transcutaneous electrical nerve stimulation and bodyweight-supported treadmill training on motor functions in children with spastic cerebral palsy. Dissertation Abstracts International: Section B: The Sciences and Engineering. 2008;68(11):7296. Exclusion: 9.

707. Meiner Z, Fisher I, Karussis D, et al. Robotassisted gait training by using the lokomat system in patients with multiple sclerosis: a pilot randomized trial. PM and R. 2011 Nov;3(10 SUPPL. 1):265. doi: 10.1016/j.pmrj.2011.08.297. Exclusion: 10. 
708. Melo FCM, de Lima KKF, Silveira A, et al. Physical training and upper limb strength of people with paraplegia: a systematic review. J Sport Rehabil. 2018 Mar 1;28(3):288-93. doi: 10.1123/jsr.2017-0062. PMID: 29364066. Exclusion: 12.

709. Methajarunon P, Eitivipart C, Diver CJ, et al. Systematic review of published studies on aquatic exercise for balance in patients with multiple sclerosis, Parkinson's disease, and hemiplegia. Hong Kong Physiother J. 2016 Dec;35:12-20. doi: 10.1016/j.hkpj.2016.03.002. PMID: 30931029. Exclusion: 12.

710. Meyer C, Weissland T, Watelain E, et al. Physiological responses in handcycling. Preliminary study. Ann Phys Rehabil Med. 2009 May;52(4):311-8. doi: 10.1016/j.rehab.2009.04.001. PMID: 19467942. Exclusion: 7.

711. Meyer-Heim A, Ammann-Reiffer C, Schmartz A, et al. Improvement of walking abilities after robotic-assisted locomotion training in children with cerebral palsy. Arch Dis Child. 2009 Aug;94(8):615-20. doi: 10.1136/adc.2008.145458. PMID: 19208675. Exclusion: 8.

712. Meyns P, Bras C, Harlaar J, et al. Does video game-based balance-training improve gait stability in children with cerebral palsy? Gait Posture. 2018;65:105-6. doi: 10.1016/j.gaitpost.2018.06.078. Exclusion: 11.

713. Meyns P, Harlaar J, Van De Pol L, et al. Can virtual reality games improve scores on clinical balance scales in children with cerebral palsy: preliminary results of a randomized controlled clinical trial. Gait Posture. 2017 Sep;57 Conference: 26th annual meeting of the european society for movement analysis in adults and children, ESMAC. 2017. Norway 204. doi: 10.1016/j.gaitpost.2017.06.390. Exclusion: 10.

714. Meyns P, Van de Walle P, Hoogkamer W, et al. Coordinating arms and legs on a hybrid rehabilitation tricycle: the metabolic benefit of asymmetrical compared to symmetrical arm movements. Eur J Appl Physiol. 2014 Apr;114(4):743-50. doi: 10.1007/s00421013-2814-5. PMID: 24384984. Exclusion: 3.
715. Mignardot JB, Le Goff CG, van den Brand $\mathrm{R}$, et al. A multidirectional gravity-assist algorithm that enhances locomotor control in patients with stroke or spinal cord injury. Sci Transl Med. 2017 Jul 19;9(399):19. doi: 10.1126/scitranslmed.aah3621. PMID: 28724575. Exclusion: 10.

716. Mikuláková W, Klímová E, Kendrová L, et al. Effect of rehabilitation on fatigue level in patients with multiple sclerosis. Med Sci Monit. 2018;24:5761-70. doi: 10.12659/MSM.909183. PMID: 30120829. Exclusion: 6.

717. Millar PJ, Rakobowchuk M, Adams MM, et al. Effects of short-term training on heart rate dynamics in individuals with spinal cord injury. Auton Neurosci. 2009 Oct 5;150(12):116-21. doi: 10.1016/j.autneu.2009.03.012. PMID: 19406691. Exclusion: 11.

718. Miller E. [Cryostimulation factor supporting rehabilitation patients with multiple sclerosis and fatigue syndrome]. Wiad Lek. 2010;63(2):41-5. PMID: 20941916. Exclusion: 13.

719. Miller L, McFadyen A, Lord AC, et al. Functional electrical stimulation for foot drop in multiple sclerosis: a systematic review and meta-analysis of the effect on gait speed. Arch Phys Med Rehabil. 2017 Jul;98(7):1435-52. doi: 10.1016/j.apmr.2016.12.007. PMID: 28088382. Exclusion: 12.

720. Millett R. Group exercise proves its worth in multiple sclerosis. Frontline (20454910). 2012;18(16):13. Exclusion: 10.

721. Mirbagheri MM, Kindig MW, Niu X. Effects of robotic-locomotor training on stretch reflex function and muscular properties in individuals with spinal cord injury. Clin Neurophysiol. 2015 May;126(5):997-1006. doi: 10.1016/j.clinph.2014.09.010. PMID: 25449559. Exclusion: 6.

722. Moghadasi A, Ghasemi G, Sadeghi-Demneh E, et al. The effect of total body resistance exercise on mobility, proprioception and muscle strength of the knee in people with multiple sclerosis. J Sport Rehabil. 2019 Apr 27:1-8. doi: 10.1123/jsr.2018-0303. PMID: 30676232. Exclusion: 11. 
723. Mohamed AS, Kamal HM, Elsied AO, et al. Universal exercise unit versus functional resisted training effect on muscle strength in spastic diaplegia. Indian J Public Health Res Dev. 2020;11(4):1139-44. Exclusion: 4.

724. Mohamed El-Shamy S. Effect of wholebody vibration on muscle strength and balance in diplegic cerebral palsy. Am J Phys Med Rehabil. 2014 Feb;93(2):114-21. doi: 10.1097/PHM.0b013e3182a541a4. PMID: 24434887. Exclusion: 14.

725. Mohammadi G, Naghibzade A, Rezaee Namjoo F, et al. The effects of massage and aqua training on Interleukin-6 levels of multiple sclerosis patients. Gazzetta Medica Italiana Archivio per le Scienze Mediche. 2016;175(10):408-12. Exclusion: 6.

726. Mokhtarzade M, Ranjbar R, Majdinasab N, et al. Effect of aerobic interval training on serum IL-10, TNFalpha, and adipokines levels in women with multiple sclerosis: possible relations with fatigue and quality of life. Endocrine. 2017 Aug;57(2):262-71. doi: 10.1007/s12020-017-1337-y. PMID: 28616851. Exclusion: 6.

727. Moore PD, Gorgey AS, Wade RC, et al. Neuromuscular electrical stimulation and testosterone did not influence heterotopic ossification size after spinal cord injury: a case series. World J Clin Cases. 2016 Jul 16;4(7):172-6. doi:

10.12998/wjcc.v4.i7.172. PMID: 27458592. Exclusion: 7.

728. Moradi M, Sahraian MA, Aghsaie A, et al. Effects of eight-week resistance training program in men with multiple sclerosis. Asian J Sports Med. 2015 Jun;6(2) doi: 10.5812/asjsm.6(2)2015.22838. PMID: 26448834. Exclusion: 11.

729. Moraes AG, Copetti F, Angelo VR, et al. The effects of hippotherapy on postural balance and functional ability in children with cerebral palsy. J Phys Ther Sci. 2016 Aug;28(8):2220-6. doi: 10.1589/jpts.28.2220. PMID: 27630401. Exclusion: 11.
730. Moraes AG, Copetti FP, Angelo VB, et al. Hippotherapy on postural balance in the sitting position of children with cerebral palsy - Longitudinal study. Physiother Theory Pract. 2018 Jun 11:1-8. doi: 10.1080/09593985.2018.1484534. PMID: 29889590. Exclusion: 11.

731. Moreau NG, Bodkin AW, Bjornson K, et al. Effectiveness of rehabilitation interventions to improve gait speed in children with cerebral palsy: systematic review and metaanalysis. Phys Ther. 2016 Dec;96(12):193854. doi: 10.2522/ptj.20150401. PMID: 27313240. Exclusion: 12.

732. Moreau NG, Holthaus K, Marlow N. Differential adaptations of muscle architecture to high-velocity versus traditional strength training in cerebral palsy. Neurorehabil Neural Repair. 2013 May;27(4):325-34. doi: 10.1177/1545968312469834. PMID: 23292847. Exclusion: 11.

733. Moreau NG, Marlow N, Holthaus K. Muscle architectural plasticity of the quadriceps in response to high velocity vs. traditional strength training in cerebral palsy: a randomized clinical. Pediatr Phys Ther. 2012 Spring;24(1):91. Exclusion: 10.

734. Morgan D, Holbrook E, Stevens SL, et al. Impact of underwater treadmill training on walking performance in youth with cerebral palsy. Dev Med Child Neurol. 2012 Sep;54:43-4. doi: $10.1111 / \mathrm{j} .1469-$ 8749.2012.04388.x. Exclusion: 10.

735. Morrison SA, Lorenz D, Eskay CP, et al. Longitudinal recovery and reduced costs after 120 sessions of locomotor training for motor incomplete spinal cord injury. Arch Phys Med Rehabil. 2018 Mar;99(3):555-62. doi: 10.1016/j.apmr.2017.10.003. PMID: 29107040. Exclusion: 8.

736. Mosalanejad F, Afrasiabifar A, Zoladl M. Investigating the combined effect of pelvic floor muscle exercise and mindfulness on sexual function in women with multiple sclerosis: a randomized controlled trial. Clin Rehabil. 2018 Oct;32(10):1340-7. doi: 10.1177/0269215518777877. PMID: 29843529. Exclusion: 4. 
737. Motl RW, Backus D, Neal WN, et al. Rationale and design of the STEP for MS Trial: comparative effectiveness of Supervised versus telerehabilitation exercise programs for multiple sclerosis. Contemp Clin Trials. 2019 Jun;81:110-22. doi: 10.1016/j.cct.2019.04.013. PMID: 31022481. Exclusion: 10.

738. Motl RW, McAuley E, Snook EM, et al. Does the relationship between physical activity and quality of life differ based on generic versus disease-targeted instruments? Ann Behav Med. 2008 Aug;36(1):93-9. doi: 10.1007/s12160-008-9049-4. PMID: 18719976. Exclusion: 4.

739. Motl RW, Sandroff BM. Randomized controlled trial of physical activity intervention effects on fatigue and depression in multiple sclerosis: Secondary analysis of data from persons with elevated symptom status. Contemp Clin Trials Commun. 2020 Mar;17:100521. doi: 10.1016/j.conctc.2020.100521. PMID: 32211556. Exclusion: 4.

740. Mueller G, Hopman MT, Perret C. Comparison of respiratory muscle training methods in individuals with motor complete tetraplegia. Top Spinal Cord Inj Rehabil. 2012;18(2):118-21. doi: 10.1310/sci1802118. PMID: 23459602. Exclusion: 4.

741. Mulroy S, Haubert LL, Eberly V, et al. Effectiveness of two intervention programs to prevent shoulder pain after spinal cord injury. Arch Phys Med Rehabil. 2017; Conference:. 2017 american congress of rehabilitation medicine annual conference, ACRM 2017. United states 98(12):e174. Exclusion: 10.

742. Mulroy SJ, Thompson L, Kemp B, et al. Strengthening and optimal movements for painful shoulders (STOMPS) in chronic spinal cord injury: a randomized controlled trial. Phys Ther. 2011 Mar;91(3):305-24. doi: 10.2522/ptj.20100182. PMID: 21292803. Exclusion: 4.

743. Munoz-Lasa S, Ferriero G, Valero R, et al. Effect of therapeutic horseback riding on balance and gait of people with multiple sclerosis. G Ital Med Lav Ergon. 2011 OctDec;33(4):462-7. PMID: 22452106.

Exclusion: 11.
744. Munoz-Lasa S, Lopez de Silanes C, AtinArratibel MA, et al. Effects of hippotherapy in multiple sclerosis: pilot study on quality of life, spasticity, gait, pelvic floor, depression and fatigue. Med Clin (Barc). 2018 Jan 18;152(2):55-8. doi: 10.1016/j.medcli.2018.02.015. PMID: 29680460. Exclusion: 11.

745. Mutluay FK, Tekeoğlu A, Saip S, et al. Group exercise training approach to multiple sclerosis rehabilitation. Nobel Medicus. 2008;4(3):20-6. Exclusion: 4.

746. Mutoh T, Mutoh T, Takada M, et al. Application of a tri-axial accelerometrybased portable motion recorder for the quantitative assessment of hippotherapy in children and adolescents with cerebral palsy. J Phys Ther Sci. 2016 Oct;28(10):2970-4. doi: 10.1589/jpts.28.2970. PMID: 27821971. Exclusion: 11.

747. Mutoh T, Mutoh T, Tsubone H, et al. Impact of serial gait analyses on long-term outcome of hippotherapy in children and adolescents with cerebral palsy. Complement Ther Clin Pract. 2018 Feb;30:19-23. doi: 10.1016/j.ctcp.2017.11.003. PMID: 29389473. Exclusion: 8.

748. Myers J, Gopalan R, Shahoumian T, et al. Effects of customized risk reduction program on cardiovascular risk in males with spinal cord injury. J Rehabil Res Dev. 2012;49(9):1355-64. PMID: 23408217. Exclusion: 11.

749. Najafi P, Moghadasi M. The effect of yoga training on enhancement of Adrenocorticotropic hormone (ACTH) and cortisol levels in female patients with multiple sclerosis. Complement Ther Clin Pract. 2017 Feb;26:21-5. doi: 10.1016/j.ctcp.2016.11.006. PMID: 28107844. Exclusion: 11.

750. Nam KY, Kim HJ, Kwon BS, et al. Robotassisted gait training (Lokomat) improves walking function and activity in people with spinal cord injury: a systematic review. J Neuroengineering Rehabil. 2017 Mar 23;14(1):24. doi: 10.1186/s12984-017-02323. PMID: 28330471. Exclusion: 12. 
751. Nardone R, Orioli A, Golaszewski S, et al. Passive cycling in neurorehabilitation after spinal cord injury: a review. J Spinal Cord Med. 2017 01;40(1):8-16. doi:

10.1080/10790268.2016.1248524. PMID: 27841091. Exclusion: 12.

752. Narimani A, Kalantari M, Dalvand H, et al. Effect of mirror therapy on dexterity and hand grasp in children aged 9-14 years with hemiplegic cerebral palsy. Iran J Child Neurol. 2019;13(4):135-42. PMID: 31645873. Exclusion: 4.

753. Nasseri NN, Ghezelbash E, Zhai Y, et al. Feasibility of a smartphone app to enhance physical activity in progressive MS: a pilot randomized controlled pilot trial over three months. PeerJ. 2020;6. Exclusion: 4.

754. Navarrete-Opazo A, Alcayaga JJ, Sepulveda $\mathrm{O}$, et al. Intermittent hypoxia and locomotor training enhances dynamic but not standing balance in patients with incomplete spinal cord injury. Arch Phys Med Rehabil. 2017 03;98(3):415-24. doi: 10.1016/j.apmr.2016.09.114. PMID: 27702556. Exclusion: 4.

755. Nedeljkovic U, Dackovic J, Basuroski ID, et al. Influence of rehabilitation on fatigue and physical activity of patients with relapse of multiple sclerosis. Mult Scler J Exp Transl Clin. 2014 Jun;20(7):983-4. doi: 10.1177/1352458514533628. Exclusion: 10.

756. Nedeljkovic U, Raspopovic ED, Ilic N, et al. Effectiveness of rehabilitation in multiple sclerosis relapse on fatigue, self-efficacy and physical activity. Acta Neurol Belg. 2016 Sep;116(3):309-15. doi: 10.1007/s13760015-0563-4. PMID: 26563405. Exclusion: 4.

757. Neefkes-Zonneveld CR, Bakkum AJ, Bishop NC, et al. Effect of long-term physical activity and acute exercise on markers of systemic inflammation in persons with chronic spinal cord injury: a systematic review. Arch Phys Med Rehabil. 2015 Jan;96(1):30-42. doi: 10.1016/j.apmr.2014.07.006. PMID: 25064781. Exclusion: 12.
758. Negahban H, Monjezi S, Mehravar M, et al. Responsiveness of postural performance measures following balance rehabilitation in multiple sclerosis patients. J Bodywork Mov Ther. 2018 Apr;22(2):502-10. doi: 10.1016/j.jbmt.2017.06.008. PMID: 29861258. Exclusion: 8.

759. Negahban H, Rezaie S, Goharpey S. Massage therapy and exercise therapy in patients with multiple sclerosis: a randomized controlled pilot study. Clin Rehabil. 2013 Dec;27(12):1126-36. doi: 10.1177/0269215513491586. PMID: 23828184. Exclusion: 5.

760. Negaresh R, Motl RW, Zimmer P, et al. Effects of exercise training on multiple sclerosis biomarkers of central nervous system and disease status: a systematic review of intervention studies. Eur J Neurol. 2019 May;26(5):711-21. doi: 10.1111/ene.13929. PMID: 30734989. Exclusion: 12.

761. Newsome S, Zackowski K, Calabresi P, et al. Evaluating the effects of functional electrical stimulation on ambulation in individuals with secondary progressive multiple sclerosis. Mult Scler J Exp Transl Clin. START: 2012 Oct 10 CONFERENCE END: 2012 Oct 13, 28th Congress of the European Committee for Treatment and Research in Multiple Sclerosis Lyon France;18(4 SUPPL. 1):484-5. Exclusion: 10.

762. Nguyen V. Efficacy of body weight supported treadmill training in a child with cerebral palsy. Dissertation Abstracts International: Section B: The Sciences and Engineering. 2018;79(4-B(E)). Exclusion: 7.

763. Nightingale TE, Bilzon J. Cardiovascular health benefits of exercise in people with spinal cord injury: more complex than a prescribed exercise intervention? Arch Phys Med Rehabil. 2016;97(6):1038. doi: 10.1016/j.apmr.2016.02.020. PMID: 27233990. Exclusion: 7.

764. Nightingale TE, Rouse PC, Walhin JP, et al. Home-based exercise enhances healthrelated quality of life in persons with spinal cord injury: a randomized controlled trial. Arch Phys Med Rehabil. 2018 Oct;99(10):1998-2006.e1. doi: 10.1016/j.apmr.2018.05.008. PMID: 29902472. Exclusion: 4. 
765. Nightingale TE, Walhin J-P, Thompson D, et al. Impact of exercise on cardiometabolic component risks in spinal cord--injured humans. Med Sci Sports Exerc.

2017;49(12):2469-77. doi: 10.1249/MSS.0000000000001390.

Exclusion: 4.

766. Nikityuk IE, Moshonkina TR, Shcherbakova NA, et al. Effects of locomotor training and functional electrical stimulation on postural function in children with severe cerebral palsy. Fiziol Cheloveka. 2016 MayJun;42(3):37-46. doi:

10.17116/kurort2016523-27. PMID: 29446895. Exclusion: 6.

767. Nilsagard YE, von Koch LK, Nilsson M, et al. Balance exercise program reduced falls in people with multiple sclerosis: a singlegroup, pretest-posttest trial. Arch Phys Med Rehabil. 2014 Dec;95(12):2428-34. doi: 10.1016/j.apmr.2014.06.016. PMID: 25004466. Exclusion: 8.

768. Nimmy A, Shanmugam S, Shilna Rani P. Effectiveness of pelvic proprioceptive neuromuscular facilitation on trunk control in children with spastic diplegia: A randomized controlled trial. Indian J Public Health Res Dev. 2020;11(2):571-6. doi: 10.37506/v11/i2/2020/ijphrd/194867. Exclusion: 4.

769. Nithiatthawanon T, Amatachaya P, Thaweewannakij $\mathrm{T}$, et al. Immediate effects of lower limb loading exercise during stepping with and without augmented loading feedback on mobility of ambulatory individuals with spinal cord injury: a singleblinded, randomized, cross-over trial. Spinal Cord. 2020 Jul 06;06:06. doi: 10.1038/s41393-020-0498-3. PMID: 32632173. Exclusion: 4.

770. Niu X, Varoqui D, Kindig M, et al. The effect of robot-assisted locomotor training on walking speed. Conf Proc IEEE Eng Med Biol Soc. 2012;2012:3858-61. doi: 10.1109/EMBC.2012.6346809. PMID: 23366770. Exclusion: 10.

771. Nooijen CF, de Groot S, Postma K, et al. A more active lifestyle in persons with a recent spinal cord injury benefits physical fitness and health. Spinal Cord. 2012 Apr;50(4):320-3. doi: 10.1038/sc.2011.152. PMID: 22143679. Exclusion: 8.
772. Nooijen CF, Stam HJ, Schoenmakers I, et al. Working mechanisms of a behavioural intervention promoting physical activity in persons with subacute spinal cord injury. J Rehabil Med. 2016 Jul 18;48(7):583-8. doi: 10.2340/16501977-2110. PMID: 27346837. Exclusion: 4.

773. Novotna K, Janatova M, Hana K, et al. Biofeedback Based Home Balance Training can Improve Balance but Not Gait in People with Multiple Sclerosis. Mult Scler Int. 2019;2019:2854130. doi: 10.1155/2019/2854130. PMID: 31934450. Exclusion: 4.

774. Novotna K, Sucha L, Kalincik T, et al. Positive impact of regular circuit training on muscle strength, stability, depression and fatigue in multiple sclerosis patients. Mult Scler J Exp Transl Clin. 2015 Apr;21(4):533. doi: 10.1177/1352458515573128. Exclusion: 10.

775. Nuara A, Avanzini P, Rizzolatti G, et al. Efficacy of a home-based platform for childto-child interaction on hand motor function in unilateral cerebral palsy. Dev Med Child Neurol. 2019 Nov;61(11):1314-22. doi: 10.1111/dmen.14262. PMID: 31115046. Exclusion: 8 .

776. Nurova B, Kurtuncu M, Coban A, et al. Computer assisted cognitive rehabilitation in patients with multiple sclerosis and parenchymal neuro-Behcet's disease. J Neurol. 2014;261(31). Exclusion: 10.

777. O'Brien TD, Noyes J, Spencer LH, et al. Systematic review of physical activity and exercise interventions to improve health, fitness and well-being of children and young people who use wheelchairs. BMJ Open Sport Exerc Med. 2016 Nov 15;2(1):e000109. doi: 10.1136/bmjsem2016-000109. PMID: 27900176. Exclusion: 12.

778. O'Neil ME, Fragala-Pinkham M, Lennon N, et al. Reliability and validity of objective measures of physical activity in youth with cerebral palsy who are ambulatory. Phys Ther. 2016 Jan;96(1):37-45. doi: 10.2522/ptj.20140201. PMID: 26089043. Exclusion: 4. 
779. Oftedal S, Bell KL, Stevenson RD, et al. Prospective cohort study of relationship between growth and diet, physical activity and time spent sedentary in preschool aged children with cerebral palsy. Dev Med Child Neurol. 2016;58:27-8. doi: 10.1111/dmcn.53_13241. Exclusion: 10.

780. Oftedal S, Davies PS, Boyd RN, et al. Body composition, diet, and physical activity: a longitudinal cohort study in preschoolers with cerebral palsy. Am J Clin Nutr. 2017 Feb;105(2):369-78. doi: 10.3945/ajen.116.137810. PMID: 28077375. Exclusion: 4.

781. Ogata H, Ogata T, Hoshikawa S, et al. Hypoventilation during passive leg movement in spinal cord-injured humans. Clin Auton Res. 2010 Apr;20(2):101-3. doi: 10.1007/s10286-009-0042-1. PMID: 19924465. Exclusion: 6.

782. Ogonowska-Slodownik A, Geigle PR, Gorman PH, et al. Aquatic, deep water peak $\mathrm{VO} 2$ testing for individuals with spinal cord injury. J Spinal Cord Med. 2019

Sep;42(5):631-8. doi: 10.1080/10790268.2018.1559494. PMID: 30632946. Exclusion: 4.

783. Ogwumike OO, Badaru UM, Adeniyi AF. Effect of task-oriented training on balance and motor function of ambulant children with cerebral palsy. Rehabilitacion. 2019;53(4):276-83. doi: 10.1016/j.rh.2019.07.003. Exclusion: 4.

784. Olama KA. Endurance exercises versus treadmill training in improving muscle strength and functional activities in hemiparetic cerebral palsy. Egypt J Med Hum Genet. 2011 Nov;12(2):193-9. doi: 10.1016/j.ejmhg.2011.07.002. Exclusion: 14.

785. Olama KA, Thabit NS. Effect of vibration versus suspension therapy on balance in children with hemiparetic cerebral palsy. Egypt J Med Hum Genet. 2012;13(2):21926. doi: 10.1016/j.ejmhg.2011.11.001. Exclusion: 14.
786. Oliveira AC, Freitas C, Eras-Garcia R, et al. Cerebral palsy in adult patients: constraintinduced movement therapy is effective to reverse the nonuse of the affected upper limb. Arq Neuropsiquiatr. 2016 Jan;74(1):18-21. doi: 10.1590/0004282X20150195. PMID: 26690842.

Exclusion: 11.

787. Orban A, Garg B, Sammi MK, et al. Effect of high-intensity exercise on multiple sclerosis function and 31P MRS outcomes. Med Sci Sports Exerc. 2019 Jan 30;30:30. doi: 10.1249/MSS.0000000000001914. PMID: 30707115. Exclusion: 11.

788. Ordonez FJ, Rosety MA, Camacho A, et al. Arm-cranking exercise reduced oxidative damage in adults with chronic spinal cord injury. Arch Phys Med Rehabil. 2013 Dec;94(12):2336-41. doi: 10.1016/j.apmr.2013.05.029. PMID: 23811316. Exclusion: 11.

789. Ozdogar AT, Ertekin O, Kahraman T, et al. Effect of videogame-based physical activity training in persons with multiple sclerosis: a randomised controlled trial. Mult Scler. 2017; Conference: 7th joint ECTRIMSACTRIMS, MSPARIS2017. France. 23(3 Supplement 1):674. Exclusion: 10.

790. Ozgen G, Karapolat H, Akkoc Y, et al. Effect of vestibular rehabilitation on balance, quality of life and depression in patients with multiple sclerosis, Multipl Skleroz Hastalarinda Vestibuler Rehabilitasyonun Denge Bozuklutu, Yasam Kalitesi ve Depresyon Uzerine Etkilerinin Deterlendirilmesi. [Turkish, English]. Turkiye Fiziksel Tip ve Rehabilitasyon Dergisi. 2013;59:229. doi: 10.4274/tftr.24.59.1. Exclusion: 10.

791. Ozgen G, Karapolat H, Akkoc Y, et al. Is customized vestibular rehabilitation effective in patients with multiple sclerosis? A randomized controlled trial. Eur J Phys Rehabil Med. 2016 Aug;52(4):466-78. PMID: 27050082. Exclusion: 4.

792. Ozkan F, Zincir H. Opinions and observations of caregivers of children with cerebral palsy about changes seen after reflexology: a qualitative study. Complement Ther Clin Pract. 2018 May;31:242-7. doi: 10.1016/j.ctcp.2018.03.011. PMID: 29705462. Exclusion: 4. 
793. Ozkul C, Guclu-Gunduz A, Irkec C, et al. Effect of combined exercise training on serum brain-derived neurotrophic factor, suppressors of cytokine signaling 1 and 3 in patients with multiple sclerosis. J

Neuroimmunol. 2018 Mar 15;316:121-9. doi: 10.1016/j.jneuroim.2018.01.002. PMID: 29329698. Exclusion: 6.

794. Ozturk ED, Lapointe MS, Kim DI, et al. Impact of 6-Month Exercise Training on Neurovascular Function in Spinal Cord Injury. Med Sci Sports Exerc. 2020 Aug 21;21:21. doi: 10.1249/MSS.0000000000002452. PMID: 32826631. Exclusion: 7.

795. Paleg G, Livingstone R. Outcomes of gait trainer use in home and school settings for children with motor impairments: a systematic review. Clin Rehabil. 2015 Nov;29(11):1077-91. doi: 10.1177/0269215514565947. PMID: 25636993. Exclusion: 12.

796. Paltiel AD, Ingvarsson E, Lee DK, et al. Demographic and clinical features of inclusion body myositis in North America. Muscle Nerve. 2015 Oct;52(4):527-33. doi: 10.1002/mus.24562. PMID: 25557419. Exclusion: 3.

797. Panisset MG, El-Ansary D, Dunlop SA, et al. Factors influencing thigh muscle volume change with cycling exercises in acute spinal cord injury - a secondary analysis of a randomized controlled trial. J Spinal Cord Med. 2020 Sep 24:1-12. doi: 10.1080/10790268.2020.1815480. PMID: 32970970. Exclusion: 6.

798. Panisset MG, Galea MP, El-Ansary D. Does early exercise attenuate muscle atrophy or bone loss after spinal cord injury? Spinal Cord. 2016 Feb;54(2):84-92. doi: 10.1038/sc.2015.150. PMID: 26345485. Exclusion: 12.

799. Panza GS, Guccione AA. Effect of repeated locomotor training on ventilatory measures, perceived exertion and walking endurance in persons with motor incomplete spinal cord injury. Spinal Cord Ser Cases. 202010 12;6(1):94. doi: 10.1038/s41394-020-003466. PMID: 33046694. Exclusion: 7.
800. Panza GS, Guccione AA, Chin LM, et al. Effects of overground locomotor training on the ventilatory response to volitional treadmill walking in individuals with incomplete spinal cord injury: a pilot study. Spinal Cord Ser Cases. 2017;3:17011. doi: 10.1038/scsandc.2017.11. PMID: 28435743. Exclusion: 11.

801. Paramonova DB, Mugerman BI. The role of therapeutic physical training and massage in the restoration of static and dynamic functions in the children presenting with the atonic-astatic form of infantile cerebral paralysis. Vopr Kurortol Fizioter Lech Fiz Kult. 2012 Sep-Oct(5):37-40. PMID: 23210361. Exclusion: 13.

802. Parent A, Raison M, Pouliot-Laforte A, et al. Impact of a short walking exercise on gait kinematics in children with cerebral palsy who walk in a crouch gait. Clin Biomech. 2016 May;34:18-21. doi: 10.1016/j.clinbiomech.2016.03.003. PMID: 27038653. Exclusion: 11.

803. Parra-Moreno M, Rodriguez-Juan JJ, RuizCardenas JD. Use of commercial video games to improve postural balance in patients with multiple sclerosis: a systematic review and meta-analysis of randomised controlled clinical trials. Neurologia. 2017 doi: 10.1016/j.nrl.2017.12.001. PMID: 29525396. Exclusion: 13.

804. Pascoe J, Thomason P, Graham HK, et al. Body mass index in ambulatory children with cerebral palsy: a cohort study. J Paediatr Child Health. 2016 Apr;52(4):41721. doi: 10.1111/jpc.13097. PMID: 27145505. Exclusion: 7.

805. Patatoukas D, Farmakides A, Aggeli V, et al. Disability-related injuries in athletes with disabilities. Folia Med (Plovdiv). 2011 JanMar;53(1):40-6. PMID: 21644404.

Exclusion: 7.

806. Patritti B, Sicari M, Deming L, et al. Enhancing robotic gait training via augmented feedback. Conf Proc IEEE Eng Med Biol Soc. 2010;2010:2271-4. doi: 10.1109/IEMBS.2010.5627707. PMID: 21097013. Exclusion: 10. 
807. Patrocinio de Oliveira CE, Moreira OC, Carrion-Yagual ZM, et al. Effects of classic progressive resistance training versus eccentric-enhanced resistance training in people with multiple sclerosis. Arch Phys Med Rehabil. 2018 May;99(5):819-25. doi: 10.1016/j.apmr.2017.10.021. PMID: 29191417. Exclusion: 4.

808. Patzer D, Vu P, Pardo V, et al. Immediate effect of whole-body vibration on gait in patients with incomplete spinal cord injury. J Spinal Cord Med. START: 2014 Oct 2 CONFERENCE END: 2014 Oct 3, 6th National Canadian Spinal Cord Injury Conference: Bioinformatics Informs SCI Toronto, ON Canada;37(5):624-5. Exclusion: 10.

809. Paul L, Coulter EH, Miller L, et al. Physiotherapy-led web-based rehabilitation for people with multiple sclerosis. Mult Scler J Exp Transl Clin. 2013 Oct;19(11 SUPPL. 1):555-6. doi: 10.1177/1352458513502429. Exclusion: 10.

810. Paul L, Coulter EH, Miller L, et al. Webbased physiotherapy for people moderately affected with multiple sclerosis; quantitative and qualitative data from a randomized, controlled pilot study. Clin Rehabil. 2014 Sep;28(9):924-35. doi: 10.1177/0269215514527995. PMID: 24691218. Exclusion: 4.

811. Paul L, Rafferty D, Young S, et al. The effect of functional electrical stimulation on the physiological cost of gait in people with multiple sclerosis. Mult Scler. 2008

Aug;14(7):954-61. doi: 10.1177/1352458508090667. PMID: 18573839. Exclusion: 11.

812. Paul L, Renfrew L, Freeman J, et al. Webbased physiotherapy for people affected by multiple sclerosis: a single blind, randomized controlled feasibility study. Clin Rehabil. 2019 Mar;33(3):473-84. doi: 10.1177/0269215518817080. PMID: 30514108. Exclusion: 4.

813. Pawik M, Kowalska J, Rymaszewska J. The effectiveness of whole-body cryotherapy and physical exercises on the psychological well-being of patients with multiple sclerosis: a comparative analysis. Adv Clin Exp Med. 2019 Apr 5;5:5. doi: 10.17219/acem/104529. PMID: 30968613. Exclusion: 5.
814. Pearson M, Dieberg G, Smart N. Exercise as a therapy for improvement of walking ability in adults with multiple sclerosis: a meta-analysis. Arch Phys Med Rehabil. 2015 Jul;96(7):1339-48.e7. doi: 10.1016/j.apmr.2015.02.011. PMID: 25712347. Exclusion: 12.

815. Pelletier CA. Incorporating physical activity into the rehabilitation process after spinal cord injury. Appl Physiol Nutr Metab. 2014 Apr;39(4):513. doi: 10.1139/apnm-20130482. Exclusion: 10.

816. Pelletier CA, Totosy de Zepetnek JO, MacDonald MJ, et al. A 16-week randomized controlled trial evaluating the physical activity guidelines for adults with spinal cord injury. Spinal Cord. 2015 May;53(5):363-7. doi: 10.1038/sc.2014.167. PMID: 25266695. Exclusion: 11.

817. Perrochon A, Borel B, Istrate D, et al. Exercise-based games interventions at home in individuals with a neurological disease: a systematic review and meta-analysis. Ann Phys Rehabil Med. 2019 Sep;62(5):366-78. doi: 10.1016/j.rehab.2019.04.004. PMID: 31078706. Exclusion: 4.

818. Peruzzi A, Cereatti A, Della Croce U, et al. Effects of a virtual reality and treadmill training on gait of subjects with multiple sclerosis: a pilot study. Mult Scler Relat Disord. 2016 Jan;5:91-6. doi: 10.1016/j.msard.2015.11.002. PMID: 26856951. Exclusion: 11.

819. Peruzzi A, Zarbo IR, Cereatti A, et al. An innovative training program based on virtual reality and treadmill: effects on gait of persons with multiple sclerosis. Disabil Rehabil. 2017 Jul;39(15):1557-63. doi: 10.1080/09638288.2016.1224935. PMID: 27808596. Exclusion: 11.

820. Petersen JL. Effects of brief aquatic exercise in multiple sclerosis on mobility and function. Dissertation Abstracts International: Section B: The Sciences and Engineering. 2016;77(5-B(E)). Exclusion: 10. 
821. Peungsuwan P, Parasin P, Siritaratiwat W, et al. Effects of Combined Exercise Training on Functional Performance in Children With Cerebral Palsy: A Randomized-Controlled Study. Pediatr Phys Ther. 2017 01;29(1):3946. doi: 10.1097/PEP.0000000000000338. PMID: 27984466. Exclusion: 11.

822. Pfalzer L, Fry D. Effects of a 10-week inspiratory muscle training program on lower-extremity mobility in people with multiple sclerosis: a randomized controlled trial. Int J MS Care. 2011;13(1):32-42. doi: 10.7224/1537-2073-13.1.32. PMID: 24453703. Exclusion: 4.

823. Phadke C, Veira L, Mathur S, et al. Impact of passive leg cycling in persons with spinal cord injury. Arch Phys Med Rehabil.

2017; Conference: 94th annual conference of the american congress of rehabilitation medicine, ACRM. 2017. United states 98(10):e135. Exclusion: 10.

824. Phadke CP, Vierira L, Mathur S, et al. Impact of passive leg cycling in persons with spinal cord injury: a systematic review. Top Spinal Cord Inj Rehabil. 2019;25(1):8396. doi: 10.1310/sci18-00020. PMID: 30774292. Exclusion: 10.

825. Pham HP, Eidem A, Hansen G, et al. Validity and responsiveness of the trunk impairment scale and trunk control measurement scale in young individuals with cerebral palsy. Phys Occup Ther Pediatr. 2016 Nov;36(4):440-52. doi: 10.3109/01942638.2015.1127867. PMID: 26890372. Exclusion: 8.

826. Phillips AA, Squair JR, Currie KD, et al. 2015 ParaPan American games: autonomic function, but not physical activity, is associated with vascular-cognitive impairment in spinal cord injury. $\mathbf{J}$ Neurotrauma. 2017 Mar 15;34(6):1283-8. doi: 10.1089/neu.2016.4751. PMID: 27998205. Exclusion: 4.

827. Pickering D, Horrocks LM, Visser KS, et al. 'Every picture tells a story': interviews and diaries with children with cerebral palsy about adapted cycling. J Paediatr Child Health. 2013 Dec;49(12):1040-4. doi: 10.1111/jpc.12289. PMID: 23781924. Exclusion: 7.
828. Piira A, Lannem AM, Gjesdal K, et al. Quality of life and psychological outcomes of body-weight supported locomotor training in spinal cord injured persons with long-standing incomplete lesions. Spinal Cord. 2020 May;58(5):560-9. doi: 10.1038/s41393-019-0401-2. PMID: 31848443. Exclusion: 11.

829. Piira A, Lannem AM, Sorensen M, et al. Robot-assisted locomotor training did not improve walking function in patients with chronic incomplete spinal cord injury: a randomized clinical trial. J Rehabil Med. 2019 May 13;51(5):385-9. doi: 10.2340/16501977-2547. PMID: 30895326. Exclusion: 11.

830. Piira A, Lannem AM, Sorensen M, et al. Manually assisted body-weight supported locomotor training does not re-establish walking in non-walking subjects with chronic incomplete spinal cord injury: a randomized clinical trial. J Rehabil Med. 2019 Feb 1;51(2):113-9. doi: 10.2340/16501977-2508. PMID: 30483724. Exclusion: 7.

831. Pillastrini P, Mugnai R, Bonfiglioli R, et al. Evaluation of an occupational therapy program for patients with spinal cord injury. Spinal Cord. 2008 Jan;46(1):78-81. doi: 10.1038/sj.sc.3102072. PMID: 17453011. Exclusion: 4.

832. Pilutti LA, Dlugonski D, Sandroff BM, et al. Randomized controlled trial of a behavioral intervention targeting symptoms and physical activity in multiple sclerosis. Mult Scler. 2014 Apr;20(5):594-601. doi: 10.1177/1352458513503391. PMID: 24009162. Exclusion: 4.

833. Pilutti LA, Dlugonski D, Sandroff BM, et al. Internet-delivered lifestyle physical activity intervention improves body composition in multiple sclerosis: preliminary evidence from a randomized controlled trial. Arch Phys Med Rehabil. 2014 Jul;95(7):1283-8. doi: 10.1016/j.apmr.2014.03.015. PMID: 24699237. Exclusion: 4.

834. Pilutti LA, Edwards TA. Is Exercise Training Beneficial in Progressive Multiple Sclerosis? Int J MS Care. 2017;19(2):10512. doi: 10.7224/1537-2073.2016-034. Exclusion: 10. 
835. Pilutti LA, Motl RW. Functional Electrical Stimulation Cycling Exercise for People with Multiple Sclerosis. Curr Treat Options Neurol. 2019 Nov 08;21(11):54. doi: 10.1007/s11940-019-0597-7. PMID: 31705304. Exclusion: 12.

836. Pilutti LA, Paulseth JE, Dove C, et al. Exercise training in progressive multiple sclerosis: a comparison of recumbent stepping and body weight-supported treadmill training. Int J MS Care. 2016 SepOct;18(5):221-9. doi: 10.7224/15372073.2015-067. PMID: 27803637. Exclusion: 11.

837. Pilutti LA, Sandroff BM, Klaren RE, et al. Physical fitness assessment across the disability spectrum in persons with multiple sclerosis: a comparison of testing modalities. J Neurol Phys Ther. 2015 Oct;39(4):241-9. doi: 10.1097/NPT.0000000000000099. PMID: 26247510. Exclusion: 4.

838. Platta ME, Ensari I, Motl RW, et al. Effect of exercise training on fitness in multiple sclerosis: a meta-analysis. Arch Phys Med Rehabil. 2016 Sep;97(9):1564-72. doi: 10.1016/j.apmr.2016.01.023. PMID: 26896750. Exclusion: 12.

839. Ploughman M, Shears J, Harris C, et al. Effectiveness of a novel community exercise transition program for people with moderate to severe neurological disabilities. NeuroRehabilitation. 2014;35(1):105-12. doi: 10.3233/NRE-141090. PMID: 24990000. Exclusion: 3.

840. Plow M, Bethoux F, Mai K, et al. A formative evaluation of customized pamphlets to promote physical activity and symptom self-management in women with multiple sclerosis. Health Educ Res. 2014 Oct;29(5):883-96. doi: 10.1093/her/cyu034. PMID: 24989822. Exclusion: 4.

841. Plow M, Bethoux F, McDaniel C, et al. Randomized controlled pilot study of customized pamphlets to promote physical activity and symptom self-management in women with multiple sclerosis. Clin Rehabil. 2014 Feb;28(2):139-48. doi: 10.1177/0269215513494229. PMID: 23864516. Exclusion: 4.
842. Plow M, Finlayson M. Potential benefits of nintendo wii fit among people with multiple sclerosis: a longitudinal pilot study. Int J MS Care. 2011;13(1):21-30. doi: 10.7224/15372073-13.1.21. PMID: 24453702. Exclusion: 8.

843. Plow M, Motl RW, Finlayson M, et al. Intervention Mediators in a Randomized Controlled Trial to Increase Physical Activity and Fatigue Self-management Behaviors Among Adults With Multiple Sclerosis. Ann Behav Med. 2019 Oct 09;09:09. doi: 10.1093/abm/kaz033. PMID: 31595300. Exclusion: 4.

844. Plow M, Motl RW, Finlayson M, et al. Response heterogeneity in a randomized controlled trial of telerehabilitation interventions among adults with multiple sclerosis. J Telemed Telecare. 2020 Oct 25:1357633X20964693. doi: 10.1177/1357633X20964693. PMID: 33100184. Exclusion: 4.

845. Plow MA, Mathiowetz V, Lowe DA. Comparing individualized rehabilitation to a group wellness intervention for persons with multiple sclerosis. Am J Health Promot. 2009 Sep-Oct;24(1):23-6. doi: 10.4278/ajhp.071211128. PMID: 19750959. Exclusion: 4.

846. Plow MA, Mathiowetz V, Resnik L. Multiple sclerosis: impact of physical activity on psychosocial constructs. Am J Health Behav. 2008 Nov-Dec;32(6):614-26. doi: 10.5555/ajhb.2008.32.6.614. PMID: 18442341. Exclusion: 4.

847. Plummer P. Critical appraisal of evidence for improving gait speed in people with multiple sclerosis: dalfampridine versus gait training. Int J MS Care. 2016 MayJun;18(3):105-15. doi: 10.7224/15372073.2014-114. PMID: 27252597.

Exclusion: 12.

848. Poehlau D, Drache M, Gerber-Grothe A, et al. Hippotherapy improves symptoms of multiple sclerosis-results of a randomised controlled multicentre study. Mult Scler J Exp Transl Clin. 2015 START: 2015 Oct 7 CONFERENCE END: 2015 Oct 10, 31st Congress of the European Committee for Treatment and Research in Multiple Sclerosis, ECTRIMS 2015 Barcelona Spain;21(11 SUPPL. 1):795-6. doi: 10.1177/1352458515604791. Exclusion: 10. 
849. Poettgen J, Moss-Morris R, Wendebourg $\mathrm{JM}$, et al. Online fatigue management program for patients with multiple sclerosisa randomized controlled trial. Mult Scler J Exp Transl Clin. START: 2015 Oct 7 CONFERENCE END: 2015 Oct 10, 31st Congress of the European Committee for Treatment and Research in Multiple Sclerosis, ECTRIMS 2015 Barcelona Spain;23(11 SUPPL. 1):41-2. Exclusion: 10.

850. Poirot I, Laudy V, Rabilloud M, et al. Rehabilitation of 190 non-ambulatory children with cerebral palsy in structures of care or in liberal sector. Ann Phys Rehabil Med. 2013 Oct;56(7-8):551-60. doi: 10.1016/j.rehab.2013.08.002. PMID: 24120244. Exclusion: 7.

851. Pool D, Blackmore AM, Bear N, et al. Effects of short-term daily community walk aide use on children with unilateral spastic cerebral palsy. Pediatr Phys Ther. 2014;26(3):308-17. doi: 10.1097/PEP.0000000000000057. PMID: 24979083. Exclusion: 8.

852. Popovic-Maneski L, Aleksic A, Metani A, et al. Assessment of spasticity by a pendulum test in SCI patients who exercise FES cycling or receive only conventional therapy. IEEE Trans Neural Syst Rehabil Eng. 2018 Jan;26(1):181-7. doi: 10.1109/TNSRE.2017.2771466. PMID: 29324409. Exclusion: 5.

853. Possover M. The LION procedure to the pelvic nerves for recovery of locomotion in 18 spinal cord injured peoples - A case series. Surg Technol Int. 2016 Oct 26;29:1925. PMID: 27728946. Exclusion: 4.

854. Postma K, Haisma JA, de Groot S, et al. Changes in pulmonary function during the early years after inpatient rehabilitation in persons with spinal cord injury: a prospective cohort study. Arch Phys Med Rehabil. 2013 Aug;94(8):1540-6. doi: 10.1016/j.apmr.2013.02.006. PMID: 23416767. Exclusion: 4.

855. Pountney T, Williams H, Bryant E. Effectiveness of an intensive six week graded exercise programmes for non ambulant children and young people with cerebral palsy. Physiotherapy. 2011;97(S1):eS1016-7. doi: 10.1016/j.physio.2011.04.002. Exclusion: 10.
856. Pourazar M, Mirakhori F, Hemayattalab R, et al. Use of virtual reality intervention to improve reaction time in children with cerebral palsy: a randomized controlled trial. Dev Neurorehabil. 2018;21(8):515-20. doi: 10.1080/17518423.2017.1368730.

Exclusion: 6.

857. Prakash RS, Snook EM, Kramer AF, et al. Correlation of physical activity with perceived cognitive deficits in relapsingremitting multiple sclerosis. Int J MS Care. 2010;12(1):1-5. Exclusion: 6 .

858. Prakash RS, Snook EM, Motl RW, et al. Aerobic fitness is associated with gray matter volume and white matter integrity in multiple sclerosis. Brain Res. 2010 Jun 23;1341:41-51. doi: 10.1016/j.brainres.2009.06.063. PMID: 19560443. Exclusion: 6.

859. Preede L, Saebu M, Perrin PB, et al. Oneyear trajectories of mental and physical functioning during and after rehabilitation among individuals with disabilities. Health Qual Life Outcomes. 2015 Aug 28;13:135. doi: 10.1186/s12955-015-0328-z. PMID: 26315445. Exclusion: 3.

860. Prenton S, Hollands KL, Kenney LPJ, et al. Functional electrical stimulation and ankle foot orthoses provide equivalent therapeutic effects on foot drop: a meta-analysis providing direction for future research. $\mathrm{J}$ Rehabil Med. 2018 Feb 13;50(2):129-39. doi: 10.2340/16501977-2289. PMID: 29227525. Exclusion: 11.

861. Priya MS, Kalpana AP. Effect of strengthening of inspiratory muscles using inspiratory muscle trainer on pulmonary function among patients with spinal cord injury-a quasi-experimental study. Indian J Physiother Occup Ther. 2018;12(1):64-9. doi: 10.5958/0973-5674.2018.00012.6. Exclusion: 14.

862. Proctor BJ, Moghaddam N, Vogt W, et al. Telephone psychotherapy in multiple sclerosis: a systematic review and metaanalysis. Rehabil Psychol. 2018 Feb;63(1):16-28. doi: 10.1037/rep0000182. PMID: 29553780. Exclusion: 4. 
863. Prosperini L, Fortuna D, Gianni C, et al. Home-based balance training using the Wii balance board: a randomized, crossover pilot study in multiple sclerosis. Neurorehabil Neural Repair. 2013 Jul-Aug;27(6):516-25. doi: $10.1177 / 1545968313478484$. PMID: 23478168. Exclusion: 4.

864. Prosperini L, Leonardi L, De Carli P, et al. Visuo-proprioceptive training reduces risk of falls in patients with multiple sclerosis. Mult Scler. 2010 Apr;16(4):491-9. doi: 10.1177/1352458509359923. PMID: 20150396. Exclusion: 3.

865. Qiu S, Alzhab S, Picard G, et al. Ventilation limits aerobic capacity after functional electrical stimulation row training in high spinal cord injury. Med Sci Sports Exerc. 2016 Jun;48(6):1111-8. doi: 10.1249/MSS.0000000000000880. PMID: 26784276. Exclusion: 11.

866. Quel de Oliveira C, Refshauge K, Middleton $\mathrm{J}$, et al. Effects of activity-based therapy interventions on mobility, independence, and quality of life for people with spinal cord injuries: a systematic review and metaanalysis. J Neurotrauma. 2017 May 1;34(9):1726-43. doi: 10.1089/neu.2016.4558. PMID: 27809702. Exclusion: 12.

867. Raab AM, Krebs J, Perret C, et al. Evaluation of a clinical implementation of a respiratory muscle training group during spinal cord injury rehabilitation. Spinal Cord Ser Cases. 2018;4:40. doi: 10.1038/s41394018-0069-4. PMID: 29736265. Exclusion: 4.

868. Raab AM, Krebs J, Pfister M, et al. Respiratory muscle training in individuals with spinal cord injury: effect of training intensity and -volume on improvements in respiratory muscle strength. Spinal Cord. 2019;57(6):482-9. doi: 10.1038/s41393-0190249-5. Exclusion: 8.

869. Rafeeyan Z, Azarbarzin M, Moosa FM, et al. Effect of aquatic exercise on the multiple sclerosis patients' quality of life. Iran J Nurs Midwifery Res. 2010;15(1):43-7. PMID: 21589749. Exclusion: 11.
870. Rahnama N, Namazizadeh M, Etemadifar $\mathrm{M}$, et al. Effects of yoga on depression in women with multiple sclerosis. Journal of isfahan medical school. 2011 Jun;29(136) doi: 10.1016/j.ejmhg.2014.02.007.

Exclusion: 13.

871. Raithatha R, Carrico C, Powell ES, et al. Non-invasive brain stimulation and robotassisted gait training after incomplete spinal cord injury: a randomized pilot study. NeuroRehabilitation. 2016;38(1):15-25. doi: 10.3233/NRE-151291. PMID: 26889794. Exclusion: 11.

872. Rameckers EA, Duysens J, Speth LA, et al. Effect of addition of botulinum toxin-A to standardized therapy for dynamic manual skills measured with kinematic aiming tasks in children with spastic hemiplegia. J Rehabil Med. 2010 Apr;42(4):332-8. doi: 10.2340/16501977-0521. PMID: 20461335. Exclusion: 5.

873. Rameckers EA, Janssen-Potten YJ, Essers IM, et al. Efficacy of upper limb strengthening in children with cerebral palsy: a critical review. Res Dev Disabil. 2015 Jan;36C:87-101. doi: 10.1016/j.ridd.2014.09.024. PMID: 25462469. Exclusion: 12.

874. Rameckers EA, Speth LA, Duysens J, et al. Botulinum toxin-a in children with congenital spastic hemiplegia does not improve upper extremity motor-related function over rehabilitation alone: a randomized controlled trial. Neurorehabil Neural Repair. 2009 Mar-Apr;23(3):218-25. doi: $10.1177 / 1545968308326629$. PMID: 19106252. Exclusion: 5.

875. Rasooli AH, Birgani PM, Azizi S, et al. Therapeutic effects of an anti-gravity locomotor training (AlterG) on postural balance and cerebellum structure in children with cerebral palsy. IEEE Int. 2017 Jul;2017:101-5. doi: 10.1109/ICORR.2017.8009229. PMID: 28813801. Exclusion: 11.

876. Ray AD, Udhoji S, Mashtare TL, et al. A combined inspiratory and expiratory muscle training program improves respiratory muscle strength and fatigue in multiple sclerosis. Arch Phys Med Rehabil. 2013 Oct;94(10):1964-70. doi: 10.1016/j.apmr.2013.05.005. PMID: 23714277. Exclusion: 11. 
877. Rayegani SM, Shojaee H, Sedighipour L, et al. The effect of electrical passive cycling on spasticity in war veterans with spinal cord injury. Front Neurol. 2011;2:39. doi: 10.3389/fneur.2011.00039. PMID: 21734906. Exclusion: 4.

878. Reedman S, Boyd RN, Sakzewski L. The efficacy of interventions to increase physical activity participation of children with cerebral palsy: a systematic review and meta-analysis. Dev Med Child Neurol. 2017 Oct;59(10):1011-8. doi:

10.1111/dmcn.13413. PMID: 28318009. Exclusion: 6.

879. Reedman SE, Boyd RN, Trost SG, et al. Efficacy of participation-focused therapy on performance of physical activity participation goals and habitual phsical activity in children with cerebral palsy: a randomized controlled trial. Arch Phys Med Rehabil. 2019 Dec 11;100(4):676-86. doi: 10.1016/j.apmr.2018.11.012. PMID: 30543803. Exclusion: 4.

880. Reedman SE, Boyd RN, Trost SG, et al. Efficacy of Participation-Focused Therapy on Performance of Physical Activity Participation Goals and Habitual Physical Activity in Children With Cerebral Palsy: A Randomized Controlled Trial. Arch Phys Med Rehabil. 2019 04;100(4):676-86. doi: 10.1016/j.apmr.2018.11.012. PMID: 30543803. Exclusion: 4.

881. Reichenfelser W, Hackl H, Hufgard J, et al. Monitoring of spasticity and functional ability in individuals with incomplete spinal cord injury with a functional electrical stimulation cycling system. J Rehabil Med. 2012 May;44(5):444-9. doi: 10.2340/16501977-0979. PMID: 22549654. Exclusion: 8.

882. Reid S, Hamer P, Alderson J, et al. Neuromuscular adaptations to eccentric strength training in children and adolescents with cerebral palsy. Dev Med Child Neurol. 2010 Apr;52(4):358-63. doi:

10.1111/j.1469-8749.2009.03409.x. PMID: 19737297. Exclusion: 11.
883. Rejc E, Angeli CA, Bryant N, et al. Effects of stand and step training with epidural stimulation on motor function for standing in chronic complete paraplegics. J Neurotrauma. 2017 May 1;34(9):1787-802. doi: 10.1089/neu.2016.4516. PMID: 27566051. Exclusion: 11.

884. Reyes ML, Hernandez M, Holmgren LJ, et al. High-frequency, low-intensity vibrations increase bone mass and muscle strength in upper limbs, improving autonomy in disabled children. J Bone Miner Res. 2011 Aug;26(8):1759-66. doi: 10.1002/jbmr.402. PMID: 21491486. Exclusion: 3.

885. Rice IM, Rice LA, Motl RW. Promoting physical activity through a manual wheelchair propulsion intervention in persons with multiple sclerosis. Arch Phys Med Rehabil. 2015 Oct;96(10):1850-8. doi: 10.1016/j.apmr.2015.06.011. PMID: 26150167. Exclusion: 11.

886. Richardson EJ, McKinley EC, Rahman A, et al. Effects of virtual walking on spinal cord injury-related neuropathic pain: a randomized, controlled trial. Rehabil Psychol. 2019 Feb;64(1):13-24. doi: 10.1037/rep0000246. PMID: 30407030. Exclusion: 4.

887. Richter WM, Kwarciak AM, Guo L, et al. Effects of single-variable biofeedback on wheelchair handrim biomechanics. Arch Phys Med Rehabil. 2011 Apr;92(4):572-7. doi: 10.1016/j.apmr.2010.11.001. PMID: 21440701. Exclusion: 4.

888. Riener R, Dislaki E, Keller U, et al. Virtual reality aided training of combined arm and leg movements of children with CP. Stud Health Technol Inform. 2013;184:349-55. PMID: 23400183. Exclusion: 7.

889. Rietberg MB, Van Wegen EEH, Eyssen ICJM, et al. Effects of multidisciplinary rehabilitation on chronic fatigue in multiple sclerosis: a randomized controlled trial. PLoS One. 2014;9(9) doi: 10.1371/journal.pone.0107710. PMID: 25232955 Exclusion: 4.

890. Rietberg MB, Veerbeek JM, Gosselink R, et al. Respiratory muscle training for multiple sclerosis. Cochrane Database Syst Rev. 2017 Dec 21;12:CD009424. doi: 10.1002/14651858.CD009424.pub2. PMID: 29267988. Exclusion: 4. 
891. Rigby BR, Gloeckner AR, Sessums S, et al. Changes in cardiorespiratory responses and kinematics with hippotherapy in youth with and without cerebral palsy. Res Q Exerc Sport. 2017 Mar;88(1):26-35. doi: 10.1080/02701367.2016.1266458. PMID: 28075704. Exclusion: 11.

892. Rigby BR, Grandjean PW. The efficacy of equine-assisted activities and therapies on improving physical function. J Altern Complement Med. 2016 Jan;22(1):9-24. doi: 10.1089/acm.2015.0171. PMID: 26654868. Exclusion: 12.

893. Rigot S, Worobey L, Boninger ML. Gait training in acute spinal cord injury rehabilitation-utilization and outcomes among nonambulatory individuals: findings from the SCIRehab project. Arch Phys Med Rehabil. 2018 Aug;99(8):1591-8. doi: 10.1016/j.apmr.2018.01.031. PMID: 29510092. Exclusion: 4.

894. Riksfjord SM, Braendvik SM, Roksund OD, et al. Ventilatory efficiency and aerobic capacity in people with multiple sclerosis: a randomized study. SAGE Open Med. 2017;5:2050312117743672. doi: 10.1177/2050312117743672. PMID: 29276588. Exclusion: 11.

895. Rimmer JH, Herman C, Wingo B, et al. Methodological and clinical implications of a three-in-one Russian doll design for tracking health trajectories and improving health and function through innovative exercise treatments in adults with disability. BMC Med Res Methodol. 2018 Mar 14;18(1):28. doi: 10.1186/s12874-018-04803. PMID: 29540164. Exclusion: 10.

896. Rimmer JH, Thirumalai M, Young HJ, et al. Rationale and design of the tele-exercise and multiple sclerosis (TEAMS) study: a comparative effectiveness trial between a clinic- and home-based telerehabilitation intervention for adults with multiple sclerosis (MS) living in the deep south. Contemp Clin Trials. 2018 Aug;71:186-93. doi: 10.1016/j.cct.2018.05.016. PMID: 29859267. Exclusion: 4.
897. Rimmer JH, Wang E, Pellegrini CA, et al. Telehealth weight management intervention for adults with physical disabilities: a randomized controlled trial. Am J Phys Med Rehabil. 2013 Dec;92(12):1084-94. doi: 10.1097/PHM.0b013e31829e780e. PMID: 24257266. Exclusion: 3.

898. Rintala A, Hakala S, Paltamaa J, et al. Effectiveness of technology-based distance physical rehabilitation interventions on physical activity and walking in multiple sclerosis: a systematic review and metaanalysis of randomized controlled trials. Disabil Rehabil. 2018 Feb;40(4):373-87. doi: 10.1080/09638288.2016.1260649. PMID: 27973919. Exclusion: 4.

899. Rios DC, Gilbertson T, McCoy SW, et al. NeuroGame Therapy to improve wrist control in children with cerebral palsy: a case series. Dev Neurorehabil. 2013

Dec;16(6):398-409. doi: 10.3109/17518423.2013.766818. PMID: 23617243. Exclusion: 11.

900. Riquelme I, Zamorano A, Montoya P. Reduction of pain sensitivity after somatosensory therapy in adults with cerebral palsy. Front Hum Neurosci. 2013;7:276. doi: 10.3389/fnhum.2013.00276. PMID: 23805086. Exclusion: 4.

901. Ritzmann R, Stark C, Krause A. Vibration therapy in patients with cerebral palsy: a systematic review. Neuropsychiatr.

2018;14:1607-25. doi: 10.2147/NDT.S152543. PMID: 29950843. Exclusion: 8.

902. Robert M, Ballaz L, Hart R, et al. Exercise intensity levels in children with cerebral palsy while playing with an active video game console. Phys Ther. 2013 Aug;93(8):1084-91. doi: 10.2522/ptj.20120204. PMID: 23580626. Exclusion: 11.

903. Robertson EK. The bottom line. On 'Effects of hand cycle training on physical capacity in individuals with tetraplegia: a clinical trial'. Phys Ther. 2009 Oct;89(10):1014-5. doi: 10.2522/ptj.20080340. Exclusion: 10. 
904. Robinson AG, Dennett AM, Snowdon DA. Treadmill training may be an effective form of task-specific training for improving mobility in people with Parkinson's disease and multiple sclerosis: a systematic review and meta-analysis. Physiotherapy. 2019 06;105(2):174-86. doi: 10.1016/j.physio.2018.11.007. PMID: 30876717. Exclusion: 12.

905. Robinson J, Dixon J, Macsween A, et al. The effects of exergaming on balance, gait, technology acceptance and flow experience in people with multiple sclerosis: a randomized controlled trial. BMC Sports Sci Med Rehabil. 2015;7:8. doi: 10.1186/s13102-015-0001-1. PMID: 25969739. Exclusion: 4.

906. Rocca MA, Filippi M, Deiva K. Promoting physical activity to control multiple sclerosis from childhood. Neurology. $2015 \mathrm{Nov}$ 10;85(19):1644-5. doi: 10.1212/WNL.0000000000001941. PMID: 26268902. Exclusion: 2.

907. Rocchi MA, Robichaud Lapointe T, Gainforth HL, et al. Delivering a tele-health intervention promoting motivation and leisure-time physical activity among adults with spinal cord injury: An implementation evaluation. Sport Exerc Perform Psychol. 2020 Mar:No Pagination Specified. doi: http://dx.doi.org/10.1037/spy0000207. PMID: 2020-17702-001. Exclusion: 11.

908. Rodrigues TA, Fischer DA, Cyrillo FN, et al. Effectiveness of virtual reality using Wii gaming in the treatment of cerebral palsy. Neurorehabil Neural Repair. START: 2012 May 16 CONFERENCE END: 2012 May 19, 7th World Congress for NeuroRehabilitation, WCNR 2012 Melbourne, VIC Australia;26(6):NP8. Exclusion: 10.

909. Roeh A, Kirchner SK, Malchow B, et al. Depression in somatic disorders: is there a beneficial effect of exercise? Front Psychiatr. 2019;10:141. doi: 10.3389/fpsyt.2019.00141. PMID: 30949079. Exclusion: 12.
910. Roels EH, Aertgeerts B, Ramaekers D, et al. Hospital- and community-based interventions enhancing (re)employment for people with spinal cord injury: a systematic review. Spinal Cord. 2016 Jan;54(1):2-7. doi: 10.1038/sc.2015.133. PMID: 26305872. Exclusion: 4.

911. Rooney S, Moffat F, Wood L, et al. Effectiveness of fatigue management interventions in reducing severity and impact of fatigue in people with progressive multiple sclerosis: a systematic review. Int J MS Care. 2019 Jan-Feb;21(1):35-46. doi: 10.7224/1537-2073.2018-019. PMID: 30833871. Exclusion: 12.

912. Roostaei M, Baharlouei H, Azadi H, et al. Effects of aquatic intervention on gross motor skills in children with cerebral palsy: a systematic review. Phys Occup Ther Pediatr. 2017 Oct 20;37(5):496-515. doi: 10.1080/01942638.2016.1247938. PMID: 27967298. Exclusion: 12.

913. Rosenbaum P. A randomized controlled trial of the impact of therapeutic horse riding on the quality of life, health, and function of children with cerebral palsy. Dev Med Child Neurol. 2009 Feb;51(2):88. doi: 10.1111/j.1469-8749.2008.03259.x. Exclusion: 10.

914. Rosety-Rodriguez M, Camacho A, Rosety I, et al. Low-grade systemic inflammation and leptin levels were improved by arm cranking exercise in adults with chronic spinal cord injury. Arch Phys Med Rehabil. 2014 Feb;95(2):297-302. doi: 10.1016/j.apmr.2013.08.246. PMID: 24060491. Exclusion: 11.

915. Rosety-Rodriguez M, Rosety I, Fornieles G, et al. A short-term arm-crank exercise program improved testosterone deficiency in adults with chronic spinal cord injury. Int Braz J Urol. 2014 May-Jun;40(3):367-72. doi: 10.1590/S1677-5538.IBJU.2014.03.10. PMID: 25010302. Exclusion: 11.

916. Ross SA, Yount M, Ankarstad S, et al. Effects of participation in sports programs on walking ability and endurance over time in children with cerebral palsy. Am J Phys Med Rehabil. 2017 Dec;96(12):843-51. doi: 10.1097/PHM.0000000000000767. PMID: 28644242. Exclusion: 8. 
917. Rostami HR, Arastoo AA, Nejad SJ, et al. Effects of modified constraint-induced movement therapy in virtual environment on upper-limb function in children with spastic hemiparetic cerebral palsy: a randomised controlled trial. NeuroRehabilitation. 2012;31(4):357-65. doi: 10.3233/NRE2012-00804. PMID: 23232158. Exclusion: 11.

918. Roth EJ, Stenson KW, Powley S, et al. Expiratory muscle training in spinal cord injury: a randomized controlled trial. Arch Phys Med Rehabil. 2010 Jun;91(6):857-61. doi: 10.1016/j.apmr.2010.02.012. PMID: 20510974. Exclusion: 4.

919. Ruck J, Chabot G, Rauch F. Vibration treatment in cerebral palsy: a randomized controlled pilot study. J Musculoskelet Neuronal Interact. 2010 Mar;10(1):77-83. PMID: 20190383. Exclusion: 11.

920. Ruiz J, Labas MP, Triche EW, et al. Combination of robot-assisted and conventional body-weight-supported treadmill training improves gait in persons with multiple sclerosis: a pilot study. $\mathrm{J}$ Neurol Phys Ther. 2013 Dec;37(4):187-93. doi: 10.1097/NPT.0000000000000018. PMID: 24189336. Exclusion: 11.

921. Rupp R, Schliesmann D, Plewa H, et al. Safety and efficacy of at-home robotic locomotion therapy in individuals with chronic incomplete spinal cord injury: a prospective, pre-post intervention, proof-ofconcept study.[Erratum appears in PLoS One. 2015;10(4):e0126999; PMID: 25894511]. PLoS One. 2015;10(3):e0119167. doi: 10.1371/journal.pone.0119167. PMID: 25803577. Exclusion: 8.

922. Ryan JM, Cassidy EE, Noorduyn SG, et al. Exercise interventions for cerebral palsy. Cochrane Database Syst Rev. 2017 Jun 11;6:CD011660. doi: 10.1002/14651858.CD011660.pub2. PMID: 28602046. Exclusion: 12.
923. Ryan JM, Fortune J, Stennett A, et al. Safety, feasibility, acceptability and effects of a behaviour-change intervention to change physical activity behaviour among people with multiple sclerosis: results from the iStep-MS randomised controlled trial. Mult Scler. 2019 Oct 31:1352458519886231. doi: 10.1177/1352458519886231. PMID: 31668138. Exclusion: 4.

924. Ryan JM, Lavelle G, Theis N, et al. Progressive resistance training for adolescents with cerebral palsy: the STAR randomized controlled trial. Dev Med Child Neurol. 2020 Jun 26 doi: 10.1111/dmcn.14601. PMID: 32588919. Exclusion: 4.

925. Ryan TE, Brizendine JT, Backus D, et al. Electrically induced resistance training in individuals with motor complete spinal cord injury. Arch Phys Med Rehabil. 2013 Nov;94(11):2166-73. doi: 10.1016/j.apmr.2013.06.016. PMID: 23816921. Exclusion: 11.

926. Ryu K, Ali A, Kwon M, et al. Effects of assisted aquatic movement and horseback riding therapies on emotion and brain activation in patients with cerebral palsy. $\mathrm{J}$ Phys Ther Sci. 2016;28(12):3283-7. PMID: 28174435. Exclusion: 4.

927. Sa-Caputo DC, Costa-Cavalcanti R, Carvalho-Lima RP, et al. Systematic review of whole body vibration exercises in the treatment of cerebral palsy: brief report. Dev Neurorehabil. 2016 Oct;19(5):327-33. doi: 10.3109/17518423.2014.994713. PMID: 25826535. Exclusion: 12.

928. Sabapathy NM, Minahan CL, Turner GT, et al. Comparing endurance- and resistanceexercise training in people with multiple sclerosis: a randomized pilot study. Clin Rehabil. 2011 Jan;25(1):14-24. doi: 10.1177/0269215510375908. PMID: 20713434. Exclusion: 11.

929. Sadeghi Bahmani D, Farnia V, Shakeri J, et al. The comparison of the effect of yoga and aquatic exercises on depression and fatigue in patients with multiple sclerosis. Pharmacopsychiatry. Conference: 29th symposium of the AGNP. Germany. Conference start. 2015;25(6). Exclusion: 10. 
930. Sadeghi Bahmani D, Kesselring J, Papadimitriou M, et al. In patients with multiple sclerosis, both objective and subjective sleep, depression, fatigue, and paresthesia improved after 3 weeks of regular exercise. Front Psychiatr. 2019;10:265. doi: 10.3389/fpsyt.2019.00265. PMID: 31130879. Exclusion: 8.

931. Sadeghi Bahmani D, Razazian N, Alikhani $\mathrm{M}$, et al. Regular exercise program impacts positively on sleep disturbances, depression and fatigue in female patients with multiple sclerosis. J Sleep Res. 2018 Sep;Conference: 24th congress of the european sleep research society, ESRS. 2018. Switzerland 27(Supplement 1):309. doi:

10.1111/jsr.12751. PMID: 30203880. Exclusion: 10.

932. Sadeghi Bahmani D, Razazian N, Motl RW, et al. Physical activity interventions can improve emotion regulation and dimensions of empathy in persons with multiple sclerosis: An exploratory study. Mult Scler Relat Disord. 2020 Jan;37:101380. doi: 10.1016/j.msard.2019.101380. PMID: 32173007. Exclusion: 6.

933. Sadeghi M, Ghasemi G, Karimi M. Effect of 12-week- rebound therapy exercise on static stability of patients with spinal cord injury. $\mathrm{J}$ Sport Rehabil. 2018 Feb 6:1-16. doi: 10.1123/jsr.2017-0303. PMID: 29405819. Exclusion: 11.

934. Sadeghi M, Sawatzky B. Effects of vibration on spasticity in individuals with spinal cord injury: a scoping systematic review. Am J Phys Med Rehabil. 2014 Nov;93(11):9951007. doi: 10.1097/PHM.0000000000000098. PMID: 24743464. Exclusion: 9.

935. Sadowska-Krepa E, Zwierzchowska A, Glowacz M, et al. Blood metabolic response to a long-term wheelchair rugby training. Spinal Cord. 2016 May;54(5):371-5. doi: 10.1038/sc.2015.178. PMID: 26481699. Exclusion: 4.
936. Sah A, Balaji G, Agrahara S. Effects of taskoriented activities based on neurodevelopmental therapy principles on trunk control, balance, and gross motor function in children with spastic diplegic cerebral palsy: a single-blinded randomized clinical trial. J Pediatr Neurosci. 2019;14(3):120-6. doi: 10.4103/jpn.JPN_35_19. Exclusion: 14.

937. Sajan JE, John JA, Grace P, et al. Wii-based interactive video games as a supplement to conventional therapy for rehabilitation of children with cerebral palsy: a pilot, randomized controlled trial. Dev Neurorehabil. 2017 Aug;20(6):361-7. doi: 10.1080/17518423.2016.1252970. PMID: 27846366. Exclusion: 11.

938. Sakzewski L. Bimanual therapy and constraint-induced movement therapy are equally effective in improving hand function in children with congenital hemiplegia. $\mathrm{J}$ Physiother. 2012;58(1):59. doi: 10.1016/S1836-9553(12)70075-9. PMID: 22341385. Exclusion: 4.

939. Sakzewski L, Provan K, Ziviani J, et al. Comparison of dosage of intensive upper limb therapy for children with unilateral cerebral palsy: how big should the therapy pill be? Res Dev Disabil. 2015 Feb;37:9-16. doi: 10.1016/j.ridd.2014.10.050. PMID: 25460215. Exclusion: 4.

940. Sakzewski L, Ziviani J, Boyd RN. Best responders after intensive upper-limb training for children with unilateral cerebral palsy. Arch Phys Med Rehabil. 2011 Apr;92(4):578-84. doi: 10.1016/j.apmr.2010.12.003. PMID: 21440702. Exclusion: 6.

941. Salbach NM, Howe JA, Brunton K, et al. Partnering to increase access to community exercise programs for people with stroke, acquired brain injury, and multiple sclerosis. J Phys Act Health. 2014 May;11(4):838-45. doi: 10.1123/jpah.2012-0183. PMID: 23676952. Exclusion: 3.

942. Salem Y, Godwin EM. Effects of taskoriented training on mobility function in children with cerebral palsy.

NeuroRehabilitation. 2009;24(4):307-13. doi: 10.3233/NRE-2009-0483. PMID: 19597267. Exclusion: 11. 
943. Salem Y, Scott AH, Karpatkin H, et al. Community-based group aquatic programme for individuals with multiple sclerosis: a pilot study. Disabil Rehabil. 2011;33(9):720-8. doi: 10.3109/09638288.2010.507855. PMID: 20726740. Exclusion: 11.

944. Salvador AF, Schubert KR, Cruz RS, et al. Bilateral muscle strength symmetry and performance are improved following walk training with restricted blood flow in an elite paralympic sprint runner: case study. Phys Ther Sport. 2016 Jul;20:1-6. doi: 10.1016/j.ptsp.2015.10.004. PMID: 27325532. Exclusion: 7.

945. Sanchez-Lastra MA, Martinez-Aldao D, Molina AJ, et al. Pilates for people with multiple sclerosis: A systematic review and meta-analysis. Mult Scler Relat Disord. 2019 Jan 03;28:199-212. doi: 10.1016/j.msard.2019.01.006. PMID: 30623859. Exclusion: 12.

946. Sandroff BM. Exercise and cognition in multiple sclerosis: the importance of acute exercise for developing better interventions. Neurosci Biobehav Rev. 2015 Dec;59:17383. doi: 10.1016/j.neubiorev.2015.10.012. PMID: 26584548. Exclusion: 4.

947. Sandroff BM, Baird JF, Silveira SL, et al. Response heterogeneity in fitness, mobility and cognition with exercise-training in MS. Acta Neurol Scand. 2019 Feb;139(2):18391. doi: 10.1111/ane.13041. PMID: 30372515. Exclusion: 8.

948. Sandroff BM, Balto JM, Klaren RE, et al. Systematically developed pilot randomized controlled trial of exercise and cognition in persons with multiple sclerosis. Neurocase. 2016 10;22(5):443-50. doi: 10.1080/13554794.2016.1237658. PMID: 27671243. Exclusion: 4.

949. Sandroff BM, Hillman CH, Benedict RH, et al. Acute effects of walking, cycling, and yoga exercise on cognition in persons with relapsing-remitting multiple sclerosis without impaired cognitive processing speed. J Clin Exp Neuropsychol. 2015;37(2):209-19. doi: 10.1080/13803395.2014.1001723. PMID: 25658772. Exclusion: 4.
950. Sandroff BM, Hillman CH, Benedict RH, et al. Acute effects of varying intensities of treadmill walking exercise on inhibitory control in persons with multiple sclerosis: a pilot investigation. Physiol Behav. 2016 Feb 01;154:20-7. doi: 10.1016/j.physbeh.2015.11.008. PMID: 26569451. Exclusion: 4.

951. Sandroff BM, Klaren RE, Pilutti LA, et al. Randomized controlled trial of physical activity, cognition, and walking in multiple sclerosis. J Neurol. 2014 Feb;261(2):363-72. doi: 10.1007/s00415-013-7204-8. PMID: 24323244. Exclusion: 4.

952. Sandroff BM, Motl RW, Scudder MR, et al. Systematic,evidence-based review of exercise, physical activity, and physical fitness effects on cognition in persons with multiple sclerosis. Neuropsychol Rev. 2016 Sep;26(3):271-94. doi: 10.1007/s11065-0169324-2. PMID: 27447980. Exclusion: 4.

953. Sangelaji B, Estebsari F, Nabavi SM, et al. The effect of exercise therapy on cognitive functions in multiple sclerosis patients: a pilot study. Med J Islam Repub Iran. 2015;29:205. PMID: 26157723. Exclusion: 11.

954. Sangelaji B, Smith CM, Paul L, et al. The effectiveness of behaviour change interventions to increase physical activity participation in people with multiple sclerosis: a systematic review and metaanalysis. Clin Rehabil. 2016 Jun;30(6):55976. doi: $10.1177 / 0269215515595274$. PMID: 26198892. Exclusion: 12.

955. Santhosh Kanna BS, Balabaskar K. A study on efficacy of respiratory exercises coupled with neuro developmental treatment on pulmonary function of children with spastic quadriplegic cerebral palsy. Biomed Pharmacol J. 2019;12(3):1519-24. doi: 10.13005/bpj/1782. Exclusion: 14.

956. Saquetto M, Carvalho V, Silva C, et al. The effects of whole body vibration on mobility and balance in children with cerebral palsy: a systematic review with meta-analysis. J Musculoskelet Neuronal Interact. 2015 Jun;15(2):137-44. PMID: 26032205. Exclusion: 12. 
957. Sattelmayer M, Chevalley O, Steuri R, et al. Over-ground walking or robot-assisted gait training in people with .multiple sclerosis: does the effect depend on baseline walking speed and disease related disabilities? A systematic review and meta-regression. BMC Neurol. 2019 May 08;19(1):93. doi: 10.1186/s12883-019-1321-7. PMID: 31068151. Exclusion: 12.

958. Saxton JM, Carter A, Daley A, et al. Pragmatic exercise for people with MS: a randomized controlled trial. Mult Scler J Exp Transl Clin. 2013 May;19(10):1402. doi: $10.1177 / 1352458513498690$.

Exclusion: 10.

959. Sayenko DG, Alekhina MI, Masani K, et al. Positive effect of balance training with visual feedback on standing balance abilities in people with incomplete spinal cord injury. Spinal Cord. 2010 Dec;48(12):886-93. doi: 10.1038/sc.2010.41. PMID: 20404833. Exclusion: 11.

960. Schasfoort F, Dallmeijer A, Pangalila R, et al. Value of botulinum toxin injections preceding a comprehensive rehabilitation period for children with spastic cerebral palsy: a cost-effectiveness study. J Rehabil Med. 2018 Jan 10;50(1):22-9. doi: 10.2340/16501977-2267. PMID: 28949368. Exclusion: 4.

961. Schindelholz M, Stoller O, Hunt KJ. A software module for cardiovascular rehabilitation in robotics-assisted treadmill exercise. Biomed Signal Process Control. 2014;10(1):296-307. doi: 10.1016/j.bspc.2012.12.006. Exclusion: 3 .

962. Schmidt S, Wonneberger M. Long-term endurance exercise improves aerobic capacity in patients with relapsing-remitting multiple sclerosis: impact of baseline fatigue. J Neurol Sci. 2014 Jan 15;336(12):29-35. doi: 10.1016/j.jns.2013.09.035. PMID: 24139841. Exclusion: 4.

963. Schneiberg S, McKinley PA, Sveistrup H, et al. The effectiveness of task-oriented intervention and trunk restraint on upper limb movement quality in children with cerebral palsy. Dev Med Child Neurol. 2010 Nov;52(11):e245-53. doi: 10.1111/j.14698749.2010.03768.x. PMID: 20813019. Exclusion: 11.
964. Schranz C, Kruse A, Belohlavek T, et al. Does home-based progressive resistance or high-intensity circuit training improve strength, function, activity or participation in children with Cerebral Palsy? Arch Phys Med Rehabil. 2018 Dec;99(12):2457-64. doi: 10.1016/j.apmr.2018.06.010. PMID: 30473019. Exclusion: 4.

965. Schranz C, Kruse A, Belohlavek T, et al. High intensity circuit training and progressive resistance training improve intervention-specific functional performance in children with cerebral palsy. Dev Med Child Neurol. 2018;Conference: 30th annual meeting of the european academy of childhood disability, EACD. 2018. Georgia 60(Supplement 2):54. Exclusion: 10.

966. Schranz C, Kruse A, Belohlavek T, et al. High intensity circuit training and progressive resistance training improve functional performance but not the gait profile score. Gait Posture. 2017; Conference: 26th annual meeting of the european society for movement analysis in adults and children, ESMAC. 2017. Norway 57(Supplement 1):364-5. Exclusion: 10.

967. Schreiber R, Souza CM, Paim LR, et al. Impact of regular physical activity on adipocytokines and cardiovascular characteristics in spinal cord-injured subjects. Arch Phys Med Rehabil. 2018 Aug;99(8):1561-7.e1. doi: 10.1016/j.apmr.2018.02.010. PMID: 29548578. Exclusion: 4.

968. Schroeder AS, Von Kries R, Riedel C, et al. Patient-specific determinants of responsiveness to robot-enhanced treadmill therapy in children and adolescents with cerebral palsy. Dev Med Child Neurol. 2014 12;56(12):1172-9. doi: 10.1111/dmcn.12564. PMID: 25154424. Exclusion: 8.

969. Schwartz I, Sajin A, Moreh E, et al. Robotassisted gait training in multiple sclerosis patients: a randomized trial. Mult Scler. 2012 Jun;18(6):881-90. doi: 10.1177/1352458511431075. PMID: 22146609. Exclusion: 11. 
970. Scott W, Geigle P, Chen K, et al.

Relationship among physical activity scale for individuals with disability (PASID), body mass index (BMI), and maximum oxygen consumption (V0MAX) in persons with motor incomplete spinal cord injury. Top Spinal Cord Inj Rehabil. START: 2013 May 6 CONFERENCE END: 2013 May 8, 40th Anniversary Annual Scientific Meeting of the American Spinal Injury Association, ASIA 2013 Chicago, IL United

States;19(1):7. Exclusion: 10.

971. Sebastiao E, McAuley E, Shigematsu R, et al. Home-based, square-stepping exercise program among older adults with multiple sclerosis: results of a feasibility randomized controlled study. Contemp Clin Trials. 2018 Oct;73:136-44. doi:

10.1016/j.cct.2018.09.008. PMID: 30243811. Exclusion: 4.

972. Sebastiao E, McAuley E, Shigematsu R, et al. Feasibility study design and methods for a home-based, square-stepping exercise program among older adults with multiple sclerosis: The SSE-MS project. Contemp Clin Trials Commun. 2017 Sep; 7:200-7. doi: 10.1016/j.conctc.2017.07.012. PMID: 29696187. Exclusion: 10.

973. Seco J, Rodriguez-Perez V, LopezRodriguez AF, et al. Effects of vibration therapy on hormone response and stress in severely disabled patients: a double-blind randomized placebo-controlled clinical trial. Rehabil Nurs. 2015 May-Jun;40(3):166-78. doi: 10.1002/rnj.116. PMID: 23922258. Exclusion: 11.

974. Seebacher B, Kuisma R, Glynn A, et al. The effect of rhythmic-cued motor imagery on walking, fatigue and quality of life in people with multiple sclerosis: a randomised controlled trial. Mult Scler. 2017

Feb;23(2):286-96. doi: 10.1177/1352458516644058. PMID: 27055804. Exclusion: 4.

975. Sexton A, McGibbon CA, Jayaraman A, et al. Evaluation of the Keeogo exoskeleton for assisting ambulatory activities in people with multiple sclerosis: an open-label, randomized, cross-over trial. J Neuroeng Rehabil. 2018;15(1) doi: 10.1186/s12984018-0468-6. Exclusion: 11.
976. Shaffer RF, Picard G, Taylor JA. Relationship of spinal cord injury level and duration to peak aerobic capacity with armsonly and hybrid functional electrical stimulation rowing. Am J Phys Med Rehabil. 2018 Jul;97(7):488-91. doi: 10.1097/PHM.0000000000000903. PMID: 29406401. Exclusion: 4.

977. Shahrbanian S. Virtual reality training or physical activity? Which one is more effective in reducing pain among persons with multiple sclerosis? A randomized controlled trial. Mult Scler. 2017; Conference: 7th joint ECTRIMSACTRIMS, MSPARIS2017. France. 23(3 Supplement 1):959-60. Exclusion: 10.

978. Sharan D, Ajeesh PS, Rameshkumar R, et al. Virtual reality based therapy for post operative rehabilitation of children with cerebral palsy. Work. 2012;41 Suppl 1:3612-5. doi: 10.3233/WOR-2012-06673612. PMID: 22317271. Exclusion: 7.

979. Sharif H, Gammage K, Chun S, et al. Effects of FES-ambulation training on locomotor function and health-related quality of life in individuals with spinal cord injury. Top Spinal Cord Inj Rehabil. 2014;20(1):58-69. doi: 10.1310/sci2001-58. PMID: 24574823. Exclusion: 11.

980. Shehata H, Elmazny A, Shalaby N. Effects of high frequency repetitive transcranial magnetic stimulation on gait and balance in patients with relapsing remitting multiple sclerosis. Mult Scler. 2017; Conference: 7th joint ECTRIMS-ACTRIMS, MSPARIS2017. France. 23(3 Supplement 1):58. Exclusion: 10.

981. Shehata HS, Shalaby NM, Elmazny A. Effects of high frequency repetitive transcranial magnetic stimulation on gait and balance in patients with chronic multiple sclerosis. Multiple sclerosis. Conference: $32 \mathrm{nd}$ congress of the european committee for treatment and research in multiple sclerosis, ECTRIMS. 2016;22(389). Exclusion: 10.

982. Shem K, Karasik D, Carufel P, et al. Seated Tai Chi to alleviate pain and improve quality of life in individuals with spinal cord disorder. J Spinal Cord Med. 2016 May;39(3):353-8. doi: 10.1080/10790268.2016.1148895. PMID: 26914968. Exclusion: 11. 
983. Sherief A, Abo Gazya AA, Abd El Gafaar MA. Integrated effect of treadmill training combined with dynamic ankle foot orthosis on balance in children with hemiplegic cerebral palsy. Egypt J Med Hum Genet. 2015;16(2):173-9. Exclusion: 4.

984. Sherrington C, Pamphlett PI, Jacka JA, et al. Group exercise can improve participants' mobility in an outpatient rehabilitation setting: a randomized controlled trial. Clin Rehabil. 2008 Jun;22(6):493-502. doi: 10.1177/0269215508087994. PMID: 18511529. Exclusion: 3.

985. Shimizu Y, Kadone H, Kubota S, et al. Voluntary ambulation by upper limbtriggered HAL in patients with complete quadri/paraplegia due to chronic spinal cord injury. Front Neurosci. 2017;11:649. doi: 10.3389/fnins.2017.00649. PMID: 29209163. Exclusion: 11.

986. Shojaei MH, Alavinia SM, Craven BC. Management of obesity after spinal cord injury: a systematic review. J Spinal Cord Med. 2017 Nov;40(6):783-94. doi: 10.1080/10790268.2017.1370207. PMID: 28929907. Exclusion: 12.

987. Shuai L, Yu GH, Feng Z, et al. Application of a paraplegic gait orthosis in thoracolumbar spinal cord injury. Nerual Regen. 2016 Dec;11(12):1997-2003. doi: 10.4103/1673-5374.197144. PMID: 28197198. Exclusion: 4.

988. Shurtleff TL, Engsberg JR. Changes in trunk and head stability in children with cerebral palsy after hippotherapy: a pilot study. Phys Occup Ther Pediatr. 2010 May;30(2):15063. doi: 10.3109/01942630903517223. PMID: 20367519. Exclusion: 5.

989. Siemonsen S, Stellmann JP, Kjolhede T, et al. Resistance training increases cortical thickness in RRMS-results of a pilot RCT. Mult Scler J Exp Transl Clin. START: 2015 Oct 7 CONFERENCE END: 2015 Oct 10, 31 st Congress of the European Committee for Treatment and Research in Multiple Sclerosis, ECTRIMS 2015 Barcelona Spain;23(11 SUPPL. 1):504. Exclusion: 10.
990. Silkwood-Sherer DJ, McGibbon NH. Can hippotherapy make a difference in the quality of life of children with cerebral palsy? A pragmatic study. Physiother Theory Pract. 2020 May 14:1-11. doi: 10.1080/09593985.2020.1759167. PMID: 32406798. Exclusion: 11.

991. Silva Ferreira AP, de Souza Pegorare ABG, Miotto Junior A, et al. A controlled clinical trial on the effects of exercise on lower uirnary tract symptoms in women with multiple sclerosis. Am J Phys Med Rehabil. 2019 Sep;98(9):777-82. doi: 10.1097/PHM.0000000000001189. PMID: 30932917. Exclusion: 4.

992. Silverman EP, Miller S, Zhang Y, et al. Effects of expiratory muscle strength training on maximal respiratory pressure and swallow-related quality of life in individuals with multiple sclerosis. Mult Scler J Exp Transl Clin. 2017 AprJun;3(2):2055217317710829. doi: 10.1177/2055217317710829. PMID: 28607760. Exclusion: 4.

993. Simis M, Uygur-Kucukseymen E, PachecoBarrios K, et al. Beta-band oscillations as a biomarker of gait recovery in spinal cord injury patients: A quantitative electroencephalography analysis. Clin Neurophysiol. 2020 May 22;131(8):180614. doi: 10.1016/j.clinph.2020.04.166. PMID: 32540720. Exclusion: 6.

994. Slaman J, Roebroeck M, Van Der Slot WM, et al. Effectiveness of a lifestyle program among adolescents and young adults with cerebral palsy: a randomized controlled trial. Dev Med Child Neurol. 2014 Sep;56(S5):65-6. doi: 10.1111/dmcn.12540. Exclusion: 10.

995. Slaman J, Roebroek M, Stam H, et al. Effectiveness of a lifestyle program on daily physical activity and fitness in adolescents and young adults with cerebral palsy: a randomized controlled trial. Dev Med Child Neurol. 2013;55(S2):30-1. doi: 10.1111/dmcn.12259. Exclusion: 10.

996. Slaman J, van den Berg-Emons R, Tan SS, et al. Cost-utility of a lifestyle intervention in adolescents and young adults with spastic cerebral palsy. J Rehabil Med. 2015 Apr;47(4):338-45. doi: 10.2340/165019771929. PMID: 25678311. Exclusion: 6. 
997. Smith M, Barker R, Williams G, et al. The effect of exercise on high-level mobility in individuals with neurodegenerative disease: a systematic literature review.

Physiotherapy. 2019 May 02;02:02. doi: 10.1016/j.physio.2019.04.003. PMID:

31477333. Exclusion: 12.

998. Soleyman-Jahi S, Yousefian A, Maheronnaghsh R, et al. Evidence-based prevention and treatment of osteoporosis after spinal cord injury: a systematic review. Eur Spine J. 2018 Aug;27(8):1798-814. doi: 10.1007/s00586-017-5114-7. PMID: 28497215. Exclusion: 3.

999. Solinsky R, Mercier H, Picard G, et al. Cardiometabolic effects of high-intensity hybrid functional electrical stimulation exercise after Spinal Cord Injury. Pm R. 2020 Oct 07;07:07. doi:

10.1002/pmrj.12507. PMID: 33027550. Exclusion: 8.

1000. Solinsky R, Vivodtzev I, Hamner JW, et al. The effect of heart rate variability on blood pressure is augmented in spinal cord injury and is unaltered by exercise training. Clin Auton Res. 2020 Mar 12;12:12. doi: 10.1007/s10286-020-00677-2. PMID: 32166421. Exclusion: 11.

1001. Sorsdahl AB, Moe-Nilssen R, Kaale HK, et al. Change in basic motor abilities, quality of movement and everyday activities following intensive, goal-directed, activityfocused physiotherapy in a group setting for children with cerebral palsy. BMC Pediatr. 2010 Apr 27;10:26. doi: 10.1186/14712431-10-26. PMID: 20423507. Exclusion: 8.

1002. Sosnoff JJ, Moon Y, Wajda DA, et al. Fall risk and incidence reduction in high risk individuals with multiple sclerosis: a pilot randomized control trial. Clin Rehabil. 2015 Oct;29(10):952-60. doi: 10.1177/0269215514564899. PMID: 25540170. Exclusion: 4.

1003. Sosnoff JJ, Sandroff BM, Pula JH, et al. Falls and physical activity in persons with multiple sclerosis. Mult Scler Int. 2012;2012:315620. doi: 10.1155/2012/315620. PMID: 22966459. Exclusion: 4.
1004. Sosnoff JJ, Sung J. Reducing falls and improving mobility in multiple sclerosis. Expert Rev Neurother. 2015 Jun;15(6):65566. doi: 10.1586/14737175.2015.1046377. PMID: 25973774. Exclusion: 12.

1005. Souto DO, Cruz TKF, Coutinho K, et al. Effect of motor imagery combined with physical practice on upper limb rehabilitation in children with hemiplegic cerebral palsy. NeuroRehabilitation. 2020;46(1):53-63. doi: 10.3233/NRE192931. Exclusion: 4.

1006. Spencer T, Aldous S, Williams G, et al. Systematic review of high-level mobility training in people with a neurological impairment. Brain Inj. 2018;32(4):403-15. doi: 10.1080/02699052.2018.1429656. PMID: 29393688. Exclusion: 12.

1007. Spina E, Carotenuto A, Aceto MG, et al. The effects of mechanical focal vibration on walking impairment in multiple sclerosis patients: a randomized, double-blinded vs placebo study. Restor Neurol Neurosci. 2016 Sep 21;34(5):869-76. doi: 10.3233/RNN-160665. PMID: 27567760. Exclusion: 11.

1008. Stampacchia G, Olivieri M, Rustici A, et al. Gait rehabilitation in persons with spinal cord injury using innovative technologies: an observational study. Spinal Cord. 2020 Apr 06;06:06. doi: 10.1038/s41393-0200454-2. PMID: 32251368. Exclusion: 8.

1009. Stark C, Herkenrath P, Hollmann H, et al. Early vibration assisted physiotherapy in toddlers with cerebral palsy - a randomized controlled pilot trial. J Musculoskelet Neuronal Interact. 2016 Sep 7;16(3):183-92. PMID: 27609033. Exclusion: 4.

1010. Steimer J, Weissert R. Effects of sport climbing on multiple sclerosis. Front Physiol. 2017;8:1021. doi: 10.3389/fphys.2017.01021. PMID: 29311957. Exclusion: 12.

1011. Stergiou A, Tzoufi M, Ntzani E, et al. Therapeutic effects of horseback riding interventions: a systematic review and metaanalysis. Am J Phys Med Rehabil. 2017 Oct;96(10):717-25. doi: 10.1097/PHM.0000000000000726. PMID: 28252520. Exclusion: 12. 
1012. Stone WJ, Stevens SL, Fuller DK, et al. Ambulation and physical function after eccentric resistance training in adults with incomplete spinal cord injury: a feasibility study. J Spinal Cord Med. 2019 Jan 23;42(4):526-33. doi: 10.1080/10790268.2017.1417804. PMID: 29360000. Exclusion: 11.

1013. Straudi S, Benedetti MG, Venturini E, et al. Does robot-assisted gait training ameliorate gait abnormalities in multiple sclerosis? A pilot randomized-control trial. NeuroRehabilitation. 2013;33(4):555-63. doi: 10.3233/NRE-130990. PMID: 24018369. Exclusion: 11.

1014. Straudi S, Martinuzzi C, Pavarelli C, et al. A task-oriented circuit training in multiple sclerosis: a feasibility study. BMC Neurol. 2014 Jun 7;14:124. doi: 10.1186/1471-237714-124. PMID: 24906545. Exclusion: 11.

1015. Streber R, Peters S, Pfeifer K. Systematic review of correlates and determinants of physical activity in persons with multiple sclerosis. Arch Phys Med Rehabil. 2016 Apr;97(4):633-45.e29. doi: 10.1016/j.apmr.2015.11.020. PMID: 26751247. Exclusion: 12.

1016. Street T, Singleton C. A clinically meaningful training effect in walking speed using functional electrical stimulation for motor-incomplete spinal cord injury. J Spinal Cord Med. 2018 May;41(3):361-6. doi: 10.1080/10790268.2017.1392106. PMID: 29108487. Exclusion: 4.

1017. Stribling K, Christy J. Dance practice improves postural control in a child with cerebral palsy. Pediatr Phys Ther. 2011 Spring;23(1):129-30. doi: 10.1097/PEP.0000000000000450. PMID: 28953185 Exclusion: 7.

1018. Sucuoglu H. Effects of robot-assisted gait training alongside conventional therapy on the development of walking in children with cerebral palsy. J Pediatr Rehabil Med. 2020 May 09;09:09. doi: 10.3233/PRM-180541. PMID: 32444570. Exclusion: 8.
1019. Suder R, Fu MJ, Knutson J, et al. Contralaterally controlled functional electrical stimulation and hand therapy video games for cerebral palsy. Am J Occup Ther. 2016 Aug;70(4):1. doi: 10.5014/ajot.2016.70S1-PO5113. Exclusion: 7.

1020. Sukal-Moulton T, Clancy T, Zhang LQ, et al. Clinical application of a robotic ankle training program for cerebral palsy compared to the research laboratory application: does it translate to practice? Arch Phys Med Rehabil. 2014 Aug;95(8):1433-40. doi: 10.1016/j.apmr.2014.04.010. PMID: 24792141. Exclusion: 4.

1021. Sung YH, Ha SY. The Vojta approach changes thicknesses of abdominal muscles and gait in children with spastic cerebral palsy: a randomized controlled trial, pilot study. Technol Health Care. 2019 Oct 16;16:16. doi: 10.3233/THC-191726. PMID: 31658070. Exclusion: 11.

1022. Surana BK, Ferre CL, Dew AP, et al. Effectiveness of lower-extremity functional training (LIFT) in young children with unilateral spastic cerebral palsy: a randomized controlled trial. Neurorehabil Neural Repair. 2019;33(10):862-72. doi: 10.1177/1545968319868719. Exclusion: 4.

1023. Swank C, Thompson M, Medley A. Aerobic exercise in people with multiple sclerosis: its feasibility and secondary benefits. Int J MS Care. 2013;15(3):138-45. doi: 10.7224/1537-2073.2012-037. PMID: 24453776. Exclusion: 11.

1024. Swank C, Trammell M, Bennett M, et al. The utilization of an overground robotic exoskeleton for gait training during inpatient rehabilitation-single-center retrospective findings. Int J Rehabil Res. 2020 Apr 08;08:08. doi: 10.1097/MRR.0000000000000409. PMID: 32282573. Exclusion: 4.

1025. Syed UE, Kamal A. Video game-based and conventional therapies in patients of neurological deficits: an experimental study. Disabil Rehabil Assist Technol. 2019 Nov 05:1-8. doi: 10.1080/17483107.2019.1679266. PMID: 31684776. Exclusion: 11. 
1026. Tafreshi AS, Riener R, KlamrothMarganska V. Distinctive steady-state heart rate and blood pressure responses to passive robotic leg exercise during head-up tilt: a pilot study in neurological patients. Front Physiol. 2017;8(JUN) doi: 10.3389/fphys.2017.00327. Exclusion: 3.

1027. Tallner A, Maeurer M, Pfeifer K. An internet-based at home training protocol enhances muscle strength and lung function in multiple sclerosis patients (\#133). Mult Scler J Exp Transl Clin. 2012 May;18(5):S23-S4. doi: 10.1177/1352458512444209. PMID: 28071271. Exclusion: 10.

1028. Tallner A, Streber R, Hentschke C, et al. Internet-supported physical exercise training for persons with multiple sclerosis-a randomised, controlled study. Int J Mol Sci. 2016 Sep 30;17(10):30. doi: 10.3390/ijms17101667. PMID: 27706046. Exclusion: 4.

1029. Tamburella F, Scivoletto G, Molinari M. Balance training improves static stability and gait in chronic incomplete spinal cord injury subjects: a pilot study. Eur J Phys Rehabil Med. 2013 Jun;49(3):353-64. PMID: 23486301. Exclusion: 11.

1030. Tan C, Battaglino R, Gupta R, et al. FESrowing training improves bone strength of the paralyzed legs in a dose-dependent fashion. J Bone Miner Res. Conference. 2015;30(Supplement 1). Exclusion: 10.

1031. Tanabe S, Koyama S, Saitoh E, et al. Clinical feasibility of gait training with a robotic exoskeleton (WPAL) in an individual with both incomplete cervical and complete thoracic spinal cord injury: a case study. NeuroRehabilitation. 2017;41(1):8595. doi: 10.3233/NRE-171460. PMID: 28527225. Exclusion: 7.

1032. Taspinar O, Aydin T, Celebi A, et al. Psychological effects of calisthenic exercises on neuroinflammatory and rheumatic diseases. Z Rheumatol. 2015 Oct;74(8):722-7. doi: 10.1007/s00393-0151570-9. PMID: 26115762. Exclusion: 4.
1033. Taylor E, Taylor-Piliae RE. The effects of Tai Chi on physical and psychosocial function among persons with multiple sclerosis: a systematic review. Complement Ther Med. 2017 Apr;31:100-8. doi: 10.1016/j.ctim.2017.03.001. PMID: 28434462. Exclusion: 12.

1034. Taylor N, Dodd KJ, Baker R, et al. The effect of individualized progressive resistance training on mobility in adolescents and young adults with cerebral palsy. Dev Med Child Neurol. 2013 Oct;55(S3):25-6. doi: 10.1111/dmcn.12247. Exclusion: 10.

1035. Taylor P, Barrett C, Mann G, et al. A feasibility study to investigate the effect of functional electrical stimulation and physiotherapy exercise on the quality of gait of people with multiple sclerosis. Neuromodulation. 2014 Jan;17(1):75-84; discussion doi: 10.1111/ner.12048. PMID: 23601128. Exclusion: 11.

1036. Taylor P, Humphreys L, Swain I. The longterm cost-effectiveness of the use of Functional Electrical Stimulation for the correction of dropped foot due to upper motor neuron lesion. J Rehabil Med. 2013 Feb;45(2):154-60. doi: 10.2340/165019771090. PMID: 23303521. Exclusion: 5.

1037. Taylor SM, Cheung EO, Sun R, et al. Applications of complementary therapies during rehabilitation for individuals with traumatic spinal cord injury: findings from the SCIRehab project. J Spinal Cord Med. 2019 Sep;42(5):571-8. doi: 10.1080/10790268.2018.1481693. PMID: 29883300. Exclusion: 4.

1038. Taylor-Schroeder S, LaBarbera J, McDowell $\mathrm{S}$, et al. The SCIRehab project: treatment time spent in SCI rehabilitation. Physical therapy treatment time during inpatient spinal cord injury rehabilitation. J Spinal Cord Med. 2011;34(2):149-61. doi: 10.1179/107902611X12971826988057. PMID: 21675354. Exclusion: 4.

1039. Tefertiller C, Hays K, Jones J, et al. Initial outcomes from a multicenter study utilizing the indego powered exoskeleton in spinal cord injury. Top Spinal Cord Inj Rehabil. 2018;24(1):78-85. doi: 10.1310/sci1700014. PMID: 29434463. Exclusion: 8. 
1040. Temcharoensuk P, Lekskulchai R, Akamanon C, et al. Effect of horseback riding versus a dynamic and static horse riding simulator on sitting ability of children with cerebral palsy: a randomized controlled trial. J Phys Ther Sci. 2015 Jan;27(1):273-7. doi: 10.1589/jpts.27.273. PMID: 25642090. Exclusion: 4.

1041. Terson de Paleville D, McKay W, Aslan S, et al. Locomotor step training with body weight support improves respiratory motor function in individuals with chronic spinal cord injury. Respir Physiolo Neurobiol. 2013 Dec 1;189(3):491-7. doi: 10.1016/j.resp.2013.08.018. PMID: 23999001. Exclusion: 11.

1042. Theis N, Korff T, Mohagheghi AA. Does long-term passive stretching alter muscletendon unit mechanics in children with spastic cerebral palsy? Clin Biomech. 2015 Dec;30(10):1071-6. doi: 10.1016/j.clinbiomech.2015.09.004. PMID: 26403361. Exclusion: 11.

1043. Thomas S, Fazakarley L, Thomas PW, et al. Mii-vitaliSe: a pilot randomised controlled trial of a home gaming system (Nintendo Wii) to increase activity levels, vitality and well-being in people with multiple sclerosis. BMJ Open. 2017 Sep 27;7(9):e016966. doi: 10.1136/bmjopen-2017-016966. PMID: 28954791. Exclusion: 4.

1044. Thongsumrit L, Nana A, Limroongreungrat W, et al. Effects of wheelchair Tai-Chi training on sitting balance of individuals with spinal cord injury. J Sci Med Sport. 2011 Dec;14(S1):e110-1. doi: 10.1016/j.jsams.2011.11.231. Exclusion: 10.

1045. To CS, Kobetic R, Bulea TC, et al. Sensorbased stance control with orthosis and functional neuromuscular stimulation for walking after spinal cord injury. J Prosthet Orthot. 2012;24(3):124-32. doi:

10.1097/JPO.0b013e3182627a13. Exclusion: 7.

1046. To CS, Kobetic R, Bulea TC, et al. Sensorbased hip control with hybrid neuroprosthesis for walking in paraplegia. $\mathrm{J}$ Rehabil Res Dev. 2014;51(2):229-44. doi: 10.1682/JRRD.2012.10.0190. PMID: 24933721. Exclusion: 7.
1047. Torhaug T, Brurok B, Hoff J, et al. The effect from maximal bench press strength training on work economy during wheelchair propulsion in men with spinal cord injury. Spinal Cord. 2016 Oct;54(10):838-42. doi: 10.1038/sc.2016.27. PMID: 26976530. Exclusion: 11.

1048. Tornberg AB, Lauruschkus K. Nonambulatory children with cerebral palsy: effects of four months of static and dynamic standing exercise on passive range of motion and spasticity in the hip. PeerJ. 2020;8:e8561. doi: 10.7717/peerj.8561. PMID: 32211225. Exclusion: 4.

1049. Tosh J, Dixon S, Carter A, et al. Cost effectiveness of a pragmatic exercise intervention (EXIMS) for people with multiple sclerosis: economic evaluation of a randomised controlled trial. Mult Scler. 2014 Jul;20(8):1123-30. doi: 10.1177/1352458513515958. PMID: 24421304. Exclusion: 6.

1050. Totosy de Zepetnek JO, Au JS, Ditor DS, et al. Lower limb conduit artery endothelial responses to acute upper limb exercise in spinal cord injured and able-bodied men. Physiol Rep. 2015 Apr;3(4) doi: 10.14814/phy2.12367. PMID: 25847920. Exclusion: 4.

1051. Totosy de Zepetnek JO, Au JS, Hol AT, et al. Predicting peak oxygen uptake from submaximal exercise after spinal cord injury. Appl Physiol Nutr Metab. 2016 Jul;41(7):775-81. doi: 10.1139/apnm-20150670. PMID: 27363732. Exclusion: 4.

1052. Totosy de Zepetnek JO, Miyatani M, Szeto $\mathrm{M}$, et al. The effects of whole body vibration on pulse wave velocity in men with chronic spinal cord injury. J Spinal Cord Med. 2017 Nov;40(6):795-802. doi: 10.1080/10790268.2017.1369248. PMID: 28868990. Exclusion: 11.

1053. Tramontano M, Cinnera AM, Manzari L, et al. Vestibular rehabilitation has positive effects on balance, fatigue and activities of daily living in highly disabled multiple sclerosis people: a preliminary randomized controlled trial. Restor Neurol Neurosci. 2018;36(6):709-18. doi: 10.3233/RNN180850. Exclusion: 11. 
1054. Troy KL. Biomechanical validation of upper extremity exercise in wheelchair users: design considerations and improvements in a prototype device. Disabil Rehabil Assist Technol. 2011;6(1):22-8. doi: 10.3109/17483107.2010.509883. PMID: 20726741. Exclusion: 4.

1055. Troy KL, Munce TA, Longworth JA. An exercise trial targeting posterior shoulder strength in manual wheelchair users: pilot results and lessons learned. Disabil Rehabil Assist Technol. 2015;10(5):415-20. doi: 10.3109/17483107.2014.905644. PMID: 24694062. Exclusion: 11.

1056. Truchon C, Fallah N, Santos A, et al. Impact of therapy on recovery during rehabilitation in patients with traumatic spinal cord injury. J Neurotrauma. 2017 Oct 15;34(20):2901-9. doi: 10.1089/neu.2016.4932. PMID: 28493787. Exclusion: 7.

1057. Tsai C-Y, Delgado AD, Weinrauch WJ, et al. Exoskeletal-Assisted Walking During Acute Inpatient Rehabilitation Leads to Motor and Functional Improvement in Persons With Spinal Cord Injury: A Pilot Study. Arch Phys Med Rehabil. 2020;101(4):607-12. doi: 10.1016/j.apmr.2019.11.010. Exclusion: 4.

1058. Tsai LC, Ren Y, Gaebler-Spira DJ, et al. Effects of an off-axis pivoting elliptical training program on gait function in persons with spastic cerebral palsy: a preliminary study. Am J Phys Med Rehabil. 2017 Jul;96(7):515-22. doi: 10.1097/PHM.0000000000000632. PMID: 28628539. Exclusion: 11.

1059. Tupimai T, Peungsuwan P, Prasertnoo J, et al. Effect of combining passive muscle stretching and whole body vibration on spasticity and physical performance of children and adolescents with cerebral palsy. J Phys Ther Sci. 2016 Jan;28(1):7-13. doi: 10.1589/jpts.28.7. PMID: 26957720. Exclusion: 11.

1060. Turbanski S, Schmidtbleicher D. Effects of heavy resistance training on strength and power in upper extremities in wheelchair athletes. J Strength Cond Res. 2010 Jan;24(1):8-16. doi: 10.1519/JSC.0b013e3181bdddda. PMID: 19996772. Exclusion: 11.
1061. Turconi AC, Biffi E, Maghini C, et al. Can new technologies improve upper limb performance in grown-up diplegic children? Eur J Phys Rehabil Med. 2016 Oct;52(5):672-81. PMID: 26554345. Exclusion: 11.

1062. Tyler ME, Kaczmarek KA, Rust KL, et al. Non-invasive neuromodulation to improve gait in chronic multiple sclerosis: a randomized double blind controlled pilot trial. J Neuroengineering Rehabil. 2014 May 1;11:79. doi: 10.1186/1743-0003-11-79. PMID: 24885412. Exclusion: 11.

1063. Ukhanova TA, Gorbunov FE. [Efficacy of reflexology in the combination with neuroprotective treatment in hemiparetic form of children cerebral palsy]. Zh Nevrol Psikhiatr Im S S Korsakova. 2012;112(7):28-31. PMID: 23011423. Exclusion: 13.

1064. Ukhanova TA, Noivikova EE, Dement'eva EV. The role of microcurrent reflexotherapy in combination with neuroprotector in the rehabilitation of the patients with infantile cerebral palsy. Vopr Kurortol Fizioter Lech Fiz Kult. 2012 Sep-Oct(5):33-6. PMID: 23210360. Exclusion: 13.

1065. Unger M, Jelsma J. Effect of a trunktargeted intervention on pelvic positioning and lower limb function in children with spastic type cerebral palsy. Physiotherapy. 2011 Jun;97(S1):eS1267. doi: 10.1016/j.physio.2011.04.002. Exclusion: 10.

1066. Unger M, Jelsma J, Stark C. Effect of a trunk-targeted intervention using vibration on posture and gait in children with spastic type cerebral palsy: a randomized control trial. Dev Neurorehabil. 2013;16(2):79-88. doi: 10.3109/17518423.2012.715313. PMID: 23477461. Exclusion: 8.

1067. Valent LJ, Dallmeijer AJ, Houdijk H, et al. Effects of hand cycle training on physical capacity in individuals with tetraplegia: a clinical trial. Phys Ther. 2009 Oct;89(10):1051-60. doi: 10.2522/ptj.20080340. PMID: 19643834. Exclusion: 11. 
1068. Valent LJ, Dallmeijer AJ, Houdijk H, et al. Influence of hand cycling on physical capacity in the rehabilitation of persons with a spinal cord injury: a longitudinal cohort study. Arch Phys Med Rehabil. 2008 Jun;89(6):1016-22. doi: 10.1016/j.apmr.2007.10.034. PMID: 18503794. Exclusion: 4.

1069. Valentin-Gudiol M, Mattern-Baxter K, Girabent-Farres M, et al. Treadmill interventions in children under six years of age at risk of neuromotor delay. Cochrane Database Syst Rev. 2017 Jul 29;7:CD009242. doi: 10.1002/14651858.CD009242.pub3. PMID: 28755534. Exclusion: 3.

1070. Van Campenhout A, Bar-On L, Aertbelien $E$, et al. Can we unmask features of spasticity during gait in children with cerebral palsy by increasing their walking velocity? Gait Posture. 2014 Mar;39(3):9537. doi: $10.1016 /$ j.gaitpost.2013.12.024. PMID: 24444653. Exclusion: 4.

1071. van den Berg-Emons RJ, Bussmann JB, Haisma JA, et al. A prospective study on physical activity levels after spinal cord injury during inpatient rehabilitation and the year after discharge. Arch Phys Med Rehabil. 2008 Nov;89(11):2094-101. doi: 10.1016/j.apmr.2008.04.024. PMID: 18996237. Exclusion: 4.

1072. Van den Broeck C, De Cat J, Molenaers G, et al. The effect of individually defined physiotherapy in children with cerebral palsy (CP). Europ J Paediatr Neurol. 2010 Nov;14(6):519-25. doi: 10.1016/j.ejpn.2010.03.004. PMID: 20434378. Exclusion: 11.

1073. van der Scheer JW, de Groot S, Postema K, et al. Design of a randomized-controlled trial on low-intensity aerobic wheelchair exercise for inactive persons with chronic spinal cord injury. Disabil Rehabil. 2013 Jun;35(13):1119-26. doi: 10.3109/09638288.2012.709301. PMID: 22900530. Exclusion: 11.
1074. van der Scheer JW, de Groot S, Vegter RJ, et al. Low-intensity wheelchair training in inactive people with long-term spinal cord injury: a randomized controlled trial on propulsion technique. Am J Phys Med Rehabil. 2015 Nov;94(11):975-86. doi: 10.1097/PHM.0000000000000289. PMID: 25802961. Exclusion: 11.

1075. van der Scheer JW, Martin Ginis KA, Ditor DS, et al. Effects of exercise on fitness and health of adults with spinal cord injury: a systematic review. Neurology. 2017 Aug 15;89(7):736-45. doi: 10.1212/WNL.0000000000004224. PMID: 28733344. Exclusion: 12.

1076. van der Woude LH, de Groot S, Postema K, et al. Active LifestyLe Rehabilitation interventions in aging spinal cord injury (ALLRISC): a multicentre research program. Disabil Rehabil. 2013 Jun;35(13):1097-103. doi: 10.3109/09638288.2012.718407. PMID: 23030594. Exclusion: 10.

1077. Van Gestel L, Molenaers G, Huenaerts C, et al. Effect of dynamic orthoses on gait: a retrospective control study in children with hemiplegia. Dev Med Child Neurol. 2008 Jan;50(1):63-7. doi: 10.1111/j.14698749.2007.02014.x. PMID: 18173633. Exclusion: 4.

1078. Van Gestel L, Wambacq H, Aertbelien E, et al. To what extent is mean EMG frequency during gait a reflection of functional muscle strength in children with cerebral palsy? Res Dev Disabil. 2012 May-Jun;33(3):916-23. doi: 10.1016/j.ridd.2011.12.010. PMID: 22245734. Exclusion: 6.

1079. van Hedel HJ, Meyer-Heim A, Rusch-Bohtz C. Robot-assisted gait training might be beneficial for more severely affected children with cerebral palsy. Dev Neurorehabil. 2016 Dec;19(6):410-5. doi: 10.3109/17518423.2015.1017661. PMID: 25837449. Exclusion: 4.

1080. van Koppenhagen CF, de Groot S, Post MW, et al. Patterns of changes in wheelchair exercise capacity after spinal cord injury. Arch Phys Med Rehabil. 2013 Jul;94(7):1260-7. doi: 10.1016/j.apmr.2013.02.025. PMID: 23510968. Exclusion: 4. 
1081. van Koppenhagen CF, Post M, de Groot S, et al. Longitudinal relationship between wheelchair exercise capacity and life satisfaction in patients with spinal cord injury: A cohort study in the Netherlands. J Spinal Cord Med. 2014 May;37(3):328-37. doi: 10.1179/2045772313Y.0000000167. PMID: 24621019. Exclusion: 5.

1082. van Middendorp JJ, Hosman AJ, Donders $\mathrm{AR}$, et al. A clinical prediction rule for ambulation outcomes after traumatic spinal cord injury: a longitudinal cohort study. Lancet. 2011 Mar 19;377(9770):1004-10. doi: 10.1016/S0140-6736(10)62276-3. PMID: 21377202. Exclusion: 4.

1083. van Middendorp JJ, Hosman AJ, Pouw MH, et al. ASIA impairment scale conversion in traumatic SCI: is it related with the ability to walk? A descriptive comparison with functional ambulation outcome measures in 273 patients. Spinal Cord. 2009

Jul;47(7):555-60. doi: 10.1038/sc.2008.162. PMID: 19104512. Exclusion: 7.

1084. van Vulpen LF, de Groot S, Rameckers E, et al. Improved walking capacity and muscle strength after functional power-training in young children with cerebral palsy.

Neurorehabil Neural Repair. 2017

Sep;31(9):827-41. doi: 10.1177/1545968317723750. PMID: 28786309. Exclusion: 8.

1085. van Vulpen LF, de Groot S, Rameckers EA, et al. Improved parent-reported mobility and achievement of individual goals on activity and participation level after functional power-training in young children with cerebral palsy: a double-baseline controlled trial. Eur J Phys Rehabil Med. 2018 Oct;54(5):730-7. doi: 10.23736/S19739087.18.04921-3. PMID: 29517188. Exclusion: 8.

1086. van Vulpen LF, de Groot S, Rameckers EAA, et al. Effectiveness of functional power training on walking ability in young children with cerebral palsy: study protocol of a double-baseline trial. Pediatr Phys Ther. 2017 Jul;29(3):275-82. doi: 10.1097/PEP.0000000000000424. PMID: 28654503. Exclusion: 8.
1087. Van Wely L, Balemans A, Becher J, et al. Effectiveness of a 6-month physical activity stimulation program for children with cerebral palsy. Dev Med Child Neurol. 2013 Oct;55(S3):8-9. doi: 10.1111/dmcn.12247. PMID: 24621033. Exclusion: 10.

1088. van Wely L, Becher JG, Balemans AC, et al. Ambulatory activity of children with cerebral palsy: which characteristics are important? Dev Med Child Neurol. 2012 May;54(5):436-42. doi: 10.1111/j.14698749.2012.04251.x. PMID: 22414202. Exclusion: 4.

1089. Vaney C, Gattlen B, Lugon-Moulin V, et al. Robotic-assisted step training (lokomat) not superior to equal intensity of over-ground rehabilitation in patients with multiple sclerosis. Neurorehabil Neural Repair. 2012 Mar-Apr;26(3):212-21. doi: 10.1177/1545968311425923. PMID: 22140197. Exclusion: 4.

1090. Veneri D, Gannotti M, Bertucco M, et al. Using the international classification of functioning, disability, and health model to gain perspective of the benefits of yoga in stroke, multiple sclerosis, and children to inform practice for children with cerebral palsy: a meta-analysis. J Altern Complement Med. 2018 May;24(5):439-57. doi: 10.1089/acm.2017.0030. PMID: 29406768. Exclusion: 12.

1091. Venturini E, Balugani L, Zarattin F, et al. The effects of robot-assisted gait training on locomotor function in subjects with multiple sclerosis: a three months follow-up study. Gait Posture. 2010;33(4). Exclusion: 10.

1092. Verschuren O, Bongers BC, Obeid J, et al. Validity of the muscle power sprint test in ambulatory youth with cerebral palsy. Pediatr Phys Ther. 2013;25(1):25-8. doi: 10.1097/PEP.0b013e3182791459. PMID: 23288003. Exclusion: 4.

1093. Verschuren O, Bosma L, Takken T. Reliability of a shuttle run test for children with cerebral palsy who are classified at Gross Motor Function Classification System level III. Dev Med Child Neurol. 2011 May;53(5):470-2. doi: 10.1111/j.14698749.2010.03893.x. PMID: 21309762. Exclusion: 4. 
1094. Verschuren O, Maltais DB, Douma-van Riet $\mathrm{D}$, et al. Anaerobic performance in children with cerebral palsy compared to children with typical development. Pediatr Phys Ther. 2013;25(4):409-13. doi: 10.1097/PEP.0b013e3182a47022. PMID: 23974826. Exclusion: 4.

1095. Verschuren O, Zwinkels M, Ketelaar M, et al. Reproducibility and validity of the 10meter shuttle ride test in wheelchair-using children and adolescents with cerebral palsy. Phys Ther. 2013 Jul;93(7):967-74. doi: 10.2522/ptj.20120513. PMID: 23580630. Exclusion: 6.

1096. Vikman T, Fielding P, Lindmark B, et al. Effects of inpatient rehabilitation in multiple sclerosis patients with moderate disability. Adv Physiother. 2008;10(2):58-65. doi: 10.1080/14038190701288785. Exclusion: 7.

1097. Villiger M, Bohli D, Kiper D, et al. Virtual reality-augmented neurorehabilitation improves motor function and reduces neuropathic pain in patients with incomplete spinal cord injury. Neurorehabil Neural Repair. 2013 Oct;27(8):675-83. doi: 10.1177/1545968313490999. PMID: 23757298. Exclusion: 11.

1098. Villiger M, Liviero J, Awai L, et al. Homebased virtual reality-augmented training improves lower limb muscle strength, balance, and functional mobility following chronic incomplete spinal cord injury. Front Neurol. 2017;8(NOV) doi: 10.3389/fneur.2017.00635. Exclusion: 11.

1099. Vitiello D, Pochon L, Malatesta D, et al. Walking-induced muscle fatigue impairs postural control in adolescents with unilateral spastic cerebral palsy. Res Dev Disabil. 2016 Jun-Jul;53-54:11-8. doi: 10.1016/j.ridd.2016.01.019. PMID: 26851383. Exclusion: 11.

1100. Vogtle LK, Malone LA, Azuero A. Outcomes of an exercise program for pain and fatigue management in adults with cerebral palsy. Disabil Rehabil. 2014;36(10):818-25. doi: 10.3109/09638288.2013.821181. PMID: 23924251. Exclusion: 8.
1101. Vore ME, Elgelid S, Bolger S, et al. Impact of a 10-week individualized exercise program on physical function and fatigue of people with multiple sclerosis: a pilot study. Int J MS Care. 2011;13(3):121-6. doi: 10.7224/1537-2073-13.3.121. PMID: 24453715. Exclusion: 11.

1102. Wall A, Borg J, Palmcrantz S. Clinical application of the Hybrid Assistive Limb (HAL) for gait training-a systematic review. Front Syst Neurosci. 2015;9:48. doi: 10.3389/fnsys.2015.00048. PMID: 25859191. Exclusion: 12.

1103. Wall T, Falvo L, Kesten A. Activity-specific aquatic therapy targeting gait for a patient with incomplete spinal cord injury. Physiother Theory Pract. 2017

Apr;33(4):331-44. doi: 10.1080/09593985.2017.1302026. PMID: 28379060. Exclusion: 7.

1104. Wall TE. The effects of the Nintendo(TM) Wii Fit on functional gait, balance and quality of life in ambulatory individuals with incomplete spinal cord injury. 2013. Exclusion: 11.

1105. Waltersson L, Rodby-Bousquet E. Physical activity in adolescents and young adults with cerebral palsy. Biomed Res Int.

2017;2017:8080473. doi: 10.1155/2017/8080473. PMID: 29423412. Exclusion: 4.

1106. Wang J, Lang YB, Du JH, et al. Effect of suspension exercise training on motor and balance functions in children with spastic cerebral palsy. Zhongguo Dang Dai Er Ke Za Zhi. 2018 Jun;20(6):465-9. PMID: 29972120. Exclusion: 13.

1107. Wang RL, Zhou ZH, Xi YC, et al. Preliminary study of robot-assisted ankle rehabilitation for children with cerebral palsy. Beijing Da Xue Xue Bao. 2018 Apr 18;50(2):207-12. PMID: 29643516. Exclusion: 13.

1108. Wang S, Wang L, Meijneke C, et al. Design and control of the MINDWALKER exoskeleton. IEEE Trans Neural Syst Rehabil Eng. 2015 Mar;23(2):277-86. doi: 10.1109/TNSRE.2014.2365697. PMID: 25373109. Exclusion: 10. 
1109. Wang TH, Peng YC, Chen YL, et al. A home-based program using patterned sensory enhancement improves resistance exercise effects for children with cerebral palsy: a randomized controlled trial. Neurorehabil Neural Repair. 2013 Oct;27(8):684-94. doi: 10.1177/1545968313491001. PMID: 23757295. Exclusion: 4.

1110. Wang TH, Peng YC, Chen YL, et al. Longterm effects of therapeutic music combined with loaded sit-to-stand resistance exercise on mobility for children with cerebral palsy. Physiotherapy. 2011;97(20). Exclusion: 10.

1111. Wang YT, Li Z, Yang Y, et al. Effects of wheelchair Tai Chi on physical and mental health among elderly with disability. Res Sports Med. 2016 Jul-Sep;24(3):157-70. doi: 10.1080/15438627.2016.1191487. PMID: 27248716. Exclusion: 3.

1112. Wang YT, Vrongistinos KD, Xu D. The relationship between consistency of propulsive cycles and maximum angular velocity during wheelchair racing. J Appl Biomech. 2008 Aug;24(3):280-7. PMID: 18843158. Exclusion: 4.

1113. Warms CA, Whitney JD, Belza B. Measurement and description of physical activity in adult manual wheelchair users. Disabil Health J. 2008 Oct;1(4):236-44. doi: 10.1016/j.dhjo.2008.07.002. PMID: 21122734. Exclusion: 4.

1114. Weightman A, Preston N, Levesley M, et al. The nature of arm movement in children with cerebral palsy when using computergenerated exercise games. Disabil Rehabil Assist Technol. 2014 May;9(3):219-25. doi: 10.3109/17483107.2013.782576. PMID: 23597314. Exclusion: 11.

1115. Wendebourg MJ, Heesen C, Finlayson M, et al. Patient education for people with multiple sclerosis-associated fatigue: a systematic review. PLoS One. 2017;12(3):e0173025. doi: 10.1371/journal.pone.0173025. PMID: 28267811. Exclusion: 4.
1116. Wens I, Dalgas U, Vandenabeele F, et al. High intensity aerobic and resistance exercise can improve glucose tolerance in persons with multiple sclerosis: a randomized controlled trial. Am J Phys Med Rehabil. 2017 Mar;96(3):161-6. doi: 10.1097/PHM.0000000000000563. PMID: 27362697. Exclusion: 4.

1117. Wens I, Keytsman C, Deckx N, et al. Brain derived neurotrophic factor in multiple sclerosis: effect of 24 weeks endurance and resistance training. Eur J Neurol. 2016 Jun;23(6):1028-35. doi: 10.1111/ene.12976. PMID: 26992038. Exclusion: 11.

1118. Wernig A, Wernig S. The trouble with "body weight support" in treadmill training. Arch Phys Med Rehabil. 2010;91(9):1478. doi: 10.1016/j.apmr.2010.05.015. Exclusion: 10.

1119. West CR, Romer LM, Krassioukov A. Autonomic function and exercise performance in elite athletes with cervical spinal cord injury. Med Sci Sports Exerc. 2013 Feb;45(2):261-7. doi: 10.1249/MSS.0b013e31826f5099. PMID: 22914247. Exclusion: 11.

1120. West CR, Taylor BJ, Campbell IG, et al. Effects of inspiratory muscle training on exercise responses in Paralympic athletes with cervical spinal cord injury. Scand J Med Sci Sports. 2014 Oct;24(5):764-72. doi: 10.1111/sms.12070. PMID: 23530708. Exclusion: 11.

1121. West CR, Wong SC, Krassioukov AV. Autonomic cardiovascular control in Paralympic athletes with spinal cord injury. Med Sci Sports Exerc. 2014 Jan;46(1):60-8. doi: 10.1249/MSS.0b013e31829e46f3. PMID: 23739527. Exclusion: 4.

1122. Westerdahl E, Wittrin A, Kånåhols M, et al. Deep breathing exercises with positive expiratory pressure in patients with multiple sclerosis - a randomized controlled trial. Clin Respir J. 2016;10(6):698-706. doi: 10.1111/crj.12272. Exclusion: 4.

1123. White L, Volfson Z, Faulkner G, et al. Reliability and validity of physical activity instruments used in children and youth with physical disabilities: a systematic review. Pediatr Exerc Sci. 2016 May;28(2):240-63. doi: 10.1123/pes.2015-0123. PMID: 26695088. Exclusion: 4. 
1124. Wiart L, Rosychuk RJ, Wright FV. Evaluation of the effectiveness of robotic gait training and gait-focused physical therapy programs for children and youth with cerebral palsy: a mixed methods RCT. BMC Neurol. 2016 Jun 2;16:86. doi: 10.1186/s12883-016-0582-7. PMID: 27255908. Exclusion: 10.

1125. Widener GL, Allen DD, Gibson-Horn C. Randomized clinical trial of balance-based torso weighting for improving upright mobility in people with multiple sclerosis. Neurorehabil Neural Repair. 2009

Oct;23(8):784-91. doi:

10.1177/1545968309336146. PMID: 19470807. Exclusion: 4.

1126. Wier LM, Hatcher MS, Triche EW, et al. Effect of robot-assisted versus conventional body-weight-supported treadmill training on quality of life for people with multiple sclerosis. J Rehabil Res Dev. 2011;48(4):483-92. PMID: 21674396. Exclusion: 11.

1127. Wilbanks SR, Rogers R, Pool S, et al. Effects of functional electrical stimulation assisted rowing on aerobic fitness and shoulder pain in manual wheelchair users with spinal cord injury. J Spinal Cord Med. 2016 Nov;39(6):645-54. doi: 10.1179/2045772315Y.0000000052. PMID: 26449780. Exclusion: 11.

1128. Willerslev-Olsen M, Andersen JB, Sinkjaer $T$, et al. Sensory feedback to ankle plantar flexors is not exaggerated during gait in spastic hemiplegic children with cerebral palsy. J Neurophysiol. 2014 Feb;111(4):74654. doi: 10.1152/jn.00372.2013. PMID: 24225545. Exclusion: 4.

1129. Willerslev-Olsen M, Petersen TH, Farmer $\mathrm{SF}$, et al. Gait training facilitates central drive to ankle dorsiflexors in children with cerebral palsy. Brain. 2015 Mar; 138(Pt 3):589-603. doi: 10.1093/brain/awu399. PMID: 25623137. Exclusion: 11.

1130. Williams SA, Elliott C, Valentine J, et al. Combining strength training and botulinum neurotoxin intervention in children with cerebral palsy: the impact on muscle morphology and strength. Disabil Rehabil. 2013 Apr;35(7):596-605. doi: 10.3109/09638288.2012.711898. PMID: 22928803. Exclusion: 11.
1131. Wilroy J, Hibberd E. Evaluation of a shoulder injury prevention program in wheelchair basketball. J Sport Rehabil. 2017 Nov 15;27(6):554-9. doi: 10.1123/jsr.20170011. PMID: 29140190. Exclusion: 4.

1132. Wilroy JD, Lai B, Davlyatov G, et al. Correlates of adherence in a home-based, self-managed exercise program tailored to wheelchair users with spinal cord injury. Spinal Cord. 2020 Jun 15;15:15. doi: 10.1038/s41393-020-0497-4. PMID: 32541883. Exclusion: 4.

1133. Winkels DG, Kottink AI, Temmink RA, et al. Wii ${ }^{\text {TM}}$-habilitation of upper extremity function in children with cerebral palsy. An explorative study. Dev Neurorehabil. 2013;16(1):44-51. doi: 10.3109/17518423.2012.713401. PMID: 23030054. Exclusion: 11.

1134. Wirz M, Bastiaenen C, de Bie R, et al. Effectiveness of automated locomotor training in patients with acute incomplete spinal cord injury: a randomized controlled multicenter trial. BMC Neurol. 2011 May 27;11:60. doi: 10.1186/1471-2377-11-60. PMID: 21619574. Exclusion: 10.

1135. Wirz M, Mac HO, Maier D, et al. Effectiveness of automated locomotor training in patients with acute incomplete spinal cord injury: a randomized, controlled, multicenter trial. J Neurotrauma.

2017;34(10):1891-6. doi: 10.1089/neu.2016.4643. PMID: 27750478. Exclusion: 11.

1136. Wittry S, Tsao E, Bjornson K. Are clinicbased walking measures associated with community walking activity in children with cerebral palsy? J Pediatr Rehabil Med. 2018;11(1):23-30. doi: 10.3233/PRM160425. Exclusion: 7.

1137. Wohl C, Siebert H, Blattner B. [Interventions for promoting physical activity in nursing homes : Systematic review of the effectiveness of universal prevention]. Z Gerontol Geriatr. 2017 Aug;50(6):475-82. doi: 10.1007/s00391016-1158-2. PMID: 27966009. Exclusion: 13. 
1138. Wolfsegger T, Assar H, Topakian R. 3-week whole body vibration does not improve gait function in mildly affected multiple sclerosis patients--a randomized controlled trial. J Neurol Sci. 2014 Dec 15;347(1-2):119-23. doi: 10.1016/j.jns.2014.09.030. PMID: 25439165. Exclusion: 11.

1139. Wonneberger M, Schmidt S. Changes of gait parameters following long-term aerobic endurance exercise in mildly disabled multiple sclerosis patients: an exploratory study. Eur J Phys Rehabil Med. 2015 Dec;51(6):755-62. PMID: 25986223. Exclusion: 4.

1140. Wood WH, Fields BE. Hippotherapy: a systematic mapping review of peer-reviewed research, 1980 to 2018. Disabil Rehabil. 2019 Sep 6:1-25. doi: 10.1080/09638288.2019.1653997. PMID: 31491353. Exclusion: 12.

1141. Woodmansee C, Hahne A, Imms C, et al. Comparing participation in physical recreation activities between children with disability and children with typical development: a secondary analysis of matched data. Res Dev Disabil. 2016 FebMar;49-50:268-76. doi: 10.1016/j.ridd.2015.12.004. PMID: 26741263. Exclusion: 4.

1142. Worobey LA, Rigot SK, Hogaboom NS, et al. Investigating the efficacy of web-based transfer training on independent wheelchair transfers through randomized controlled trials. Arch Phys Med Rehabil. 2018 Jan;99(1):9-16.e0. doi: 10.1016/j.apmr.2017.06.025. PMID: 28782541. Exclusion: 4.

1143. Wouda MF, Lundgaard E, Becker F, et al. Effects of moderate- and high-intensity aerobic training program in ambulatory subjects with incomplete spinal cord injurya randomized controlled trial. Spinal Cord. 2018 Oct;56(10):955-63. doi: 10.1038/s41393-018-0140-9. PMID: 29795172. Exclusion: 8.

1144. Wouda MF, Lundgaard E, Becker F, et al. Changes in cardiorespiratory fitness and activity levels over the first year after discharge in ambulatory persons with recent incomplete spinal cord injury. Spinal Cord. 2020 Jul 09;09:09. doi: 10.1038/s41393020-0514-7. PMID: 32647328. Exclusion: 4.
1145. Wren TA, Dryden JW, Mueske NM, et al. Comparison of 2 orthotic approaches in children with cerebral palsy. Pediatr Phys Ther. 2015;27(3):218-26. doi: 10.1097/PEP.0000000000000153. PMID: 26035652. Exclusion: 11.

1146. Wren TA, Lee DC, Hara R, et al. Effect of high-frequency, low-magnitude vibration on bone and muscle in children with cerebral palsy. J Pediatr Orthop. 2010 OctNov;30(7):732-8. doi: 10.1097/BPO.0b013e3181efbabc. PMID: 20864862. Exclusion: 4.

1147. Wren TA, Lening C, Rethlefsen SA, et al. Impact of gait analysis on correction of excessive hip internal rotation in ambulatory children with cerebral palsy: a randomized controlled trial. Dev Med Child Neurol. 2013 Oct;55(10):919-25. doi: 10.1111/dmcn.12184. PMID: 23738949. Exclusion: 4.

1148. Wright V, Fehlings D, Avery L, et al. Walking with my robot: results of a randomized crossover trial evaluating the impact of robotic-assisted gait training on the walking-related gross motor skills and goal accomplishment of children with cerebral palsy. Dev Med Child Neurol. 2017;59:77. doi: 10.1111/dmcn.118_13511. Exclusion: 10.

1149. Wu M, Hornby TG, Landry JM, et al. A cable-driven locomotor training system for restoration of gait in human SCI. Gait Posture. 2011 Feb;33(2):256-60. doi: 10.1016/j.gaitpost.2010.11.016. PMID: 21232961. Exclusion: 11.

1150. Wu M, Kim J, Arora P, et al. Kinematic and EMG responses to pelvis and leg assistance force during treadmill walking in children with cerebral palsy. Neural Plast. 2016;2016:5020348. doi: 10.1155/2016/5020348. PMID: 27651955. Exclusion: 11.

1151. Wu M, Kim J, Gaebler-Spira D, et al. Robotic resistance treadmill training improves locomotor function in children with cerebral palsy: a randomized controlled study. Dev Med Child Neurol. 2015;57:667. doi: 10.1111/dmcn.108_12887. Exclusion: 10. 
1152. Wu M, Kim J, Wei F. Facilitating weight shifting during treadmill training improves walking function in humans with spinal cord injury: a randomized controlled pilot study. Am J Phys Med Rehabil. 2018

Aug;97(8):585-92. doi: 10.1097/PHM.0000000000000927. PMID: 29547448. Exclusion: 11.

1153. Wu M, Landry JM, Kim J, et al. Repeat exposure to leg swing perturbations during treadmill training induces long-term retention of increased step length in human SCI: a pilot randomized controlled study.

Am J Phys Med Rehabil. 2016

Dec;95(12):911-20. doi:

10.1097/PHM.0000000000000517. PMID: 27149587. Exclusion: 11.

1154. Wu M, Landry JM, Schmit BD, et al. Robotic resistance treadmill training improves locomotor function in human spinal cord injury: a pilot study. Arch Phys Med Rehabil. 2012 May;93(5):782-9. doi: 10.1016/j.apmr.2011.12.018. PMID: 22459697. Exclusion: 11.

1155. Wu YN, Hwang M, Ren Y, et al. Combined passive stretching and active movement rehabilitation of lower-limb impairments in children with cerebral palsy using a portable robot. Neurorehabil Neural Repair. 2011 May;25(4):378-85. doi: 10.1177/1545968310388666. PMID: 21343525. Exclusion: 11.

1156. Xiang Y, Lu L, Chen X, et al. Does Tai Chi relieve fatigue? A systematic review and meta-analysis of randomized controlled trials. PLoS One. 2017;12(4):e0174872. doi: 10.1371/journal.pone.0174872. PMID: 28380067. Exclusion: 12.

1157. Xu K, He L, Mai J, et al. Muscle recruitment and coordination following constraintinduced movement therapy with electrical stimulation on children with hemiplegic cerebral palsy: a randomized controlled trial. PLoS One. 2015;10(10):e0138608. doi: 10.1371/journal.pone.0138608. PMID: 26452230. Exclusion: 4.
1158. Xu K, Wang L, Mai J, et al. Efficacy of constraint-induced movement therapy and electrical stimulation on hand function of children with hemiplegic cerebral palsy: a controlled clinical trial. Disabil Rehabil. 2012;34(4):337-46. doi: 10.3109/09638288.2011.607213. PMID: 21961441. Exclusion: 4.

1159. Ya L, Petrini MA. Effects of a home-based resistance exercise in Chinese individuals living with physical disability: resistance exercise on PWPD. Rehabil Nurs. 2018 May/Jun;43(3):174-82. doi: 10.1097/rnj.0000000000000010. PMID: 29710062. Exclusion: 3.

1160. Yamada M, Arai H. Self-management group exercise extends healthy life expectancy in frail community-dwelling older adults. Int J Environ Res Public Health. 2017 May 15;14(5):15. doi: 10.3390/ijerph14050531. PMID: 28505140. Exclusion: 3.

1161. Yanci J, Castillo D, Iturricastillo A, et al. Evaluation of the official match external load in soccer players with cerebral palsy. J Strength Cond Res. 2019 Mar;33(3):866-73. doi: 10.1519/JSC.0000000000002085. PMID: 28658075. Exclusion: 4.

1162. Yang F, Estrada EF, Sanchez MC. Vibration training improves disability status in multiple sclerosis: a pretest-posttest pilot study. J Neurol Sci. 2016 Oct 15;369:96101. doi: 10.1016/j.jns.2016.08.013. PMID: 27653872. Exclusion: 11.

1163. Yang F, Finlayson M, Bethoux F, et al. Effects of controlled whole-body vibration training in improving fall risk factors among individuals with multiple sclerosis: a pilot study. Disabil Rehabil. 2018 Mar;40(5):55360. doi: 10.1080/09638288.2016.1262466. PMID: 27976932. Exclusion: 11.

1164. Yang JF, Norton J, Nevett-Duchcherer J, et al. Volitional muscle strength in the legs predicts changes in walking speed following locomotor training in people with chronic spinal cord injury. Phys Ther. 2011 Jun;91(6):931-43. doi: 10.2522/ptj.20100163. PMID: 21511993. Exclusion: 11. 
1165. Yarar C. Acute effects of whole body vibration on central and peripheral hemodynamics and oxygen consumption in individuals with spinal cord injury. 2011. Exclusion: 4.

1166. Yarar-Fisher C, Pascoe DD, Gladden LB, et al. Acute physiological effects of whole body vibration (WBV) on central hemodynamics, muscle oxygenation and oxygen consumption in individuals with chronic spinal cord injury. Disabil Rehabil. 2014;36(2):136-45. doi: 10.3109/09638288.2013.782358. PMID: 23651125. Exclusion: 11.

1167. Yarar-Fisher C, Polston KFL, Eraslan M, et al. Paralytic and nonparalytic muscle adaptations to exercise training versus highprotein diet in individuals with longstanding spinal cord injury. J Appl Physiol. 2018 Jul 1;125(1):64-72. doi: 10.1152/japplphysiol.01029.2017. PMID: 29494292. Exclusion: 11.

1168. Yasar E, Yilmaz B, Goktepe S, et al. The effect of functional electrical stimulation cycling on late functional improvement in patients with chronic incomplete spinal cord injury.[Erratum appears in Spinal Cord. 2015 Dec;53(12):900; PMID: 26634319]. Spinal Cord. 2015 Dec;53(12):866-9. doi: 10.1038/sc.2015.19. PMID: 25687513. Exclusion: 11.

1169. Yen CL, McHenry CL, Petrie MA, et al. Vibration training after chronic spinal cord injury: evidence for persistent segmental plasticity. Neurosci Lett. 2017 Apr 24;647:129-32. doi: 10.1016/j.neulet.2017.03.019. PMID: 28315725. Exclusion: 11.

1170. Yen SC, Schmit BD, Landry JM, et al. Locomotor adaptation to resistance during treadmill training transfers to overground walking in human SCI. Exp Brain Res. 2012 Feb;216(3):473-82. doi: 10.1007/s00221011-2950-2. PMID: 22108702. Exclusion: 11.

1171. Yeo SS, Kwon JW. Wheelchair skills training for functional activity in adults with cervical spinal cord injury. Int J Sports Med. 2018 Sep 11;39(12):924-8. doi: 10.1055/a0635-0941. PMID: 30206918. Exclusion: 6.
1172. Yildirim A, Surucu GD, Karamercan A, et al. Short-term effects of upper extremity circuit resistance training on muscle strength and functional independence in patients with paraplegia. J Back Musculoskeletal Rehabil. 2016 Nov 21;29(4):817-23. doi: 10.3233/BMR-160694. PMID: 27002667. Exclusion: 11.

1173. Yildirim Şik B, Çekmece C, Dursun N, et al. Is hyppotherapy beneficial for rehabilitation of children with cerebral palsy? Turkiye Klinikleri Journal of Medical Sciences. 2012;32(3):601-8. doi: 10.5336/medsci.2011-22465. Exclusion: 13.

1174. You JS, Kim YL, Lee SM. Effects of a standard transfer exercise program on transfer quality and activities of daily living for transfer-dependent spinal cord injury patients. J Phys Ther Sci. 2017 Mar;29(3):478-83. doi: 10.1589/jpts.29.478. PMID: 28356635. Exclusion: 4.

1175. Yu Y, Wang D, Ma K, et al. Research hot spots in the treatment of pediatric cerebral palsy based on bibliometric analysis. Int $\mathrm{J}$ Clin Exp Med. 2019;12(7):8239-50. Exclusion: 7.

1176. Zabay Neiro C, Martínez Lerín N. How to decrease cardiovascular risk factors in people with multiple sclerosis. Revista Cientifica de la Sociedad Espanola de Enfermeria Neurologica. 2018;47:11-7. doi: 10.1016/j.sedene.2017.10.003. PMID: 8112704 Exclusion: 11.

1177. Zackowski KM, Wang JI, McGready J, et al. Quantitative sensory and motor measures detect change overtime and correlate with walking speed in individuals with multiple sclerosis. Mult Scler Relat Disord. 2015 Jan;4(1):67-74. doi: 10.1016/j.msard.2014.11.001. PMID: 25692092. Exclusion: 4.

1178. Zaenker P, Favret F, Lonsdorfer E, et al. High-intensity interval training combined with resistance training improves physiological capacities, strength and quality of life in multiple sclerosis patients: a pilot study. Eur J Phys Rehabil Med. 2018 Feb;54(1):58-67. doi: 10.23736/S19739087.17.04637-8. PMID: 28681596. Exclusion: 11. 
1179. Žalienė L, Mockevičienė D, Kreivinienė B, et al. Short-term and long-term effects of riding for children with cerebral palsy gross motor functions. Biomed Res Int. 2018;2018:1-6. doi: 10.1155/2018/4190249. Exclusion: 11.

1180. Zamuner AR, Silva E, Teodori RM, et al. Autonomic modulation of heart rate in paraplegic wheelchair basketball players: linear and nonlinear analysis. J Sports Sci. 2013;31(4):396-404. doi: 10.1080/02640414.2012.734917. PMID: 23088300. Exclusion: 11.

1181. Zanca JM, Natale A, Labarbera J, et al. Group physical therapy during inpatient rehabilitation for acute spinal cord injury: findings from the SCIRehab Study. Phys Ther. 2011 Dec;91(12):1877-91. doi: 10.2522/ptj.20100392. PMID: 22003169. Exclusion: 7.

1182. Zariffa J, Kapadia N, Kramer JL, et al. Feasibility and efficacy of upper limb robotic rehabilitation in a subacute cervical spinal cord injury population. Spinal Cord. 2012 Mar;50(3):220-6. doi: 10.1038/sc.2011.104. PMID: 21912402. Exclusion: 11.

1183. Zbogar D, Eng JJ, Miller WC, et al. Reliability and validity of daily physical activity measures during inpatient spinal cord injury rehabilitation. SAGE Open Med. 2016;4 doi: 10.1177/2050312116666941. PMID: 27635252. Exclusion: 4.

1184. Zewdie ET, Roy FD, Yang JF, et al. Facilitation of descending excitatory and spinal inhibitory networks from training of endurance and precision walking in participants with incomplete spinal cord injury. Prog Brain Res. 2015;218:127-55. doi: 10.1016/bs.pbr.2014.12.005. PMID: 25890135. Exclusion: 11.

1185. Zhang B, Zhu Y, Jiang C, et al. Effects of transcutaneous electrical acupoint stimulation on motor functions and self-care ability in children with cerebral palsy. J Altern Complement Med. 2018 Jan;24(1):55-61. doi: 10.1089/acm.2016.0111. PMID: 28767271. Exclusion: 4.
1186. Zhang ZM, Hu XM, Yang CM, et al. Early age at menarche is associated with insulin resistance: a systemic review and metaanalysis. Postgrad Med. 2018 Dec 18;18:18. doi: $10.1080 / 00325481.2019 .1559429$. PMID: 30560708. Exclusion: 6.

1187. Zhao H, Wu YN, Liu J, et al. Changes of calf muscle-tendon properties due to stretching and active movement of children with cerebral palsy--a pilot study. Conf Proc IEEE Eng Med Biol Soc. 2009;2009:528790. doi: 10.1109/IEMBS.2009.5333518. PMID: 19964117. Exclusion: 10.

1188. Zhou H, Xu Q, Zhao S, et al. Distinct clinical characteristics of atypical optic neuritis with seronegative aquaporin- 4 antibody among Chinese patients. $\mathrm{Br} \mathrm{J}$ Ophthalmol. 2017 12;101(12):1720-4. doi: 10.1136/bjophthalmol-2017-310157. PMID: 28404667. Exclusion: 3.

1189. Zhou R, Alvarado L, Ogilvie R, et al. Nongait-specific intervention for the rehabilitation of walking after SCI: Role of the arms. J Neurophysiol. 2018;119(6):2194-212. doi: 10.1152/jn.00569.2017. PMID: 29364074 Exclusion: 11.

1190. Zhou R, Parhizi B, Assh J, et al. Effect of cervicolumbar coupling on spinal reflexes during cycling after incomplete spinal cord injury. J Neurophysiol. 2018;120(6):317286. doi: 10.1152/jn.00509.2017. PMID: 30207867. Exclusion: 6.

1191. Zimmer P, Bloch W, Schenk A, et al. Highintensity interval exercise improves cognitive performance and reduces matrix metalloproteinases- 2 serum levels in persons with multiple sclerosis: a randomized controlled trial. Mult Scler. 2018 Oct;24(12):1635-44. doi: 10.1177/1352458517728342. PMID: 28825348. Exclusion: 4.

1192. Zollinger M, Degache F, Currat G, et al. External mechanical work and pendular energy transduction of overground and treadmill walking in adolescents with unilateral cerebral palsy. Front Physiol. 2016;7(APR) doi: 10.3389/fphys.2016.00121. PMID: 27148062 Exclusion: 5. 
1193. Zou L, Wang H, Xiao Z, et al. Tai chi for health benefits in patients with multiple sclerosis: a systematic review. PLoS One. 2017;12(2):e0170212. doi:

10.1371/journal.pone.0170212. PMID: 28182629. Exclusion: 12.

1194. Zwinkels M, Takken T, Ruyten T, et al. Body mass index and fitness in highfunctioning children and adolescents with cerebral palsy: what happened over a decade? Res Dev Disabil. 2017 Dec;71:706. doi: 10.1016/j.ridd.2017.09.021. PMID: 29024824. Exclusion: 4.
1195. Zwinkels M, Verschuren O, Lankhorst K, et al. Sport-2-stay-fit study: health effects of after-school sport participation in children and adolescents with a chronic disease or physical disability. BMC Sports Sci Med Rehabil. 2015;7:22. doi: 10.1186/s13102015-0016-7. PMID: 26445674. Exclusion: 3. 


\section{Appendix D. Included and Excluded Study Details and Systematic Reviews Evaluated}

Table D-1. Included and excluded study definitions and characteristics

\begin{tabular}{|c|c|c|}
\hline Study Design & Definition/Characteristics & Include/Exclude \\
\hline Systematic Reviews & $\begin{array}{l}\text { A literature review that uses systematic methods } \\
\text { to synthesize, summarize, and grade evidence, on } \\
\text { outcomes to address specific research questions. }\end{array}$ & $\begin{array}{l}\text { Include systematic } \\
\text { reviews published since } \\
2014 \text { for examination of } \\
\text { reference lists for relevant } \\
\text { studies }\end{array}$ \\
\hline Randomized Controlled Trial & $\begin{array}{l}\text { Participants randomized to two or more groups } \\
\text { where each group receives a different intervention. }\end{array}$ & Include \\
\hline Comparative Cohort Study & $\begin{array}{l}\text { An observational study of } 2 \text { groups of participants } \\
\text { where one group received an intervention and } \\
\text { second group received a control or other } \\
\text { intervention and participants are followed forward; } \\
\text { participants were not randomized to different } \\
\text { groups. }\end{array}$ & Include \\
\hline Pre-Post Study & $\begin{array}{l}\text { An observational study of one group of participants } \\
\text { where baseline values are compared with values } \\
\text { after an intervention and all participants received } \\
\text { the same intervention; the study reports results } \\
\text { from the group of participants (reporting is not } \\
\text { selective). }\end{array}$ & $\begin{array}{l}\text { Exclude unless need to } \\
\text { include due to limited or } \\
\text { no evidence from RCTs } \\
\text { and cohort studies }\end{array}$ \\
\hline Case Series & $\begin{array}{l}\text { A publication that reports findings from more than } \\
\text { one individual; if individuals were involved in a } \\
\text { study, the publication does not report results from } \\
\text { all participants but from those who demonstrate } \\
\text { some finding (reporting is selective). }\end{array}$ & Exclude \\
\hline Case Report & $\begin{array}{l}\text { A publication that reports findings from one } \\
\text { individual (reporting is selective). }\end{array}$ & Exclude \\
\hline
\end{tabular}

\section{Systematic Reviews: Reference Lists Evaluated for Eligibility for Inclusion in the Review}

1. Adamson BC, Ensari I, Motl RW. Effect of exercise on depressive symptoms in adults with neurologic disorders: a systematic review and meta-analysis. Arch Phys Med Rehabil. 2015 Jul;96(7):1329-38. doi: 10.1016/j.apmr.2015.01.005. PMID: 25596001 .

2. Afkar A, Ashouri A, Rahmani M, et al. Effect of exercise therapy on quality of life of patients with multiple sclerosis in Iran: a systematic review and meta-analysis. Neurol Sci. 2017 Nov;38(11):1901-11. doi: 10.1007/s10072-017-3047-x. PMID: 28687973 .
3. Aguirre-Guemez AV, Perez-Sanpablo AI, Quinzanos-Fresnedo J, et al. Walking speed is not the best outcome to evaluate the effect of robotic assisted gait training in people with motor incomplete spinal cord injury: a systematic review with meta-analysis. J Spinal Cord Med. 2017 Oct 25;42(2):14254. doi: 10.1080/10790268.2017.1390644. PMID: 29065788.

4. Alashram AR, Padua E, Annino G. Effects of whole body vibration on motor impairments in patients with neurological disorders: a systematic review. Am J Phys Med Rehabil. 2019 Jun 21;21:21. doi: 10.1097/PHM.0000000000001252. PMID: 31246611 . 
5. Alphonsus KB, Su Y, D'Arcy C. The effect of exercise, yoga and physiotherapy on the quality of life of people with multiple sclerosis: systematic review and metaanalysis. Complement Ther Med. 2019 Apr;43:188-95. doi: 10.1016/j.ctim.2019.02.010. PMID: 30935529 .

6. Aravind N, Harvey LA, Glinsky JV. Physiotherapy interventions for increasing muscle strength in people with spinal cord injuries: a systematic review. Spinal Cord. 2019 Jun;57(6):449-60. doi: 10.1038/s41393-019-0242-z. PMID: 30723256 .

7. Armstrong EL, Spencer S, Kentish MJ, et al. Efficacy of cycling interventions to improve function in children and adolescents with cerebral palsy: a systematic review and meta-analysis. Clin Rehabil. 2019 Jul;33(7):1113-29. doi: 10.1177/0269215519837582. PMID: 30935240 .

8. Baxter K. The effect of core strengthening versus extremity strengthening on mobility measures in patients with multiple sclerosis. Dissertation Abstracts International: Section B: The Sciences and Engineering. 2018;78(10-B(E)):No Pagination Specified.

9. Bisson EJ, Fakolade A, Petrin J, et al. Exercise interventions in multiple sclerosis rehabilitation need better reporting on comorbidities: a systematic scoping review. Clin Rehabil. 2017 Oct;31(10):1305-12. doi: 10.1177/0269215517698734. PMID: 28933610 .

10. Bloemen M, Van Wely L, Mollema J, et al. Evidence for increasing physical activity in children with physical disabilities: a systematic review. Dev Med Child Neurol. 2017 10;59(10):1004-10. doi: 10.1111/dmcn.13422. PMID: 28374442.

11. Bochkezanian V, Raymond J, de Oliveira $\mathrm{CQ}$, et al. Can combined aerobic and muscle strength training improve aerobic fitness, muscle strength, function and quality of life in people with spinal cord injury? A systematic review. Spinal Cord. 2015 Jun;53(6):418-31. doi: 10.1038/sc.2015.48. PMID: 25823799.
12. Booth ATC, Buizer AI, Meyns P, et al. The efficacy of functional gait training in children and young adults with cerebral palsy: a systematic review and metaanalysis. Dev Med Child Neurol. 2018 Sep;60(9):866-83. doi: 10.1111/dmcn.13708. PMID: 29512110.

13. Buker DB, Oyarce CC, Plaza RS. Effects of spinal cord injury in heart rate variability after acute and chronic exercise: a systematic review. Top Spinal Cord Inj Rehabil. 2018;24(2):167-76. doi: 10.1310/sci17-00028. PMID: 29706761.

14. Burns AS, Marino RJ, Kalsi-Ryan S, et al. Type and timing of rehabilitation following acute and subacute spinal cord injury: a systematic review. Global Spine J. 2017 Sep;7(3 ):175S-94S. doi: 10.1177/2192568217703084. PMID: 29164023.

15. Byrnes $\mathrm{K}, \mathrm{Wu} \mathrm{PJ}$, Whillier $\mathrm{S}$. Is pilates an effective rehabilitation tool? A systematic review. J Bodywork Mov Ther. 2018 Jan;22(1):192-202. doi: 10.1016/j.jbmt.2017.04.008. PMID: 29332746.

16. Campbell E, Coulter EH, Mattison PG, et al. Physiotherapy rehabilitation for people with progressive multiple sclerosis: a systematic review. Arch Phys Med Rehabil. 2016 Jan;97(1):141-51. doi: 10.1016/j.apmr.2015.07.022. PMID: 26281954.

17. Carter AM. The effects of a pragmatic exercise intervention in people with multiple sclerosis. Dissertation Abstracts International Section C: Worldwide. 2018 Apr;75(4-C):No Pagination Specified. doi: 10.1177/1352458513519354. PMID: 24421303.

18. Carvalho I, Pinto SM, Chagas DDV, et al. Robotic gait training for individuals with cerebral palsy: a systematic review and meta-analysis. Arch Phys Med Rehabil. 2017 11;98(11):2332-44. doi: 10.1016/j.apmr.2017.06.018. PMID: 28751254. 
19. Castro O, Ng K, Novoradovskaya E, et al. A scoping review on interventions to promote physical activity among adults with disabilities. Disabil Health J. 2018

Apr;11(2):174-83. doi:

10.1016/j.dhjo.2017.10.013. PMID: 29132847.

20. Charron S, McKay KA, Tremlett H. Physical activity and disability outcomes in multiple sclerosis: a systematic review (2011-2016). Mult Scler Relat Disord. 2018 Feb;20:169-77. doi: 10.1016/j.msard.2018.01.021. PMID: 29414293.

21. Clutterbuck G, Auld M, Johnston L. Active exercise interventions improve gross motor function of ambulant/semi-ambulant children with cerebral palsy: a systematic review. Disabil Rehabil. 2019 Jan 05;41(10):1131-51. doi: 10.1080/09638288.2017.1422035. PMID: 29303007.

22. Corsi C, Santos MM, Moreira RFC, et al. Effect of physical therapy interventions on spatiotemporal gait parameters in children with cerebral palsy: a systematic review. Disabil Rehabil. 2019 Oct 7:1-10. doi: 10.1080/09638288.2019.1671500. PMID: 31588810 .

23. Corvillo I, Varela E, Armijo F, et al. Efficacy of aquatic therapy for multiple sclerosis: a systematic review. Eur J Phys Rehabil Med. 2017 Dec;53(6):944-52. doi: 10.23736/S1973-9087.17.04570-1. PMID: 28215060 .

24. Cruickshank TM, Reyes AR, Ziman MR. A systematic review and meta-analysis of strength training in individuals with multiple sclerosis or Parkinson disease. Medicine (Baltimore). 2015 Jan;94(4):e411. doi: 10.1097/MD.0000000000000411. PMID: 25634170 .

25. Dalgas U, Stenager E, Sloth M, et al. The effect of exercise on depressive symptoms in multiple sclerosis based on a meta-analysis and critical review of the literature. Eur J Neurol. 2015 Mar;22(3):443-e34. doi: 10.1111/ene.12576. PMID: 25327395.
26. Dauwan M, Begemann MJH, Slot MIE, et al. Physical exercise improves quality of life, depressive symptoms, and cognition across chronic brain disorders: a transdiagnostic systematic review and metaanalysis of randomized controlled trials. J Neurol. 2019 Aug 14;14:14. doi: 10.1007/s00415-019-09493-9. PMID: 31414194 .

27. de Freitas GR, Szpoganicz C, Ilha J. Does neuromuscular electrical stimulation therapy increase voluntary muscle strength after spinal cord injury? A systematic review. Top Spinal Cord Inj Rehabil. 2018;24(1):617. doi: 10.1310/sci16-00048. PMID: 29434456.

28. De Keersmaecker E, Lefeber N, Geys M, et al. Virtual reality during gait training: does it improve gait function in persons with central nervous system movement disorders? A systematic review and meta-analysis. NeuroRehabilitation. 2019;44(1):43-66. doi: 10.3233/NRE-182551. PMID: 30814368.

29. de Paz RH, Serrano-Munoz D, PerezNombela $\mathrm{S}$, et al. Combining transcranial direct-current stimulation with gait training in patients with neurological disorders: a systematic review. J Neuroengineering Rehabil. 2019 Sep 14;16(1):114. doi: 10.1186/s12984-019-0591-z. PMID: 31521179 .

30. Dehghansai N, Lemez S, Wattie N, et al. A systematic review of influences on development of athletes with disabilities. Adapt Phys Act Q. 2017 Jan;34(1):72-90. doi: 10.1123/APAQ.2016-0030. PMID: 28218871

31. Deutsch JE, Guarrera-Bowlby P, Myslinski $\mathrm{MJ}$, et al. Is there evidence that active videogames increase energy expenditure and exercise intensity for people poststroke and with cerebral palsy? Games Health J. 2015 Feb;4(1):31-7. doi: 10.1089/g4h.2014.0082. PMID: 26181678.

32. Devasahayam AJ, Downer MB, Ploughman $M$. The effects of aerobic exercise on the recovery of walking ability and neuroplasticity in people with multiple sclerosis: a systematic review of animal and clinical studies. Mult Scler Int. 2017;2017 doi: 10.1155/2017/4815958. PMID: 29181199. 
33. Dewar R, Love S, Johnston LM. Exercise interventions improve postural control in children with cerebral palsy: a systematic review. Dev Med Child Neurol. 2015 Jun;57(6):504-20. doi: 10.1111/dmcn.12660. PMID: 25523410.

34. Dolbow JD, Dolbow DR, Stevens SL, et al. Restorative effects of aquatic exercise therapies on motor, gait, and cardiovascular function in children with cerebral palsy: a review of literature. Journal of Aquatic Physical Therapy. 2017 Summer;25(1):22-9.

35. Donenberg JG, Fetters L, Johnson R. The effects of locomotor training in children with spinal cord injury: a systematic review. Dev Neurorehabil. 2019 Jun 19;22(4):27287. doi: 10.1080/17518423.2018.1487474. PMID: 29920126.

36. Duquette SA, Guiliano AM, Starmer DJ. Whole body vibration and cerebral palsy: a systematic review. J Can Chiropractic Assoc. 2015 Sep;59(3):245-52. PMID: 26500358.

37. Edwards T, Pilutti LA. The effect of exercise training in adults with multiple sclerosis with severe mobility disability: a systematic review and future research directions. Mult Scler Relat Disord. 2017 Aug;16:31-9. doi:

10.1016/j.msard.2017.06.003. PMID: 28755682

38. Eerden S, Dekker R, Hettinga FJ. Maximal and submaximal aerobic tests for wheelchair-dependent persons with spinal cord injury: a systematic review to summarize and identify useful applications for clinical rehabilitation. Disabil Rehabil. 2018 Mar;40(5):497-521. doi: 10.1080/09638288.2017.1287623. PMID: 28637157.

39. Ellapen TJ, Hammill HV, Swanepoel M, et al. The health benefits and constraints of exercise therapy for wheelchair users: a clinical commentary. Afr J Disabil. 2017;6:337. doi: 10.4102/ajod.v6i0.337. PMID: 28936414.

40. Ellapen TJ, Hammill HV, Swanepoel M, et al. The benefits of hydrotherapy to patients with spinal cord injuries. Afr J Disabil. 2018 May 16;7(0):450. doi:

10.4102/ajod.v7i0.450. PMID: 29850439.
41. Elnahhas AM, Elshennawy S, Aly MG. Effects of backward gait training on balance, gross motor function, and gait in children with cerebral palsy: a systematic review. Clin Rehabil. 2018 Jul 25;33(1):3-12. doi: 10.1177/0269215518790053. PMID: 30043634.

42. Etoom M, Khraiwesh Y, Lena F, et al. Effectiveness of physiotherapy interventions on spasticity in people with multiple sclerosis: a systematic review and metaanalysis. Am J Phys Med Rehabil. 2018 Nov;97(11):793-807. doi: 10.1097/PHM.0000000000000970. PMID: 29794531.

43. Fakolade A, Bisson EJ, Petrin J, et al. Effect of comorbidities on outcomes of neurorehabilitation interventions in multiple sclerosis: A scoping review. Int J MS Care. 2016 Nov-Dec;18(6):282-90. doi: 10.7224/1537-2073.2016-015. PMID: 27999522.

44. Federici S, Meloni F, Bracalenti M, et al. The effectiveness of powered, active lower limb exoskeletons in neurorehabilitation: a systematic review. NeuroRehabilitation. 2015;37(3):321-40. doi: 10.3233/NRE151265. PMID: 26529583.

45. Fischer M, Vialleron T, Laffaye G, et al. Long-term effects of whole-body vibration on human gait: a systematic review and meta-analysis. Front Neurol. 2019;10:627. doi: 10.3389/fneur.2019.00627. PMID: 31316447.

46. Frank R, Larimore J. Yoga as a method of symptom management in multiple sclerosis. Front Neurosci. 2015;9:133. doi: 10.3389/fnins.2015.00133. PMID: 25983675.

47. Gandhi P, Chan K, Verrier MC, et al. Training to improve walking after pediatric spinal cord injury: a systematic review of parameters and walking outcomes. J Neurotrauma. 2017 May 1;34(9):1713-25. doi: 10.1089/neu.2016.4501. PMID: 27869534.

48. Ghai S, Ghai I, Effenberg AO. Effects of dual tasks and dual-task training on postural stability: a systematic review and metaanalysis. Clin Interv Aging. 2017;12:557-77. doi: 10.2147/CIA.S125201. PMID: 28356727. 
49. Gibbs JC, Gagnon DH, Bergquist AJ, et al. Rehabilitation interventions to modify endocrine-metabolic disease risk in individuals with chronic spinal cord injury living in the community (RIISC): a systematic review and scoping perspective. J Spinal Cord Med. 2017 Nov;40(6):733-47. doi: 10.1080/10790268.2017.1350341. PMID: 28703038.

50. Gillett JG, Boyd RN, Carty CP, et al. The impact of strength training on skeletal muscle morphology and architecture in children and adolescents with spastic cerebral palsy: a systematic review. Res Dev Disabil. 2016 Sep;56:183-96. doi: 10.1016/j.ridd.2016.06.003. PMID: 27337690 .

51. Herring MP, Fleming KM, Hayes SP, et al. Moderators of exercise effects on depressive symptoms in multiple sclerosis: a metaregression. Am J Prev Med. 2017 Oct;53(4):508-18. doi: 10.1016/j.amepre.2017.04.011. PMID: 28602542.

52. Hoyme D. Body weight supported treadmill training versus gait trainer in young children with cerebral palsy. Dissertation Abstracts International: Section B: The Sciences and Engineering. 2017;78(4-B(E)):No Pagination Specified.

53. Hsu CW, Kang YN, Tseng SH. Effects of therapeutic exercise intensity on cerebral palsy outcomes: a systematic review with meta-regression of randomized clinical trials. Front Neurol. 2019;10:657. doi: 10.3389/fneur.2019.00657. PMID: 31293501 .

54. Huang M, Liao LR, Pang MY. Effects of whole body vibration on muscle spasticity for people with central nervous system disorders: a systematic review. Clin Rehabil. 2017 Jan;31(1):23-33. doi: 10.1177/0269215515621117. PMID: 26658333.

55. Lai B, Cederberg K, Vanderbom KA, et al. Characteristics of adults with neurologic disability recruited for exercise trials: a secondary analysis. Adapt Phys Act Q. 2018;35(4):476-97. doi: 10.1123/apaq.20170109 .
56. Lai B, Young HJ, Bickel CS, et al. Current trends in exercise intervention research, technology, and behavioral change strategies for people with disabilities: a scoping review. Am J Phys Med Rehabil. 2017 Oct;96(10):748-61. doi: 10.1097/PHM.0000000000000743. PMID: 28398967.

57. Lajeunesse V, Vincent C, Routhier F, et al. Exoskeletons' design and usefulness evidence according to a systematic review of lower limb exoskeletons used for functional mobility by people with spinal cord injury.

Disabil Rehabil Assist Technol. 2016

Oct;11(7):535-47. doi: 10.3109/17483107.2015.1080766. PMID: 26340538 .

58. Langeskov-Christensen M, Heine M, Kwakkel G, et al. Aerobic capacity in persons with multiple sclerosis: a systematic review and meta-analysis. Sports Med. 2015 Jun;45(6):905-23. doi: 10.1007/s40279-0150307-x. PMID: 25739555.

59. Lawrence H, Hills S, Kline N, et al. Effectiveness of exercise on functional mobility in adults with cerebral palsy: a systematic review. Physiother Can. 2016;68(4):398-407. doi: 10.3138/ptc.201538LHC. PMID: 27904240.

60. Lefmann S, Russo R, Hillier S. The effectiveness of robotic-assisted gait training for paediatric gait disorders: systematic review. J Neuroengineering Rehabil. 2017 Jan 5;14(1):1. doi: 10.1186/s12984-0160214-x. PMID: 28057016.

61. Li C, Khoo S, Adnan A. Effects of aquatic exercise on physical function and fitness among people with spinal cord injury: a systematic review. Medicine (Baltimore). 2017 Mar;96(11) doi: 10.1097/MD.0000000000006328. PMID: 28296754.

62. Lopes S, Magalhaes P, Pereira A, et al. Games used with serious purposes: a systematic review of interventions in patients with cerebral palsy. Front Psychol. 2018 Sep 19;9(1712) doi: 10.3389/fpsyg.2018.01712. PMID: 30283377 . 
63. Lu X, Battistuzzo CR, Zoghi M, et al. Effects of training on upper limb function after cervical spinal cord injury: a systematic review. Clin Rehabil. 2015 Jan;29(1):3-13. doi: 10.1177/0269215514536411. PMID: 25575932 .

64. Ma DN, Zhang XQ, Ying J, et al. Efficacy and safety of 9 nonoperative regimens for the treatment of spinal cord injury: a network meta-analysis. Medicine (Baltimore). 2017 Nov;96(47):e8679. doi: 10.1097/MD.0000000000008679. PMID: 29381946.

65. Manago MM, Glick S, Hebert JR, et al. Strength training to improve gait in people with multiple sclerosis: a critical review of exercise parameters and intervention approaches. Int J MS Care. 2019 MarApr;21(2):47-56. doi: 10.7224/15372073.2017-079. PMID: 31049034.

66. Manca A, Dvir Z, Deriu F. Meta-analytic and scoping study on strength training in people with multiple sclerosis. J Strength Cond Res. 2019 Mar;33(3):874-89. doi: 10.1519/JSC.000000000002381. PMID: 30192317.

67. Marinho-Buzelli AR, Bonnyman AM, Verrier MC. The effects of aquatic therapy on mobility of individuals with neurological diseases: a systematic review. Clin Rehabil. 2015 Aug;29(8):741-51. doi: 10.1177/0269215514556297. PMID: 25394397.

68. Marques Catelli A, Ferreira Gattino LA, da Campo LA, et al. Cycle ergometer in the improvement of gross motor function of children with cerebral palsy: a systematic review with meta-analysis. Fisioterapia e Pesquisa. 2019;26(1):101-9. doi: 10.1590/1809-2950/18011026012019.

69. Martin Ginis KA, van der Scheer JW, Latimer-Cheung AE, et al. Evidence-based scientific exercise guidelines for adults with spinal cord injury: an update and a new guideline. Spinal Cord. 2018 Apr;56(4):30821. doi: 10.1038/s41393-017-0017-3. PMID: 29070812 .
70. Martins E, Cordovil R, Oliveira R, et al. Efficacy of suit therapy on functioning in children and adolescents with cerebral palsy: a systematic review and meta-analysis. Dev Med Child Neurol. 2016 Apr;58(4):348-60. doi: 10.1111/dmen.12988. PMID: 26613800 .

71. Mat Rosly M, Mat Rosly H, Davis Oam $\mathrm{GM}$, et al. Exergaming for individuals with neurological disability: a systematic review. Disabil Rehabil. 2017 04;39(8):727-35. doi: 10.3109/09638288.2016.1161086. PMID: 27108475.

72. Mathevon L, Bonan I, Barnais JL, et al. Adjunct therapies to improve outcomes after botulinum toxin injection in children: a systematic review. Ann Phys Rehabil Med. 2019 Jul 29;62(4):283-90. doi: 10.1016/j.rehab.2018.06.010. PMID: 30063979 .

73. Mehrholz J, Harvey LA, Thomas S, et al. Is body-weight-supported treadmill training or robotic-assisted gait training superior to overground gait training and other forms of physiotherapy in people with spinal cord injury? A systematic review. Spinal Cord. 2017 Aug;55(8):722-9. doi: 10.1038/sc.2017.31. PMID: 28398300.

74. Melo FCM, de Lima KKF, Silveira A, et al. Physical training and upper limb strength of people with paraplegia: a systematic review. J Sport Rehabil. 2018 Mar 1;28(3):288-93. doi: 10.1123/jsr.2017-0062. PMID: 29364066.

75. Methajarunon P, Eitivipart C, Diver CJ, et al. Systematic review of published studies on aquatic exercise for balance in patients with multiple sclerosis, Parkinson's disease, and hemiplegia. Hong Kong Physiother J. 2016 Dec;35:12-20. doi: 10.1016/j.hkpj.2016.03.002. PMID: 30931029.

76. Miller L, McFadyen A, Lord AC, et al. Functional electrical stimulation for foot drop in multiple sclerosis: a systematic review and meta-analysis of the effect on gait speed. Arch Phys Med Rehabil. 2017 Jul;98(7):1435-52. doi: 10.1016/j.apmr.2016.12.007. PMID: 28088382. 
77. Moreau NG, Bodkin AW, Bjornson K, et al. Effectiveness of rehabilitation interventions to improve gait speed in children with cerebral palsy: systematic review and metaanalysis. Phys Ther. 2016 Dec;96(12):193854. doi: 10.2522/ptj.20150401. PMID: 27313240 .

78. Nam KY, Kim HJ, Kwon BS, et al. Robotassisted gait training (Lokomat) improves walking function and activity in people with spinal cord injury: a systematic review. J Neuroengineering Rehabil. 2017 Mar 23;14(1):24. doi: 10.1186/s12984-017-02323. PMID: 28330471.

79. Nardone R, Orioli A, Golaszewski S, et al. Passive cycling in neurorehabilitation after spinal cord injury: a review. J Spinal Cord Med. 2017 01;40(1):8-16. doi: 10.1080/10790268.2016.1248524. PMID: 27841091 .

80. Neefkes-Zonneveld CR, Bakkum AJ, Bishop NC, et al. Effect of long-term physical activity and acute exercise on markers of systemic inflammation in persons with chronic spinal cord injury: a systematic review. Arch Phys Med Rehabil. 2015 Jan;96(1):30-42. doi: 10.1016/j.apmr.2014.07.006. PMID: 25064781 .

81. Negaresh R, Motl RW, Zimmer P, et al. Effects of exercise training on multiple sclerosis biomarkers of central nervous system and disease status: a systematic review of intervention studies. Eur J Neurol. 2019 May;26(5):711-21. doi: 10.1111/ene.13929. PMID: 30734989.

82. O'Brien TD, Noyes J, Spencer LH, et al. Systematic review of physical activity and exercise interventions to improve health, fitness and well-being of children and young people who use wheelchairs. BMJ Open Sport Exerc Med. 2016 Nov 15;2(1):e000109. doi: 10.1136/bmjsem2016-000109. PMID: 27900176.

83. Paleg G, Livingstone R. Outcomes of gait trainer use in home and school settings for children with motor impairments: a systematic review. Clin Rehabil. 2015

Nov;29(11):1077-91. doi: 10.1177/0269215514565947. PMID: 25636993.
84. Panisset MG, Galea MP, El-Ansary D. Does early exercise attenuate muscle atrophy or bone loss after spinal cord injury? Spinal Cord. 2016 Feb;54(2):84-92. doi: 10.1038/sc.2015.150. PMID: 26345485.

85. Pearson M, Dieberg G, Smart N. Exercise as a therapy for improvement of walking ability in adults with multiple sclerosis: a meta-analysis. Arch Phys Med Rehabil. 2015 Jul;96(7):1339-48.e7. doi: 10.1016/j.apmr.2015.02.011. PMID: 25712347.

86. Pilutti LA, Motl RW. Functional Electrical Stimulation Cycling Exercise for People with Multiple Sclerosis. Curr Treat Options Neurol. 2019 Nov 08;21(11):54. doi: 10.1007/s11940-019-0597-7. PMID: 31705304.

87. Platta ME, Ensari I, Motl RW, et al. Effect of exercise training on fitness in multiple sclerosis: a meta-analysis. Arch Phys Med Rehabil. 2016 Sep;97(9):1564-72. doi: 10.1016/j.apmr.2016.01.023. PMID: 26896750.

88. Plummer P. Critical appraisal of evidence for improving gait speed in people with multiple sclerosis: dalfampridine versus gait training. Int J MS Care. 2016 MayJun;18(3):105-15. doi: 10.7224/15372073.2014-114. PMID: 27252597.

89. Quel de Oliveira C, Refshauge K, Middleton J, et al. Effects of activity-based therapy interventions on mobility, independence, and quality of life for people with spinal cord injuries: a systematic review and metaanalysis. J Neurotrauma. 2017 May 1;34(9):1726-43. doi: 10.1089/neu.2016.4558. PMID: 27809702.

90. Rameckers EA, Janssen-Potten YJ, Essers IM, et al. Efficacy of upper limb strengthening in children with cerebral palsy: a critical review. Res Dev Disabil. 2015 Jan;36C:87-101. doi: 10.1016/j.ridd.2014.09.024. PMID: 25462469.

91. Rigby BR, Grandjean PW. The efficacy of equine-assisted activities and therapies on improving physical function. J Altern Complement Med. 2016 Jan;22(1):9-24. doi: 10.1089/acm.2015.0171. PMID: 26654868. 
92. Robinson AG, Dennett AM, Snowdon DA. Treadmill training may be an effective form of task-specific training for improving mobility in people with Parkinson's disease and multiple sclerosis: a systematic review and meta-analysis. Physiotherapy. 2019 06;105(2):174-86. doi: https://dx.doi.org/10.1016/j.physio.2018.11. 007. PMID: 30876717.

93. Roeh A, Kirchner SK, Malchow B, et al. Depression in somatic disorders: is there a beneficial effect of exercise? Front Psychiatr. 2019;10:141. doi: 10.3389/fpsyt.2019.00141. PMID: 30949079.

94. Rooney S, Moffat F, Wood L, et al. Effectiveness of fatigue management interventions in reducing severity and impact of fatigue in people with progressive multiple sclerosis: a systematic review. Int J MS Care. 2019 Jan-Feb;21(1):35-46. doi: 10.7224/1537-2073.2018-019. PMID: 30833871 .

95. Roostaei M, Baharlouei H, Azadi H, et al. Effects of aquatic intervention on gross motor skills in children with cerebral palsy: a systematic review. Phys Occup Ther Pediatr. 2017 Oct 20;37(5):496-515. doi: 10.1080/01942638.2016.1247938. PMID: 27967298.

96. Ryan JM, Cassidy EE, Noorduyn SG, et al. Exercise interventions for cerebral palsy. Cochrane Database Syst Rev. 2017 Jun 11;6:CD011660. doi: 10.1002/14651858.CD011660.pub2. PMID: 28602046.

97. Sa-Caputo DC, Costa-Cavalcanti R, Carvalho-Lima RP, et al. Systematic review of whole body vibration exercises in the treatment of cerebral palsy: brief report. Dev Neurorehabil. 2016 Oct;19(5):327-33. doi: 10.3109/17518423.2014.994713. PMID: 25826535 .

98. Sanchez-Lastra MA, Martinez-Aldao D, Molina AJ, et al. Pilates for people with multiple sclerosis: A systematic review and meta-analysis. Mult Scler Relat Disord. 2019 Jan 03;28:199-212. doi: https://dx.doi.org/10.1016/j.msard.2019.01.0 06. PMID: 30623859.
99. Sangelaji B, Smith CM, Paul L, et al. The effectiveness of behaviour change interventions to increase physical activity participation in people with multiple sclerosis: a systematic review and metaanalysis. Clin Rehabil. 2016 Jun;30(6):55976. doi: $10.1177 / 0269215515595274$. PMID: 26198892.

100. Saquetto M, Carvalho V, Silva C, et al. The effects of whole body vibration on mobility and balance in children with cerebral palsy: a systematic review with meta-analysis. $\mathbf{J}$ Musculoskelet Neuronal Interact. 2015 Jun;15(2):137-44. PMID: 26032205.

101. Sattelmayer M, Chevalley O, Steuri R, et al. Over-ground walking or robot-assisted gait training in people with .multiple sclerosis: does the effect depend on baseline walking speed and disease related disabilities? A systematic review and meta-regression. BMC Neurol. 2019 May 08;19(1):93. doi: https://dx.doi.org/10.1186/s12883-0191321-7. PMID: 31068151.

102. Shojaei MH, Alavinia SM, Craven BC. Management of obesity after spinal cord injury: a systematic review. J Spinal Cord Med. 2017 Nov;40(6):783-94. doi: 10.1080/10790268.2017.1370207. PMID: 28929907.

103. Smith M, Barker R, Williams G, et al. The effect of exercise on high-level mobility in individuals with neurodegenerative disease: a systematic literature review. Physiotherapy. 2019 May 02;02:02. doi: https://dx.doi.org/10.1016/j.physio.2019.04. 003. PMID: 31477333.

104. Sosnoff JJ, Sung J. Reducing falls and improving mobility in multiple sclerosis. Expert Rev Neurother. 2015 Jun;15(6):65566. doi: $10.1586 / 14737175.2015 .1046377$. PMID: 25973774.

105. Spencer T, Aldous S, Williams G, et al. Systematic review of high-level mobility training in people with a neurological impairment. Brain Inj. 2018;32(4):403-15. doi: 10.1080/02699052.2018.1429656. PMID: 29393688.

106. Steimer J, Weissert R. Effects of sport climbing on multiple sclerosis. Front Physiol. 2017;8:1021. doi: 10.3389/fphys.2017.01021. PMID: 29311957. 
107. Stergiou A, Tzoufi M, Ntzani E, et al. Therapeutic effects of horseback riding interventions: a systematic review and metaanalysis. Am J Phys Med Rehabil. 2017 Oct;96(10):717-25. doi: 10.1097/PHM.0000000000000726. PMID: 28252520.

108. Streber R, Peters S, Pfeifer K. Systematic review of correlates and determinants of physical activity in persons with multiple sclerosis. Arch Phys Med Rehabil. 2016 Apr;97(4):633-45.e29. doi: 10.1016/j.apmr.2015.11.020. PMID: 26751247.

109. Taylor E, Taylor-Piliae RE. The effects of Tai Chi on physical and psychosocial function among persons with multiple sclerosis: a systematic review. Complement Ther Med. 2017 Apr;31:100-8. doi: 10.1016/j.ctim.2017.03.001. PMID: 28434462.

110. van der Scheer JW, Martin Ginis KA, Ditor DS, et al. Effects of exercise on fitness and health of adults with spinal cord injury: a systematic review. Neurology. 2017 Aug 15;89(7):736-45. doi:

10.1212/WNL.0000000000004224. PMID: 28733344.
111. Veneri D, Gannotti M, Bertucco M, et al. Using the international classification of functioning, disability, and health model to gain perspective of the benefits of yoga in stroke, multiple sclerosis, and children to inform practice for children with cerebral palsy: a meta-analysis. J Altern Complement Med. 2018 May;24(5):439-57. doi: 10.1089/acm.2017.0030. PMID: 29406768.

112. Wall A, Borg J, Palmcrantz S. Clinical application of the Hybrid Assistive Limb (HAL) for gait training-a systematic review. Front Syst Neurosci. 2015;9:48. doi: 10.3389/fnsys.2015.00048. PMID: 25859191.

113. Wood WH, Fields BE. Hippotherapy: a systematic mapping review of peer-reviewed research, 1980 to 2018. Disabil Rehabil. 2019 Sep 6:1-25. doi: 10.1080/09638288.2019.1653997. PMID: 31491353.

114. Xiang Y, Lu L, Chen X, et al. Does Tai Chi relieve fatigue? A systematic review and meta-analysis of randomized controlled trials. PLoS One. 2017;12(4):e0174872. doi: 10.1371/journal.pone.0174872. PMID: 28380067.

115. Zou L, Wang H, Xiao Z, et al. Tai chi for health benefits in patients with multiple sclerosis: a systematic review. PLoS One. 2017;12(2):e0170212. doi: 10.1371/journal.pone.0170212. PMID: 28182629. 


\section{Appendix E. Criteria for Assessing Quality and Internal Validity of Individual Studies}

\section{Quality Assessment Criteria: Randomized Controlled Trials}

Table E-1. Methods to assess quality of trials: assessment of internal validity

\begin{tabular}{|c|c|c|}
\hline $\begin{array}{l}\text { Assessment } \\
\text { Question }\end{array}$ & $\begin{array}{l}\text { Yes/ } \\
\text { Nol } \\
\text { Unclear }\end{array}$ & Description \\
\hline \multirow[t]{3}{*}{$\begin{array}{l}\text { 1. Was the } \\
\text { assignment to } \\
\text { the treatment } \\
\text { groups really } \\
\text { random? }\end{array}$} & Yes & $\begin{array}{l}\text { Use of the term "randomized" alone is not sufficient for a judgment of "Yes". } \\
\text { Explicit description of method for sequence generation must be provided. } \\
\text { Adequate approaches include: Computer-generated random numbers, random } \\
\text { numbers tables }\end{array}$ \\
\hline & No & $\begin{array}{l}\text { Randomization was either not attempted or was based on an inferior approach } \\
\text { (e.g., alternation, case record number, birth date, or day of week) }\end{array}$ \\
\hline & Unclear & Insufficient detail provided to make a judgment of yes or no. \\
\hline \multirow[t]{3}{*}{$\begin{array}{l}\text { 2. Was the } \\
\text { treatment } \\
\text { allocation } \\
\text { concealed? }\end{array}$} & Yes & $\begin{array}{l}\text { Adequate approaches to concealment of randomization: Centralized or } \\
\text { pharmacy-controlled randomization, serially-numbered identical containers, on- } \\
\text { site computer based system with a randomization sequence that is not readable } \\
\text { until allocation } \\
\text { Note: If a trial did not use adequate allocation concealment methods, the highest } \\
\text { rating it can receive is "Fair". }\end{array}$ \\
\hline & No & $\begin{array}{l}\text { Inferior approaches to concealment of randomization: Use of alternation, case } \\
\text { record number, birth date, or day of week, open random numbers lists, serially } \\
\text { numbered envelopes (even sealed opaque envelopes can be subject to } \\
\text { manipulation) }\end{array}$ \\
\hline & Unclear & $\begin{array}{l}\text { No details about allocation methods. A statement that "allocation was concealed" } \\
\text { is not sufficient; details must be provided. }\end{array}$ \\
\hline \multirow[t]{3}{*}{$\begin{array}{l}\text { 3. Were } \\
\text { groups similar } \\
\text { at baseline in } \\
\text { terms of } \\
\text { prognostic } \\
\text { factors? }\end{array}$} & Yes & $\begin{array}{l}\text { Parallel design: No clinically important differences } \\
\text { Crossover design: Comparison of baseline characteristics must be made based } \\
\text { on order of randomization. } \\
\text { Note: Determine beforehand which prognostic factors are important to consider. } \\
\text { A statistically significant difference does not automatically constitute a clinically } \\
\text { important difference. }\end{array}$ \\
\hline & No & Clinically important differences \\
\hline & Unclear & $\begin{array}{l}\text { Statement of "no differences at baseline", but data not reported; or data not } \\
\text { reported by group, or no mention at all of baseline characteristics. For crossover } \\
\text { design, only reported baseline characteristics of the overall group. }\end{array}$ \\
\hline \multirow{4}{*}{$\begin{array}{l}\text { 4. Were } \\
\text { outcome } \\
\text { assessors } \\
\text { blinded to } \\
\text { treatment } \\
\text { allocation? } \\
\text { 5. Was the } \\
\text { care provider } \\
\text { blinded? } \\
\text { 6. Was the } \\
\text { patient } \\
\text { blinded? }\end{array}$} & Yes & $\begin{array}{l}\text { Explicit statement(s) that outcome assessors/care provider/patient were blinded. } \\
\text { Double-dummy studies and use of identically-appearing treatments are also } \\
\text { considered sufficient blinding methods for patients and care providers. }\end{array}$ \\
\hline & No & No blinding used, open-label \\
\hline & $\begin{array}{l}\text { Unclear, } \\
\text { described } \\
\text { as double- } \\
\text { blind }\end{array}$ & Study described as double-blind but no details provided. \\
\hline & $\begin{array}{l}\text { Not } \\
\text { reported }\end{array}$ & No information about blinding \\
\hline
\end{tabular}




\begin{tabular}{|c|c|c|}
\hline $\begin{array}{l}\text { Assessment } \\
\text { Question }\end{array}$ & $\begin{array}{l}\text { Yesl } \\
\text { Nol } \\
\text { Unclear }\end{array}$ & Description \\
\hline \multirow{3}{*}{$\begin{array}{l}\text { 7. Did the } \\
\text { article include } \\
\text { an intention- } \\
\text { to-treat } \\
\text { analysis or } \\
\text { provide the } \\
\text { data needed to } \\
\text { calculate it } \\
\text { (i.e., number } \\
\text { assigned to } \\
\text { each group, } \\
\text { number of } \\
\text { subjects who } \\
\text { finished in } \\
\text { each group, } \\
\text { and their } \\
\text { results)? }\end{array}$} & Yes & $\begin{array}{l}\text { All patients that were randomized were included in the analysis. Specify if } \\
\text { imputation methods (e.g., last-observation carried forward) were used. } \\
\text { OR } \\
\text { Exclusion of } 5 \% \text { of patients or less is acceptable, given that the reasons for } \\
\text { exclusion are not related to outcome (e.g., did not take study medication) and } \\
\text { that the exclusions would not be expected to have an important impact on the } \\
\text { effect size }\end{array}$ \\
\hline & No & $\begin{array}{l}\text { Exclusion of greater than } 5 \% \text { of patients from analysis OR less than } 5 \% \text {, with } \\
\text { reasons that may affect the outcome (e.g., adverse events, lack of efficacy) or } \\
\text { reasons that may be due to bias (e.g., investigator decision) }\end{array}$ \\
\hline & Unclear & Numbers analyzed are not reported \\
\hline \multirow{4}{*}{$\begin{array}{l}\text { 8. Was the rate } \\
\text { of overall } \\
\text { attrition and } \\
\text { the difference } \\
\text { between } \\
\text { groups in } \\
\text { attrition within } \\
\text { acceptable } \\
\text { levels? }\end{array}$} & Yes & $\begin{array}{l}\text { Overall attrition }{ }^{\text {a }} \text { The overall attrition rate was below the level that was } \\
\text { established by the review team. } \\
\text { Differential attrition: The absolute difference between groups in rate of attrition } \\
\text { was below } 10 \% \text {. }\end{array}$ \\
\hline & No & $\begin{array}{l}\text { Overall attrition: The overall attrition rate was above the level that was } \\
\text { established by the review team. } \\
\text { Differential attrition: The difference between groups in the overall attrition rate } \\
\text { or in the rate of attrition for a specific reason (e.g., adverse events, protocol } \\
\text { violations, etc.) was } 10 \% \text { or more. }\end{array}$ \\
\hline & Unclear & $\begin{array}{l}\text { Overall attrition: Insufficient information provided to determine the level of } \\
\text { attrition }\end{array}$ \\
\hline & & $\begin{array}{l}\text { Differential attrition: Insufficient information provided to determine the level of } \\
\text { attrition }\end{array}$ \\
\hline
\end{tabular}

${ }^{a}$ Overall attrition: There is no empirical evidence to support establishment of a specific level of attrition that is universally considered "important". The level of attrition considered important will vary by review and should be determined a priori by the review teams. Attrition refers to discontinuation for ANY reason, including lost to followup, lack of efficacy, adverse events, investigator decision, protocol violation, consent withdrawal, etc.

\section{Quality Assessment Criteria: Nonrandomized Studies}

Table E-2. Nonrandomized studies: guidance for quality assessment

\begin{tabular}{|c|c|c|}
\hline $\begin{array}{l}\text { Assessment } \\
\text { Question }\end{array}$ & $\begin{array}{l}\text { Yes/ } \\
\text { Nol } \\
\text { Unclear }\end{array}$ & Description \\
\hline \multirow[t]{3}{*}{$\begin{array}{l}\text { 1. Was the } \\
\text { selection of } \\
\text { patients for } \\
\text { inclusion } \\
\text { unbiased? }\end{array}$} & Yes & $\begin{array}{l}\text { - Same pre-specified eligibility criteria for all groups } \\
\text { - Same strategy for obtaining groups } \\
\text { - Enroll random or consecutive sample of all patients meeting eligibility criteria } \\
\text { - Samples drawn from same source and same timeperiod }\end{array}$ \\
\hline & No & - One or more of the above were not met \\
\hline & Unclear & Insufficient detail provided to make a judgment of yes or no. \\
\hline \multirow{3}{*}{$\begin{array}{l}\text { 2. Differences } \\
\text { in } \\
\text { predetermined } \\
\text { prognostic } \\
\text { factors at } \\
\text { baseline? }\end{array}$} & Yes & $\begin{array}{l}\text { - } 10 \% \text { difference in dichotomous outcomes } \\
\text { - Clinically meaningful differences in continuous outcomes }\end{array}$ \\
\hline & No & - Differences above limits set a priori \\
\hline & Unclear & Insufficient detail provided to make a judgment of yes or no. \\
\hline
\end{tabular}




\begin{tabular}{|c|c|c|}
\hline $\begin{array}{l}\text { Assessment } \\
\text { Question }\end{array}$ & $\begin{array}{l}\text { Yes/ } \\
\text { Nol } \\
\text { Unclear }\end{array}$ & Description \\
\hline \multirow{6}{*}{$\begin{array}{l}\text { 3. Was the } \\
\text { rate of overall } \\
\text { attrition and } \\
\text { the difference } \\
\text { between } \\
\text { groups in } \\
\text { attrition within } \\
\text { acceptable } \\
\text { levels? }\end{array}$} & Yes & $\begin{array}{l}\text { Overall attrition }{ }^{\mathrm{a}} \text { : The overall attrition rate was below the level that was } \\
\text { established by the review team }\end{array}$ \\
\hline & & $\begin{array}{l}\text { Differential attrition: The absolute difference between groups in rate of attrition } \\
\text { was below } 10 \%\end{array}$ \\
\hline & No & $\begin{array}{l}\text { Overall attrition: The overall attrition rate was above the level that was } \\
\text { established by the review team. }\end{array}$ \\
\hline & & $\begin{array}{l}\text { Differential attrition: The difference between groups in the overall attrition rate } \\
\text { was } 10 \% \text { or more }\end{array}$ \\
\hline & Unclear & $\begin{array}{l}\text { Overall attrition: Insufficient information provided to determine the level of } \\
\text { attrition }\end{array}$ \\
\hline & & $\begin{array}{l}\text { Differential attrition: Insufficient information provided to determine the level of } \\
\text { attrition }\end{array}$ \\
\hline \multirow{2}{*}{$\begin{array}{l}\text { 4. Were the } \\
\text { events } \\
\text { investigated } \\
\text { prespecified } \\
\text { and defined? }\end{array}$} & Yes & $\begin{array}{l}\text { Events were explicitly defined, including methods for categorizing continuous } \\
\text { variables. }\end{array}$ \\
\hline & No & \\
\hline \multirow{2}{*}{$\begin{array}{l}\text { 5. Was there a } \\
\text { clear } \\
\text { description of } \\
\text { the } \\
\text { techniques } \\
\text { used to } \\
\text { identify the } \\
\text { events? }\end{array}$} & Yes & $\begin{array}{l}\text { Techniques used to identify the events were clearly described, including who } \\
\text { ascertained, timing and methods used }\end{array}$ \\
\hline & No & \\
\hline \multirow{3}{*}{$\begin{array}{l}\text { 6. Was there } \\
\text { unbiased and } \\
\text { accurate } \\
\text { ascertainment } \\
\text { of events? }\end{array}$} & Yes & $\begin{array}{l}\text { Ascertainment was conducted by an independent individual or endpoint } \\
\text { committee using appropriate data sources and validated techniques with limited } \\
\text { reliance on patient recall. Ascertainment should be blinded where possible and } \\
\text { appropriate. }\end{array}$ \\
\hline & No & \\
\hline & Unclear & Insufficient detail provided to make a judgment of yes or no \\
\hline \multirow{3}{*}{$\begin{array}{l}\text { 7. Were } \\
\text { potential } \\
\text { confounding } \\
\text { variables and } \\
\text { risk factors } \\
\text { identified and } \\
\text { examined } \\
\text { using } \\
\text { acceptable } \\
\text { statistical } \\
\text { techniques? } \\
\text { Such } \\
\text { techniques } \\
\text { include: } \\
\text { stratification, } \\
\text { multivariable } \\
\text { regression, } \\
\text { propensity } \\
\text { score } \\
\text { matching. }\end{array}$} & Yes & $\begin{array}{l}\text { Use one of the acceptable techniques to address multiple variables considered } \\
\text { important. Not all variables must be considered to achieve "yes", but multiple } \\
\text { should be addressed. }\end{array}$ \\
\hline & No & \\
\hline & Unclear & $\begin{array}{l}\text { Insufficient detail provided to make a judgment of yes or no, or too few variables } \\
\text { considered, or variables considered not deemed important. }\end{array}$ \\
\hline
\end{tabular}




\begin{tabular}{|l|l|l|}
\hline $\begin{array}{l}\text { Assessment } \\
\text { Question }\end{array}$ & $\begin{array}{l}\text { Yes/ } \\
\text { Nol } \\
\text { Unclear }\end{array}$ & Description \\
\hline $\begin{array}{l}\text { 8. Was the } \\
\text { duration of }\end{array}$ & Yes & \\
followup & No & \\
\cline { 2 - 2 } $\begin{array}{l}\text { reasonable for } \\
\text { investigated } \\
\text { events? }\end{array}$ & Unclear & Insufficient detail provided to make a judgment of yes or no. \\
$\begin{array}{l}\text { Should be } \\
\text { determined a }\end{array}$ & & \\
priori, by & & \\
outcome-may & & \\
differ for & & \\
adverse & & \\
events, for & & \\
example. & & \\
\hline
\end{tabular}

${ }^{a}$ Overall attrition: The level of attrition considered important will vary by review and should be determined a priori by the review teams. Attrition refers to discontinuation for ANY reason, including lost to followup, lack of efficacy, adverse events, investigator decision, consent withdrawal, etc. Generally $\leq 20 \%$ is considered a reasonable cutoff for acceptable attrition, but greater levels may be acceptable depending on the duration of study and population characteristics.

\section{Appendix E References}

1. Methods Guide for Effectiveness and Comparative Effectiveness Reviews. AHRQ

Publication No. 10(14)-EHC063-EF.

Rockville, MD: Agency for Healthcare

Research and Quality; January 2014.

Current chapters available at

https://effectivehealthcare.ahrq.gov/topics/ce r-methods-guide/overview/.
2. Nelson HD. Systematic Reviews to Answer Health Care Questions. In: Gaertner R, editor. Philadelphia, PA: Wolters Kluwer; 2014.

3. U.S. Preventive Services Task Force Procedure Manual. Rockville, MD: Agency for Health Care Research and Quality; 2018. https://www.uspreventiveservicestaskforce.o rg/Page/Name/procedure-manual. Accessed February 27, 2019. 


\section{Appendix F. Evidence Tables}

Table F-1. Study description and results (continued in Table F-2)

\begin{tabular}{|c|c|c|c|c|}
\hline $\begin{array}{l}\text { Author, Year } \\
\text { Intervention Type } \\
\text { Duration of } \\
\text { Postintervention } \\
\text { Followup } \\
\text { Quality } \\
\text { (See Appendix B } \\
\text { for Full Citation) }\end{array}$ & $\begin{array}{l}\text { Intervention and } \\
\text { Comparison }\end{array}$ & $\begin{array}{l}\text { Population } \\
\text { Age (Mean) } \\
\text { Gender (\% Female) } \\
\text { Race (\%) } \\
\text { Ambulatory (\%) } \\
\text { Wheelchair User (\%) } \\
\text { Condition Specific (\%) } \\
\text { Other (\%) }\end{array}$ & Prioritized Outcomes & Other Outcomes \\
\hline $\begin{array}{l}\text { Acar, } 2016 \\
\text { Postural Control } \\
\text { Motion gaming } \\
\text { Postintervention, } 6 \\
\text { weeks } \\
\text { Poor }\end{array}$ & $\begin{array}{l}\text { A. Nintendo } \\
\text { Wii gaming plus } \\
\text { neuro-developmental } \\
\text { treatment, } 12 \\
\text { sessions over } 6 \\
\text { weeks }(n=15) \\
\\
\text { B. } \\
\text { Neurodevelopmental } \\
\text { treatment, } 12 \\
\text { sessions over } 6 \\
\text { weeks }(n=15)\end{array}$ & $\begin{array}{l}\text { A vs. B } \\
\text { Age (mean years): } 9.53 \text { vs. } \\
9.73 \\
\text { Female: } 7(47 \%) \text { vs. } 9(60 \%) \\
\text { Race: NR } \\
\text { Ambulatory: NR } \\
\text { Wheelchair user: NR } \\
\text { GMFCS I: } 40 \% \text { vs. } 40 \% \\
\text { GMFCS II: } 60 \% \text { vs. } 60 \% \\
\text { Spastic hemiparesis: } 100 \% \\
\text { Manual Ability Classification } \\
\text { System median score, } \\
\text { (range): } \\
2 \text { (range } 1-3 \text { ) vs. } 2 \text { (range } \\
\text { 1-3) } \\
\text { GMFCS, levels I-V: } \\
\text { Level 1: } 6 \text { cases vs. } 6 \text { cases } \\
\text { Level 2: } 9 \text { cases vs. } 9 \text { cases }\end{array}$ & $\begin{array}{l}\text { A vs. B, mean (SD) } \\
\text { Baseline } \\
\text { QUEST (Minimum score=0, Maximum score=100) } \\
\text { QUEST Dissociated movements (score): } 80.1 \text { (7.73) vs. } \\
81.4(10.70) \\
\text { QUEST Grasps (score): } 42.2(18.76) \text { vs. } 53(16.45) \\
\text { QUEST Weight bearing (score): } 69.2(19.46) \text { vs. } 75.4 \\
(17.07) \\
\text { QUEST Protective extension (score): } 72.9(14.78) \text { vs. } 71 \\
\text { (23.52) } \\
\text { Postintervention } \\
\text { QUEST (Minimum score=0, Maximum score=100) } \\
\text { QUEST Dissociated movements: } 85.6 \text { ( } 8.54) \text { vs. } 86.4 \\
(8.78) \\
\text { QUEST Grasps (score): } 47.1 \text { (16.64) vs. } 55.7(15.30) \\
\text { QUEST Weight bearing (score): } 72.7(19.60) \text { vs. } 77.3 \\
\text { (15.43) } \\
\text { QUEST Protective extension (score): } 77 \text { (12.66) vs. } 74 \\
\text { (23.36) } \\
\text { *Mean Change Data - not included? } \\
\text { WeeFIM, (18 items, 7-point scale, } 1=\text { total assistance } \\
\text { required to 7=complete independence, } 7 \text { to 126-point total } \\
\text { range) } \\
\text { WeeFIM: } 46 \text { (8.23) vs. } 48.3 \text { (7.27) (baseline) } \\
\text { WeeFIM: } 46.7 \text { (7.51) vs. } 48.9 \text { (7.14) (postintervention) }\end{array}$ & NA \\
\hline
\end{tabular}




\begin{tabular}{|c|c|c|c|c|}
\hline $\begin{array}{l}\text { Author, Year } \\
\text { Intervention Type } \\
\text { Duration of } \\
\text { Postintervention } \\
\text { Followup } \\
\text { Quality } \\
\text { (See Appendix B } \\
\text { for Full Citation) } \\
\end{array}$ & $\begin{array}{l}\text { Intervention and } \\
\text { Comparison }\end{array}$ & $\begin{array}{l}\text { Population } \\
\text { Age (Mean) } \\
\text { Gender (\% Female) } \\
\text { Race (\%) } \\
\text { Ambulatory (\%) } \\
\text { Wheelchair User (\%) } \\
\text { Condition Specific (\%) } \\
\text { Other (\%) }\end{array}$ & Prioritized Outcomes & Other Outcomes \\
\hline $\begin{array}{l}\text { Abbasi, } 2019 \\
\text { Postural Control } \\
\text { Whole body } \\
\text { vibration } \\
\text { Immediately } \\
\text { postintervention, } \\
6 \text { weeks } \\
\text { Fair }\end{array}$ & $\begin{array}{l}\text { A. Whole body } \\
\text { vibration, } 18 \\
\text { sessions over } 6 \\
\text { weeks }(n=22) \\
\text { B. No treatment, no } \\
\text { exercise }(n=24)\end{array}$ & $\begin{array}{l}\text { A vs. B } \\
\text { Age: } 37 \text { vs. } 39 \\
\text { Female: } 5 \% \text { vs. } 17 \% \\
\text { EDSS: } 1.54 \text { vs. } 1.55\end{array}$ & $\begin{array}{l}\text { A vs. B, Median (Interquartile range), p-value is between } \\
\text { groups } \\
\text { MSQOL-54 (PCS): } \\
4.20(1.73,8.40) \text { vs. }-1.26(-3.28,0), p<0.001 \\
\text { MSQOL-54 (MCS): } \\
5.96(2.71,11.89) \text { vs. }-0.17(-2.20,0.07), p<0.001\end{array}$ & $\begin{array}{l}\text { A vs. B, Median (Interquartile range) followup- } \\
\text { baseline scores, } p=\text { between groups: } \\
\text { Trunk Flexor: Med Diff } 25.83 \text { ( } 8.83 \text { to } 46.41) \\
\text { vs. }-0.33 \text { ( }-5.67 \text { to } 6.75), p<0.001 \\
\text { Trunk Extensor: Med Diff } 38.17(20.75 \text { to } 70) \\
\text { vs. }-1.49 \text { ( }-11.83 \text { to } 3.49), p<0.001\end{array}$ \\
\hline $\begin{array}{l}\text { Adar, } 2017 \\
\text { Aerobic Exercise } \\
\text { Aquatics } \\
\text { Postintervention, } 0 \\
\text { weeks } \\
\text { Fair }\end{array}$ & $\begin{array}{l}\text { A. Aquatic exercise, } \\
30 \text { sessions over } 6 \\
\text { weeks }(n=17) \\
\text { B. Land-based } \\
\text { exercise, } 30 \text { sessions } \\
\text { over } 6 \text { weeks }(n=15)\end{array}$ & $\begin{array}{l}\text { A vs. B } \\
\text { Age (mean years): } \\
10.1 \text { vs. } 9.3 \\
\text { Female: } \\
9(53 \%) \text { vs. } 6(40 \%) \\
\text { Race: NR } \\
\text { Ambulatory: NR } \\
\text { Wheelchair user: NR } \\
\text { Other: } \\
\text { A: Spastic Diplegia (65\%) } \\
\text { vs. Hemiplegia (35\%) } \\
\text { B: Spastic Diplegia }(67 \%) \\
\text { vs. Hemiplegia (33\%) }\end{array}$ & $\begin{array}{l}\text { A vs. B, Mean change scores: } \\
\text { TUG: }-0.13(0.14) \text { vs. }-0.16(0.13), p=0.664 \\
\text { GMFM-88: } 0.05(0.05) \text { vs. } 0.05(0.03), p=0.451 \\
\text { WeeFIM motor: } 0.04(0.04) \text { vs. } 0.06(0.06), p=0.860 \\
\text { WeeFIM total: }-0.13(0.14) \text { vs. }-0.16(0.13), p=0.287\end{array}$ & NA \\
\hline
\end{tabular}




\begin{tabular}{|c|c|c|c|c|}
\hline $\begin{array}{l}\text { Author, Year } \\
\text { Intervention Type } \\
\text { Duration of } \\
\text { Postintervention } \\
\text { Followup } \\
\text { Quality } \\
\text { (See Appendix B } \\
\text { for Full Citation) }\end{array}$ & $\begin{array}{l}\text { Intervention and } \\
\text { Comparison }\end{array}$ & $\begin{array}{l}\text { Population } \\
\text { Age (Mean) } \\
\text { Gender (\% Female) } \\
\text { Race (\%) } \\
\text { Ambulatory (\%) } \\
\text { Wheelchair User (\%) } \\
\text { Condition Specific (\%) } \\
\text { Other (\%) }\end{array}$ & Prioritized Outcomes & Other Outcomes \\
\hline $\begin{array}{l}\text { Afrasiabifar, } 2018 \\
\text { Postural Control } \\
\text { Balance } \\
\text { Postintervention, } \\
12 \text { weeks } \\
\text { Good }\end{array}$ & $\begin{array}{l}\text { A. Cawthorne- } \\
\text { Cooksey exercise: } 36 \\
\text { sessions, } 3 \text { times a } \\
\text { week, over } 12 \\
\text { weeks, }(n=24) \\
\text { B. Frankel exercises: } \\
\text { number of sessions } \\
\text { NR, over } 12 \text { weeks, } \\
(n=23) \\
\text { C. Usual care control } \\
(n=25)\end{array}$ & $\begin{array}{l}\text { A vs. B vs. C } \\
\text { Age (mean years): } 32.4 \text { vs. } \\
32 \text { vs. } 33.6 \\
\text { Female: } 20(83 \%) \text { vs. } 17 \\
\text { (74\%) vs. } 19(76 \%) \\
\text { Race: NR } \\
\text { Ambulatory: NR } \\
\text { Wheelchair user: NR } \\
\\
\text { Body mass index (kg/m², } \\
\text { mean): } 23.4 \text { vs. } 23.6 \text { vs. } \\
23.3 \\
\text { MS subtype } \\
\text { Relapsing-remitting: } 23 \\
(95.8 \%) \text { vs. } 22(95.7 \%) \text { vs. } \\
23(92 \%) \\
\text { Primary and secondary } \\
\text { progressive: } 1(4.2 \%) \text { vs. } 1 \\
(4.3 \%) \text { vs. } 2(8 \%)\end{array}$ & NA & $\begin{array}{l}\text { A vs. B vs. C, mean (SD) } \\
\text { Balance score (BBS 0-56, higher } \\
\text { scores=better balance) } \\
\text { A vs. B } \\
\text { BBS: } \\
30.9 \text { (5.6) vs. } 31.6 \text { (5.1), (baseline) } \\
33.9 \text { (6.1) vs. } 32.5 \text { (5.1), (6 weeks) } \\
39.8 \text { (4.5) vs. } 33.9 \text { (5.6), (postintervention) } \\
\text { A vs. C } \\
\text { BBS: } \\
30.9(5.6) \text { vs. } 30.3 \text { (6), (baseline) } \\
\text { BBS: } \\
33.9(6.1) \text { vs. } 29.8 \text { (6.2), (6 weeks) } \\
\text { BBS: } \\
39.8(4.5) \text { vs. } 29.1 \text { (6.5), (postintervention) } \\
\text { B vs. C } \\
\text { BBS: } \\
31.6(5.1) \text { vs. } 30.3 \text { (6), (baseline) } \\
\text { BBS: } \\
32.5(5.1) \text { vs. } 29.8 \text { (6.2), (6 weeks) } \\
\text { BBS: } \\
33.9(5.6) \text { vs. } 29.1 \text { (6.5), (postintervention) } \\
\text { Mean differences, paired comparisons } \\
\text { A + B } \\
\text { BBS: } \\
-0.7(95 \% \text { Cl }-4.8 \text { to 3.4), p=0.9, (baseline) } \\
\text { BBS: } \\
1.4(95 \% \text { Cl }-2.8 \text { to 5.5), p=0.7, (6 weeks) } \\
\text { BBS: } \\
5.9(95 \% \text { Cl } 1.9 \text { to } 9.9), \text { p=0.001, } \\
\text { (postintervention) }\end{array}$ \\
\hline
\end{tabular}




\begin{tabular}{|c|c|c|c|c|}
\hline \begin{tabular}{|l} 
Author, Year \\
Intervention Type \\
Duration of \\
Postintervention \\
Followup \\
Quality \\
(See Appendix B \\
for Full Citation) \\
\end{tabular} & $\begin{array}{l}\text { Intervention and } \\
\text { Comparison }\end{array}$ & $\begin{array}{l}\text { Population } \\
\text { Age (Mean) } \\
\text { Gender (\% Female) } \\
\text { Race (\%) } \\
\text { Ambulatory (\%) } \\
\text { Wheelchair User (\%) } \\
\text { Condition Specific (\%) } \\
\text { Other (\%) } \\
\end{array}$ & Prioritized Outcomes & Other Outcomes \\
\hline $\begin{array}{l}\text { Afrasiabifar, } 2018 \\
\text { (Continued) }\end{array}$ & & & & $\begin{array}{l}\text { A +C } \\
\text { BBS: } 0.6(95 \% \mathrm{Cl}-3.3 \text { to } 4.6), p=0.9, \\
\text { (baseline) } \\
\text { BBS: } 4.1(95 \% \mathrm{Cl}-0.02 \text { to } 8.2), p=0.05,(6 \\
\text { weeks) } \\
\text { BBS: }(95 \% \mathrm{Cl} 10.7 \text { to } 6.814 .6), p=0.001, \\
\text { (postintervention) } \\
\text { B +C } \\
\text { BBS: } 1.3(95 \% \mathrm{Cl}-2.75 .2), p=0.7 \text {, (baseline) } \\
\text { BBS: } 2.7(95 \% \mathrm{Cl}-1.4 \text { to } 6.9), p=0.2,(6 \\
\text { weeks) } \\
\text { BBS: } 4.8(95 \% \mathrm{Cl} 0.9 \text { to } 8.8), p=0.01, \\
\text { (postintervention) }\end{array}$ \\
\hline $\begin{array}{l}\text { Ahmadi, } 2013 \\
\text { Aerobic Exercise } \\
\text { Treadmill } \\
\text { Postural Control } \\
\text { Yoga } \\
\text { Immediately } \\
\text { postintervention, } \\
8 \text { weeks } \\
\text { Fair }\end{array}$ & $\begin{array}{l}\text { A. Treadmill, } 24 \\
\text { sessions over } 8 \\
\text { weeks }(n=10) \\
\text { C. Yoga, } 24 \text { sessions } \\
\text { over } 8 \text { weeks }(n=11) \\
\text { B. Waitlist control } \\
(n=10)\end{array}$ & $\begin{array}{l}\text { A vs. B vs. C } \\
\text { Age: } 37 \text { vs. } 32 \text { vs. } 37 \\
\text { Female: } 100 \% \\
\text { EDSS: } 2.40 \text { vs. } 2.00 \text { vs. } 2.25\end{array}$ & 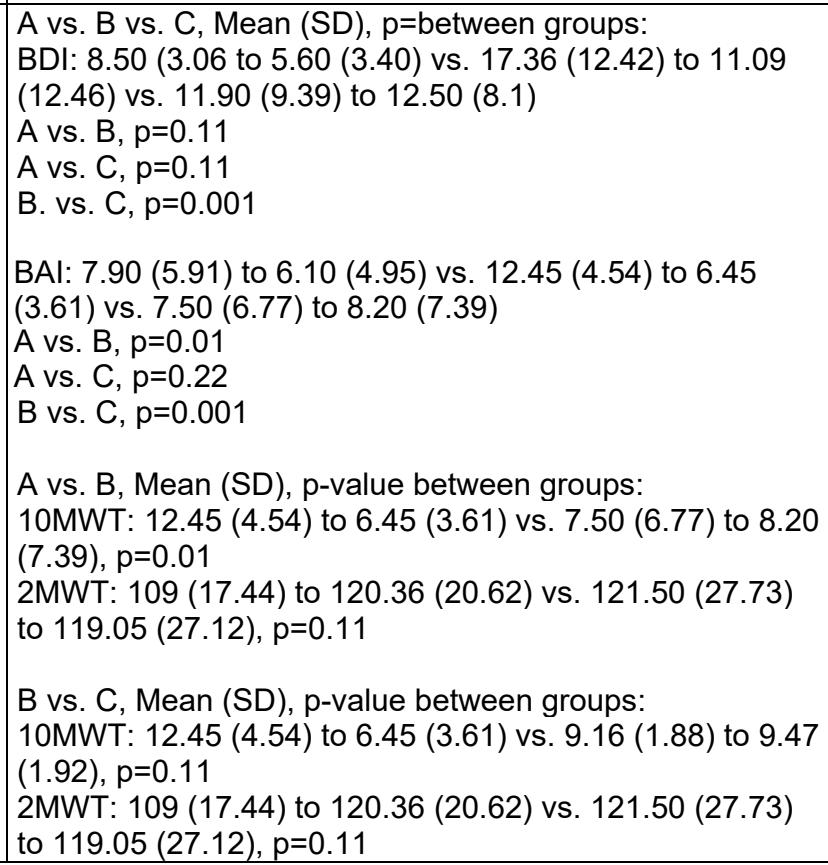 & $\begin{array}{l}\text { A vs. B, Mean (SD), p-value between groups: } \\
\text { BBS: } 47.72(6.78) \text { to } 53.81(3.40) \text { vs. } 44.50 \\
(8.48) \text { to } 41.70(8.48), p=0.07 \\
\text { B vs. C, Mean (SD), p-value between groups: } \\
\text { BBS: } 47.72(6.78) \text { to } 53.81(3.40) \text { vs. } 44.50 \\
(8.48) \text { to } 41.70(8.48), p=0.07\end{array}$ \\
\hline
\end{tabular}




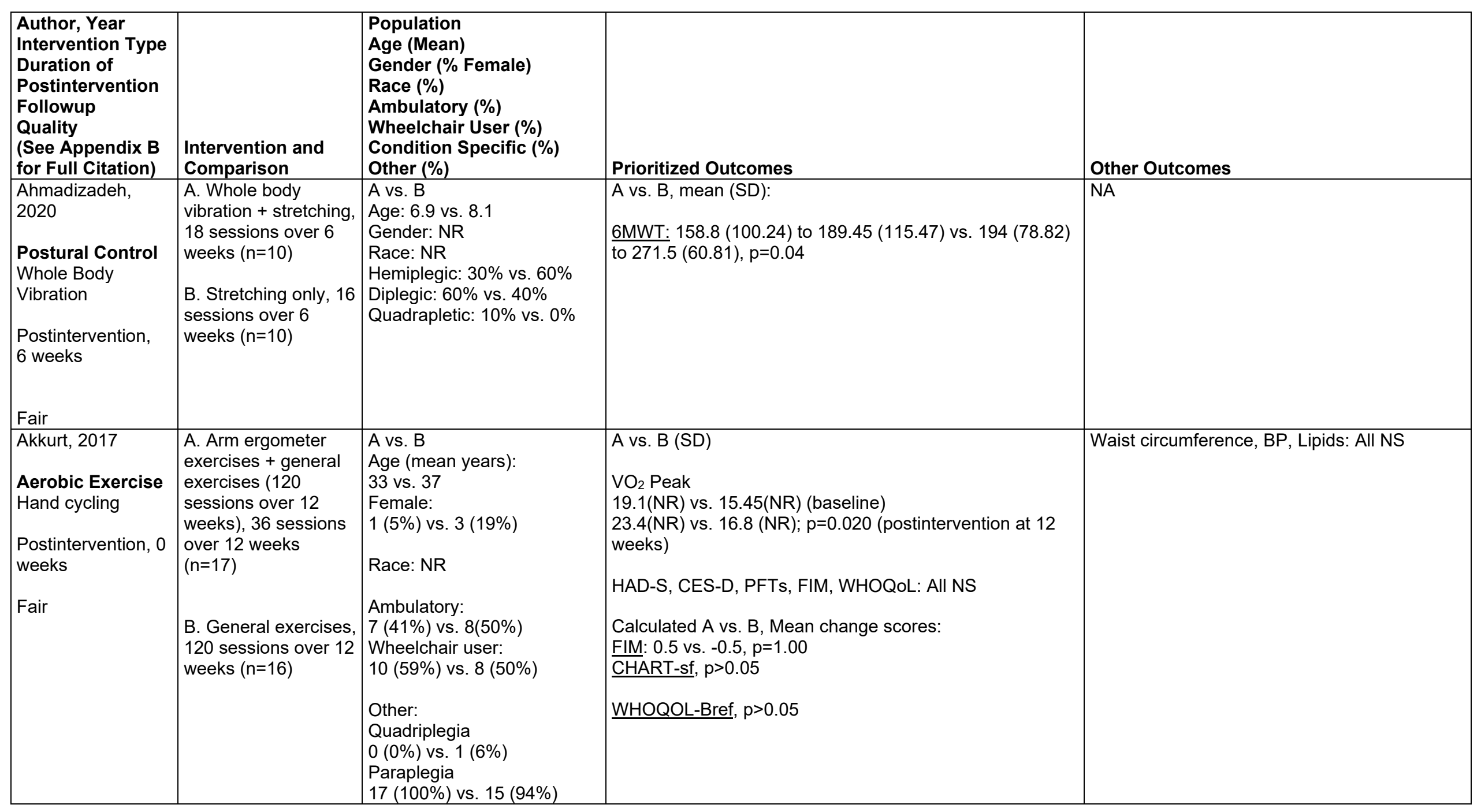




\begin{tabular}{|c|c|c|c|c|}
\hline $\begin{array}{l}\text { Author, Year } \\
\text { Intervention Type } \\
\text { Duration of } \\
\text { Postintervention } \\
\text { Followup } \\
\text { Quality } \\
\text { (See Appendix B } \\
\text { for Full Citation) } \\
\end{array}$ & $\begin{array}{l}\text { Intervention and } \\
\text { Comparison }\end{array}$ & $\begin{array}{l}\text { Population } \\
\text { Age (Mean) } \\
\text { Gender (\% Female) } \\
\text { Race (\%) } \\
\text { Ambulatory (\%) } \\
\text { Wheelchair User (\%) } \\
\text { Condition Specific (\%) } \\
\text { Other (\%) }\end{array}$ & Prioritized Outcomes & Other Outcomes \\
\hline $\begin{array}{l}\text { Alexeeva } 2011 \\
\text { Aerobic Exercise } \\
\text { Treadmill } \\
\text { Pre to post } \\
\text { Fair }\end{array}$ & $\begin{array}{l}\text { A. Body weight } \\
\text { supported treadmill } \\
\text { training maximum } 60 \\
\text { minutes for } 3 \text { days a } \\
\text { week for } 13 \text { weeks } \\
\text { (39 sessions) } \\
\\
\text { B. Body weight } \\
\text { supported track } \\
\text { training maximum } 60 \\
\text { minutes for } 3 \text { days a } \\
\text { week for } 13 \text { weeks } \\
\text { (39 sessions) } \\
\\
\text { C. Structured } \\
\text { physical therapy } \\
\text { maximum } 60 \text { minutes } \\
\text { for } 3 \text { days a week for } \\
13 \text { weeks (39 } \\
\text { sessions) }\end{array}$ & $\begin{array}{l}\text { International Standards for } \\
\text { Neurological Classification of } \\
\text { Spinal Cord Injury } \\
\text { C or D } \\
\text { Mean age } 36.0 \text { years } \\
30 \text { males and } 5 \text { females } \\
(86 \% \text { male })\end{array}$ & $\begin{array}{l}\text { Pre, post } \\
10 \text { minute walk test (meters/second) } \\
\text { A. } 0.30(0.26) \text { to } 0.46(0.40) \\
\text { B. } 0.22(0.20) \text { to } 0.44(0.33) \\
\text { C. } 0.41((0.34) \text { to } 0.51(0.36) \\
\text { Combined there was an increase in walking speed } \\
\text { ( } p=0.001) \text { but no group by time effect (no difference } \\
\text { among groups) } \\
\text { Tinetti Balance } \\
\text { A. } 9.8(5.4) \text { to } 19.4(5.0) \\
\text { B. } 10.5(3.4) \text { to } 11.9(2.5) \\
\text { C. } 10.1(3.6) \text { to } 12.9(2.7) \\
\text { Significant time by group interaction ( } p<0.05) \text {, with post- } \\
\text { hoc group C improving ( } p<0.001) \text { and B improving } \\
\text { (p<0.01) but not } A \text { ( } p=0.23) \\
\text { Peak oxygen uptake } \\
\text { Baseline ranged from } 10 \text { to } 26 \mathrm{ml} / \mathrm{kg} / \text { minute and overall } \\
\text { modest } 12 \% \text { increase in each group but no differences } \\
\text { among groups }\end{array}$ & $\begin{array}{l}\text { MMT (Maximal Muscle Testing combined } \\
\text { upper and lower limb strength) } \\
\text { A. } 71.5(15.1) \text { to } 78.1(15.3) \\
\text { B. } 69.5(12.1) \text { to } 73.3(11.5) \\
\text { C. } 76.3(11.6) \text { to } 81.8(11.0) \\
\text { Overall } 6 \%-9 \% \text { increase across groups; each } \\
\text { group achieved a significant increase ( } p<0.05 \\
\text { for each), no difference among groups } \\
\text { SAWS (13 components) (lower score is } \\
\text { improvement) } \\
\text { Completed pre, immediately post and } 1 \text { month } \\
\text { later (28 of } 35 \text { participants completed surveys) } \\
\text { A. } 39.3 \text { (8.3) to } 35.2 \text { (8.7) to } 31.2(7.8) \\
\text { B. } 35.9 \text { (6.9) to } 32.4 \text { (7.6) to } 32.4(6.4) \\
\text { C. } 36.6 \text { (9.9) to } 29.0(7.9) \text { to } 31.4(5.5) \\
\text { Across groups significant improvement } \\
\text { (p=0.03) }\end{array}$ \\
\hline $\begin{array}{l}\text { Al-Sharman, } 2019 \\
\text { Aerobic Exercise } \\
\text { Aerobics } \\
\text { Postintervention, } 6 \\
\text { weeks } \\
\text { Poor }\end{array}$ & $\begin{array}{l}\text { A. Moderate intensity } \\
\text { exercise with stair } \\
\text { stepper, } 18 \text { sessions } \\
\text { over } 6 \text { weeks }(n=17) \\
\begin{array}{l}\text { B. Home exercises } \\
(n=13)\end{array}\end{array}$ & $\begin{array}{l}\text { A vs. } B \\
\text { Age: } 39 \text { vs. } 32 \\
\text { Female: } 76 \% \text { vs. } 77 \% \\
\text { EDSS: } 2.1 \text { vs. } 1.9\end{array}$ & $\begin{array}{l}\text { A vs. B, Mean (SD), p-value is between groups: } \\
\text { PSQI: } \\
8.0(3.8) \text { to } 4.6(2.3) \text { vs. } 8.9(4.3) \text { to } 7.1(3.2), p<0.001 \\
\text { ISI: } \\
12.8(5.3) \text { to } 6.6(4.08) \text { vs. } 10.3(3.3) \text { to } 8.7(5.1), p=0.04\end{array}$ & $\begin{array}{l}\text { Total Sleep Time: } \\
333.38(84.6) \text { to } 372.4(59.4) \text { vs. } 325.9(84.5) \\
\text { to } 320(54), p=0.05\end{array}$ \\
\hline
\end{tabular}




\begin{tabular}{|c|c|c|c|c|}
\hline $\begin{array}{l}\text { Author, Year } \\
\text { Intervention Type } \\
\text { Duration of } \\
\text { Postintervention } \\
\text { Followup } \\
\text { Quality } \\
\text { (See Appendix B } \\
\text { for Full Citation) } \\
\end{array}$ & $\begin{array}{l}\text { Intervention and } \\
\text { Comparison }\end{array}$ & $\begin{array}{l}\text { Population } \\
\text { Age (Mean) } \\
\text { Gender (\% Female) } \\
\text { Race (\%) } \\
\text { Ambulatory (\%) } \\
\text { Wheelchair User (\%) } \\
\text { Condition Specific (\%) } \\
\text { Other (\%) }\end{array}$ & Prioritized Outcomes & Other Outcomes \\
\hline $\begin{array}{l}\text { Amiri, } 2019 \\
\text { Postural Control } \\
\text { Balance exercises } \\
\text { Postintervention, } \\
10 \text { weeks } \\
\text { Fair }\end{array}$ & $\begin{array}{l}\text { A. Core Stability } \\
\text { Training, } 30 \text { sessions } \\
\text { over } 10 \text { weeks } \\
(n=35) \\
\text { B. Conventional care } \\
\text { including stretching } \\
\text { and range of motion } \\
\text { exercises }(n=34)\end{array}$ & $\begin{array}{l}\text { A vs. B } \\
\text { Age: } 32 \text { vs. } 31 \\
\text { Female: } 100 \% \\
\text { EDSS: } 3.58 \text { vs. } 3.74 \\
\text { RRMS: } 100 \%\end{array}$ & $\begin{array}{l}\text { Significant interaction between time and group according } \\
\text { to baseline EDSS score for core muscle function (i.e., } \\
\text { core endurance and core strength tests) and static and } \\
\text { dynamic stability ( } p<0.05) \\
\text { Core strength tests (R/L hip abduction, R/L external } \\
\text { rotation) demonstrate significant differences in strength } \\
\text { based on baseline EDSS score }(2.5-3.5 ; 3.5-4.5 ; 4.5-5.5) \text {, } \\
p<0.001\end{array}$ & $\begin{array}{l}\text { Plank test: significant differences between } \\
\text { groups based on EDSS score, } p<0.001 \\
\text { Overall static balance tests demonstrate } \\
\text { significant differences in strength based on } \\
\text { baseline EDSS score and significant } \\
\text { differences compared with the control group, } \\
p<0.001 \\
\text { Greatest improvements seen in those with } \\
\text { greatest disability (least strong) }\end{array}$ \\
\hline $\begin{array}{l}\text { Aras, } 2019 \\
\text { Aerobic Exercise } \\
\text { Robot-Assisted } \\
\text { Gait Training } \\
\text { Postintervention, } \\
4 \text { weeks, and } 6 \\
\text { month followup } \\
\text { Fair }\end{array}$ & $\begin{array}{l}\text { A. RAGT, } 20 \\
\text { sessions over } 4 \\
\text { weeks }(n=10) \\
\text { B. Partial body- } \\
\text { weight supported } \\
\text { treadmill training, } 20 \\
\text { sessions over } 4 \\
\text { weeks ( } n=10) \\
\\
\text { C. Anti-gravity } \\
\text { treadmill training, } 20 \\
\text { sessions over } 4 \\
\text { weeks (n=9) }\end{array}$ & $\begin{array}{l}\text { A vs. B } \\
\text { Age: NR } \\
\text { Female: } 40 \% \text { vs. } 40 \% \text { vs. } \\
33.3 \% \\
\text { Race: NR } \\
\text { GMFCS II: } 90 \% \text { vs. } 70 \% \text { vs. } \\
88.9 \% \\
\text { Hemiplegic: } 30 \% \text { vs. } 30 \% \text { vs. } \\
33.3 \%\end{array}$ & $\begin{array}{l}\text { A vs. B vs. C, mean change (SD): } \\
\text { 6MWT: } 39.6(40.4) \text { vs. } 37.6(20.2) \text { vs. } 48.3(25.1), p>0.05 \\
\text { for all pairwise comparisons } \\
\text { 6MWT ( } 3 \text { mo followup): } 45.2(44.4) \text { vs. } 48.6(37.8) \text { vs. } \\
\text { 58.2 }(22.9), p>0.05 \text { for all pairwise comparisons } \\
\text { GMFM-D: } 3.6(2.5) \text { vs. } 4.6(4.6) \text { vs. } 3.5(2.5), p>0.05 \text { for } \\
\text { all pairwise comparisons } \\
\text { GMFM-D ( } 3 \text { month } \\
\text { followup): } 3.6(2.5) \text { vs. } 4.6(4.6) \text { vs. } 3.5(2.5), p>0.05 \text { for } \\
\text { all pairwise comparisons } \\
\text { GMFM-E: } 2.4(2.0) \text { vs. } 2.6(1.7) \text { vs. } 3.7(1.9), p>0.05 \text { for all } \\
\text { pairwise comparisons } \\
\text { GMFM-E ( } 3 \text { month followup): } 2.6(1.8) \text { vs. } 2.6(1.7) \text { vs. } 3.7 \\
\text { (1.9), p>0.05 for all pairwise comparisons }\end{array}$ & NA \\
\hline $\begin{array}{l}\text { Arntzen, } 2019 \\
\text { Arntzen, } 2020 \\
\text { Postural Control } \\
\text { Balance exercises } \\
7 \text { weeks, plus } 18, \\
\text { and } 30 \text { weeks } \\
\text { Good }\end{array}$ & $\begin{array}{l}\text { A. GroupCoreDIST, } \\
18 \text { sessions over } 6 \\
\text { weeks }+ \text { home } \\
\text { exercises }(n=39) \\
\text { B. Usual care }(n=40)\end{array}$ & $\begin{array}{l}\text { A vs. B } \\
\text { Age: } 52 \text { vs. } 48 \\
\text { Female: } 69 \% \text { vs. } 73 \% \\
\text { EDSS: } 2.45 \text { vs. } 2.28 \\
\text { RRMS: } 82 \% \text { vs. } 90 \% \\
\text { PPMS: } 13 \% \text { vs. } 5 \% \\
\text { SPMS: } 5 \% \text { vs. } 5 \%\end{array}$ & $\begin{array}{l}\text { A vs. B, Mean Difference between groups: } \\
\text { Mini-BEST: } \\
\text { MD } 1.91,95 \% \mathrm{Cl} 1.07 \text { to } 2.76, \mathrm{p}<0.001 \\
\text { MWT at } 7 \text { weeks: MD } 16.7,95 \% \mathrm{Cl} 8.15 \text { to } 25.25 \\
\text { 2MWT at } 30 \text { weeks: MD } 16.38,95 \% \mathrm{Cl} 7.65 \text { to } 25.12 \\
\text { 10MWT at } 7 \text { weeks: MD } 0.48,95 \% \mathrm{Cl} 0.11 \text { to } 0.85 \\
\text { 10MWT at } 30 \text { weeks: MD } 0.33,95 \% \mathrm{Cl}-0.04 \text { to } 0.71 \\
\text { MSWS-12 at } 7 \text { weeks: MD } 9.77,95 \% \mathrm{Cl} 3.19 \text { to } 16.35 \\
\text { MSWS-12 at } 30 \text { weeks: MD } 3.87,95 \% \mathrm{Cl}-2.80 \text { to } 10.54\end{array}$ & NA \\
\hline
\end{tabular}




\begin{tabular}{|c|c|c|c|c|}
\hline $\begin{array}{l}\text { Author, Year } \\
\text { Intervention Type } \\
\text { Duration of } \\
\text { Postintervention } \\
\text { Followup } \\
\text { Quality } \\
\text { (See Appendix B } \\
\text { for Full Citation) } \\
\end{array}$ & $\begin{array}{l}\text { Intervention and } \\
\text { Comparison }\end{array}$ & $\begin{array}{l}\text { Population } \\
\text { Age (Mean) } \\
\text { Gender (\% Female) } \\
\text { Race (\%) } \\
\text { Ambulatory (\%) } \\
\text { Wheelchair User (\%) } \\
\text { Condition Specific (\%) } \\
\text { Other (\%) } \\
\end{array}$ & Prioritized Outcomes & Other Outcomes \\
\hline $\begin{array}{l}\text { Aviram } 2017 \\
\text { Aerobic Exercise } \\
\text { Treadmill } \\
\text { Postintervention, } 6 \\
\text { months } \\
\text { Fair }\end{array}$ & $\begin{array}{l}\text { A. Treadmill walking } \\
60 \text {-minute session } \\
\text { twice a week over } 3 \\
\text { months (total } 30 \\
\text { sessions) } \\
\text { B. } 8 \text { stations circuit } \\
\text { resistance training, } \\
\text { including stair } \\
\text { climbing and balance } \\
\text { sessions similar } \\
\text { duration and number } \\
\text { of sessions }\end{array}$ & 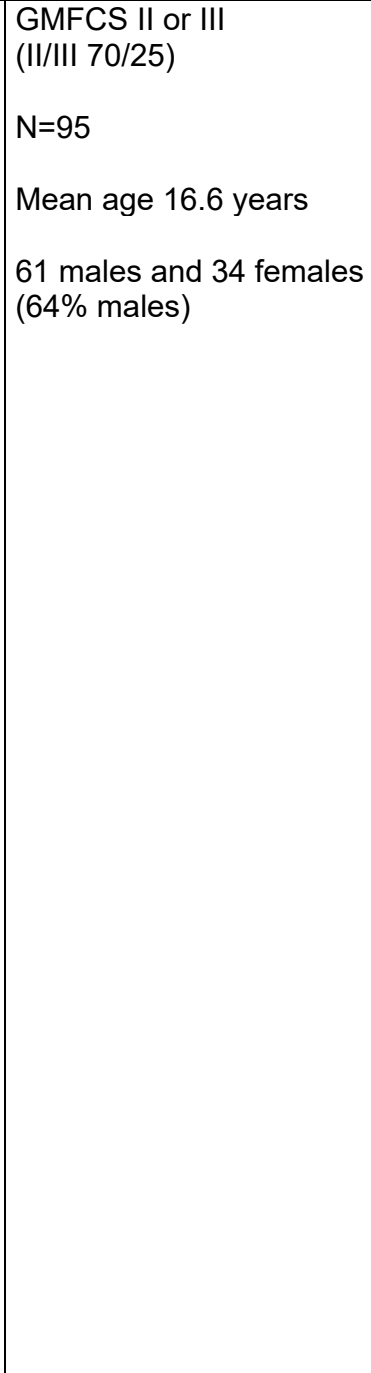 & 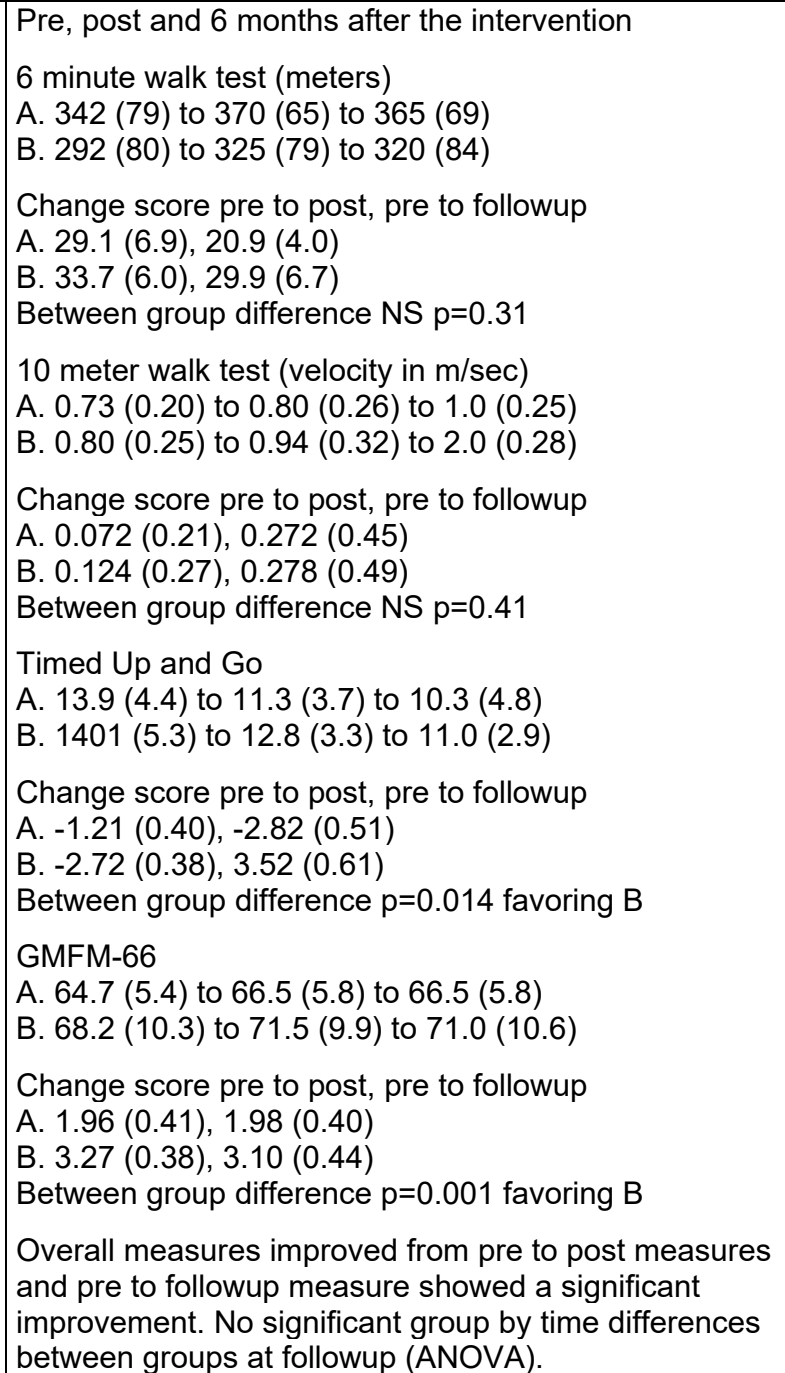 & NA \\
\hline
\end{tabular}




\begin{tabular}{|c|c|c|c|c|}
\hline $\begin{array}{l}\text { Author, Year } \\
\text { Intervention Type } \\
\text { Duration of } \\
\text { Postintervention } \\
\text { Followup } \\
\text { Quality } \\
\text { (See Appendix B } \\
\text { for Full Citation) }\end{array}$ & $\begin{array}{l}\text { Intervention and } \\
\text { Comparison }\end{array}$ & $\begin{array}{l}\text { Population } \\
\text { Age (Mean) } \\
\text { Gender (\% Female) } \\
\text { Race (\%) } \\
\text { Ambulatory (\%) } \\
\text { Wheelchair User (\%) } \\
\text { Condition Specific (\%) } \\
\text { Other (\%) }\end{array}$ & Prioritized Outcomes & Other Outcomes \\
\hline $\begin{array}{l}\text { Aydin, } 2014 \\
\text { Aerobic Exercise } \\
\text { Aerobic Exercise } \\
\text { Postintervention, } \\
12 \text { weeks } \\
\text { Fair }\end{array}$ & $\begin{array}{l}\text { A. Callisthenic } \\
\text { exercises (in clinic): } \\
60 \text { sessions, over } 12 \\
\text { weeks, }(n=16) \\
\text { B. Callisthenic } \\
\text { exercises (home- } \\
\text { based): } 60 \text { sessions, } \\
\text { over } 12 \text { weeks, } \\
(n=20)\end{array}$ & $\begin{array}{l}\text { A vs. B } \\
\text { Age (mean years): } 32.6 \text { vs. } \\
33 \\
\text { Female: } 9(56 \%) \text { vs. } 11 \\
(55 \%) \\
\text { Race: NR } \\
\text { Ambulatory: NR } \\
\text { Wheelchair user: NR } \\
\text { MS duration (years): } 6.4 \text { vs. } \\
7.4 \\
\text { BMI (cm/Kg2): } 26.12 \text { vs. } \\
25.25 \\
\text { EDSS: } 3.6 \text { vs. } 3.4\end{array}$ & 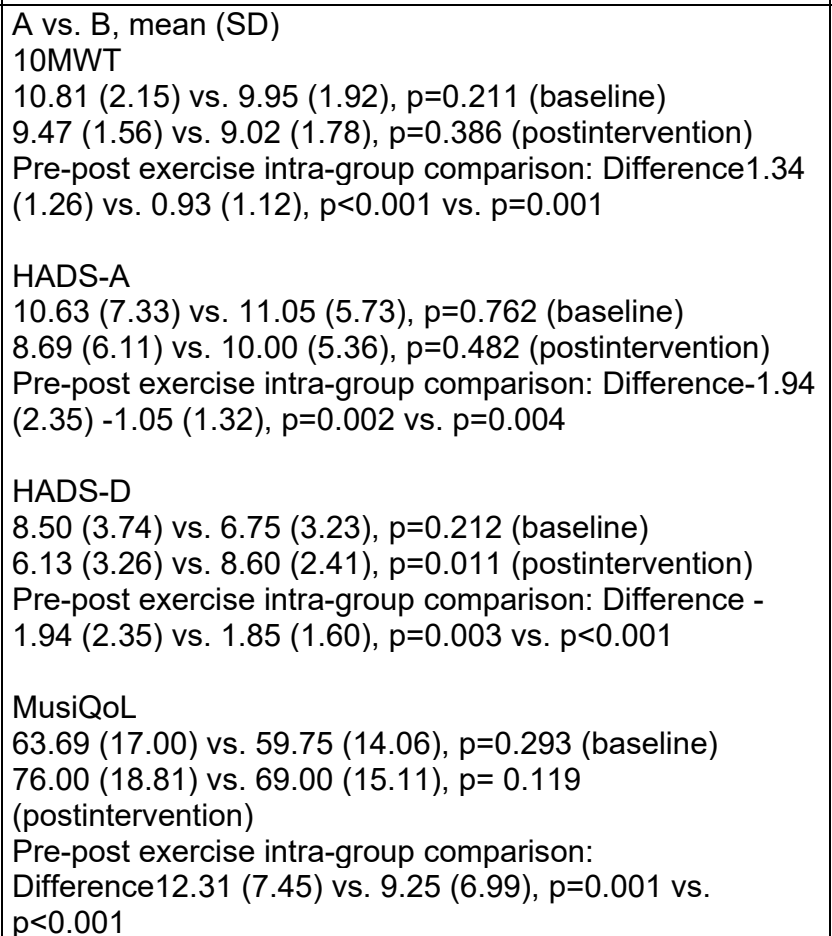 & $\begin{array}{l}2.68 \\
\text { A vs. B, mean (SD) } \\
\text { BBS } \\
47.56(6.57) \text { vs. } 48.95(5.38), p=0.369 \\
\text { (baseline) } \\
50.94(4.97) \text { vs. } 50.40(5.27), p=0.700 \\
\text { (postintervention) } \\
\text { Pre-post exercise intra-group comparison: } \\
\text { Difference } 3.38(2.78) \text { vs. } 1.45(1.85), p=0.001 \\
\text { vs. } p=0.003\end{array}$ \\
\hline
\end{tabular}




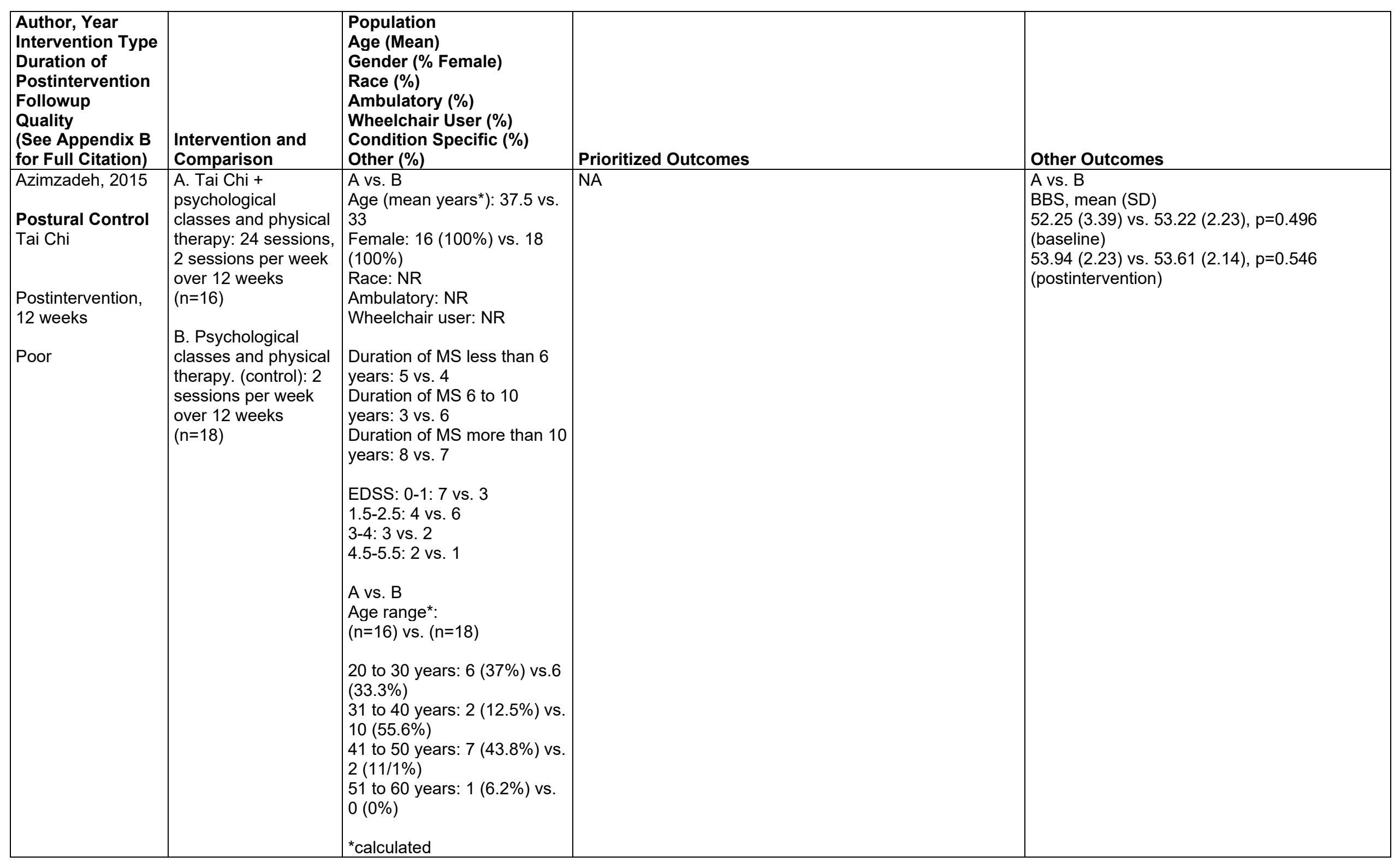




\begin{tabular}{|c|c|c|c|c|}
\hline $\begin{array}{l}\text { Author, Year } \\
\text { Intervention Type } \\
\text { Duration of } \\
\text { Postintervention } \\
\text { Followup } \\
\text { Quality } \\
\text { (See Appendix B } \\
\text { for Full Citation) } \\
\end{array}$ & $\begin{array}{l}\text { Intervention and } \\
\text { Comparison }\end{array}$ & $\begin{array}{l}\text { Population } \\
\text { Age (Mean) } \\
\text { Gender (\% Female) } \\
\text { Race (\%) } \\
\text { Ambulatory (\%) } \\
\text { Wheelchair User (\%) } \\
\text { Condition Specific (\%) } \\
\text { Other (\%) }\end{array}$ & Prioritized Outcomes & Other Outcomes \\
\hline $\begin{array}{l}\text { Bahrami, 2019a } \\
\text { Aerobic Exercise } \\
\text { Treadmill } \\
\text { Immediately } \\
\text { postintervention, } \\
8 \text { weeks } \\
\text { Fair }\end{array}$ & $\begin{array}{l}\text { A. Treadmill, } 16 \\
\text { sessions over } 8 \\
\text { weeks }(n=15) \\
\text { B. Physiotherapy, } 16 \\
\text { sessions over } 8 \\
\text { weeks }(n=15)\end{array}$ & $\begin{array}{l}\text { A vs. B } \\
\text { Age: } 30 \text { vs. } 25 \\
\text { Female: } 47 \% \text { vs. } 40 \% \\
\text { GMFCS I; } 47 \% \text { vs. } 53 \% \\
\text { GMFCS II: } 13 \% \text { vs. } 13 \% \\
\text { GMFCS III: } 40 \% \text { vs. } 33 \%\end{array}$ & $\begin{array}{l}\text { A vs. B, Mean (SD); percentage change score, } \\
\text { p=between groups } \\
\text { 10MWT: } \\
1.080(0.47) \text { to } 1.22(0.50)[22.46 \% \text { change] vs. } 0.99 \\
(0.56) \text { to } 1.02(0.61)[1.28 \% \text { change], \% change } p<0.05 \\
\text { 6MWT: } \\
291.13(160.28) \text { to } 342.63(174.62)[23.68 \% \text { change] vs. } \\
276.10(167.19) \text { to } 308.57(181.22)[16.54 \% \text { change], } \% \\
\text { change p>0.05 } \\
\text { WHOQOL-Brief: } \\
3.55(.55) \text { to } 3.66(0.59)[3.83 \% \text { change] vs. } 3.33(0.69) \\
3.57(0.67)[8.94 \% \text { change], } \% \text { change p>0.05 }\end{array}$ & NA \\
\hline $\begin{array}{l}\text { Baquet, } 2018 \\
\text { Aerobic Exercise } \\
\text { Cycling } \\
\text { Postintervention, } \\
12 \text { weeks } \\
\text { Fair }\end{array}$ & $\begin{array}{l}\text { A. Bicycle ergometry, } \\
24-36 \text { sessions over } \\
12 \text { weeks }(n=34) \\
\text { B. Waitlist control } \\
\text { group }(n=34)\end{array}$ & $\begin{array}{l}\text { A vs. B } \\
\text { Age (mean years): } \\
38.2 \text { vs. } 39.6 \\
\text { Female: } \\
21(62 \%) \text { vs. } 25(74 \%) \\
\text { Race: NR } \\
\text { Ambulatory: NR } \\
\text { Wheelchair user: NR } \\
\text { Other: } \\
\text { RRMS } \\
34(100 \%) \text { vs. } 34(100 \%)\end{array}$ & $\begin{array}{l}\text { A vs. B Mean Difference between groups: } \\
\text { 6MWT: } 4.0,95 \% \mathrm{Cl}-36.5 \text { to } 44.5, p=0.85 \\
\text { 25 foot walk: }-0.1,95 \% \mathrm{Cl}-0.4 \text { to } 0.2, p=0.49 \\
\text { MSWS: }-0.3,95 \% \mathrm{Cl}-2.1 \text { to } 1.6, p=0.78 \\
\text { HAQUAMS: }-0.4,95 \% \mathrm{Cl}-4.5 \text { to } 3.7, p=0.84\end{array}$ & NA \\
\hline
\end{tabular}




\begin{tabular}{|c|c|c|c|c|}
\hline $\begin{array}{l}\text { Author, Year } \\
\text { Intervention Type } \\
\text { Duration of } \\
\text { Postintervention } \\
\text { Followup } \\
\text { Quality } \\
\text { (See Appendix B } \\
\text { for Full Citation) } \\
\end{array}$ & $\begin{array}{l}\text { Intervention and } \\
\text { Comparison }\end{array}$ & $\begin{array}{l}\text { Population } \\
\text { Age (Mean) } \\
\text { Gender (\% Female) } \\
\text { Race (\%) } \\
\text { Ambulatory (\%) } \\
\text { Wheelchair User (\%) } \\
\text { Condition Specific (\%) } \\
\text { Other (\%) } \\
\end{array}$ & Prioritized Outcomes & Other Outcomes \\
\hline $\begin{array}{l}\text { Bleyenheuft, } 2017 \\
\text { Postural Control } \\
\text { Balance } \\
\text { Postintervention, } \\
12 \text { weeks } \\
\text { Poor }\end{array}$ & $\begin{array}{l}\text { A. Virtual reality } \\
\text { (HABIT-ILE): } 136.4 \\
\text { hour sessions over } \\
13 \text { days }(n=10) \\
\text { B. Usual care control } \\
\text { (physical therapy): } 2 \\
\text { weeks }(n=10)\end{array}$ & $\begin{array}{l}\text { A vs. B } \\
\text { Age (mean years): } 10.5 \text { vs. } \\
11.4 \\
\text { Female: } 4(40 \%) \text { vs. } 5(50 \%) \\
\text { Race: NR } \\
\text { Ambulatory: NR } \\
\text { Wheelchair user: NR } \\
\\
\text { GMFCS level II: } 2 \text { vs. } 2 \\
\text { GMFCS level III: } 7 \text { vs. } 7 \\
\text { GMFCS level IV: } 1 \text { vs. } 1\end{array}$ & $\begin{array}{l}\text { A vs. B, mean (SD) } \\
\text { GMFM-66 } \\
55(5.9) \text { vs. } 55(8.7), p=0.894, \text { (baseline) } \\
62 \text { (6.4) vs. } 57 \text { (6.6), } p<0.001 \text {, (postintervention) } \\
\text { 6MWT } \\
190(108.5) \text { vs. } 194(101.1), p=0.940 \text { (baseline) } \\
236(105.1) \text { vs. } 182 \text { (101.1), } p=0.026, p \text {-value NS } \\
\text { (postintervention) }\end{array}$ & $\begin{array}{l}\text { A vs. B, mean (SD) } \\
\text { Pediatric Evaluation of Disability Inventory } \\
\text { (PEDI) } \\
52(12.4) \text { vs. } 51(14.6), p=0.987, \text { (baseline) } \\
60(10.7) \text { vs. } 51(15.8), p=0.001, \\
\text { (postintervention) } \\
\text { Pediatric Balance Scale (PBS) } \\
33(17.5) \text { vs. } 30(23.9), p=0.749, \text { (baseline) } \\
42(21.3) \text { vs. } 26(23.2), p=0.002 \text { NS, } \\
\text { (postintervention) } \\
\\
\text { ABILOCO-Kids (Disability Inventory), logit } \\
(\text { SD) } \\
-2.5(2.1) \text { vs. }-1.4(2.2), p=0.291, \text { (baseline) }( \\
0.4(1.7 ; 0.1), p=0.072 \text { vs. } 1.4 \text { ( } 2.6 ; 0.4), \\
\text { p=0.236 (postintervention) }\end{array}$ \\
\hline $\begin{array}{l}\text { Brichetto, } 2015 \\
\text { Postural Control } \\
\text { Balance } \\
\text { Postintervention, } 4 \\
\text { weeks } \\
\text { Good }\end{array}$ & $\begin{array}{l}\text { A. Personalized } \\
\text { rehab (tailored to } \\
\text { sensory impairment): } \\
12 \text { sessions over } 4 \\
\text { weeks ( } n=16) \\
\\
\text { B. Traditional rehab } \\
\text { (visual rehab for } \\
\text { balance disorders): } \\
12 \text { sessions over } 4 \\
\text { weeks }(n=16)\end{array}$ & $\begin{array}{l}\text { A vs. B } \\
\text { Age (mean years): } 50.1 \text { vs. } \\
51.0 \\
\text { Female: } 11 \text { (69\%) vs. } 12 \\
(75 \%) \\
\text { Race: NR } \\
\text { Ambulatory: NR } \\
\text { Wheelchair user: NR } \\
\\
\text { Relapsing-remitting: } 9 \\
(56 \%) \text { vs. } 10 \text { (63\%) } \\
\text { Secondary progressive: } 5 \\
\text { (31\%) vs. } 4 \text { (25\%) } \\
\text { Primary progressive: } 2 \\
(13 \%) \text { vs. } 2 \text { (13\%) } \\
\text { Disease duration (years): } 9.5 \\
\text { vs. } 12 \\
\text { EDSs: } 3.7 \text { vs. } 3.7 \\
\end{array}$ & NA & $\begin{array}{l}\text { BBS, mean (SD) } \\
46.5(3.6) \text { vs. } 45.8(6.6) \text { (baseline) } \\
52.8(2.8) \text { vs. } 47.8(6.1) \text { (postintervention), } \\
p<0.001\end{array}$ \\
\hline
\end{tabular}




\begin{tabular}{|c|c|c|c|c|}
\hline $\begin{array}{l}\text { Author, Year } \\
\text { Intervention Type } \\
\text { Duration of } \\
\text { Postintervention } \\
\text { Followup } \\
\text { Quality } \\
\text { (See Appendix B } \\
\text { for Full Citation) } \\
\end{array}$ & $\begin{array}{l}\text { Intervention and } \\
\text { Comparison }\end{array}$ & \begin{tabular}{|l} 
Population \\
Age (Mean) \\
Gender (\% Female) \\
Race (\%) \\
Ambulatory (\%) \\
Wheelchair User (\%) \\
Condition Specific (\%) \\
Other (\%) \\
\end{tabular} & Prioritized Outcomes & Other Outcomes \\
\hline $\begin{array}{l}\text { Bryant, } 2013 \\
\text { Aerobic Exercise } \\
\text { Cycling } \\
\text { Postintervention, } \\
12 \text { weeks }\end{array}$ & $\begin{array}{l}\text { A: Static bike group, } \\
18 \text { sessions over } 6 \\
\text { weeks }(n=11) \\
\\
\text { B: Treadmill group, } \\
18 \text { sessions over } 6 \\
\text { weeks }(n=12)\end{array}$ & $\begin{array}{l}\text { A vs. C } \\
\text { Age (mean years): } \\
14.3 \text { vs. } 13.8 \\
\text { Female: } \\
5(45 \%) \text { vs. } 7(58 \%) \\
\text { Race: NR }\end{array}$ & $\begin{array}{l}\text { A vs. C, mean (SD) } \\
\text { GMFM-66: NS } \\
\text { B vs. C, mean (SD) } \\
\text { GMFM-66: NS }\end{array}$ & NA \\
\hline Fair & $\begin{array}{l}\text { C: Control group no } \\
\text { intervention }(n=12)\end{array}$ & $\begin{array}{l}\text { Ambulatory: } \\
0 \text { (0\%) vs. } 0(0 \%) \\
\text { Wheelchair user: } \\
11(100 \%) \text { vs. } 12(100 \%) \\
\text { Other: } \\
\text { CP: } 100 \% \text { had bilateral CP } \\
\text { B vs. C } \\
\text { Age (mean years): } \\
13.5 \text { vs. } 13.8 \\
\text { Female: } \\
3(25 \%) \text { vs. } 7(58 \%) \\
\text { Race: NR } \\
\text { Ambulatory: } \\
12(100 \%) \text { vs. } 12(1000 \%) \\
\text { Wheelchair user: } \\
12(100 \%) \text { vs. } 12(100 \%) \\
\text { Other: } \\
\text { CP: } 100 \% \text { had bilateral CP }\end{array}$ & & \\
\hline
\end{tabular}




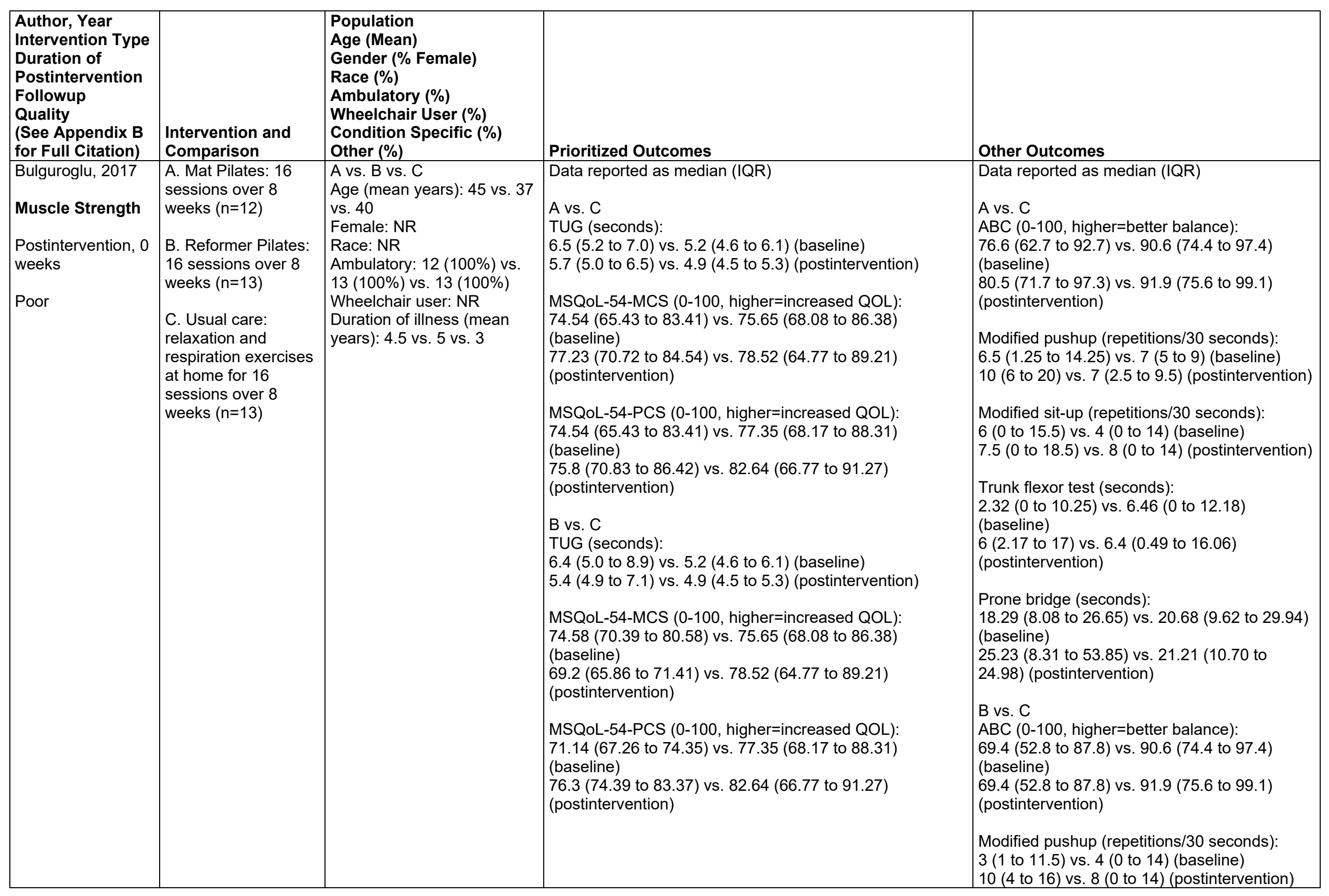




\begin{tabular}{|c|c|c|c|c|}
\hline $\begin{array}{l}\text { Author, Year } \\
\text { Intervention Type } \\
\text { Duration of } \\
\text { Postintervention } \\
\text { Followup } \\
\text { Quality } \\
\text { (See Appendix B } \\
\text { for Full Citation) } \\
\end{array}$ & $\begin{array}{l}\text { Intervention and } \\
\text { Comparison }\end{array}$ & $\begin{array}{l}\text { Population } \\
\text { Age (Mean) } \\
\text { Gender (\% Female) } \\
\text { Race (\%) } \\
\text { Ambulatory (\%) } \\
\text { Wheelchair User (\%) } \\
\text { Condition Specific (\%) } \\
\text { Other (\%) } \\
\end{array}$ & Prioritized Outcomes & Other Outcomes \\
\hline $\begin{array}{l}\text { Bulguroglu, } 2017 \\
\text { (Continued) }\end{array}$ & & & 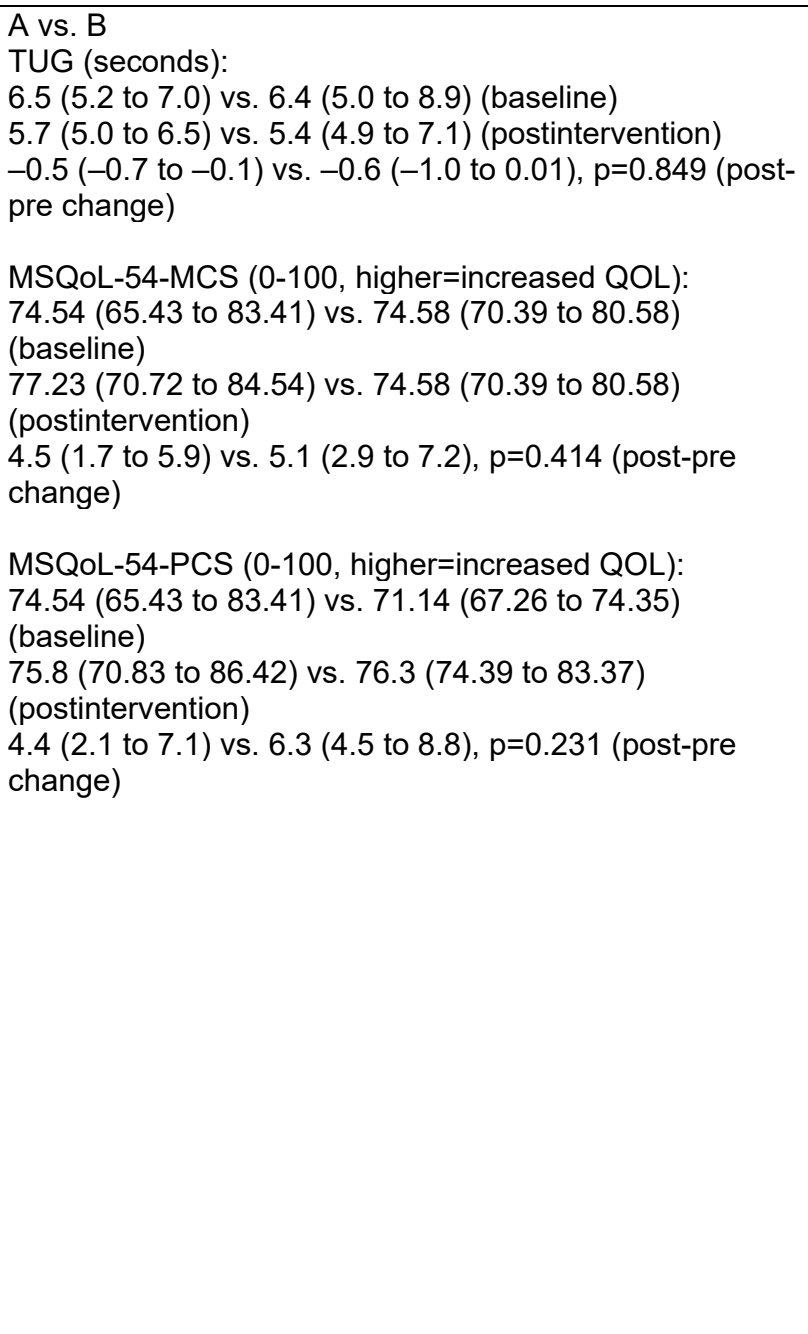 & 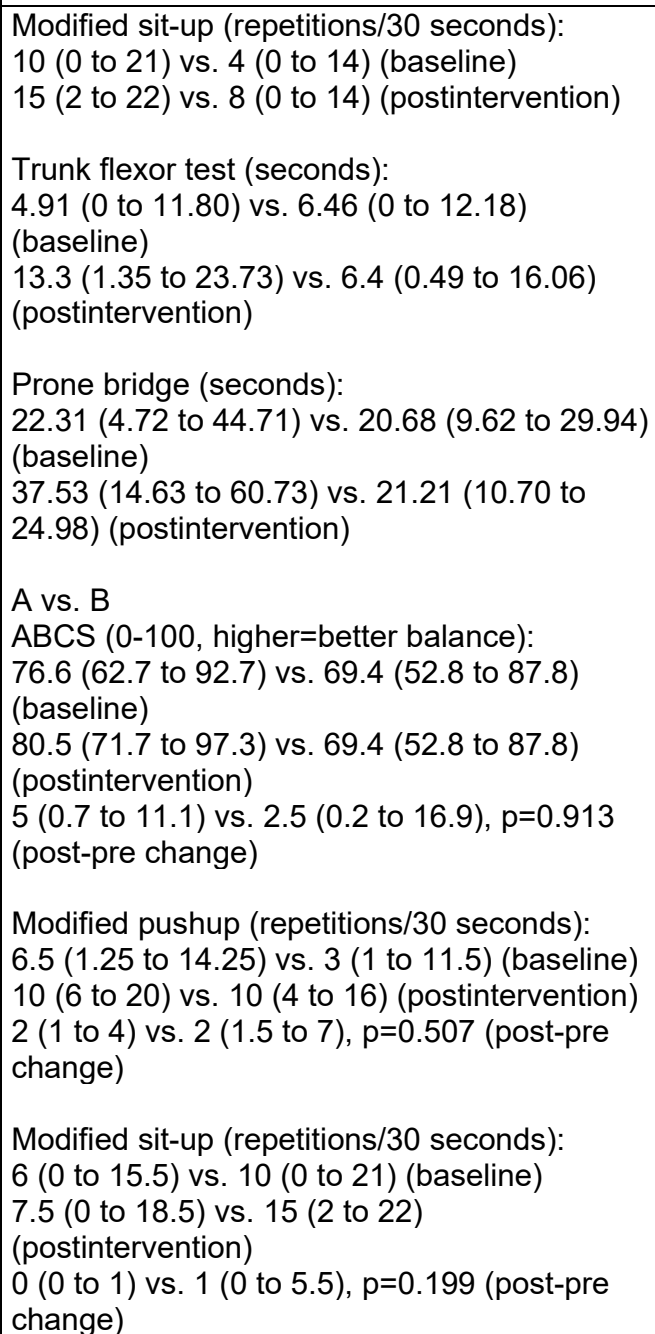 \\
\hline
\end{tabular}




\begin{tabular}{|c|c|c|c|c|}
\hline $\begin{array}{l}\text { Author, Year } \\
\text { Intervention Type } \\
\text { Duration of } \\
\text { Postintervention } \\
\text { Followup } \\
\text { Quality } \\
\text { (See Appendix B } \\
\text { for Full Citation) } \\
\end{array}$ & $\begin{array}{l}\text { Intervention and } \\
\text { Comparison }\end{array}$ & $\begin{array}{l}\text { Population } \\
\text { Age (Mean) } \\
\text { Gender (\% Female) } \\
\text { Race (\%) } \\
\text { Ambulatory (\%) } \\
\text { Wheelchair User (\%) } \\
\text { Condition Specific (\%) } \\
\text { Other (\%) }\end{array}$ & Prioritized Outcomes & Other Outcomes \\
\hline $\begin{array}{l}\text { Bulguroglu, } 2017 \\
\text { (Continued) }\end{array}$ & & & & 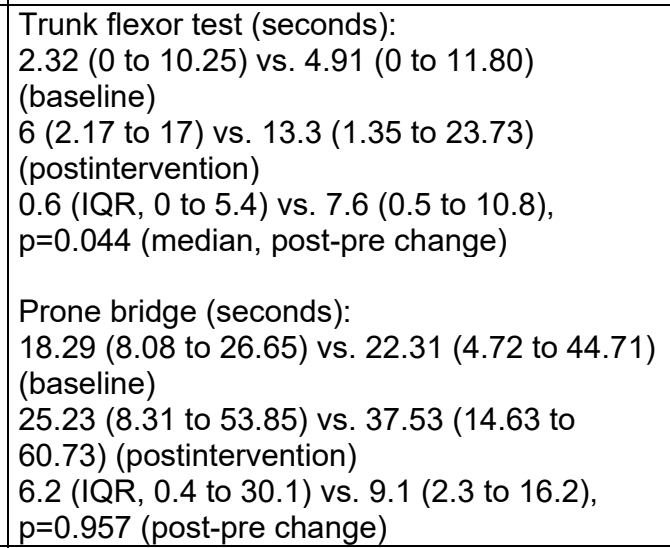 \\
\hline $\begin{array}{l}\text { Burschka, } 2014 \\
\text { Postural Control } \\
\text { Tai Chi } \\
\text { Postintervention, } \\
24 \text { weeks } \\
\text { Poor }\end{array}$ & \begin{tabular}{|l|} 
A. Tai Chi: 48 \\
sessions ( 2 sessions \\
per week) over 24 \\
weeks, 6 months \\
$(n=15)$ \\
B. Usual care control: \\
$(n=17)$
\end{tabular} & $\begin{array}{l}\text { A vs. B } \\
\text { Age (mean years): } 42 \text { vs. } 43 \\
\text { Female: } 10 \text { (66\%) vs. } 12 \\
\text { (71\%) } \\
\text { Race: NR } \\
\text { Ambulatory: } 100 \% \\
\text { Wheelchair user: NR } \\
\text { BMI: } 24.2 \text { vs. } 25.5 \\
\text { MS duration (mean years): } 6 \\
\text { vs. } 7.8 \\
\text { MS Course } \\
\text { Relapsing-remitting: } 14 \text { vs. } \\
13 \\
\text { Secondary progressive: } 0 \\
\text { vs. } 4 \\
\text { Clinically isolated syndrome: } \\
1 \text { vs. } 0 \\
\text { EDSS score <5: } 100 \% \text { vs. } \\
100 \% \\
\text { EDSS (range, median): } 1-4, \\
\text { median=2 vs. } \\
1-4.5, \text { median=4 }\end{array}$ & $\begin{array}{l}\text { A vs. B, mean (SD) } \\
\text { Depression (15-item questionnaire Center for } \\
\text { Epidemiological Studies Depression Scale CES-D) (ADS } \\
\text { score) } \\
12.21(6.66) \text { vs. } 13.87(10.82) \text { (baseline) } \\
7.67(5.12) \text { vs. } 16.13(11.99) \text { (postintervention) } \\
\text { Depression, main effect of time } \\
\text { [F }(1,27)=6.61, p<0.05, \text { partial } \eta 2=0.19] \\
\text { Time by Group interaction [F }(1,27)=6.55, p<0.05 \text {, partial } \\
\eta 2=0.20] \\
\text { Quality of Life } \\
\text { Questionnaire of Life Satisfaction (QLS - } 7 \text { item, } 1-7 \\
\text { rating scale, max score } 420 \text { points) } \\
215.77(25.55) \text { vs. } 204.46(27.77) \text { (baseline) } \\
232.57(25.62) \text { vs. } 193.81(36.2) \text { (postintervention) } \\
\text { QSL significant main effect Group [F }(1,24)=8.64, p<0.01 \text {, } \\
\text { partial } \eta 2=0.19]\end{array}$ & $\begin{array}{l}\text { A vs. } B, \text { mean }(\mathrm{SD}) \\
\text { Balance }(14 \text { tasks, measured } 1=\text { achieved task, } \\
0=\text { failed task) } \\
\text { Balance, } \\
8.00(2.83) \text { vs. } 6.88(4.09) \text { (baseline) } \\
9.33(2.26) \text { vs. } 6.53(4.49) \text { (postintervention) } \\
\\
\text { Coordination, main effect of time } \\
{[F(1,30)=4.89, p<0.05, \text { partial } \eta 2=0.14]} \\
\text { Time by group interaction }[F(1,30)=6.57, \\
p<0.05, \text { partial } \eta 2=0.18]\end{array}$ \\
\hline
\end{tabular}




\begin{tabular}{|c|c|c|c|c|}
\hline $\begin{array}{l}\text { Author, Year } \\
\text { Intervention Type } \\
\text { Duration of } \\
\text { Postintervention } \\
\text { Followup } \\
\text { Quality } \\
\text { (See Appendix B } \\
\text { for Full Citation) }\end{array}$ & $\begin{array}{l}\text { Intervention and } \\
\text { Comparison }\end{array}$ & $\begin{array}{l}\text { Population } \\
\text { Age (Mean) } \\
\text { Gender (\% Female) } \\
\text { Race (\%) } \\
\text { Ambulatory (\%) } \\
\text { Wheelchair User (\%) } \\
\text { Condition Specific (\%) } \\
\text { Other (\%) }\end{array}$ & Prioritized Outcomes & Other Outcomes \\
\hline $\begin{array}{l}\text { Cakit, } 2010 \\
\text { Multimodal } \\
\text { Exercise } \\
\text { Postintervention, } 0 \\
\text { weeks }\end{array}$ & $\begin{array}{l}\text { A. Progressive } \\
\text { resistance cycling + } \\
\text { balance exercises } \\
\text { (lower extremity } \\
\text { strengthening): } 16 \\
\text { sessions over } 8 \\
\text { weeks ( } n=14)\end{array}$ & $\begin{array}{l}\text { A vs. B } \\
\text { (data only for those that } \\
\text { completed the study: } n=14 \\
\text { vs. } 9 \text { ) } \\
\text { Age (mean years): } 36.4 \text { vs. } \\
35.5 \\
\text { Female: } 9(64 \%) \text { vs. } 6(67 \%) \\
\text { Race: NR }\end{array}$ & $\begin{array}{l}\text { Data reported as mean (SD) } \\
\text { A vs. B } \\
\text { BDI (0-63, higher=worse depression): } \\
22.8(12.7) \text { vs. } 27.0(17.6), p=N R \text { (baseline) } \\
17.2(12.3) \text { vs. } 25.4(22.8), p=N R \text { (postintervention) } \\
-5.5(5.3) \text { vs. }-1.6(6.0), p=<0.05 \text { (pre-post change) }\end{array}$ & $\begin{array}{l}\text { Data reported as mean (SD) } \\
\text { A vs. B } \\
\text { FES (0 to 100; higher scores=increased } \\
\text { confidence in performing ADL): } \\
19.7(11.7) \text { vs. } 32.4(24.1), p=N R \text { (baseline) } \\
8.3(5.6) \text { vs. } 29.8(24.1), p=N R \\
\text { (postintervention) }\end{array}$ \\
\hline Poor & B. Usual care $(n=9)$ & $\begin{array}{l}\text { Ambulatory: NR } \\
\text { Assistive device: } 4(28.5 \%) \\
\text { vs. } 3(37.5 \%) \\
\text { Duration of MS (mean } \\
\text { years): } 9.2 \text { vs. } 6.6 \\
\text { Fall frequency last year } \\
\text { (mean): } 2.0 \text { vs. } 2.4\end{array}$ & $\begin{array}{l}\text { TUG (seconds): } \\
10.7(1.4) \text { vs. } 14.6(9.1), p=N R \text { (baseline) } \\
9.3(0.8) \text { vs. } 14.4(9.5), p=N R \text { (postintervention) } \\
-1.3(1.2) \text { vs. }-0.2(0.8), p<0.05 \text { (pre-post change) } \\
\text { 10MWT (seconds): } \\
12.0(2.4) \text { vs. vs. } 12.2(3.1), p=N R \text { (baseline) } \\
10.0(1.6) \text { vs. } 12.3(3.2), p=N R \text { (postintervention) } \\
-1.9(1.2) \text { vs. } 0.1(0.8), p<0.05 \text { (pre-post change) } \\
\text { DGI (0-24; } \geq 16 \text { high risk for falls, <19 decreased risk for } \\
\text { falls): } \\
17.4(4.4) \text { vs. } 16.4(4.9), p=N R \text { (baseline) } \\
20.1(3.8) \text { vs. } 16.8(5.7), p=N R \text { (postintervention) } \\
2.7(0.5) \text { vs. } 0.4 \text { (0.4), p<0.01 (pre-post change) } \\
\text { SF-36 subscale - Physical Functioning } \\
43.3(16.6) \text { vs. } 43.2 \text { (17.7) (baseline) } \\
64.6(18.6) \text { vs. } 51.0 \text { (20.5) (postintervention) } \\
21.2(14.4) \text { vs. } 7.7 \text { (7.4) (pre-post change) } \\
\text { SF-36 subscale }- \text { Role-physical Function } \\
15.9(23.1) \text { vs. } 30.0 \text { (20.9) (baseline) } \\
50.0(43.3) \text { vs. } 35.0 \text { (37.1) (postintervention) } \\
34.0 \text { (30.1) vs. } 5.0 \text { (44.7) (pre-post change) }\end{array}$ & $\begin{array}{l}-11.3(7.8) \text { vs. }-2.6(3.1), p<0.01 \text { (pre-post } \\
\text { change) }\end{array}$ \\
\hline
\end{tabular}




\begin{tabular}{|c|c|c|c|c|}
\hline $\begin{array}{l}\text { Author, Year } \\
\text { Intervention Type } \\
\text { Duration of } \\
\text { Postintervention } \\
\text { Followup } \\
\text { Quality } \\
\text { (See Appendix B } \\
\text { for Full Citation) } \\
\end{array}$ & $\begin{array}{l}\text { Intervention and } \\
\text { Comparison }\end{array}$ & $\begin{array}{l}\text { Population } \\
\text { Age (Mean) } \\
\text { Gender (\% Female) } \\
\text { Race (\%) } \\
\text { Ambulatory (\%) } \\
\text { Wheelchair User (\%) } \\
\text { Condition Specific (\%) } \\
\text { Other (\%) }\end{array}$ & Prioritized Outcomes & Other Outcomes \\
\hline $\begin{array}{l}\text { Cakit, } 2010 \\
\text { (Continued) }\end{array}$ & & & 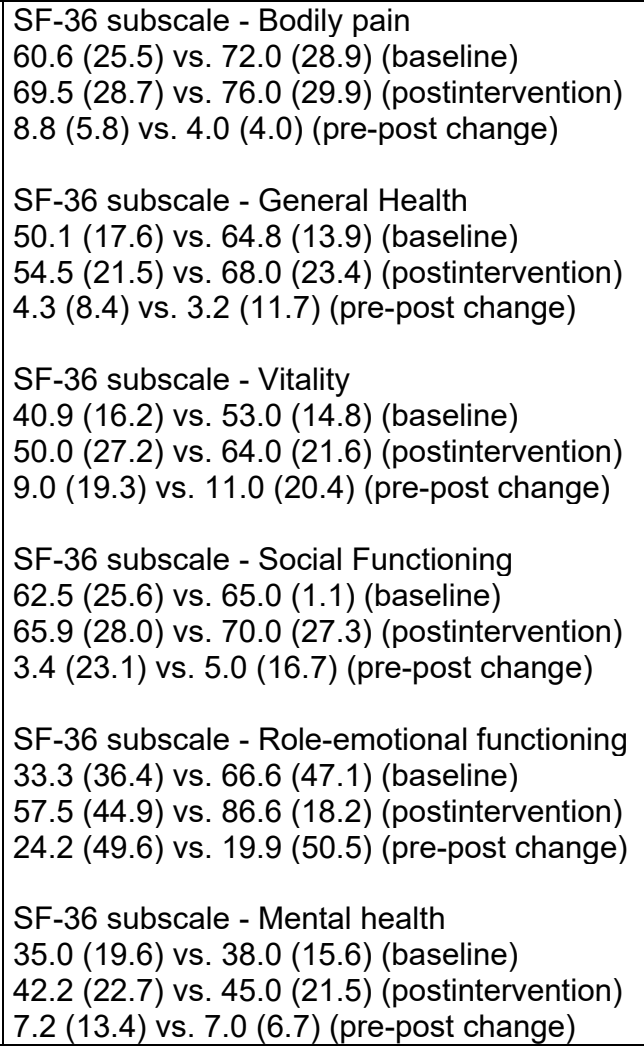 & \\
\hline
\end{tabular}




\begin{tabular}{|c|c|c|c|c|}
\hline $\begin{array}{l}\text { Author, Year } \\
\text { Intervention Type } \\
\text { Duration of } \\
\text { Postintervention } \\
\text { Followup } \\
\text { Quality } \\
\text { (See Appendix B } \\
\text { for Full Citation) } \\
\end{array}$ & $\begin{array}{l}\text { Intervention and } \\
\text { Comparison }\end{array}$ & $\begin{array}{l}\text { Population } \\
\text { Age (Mean) } \\
\text { Gender (\% Female) } \\
\text { Race (\%) } \\
\text { Ambulatory (\%) } \\
\text { Wheelchair User (\%) } \\
\text { Condition Specific (\%) } \\
\text { Other (\%) }\end{array}$ & Prioritized Outcomes & Other Outcomes \\
\hline $\begin{array}{l}\text { Calabro, } 2017 \\
\text { Intervention type: } \\
\text { Aerobic Exercise } \\
\text { Robot-Assisted } \\
\text { Gait Training } \\
\text { Postintervention, } 8 \\
\text { weeks } \\
\text { Good }\end{array}$ & $\begin{array}{l}\text { A. Lokomat-Nanos } \\
\text { (RAGT), } 40 \text { sessions } \\
\text { over } 8 \text { weeks }(n=20) \\
\text { B. } \\
\text { Lokomat-Pros } \\
\text { (RAGT + VR, } 40 \\
\text { sessions over } 8 \\
\text { weeks }(n=20)\end{array}$ & $\begin{array}{l}\text { A vs. B } \\
\text { Age (mean years): } \\
41 \text { vs. } 44 \\
\text { Female: } \\
60(\%) \text { vs. } 65(\%) \\
\text { Race: NR } \\
\text { Ambulatory: } \\
\text { NR } \\
\text { Wheelchair user: } \\
\text { NR } \\
\text { Other: } \\
\text { disease duration mean: } 11.5 \\
\text { years vs. } 11.5 \text { years } \\
\text { EDSS } 4.75 \text { vs. } 4.4 \\
\text { years of education } 10 \text { vs. } 11\end{array}$ & $\begin{array}{l}\text { A. } \\
\text { TUG } \\
\text { T0: } 9.8, T 1: 8, p=0.002 \\
\text { Initial } 10, T 17.9, p=0.001 \\
\text { BBS } \\
\text { T0: } 36, T 1: 44, p=0.003 \\
\text { T0:35, T1:50, } p<0.001 \\
\text { MAS } \\
\text { T0:1.5, T1:0.5, } p=0.2 \\
\text { T0:2, T1:1, } p=0.1 \\
\text { FIM } \\
\text { T0:89, T1:92, } p=0.3 \\
\text { T0:87, T2:89, } p=0.4\end{array}$ & $\begin{array}{l}\text { A. } \\
\text { HRSD } \\
\text { T0: } 12, T 1: 7, p=0.003 \\
\text { T0: } 10, \text { T1: } 6, p<0.001\end{array}$ \\
\hline
\end{tabular}




\begin{tabular}{|c|c|c|c|c|}
\hline $\begin{array}{l}\text { Author, Year } \\
\text { Intervention Type } \\
\text { Duration of } \\
\text { Postintervention } \\
\text { Followup } \\
\text { Quality } \\
\text { (See Appendix B } \\
\text { for Full Citation) } \\
\end{array}$ & $\begin{array}{l}\text { Intervention and } \\
\text { Comparison }\end{array}$ & $\begin{array}{l}\text { Population } \\
\text { Age (Mean) } \\
\text { Gender (\% Female) } \\
\text { Race (\%) } \\
\text { Ambulatory (\%) } \\
\text { Wheelchair User (\%) } \\
\text { Condition Specific (\%) } \\
\text { Other (\%) }\end{array}$ & Prioritized Outcomes & Other Outcomes \\
\hline $\begin{array}{l}\text { Callesen, } 2019 \\
\text { Postural Control } \\
\text { Balance exercises } \\
\text { Strength } \\
\text { interventions } \\
\text { Muscle Strength } \\
\text { Exercises }\end{array}$ & $\begin{array}{l}\text { A. Progressive } \\
\text { resistance training } \\
\text { ( } n=23 \text { ): } 20 \text { sessions } \\
\text { over } 10 \text { weeks } \\
\text {-Median number of } \\
\text { sessions completed } \\
\text { (range): } 17 \text { (8 to 19) } \\
\text { B. Balance training } \\
\text { ( } n=28): 20 \text { sessions } \\
\text { over } 10 \text { weeks } \\
- \text { Median number of } \\
\text { sessions completed } \\
\text { (range): } 16 \text { (6 to 20) } \\
\text { C. Waitlist control } \\
\text { ( } n=20)\end{array}$ & $\begin{array}{l}\text { A vs. B vs. C } \\
\text { Median age: } 52 \text { vs. } 51 \text { vs. } 56 \\
\text { years } \\
\text { Female: } 70 \% \text { vs. } 82 \% \text { vs. } \\
80 \% \\
\text { Race: NR } \\
\text { Ambulatory: } 100 \% \text { vs. } 100 \% \\
\text { vs. } 100 \% \\
\text { Gait assistive devices: } 17 \% \\
\text { vs. } 11 \% \text { vs. } 10 \% \\
\text { Median duration of illness: } \\
15 \text { vs. } 10 \text { vs. } 11 \text { years } \\
\text { MS type } \\
\text { - Relapsing remitting: } 70 \% \\
\text { vs. } 75 \% \text { vs. } 65 \% \\
\text { - Secondary progressive: } \\
22 \% \text { vs. } 14 \% \text { vs. } 15 \% \\
\text { - Primary progressive: } 70 \% \\
\text { vs. } 9 \% \text { vs. } 20 \% \\
\text { Median EDSS: } 4 \text { vs. } 4 \text { vs. } \\
3.5\end{array}$ & 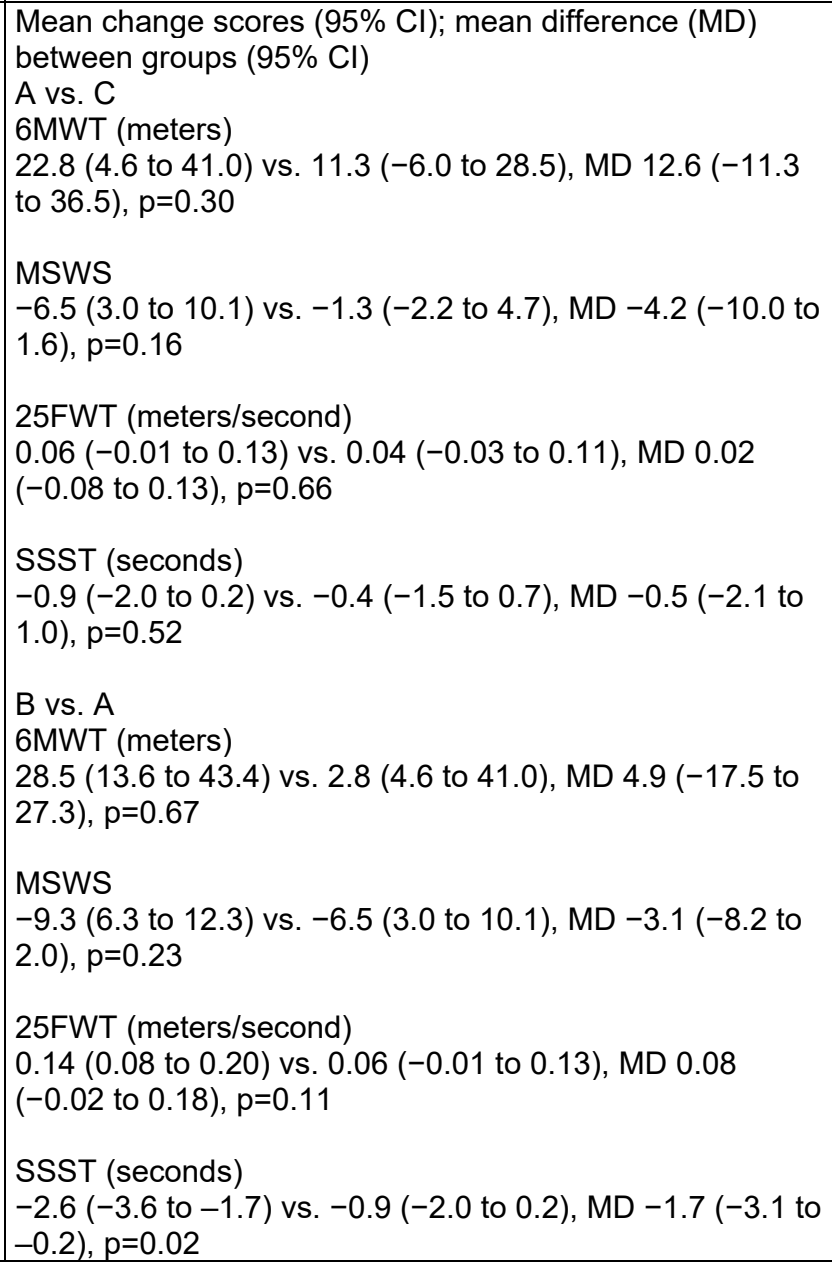 & $\begin{array}{l}\text { MiniBEST } \\
2.1(0.8 \text { to } 3.4) \text { vs. } 0.9(-0.4 \text { to } 2.2), \text { MD } 1.1 \\
(-0.7 \text { to } 2.9), p=0.24 \\
\text { MiniBEST } \\
4.1(3.0 \text { to } 5.2) \text { vs. } 2.1(0.8 \text { to } 3.4), \text { MD } 2.2(0.5 \\
\text { to } 3.9), p=0.01\end{array}$ \\
\hline
\end{tabular}




\begin{tabular}{|c|c|c|c|c|}
\hline $\begin{array}{l}\text { Author, Year } \\
\text { Intervention Type } \\
\text { Duration of } \\
\text { Postintervention } \\
\text { Followup } \\
\text { Quality } \\
\text { (See Appendix B } \\
\text { for Full Citation) } \\
\end{array}$ & $\begin{array}{l}\text { Intervention and } \\
\text { Comparison }\end{array}$ & $\begin{array}{l}\text { Population } \\
\text { Age (Mean) } \\
\text { Gender (\% Female) } \\
\text { Race (\%) } \\
\text { Ambulatory (\%) } \\
\text { Wheelchair User (\%) } \\
\text { Condition Specific (\%) } \\
\text { Other (\%) }\end{array}$ & Prioritized Outcomes & Other Outcomes \\
\hline $\begin{array}{l}\text { Carling, } 2017 \\
\text { Postural Control } \\
\text { Balance } \\
\\
\text { Postintervention, } 7 \\
\text { weeks } \\
\text { Fair }\end{array}$ & $\begin{array}{l}\text { A. Group CoDuSe } \\
\text { balance exercises: } \\
14 \text { sessions over } 7 \\
\text { weeks }(n=23) \\
\begin{array}{l}\text { B. Waitlist (Late start) } \\
\text { controls: }(n=25)\end{array}\end{array}$ & $\begin{array}{l}\text { A vs. B } \\
\text { Age (mean years): } 62 \text { vs. } 55 \\
\text { Female: } 19 \text { (76\%) vs. } 16 \\
(62 \%) \\
\text { Race: NR } \\
\text { Ambulatory: } 100 \% \\
\text { RRMS: } 0 \% \text { vs. } 23 \% \\
\text { SPMS: } 68 \% \text { vs. } 58 \% \\
\text { PPMS: } 32 \% \text { vs. } 19 \% \\
\text { Baseline EDSS: } 6.16 \text { vs. } \\
6.06 \\
\text { Baseline No Falls: } 48 \% \text { vs. } \\
46 \% \\
\text { Baseline Multiple Falls: } 20 \% \\
\text { vs. } 35 \%\end{array}$ & $\begin{array}{l}\text { A vs. B } \\
\text { TUG: MD } 4.41 \text { SE } 3.17, p=0.17 \\
\text { 2MWT: }-3.24 \text { SE } 3.37, p=0.34 \\
\text { Sit to Stand: } 0.24 \text { SE } 2.12, p=0.91 \\
\text { 10MWT: } 1.49 \text { SE } 3.84, p=0.70 \\
\text { Falls Efficacy Scale International: }-1.66 \text { SE } 2.39, p=0.49 \\
\text { MS Walking Scale: }-7.21 \text { SE } 3.60, p=0.051 \\
\text { Trend for falls before treatment, during treatment, after } \\
\text { treatment in control group only: }-1.24(1.66), p<0.001\end{array}$ & BBS: MD 3.65 SE 1.44, $p=0.015$ \\
\hline $\begin{array}{l}\text { Castro-Sanchez, } \\
2012 \\
\text { Aerobic Exercise } \\
\text { Aquatics } \\
\text { Postintervention, } \\
20 \text { weeks, and } 30 \\
\text { weeks } \\
\text { Good }\end{array}$ & $\begin{array}{l}\text { A. Ai-Chi aqua } \\
\text { therapy with Tai-Chi } \\
\text { music, } 40 \text { sessions } \\
\text { over } 20 \text { weeks } \\
(n=36) \\
\\
\text { B. Same exercises } \\
\text { as group A on } \\
\text { exercise mat without } \\
\text { music, } 40 \text { sessions } \\
\text { over } 20 \text { weeks } \\
(n=37)\end{array}$ & $\begin{array}{l}\text { A vs. B } \\
\text { Age: } 46 \text { vs. } 50 \\
\text { Female: } 72 \% \text { vs. } 65 \% \\
\text { EDSS: } 6.3 \text { vs. } 5.9 \\
\text { PPMS: } 17 \% \text { vs. } 24 \% \\
\text { SPMS: } 25 \% \text { vs. } 32 \%\end{array}$ & $\begin{array}{l}\text { A vs. B, Median (SD), p-value=between groups: } \\
\text { MSIS-29 Physical: } \\
48(15.91) \text { to } 41 \text { (12.37) vs. } 46(18.34) \text { to } 45(17.14), \\
p=0.014 \\
\text { MSIS-29 Psychological: } 34(29.47) \text { to } 21 \text { (15.73) vs. } 30 \\
(23.53) \text { to } 25(19.36), p=0.023 \\
\text { Differences in MSIS-29 maintained at } 30 \text { weeks } \\
\text { BDI: } \\
14 \text { (7.72) to } 5 \text { (3.2) vs. } 15 \text { (8.68) to } 13(5.91), p<0.05 \\
\text { Mean (SD) baseline to post-intervention, p-value between } \\
\text { groups: } \\
\text { Spasm VAS: } \\
5 \text { (2.8) to } 2 \text { (4.3) vs. } 6(3.1) \text { to } 4(4.5), 91 \% \text { improvement } \\
\text { vs. } 10 \% \text { improvement, } p<0.05\end{array}$ & $\begin{array}{l}\text { Barthel Index: } \\
91(7.12) \text { to } 86(9.23) \text { vs. } 87(10.34) \text { to } 88 \\
(8.92), p>0.05\end{array}$ \\
\hline
\end{tabular}




\begin{tabular}{|c|c|c|c|c|}
\hline $\begin{array}{l}\text { Author, Year } \\
\text { Intervention Type } \\
\text { Duration of } \\
\text { Postintervention } \\
\text { Followup } \\
\text { Quality } \\
\text { (See Appendix B } \\
\text { for Full Citation) } \\
\end{array}$ & $\begin{array}{l}\text { Intervention and } \\
\text { Comparison }\end{array}$ & $\begin{array}{l}\text { Population } \\
\text { Age (Mean) } \\
\text { Gender (\% Female) } \\
\text { Race (\%) } \\
\text { Ambulatory (\%) } \\
\text { Wheelchair User (\%) } \\
\text { Condition Specific (\%) } \\
\text { Other (\%) }\end{array}$ & Prioritized Outcomes & Other Outcomes \\
\hline $\begin{array}{l}\text { Chen, } 2016 \\
\text { Muscle Strength } \\
8 \text { weeks - mid } \\
\text { intervention } \\
16 \text { weeks - mid } \\
\text { intervention } \\
\text { Post-52-week } \\
\text { intervention } \\
4 \text { weeks } \\
\text { Fair }\end{array}$ & $\begin{array}{l}\text { A. Pulmonary } \\
\text { rehabilitation: } 365 \\
\text { sessions over } 52 \\
\text { weeks }(n=49) \\
\text { B. Usual care }(n=49)\end{array}$ & $\begin{array}{l}\text { A vs. B } \\
\text { Age (mean years): } 62.3 \text { vs. } \\
63.1 \\
\text { Female: } 0(0 \%) \text { vs. } 0(0 \%) \\
\text { Race: NR } \\
\text { Ambulatory: NR } \\
\text { Wheelchair user: NR } \\
\text { Injury Level } \\
- \text { T1-2: } 17(35 \%) \text { vs. } 17 \\
(35 \%) \\
- \text { T3-4: } 16(33 \%) \text { vs. } 16 \\
(33 \%) \\
- \text { T5-6: } 16(33 \%) \text { vs. } 16 \\
(33 \%)\end{array}$ & 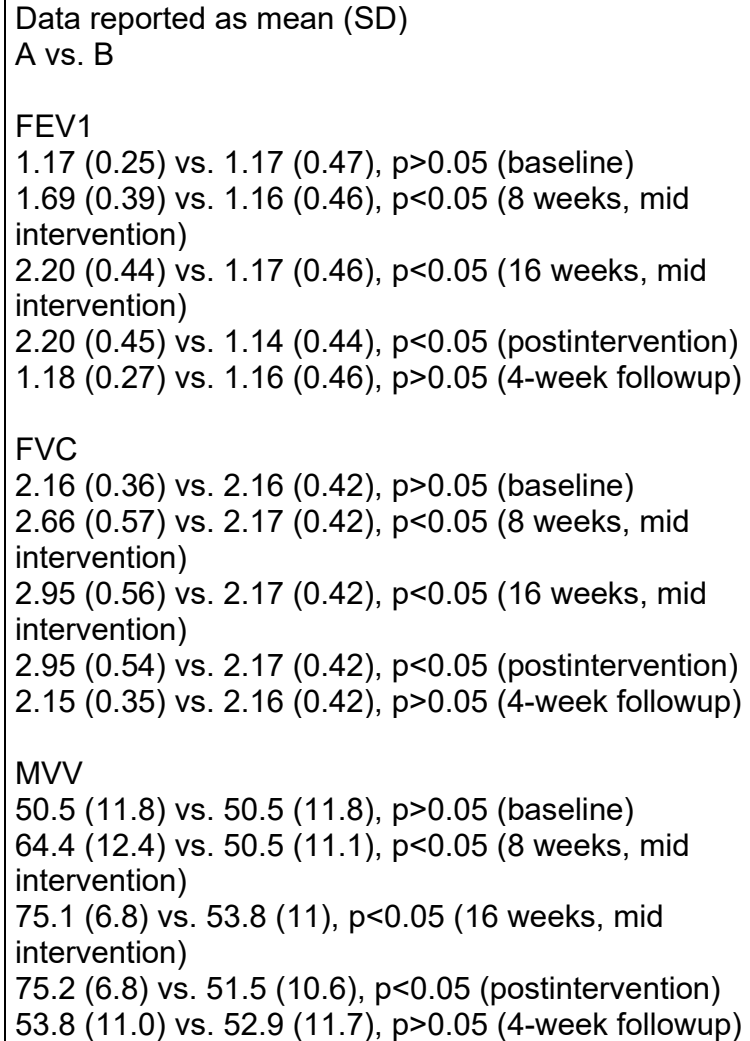 & NR \\
\hline
\end{tabular}




\begin{tabular}{|c|c|c|c|c|}
\hline $\begin{array}{l}\text { Author, Year } \\
\text { Intervention Type } \\
\text { Duration of } \\
\text { Postintervention } \\
\text { Followup } \\
\text { Quality } \\
\text { (See Appendix B } \\
\text { for Full Citation) } \\
\end{array}$ & $\begin{array}{l}\text { Intervention and } \\
\text { Comparison }\end{array}$ & \begin{tabular}{|l|} 
Population \\
Age (Mean) \\
Gender (\% Female) \\
Race (\%) \\
Ambulatory (\%) \\
Wheelchair User (\%) \\
Condition Specific (\%) \\
Other (\%) \\
\end{tabular} & Prioritized Outcomes & Other Outcomes \\
\hline $\begin{array}{l}\text { Chen, } 2016 \\
\text { (Continued) }\end{array}$ & & & 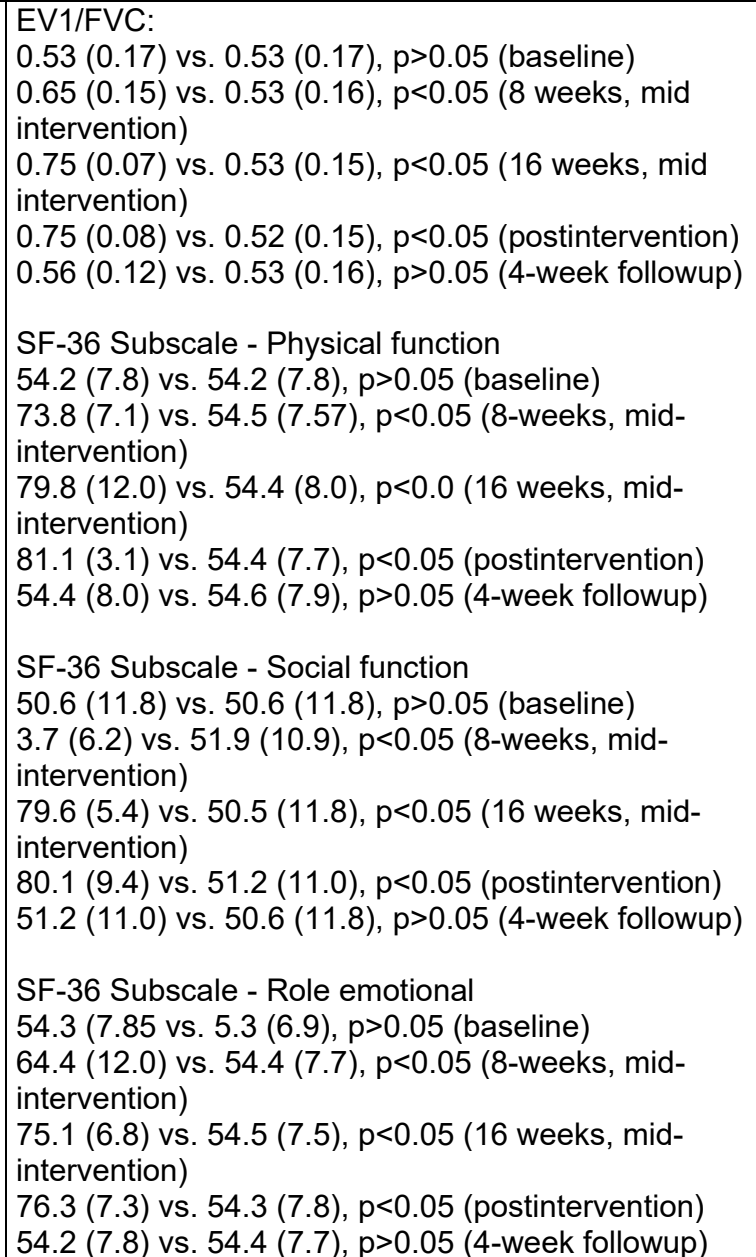 & \\
\hline
\end{tabular}




\begin{tabular}{|c|c|c|c|c|}
\hline $\begin{array}{l}\text { Author, Year } \\
\text { Intervention Type } \\
\text { Duration of } \\
\text { Postintervention } \\
\text { Followup } \\
\text { Quality } \\
\text { (See Appendix B } \\
\text { for Full Citation) } \\
\end{array}$ & $\begin{array}{l}\text { Intervention and } \\
\text { Comparison }\end{array}$ & \begin{tabular}{|l|} 
Population \\
Age (Mean) \\
Gender (\% Female) \\
Race (\%) \\
Ambulatory (\%) \\
Wheelchair User (\%) \\
Condition Specific (\%) \\
Other (\%) \\
\end{tabular} & Prioritized Outcomes & Other Outcomes \\
\hline $\begin{array}{l}\text { Chen, } 2016 \\
\text { (Continued) }\end{array}$ & & & $\begin{array}{l}\text { SF-36 Subscale - Mental health } \\
54.1 \text { ( } 7.7) \text { vs. } 54.2 \pm 7.8, p>0.05 \text { (baseline) } \\
64.3 \text { (12.0) vs. } 54.6 \pm 7.9, p<0.05 \text { ( } 8 \text {-weeks, mid- } \\
\text { intervention) } \\
75.3 \text { (6.7) vs. } 54.1 \pm 7.7, p<0.05 \text { ( } 16 \text { weeks, mid- } \\
\text { intervention) } \\
75.1 \text { (6.8) vs. } 54.2 \pm 7.8, p<0.05 \text { (postintervention) } \\
54.2 \text { (7.8) vs. } 54.2 \pm 7.8, p>0.05 \text { (4-week followup) } \\
\\
\text { SF-36 Subscale - Body pain } \\
51.6 \text { (11.3) vs. } 51.2(11.0), p>0.05 \text { (baseline) } \\
52.7 \text { (11.9) vs. } 50.6 \text { (11.8), } p>0.05 \text { (8-weeks, mid- } \\
\text { intervention) } \\
52.2 \text { (10.5) vs. } 51.6(11.3), p>0.05 \text { (16 weeks, mid- } \\
\text { intervention) } \\
51.9 \text { (10.8) vs. } 51.5(10.6), p>0.05 \text { (postintervention) } \\
51.5 \text { (10.6) vs. } 51.9 \text { (10.8), } p>0.05 \text { (4-week followup) }\end{array}$ & \\
\hline
\end{tabular}




\begin{tabular}{|c|c|c|c|c|}
\hline $\begin{array}{l}\text { Author, Year } \\
\text { Intervention Type } \\
\text { Duration of } \\
\text { Postintervention } \\
\text { Followup } \\
\text { Quality } \\
\text { (See Appendix B } \\
\text { for Full Citation) }\end{array}$ & $\begin{array}{l}\text { Intervention and } \\
\text { Comparison }\end{array}$ & $\begin{array}{l}\text { Population } \\
\text { Age (Mean) } \\
\text { Gender (\% Female) } \\
\text { Race (\%) } \\
\text { Ambulatory (\%) } \\
\text { Wheelchair User (\%) } \\
\text { Condition Specific (\%) } \\
\text { Other (\%) }\end{array}$ & Prioritized Outcomes & Other Outcomes \\
\hline $\begin{array}{l}\text { Muscle Strength } \\
\text { Immediately } \\
\text { Postintervention, } 6 \\
\text { weeks } \\
\text { Poor }\end{array}$ & $\begin{array}{l}\text { A. Functional } \\
\text { progressive } \\
\text { resistance exercise } \\
\text { (FPRE), } 12 \text { sessions } \\
\text { over } 6 \text { weeks }(n=13) \\
\\
\text { B. Control group, } \\
\text { Conventional } \\
\text { therapy, } 18 \text { sessions; } \\
3 \text { times a week, over } \\
6 \text { weeks, }(n=12)\end{array}$ & $\begin{array}{l}\text { A vs. B } \\
\text { Age (mean years): } 5.54 \text { vs. } \\
7.17 \\
\text { Female: } 9 \text { (69\%) vs. } 4 \text { (33\%) } \\
\text { Race: NR } \\
\text { Ambulatory: } 100 \% \\
\text { Wheelchair user: NR } \\
\text { GMFM Classification Level } \\
\text { (1-4 higher scores=physical } \\
\text { impairments): } 2.08 \text { vs. } 2.33 \\
\text { GMFM score: } 69.98 \text { vs. } \\
68.15 \\
\text { BMI (Z-score, mean): } 0.14 \\
\text { vs. } 0.60\end{array}$ & $\begin{array}{l}\text { A vs. B, mean (SD) } \\
\text { GMFM-88 score } \\
69.98(21.55) \text { vs. } 68.15(27.15) \text { (baseline) } \\
71.78(21.05) \text { vs. } 63.48(27.48) \text { (postintervention), } \\
p=0.019 \text { for group } A \text { and } 0.375 \text { for group } B \text { for change } \\
\text { from baseline } \\
\text { Increase pre-post for FPRE group } p=0.019 \text {; control group } \\
\text { showed no significant difference, } p=0.375 \text {. }\end{array}$ & $\begin{array}{l}\text { A vs. B, mean (SD) } \\
\text { Dynamic Balance, Forward functional reach } \\
\text { test (F-FRT): } \\
21.62(6.87) \text { vs. } 28.17 \text { (14.49) (baseline) } \\
26.65(7.92), p=0.000 \text { vs. } 25.37 \text { (10.20), } \\
p=0.261 \text { (postintervention) } \\
\\
\text { Dynamic Balance, Side functional reach test } \\
\text { (S-FRT): } \\
11.57(5.72) \text { vs. } 15.52 \text { (10.43) (baseline) } \\
16.21(5.37), p=0.003 \text { vs. } 15.95 \text { (8.26), } \\
p=0.793 \text { (postintervention) } \\
\text { Knee extensor muscle strength, } \\
\text { non-dominant side: } \\
40.62 \text { (30.61) vs. } 34.54 \text { (28.55) (baseline) } \\
51.24 \text { (33.58), } p=0.048 \text { vs. 40.59 (29.50), } \\
p=0.062 \text { (postintervention) } \\
\text { Knee extensor muscle strength, } \\
\text { dominant side } \\
30.45 \text { (27.57) vs. } 41.61 \text { (34.00) (baseline) } \\
52.39 \text { (33.13), } p=0.010) \text { vs. 43.12 (32.17), } \\
p=0.567 \text { (postintervention) }\end{array}$ \\
\hline
\end{tabular}




\begin{tabular}{|c|c|c|c|c|}
\hline $\begin{array}{l}\text { Author, Year } \\
\text { Intervention Type } \\
\text { Duration of } \\
\text { Postintervention } \\
\text { Followup } \\
\text { Quality } \\
\text { (See Appendix B } \\
\text { for Full Citation) } \\
\end{array}$ & $\begin{array}{l}\text { Intervention and } \\
\text { Comparison }\end{array}$ & $\begin{array}{l}\text { Population } \\
\text { Age (Mean) } \\
\text { Gender (\% Female) } \\
\text { Race (\%) } \\
\text { Ambulatory (\%) } \\
\text { Wheelchair User (\%) } \\
\text { Condition Specific (\%) } \\
\text { Other (\%) }\end{array}$ & Prioritized Outcomes & Other Outcomes \\
\hline $\begin{array}{l}\text { Chrysagis } 2012 \\
\text { Aerobic Exercise } \\
\text { Treadmill } \\
\text { Postintervention, } 0 \\
\text { weeks } \\
\text { Fair }\end{array}$ & $\begin{array}{l}\text { A. Treadmill training } \\
30 \text { minutes at } \\
\text { comfortable speed } 3 \\
\text { times a week for } 12 \\
\text { weeks } \\
\text { B. Conventional } \\
\text { physical therapy } 3 \\
\text { times a week for } 12 \\
\text { weeks }\end{array}$ & $\begin{array}{l}\text { GMFCS I-III } \\
n=22 \\
\text { mean age } 16.9 \text { years } \\
13 \text { males and } 9 \text { females } \\
(59 \% \text { male })\end{array}$ & $\begin{array}{l}\text { GMFM (Gross Motor Function Measure) (average D } \\
\text { [standing] and E [walking] subscales) } \\
\text { A. } 67.81 \text { (18.22) to } 71.67(18.22) \\
\text { B. } 64.45(18.61) \text { to } 65.1(16.53) \\
\text { Difference between groups } \mathrm{F}=9.088, \mathrm{p}=0.007 \\
\text { Medium effect size } \mathrm{d}=0.3895 \% \mathrm{Cl}-0.50 \text { to } 1.26 \\
\text { MAS (spasticity) } \\
\text { Knee extensors } \\
\text { A. } 2.59(0.62) \text { to } 2.27(0.60) \\
\text { B. } 2.00(0.54) \text { to } 1.51(0.56) \\
\text { No difference between groups } \mathrm{F}=0.237, \mathrm{p}=0.827 \\
\text { Knee flexors } \\
\text { A. } 2.45(0.68) \text { to } 2.12(0.67) \\
\text { B. } 2.40(0.66) \text { to } 2.18(0.71) \\
\text { No difference between groups } \mathrm{F}=0.237, \mathrm{p}=0.632 \\
\text { Foot plantar flexors } \\
\text { A. } 2.50(0.50) \text { to } 2.18(0.56) \\
\text { B. } 2.40 \text { (0.62) to } 2.22(0.56) \\
\text { No difference between groups } \mathrm{F}=0.570, \mathrm{p}=0.046\end{array}$ & NA \\
\hline
\end{tabular}




\begin{tabular}{|c|c|c|c|c|}
\hline $\begin{array}{l}\text { Author, Year } \\
\text { Intervention Type } \\
\text { Duration of } \\
\text { Postintervention } \\
\text { Followup } \\
\text { Quality } \\
\text { (See Appendix B } \\
\text { for Full Citation) }\end{array}$ & $\begin{array}{l}\text { Intervention and } \\
\text { Comparison }\end{array}$ & $\begin{array}{l}\text { Population } \\
\text { Age (Mean) } \\
\text { Gender (\% Female) } \\
\text { Race (\%) } \\
\text { Ambulatory (\%) } \\
\text { Wheelchair User (\%) } \\
\text { Condition Specific (\%) } \\
\text { Other (\%) }\end{array}$ & Prioritized Outcomes & Other Outcomes \\
\hline $\begin{array}{l}\text { Claerbout, } 2012 \\
\text { Postural Control } \\
\text { Whole Body } \\
\text { Vibration } \\
\text { Postintervention, } 0 \\
\text { weeks } \\
\text { Fair }\end{array}$ & $\begin{array}{l}\text { A. Whole body } \\
\text { vibration + } \\
\text { conventional therapy: } \\
10 \text { sessions over } 3 \\
\text { weeks ( } n=20) \\
\\
\text { B. Whole body light } \\
\text { vibration + } \\
\text { conventional therapy: } \\
10 \text { sessions over } 3 \\
\text { weeks ( } n=18 \text { ) } \\
\text { C. Conventional } \\
\text { therapy ( }=17 \text { ) } \\
\text { [All patients } \\
\text { participated in a } \\
\text { minimum 3-week } \\
\text { multi-disciplinary } \\
\text { rehabilitation } \\
\text { program which } \\
\text { included daily PT/OT } \\
\text { sessions (+ other } \\
\text { therapies)] }\end{array}$ & $\begin{array}{l}\text { A vs. B vs. C } \\
\text { Age (mean years): } 39.1 \text { vs. } \\
\text { 43.8 vs. } 47.6 \\
\text { Female: } 6(28.6 \%) \text { vs. } 4 \\
\text { (22.2\%) vs. } 11(64.7 \%) \\
\text { Race: NR } \\
\text { Ambulatory: NR } \\
\text { Wheelchair user: NR } \\
\text { Disease duration (mean } \\
\text { years): } 12.1 \text { vs. } 12.5 \text { vs. } 10.3 \\
\text { EDSS: } 5.3 \text { vs. } 5.1 \text { vs. } 5.2\end{array}$ & 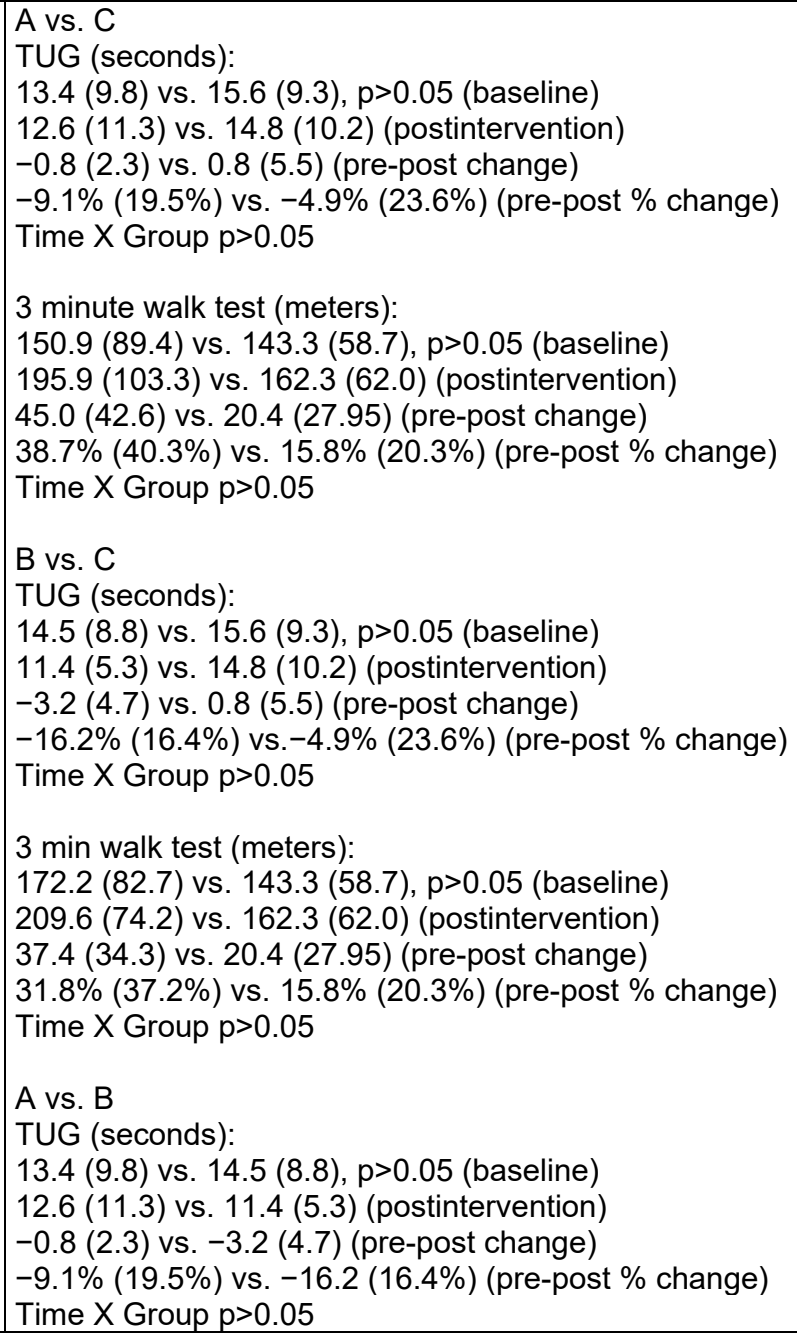 & $\begin{array}{l}\text { A vs. C } \\
\text { BBS: } \\
45.1(12.2) \text { vs. } 46.7 \text { (7.0), p>0.05 (baseline) } \\
49.0(11.5) \text { vs. } 48.5 \text { (7.7) (postintervention) } \\
3.9(4.4) \text { vs. } 0.2(7.5) \text { (pre-post change) } \\
10.0 \%(11.8 \%) \text { vs. } 3.9 \% \text { (5.8\%) (pre-post \% } \\
\text { change) } \\
\text { Time X Group p>0.05 } \\
\text { B vs. C } \\
\text { BBS: } \\
43.0(13.3) \text { vs. } 46.7 \text { (7.0), p>0.05 (baseline) } \\
47.2(12.7) \text { vs. } 48.5 \text { (7.7) (postintervention) } \\
4.2(6.1) \text { vs. } 0.2(7.5) \text { (pre-post change) } \\
12.0(19.4) \text { vs. } 3.9 \% \text { (5.8\%) (pre-post } \% \\
\text { change) } \\
\text { Time X Group p>0.05 } \\
\text { A vs. B } \\
\text { BBS: } \\
45.1(12.2) \text { vs. } 43.0 \text { (13.3), p>0.05 (baseline) } \\
49.0(11.5) \text { vs. } 47.2(12.7) \text { (postintervention) } \\
3.9(4.4) \text { vs. } 4.2 \text { (6.1) (pre-post change) } \\
10.0 \%(11.8 \%) \text { vs. } 12.0 \text { (19.4) (pre-post \% } \\
\text { change) } \\
\text { Time X Group p>0.05 }\end{array}$ \\
\hline
\end{tabular}




\begin{tabular}{|c|c|c|c|c|}
\hline $\begin{array}{l}\text { Author, Year } \\
\text { Intervention Type } \\
\text { Duration of } \\
\text { Postintervention } \\
\text { Followup } \\
\text { Quality } \\
\text { (See Appendix B } \\
\text { for Full Citation) }\end{array}$ & $\begin{array}{l}\text { Intervention and } \\
\text { Comparison }\end{array}$ & $\begin{array}{l}\text { Population } \\
\text { Age (Mean) } \\
\text { Gender (\% Female) } \\
\text { Race (\%) } \\
\text { Ambulatory (\%) } \\
\text { Wheelchair User (\%) } \\
\text { Condition Specific (\%) } \\
\text { Other (\%) }\end{array}$ & Prioritized Outcomes & Other Outcomes \\
\hline $\begin{array}{l}\text { Claerbout, } 2012 \\
\text { (Continued) }\end{array}$ & & & $\begin{array}{l}3 \text { min walk test (meters): } \\
150.9(89.4) \text { vs. } 143.3(58.7), p>0.05 \text { (baseline) } \\
195.9(103.3) \text { vs. } 209.6(74.2) \text { (postintervention) } \\
45.0(42.6) \text { vs. } 37.4(34.3) \text { (pre-post change) } \\
38.7 \%(40.3 \%) \text { vs. } 31.8 \%(37.2 \%) \text { (pre-post \% change) } \\
\text { Time X Group p>0.05 }\end{array}$ & \\
\hline $\begin{array}{l}\text { Collett, } 2010 \\
\text { Aerobic Exercise } \\
\text { Cycling } \\
\text { Postintervention, } \\
12 \text { weeks } \\
\text { Poor }\end{array}$ & $\begin{array}{l}\text { A. Combined } \\
\text { intermittent and } \\
\text { continuous static } \\
\text { cycling, } 24 \text { sessions } \\
\text { over } 12 \text { weeks } \\
(\mathrm{n}=20) \\
\text { B. Intermittent static } \\
\text { cycling, } 24 \text { sessions } \\
\text { over } 12 \text { weeks } \\
(\mathrm{n}=21) \\
\text { C. Continuous static } \\
\text { cycling, } 24 \text { sessions } \\
\text { over } 12 \text { weeks } \\
\text { ( } n=20)\end{array}$ & $\begin{array}{l}\text { A vs. B vs. C } \\
\text { Age (mean years): } \\
55 \text { vs. } 50 \text { vs. } 52 \\
\text { Female: } \\
9(53 \%) \text { vs. } 14(78 \%) \text { vs. } 16 \\
(80 \%) \\
\text { Race: NR } \\
\text { Ambulatory: } \\
20(100 \%) \text { vs. } 21(100 \%) \text { vs. } \\
20(100 \%) \\
\text { Wheelchair user: NR } \\
\text { Other: } \\
\text { MS subtypes NR }\end{array}$ & $\begin{array}{l}\text { A vs. B vs. C (SD) } \\
\text { Change post-intervention: no data provided } \\
\text { 2MWT, SF-36 total, TUG: All NS }\end{array}$ & Barthel Index Total, Leg Power: All NS \\
\hline
\end{tabular}




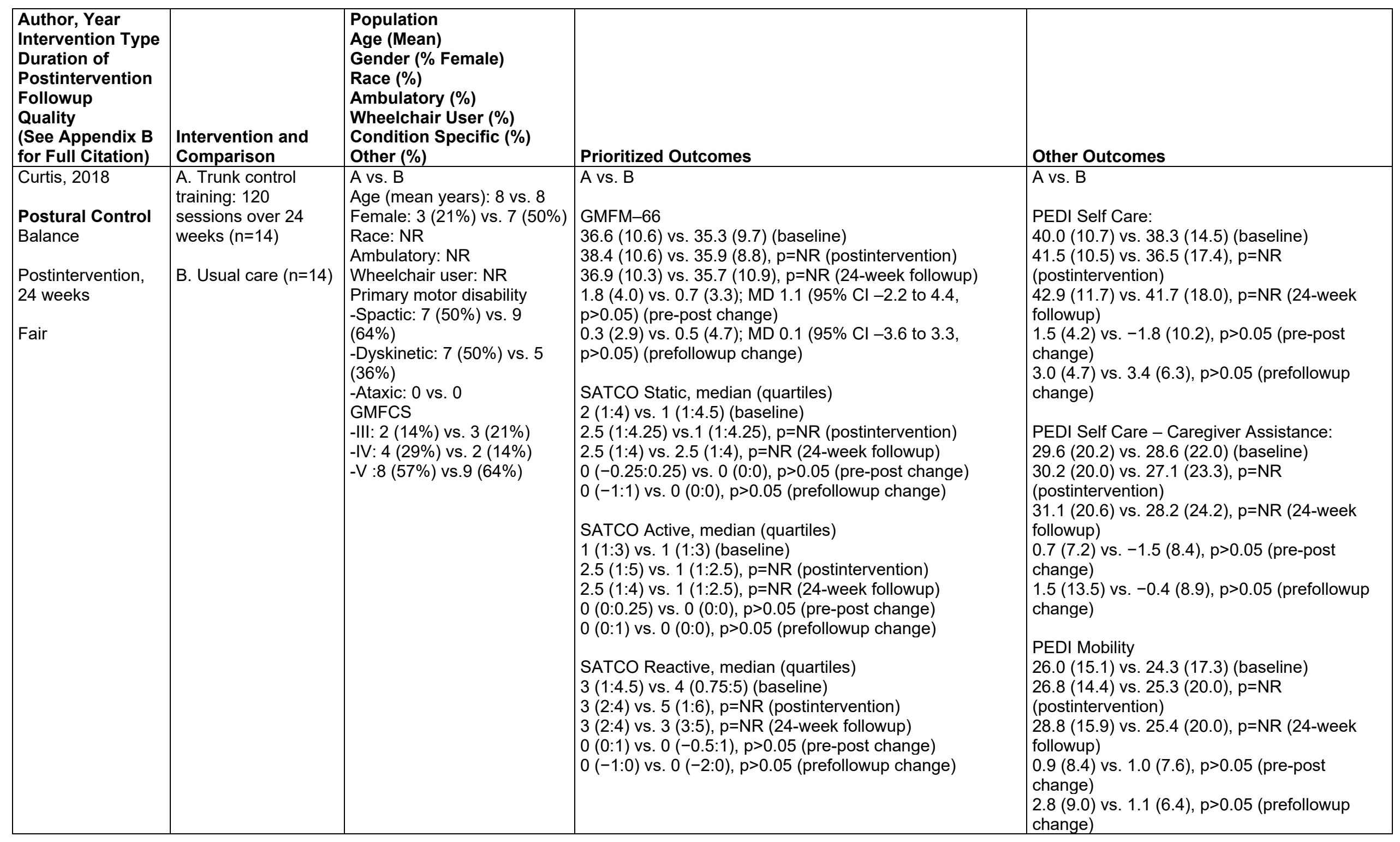




\begin{tabular}{|c|c|c|c|c|}
\hline $\begin{array}{l}\text { Author, Year } \\
\text { Intervention Type } \\
\text { Duration of } \\
\text { Postintervention } \\
\text { Followup } \\
\text { Quality } \\
\text { (See Appendix B } \\
\text { for Full Citation) } \\
\end{array}$ & $\begin{array}{l}\text { Intervention and } \\
\text { Comparison }\end{array}$ & $\begin{array}{l}\text { Population } \\
\text { Age (Mean) } \\
\text { Gender (\% Female) } \\
\text { Race (\%) } \\
\text { Ambulatory (\%) } \\
\text { Wheelchair User (\%) } \\
\text { Condition Specific (\%) } \\
\text { Other (\%) }\end{array}$ & Prioritized Outcomes & Other Outcomes \\
\hline $\begin{array}{l}\text { Curtis, } 2018 \\
\text { (Continued) }\end{array}$ & & & & $\begin{array}{l}\text { PEDI Mobility - Caregiver Assistance } \\
29.2(18.3) \text { vs. } 23.7 \text { (22.8) (baseline) } \\
30.3(20.0) \text { vs. } 22.8 \text { (26.5), p=NR } \\
\text { (postintervention) } \\
25.0(19.5) \text { vs. } 23.0 \text { (25.3), p=NR (24-week } \\
\text { followup) } \\
1.1(12.6) \text { vs. }-0.9(6.6), p>0.05 \text { (pre-post } \\
\text { change) } \\
-4.2(17.2) \text { vs. }-0.6(5.8), p>0.05 \text { (prefollowup } \\
\text { change) }\end{array}$ \\
\hline
\end{tabular}




\begin{tabular}{|c|c|c|c|c|}
\hline $\begin{array}{l}\text { Author, Year } \\
\text { Intervention Type } \\
\text { Duration of } \\
\text { Postintervention } \\
\text { Followup } \\
\text { Quality } \\
\text { (See Appendix B } \\
\text { for Full Citation) }\end{array}$ & $\begin{array}{l}\text { Intervention and } \\
\text { Comparison }\end{array}$ & $\begin{array}{l}\text { Population } \\
\text { Age (Mean) } \\
\text { Gender (\% Female) } \\
\text { Race (\%) } \\
\text { Ambulatory (\%) } \\
\text { Wheelchair User (\%) } \\
\text { Condition Specific (\%) } \\
\text { Other (\%) }\end{array}$ & Prioritized Outcomes & Other Outcomes \\
\hline $\begin{array}{l}\text { Dalgas, } 2009 \\
\text { Dalgas, } 2010 \\
\text { Muscle Strength } \\
\text { Postintervention, } 0 \\
\text { weeks } \\
\text { Dalgas, 2009: Fair } \\
\text { to Good } \\
\text { Dalgas, 2010: Poor }\end{array}$ & $\begin{array}{l}\text { A. Progressive } \\
\text { resistance: } 24 \\
\text { sessions over } 12 \\
\text { weeks ( } n=15) \\
\text { B. Usual care } \\
\text { (continue previous } \\
\text { activity level) }(n=16)\end{array}$ & $\begin{array}{l}\text { A vs. B (according per- } \\
\text { protocol analysis) } \\
\text { Age (mean years): } 45 \text { vs. } 48 \\
\text { Female: } 10(63 \%) \text { vs. } 10 \\
(67 \%) \\
\text { Race: NR } \\
\text { Ambulatory: } 15(100 \%) \text { vs. } \\
16(100 \%) \\
\text { Wheelchair user: NR }\end{array}$ & 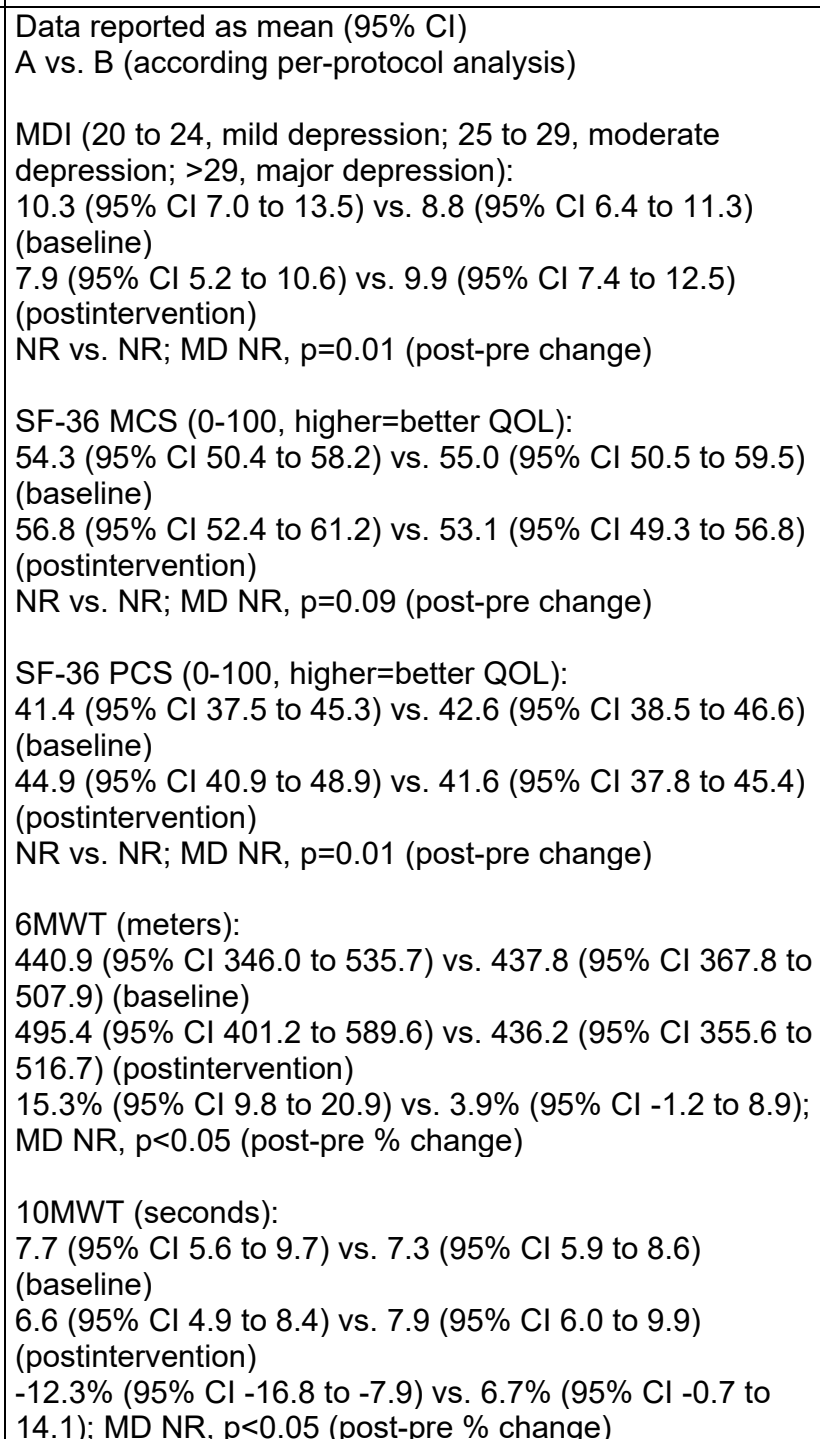 & $\begin{array}{l}\text { Data reported as mean }(95 \% \mathrm{Cl}) \\
\text { A vs. B (according per-protocol analysis) } \\
\text { Max leg press (pounds): } \\
102.4(95 \% \mathrm{Cl} 76.7 \text { to } 128.1) \text { vs. NR } \\
\text { (baseline) } \\
140.1(95 \% \mathrm{Cl} 112.1 \text { to } 168.1) \text { vs. } 86.4 \text { (72.4 } \\
\text { to } 100.4) \text { (postintervention) } \\
37.1 \% \text { (95\% Cl } 26.6 \text { to } 47.6) \text { vs. NR; MD NR, } \\
\text { p=NR (post-pre \% change) } \\
\text { EDSS (0-10, higher=greater disability): } \\
3.7(95 \% \mathrm{Cl} 3.2 \text { to } 4.2) \text { vs. } 3.9 \text { (95\% Cl } 3.5 \text { to } \\
4.4), p>0.05 \text { (baseline) } \\
3.9(95 \% \mathrm{Cl} 3.3 \text { to } 4.6) \text { vs. } 4.0 \text { (95\% Cl } 3.4 \text { to } \\
4.6)(\text { postintervention) } \\
3.9 \%(95 \% \mathrm{Cl}-3.4 \% \text { to } 11.2 \%) \text { vs. }-0.7 \%(95 \% \\
\text { Cl }-9.3 \text { to } 7.9) ; \text { MD NR, p=NR (post-pre } \% \\
\text { change) }\end{array}$ \\
\hline
\end{tabular}




\begin{tabular}{|c|c|c|c|c|}
\hline $\begin{array}{l}\text { Author, Year } \\
\text { Intervention Type } \\
\text { Duration of } \\
\text { Postintervention } \\
\text { Followup } \\
\text { Quality } \\
\text { (See Appendix B } \\
\text { for Full Citation) } \\
\end{array}$ & $\begin{array}{l}\text { Intervention and } \\
\text { Comparison }\end{array}$ & $\begin{array}{l}\text { Population } \\
\text { Age (Mean) } \\
\text { Gender (\% Female) } \\
\text { Race (\%) } \\
\text { Ambulatory (\%) } \\
\text { Wheelchair User (\%) } \\
\text { Condition Specific (\%) } \\
\text { Other (\%) } \\
\end{array}$ & Prioritized Outcomes & Other Outcomes \\
\hline $\begin{array}{l}\text { Demuth, } 2012 \\
\text { Companion to: } \\
\text { Fowler, } 2010 \\
\text { Aerobic Exercise } \\
\text { Cycling } \\
\text { Postintervention, } 0 \\
\text { weeks } \\
\text { Fair }\end{array}$ & $\begin{array}{l}\text { A. Stationary cycling, } \\
30 \text { sessions over } 12 \\
\text { weeks }(n=33) \\
\text { B. } \\
\text { Control No } \\
\text { Intervention }(n=31)\end{array}$ & $\begin{array}{l}\text { A vs. B } \\
\text { Age (mean years): } \\
10.7 \text { vs. } 11.2 \\
\text { Female: } \\
13(42 \%) \text { vs. } 20(65 \%) \\
\\
\text { Race: } \\
\text { African-American } \\
5(16 \%) \text { vs. } 3(10 \%) \\
\text { White } \\
18(58 \%) \text { vs. } 15(48 \%) \\
\text { Asian } \\
1(3 \%) \text { vs. } 516(\%) \\
\text { Other } \\
7(23 \%) \text { vs. } 8(26 \%) \\
\text { Ambulatory: } \\
33(100 \%) \text { vs. } 31(100 \%) \\
\text { Wheelchair user: NR } \\
\\
\text { Other: } \\
\text { CP type NR }\end{array}$ & $\begin{array}{l}\text { A vs. B (SD) } \\
\text { GMFM-66: } \\
\text { Change from baseline: } 1.2,95 \% \mathrm{Cl} 0.5 \text { to } 1.8 \text { ) vs. } 0.5 \text {, } \\
95 \% \mathrm{Cl}-0.2 \text { to } 1.3 \text {, between groups } \mathrm{p}=0.23 \\
600-\text { Yard Walk-Run Test: } \\
\text { Change from baseline: } 5.6,95 \% \mathrm{Cl} 1.6 \text { to } 9.5 \text { vs. } 2.5,95 \% \\
\mathrm{Cl}-1.1 \text { to } 6.0, p=0.24 \\
\text { Peds Quality of Life Total Score: } \\
\text { Mean difference between groups: } \\
3.5,95 \% \mathrm{Cl}-2.0 \text { to } 8.8, p=0.21 \\
\text { PedsQL Emotional Functioning } \\
55.6 \text { (NR) vs. } 68.1 \text { (NR) (baseline) } \\
64.7 \text { (NR) vs. } 68.3 \text { (NR); } p=0.046 \text { (postintervention) } \\
\text { PedsQL Physical Functioning, Psychosocial Health } \\
\text { Summary, Social Functioning, School Functioning, Total } \\
\text { Score: All NS }\end{array}$ & NA \\
\hline
\end{tabular}




\begin{tabular}{|c|c|c|c|c|}
\hline $\begin{array}{l}\text { Author, Year } \\
\text { Intervention Type } \\
\text { Duration of } \\
\text { Postintervention } \\
\text { Followup } \\
\text { Quality } \\
\text { (See Appendix B } \\
\text { for Full Citation) } \\
\end{array}$ & $\begin{array}{l}\text { Intervention and } \\
\text { Comparison }\end{array}$ & $\begin{array}{l}\text { Population } \\
\text { Age (Mean) } \\
\text { Gender (\% Female) } \\
\text { Race (\%) } \\
\text { Ambulatory (\%) } \\
\text { Wheelchair User (\%) } \\
\text { Condition Specific (\%) } \\
\text { Other (\%) }\end{array}$ & Prioritized Outcomes & Other Outcomes \\
\hline $\begin{array}{l}\text { Deutz, } 2017 \\
\text { Postural Control } \\
\text { Hippotherapy } \\
\text { Middle of treatment } \\
\text { (after 8-week } \\
\text { observational } \\
\text { phase and 16- to } \\
20 \text {-week } \\
\text { intervention) and } \\
\text { end of treatment } \\
\text { (after 16-week } \\
\text { washout period, } \\
\text { 16- to 20-week } \\
\text { intervention, and 8- } \\
\text { week observational } \\
\text { phase) } \\
\text { Poor }\end{array}$ & $\begin{array}{l}\text { A. Early treatment } \\
\text { group (ETG), } 16 \text { to } \\
32 \text { units of } \\
\text { hippotherapy over } 16 \\
\text { to } 20 \text { weeks in } \\
\text { addition to usual } \\
\text { conventional } \\
\text { physiotherapy ( } n=35 \text { ) } \\
\text { B. Late treatment } \\
\text { group (LTG), usual } \\
\text { conventional } \\
\text { physiotherapy over } \\
16 \text { to } 20 \text { weeks } \\
\text { (n=38) } \\
\text { Crossover trial with a } \\
\text { washout period of } 16 \\
\text { weeks between two } \\
\text { intervention periods }\end{array}$ & $\begin{array}{l}\text { A vs. B } \\
\text { Age (mean years): } 9.29 \text { vs. } \\
8.87 \\
\text { Female: } 12(34 \%) \text { vs. } 17 \\
\text { (45\%) } \\
\text { Race: NR } \\
\text { Ambulatory: NR } \\
\text { Wheelchair user: NR } \\
\\
\text { GMFCS level: } \\
\text { GMFCS level II: } 10(29 \%) \\
\text { vs. } 17 \text { (45\%) } \\
\text { GMFCS level III: } 7(20 \%) \text { vs. } \\
10(26 \%) \\
\text { GMFCS level IV: } 18(51 \%) \\
\text { vs. } 11(29 \%) \\
\\
\text { Preterm children: } 26(74 \%) \\
\text { vs. } 28 \text { ( } 74 \%) \\
\text { Nonpreterm children: } 9 \\
\text { (26\%) vs. } 10(26 \%)\end{array}$ & $\begin{array}{l}\text { A vs. B } \\
16 \text { to } 20 \text { weeks } \\
\text { GMFM-66: } \\
\text { Direct treatment effect resulting from the mixed model } \\
\text { approach: } p=0.3193, F=1.01, \mathrm{DF}=(1,47) \\
\text { Difference in means between the two treatments: } 0.52 \\
(95 \% \mathrm{Cl}-0.52 \text { to } 1.55) \\
\text { CHQ-28: } \\
\text { Psychosocial: } \\
\text { Difference in means: }-0.21(95 \% \mathrm{Cl}-3.89 \text { to } 3.47) \\
\text { Direct treatment effect: } p=0.9089 \\
\text { Physical: } \\
\text { Difference in means: } 4.77(95 \% \mathrm{Cl}-1.12 \text { to } 10.66) \\
\text { Direct treatment effect: } p=0.1092 \\
\text { KIDSCREEN-27: } \\
\text { Difference in means: } 1.07(95 \% \mathrm{Cl}-2.53 \text { to } 4.68) \\
\text { Direct treatment effect: } p=0.5483\end{array}$ & NR \\
\hline
\end{tabular}




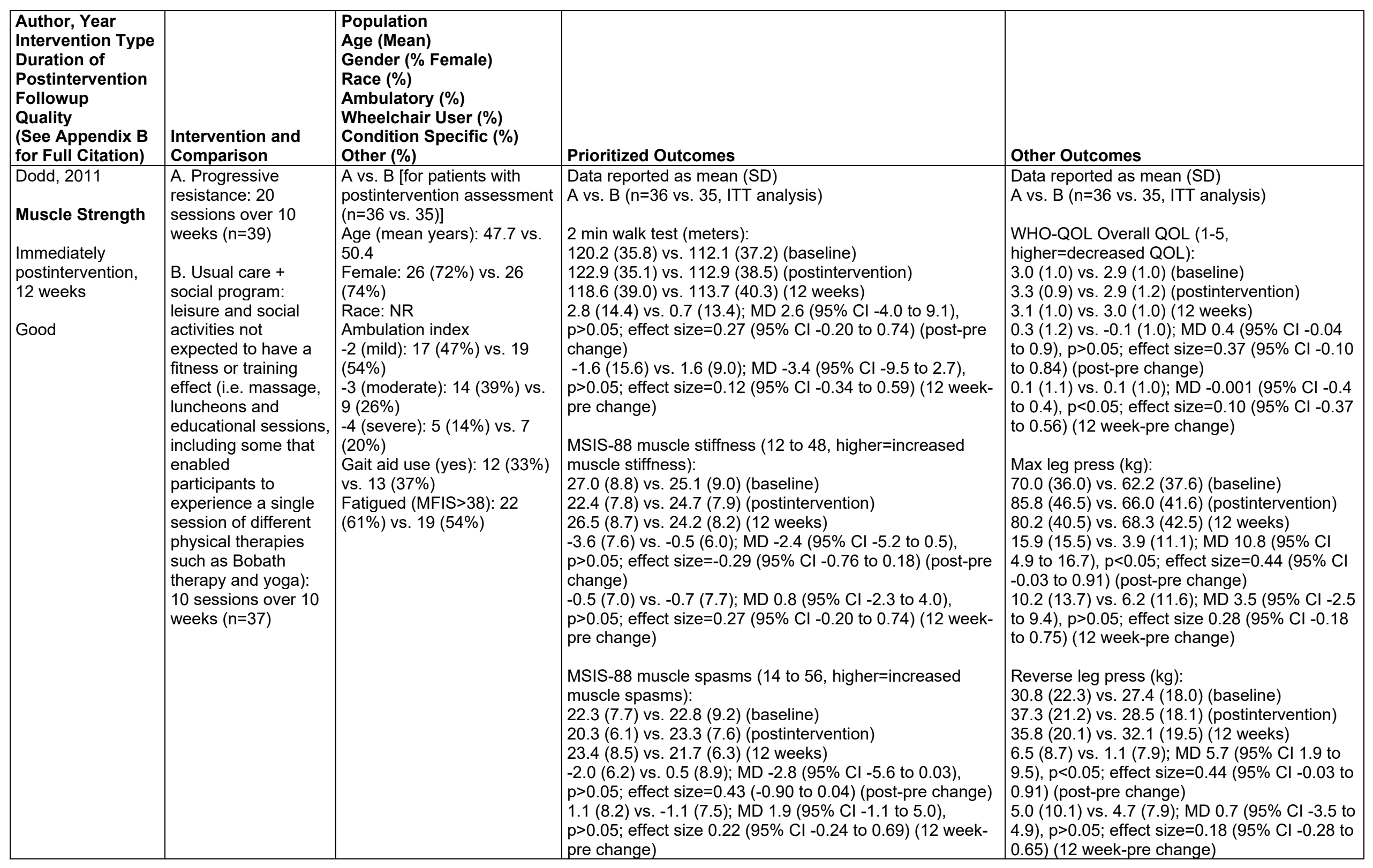




\begin{tabular}{|c|c|c|c|c|}
\hline $\begin{array}{l}\text { Author, Year } \\
\text { Intervention Type } \\
\text { Duration of } \\
\text { Postintervention } \\
\text { Followup } \\
\text { Quality } \\
\text { (See Appendix B } \\
\text { for Full Citation) } \\
\end{array}$ & $\begin{array}{l}\text { Intervention and } \\
\text { Comparison }\end{array}$ & $\begin{array}{l}\text { Population } \\
\text { Age (Mean) } \\
\text { Gender (\% Female) } \\
\text { Race (\%) } \\
\text { Ambulatory (\%) } \\
\text { Wheelchair User (\%) } \\
\text { Condition Specific (\%) } \\
\text { Other (\%) }\end{array}$ & Prioritized Outcomes & Other Outcomes \\
\hline $\begin{array}{l}\text { Doulatabad, } 2013 \\
\text { Postural Control } \\
\text { Yoga } \\
\text { Postintervention, } \\
12 \text { weeks } \\
\text { Poor }\end{array}$ & $\begin{array}{l}\text { A. Yoga: } 24 \text { sessions } \\
\text { over } 12 \text { weeks } \\
(n=30) \\
\text { B. Usual care control: } \\
\text { no intervention, over } \\
12 \text { weeks }(n=30)\end{array}$ & $\begin{array}{l}\text { A vs. B } \\
\text { Age (average): } 31.6 \text { (range: } \\
18 \text { to } 45) \\
\text { Female: } 30(100 \%) \text { vs. } 30 \\
(100 \%) \\
\text { Race: NR } \\
\text { Ambulatory: NR } \\
\text { Wheelchair user: NR }\end{array}$ & $\begin{array}{l}\text { A. vs. B., mean (SD) } \\
\text { MSQoL-54 (10 indicates best quality of life): } \\
4.9 \pm 1.9 \text { vs. } 6.9 \pm 1.5 \text { (baseline) } \\
7.4 \pm 2.16 \text { vs. } 6.8 \pm 1.9 \text { (postintervention), } p=0.001\end{array}$ & NA \\
\hline $\begin{array}{l}\text { Duarte Nde, } 2014 \\
\text { Aerobic Exercise } \\
\text { Treadmill } \\
\text { Postintervention, } 3 \\
\text { weeks and } 5 \\
\text { weeks } \\
\text { Fair } \\
\text { Note: May share } \\
\text { participants with } \\
\text { Grecco, } 2014\end{array}$ & $\begin{array}{l}\text { A. Treadmill + tDCS, } \\
10 \text { sessions over } 2 \\
\text { weeks }(n=12) \\
\text { B. Treadmill + sham } \\
\text { tDCS, } 10 \text { sessions } \\
\text { over } 2 \text { weeks, }(n=12)\end{array}$ & $\begin{array}{l}\text { A vs. B } \\
\text { Age: } 8 \text { vs. } 8 \\
\text { Female: NR } \\
\text { GMFCS I: } 25 \% \text { vs. } 17 \% \\
\text { GMFCS II: } 50 \% \text { vs. } 57 \% \\
\text { GMFCS III: } 25 \% \text { vs. } 25 \%\end{array}$ & $\begin{array}{l}\text { A vs. B, Mean (SD), p-value=between groups: } \\
\text { PEDI self-care: } \\
46.1(10) \text { to } 48.0(9.5) \text { vs. } 45.0(9.2) \text { to } 45.5(9.3) \text {; MD } 1.4 \\
95 \% \mathrm{Cl}-6.21 \text { to } 9.01, p=0.718 \\
\text { PEDI mobility: } \\
38.0(8.5) \text { to } 41.7(7.4) \text { vs. } 38.3(7.4) \text { to } 39.5(7.6) \text {; MD } 2.5 \\
95 \% \mathrm{Cl}-3.71 \text { to } 8.71, p=0.430\end{array}$ & $\begin{array}{l}\text { A vs. B, Mean (SD), p-value=between groups: } \\
\text { PBS: } \\
40.5(9.4) \text { to } 45.3(7.9) \text { vs.39.1 (9.8) to } 39.7 \\
\text { (8.4); MD 4.2, } 95 \% \mathrm{Cl}-2.88 \text { to } 11.28, p=0.245\end{array}$ \\
\hline
\end{tabular}




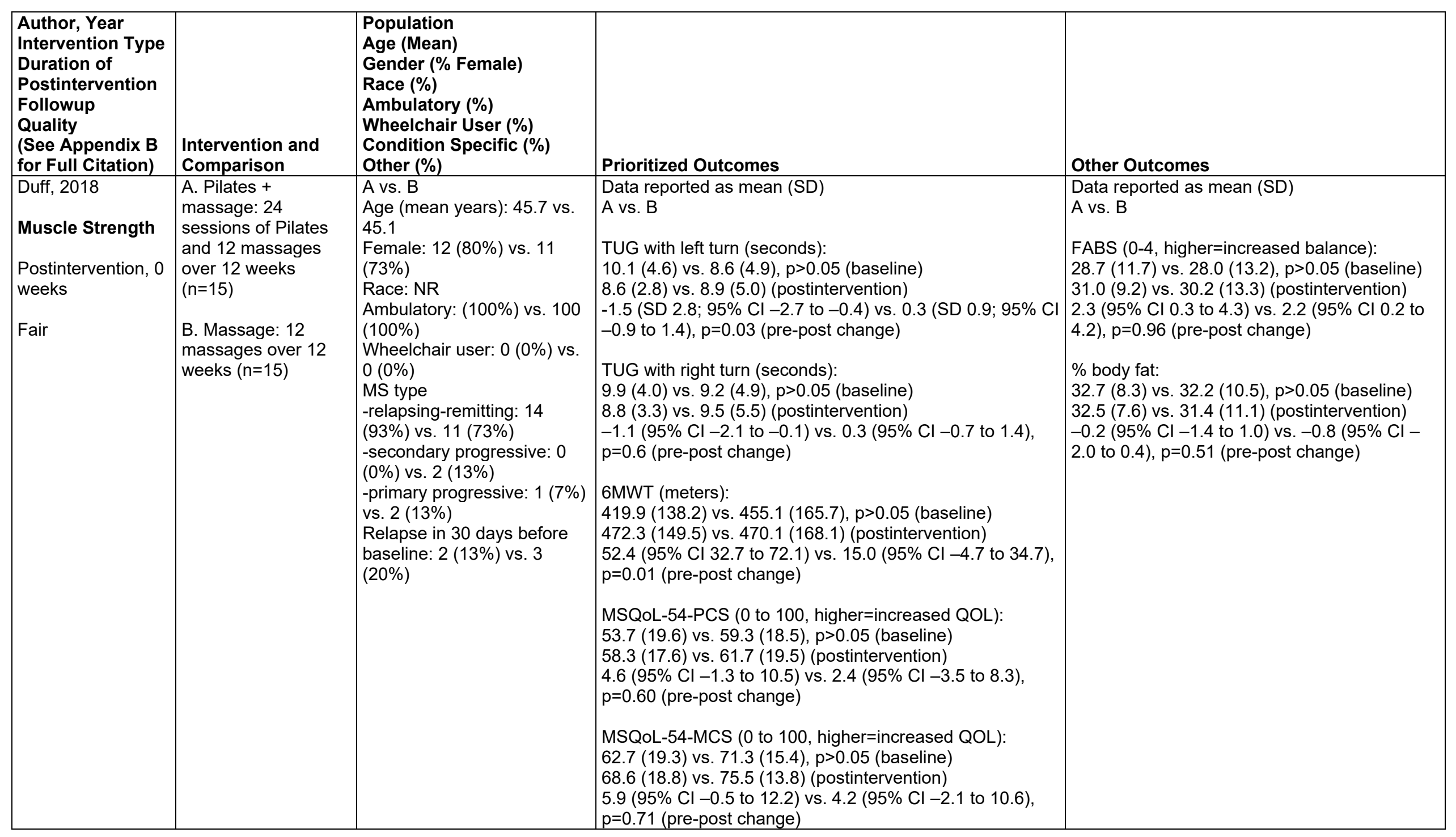




\begin{tabular}{|c|c|c|c|c|}
\hline $\begin{array}{l}\text { Author, Year } \\
\text { Intervention Type } \\
\text { Duration of } \\
\text { Postintervention } \\
\text { Followup } \\
\text { Quality } \\
\text { (See Appendix B } \\
\text { for Full Citation) } \\
\end{array}$ & $\begin{array}{l}\text { Intervention and } \\
\text { Comparison }\end{array}$ & $\begin{array}{l}\text { Population } \\
\text { Age (Mean) } \\
\text { Gender (\% Female) } \\
\text { Race (\%) } \\
\text { Ambulatory (\%) } \\
\text { Wheelchair User (\%) } \\
\text { Condition Specific (\%) } \\
\text { Other (\%) }\end{array}$ & Prioritized Outcomes & Other Outcomes \\
\hline $\begin{array}{l}\text { Duffell, } 2014 \\
\text { Aerobic Exercise } \\
\text { Robot-Assisted } \\
\text { Gait Training } \\
\text { Postintervention, } 4 \\
\text { weeks } \\
\text { Poor }\end{array}$ & $\begin{array}{l}\text { A. Robot-assisted } \\
\text { gait training (RAGT), } \\
12 \text { sessions over } 4 \\
\text { weeks }(n=23) \\
\text { B. No intervention } \\
(n=29)\end{array}$ & $\begin{array}{l}\text { A vs. B } \\
\text { Age: NR } \\
\text { Female: NR } \\
\text { Incomplete: } 100 \%\end{array}$ & $\begin{array}{l}\text { A vs. } B, p=\text { between groups } \\
\text { 10MWT achieved MID }(0.13 \mathrm{~m} / \mathrm{s}) \text { : } \\
13 \% \text { vs. } 8 \%, p>0.05 \\
6 \mathrm{MWT} \text { and TUG: } p>0.05\end{array}$ & NA \\
\hline $\begin{array}{l}\text { Ebrahimi, } 2015 \\
\text { Multimodal } \\
\text { Postintervention, } 0 \\
\text { weeks } \\
\text { Poor }\end{array}$ & $\begin{array}{l}\text { A. Whole body } \\
\text { vibration + low } \\
\text { intensity exercise: } 30 \\
\text { sessions over } 10 \\
\text { weeks }(n=16) \\
\text { B. Usual care }(n=14)\end{array}$ & $\begin{array}{l}\text { A vs. B (Data are for those } \\
\text { with complete followup data) } \\
\text { Age (mean years): } 37.06 \text { vs. } \\
40.75 \\
\text { Female: } 11(69 \%) \text { vs. } 12 \\
(86 \%) \\
\text { Race: NR } \\
\text { Ambulatory: } 16(100 \%) \text { vs. } \\
14(100 \%) \\
\text { Wheelchair user: NR } \\
\text { Baseline EDSS: } 3.12 \text { vs. } \\
3.10 \\
\text { Use of disease-modifying } \\
\text { drugs: } 10 \text { (62.5\%) vs. } 8 \\
\text { (57.1\%) } \\
\text { Duration of disease (mean } \\
\text { years): } 6.5 \text { vs. } 10.5\end{array}$ & $\begin{array}{l}\text { Data reported as mean (SD) } \\
\text { A vs. B } \\
\text { TUG (seconds) } \\
11.32(5.21) \text { vs. } 14.43 \text { (3.20) (baseline) } \\
11.16(8.82) \text { vs. } 14.57 \text { (4.02) (postintervention) } \\
\text { Group p=0.05 } \\
\text { 10MWT (seconds) } \\
17.67 \text { (8.92) vs. } 21.16(6.36) \text { (baseline) } \\
13.37 \text { (4.59) vs. } 19.39 \text { (6.52) (postintervention) } \\
\text { Group p=0.56 } \\
6 \text { MWT (meters) } \\
184.01 \text { (101.04) vs. } 150.37 \text { (65.18) (baseline) } \\
272.32(105.60) \text { vs. } 162.80 \text { (60.57) (postintervention) } \\
47.99 \% \text { vs. NR (pre-post } \% \text { change) } \\
\text { Group p=0.01 } \\
\text { MSQ0L-54-PCS (0-100, higher=increased QOL) } \\
45.80 \pm 9.70 \text { vs. } 43.38 \pm 15.43 \text { (baseline) } \\
53.36 \pm 11.9 \text { vs. } 45.53 \pm 7.30 \text { (postintervention) } \\
\text { Group p=0.40 } \\
\text { MSQOL-54-MCS }(0-100, \text { higher=increased QOL) } \\
50.87 \pm 15.46 \text { vs. } 41.66 \pm 17.07 \text { (baseline) } \\
58.34 \pm 14.89 \text { vs. } 50.10 \pm 14.72 \text { (postintervention) } \\
\text { Group p=0.42 }\end{array}$ & $\begin{array}{l}\text { Data reported as mean (SD) } \\
\text { A vs. B } \\
\text { Modified pushup } \\
5.31(4.75) \text { vs. } 2.42(3.99) \text { (baseline) } \\
12.12(6.54) \text { vs. } 2.92(3.83) \text { (postintervention) } \\
\text { Group p=0.07 } \\
\text { EDSS }(1-10, \text { higher scores=greater disability) } \\
3.12(1.19) \text { vs. } 3.10(0.76) \text { (baseline) } \\
2.65(1.20) \text { vs. } 3.03 \text { (0.69) (postintervention) } \\
-15.06 \% \text { vs. NR (pre-post \% change) } \\
\text { Group p=0.01 } \\
\text { BBS }(0-56, \text { higher scores=better balance) } \\
40.37(9.97) \text { vs. } 34.00(9.13) \text { (baseline) } \\
46.43(8.34) \text { vs. } 35.85(7.22) \text { (postintervention) } \\
15.00 \% \text { vs. NR (pre-post \% change) } \\
\text { Group p=0.01 }\end{array}$ \\
\hline
\end{tabular}




\begin{tabular}{|c|c|c|c|c|}
\hline $\begin{array}{l}\text { Author, Year } \\
\text { Intervention Type } \\
\text { Duration of } \\
\text { Postintervention } \\
\text { Followup } \\
\text { Quality } \\
\text { (See Appendix B } \\
\text { for Full Citation) }\end{array}$ & $\begin{array}{l}\text { Intervention and } \\
\text { Comparison }\end{array}$ & $\begin{array}{l}\text { Population } \\
\text { Age (Mean) } \\
\text { Gender (\% Female) } \\
\text { Race (\%) } \\
\text { Ambulatory (\%) } \\
\text { Wheelchair User (\%) } \\
\text { Condition Specific (\%) } \\
\text { Other (\%) }\end{array}$ & Prioritized Outcomes & Other Outcomes \\
\hline $\begin{array}{l}\text { Elnaggar } 2019 \\
\text { Strength } \\
\text { Plyometric training } \\
\text { Postintervention, } 8 \\
\text { weeks } \\
\text { Fair }\end{array}$ & $\begin{array}{l}\text { A. Plyometric } \\
\text { training, } 16 \text { sessions } \\
\text { over } 8 \text { weeks }(n=19) \\
\text { B. Usual care }(n=20)\end{array}$ & $\begin{array}{l}\text { Age: } 9.47 \text { vs. } 10.3 \\
\text { Female: } 32 \% \text { vs. } 45 \% \\
\text { Race: NR } \\
\text { Abulatory: } 100 \% \text { vs. } 100 \% \\
\text { Wheelchair user: NR } \\
\text { All patients were considered } \\
\text { to have mildly spastic CP }\end{array}$ & $\begin{array}{l}\text { A vs. B, Mean (SD) } \\
\text { [pre-post change score and MD calculated by EPC] } \\
\text { 10MWT (m/s): } \\
1.18(0.08) \text { vs. } 1.21(0.09) \text { (baseline) } \\
1.29(0.06) \text { vs. } 1.25(0.05) \text { (post-intervention) } \\
0.11(0.05) \text { vs. } 0.04(0.06), \text { MD } 0.07 \text { (95\% Cl } 0.04 \text { to } 0.10) \\
\text { (pre-post change score) }\end{array}$ & NA \\
\hline $\begin{array}{l}\text { El-Shamy, } 2018 \\
\text { Postural Control } \\
\text { Motion gaming } \\
\text { Postintervention, } \\
12 \text { weeks } \\
\text { Fair }\end{array}$ & $\begin{array}{l}\text { A. Robotic upper- } \\
\text { limb therapy, } 36 \\
\text { sessions over } 12 \\
\text { weeks ( } n=15) \\
\\
\text { B. Conventional } \\
\text { therapy of stretching } \\
\text { and strength } \\
\text { exercises, } 36 \\
\text { sessions over } 12 \\
\text { weeks ( } n=15)\end{array}$ & $\begin{array}{l}\text { A vs. B } \\
\text { Age: } 6.9 \text { vs. } 6.8 \\
\text { Female: } 40 \% \text { vs. } 27 \% \\
\text { MACS I: } 33 \% \text { vs. } 40 \% \\
\text { MACS II: } 53 \% \text { vs. } 40 \% \\
\text { MACS III: } 13 \% \text { vs. } 20 \%\end{array}$ & $\begin{array}{l}\text { A vs. B, Mean (SD), } p=\text { between groups } \\
\text { Spasticity MAS: } \\
-0.4,95 \% \mathrm{Cl}-0.8 \text { to }-0.1, p<0.05 \\
\text { QUEST total: } \\
61.9 \text { (2) to } 84.6(2.7) \text { vs. } 62.3(1.8) \text { to } 79.1 \text { (2); MD } 5.9 \text {, } \\
95 \% \mathrm{Cl} 3.7 \text { to } 7.3, p<0.05\end{array}$ & NA \\
\hline
\end{tabular}




\begin{tabular}{|c|c|c|c|c|}
\hline $\begin{array}{l}\text { Author, Year } \\
\text { Intervention Type } \\
\text { Duration of } \\
\text { Postintervention } \\
\text { Followup } \\
\text { Quality } \\
\text { (See Appendix B } \\
\text { for Full Citation) }\end{array}$ & $\begin{array}{l}\text { Intervention and } \\
\text { Comparison }\end{array}$ & $\begin{array}{l}\text { Population } \\
\text { Age (Mean) } \\
\text { Gender (\% Female) } \\
\text { Race (\%) } \\
\text { Ambulatory (\%) } \\
\text { Wheelchair User (\%) } \\
\text { Condition Specific (\%) } \\
\text { Other (\%) }\end{array}$ & Prioritized Outcomes & Other Outcomes \\
\hline $\begin{array}{l}\text { Emara, } 2016 \\
\text { Aerobic Exercise } \\
\text { Treadmill } \\
\text { Postintervention, } \\
12 \text { weeks } \\
\text { Fair }\end{array}$ & $\begin{array}{l}\text { A. Treadmill training, } \\
36 \text { sessions over } 12 \\
\text { weeks }(n=11) \\
\\
\text { B. Suspension } \\
\text { training, (dynamic } \\
\text { spider cage) } 36 \\
\text { sessions over } 12 \\
\text { weeks }(n=11)\end{array}$ & $\begin{array}{l}\text { A vs. B } \\
\text { Age (mean years): } 6.6 \text { vs. } \\
6.9 \\
\text { Female: } 7 \text { (64\%) vs. } 6 \text { (55\%) } \\
\text { Race: NR } \\
\text { Ambulatory: NR } \\
\text { Wheelchair user: NR } \\
\text { Baseline GMFM-d: gross } \\
\text { motor functional measure } \\
\text { dimension D (standing): } \\
\text { GMFM-d: } 12 \text { (1.6) vs. } 12.0 \\
\text { (0.7) } \\
\text { Baseline GMFM-e: gross } \\
\text { motor function measure } \\
\text { dimension E (walking): } \\
\text { GMFM-e: } 10.9 \text { (1.3) vs. } 10.4 \\
\text { (0.8) } \\
\text { Baseline 10-m Walking Test: } \\
0.4 \text { (0.04) vs. } 0.4 \text { (0.03) } \\
\text { Baseline Five times sit to } \\
\text { stand: } \\
\text { 21.5 (1.3) vs. } 21.7 \text { (1.5) }\end{array}$ & $\begin{array}{l}\text { A vs. B, mean (SD) } \\
6 \text { weeks (18-sessions) } \\
\text { GMFM-d: } 13.7(1.2) \text { vs. } 15.3(1.9), p=0.04 \\
\text { GMFM-e: } 13.2 \text { (1.9 vs. } 14.3(1.9), p=0.21 \\
\text { 10-m Walking Test: } 0.4(0.05) \text { vs. } 0.5(0.04), p=0.12 \\
\text { Five times sit to stand: } 20.1 \text { (1.0 vs. } 19.5(0.9), p=0.26 \\
\text { 12 weeks (36-sessions) } \\
\text { GMFM-d: } 15.8(1.5) \text { vs. } 19.2(2.1), p=0.001 \\
\text { GMFM-e: } 14.8(1.5) \text { vs. } 17.2(2.1), p=0.008 \\
\text { 10-m Walking Test: } 0.5(0.04) \text { vs. } 0.6(0.04) \\
\text { Five times sit to stand: } 18.9(1.0) \text { vs. } 17.7(0.8)\end{array}$ & NR \\
\hline
\end{tabular}




\begin{tabular}{|c|c|c|c|c|}
\hline $\begin{array}{l}\text { Author, Year } \\
\text { Intervention Type } \\
\text { Duration of } \\
\text { Postintervention } \\
\text { Followup } \\
\text { Quality } \\
\text { (See Appendix B } \\
\text { for Full Citation) } \\
\end{array}$ & $\begin{array}{l}\text { Intervention and } \\
\text { Comparison }\end{array}$ & $\begin{array}{l}\text { Population } \\
\text { Age (Mean) } \\
\text { Gender (\% Female) } \\
\text { Race (\%) } \\
\text { Ambulatory (\%) } \\
\text { Wheelchair User (\%) } \\
\text { Condition Specific (\%) } \\
\text { Other (\%) }\end{array}$ & Prioritized Outcomes & Other Outcomes \\
\hline $\begin{array}{l}\text { Esclarin-Ruz, } 2014 \\
\text { Aerobic Exercise } \\
\text { Robot-Assisted } \\
\text { Gait Training } \\
\text { Postintervention, } 8 \\
\text { weeks } \\
\text { Fair }\end{array}$ & $\begin{array}{l}\text { A. Robotic locomotor } \\
\text { training plus } \\
\text { overground therapy } \\
40 \text { sessions over } 8 \\
\text { weeks }(n=44) \\
\text { B. Overground } \\
\text { therapy, } 40 \text { sessions } \\
\text { over } 8 \text { weeks }(n=44)\end{array}$ & $\begin{array}{l}\text { A vs. B } \\
\text { Age (mean years): } \\
\text { UMN injury: } 43.6 \text { vs. } 44.9 \\
\text { LMN injury: } 36.4 \text { vs. } 42.7 \\
\text { Female: } \\
\text { UMN } 29 \% \text { vs. } 29 \% \\
\text { LMN } 30 \% \text { vs. } 29 \% \\
\text { Race: NR } \\
\text { Ambulatory: } \\
\text { NR } \\
\text { Wheelchair user: } \\
\text { NR } \\
\text { Other: } \\
\text { N (\%) vs. N (\%) }\end{array}$ & $\begin{array}{l}\text { A vs. B, mean (SD) } \\
\text { 10MWT } \\
\text { UMN: } 0.48(0.25) \text { to } 0.54(0.31) \text { vs. } 0.36(0.25) \text { to } 0.39 \\
\text { (0.31) } \\
\text { LMN: } 0.24(0.11) \text { to } 0.46(0.25) \text {, vs. } 0.28(0.27) \text { to } 0.45 \\
(0.25) \\
\text { p=0.09 } \\
\text { 6MWT } \\
\text { UMN: } 122.3(49.2) \text { to } 187.48(103.78) \text { vs. } 93.3(53.1) \text { to } \\
\text { 119.41 }(89.25) \\
\text { LMN: } 82.7(45.5) \text { to } 157.54(89.51) \text { vs. } 94.3(75.1) \text { to } \\
\text { 145.62 }(125.15) \\
\text { PGIC Scale } \\
\text { UMN: } \\
\text { LMN: } \\
\text { LEMS } \\
\text { UMN: } 30(10.4) \text { to } 38.33(10.6) \text { vs. } 27(10.9) \text { to } 32.28 \\
(11.04) \\
\text { LMN: } 21(10.3) \text { to } 27.15(11.10) \text { vs. } 20(9.9) \text { to } 22.57 \\
\text { (10.8) } \\
\text { WISCI-II } \\
\text { UMN: } 5.9(4.5) \text { to } 13.47(5.65) \text { vs. } 4.9(4.1) \text { to } 11.04(5.09) \\
\text { LMN: } 6(3.2) \text { to } 12.45(4.17) \text { vs. } 5(3.7) \text { to } 10.8(4.54) \\
\text { FIM/Motor } \\
\text { UMN: } 5(2.7) \text { to } 8.95(2.96) \text { vs. } 4.9(4.1) \text { to } 7.05(2.62) \\
\text { LMN: } 6(2.9) \text { to } 8.9(2.61) \text { vs. } 5(2.8) \text { to } 8.67(2.65)\end{array}$ & A vs. B, mean (SD) \\
\hline
\end{tabular}




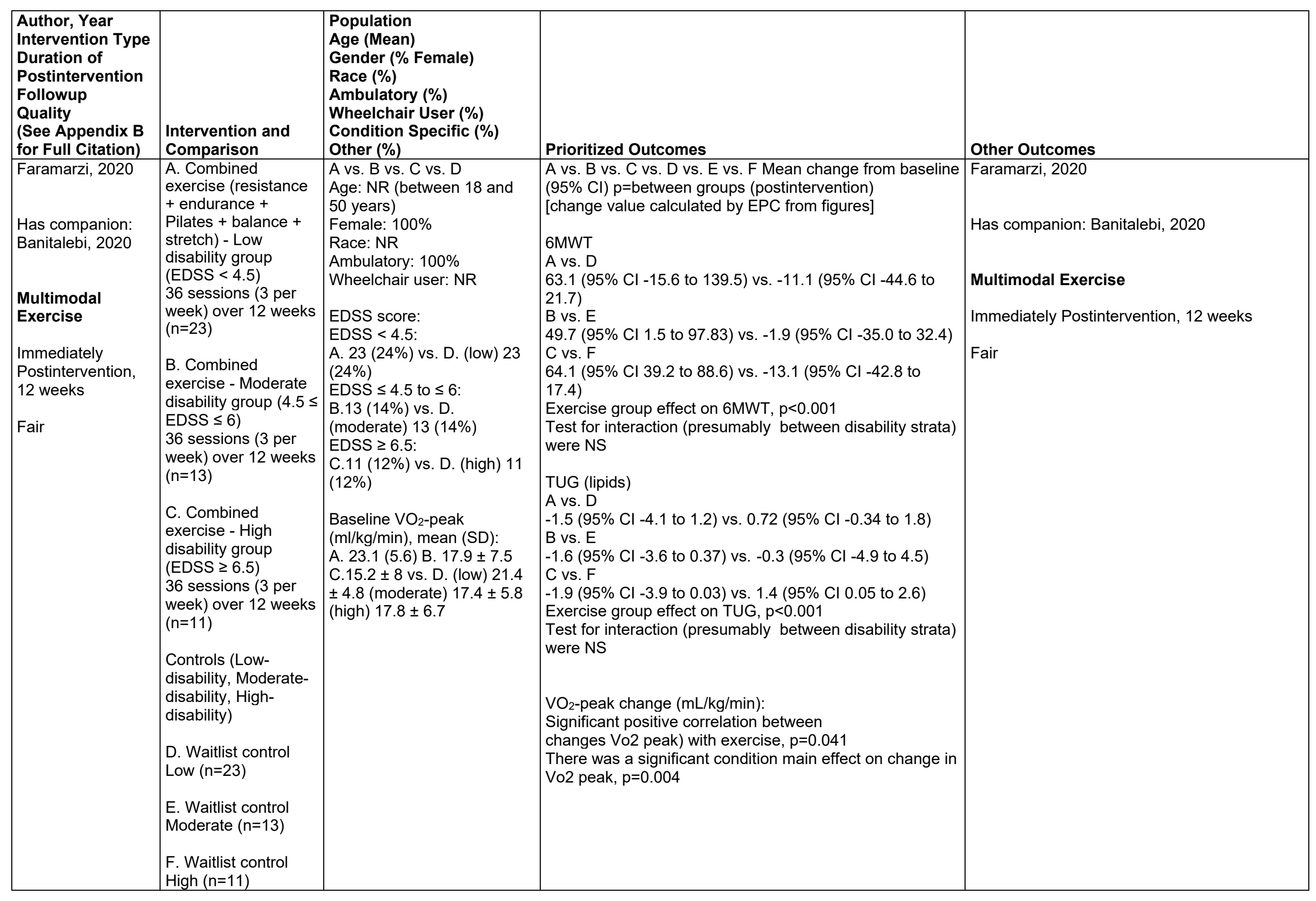




\begin{tabular}{|c|c|c|c|c|}
\hline $\begin{array}{l}\text { Author, Year } \\
\text { Intervention Type } \\
\text { Duration of } \\
\text { Postintervention } \\
\text { Followup } \\
\text { Quality } \\
\text { (See Appendix B } \\
\text { for Full Citation) } \\
\end{array}$ & $\begin{array}{l}\text { Intervention and } \\
\text { Comparison }\end{array}$ & $\begin{array}{l}\text { Population } \\
\text { Age (Mean) } \\
\text { Gender (\% Female) } \\
\text { Race (\%) } \\
\text { Ambulatory (\%) } \\
\text { Wheelchair User (\%) } \\
\text { Condition Specific (\%) } \\
\text { Other (\%) }\end{array}$ & Prioritized Outcomes & Other Outcomes \\
\hline $\begin{array}{l}\text { Field-Fote, } 2011 \\
\text { Has companions: } \\
\text { Kressler, } 2013 \\
\text { Sandler, } 2017 \\
\text { Aerobic Exercise } \\
\text { Robot-Assisted } \\
\text { Gait Training } \\
\text { Postintervention, } \\
12 \text { weeks } \\
\text { Fair }\end{array}$ & $\begin{array}{l}\text { A. Treadmill BWS } \\
\text { Training with Manual } \\
\text { Assistance, } 60 \\
\text { sessions over } 12 \\
\text { weeks ( } n=17 \text { ) (TM) } \\
\\
\text { B. Treadmill BWS } \\
\text { Training with } \\
\text { Electrical Stimulation, } \\
60 \text { sessions over } 12 \\
\text { weeks ( } n=18 \text { ) (TS) } \\
\text { C. Overground BWS } \\
\text { Training with } \\
\text { Electrical Stimulation, } \\
60 \text { sessions over } 12 \\
\text { weeks ( } n=15 \text { ) (OG) } \\
\text { D. Treadmill BWS } \\
\text { Training with Robotic } \\
\text { Assistance, 60 } \\
\text { sessions over 12 } \\
\text { weeks ( } n=14 \text { ) (LR) }\end{array}$ & $\begin{array}{l}\text { A vs. B } \\
\text { Age (mean years): } \\
39.3 \text { vs. } 38.5 \text { vs. } 42.2 \text { vs. } 45 \\
\text { Female: } \\
17.7 \% \text { vs. } 22.2 \% \text { vs. } 13.9 \% \\
\text { vs. } 18 \% \\
\text { Race: } \\
\text { White or non-Hispanic } \\
58.8 \% \text { vs. } 44.4 \% \text { vs. } 40.0 \% \\
\text { vs. } 42.9 \% \\
\text { Hispanic } 29.4 \% \text { vs. } 38.9 \% \\
\text { vs. } 40 \% \text { vs. } 35.7 \% \\
\text { African American } 11.8 \% \text { vs. } \\
16.7 \% \text { vs. } 20 \% \text { vs. } 21.4 \% \\
\text { Ambulatory: } \\
\text { NR } \\
\text { Wheelchair user: } \\
\text { NR } \\
\text { Other: } \\
2 \text { min walk, in meters } \\
\text { TM: } 22.1(21.4) \\
\text { TS: } 20.6(23.1) \\
\text { OG: } 24.0 \text { (35.3) } \\
\text { LR:16.8 (11.3) } \\
\text { Short distance overground } \\
\text { walking speed, m/s } \\
0.17 \text { (0.14) vs. } 0.18 \text { (0.18) } \\
\text { vs. } 0.19(0.20) \text { vs. } 0.17 \\
\text { (0.10) }\end{array}$ & $\begin{array}{l}\text { A vs. } \mathrm{B}, \text { mean (SD) } \\
\text { Short distance over ground speed, change in } \mathrm{m} / \mathrm{s} \\
\text { TM: } 0.04(0.07) \\
\text { TS: } 0.05(0.09) \\
\text { OG: } 0.09(0.11) \\
\text { LR: } 0.01 \text { (0.05) } \\
\text { Distance walked (2 min), change in meters } \\
\text { TM:0.8 (7.7) } \\
\text { TS: } 3.8(6.3) \\
\text { OG: } 14.2(15.2) \\
\text { LR: } 1.2(5.1) \\
\\
\text { LEMS, left leg, change in score } \\
\text { TM: } 1.7(1.8) \\
\text { TS: } 1.5(2.7) \\
\text { OG: } 1.1(1.5) \\
\text { LR: } 1.2(3.2) \\
\text { LEMS, right leg, change in score } \\
\text { TM: } 1.5(2.1) \\
\text { TS: } 1.6(2.0) \\
\text { OG: } 1.7(2.3) \\
\text { LR: } 1.3(1.5)\end{array}$ & A vs. B, mean (SD) \\
\hline
\end{tabular}




\begin{tabular}{|c|c|c|c|c|}
\hline $\begin{array}{l}\text { Author, Year } \\
\text { Intervention Type } \\
\text { Duration of } \\
\text { Postintervention } \\
\text { Followup } \\
\text { Quality } \\
\text { (See Appendix B } \\
\text { for Full Citation) }\end{array}$ & $\begin{array}{l}\text { Intervention and } \\
\text { Comparison }\end{array}$ & $\begin{array}{l}\text { Population } \\
\text { Age (Mean) } \\
\text { Gender (\% Female) } \\
\text { Race (\%) } \\
\text { Ambulatory (\%) } \\
\text { Wheelchair User (\%) } \\
\text { Condition Specific (\%) } \\
\text { Other (\%) }\end{array}$ & Prioritized Outcomes & Other Outcomes \\
\hline $\begin{array}{l}\text { Forsberg, } 2016 \\
\text { Postural Control } \\
\text { Balance } \\
\text { Postintervention, } 8 \\
\text { weeks } \\
\text { Fair }\end{array}$ & $\begin{array}{l}\text { A. CoDuSe balance } \\
\text { exercises: } 14 \\
\text { sessions over } 7 \\
\text { weeks }(n=35) \\
\begin{array}{l}\text { B. Usual care control: } \\
(n=38)\end{array}\end{array}$ & $\begin{array}{l}\text { A vs. B } \\
\text { Age (mean years): } 52 \text { vs. } 56 \\
\text { Female: } 28 \text { (80\%) vs. } 31 \\
\text { (82\%) } \\
\text { Race: NR } \\
\text { Ambulatory: NR } \\
\text { Wheelchair use/assistive } \\
\text { walking device: } 18 \text { (51\%) vs. } \\
26 \text { (68\%) } \\
\text { Wheelchair use/assistive } \\
\text { walking device indoors: } 5 \\
\text { (14\%) vs. } 7 \text { (18\%) } \\
\text { MSIS physical subscale (0- } \\
100): 54 \text { (18\%) vs. } 56(14 \%) \\
\text { MSIS psychosocial subscale } \\
(0-100): 22 \text { (10\%) vs. } 22 \\
(8 \%) \\
\text { Type of MS - Relapsing- } \\
\text { remitting: } 20 \text { (57\%) } 13 \text { (34\%) } \\
\text { Type of MS -Primary } \\
\text { progressive: } 4 \text { (11\%) vs. } 5 \\
\text { (13\%) } \\
\text { Type of MS -Secondary } \\
\text { progressive: } 11 \text { (31\%) vs. } 20 \\
\text { (53\%) }\end{array}$ & 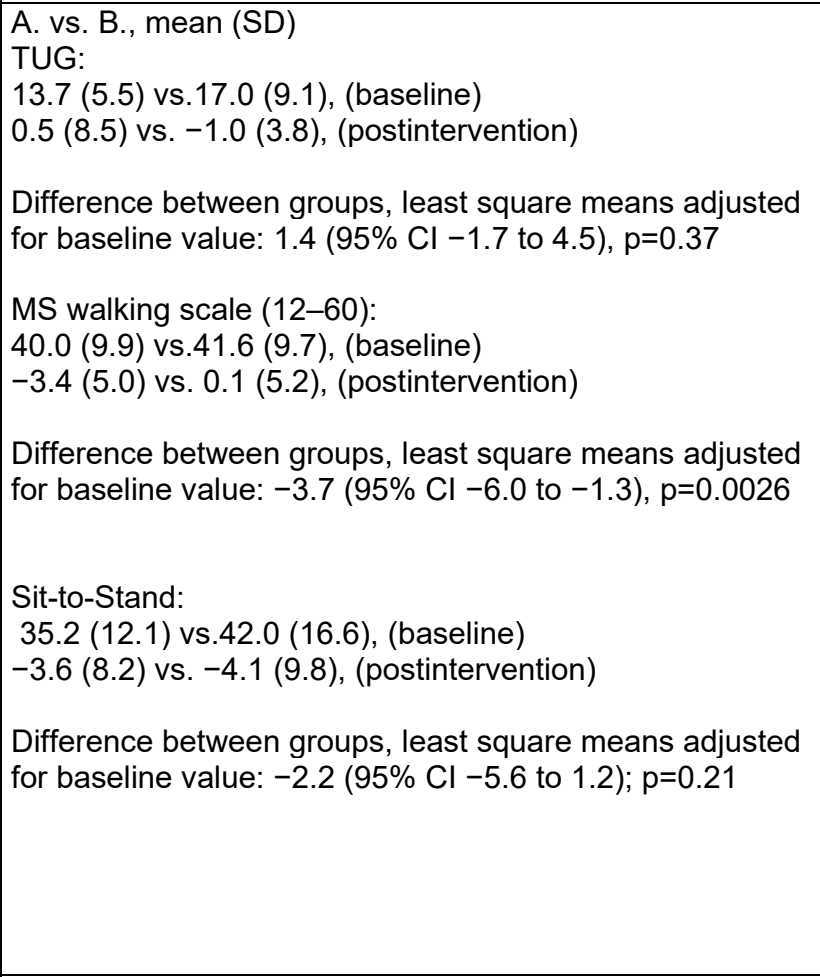 & $\begin{array}{l}\text { A. vs. B., mean (SD) } \\
\text { BBS: } \\
48.9(5.8) \text { vs. } 45.1(9.0) \text {, (baseline) } \\
2.6(4.1) \text { vs. } 1.6(4.1), \text { (postintervention) } \\
\\
\text { Difference between groups, least square } \\
\text { means adjusted for baseline value: } 2.1 \text { (95\% } \\
\text { Cl } 0.5 \text { to } 3.8) ; p=0.011\end{array}$ \\
\hline $\begin{array}{l}\text { Fosdahl, 2019b } \\
\text { Multimodal } \\
\text { Exercise } \\
\text { Postintervention, } \\
16 \text { weeks and } 32 \\
\text { weeks } \\
\text { Fair }\end{array}$ & $\begin{array}{l}\text { A. Strength training } \\
\text { (progressive } \\
\text { resistance exercise) } \\
+ \text { stretching, } 48 \\
\text { sessions over } 16 \\
\text { weeks }(n=17) \\
\text { B. Usual care }(n=20)\end{array}$ & $\begin{array}{l}\text { A vs. B } \\
\text { Age: } 10.4 \text { vs. } 10.0 \\
\text { Female: } 59 \% \text { vs. } 30 \% \\
\text { Ambulatory: } 100 \% \\
\text { GMFM: } \\
\text { I: } 59 \% \text { vs. } 60 \% \\
\text { II: } 41 \% \text { vs. } 35 \% \\
\text { III: } 0 \% \text { vs. } 5 \%\end{array}$ & $\begin{array}{l}\text { A vs. B, Mean change score (SD) } \\
6 \mathrm{MWT} \text { (meters) } \\
-45.7 \text { (55.4) vs. }-55.4 \text { (55.5), adj. MD10.6 }(95 \% \mathrm{Cl}-29.3 \\
\text { to } 50.6), p=0.590 \text { (pre-post change) } \\
-51.1 \text { ( } 72.8) \text { vs. }-56.6 \text { (59.6), adj. MD } 7.2(95 \% \mathrm{Cl}-43.3 \\
\text { to } 57.7), p=0.772 \text { (16-week change) } \\
\text { GDI (Gait Deviation Index) } \\
-0.4(4.4) \text { vs. }-0.8 \text { (7.14), adj. MD }-1.0(95 \% \mathrm{Cl}-5.3 \text { to } \\
3.3), p=0.650 \text { (pre-post change) } \\
-0.7(6.0) \text { vs. } 1.01 \text { (5.9), adj. MD }-1.4(95 \% \mathrm{Cl}-5.6 \text { to } \\
2.8), p=0.504 \text { (16-week change) }\end{array}$ & NA \\
\hline
\end{tabular}




\begin{tabular}{|c|c|c|c|c|}
\hline $\begin{array}{l}\text { Author, Year } \\
\text { Intervention Type } \\
\text { Duration of } \\
\text { Postintervention } \\
\text { Followup } \\
\text { Quality } \\
\text { (See Appendix B } \\
\text { for Full Citation) } \\
\end{array}$ & $\begin{array}{l}\text { Intervention and } \\
\text { Comparison }\end{array}$ & $\begin{array}{l}\text { Population } \\
\text { Age (Mean) } \\
\text { Gender (\% Female) } \\
\text { Race (\%) } \\
\text { Ambulatory (\%) } \\
\text { Wheelchair User (\%) } \\
\text { Condition Specific (\%) } \\
\text { Other (\%) }\end{array}$ & Prioritized Outcomes & Other Outcomes \\
\hline $\begin{array}{l}\text { Fowler, } 2010 \\
\text { Has companion: } \\
\text { Demuth, } 2012 \\
\text { Aerobic Exercise } \\
\text { Cycling } \\
\text { Postintervention, } 0 \\
\text { weeks } \\
\text { Fair }\end{array}$ & $\begin{array}{l}\text { A. Stationary cycling } \\
30 \text { sessions over } 12 \\
\text { weeks }(n=29) \\
\text { B. } \\
\text { Control No } \\
\text { Intervention }(n=29)\end{array}$ & $\begin{array}{l}\text { A vs. B } \\
\text { Age (mean years): } \\
11.1 \text { vs. } 11.6 \\
\text { Female: } \\
13(42 \%) \text { vs. } 20(64 \%) \\
\text { Ambulatory: } \\
31(100 \%) \text { vs. } 31(100 \%) \\
\text { Wheelchair user: NR } \\
\text { Race: } \\
\text { African American } \\
5(16 \%) \text { vs. } 3(10 \%) \\
\text { White } \\
18(58 \%) \text { vs. } 15(48 \%) \\
\text { Asian } \\
1(3 \%) \text { vs. } 5(16 \%) \\
\text { Other } \\
7(23 \%) \text { vs. } 8(26 \%) \\
\text { CP subtype NR }\end{array}$ & $\begin{array}{l}\text { A vs. B (SD) } \\
\text { GMFM-66 } \\
69.6 \text { (NR) vs. } 68.8 \text { (NR) (baseline) } \\
70.8 \text { (NR) vs. } 69.3 \text { (NR); } p=0.002 \text { in A (postintervention) } \\
600 \text { yard walk-run test speed (m/min) } \\
85.0 \text { (NR) vs. } 81.6 \text { (NR) (baseline) } \\
90.6 \text { (NR) vs. } 84.1 \text { (NR); } p=0.008 \text { in A (postintervention) } \\
30 \text { sec walk test speed (m/min): NS }\end{array}$ & NA \\
\hline
\end{tabular}




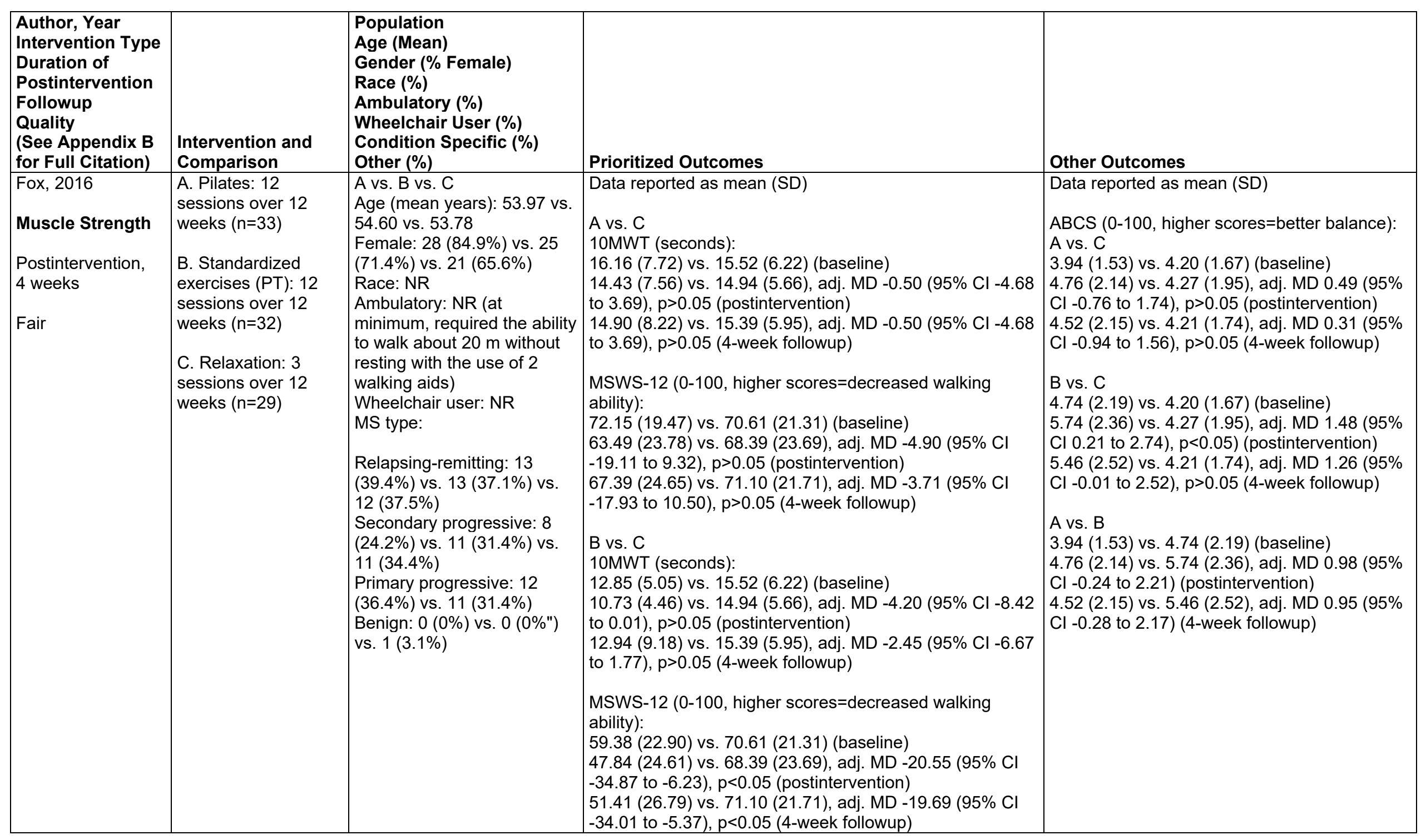




\begin{tabular}{|c|c|c|c|c|}
\hline $\begin{array}{l}\text { Author, Year } \\
\text { Intervention Type } \\
\text { Duration of } \\
\text { Postintervention } \\
\text { Followup } \\
\text { Quality } \\
\text { (See Appendix B } \\
\text { for Full Citation) } \\
\end{array}$ & $\begin{array}{l}\text { Intervention and } \\
\text { Comparison }\end{array}$ & \begin{tabular}{|l|} 
Population \\
Age (Mean) \\
Gender (\% Female) \\
Race (\%) \\
Ambulatory (\%) \\
Wheelchair User (\%) \\
Condition Specific (\%) \\
Other (\%) \\
\end{tabular} & Prioritized Outcomes & Other Outcomes \\
\hline $\begin{array}{l}\text { Fox, } 2016 \\
\text { (Continued) }\end{array}$ & & & 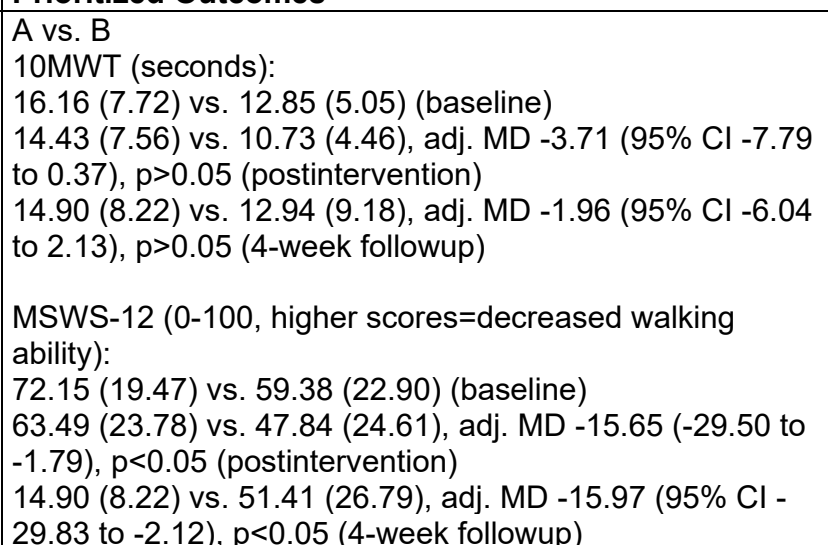 & \\
\hline
\end{tabular}




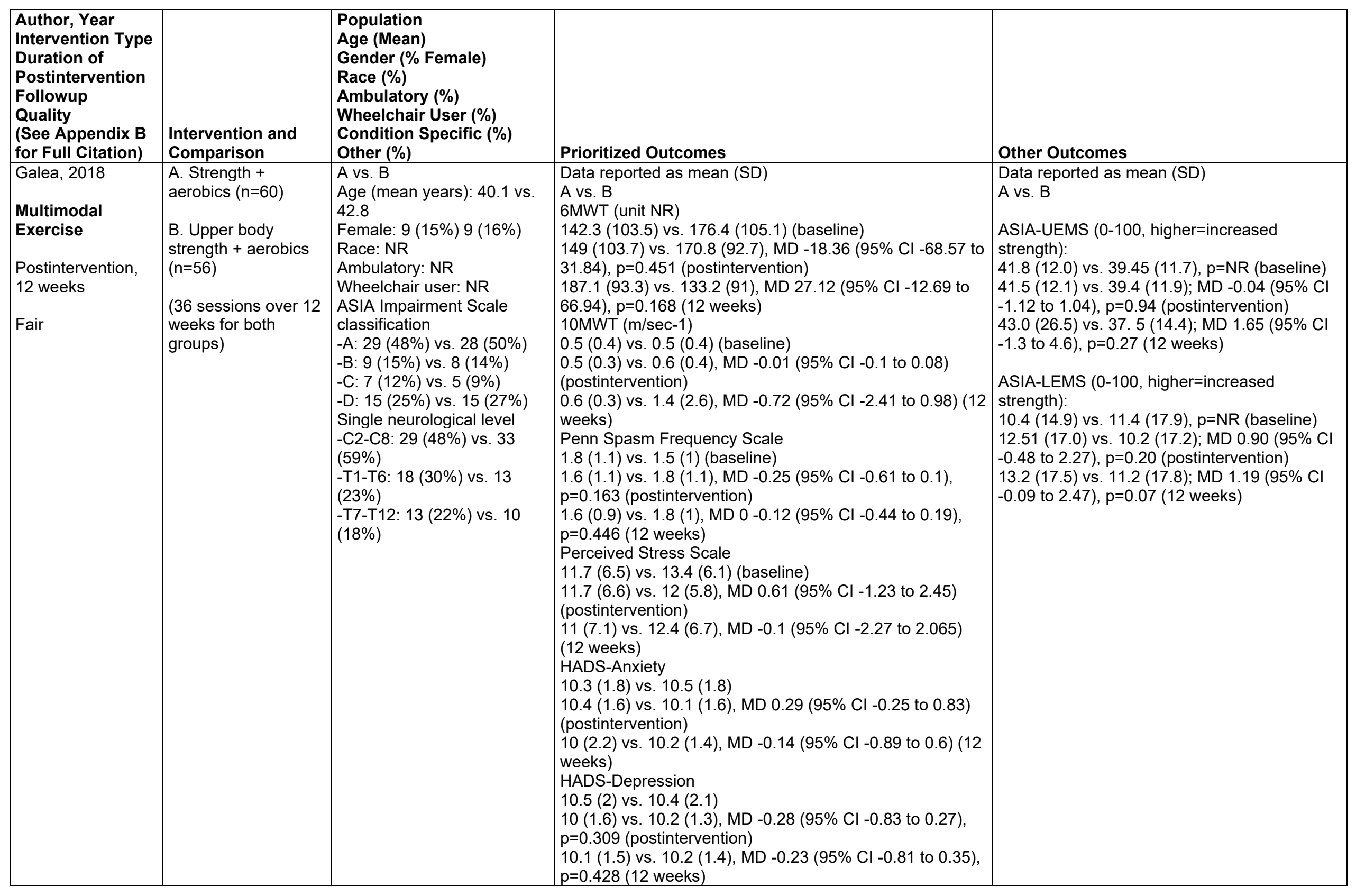




\begin{tabular}{|c|c|c|c|c|}
\hline $\begin{array}{l}\text { Author, Year } \\
\text { Intervention Type } \\
\text { Duration of } \\
\text { Postintervention } \\
\text { Followup } \\
\text { Quality } \\
\text { (See Appendix B } \\
\text { for Full Citation) } \\
\end{array}$ & $\begin{array}{l}\text { Intervention and } \\
\text { Comparison }\end{array}$ & $\begin{array}{l}\text { Population } \\
\text { Age (Mean) } \\
\text { Gender (\% Female) } \\
\text { Race (\%) } \\
\text { Ambulatory (\%) } \\
\text { Wheelchair User (\%) } \\
\text { Condition Specific (\%) } \\
\text { Other (\%) }\end{array}$ & Prioritized Outcomes & Other Outcomes \\
\hline $\begin{array}{l}\text { Gandolfi, } 2015 \\
\text { Postural Control } \\
\text { Balance } \\
\text { RCT } \\
\text { Fair }\end{array}$ & $\begin{array}{l}\text { A. Balance training } \\
\text { (sensory integration), } \\
15 \text { sessions over } 5 \\
\text { weeks ( } n=39) \\
\\
\text { B. Conventional } \\
\text { rehabilitation, } 15 \\
\text { sessions over } 5 \\
\text { weeks ( } n=41)\end{array}$ & $\begin{array}{l}\text { A vs. B } \\
\text { Age (mean years): } 47.21 \text { vs. } \\
49.56 \\
\text { Female: } 28 \text { (72\%) vs. } 31 \\
(76 \%) \\
\text { Race: NR } \\
\text { Ambulatory: NR } \\
\text { Wheelchair user: NR } \\
\text { EDSS score (median) } 3.00 \\
\text { vs. } 3.66 \\
\text { Q1-Q3: } 2-4 \text { vs. } 2.50-4.25 \\
\text { MS duration (mean years): } \\
12.25 \text { vs. } 15.24\end{array}$ & $\begin{array}{l}\text { A. vs. B., mean (SD) } \\
\text { MSQOL-54 (0-100; higher=better performance) } \\
63.09(11.09) \text { vs. } 58.77(11.05) \text { (baseline) } \\
65.56(10.31) \text { vs. } 59.64(9.80) \text { (postintervention) } \\
63.56(10.27) 58.54 \text { vs. }(11.64)(1 \text {-month followup) } \\
\text { Between-group difference }(95 \% \mathrm{Cl}) \text { mean: } \\
\text { Before: } 4.32(95 \% \mathrm{Cl}-0.61 \text { to } 9.25) \\
\text { After: } 5.92(95 \% \mathrm{Cl} 1.44 \text { to } 10.40) \\
\text { Followup: } 5.02(95 \% \mathrm{Cl}-1.12 \text { to } 9.92), \mathrm{p}<0.001 \\
\text { Number of falls } \\
0.59 \text { (.99) vs. } 0.37(0.54) \text { (baseline) } \\
0.03(0.16) \text { vs. } 0.29(0.34) \text { (postintervention) } \\
0.08(0.27) \text { vs. } 0.27(0.55)(1-\text { month followup) } \\
\text { Between-group difference }(95 \% \mathrm{Cl}) \text { mean: } \\
\text { Before: } 0.22(95 \% \mathrm{Cl}-0.129 \text { to } 0.577) \\
\text { After:. }-0.30(95 \% \mathrm{Cl}-0.452 \text { to }-0.08) \\
\text { Followup: }-0.191(95 \% \mathrm{Cl}-0.385 \text { to } 0.003)\end{array}$ & $\begin{array}{l}\text { A. vs. B., mean (SD) } \\
\text { BBS }(0-56, \text { higher=better performance) } \\
47.97(4.89) \text { vs. } 46.49(5.21) \text { (baseline) } \\
52.77(3.15) \text { vs. } 47.79(6.05) \text { (postintervention) } \\
52.92(2.97) \text { vs. } 48.33 \text { (5.88) (1-month } \\
\text { followup) } \\
\text { Between-group difference }(95 \% \mathrm{Cl}) \text { mean } \\
\text { before: } 1.49(95 \% \mathrm{Cl}-0.76 \text { to } 3.74) \text {, after: } 4.99 \\
(95 \% \mathrm{Cl} 2.83 \text { to } 7.15) \text { followup: } 4.60 \text { (95\% Cl } \\
2.50 \text { to } 6.69), p<0.001\end{array}$ \\
\hline
\end{tabular}




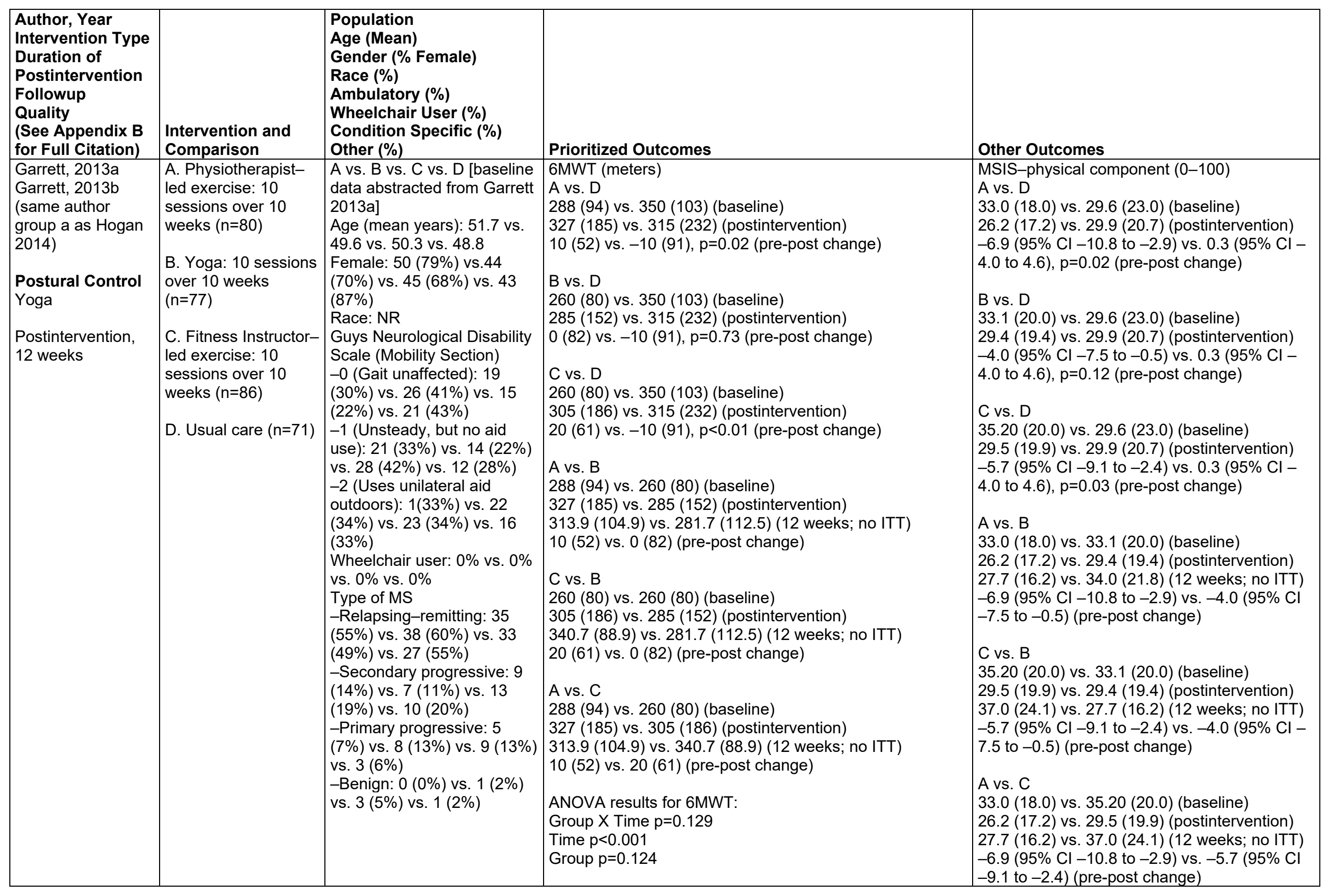




\begin{tabular}{|c|c|c|c|c|}
\hline $\begin{array}{l}\text { Author, Year } \\
\text { Intervention Type } \\
\text { Duration of } \\
\text { Postintervention } \\
\text { Followup } \\
\text { Quality } \\
\text { (See Appendix B } \\
\text { for Full Citation) } \\
\end{array}$ & $\begin{array}{l}\text { Intervention and } \\
\text { Comparison }\end{array}$ & $\begin{array}{l}\text { Population } \\
\text { Age (Mean) } \\
\text { Gender (\% Female) } \\
\text { Race (\%) } \\
\text { Ambulatory (\%) } \\
\text { Wheelchair User (\%) } \\
\text { Condition Specific (\%) } \\
\text { Other (\%) } \\
\end{array}$ & Prioritized Outcomes & Other Outcomes \\
\hline $\begin{array}{l}\text { Garrett, 2013a } \\
\text { Garrett, 2013b } \\
\text { (same author } \\
\text { group a as Hogan } \\
\text { 2014) } \\
\text { (Continued) }\end{array}$ & & & & 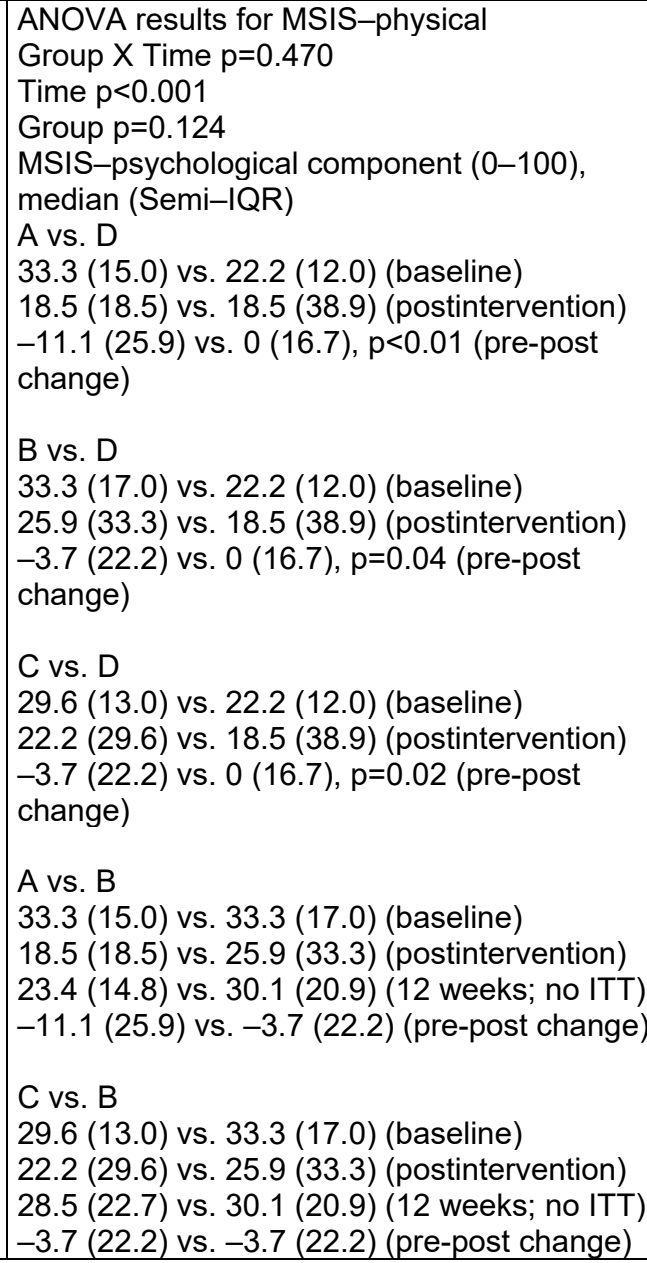 \\
\hline
\end{tabular}




\begin{tabular}{|c|c|c|c|c|}
\hline $\begin{array}{l}\text { Author, Year } \\
\text { Intervention Type } \\
\text { Duration of } \\
\text { Postintervention } \\
\text { Followup } \\
\text { Quality } \\
\text { (See Appendix B } \\
\text { for Full Citation) } \\
\end{array}$ & $\begin{array}{l}\text { Intervention and } \\
\text { Comparison }\end{array}$ & $\begin{array}{l}\text { Population } \\
\text { Age (Mean) } \\
\text { Gender (\% Female) } \\
\text { Race (\%) } \\
\text { Ambulatory (\%) } \\
\text { Wheelchair User (\%) } \\
\text { Condition Specific (\%) } \\
\text { Other (\%) }\end{array}$ & Prioritized Outcomes & Other Outcomes \\
\hline $\begin{array}{l}\text { Garrett, 2013a } \\
\text { Garrett, 2013b } \\
\text { (same author } \\
\text { group a as Hogan } \\
2014 \text { ) } \\
\text { (Continued) }\end{array}$ & & & & $\begin{array}{l}\text { A vs. C } \\
33.3(15.0) \text { vs. } 29.6(13.0) \text { (baseline) } \\
18.5(18.5) \text { vs. } 22.2(29.6) \text { (postintervention) } \\
23.4(14.8) \text { vs. } 28.5(22.7) \text { (12 weeks; no ITT) } \\
-11.1(25.9) \text { vs. }-3.7(22.2) \text { (pre-post change) } \\
\text { ANOVA results for MSIS-psychological } \\
\text { Time } X \text { Group } p=0.446 \\
\text { Time } p<0.0001 \\
\text { Group } p=0.246\end{array}$ \\
\hline $\begin{array}{l}\text { Gervasoni } 2014 \\
\text { Aerobic Exercise } \\
\text { Treadmill } \\
\text { Postintervention, } 0 \\
\text { weeks } \\
\text { Fair }\end{array}$ & $\begin{array}{l}\text { A. Treadmill } 30 \\
\text { minutes }+15 \text { minutes } \\
\text { conventional physical } \\
\text { therapy, } 12 \text { sessions } \\
\text { over } 2 \text { weeks } \\
\text { B. } 45 \text { minutes } \\
\text { conventional physical } \\
\text { therapy }\end{array}$ & $\begin{array}{l}\mathrm{n}=30 \\
18 \text { male } / 12 \text { female }(60 \% \\
\text { male) } \\
\text { Mean age }=48.75 \text { years } \\
\text { (range NR) } \\
\text { EDSS } \\
\text { Mean } 5.25 \\
(3-6.5)\end{array}$ & $\begin{array}{l}\text { pre, post } \\
\text { Dynamic Gait Index (walking + balance) } \\
\text { A. } 15.38 \text { to } 12.54 \\
\text { B. } 16.00 \text { to } 18.07 \\
\text { p=0.51 } \\
\text { Positive and Negative Affect Schedule } \\
\text { Positive } \\
\text { A. } 29.0 \text { to } 30.0 \\
\text { B. } 28.0 \text { to } 33.0 \\
\text { p=0.89 } \\
\text { Negative } \\
\text { A. } 26.0 \text { to } 21.0 \\
\text { B. } 23.0 \text { to } 21.0 \\
\text { p=0.48 }\end{array}$ & NA \\
\hline
\end{tabular}




\begin{tabular}{|c|c|c|c|c|}
\hline \begin{tabular}{l|} 
Author, Year \\
Intervention Type \\
Duration of \\
Postintervention \\
Followup \\
Quality \\
(See Appendix B \\
for Full Citation)
\end{tabular} & $\begin{array}{l}\text { Intervention and } \\
\text { Comparison }\end{array}$ & $\begin{array}{l}\text { Population } \\
\text { Age (Mean) } \\
\text { Gender (\% Female) } \\
\text { Race (\%) } \\
\text { Ambulatory (\%) } \\
\text { Wheelchair User (\%) } \\
\text { Condition Specific (\%) } \\
\text { Other (\%) }\end{array}$ & Prioritized Outcomes & Other Outcomes \\
\hline $\begin{array}{l}\begin{array}{l}\text { Giangregorio } 2012 \\
\text { (Body composition) }\end{array} \\
\text { Hitzig } 2013 \text { (quality } \\
\text { of life) } \\
\text { Kapadia } 2014 \\
\text { (walking capacity) } \\
\text { Craven } 2017 \text { (bone } \\
\text { markers) } \\
\text { Aerobic Exercise } \\
\text { Treadmill } \\
\text { Postintervention, } 6 \\
\text { months } \\
\text { Fair }\end{array}$ & $\begin{array}{l}\text { A. Functional } \\
\text { electrical stimulation } \\
\text { walking while on } \\
\text { body weight assisted } \\
\text { treadmill } 45 \text { minutes, } \\
3 \text { times a week for } 16 \\
\text { weeks } \\
\text { B. Aerobic and } \\
\text { resistance training for } \\
40 \text { to } 50 \text { minutes } 3 \\
\text { times a week for } 16 \\
\text { weeks }\end{array}$ & $\begin{array}{l}\text { American Spinal Injury } \\
\text { Association Impairment } \\
\text { Scale } C \text { or } D \\
\text { n=34 randomized and } \\
\text { analyzed } 27 \\
\text { Mean age } 55.3 \text { years } \\
26 \text { males and } 8 \text { females } \\
\text { ( } 76 \% \text { males) }\end{array}$ & $\begin{array}{l}\text { Pre, post and } 8 \text { months after intervention } \\
10 \text { meter walk test (seconds) } \\
\text { A. } 42.8(46.2) \text { to } 35.2(40.8) \text { to } 42.2(67.7) \\
\text { B. } 49.1(41.7) \text { to } 28.7(8.3) \text { to } 35.1(18.8) \\
\text { No significant change over time } p=0.084 \text { and no } \\
\text { difference between groups } p=0.829 \\
\\
6 \text { minute walk test (meters) } \\
\text { A. } 187.9(123.4) \text { to } 217.1(134.4) \text { to } 232.5(138.9) \\
\text { B. } 79.4(83.9) \text { to } 130(46.0) \text { to } 126.4(63.8) \\
\text { Overall increase in distance walked } p=0.002 \\
\text { No significant difference between groups } p=0.096 \\
\text { Timed up and go (seconds) } \\
\text { A. } 43.6 \text { (25.5) to } 33.0(15.7) \text { to } 32.2(19.1) \\
\text { B. } 61.6 \text { (36.2) to } 49.5(21.9) \text { to } 51.3(19.6) \\
\text { Overall change over time } p=0.016 \text { and no difference } \\
\text { between the groups } p=0.138\end{array}$ & 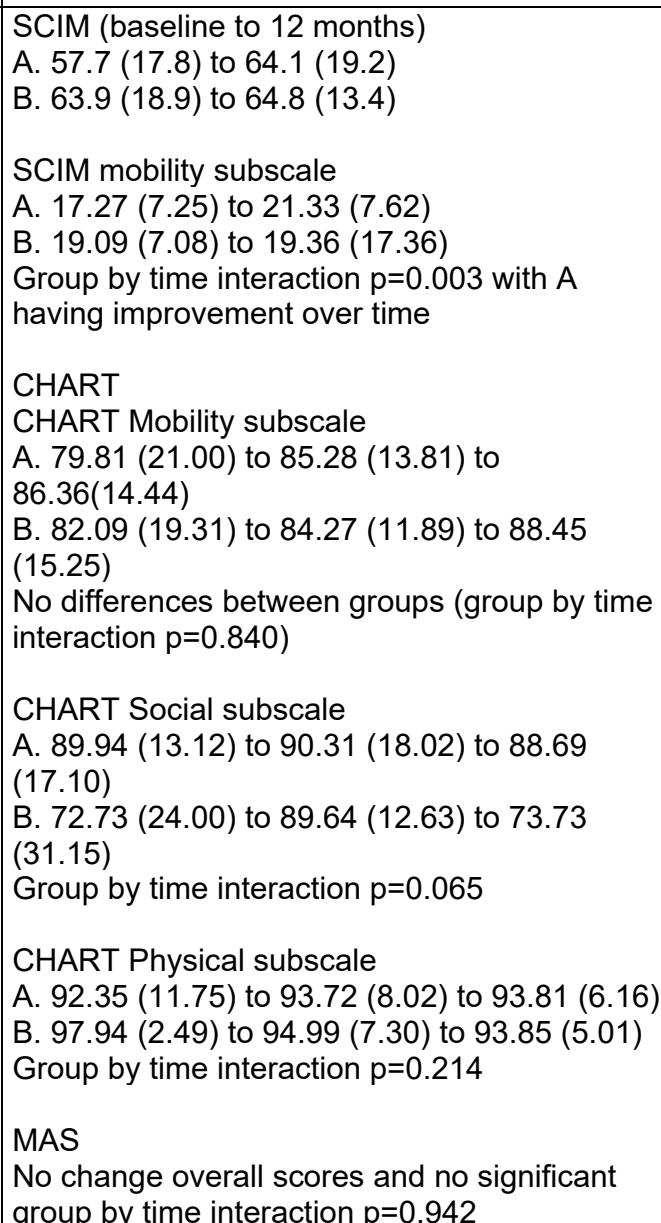 \\
\hline
\end{tabular}




\begin{tabular}{|c|c|c|c|c|}
\hline \begin{tabular}{|l|} 
Author, Year \\
Intervention Type \\
Duration of \\
Postintervention \\
Followup \\
Quality \\
(See Appendix B \\
for Full Citation)
\end{tabular} & $\begin{array}{l}\text { Intervention and } \\
\text { Comparison }\end{array}$ & $\begin{array}{l}\text { Population } \\
\text { Age (Mean) } \\
\text { Gender (\% Female) } \\
\text { Race (\%) } \\
\text { Ambulatory (\%) } \\
\text { Wheelchair User (\%) } \\
\text { Condition Specific (\%) } \\
\text { Other (\%) }\end{array}$ & Prioritized Outcomes & Other Outcomes \\
\hline $\begin{array}{l}\text { Giangregorio } 2012 \\
\text { (Body composition) } \\
\text { Hitzig } 2013 \text { (quality } \\
\text { of life) } \\
\text { Kapadia } 2014 \\
\text { (walking capacity) } \\
\text { Craven } 2017 \text { (bone } \\
\text { markers) } \\
\text { (Continued) }\end{array}$ & & & & $\begin{array}{l}\text { BMD mean change left total hip } \text { g } / \mathrm{cm} 2]) \\
\text { A. } 0.90(0.20) \text { to } 0.88(0.20) \text { to } 0.89(0.20) \\
\text { ( } p=0.41 \\
\text { B. } 0.86(0.21) \text { to } 0.87(0.23) \text { to } 0.90(0.21) \\
p=0.06 \\
\text { No significant differences between groups in } \\
\text { BMD or in any bone architecture indices } \\
\text { (pQCT) at any time point } \\
\text { Fat mass (kilogram) } \\
\text { A. } 25.4(9.5) \text { to } 24.3(9.5) \text { to } 25.2(9.0) \\
\text { B. } 23.4(10.8) \text { to } 23.0(10.7) \text { to } 23.3(11.1) \\
\text { No differences over time or between groups }\end{array}$ \\
\hline $\begin{array}{l}\text { Gibson, } 2018 \\
\text { Aerobic Exercise } \\
\text { Aerobics } \\
\text { Postintervention, } \\
12 \text { weeks } \\
\text { Good }\end{array}$ & $\begin{array}{l}\text { A. Running and } \\
\text { running exercises, } 48 \\
\text { sessions over } 12 \\
\text { weeks }(n=21) \\
\text { B. Usual care }(n=21)\end{array}$ & $\begin{array}{l}\text { A vs. B } \\
\text { Age: } 12.4 \text { vs. } 12.5 \\
\text { Female: } 33 \% \text { vs. } 38 \% \\
\text { GMFCS I: } 57 \% \text { vs. } 60 \% \\
\text { GMFCS II: } 38 \% \text { vs. } 40 \% \\
\text { GMFCS III: } 5 \% \text { vs. } 0 \%\end{array}$ & $\begin{array}{l}\text { A vs. B, Mean Difference between groups: } \\
\text { Shuttle Run Test (min): } 0.9,95 \% \mathrm{Cl}-0.3 \text { to } 2.2, \mathrm{p}=0.142 \\
\text { HiMat: } 0.8,95 \% \mathrm{Cl}-2.7 \text { to } 4.3, \mathrm{p}=0.651 \\
\text { 10X5 sprint }(\mathrm{sec}):-1.3,95 \% \mathrm{Cl}-5.4 \text { to } 2.8, \mathrm{p}=0.535\end{array}$ & NA \\
\hline
\end{tabular}




\begin{tabular}{|c|c|c|c|c|}
\hline $\begin{array}{l}\text { Author, Year } \\
\text { Intervention Type } \\
\text { Duration of } \\
\text { Postintervention } \\
\text { Followup } \\
\text { Quality } \\
\text { (See Appendix B } \\
\text { for Full Citation) }\end{array}$ & $\begin{array}{l}\text { Intervention and } \\
\text { Comparison }\end{array}$ & $\begin{array}{l}\text { Population } \\
\text { Age (Mean) } \\
\text { Gender (\% Female) } \\
\text { Race (\%) } \\
\text { Ambulatory (\%) } \\
\text { Wheelchair User (\%) } \\
\text { Condition Specific (\%) } \\
\text { Other (\%) }\end{array}$ & Prioritized Outcomes & Other Outcomes \\
\hline $\begin{array}{l}\text { Gorman, } 2019 \\
\text { Aerobic Exercise } \\
\text { Aquatics } \\
\text { Postintervention, } \\
12 \text { weeks } \\
\text { Fair }\end{array}$ & $\begin{array}{l}\text { A. RAGT, } 36 \\
\text { sessions over } 3 \\
\text { months }(n=18) \\
\\
\text { B. Aquatic therapy, } \\
36 \text { sessions over } 3 \\
\text { months }(n=15)\end{array}$ & $\begin{array}{l}\text { A vs. B } \\
\text { Age (mean years): } \\
45.4 \text { vs. } 46.9 \\
\text { Female: } \\
\text { NR } \\
\\
\text { Race: NR } \\
\text { Ambulatory: } \\
\text { Community Ambulation } \\
16 \text { (83\%) vs. } 10 \text { (67\%) } \\
\text { Wheelchair user: } \\
\text { NR } \\
\\
\text { Other: } \\
\text { Time since injury (years): } \\
6.6 \text { vs. } 12.2 \\
\text { Tetraplegic: } 12(67 \%) \text { vs. } 11 \\
\text { (73\%) } \\
\text { Paraplegic: } 6(33 \%) \text { vs. } 4 \\
\text { (27\%) } \\
\text { WISCl:9.5 } \pm 7.611 .7 \pm 6.5\end{array}$ & $\begin{array}{l}\text { A vs. } B, \text { mean }(S D) \\
\text { Robotic Peak VO2 change: } \\
2.07(p=0.03) \text { vs. } \\
\text { Arm ergometer peak } \mathrm{VO}_{2} \text { change } \\
\text { Robotic: }-0.30(p=033) \\
\text { Aquatic: } 0.98(p=0.14)\end{array}$ & NA \\
\hline
\end{tabular}




\begin{tabular}{|c|c|c|c|c|}
\hline $\begin{array}{l}\text { Author, Year } \\
\text { Intervention Type } \\
\text { Duration of } \\
\text { Postintervention } \\
\text { Followup } \\
\text { Quality } \\
\text { (See Appendix B } \\
\text { for Full Citation) } \\
\end{array}$ & $\begin{array}{l}\text { Intervention and } \\
\text { Comparison }\end{array}$ & $\begin{array}{l}\text { Population } \\
\text { Age (Mean) } \\
\text { Gender (\% Female) } \\
\text { Race (\%) } \\
\text { Ambulatory (\%) } \\
\text { Wheelchair User (\%) } \\
\text { Condition Specific (\%) } \\
\text { Other (\%) }\end{array}$ & Prioritized Outcomes & Other Outcomes \\
\hline $\begin{array}{l}\text { Grecco } 2013 \\
\text { Aerobic Exercise } \\
\text { Treadmill } \\
\text { RCT } \\
\text { Postintervention, } 3 \\
\text { weeks } \\
\text { Fair }\end{array}$ & $\begin{array}{l}\text { A. Transcranial motor } \\
\text { cortex stimulation } \\
\text { while treadmill } \\
\text { training } 5 \text { times a } \\
\text { week for } 2 \text { weeks (no } \\
\text { body weight support) } \\
\text { B. Treadmill training } \\
\text { with placebo } \\
\text { stimulation }\end{array}$ & $\begin{array}{l}\text { GMFCS II or III } \\
(\mathrm{II} / \mathrm{III} 16 / 8) \\
\mathrm{N}=24 \text { randomized and all } \\
\text { completed } \\
\text { Mean age } 7.9 \text { years } \\
7 \text { males and } 17 \text { females } \\
(29 \% \text { males })\end{array}$ & $\begin{array}{l}\text { Pre, post and } 3 \text { weeks later } \\
6 \text { minute walk (meters) } \\
\text { A. } 223(58) \text { to } 448.2(100.5) \text { to } 409.6 \text { ( } 81.6) \text { (within group } \\
\text { increase F=9.966, p<0.001) } \\
\text { B. } 255.4(62.8) \text { to } 367.2(97.6) \text { to } 345.4 \text { (97.7) NS within } \\
\text { group difference } \\
\text { Change distance baseline to post, baseline to followup } \\
\text { A. } 199.6,186.4 \\
\text { B. } 111.8,90.0 \\
\text { Between group comparison effect size pre to post } 87.8 \\
\text { (p<0.05) and pre to followup } 96.4 \text { ( } p<0.05) \\
\text { GMFM-88 D scale } \\
\text { A. } 63.7(7.0) \text { to } 75.3 \text { (11.6) to } 72.6 \text { (12.4) (no significant } \\
\text { change) } \\
\text { B. } 66.2(6.2) \text { to } 70.0 \text { ( } 9.2) \text { to } 68.4 \text { ( } 9.8) \text { (no significant } \\
\text { change) } \\
\text { Change score baseline to post, baseline to followup } \\
\text { A. } 11.5,8.8 \\
\text { B. } 3.7,2.1 \\
\text { NS between group comparison effect sizes } \\
\text { GMFM-88 E } \\
\text { A. } 54.1(7.7) \text { to } 59.9 \text { (11.1) to } 60.7 \text { (10.5) (no significant } \\
\text { change) } \\
\text { B. } 60.7 \text { (10.5) to } 61.7 \text { (10.7) to } 60.1 \text { (10.7) (no significant } \\
\text { change) } \\
\text { Change baseline to post, baseline to followup } \\
\text { A. } 0.8,0.4 \\
\text { B. } 1.0,0.7 \\
\text { NS between group comparison effect sizes }\end{array}$ & NA \\
\hline
\end{tabular}




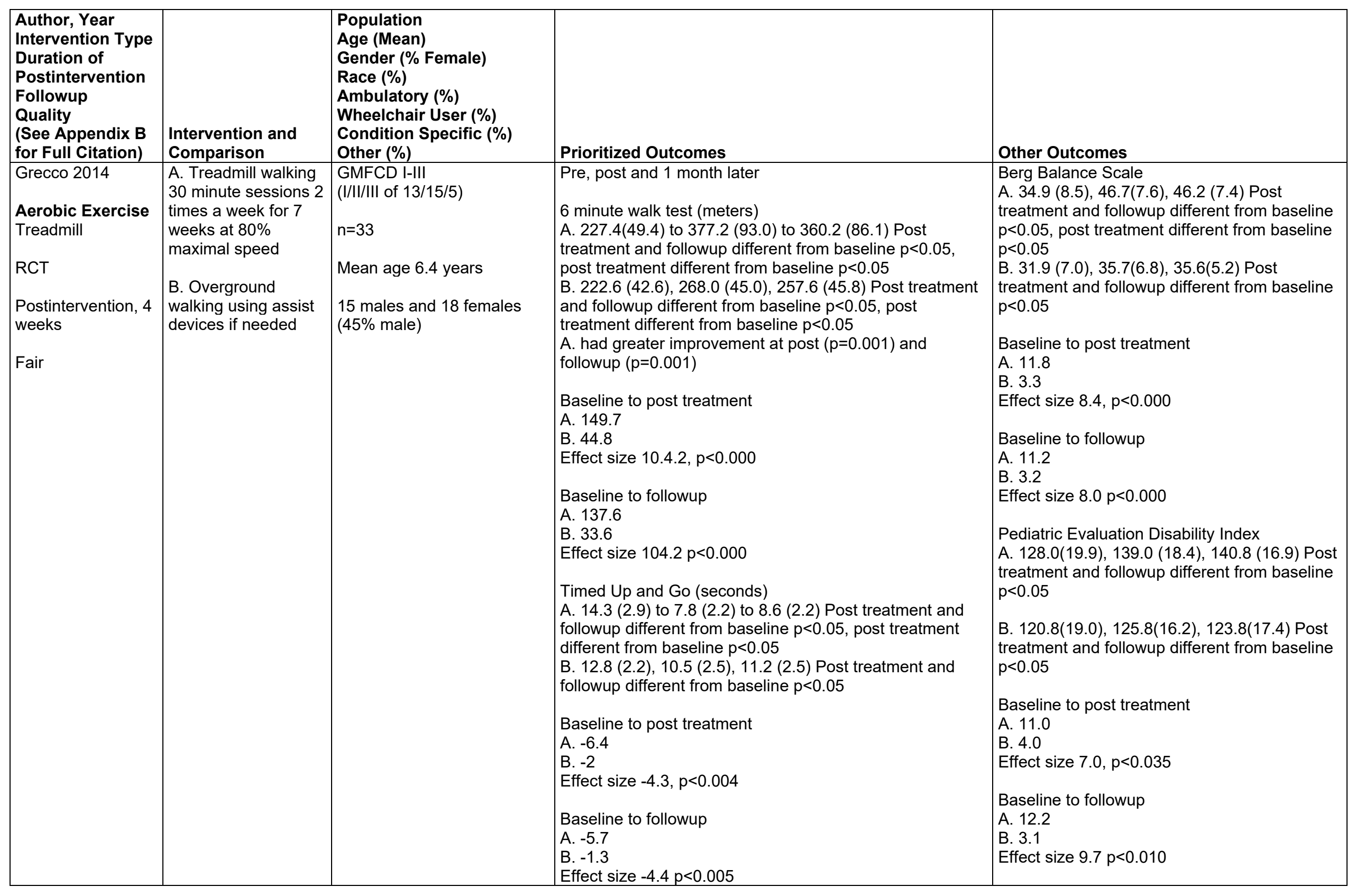




\begin{tabular}{|c|c|c|c|c|}
\hline $\begin{array}{l}\text { Author, Year } \\
\text { Intervention Type } \\
\text { Duration of } \\
\text { Postintervention } \\
\text { Followup } \\
\text { Quality } \\
\text { (See Appendix B } \\
\text { for Full Citation) } \\
\end{array}$ & $\begin{array}{l}\text { Intervention and } \\
\text { Comparison }\end{array}$ & $\begin{array}{l}\text { Population } \\
\text { Age (Mean) } \\
\text { Gender (\% Female) } \\
\text { Race (\%) } \\
\text { Ambulatory (\%) } \\
\text { Wheelchair User (\%) } \\
\text { Condition Specific (\%) } \\
\text { Other (\%) }\end{array}$ & Prioritized Outcomes & Other Outcomes \\
\hline $\begin{array}{l}\text { Grecco } 2014 \\
\text { (Continued) }\end{array}$ & & & $\begin{array}{l}\text { GMFM-88 (\%) } \\
\text { A. } 81.6(8.7) \text { to } 93.0(5.7) \text {, to } 91.7(5.0) \text {; post treatment } \\
\text { and followup different from baseline } p<0.05 \text {, post } \\
\text { treatment different from baseline } p<0.05 \\
\text { B. } 77.3(7.0) \text { to } 80.8(7.2) \text { to } 80.7(7.5) ; \text { post treatment and } \\
\text { followup different from baseline } p<0.05 \\
\\
\text { Baseline to post treatment } \\
\text { A. } 11.3 \\
\text { B } 3.6 \\
\text { Effect size } 7.7, p<0.000 \\
\text { Baseline to followup } \\
\text { A. } 10.0 \\
\text { B. } 3.5 \\
\text { Effect size } 6.5 ; p<0.000\end{array}$ & NA \\
\hline $\begin{array}{l}\text { Harness, } 2008 \\
\text { Multimodal } \\
\text { Exercise } \\
\text { Postintervention, } 0 \\
\text { weeks } \\
\text { Fair }\end{array}$ & $\begin{array}{l}\text { A. Strength + cycling } \\
+ \text { vibration: } \\
\text { averaging } 7.3 \text { hours } \\
\text { per week of exercise } \\
\text { over } 6 \text { months }(n=22) \\
\text { B. Self-regulated } \\
\text { exercise: averaging } \\
5.2 \text { hours per week } \\
\text { of exercise over } 6 \\
\text { months }(n=9)\end{array}$ & $\begin{array}{l}\text { A vs. B } \\
\text { Age (mean years): } 37.8 \text { vs. } \\
34.5 \\
\text { Female: }(13.6 \%) \text { vs. } 0(0 \%) \\
\text { Race: NR } \\
\text { Ambulatory: NR } \\
\text { Wheelchair user: NR } \\
\text { Time postinjury (mean } \\
\text { months): } 40.0 \text { vs. } 97.0, \\
\text { p=0.0057 } \\
\text { Baseline ASIA-UEMS: } 31.0 \\
\text { vs. } 38.0, p=0.37\end{array}$ & $\begin{array}{l}\text { Data reported as mean (SEM) } \\
\text { A vs. B } \\
\text { EQ-5D (0-100, higher=increased QOL): } \\
65.0(4.0) \text { vs. } 67.0(6.0), p=0.93 \text { (baseline) } \\
14.0(5.0) \text { vs. } 3.0(5.0), p=0.14 \text { (post-pre change) }\end{array}$ & $\begin{array}{l}\text { Data reported as mean (SEM) } \\
\text { A vs. B } \\
\text { ASIA-LEMS: } \\
8.0(2.0) \text { vs. } 4.0(4.0), p=0.37 \text { (baseline) } \\
3.3(0.9) \text { vs. } 0.0(0.2), p=0.035 \text { (post-pre } \\
\text { change) } \\
\text { ASIA-Total Motor } \\
39.0(3.0) \text { vs. } 42.0(5.0), p=0.54 \text { (baseline) } \\
4.8(1.0) \text { vs. }-0.1(0.5), p=0.0001 \text { (post-pre } \\
\text { change) } \\
\text { CHART (0-100, higher=increased handicap): } \\
444.0(19.0) \text { vs. } 521.0(23.0), p=0.017 \\
\text { (baseline) } \\
12.0(15.0) \text { vs. } 0.1(18.0), p=0.60 \text { (post-pre } \\
\text { change) }\end{array}$ \\
\hline
\end{tabular}




\begin{tabular}{|c|c|c|c|c|}
\hline $\begin{array}{l}\text { Author, Year } \\
\text { Intervention Type } \\
\text { Duration of } \\
\text { Postintervention } \\
\text { Followup } \\
\text { Quality } \\
\text { (See Appendix B } \\
\text { for Full Citation) } \\
\end{array}$ & $\begin{array}{l}\text { Intervention and } \\
\text { Comparison }\end{array}$ & $\begin{array}{l}\text { Population } \\
\text { Age (Mean) } \\
\text { Gender (\% Female) } \\
\text { Race (\%) } \\
\text { Ambulatory (\%) } \\
\text { Wheelchair User (\%) } \\
\text { Condition Specific (\%) } \\
\text { Other (\%) }\end{array}$ & Prioritized Outcomes & Other Outcomes \\
\hline $\begin{array}{l}\text { Hasanpour- } \\
\text { Dehkordi, } 2014 \\
\text { "Comparison of } \\
\text { regular aerobic } \\
\text { and yoga on the } \\
\text { quality of life in } \\
\text { patients" } \\
\text { Has companions: } \\
\text { Hasanpour- } \\
\text { Dehkordi, 2016; } \\
\text { Hasanpour- } \\
\text { Dehkordi, 2016 (2) } \\
\text { Postural Control } \\
\text { Yoga } \\
\text { Postintervention, } \\
12 \text { weeks } \\
\text { Poor }\end{array}$ & $\begin{array}{l}\text { A. Yoga: } 36 \text { sessions } \\
\text { over } 12 \text { weeks } \\
(n=20) \\
\text { B. Aerobics: } X \\
\text { sessions over } 12 \\
\text { weeks ( } n=20) \\
\text { C. Usual care } \\
\text { control: } \\
(n=21)\end{array}$ & $\begin{array}{l}\text { A vs. B } \\
\text { Age (mean years): } 31.9 \text { (A } \\
\text { vs. B vs. C, NR) } \\
\text { Female: } 60 \text { (98\%) (A vs. B } \\
\text { vs. C, NR) } \\
\text { Race: NR } \\
\text { Ambulatory: NR } \\
\text { Wheelchair user: NR }\end{array}$ & $\begin{array}{l}\text { A. vs. B. vs. C mean (SD) } \\
\text { SF-36 QOL } \\
\text { A. } 1533(759.10) \text { (baseline) } \\
2446(540.76) \text { (postintervention), } p=0.05 \\
\text { B. } 1240.24(527.32) \text { (baseline) } \\
2050(527.32) \text { (postintervention), } p=0.05 \\
\text { C. } 1385.75(600.04) \text { (baseline) } \\
1255.75(600.22) \text { (postintervention), } p=0.05 \\
\text { SF-36 QOL mean difference between groups } \\
\text { A vs. B } \\
229.32, p=0.07 \\
\text { A vs. C } \\
1106.41, p=0.000 \\
\text { B vs. C } \\
877.10, p-0.000\end{array}$ & NA \\
\hline
\end{tabular}




\begin{tabular}{|c|c|c|c|c|}
\hline $\begin{array}{l}\text { Author, Year } \\
\text { Intervention Type } \\
\text { Duration of } \\
\text { Postintervention } \\
\text { Followup } \\
\text { Quality } \\
\text { (See Appendix B } \\
\text { for Full Citation) } \\
\end{array}$ & $\begin{array}{l}\text { Intervention and } \\
\text { Comparison }\end{array}$ & $\begin{array}{l}\text { Population } \\
\text { Age (Mean) } \\
\text { Gender (\% Female) } \\
\text { Race (\%) } \\
\text { Ambulatory (\%) } \\
\text { Wheelchair User (\%) } \\
\text { Condition Specific (\%) } \\
\text { Other (\%) } \\
\end{array}$ & Prioritized Outcomes & Other Outcomes \\
\hline $\begin{array}{l}\text { Hasanpour- } \\
\text { Dehkordi, } 2016 \text { (2) } \\
\text { "Influence of yoga } \\
\text { and aerobics } \\
\text { exercise on } \\
\text { fatigue, pain and } \\
\text { psychosocial } \\
\text { status..." } \\
\text { Postural Control } \\
\text { Yoga } \\
\text { Companion to: } \\
\text { Hasanpour- } \\
\text { Dehkordi, 2014 } \\
\text { Postintervention, } \\
12 \text { weeks } \\
\text { Poor }\end{array}$ & $\begin{array}{l}\text { A. Yoga: } 36 \text { sessions } \\
\text { over } 12 \text { weeks } \\
(n=20) \\
\text { B. Group exercise: } X \\
\text { sessions over } 12 \\
\text { weeks }(n=20) \\
\begin{array}{l}\text { C. Usual care } \\
\text { control: } \\
(n=21)\end{array}\end{array}$ & $\begin{array}{l}\text { A vs. B vs. C } \\
\text { Age (mean years): } 31.9 \text { (A } \\
\text { vs. B vs. C, NR) } \\
\text { Female: } 60 \text { (98\%) (A vs. B } \\
\text { vs. C, NR) } \\
\text { Race: NR } \\
\text { Ambulatory: NR } \\
\text { Wheelchair user: NR }\end{array}$ & $\begin{array}{l}\text { A vs. B vs. C } \\
\text { SF-36 QOL } \\
\text { Mental health } \\
\text { (baseline) } \\
\text { (postintervention) } \\
\\
\text { Limited activities following emotional problems } \\
41.9 \pm 9.16 \text { vs. vs. (baseline) } \\
35.65 \pm 12.3 \text { (postintervention) }\end{array}$ & NA \\
\hline $\begin{array}{l}\text { Hasanpour- } \\
\text { Dehkordi, } 2016 \\
\text { "Effects of Yoga on } \\
\text { Physiological } \\
\text { Indices, Anxiety } \\
\text { and Social } \\
\text { Functioning" } \\
\text { Companion to: } \\
\text { Hasanpour- } \\
\text { Dehkordi, 2014 } \\
\text { Postural Control } \\
\text { Yoga } \\
\text { Postintervention, } \\
12 \text { weeks } \\
\text { Poor }\end{array}$ & $\begin{array}{l}\text { A. Yoga: } 36 \text { sessions } \\
\text { over } 12 \text { weeks } \\
(n=30) \\
\text { B. Usual care control: } \\
(n=30) \\
\text { Yoga vs. usual care }\end{array}$ & $\begin{array}{l}\text { A vs. B } \\
\text { Age (mean years): } 30 \text { vs. } 30 \\
\text { Female: NR } \\
\text { Race: NR } \\
\text { Ambulatory: NR } \\
\text { Wheelchair user: NR }\end{array}$ & $\begin{array}{l}\text { SF-36 QOL, mean1533 (759.10) vs. } 1385.75 \text { (600.04) } \\
\text { (baseline), } p=0.5 \\
2446(540.76) \text { vs. } 1255 \text { (600.22) (postintervention), } p=0.5 \\
\begin{array}{l}3.3(5.63 \text { SD) vs. } 3.9(4.4) \text { (before and after score) } \\
\text { (postintervention), } p=0.05\end{array}\end{array}$ & NA \\
\hline
\end{tabular}




\begin{tabular}{|c|c|c|c|c|}
\hline $\begin{array}{l}\text { Author, Year } \\
\text { Intervention Type } \\
\text { Duration of } \\
\text { Postintervention } \\
\text { Followup } \\
\text { Quality } \\
\text { (See Appendix B } \\
\text { for Full Citation) } \\
\end{array}$ & $\begin{array}{l}\text { Intervention and } \\
\text { Comparison }\end{array}$ & $\begin{array}{l}\text { Population } \\
\text { Age (Mean) } \\
\text { Gender (\% Female) } \\
\text { Race (\%) } \\
\text { Ambulatory (\%) } \\
\text { Wheelchair User (\%) } \\
\text { Condition Specific (\%) } \\
\text { Other (\%) }\end{array}$ & Prioritized Outcomes & Other Outcomes \\
\hline $\begin{array}{l}\text { Hebert, } 2011 \\
\text { Aerobic Exercise } \\
\text { Cycling } \\
\text { Postintervention, } 4 \\
\text { weeks } \\
\text { Fair }\end{array}$ & $\begin{array}{l}\text { A. Bicycle } \\
\text { ergometry plus } \\
\text { stretching, } 12 \\
\text { sessions for } 6 \text { weeks } \\
(n=12) \\
\text { B. Vestibular } \\
\text { rehabilitation }(n=13) \\
\text { C. Waitlist control } \\
(n=13)\end{array}$ & $\begin{array}{l}\text { A vs. B vs. C } \\
\text { Age: } 46.8 \text { vs. } 42.6 \text { vs. } 50.2 \\
\text { Female: } \\
75 \% \text { vs. } 85 \% \text { vs. } 85 \% \\
\text { Race: NR } \\
\text { Ambulatory: } 100 \% \\
\text { Wheelchair user: NR } \\
\\
\text { BDI-II total score, mean } \\
\text { (SD): } \\
16.5(9.1) \text { vs. } 17.3(8.6) \text { vs. } \\
18.5(6.4)\end{array}$ & $\begin{array}{l}\text { From start to end of intervention } \\
\text { Mean difference between groups } \\
\text { 6MWT: } \\
\text { A vs. B: effect size } 39.1,95 \% \mathrm{Cl}-105 \text { to } 183, p=1.00 \\
\text { A vs. C: effect size } 62.7,95 \% \mathrm{Cl}-81 \text { to } 2.7, p=1.00 \\
\text { B vs. C: effect size } 23.6,95 \% \mathrm{Cl}-117 \text { to } 165, p=1.00 \\
\text { BDI-II } \\
\text { At } 14 \text { weeks-end of intervention phase to end of followup } \\
\text { phase }(14 \text { weeks) (change from end of intervention) } \\
\text { A vs. B } \\
\text { BDI-II: } \\
\text { 0.7 vs. } 2.6(p=1.000) \\
6 M W T: \\
-58.2 \text { vs. } 38.9 \\
\text { (p=0.731) } \\
\text { A vs. C } \\
\text { BDI-II: } \\
4.6 \text { vs. } 0.7(p=0.385) \\
6 \text { MWT: } \\
-58.2 \text { vs. }-24.6(p=1.000) \\
\end{array}$ & NA \\
\hline $\begin{array}{l}\text { Hebert, } 2009 \\
\text { Companion to: } \\
\text { Hebert, } 2011 \\
\text { End of treatment: } \\
14 \text { weeks } \\
\text { Fair }\end{array}$ & $\begin{array}{l}\text { A. Balance + Eye } \\
\text { movement exercises: } \\
20 \text { sessions over } 14 \\
\text { weeks }(n=44) \\
\text { B. No treatment } \\
(n=44)\end{array}$ & $\begin{array}{l}\text { A vs. B } \\
\text { Age (mean years): } 47 \text { vs. } 43 \\
\text { Female: } 37 \text { (84\%) vs. } 38 \\
(86 \%) \\
\text { Race: NR } \\
\text { Ambulatory: } 100 \% \\
\text { Baseline: } 3.50 \text { vs. } 3.34 \\
\text { Baseline PHQ-9: } 37.8 \text { vs. } \\
37.6 \\
\text { Baseline T25W: } 6.19 \text { vs. } \\
5.53 \\
\text { Baseline SF-36 PCS: } 35.8 \\
\text { vs. } 35.4 \\
\text { Baseline SF-36 MCS: } 42.6 \\
\text { vs. } 42.9\end{array}$ & $\begin{array}{l}\text { A vs. B } \\
\text { 6 weeks } \\
\text { SF-36 PCS: MD } 2.39(95 \% \mathrm{Cl}-0.99 \text { to } 5.78, p=0.16) \\
\text { SF-36 MCS: MD } 2.11(95 \% \mathrm{Cl}-2.24 \text { to } 6.46, p=0.34) \\
\text { T25W: MD }-0.02(95 \% \mathrm{Cl}-0.27 \text { to } 0.23, p=0.86) \\
14 \text { weeks } \\
\text { SF-36 PCS: MD } 1.92(95 \% \mathrm{Cl}-1.51 \text { to } 5.34, p=0.27) \\
\text { SF-36 MCS: MD } 1.82(95 \% \mathrm{Cl}-2.58 \text { to } 6.23, p=0.41) \\
\text { T25W: MD }-0.05(95 \% \mathrm{Cl}-0.63 \text { to } 0.53, p=0.86)\end{array}$ & NA \\
\hline
\end{tabular}




\begin{tabular}{|c|c|c|c|c|}
\hline $\begin{array}{l}\text { Author, Year } \\
\text { Intervention Type } \\
\text { Duration of } \\
\text { Postintervention } \\
\text { Followup } \\
\text { Quality } \\
\text { (See Appendix B } \\
\text { for Full Citation) }\end{array}$ & $\begin{array}{l}\text { Intervention and } \\
\text { Comparison }\end{array}$ & $\begin{array}{l}\text { Population } \\
\text { Age (Mean) } \\
\text { Gender (\% Female) } \\
\text { Race (\%) } \\
\text { Ambulatory (\%) } \\
\text { Wheelchair User (\%) } \\
\text { Condition Specific (\%) } \\
\text { Other (\%) }\end{array}$ & Prioritized Outcomes & Other Outcomes \\
\hline $\begin{array}{l}\text { Heine, } 2017 \\
\text { Aerobic Exercise } \\
\text { Cycling } \\
16 \text { weeks } \\
\text { Postintervention, } \\
36 \text { weeks } \\
\text { Fair }\end{array}$ & $\begin{array}{l}\text { A. Leg cycling, } 48 \\
\text { sessions over } 16 \\
\text { weeks (43) } \\
\text { B. MS nurse } \\
\text { consultation, } 3 \\
\text { consultations over } 16 \\
\text { weeks (46) }\end{array}$ & $\begin{array}{l}\text { A vs. B } \\
\text { Age: } 43.1 \text { vs. } 48.2 \\
\text { Female: } 74 \% \text { vs. } 72 \% \\
\text { EDSS: } 2.5 \text { vs. } 3.0 \\
\text { RRMS: } 72 \% \text { vs. } 74 \% \\
\text { SPMS: } 7 \% \text { vs. } 11 \% \\
\text { PPMS: } 21 \% \text { vs. } 15 \% \\
\text { VO } 2 \text { peak (L/min): } 1.75 \text { vs. } \\
1.53 \\
\text { Ambulatory: } 100 \%\end{array}$ & $\begin{array}{l}\text { A vs. B } \\
\text { VO } 2 \text { peak end of treatment: MD } 0.048(0.082), p=0.561 \\
\text { VO2 peak end of followup: } \\
\text { MD }-0.046(0.082), 0.579 \\
\text { Calculated A vs. B, Mean Difference (SE) between } \\
\text { groups: } \\
\text { IPA autonomy indoors: }-0.11(0.088), p=0.203 \\
\text { IPA family role: }-0.082(0.1222), p=0.502 \\
\text { IPA autonomy outdoors: }-0.097(0.125), p=0.438 \\
\text { IPA Social Relations: }-0.138(0.092), p=0.135 \\
\text { IPA Work/education: } 0.225(0.167), p=0.181\end{array}$ & $\begin{array}{l}\text { Impact on participation and autonomy: no } \\
\text { significant difference on any subscale at end } \\
\text { of treatment or at end of followup }\end{array}$ \\
\hline
\end{tabular}




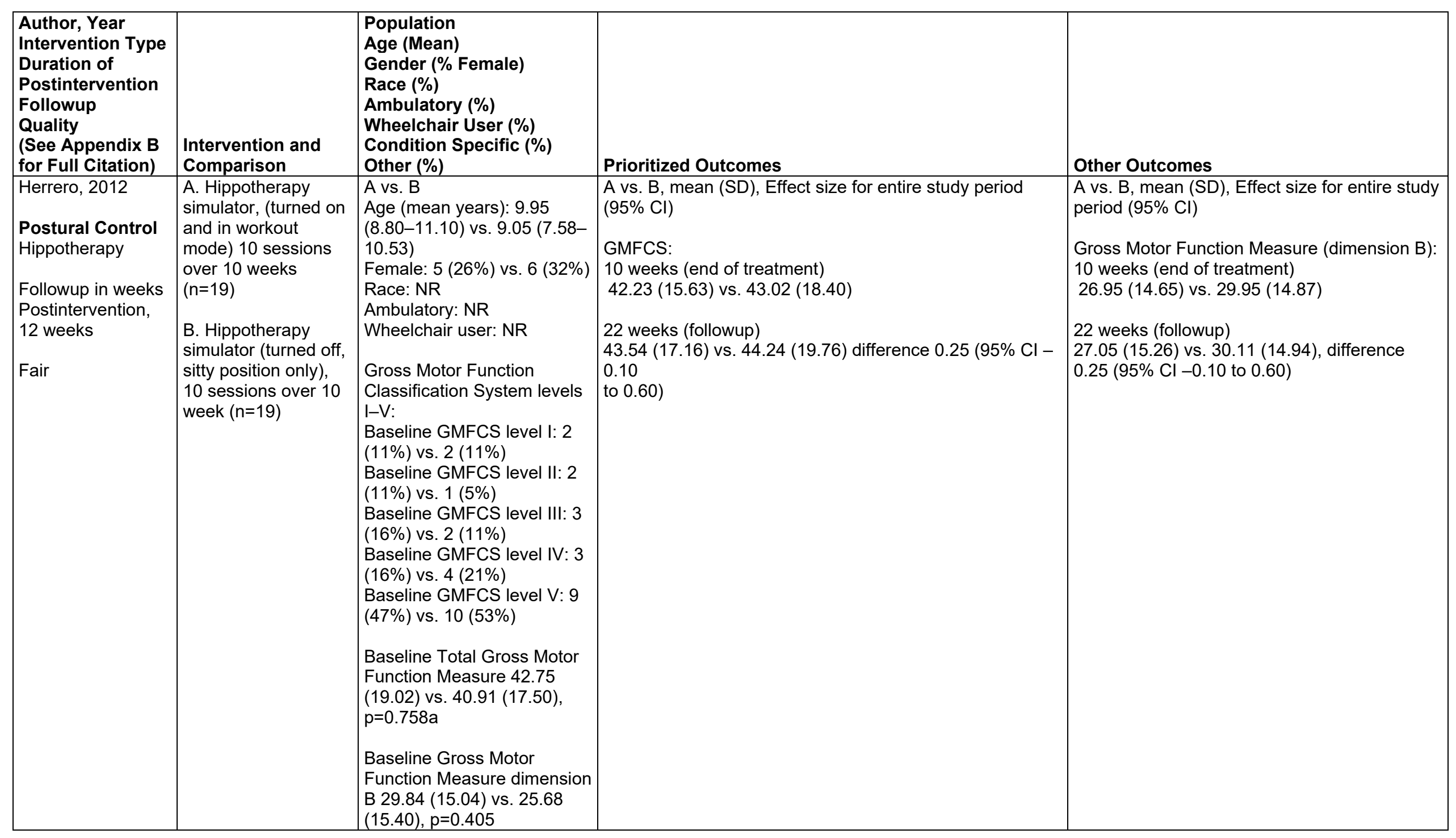




\begin{tabular}{|c|c|c|c|c|}
\hline $\begin{array}{l}\text { Author, Year } \\
\text { Intervention Type } \\
\text { Duration of } \\
\text { Postintervention } \\
\text { Followup } \\
\text { Quality } \\
\text { (See Appendix B } \\
\text { for Full Citation) }\end{array}$ & $\begin{array}{l}\text { Intervention and } \\
\text { Comparison }\end{array}$ & $\begin{array}{l}\text { Population } \\
\text { Age (Mean) } \\
\text { Gender (\% Female) } \\
\text { Race (\%) } \\
\text { Ambulatory (\%) } \\
\text { Wheelchair User (\%) } \\
\text { Condition Specific (\%) } \\
\text { Other (\%) }\end{array}$ & Prioritized Outcomes & Other Outcomes \\
\hline $\begin{array}{l}\text { Hochsprung, } 2017 \\
\text { Aerobic Exercise } \\
\text { Cycling } \\
\text { Postintervention, } 0 \\
\text { weeks } \\
\text { Poor }\end{array}$ & $\begin{array}{l}\text { A. Visual } \\
\text { biofeedback cycling } \\
\text { training, } 12 \text { sessions } \\
\text { over } 12 \text { weeks } \\
(n=30) \\
\\
\text { B. Home exercise } \\
\text { program, sessions } \\
\text { not stated }(n=31)\end{array}$ & $\begin{array}{l}\text { A vs. B } \\
\text { Age (mean years): NR } \\
\text { Female: } \\
20(66 \%) \text { vs. } 16(50 \%) \\
\text { Race: NR } \\
\text { Ambulatory: } \\
30 \text { (100\%) vs. } 31(100 \%) \\
\text { Wheelchair user: NR }\end{array}$ & $\begin{array}{l}\text { A vs. B (SD) } \\
\text { FAP } \\
(0.820) \text { vs. }(0.929) \text { (baseline) } \\
(0.792) \text { vs. }(0.942) ; p=0.002 \text { (postintervention) } \\
\text { Calculated A vs. B Mean change scores: } \\
\text { FFAP: } \\
3.036(p=0.002) \text { vs. }-1.06(p=0.289) \\
\text { No comparison between groups provided }\end{array}$ & NA \\
\hline
\end{tabular}




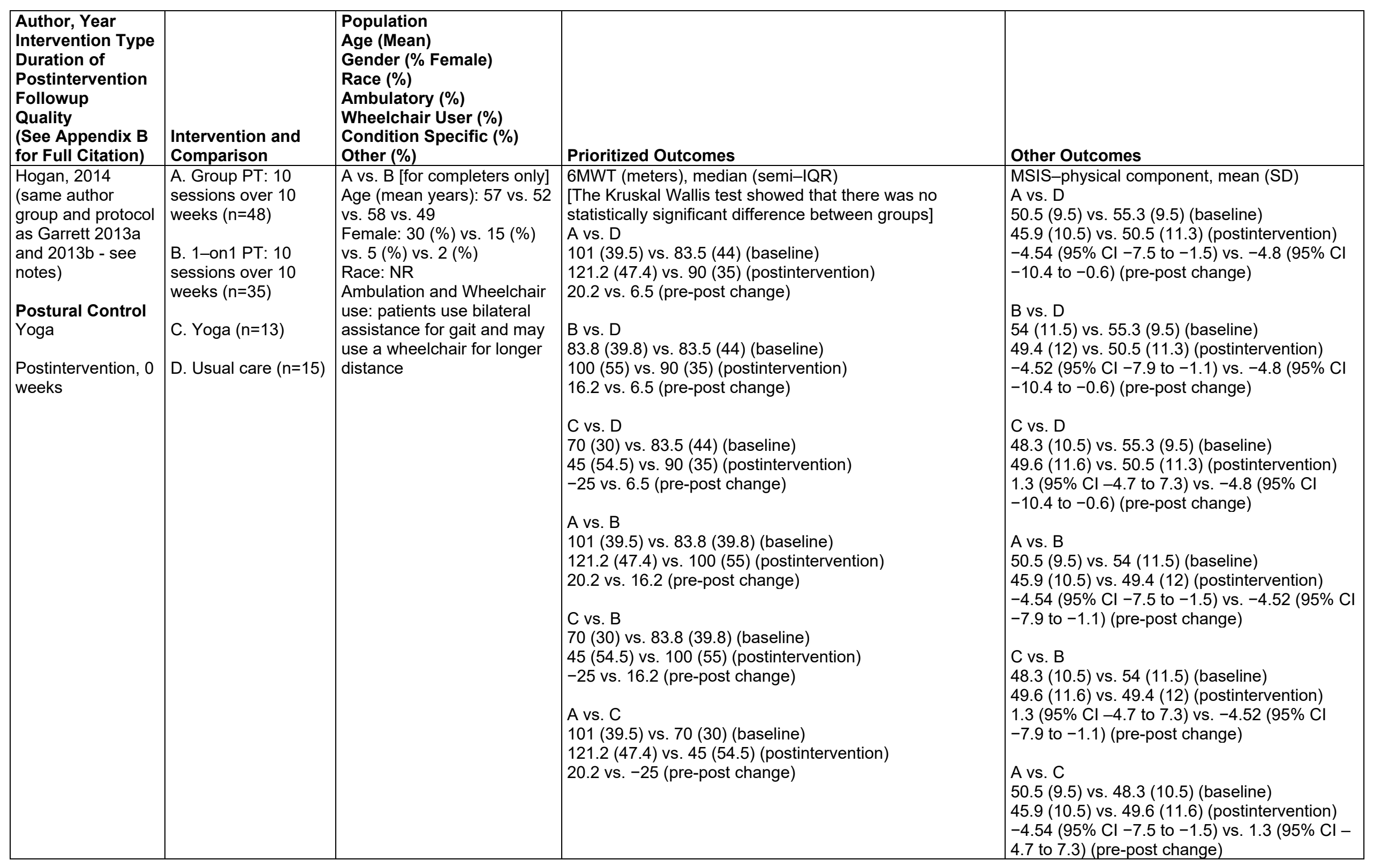




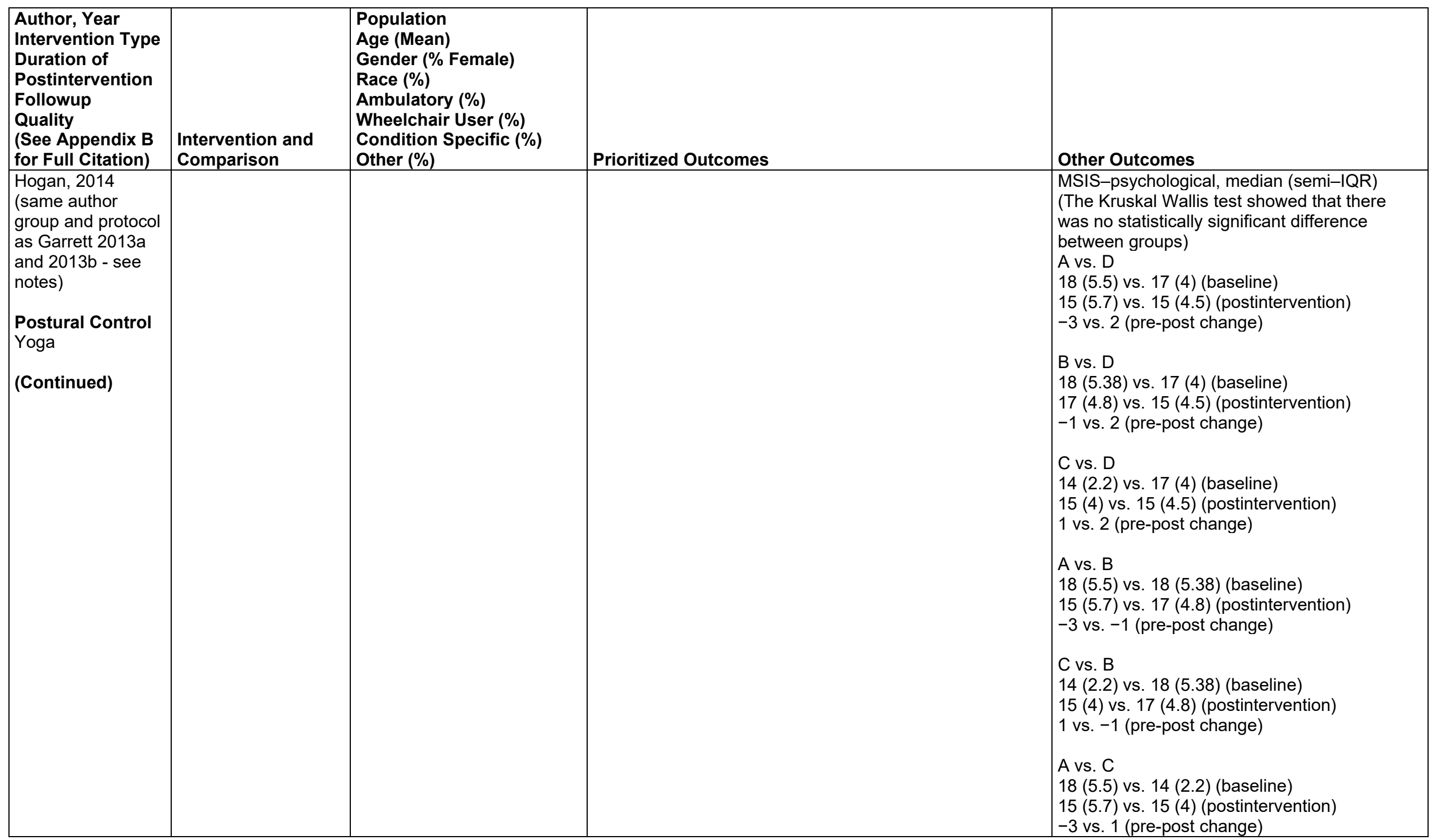




\begin{tabular}{|c|c|c|c|c|}
\hline $\begin{array}{l}\text { Author, Year } \\
\text { Intervention Type } \\
\text { Duration of } \\
\text { Postintervention } \\
\text { Followup } \\
\text { Quality } \\
\text { (See Appendix B } \\
\text { for Full Citation) } \\
\end{array}$ & $\begin{array}{l}\text { Intervention and } \\
\text { Comparison }\end{array}$ & $\begin{array}{l}\text { Population } \\
\text { Age (Mean) } \\
\text { Gender (\% Female) } \\
\text { Race (\%) } \\
\text { Ambulatory (\%) } \\
\text { Wheelchair User (\%) } \\
\text { Condition Specific (\%) } \\
\text { Other (\%) }\end{array}$ & Prioritized Outcomes & Other Outcomes \\
\hline $\begin{array}{l}\text { Hota, } 2020 \\
\text { Postural Control } \\
\text { Balance Exercises } \\
\text { Postintervention, } \\
4 \text { weeks } \\
\text { Fair }\end{array}$ & $\begin{array}{l}\text { A. Dual task } \\
\text { exercises for upper } \\
\text { and lower limbs, } 24 \\
\text { sessions over } 4 \\
\text { weeks }(n=20) \\
\text { B. Control group - } \\
\text { details NR, }(n=20)\end{array}$ & $\begin{array}{l}\text { A vs. B } \\
\text { Age } 11-25: 40 \% \text { vs. } 30 \% \\
\text { Age } 26-40: 25 \% \text { vs. } 45 \% \\
\text { Age } 41-55: 25 \% \text { vs. } 25 \% \\
\text { Age } 56-70: 10 \% \text { vs. } 0 \% \\
\text { Female: } 10 \% \text { vs. } 10 \% \\
\text { Race: NR }\end{array}$ & $\begin{array}{l}\text { A vs. B, mean (SD): } \\
\text { BBS: MD } 4.55,95 \% \text { Cl } 2.16 \text { to } 6.94 \\
\text { Motor Assessment Scale: MD } 3.82,95 \% \text { Cl } 1.09 \text { to } 6.55 \text {, } \\
p=0.006\end{array}$ & NA \\
\hline $\begin{array}{l}\text { Hsieh, } 2018 \\
\text { Postural Control } \\
\text { Motion gaming } \\
\text { Postintervention, } \\
12 \text { weeks } \\
\text { Fair }\end{array}$ & $\begin{array}{l}\text { A. PC gaming using } \\
\text { arm and trunk, } 60 \\
\text { sessions over } 12 \\
(n=20) \\
\text { B PC gaming using } \\
\text { mouse, } 60 \text { sessions } \\
\text { over } 12 \text { weeks } \\
(n=20)\end{array}$ & $\begin{array}{l}\text { A vs. B } \\
\text { Age (mean years): } 7.33 \text { vs. } \\
7.41 \\
\text { Female: } 6 \text { (30\%) vs. } 5(25 \%) \\
\text { Race: NR } \\
\text { Ambulatory: NR } \\
\text { Wheelchair user: NR } \\
\text { CP subtype } \\
\text { Spastic quadriplegia } 11 \\
\text { (55\%) vs. } 12(60 \%) \\
\text { Spastic diplegic } 4(20 \%) \text { vs. } \\
3(15 \%) \\
\text { Athetoid } 2(10 \%) \text { vs. } 2(10 \%) \\
\text { Ataxic } 3(15 \%) \text { vs. } 3(15 \%) \\
\text { GMFCS level } \\
\text { Level II } 10(50 \%) \text { vs. } 10 \\
(50 \%) \\
\text { Level III } 6(30 \%) \text { vs. } 5(25 \%) \\
\text { Level IV } 4(20 \%) \text { vs. } 5(25 \%)\end{array}$ & $\begin{array}{l}\text { A vs. B } \\
\text { TUG (score) } \\
16.43(2.12) \text { vs. } 15.60(1.10) \text { (baseline) } \\
17.51(1.70) \text { vs. } 15.91(1.87) \text { (postintervention) }\end{array}$ & $\begin{array}{l}\text { A vs. B, mean (SD) } \\
\text { BBS (score) } \\
44.74(2.75) \text { vs. } 44.39 \text { (2.33) (baseline) } \\
48.81 \text { (4.74) vs. } 45.37 \text { (2.68) (postintervention) }\end{array}$ \\
\hline $\begin{array}{l}\text { Hsieh, } 2020 \\
\text { Postural Control } \\
\text { Motion Gaming } \\
\text { Postintervention, } \\
0 \text { weeks } \\
\text { Fair }\end{array}$ & $\begin{array}{l}\text { A. PC gaming using } \\
\text { balance board, } 36 \\
\text { sessions over } 12 \\
\text { weeks ( } n=28) \\
\text { B. PC gaming using } \\
\text { mouse, } 36 \text { sessions } \\
\text { over } 12 \text { weeks } \\
(n=28)\end{array}$ & $\begin{array}{l}\text { A vs. B } \\
\text { Age: } 7.9 \text { vs. } 8.1 \\
\text { Female: } 32 \% \text { vs. } 31.5 \% \\
\text { Race: NR } \\
\text { GMFCS I: } 53.5 \% \text { vs. } 50 \% \\
\text { GMFCS II: } 28.6 \% \text { vs. } 32.1 \% \\
\text { GMFCS III: } 17.9 \% \text { vs. } 17.9 \% \\
\text { Deplegic: } 57.1 \% \text { vs. } 42.9 \%\end{array}$ & $\begin{array}{l}\text { A vs. B, mean (SD) } \\
\text { 2MWT: } 103.4(16.6) \text { to } 120.1(20.2) \text { vs. } 101.4(23.1) \text { to } \\
106.1(22.8), p=0.002 \\
\text { PBS-total: } 29.9(5.3) \text { to } 35.8(5.5) \text { vs. } 32.3(7.5) \text { to } 34.4 \\
(5.9), p=0.002\end{array}$ & NA \\
\hline
\end{tabular}




\begin{tabular}{|c|c|c|c|c|}
\hline $\begin{array}{l}\text { Author, Year } \\
\text { Intervention Type } \\
\text { Duration of } \\
\text { Postintervention } \\
\text { Followup } \\
\text { Quality } \\
\text { (See Appendix B } \\
\text { for Full Citation) } \\
\end{array}$ & $\begin{array}{l}\text { Intervention and } \\
\text { Comparison }\end{array}$ & $\begin{array}{l}\text { Population } \\
\text { Age (Mean) } \\
\text { Gender (\% Female) } \\
\text { Race (\%) } \\
\text { Ambulatory (\%) } \\
\text { Wheelchair User (\%) } \\
\text { Condition Specific (\%) } \\
\text { Other (\%) } \\
\end{array}$ & Prioritized Outcomes & Other Outcomes \\
\hline $\begin{array}{l}\text { Huang, } 2015 \\
\text { Aerobic Exercise } \\
\text { Robot-Assisted } \\
\text { Gait Training } \\
\text { Postintervention, } 4 \\
\text { weeks } \\
\text { Fair }\end{array}$ & $\begin{array}{l}\text { A. RAGT, } 16 \\
\text { sessions over } 4 \\
\text { weeks }(n=12) \\
\\
\text { B. Body Weight } \\
\text { Support Treadmill } \\
\text { Training, } 16 \text { sessions } \\
\text { over } 4 \text { weeks }(n=12)\end{array}$ & $\begin{array}{l}\text { A vs. B } \\
\text { Age (mean years): } \\
41.7 \text { vs. } 38.4 \\
\text { Female: } \\
5(42 \%) \text { vs. } 3(25 \%) \\
\text { Race: NR } \\
\text { Ambulatory: NR } \\
\text { Wheelchair user: NR } \\
\text { Other: } \\
\text { height } 168.8 \text { cm vs. } 169.8 \\
\text { cm } \\
\text { weight }(k g) 66.1 \text { vs. } 65.3\end{array}$ & $\begin{array}{l}\text { A vs. B, mean (SD) } \\
\text { A. Defecation time } \\
\text { Before } 93.0+/-14.7 \\
\text { After } 64.5+/-11.6 \\
\text { B. Before } 84.0+/-15.2 \\
\text { After } 69.5+/-15.6\end{array}$ & NA \\
\hline
\end{tabular}




\begin{tabular}{|c|c|c|c|c|}
\hline $\begin{array}{l}\text { Author, Year } \\
\text { Intervention Type } \\
\text { Duration of } \\
\text { Postintervention } \\
\text { Followup } \\
\text { Quality } \\
\text { (See Appendix B } \\
\text { for Full Citation) } \\
\end{array}$ & $\begin{array}{l}\text { Intervention and } \\
\text { Comparison }\end{array}$ & $\begin{array}{l}\text { Population } \\
\text { Age (Mean) } \\
\text { Gender (\% Female) } \\
\text { Race (\%) } \\
\text { Ambulatory (\%) } \\
\text { Wheelchair User (\%) } \\
\text { Condition Specific (\%) } \\
\text { Other (\%) }\end{array}$ & Prioritized Outcomes & Other Outcomes \\
\hline $\begin{array}{l}\text { In, } 2018 \\
\text { Postural Control } \\
\text { Whole body } \\
\text { vibration } \\
\text { Postintervention, } 0 \\
\text { weeks } \\
\text { Fair }\end{array}$ & $\begin{array}{l}\text { A. Whole body } \\
\text { vibration }+ \\
\text { conventional physical } \\
\text { therapy: } 80 \text { sessions } \\
\text { over } 8 \text { weeks }(n=14) \\
\text { B. Placebo whole } \\
\text { body vibration }+ \\
\text { conventional physical } \\
\text { training }(n=14)\end{array}$ & $\begin{array}{l}\text { A vs. B (Data for completers } \\
\text { only) } \\
\text { Age (mean years): } 46.1 \text { vs. } \\
49.9 \\
\text { Female: } 5(36 \%) \text { vs. } 4 \text { (29\%) } \\
\text { Race: NR } \\
\text { Ambulatory: } 14(100 \%) \text { vs. } \\
14(100 \%) \\
\text { Wheelchair user: NR } \\
\text { Duration (mean months): } \\
13.7 \text { vs. } 14.3\end{array}$ & $\begin{array}{l}\text { A vs. B } \\
\text { TUG (seconds) } \\
13.7(3.1) \text { vs. } 14.7(4.5), p=0.608 \text { (baseline) } \\
11.4(2.8) \text { vs. } 13.7(4.1), p=N R \text { (postintervention) } \\
-2.3(1.3) \text { vs. }-1.0(1.0), p=0.016 \text { (post-pre change) } \\
\text { Time X Group } p=0.016 \\
\text { 10MWT (seconds) } \\
29.3(9.0) \text { vs. } 28.8(7.2), p=0.868 \text { (baseline) } \\
25.8(8.1) \text { vs. } 27.5(6.3), p=N R \text { (postintervention) } \\
-3.5(2.3) \text { vs. }-1.3(1.4), p=0.005 \text { (post-pre change) } \\
\text { Time X Group } p=0.005 \\
\text { Spasticity measured by manual muscle tester (kg) } \\
- \text { Right ankle } \\
11.9(3.5) \text { vs. } 12.2(3.2), p=0.785 \text { (baseline) } \\
\text { 8.8 (2.9) vs. } 11.1(2.9), p=N R \text { (postintervention) } \\
-3.1(1.9) \text { vs. }-1.1(0.6), p=0.001 \text { (post-pre change) } \\
\text { Time X Group } p=0.001 \\
\text {-Left ankle } \\
13.2(2.3) \text { vs. } 12.5(3.1), p=0.526 \text { (baseline) } \\
10.1(2.2) \text { vs. } 11.6(2.3), p=N R \text { (postintervention) } \\
-3.0(1.7) \text { vs. }-0.9(1.2), p=0.001 \text { (post-pre change) } \\
\text { Time X Group } p=0.001\end{array}$ & NA \\
\hline
\end{tabular}




\begin{tabular}{|c|c|c|c|c|}
\hline $\begin{array}{l}\text { Author, Year } \\
\text { Intervention Type } \\
\text { Duration of } \\
\text { Postintervention } \\
\text { Followup } \\
\text { Quality } \\
\text { (See Appendix B } \\
\text { for Full Citation) }\end{array}$ & $\begin{array}{l}\text { Intervention and } \\
\text { Comparison }\end{array}$ & $\begin{array}{l}\text { Population } \\
\text { Age (Mean) } \\
\text { Gender (\% Female) } \\
\text { Race (\%) } \\
\text { Ambulatory (\%) } \\
\text { Wheelchair User (\%) } \\
\text { Condition Specific (\%) } \\
\text { Other (\%) }\end{array}$ & Prioritized Outcomes & Other Outcomes \\
\hline $\begin{array}{l}\text { Johnston } 2011 \\
\text { Aerobic Exercise } \\
\text { Treadmill } \\
\text { Postintervention, } 4 \\
\text { weeks } \\
\text { Fair }\end{array}$ & $\begin{array}{l}\text { A. Partial body } \\
\text { weight supported } \\
\text { treadmill training with } \\
\text { two } 30 \text { minute } \\
\text { sessions } 5 \text { days a } \\
\text { week for } 2 \text { weeks, } \\
\text { followed by } 30 \\
\text { minutes } 5 \text { days a } \\
\text { week of home } \\
\text { training for } 10 \text { weeks } \\
\text { B. Individualized } \\
\text { physical therapy } \\
\text { sessions strength } \\
\text { and weigh-bearing } \\
\text { activities comparable } \\
\text { session duration and } \\
\text { number }\end{array}$ & $\begin{array}{l}\text { Marginal ambulatory function } \\
\text { and GMFCD III or IV } \\
n=34 \text { randomized and } 26 \\
\text { completed the study } \\
\text { Mean age } 9.5 \text { years } \\
14 \text { males and } 12 \text { females } \\
(39 \% \text { male })\end{array}$ & $\begin{array}{l}\text { Pre, post and } 1 \text { month later } \\
\text { Gait speed (meters/second) } \\
\text { A. } 0.50(0.26) \text { to } 0.62(0.31) \text { to } 0.63(0.28) \\
\text { B. } 0.44(0.35) \text { to } 0.32(0.50) \text { to } 0.44(0.34) \\
\text { within group differences pre to post for both groups [body } \\
\text { weight supported treadmill } p=0.008 \text {, physical therapy } \\
p=0.007] \text {; but gains only maintained in treadmill group } \\
\text { GMFM } \\
\text { A. } 62.7(17.5) \text { to } 63.3(16.2) \text { to } 65.3(16.5) \\
\text { B. } 58.4(26.9) \text { to } 60.1(25.1) \text { to } 60.6(26.7) \\
\text { no significant change in either group and no difference } \\
\text { between groups } \\
\text { Pediatric Outcomes Data Collection Instrument (global) } \\
\text { A. } 50.4(11.2) \text { to } 59.3(11.4) \text { to } 60.0(10.0) \\
\text { B. } 50.9 \text { (14.9) to, } 52.0(22.6) \text { to } 55.4(21.7) \\
\text { score improved for all participants ( } p=0.003) \text { but no } \\
\text { difference between groups ( } p=0.73) \text {; only the treadmill } \\
\text { group maintained the improvement }\end{array}$ & $\begin{array}{l}\text { Plantar flexor spasticity } \\
\text { A. } 0.0013(0.0012) \text { to } 0.0016(0.0024) \text { to } \\
0.0012(0.0018) \\
\text { B. } 0.0030(0.0024) \text { to } 0.003 .(0.0021) \text { to } \\
0.0026(0.0013) \\
\text { Knee flexor spasticity } \\
\text { A. } 0.0088(0.0114), 0.0074(0.0133), 0.0083 \\
(0.0139) \\
\text { B. } 0.0032(0.0044), 0.0072(0.0137), 0.0053 \\
(0.0044) \\
\text { Knee extension strength } \\
\text { A. } 3.90 \text { (3.09) to } 3.58(2.82) \text { to } 3.06(3.25) \\
\text { B. } 3.09 \text { (3.15) to } 3.80 \text { (4.22) to } 3.69(3.66) \\
\text { Knee flexion strength } \\
\text { A. } 2.47 \text { (1.45) to } 2.43(1.54) \text { to } 2.57(1.65) \\
\text { B. } 2.35 \text { (2.04) to } 2.98 \text { (3.26) to } 2.54(2.09) \\
\text { No significant within or between group } \\
\text { differences in spasticity or strength }\end{array}$ \\
\hline
\end{tabular}




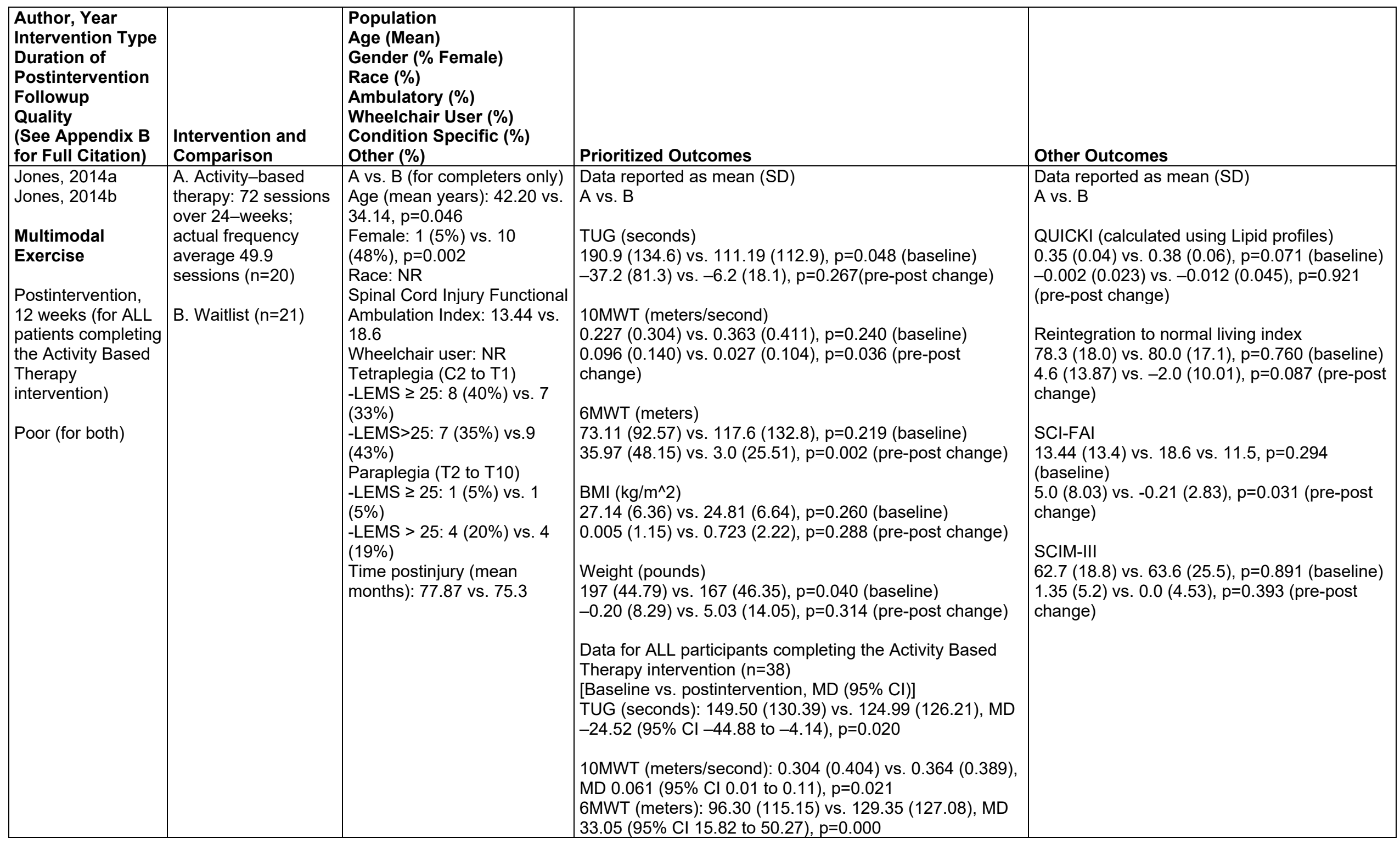




\begin{tabular}{|c|c|c|c|c|}
\hline $\begin{array}{l}\text { Author, Year } \\
\text { Intervention Type } \\
\text { Duration of } \\
\text { Postintervention } \\
\text { Followup } \\
\text { Quality } \\
\text { (See Appendix B } \\
\text { for Full Citation) }\end{array}$ & $\begin{array}{l}\text { Intervention and } \\
\text { Comparison }\end{array}$ & $\begin{array}{l}\text { Population } \\
\text { Age (Mean) } \\
\text { Gender (\% Female) } \\
\text { Race (\%) } \\
\text { Ambulatory (\%) } \\
\text { Wheelchair User (\%) } \\
\text { Condition Specific (\%) } \\
\text { Other (\%) }\end{array}$ & Prioritized Outcomes & Other Outcomes \\
\hline $\begin{array}{l}\text { Jones, 2014a } \\
\text { Jones, 2014b } \\
\text { (Continued) }\end{array}$ & & & 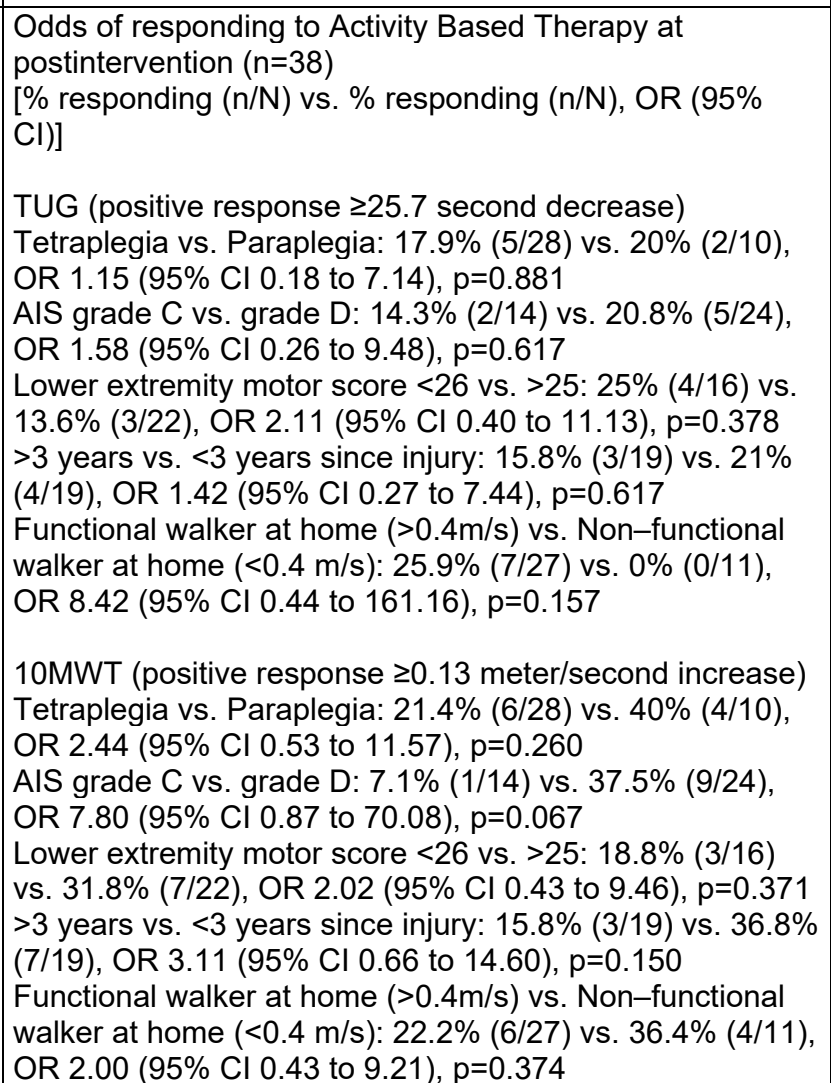 & \\
\hline
\end{tabular}




\begin{tabular}{|c|c|c|c|c|}
\hline $\begin{array}{l}\text { Author, Year } \\
\text { Intervention Type } \\
\text { Duration of } \\
\text { Postintervention } \\
\text { Followup } \\
\text { Quality } \\
\text { (See Appendix B } \\
\text { for Full Citation) }\end{array}$ & $\begin{array}{l}\text { Intervention and } \\
\text { Comparison }\end{array}$ & $\begin{array}{l}\text { Population } \\
\text { Age (Mean) } \\
\text { Gender (\% Female) } \\
\text { Race (\%) } \\
\text { Ambulatory (\%) } \\
\text { Wheelchair User (\%) } \\
\text { Condition Specific (\%) } \\
\text { Other (\%) }\end{array}$ & Prioritized Outcomes & Other Outcomes \\
\hline $\begin{array}{l}\text { Jones, 2014a } \\
\text { Jones, 2014b } \\
\text { (Continued) }\end{array}$ & & & 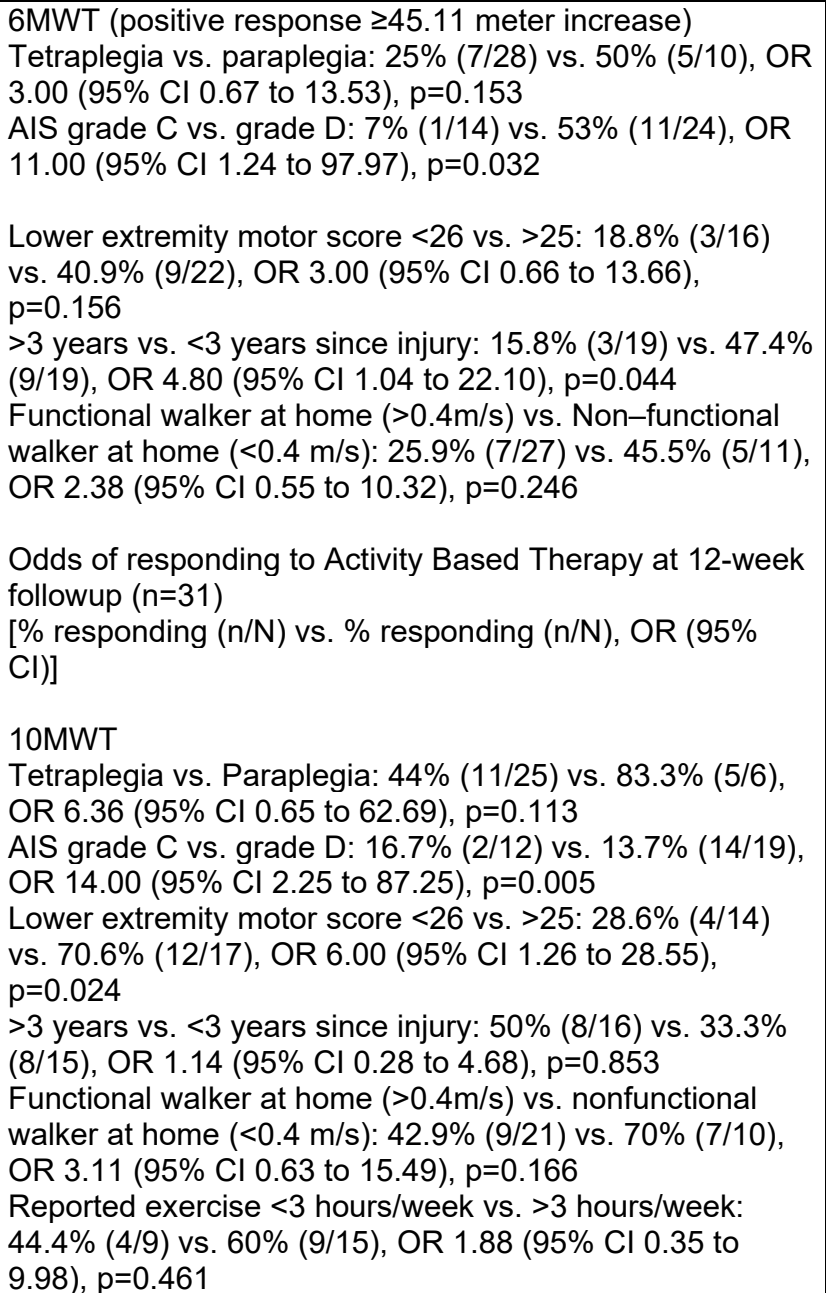 & \\
\hline
\end{tabular}




\begin{tabular}{|c|c|c|c|c|}
\hline $\begin{array}{l}\text { Author, Year } \\
\text { Intervention Type } \\
\text { Duration of } \\
\text { Postintervention } \\
\text { Followup } \\
\text { Quality } \\
\text { (See Appendix B } \\
\text { for Full Citation) } \\
\end{array}$ & $\begin{array}{l}\text { Intervention and } \\
\text { Comparison }\end{array}$ & $\begin{array}{l}\text { Population } \\
\text { Age (Mean) } \\
\text { Gender (\% Female) } \\
\text { Race (\%) } \\
\text { Ambulatory (\%) } \\
\text { Wheelchair User (\%) } \\
\text { Condition Specific (\%) } \\
\text { Other (\%) }\end{array}$ & Prioritized Outcomes & Other Outcomes \\
\hline $\begin{array}{l}\text { Jones, 2014a } \\
\text { Jones, 2014b } \\
\text { (Continued) }\end{array}$ & & & 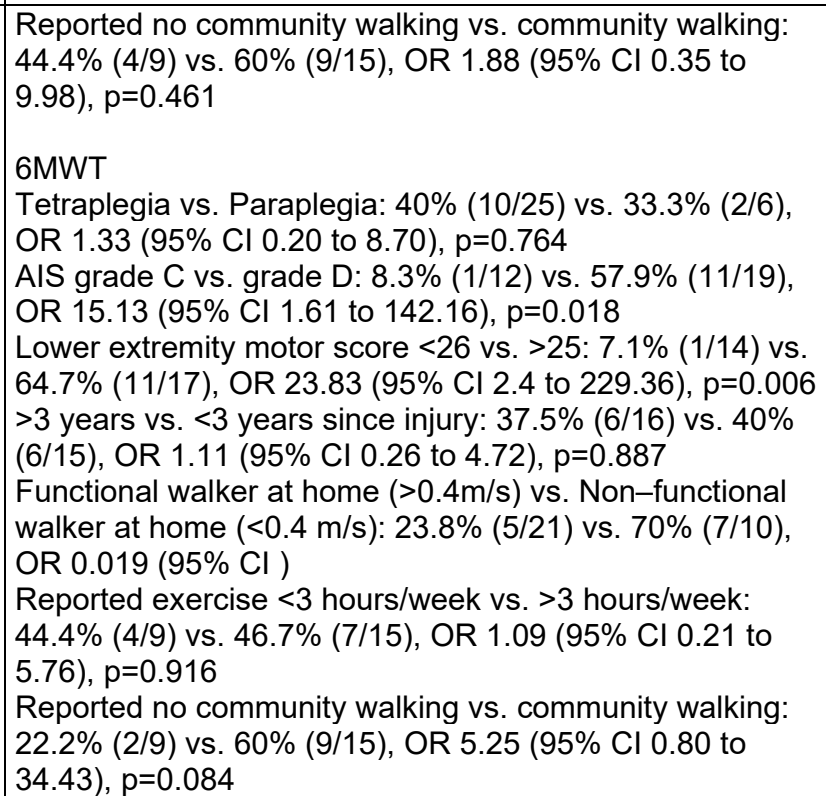 & \\
\hline $\begin{array}{l}\text { Jung, } 2014 \\
\text { Aerobic Exercise } \\
\text { Aquatics } \\
\text { Postintervention, } 0 \\
\text { weeks } \\
\text { Fair }\end{array}$ & $\begin{array}{l}\text { A. Aquatic exercise, } \\
24 \text { sessions over } 8 \\
\text { weeks }(n=10) \\
\text { B. Land exercise, } 24 \\
\text { sessions over } 8 \\
\text { weeks }(n=10)\end{array}$ & $\begin{array}{l}\text { A vs. B } \\
\text { Age (mean years): } \\
42.1 \text { vs. } 51.1 \\
\text { Female: } \\
3(30 \%) \text { vs. } 5(50 \%) \\
\text { Race: NR } \\
\text { Ambulatory: NR } \\
\text { Wheelchair user: NR }\end{array}$ & $\begin{array}{l}\text { A vs. B } \\
\text { FVC(L): } \\
2.5(0.7) \text { vs. } 3.0(0.9) \text { baseline } \\
4.3(1.4) \text { vs. } 3.4(1.4) \text {; change values }-1.8(1.3) \text { vs. }-0.31 \\
(1.6), p<0.01 \text { (postintervention) } \\
\text { FEV1(L): } \\
2.1(0.9) \text { vs. } 2.7(1.0) \text { baseline } \\
3.2(1.2) \text { vs. } 2.9(1.0) \text {; change values }-1.1(1.2) \text { vs. }-0.21 \\
(0.3) ; p<0.05 \text { (postintervention) } \\
\text { FER(L/sec) and FEV1/FVC: all NS }\end{array}$ & NA \\
\hline
\end{tabular}




\begin{tabular}{|c|c|c|c|c|}
\hline $\begin{array}{l}\text { Author, Year } \\
\text { Intervention Type } \\
\text { Duration of } \\
\text { Postintervention } \\
\text { Followup } \\
\text { Quality } \\
\text { (See Appendix B } \\
\text { for Full Citation) } \\
\end{array}$ & $\begin{array}{l}\text { Intervention and } \\
\text { Comparison }\end{array}$ & \begin{tabular}{|l|} 
Population \\
Age (Mean) \\
Gender (\% Female) \\
Race (\%) \\
Ambulatory (\%) \\
Wheelchair User (\%) \\
Condition Specific (\%) \\
Other (\%) \\
\end{tabular} & Prioritized Outcomes & Other Outcomes \\
\hline $\begin{array}{l}\text { Jonsdottir, } 2018 \\
\text { Aerobic Exercise } \\
\text { Treadmill } \\
\text { Postintervention, } 0 \\
\text { weeks } \\
\text { Fair }\end{array}$ & $\begin{array}{l}\text { A. } 30 \text { minutes } \\
\text { treadmill walking, } 5 \\
\text { days a week for } 8 \\
\text { weeks; } 10 \text { of the } 30 \\
\text { minutes was doing } \\
\text { other cognitive or } \\
\text { motor tasks (dual } \\
\text { tasking treadmill) } \\
\\
\text { B. Strength training } 4 \\
\text { or } 5 \text { days a week, } 3 \\
\text { sets with } 10 \text { reps/set } \\
\text { multiple lifts }\end{array}$ & $\begin{array}{l}\text { EDSS } 5.53 \\
(3.5-7) \\
n=42 \\
\text { Mean age } 54.05 \text { years } \\
(n=38) \\
\\
28 \text { females and } 10 \text { males } \\
(26.3 \% \text { males })\end{array}$ & 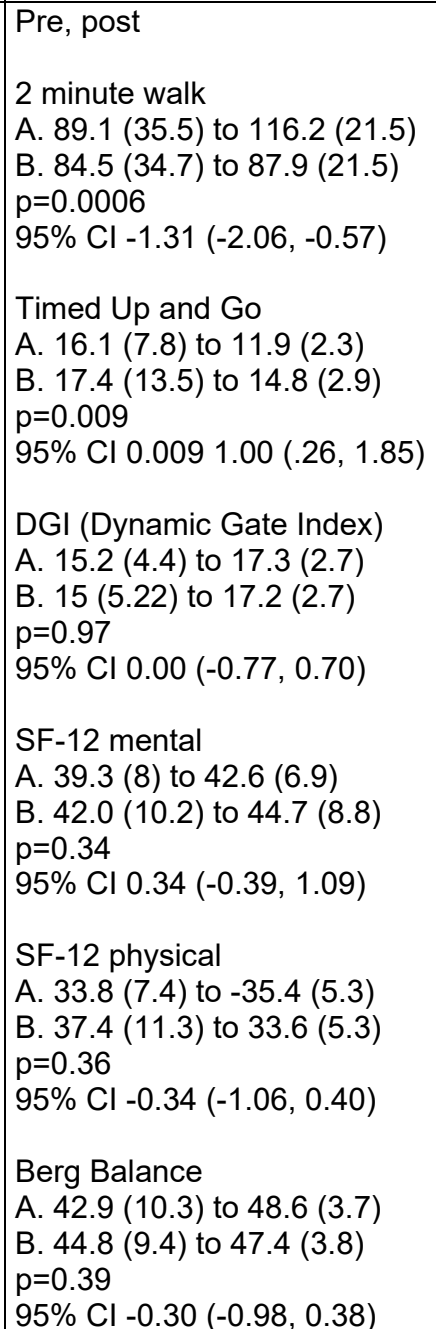 & NA \\
\hline
\end{tabular}




\begin{tabular}{|c|c|c|c|c|}
\hline $\begin{array}{l}\text { Author, Year } \\
\text { Intervention Type } \\
\text { Duration of } \\
\text { Postintervention } \\
\text { Followup } \\
\text { Quality } \\
\text { (See Appendix B } \\
\text { for Full Citation) }\end{array}$ & $\begin{array}{l}\text { Intervention and } \\
\text { Comparison }\end{array}$ & $\begin{array}{l}\text { Population } \\
\text { Age (Mean) } \\
\text { Gender (\% Female) } \\
\text { Race (\%) } \\
\text { Ambulatory (\%) } \\
\text { Wheelchair User (\%) } \\
\text { Condition Specific (\%) } \\
\text { Other (\%) }\end{array}$ & Prioritized Outcomes & Other Outcomes \\
\hline $\begin{array}{l}\text { Kalron, } 2016 \\
\text { Postural Control } \\
\text { Motion gaming } \\
\text { Postintervention, } 6 \\
\text { weeks } \\
\text { Fair }\end{array}$ & $\begin{array}{l}\text { A. Virtual reality } \\
\text { (motion platform, VR } \\
\text { 3D visuals and } \\
\text { sound, plus balance } \\
\text { training): } 12 \text { sessions } \\
\text { over } 6 \text { weeks }(n=16) \\
\\
\text { B. Usual care control } \\
\text { (conventional } \\
\text { exercise, plus } \\
\text { balance training): } 12 \\
\text { sessions over } 6 \\
\text { weeks ( } n=16 \text { ) }\end{array}$ & $\begin{array}{l}\text { A vs. B NR } \\
\text { Age (mean years): } 45.2 \\
\text { Female: } 19 \text { (63\%) vs. } 11 \\
(37 \%) \\
\text { Race: NR } \\
\text { Ambulatory: NR } \\
\text { Wheelchair user: NR } \\
\text { EDSS, mean (SD): } 4.1(1.3)\end{array}$ & $\begin{array}{l}\text { A. vs. B., mean (SD) } \\
\text { FSST: } \\
16.2(7.0) \text { vs. } 14.2(7.1), \text { (baseline) } \\
12.7(6.4) \text { vs. } 11.7(5.9) \text { (postintervention) } \\
\text { Mean difference }-3.5(6.1), F=9.011, p=0.031 \\
\text { FES-I: } \\
36.4(9.7) \text { vs. } 32.9(10.3) \text { (baseline) } \\
29.4(7.8) \text { vs. } 28.6(5.8) \text { (postintervention) } \\
\text { Mean difference }-4.3(6.3), F=17.815, p=0.023\end{array}$ & $\begin{array}{l}\text { A. vs. B, mean (SD) } \\
\text { BBS* } \\
46.8(9.6) \text { vs. } 43.3(7.1) \text { (baseline) } \\
47.9(6.4) \text { vs. } 44.6(4.9) \text { (postintervention) } \\
\text { Mean difference } 1.3(5.2), F=1.541, p=0.215 \\
\\
\text { *Labeled in the study as "BBT- Berg Balance } \\
\text { Test" }\end{array}$ \\
\hline
\end{tabular}




\begin{tabular}{|c|c|c|c|c|}
\hline $\begin{array}{l}\text { Author, Year } \\
\text { Intervention Type } \\
\text { Duration of } \\
\text { Postintervention } \\
\text { Followup } \\
\text { Quality } \\
\text { (See Appendix B } \\
\text { for Full Citation) } \\
\end{array}$ & $\begin{array}{l}\text { Intervention and } \\
\text { Comparison }\end{array}$ & $\begin{array}{l}\text { Population } \\
\text { Age (Mean) } \\
\text { Gender (\% Female) } \\
\text { Race (\%) } \\
\text { Ambulatory (\%) } \\
\text { Wheelchair User (\%) } \\
\text { Condition Specific (\%) } \\
\text { Other (\%) } \\
\end{array}$ & Prioritized Outcomes & Other Outcomes \\
\hline $\begin{array}{l}\text { Kalron, } 2017 \\
\text { Muscle Strength } \\
\text { Postintervention, } 0 \\
\text { weeks } \\
\text { Fair }\end{array}$ & $\begin{array}{l}\text { A. Pilates: } 12 \\
\text { sessions over } 12 \\
\text { weeks }+15 \text {-minute } \\
\text { daily home exercise } \\
\text { program ( } n=22) \\
\\
\text { B. Standardized } \\
\text { physical therapy } \\
\text { (Usual care ?): } 12 \\
\text { sessions over } 12 \\
\text { weeks + 15-minute } \\
\text { daily home exercise } \\
\text { program }(n=23)\end{array}$ & $\begin{array}{l}\text { A vs. B [not accounting for } \\
\text { those lost to followup] } \\
\text { Age (mean years): } 42.9 \text { vs. } \\
44.3 \\
\text { Female: } 14 \text { (60.9\%) vs. } 15 \\
(68.2 \%) \\
\text { Race: NR } \\
\text { Ambulatory: NR (minimum } \\
\text { ability to walk } 100 \text { m with or } \\
\text { without resting with the } \\
\text { assistance of a walking aid } \\
\text { was required for inclusion) } \\
\text { Wheelchair user: NR } \\
\text { Baseline EDSS (mean): } 4.1 \\
\text { vs. } 4.6 \\
\text { Disease duration (mean } \\
\text { years): } 12.4 \text { vs. } 11.3\end{array}$ & 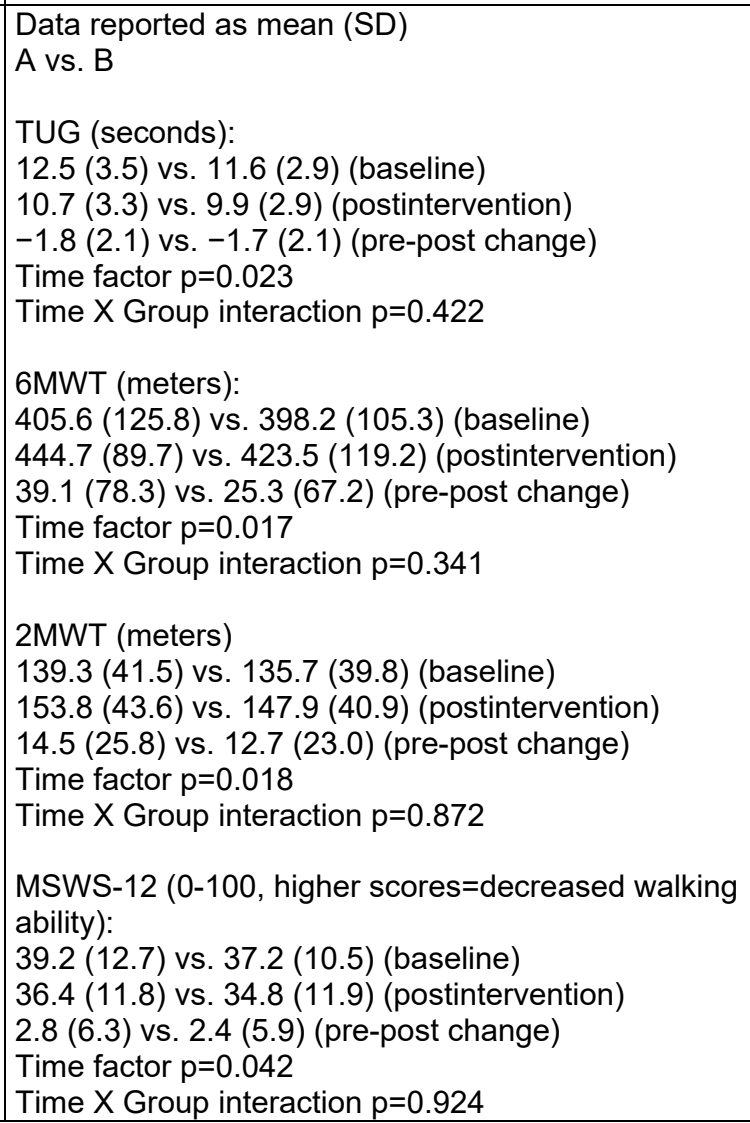 & $\begin{array}{l}\text { Data reported as mean (SD) } \\
\text { A vs. B } \\
\text { BBS }(0-56, \text { higher scores=better balance): } \\
46.8(9.6) \text { vs. } 43.3(7.1) \text { (baseline) } \\
47.9(6.4) \text { vs. } 44.6(4.9) \text { (postintervention) } \\
1.1(4.2) \text { vs. } 1.3(5.2) \text { (pre-post change) } \\
\text { Time factor } p=0.215 \\
\text { Time } X \text { Group interaction } p=0.561\end{array}$ \\
\hline
\end{tabular}




\begin{tabular}{|c|c|c|c|c|}
\hline $\begin{array}{l}\text { Author, Year } \\
\text { Intervention Type } \\
\text { Duration of } \\
\text { Postintervention } \\
\text { Followup } \\
\text { Quality } \\
\text { (See Appendix B } \\
\text { for Full Citation) } \\
\end{array}$ & $\begin{array}{l}\text { Intervention and } \\
\text { Comparison }\end{array}$ & $\begin{array}{l}\text { Population } \\
\text { Age (Mean) } \\
\text { Gender (\% Female) } \\
\text { Race (\%) } \\
\text { Ambulatory (\%) } \\
\text { Wheelchair User (\%) } \\
\text { Condition Specific (\%) } \\
\text { Other (\%) }\end{array}$ & Prioritized Outcomes & Other Outcomes \\
\hline $\begin{array}{l}\text { Kara, } 2017 \\
\text { Aerobic Exercise } \\
\text { Aerobics } \\
\text { Postintervention, } 0 \\
\text { weeks } \\
\text { Poor }\end{array}$ & $\begin{array}{l}\text { A. Pilates: } 16 \\
\text { sessions over } 8 \\
\text { weeks }(n=27) \\
\text { B. Aerobic exercise: } \\
16 \text { sessions over } 8 \\
\text { weeks }(n=28)\end{array}$ & $\begin{array}{l}\text { A vs. B } \\
\text { Age: } 50 \text { vs. } 43 \\
\text { Female: } 67 \% \text { vs. } 65 \% \\
\text { EDSS: } 2.85 \text { vs. } 3.2\end{array}$ & $\begin{array}{l}\text { Data reported as mean (SD) } \\
\text { A vs. B } \\
\text { TUG right } \\
11.75(3.38) \text { vs. } 10.33(6.32), p<0.001 \text { (baseline) } \\
10.51(2.69) \text { vs. } 9.56(6.04), p=0.075 \text { (postintervention) } \\
\text { TUG left: } \\
12.74(3.32) \text { vs. } 10.33(6.28), p=0.001 \text { (baseline) } \\
9.73(3.17) \text { vs. } 10.39(7.09), p=0.515 \text { (postintervention) } \\
\text { BDI: } \\
11.44(6.52) \text { vs. } 8.92(6.49), p=0.001 \text { (baseline) } \\
9.77(5.26) \text { vs. } 7.15(6.35), p=0.156 \text { (postintervention) }\end{array}$ & $\begin{array}{l}\text { Data reported as mean (SD) } \\
\text { A vs. B } \\
44.66(10.98) \text { vs. } 46.11 \text { (12.44), p=0.028 } \\
\text { (baseline) } \\
47.77(13.89) \text { vs. } 48.57 \text { (16.02), p=0.243 } \\
\text { (postintervention) } \\
3.11(\mathrm{NR}) \text { vs. } 2.46 \text { (NR), p=NR (pre-post } \\
\text { change) }\end{array}$ \\
\hline $\begin{array}{l}\text { Kara, } 2020 \\
\text { Strength } \\
\text { Immediately } \\
\text { postintervention, } \\
12 \text { weeks } \\
\text { Fair }\end{array}$ & $\begin{array}{l}\text { A. Strength and } \\
\text { power training, } 36 \\
\text { sessions over } 12 \\
\text { weeks ( } n=15) \\
\text { B. Usual care; } \\
\text { occupational therapy, } \\
36 \text { sessions over } 12 \\
\text { weeks ( } n=15)\end{array}$ & $\begin{array}{l}\text { A vs. B } \\
\text { Age: } 12.3 \text { vs. } 11.8 \\
\text { Female: } 53 \% \text { vs. } 53 \% \\
\text { Race: NR } \\
\text { Ambulatory: NR } \\
\text { Wheelchair: NR } \\
\text { Manual ability classification } \\
\text { system (MACS) Level } \\
\text { I: } 47 \% \text { vs. } 40 \% \\
\text { II: } 27 \% \text { vs. } 33 \% \\
\text { III: } 27 \% \text { vs. } 27 \% \\
\text { GMFCS Level } \\
\text { I: } 87 \% \text { vs. } 87 \% \\
\text { II: } 13 \% \text { vs. } 13 \%\end{array}$ & $\begin{array}{l}\text { A vs. B, Mean (SD), p-value for between group difference } \\
\text { QUEST total } \\
\text { 8.88 (6.51) vs. } 2.22 \text { (4.74), MD } 6.65(95 \% \mathrm{Cl} 2.4 \text { to } 10.9) \\
p=0.001 \text { (pre-post change) }\end{array}$ & $\begin{array}{l}\text { A vs. B, Mean (SD), p-value for between group } \\
\text { difference } \\
\text { COMP total } \\
6.12(2.33) \text { vs. } 0.41 \text { (1.56), MD } 5.71(95 \% \mathrm{Cl} \\
4.2 \text { to } 7.2), p<0.001 \text { (pre-post change) }\end{array}$ \\
\hline
\end{tabular}




\begin{tabular}{|c|c|c|c|c|}
\hline $\begin{array}{l}\text { Author, Year } \\
\text { Intervention Type } \\
\text { Duration of } \\
\text { Postintervention } \\
\text { Followup } \\
\text { Quality } \\
\text { (See Appendix B } \\
\text { for Full Citation) } \\
\end{array}$ & $\begin{array}{l}\text { Intervention and } \\
\text { Comparison }\end{array}$ & $\begin{array}{l}\text { Population } \\
\text { Age (Mean) } \\
\text { Gender (\% Female) } \\
\text { Race (\%) } \\
\text { Ambulatory (\%) } \\
\text { Wheelchair User (\%) } \\
\text { Condition Specific (\%) } \\
\text { Other (\%) } \\
\end{array}$ & Prioritized Outcomes & Other Outcomes \\
\hline $\begin{array}{l}\text { Kargarfard, } 2017 \\
\text { Aerobic Exercise } \\
\text { Aquatics } \\
\text { Postintervention, } 0 \\
\text { weeks } \\
\text { Fair }\end{array}$ & \begin{tabular}{|l|} 
A. Aquatic exercise, \\
24 sessions over 8 \\
weeks $(n=17)$, plus \\
$2-3$ sessions per \\
week with neurologic \\
PTs and once weekly \\
educational session \\
\\
B. $2-3$ sessions per \\
week with neurologic \\
PTs and once weekly \\
educational session, \\
$16-24$ sessions over \\
8 weeks $(n=15)$
\end{tabular} & $\begin{array}{l}\text { A vs. B } \\
\text { Age (mean years): } \\
36.5(9.0) \text { vs. } 36.2(7.4) \\
\text { Female: } \\
20(100 \%) \text { vs. } 15(100 \%) \\
\text { Race: NR } \\
\text { Ambulatory: NR } \\
\text { Wheelchair user: NR } \\
\text { Other: } \\
\text { MS: subtypes NR }\end{array}$ & $\begin{array}{l}\text { A vs. B, mean (SD) } \\
\text { 6MWT: } \\
451 \text { (58) vs. } 447 \text { (30) (baseline) } \\
503 \text { (57) vs. } 418(29) ; p<0.001 \text { (postintervention) } \\
\text { Sit to Stand: } \\
21.0(5.7) \text { vs. } 21.4(4.7) \text { (baseline) } \\
16.8(5.1) \text { vs. } 27.3 \text { (4.8); } p<0.001 \text { (postintervention) } \\
\text { Calculated A vs. B, Mean change scores: } \\
\text { 6MWT: }-52 \text { vs. } 29, p<0.001 \\
\text { Sit to Stand: } 4.2 \text { vs. }-5.9, p<0.001 \\
\text { BBS: }-1.6 \text { vs. } 2.1, p<0.001\end{array}$ & $\begin{array}{l}\text { A vs. B, mean (SD) } \\
\text { Pushup: } \\
17 \text { (9) vs. } 18(7) \text { (baseline) } \\
26 \text { (11) vs. } 10(5) ; p<0.001 \text { (postintervention) } \\
\text { BBS: } \\
53.6(1.7) \text { vs. } 52.3(3.3) \text { (baseline) } \\
55.2(1.2) \text { vs. } 50.2(4.6) ; p<0.001 \\
\text { (postintervention) }\end{array}$ \\
\hline $\begin{array}{l}\text { Kaya Kara, } 2019 \\
\text { Multimodal } \\
\text { Exercise } \\
\text { Immediately } \\
\text { postintervention, } \\
12 \text { weeks } \\
\text { Fair }\end{array}$ & $\begin{array}{l}\text { A. Strength training } \\
\text { (progressive } \\
\text { resistance exercise) } \\
+ \text { balance, } 36 \\
\text { sessions over } 12 \\
\text { weeks ( } n=17) \\
\\
\text { B. Usual care, } \\
36 \text { sessions over } 12 \\
\text { weeks }(n=16)\end{array}$ & $\begin{array}{l}\text { A vs. B } \\
\text { Age: } 11.8 \text { vs. } 11.3 \\
\text { Female: } 53 \% \text { vs. } 60 \% \\
\text { Ambulatory: } 100 \% \\
\text { Manual ability classification } \\
\text { system level: } \\
\text { I: } 47 \% \text { vs. } 47 \% \\
\text { II: } 33 \% \text { vs. } 27 \% \\
\text { III: } 20 \% \text { vs. } 27 \%\end{array}$ & $\begin{array}{l}\text { A vs. B, Mean change from baseline (SD) } \\
\text { (data are for completers only; } n=15 \text { vs. } 15) \\
\text { GMFM-88D } \\
0.17(0.67) \text { vs. } 0.32(1.42), M D-0.15(95 \% \mathrm{Cl}-0.93 \text { to } \\
0.63), p=0.632 \text {; effect size } 0.13 \\
\text { GMFM-88E } \\
2.31(2.20) \text { vs. }-0.37(2.59), M D 2.68(95 \% \mathrm{Cl} 0.98 \text { to } \\
4.38), p=0.004 ; \text { effect size } 1.11 \\
1 \text { minute walk } \\
7.76(7.03) \text { vs. } 0.53(3.37), \mathrm{MD} 7.23(95 \% \mathrm{Cl} \mathrm{NR}), \\
p=0.001 ; \text { effect size } 1.31 \\
\text { TUG } \\
-1.02(0.45) \text { vs. } 0.08(0.45), \mathrm{MD}-1.10(95 \% \mathrm{Cl}-1.42 \text { to } \\
-0.78), p<0.001 ; \text { effect size } 2.42\end{array}$ & $\begin{array}{l}\text { A vs. B, mean difference, Effect size, p-value } \\
\text { is between groups } \\
\text { Affected lower leg } 1 \mathrm{RM}(\mathrm{kg}): 54.33 \text {, ES } 3.23 \text {, } \\
p<0.001 \\
\text { Unaffected lower leg } 1 \mathrm{RM}(\mathrm{kg}): 44.33, \text { ES } \\
2.74, p<0.001\end{array}$ \\
\hline
\end{tabular}




\begin{tabular}{|c|c|c|c|c|}
\hline $\begin{array}{l}\text { Author, Year } \\
\text { Intervention Type } \\
\text { Duration of } \\
\text { Postintervention } \\
\text { Followup } \\
\text { Quality } \\
\text { (See Appendix B } \\
\text { for Full Citation) } \\
\end{array}$ & $\begin{array}{l}\text { Intervention and } \\
\text { Comparison }\end{array}$ & $\begin{array}{l}\text { Population } \\
\text { Age (Mean) } \\
\text { Gender (\% Female) } \\
\text { Race (\%) } \\
\text { Ambulatory (\%) } \\
\text { Wheelchair User (\%) } \\
\text { Condition Specific (\%) } \\
\text { Other (\%) } \\
\end{array}$ & Prioritized Outcomes & Other Outcomes \\
\hline $\begin{array}{l}\text { Kerling, } 2015 \\
\text { Multimodal } \\
\text { Exercise } \\
\text { Postintervention, } 0 \\
\text { weeks }\end{array}$ & $\begin{array}{l}\text { A. Full body } \\
\text { progressive } \\
\text { resistance }+ \text { aerobic } \\
\text { training }(n=30) \\
\text { B. Aerobic training } \\
(n=30)\end{array}$ & $\begin{array}{l}\text { A vs. B } \\
\text { Age (mean years): } 42.3 \text { vs. } \\
45.6 \\
\text { Female: } 24(80 \%) \text { vs. } 20 \\
(67 \%) \\
\text { Race: NR } \\
\text { Ambulatory: NR } \\
\text { Wheelchair user: NR }\end{array}$ & $\begin{array}{l}\text { Data reported as mean (SD) } \\
\text { A vs. B } \\
\text { SF-36 PCS }(0-100, \text { higher=greater QOL) } \\
44.9(9.1) \text { vs. } 39.0(10.8), p=N R \text { (baseline) } \\
46.2(9.1) \text { vs. } 39.6(11.3), p=N R \text { (postintervention) } \\
\text { Time X Group } p=0.56\end{array}$ & $\begin{array}{l}\text { Data reported as mean (SD) } \\
\text { A vs. B } \\
\text { Resting HR (bpm): } \\
92(12) \text { vs. } 88(12), p=N R \text { (baseline) } \\
90(11) \text { vs. } 85(13), p=N R \text { (postintervention) } \\
\text { Time X Group } p=0.63\end{array}$ \\
\hline Fair & $\begin{array}{l}\text { [36 sessions over } 12 \\
\text { weeks for both } \\
\text { groups] }\end{array}$ & $\begin{array}{l}\text { MS specific medication } \\
\text { (yes): } 20(67 \%) \text { vs. } 20(67 \%)\end{array}$ & $\begin{array}{l}\text { SF-36 MCS }(0-100, \text { higher=greater QOL) } \\
44.9(13.6) \text { vs. } 46.7(11.7), p=N R \text { (baseline) } \\
45.4(13.4) \text { vs. } 51.4(8.6), p=N R \text { (postintervention) } \\
\text { Time X Group } p=0.01 \\
\text { VO2-peak (mL/min): } \\
1684(601) \text { vs. } 1632(539), p=N R \text { (baseline) } \\
1756(599) \text { vs. } 1676(494), p=N R \text { (postintervention) } \\
\text { Time X Group } p=0.71 \\
\text { VO }{ }_{2} \text {-peak (ml/min/kg): } \\
23.8(7.8) \text { vs. } 23.5(8.2), p=N R \text { (baseline) } \\
24.6(7.4) \text { vs. } 23.7(7.1), p=N R \text { (postintervention) } \\
\text { Time X Group } p=0.72\end{array}$ & 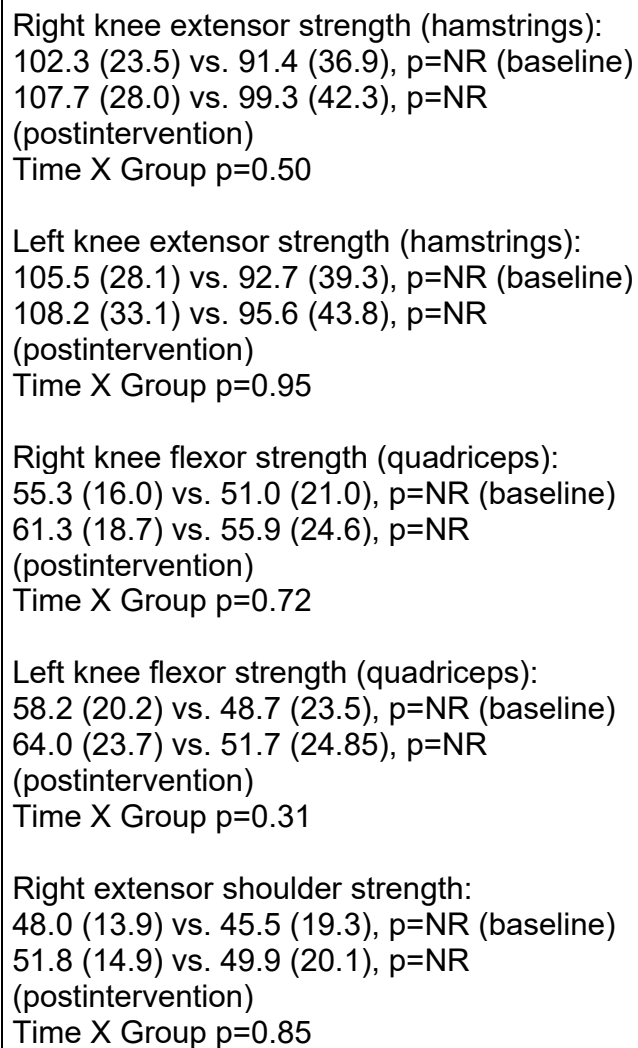 \\
\hline
\end{tabular}




\begin{tabular}{|c|c|c|c|c|}
\hline $\begin{array}{l}\text { Author, Year } \\
\text { Intervention Type } \\
\text { Duration of } \\
\text { Postintervention } \\
\text { Followup } \\
\text { Quality } \\
\text { (See Appendix B } \\
\text { for Full Citation) }\end{array}$ & $\begin{array}{l}\text { Intervention and } \\
\text { Comparison }\end{array}$ & $\begin{array}{l}\text { Population } \\
\text { Age (Mean) } \\
\text { Gender (\% Female) } \\
\text { Race (\%) } \\
\text { Ambulatory (\%) } \\
\text { Wheelchair User (\%) } \\
\text { Condition Specific (\%) } \\
\text { Other (\%) }\end{array}$ & Prioritized Outcomes & Other Outcomes \\
\hline $\begin{array}{l}\text { Kerling, } 2015 \\
\text { (Continued) }\end{array}$ & & & & $\begin{array}{l}\text { Left extensor shoulder strength: } \\
46.3(17.5) \text { vs. } 43.3(17.3), p=N R \text { (baseline) } \\
50.0(18.9) \text { vs. } 46.9(18.6), p=N R \\
\text { (postintervention) } \\
\text { Time X Group } p=0.98 \\
\text { Right flexor shoulder strength: } \\
34.2(9.6) \text { vs. } 35.3(12.6), p=N R \text { (baseline) } \\
36.5(10.0) \text { vs. } 36.9(14.1), p=N R \\
\text { (postintervention) } \\
\text { Time X Group } p=0.67 \\
\\
\text { Left flexor shoulder strength: } \\
35.8(13.9) \text { vs. } 34.0(12.1), p=N R \text { (baseline) } \\
36.9(12.4) \text { vs. } 35.9(12.5), p=N R \\
\text { (postintervention) } \\
\text { Time X Group } p=0.60\end{array}$ \\
\hline $\begin{array}{l}\text { Keser, } 2011 \\
\text { Aerobic Exercise } \\
\text { Aerobics } \\
\text { Postintervention, } 0 \\
\text { weeks } \\
\text { Poor }\end{array}$ & $\begin{array}{l}\text { A. Calisthenics, } 18 \\
\text { sessions over } 6 \\
\text { weeks (15) } \\
\text { B. Routine } \\
\text { neurorehabilitation } \\
\text { (strength, balance, } \\
\text { coordination, anti- } \\
\text { spasticity exercises) } \\
18 \text { sessions over } 6 \\
\text { weeks (15) }\end{array}$ & $\begin{array}{l}\text { A vs. B } \\
\text { Age: } 36 \text { vs. } 35 \\
\text { Female: } 53 \% \text { vs. } 47 \% \\
\text { EDSS: } 2.9 \text { vs. } 2.8\end{array}$ & $\begin{array}{l}\text { A vs. B mean Difference } \\
\text { MSFC: }-0.002(0.44) \text { vs. } 0.02(0.23), p>0.05 \\
\text { SF-36: } 0.20(5.67) \text { vs. } 1.73(7.75), p>0.05 \\
\text { HADS-A: }-2.26(3.23) \text { vs. }-0.80(2.40), p>0.05 \\
\text { HADS-D: } 0.20(2.65) \text { vs. } 1.46(2.19), p>0.05\end{array}$ & $\begin{array}{l}\text { A vs. B mean Difference } \\
\text { BBS: }-1.73 \text { (3.03) vs. }-1.80(2.67), p>0.05 \\
\text { Strength UE right: } \\
8.67 \text { (10.17) vs. } 15.19 \text { (7.77), } p<0.05 \\
\text { Strength UE left: } \\
7.86 \text { (11.97) vs. } 16.25 \text { (10.95), } p<0.05 \\
\text { Strength LE right: } \\
15.76 \text { (11.17) vs. } 20.66 \text { (6.18), } p>0.05 \\
\text { Strength LE left: } \\
18.54 \text { (7.59) vs. } 24.17 \text { (16.69), } p>0.05\end{array}$ \\
\hline
\end{tabular}




\begin{tabular}{|c|c|c|c|c|}
\hline $\begin{array}{l}\text { Author, Year } \\
\text { Intervention Type } \\
\text { Duration of } \\
\text { Postintervention } \\
\text { Followup } \\
\text { Quality } \\
\text { (See Appendix B } \\
\text { for Full Citation) } \\
\end{array}$ & $\begin{array}{l}\text { Intervention and } \\
\text { Comparison }\end{array}$ & $\begin{array}{l}\text { Population } \\
\text { Age (Mean) } \\
\text { Gender (\% Female) } \\
\text { Race (\%) } \\
\text { Ambulatory (\%) } \\
\text { Wheelchair User (\%) } \\
\text { Condition Specific (\%) } \\
\text { Other (\%) }\end{array}$ & Prioritized Outcomes & Other Outcomes \\
\hline $\begin{array}{l}\text { Khalil, } 2018 \\
\text { Postural Control } \\
\text { Motion gaming } \\
\text { Postintervention, } 6 \\
\text { weeks } \\
\text { Fair }\end{array}$ & $\begin{array}{l}\text { A. Nintendo Wii } \\
\text { balance board and } \\
\text { VR scenarios with } \\
\text { tasks to complete, } 12 \\
\text { sessions over } 6 \\
\text { weeks }(n=16) \\
\\
\text { B. Balance training at } \\
\text { home, } 18 \text { sessions } \\
\text { over } 6 \text { weeks }(n=16)\end{array}$ & $\begin{array}{l}\text { A vs. B } \\
\text { Age (mean years): } 39.8 \text { vs. } \\
34.9 \\
\text { Female: } 12(75 \%) \text { vs. } 10 \\
(63 \%) \\
\text { Race: NR } \\
\text { Ambulatory: NR } \\
\text { Wheelchair user: NR } \\
\text { Duration of MS (mean years) } \\
8.38 \text { vs. } 10.43 \\
\text { EDSS: } 2.9(1.4) \text { vs. } 3.1(1.1) \\
\\
\text { *EDSS = a lower score } \\
\text { indicates a better } \\
\text { performance }\end{array}$ & 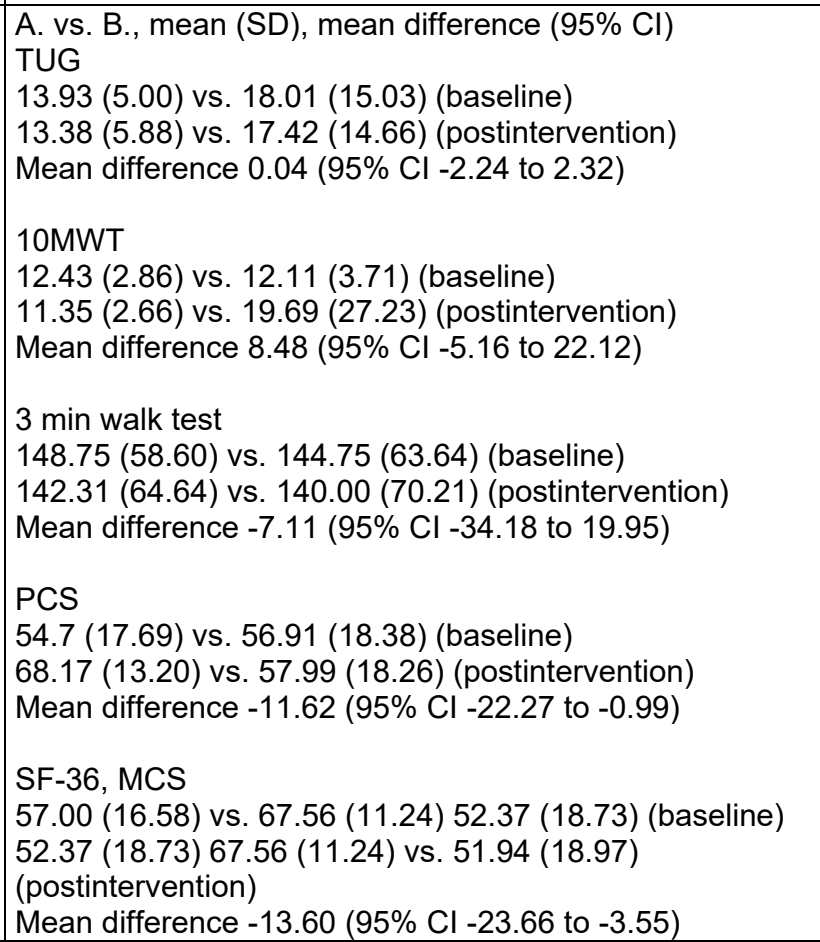 & $\begin{array}{l}\text { A. vs. B., mean (SD), mean difference }(95 \% \\
\text { CI) } \\
\text { BBS } \\
43.69(6.58) \text { vs. } 42.31 \text { (10.82) (baseline) } \\
50.44(3.76) \text { vs. } 45.19 \text { (8.64) (postintervention) } \\
\text { Mean difference }-4.52 \text { (95\% Cl }-7.90 \text { to }-1.09)\end{array}$ \\
\hline $\begin{array}{l}\text { Kim } 2015 \\
\text { Aerobic Exercise } \\
\text { Treadmill } \\
\text { Postintervention, } 0 \\
\text { weeks } \\
\text { Fair }\end{array}$ & $\begin{array}{l}\text { A. Conventional } \\
\text { physical therapy plus } \\
30 \text { minutes of } \\
\text { treadmill walking for } \\
20 \text { sessions ( } 3 \text { to } 5 \\
\text { sessions a week for } \\
1 \text { to } 2 \text { months) } \\
\text { B. Conventional } \\
\text { physical therapy for } \\
\text { similar number of } \\
\text { sessions }\end{array}$ & $\begin{array}{l}\text { Mean age } 27.2 \text { years } \\
11 \text { males and } 10 \text { females } \\
(52 \% \text { male }) \\
\text { A. } n=14 \\
\text { B. } n=7\end{array}$ & $\begin{array}{l}\text { Pre, post } \\
6 \text { minute walk (meters) } \\
\text { A. } 151.29(91.79) \text { to } 193.93(79.01) \\
\text { B. } 162.14(81.85) \text { to } 180.71(61.40) \\
\text { A. Significantly increased after training } p<0.05 \text { and B. Did } \\
\text { not significantly change; direct comparison changes in A } \\
\text { and B NR }\end{array}$ & NR \\
\hline
\end{tabular}




\begin{tabular}{|c|c|c|c|c|}
\hline $\begin{array}{l}\text { Author, Year } \\
\text { Intervention Type } \\
\text { Duration of } \\
\text { Postintervention } \\
\text { Followup } \\
\text { Quality } \\
\text { (See Appendix B } \\
\text { for Full Citation) } \\
\end{array}$ & $\begin{array}{l}\text { Intervention and } \\
\text { Comparison }\end{array}$ & $\begin{array}{l}\text { Population } \\
\text { Age (Mean) } \\
\text { Gender (\% Female) } \\
\text { Race (\%) } \\
\text { Ambulatory (\%) } \\
\text { Wheelchair User (\%) } \\
\text { Condition Specific (\%) } \\
\text { Other (\%) }\end{array}$ & Prioritized Outcomes & Other Outcomes \\
\hline $\begin{array}{l}\text { Kim, } 2017 \\
\text { Postural Control } \\
\text { Balance } \\
\text { Social } \\
\text { activity/exercise } \\
\text { (Boccia) } \\
\text { Postintervention, } 0 \\
\text { weeks } \\
\text { Poor }\end{array}$ & $\begin{array}{l}\text { A. Group boccia: } 12 \\
\text { sessions over } 6 \\
\text { weeks }(n=11) \\
\text { B. Usual care }(n=12)\end{array}$ & $\begin{array}{l}\text { A vs. B } \\
\text { Age (mean years): } 22.36 \text { vs. } \\
21.83 \\
\text { Female: } 5(45 \%) \text { vs. } 5(42 \%) \\
\text { Race: NR } \\
\text { Ambulatory: NR } \\
\text { Wheelchair user: NR }\end{array}$ & NR & $\begin{array}{l}\text { Data reported as mean (SD) } \\
\text { A vs. B } \\
\text { Modified Barthel Index (0-100, higher=greater } \\
\text { independence completing ADLs) } \\
39.00(9.34) \text { vs. } 35.67(11.41), p=N R \\
\text { (baseline) } \\
41.81(10.24) \text { vs. } 37.25(11.77), p=N R \\
\text { (postintervention) } \\
2.82(1.25) \text { vs. } 1.58(1.38), p<0.05, \text { MD } 1.24 \\
(95 \% \mathrm{Cl} 0.09 \text { to } 2.34), p=0.0352 \text { (post-pre } \\
\text { change) }\end{array}$ \\
\hline
\end{tabular}




\begin{tabular}{|c|c|c|c|c|}
\hline $\begin{array}{l}\text { Author, Year } \\
\text { Intervention Type } \\
\text { Duration of } \\
\text { Postintervention } \\
\text { Followup } \\
\text { Quality } \\
\text { (See Appendix B } \\
\text { for Full Citation) } \\
\end{array}$ & $\begin{array}{l}\text { Intervention and } \\
\text { Comparison }\end{array}$ & $\begin{array}{l}\text { Population } \\
\text { Age (Mean) } \\
\text { Gender (\% Female) } \\
\text { Race (\%) } \\
\text { Ambulatory (\%) } \\
\text { Wheelchair User (\%) } \\
\text { Condition Specific (\%) } \\
\text { Other (\%) }\end{array}$ & Prioritized Outcomes & Other Outcomes \\
\hline $\begin{array}{l}\text { Kirk, } 2016 \\
\text { Muscle Strength } \\
\text { Postintervention, } 0 \\
\text { weeks }\end{array}$ & $\begin{array}{l}\text { A. Progressive } \\
\text { resistance: } 36 \\
\text { sessions over } 12 \\
\text { weeks }(n=11) \\
\text { B. Usual care }(n=21)\end{array}$ & $\begin{array}{l}\text { A+B (data across all } \\
\text { patients) } \\
\text { Age (mean years): } 36.5 \\
\text { Female: } 15(43 \%) \\
\text { Race: NR } \\
\text { Ambulatory: NR } \\
\text { Wheelchair user: } 6(17 \%)\end{array}$ & $\begin{array}{l}\text { Data reported as mean (SD) } \\
\text { A vs. B } \\
\text { 10MWT (seconds) } \\
7.76(1.23) \text { vs. } 8.83(0.78) \text { (baseline) } \\
7.49(1.10) \text { vs. } 8.47(0.86) \text { (postintervention) }\end{array}$ & $\begin{array}{l}\text { Data reported as mean (SD) } \\
\text { A vs. B } \\
\text { There was a statistically significant Groups X } \\
\text { Time interaction for the 1RM measurements of } \\
\text { all exercises. }\end{array}$ \\
\hline Poor & & & $\begin{array}{l}\text { 6MWT (meters) } \\
481 \text { (30) vs. } 400 \text { (32) (baseline) } \\
510(33) \text { vs. } 416 \text { (33) (postintervention) } \\
\text { Timed Stair Test (seconds) } \\
30.69 \text { (4.92) vs. } 49.82 \text { (7.27) (baseline) } \\
29.15(4.62) \text { vs. } 45.01 \text { (6.57) (postintervention) }\end{array}$ & $\begin{array}{l}\text { Ankle dorsiflexion 1RM for most affected leg } \\
\text { (kg) } \\
5.7(0.6) \text { vs. NR (baseline) } \\
10.4 \text { (1.1) vs. NR (postintervention) } \\
83 \% \text { vs. NR (pre-post \% change) } \\
\text { Ankle plantarflexion 1RM for most affected leg } \\
\text { (kg) } \\
30.3(4.9) \text { vs. NR (baseline) } \\
71.8(6.7) \text { vs. NR (postintervention) } \\
137 \% \text { vs. NR (pre-post \% change) } \\
\text { Knee flexion 1RM for most affected leg (kg) } \\
16.3 \text { (2.0) vs. NR (baseline) } \\
29.5 \text { (3.1) vs. NR (postintervention) } \\
82 \% \text { vs. NR (pre-post \% change) } \\
\text { Knee extension 1RM for most affected leg (kg) } \\
72.3(5.8) \text { vs. NR (baseline) } \\
104.5 \text { (6.7) vs. NR (postintervention) } \\
45 \% \text { vs. NR (pre-post \% change) }\end{array}$ \\
\hline
\end{tabular}




\begin{tabular}{|c|c|c|c|c|}
\hline $\begin{array}{l}\text { Author, Year } \\
\text { Intervention Type } \\
\text { Duration of } \\
\text { Postintervention } \\
\text { Followup } \\
\text { Quality } \\
\text { (See Appendix B } \\
\text { for Full Citation) } \\
\end{array}$ & $\begin{array}{l}\text { Intervention and } \\
\text { Comparison }\end{array}$ & $\begin{array}{l}\text { Population } \\
\text { Age (Mean) } \\
\text { Gender (\% Female) } \\
\text { Race (\%) } \\
\text { Ambulatory (\%) } \\
\text { Wheelchair User (\%) } \\
\text { Condition Specific (\%) } \\
\text { Other (\%) }\end{array}$ & Prioritized Outcomes & Other Outcomes \\
\hline $\begin{array}{l}\text { Kjolhede, } 2016 \\
\text { Muscle Strength } \\
\text { Postintervention, } 0 \\
\text { weeks } \\
\text { Fair }\end{array}$ & $\begin{array}{l}\text { A. Progressive } \\
\text { resistance: } 48 \\
\text { sessions over } 24 \\
\text { weeks ( } n=16) \\
\\
\text { B. Usual care } \\
\text { (habitual lifestyle) } \\
(n=14)\end{array}$ & $\begin{array}{l}\text { A vs. B } \\
\text { Age (mean years): } 44.6 \text { vs. } \\
42.2 \\
\text { Female: } 12(75 \%) \text { vs. } 12 \\
(75 \%) \\
\text { Race: NR } \\
\text { Ambulatory: NR } \\
\text { Wheelchair user: NR } \\
\text { EDSS: } 2.9(1) \text { vs. } 2.9(1) \\
\text { Disease Duration (mean } \\
\text { years): } 6.7(7.8) \text { vs. } 7.2(6) \\
\text { Medication ( } n \text { ) } \\
\text { (Rebif/Avonex/Extavia/Betaf } \\
\text { eron): } 5 / 7 / 4 / 0 \text { vs. 8/6/1/0 }\end{array}$ & $\begin{array}{l}\text { Data reported as mean (SD) } \\
\text { A vs. B } \\
25 \text { foot walk test (m/s): } \\
1.66(95 \% \mathrm{Cl} 1.5 \text { to } 1.8) \text { vs. } 1.79(95 \% \mathrm{Cl} 1.6 \text { to } 2.0) \\
\text { (baseline) } \\
1.82(95 \% \mathrm{Cl} 1.7 \text { to } 2.0) \text { vs. } 1.80(95 \% \mathrm{Cl} 1.6 \text { to } 2.0) \\
\text { (postintervention) } \\
\text { Time } \times \text { group interaction p-value }=0.0009 \\
2 \text { minute walk test (m/s): } \\
1.61(95 \% \mathrm{Cl} 1.4 \text { to } 1.8) \text { vs. } 1.66(95 \% \mathrm{Cl} 1.5 \text { to } 1.8) \\
\text { (baseline) } \\
1.77(95 \% \mathrm{Cl} 1.6 \text { to } 2.0) \text { vs. } 1.69(95 \% \mathrm{Cl} 1.5 \text { to } 1.9) \\
\text { (postintervention) } \\
\text { Time } \times \text { group interaction p-value=0.0111 } \\
2 \text { minute walk test (meters) - calculated by } \mathrm{AAl} \\
193.2(95 \% \mathrm{Cl} 168 \text { to } 216) \text { vs. } 199.2(95 \% \mathrm{Cl} 180 \text { to } 216) \\
\text { (baseline) } \\
212.2(95 \% \mathrm{Cl} 192 \text { to } 240) \text { vs. } 202.8(95 \% \mathrm{Cl} 180 \text { to } 228) \\
\text { (postintervention) }\end{array}$ & NA \\
\hline
\end{tabular}




\begin{tabular}{|c|c|c|c|c|}
\hline $\begin{array}{l}\text { Klobucka, } 2020 \\
\text { Aerobic Exercise } \\
\text { Robot-Assisted } \\
\text { Gait Training } \\
\text { Immediately } \\
\text { Postintervention, } \\
\text { and 12-16 weeks } \\
\text { Poor }\end{array}$ & $\begin{array}{l}\text { A. RAGT, } 20 \\
\text { sessions, over } 12 \\
\text { weeks, }(n=21) \\
\\
\text { B. Usual care, } \\
\text { conventional therapy: } \\
20 \text { sessions, over } 12 \\
\text { weeks, }(n=26)\end{array}$ & $\begin{array}{l}\text { A vs. B } \\
\text { Age (mean years): } 18 \text { vs. } 23 \\
\text { Female: } 47 \% \text { vs. } 38 \% \\
\text { Race: NR } \\
\text { Ambulatory: } 4.8 \% \text { vs. } 11.5 \% \\
\text { Wheelchair user: } 23.8 \% \text { vs. } \\
53.8 \% \\
\text { Mechanical wheelchair: } \\
23.8 \% \text { vs. } 53.8 \% \\
\text { Electric wheelchair: } 0 \% \text { vs. } \\
15.3 \% \\
\\
\text { GMFCS levels I-IV }(\%): \\
\text { Level I: } 4.8 \% \text { vs. } 0 \% \\
\text { Level II: } 14.3 \% \text { vs. } 15.4 \% \\
\text { Level III: } 42.9 \% \text { vs. } 46.2 \% \\
\text { Level IV: } 38.1 \% \text { vs. } 38.5 \%\end{array}$ & 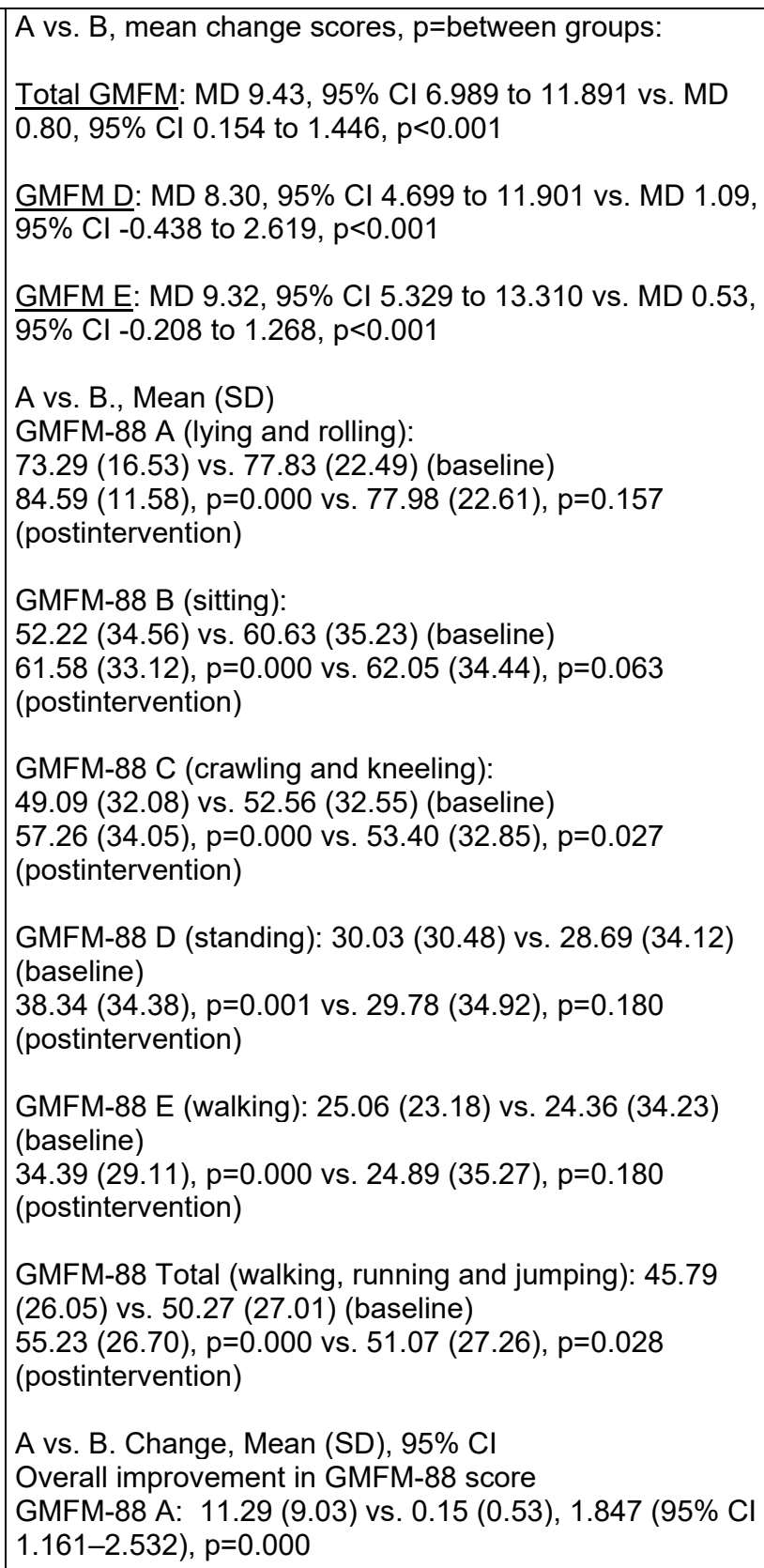 & NA \\
\hline
\end{tabular}




\begin{tabular}{|c|c|c|c|c|}
\hline $\begin{array}{l}\text { Author, Year } \\
\text { Intervention Type } \\
\text { Duration of } \\
\text { Postintervention } \\
\text { Followup } \\
\text { Quality } \\
\text { (See Appendix B } \\
\text { for Full Citation) } \\
\end{array}$ & $\begin{array}{l}\text { Intervention and } \\
\text { Comparison }\end{array}$ & $\begin{array}{l}\text { Population } \\
\text { Age (Mean) } \\
\text { Gender (\% Female) } \\
\text { Race (\%) } \\
\text { Ambulatory (\%) } \\
\text { Wheelchair User (\%) } \\
\text { Condition Specific (\%) } \\
\text { Other (\%) }\end{array}$ & Prioritized Outcomes & Other Outcomes \\
\hline & & & $\begin{array}{l}\text { GMFM-88 B: } 9.36(7.48) \text { vs.1.42 (3.38), } 1.421(95 \% \mathrm{Cl} \\
0.778-2.064), p=0.000 \\
\text { GMFM-88 C: } 8.17(8.69) \text { vs.0.84 (1.89), } 1.229(95 \% \mathrm{Cl} \\
0.603-1.856), p=0.000 \\
\text { GMFM-88 D: } 8.30(8.42) \text { vs. } 1.09(3.98), 1.136(95 \% \mathrm{Cl} \\
0.516-1.755), p=0.000 \\
\text { GMFM-88 E: } 9.32(9.33) \text { vs.0.53 (1.92), } 1.377(95 \% \mathrm{Cl} \\
0.738-2.016), p=0.000 \\
\text { GMFM-88 Total: } 9.43(5.73) \text { vs. } 0.80(1.68), 2.147(95 \% \\
\text { Cl } 1.426-2.867), p=0.000\end{array}$ & \\
\hline $\begin{array}{l}\text { Kooshiar, } 2015 \\
\text { Aerobic Exercise } \\
\text { Aquatics } \\
\text { Postintervention, } 0 \\
\text { weeks } \\
\text { Fair }\end{array}$ & $\begin{array}{l}\text { A. Aquatic exercise, } \\
24 \text { sessions over } 8 \\
\text { weeks }(n=20) \\
\text { B. Usual care }(n=20)\end{array}$ & $\begin{array}{l}\text { A vs. B } \\
\text { Age (mean years): Only } \\
\text { given as mean of all } \\
\text { participants, } 29.24 \\
\text { Female: } \\
20(100 \%) \text { vs. } 20(100 \%) \\
\text { Race: NR } \\
\text { Ambulatory: NR } \\
\text { Wheelchair user: NR } \\
\text { MS: all participants, RRMS } \\
28(75.7 \%), \text { PPMS } 6 \\
(16.2 \%), \text { SPMS } 3(8.1 \%)\end{array}$ & $\begin{array}{l}\text { A vs. B, mean (SD) } \\
\text { MQLIM: } \\
80.06(11.53) \text { vs. } 66.52(6.22) \\
65.48(9.74) \text { vs. } 63.13(13.02) \text { baseline; } p<0.001 \\
\text { (postintervention) } \\
\text { Calculated A vs. B, Mean change scores: } \\
\text { MQLIM: }-16.93 \text { vs. }-1.04, p<0.001 \\
\end{array}$ & NA \\
\hline
\end{tabular}




\begin{tabular}{|c|c|c|c|c|}
\hline $\begin{array}{l}\text { Author, Year } \\
\text { Intervention Type } \\
\text { Duration of } \\
\text { Postintervention } \\
\text { Followup } \\
\text { Quality } \\
\text { (See Appendix B } \\
\text { for Full Citation) } \\
\end{array}$ & $\begin{array}{l}\text { Intervention and } \\
\text { Comparison }\end{array}$ & $\begin{array}{l}\text { Population } \\
\text { Age (Mean) } \\
\text { Gender (\% Female) } \\
\text { Race (\%) } \\
\text { Ambulatory (\%) } \\
\text { Wheelchair User (\%) } \\
\text { Condition Specific (\%) } \\
\text { Other (\%) } \\
\end{array}$ & Prioritized Outcomes & Other Outcomes \\
\hline $\begin{array}{l}\text { Kressler, } 2013 \\
\text { Companion to: } \\
\text { Field-Fote, } 2011 \\
\text { Aerobic Exercise } \\
\text { Robot-Assisted } \\
\text { Gait Training } \\
\text { Postintervention, } \\
12 \text { weeks } \\
\text { Poor }\end{array}$ & $\begin{array}{l}\text { A. RAGT (DGO), } 60 \\
\text { sessions over } 12 \\
\text { weeks ( } n=14 \text { ) } \\
{ }^{*} \text { guidance force at } \\
100 \% \\
\text { B. Treadmill Gait } \\
\text { Training with E-Stim } \\
\text { (TS), } 6 \text { sessions over } \\
12 \text { weeks ( } n=18 \text { ) } \\
\text { C. Manual Assisted } \\
\text { Treadmill Gait } \\
\text { Training (TM), } 6 \\
\text { sessions over } 12 \\
\text { weeks (N=17) } \\
\\
\text { D. Overground Gait } \\
\text { Training with E-stim } \\
\text { (OG), } 60 \text { sessions } \\
\text { over } 12 \text { weeks } \\
\text { ( } n=15 \text { ) }\end{array}$ & NR (no demographics table) & $\begin{array}{l}\text { Walking speed pertaining to post training }(\mathrm{m} / \mathrm{s}) \text { : } \\
\text { Slow: DGO: } 0.08(0.05) \text { to } 0.09(0.06), p=0.233 \\
\text { TS: } 0.09(0.07) \text { to } 0.10(0.07), p=0.170 \\
\text { TM: } 0.10(0.07) \text { to } 0.10(0.06), p=0.955 \\
\text { OG: } 0.06(0.04) \text { to } 0.11(0.09), p=0.001 \\
\text { Moderate: DGO: } 0.14 \text { to } 0.14(0.07), p=0.572 \\
\text { TS: } 0.14(0.12) \text { to } 0.20(0.15), p=0.007 \\
\text { TM: } 0.17(0.13) \text { to } 0.19(0.14), p=0.194 \\
\text { OG: } 0.13(0.15) \text { to } 0.25(0.27) p=0.002 \\
\text { Fast: DGO: } 0.20(0.13) \text { to } 0.20(0.11), p=0.814 \\
\text { TS: } 0.22(0.22) \text { to } 0.28(0.27), p=0.003 \\
\text { TM: } 0.23(0.18) \text { to } 0.26(0.19), p=0.232 \\
\text { OG: } 0.32(0.62) \text { to } 0.35(0.42), p=0.084) \\
\text { VO: }(p e a k) \text { Ln[L/m] } \\
\text { Moderate Pace: } \\
\text { DGO: } 1.11(0.37) \text { to } 1.05(0.40), p=00.046 \\
\text { TS: } 0.91(0.28) \text { to } 1.01(0.28), p=0.041 \\
\text { TM: } 0.90(0.27) \text { to } 1.07(0.34), p=0.035 \\
\text { OG: } 0.90(0.27) \text { to } 1.07(0.34), p=0.033 \\
\text { Maximal Pace: } \\
\text { DGO: } 1.32(0.40) \text { to } 1.28(0.40), p=0.439 \\
\text { TS: } 1.07(0.36) \text { to } 1.17(0.44), p=0.060 \\
\text { TM: } 0.97(0.25) \text { to } 1.17(0.35), p=0.017 \\
\text { OG: } 1.00(0.39) \text { to } 1.13(0.45), p=0.038\end{array}$ & NA \\
\hline
\end{tabular}




\begin{tabular}{|c|c|c|c|c|}
\hline $\begin{array}{l}\text { Author, Year } \\
\text { Intervention Type } \\
\text { Duration of } \\
\text { Postintervention } \\
\text { Followup } \\
\text { Quality } \\
\text { (See Appendix B } \\
\text { for Full Citation) }\end{array}$ & $\begin{array}{l}\text { Intervention and } \\
\text { Comparison }\end{array}$ & $\begin{array}{l}\text { Population } \\
\text { Age (Mean) } \\
\text { Gender (\% Female) } \\
\text { Race (\%) } \\
\text { Ambulatory (\%) } \\
\text { Wheelchair User (\%) } \\
\text { Condition Specific (\%) } \\
\text { Other (\%) }\end{array}$ & Prioritized Outcomes & Other Outcomes \\
\hline $\begin{array}{l}\text { Kumru, } 2016 \\
\text { Aerobic Exercise } \\
\text { Robot-Assisted } \\
\text { Gait Training } \\
\text { Postintervention, } 8 \\
\text { weeks } \\
\text { Fair }\end{array}$ & $\begin{array}{l}\text { A. RAGT with rTMS, } \\
20 \text { sessions over } 4 \\
\text { weeks, then } 20 \\
\text { sessions without } \\
\text { rTMS for } 4 \text { weeks } \\
(n=15) \\
\\
\text { B. RAGT alone, } 40 \\
\text { sessions over } 8 \\
\text { weeks }(n=16)\end{array}$ & $\begin{array}{l}\text { A vs. B } \\
\text { Age (mean years): } \\
51 \text { vs. } 49 \\
\text { Female: } \\
5(33 \%) \text { vs. } 2(13 \%) \\
\text { Race: NR } \\
\text { Ambulatory: } \\
\text { NR } \\
\text { Wheelchair user: } \\
\text { NR } \\
\text { Other: } \\
\text { Time since SCl (months) } 2.8 \\
\text { vs. } 2.8 \\
\text { Level of Injury } \\
\text { Cervical or Thoracic } \\
\text { Cervical: } 8 \text { (53\%) vs. } 6 \\
\text { (38\%) }\end{array}$ & $\begin{array}{l}\text { 10MWT } \\
\text { number able to perform test } \\
2 \text { at baseline, } 6 \text { after last session, } 10 \text { at followup } \\
2 \text { at baseline, } 4 \text { after last session, } 6 \text { at followup } \\
\text { LEMS mean change score after last stimulation session ( } 4 \\
\text { weeks) } \\
8 \text { vs. } 4 \\
\text { at followup } \\
10 \text { vs. } 6 \\
\text { UEMS mean change score after last stimulation session } \\
(4 \text { weeks) } \\
5 \text { vs. } 1 \\
\text { at followup } \\
8 \text { vs. } 5 \\
\text { Modified Ashworth Score } \\
1.1(0.8) \text { to } 1.1 \text { (0.9) vs. } 1.3 \text { (1.3) to } 1.1 \text { (1.1) }\end{array}$ & NA \\
\hline
\end{tabular}




\begin{tabular}{|c|c|c|c|c|}
\hline $\begin{array}{l}\text { Author, Year } \\
\text { Intervention Type } \\
\text { Duration of } \\
\text { Postintervention } \\
\text { Followup } \\
\text { Quality } \\
\text { (See Appendix B } \\
\text { for Full Citation) } \\
\end{array}$ & $\begin{array}{l}\text { Intervention and } \\
\text { Comparison }\end{array}$ & $\begin{array}{l}\text { Population } \\
\text { Age (Mean) } \\
\text { Gender (\% Female) } \\
\text { Race (\%) } \\
\text { Ambulatory (\%) } \\
\text { Wheelchair User (\%) } \\
\text { Condition Specific (\%) } \\
\text { Other (\%) } \\
\end{array}$ & Prioritized Outcomes & Other Outcomes \\
\hline $\begin{array}{l}\text { Kwon, } 2011 \\
\text { Postural Control } \\
\text { Hippotherapy } \\
\text { Postintervention, } 0 \\
\text { weeks } \\
\text { (End of treatment } \\
\text { after 8-week } \\
\text { intervention) } \\
\text { Fair }\end{array}$ & $\begin{array}{l}\text { A. Hippotherapy- } \\
\text { plus-conventional- } \\
\text { physiotherapy group } \\
\text { (hippotherapy group), } \\
16 \text { hippotherapy } \\
\text { sessions over } 8 \\
\text { weeks and } \\
\text { conventional } \\
\text { physiotherapy ( } n=16 \text { ) } \\
\text { B. Conventional- } \\
\text { physiotherapy group } \\
\text { (Control group), } 2 \\
\text { sessions per week } \\
\text { (n=16) }\end{array}$ & $\begin{array}{l}\text { A vs. B } \\
\text { Age (mean years): } 6.4 \text { vs. } \\
6.1 \\
\text { Female: } 5(31 \%) \text { vs. } 6(38 \%) \\
\text { Race: NR } \\
\text { Ambulatory: } 100 \% \\
\text { Wheelchair user: NR } \\
\\
\text { GMFCS level: } \\
\text { GMFCS level I: } 4 \text { (25\%) vs. } \\
4 \text { (25\%) } \\
\text { GMFCS level II: } 12(75 \%) \\
\text { vs. } 12 \text { ( } 75 \%) \\
\text { Body weight (mean kg): } 21.8 \\
\text { vs. } 19.8 \\
\text { Height (mean cm): } 113.5 \text { vs. } \\
111.0 \\
\text { Previous surgery: } 3(19 \%) \\
\text { vs. } 4 \text { (25\%) }\end{array}$ & $\begin{array}{l}\text { A vs. B, mean (SD) } \\
\text { 8 weeks } \\
\text { GMFM-66: } \\
70.4(7.4) \text { vs. } 69.8 \text { (8.7) (baseline) } \\
73.7(8.3) \text { vs. } 70.1 \text { (8.1), } p=0.003 \text { (postintervention) }\end{array}$ & $\begin{array}{l}\text { A vs. B, mean (SD) } \\
8 \text { weeks } \\
\text { PBS: } \\
41.7(8.8) \text { vs. } 41.0(10.4) \text { (baseline) } \\
45.8 \text { (8.6) vs. } 41.5(10.6), p=0.004 \\
\text { (postintervention) }\end{array}$ \\
\hline
\end{tabular}




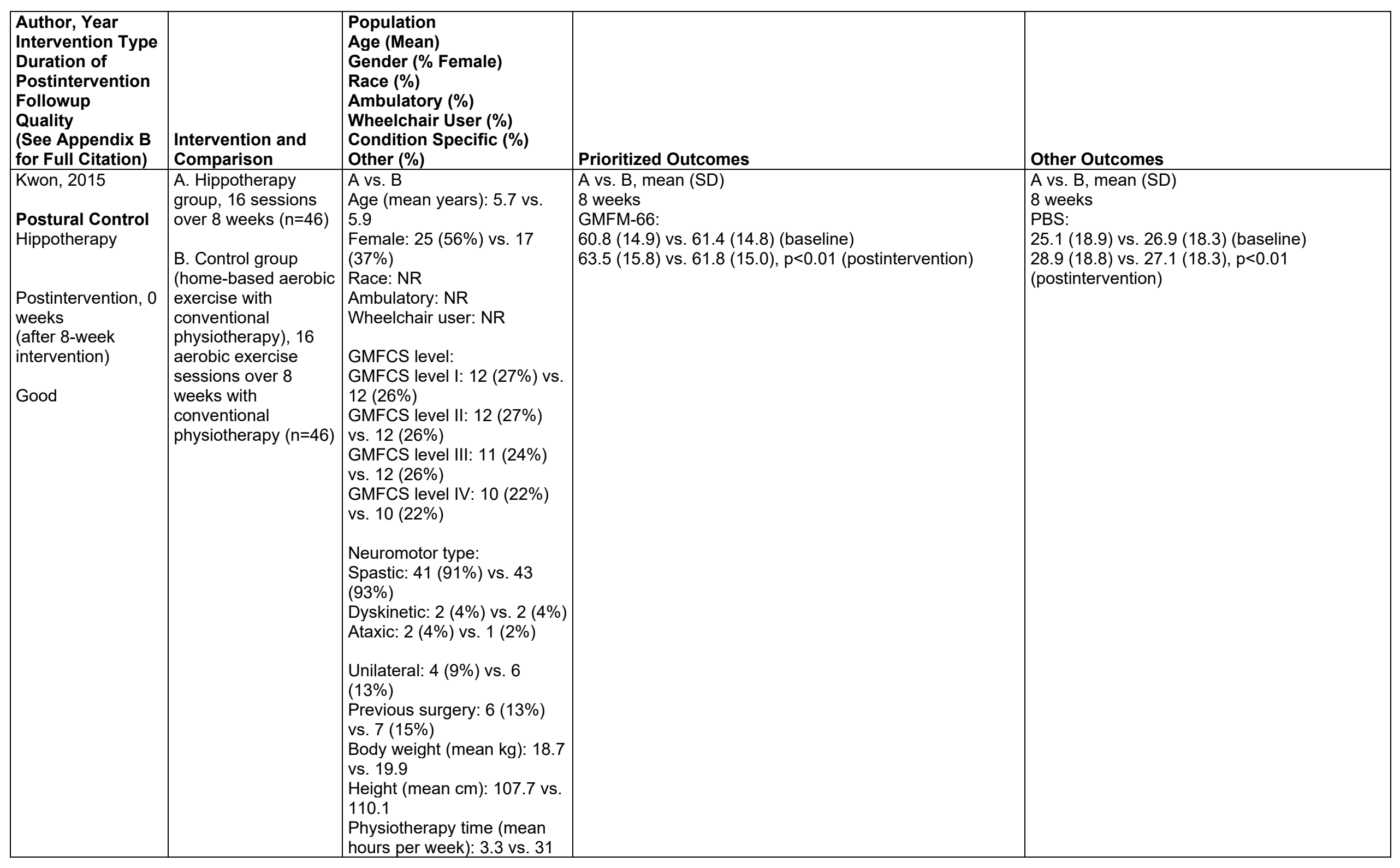




\begin{tabular}{|c|c|c|c|c|}
\hline $\begin{array}{l}\text { Author, Year } \\
\text { Intervention Type } \\
\text { Duration of } \\
\text { Postintervention } \\
\text { Followup } \\
\text { Quality } \\
\text { (See Appendix B } \\
\text { for Full Citation) } \\
\end{array}$ & $\begin{array}{l}\text { Intervention and } \\
\text { Comparison }\end{array}$ & $\begin{array}{l}\text { Population } \\
\text { Age (Mean) } \\
\text { Gender (\% Female) } \\
\text { Race (\%) } \\
\text { Ambulatory (\%) } \\
\text { Wheelchair User (\%) } \\
\text { Condition Specific (\%) } \\
\text { Other (\%) }\end{array}$ & Prioritized Outcomes & Other Outcomes \\
\hline $\begin{array}{l}\text { Lai, } 2010 \\
\text { Aerobic Exercise } \\
\text { Cycling } \\
\text { Postintervention, } \\
12 \text { weeks } \\
\text { Fair }\end{array}$ & $\begin{array}{l}\text { A. Functional } \\
\text { electrical stimulation } \\
\text { cycling exercises, } 36 \\
\text { sessions over } 12 \\
\text { weeks }(n=12) \\
\\
\begin{array}{l}\text { B. Control group } \\
(n=12)\end{array}\end{array}$ & $\begin{array}{l}\text { A vs. B } \\
\text { Age (mean years): } \\
28.9 \text { vs. } 28.3 \\
\text { Female: } \\
2 \text { (17\%) vs. } 2(17 \%) \\
\text { Race: NR } \\
\text { Ambulatory: } \\
0 \text { (0\%) vs. } 0 \text { (0\%) } \\
\text { Wheelchair user: NR } \\
\text { Other: } \\
\text { SCl: } \\
\text { Quadriplegia: } \\
5(33 \%) \text { vs. } 5(33 \%) \\
\text { Paraplegia: } \\
10(67 \%) \text { vs. } 10(67 \%) \\
\end{array}$ & NA & $\begin{array}{l}\text { A vs. B (SD) } \\
\text { BMD femoral neck (g/cm2) } \\
0.927(0.189) \text { vs. } 0.913(0.097) \text { (baseline) } \\
0.884(0.171) \text { vs. } 0.867(0.095) ; p<0.050 \text { for } \\
\text { difference between } 1 \mathrm{st} \text { and } 2 \text { nd } \\
\text { measurements (postintervention) } \\
0.842(0.168) \text { vs. } 0.825(0.092) ; p<0.050 \text { for } \\
\text { difference between } 2 \text { nd and 3rd } \\
\text { measurements ( } 3 \text { months postintervention) } \\
\\
\text { BMD distal femur ( } / \mathrm{cm} 2) \\
1.003(0.064) \text { vs. } 1.003(0.110) \text { (baseline) } \\
0.981 \text { (0.063) vs. } 0.936(0.103) ; p<0.050 \text { for } \\
\text { difference between } 1 \mathrm{st} \text { and } 2 \text { nd } \\
\text { measurements (postintervention) } \\
0.913 \text { (0.058) vs. } 0.868 \text { (0.097); } p<0.050 \text { for } \\
\text { difference between 2nd and 3rd } \\
\text { measurements ( } 3 \text { months postintervention) }\end{array}$ \\
\hline $\begin{array}{l}\text { Lai, } 2015 \\
\text { Aerobic Exercise } \\
\text { Aquatics } \\
\text { Postintervention, } 0 \\
\text { weeks } \\
\text { Fair }\end{array}$ & \begin{tabular}{|l|} 
A. Aquatic therapy \\
plus traditional \\
rehabilitation, 24 \\
sessions over 12 \\
weeks ( $n=11)$ \\
\\
B. Traditional \\
rehabilitation, \\
average $2-3$ sessions \\
over 12 weeks \\
$(n=13)$
\end{tabular} & $\begin{array}{l}\text { A vs. B } \\
\text { Age (mean years): } \\
7.6 \text { vs. } 6.6 \\
\text { Female: } \\
7 \text { (64\%) vs. } 4(31 \%) \\
\text { Race: NR } \\
\text { Ambulatory: NR } \\
\text { Wheelchair user: NR } \\
\text { N (\%) vs. N (\%) } \\
\\
\text { Other: } \\
\text { CP subtypes: } \\
\text { Diplegia: } 3 \text { (27.3) vs. } 6 \\
\text { (46.2) } \\
\text { Quadriplegia } 5 \text { (45.5) vs. } 4 \\
\text { (30.8) } \\
\text { Hemiplegia } 3 \text { (27.3) vs. } 3 \\
\text { (23.1) }\end{array}$ & $\begin{array}{l}\text { A vs. B, Mean difference between groups: } \\
\text { GMFM-66: } 5.0 \text { vs. } 0.7, p=0.007 \\
\text { GMFM-66: } \\
61.2(18.7) \text { vs. } 64.6(19.4) \text { (baseline) } \\
66.2 \text { (18.2) vs. } 65.3(19.1) ; p=0.007 \text { (postintervention) } \\
\text { CPQoL scales for Social, Functioning, Participation, } \\
\text { Emotional, Access, Pain and Disability, and Family } \\
\text { Health: All NS }\end{array}$ & $\begin{array}{l}\text { A vs. B. mean (SD) } \\
\text { MAS: } \\
\text { Ankle, Knee, Wrist, Elbow: all NS } \\
\text { Vineline Adaptive Beh Scale for Daily Living: } \\
\text { NS }\end{array}$ \\
\hline
\end{tabular}




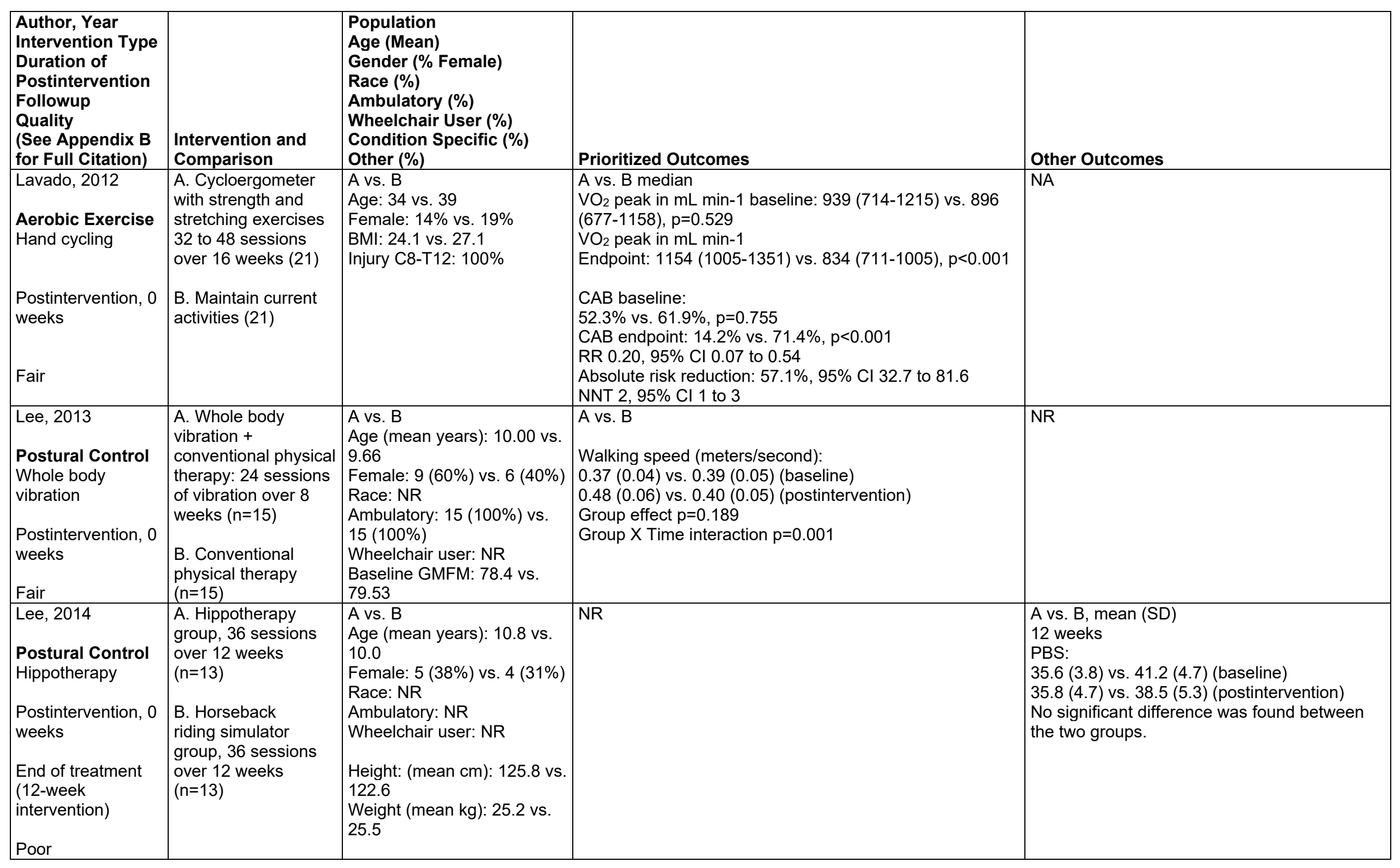




\begin{tabular}{|c|c|c|c|c|}
\hline $\begin{array}{l}\text { Author, Year } \\
\text { Intervention Type } \\
\text { Duration of } \\
\text { Postintervention } \\
\text { Followup } \\
\text { Quality } \\
\text { (See Appendix B } \\
\text { for Full Citation) }\end{array}$ & $\begin{array}{l}\text { Intervention and } \\
\text { Comparison }\end{array}$ & $\begin{array}{l}\text { Population } \\
\text { Age (Mean) } \\
\text { Gender (\% Female) } \\
\text { Race (\%) } \\
\text { Ambulatory (\%) } \\
\text { Wheelchair User (\%) } \\
\text { Condition Specific (\%) } \\
\text { Other (\%) }\end{array}$ & Prioritized Outcomes & Other Outcomes \\
\hline $\begin{array}{l}\text { Liu, } 2019 \\
\text { Multimodal } \\
\text { Exercise } \\
\text { Immediately } \\
\text { postintervention, } \\
12 \text { weeks } \\
\text { Fair }\end{array}$ & $\begin{array}{l}\text { A. Strength exercise } \\
+ \text { treadmill }+ \text { core } \\
\text { stability training on a } \\
\text { stable support } \\
\text { surface, } 60 \text { sessions } \\
\text { over } 12 \text { weeks } \\
(n=20) \\
\\
\text { B. Strength exercise } \\
+ \text { treadmill + core } \\
\text { stability training on } \\
\text { an unstable support } \\
\text { surface, } 60 \text { sessions } \\
\text { over } 12 \text { weeks } \\
(n=20)\end{array}$ & $\begin{array}{l}\text { A vs. } B \\
\text { (data are for completers } \\
\text { only; } n=14 \text { vs. } 15 \text { ) } \\
\text { Age: } 43 \text { vs. } 46 \\
\text { Female: } 21 \% \text { vs. } 27 \% \\
\text { Ambulatory: } 100 \% \\
\text {-paraplegia: } 36 \% \text { vs. } 40 \% \\
\text {-tetraplegia: } 64 \% \text { vs. } 60 \%\end{array}$ & $\begin{array}{l}\text { A vs. B, Mean (SD), data for completers only): } \\
\text { Stride length (units NR) } \\
0.564(0.189) \text { vs. } 0.454(0.173), p=0.025 \text { (post- } \\
\text { intervention) } \\
0.09(0.26) \text { vs. } 0.06(0.24), \mathrm{MD} 0.03(95 \% \mathrm{Cl}-0.16 \text { to } \\
0.22), p=N R \text { (pre-post change) } \\
\text { Walking speed (units NR) } \\
0.350(0.226) \text { vs. } 0.209(0.171), p=0.0196 \text { (post- } \\
\text { intervention) } \\
0.09(0.30) \text { vs. } 0.03(0.23), \mathrm{MD} 0.06(95 \% \mathrm{Cl}-0.14 \text { to } \\
0.26), p=N R \text { (pre-post change) }\end{array}$ & NA \\
\hline
\end{tabular}




\begin{tabular}{|c|c|c|c|c|}
\hline $\begin{array}{l}\text { Author, Year } \\
\text { Intervention Type } \\
\text { Duration of } \\
\text { Postintervention } \\
\text { Followup } \\
\text { Quality } \\
\text { (See Appendix B } \\
\text { for Full Citation) }\end{array}$ & $\begin{array}{l}\text { Intervention and } \\
\text { Comparison }\end{array}$ & $\begin{array}{l}\text { Population } \\
\text { Age (Mean) } \\
\text { Gender (\% Female) } \\
\text { Race (\%) } \\
\text { Ambulatory (\%) } \\
\text { Wheelchair User (\%) } \\
\text { Condition Specific (\%) } \\
\text { Other (\%) }\end{array}$ & Prioritized Outcomes & Other Outcomes \\
\hline $\begin{array}{l}\text { Lorentzen, } 2015 \\
\text { Postural Control } \\
\text { Balance } \\
\text { Postintervention, } \\
20 \text { weeks } \\
\text { Poor }\end{array}$ & $\begin{array}{l}\text { Interactive computer } \\
\text { training vs. usual } \\
\text { care } \\
\text { A. Interactive } \\
\text { computer training } \\
\text { (home-based): } 140 \\
\text { sessions over } 20 \\
\text { weeks, } 40 \text { hours of } \\
\text { total training } \\
\text { time. ( } n=34) \\
\text { B. Usual care control: } \\
(n=12)\end{array}$ & $\begin{array}{l}\text { A vs. B } \\
\text { Age (mean years): } 10.9 \text { vs. } \\
11.3 \\
\text { Female: } 11(32 \%) \text { vs. } 5 \\
(42 \%) \\
\text { Race: NR } \\
\text { Ambulatory: NR } \\
\text { Wheelchair user: NR }\end{array}$ & 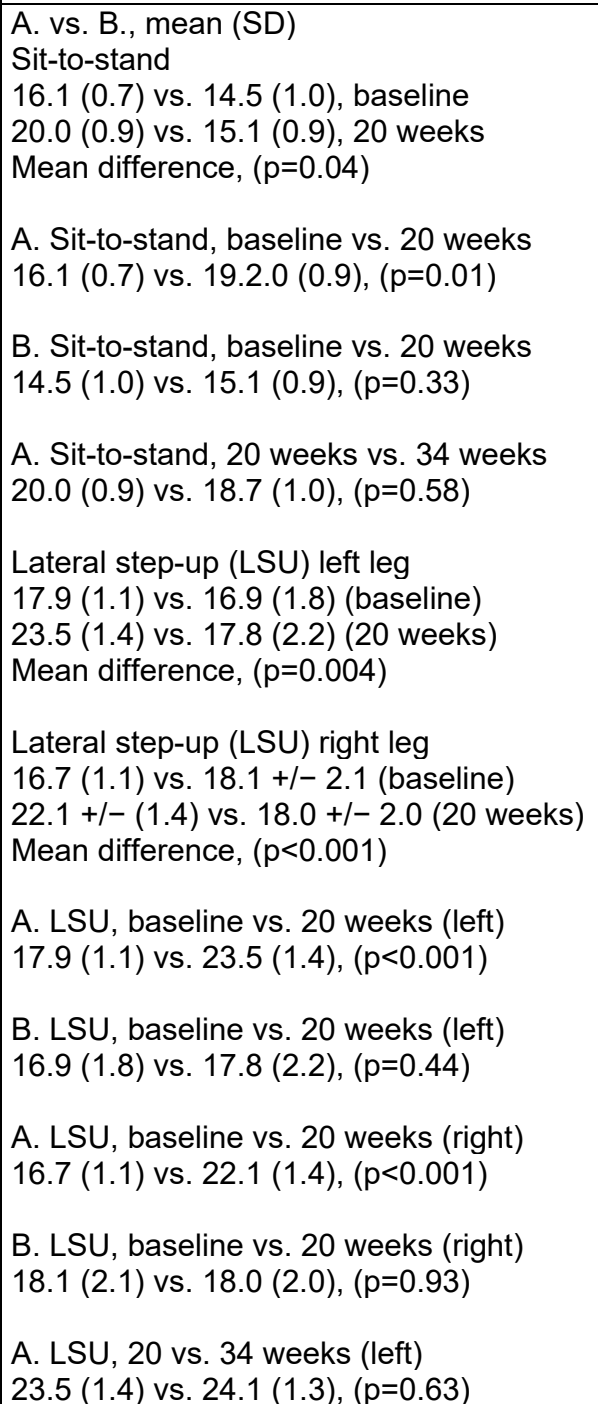 & $\begin{array}{l}\text { A. vs. B., mean (SD) } \\
\text { Romberg Balance Test: C90 (mm2) } \\
427.2(57.6) \text { vs. } 310.9(131.9) \text { (intro) } \\
462.2(62.5) \text { vs. } 314.6(104.9) \text { (test1) } \\
\text { Mean difference, }(p=0.18) \\
\text { Romberg Balance Test: velocity }(\mathrm{mm} / \mathrm{s}) \\
13.4(0.7) \text { vs. } 10.6(1.4) \\
14.1(0.7) \text { vs. } 11.7(1.7) \\
\text { Mean difference, }(p=0.59) \\
\text { Romberg Balance Test: trace length }(\mathrm{mm}) \\
403.1(21.3) \text { vs. } 317.4(43.0) \\
422.8(19.9) \text { vs. } 351.6(50.2) \\
\text { Mean difference, }(p=0.9)\end{array}$ \\
\hline
\end{tabular}




\begin{tabular}{|c|c|c|c|c|}
\hline $\begin{array}{l}\text { Author, Year } \\
\text { Intervention Type } \\
\text { Duration of } \\
\text { Postintervention } \\
\text { Followup } \\
\text { Quality } \\
\text { (See Appendix B } \\
\text { for Full Citation) }\end{array}$ & $\begin{array}{l}\text { Intervention and } \\
\text { Comparison }\end{array}$ & $\begin{array}{l}\text { Population } \\
\text { Age (Mean) } \\
\text { Gender (\% Female) } \\
\text { Race (\%) } \\
\text { Ambulatory (\%) } \\
\text { Wheelchair User (\%) } \\
\text { Condition Specific (\%) } \\
\text { Other (\%) }\end{array}$ & Prioritized Outcomes & Other Outcomes \\
\hline $\begin{array}{l}\text { Lorentzen, } 2015 \\
\text { (Continued) }\end{array}$ & & & 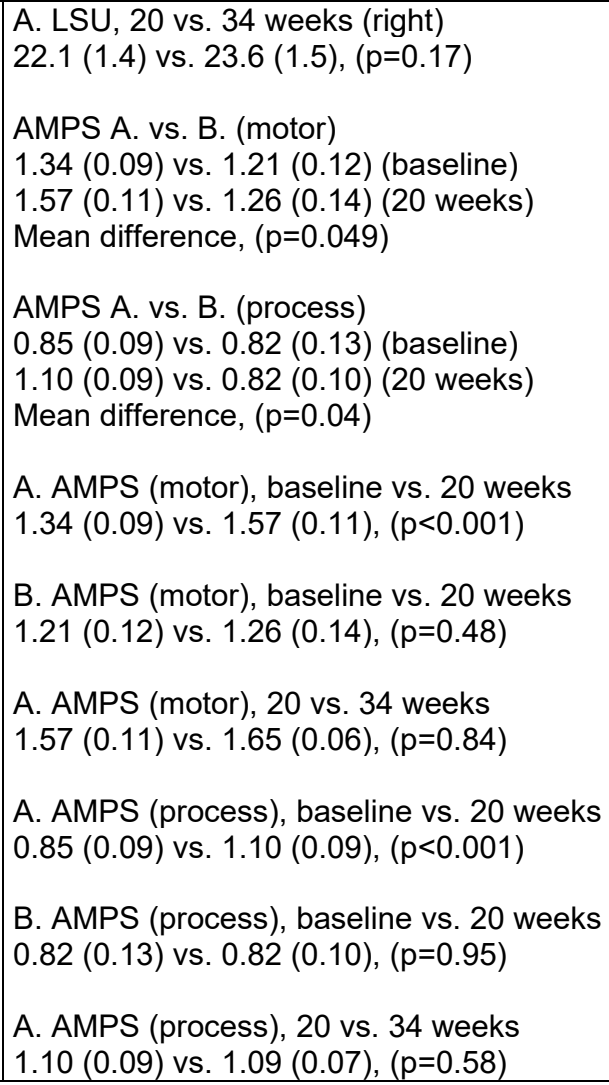 & NA \\
\hline
\end{tabular}




\begin{tabular}{|c|c|c|c|c|}
\hline $\begin{array}{l}\text { Author, Year } \\
\text { Intervention Type } \\
\text { Duration of } \\
\text { Postintervention } \\
\text { Followup } \\
\text { Quality } \\
\text { (See Appendix B } \\
\text { for Full Citation) }\end{array}$ & $\begin{array}{l}\text { Intervention and } \\
\text { Comparison }\end{array}$ & $\begin{array}{l}\text { Population } \\
\text { Age (Mean) } \\
\text { Gender (\% Female) } \\
\text { Race (\%) } \\
\text { Ambulatory (\%) } \\
\text { Wheelchair User (\%) } \\
\text { Condition Specific (\%) } \\
\text { Other (\%) }\end{array}$ & Prioritized Outcomes & Other Outcomes \\
\hline $\begin{array}{l}\text { Lucena-Anton, } \\
2018 \\
\text { Postural Control } \\
\text { Hippotherapy } \\
\text { Postintervention, } 1 \\
\text { week (13 weeks } \\
\text { total including 12- } \\
\text { week intervention) } \\
\text { Fair }\end{array}$ & $\begin{array}{l}\text { A. Intervention group } \\
\text { (hippotherapy and } \\
\text { conventional } \\
\text { therapy), } 12 \text { sessions } \\
\text { over } 12 \text { weeks } \\
\text { ( } n=22) \\
\\
\text { B. Control group } \\
\text { (conventional } \\
\text { therapy), } 24 \text { sessions } \\
\text { over } 12 \text { weeks } \\
\text { (n=22) }\end{array}$ & $\begin{array}{l}\text { A vs. B } \\
\text { Age (mean years): } 9.500 \text { vs. } \\
8.227 \\
\text { Female: } 9(41 \%) \text { vs. } 7(32 \%) \\
\text { Race: NR } \\
\text { Ambulatory: } 0 \% \\
\text { Wheelchair user: NR } \\
\\
\text { SLLA: } 2.773 \text { vs. } 2.591 \\
\text { SLRA: } 2.227 \text { vs. } 2.409\end{array}$ & $\begin{array}{l}\text { A vs. B, mean (SD) } \\
13 \text { weeks } \\
\text { MAS: } \\
\text { Left adductors: } \\
2.77(1.15) \text { vs. } 2.59(1.22) \text { (baseline) } \\
2.50(1.05) \text { vs. } 2.54(1.22), p=0.040 \text { (postintervention) } \\
\text { Right adductors: } \\
2.22(1.26) \text { vs. } 2.40(1.14) \text { (baseline) } \\
1.77(1.26) \text { vs. } 2.31(1.24), p=0.047 \text { (postintervention) }\end{array}$ & NR \\
\hline
\end{tabular}




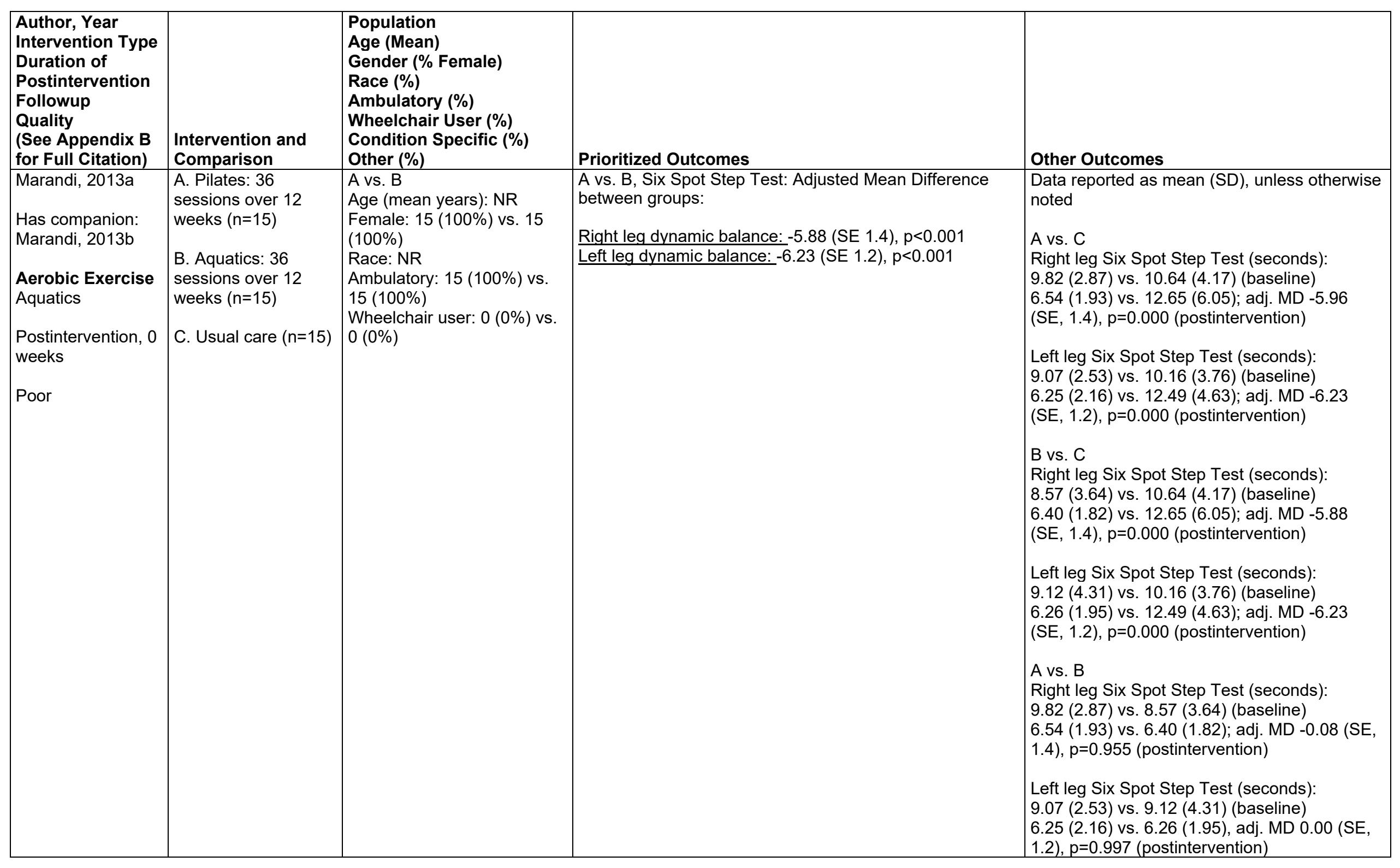




\begin{tabular}{|c|c|c|c|c|}
\hline $\begin{array}{l}\text { Author, Year } \\
\text { Intervention Type } \\
\text { Duration of } \\
\text { Postintervention } \\
\text { Followup } \\
\text { Quality } \\
\text { (See Appendix B } \\
\text { for Full Citation) } \\
\end{array}$ & $\begin{array}{l}\text { Intervention and } \\
\text { Comparison }\end{array}$ & $\begin{array}{l}\text { Population } \\
\text { Age (Mean) } \\
\text { Gender (\% Female) } \\
\text { Race (\%) } \\
\text { Ambulatory (\%) } \\
\text { Wheelchair User (\%) } \\
\text { Condition Specific (\%) } \\
\text { Other (\%) }\end{array}$ & Prioritized Outcomes & Other Outcomes \\
\hline $\begin{array}{l}\text { Marandi, 2013b } \\
\text { Companion to: } \\
\text { Marandi, 2013a } \\
\text { Aerobic Exercise } \\
\text { Aquatics } \\
\text { Postintervention, } 0 \\
\text { weeks } \\
\text { Poor }\end{array}$ & $\begin{array}{l}\text { A. Pilates: } 36 \\
\text { sessions over } 12 \\
\text { weeks }(n=15) \\
\text { B. Aquatics: } 36 \\
\text { sessions over } 12 \\
\text { weeks }(n=15) \\
\text { C. Usual care }(n=15)\end{array}$ & $\begin{array}{l}\text { B vs. C } \\
\text { Age (mean years): } 40 \\
\text { Female: } 15(100 \%) \text { vs. } 15 \\
(100 \%) \\
\text { Race: NR } \\
\text { Ambulatory: } 15(100 \%) \text { vs. } \\
15(100 \%) \\
\text { Wheelchair user: } 0(0 \%) \text { vs. } \\
0(0 \%)\end{array}$ & $\begin{array}{l}\text { B vs. C } \\
\text { Right leg dynamic balance: } \\
8.57 \text { (3.64) vs. } 10.64 \text { (4.17) (baseline) } \\
6.40 \text { (1.82) vs. } 12.65 \text { (6.05); adj. MD -5.88 (SE, 1.4), } \\
p=0.000 \text { (postintervention) } \\
\\
\text { Left leg dynamic balance: } \\
9.12 \text { (4.31) vs. } 10.16 \text { (3.76) (baseline) } \\
6.26(1.95) \text { vs. } 12.49 \text { (4.63); adj. MD }-6.23 \text { (SE, 1.2), } \\
p=0.000 \text { (postintervention) }\end{array}$ & NR \\
\hline
\end{tabular}




\begin{tabular}{|c|c|c|c|c|}
\hline $\begin{array}{l}\text { Author, Year } \\
\text { Intervention Type } \\
\text { Duration of } \\
\text { Postintervention } \\
\text { Followup } \\
\text { Quality } \\
\text { (See Appendix B } \\
\text { for Full Citation) }\end{array}$ & $\begin{array}{l}\text { Intervention and } \\
\text { Comparison }\end{array}$ & $\begin{array}{l}\text { Population } \\
\text { Age (Mean) } \\
\text { Gender (\% Female) } \\
\text { Race (\%) } \\
\text { Ambulatory (\%) } \\
\text { Wheelchair User (\%) } \\
\text { Condition Specific (\%) } \\
\text { Other (\%) }\end{array}$ & Prioritized Outcomes & Other Outcomes \\
\hline $\begin{array}{l}\text { Matusiak- } \\
\text { Wieczorek, } 2020 \\
\text { Postural Control } \\
\text { Hippotherapy } \\
\text { Immediately } \\
\text { Postintervention, } \\
12 \text { weeks } \\
\text { Fair }\end{array}$ & $\begin{array}{l}\text { A. Hippotherapy, } 24 \\
\text { sessions over } 12 \\
\text { weeks ( } n=15) \\
\text { B. Hippotherapy, } 12 \\
\text { sessions over } 12 \\
\text { weeks ( } n=15) \\
\begin{array}{l}\text { C. Waitlist control } \\
(n=15)\end{array}\end{array}$ & $\begin{array}{l}\text { A vs. B vs. C } \\
\text { Age (mean years): } 7.9 \text { vs. } \\
8.6 \text { vs. } 8.3 \\
\text { Female: } 40 \% \text { vs. } 46 \% \text { vs. } \\
46 \% \\
\text { GMFCS, level I: } 67 \% \text { vs. } \\
80 \% \text { vs. } 47 \% \\
\text { GMFCS, level II: } 33 \% \text { vs. } \\
20 \% \text { vs. } 53 \%\end{array}$ & NA & $\begin{array}{l}\text { A vs. B vs. C, mean (SD), p=between groups } \\
\text { SAS: } \\
10.93(3.97) \text { vs. } 15.93(4.17) \text { vs. } 14.87 \text { (3.27) } \\
\text { (baseline) } \\
11.53(3.74) \text { vs. } 16.53(3.50) \text { vs. } 14.93(3.35) \\
(4 \text { weeks) } \\
12.40(3.70) \text { vs. } 16.93(3.24) \text { vs. } 15.00(3.30) \\
(8 \text { weeks) } \\
13.13(3.46) \text { vs. } 17.27(2.76) \text { vs. } 15.13(3.36) \\
\text { (postintervention) } \\
2.20(1.42) \text { vs. } 1.33(0.76) \text { vs. } 0.27(0.46) \\
\text { (difference in pre- and postintervention scores) } \\
\text { A vs. C: MD } 1.93,95 \% \text { Cl } 0.94 \text { to } 2.92, \\
p<0.001 \\
B \text { vs. C: MD } 1.06,95 \% \text { Cl } 0.61 \text { to } 1.51, \\
\text { p<0.001 } \\
\text { A vs. B: MD } 0.87,95 \% \text { Cl } 0.06 \text { to } 1.69, \\
\text { p=0.036 }\end{array}$ \\
\hline $\begin{array}{l}\text { Midik, } 2020 \\
\text { Aerobic Exercise } \\
\text { Robot-Assisted } \\
\text { Gait Training } \\
\text { Postintervention, } \\
\text { and } 12 \text { weeks } \\
\text { Fair }\end{array}$ & $\begin{array}{l}\text { A. RAGT plus } \\
\text { conventional rehab, } \\
25 \text { sessions over } 5 \\
\text { weeks }(n=15) \\
\text { B. Conventional } \\
\text { rehab only, } 25 \\
\text { sessions over } 5 \\
\text { weeks ( } n=15)\end{array}$ & $\begin{array}{l}\text { A vs. B } \\
\text { Age: } 35.4 \text { vs. } 37.9 \\
\text { Female: } 0 \% \\
\text { Race: NR } \\
\text { AIS C: } 40 \% \text { vs. } 67 \% \\
\text { AIS D: } 60 \% \text { vs. } 33 \%\end{array}$ & $\begin{array}{l}\text { A vs. B, mean change (SE), } p=\text { between groups: } \\
\text { WISCl: } 3.9(0.8) \text { vs. } 2.5(0.5), p=0.178 \\
\text { SCIM: } 9.9(2.5) \text { vs. } 7.0(1.3), p=0.326 \\
\text { LEMS: } 1.8(0.4) \text { vs. } 0.6(0.2), p=0.061 \\
\text { At } 3 \text { month followup, change from baseline: } \\
\text { WISC: } 4.3(1.0) \text { vs. } 2.5(0.5), p=0.139 \\
\text { SCIM: } 16.5(3.2) \text { vs. } 7.6(1.5), p=0.127 \\
\text { LEMS: } 2.1(0.5) \text { vs. } 0.6(0.2), p=0.049\end{array}$ & NA \\
\hline
\end{tabular}




\begin{tabular}{|c|c|c|c|c|}
\hline $\begin{array}{l}\text { Author, Year } \\
\text { Intervention Type } \\
\text { Duration of } \\
\text { Postintervention } \\
\text { Followup } \\
\text { Quality } \\
\text { (See Appendix B } \\
\text { for Full Citation) } \\
\end{array}$ & $\begin{array}{l}\text { Intervention and } \\
\text { Comparison }\end{array}$ & $\begin{array}{l}\text { Population } \\
\text { Age (Mean) } \\
\text { Gender (\% Female) } \\
\text { Race (\%) } \\
\text { Ambulatory (\%) } \\
\text { Wheelchair User (\%) } \\
\text { Condition Specific (\%) } \\
\text { Other (\%) }\end{array}$ & Prioritized Outcomes & Other Outcomes \\
\hline $\begin{array}{l}\text { Mogharnasi, } 2018 \\
\text { Muscle Strength } \\
\text { exercise } \\
\text { Postintervention, } 0 \\
\text { weeks } \\
\text { Poor }\end{array}$ & $\begin{array}{l}\text { A. Upper body } \\
\text { resistance training: } \\
24 \text { sessions over } 8 \\
\text { weeks }(n=10) \\
\text { B. Usual care }(n=10)\end{array}$ & $\begin{array}{l}\text { A vs. B } \\
\text { Age (mean years): } 25.33 \text { vs. } \\
25.50 \\
\text { Female: } 0(0 \%) \text { vs. } 0(0 \%) \\
\text { Race: NR } \\
\text { Ambulatory: } 0(0 \%) \text { vs. } 0 \\
(0 \%) \\
\text { Wheelchair user: } 100 \\
(100 \%) \text { vs. } 100(100 \%) \\
\text { Duration of paralysis (mean } \\
\text { months): } 117 \text { vs. } 111 \\
\text { Smoking (yes): } 1(10 \%) \text { vs. } 3 \\
(30 \%) \\
\text { Etiology } \\
- \text { Motor vehicle accident: } 8 \\
(80 \%) \text { vs. } 9(90 \%) \\
- \text { Fall: } 2(20 \%) \text { vs. } 1(10 \%) \\
\text { Neurological status } \\
- \text {-T9: } 1(10 \%) \text { vs. } 2(20 \%) \\
- \text { T10: } 2(20 \%) \text { vs. } 2(20 \%) \\
- \text {-T11: } 2(20 \%) \text { vs. } 0(0 \%) \\
- \text { T12: } 5(50 \%) \text { vs. } 6(60 \%)\end{array}$ & $\begin{array}{l}\text { Data reported as mean (SD) } \\
\text { A vs. B } \\
\text { BMl }\left(\mathrm{kg} / \mathrm{m}^{\wedge} 2\right) \text { : } \\
25.33(1.37) \text { vs. } 24.91(0.98), \mathrm{p}>0.05 \text { (baseline) } \\
24.73(1.24) \text { vs. } 25.14(1.01), \mathrm{p}>0.05 \text { (postintervention) }\end{array}$ & 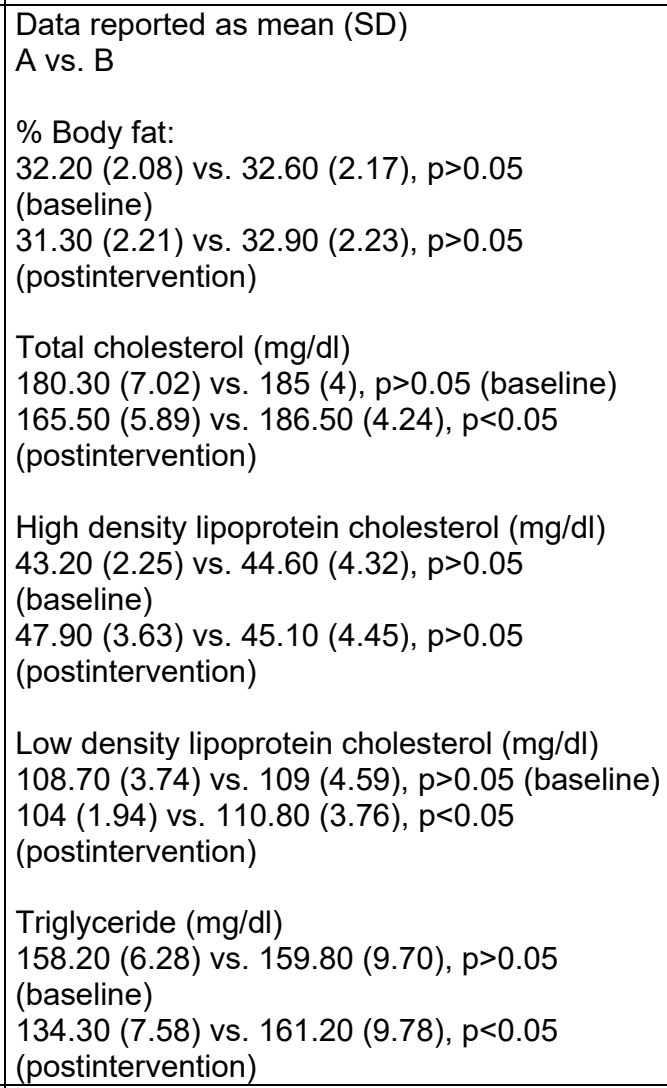 \\
\hline $\begin{array}{l}\text { Moraes, } 2020 \\
\text { Postural Control } \\
\text { Hippotherapy } \\
\text { Postintervention, } \\
0 \text { weeks } \\
\text { Fair }\end{array}$ & $\begin{array}{l}\text { A. Hippotherapy, } 16 \\
\text { sessions over } 8 \\
\text { weeks }(n=17) \\
\begin{array}{l}\text { B. Waitlist control } \\
(n=16)\end{array}\end{array}$ & $\begin{array}{l}\text { A vs. B } \\
\text { Age: } 45.5 \text { vs. } 48.4 \\
\text { Female: } 94 \% \text { vs. } 94 \% \\
\text { Race: NR } \\
\text { EDSS, median: } 2.0 \text { vs. } 1.75 \\
\text { RRMS: } 100 \%\end{array}$ & $\begin{array}{l}\text { A vs. B, mean (SD): } \\
\text { 6MWT: } 459.06(118.34) \text { to } 503.59(126.38) \text { vs. } 513.00 \\
\text { (101.97) to } 497.13(88.88), p<0.001 \\
\text { 25FWT: } 6.37(1.70) \text { to } 5.36(1.43) \text { vs. } 5.82(1.29) \text { to } 5.84 \\
(1.08), p<0.001\end{array}$ & NA \\
\hline
\end{tabular}




\begin{tabular}{|c|c|c|c|c|}
\hline $\begin{array}{l}\text { Author, Year } \\
\text { Intervention Type } \\
\text { Duration of } \\
\text { Postintervention } \\
\text { Followup } \\
\text { Quality } \\
\text { (See Appendix B } \\
\text { for Full Citation) }\end{array}$ & $\begin{array}{l}\text { Intervention and } \\
\text { Comparison }\end{array}$ & $\begin{array}{l}\text { Population } \\
\text { Age (Mean) } \\
\text { Gender (\% Female) } \\
\text { Race (\%) } \\
\text { Ambulatory (\%) } \\
\text { Wheelchair User (\%) } \\
\text { Condition Specific (\%) } \\
\text { Other (\%) }\end{array}$ & Prioritized Outcomes & Other Outcomes \\
\hline $\begin{array}{l}\text { Mutoh, } 2019 \\
\text { Postural Control } \\
\text { Hippotherapy } \\
\text { Postintervention, } \\
12 \text { weeks (post 48- } \\
\text { week intervention) } \\
\text { Fair }\end{array}$ & $\begin{array}{l}\text { A. Hippotherapy, } 48 \\
\text { sessions over } 48 \\
\text { weeks }(n=12) \\
\text { B. Outdoor recreation } \\
48 \text { sessions over } 48 \\
\text { weeks }(n=12)\end{array}$ & $\begin{array}{l}\text { A vs. B } \\
\text { Age: } 8 \text { vs. } 9 \\
\text { Female: } 58 \% \text { vs. } 50 \% \\
\text { GMFCS II: } 42 \% \text { vs. } 42 \% \\
\text { GMFCS III: } 58 \% \text { vs. } 58 \%\end{array}$ & $\begin{array}{l}\text { A vs. B, Mean (SD), p=between groups } \\
\text { GMFM-66: } \\
56.6(9.2) \text { to } 62.8(10.8) \text { vs. } 57.4(7.9) \text { to } 57.9(9.2), \\
p<0.05 \\
\text { GMFM-66E: } \\
45.4(7.0) \text { to } 49.7(7.6) \text { vs. } 46.0(6.3) \text { to } 46.5(6.6), p<0.05 \\
\text { 5MWT (m/min): } \\
31.9(10.7) \text { to } 38.8(13.5) \text { vs. } 31.1(11.3) \text { to } 32.3(11.6) \text {, } \\
\text { p<0.05 } \\
\text { WHOQOL (positive feelings): } \\
3.1(1) \text { to } 4.1(1) \text { vs. } 3.1(0.9) \text { to } 3.4(1), p<0.05 \\
\text { WHOQOL (self-esteem): } \\
2.9(1.2) \text { to } 4.0(0.7) \text { vs. } 3.3(1.1) \text { to } 3.7(0.7), p<0.05 \\
\text { WHOQOL (negative feelings): } \\
2.9(0.8) \text { to } 2.8(0.7) \text { vs. } 2.8\left(0.8^{*} \text { to } 2.8(0.8), p>0.05\right.\end{array}$ & NA \\
\hline $\begin{array}{l}\text { Najafidoulataba, } \\
2014 \\
\text { Postural Control } \\
\text { Postintervention, } \\
12 \text { weeks } \\
\text { Poor }\end{array}$ & $\begin{array}{l}\text { Yoga vs. usual care } \\
\begin{array}{l}\text { A. Yoga: } 24 \text { sessions } \\
\text { over } 12 \text { weeks } \\
(n=30)\end{array} \\
\begin{array}{l}\text { B. Usual care control: } \\
(n=30)\end{array}\end{array}$ & $\begin{array}{l}\text { A vs. B } \\
\text { Age (years): "mean age in } \\
\text { the case group 31.6" } \\
\text { Female: } 30(100 \%) \text { vs. } 30 \\
(100 \%) \\
\text { Race: NR } \\
\text { Ambulatory: NR } \\
\text { Wheelchair user: NR }\end{array}$ & $\begin{array}{l}\text { A. vs. B., mean (SD) } \\
\text { Physical activity QoL } \\
23.7(4.25) \text { vs. } 20.55(3.5)(95 \% \mathrm{Cl}-2.42 \text { to } 0.42), \\
\text { p=0.001 (baseline) } \\
24.7(3.94) \text { vs. } 19.45(4.1)(95 \% \mathrm{Cl} 0.49 \text { to } 1.7), p=0.00 \\
\text { (postintervention) } \\
\text { Sexual satisfaction QoL } \\
1.8(2.0 \text { vs. } 2.1(1.2)(95 \% \mathrm{Cl}-0.09 \text { to } 0.89), \mathrm{p}=0.01 \\
\text { (baseline) } \\
1.4(1.5) \text { vs. } 2.1(1.2)(95 \% \mathrm{Cl} \mathrm{NR}), \mathrm{p}=\mathrm{NR} \\
\text { (postintervention)* } \\
\text { * stated as not significant }\end{array}$ & NA \\
\hline
\end{tabular}




\begin{tabular}{|c|c|c|c|c|}
\hline $\begin{array}{l}\text { Author, Year } \\
\text { Intervention Type } \\
\text { Duration of } \\
\text { Postintervention } \\
\text { Followup } \\
\text { Quality } \\
\text { (See Appendix B } \\
\text { for Full Citation) }\end{array}$ & $\begin{array}{l}\text { Intervention and } \\
\text { Comparison }\end{array}$ & $\begin{array}{l}\text { Population } \\
\text { Age (Mean) } \\
\text { Gender (\% Female) } \\
\text { Race (\%) } \\
\text { Ambulatory (\%) } \\
\text { Wheelchair User (\%) } \\
\text { Condition Specific (\%) } \\
\text { Other (\%) }\end{array}$ & Prioritized Outcomes & Other Outcomes \\
\hline $\begin{array}{l}\text { Negaresh, } 2018 \\
\text { Aerobic Exercise } \\
\text { Cycling } \\
\text { Postintervention, } 0 \\
\text { weeks } \\
\text { Fair }\end{array}$ & $\begin{array}{l}\text { A. vs. B. } \\
\text { A. Normal BMI } \\
\text { cycling UE/LE, } 24 \\
\text { sessions over } 8 \\
\text { weeks ( } n=18) \\
\\
\text { B. } \\
\text { Normal BMI Control } \\
(n=15) \\
\text { C vs. D: } \\
\text { C: Overweight BMI } \\
\text { cycling UE/LE, } 24 \\
\text { sessions over } 8 \\
\text { weeks ( } n=18) \\
\text { D: Overweight BMI } \\
\text { Control ( } n=15)\end{array}$ & $\begin{array}{l}\text { A vs. B } \\
\text { Age (mean years): } \\
31.2 \text { vs. } 29.1 \\
\text { Female: } \\
6 \text { (35\%) vs. } 9(64 \%) \\
\text { Race: NR } \\
\text { Ambulatory: } \\
17(100 \%) \text { vs. } 14 \text { (100\%) } \\
\text { Wheelchair user: NR } \\
\text { Other: } \\
\text { MS RRMS } \\
18(100 \%) \text { vs. } 15(100 \%) \\
\\
\text { C vs. D } \\
\text { Age (mean years): } \\
32.1 \text { vs. } 32.2 \\
\text { Female: } \\
6 \text { (35\%) vs. } 4 \text { (29\%) } \\
\text { Race: NR } \\
\text { Ambulatory: } \\
17(100 \%) \text { vs. } 13(100 \%) \\
\text { Wheelchair user: NR } \\
\\
\text { Other: } \\
\text { RRMS } \\
17(100 \%) \text { vs. 13 (100\%) }\end{array}$ & $\begin{array}{l}\text { A vs. } B \text { vs. C vs. } D \text {, Mean difference between groups } \\
\text { (scores are estimates from graph): } \\
\text { TUG: }-3.8 \text { vs. }-0.1 \text { vs. }-2.5 \text { vs. } 0, p=0.001 \\
\text { Interaction between Weight and Exercise } p=0.52 \\
\text { A vs. } B \\
\text { (scores are estimates from graph) } \\
\text { BDI changes (score): } \\
-5 \text { vs. } 0, p=0.005 \\
\text { TUG changes (sec): } \\
-4 \text { vs. } 0, p=0.001 \\
\text { VO } 2 \text { peak changes (mg/kg/min): } \\
2.5 \text { vs. } 0 \text { ), } p=0.001\end{array}$ & $\begin{array}{l}\text { A vs. B } \\
\text { BMI: NS }\end{array}$ \\
\hline
\end{tabular}




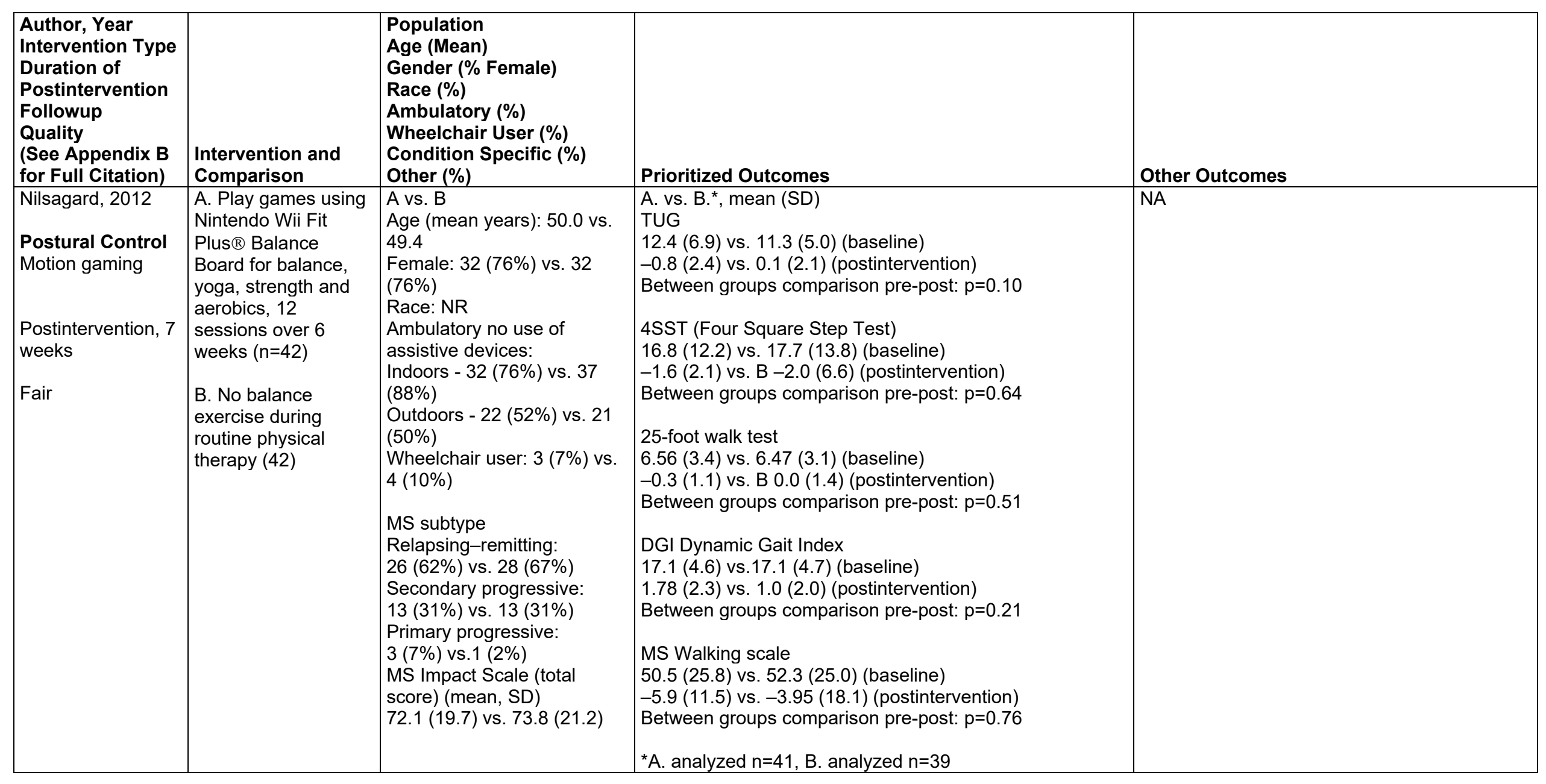




\begin{tabular}{|c|c|c|c|c|}
\hline $\begin{array}{l}\text { Author, Year } \\
\text { Intervention Type } \\
\text { Duration of } \\
\text { Postintervention } \\
\text { Followup } \\
\text { Quality } \\
\text { (See Appendix B } \\
\text { for Full Citation) } \\
\end{array}$ & $\begin{array}{l}\text { Intervention and } \\
\text { Comparison }\end{array}$ & $\begin{array}{l}\text { Population } \\
\text { Age (Mean) } \\
\text { Gender (\% Female) } \\
\text { Race (\%) } \\
\text { Ambulatory (\%) } \\
\text { Wheelchair User (\%) } \\
\text { Condition Specific (\%) } \\
\text { Other (\%) } \\
\end{array}$ & Prioritized Outcomes & Other Outcomes \\
\hline $\begin{array}{l}\text { Niwald, } 2017 \\
\text { Aerobic Exercise } \\
\text { Cycling } \\
\text { Postintervention, } 0 \\
\text { weeks } \\
\text { Fair }\end{array}$ & $\begin{array}{l}\text { A. Cycle ergometry } \\
\text { Intervention }+ \\
\text { complex } \\
\text { rehabilitation } 120 \\
\text { min/wk, } 20 \text { sessions } \\
\text { over } 4 \text { weeks }(n=21) \\
\\
\text { B. Control } \\
\text { intervention of } \\
\text { complex } \\
\text { rehabilitation } 120 \\
\text { min/wk }(n=32)\end{array}$ & $\begin{array}{l}\text { A vs. B } \\
\text { Age (mean years): } \\
57 \text { vs. } 60 \\
\text { Female: } \\
13(62 \%) \text { vs. } 21(65 \%) \\
\text { Race: NR } \\
\text { Ambulatory: } \\
21(100 \%) \text { vs. } 32(100 \%) \\
\text { Wheelchair user: NR } \\
\text { Other: } \\
\text { MS subtypes NR }\end{array}$ & 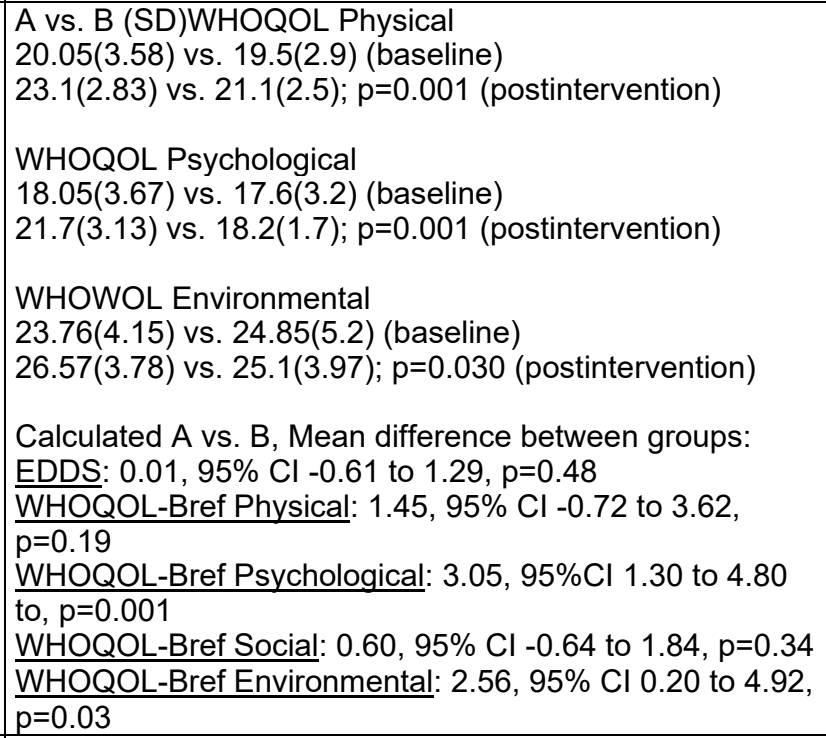 & EDSS: NS \\
\hline $\begin{array}{l}\text { Norouzi, } 2019 \\
\text { Postural Control } \\
\text { Balance exercises } \\
\text { Immediately } \\
\text { postintervention, } \\
4 \text { weeks } \\
\text { Fair }\end{array}$ & $\begin{array}{l}\text { A. Cawthorne/ } \\
\text { Cooksey exercises, } \\
12 \text { sessions over } 4 \\
\text { weeks }(n=10) \\
\\
\text { B. Usual care, } 4 \\
\text { sessions over } 4 \\
\text { weeks }(n=10)\end{array}$ & $\begin{array}{l}\text { A vs. B } \\
\text { Age: NR } \\
\text { Female: } 0 \% \\
\text { L3-L4: } 100 \%\end{array}$ & NA & $\begin{array}{l}\text { A vs. B, Mean (SD), p-value=between groups } \\
\text { BBS: } \\
38.36(6.01) \text { to } 48.39(4.01) \text { vs. } 37.67(6.07) \text { to } \\
43.20(4.05), M D 4.5,95 \% \mathrm{Cl}-0.17 \text { to } 9.17, \\
p=0.059^{*} \\
\text { *authors report } p<0.05 \text { but unclear if this value } \\
\text { also includes a third, neurofeedback group }\end{array}$ \\
\hline
\end{tabular}




\begin{tabular}{|c|c|c|c|c|}
\hline $\begin{array}{l}\text { Author, Year } \\
\text { Intervention Type } \\
\text { Duration of } \\
\text { Postintervention } \\
\text { Followup } \\
\text { Quality } \\
\text { (See Appendix B } \\
\text { for Full Citation) } \\
\end{array}$ & $\begin{array}{l}\text { Intervention and } \\
\text { Comparison }\end{array}$ & $\begin{array}{l}\text { Population } \\
\text { Age (Mean) } \\
\text { Gender (\% Female) } \\
\text { Race (\%) } \\
\text { Ambulatory (\%) } \\
\text { Wheelchair User (\%) } \\
\text { Condition Specific (\%) } \\
\text { Other (\%) }\end{array}$ & Prioritized Outcomes & Other Outcomes \\
\hline $\begin{array}{l}\text { Nsenga, } 2013 \\
\text { Aerobic Exercise } \\
\text { Cycling } \\
\text { Postintervention, } 0 \\
\text { weeks } \\
\text { Fair }\end{array}$ & $\begin{array}{l}\text { A. Cycle ergometry, } \\
24 \text { sessions over } 8 \\
\text { weeks }(n=10) \\
\text { B. Control with CP, } \\
\text { no training }(n=10)\end{array}$ & $\begin{array}{l}\text { A vs. B } \\
\text { Age (mean years): } \\
14.2 \text { vs. } 14.2 \\
\text { Female: } \\
4 \text { (40\%) vs. } 4(40 \%) \\
\text { Race: NR } \\
\text { Ambulatory: } \\
10(100 \%) \text { vs. } 10(100 \%) \\
\text { Wheelchair user: NR } \\
\text { Other: } \\
\text { CP: } \\
\text { Hemiplegia: } \\
8(80 \%) \text { vs. } 8(80 \%) \\
\text { Diplegia: } \\
2 \text { (20\%) vs. } 2(20 \%)\end{array}$ & $\begin{array}{l}\text { A Baseline vs. A After Training Period (SD) } \\
\text { VO2 peak ( } \mathrm{ml} / \mathrm{kg} / \mathrm{min}): \\
35.6(5.6) \text { vs. } 43.7(4.7) \\
(p<0.050)\end{array}$ & NA \\
\hline $\begin{array}{l}\text { Nsenga Leunkeu, } \\
2012 \\
\text { Aerobic Exercise } \\
\text { Treadmill } \\
\text { Postintervention, } 0 \\
\text { weeks } \\
\text { Fair }\end{array}$ & $\begin{array}{l}\text { A. } 40 \text { minutes } \\
\text { treadmill walking } 3 \\
\text { times a week for } 8 \\
\text { weeks } \\
\text { Walking ( } n=12) \\
\text { B. Usual care (no } \\
\text { training) }(n=12)\end{array}$ & $\begin{array}{l}\text { A vs. B } \\
\text { Age (mean years): } 14.2 \text { vs. } \\
14.2 \\
\text { Female: } 6(50 \%) \text { vs. } 6(50 \%) \\
\text { Race: NR } \\
\text { Ambulatory: } 12(100 \%) \text { vs. } \\
12(100 \%) \\
\text { Wheelchair user: } 0(0 \%) \text { vs. } \\
0(0 \%) \\
\text { Hemiplegia: } 10(83 \%) \text { vs. } 10 \\
(83 \%) \\
\text { Diplegia: } 2(17 \%) \text { vs. } 2 \\
\text { (17\%) } \\
\text { GMFCS } \\
- \text { I: } 8(67 \%) \text { vs. } 8(67 \%) \\
\text {-II: } 4(33 \%) \text { vs. } 4(33 \%)\end{array}$ & $\begin{array}{l}\text { A vs. B } \\
6 \mathrm{MWT} \text { (meters): } \\
490 \text { (NR) vs. } 450 \text { (NR), } \mathrm{p}>0.05 \text { (baseline) } \\
600 \text { (NR) vs. } 450 \text { (NR), } \mathrm{p}<0.05 \text { (postintervention) } \\
\text { VO2 peak: } \\
32 \text { (NR) vs. } 32.5 \text { (NR), } p>0.05 \text { (baseline) } \\
40 \text { (NR) vs. } 32.5 \text { (NR), p<0.05 (postintervention) } \\
\text { Measured peak oxygen uptake during incremental cycle } \\
\text { ergometry (ml/kg/min) } \\
\text { A. } 32.5 \text { to } 39.0 \\
\text { B. } 32.5 \text { to } 32.5 \\
\text { (data estimated from bar graph) } \\
\text { Significant increase for those trained }(A) p=0.046 \text { ) and no } \\
\text { significant change in B; comparison beween groups NR }\end{array}$ & NR \\
\hline
\end{tabular}




\begin{tabular}{|c|c|c|c|c|}
\hline $\begin{array}{l}\text { Author, Year } \\
\text { Intervention Type } \\
\text { Duration of } \\
\text { Postintervention } \\
\text { Followup } \\
\text { Quality } \\
\text { (See Appendix B } \\
\text { for Full Citation) } \\
\end{array}$ & $\begin{array}{l}\text { Intervention and } \\
\text { Comparison }\end{array}$ & $\begin{array}{l}\text { Population } \\
\text { Age (Mean) } \\
\text { Gender (\% Female) } \\
\text { Race (\%) } \\
\text { Ambulatory (\%) } \\
\text { Wheelchair User (\%) } \\
\text { Condition Specific (\%) } \\
\text { Other (\%) }\end{array}$ & Prioritized Outcomes & Other Outcomes \\
\hline $\begin{array}{l}\text { Ortiz-Rubio, } 2016 \\
\text { Muscle Strength } \\
\text { Postintervention, } 0 \\
\text { weeks } \\
\text { Good }\end{array}$ & $\begin{array}{l}\text { A. Upper extremity } \\
\text { strength }+ \\
\text { coordination: } 16 \\
\text { sessions over } 8 \\
\text { weeks }(n=19) \\
\text { B. Booklet with } \\
\text { exercise info }(n=18)\end{array}$ & $\begin{array}{l}\text { A vs. B } \\
\text { Age (mean years):42.21 vs. } \\
44.89 \\
\text { Female: } 5 \text { (26\%) vs. } 6(33 \%) \\
\text { Race: NR } \\
\text { Ambulatory: NR } \\
\text { Wheelchair user: NR } \\
\text { MS type } \\
\text {-Relapsing-remitting } 4 \\
\text { (21.05\%) vs. } 4(22.22 \%) \\
\text {-Primary progressive } 3 \\
\text { (15.79\%) vs. } 2(11.11 \%) \\
\text {-Secondary progressive } 12 \\
\text { (63.16\%) vs. } 12 \text { (66.67\%) } \\
\text { EDSS (mean): } 5.71 \text { vs. } 6.04 \\
\end{array}$ & $\begin{array}{l}\text { Data reported as mean (SD) } \\
\text { A vs. B } \\
\text { ARAT most affected upper limb (scale unclear): } \\
54.68(1.82) \text { vs. } 54.27(0.95), p=0.803 \text { (baseline) } \\
56.89(0.31) \text { vs. } 54.11(1.07), p<0.001 \text { (postintervention) } \\
2.21(95 \% \mathrm{Cl}-2.95 \text { to }-1.46) \text { vs. } 0.16 \text { (95\% Cl }-0.29 \text { to } \\
0.62), p=\mathrm{NR} \text { (post-pre change) } \\
\text { ARAT least affected upper limb (scale unclear): } \\
56.31(1.24) \text { vs. } 56.33(0.68), p=0.895 \text { (baseline) } \\
57.17(0.00) \text { vs. } 56.16(0.78), p<0.001 \text { (postintervention) } \\
0.68(95 \% \mathrm{Cl}-1.28 \text { to }-0.08) \text { vs. } 0.16 \text { ( } 95 \% \mathrm{Cl}-0.08 \text { to } \\
0.42), p=\mathrm{NR} \text { (post-pre change) }\end{array}$ & $\begin{array}{l}\text { No adverse effects were reported by any } \\
\text { patient. }\end{array}$ \\
\hline $\begin{array}{l}\text { Ozkul, } 2020 \\
\text { Postural Control } \\
\text { Balance Exercises } \\
\text { Motion Gaming } \\
\text { Postintervention, } \\
0 \text { weeks } \\
\text { Fair }\end{array}$ & $\begin{array}{l}\text { A. Immersive virtual } \\
\text { reality, } 16 \text { sessions } \\
\text { over } 8 \text { weeks }(n=13) \\
\text { B. Relaxation } \\
\text { exercises at home, } \\
16 \text { sessions over } 8 \\
\text { weeks }(n=13)\end{array}$ & $\begin{array}{l}\text { A vs. B } \\
\text { Age: } 29 \text { vs. } 34 \\
\text { Female: } 69 \% \text { vs. } 77 \% \\
\text { Race: NR } \\
\text { EDSS median: } 1 \text { vs. } 2 \\
\text { Number of relapses: } 3 \text { vs. } 2\end{array}$ & $\begin{array}{l}\text { Pre-post median (IQR): } \\
\text { BBS: } 52(42.5,56) \text { to } 54(44.5,56) \text { vs. } 55(53,56) \text { to } 56 \\
(53.5,56), p>0.05 \\
\frac{\text { TUG: } 7.6(6.9,8) \text { to } 6.3(5.7,7.2) \text { vs. } 6.9(6.5,7.5) \text { to } 7.4}{(6.4,7.7), p<0.017}\end{array}$ & NA \\
\hline
\end{tabular}




\begin{tabular}{|c|c|c|c|c|}
\hline $\begin{array}{l}\text { Author, Year } \\
\text { Intervention Type } \\
\text { Duration of } \\
\text { Postintervention } \\
\text { Followup } \\
\text { Quality } \\
\text { (See Appendix B } \\
\text { for Full Citation) }\end{array}$ & $\begin{array}{l}\text { Intervention and } \\
\text { Comparison }\end{array}$ & $\begin{array}{l}\text { Population } \\
\text { Age (Mean) } \\
\text { Gender (\% Female) } \\
\text { Race (\%) } \\
\text { Ambulatory (\%) } \\
\text { Wheelchair User (\%) } \\
\text { Condition Specific (\%) } \\
\text { Other (\%) }\end{array}$ & Prioritized Outcomes & Other Outcomes \\
\hline $\begin{array}{l}\text { Ozkul, } 2020 \mathrm{~b} \\
\text { Multimodal } \\
\text { Exercise } \\
\text { Immediately } \\
\text { Postintervention, } 8 \\
\text { weeks } \\
\text { Fair }\end{array}$ & $\begin{array}{l}\text { A. Aerobics + Pilates, } \\
24 \text { sessions ( } 3 \text { per } \\
\text { week) over } 8 \text { weeks } \\
(n=17) \\
\text { B. Control group, } \\
\text { relaxation exercise at } \\
\text { home, } 24 \text { sessions ( } 3 \\
\text { per week) over } 8 \\
\text { weeks }(n=17)\end{array}$ & $\begin{array}{l}\text { A vs. B } \\
\text { Age (mean years): } 35.8 \text { vs. } \\
36.7 \text { (mean years): } \\
\text { Female: } 76 \% \text { vs. } 76 \% \\
\text { Race: NR } \\
\text { Ambulatory: } 100 \% \\
\text { Wheelchair user: NR } \\
\text { EDSS (score): } 1.5 \text { vs. } 1.71 \\
\text { GMFM: NR } \\
\\
\text { BMI (kg/m2) } 23.75 \text { vs. } 25.39 \\
\text { Disease duration (years): } \\
7.18 \text { vs. } 5.71 \\
\text { Number of relapses } 2.88 \text { vs. } \\
2.71\end{array}$ & 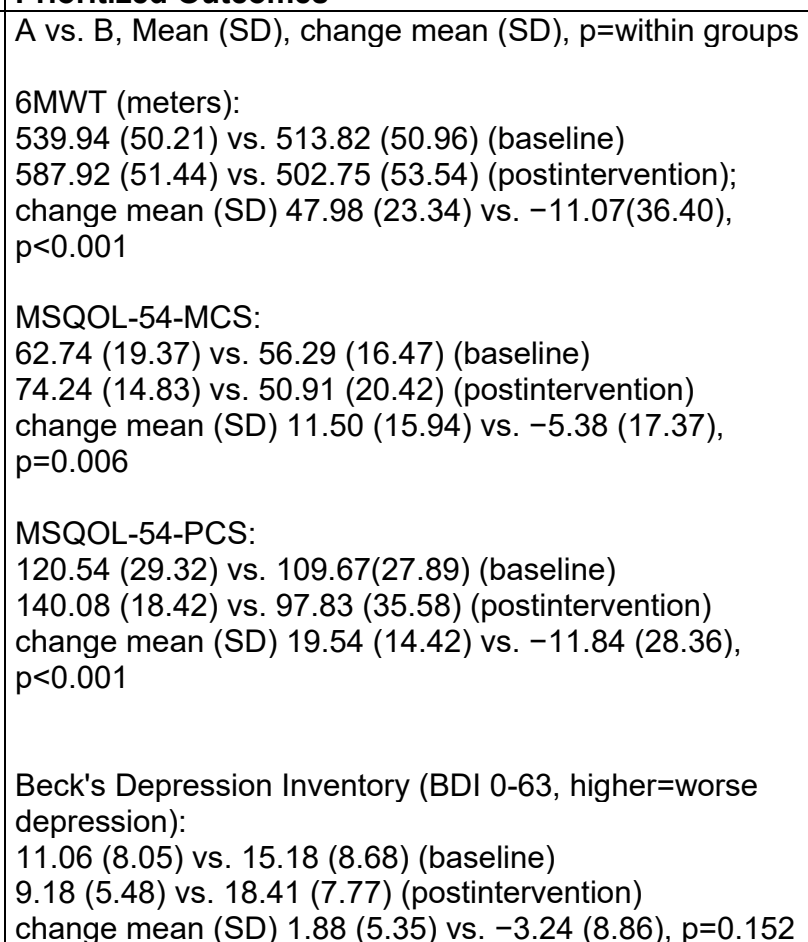 & $\begin{array}{l}\text { Fatigue Impact Scale (FIS-Total score): } \\
53.35(29.64) \text { vs. } 68.12(36.84) \text { (baseline) } \\
36.18(21.57) \text { vs. } 78.88(39.72) \\
\text { (postintervention) } \\
\text { change mean (SD) }-17.18(22.24) \text { vs. } 10.76 \\
(28.01), p=0.006\end{array}$ \\
\hline
\end{tabular}




\begin{tabular}{|c|c|c|c|c|}
\hline $\begin{array}{l}\text { Author, Year } \\
\text { Intervention Type } \\
\text { Duration of } \\
\text { Postintervention } \\
\text { Followup } \\
\text { Quality } \\
\text { (See Appendix B } \\
\text { for Full Citation) }\end{array}$ & $\begin{array}{l}\text { Intervention and } \\
\text { Comparison }\end{array}$ & $\begin{array}{l}\text { Population } \\
\text { Age (Mean) } \\
\text { Gender (\% Female) } \\
\text { Race (\%) } \\
\text { Ambulatory (\%) } \\
\text { Wheelchair User (\%) } \\
\text { Condition Specific (\%) } \\
\text { Other (\%) }\end{array}$ & Prioritized Outcomes & Other Outcomes \\
\hline $\begin{array}{l}\text { Park, } 2014 \\
\text { Postintervention, } 8 \\
\text { weeks } \\
\text { (within } 2 \text { months } \\
\text { after 8-week } \\
\text { intervention) } \\
\text { Postural Control } \\
\text { Hippotherapy } \\
\text { Poor }\end{array}$ & $\begin{array}{l}\text { A. Intervention group } \\
\text { (hippotherapy), } 16 \\
\text { sessions over } 8 \\
\text { weeks ( } \mathrm{n}=34 \text { ) } \\
\begin{array}{l}\text { B. Control group } \\
\text { (waitlist) }\end{array}\end{array}$ & $\begin{array}{l}\text { A vs. B } \\
\text { Age (mean years): } 6.68 \text { vs. } \\
7.76 \\
\text { Female: } 19 \text { (56\%) vs. } 11 \\
(52 \%) \\
\text { Race: NR } \\
\text { Ambulatory: NR } \\
\text { Wheelchair user: NR } \\
\text { Bilateral CP: } 32 \text { (94\%) vs. } 19 \\
\text { (90\%) } \\
\text { Unilateral CP: } 2 \text { 6\%) vs. } 2 \\
\text { (10\%) } \\
\text { GMFCS level: } \\
\text { GMFCS level I: } 8 \text { (24\%) vs. } \\
6 \text { (29\%) } \\
\text { GMFCS level II: } 11 \text { (32\%) } \\
\text { vs. } 4(19 \%) \\
\text { GMFCS level III: } 5 \text { (15\%) vs. } \\
6 \text { (29\%) } \\
\text { GMFCS level IV: } 10 \text { (29\%) } \\
\text { vs. } 5 \text { (24\%) }\end{array}$ & $\begin{array}{l}\text { A vs. B, mean (SD) } \\
\text { within } 2 \text { months after } 8 \text {-week intervention } \\
\text { GMFM-66: } \\
58.49(13.40) \text { vs. } 61.20(21.69) \text { (baseline) } \\
61.43(14.78) \text { vs. } 62.46(21.70) \text { (postintervention) } \\
2.93(3.95) \text { vs. } 1.25(1.99), p<0.05 \text { (pre-postintervention } \\
\text { difference) } \\
\text { PEDI: } \\
\text { Intervention group: } n=28 ; \text { Control group: } n=21 \\
116.32(48.61) \text { vs. } 112.52(64.98) \text { (baseline) } \\
127.21(46.89) \text { vs. } 114.52(64.53) \text { (postintervention) } \\
10.89(11.94) \text { vs. } 2.00(4.93), p<0.05 \text { (pre-post } \\
\text { intervention difference) }\end{array}$ & NR \\
\hline
\end{tabular}




\begin{tabular}{|c|c|c|c|c|}
\hline $\begin{array}{l}\text { Author, Year } \\
\text { Intervention Type } \\
\text { Duration of } \\
\text { Postintervention } \\
\text { Followup } \\
\text { Quality } \\
\text { (See Appendix B } \\
\text { for Full Citation) }\end{array}$ & $\begin{array}{l}\text { Intervention and } \\
\text { Comparison }\end{array}$ & $\begin{array}{l}\text { Population } \\
\text { Age (Mean) } \\
\text { Gender (\% Female) } \\
\text { Race (\%) } \\
\text { Ambulatory (\%) } \\
\text { Wheelchair User (\%) } \\
\text { Condition Specific (\%) } \\
\text { Other (\%) }\end{array}$ & Prioritized Outcomes & Other Outcomes \\
\hline $\begin{array}{l}\text { Peri, } 2017 \\
\text { Aerobic Exercise } \\
\text { Robot-Assisted }\end{array}$ & $\begin{array}{l}\text { A. RAGT plus TOP } \\
\text { (task oriented } \\
\text { physical therapy), } \\
2+2 \text { sessions/week } \\
\text { over } 10 \text { weeks } \\
(n=10)\end{array}$ & $\begin{array}{l}\text { A vs. B vs. C vs. D } \\
\text { Age (mean years): } \\
6.8 \text { vs. } 10.8 \text { vs. } 9.3 \text { vs. } 8 \\
\text { Female: } \\
6(60 \%) \text { vs. } 5(42 \%) \text { vs. } 5 \\
(50 \%) \text { vs. } 6(50 \%)\end{array}$ & $\begin{array}{l}\text { A vs. B vs. C vs. D, mean (SD) } \\
6 \text { MWT (meters, T0 to T1 to T2) } \\
285.2(219.2) \text { to } 300.9(201.9) \text { to } 309.0(214.9) \text { vs. } 222.1 \\
(237.6) \text { to } 208.5(252.7) \text { to } 225.0(193.7) \text { vs. } 378.2(182.6) \\
\text { to } 381.7(159.3) \text { to } 364.1(179.8) \text { vs. } 324.4(110.2) \text { to } \\
345.0(92.4) \text { to } 346.5(84.3)\end{array}$ & A vs. B, mean (SD) \\
\hline $\begin{array}{l}\text { Postintervention, 4- } \\
10 \text { weeks } \\
\text { Poor }\end{array}$ & $\begin{array}{l}\text { B. RAGT plus TOP } \\
\text { (task oriented } \\
\text { physical therapy), } \\
5+5 \text { sessions/week } \\
\text { over } 4 \text { weeks }(n=10) \\
\text { C. TOP } 4 \\
\text { sessions/wk over } 10 \\
\text { weeks }(n=10) \\
\text { D. RAGT } 4 \\
\text { sessions/wk over } 10 \\
\text { weeks }\end{array}$ & $\begin{array}{l}\text { Race: NR } \\
\text { Ambulatory: } \\
\text { NR } \\
\text { Wheelchair user: } \\
\text { NR } \\
\\
\text { Other: } \\
\text { GMFCS }(\mathrm{I} / \mathrm{II} / \mathrm{III}) \\
3 / 4 / 3 \text { vs. } 5 / 2 / 5 \text { vs. } 3 / 5 / 2 \text { vs. } \\
3 / 5 / 4\end{array}$ & $\begin{array}{l}\text { GMFM-66 } \\
66.0(12.1) \text { to } 67.0(12.7) \text { to } 69.2(10.4) \text { vs. } 66.2(6.3) \text { to } \\
67.1(6.2) \text { to } 68.1(6.3) \text { vs. } 66.4(13.4) \text { to } 68.2(11.9) \text { to } \\
69.2(9.7) \text { vs. } 68.5(8.8) \text { to } 68.9(8.6) \text { to } 69.2(9.7)\end{array}$ & \\
\hline
\end{tabular}




\begin{tabular}{|c|c|c|c|c|}
\hline $\begin{array}{l}\text { Author, Year } \\
\text { Intervention Type } \\
\text { Duration of } \\
\text { Postintervention } \\
\text { Followup } \\
\text { Quality } \\
\text { (See Appendix B } \\
\text { for Full Citation) } \\
\end{array}$ & $\begin{array}{l}\text { Intervention and } \\
\text { Comparison }\end{array}$ & $\begin{array}{l}\text { Population } \\
\text { Age (Mean) } \\
\text { Gender (\% Female) } \\
\text { Race (\%) } \\
\text { Ambulatory (\%) } \\
\text { Wheelchair User (\%) } \\
\text { Condition Specific (\%) } \\
\text { Other (\%) }\end{array}$ & Prioritized Outcomes & Other Outcomes \\
\hline $\begin{array}{l}\text { Pompa, } 2017 \\
\text { Aerobic Exercise } \\
\text { Robot-Assisted } \\
\text { Gait Training } \\
\text { Postintervention, } 4 \\
\text { weeks } \\
\text { Fair }\end{array}$ & $\begin{array}{l}\text { A. RAGT, } 12 \\
\text { sessions over } 4 \\
\text { weeks }(n=21) \\
\text { B. Conventional } \\
\text { Walking Training, } 12 \\
\text { sessions over } 4 \\
\text { weeks }(n=22)\end{array}$ & $\begin{array}{l}\text { A vs. B } \\
\text { Age (mean years): } \\
47 \text { vs. } 50 \\
\text { Female: } \\
10(48 \%) \text { vs. } 12(55 \%) \\
\text { Race: NR } \\
\text { Ambulatory: NR } \\
\text { Wheelchair user: NR } \\
\text { Other: } \\
\text { disease duration (years) } \\
17.05 \text { vs. } 14.09 \\
\text { Primary } \\
\text { Progressive/Secondary } \\
\text { Progressive MS } \\
\text { 0/21 vs. } 3 / 22 \\
\text { EDSS } \\
6.62 \text { vs. } 6.50\end{array}$ & $\begin{array}{l}2 \text { MWT } \\
33.71(15.43) \text { to } 42.59(20.79) p=0.001 \text { vs. } 40.91 \text { (22.45) } \\
\text { to } 43.72(24.50) p=0.076 \\
\text { FAC } \\
3.10(1.51) \text { to } 3.76(1.04) p=0.017 \text { vs. } 3.50(1.10) \text { to } 3.50 \\
(1.10) p=0.999 \\
\text { EDSS } \\
6.62(0.42) \text { to } 6.48(0.37) p=0.014 \text { vs. } 6.50(1.10) \text { to } 6.50 \\
(0.49) p=0.999 \\
\text { Rivername Mobility index } \\
5.76(2.05) \text { to } 7.76(2.62) p<0.001 \text { vs. } 6.14(3.11) \text { to } 7.41 \\
(2.58) p<0.001 \\
\text { LE Spasticity VAS } \\
5.05(1.01) \text { to } 3.40(1.24) p=0.007 \text { vs. } 5.31(2.52) \text { to } 5.23 \\
(2.29) p=0.693 \\
\text { Modified Barthel Index } \\
63.43(18.51) \text { to } 77.43(15.91) p<0.001 \text { vs. } 64.09 \text { (20.60) } \\
\text { to } 74.10 \text { (14.72) } p<0.001\end{array}$ & A vs. B, mean (SD) \\
\hline $\begin{array}{l}\text { Pourazar, } 2020 \\
\text { Postural Control } \\
\text { Motion Gaming } \\
\text { Postintervention, } \\
0 \text { weeks } \\
\text { Fair }\end{array}$ & $\begin{array}{l}\text { A. Virtual reality Xbox } \\
360 \text { Kinect, } 20 \\
\text { sessions over } 6 \\
\text { weeks ( } n=10) \\
\text { B. Encouraged to do } \\
\text { typical physical } \\
\text { activity at home } \\
(n=10)\end{array}$ & $\begin{array}{l}\text { A vs. B } \\
\text { Age: } 9.2 \text { vs. } 9.6 \\
\text { Female: } 100 \% \\
\text { Race: NR } \\
\text { GMFCS !: } 50 \% \text { vs. } 60 \% \\
\text { GMFCS II: } 20 \% \text { vs. } 30 \% \\
\text { GMFCS III: } 30 \% \text { vs. } 10 \%\end{array}$ & $\begin{array}{l}\text { Dynamic balance was improved in the anterior, } \\
\text { posterolateral, and posteromedial directions with virtual } \\
\text { reality dance game compare with the control group, } \\
p=0.001 \text { all comparisons }\end{array}$ & NA \\
\hline
\end{tabular}




\begin{tabular}{|c|c|c|c|c|}
\hline $\begin{array}{l}\text { Author, Year } \\
\text { Intervention Type } \\
\text { Duration of } \\
\text { Postintervention } \\
\text { Followup } \\
\text { Quality } \\
\text { (See Appendix B } \\
\text { for Full Citation) }\end{array}$ & $\begin{array}{l}\text { Intervention and } \\
\text { Comparison }\end{array}$ & $\begin{array}{l}\text { Population } \\
\text { Age (Mean) } \\
\text { Gender (\% Female) } \\
\text { Race (\%) } \\
\text { Ambulatory (\%) } \\
\text { Wheelchair User (\%) } \\
\text { Condition Specific (\%) } \\
\text { Other (\%) }\end{array}$ & Prioritized Outcomes & Other Outcomes \\
\hline $\begin{array}{l}\text { Qi, } 2018 \\
\text { Postural Control } \\
\text { Tai Chi } \\
\text { Postintervention, } 6 \\
\text { weeks } \\
\text { Fair }\end{array}$ & $\begin{array}{l}\text { Tai Chi vs. usual } \\
\text { care } \\
\text { A. Tai Chi: } 60 \\
\text { sessions }(2 \\
\text { sessions/day, } 5 \\
\text { days/week) over } 6 \\
\text { weeks ( } n=20) \\
\text { B. Usual care control: } \\
(n=20)\end{array}$ & $\begin{array}{l}\text { A vs. B } \\
\text { Age (mean years): } 38.3 \text { vs. } \\
43.05 \\
\text { Female: } 5(25 \%) \text { vs. } 4(20 \%) \\
\text { Race: NR } \\
\text { Ambulatory: NR } \\
\text { Wheelchair user: } 100 \% \\
\text { BMl: } 24.46 \text { vs. } 24.28 \\
\text { Injury level } \\
\text { C6-T1: } 3 \text { vs. } 4 \\
\text { T2-T5: } 5 \text { vs. } 6 \\
\text { T6-T12: } 8 \text { vs. } 7 \\
\text { Below L1: } 4 \text { vs. } 3\end{array}$ & $\begin{array}{l}\text { A. vs. B., mean (SD) } \\
\text { WHOQOL-BREF (5-point scale, higher score=higher } \\
\text { QOL) } \\
\text { Quality of life - Physical: } 11.40 \text { (1.25) vs. } 10.94 \text { (1.15), } \\
\text { p=0.24 (baseline) } \\
\text { Quality of life - Psychological } 10.95 \text { (1.57) vs. } 10.87 \\
\text { (1.08), p=0.09 (postintervention) } \\
\text { Quality of life - Physical: } 11.80(1.33) \text { vs. } 11.09(1.29) \text {, } \\
p=0.85 \text { (baseline) } \\
\text { Quality of life - Psychological: } 12.23(1.65) \text { vs. } 10.87 \\
(1.08), p=0.04 \text { (postintervention) }\end{array}$ & NA \\
\hline $\begin{array}{l}\text { Qi, 2018a } \\
\text { Muscle Strength } \\
\text { Immediately } \\
\text { postintervention, } \\
6 \text { weeks } \\
\text { Fair }\end{array}$ & $\begin{array}{l}\text { A. Strength + } \\
\text { neuromuscular } \\
\text { electrical stimulation: } \\
30 \text { sessions over } 6 \\
\text { weeks, electrodes } \\
\text { were placed on } \\
\text { extensor of } \\
\text { acrotarsium with a } \\
\text { current intensity used } \\
\text { just strong enough to } \\
\text { cause muscle } \\
\text { contraction, which } \\
\text { continued for } 20 \\
\text { minutes ( } n=50 \text { ) } \\
\\
\text { B. Neuromuscular } \\
\text { electrical stimulation: } \\
\text { same stimulation as } \\
\text { above ( } n=50 \text { ) }\end{array}$ & $\begin{array}{l}\text { A vs. B } \\
\text { Age (mean years): } 5.8 \text { vs. } \\
6.0 \\
\text { Female: } 24(48 \%) \text { vs. } 23 \\
(46 \%) \\
\text { Race: NR } \\
\text { Ambulatory: NR } \\
\text { Wheelchair user: NR }\end{array}$ & $\begin{array}{l}\text { Data reported as mean (SD) } \\
\text { A vs. B } \\
\text { GMFM-D/E (0-100, higher=increased motor function): } \\
44.5(13.2) \text { vs. } 44(12.6), p>0.05 \text { (baseline) } \\
70.6(15.2) \text { vs. } 56.7(14.3), p<0.05 \text { (postintervention) } \\
71.0(16.4) \text { vs. } 58.0(15.6), p<0.05 \text { (6 weeks) } \\
\text { CSS (<7=spasm, } 7-9=\text { mild spasm, 10-12=moderate } \\
\text { spasm, } 13-16=\text { severe spasm): } \\
12.0(3.4) \text { vs. } 12.3(3.6), p>0.05 \text { (baseline) } \\
7.6(3.0) \text { vs. } 9.5(2.8), p<0.05 \text { (postintervention) } \\
7.4(2.4) \text { vs. } 9.4(2.6), p<0.05 \text { (6 weeks) }\end{array}$ & NR \\
\hline
\end{tabular}




\begin{tabular}{|c|c|c|c|c|}
\hline $\begin{array}{l}\text { Author, Year } \\
\text { Intervention Type } \\
\text { Duration of } \\
\text { Postintervention } \\
\text { Followup } \\
\text { Quality } \\
\text { (See Appendix B } \\
\text { for Full Citation) } \\
\end{array}$ & $\begin{array}{l}\text { Intervention and } \\
\text { Comparison }\end{array}$ & $\begin{array}{l}\text { Population } \\
\text { Age (Mean) } \\
\text { Gender (\% Female) } \\
\text { Race (\%) } \\
\text { Ambulatory (\%) } \\
\text { Wheelchair User (\%) } \\
\text { Condition Specific (\%) } \\
\text { Other (\%) } \\
\end{array}$ & Prioritized Outcomes & Other Outcomes \\
\hline $\begin{array}{l}\text { Razazian, } 2016 \\
\text { Aerobic Exercise } \\
\text { Aquatics } \\
\text { Postintervention, } 8 \\
\text { weeks } \\
\text { Poor }\end{array}$ & $\begin{array}{l}\text { Yoga vs. Aquatics } \\
\text { A. Yoga: } 24 \text { sessions } \\
\text { over } 8 \text { weeks }(n=18) \\
\text { B. Aquatic exercise: } \\
24 \text { sessions over } 8 \\
\text { weeks }(n=18) \\
\\
\text { C. Usual care } \\
\text { control: met } 2-3 \\
\text { times a week in } \\
\text { hospital for usual } \\
\text { care, over } 8 \text { weeks } \\
(n=18)\end{array}$ & $\begin{array}{l}\text { A vs. B vs. C } \\
\text { Age (mean years): } 33 \text { vs. } 35 \\
\text { vs. } 33 \\
\text { Female: } 18 \text { (100\%) vs. } 18 \\
\text { (100\%) vs. } 18(100 \%) \\
\text { Race: NR } \\
\text { Ambulatory: NR } \\
\text { Wheelchair user: NR } \\
\\
\text { Duration of MS (years) } 6.90 \\
\text { vs. } 7.11 \text { vs. } 6.78 \\
\text { EDDS: } 3.89 \text { vs. } 3.44 \text { vs. } 3.25 \\
\text { MS (primary-progressive): } 0 \\
(0 \%) \text { vs. } 0 \text { (0\%) vs. (0\%) } \\
\text { MS (secondary-progressive): } \\
1(.5 \%) \text { vs. } 2 \text { (11\%) vs. } 2 \\
(11 \%) \\
\text { MS (relapsing-remitting): } 13 \\
(72 \%) \text { vs. } 11(61 \%) \text { vs. } 12 \\
(66 \%) \\
\text { MS (progressive-relapsing): } \\
4(22 \%) \text { vs. } 5 \text { (27\%) vs. } 4 \\
\text { (22\%) }\end{array}$ & $\begin{array}{l}\text { A. vs. B. vs. C, mean (SD) } \\
\text { Beck Depression Inventory (BDI: } 0-9=\text { no or minimal to } \\
30-63=\text { severe depression) } \\
19.72(7.04) \text { vs. } 19.17(7.83) \text { vs. } 20.78(6.22) \text {, (baseline) } \\
5.06(2.92) \text { vs. } 4.78(3.42) \text { vs. } 21.33(6.88), \\
\text { (postintervention), } p=0.000\end{array}$ & NA \\
\hline $\begin{array}{l}\text { Roppolo, } 2013 \\
\text { Multimodal } \\
\text { Exercise } \\
\text { Immediately } \\
\text { postintervention, } \\
12 \text { weeks } \\
\text { Fair }\end{array}$ & $\begin{array}{l}\text { A. Combination } \\
\text { therapy (aerobic }+ \\
\text { strength training) } 12 \\
\text { weeks } 24 \text { sessions } \\
\text { over } 12 \text { weeks } \\
(n=17) \\
\text { B. Control group } \\
\text { (activity not } \\
\text { specified) }(n=18)\end{array}$ & $\begin{array}{l}\text { A vs. B } \\
\text { Age: } 40 \text { vs. } 40 \\
\text { Female: } 100 \% \\
\text { EDSS: } 1.5 \text { vs. } 2.0\end{array}$ & $\begin{array}{l}\text { A vs. B, Mean (SD), p=between groups } \\
\text { BDI: } \\
8.8(5.8) \text { to } 3.4(2.9) \text { vs. } 9.2(3.7) \text { to } 17.0(7.0), M D 13.2 \text {, } \\
95 \% \text { CI } 9.86 \text { to } 16.55, p<0.001 \\
\text { MSQOL-54: } \\
202.7(7.9) \text { vs. } 139.3 \text { (32.4), MD } 63.4(7.86)(95 \% \mathrm{Cl} \\
47.43 \text { to } 79.4), p<0.001 \text { (post-intervention); } \\
29.5(36.17) \text { vs. }-22.5 \text { (55.57), MD } 52.0,95 \% \mathrm{Cl} 20.8 \text { to } \\
83.2, p=N R \text { (pre-post change) }\end{array}$ & NA \\
\hline
\end{tabular}




\begin{tabular}{|c|c|c|c|c|}
\hline $\begin{array}{l}\text { Author, Year } \\
\text { Intervention Type } \\
\text { Duration of } \\
\text { Postintervention } \\
\text { Followup } \\
\text { Quality } \\
\text { (See Appendix B } \\
\text { for Full Citation) } \\
\end{array}$ & $\begin{array}{l}\text { Intervention and } \\
\text { Comparison }\end{array}$ & $\begin{array}{l}\text { Population } \\
\text { Age (Mean) } \\
\text { Gender (\% Female) } \\
\text { Race (\%) } \\
\text { Ambulatory (\%) } \\
\text { Wheelchair User (\%) } \\
\text { Condition Specific (\%) } \\
\text { Other (\%) } \\
\end{array}$ & Prioritized Outcomes & Other Outcomes \\
\hline $\begin{array}{l}\text { Russo, } 2018 \\
\text { Aerobic Exercise } \\
\text { Robot-Assisted } \\
\text { Gait Training } \\
\text { Postintervention, } \\
18 \text { weeks } \\
\text { Fair }\end{array}$ & $\begin{array}{l}\text { A. RAGT, } 18 \\
\text { sessions over } 6 \\
\text { weeks followed by } \\
\text { usual } 12 \text { weeks ( } 36 \\
\text { sessions) of } \\
\text { traditional training } \\
\text { ( } n=30) \\
\\
\text { B. Usual care } \\
\text { (traditional } \\
\text { rehabilitation } \\
\text { training), } 54 \text { sessions } \\
\text { over } 18 \text { weeks } \\
\text { ( } n=15)\end{array}$ & $\begin{array}{l}\text { A vs. B } \\
\text { Age (mean years): } \\
42 \text { vs. } 41 \\
\text { Female: } \\
16(53 \%) \text { vs. } 10(67 \%) \\
\text { Race: NR } \\
\text { Ambulatory: } \\
\mathrm{N}(\%) \text { vs. } \mathrm{N}(\%) \\
\text { Wheelchair user: } \\
\mathrm{N}(\%) \text { vs. } \mathrm{N}(\%) \\
\\
\text { Other: } \\
\text { Disease duration } \\
11.4 \text { years vs. } 12.3 \text { years }\end{array}$ & $\begin{array}{l}\text { TUG change in score (seconds) } \\
\text { Post training } \\
11.4, p<0.001 \text { vs. } 11.2, p<0.001 \\
\text { At followup } \\
8.9, p<0.001 \text { vs. } 5.1, p<0.001 \\
\text { EDSS } \\
5.5 \text { to } 5.0, p=0.026 \text { vs. } 4.5 \text { to } 4.0, p=0.003 \\
\text { Tinetti Balance Scale change in score } \\
\text { Post training } \\
-1.2, p<0.001 \text { vs. }-.7, p<0.01 \\
\text { At followup } \\
-1.0, p<0.001 \text { vs. }-0.1 p=0.71 \\
\text { FIM } \\
\text { Change in score } \\
\text { Post training } \\
-2.2, p<0.001 \text { vs. }-1.7, p<0.001 \\
\text { At followup } \\
-1.8, p<0.001 \text { vs. }-1.5, p<0.001\end{array}$ & $\begin{array}{l}\text { HRSD } \\
10.0 \text { to } 7.0, p=0.004 \\
\text { vs. } 12.5 \text { to } 7.0, p=0.004\end{array}$ \\
\hline $\begin{array}{l}\text { Sadeghi Bahmani, } \\
2019 \\
\text { Aerobic Exercise } \\
\text { Aerobics } \\
\text { Postural Control } \\
\text { Balance } \\
\text { Postintervention, } 8 \\
\text { weeks }\end{array}$ & $\begin{array}{l}\text { A. Endurance } \\
\text { training (treadmill, } \\
\text { cycling, walking, } \\
\text { jogging), } 24 \text { sessions } \\
\text { over } 8 \text { weeks ( } n=26) \\
\text { B. Balance and } \\
\text { coordination } \\
\text { exercises, } 24 \\
\text { sessions over } 8 \\
\text { weeks ( } n=24) \\
\text { C. Attention control, } \\
24 \text { sessions over } 8 \\
\text { weeks }(n=21)\end{array}$ & $\begin{array}{l}\text { A vs. B vs. C } \\
\text { Age: } 38 \text { vs. } 39 \text { vs. } 38 \\
\text { Female: } 100 \% \\
\text { EDSS: } 2.46 \text { vs. } 3.38 \text { vs. } 2.02\end{array}$ & $\begin{array}{l}\text { A vs. B vs. C, Mean (SD), p=between groups: } \\
\text { BDI-FS: } \\
7.92(5.11) \text { to } 5.12(4.65) \text { vs. } 7.96(6.67) \text { to } 5.29(5.75) \text { vs. } \\
6.24(4.47) \text { to } 6.52(4.91) \\
\text { A vs. C: } \\
\text { MD } 3.08,95 \% \mathrm{Cl} 0.33 \text { to } 5.84, p=0.028 \\
\text { B vs. C: } \\
\text { MD } 2.95,95 \% \mathrm{Cl}-0.26 \text { to } 6.16, p=0.072 \\
\text { A vs. } \mathrm{B}: \\
\text { MD } 0.13,95 \% \mathrm{Cl}-3.00 \text { to } 3.26, p=0.935\end{array}$ & $\begin{array}{l}\text { A vs. C, Mean (SD), } p=\text { between groups: } \\
\text { EDSS }- \text { Expanded Disability status: } \\
3.38(1.87) \text { to } 3.10(1.86) \text { vs. } 2.02(1.84) \text { to } \\
1.98(1.70), p>0.05 \\
\\
\text { ISI = Insomnia Severity Index; } \\
13.46(5.81) \text { to } 10.13(4.92) \text { vs. } 1.71(5.43) \text { to } \\
11.14(5.39), p>0.05\end{array}$ \\
\hline
\end{tabular}




\begin{tabular}{|c|c|c|c|c|}
\hline $\begin{array}{l}\text { Author, Year } \\
\text { Intervention Type } \\
\text { Duration of } \\
\text { Postintervention } \\
\text { Followup } \\
\text { Quality } \\
\text { (See Appendix B } \\
\text { for Full Citation) } \\
\end{array}$ & $\begin{array}{l}\text { Intervention and } \\
\text { Comparison }\end{array}$ & $\begin{array}{l}\text { Population } \\
\text { Age (Mean) } \\
\text { Gender (\% Female) } \\
\text { Race (\%) } \\
\text { Ambulatory (\%) } \\
\text { Wheelchair User (\%) } \\
\text { Condition Specific (\%) } \\
\text { Other (\%) }\end{array}$ & Prioritized Outcomes & Other Outcomes \\
\hline $\begin{array}{l}\text { Sadeghi Bahmani, } \\
\text { 2020a (aquatic) } \\
\text { Aerobic Exercise } \\
\text { Aquatic } \\
\text { Postintervention, } \\
5 \text { weeks } \\
\text { Fair }\end{array}$ & $\begin{array}{l}\text { A. Aquatic exercise } \\
\text { for two weeks }(n=21) \\
\text { B. Aquatic exercise } \\
\text { for three weeks } \\
(n=19) \\
\text { C. Control }(n=22)\end{array}$ & $\begin{array}{l}\text { Age: } 39 \text { vs. } 41 \text { vs. } 34 \\
\text { Female: } 100 \% \\
\text { Race: NR } \\
\text { Ambulatory: } 100 \% \text { vs. } 100 \% \\
\text { vs. } 100 \% \\
\text { Wheelchair user: NR } \\
\\
\text { Median EDSS score: } 3.00 \\
\text { vs. } 1.50 \text { vs. } 1.50 \\
\text { Baseline Female Sexual } \\
\text { Function Index (FSFI), } \\
\text { mean: } 41.40 \text { vs. } 45.67 \text { vs. } \\
50.59\end{array}$ & $\begin{array}{l}\text { A vs. B, adjusted mean (SE): } \\
\text { FSFI: } 52.14(1.2) \text { vs. } 48.80(1.2) \text { vs. } 42.80(1.1) \\
\text { After controlling for baseline values, the highest scores } \\
\text { were achieved in the group that exercised } 2 \text { times weekly } \\
(52.14) \text { followed by three times weekly }(48.80) \text { and active } \\
\text { control }(42.80), p<0.001 \\
\text { Correlation coefficients between sexual function, EDSS } \\
\text { EDSS: }-0.29, p<0.05 \text { (baseline) } \\
-0.4, p=N R \text { (postintervention) } \\
\text { Correlation coefficients between sexual function, } \\
\text { Depression } \\
\text { Depression: }-0.17 \text { (baseline) } \\
-0.09 ; p<0.01 \text { (postintervention) }\end{array}$ & $\begin{array}{l}\text { Correlation coefficients between sexual } \\
\text { function, Fatigue } \\
\text { Fatigue: }-0.33 ; p<0.01 \text { (baseline) } \\
-0.14 \text { (postintervention) } \\
\text { Correlation coefficients between sexual } \\
\text { function, Couple satisfaction } \\
\text { Couple satisfaction: } 0.48 \text { (baseline) } \\
0.64 ; p<0.001 \text { (postintervention) }\end{array}$ \\
\hline $\begin{array}{l}\text { Sadowsky, } 2013 \\
\text { Aerobic Exercise } \\
\text { Cycling } \\
\text { Postintervention, } 0 \\
\text { weeks } \\
\text { Poor }\end{array}$ & $\begin{array}{l}\text { A. FES cycle } \\
\text { ergometry, } 3 \\
\text { sessions per week } \\
\text { over range of } 3-168 \\
\text { months }(n=25) \\
\text { B. Standard } \\
\text { rehabilitation care, } \\
\text { not specified ( } n=20)\end{array}$ & $\begin{array}{l}\text { A vs. B } \\
\text { Age (mean years): } \\
37.2 \text { vs. } 34.6 \\
\text { Female: } \\
4(12 \%) \text { vs. } 4(20 \%) \\
\text { Race: NR } \\
\text { Ambulatory: NR } \\
\text { Wheelchair user: NR } \\
\\
\text { Other: } \\
\text { Quadriplegia } \\
\text { 13 (52\%) vs. } 15(75 \%) \\
\text { Paraplegia } \\
\text { 12(48\%) vs. } 5(25 \%) \\
\end{array}$ & $\begin{array}{l}\text { Calculated A vs. B, Mean change scores: } \\
\text { Total FIM: } 80 \% \text { vs. } 60 \%, p<0.001 \\
\text { With significant improvement with FES in subscales: self- } \\
\text { care, sphincter control, transfer, and locomotion } \\
\text { SF-36: total and composite scores NR } \\
\text { Significant improvement in physical function and role limit } \\
\text { physical with FES, no difference in mental health } \\
\text { subscales }\end{array}$ & $\begin{array}{l}\text { A vs. B (SD) } \\
\text { Body Fat Volume (cc): } \\
450 \text { vs. } 800 \text { (est), } p<0.010\end{array}$ \\
\hline
\end{tabular}




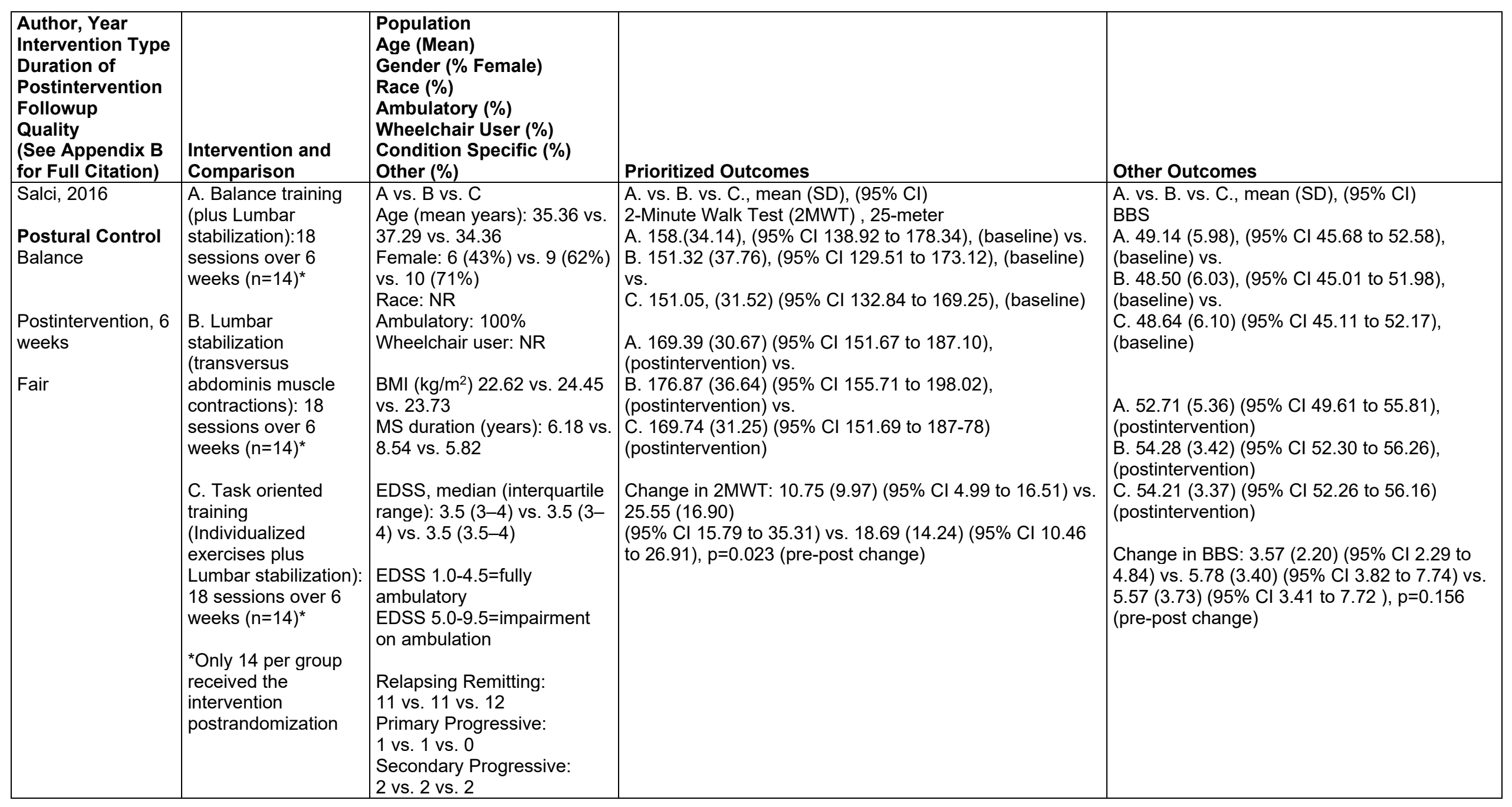




\begin{tabular}{|c|c|c|c|c|}
\hline $\begin{array}{l}\text { Author, Year } \\
\text { Intervention Type } \\
\text { Duration of } \\
\text { Postintervention } \\
\text { Followup } \\
\text { Quality } \\
\text { (See Appendix B } \\
\text { for Full Citation) }\end{array}$ & $\begin{array}{l}\text { Intervention and } \\
\text { Comparison }\end{array}$ & \begin{tabular}{|l|} 
Population \\
Age (Mean) \\
Gender (\% Female) \\
Race (\%) \\
Ambulatory (\%) \\
Wheelchair User (\%) \\
Condition Specific (\%) \\
Other (\%)
\end{tabular} & Prioritized Outcomes & Other Outcomes \\
\hline $\begin{array}{l}\text { Samaei } \\
2014 \\
\text { Aerobic Exercise } \\
\text { Treadmill } \\
\text { Postintervention, } 0 \\
\text { weeks } \\
\text { Fair }\end{array}$ & $\begin{array}{l}\text { A. } 30 \text { minutes } \\
\text { walking at } 10 \\
\text { degrees downgrade } \\
3 \text { times a week for } 4 \\
\text { weeks } \\
\\
\text { B. } 30 \text { minutes } \\
\text { walking at } 10 \\
\text { degrees elevation } 3 \\
\text { times a week for } 4 \\
\text { weeks }\end{array}$ & $\begin{array}{l}\text { Score greater than } 3 \text { on } \\
\text { GNDS limb score } \\
n=34 \\
\text { Mean age } 33.03 \text { years } \\
28 \text { females and } 6 \text { males } \\
\text { (17.6\% males) }\end{array}$ & $\begin{array}{l}\text { Pre, post } \\
25 \text { foot walk } \\
\text { A. } 8.7(2.4) \text { to } 6.1(1.8) p=0.002 \text { (within group differences) } \\
\text { B. } 7.9(1.1) \text { to } 7.0(1.6) p=0.048 \\
p=0.001 \\
2 \text { minute walk } \\
\text { A. } 120.01(23.6) \text { to } 160.1(35.7) p=0.001 \\
\text { B. } 132.6(32.3) \text { to } 147.5(29.8) p=0.026 \\
p=0.0001 \\
\text { Timed Up and Go } \\
\text { A. } 98 .(1.7) \text { to } 7.5(1.8) p=0.008 \\
\text { B. } 9.4(2.3) \text { to } 8.9(0.9) p=0.039 \\
p=0.041 \\
\text { GNDS } \\
\text { A. } 35.4(9.1) \text { to } 21.8(5.3) p=0.006 \\
\text { B. } 32.1(8.6) \text { to } 27.5 \text { (6.1) } p=0.041 \\
p=0.12 \\
\text { Modified Riverman Mobility Index } \\
\text { A. } 10.6(3.2) \text { to } 14.3(2.7) p=0.009 \\
\text { B. } 10.5(2.3) \text { to } 11.9 \text { (2.1) } p=0.038 \\
p=0.005\end{array}$ & NA \\
\hline
\end{tabular}




\begin{tabular}{|c|c|c|c|c|}
\hline $\begin{array}{l}\text { Author, Year } \\
\text { Intervention Type } \\
\text { Duration of } \\
\text { Postintervention } \\
\text { Followup } \\
\text { Quality } \\
\text { (See Appendix B } \\
\text { for Full Citation) } \\
\end{array}$ & $\begin{array}{l}\text { Intervention and } \\
\text { Comparison }\end{array}$ & $\begin{array}{l}\text { Population } \\
\text { Age (Mean) } \\
\text { Gender (\% Female) } \\
\text { Race (\%) } \\
\text { Ambulatory (\%) } \\
\text { Wheelchair User (\%) } \\
\text { Condition Specific (\%) } \\
\text { Other (\%) } \\
\end{array}$ & Prioritized Outcomes & Other Outcomes \\
\hline $\begin{array}{l}\text { Sandroff, } 2017 \\
\text { Multimodal } \\
\text { Exercise } \\
\text { Mid-intervention } \\
\text { (12 weeks into } \\
\text { intervention); } \\
\text { Immediately } \\
\text { postintervention } \\
\text { Fair }\end{array}$ & $\begin{array}{l}\text { A. Resistance + } \\
\text { aerobics + balance: } \\
72 \text { sessions over } 24 \\
\text { weeks. } \\
\text { Approximately } \\
\text { equal durations of } \\
\text { aerobic, lower- } \\
\text { extremity resistance, } \\
\text { and balance training } \\
(n=43) \\
\text { B. Stretching and } \\
\text { toning ( } n=40) \\
72 \text { sessions over } 24 \\
\text { weeks for both } \\
\text { groups }\end{array}$ & $\begin{array}{l}\text { A vs. B } \\
\text { Age (mean years): } 49.8 \text { vs. } \\
51.2 \\
\text { Female: } 36(83.7 \%) \text { vs. } 35 \\
(87.5 \%) \\
\text { Race: NR } \\
\text { Ambulatory: NR } \\
\text { Wheelchair user: NR } \\
\text { BMI: } 29.2 \text { vs. } 31.2\end{array}$ & 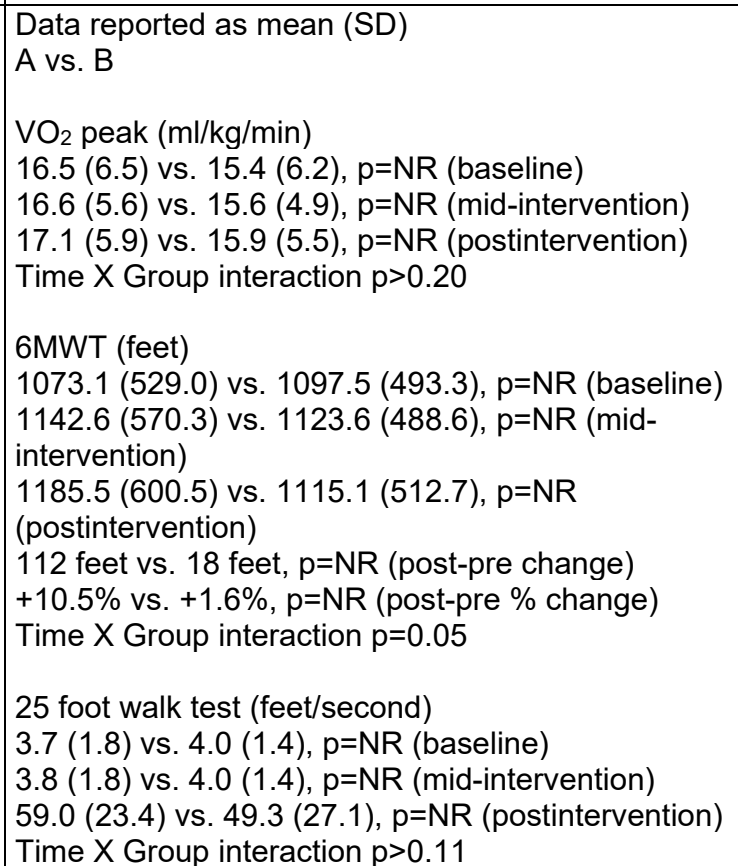 & NR \\
\hline
\end{tabular}




\begin{tabular}{|c|c|c|c|c|}
\hline $\begin{array}{l}\text { Author, Year } \\
\text { Intervention Type } \\
\text { Duration of } \\
\text { Postintervention } \\
\text { Followup } \\
\text { Quality } \\
\text { (See Appendix B } \\
\text { for Full Citation) } \\
\end{array}$ & $\begin{array}{l}\text { Intervention and } \\
\text { Comparison }\end{array}$ & $\begin{array}{l}\text { Population } \\
\text { Age (Mean) } \\
\text { Gender (\% Female) } \\
\text { Race (\%) } \\
\text { Ambulatory (\%) } \\
\text { Wheelchair User (\%) } \\
\text { Condition Specific (\%) } \\
\text { Other (\%) }\end{array}$ & Prioritized Outcomes & Other Outcomes \\
\hline $\begin{array}{l}\text { Sangelaji, } 2014 \\
\text { Multimodal } \\
\text { Exercise } \\
\text { Postintervention, } 0 \\
\text { weeks } \\
\text { Poor }\end{array}$ & $\begin{array}{l}\text { A. Strength }+ \\
\text { aerobics + balance: } \\
30 \text { sessions over } 10 \\
\text { weeks }(n=35) \\
\text { B. Usual care }(n=20)\end{array}$ & $\begin{array}{l}\text { A vs. B [according to those } \\
\text { with followup data] } \\
\text { Age (mean years): } 33.05 \text { vs. } \\
7.68 \\
\text { Female: } 24(61.5 \%) \text { vs. } 15 \\
(68.2 \%) \\
\text { Race: NR } \\
\text { Ambulatory: NR } \\
\text { Wheelchair user: NR }\end{array}$ & 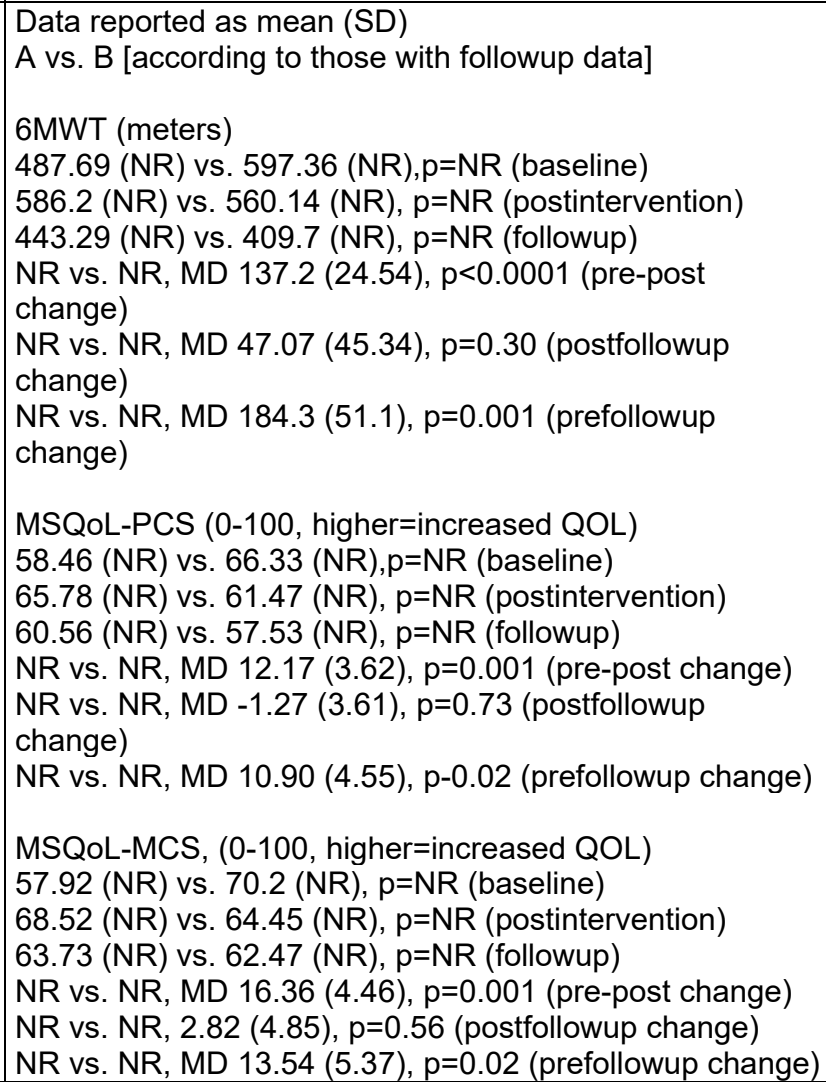 & $\begin{array}{l}\text { Data reported as mean (SD) } \\
\text { A vs. B [according to those with followup data] } \\
\text { EDSS (1-10, higher scores=greater disability) } \\
1.7 \text { (NR) v. } 1.96 \text { (NR),p=NR (baseline) } \\
1.7 \text { (NR) vs. } 2.06 \text { (NR), p=NR } \\
\text { (postintervention) } \\
2.2 \text { (NR) vs. } 2.74 \text { (NR), } p=N R \text { (followup) } \\
\text { NR vs. NR, MD }-0.13(0.23), p=0.60 \text { (pre-post } \\
\text { change) } \\
\text { NR vs. NR, MD }-0.15(0.21), p=0.50 \\
\text { (postfollowup change) } \\
\text { NR vs. NR, MD }-0.28(0.29), p=0.35 \\
\text { (prefollowup change) } \\
\text { BBS (0-56, higher scores=better balance) } \\
48.47 \text { (NR) vs. } 46.68 \text { (NR), } p=N R \text { (baseline) } \\
51.41 \text { (NR) vs. } 46.28 \text { (NR), } p=N R \\
\text { (postintervention) } \\
48.52 \text { (NR) vs. } 42.53(N R), p=N R \text { (followup) } \\
\text { NR vs. NR, MD } 3.34(0.87), p<0.0001 \text { (pre- } \\
\text { post change) } \\
\text { NR vs. NR, MD }-0.14(1.32), p=0.92 \\
\text { (postfollowup change) } \\
\text { NR vs. NR, MD } 3.21(1.44), p=0.03 \\
\text { (prefollowup change) }\end{array}$ \\
\hline
\end{tabular}




\begin{tabular}{|c|c|c|c|c|}
\hline $\begin{array}{l}\text { Author, Year } \\
\text { Intervention Type } \\
\text { Duration of } \\
\text { Postintervention } \\
\text { Followup } \\
\text { Quality } \\
\text { (See Appendix B } \\
\text { for Full Citation) } \\
\end{array}$ & $\begin{array}{l}\text { Intervention and } \\
\text { Comparison }\end{array}$ & $\begin{array}{l}\text { Population } \\
\text { Age (Mean) } \\
\text { Gender (\% Female) } \\
\text { Race (\%) } \\
\text { Ambulatory (\%) } \\
\text { Wheelchair User (\%) } \\
\text { Condition Specific (\%) } \\
\text { Other (\%) }\end{array}$ & Prioritized Outcomes & Other Outcomes \\
\hline $\begin{array}{l}\text { Sangelaji, } 2016 \\
\text { Multimodal } \\
\text { Exercise } \\
\text { Postintervention, } 0 \\
\text { weeks } \\
\text { Fair }\end{array}$ & $\begin{array}{l}\text { A. } 1 \text { aerobic }+3 \\
\text { resistance training } \\
\text { exercises per week } \\
\text { for } 8 \text { weeks }(32 \text { total } \\
\text { sessions) }(n=10) \\
\\
\text { B. } 2 \text { aerobic }+2 \\
\text { resistance training } \\
\text { exercises per week } \\
\text { for } 8 \text { weeks ( } 32 \text { total } \\
\text { sessions) ( } n=10) \\
\text { C. } 3 \text { aerobic vs. } 1 \\
\text { resistance training } \\
\text { exercises per week } \\
\text { for } 8 \text { weeks (32 total } \\
\text { sessions) ( } n=10) \\
\text { D. Control group } \\
\text { ( } n=10)\end{array}$ & $\begin{array}{l}\text { A vs. B vs. C vs. D } \\
\text { Age (mean years): } 35.80 \text { vs. } \\
31.33 \text { vs. } 33.91 \text { vs. } 33.63 \\
\text { Female: } 4(60 \%) \text { vs. } 4(60 \%) \\
\text { vs. } 4(60 \%) \text { vs. } 4(60 \%) \\
\text { Race: NR } \\
\text { Ambulatory: NR } \\
\text { Wheelchair user: NR } \\
\text { Baseline EDSS (mean): } 1.33 \\
\text { vs. } 2.06 \text { vs. } 1.95 \text { vs. } 1.81\end{array}$ & $\begin{array}{l}\text { Data reported as mean (SD) } \\
\text { A vs. D } \\
\text { 10MWT (seconds): } \\
9.828(4.89645) \text { vs. } 15.217 \text { (18.94777), p=NR (baseline) } \\
7.422 \text { (2.42591) vs. } 15.122 \text { (19.02946), p=NR } \\
\text { (postintervention) } \\
\text {-2.4056 (NR) vs. }-0.095 \text { (NR); MD } 2.31 \text { (SE, 1.04), } \\
\text { p=0.030 (post-pre change) } \\
-0.624281255 \% \text { (NR) vs. }-24.47750259 \% \text { (NR), p=NR } \\
\text { (post-pre \% change) } \\
\text { 6MWT (meters): } \\
380.222 \text { (136.77790) vs. } 361.500(238.86757), p=N R \\
\text { (baseline) } \\
461.444 \text { (139.61206) vs. } 367.500(258.75692), p=N R \\
\text { (postintervention) } \\
81.2222 \text { (NR) vs. } 6.0000 \text { (NR); MD }-75.22 \text { (SE, 28.21), } \\
\text { p=0.010 (post-pre change) } \\
21.36177674 \% \text { (NR) vs. } 1.659751037 \% \text { (NR), p=NR } \\
\text { (post-pre \% change) }\end{array}$ & 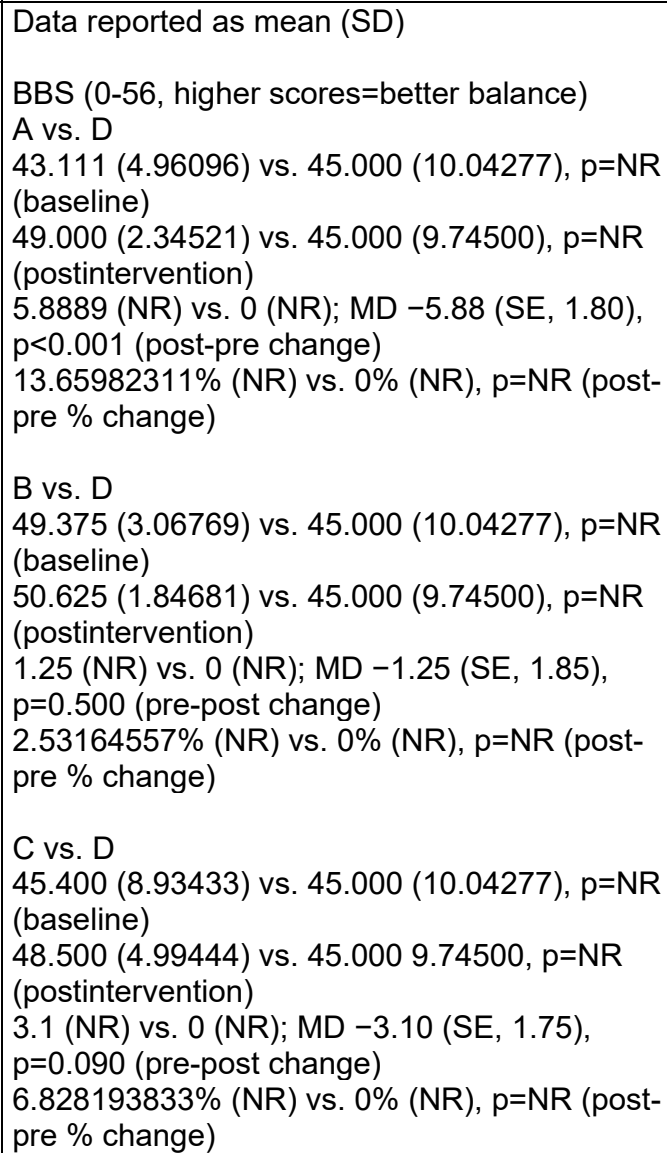 \\
\hline
\end{tabular}




\begin{tabular}{|c|c|c|c|c|}
\hline $\begin{array}{l}\text { Author, Year } \\
\text { Intervention Type } \\
\text { Duration of } \\
\text { Postintervention } \\
\text { Followup } \\
\text { Quality } \\
\text { (See Appendix B } \\
\text { for Full Citation) }\end{array}$ & $\begin{array}{l}\text { Intervention and } \\
\text { Comparison }\end{array}$ & $\begin{array}{l}\text { Population } \\
\text { Age (Mean) } \\
\text { Gender (\% Female) } \\
\text { Race (\%) } \\
\text { Ambulatory (\%) } \\
\text { Wheelchair User (\%) } \\
\text { Condition Specific (\%) } \\
\text { Other (\%) }\end{array}$ & Prioritized Outcomes & Other Outcomes \\
\hline $\begin{array}{l}\text { Sangelaji, } 2016 \\
\text { (Continued) }\end{array}$ & & & 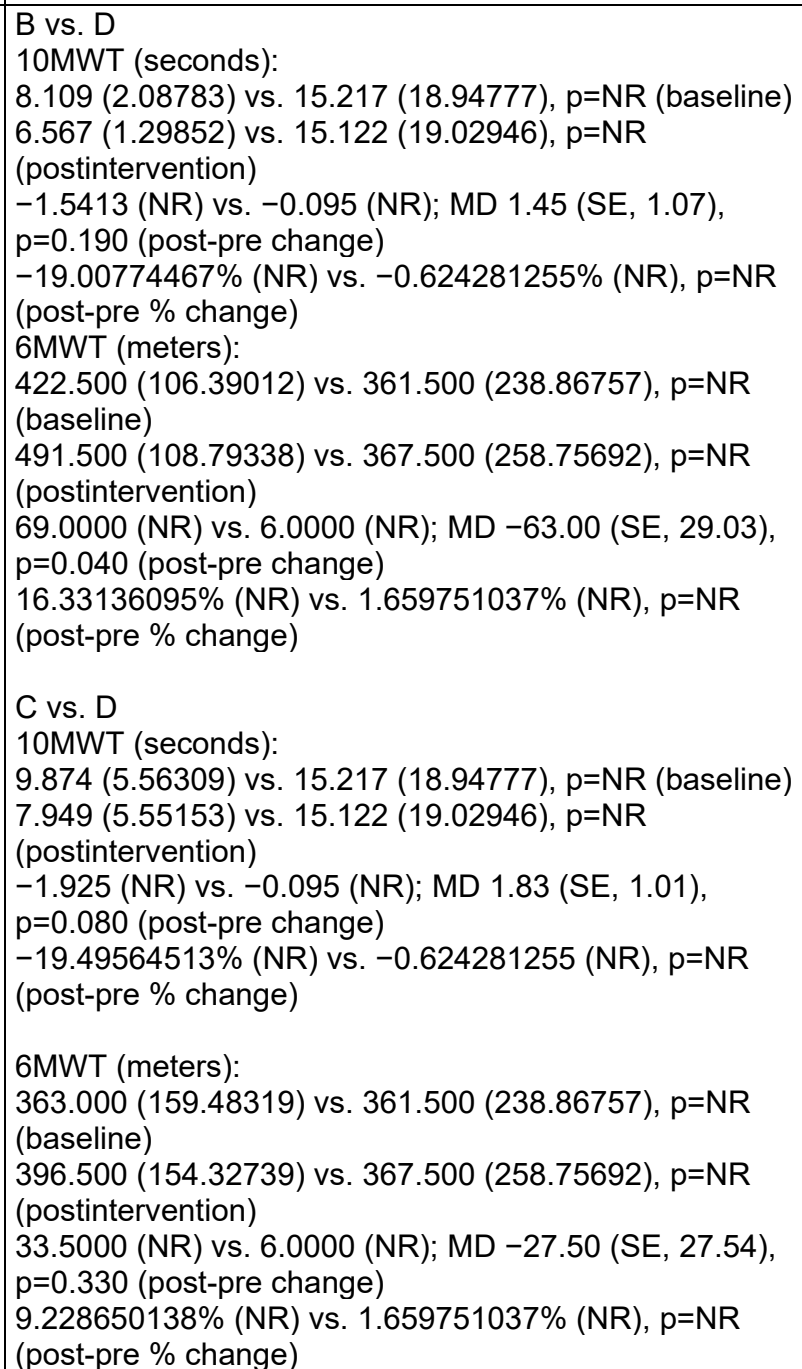 & $\begin{array}{l}\text { Left knee extension strength (kg) } \\
\text { A vs. D } \\
12.000 \text { (5.3619) vs. } 10.667 \text { (5.04645) } \\
\text { (baseline) } \\
20.444(6.12599) \text { vs. } 11.333(6.43946) \\
\text { (postintervention) } \\
8.4444 \text { vs. } 0.6666 \text { (pre-post change) } \\
70.37 \% \text { vs. } 6.249355471 \% \text { (pre-post \% } \\
\text { change)B vs. D } \\
19.000 \text { (10.01428) vs. } 10.667 \text { (5.04645) } \\
\text { (baseline) } \\
24.750(10.93814) \text { vs. } 11.333(6.43946) \\
\text { (postintervention) } \\
5.75 \text { vs. } 0.6666 \text { (pre-post change) } \\
30.26315789 \text { vs. } 6.249355471 \% \text { (pre-post \% } \\
\text { change) } \\
\text { C vs. D } \\
14.580(7.16377) \text { vs. } 10.667 \text { (5.04645) } \\
\text { (baseline) } \\
23.200(8.70249) \text { vs. } 11.333(6.43946) \\
\text { (postintervention) } \\
8.62 \text { vs. } 0.6666 \text { (pre-post change) } \\
59.12208505 \% \text { vs. } 6.249355471 \% \text { (pre-post \% } \\
\text { change) } \\
\text { Left knee flexion strength (kg) } \\
\text { A vs. D } \\
7.422 \text { (3.50955) vs. } 5.3462 .761 \text { (baseline) } \\
13.000(4.03113) \text { vs. } 4.9172 .61566 \\
\text { (postintervention) } \\
5.5778 \text { vs. }-0.42897 \text { (pre-post change) } \\
75.150225 \text { vs. }-8.024625538 \% \text { (pre-post \% } \\
\text { change) }\end{array}$ \\
\hline
\end{tabular}




\begin{tabular}{|c|c|c|c|c|}
\hline $\begin{array}{l}\text { Author, Year } \\
\text { Intervention Type } \\
\text { Duration of } \\
\text { Postintervention } \\
\text { Followup } \\
\text { Quality } \\
\text { (See Appendix B } \\
\text { for Full Citation) } \\
\end{array}$ & $\begin{array}{l}\text { Intervention and } \\
\text { Comparison }\end{array}$ & $\begin{array}{l}\text { Population } \\
\text { Age (Mean) } \\
\text { Gender (\% Female) } \\
\text { Race (\%) } \\
\text { Ambulatory (\%) } \\
\text { Wheelchair User (\%) } \\
\text { Condition Specific (\%) } \\
\text { Other (\%) }\end{array}$ & Prioritized Outcomes & Other Outcomes \\
\hline $\begin{array}{l}\text { Sangelaji, } 2016 \\
\text { (Continued) }\end{array}$ & & & & $\begin{array}{l}\text { B vs. D } \\
12.375 \text { (4.89716) vs. } 5.3462 .761 \text { (baseline) } \\
15.500(5.47723) \text { vs. } 4.9172 .61566 \\
\text { (postintervention) } \\
3.125 \text { vs. }-0.42897 \text { (pre-post change) } \\
25.25252525 \% \text { vs. }-8.024625538 \% \text { (pre-post } \\
\% \text { change) } \\
\\
\text { C vs. D } \\
7.060 \text { (2.49275) vs. } 5.3462 .761 \text { (baseline) } \\
12.600(2.79682) \text { vs. } 4.9172 .61566 \\
\text { (postintervention) } \\
5.54 \text { vs. }-0.42897 \text { (pre-post change) } \\
78.47025496 \% \text { vs. }-8.024625538 \% \text { (pre-post } \\
\% \text { change) } \\
\text { Right knee extension strength (kg) } \\
\text { A vs. D } \\
12.111(5.1099) \text { vs. } 14.667 \text { (3.26599) } \\
\text { (baseline) } \\
19.000(6.61438) \text { vs. } 16.667 \text { (7.44759) } \\
\text { (postintervention) } \\
6.8889 \text { vs. } 2 \text { (pre-post change) } \\
56.88087787 \% \text { vs. } 13.63633264 \% \text { (pre-post \% } \\
\text { change) } \\
\\
\text { B vs. D } \\
21.375 \text { (9.31876) vs. } 14.667 \text { (3.26599) } \\
\text { (baseline) } \\
25.000(10.91526) \text { vs. } 16.667 \text { (7.44759) } \\
\text { (postintervention) } \\
3.625 \text { vs. } 2 \text { (pre-post change) } \\
16.95906433 \% \text { vs. } 13.63633264 \% \text { (pre-post \% } \\
\text { change) }\end{array}$ \\
\hline
\end{tabular}




\begin{tabular}{|c|c|c|c|c|}
\hline $\begin{array}{l}\text { Author, Year } \\
\text { Intervention Type } \\
\text { Duration of } \\
\text { Postintervention } \\
\text { Followup } \\
\text { Quality } \\
\text { (See Appendix B } \\
\text { for Full Citation) } \\
\end{array}$ & $\begin{array}{l}\text { Intervention and } \\
\text { Comparison }\end{array}$ & $\begin{array}{l}\text { Population } \\
\text { Age (Mean) } \\
\text { Gender (\% Female) } \\
\text { Race (\%) } \\
\text { Ambulatory (\%) } \\
\text { Wheelchair User (\%) } \\
\text { Condition Specific (\%) } \\
\text { Other (\%) }\end{array}$ & Prioritized Outcomes & Other Outcomes \\
\hline $\begin{array}{l}\text { Sangelaji, } 2016 \\
\text { (Continued) }\end{array}$ & & & & $\begin{array}{l}\text { C vs. D } \\
16.000(6.8313) \text { vs. } 14.667 \text { (3.26599) } \\
\text { (baseline) } \\
24.300(8.53815) \text { vs. } 16.667 \text { (7.44759) } \\
\text { (postintervention) } \\
8.3 \text { vs. } 2 \text { (pre-post change) } \\
51.875 \% \text { vs. } 13.63633264 \% \text { (pre-post \% } \\
\text { change) } \\
\text { Right knee flexion strength (kg) } \\
\text { A vs. D } \\
7.7223 .(64958) \text { vs. } 8.205 \text { (3.55624) (baseline) } \\
12.333(4.74342) \text { vs. } 7.750(2.80624) \\
\text { (postintervention) } \\
4.6111 \text { vs. }-0.4555 \text { (pre-post change) } \\
59.71225816 \% \text { vs. }-5.551154713 \% \text { (pre-post } \\
\% \text { change) } \\
\text { B vs. D } \\
13.375(5.15302) \text { vs. } 8.205 \text { (3.55624) } \\
\text { (baseline) } \\
17.250(5.94619) \text { vs. } 7.750(2.80624) \\
\text { (postintervention) } \\
3.875 \text { vs. }-0.4555 \text { (pre-post change) } \\
28.97196262 \% \text { vs. }-5.551154713 \% \text { (pre-post } \\
\% \text { change) } \\
\text { C vs. D } \\
8.850(2.80921) \text { vs. } 8.205 \text { (3.55624) (baseline) } \\
12.900(3.38132) \text { vs. } 7.750 \text { (2.80624) } \\
\text { (postintervention) } \\
4.05 \text { vs. }-0.4555 \text { (pre-post change) } \\
45.76271186 \% \text { vs. }-5.551154713 \% \text { (pre-post } \\
\% \text { change) }\end{array}$ \\
\hline
\end{tabular}




\begin{tabular}{|c|c|c|c|c|}
\hline $\begin{array}{l}\text { Author, Year } \\
\text { Intervention Type } \\
\text { Duration of } \\
\text { Postintervention } \\
\text { Followup } \\
\text { Quality } \\
\text { (See Appendix B } \\
\text { for Full Citation) } \\
\end{array}$ & $\begin{array}{l}\text { Intervention and } \\
\text { Comparison }\end{array}$ & $\begin{array}{l}\text { Population } \\
\text { Age (Mean) } \\
\text { Gender (\% Female) } \\
\text { Race (\%) } \\
\text { Ambulatory (\%) } \\
\text { Wheelchair User (\%) } \\
\text { Condition Specific (\%) } \\
\text { Other (\%) }\end{array}$ & Prioritized Outcomes & Other Outcomes \\
\hline $\begin{array}{l}\text { Scholtes, } 2010 \\
\text { Scholtes, } 2011 \\
\text { Scholtes, } 2008 \\
\text { Muscle Strength } \\
\text { Postintervention, } \\
6 \text { weeks } \\
\text { Fair }\end{array}$ & $\begin{array}{l}\text { A. Progressive } \\
\text { resistance }(n=26) \text { : } 36 \\
\text { sessions over } 12 \\
\text { weeks } \\
\text { B. Usual care }(n=25)\end{array}$ & $\begin{array}{l}\text { A vs. B [data for completers } \\
\text { only] } \\
\text { Age (mean years): } 10.33 \text { vs. } \\
10.25 \\
\text { Female: } 8(33 \%) \text { vs. } 12 \\
(50 \%) \\
\text { Race: NR } \\
\text { Ambulatory: } 24(100 \%) \text { vs. } \\
25(100 \%) \\
\text { Wheelchair user: NR } \\
\text { Limb distribution } \\
\text {-unilateral: } 29 \% \text { vs. } 40 \% \\
\text {-bilateral: } 71 \% \text { vs. } 60 \% \\
\text { GMFM Classification Level } \\
\text {-I: } 54 \% \text { vs. } 48 \% \\
\text {-II: } 33 \% \text { vs. } 36 \% \\
\text {-III: } 13 \% \text { vs. } 16 \%\end{array}$ & 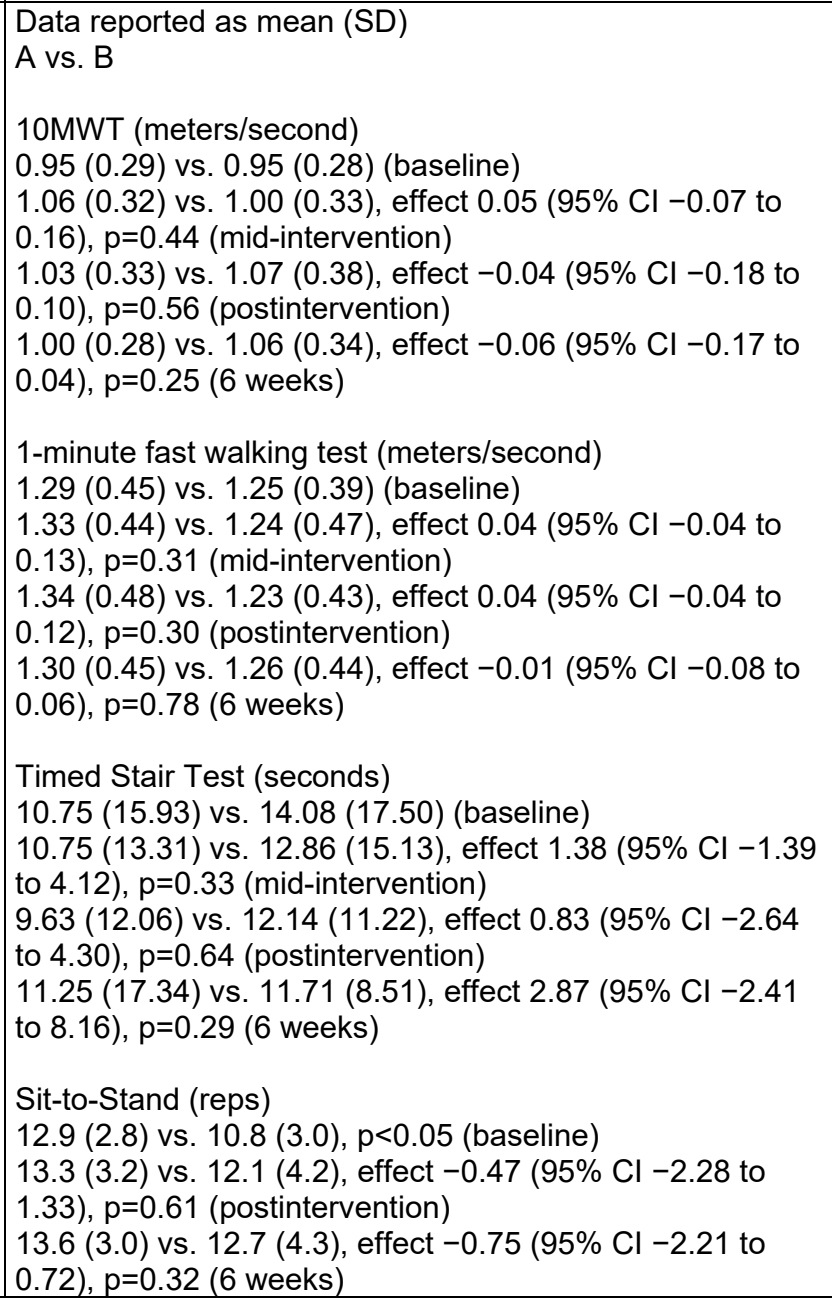 & 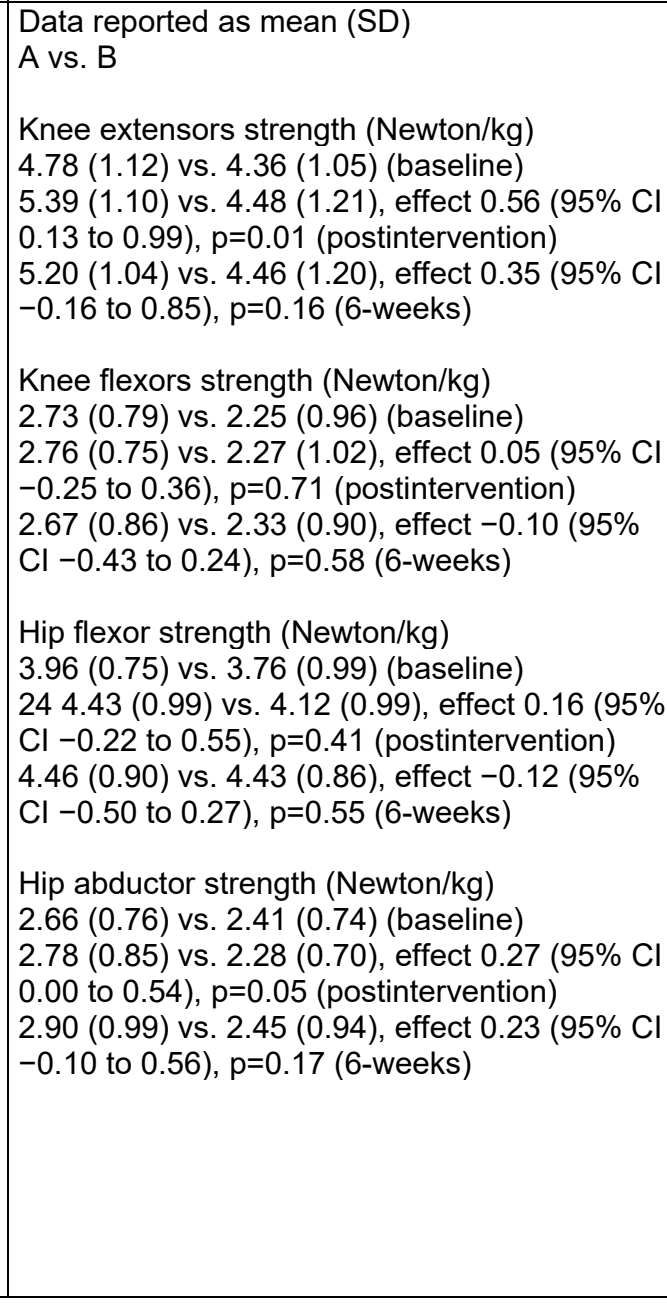 \\
\hline
\end{tabular}




\begin{tabular}{|c|c|c|c|c|}
\hline $\begin{array}{l}\text { Author, Year } \\
\text { Intervention Type } \\
\text { Duration of } \\
\text { Postintervention } \\
\text { Followup } \\
\text { Quality } \\
\text { (See Appendix B } \\
\text { for Full Citation) } \\
\end{array}$ & $\begin{array}{l}\text { Intervention and } \\
\text { Comparison }\end{array}$ & $\begin{array}{l}\text { Population } \\
\text { Age (Mean) } \\
\text { Gender (\% Female) } \\
\text { Race (\%) } \\
\text { Ambulatory (\%) } \\
\text { Wheelchair User (\%) } \\
\text { Condition Specific (\%) } \\
\text { Other (\%) }\end{array}$ & Prioritized Outcomes & Other Outcomes \\
\hline $\begin{array}{l}\text { Scholtes, } 2010 \\
\text { Scholtes, } 2011 \\
\text { Scholtes, } 2008 \\
\\
\text { (Continued) }\end{array}$ & & & 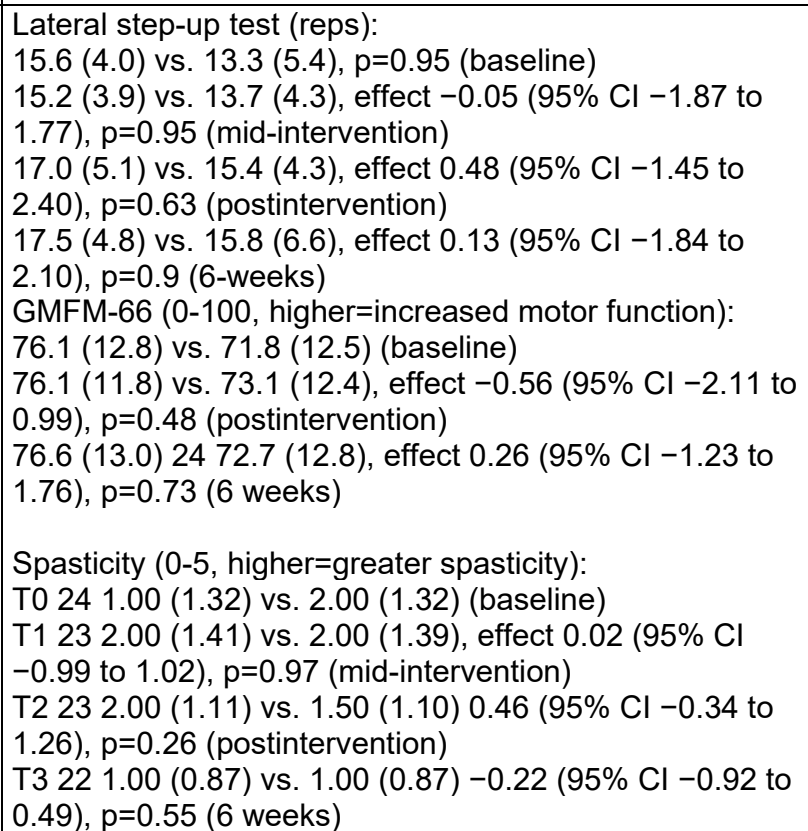 & $\begin{array}{l}\text { Leg power - Six-repetition maximum on leg } \\
\text { press ( } \% \text { body weight) } \\
112.78(21.28) \text { vs. } 93.76(20.18), p<0.05 \\
\text { (baseline) } \\
119.38(26.61) \text { vs. } 100.80 \text { (23.72), effect } 1.97 \\
\text { ( } 95 \% \mathrm{Cl}-8.45 \text { to } 12.41), p=0.71 \text { (mid- } \\
\text { intervention) } \\
135.63(31.87) \text { vs. } 102.88(26.76) \text {, effect } 14.17 \\
\text { (95\% Cl } 1.99 \text { to } 26.35), p=0.02 \\
\text { (postintervention) } \\
129.90(32.15) \text { vs. } 111.99(26.17) \text {, effect } 3.42 \\
\text { (95\% Cl }-8.62 \text { to } 15.46), p=0.58 \text { (6 weeks) }\end{array}$ \\
\hline
\end{tabular}




\begin{tabular}{|c|c|c|c|c|}
\hline $\begin{array}{l}\text { Author, Year } \\
\text { Intervention Type } \\
\text { Duration of } \\
\text { Postintervention } \\
\text { Followup } \\
\text { Quality } \\
\text { (See Appendix B } \\
\text { for Full Citation) } \\
\end{array}$ & $\begin{array}{l}\text { Intervention and } \\
\text { Comparison }\end{array}$ & $\begin{array}{l}\text { Population } \\
\text { Age (Mean) } \\
\text { Gender (\% Female) } \\
\text { Race (\%) } \\
\text { Ambulatory (\%) } \\
\text { Wheelchair User (\%) } \\
\text { Condition Specific (\%) } \\
\text { Other (\%) } \\
\end{array}$ & Prioritized Outcomes & Other Outcomes \\
\hline $\begin{array}{l}\text { Shin, } 2014 \\
\text { Aerobic Exercise } \\
\text { Robot-Assisted } \\
\text { Gait Training } \\
\text { Postintervention, } 4 \\
\text { weeks } \\
\text { Fair }\end{array}$ & \begin{tabular}{|l|} 
A. RAGT, 12 \\
sessions over 4 \\
weeks, plus usual \\
physiotherapy 2 \\
times per day 5 days \\
per week, 40 \\
sessions over 4 \\
weeks $(\mathrm{N}=27)$ \\
\\
B. Conventional \\
Overground Training, \\
2 times per day 5 \\
days per week, 40 \\
sessions over 4 \\
weeks ( $n=26)$
\end{tabular} & $\begin{array}{l}\text { A vs. B } \\
\text { Age (mean years): } \\
43 \text { vs. } 48 \\
\text { Female: } \\
7 \text { (26\%) vs. } 12(46 \%) \\
\\
\text { Race: NR } \\
\\
\text { Ambulatory: } \\
\mathrm{N}(\%) \text { vs. } \mathrm{N}(\%) \\
\text { Wheelchair user: } \\
\mathrm{N}(\%) \text { vs. } \mathrm{N}(\%) \\
\\
\text { Other: } \\
\text { Cervical SCl } \\
15 \text { (52\%) vs. } 16(62 \%) \\
\text { Months since injury } \\
3.33 \text { vs. } 2.73\end{array}$ & $\begin{array}{l}\text { A vs. B, mean } \\
\text { WISCI-II change in score } \\
8 \text { vs. } 5 \text { (intergroup } p=0.01 \text { ) } \\
\text { LEMS change in score } \\
6 \text { vs. } 4 \text { (intergroup } p=0.24 \text { ) } \\
\text { SCiM3-M } \\
6 \text { vs. } 3 \text { (intergroup } p=0.13 \text { ) } \\
\text { All intragroup p-values }<0.001\end{array}$ & A vs. B, mean (SD) \\
\hline $\begin{array}{l}\text { Silva e Borges, } \\
2011 \\
\text { Postintervention, } 0 \\
\text { weeks (after 6- } \\
\text { week intervention) } \\
\text { Postural Control } \\
\text { Hippotherapy } \\
\text { Fair }\end{array}$ & \begin{tabular}{|l|} 
A. Riding simulator \\
(RS) group, 12 \\
sessions over 6 \\
weeks $(n=20)$ \\
\\
B. Conventional \\
physical therapy (CT) \\
group, 12 sessions \\
over 6 weeks $(n=20)$
\end{tabular} & $\begin{array}{l}\text { A vs. B } \\
\text { Age (mean years): } 5.65 \text { vs. } \\
5.77 \\
\text { Female: } 12(60 \%) \text { vs. } 11 \\
(55 \%) \\
\text { Race: NR } \\
\text { Ambulatory: NR } \\
\text { Wheelchair user: NR }\end{array}$ & $\begin{array}{l}\text { A vs. B, number of people (\%) } \\
\text { GMFCS: } \\
\text { GMFCS level III (baseline) to level II (postintervention): } 2 \\
(10 \%) \text { vs. } 1 \text { ( } 5 \%) \\
\text { GMFCS level IV (baseline) to level III (postintervention): } 1 \\
(5 \%) \text { vs. } 1(5 \%) \\
\text { GMFCS level V (baseline) to level IV (postintervention): } 2 \\
(10 \%) \text { vs. } 0(0 \%) \\
\text { Children in RS group had } 1.63 \text { times more chances to } \\
\text { show a better GMFCS score after the treatment than } \\
\text { before the treatment ( } p=0.0110) \text {. } \\
\text { Children in CT group had } 1.22 \text { times more chances to } \\
\text { obtain a better score after treatment than before the } \\
\text { treatment }(p=0.1510) .\end{array}$ & NR \\
\hline
\end{tabular}




\begin{tabular}{|c|c|c|c|c|}
\hline $\begin{array}{l}\text { Author, Year } \\
\text { Intervention Type } \\
\text { Duration of } \\
\text { Postintervention } \\
\text { Followup } \\
\text { Quality } \\
\text { (See Appendix B } \\
\text { for Full Citation) } \\
\end{array}$ & $\begin{array}{l}\text { Intervention and } \\
\text { Comparison }\end{array}$ & $\begin{array}{l}\text { Population } \\
\text { Age (Mean) } \\
\text { Gender (\% Female) } \\
\text { Race (\%) } \\
\text { Ambulatory (\%) } \\
\text { Wheelchair User (\%) } \\
\text { Condition Specific (\%) } \\
\text { Other (\%) }\end{array}$ & Prioritized Outcomes & Other Outcomes \\
\hline $\begin{array}{l}\text { Slaman, 2014a } \\
\text { Slaman, 2014b } \\
\text { Slaman, 2015 } \\
\text { Slaman, } 2010 \\
\text { Multimodal } \\
\text { Exercise } \\
\text { Mid-intervention } \\
\text { (12 weeks into } \\
\text { intervention); } \\
\text { Immediately } \\
\text { postintervention; } \\
24 \text { weeks } \\
\text { Fair }\end{array}$ & $\begin{array}{l}\text { A. Strength training + } \\
\text { cardiopulmonary } \\
\text { fitness: ( } n=29 \text { ) } \\
\text { The intervention } \\
\text { consisted of } 3 \text { parts } \\
\text { over } 6 \text { months. } \\
\text { 1) Supervised center } \\
\text { and home-based } \\
\text { physical fitness } \\
\text { training (24 sessions } \\
\text { over } 12 \text { weeks) } \\
\text { 2) Counseling on } \\
\text { daily physical activity, } \\
\text { based on } \\
\text { motivational } \\
\text { interviewing (6 } \\
\text { sessions over } 24 \\
\text { weeks) } \\
3 \text { ) Counseling on } \\
\text { sports participation, } \\
\text { (2 required sessions } \\
\text { - up to } 4 \text { offered- } \\
\text { over } 24 \text { weeks) } \\
\text { B. Usual care ( } n=28 \text { ) } \\
\text { (Entire intervention } \\
\text { period was } 24 \\
\text { weeks) }\end{array}$ & $\begin{array}{l}\text { A vs. B } \\
\text { Age (mean years): } 20 \text { vs. } 20 \\
\text { Female: } 14 \text { (48.3\%) vs. } 16 \\
(57.1 \%) \\
\text { Race: NR } \\
\text { Ambulatory: } 28(96.6 \%) \text { vs. } \\
25(89.3 \%) \\
\text { Wheelchair user: } 1(3.3 \%) \\
\text { vs. } 3(10.7 \%) \\
\text { CP distribution } \\
\text {-unilateral: } 15 \text { (51.7\%) vs. } 14 \\
(50 \%) \\
\text {-bilateral: } 14 \text { (48.3\%) vs. } 13 \\
\text { (46.4\%) } \\
\text {-unknown: } 0 \text { (0\%) vs. } 1 \\
(3.4 \%) \\
\text { baseline VO } 2 \text { peak (mean } \\
\text { mL/min): } 2533 \text { vs. } 2260 \\
\text { Waist circumference (mean } \\
\text { cm): } 79 \text { vs. } 87, \text { p<0.04 } \\
\text { Total lower-extremity muscle } \\
\text { strength (mean): } 1482 \text { vs. } \\
1307 \\
\text { Total upper-extremity } \\
\text { muscle strength (mean): } 466 \\
\text { vs. } 448 \\
\text { Gross Motor Function } \\
\text { Classification System Level } \\
\text { (I/II/III/IV): } 16 / 9 / 3 / 1 \text { vs. } \\
17 / 9 / 2 / 0\end{array}$ & 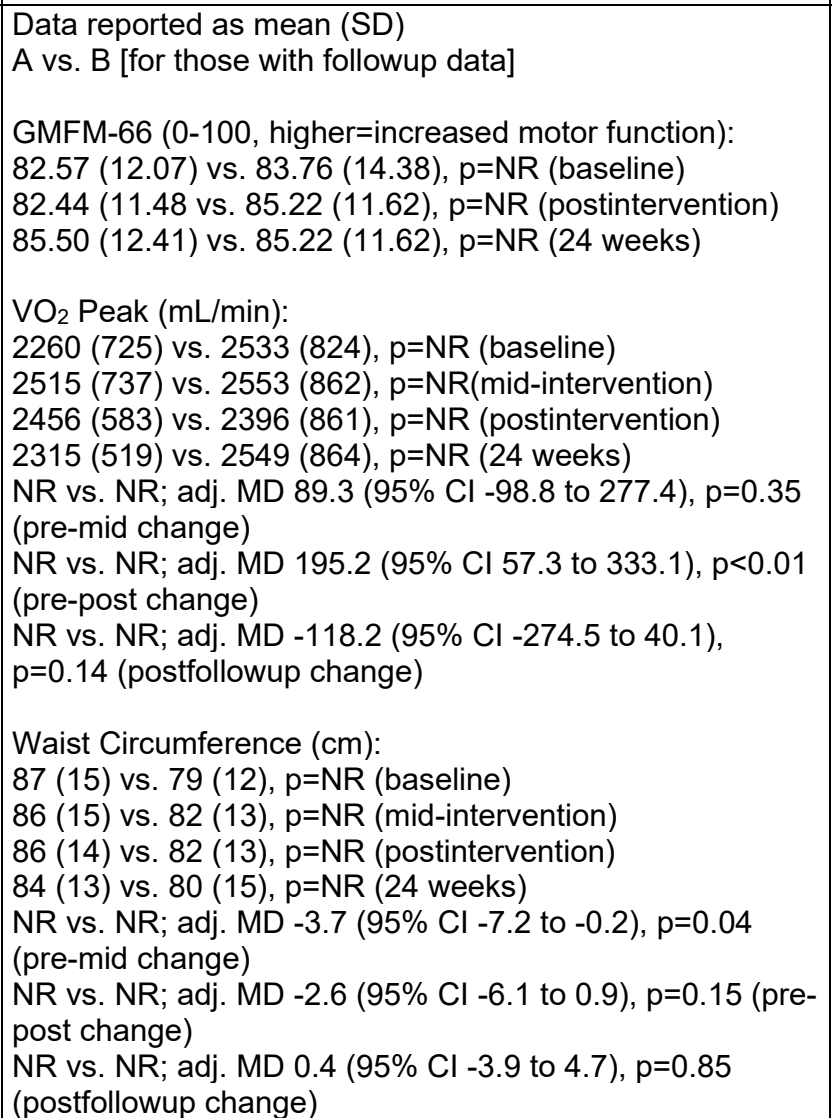 & 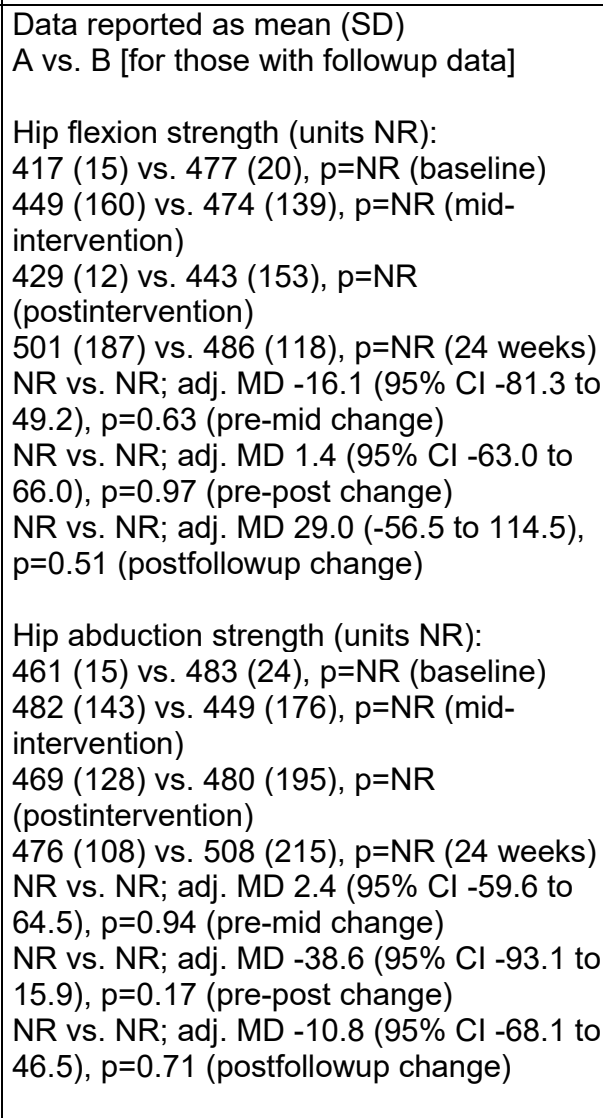 \\
\hline
\end{tabular}




\begin{tabular}{|c|c|c|c|c|}
\hline $\begin{array}{l}\text { Author, Year } \\
\text { Intervention Type } \\
\text { Duration of } \\
\text { Postintervention } \\
\text { Followup } \\
\text { Quality } \\
\text { (See Appendix B } \\
\text { for Full Citation) } \\
\end{array}$ & $\begin{array}{l}\text { Intervention and } \\
\text { Comparison }\end{array}$ & \begin{tabular}{|l|} 
Population \\
Age (Mean) \\
Gender (\% Female) \\
Race (\%) \\
Ambulatory (\%) \\
Wheelchair User (\%) \\
Condition Specific (\%) \\
Other (\%) \\
\end{tabular} & Prioritized Outcomes & Other Outcomes \\
\hline $\begin{array}{l}\text { Slaman, 2014a } \\
\text { Slaman, 2014b } \\
\text { Slaman, 2015 } \\
\text { Slaman, } 2010 \\
\\
\text { (Continued) }\end{array}$ & & & 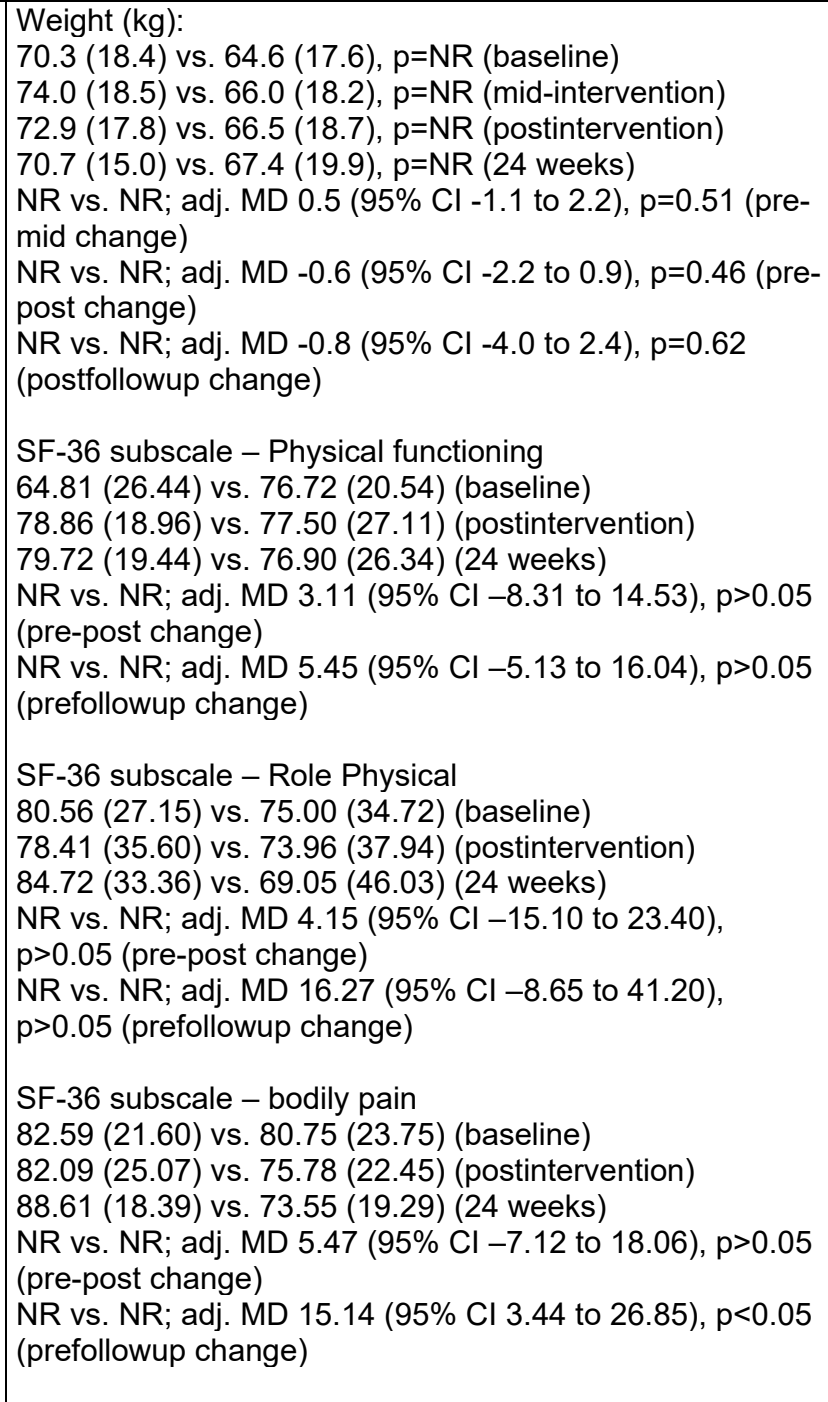 & 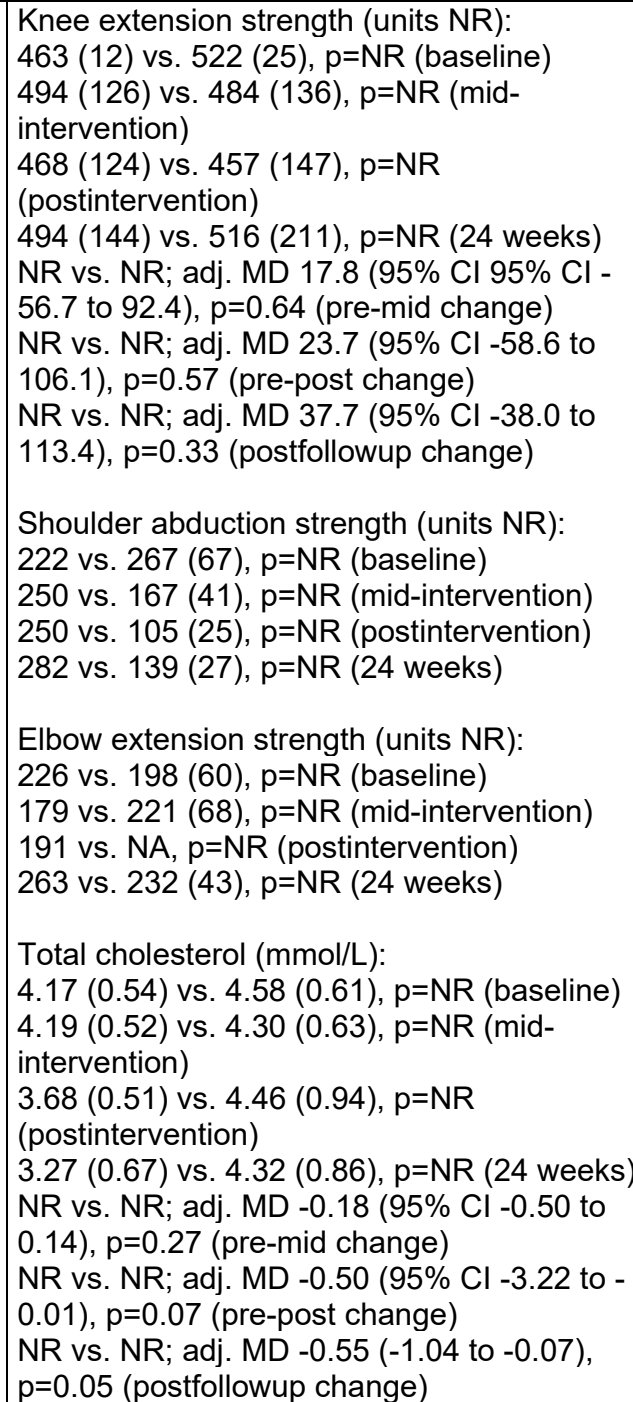 \\
\hline
\end{tabular}




\begin{tabular}{|c|c|c|c|c|}
\hline $\begin{array}{l}\text { Author, Year } \\
\text { Intervention Type } \\
\text { Duration of } \\
\text { Postintervention } \\
\text { Followup } \\
\text { Quality } \\
\text { (See Appendix B } \\
\text { for Full Citation) }\end{array}$ & $\begin{array}{l}\text { Intervention and } \\
\text { Comparison }\end{array}$ & $\begin{array}{l}\text { Population } \\
\text { Age (Mean) } \\
\text { Gender (\% Female) } \\
\text { Race (\%) } \\
\text { Ambulatory (\%) } \\
\text { Wheelchair User (\%) } \\
\text { Condition Specific (\%) } \\
\text { Other (\%) }\end{array}$ & Prioritized Outcomes & Other Outcomes \\
\hline \begin{tabular}{|l|} 
Slaman, 2014a \\
Slaman, 2014b \\
Slaman, 2015 \\
Slaman, 2010 \\
\\
(Continued)
\end{tabular} & & & 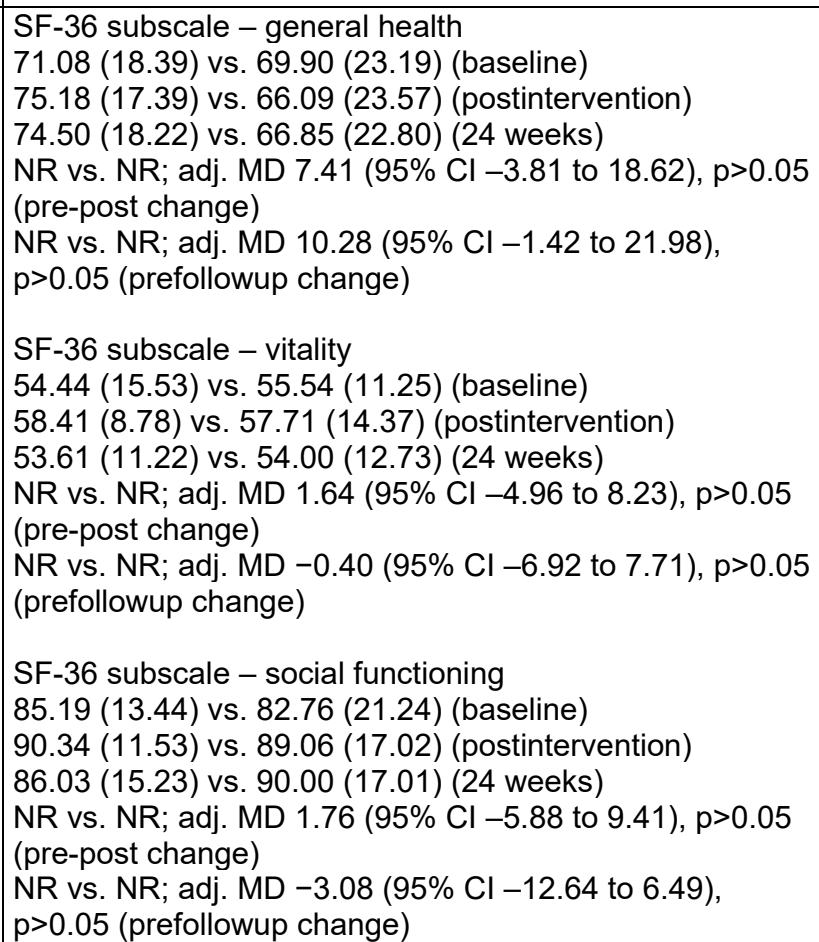 & 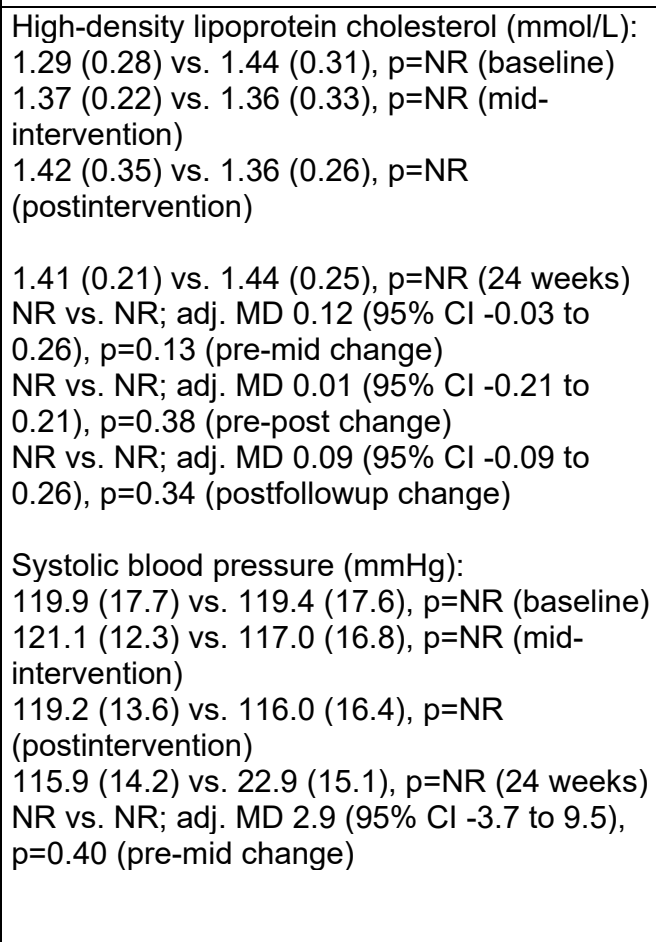 \\
\hline
\end{tabular}




\begin{tabular}{|c|c|c|c|c|}
\hline $\begin{array}{l}\text { Author, Year } \\
\text { Intervention Type } \\
\text { Duration of } \\
\text { Postintervention } \\
\text { Followup } \\
\text { Quality } \\
\text { (See Appendix B } \\
\text { for Full Citation) }\end{array}$ & $\begin{array}{l}\text { Intervention and } \\
\text { Comparison }\end{array}$ & $\begin{array}{l}\text { Population } \\
\text { Age (Mean) } \\
\text { Gender (\% Female) } \\
\text { Race (\%) } \\
\text { Ambulatory (\%) } \\
\text { Wheelchair User (\%) } \\
\text { Condition Specific (\%) } \\
\text { Other (\%) }\end{array}$ & Prioritized Outcomes & Other Outcomes \\
\hline $\begin{array}{l}\text { Slaman, 2014a } \\
\text { Slaman, 2014b } \\
\text { Slaman, 2015 } \\
\text { Slaman, 2010 } \\
\\
\text { (Continued) }\end{array}$ & & & 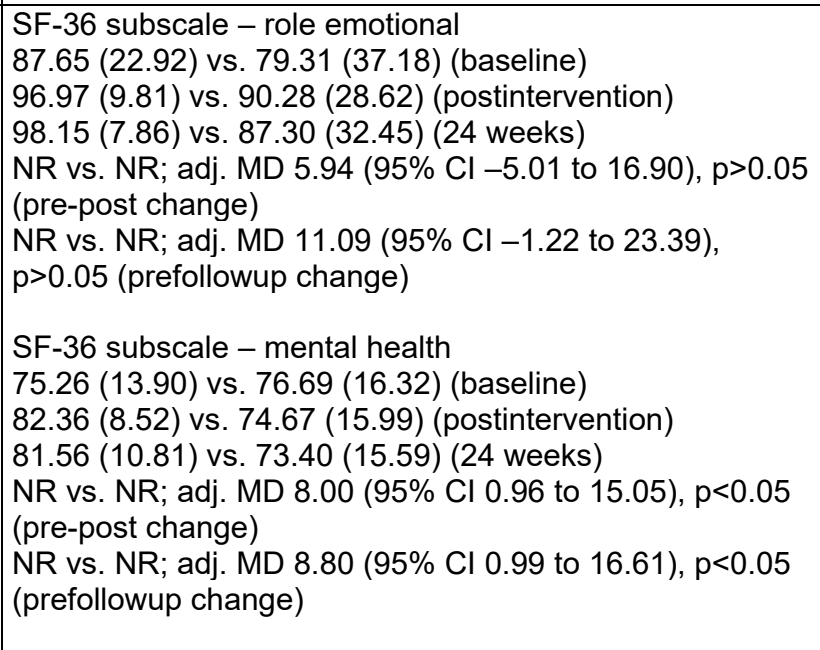 & 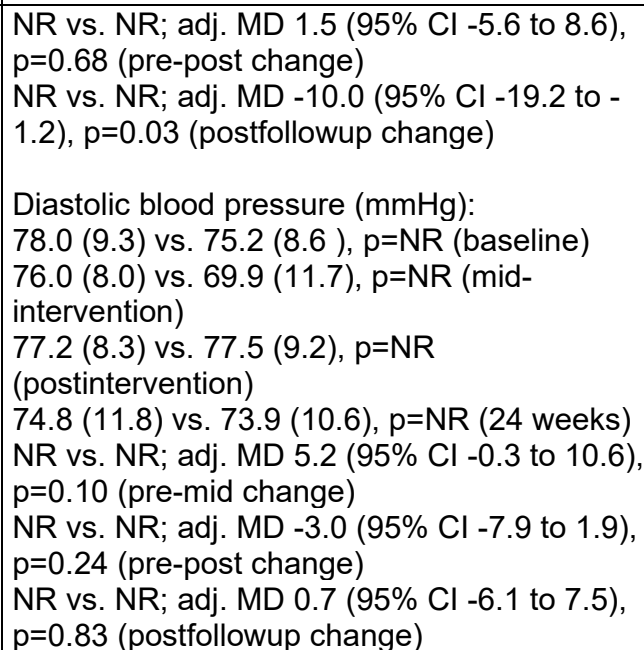 \\
\hline
\end{tabular}




\begin{tabular}{|c|c|c|c|c|}
\hline $\begin{array}{l}\text { Author, Year } \\
\text { Intervention Type } \\
\text { Duration of } \\
\text { Postintervention } \\
\text { Followup } \\
\text { Quality } \\
\text { (See Appendix B } \\
\text { for Full Citation) } \\
\end{array}$ & $\begin{array}{l}\text { Intervention and } \\
\text { Comparison }\end{array}$ & $\begin{array}{l}\text { Population } \\
\text { Age (Mean) } \\
\text { Gender (\% Female) } \\
\text { Race (\%) } \\
\text { Ambulatory (\%) } \\
\text { Wheelchair User (\%) } \\
\text { Condition Specific (\%) } \\
\text { Other (\%) }\end{array}$ & Prioritized Outcomes & Other Outcomes \\
\hline $\begin{array}{l}\text { Straudi, } 2016 \\
\text { Aerobic Exercise } \\
\text { Robot-Assisted } \\
\text { Gait Training } \\
\text { Postintervention, } 6 \\
\text { weeks } \\
\text { Fair }\end{array}$ & $\begin{array}{l}\text { A. RAGT, } 12 \\
\text { sessions over } 6 \\
\text { weeks }(n=27) \\
\\
\text { B. Conventional } \\
\text { physiotherapy, } 12 \\
\text { sessions over } 6 \\
\text { weeks }(n=25)\end{array}$ & $\begin{array}{l}\text { A vs. B } \\
\text { Age (mean years): } \\
52 \text { vs. } 54 \\
\text { Female: } \\
17(63 \%) \text { vs. } 17(68 \%) \\
\text { Race: NR } \\
\text { Ambulatory: } \\
\text { NR } \\
\text { Wheelchair user: } \\
\text { NR } \\
\text { Other: } \\
\text { EDSS (mean): } \\
6.43 \text { vs. } 6.46 \\
\text { MS type: } \\
\text { primary progressive } 9(33 \%) \\
\text { vs. } 7 \text { (28\%), remainder } \\
\text { secondary progressive }\end{array}$ & $\begin{array}{l}\text { PHQ-9 } \\
\text { T1-T0: }-1.19(3.26) \text { vs. }-1.88(5.92), p=0.25 \\
\text { T2-T0: }-1.7(3.24) \text { vs. }-3.04(4.66), p=0.213 \\
\text { T3-T0: }-0.78(3.31) \text { vs. }-2.20(4.49), p=0.44 \\
\text { SF 36-PCS } \\
\text { T1-T0: } 1.85(6.92) \text { vs. } 0.72(5.63), p=0.50 \\
\text { T2-T0: } 1.67(7.74) \text { vs. } 1.84(6.77), p=0.99 \\
\text { T3-T0: } 5.11 \text { (16.60) vs. } 1.04(6.24), p=0.91 \\
\text { SF 36-MCS } \\
\text { T1-T0: } 3.33(8.77) \text { vs. }-1.16(8.88), p=0.08 \\
\text { T2-T0: } 5.37(9.58) \text { vs. } 1.60(9.41), p=0.14 \\
\text { T3-T0: }-2.52(14.11) \text { vs. } 1.08(8.74) p=0.34 \\
\text { TUG (s) } \\
\text { T1-T0: }-1.11(6.73) \text { vs. }-0.09(7.04), p=0.76 \\
\text { T2-T0: } 2.66(13.79) \text { vs. }-3.96(10.50), p=0.95 \\
\text { T3-T0: }-4.16(15.30) \text { vs. }-3.63(10.61) p=0.24 \\
\text { 6MWT }(m) \\
\text { T1-T0: } 16.94(18.96) \text { vs. }-6.02(27.70), p=0.003 \\
\text { T2-T0: } 23.22(32.23) \text { vs. }-0.75(26.40), p=0.01 \\
\text { T3-T0: } 10.64(35.07) \text { vs. } 4.51(33.59) p=0.55 \\
\text { BBS } \\
\text { T1-T0: } 2.44(3.98) \text { vs. }-0.22(4.48), p=0.043 \\
\text { T2-T0: } 3.24(4.99) \text { vs. } 0.87(6.45), p=0.19 \\
\text { T3-T0: } 1.72(6.05) \text { vs. }-0.17(6.04) p=0.37\end{array}$ & NA \\
\hline
\end{tabular}




\begin{tabular}{|c|c|c|c|c|}
\hline $\begin{array}{l}\text { Author, Year } \\
\text { Intervention Type } \\
\text { Duration of } \\
\text { Postintervention } \\
\text { Followup } \\
\text { Quality } \\
\text { (See Appendix B } \\
\text { for Full Citation) }\end{array}$ & $\begin{array}{l}\text { Intervention and } \\
\text { Comparison }\end{array}$ & $\begin{array}{l}\text { Population } \\
\text { Age (Mean) } \\
\text { Gender (\% Female) } \\
\text { Race (\%) } \\
\text { Ambulatory (\%) } \\
\text { Wheelchair User (\%) } \\
\text { Condition Specific (\%) } \\
\text { Other (\%) }\end{array}$ & Prioritized Outcomes & Other Outcomes \\
\hline $\begin{array}{l}\text { Straudi, } 2019 \\
\text { Aerobic Exercise } \\
\text { Robot-Assisted } \\
\text { Gait Training } \\
\text { Postintervention, } \\
12 \text { weeks } \\
\text { Good }\end{array}$ & $\begin{array}{l}\text { A. Robot-assisted } \\
\text { gait training (RAGT), } \\
12 \text { sessions over } 4 \\
\text { weeks }(n=36) \\
\text { B. Overground } \\
\text { walking, } 12 \text { sessions } \\
\text { over } 4 \text { weeks }(n=36)\end{array}$ & $\begin{array}{l}\text { A vs. B } \\
\text { Age: } 56 \text { vs. } 55 \\
\text { Female: } 67 \% \text { vs. } 69 \% \\
\text { EDSS: } 6.5 \text { vs. } 6.5 \\
\text { PPMS: } 50 \% \text { vs. } 45 \% \\
\text { SPMS: } 50 \% \text { vs. } 55 \%\end{array}$ & $\begin{array}{l}\text { A vs. B, mean difference between groups: } \\
\text { 6MWT: } \\
\text { 4, } 95 \% \mathrm{Cl}-10 \text { to } 18, p=0.86 \\
\text { 25FWT: } \\
\text { 0, } 95 \% \mathrm{Cl}-0.06 \text { to } 0.05, p=0.98 \\
\text { TUG: } \\
7.8,-0.2 \text { to } 15.8, p=0.25 \\
\text { MSIS- } 29 \text { motor: } \\
-3,95 \% \mathrm{Cl}-9 \text { to } 3, p=0.31 \\
\text { MSIS-29 psychological: } \\
-2,95 \% \mathrm{Cl}-5 \text { to } 1, p=0.22 \mathrm{~S} \\
\text { SF-36 PCS: } \\
-1,95 \% \mathrm{Cl}-4 \text { to } 3, p=0.13 \\
\text { SF-36 } \mathrm{MCS}: \\
\text { 1, } 95 \% \mathrm{Cl}-2 \text { to } 4, p=0.94\end{array}$ & $\begin{array}{l}\text { A vs. } \mathrm{B}, \text { Mean difference between groups: } \\
\text { BBS: } \\
0,95 \% \mathrm{Cl}-2 \text { to } 2, p=0.91 \\
\text { PHQ-9: } \\
-0.4,95 \% \mathrm{Cl}-2.3 \text { to } 1.4, p=0.86\end{array}$ \\
\hline $\begin{array}{l}\text { Swe } 2015 \\
\text { Aerobic Exercise } \\
\text { Treadmill } \\
\text { Pre to post only } \\
\text { Good }\end{array}$ & \begin{tabular}{|l|} 
A. Partial body \\
weight supported \\
treadmill walking 30 \\
minutes twice a week \\
for 8 weeks \\
B. Overground \\
walking similar \\
duration and number \\
of sessions
\end{tabular} & $\begin{array}{l}\text { GMFCS II or III } \\
(\text { II/III } 70 / 25) \\
\mathrm{N}=95 \\
\text { Mean age } 16.6 \text { years } \\
61 \text { males and } 34 \text { females } \\
(64 \% \text { males })\end{array}$ & $\begin{array}{l}\text { Pre to week } 8 \\
10 \text { meter walk test (meters/second) } \\
\text { A } 0.922(0.316) \text { to } 1.082(0.352) \\
\text { B. } 0.805(0.248) \text { to } 0.978(0.299) \\
6 \text { minute walk test (meters) } \\
\text { A. } 223.33(94.62) \text { to } 250.60(110.86) \\
\text { B. } 205.00 \text { (88.58) to } 249.27(107.84) \\
\text { Gross Motor Function Measure-88 (D) (standing) } \\
\text { A. } 66.07(22.28) \text { to } 77.73(21.73) \\
\text { B. } 64.53 \text { (16.29) } 79.13(14.22) \\
\text { Gross Motor Function Measure-88 (E) (walking) } \\
\text { A. } 41.07(24.60) \text { to } 54.13(28.25) \\
\text { B. } 40.47 \text { (19.17) to } 56.33(23.05) \\
\text { Outcome measures all showed an improvement over time } \\
\text { (p<0.001); no effect of group allocation on any parameter }\end{array}$ & NA \\
\hline
\end{tabular}




\begin{tabular}{|c|c|c|c|c|}
\hline $\begin{array}{l}\text { Author, Year } \\
\text { Intervention Type } \\
\text { Duration of } \\
\text { Postintervention } \\
\text { Followup } \\
\text { Quality } \\
\text { (See Appendix B } \\
\text { for Full Citation) }\end{array}$ & $\begin{array}{l}\text { Intervention and } \\
\text { Comparison }\end{array}$ & $\begin{array}{l}\text { Population } \\
\text { Age (Mean) } \\
\text { Gender (\% Female) } \\
\text { Race (\%) } \\
\text { Ambulatory (\%) } \\
\text { Wheelchair User (\%) } \\
\text { Condition Specific (\%) } \\
\text { Other (\%) }\end{array}$ & Prioritized Outcomes & Other Outcomes \\
\hline $\begin{array}{l}\text { Tak, } 2015 \\
\text { Postural Control } \\
\text { Motion gaming } \\
\text { Immediately } \\
\text { postintervention, } \\
6 \text { weeks } \\
\text { Fair }\end{array}$ & $\begin{array}{l}\text { A. Nintendo-Wii, } 18 \\
\text { sessions over } 6 \\
\text { weeks }+ \\
\text { conventional } \\
\text { rehabilitation }(n=13) \\
\text { B. Conventional } \\
\text { rehabilitation }(n=13)\end{array}$ & $\begin{array}{l}\text { A vs. B } \\
\text { Age: } 50 \text { vs. } 43 \\
\text { Cervical: } 31 \% \text { vs. } 38 \% \\
\text { ASIA (A): } 77 \% \text { vs. } 77 \% \\
\text { ASIA (B): } 23 \% \text { vs. } 23 \%\end{array}$ & $\begin{array}{l}\text { A vs. B Mean (SD), p=between groups } \\
\text { T-shirt test (s): } 29.5(10.95) \text { to } 22.60(8.28) \text { vs. } 23.59 \\
(11.35) \text { to } 22.15(12.28), p<0.05 \\
\text { Change } \\
6.90(3.55) \text { vs. } 1.44(1.51), p<0.05\end{array}$ & NA \\
\hline
\end{tabular}




\begin{tabular}{|c|c|c|c|c|}
\hline $\begin{array}{l}\text { Author, Year } \\
\text { Intervention Type } \\
\text { Duration of } \\
\text { Postintervention } \\
\text { Followup } \\
\text { Quality } \\
\text { (See Appendix B } \\
\text { for Full Citation) } \\
\end{array}$ & $\begin{array}{l}\text { Intervention and } \\
\text { Comparison }\end{array}$ & $\begin{array}{l}\text { Population } \\
\text { Age (Mean) } \\
\text { Gender (\% Female) } \\
\text { Race (\%) } \\
\text { Ambulatory (\%) } \\
\text { Wheelchair User (\%) } \\
\text { Condition Specific (\%) } \\
\text { Other (\%) }\end{array}$ & Prioritized Outcomes & Other Outcomes \\
\hline $\begin{array}{l}\text { Tarakci, } 2013 \\
\text { Multimodal } \\
\text { Exercise } \\
\text { Postintervention, } 0 \\
\text { weeks } \\
\text { Fair }\end{array}$ & $\begin{array}{l}\text { A. Group exercise: } \\
36 \text { sessions over } 12 \\
\text { weeks }(n=55) \\
\text { B. Waitlist }(n=55)\end{array}$ & $\begin{array}{l}\text { A vs. B } \\
\text { Age (mean years): } 41.49 \text { vs. } \\
39.65 \\
\text { Female: } 34 \text { (67\%) vs. } 30 \\
(48 \%) \\
\text { Race: NR } \\
\text { Ambulatory: NR } \\
\text { Wheelchair user: NR } \\
\text { Mean EDSS: } 4.38 \text { vs. } 4.21 \\
\text { MS type } \\
\text {-Relapsing Remitting: } 32 \\
\text { (62.7\%) vs. } 33 \text { (68.7\%) } \\
\text {-Primary Progressive: } 10 \\
\text { (19.6\%) vs. } 8 \text { (16.6\%) } \\
\text {-Secondary Progressive: } 9 \\
\text { (17.6\%) vs. } 7 \text { (14.5\%) }\end{array}$ & 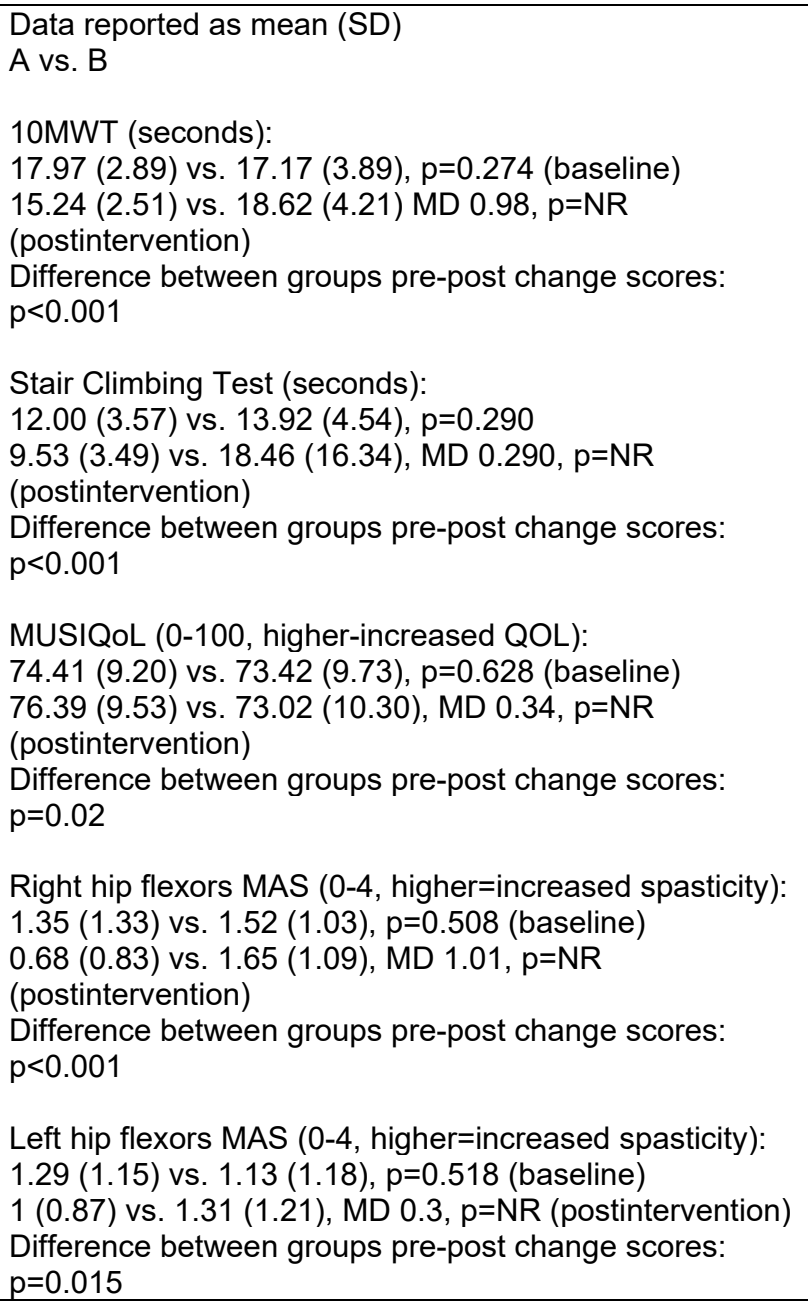 & $\begin{array}{l}\text { Data reported as mean (SD) } \\
\text { A vs. B } \\
\text { BBS }(0-56, \text { higher scores=better balance): } \\
37.68(9.91) \text { vs. } 36.94(12.55), p=0.757 \\
\text { (baseline) } \\
42.01(9.32) \text { vs. } 34.81 \text { (12.85), MD } 0.64, p=N R \\
\text { (postintervention) } \\
\text { Difference between groups pre-post change } \\
\text { scores: } p=0.003\end{array}$ \\
\hline
\end{tabular}




\begin{tabular}{|c|c|c|c|c|}
\hline $\begin{array}{l}\text { Author, Year } \\
\text { Intervention Type } \\
\text { Duration of } \\
\text { Postintervention } \\
\text { Followup } \\
\text { Quality } \\
\text { (See Appendix B } \\
\text { for Full Citation) } \\
\end{array}$ & $\begin{array}{l}\text { Intervention and } \\
\text { Comparison }\end{array}$ & \begin{tabular}{|l|} 
Population \\
Age (Mean) \\
Gender (\% Female) \\
Race (\%) \\
Ambulatory (\%) \\
Wheelchair User (\%) \\
Condition Specific (\%) \\
Other (\%) \\
\end{tabular} & Prioritized Outcomes & Other Outcomes \\
\hline $\begin{array}{l}\text { Tarakci, } 2013 \\
\text { (Continued) }\end{array}$ & & & 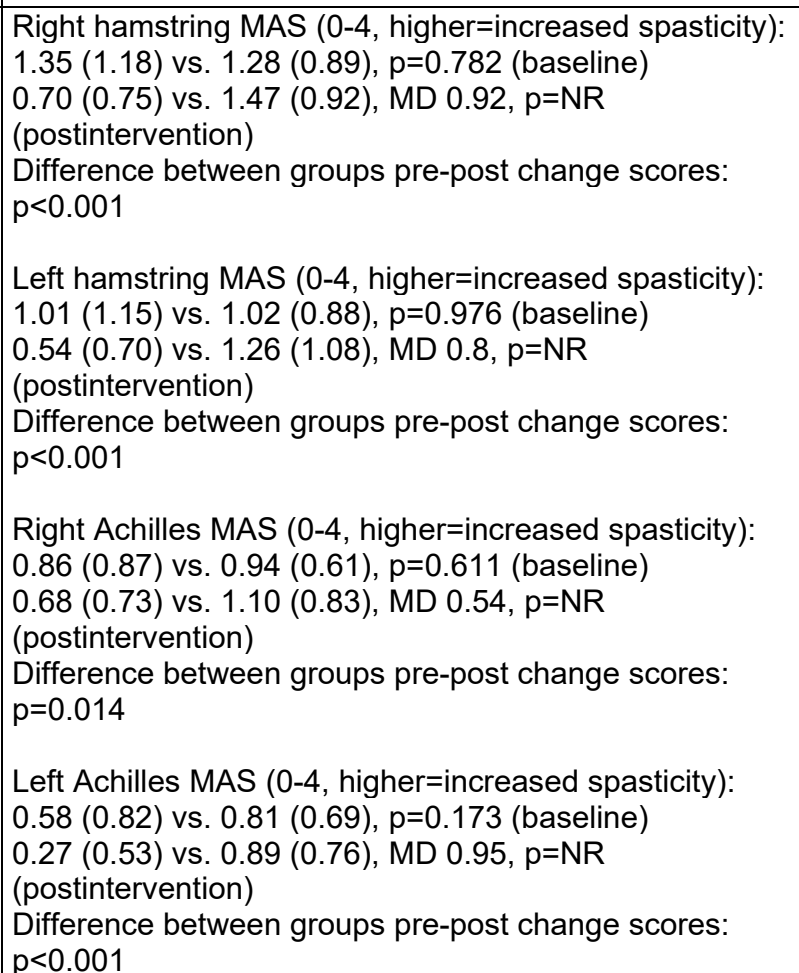 & NA \\
\hline
\end{tabular}




\begin{tabular}{|c|c|c|c|c|}
\hline $\begin{array}{l}\text { Author, Year } \\
\text { Intervention Type } \\
\text { Duration of } \\
\text { Postintervention } \\
\text { Followup } \\
\text { Quality } \\
\text { (See Appendix B } \\
\text { for Full Citation) } \\
\end{array}$ & $\begin{array}{l}\text { Intervention and } \\
\text { Comparison }\end{array}$ & $\begin{array}{l}\text { Population } \\
\text { Age (Mean) } \\
\text { Gender (\% Female) } \\
\text { Race (\%) } \\
\text { Ambulatory (\%) } \\
\text { Wheelchair User (\%) } \\
\text { Condition Specific (\%) } \\
\text { Other (\%) }\end{array}$ & Prioritized Outcomes & Other Outcomes \\
\hline $\begin{array}{l}\text { Tarakci, } 2016 \\
\text { Postural Control } \\
\text { Motion gaming } \\
\text { Postintervention, } \\
12 \text { weeks } \\
\text { Fair }\end{array}$ & $\begin{array}{l}\text { A. Nintendo Wii-Fit } \\
\text { balanced gaming, } 24 \\
\text { sessions over } 12 \\
\text { weeks }(n=15) \\
\text { B. Conventional } \\
\text { balance training, } 24 \\
\text { sessions over } 12 \\
\text { weeks }(n=15)\end{array}$ & $\begin{array}{l}\text { A vs. B } \\
\text { Age (mean years): } 10.46 \text { vs. } \\
10.53 \\
\text { Female: } 5 \text { (33\%) vs. } 6 \text { (40\%) } \\
\text { Race: NR } \\
\text { Ambulatory: NR } \\
\text { Wheelchair user: NR } \\
\text { Assistive devices: } 0 \text { (0\%) vs. } \\
3 \text { (20\%) } \\
\text { Orthesis: } 9 \text { (16\%) vs. } 10 \text { (67 } \\
\%) \\
\text { Type of CP: } \\
\text { Hemiplegic: } 7 \text { (47\%) vs. } 7 \\
\text { (47\%) } \\
\text { Diplegic: } 7 \text { (47\%) vs. } 5 \\
\text { (33\%) } \\
\text { Dyskinetic: } 1 \text { (.06\%) vs. } 3 \\
\text { (20\%) } \\
\text { GMFCS median (min-max): } \\
2 \text { vs. } 2\end{array}$ & $\begin{array}{l}\text { A. vs. B., mean (SD) } \\
\text { TUG (seconds) } \\
12.96(3.65) \text { vs. } 15.77(4.52) \text { (baseline) } \\
10.62 \text { (3.30) vs. } 14.67(4.54) \text { (postintervention): } \\
\text { Difference between groups pre-post change scores: } \\
p=0.001 \\
10 \text { MWT 10MWT (seconds) } \\
13.25(3.56) \text { vs. } 13.77(4.72) \text { (baseline) } \\
11.04 \text { (3.46) vs. } 12.96 \text { (4.64) (postintervention) } \\
\text { Difference between groups pre-post change scores: } \\
p=0.001 \\
\text { Wee FIM } \\
95.73(10.10) \text { vs. } 94.40 \text { (10.70) (baseline) } \\
100.26(8.75) \text { vs. } 95.50(10.47) \text { (postintervention) } \\
\text { Difference between groups pre-post change scores: } \\
p=0.001 \\
\text { Sit-to-Stand Test (number of stands in } 30 \text { seconds) } \\
6.13(1.55) \text { vs. } 5.60 \text { (1.50) (baseline) } \\
8.73(2.08) \text { vs. } 6.13 \text { (1.68) (postintervention) } \\
\text { Difference between groups pre-post change scores: } \\
p=0.001 \\
10-\text { stair climbing test } \\
10.32 \text { (3.81) vs. } 12.03 \text { (4.91) (baseline) } \\
8.42(3.57) \text { vs. } 11.12 \text { (4.27) (postintervention) } \\
\text { Difference between groups pre-post change scores: } \\
p=0.001\end{array}$ & NA \\
\hline
\end{tabular}




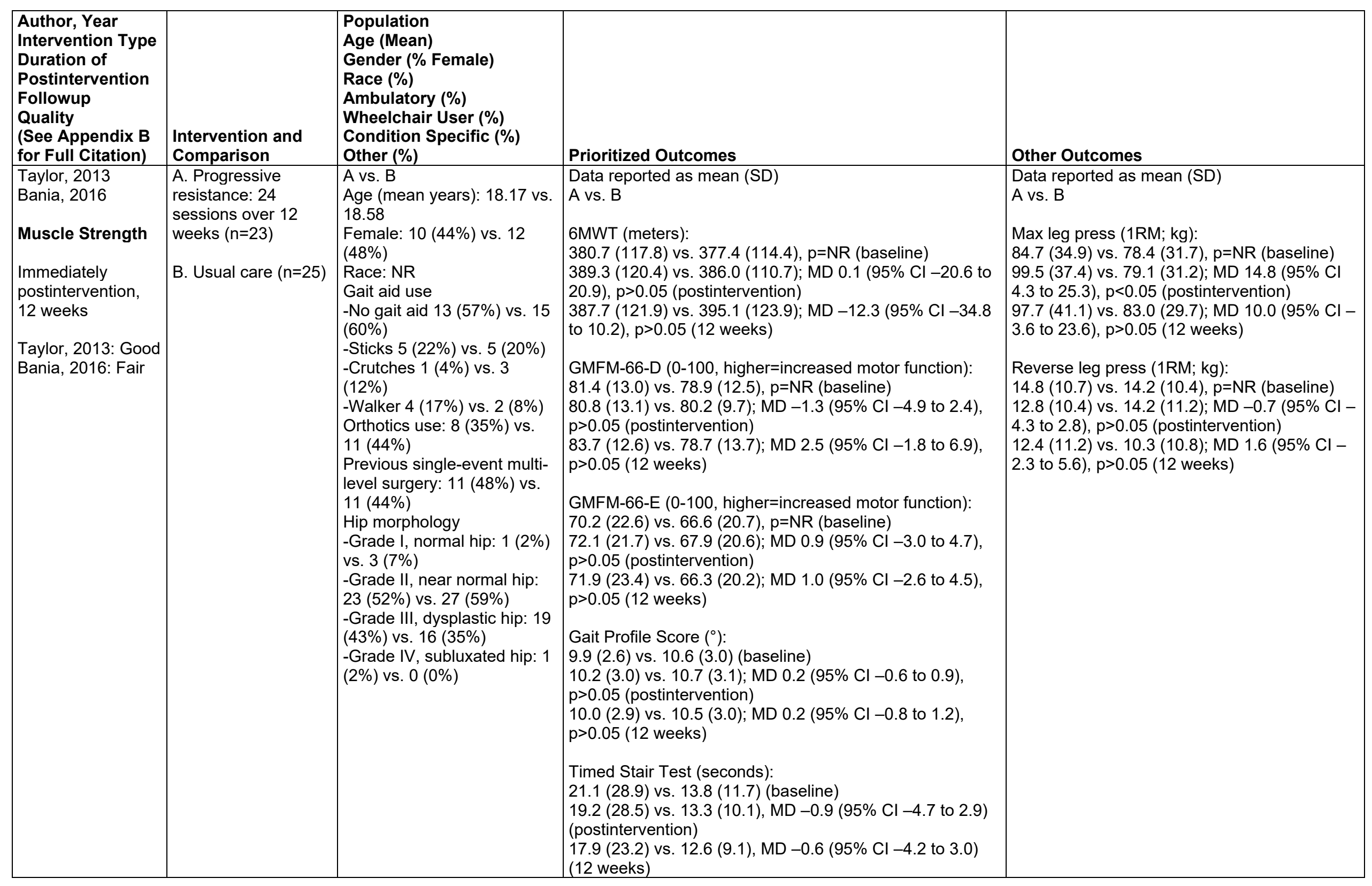




\begin{tabular}{|c|c|c|c|c|}
\hline $\begin{array}{l}\text { Author, Year } \\
\text { Intervention Type } \\
\text { Duration of } \\
\text { Postintervention } \\
\text { Followup } \\
\text { Quality } \\
\text { (See Appendix B } \\
\text { for Full Citation) }\end{array}$ & $\begin{array}{l}\text { Intervention and } \\
\text { Comparison }\end{array}$ & $\begin{array}{l}\text { Population } \\
\text { Age (Mean) } \\
\text { Gender (\% Female) } \\
\text { Race (\%) } \\
\text { Ambulatory (\%) } \\
\text { Wheelchair User (\%) } \\
\text { Condition Specific (\%) } \\
\text { Other (\%) }\end{array}$ & Prioritized Outcomes & Other Outcomes \\
\hline $\begin{array}{l}\text { Tedla, } 2014 \\
\text { Strength } \\
\text { interventions } \\
\text { Muscle Strength } \\
\text { Exercises } \\
\text { Immediately } \\
\text { postintervention, } \\
6 \text { weeks } \\
\text { Poor }\end{array}$ & $\begin{array}{l}\text { A. Strength training } \\
18 \text { sessions over } 6 \\
\text { weeks + } \\
\text { conventional PT 1-2 } \\
\text { days/week ( } n=31) \\
\text { B. Conventional } \\
\text { physical therapy 3-5 } \\
\text { sessions/per for } 6 \\
\text { weeks }(n=31)\end{array}$ & $\begin{array}{l}\text { A vs. B (data are for } \\
\text { completers only; } n=30 \text { vs. } \\
30 \text { ) } \\
\text { Age: } 9.1 \text { vs. } 8.9 \text { years } \\
\text { Female: } 33 \% \text { vs. } 33 \% \\
\text { Gross motor function } \\
\text { classification system: } \\
\text { I: } 7 \% \text { vs. } 3 \% \\
\text { II: } 20 \% \text { vs. } 27 \% \\
\text { III: } 37 \% \text { vs. } 27 \% \\
\text { IV: } 37 \% \text { vs. } 43 \%\end{array}$ & $\begin{array}{l}\text { A vs. B, Mean change from baseline (SD): } \\
\text { PBS total score } \\
7.23(3.350) \text { vs. } 1.87(1.074), p<0.001 \\
\text { GMFM-total score } \\
9.9 \text { (NR) vs. } 2.2 \text { (NR), p=NR }\end{array}$ & $\begin{array}{l}\text { Change in Strength of Trunk, Hip, Knee, } \\
\text { Ankle: } \\
\text { significantly better in group A than B, p<0.05 }\end{array}$ \\
\hline
\end{tabular}




\begin{tabular}{|c|c|c|c|c|}
\hline $\begin{array}{l}\text { Author, Year } \\
\text { Intervention Type } \\
\text { Duration of } \\
\text { Postintervention } \\
\text { Followup } \\
\text { Quality } \\
\text { (See Appendix B } \\
\text { for Full Citation) } \\
\end{array}$ & $\begin{array}{l}\text { Intervention and } \\
\text { Comparison }\end{array}$ & $\begin{array}{l}\text { Population } \\
\text { Age (Mean) } \\
\text { Gender (\% Female) } \\
\text { Race (\%) } \\
\text { Ambulatory (\%) } \\
\text { Wheelchair User (\%) } \\
\text { Condition Specific (\%) } \\
\text { Other (\%) }\end{array}$ & Prioritized Outcomes & Other Outcomes \\
\hline $\begin{array}{l}\text { Teixeira-Machado, } \\
2017 \\
\text { Aerobic Exercise } \\
\text { Aerobics (Dance) } \\
\text { Postintervention, } \\
12 \text { weeks } \\
\text { Fair }\end{array}$ & $\begin{array}{l}\text { A. Dance (somatic } \\
\text { dance therapy): } 24 \\
\text { sessions, over } 12 \\
\text { weeks, }(n=13) \\
\text { B. Usual care control } \\
(n=13)\end{array}$ & $\begin{array}{l}\text { A vs. B } \\
\text { Age (mean years): } 18 \text { vs. } 17 \\
\text { Female: } 54 \% \text { vs. } 62 \% \\
\text { Race: NR } \\
\text { Ambulatory: NR } \\
\text { Wheelchair user: NR } \\
\\
\text { BMI }\left(\mathrm{cm} / \mathrm{Kg}^{2}\right) \text { : } \\
\\
\text { GMFCS } \\
\text { Level II: } 6 \text { vs. } 3 \\
\text { Level III: } 3 \text { vs. } 5 \\
\text { Level IV: } 3 \text { vs. } 4 \\
\text { Level V: } 1 \text { vs. } 1\end{array}$ & $\begin{array}{l}\text { A vs. B, mean (SD) } \\
\text { FIM } \\
3.68(0.50) \text { vs. } 3.61(0.38) \text { (baseline) } \\
5.38(0.50) \text { vs. } 3.64(0.38) \text { (postintervention) } \\
\text { Total scores post intervention, p=0.0006 } \\
\text { FIM - Self care } \\
3.17(0.48) \text { vs. } 3.21(0.49) \text { (baseline) } \\
4.50 \text { (0.59) vs. } 3.28 \text { (0.50) (postintervention) } \\
\text { FIM - Sphincter control } \\
5.00(0.78) \text { vs. } 4.84(0.77) 4.84 \text { (0.77) (baseline) } \\
5.30 \text { (0.66) vs. (postintervention) } \\
\text { FIM - Mobility } \\
3.25(0.44) \text { vs. } 3.23(0.45) \text { (baseline) } \\
4.71(0.67) \text { vs. } 3.30(0.41) \text { (postintervention) } \\
\text { FIM - Locomotion } \\
3.19(0.42) \text { vs. } 3.07(0.43) \text { (baseline) } \\
4.50(0.55) \text { vs. } 3.11(0.41) \text { (postintervention) } \\
\text { FIM - Communication } \\
4.76(0.66) \text { vs. } 4.61(0.58) \text { (baseline) } \\
5.57(0.46) \text { vs. } 2.69(0.41) \text { (postintervention) } \\
\text { FIM - Psychosocial adjustments } \\
2.71(0.46) \text { vs. } 2.69(0.41) \text { (baseline) } \\
5.38(0.50) \text { vs. } 2.69(0.41) \text { (postintervention) } \\
\text { FIM - Cognitive function } \\
2.71(0.46) \text { vs. } 2.69(0.41) \text { (baseline) } \\
5.38(0.50) \text { vs. } 2.69(0.41) \text { (postintervention) } \\
\\
\text { WHODAS-IFC, overall scores } \\
84.56(4.62) \text { vs. } 84.45(4.05) \text { (baseline) } \\
39.90(5.80) \text { vs. } 69.55(4.39) \text { (postintervention) } \\
\text { Overall scores, p=0.0002 }\end{array}$ & NA \\
\hline
\end{tabular}




\begin{tabular}{|c|c|c|c|c|}
\hline $\begin{array}{l}\text { Author, Year } \\
\text { Intervention Type } \\
\text { Duration of } \\
\text { Postintervention } \\
\text { Followup } \\
\text { Quality } \\
\text { (See Appendix B } \\
\text { for Full Citation) }\end{array}$ & $\begin{array}{l}\text { Intervention and } \\
\text { Comparison }\end{array}$ & $\begin{array}{l}\text { Population } \\
\text { Age (Mean) } \\
\text { Gender (\% Female) } \\
\text { Race (\%) } \\
\text { Ambulatory (\%) } \\
\text { Wheelchair User (\%) } \\
\text { Condition Specific (\%) } \\
\text { Other (\%) }\end{array}$ & Prioritized Outcomes & Other Outcomes \\
\hline $\begin{array}{l}\text { Tollar, } 2020 \\
\text { Aerobic Exercise } \\
\text { Cycling } \\
\text { Postural Control } \\
\text { Balance Exercises } \\
\text { Motion Gaming } \\
\text { Strength } \\
\text { proprioceptive } \\
\text { neuromuscular } \\
\text { facilitation (PNF) } \\
\text { Immediately } \\
\text { postintervention,5 } \\
\text { weeks } \\
\text { Fair }\end{array}$ & $\begin{array}{l}\text { A. Exergaming: Xbox } \\
360, \text { Adventure video } \\
\text { game, } 25 \text { sessions } \\
\text { over } 5 \text { weeks ( } n=14 \text { ) } \\
\text { Sensorimotor and } \\
\text { visuomotor agility } \\
\text { training using } \\
\text { each of the three } \\
\text { modules of the Xbox } \\
360 \text { core system } \\
\text { ( } n=14 \text { ) } \\
\text { B. Balance } \\
\text { exercises: dynamic } \\
\text { and static balance } \\
\text { and stepping } \\
\text { exercises performed } \\
\text { in multiple directions } \\
\text { ( } n=14 \text { ) } \\
\text { C. Cycling ( } n=14 \text { ) } \\
\text { D. Proprioceptive } \\
\text { neuromuscular } \\
\text { facilitation ( } n=14 \text { ) } \\
\text { E. Usual care: } \\
\text { continuation of } \\
\text { standard physical } \\
\text { therapy and habitual } \\
\text { activity ( } n=12 \text { ) } \\
\text { All interventions } \\
\text { consisted of } 25,1 \\
\text { hour sessions over } 5 \\
\text { weeks }\end{array}$ & $\begin{array}{l}\text { Age: } 48 \text { vs. } 47 \text { vs. } 48 \text { vs. } 47 \\
\text { vs. } 44 \\
\text { Female: } 86 \% \text { vs. } 86 \% \text { vs. } \\
93 \% \text { vs. } 93 \% \text { vs. } 92 \% \\
\text { Race: NR } \\
\text { Ambulatory: } 100 \% \text { vs. } 100 \% \\
\text { vs. } 100 \% \text { vs. } 100 \% \text { vs. } \\
100 \% \\
\text { Wheelchair user: NR } \\
\text { RRMS: } 50 \% \text { vs. } 64 \% \text { vs. } \\
64 \% \text { vs. } 64 \% \text { vs. } 66 \% \\
\text { PPMS: } 50 \% \text { vs. } 36 \% \text { vs. } \\
36 \% \text { vs. } 36 \% 34 \% \\
\text { Median EDSS score: } 5.0 \text { vs. } \\
5.0 \text { vs. } 5.0 \text { vs. } 5.0 \text { vs. } 5.0\end{array}$ & 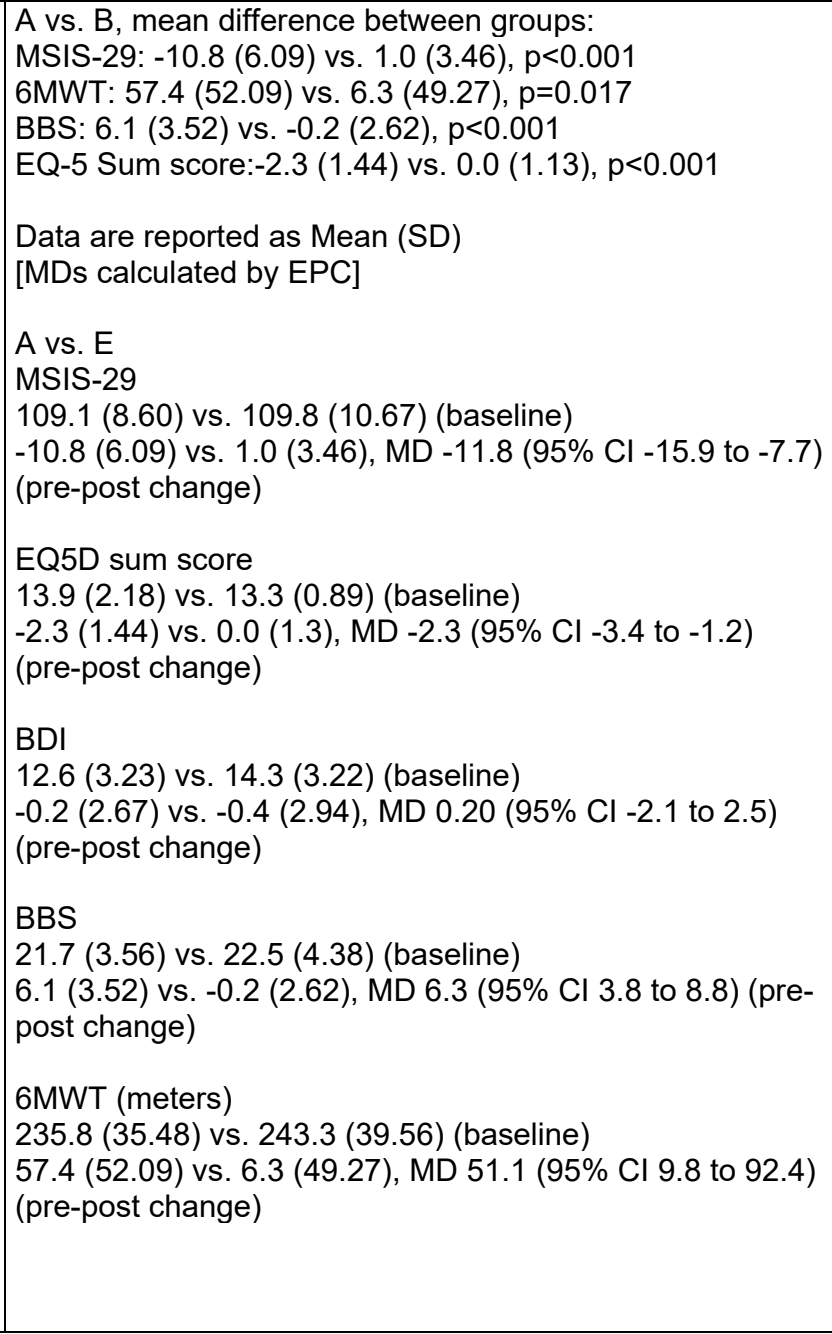 & $\begin{array}{l}\text { A. Exergaming: sensorimotor and visuomotor } \\
\text { agility training using } \\
\text { each of the three modules of the Xbox } 360 \\
\text { core system }(n=14) \\
\text { B. Balance exercises: dynamic and static } \\
\text { balance and stepping } \\
\text { exercises performed in multiple directions } \\
\text { ( } n=14) \\
\text { C. Cycling ( } n=14) \\
\begin{array}{l}\text { D. Proprioceptive neuromuscular facilitation } \\
(n=14)\end{array} \\
\text { E. Usual care: continuation of standard } \\
\text { physical therapy and habitual activity ( } n=12) \\
\text { All interventions consisted of } 25,1 \text { hour } \\
\text { sessions over } 5 \text { weeks }\end{array}$ \\
\hline
\end{tabular}




\begin{tabular}{|c|c|c|c|c|}
\hline $\begin{array}{l}\text { Author, Year } \\
\text { Intervention Type } \\
\text { Duration of } \\
\text { Postintervention } \\
\text { Followup } \\
\text { Quality } \\
\text { (See Appendix B } \\
\text { for Full Citation) } \\
\end{array}$ & $\begin{array}{l}\text { Intervention and } \\
\text { Comparison }\end{array}$ & \begin{tabular}{|l|} 
Population \\
Age (Mean) \\
Gender (\% Female) \\
Race (\%) \\
Ambulatory (\%) \\
Wheelchair User (\%) \\
Condition Specific (\%) \\
Other (\%) \\
\end{tabular} & Prioritized Outcomes & Other Outcomes \\
\hline $\begin{array}{l}\text { Tollar, 2020 } \\
\text { (Continued) }\end{array}$ & & & 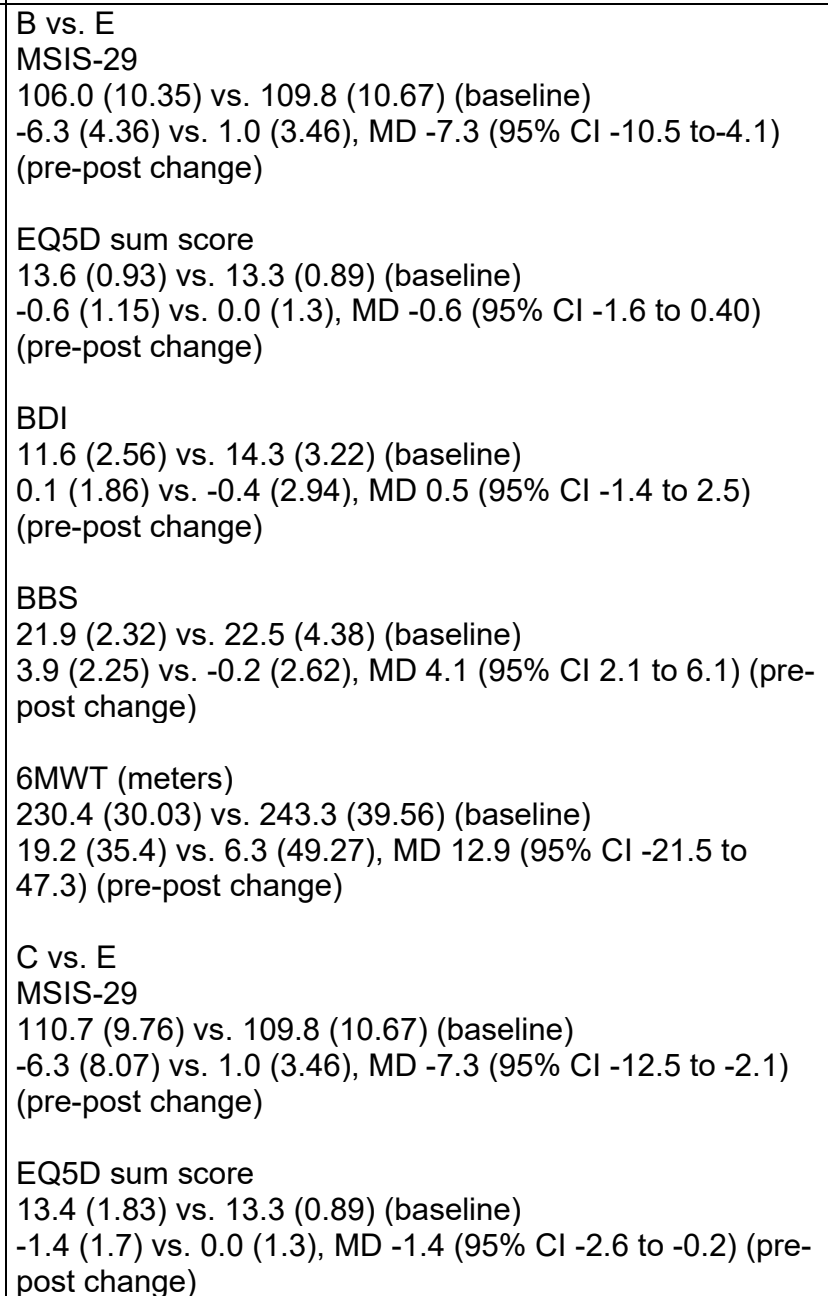 & \\
\hline
\end{tabular}




\begin{tabular}{|c|c|c|c|c|}
\hline $\begin{array}{l}\text { Author, Year } \\
\text { Intervention Type } \\
\text { Duration of } \\
\text { Postintervention } \\
\text { Followup } \\
\text { Quality } \\
\text { (See Appendix B } \\
\text { for Full Citation) } \\
\end{array}$ & $\begin{array}{l}\text { Intervention and } \\
\text { Comparison }\end{array}$ & \begin{tabular}{|l|} 
Population \\
Age (Mean) \\
Gender (\% Female) \\
Race (\%) \\
Ambulatory (\%) \\
Wheelchair User (\%) \\
Condition Specific (\%) \\
Other (\%) \\
\end{tabular} & Prioritized Outcomes & Other Outcomes \\
\hline $\begin{array}{l}\text { Tollar, } 2020 \\
\text { (Continued) }\end{array}$ & & & 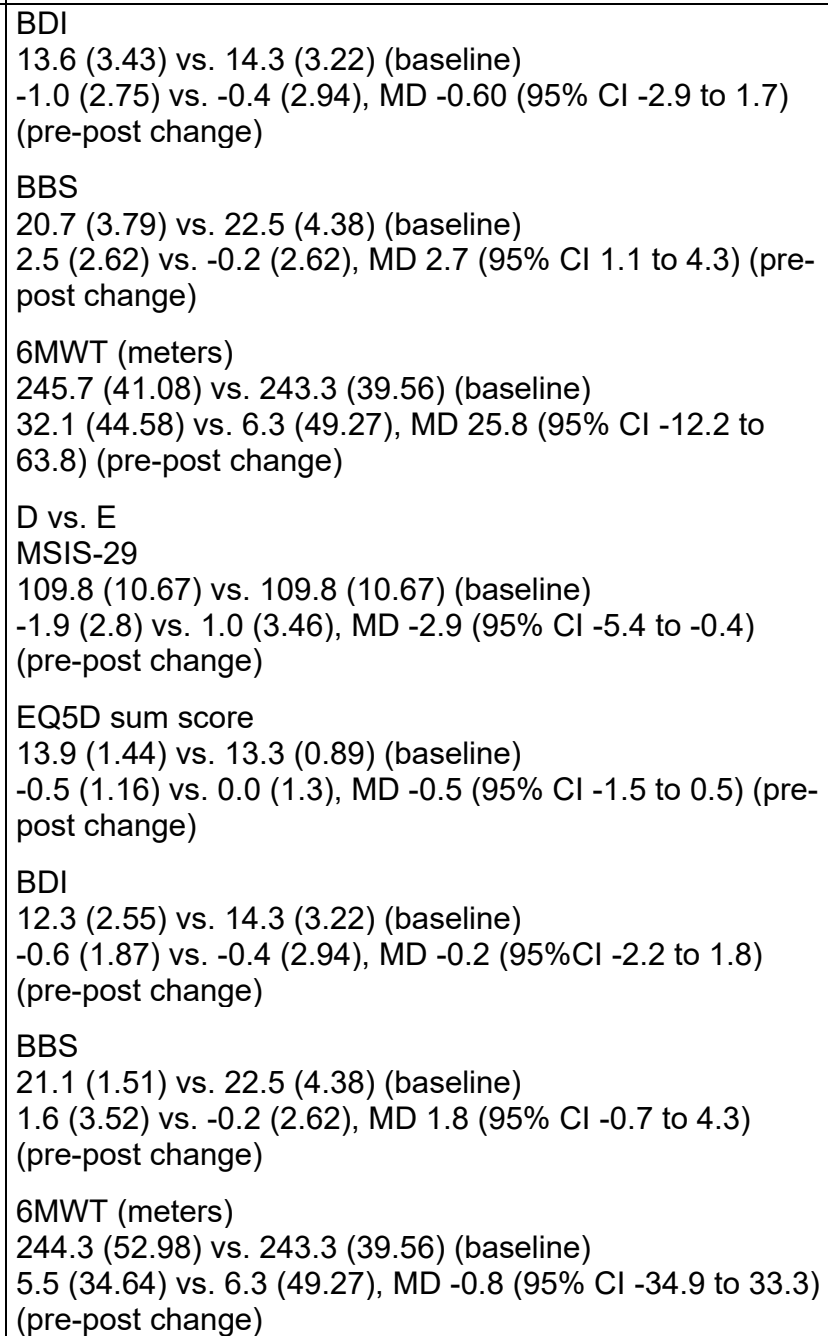 & \\
\hline
\end{tabular}




\begin{tabular}{|c|c|c|c|c|}
\hline $\begin{array}{l}\text { Author, Year } \\
\text { Intervention Type } \\
\text { Duration of } \\
\text { Postintervention } \\
\text { Followup } \\
\text { Quality } \\
\text { (See Appendix B } \\
\text { for Full Citation) } \\
\end{array}$ & $\begin{array}{l}\text { Intervention and } \\
\text { Comparison }\end{array}$ & $\begin{array}{l}\text { Population } \\
\text { Age (Mean) } \\
\text { Gender (\% Female) } \\
\text { Race (\%) } \\
\text { Ambulatory (\%) } \\
\text { Wheelchair User (\%) } \\
\text { Condition Specific (\%) } \\
\text { Other (\%) }\end{array}$ & Prioritized Outcomes & Other Outcomes \\
\hline $\begin{array}{l}\text { Totosy de } \\
\text { Zepetnek, } 2015 \\
\text { Multimodal } \\
\text { Exercise } \\
\text { Immediately } \\
\text { postintervention, } \\
16 \text { weeks } \\
\text { Fair }\end{array}$ & $\begin{array}{l}\text { A. Progressive } \\
\text { resistance }+ \text { aerobic } \\
\text { training, } 32 \text { sessions } \\
\text { over } 16 \text { weeks } \\
(n=12) \\
\text { B. Maintain existing } \\
\text { physical activity } \\
\text { levels }(n=11)\end{array}$ & $\begin{array}{l}\text { A vs. B } \\
\text { Age: } 39 \text { vs. } 42 \\
\text { Female: } 0 \% \text { vs. } 18 \% \\
\text { AIS A-B: } 25 \% \text { vs. } 45 \% \\
\text { AIS C-D: } 75 \% \text { vs. } 55 \%\end{array}$ & $\begin{array}{l}\text { A vs. B, Mean (SD), p-value between groups: } \\
\text { Systolic blood pressure: } \\
116(18) \text { to } 116(15) \text { vs. } 118(18) \text { to } 116(17), p>0.05 \\
\text { Diastolic blood pressure: } \\
68 \text { (9) to } 67(9) \text { vs. } 74(13) \text { to } 72(11), p>0.05 \\
\text { Heart rate: } \\
75(13) \text { to } 71(13) \text { vs. } 75(10) \text { to } 74(10), p>0.05 \\
\text { HbA1c (mmol/mol): } \\
35.7(11.6) \text { to } 36.6(11.2) \text { vs. } 34.9(4.8) \text { to } 34.7(3.9), \\
\text { p>0.05 } \\
\text { BMl: } \\
27.3(5.2) \text { to } 27.0(5.0) \text { vs. } 25.7(4.9) \text { to } 26.6(4.7), p<0.05\end{array}$ & $\begin{array}{l}\text { A vs. B, Mean (SD), p-value between groups: } \\
\text { Total cholesterol (mmol/L): } 1.5(0.9) \text { to } 4.3 \\
(1.0) \text { vs. } 4.1(0.9) \text { to } 4.1(0.9), p>0.05 \\
\text { Low-density lipoprotein cholesterol (mmol/L): } \\
2.9(0.9) \text { to } 2.7(0.7) \text { vs. } 2.5(0.7) \text { to } 2.4(0.6) \text {, } \\
p>0.05 \\
\text { High density lipoprotein cholesterol (mmol/L): } \\
1.01(0.2) \text { to } 1.01(0.3) \text { vs. } 1.13(0.2) \text { to } 1.17 \\
(0.3), p>0.05 \\
\text { Triglyceride: } 1.3(0.6) \text { to } 1.4(0.6) \text { vs. } 1.1(0.7) \\
\text { to } 1.0(0.7), p>0.05\end{array}$ \\
\hline
\end{tabular}




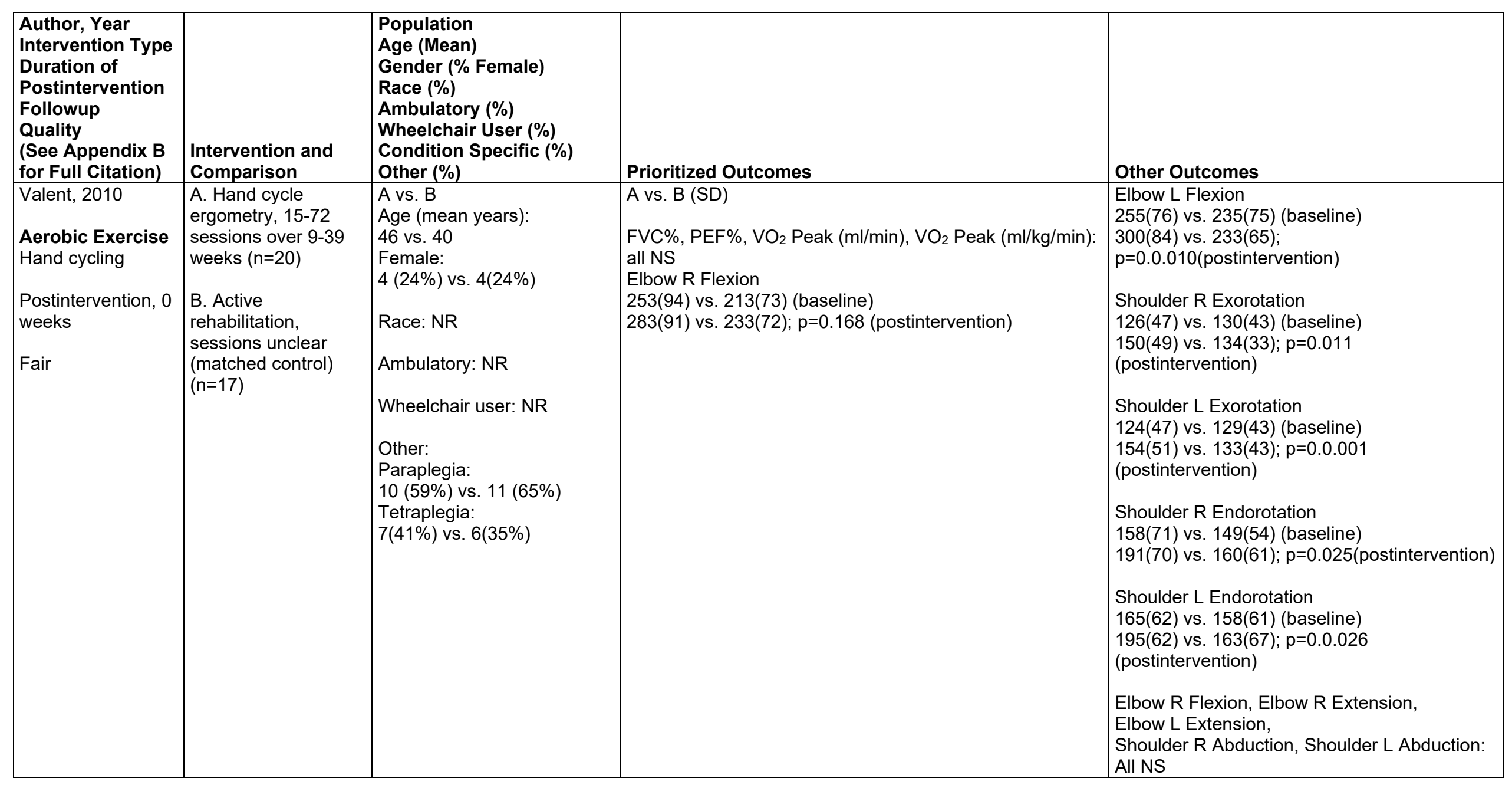




\begin{tabular}{|c|c|c|c|c|}
\hline $\begin{array}{l}\text { Author, Year } \\
\text { Intervention Type } \\
\text { Duration of } \\
\text { Postintervention } \\
\text { Followup } \\
\text { Quality } \\
\text { (See Appendix B } \\
\text { for Full Citation) }\end{array}$ & $\begin{array}{l}\text { Intervention and } \\
\text { Comparison }\end{array}$ & $\begin{array}{l}\text { Population } \\
\text { Age (Mean) } \\
\text { Gender (\% Female) } \\
\text { Race (\%) } \\
\text { Ambulatory (\%) } \\
\text { Wheelchair User (\%) } \\
\text { Condition Specific (\%) } \\
\text { Other (\%) } \\
\end{array}$ & $x^{2}$ & Other Outcomes \\
\hline $\begin{array}{l}\text { van der Scheer } \\
2016 \\
\text { Aerobic Exercise } \\
\text { Treadmill } \\
16 \text { week } \\
\text { intervention } \\
\text { Postintervention, } 0 \\
\text { weeks } \\
\text { Fair }\end{array}$ & $\begin{array}{l}\text { A. Low-intensity } \\
\text { wheelchair training in } \\
\text { a treadmill for } 30 \\
\text { minutes } 2 \text { times a } \\
\text { week for } 16 \text { weeks } \\
\text { B. Usual care }\end{array}$ & $\begin{array}{l}\text { Mean age } 56.0 \text { years } \\
22 \text { males and } 7 \text { females } \\
\text { ( } 76 \% \text { males })\end{array}$ & $\begin{array}{l}\text { Peak oxygen uptake (median) } \\
\text { A. } 1.02 \text { to } 1.01 \\
\text { B. } 1.09 \text { to } 1.07 \\
\text { No differences }\end{array}$ & NA \\
\hline
\end{tabular}




\begin{tabular}{|c|c|c|c|c|}
\hline $\begin{array}{l}\text { Author, Year } \\
\text { Intervention Type } \\
\text { Duration of } \\
\text { Postintervention } \\
\text { Followup } \\
\text { Quality } \\
\text { (See Appendix B } \\
\text { for Full Citation) } \\
\end{array}$ & $\begin{array}{l}\text { Intervention and } \\
\text { Comparison }\end{array}$ & $\begin{array}{l}\text { Population } \\
\text { Age (Mean) } \\
\text { Gender (\% Female) } \\
\text { Race (\%) } \\
\text { Ambulatory (\%) } \\
\text { Wheelchair User (\%) } \\
\text { Condition Specific (\%) } \\
\text { Other (\%) }\end{array}$ & Prioritized Outcomes & Other Outcomes \\
\hline $\begin{array}{l}\text { Van Wely, 2014a } \\
\text { Van Wely, 2014b } \\
\text { Van Wely, 2010 } \\
\text { Multimodal } \\
\text { Exercise } \\
\text { Mid-intervention } \\
\text { (16 weeks into } \\
\text { trial); } \\
\text { Immediately } \\
\text { postintervention, } \\
24 \text { weeks } \\
\text { Van Wely, 2014a: } \\
\text { Good } \\
\text { Van Wely, 2014b: } \\
\text { Fair }\end{array}$ & $\begin{array}{l}\text { A. Strength + } \\
\text { aerobics }+ \\
\text { physiotherapy + } \\
\text { counseling: } 24 \\
\text { sessions of fitness } \\
\text { training over } 16 \\
\text { weeks }(n=25) \\
\text { B. Physiotherapy } \\
(n=25)\end{array}$ & $\begin{array}{l}\text { A vs. B } \\
\text { Age (mean years): } 9.5 \text { vs. } \\
10.0 \\
\text { Female: } 13 \text { (52\%) vs. } 8 \\
(33 \%) \\
\text { Race: NR } \\
\text { Ambulatory: } 25 \text { (100\%) vs. } \\
24(100 \%) \\
\text { Wheelchair user for long } \\
\text { distances: } 5 \text { (20\%) vs. } 5 \\
(21 \%) \\
\text { GMFCS } \\
- \text { I: } 15(60 \%) \text { vs. } 13(54 \%) \\
- \text { II: } 6(24 \%) \text { vs. } 6(25 \%) \\
- \text { III: } 4(16 \%) \text { vs. } 5(21 \%) \\
\text { Laterality } \\
- \text { Unilateral: } 12(48 \%) \text { vs. } 11 \\
(46 \%) \\
\text {-Bilateral: } 13(52 \%) \text { vs. } 13 \\
(54 \%) \\
\text { Orthoses (yes): } 17 \text { (68\%) vs. } \\
15 \text { (62\%) }\end{array}$ & 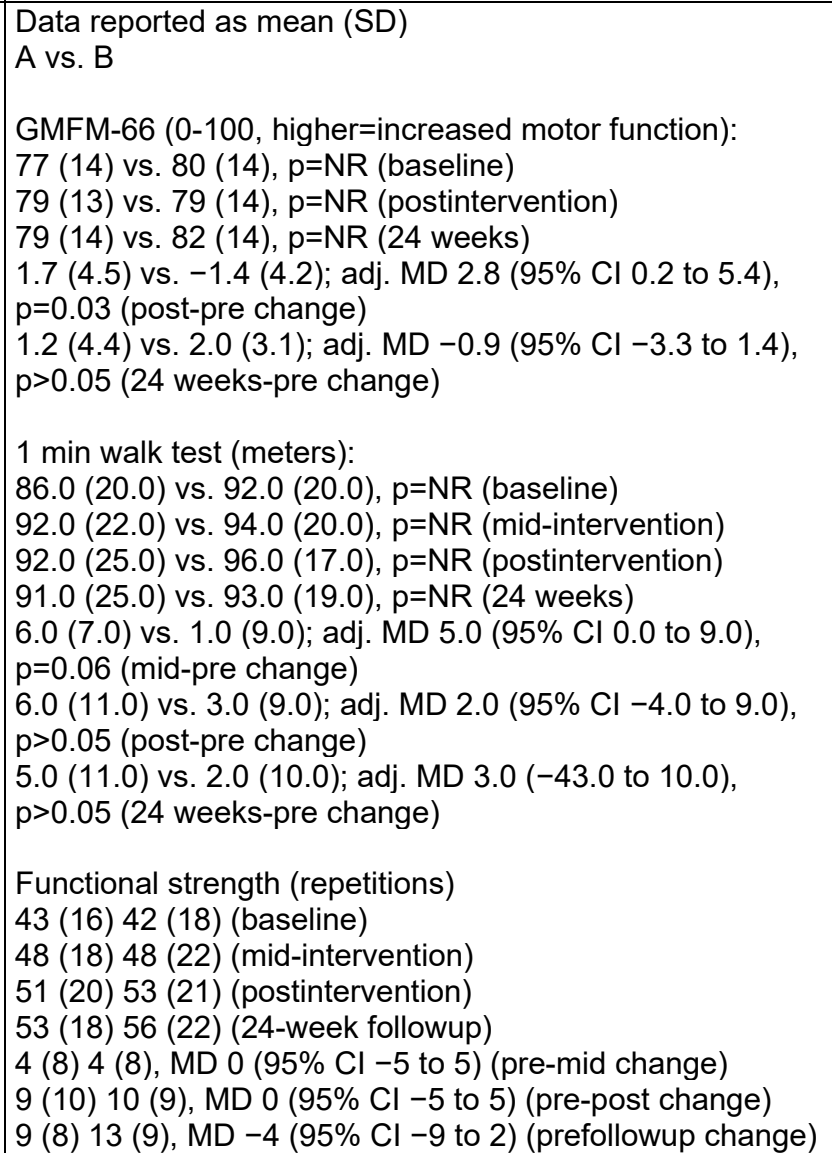 & NR \\
\hline
\end{tabular}




\begin{tabular}{|c|c|c|c|c|}
\hline $\begin{array}{l}\text { Author, Year } \\
\text { Intervention Type } \\
\text { Duration of } \\
\text { Postintervention } \\
\text { Followup } \\
\text { Quality } \\
\text { (See Appendix B } \\
\text { for Full Citation) }\end{array}$ & $\begin{array}{l}\text { Intervention and } \\
\text { Comparison }\end{array}$ & $\begin{array}{l}\text { Population } \\
\text { Age (Mean) } \\
\text { Gender (\% Female) } \\
\text { Race (\%) } \\
\text { Ambulatory (\%) } \\
\text { Wheelchair User (\%) } \\
\text { Condition Specific (\%) } \\
\text { Other (\%) }\end{array}$ & Prioritized Outcomes & Other Outcomes \\
\hline $\begin{array}{l}\text { Van Wely, 2014a } \\
\text { Van Wely, 2014b } \\
\text { Van Wely, 2010 } \\
\text { (Continued) }\end{array}$ & & & 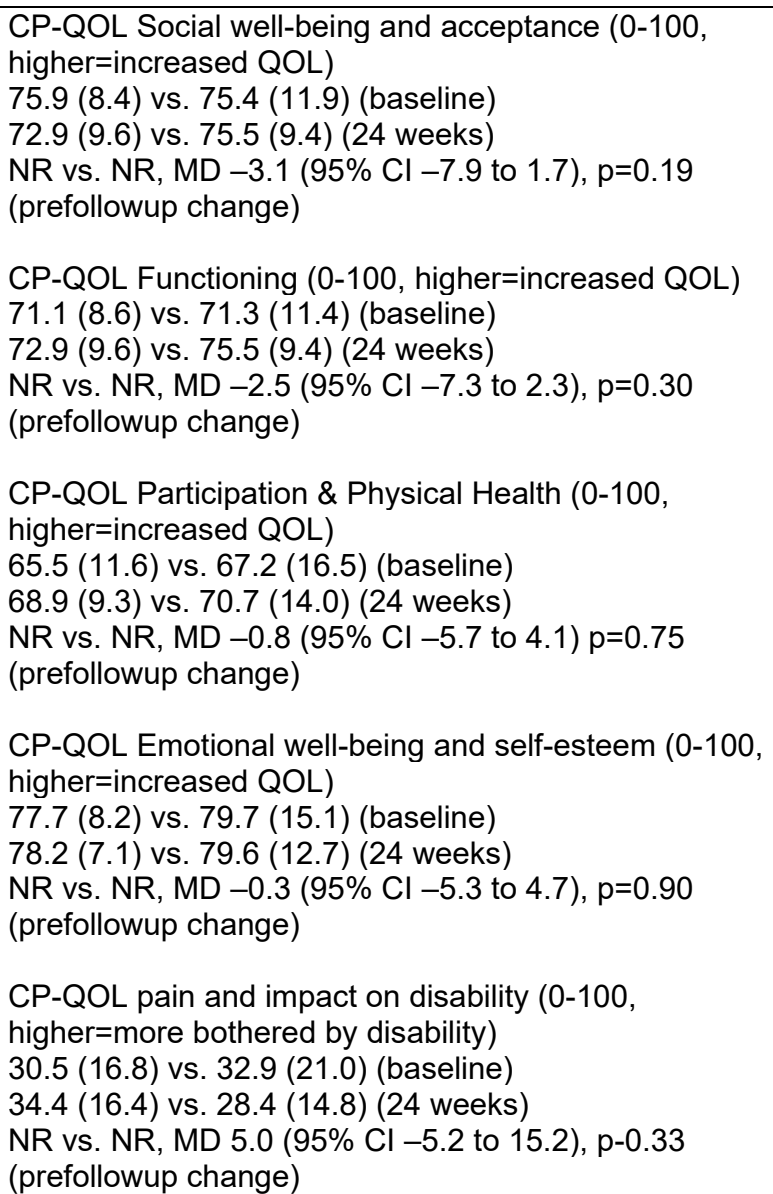 & \\
\hline
\end{tabular}




\begin{tabular}{|c|c|c|c|c|}
\hline $\begin{array}{l}\text { Author, Year } \\
\text { Intervention Type } \\
\text { Duration of } \\
\text { Postintervention } \\
\text { Followup } \\
\text { Quality } \\
\text { (See Appendix B } \\
\text { for Full Citation) } \\
\end{array}$ & $\begin{array}{l}\text { Intervention and } \\
\text { Comparison }\end{array}$ & $\begin{array}{l}\text { Population } \\
\text { Age (Mean) } \\
\text { Gender (\% Female) } \\
\text { Race (\%) } \\
\text { Ambulatory (\%) } \\
\text { Wheelchair User (\%) } \\
\text { Condition Specific (\%) } \\
\text { Other (\%) }\end{array}$ & Prioritized Outcomes & Other Outcomes \\
\hline $\begin{array}{l}\text { Vermohlen, } 2018 \\
\text { Protocol: } \\
\text { Wollenweber, } 2016 \\
\text { Postural Control } \\
\text { Hippotherapy } \\
\text { End of treatment } \\
\text { (after 12-week } \\
\text { intervention) }\end{array}$ & $\begin{array}{l}\text { A. Hippotherapy plus } \\
\text { standard care, } 12 \\
\text { sessions over } 12 \\
\text { weeks ( } n=32 \text { ) } \\
\text { B. Control group } \\
\text { (standard care), } 12 \\
\text { weeks ( } n=38 \text { ) }\end{array}$ & $\begin{array}{l}\text { A ( } n=30) \text { vs. } B(n=37) \\
\text { Age (median years): } 50 \text { vs. } \\
51 \\
\text { Female: } 27(90 \%) \text { vs. } 27 \\
(73 \%) \\
\text { Race: NR } \\
\text { Ambulatory: NR } \\
\text { Wheelchair user: NR } \\
\\
\text { EDSS at inclusion (mean): } \\
5.4 \text { vs. } 5.3 \\
\text { Weight (mean kg): } 67 \text { vs. } \\
70.6 \\
\text { Median time from onset of } \\
\text { MS to inclusion (IQR years): } \\
16.5 \text { vs. } 17.6 \\
\text { Physiotherapy: } 29 \text { (97\%) vs. } \\
35 \text { (95\%) }\end{array}$ & $\begin{array}{l}\text { A vs. B } \\
12 \text { weeks } \\
\text { MSQoL-54: } \\
\text { A ( } n=30) \text { vs. B ( } n=36) \text {, mean (SD) } \\
\text { Mental health subscale score: } \\
62.6(18.0) \text { vs. } 67.1(17.2) \text { (baseline) } \\
75.7(15.0) \text { vs. } 64.2(19.9) \text { (postintervention) } \\
\text { Mean difference in change between groups at } 12 \text { weeks: } \\
14.4(95 \% \mathrm{Cl} 7.5 \text { to } 21.3), p<0.001 \\
\text { A ( } n=25) \text { vs. B ( } n=31), \text { mean (SD) } \\
\text { Physical health subscale score: } \\
46.0(14.2) \text { vs. } 53.7(14.6) \text { (baseline) } \\
57.0(15.1) \text { vs. } 51.3(15.9) \text { (postintervention) } \\
\text { Mean difference in change between groups at } 12 \text { weeks: } \\
12.0(95 \% \text { Cl } 6.2 \text { to } 17.7), p<0.001 \\
\text { A ( } n=30) \text { vs. B ( } n=36) \text {, mean (SD) } \\
\text { NRS: } \\
4.6(2.1) \text { vs. } 4.4(2.2) \text { (baseline) } \\
2.9(2.1) \text { vs. } 3.8(2.3) \text { (postintervention) } \\
\text { Mean difference in change between groups at } 12 \text { weeks: } \\
-0.9(95 \% \mathrm{Cl}-1.9 \text { to }-0.1), p=0.031\end{array}$ & $\begin{array}{l}\text { A }(n=30) \text { vs. B ( } n=37) \text {, mean (SD) } \\
12 \text { weeks } \\
\text { BBS: } \\
\text { LOCF ANCOVA: } \\
40.6(11.5) \text { vs. } 42.1(10.9) \text { (baseline) } \\
47.0 \text { (8.7) vs. } 45.1(10.9) \text { (postintervention) } \\
\text { Mean difference in change between groups at } \\
12 \text { weeks: } 2.33 \text { ( } 95 \% \text { Cl } 0.03 \text { to } 4.63), p=0.047 \\
\text { MMRM: } \\
\text { Mean difference in change between groups at } \\
12 \text { weeks: } 3.07 \text { ( } 95 \% \text { Cl } 1.00 \text { to } 5.14), p=0.004\end{array}$ \\
\hline $\begin{array}{l}\text { Wallard, } 2017 \\
\text { Wallard, } 2018 \\
\text { Aerobic Exercise } \\
\text { Robot-Assisted } \\
\text { Gait Training } \\
\text { Postintervention, } 4 \\
\text { weeks } \\
\text { Poor }\end{array}$ & $\begin{array}{l}\text { A. RAGT, } 20 \\
\text { sessions over } 4 \\
\text { weeks }(n=14) \\
\text { B. Usual care, } 20 \\
\text { sessions over } 4 \\
\text { weeks }(n=16)\end{array}$ & $\begin{array}{l}\text { A vs. B } \\
\text { Age (mean years): } \\
8.3 \text { vs. } 9.6 \\
\text { Female: } \\
6(43 \%) \text { vs. } 9(56 \%) \\
\text { Race: NR } \\
\text { Ambulatory: } \\
\text { NR } \\
\text { Wheelchair user: } \\
\text { NR }\end{array}$ & $\begin{array}{l}\text { GMFM-66 D (\%), mean (SD) } \\
53.89(16.02) \text { to } 60.68(14.71) \text { vs. } 53.81(14.67) \text { to } 55.74 \\
(15.02), p=0.048 \\
\text { GMFM-66 E } \\
42.23(14.65) \text { to } 50.87(15.82) \text { vs. } 42.51(13.09) \text { to } 43.61 \\
(12.59), p=0.026\end{array}$ & A vs. B, mean (SD) \\
\hline
\end{tabular}




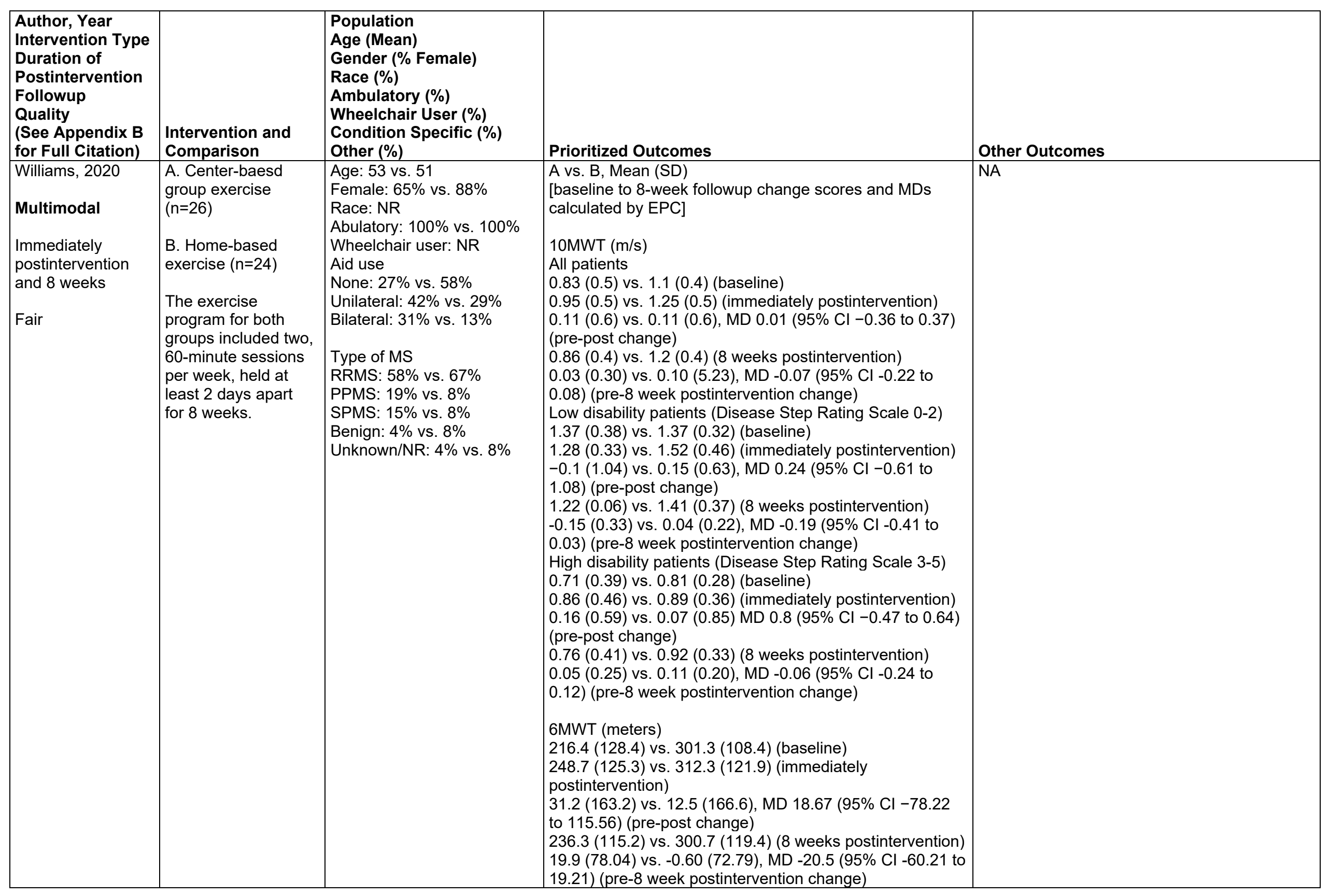




\begin{tabular}{|c|c|c|c|c|}
\hline $\begin{array}{l}\text { Author, Year } \\
\text { Intervention Type } \\
\text { Duration of } \\
\text { Postintervention } \\
\text { Followup } \\
\text { Quality } \\
\text { (See Appendix B } \\
\text { for Full Citation) } \\
\end{array}$ & $\begin{array}{l}\text { Intervention and } \\
\text { Comparison }\end{array}$ & $\begin{array}{l}\text { Population } \\
\text { Age (Mean) } \\
\text { Gender (\% Female) } \\
\text { Race (\%) } \\
\text { Ambulatory (\%) } \\
\text { Wheelchair User (\%) } \\
\text { Condition Specific (\%) } \\
\text { Other (\%) } \\
\end{array}$ & Prioritized Outcomes & Other Outcomes \\
\hline $\begin{array}{l}\text { Williams, } 2020 \\
\text { (Continued) }\end{array}$ & & & 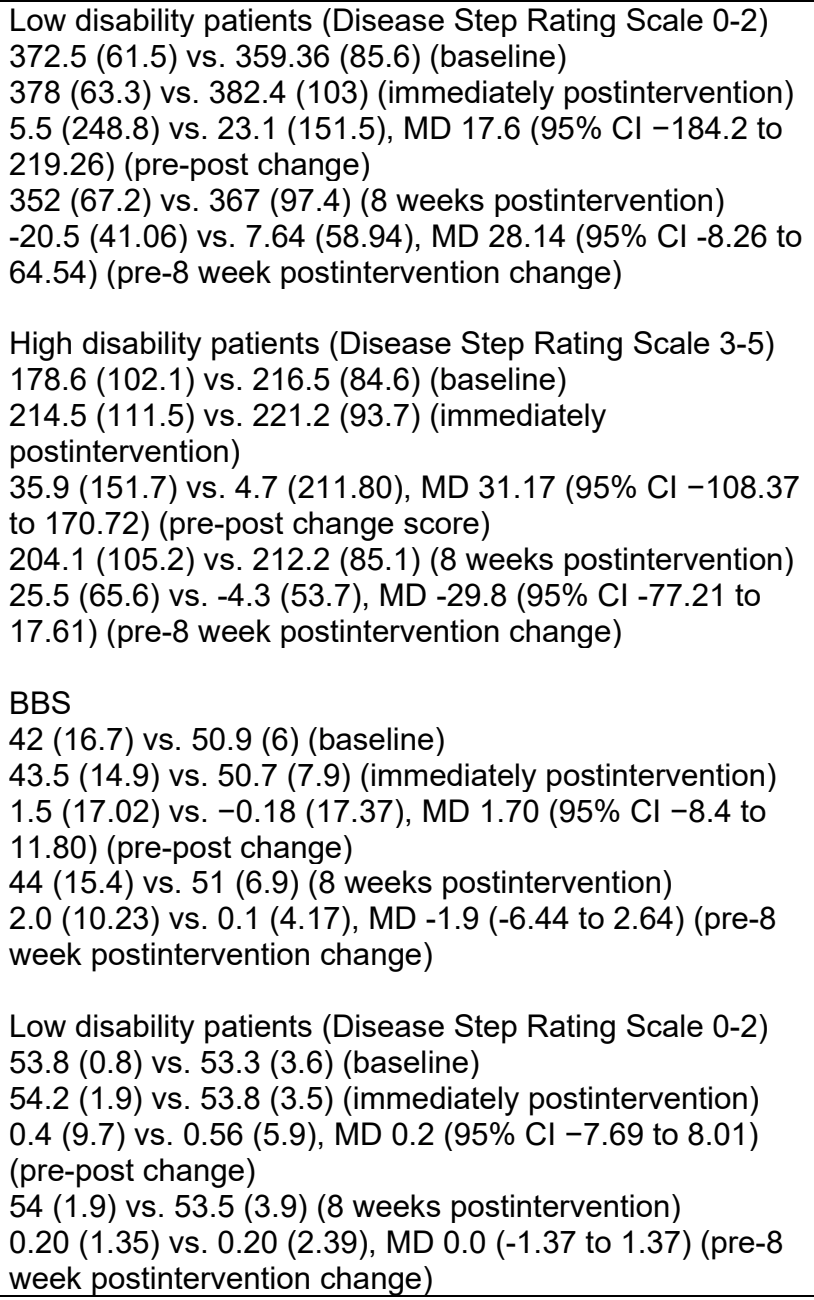 & \\
\hline
\end{tabular}




\begin{tabular}{|c|c|c|c|c|}
\hline $\begin{array}{l}\text { Author, Year } \\
\text { Intervention Type } \\
\text { Duration of } \\
\text { Postintervention } \\
\text { Followup } \\
\text { Quality } \\
\text { (See Appendix B } \\
\text { for Full Citation) } \\
\end{array}$ & $\begin{array}{l}\text { Intervention and } \\
\text { Comparison }\end{array}$ & $\begin{array}{l}\text { Population } \\
\text { Age (Mean) } \\
\text { Gender (\% Female) } \\
\text { Race (\%) } \\
\text { Ambulatory (\%) } \\
\text { Wheelchair User (\%) } \\
\text { Condition Specific (\%) } \\
\text { Other (\%) }\end{array}$ & Prioritized Outcomes & Other Outcomes \\
\hline $\begin{array}{l}\text { Williams, } 2020 \\
\text { (Continued) }\end{array}$ & & & $\begin{array}{l}\text { High disability patients (Disease Step Rating Scale 3-5) } \\
39.1 \text { (17.5) vs. } 47.6(7.3) \text { (baseline) } \\
40.7(15.5) \text { vs. } 46.7(10.2) \text { (immediately postintervention) } \\
1.6(22.3) \text { vs. }-0.9(31.2) \text {, MD } 2.54 \text { (95\% } \mathrm{Cl}-18.01 \text { to } \\
23.08) \text { (pre-post change) } \\
41.2 \text { (16.4) vs. } 47.7(8.7) \text { (8 weeks postintervention) } \\
2.10 \text { (10.77) vs. } 0.10(5.23) \text {, MD }-2.0(95 \% \mathrm{Cl}-9.31 \text { to } \\
5.31) \text { (pre-8 week postintervention change) }\end{array}$ & \\
\hline $\begin{array}{l}\text { Wens, } 2015 a \\
\text { "Impact of } 24 \\
\text { weeks of } \\
\text { resistance and } \\
\text { endurance } \\
\text { exercise on } \\
\text { glucose tolerance } \\
\text { in persons with } \\
\text { multiple sclerosis" } \\
\text { Multimodal } \\
\text { Exercise } \\
\text { Postintervention, } 0 \\
\text { weeks } \\
\text { Poor }\end{array}$ & $\begin{array}{l}\text { A. Progressive } \\
\text { resistance }+ \\
\text { aerobics, } 60 \\
\text { sessions over } 24 \\
\text { weeks }(n=29) \\
\begin{array}{l}\text { B. Nonexercise } \\
\text { control }(n=15)\end{array}\end{array}$ & $\begin{array}{l}\text { A vs. B } \\
\text { Age (mean years): } 48 \text { vs. } 49 \\
\text { Female: } 17(59 \%) \text { vs. } 8 \\
\text { (53\%) } \\
\text { Race: NR } \\
\text { Ambulatory: NR } \\
\text { Wheelchair user: NR } \\
\text { MS type: } \\
\text { RRMS: } 17(59 \%) \text { vs. } 11 \\
\text { (73\%) } \\
\text { CPMS: } 12(41 \%) \text { vs. } 4(27 \%) \\
\text { EDSS (mean): } 3.25 \text { vs. } 3.36\end{array}$ & $\begin{array}{l}\text { A vs. B, Mean difference between groups: } \\
24 \text { weeks (end of treatment) } \\
\text { Body weight }(\mathrm{kg}): 1.9,95 \% \mathrm{Cl}-0.124 \text { to } 0.07 \\
\text { No differences in glucose and insulin }\end{array}$ & $\begin{array}{l}\text { A vs. B, Mean difference between groups: } \\
24 \text { weeks (end of treatment) } \\
\text { Resting HR: } 9.0,95 \% \mathrm{Cl} 6.57 \text { to } 11.43, \\
p<0.001 \\
\text { Body fat } \%: 2.0,95 \% \mathrm{Cl} 0.67 \text { to } 3.33, p=0.003 \\
\text { Knee extension and flexion improved with } \\
\text { exercise. Group X Time interaction } p<0.05\end{array}$ \\
\hline
\end{tabular}




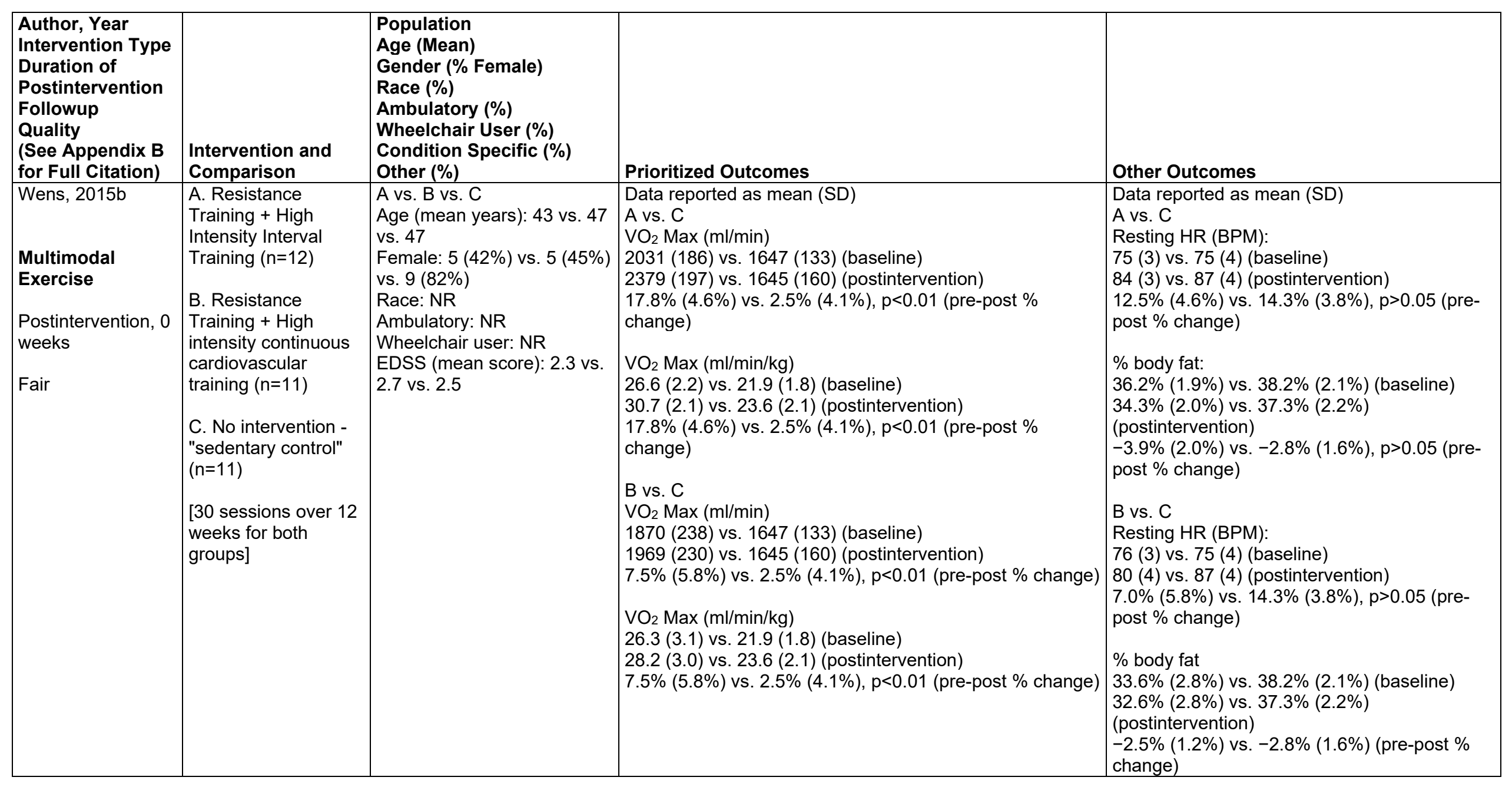




\begin{tabular}{|c|c|c|c|c|}
\hline \begin{tabular}{l|} 
Author, Year \\
Intervention Type \\
Duration of \\
Postintervention \\
Followup \\
Quality \\
(See Appendix B \\
for Full Citation) \\
\end{tabular} & $\begin{array}{l}\text { Intervention and } \\
\text { Comparison }\end{array}$ & $\begin{array}{l}\text { Population } \\
\text { Age (Mean) } \\
\text { Gender (\% Female) } \\
\text { Race (\%) } \\
\text { Ambulatory (\%) } \\
\text { Wheelchair User (\%) } \\
\text { Condition Specific (\%) } \\
\text { Other (\%) }\end{array}$ & Prioritized Outcomes & Other Outcomes \\
\hline $\begin{array}{l}\text { Willoughby, } 2010 \\
\text { Aerobic Exercise } \\
\text { Treadmill } \\
\text { RCT } \\
\text { Postintervention, } \\
10 \text { weeks after } \\
\text { intervention } \\
\text { Fair }\end{array}$ & $\begin{array}{l}\text { A. Partial body } \\
\text { weight supported } \\
\text { treadmill training } 30 \\
\text { minutes } 2 \text { times a } \\
\text { week for } 9 \text { weeks } \\
\text { B. Overground } \\
\text { walking with walker } \\
\text { or assist device } \\
\text { comparable duration } \\
\text { and number of } \\
\text { sessions }\end{array}$ & $\begin{array}{l}\text { GMFCD III or IV } \\
n=33 \text { randomized and } 26 \\
\text { analyzed } \\
\text { Mean age } 10.8 \text { years } \\
15 \text { males and } 11 \text { females } \\
(54 \% \text { males) }\end{array}$ & $\begin{array}{l}\text { Pre, post, } 10 \text { weeks after training } \\
10 \text { minute walk test (10MWT) (walking endurance) } \\
\text { (meters) } \\
\text { A. } 244.33(115.41) \text { to } 218.38(123.71) \text { to } 215.67(142.99) \\
\text { B. } 118.36(89.89) \text { to } 135.82(95.65) \text { to } 148.43(103.52) \\
\text { Pre to post trend for between groups } F=3.004 p=0.097 \\
\text { favoring } B \\
\text { Pre to } 24 \text { week trend for between groups } F=2.992 \\
p=0.098 \text { favoring } B\end{array}$ & NA \\
\hline
\end{tabular}




\begin{tabular}{|c|c|c|c|c|}
\hline $\begin{array}{l}\text { Author, Year } \\
\text { Intervention Type } \\
\text { Duration of } \\
\text { Postintervention } \\
\text { Followup } \\
\text { Quality } \\
\text { (See Appendix B } \\
\text { for Full Citation) } \\
\end{array}$ & $\begin{array}{l}\text { Intervention and } \\
\text { Comparison }\end{array}$ & $\begin{array}{l}\text { Population } \\
\text { Age (Mean) } \\
\text { Gender (\% Female) } \\
\text { Race (\%) } \\
\text { Ambulatory (\%) } \\
\text { Wheelchair User (\%) } \\
\text { Condition Specific (\%) } \\
\text { Other (\%) }\end{array}$ & Prioritized Outcomes & Other Outcomes \\
\hline $\begin{array}{l}\text { Wu, 2017b } \\
\text { "The effects of the } \\
\text { integration of } \\
\text { dynamic weight } \\
\text { shifting training into } \\
\text { treadmill training } \\
\text { on walking function } \\
\text { of children with } \\
\text { cerebral palsy- a } \\
\text { randomized } \\
\text { controlled study" } \\
\text { Aerobic Exercise } \\
\text { Robot-Assisted } \\
\text { Gait Training } \\
\text { Postintervention,8 } \\
\text { weeks (after } 6 \\
\text { weeks intervention) } \\
\text { Fair }\end{array}$ & $\begin{array}{l}\text { A. Robotic treadmill } \\
\text { training, } 18 \text { sessions } \\
\text { over } 6 \text { weeks }(n=11) \\
\text { B. Treadmill only } \\
\text { training, } 18 \text { sessions } \\
\text { over } 6 \text { weeks }(n=12)\end{array}$ & $\begin{array}{l}\text { A vs. B } \\
\text { Age (mean years): } \\
11.3 \text { vs. } 10.5 \\
\text { Female: } \\
5(45 \%) \text { vs. } 4(33 \%) \\
\text { Race: -week followup } \\
\text { white: } 6(55 \%) \text { vs. } 7 \text { (58\%) } \\
\text { Ambulatory: } 100 \% \\
\text { Wheelchair user: NR } \\
\text { Other: } \\
\text { GMFCS I: } 1(9 \%) \text { vs. } 2 \\
\text { (17\%) } \\
\text { GMFCS II: } 6(55 \%) \text { vs. } 3 \\
\text { (25\%) } \\
\text { GMFCS III: } 3(27 \%) \text { vs. } 5 \\
\text { (42\%) } \\
\text { GMFCS IV: } 1(9 \%) \text { vs. } 2 \\
\text { (17\%) }\end{array}$ & $\begin{array}{l}\text { A vs. B, pre to post to followup mean (SD), p-value } \\
\text { GMFM-66B } \\
64.0(8.3) \text { to } 64.7(9.2) \text { to } 64.7(9.4), p=0.57 \text { vs. } 62.6 \\
(10.7) \text { to } 64.5(11.1) \text { to } 63.8(10.5), p=0.08 \\
\text { PODCl self } \\
10.0(14.6) \text { to }-1.0(24.8) \text { to } 16.0(17.45), p=0.52 \text { vs. } 23.0 \\
(23.6) \text { to } 19.5(12.1) \text { to } 24.0(16.0), p=0.73 \\
\text { PODCI parent } \\
12.9(16.2) \text { to } 19.4(12.9) \text { to } 17.2(16.0), p=0.17 \text { vs. } 17.2 \\
(17.6) \text { to } 18.2(19.4) \text { to } 21.4(21.2), p=0.34 \\
\text { MAS } \\
0.62(0.46) \text { to } 0.67(0.60) \text { to } 0.41(0.38), p=0.18 \text { vs. } 0.65 \\
(0.36) \text { to } 0.48(0.47) \text { to } 0.58(0.44), p=0.19\end{array}$ & NA \\
\hline
\end{tabular}




\begin{tabular}{|c|c|c|c|c|}
\hline \begin{tabular}{l|} 
Author, Year \\
Intervention Type \\
Duration of \\
Postintervention \\
Followup \\
Quality \\
(See Appendix B \\
for Full Citation) \\
\end{tabular} & $\begin{array}{l}\text { Intervention and } \\
\text { Comparison }\end{array}$ & $\begin{array}{l}\text { Population } \\
\text { Age (Mean) } \\
\text { Gender (\% Female) } \\
\text { Race (\%) } \\
\text { Ambulatory (\%) } \\
\text { Wheelchair User (\%) } \\
\text { Condition Specific (\%) } \\
\text { Other (\%) }\end{array}$ & Prioritized Outcomes & Other Outcomes \\
\hline $\begin{array}{l}\text { Yang } 2013 \\
\text { Aerobic Exercise } \\
\text { Treadmill } \\
\text { Pre to post, } \\
\text { crossover with } 2 \\
\text { months rest } \\
\text { between } \\
8 \text { week intervention } \\
\text { Fair }\end{array}$ & $\begin{array}{l}\text { A. Walking on a track } \\
\text { while stepping over } \\
\text { individualized series } \\
\text { of obstacles and on } \\
\text { targets (precision } \\
\text { training) one hour a } \\
\text { day for } 5 \text { days a } \\
\text { week for } 8 \text { weeks } \\
\\
\text { B. Walking on a } \\
\text { treadmill with body } \\
\text { weight support if } \\
\text { needed (used by } 5 \text { of } \\
10 \text { participants) at } \\
\text { faster than their } \\
\text { overground walking } \\
\text { speed for one hour a } \\
\text { day for } 5 \text { days a } \\
\text { week for } 8 \text { weeks } \\
\text { Crossover trial with } 2 \\
\text { months rest between }\end{array}$ & $\begin{array}{l}\text { Spinal cord injury } \\
n=22 \text { randomized and } 20 \\
\text { analyzed } \\
\text { Mean age } 46 \text { years } \\
14 \text { males and } 6 \text { females } \\
\text { ( } 70 \% \text { males) }\end{array}$ & $\begin{array}{l}\text { Pre to post change scores } \\
6 \text { minute walk test (meters) (change scores) } \\
\text { A. } 10 \\
\text { B. } 29 \\
\text { Both groups achieved significant improvement } p<0.05 \\
\text { Improvement significantly greater with treadmill training } \\
p=0.045 \\
10 \text { meter walk test (meters/second) } \\
\text { A. } 0.025 \\
\text { B. } 0.070 \\
\text { Both groups achieved significant improvement } p<0.05 \\
\text { No difference between groups } \\
\text { Spinal Cord Injury Functional Ambulatory Profile } \\
\text { (measures walking skills in daily life) } \\
\text { A. }-42 \\
\text { B. }-75 \\
\text { Both groups achieved significant improvement } p<0.05 \\
\text { No difference between groups }\end{array}$ & $\begin{array}{l}\text { CES-D (change scores) } \\
A=-2.5 \\
B=-2.3 \\
\text { Both groups achieved significant improvement } \\
p<0.05 \\
\text { No difference between groups }\end{array}$ \\
\hline $\begin{array}{l}\text { Yazgan } 2020 \\
\text { Postural Control } \\
\text { Motion Gaming } \\
\text { Postintervention, } \\
0 \text { weeks }\end{array}$ & $\begin{array}{l}\text { A. Nintendo Wii Fit, } \\
16 \text { sessions over } 8 \\
\text { weeks }(n=15) \\
\text { B. Balance Trainer } \\
\text { motion gaming, } 16 \\
\text { sessions over } 8 \\
\text { weeks ( } n=12) \\
\text { C. Waitlist control } \\
(n=15)\end{array}$ & $\begin{array}{l}\text { A vs. B vs. C } \\
\text { Age: } 47.5 \text { vs. } 43.1 \text { vs. } 40.7 \\
\text { Female: } 86.7 \% \text { vs. } 100 \% \text { vs. } \\
86.7 \% \\
\text { EDSS: } 4.16 \text { vs. } 3.83 \text { vs. } 4.06 \\
\text { RRMS: } 73.3 \% \text { vs. } 66.7 \% \text { vs. } \\
93.3 \%\end{array}$ & $\begin{array}{l}\text { A vs. C, Mean change scores: } \\
\text { BBS: } 5.8 \text { vs. } 0.93, p<0.05 \\
\text { TUG: }-1.54 \text { vs; } 0.05, p<0.05 \\
\text { 6MWT: } 42.71 \text { vs. } 7.59 p<0.05 \\
\text { MusiQoL: } 12.61 \text { vs. }-0.19, p<0.05 \\
\text { B vs. C, Mean change scores: } \\
\text { BBS: } 2.66 \text { vs. } 0.93, p<0.05 \\
\underline{\text { TUG: }}-0.64 \text { vs; } 0.05, p<0.05 \\
\text { 6MWT: } 23.25 \text { vs. } 7.59 p>0.05 \\
\text { MusiQoL: } 5.32 \text { vs. }-0.19, p<0.05 \\
\text { A vs. C, Mean change scores: } p<0.05 \text { in favor of group A } \\
\text { for BBS and MusiQoL }\end{array}$ & NA \\
\hline
\end{tabular}




\begin{tabular}{|c|c|c|c|c|}
\hline $\begin{array}{l}\text { Author, Year } \\
\text { Intervention Type } \\
\text { Duration of } \\
\text { Postintervention } \\
\text { Followup } \\
\text { Quality } \\
\text { (See Appendix B } \\
\text { for Full Citation) } \\
\end{array}$ & $\begin{array}{l}\text { Intervention and } \\
\text { Comparison }\end{array}$ & $\begin{array}{l}\text { Population } \\
\text { Age (Mean) } \\
\text { Gender (\% Female) } \\
\text { Race (\%) } \\
\text { Ambulatory (\%) } \\
\text { Wheelchair User (\%) } \\
\text { Condition Specific (\%) } \\
\text { Other (\%) } \\
\end{array}$ & Prioritized Outcomes & Other Outcomes \\
\hline $\begin{array}{l}\text { Yazici, } 2019 \\
\text { Aerobic Exercise } \\
\text { Robot-Assisted } \\
\text { Gait Training } \\
\text { Immediately } \\
\text { postintervention, } \\
12 \text { weeks } \\
\text { Poor }\end{array}$ & $\begin{array}{l}\text { A. Robot-assisted } \\
\text { gate (RAGT), } 36 \\
\text { sessions over } 12 \\
\text { weeks ( } n=12) \\
\\
\text { B. Physiotherapy } \\
\text { assumed, } 36 \\
\text { sessions over } 12 \\
\text { weeks assumed } \\
(n=12)\end{array}$ & $\begin{array}{l}\text { A vs. B } \\
\text { Age: } 8.8 \text { vs. } 9.5 \\
\text { Female: } 50 \% \text { vs. } 50 \% \\
\text { GMFCS I or II: } 100 \%\end{array}$ & $\begin{array}{l}\text { A vs. B, Mean or Median (SD), MD calculated as if all are } \\
\text { means, p=between groups } \\
\text { 6MWT: } \\
\text { 409.58 (49.1) to } 475.17(47.7) \text { vs. } 437.00(55.0) \text { to } 459.17 \\
(53.75) \text {; MD } 43.42,95 \% \mathrm{Cl} 19.64 \text { to } 67.21, p<0.001 \\
\text { GMFM-88: } \\
\text { 253.00 (8.81) to } 256.17(8.23) \text { vs. } 253.67(7.70) \text { to } 255.25 \\
(7.94), M D 1.59,95 \% \mathrm{Cl}-2.19 \text { to } 5.37, p=0.410 \\
\text { GMFM-88D: } \\
\text { 36.08 (2.27) to } 36.92(1.73) \text { vs. } 36.75(2.22) \text { to } 37.42 \\
(1.98), M D 0.17,95 \% \mathrm{Cl}-0.79 \text { to } 1.13, p=0.729 \\
\text { GMFM-88E: } 6 \\
4.00(6.90) \text { to } 66.25(6.78) \text { vs. } 64.08(6.43) \text { to } 64.92 \\
(6.72), M D 1.14,95 \% \mathrm{Cl}-1.69 \text { to } 4.51, p=0.373\end{array}$ & $\begin{array}{l}\text { BBS: } 50.08(2.43) \text { to } 52.08(2.68) \text { vs. } 50.25 \\
(2.93) \text { to } 51.00(3.30), \mathrm{MD} 1.25,95 \% \mathrm{Cl}-0.07 \\
\text { to } 2.57, \mathrm{p}=0.064\end{array}$ \\
\hline $\begin{array}{l}\text { Yildirim, } 2019 \\
\text { Aerobic Exercise } \\
\text { Robot-Assisted } \\
\text { Gait Training } \\
\text { Immediately } \\
\text { postintervention, } \\
8 \text { weeks } \\
\text { Fair }\end{array}$ & $\begin{array}{l}\text { A. Robot-assisted } \\
\text { gate (RAGT), } 16 \\
\text { sessions over } 8 \\
\text { weeks }+ \\
\text { conventional therapy } \\
(n=44) \\
\text { B. Conventional } \\
\text { therapy }(n=44)\end{array}$ & $\begin{array}{l}\text { A vs. B } \\
\text { Age: } 32 \text { vs. } 37 \\
\text { Female: } 39 \% \text { vs. } 36 \% \\
\text { Tetraplegia: } 20 \% \text { vs. } 16 \% \\
\text { ASIA Complete: } 48 \% \text { vs. } \\
41 \%\end{array}$ & $\begin{array}{l}\text { A vs. B, Median (Interquartile range), p-value=between } \\
\text { groups: } \\
\text { Functional Independence Measure (FIM): } 69 \text { (31) to } 85 \\
\text { (35) vs. } 67 \text { (36) to } 77 \text { (24), p=0.022 } \\
\text { Walking Index for Spinal Cord Injury (WISCI II): } 5 \text { (9) to } 9 \\
\text { (7) vs. } 5 \text { (6.7) to } 6.5 \text { (5), p=0.011 }\end{array}$ & NA \\
\hline
\end{tabular}




\begin{tabular}{|c|c|c|c|c|}
\hline $\begin{array}{l}\text { Author, Year } \\
\text { Intervention Type } \\
\text { Duration of } \\
\text { Postintervention } \\
\text { Followup } \\
\text { Quality } \\
\text { (See Appendix B } \\
\text { for Full Citation) } \\
\end{array}$ & $\begin{array}{l}\text { Intervention and } \\
\text { Comparison }\end{array}$ & $\begin{array}{l}\text { Population } \\
\text { Age (Mean) } \\
\text { Gender (\% Female) } \\
\text { Race (\%) } \\
\text { Ambulatory (\%) } \\
\text { Wheelchair User (\%) } \\
\text { Condition Specific (\%) } \\
\text { Other (\%) } \\
\end{array}$ & Prioritized Outcomes & Other Outcomes \\
\hline $\begin{array}{l}\text { Young, } 2019 \\
\text { Aerobic Exercise } \\
\text { Aerobics } \\
\text { Postintervention, } 0 \\
\text { weeks }\end{array}$ & $\begin{array}{l}\text { A. Movement to } \\
\text { Music } 36 \text { sessions } \\
\text { over } 12 \text { weeks (27) } \\
\text { B. Adapted Yoga } \\
\text { (26) } \\
\text { C. Waitlist control } \\
(28)\end{array}$ & $\begin{array}{l}\text { A vs. B vs. C } \\
\text { Age: } 50 \text { vs. } 48 \text { vs. } 47 \\
\text { Female: } 81 \% \text { vs. } 77 \% \text { vs. } \\
86 \% \\
\text { White: } 44 \text { vs. } 58 \% \text { vs. } 61 \% \\
\text { Patient Determined Disease } \\
\text { Steps (PDDS): } \\
\text { PDDS } 0: 30 \% \text { vs. } 46 \% \text { vs. } \\
21 \% \\
\text { PDDS } 3: 15 \% \text { vs. } 8 \% \text { vs. } \\
14 \% \\
\text { PDDS } 6: 11 \% \text { vs. } 4 \% \text { vs. } \\
11 \%\end{array}$ & $\begin{array}{l}\text { A vs. B vs. C } \\
\text { TUG A vs. C: }-1.89,95 \% \mathrm{Cl}-3.30 \text { to }-0.48, p=0.01 \\
\text { TUG B vs. C: }-1.20,95 \% \mathrm{Cl}-2.58 \text { to } 0.18, p=0.09 \\
\text { TUG B vs. A: } 0.69,95 \% \mathrm{Cl}-0.71 \text { to } 2.08, p=0.33 \\
\text { 6MWT A vs. C: } 40.98,95 \% \mathrm{Cl} 2.21 \text { to } 79.75 \\
\text { 6MWT B vs. C: } 22.83,95 \% \mathrm{Cl}-16.67 \text { to } 6.2, p=0.25 \\
\text { 6MWT B vs. A: }-18.15,95 \% \mathrm{Cl}-56.36 \text { to } 20.05 \\
\text { 5xSit-to-Stand A vs. C: }-1.00,95 \% \mathrm{Cl}-2.58 \text { to } 0.55, \\
p=0.20 \\
\text { 5xSit-to-Stand B vs. C: }-0.70,95 \% \mathrm{Cl}-2.17 \text { to } 0.77, \\
p=0.34 \\
\text { 5xSit-to-Stand B vs. A: } 0.30,95 \%-1.21 \text { to } 1.82, p=0.69\end{array}$ & NA \\
\hline $\begin{array}{l}\text { Zoccolillo, } 2015 \\
\text { Postural Control } \\
\text { Motion gaming } \\
\text { Postintervention, } 8 \\
\text { weeks } \\
\text { Poor }\end{array}$ & $\begin{array}{l}\text { A. X-box with Kinect } \\
\text { (3D motion capture) } \\
\text { gaming plus neuro- } \\
\text { developmental } \\
\text { treatment, } 16 \\
\text { sessions over } 8 \\
\text { weeks ( }=15) \\
\\
\text { B.Neurodevelopment } \\
\text { al treatment, } 16 \\
\text { sessions over } 8 \\
\text { weeks }(n=16)\end{array}$ & $\begin{array}{l}\text { A and B (combined) } \\
\text { Age (mean years): } 6.89 \\
\text { Female: NR } \\
\text { Race: NR } \\
\text { Ambulatory: NR } \\
\text { Wheelchair user: NR } \\
\text { GMFM88=84.6 } \pm 19.8 \%\end{array}$ & (2) & $\begin{array}{l}\text { A. vs. B., mean (SD) } \\
\text { QUEST (Quality of Upper Extremities Skills } \\
\text { Test) } \\
\text { A. } 76(21) \text { (baseline) } \\
81(20), p=0.003 \text { (postintervention) } \\
\text { B. NR (baseline) } \\
\text { (postintervention) NR, } p=0.056\end{array}$ \\
\hline
\end{tabular}

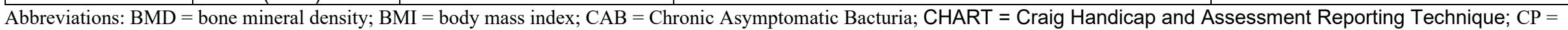

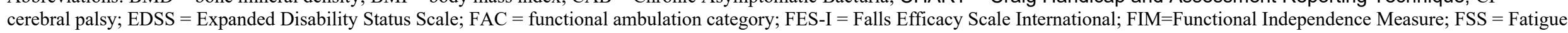

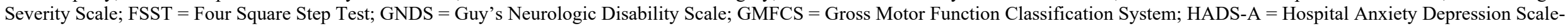

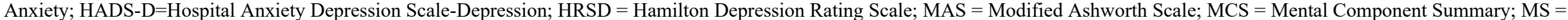

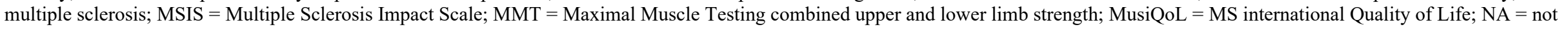

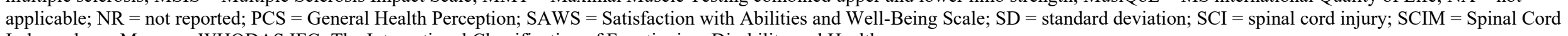
Independence Measure; WHODAS IFC=The International Classification of Functioning, Disability and Health

See Appendix B. Included Studies for full study citation. 
Table F-2. Harms and study characteristics (continuation of Table F-1 results by study)

\begin{tabular}{|c|c|c|c|c|c|c|}
\hline $\begin{array}{l}\text { Author, Year } \\
\text { Intervention Type } \\
\text { Duration of } \\
\text { Postintervention } \\
\text { Followup } \\
\text { Quality } \\
\text { (See Appendix B for } \\
\text { Full Citation) }\end{array}$ & Harms & Condition & Inclusion Criteria & $\begin{array}{l}\text { Number } \\
\text { Randomized } \\
\text { Analyzed } \\
\text { Attrition }\end{array}$ & $\begin{array}{l}\text { Country } \\
\text { Setting } \\
\text { Study Design }\end{array}$ & Funding Source \\
\hline $\begin{array}{l}\text { Acar, } 2016 \\
\text { Postural Control } \\
\text { Motion gaming } \\
\text { Postintervention, } 6 \\
\text { weeks } \\
\text { Poor }\end{array}$ & NR & Cerebral palsy & $\begin{array}{l}\text { Children with spastic } \\
\text { hemiparesis, } 6 \text { and } 15 \\
\text { years old, } 1-3 \text { Manual } \\
\text { Ability Classification } \\
\text { System, level 1or } 2 \text { of the } \\
\text { GMFCS and ability to } \\
\text { grasp and release an } \\
\text { object. }\end{array}$ & $\begin{array}{l}\text { Randomized:30 } \\
\text { Analyzed:3 } 0 \\
\text { Attrition: } 0 \% \\
(0 / 30)\end{array}$ & $\begin{array}{l}\text { Turkey } \\
\text { Outpatient clinic } \\
\text { RCT }\end{array}$ & NR \\
\hline $\begin{array}{l}\text { Abbasi, } 2019 \\
\text { Postural Control } \\
\text { Whole body vibration } \\
\text { Immediately } \\
\text { postintervention, } \\
6 \text { weeks } \\
\text { Fair }\end{array}$ & NR & Multiple sclerosis & $\begin{array}{l}\text { Inclusion Criteria: } 20-50 \\
\text { years old with MS, } \\
\text { ambulatory, EDSS 1-4.5 } \\
\text { with no relapse in the past } \\
2 \text { months }\end{array}$ & $\begin{array}{l}\text { Randomized: } \\
50 \\
\text { Analyzed:46 } \\
\text { Attrition: } 8.7 \% \\
(4 / 46)\end{array}$ & $\begin{array}{l}\text { Iran } \\
\text { Rehabilitation } \\
\text { Clinic } \\
\text { RCT }\end{array}$ & NR \\
\hline $\begin{array}{l}\text { Adar, } 2017 \\
\text { Aerobic Exercise } \\
\text { Aquatics } \\
\text { Postintervention, } 0 \\
\text { weeks } \\
\text { Fair }\end{array}$ & NR & Cerebral palsy & $\begin{array}{l}\text { Inclusion Criteria: } \\
\text { Diagnosis of CP, Age 4-18 } \\
\text { years. Grade }>1 \text { in lower } \\
\text { extremities according to } \\
\text { MAS, being able to being } \\
\text { medically able to } \\
\text { participate in an exercise } \\
\text { program (no severe } \\
\text { medical illness other than } \\
\mathrm{CP} \text { ), being able to follow } \\
\text { directions, and adherence } \\
\text { to the exercise program. }\end{array}$ & $\begin{array}{l}\text { Randomized: } \\
\mathrm{N}=32 \\
\text { Analyzed: } 32 \\
\text { Attrition: } 0 \% \\
(0 / 32)\end{array}$ & $\begin{array}{l}\text { Turkey } \\
\text { Outpatient clinic } \\
\text { RCT }\end{array}$ & None \\
\hline
\end{tabular}




\begin{tabular}{|c|c|c|c|c|c|c|}
\hline $\begin{array}{l}\text { Author, Year } \\
\text { Intervention Type } \\
\text { Duration of } \\
\text { Postintervention } \\
\text { Followup } \\
\text { Quality } \\
\text { (See Appendix B for } \\
\text { Full Citation) }\end{array}$ & Harms & Condition & Inclusion Criteria & $\begin{array}{l}\text { Number } \\
\text { Randomized } \\
\text { Analyzed } \\
\text { Attrition }\end{array}$ & $\begin{array}{l}\text { Country } \\
\text { Setting } \\
\text { Study Design }\end{array}$ & Funding Source \\
\hline $\begin{array}{l}\text { Afrasiabifar, } 2018 \\
\text { Postural Control } \\
\text { Balance } \\
\text { Postintervention, } 12 \\
\text { weeks } \\
\text { Good }\end{array}$ & $\begin{array}{l}\text { No adverse events reported } \\
\text { Cawthorne-Cooksey Group: Lost to } \\
\text { followup after } 8 \text { weeks }(n=1) \\
\text {-Unable to participate regularly }(n=1) \\
\text { Frankel Group: Lost to followup after } 8 \\
\text { weeks ( } n=2) \\
\text {-Unable to participate regularly }(n=1) \\
\text {-Disease relapses }(n=1)\end{array}$ & Multiple sclerosis & $\begin{array}{l}\text { Patients with confirmed } \\
\text { MS for } 6 \text { months, in } \\
\text { remission, aged } 15 \text { to } 55 \\
\text { years, ability to stand for } \\
30 \text { seconds and to walk a } \\
\text { distance of } 6 \text { meters } \\
\text { without any assistance, } \\
\text { and BBS score of } 21-40 .\end{array}$ & $\begin{array}{l}\text { Randomized: } \\
75 \\
\text { Analyzed: } 72 \\
\text { Attrition: } 4 \% \\
(3 / 75)\end{array}$ & $\begin{array}{l}\text { Iran } \\
\text { Outpatient clinic } \\
\text { RCT }\end{array}$ & $\begin{array}{l}\text { University } \\
\text { This work was } \\
\text { supported by a Master } \\
\text { thesis grant from the } \\
\text { Deputy of Research } \\
\text { and Technology of } \\
\text { Yasuj University of } \\
\text { Medical Sciences, } \\
\text { Iran }\end{array}$ \\
\hline $\begin{array}{l}\text { Ahmadi, } 2013 \\
\text { Aerobic Exercise } \\
\text { Treadmill } \\
\text { Postural Control } \\
\text { Yoga } \\
\text { Immediately } \\
\text { postintervention, } \\
8 \text { weeks } \\
\text { Fair }\end{array}$ & None & Multiple sclerosis & $\begin{array}{l}\text { Inclusion Criteria: Women } \\
\text { aged } 19 \text { to } 54 \text { with EDSS } \\
\text { score } 1-4\end{array}$ & $\begin{array}{l}\text { Randomized: } \\
31 \\
\text { Analyzed: } 31 \\
\text { Attrition: NR }\end{array}$ & $\begin{array}{l}\text { Iran } \\
\text { Outpatient clinic } \\
\text { RCT }\end{array}$ & NR \\
\hline $\begin{array}{l}\text { Ahmadizadeh, } 2020 \\
\text { Postural Control } \\
\text { Whole Body Vibration } \\
\text { Postintervention, } 6 \\
\text { weeks }\end{array}$ & NR & Cerebral Palsy & $\begin{array}{l}\text { Children with CP, up to } 12 \\
\text { years old; able to walk } \\
\text { without falling or without } \\
\text { walking aids, ability to } \\
\text { follow orders and to be at } \\
\text { the level of } \\
1,2 \text { and } 3 \text { GMFCS }\end{array}$ & $\begin{array}{l}\text { Randomized: } \\
20 \\
\text { Analyzed: } 20 \\
\text { Attrition: } 0 \% \\
(0 / 20)\end{array}$ & $\begin{array}{l}\text { Iran } \\
\text { Outpatient } \\
\text { rehabilitation } \\
\text { RCT }\end{array}$ & $\begin{array}{l}\text { Neuromuscular } \\
\text { Rehabilitation } \\
\text { Research Center of } \\
\text { Semnan University } \\
\text { of Medical Sciences }\end{array}$ \\
\hline Fair & & & & & & \\
\hline
\end{tabular}




\begin{tabular}{|c|c|c|c|c|c|c|}
\hline $\begin{array}{l}\text { Author, Year } \\
\text { Intervention Type } \\
\text { Duration of } \\
\text { Postintervention } \\
\text { Followup } \\
\text { Quality } \\
\text { (See Appendix B for } \\
\text { Full Citation) }\end{array}$ & Harms & Condition & Inclusion Criteria & $\begin{array}{l}\text { Number } \\
\text { Randomized } \\
\text { Analyzed } \\
\text { Attrition } \\
\end{array}$ & \begin{tabular}{|l|} 
Country \\
Setting \\
Study Design \\
\end{tabular} & Funding Source \\
\hline $\begin{array}{l}\text { Akkurt, } 2017 \\
\text { Aerobic Exercise } \\
\text { Hand cycling } \\
\text { Postintervention, } 0 \\
\text { weeks } \\
\text { Fair }\end{array}$ & NR & Spinal cord injury & $\begin{array}{l}\text { Participants were included } \\
\text { if: } 1 \text { ) they were aged } \\
\text { between } 15 \text { and } 65 \text { years; } \\
\text { 2) all lesions were } \\
\text { traumatic; 3) lesion levels } \\
\text { were C7-L5; 4) they were } \\
\text { at least } 1 \text { month } \\
\text { postinjury; 5) they were } \\
\text { physically active in } \\
\text { training and outdoor } \\
\text { mobility less than two } \\
\text { hours a week; } 6 \text { ) they } \\
\text { received medical approval } \\
\text { for participation in physical } \\
\text { activity; } 7 \text { ) they had the } \\
\text { ability to read and write } \\
\text { the Turkish language. }\end{array}$ & $\begin{array}{l}\text { Randomized: } \\
\mathrm{N}=40 \\
\text { Analyzed:33 } \\
\text { Attrition: } 18 \% \\
(7 / 40)\end{array}$ & $\begin{array}{l}\text { Turkey } \\
\text { Outpatient clinic } \\
\text { RCT }\end{array}$ & NR \\
\hline $\begin{array}{l}\text { Alexeeva } 2011 \\
\text { Aerobic Exercise } \\
\text { Treadmill } \\
\text { Pre to post } \\
\text { Fair }\end{array}$ & $\begin{array}{l}2 \text { experienced an increase in spasticity with } \\
\text { slower walking times after training }\end{array}$ & Spinal cord injury & $\begin{array}{l}\text { Injury at T10 or below } \\
17-60 \text { years old } \\
\text { Able to rise from sitting } \\
\text { with minimal assistance } \\
\text { and independently } \\
\text { advance at least one leg }\end{array}$ & \begin{tabular}{|l|} 
Randomized 40 \\
Analyzed 35 \\
Attrition \\
$5 / 35=12.5 \%$ \\
(13 w \\
intervention)
\end{tabular} & $\begin{array}{l}\text { USA (author), } \\
\text { states recruited } \\
\text { nationally to } \\
\text { internationally but } \\
\text { does not provide } \\
\text { details } \\
\text { Coordinated at } \\
\text { large spinal cord } \\
\text { injury rehabilitation } \\
\text { hospital } \\
\text { RCT }\end{array}$ & Government funding \\
\hline $\begin{array}{l}\text { Al-Sharman, } 2019 \\
\text { Aerobic Exercise } \\
\text { Aerobics } \\
\text { Postintervention, } 6 \\
\text { weeks } \\
\text { Poor }\end{array}$ & $\mathrm{N}=1$ patient broke a leg. & Multiple sclerosis & $\begin{array}{l}\text { Multiple sclerosis patients } \\
\text { attending neurology clinics } \\
\text { with EDSS } 3-5.5>5\end{array}$ & $\begin{array}{l}\text { Randomized: } \\
40 \\
\text { Analyzed: } 30 \\
\text { Attrition: } 25 \% \\
(10 / 40)\end{array}$ & $\begin{array}{l}\text { Jordan } \\
\text { Neurological } \\
\text { hospital clinic } \\
\text { RCT }\end{array}$ & NR \\
\hline
\end{tabular}




\begin{tabular}{|c|c|c|c|c|c|c|}
\hline $\begin{array}{l}\text { Author, Year } \\
\text { Intervention Type } \\
\text { Duration of } \\
\text { Postintervention } \\
\text { Followup } \\
\text { Quality } \\
\text { (See Appendix B for } \\
\text { Full Citation) }\end{array}$ & Harms & Condition & Inclusion Criteria & $\begin{array}{l}\text { Number } \\
\text { Randomized } \\
\text { Analyzed } \\
\text { Attrition }\end{array}$ & $\begin{array}{l}\text { Country } \\
\text { Setting } \\
\text { Study Design }\end{array}$ & Funding Source \\
\hline $\begin{array}{l}\text { Amiri, } 2019 \\
\text { Postural Control } \\
\text { Balance exercises } \\
\text { Strength interventions } \\
\text { Muscle Strength } \\
\text { Exercises } \\
\text { Postintervention, } 10 \\
\text { weeks } \\
\text { Fair }\end{array}$ & NR & Multiple sclerosis & $\begin{array}{l}\text { Inclusion Criteria: Women } \\
\text { with relapse MS, } \\
\text { subgroup scores of EDSS } \\
2.5 \text { to } 5.5\end{array}$ & $\begin{array}{l}\text { Randomized: } \\
69 \text { (in abstract } \\
\text { (72, Figure 1) } \\
\text { Analyzed: } 69 \\
\text { Attrition: } 4 \% \\
(3 / 69)\end{array}$ & \begin{tabular}{|l|} 
Iran \\
Outpatient clinic for \\
sports injury \\
RCT
\end{tabular} & NR \\
\hline $\begin{array}{l}\text { Aras, } 2019 \\
\text { Aerobic Exercise } \\
\text { Robot-Assisted Gait } \\
\text { Training } \\
\text { Postintervention, } \\
4 \text { weeks, and } 6 \text { month } \\
\text { followup } \\
\text { Fair }\end{array}$ & None reported & Cerebral Palsy & $\begin{array}{l}\text { Children with CP, } 6 \text { to } 14 \\
\text { years; } \\
\text { Level II-III GMFCS and } \\
\text { able to ambulate at least } \\
10 \text { meters } \\
\text { with or without an } \\
\text { assistive device }\end{array}$ & $\begin{array}{l}\text { Randomized: } \\
30 \\
\text { Analyzed: } 29 \\
\text { Attrition: } 3 \% \\
(1 / 30)\end{array}$ & $\begin{array}{l}\text { Turkey } \\
\text { Outpatient } \\
\text { rehabilitation } \\
\text { RCT }\end{array}$ & No funding received \\
\hline $\begin{array}{l}\text { Arntzen, } 2019 \\
\text { Arntzen, } 2020 \\
\text { Postural Control } \\
\text { Balance exercises } \\
\text { Postintervention, } 7 \\
\text { weeks, plus } 18 \text { and } 30 \\
\text { weeks } \\
\text { Good }\end{array}$ & None & Multiple sclerosis & $\begin{array}{l}\text { Diagnosis of MS; Patients } \\
\text { with expanded disability } \\
\text { scores of } 1 \text { to } 6.5\end{array}$ & $\begin{array}{l}\text { Randomized: } \\
80 \\
\text { Analyzed: } 80 \\
\text { Attrition: } 0 \% \\
(80 / 80) \text { post } \\
\text { intervention }\end{array}$ & $\begin{array}{l}\text { Norway } \\
\text { Setting Outpatient } \\
\text { clinic } \\
\text { RCT }\end{array}$ & $\begin{array}{l}\text { Norway Regional } \\
\text { Health Authority }\end{array}$ \\
\hline
\end{tabular}




\begin{tabular}{|c|c|c|c|c|c|c|}
\hline $\begin{array}{l}\text { Author, Year } \\
\text { Intervention Type } \\
\text { Duration of } \\
\text { Postintervention } \\
\text { Followup } \\
\text { Quality } \\
\text { (See Appendix B for } \\
\text { Full Citation) } \\
\end{array}$ & Harms & Condition & Inclusion Criteria & $\begin{array}{l}\text { Number } \\
\text { Randomized } \\
\text { Analyzed } \\
\text { Attrition } \\
\end{array}$ & \begin{tabular}{|l|} 
Country \\
Setting \\
Study Design \\
\end{tabular} & Funding Source \\
\hline $\begin{array}{l}\text { Aviram } 2017 \\
\text { Aerobic Exercise } \\
\text { Treadmill } \\
\text { Postintervention, } 6 \\
\text { months } \\
\text { Fair }\end{array}$ & None reported & Cerebral palsy & 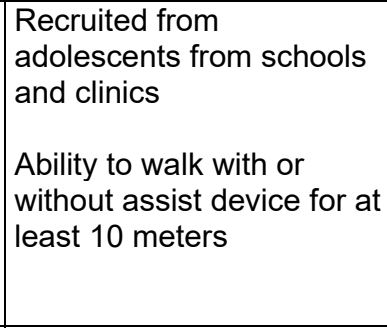 & $\begin{array}{l}95 \text { randomized } \\
95 \text { assessed }\end{array}$ & $\begin{array}{l}\text { Israel, Jordan, } \\
\text { Palestine }\end{array}$ & Funding NR \\
\hline $\begin{array}{l}\text { Aydin, } 2014 \\
\text { Aerobic Exercise } \\
\text { Aerobics } \\
\text { Postintervention, } 12 \\
\text { weeks } \\
\text { Fair }\end{array}$ & $\begin{array}{l}\text { No harms reported. } \\
\text { Note: Two patients discontinued due to } \\
\text { "failure to adapt to the exercise" }\end{array}$ & Multiple sclerosis & $\begin{array}{l}\text { Patients with relapsing- } \\
\text { remitting type of MS and } \\
\text { EESS scores above } 4.5\end{array}$ & $\begin{array}{l}\text { Randomized: } \\
40 \\
\text { Analyzed: } 36 \\
\text { Attrition: } 10 \% \\
(4 / 40)\end{array}$ & $\begin{array}{l}\text { Germany } \\
\text { Outpatient clinic } \\
\text { RCT }\end{array}$ & NR \\
\hline $\begin{array}{l}\text { Azimzadeh, } 2015 \\
\text { Postural Control } \\
\text { Tai Chi } \\
\text { Postintervention, } 12 \\
\text { weeks } \\
\text { Poor }\end{array}$ & NR & Multiple sclerosis & $\begin{array}{l}\text { Women between } 20 \text { and } \\
60 \text { years old; diagnosed } \\
\text { with MS by a physician } \\
\text { specialist based on their } \\
\text { medical records; EDSS } \\
\text { scores equal to or less } \\
\text { than } 5 / 5 \text { based on medical } \\
\text { records; No other acute or } \\
\text { chronic debilitating } \\
\text { conditions such as lung } \\
\text { and heart diseases, } \\
\text { musculoskeletal disorders, } \\
\text { mental or psychological } \\
\text { problems based on } \\
\text { patients' statements and } \\
\text { medical records; Absence } \\
\text { of any stage of pregnancy }\end{array}$ & $\begin{array}{l}\text { Randomized: } \\
36 \\
\text { Analyzed:34 } \\
\text { Attrition: } 5.5 \% \\
(2 / 36)\end{array}$ & \begin{tabular}{|l} 
Iran \\
Group setting - \\
location NR \\
RCT
\end{tabular} & Unclear \\
\hline
\end{tabular}




\begin{tabular}{|c|c|c|c|c|c|c|}
\hline $\begin{array}{l}\text { Author, Year } \\
\text { Intervention Type } \\
\text { Duration of } \\
\text { Postintervention } \\
\text { Followup } \\
\text { Quality } \\
\text { (See Appendix B for } \\
\text { Full Citation) }\end{array}$ & Harms & Condition & Inclusion Criteria & $\begin{array}{l}\text { Number } \\
\text { Randomized } \\
\text { Analyzed } \\
\text { Attrition } \\
\end{array}$ & $\begin{array}{l}\text { Country } \\
\text { Setting } \\
\text { Study Design }\end{array}$ & Funding Source \\
\hline $\begin{array}{l}\text { Bahrami, 2019a } \\
\text { Aerobic Exercise } \\
\text { Treadmill } \\
\text { Immediately } \\
\text { postintervention, } \\
8 \text { weeks } \\
\text { Fair }\end{array}$ & $\begin{array}{l}\text { None - reported that no injury occurred that } \\
\text { was due to the intervention. }\end{array}$ & Cerebral palsy & $\begin{array}{l}\text { Inclusion Criteria: spastic } \\
\text { CP patients aged 18-45 } \\
\text { year old with GMFCS } \\
\text { level I to III }\end{array}$ & $\begin{array}{l}\text { Randomized: } \\
35 \\
\text { Analyzed: } 29 \\
\text { Attrition: } 17 \% \\
(6 / 35)\end{array}$ & $\begin{array}{l}\text { Iran } \\
\text { Outpatient rehab } \\
\text { clinic } \\
\text { RCT }\end{array}$ & Iran University Grant \\
\hline $\begin{array}{l}\text { Baquet, } 2018 \\
\text { Aerobic Exercise } \\
\text { Cycling } \\
\text { Postintervention, } 12 \\
\text { weeks } \\
\text { Fair }\end{array}$ & NR & Multiple sclerosis & $\begin{array}{l}\text { Patients had to be } \\
\text { diagnosed with RRMS } \\
\text { according to } \\
\text { the McDonald criteria } \\
2010, \text { an } \\
\text { EDSS score }<3.5 \text {, and } \\
\text { currently in remission with } \\
\text { no relapse or } \\
\text { progression during the last } \\
3 \text { months. Patients had to } \\
\text { be on stable } \\
\text { immunotherapy for } \\
\text { more than } 3 \text { months or } \\
\text { without any planned } \\
\text { change in disease- } \\
\text { modifying therapies for the } \\
\text { next } 6 \text { months. }\end{array}$ & $\begin{array}{l}\text { Randomized: } \\
\mathrm{N}=64\end{array}$ & $\begin{array}{l}\text { Germany } \\
\text { Outpatient clinic } \\
\text { RCT with } 12 \text { week } \\
\text { extension }\end{array}$ & $\begin{array}{l}\text { German Ministry of } \\
\text { Research and } \\
\text { Education }\end{array}$ \\
\hline
\end{tabular}




\begin{tabular}{|c|c|c|c|c|c|c|}
\hline $\begin{array}{l}\text { Author, Year } \\
\text { Intervention Type } \\
\text { Duration of } \\
\text { Postintervention } \\
\text { Followup } \\
\text { Quality } \\
\text { (See Appendix B for } \\
\text { Full Citation) }\end{array}$ & Harms & Condition & Inclusion Criteria & $\begin{array}{l}\text { Number } \\
\text { Randomized } \\
\text { Analyzed } \\
\text { Attrition }\end{array}$ & \begin{tabular}{|l} 
Country \\
Setting \\
Study Design
\end{tabular} & Funding Source \\
\hline $\begin{array}{l}\text { Bleyenheuft, } 2017 \\
\text { Postural Control } \\
\text { Balance } \\
\text { Postintervention, } 12 \\
\text { weeks } \\
\text { Poor }\end{array}$ & NR & Cerebral palsy & $\begin{array}{l}\text { Patients diagnosed with } \\
\text { bilateral CP in GMFCS } \\
\text { levels II to IV; age } 6 \text { to } 16 \\
\text { years; an ability to grasp } \\
\text { light objects and lift the } \\
\text { more affected arm } 15 \mathrm{~cm} \\
\text { above a table surface, } \\
\text { school level equal to that } \\
\text { of typically developing } \\
\text { peers; ability to follow } \\
\text { instructions and complete } \\
\text { testing }\end{array}$ & $\begin{array}{l}\text { Randomized: } \\
20 \\
\text { Analyzed:20 } \\
\text { Attrition: } 0 \% \\
(0 / 20)\end{array}$ & $\begin{array}{l}\text { Belgium, USA } \\
\text { Day camp } \\
\text { Non randomized } \\
\text { study } \\
\text { (quasirandomized) }\end{array}$ & $\begin{array}{l}\text { This study was a pilot } \\
\text { for a larger trial } \\
\text { (NCT02667613). The } \\
\text { work was supported in } \\
\text { part by Goldman } \\
\text { Sachs Gives and by } \\
\text { Mindy and Mark } \\
\text { Dehnert. YB had a } \\
\text { research grant from } \\
\text { the Fonds de la } \\
\text { recherche clinique, } \\
\text { cliniques } \\
\text { universitaires Saint- } \\
\text { Luc, Brussels, } \\
\text { Belgium. }\end{array}$ \\
\hline $\begin{array}{l}\text { Brichetto, } 2015 \\
\text { Postural Control } \\
\text { Balance } \\
\text { Postintervention, } 4 \\
\text { weeks } \\
\text { Good }\end{array}$ & NR & Multiple sclerosis & $\begin{array}{l}\text { Patients } 18 \text { years or older; } \\
\text { stable without relapses or } \\
\text { worsening in the last } 3 \\
\text { months; history of falls (at } \\
\text { least one fall in the last } \\
\text { year) }\end{array}$ & $\begin{array}{l}\text { Randomized: } \\
32 \\
\text { Analyzed:32 } \\
\text { Attrition: } 0 \% \\
(0 / 32)\end{array}$ & $\begin{array}{l}\text { Italy } \\
\text { Outpatient clinic } \\
\text { RCT }\end{array}$ & None \\
\hline $\begin{array}{l}\text { Bryant, } 2013 \\
\text { Aerobic Exercise } \\
\text { Cycling } \\
\text { Postintervention, } 12 \\
\text { weeks } \\
\text { Fair }\end{array}$ & NR & Cerebral palsy & $\begin{array}{l}\text { Children aged 8-17 years, } \\
\text { with cerebral palsy at } \\
\text { GMFCS levels IV and V, } \\
\text { able to pedal on an } \\
\text { adapted static bicycle and } \\
\text { walk with partial body } \\
\text { weight support on a } \\
\text { treadmill }\end{array}$ & $\begin{array}{l}\text { Randomized: } \\
\mathrm{N}=23 \\
\text { Analyzed: } 21 \\
\text { Attrition: } 10 \% \\
(2 / 21)\end{array}$ & $\begin{array}{l}\text { UK } \\
\text { School } \\
\text { RCT }\end{array}$ & $\begin{array}{l}\text { National Institute for } \\
\text { Health Research }\end{array}$ \\
\hline
\end{tabular}




\begin{tabular}{|c|c|c|c|c|c|c|}
\hline $\begin{array}{l}\text { Author, Year } \\
\text { Intervention Type } \\
\text { Duration of } \\
\text { Postintervention } \\
\text { Followup } \\
\text { Quality } \\
\text { (See Appendix B for } \\
\text { Full Citation) }\end{array}$ & Harms & Condition & Inclusion Criteria & $\begin{array}{l}\text { Number } \\
\text { Randomized } \\
\text { Analyzed } \\
\text { Attrition }\end{array}$ & $\begin{array}{l}\text { Country } \\
\text { Setting } \\
\text { Study Design }\end{array}$ & Funding Source \\
\hline $\begin{array}{l}\text { Bryant, } 2013 \\
\text { Aerobic Exercise } \\
\text { Cycling } \\
\text { Postintervention, } 12 \\
\text { weeks } \\
\text { Fair }\end{array}$ & None reported & Cerebral palsy & $\begin{array}{l}\text { Children aged 8-17 years, } \\
\text { with cerebral palsy at } \\
\text { GMFCS levels IV and V, } \\
\text { able to pedal on an } \\
\text { adapted static bicycle and } \\
\text { walk with partial body } \\
\text { weight support on a } \\
\text { treadmill }\end{array}$ & $\begin{array}{l}\text { Randomized: } \\
\mathrm{N}=24 \\
\text { Analyzed: } 22 \\
\text { Attrition: } 8 \% \\
(2 / 24)\end{array}$ & $\begin{array}{l}\text { UK } \\
\text { School } \\
\text { RCT }\end{array}$ & $\begin{array}{l}\text { National Institute for } \\
\text { Health Research }\end{array}$ \\
\hline $\begin{array}{l}\text { Bulguroglu, } 2017 \\
\text { Muscle Strength } \\
\text { Postintervention, } 0 \\
\text { weeks } \\
\text { Poor }\end{array}$ & NR & Multiple sclerosis & $\begin{array}{l}\text { Being over } 18 \text { years of } \\
\text { age; not having had an } \\
\text { MS attack or any surgery } \\
\text { in the last } 6 \text { months, being } \\
\text { below } 4.5 \text { EDSS score }\end{array}$ & $\begin{array}{l}\text { Randomized: } \\
45 \\
\text { Analyzed: } 38 \\
\text { Attrition: } 15.6 \% \\
\text { (7/45) }\end{array}$ & $\begin{array}{l}\text { Turkey } \\
\text { Outpatient } \\
\text { RCT }\end{array}$ & NR \\
\hline $\begin{array}{l}\text { Burschka, } 2014 \\
\text { Postural Control } \\
\text { Tai Chi } \\
\text { Postintervention, } 24 \\
\text { weeks } \\
\text { Poor }\end{array}$ & $\begin{array}{l}\text { NR } \\
\text { (One patient in the Tai Chi withdrew due to } \\
\text { unspecified health issues.) }\end{array}$ & Multiple sclerosis & $\begin{array}{l}\text { MS patients able to walk } \\
\text { without a walking aid, an } \\
\text { EDSS score }<5 \text {, relapse- } \\
\text { free for the past } 4 \text { weeks }\end{array}$ & $\begin{array}{l}\text { Randomized: } \\
38 \\
\text { Analyzed: } 32 \\
\text { Attrition: } 15 \% \\
(6 / 38)^{*} \\
\\
\text { *Six patients } \\
\text { from the Tai } \\
\text { Chi group } \\
\text { withdrew from } \\
\text { the study due } \\
\text { to time issues } \\
(\mathrm{N}=5) \text { and } \\
(\mathrm{N}=1) \text { health } \\
\text { problems, } 32 \\
\text { patients was } \\
\text { included in the } \\
\text { final analysis } \\
\end{array}$ & $\begin{array}{l}\text { Germany } \\
\text { Outpatient clinic } \\
\text { RCT (two-arm trial) }\end{array}$ & $\begin{array}{l}\text { Industry } \\
\text { Novartis Pharma } \\
\text { GmbH. }\end{array}$ \\
\hline
\end{tabular}




\begin{tabular}{|c|c|c|c|c|c|c|}
\hline $\begin{array}{l}\text { Author, Year } \\
\text { Intervention Type } \\
\text { Duration of } \\
\text { Postintervention } \\
\text { Followup } \\
\text { Quality } \\
\text { (See Appendix B for } \\
\text { Full Citation) }\end{array}$ & Harms & Condition & Inclusion Criteria & $\begin{array}{l}\text { Number } \\
\text { Randomized } \\
\text { Analyzed } \\
\text { Attrition }\end{array}$ & $\begin{array}{l}\text { Country } \\
\text { Setting } \\
\text { Study Design }\end{array}$ & Funding Source \\
\hline $\begin{array}{l}\text { Cakit, } 2010 \\
\text { Multimodal Exercise } \\
\text { Postintervention, } 0 \\
\text { weeks } \\
\text { Poor }\end{array}$ & NR & Multiple sclerosis & $\begin{array}{l}\text { Clinically or laboratorially } \\
\text { definite relapsing-remitting } \\
\text { or secondary progressive } \\
\text { MS, mild or moderate MS } \\
\text { determined by Kurtzke } \\
\text { Expanded Disability } \\
\text { Status Scale scores of } \\
6.0, \text { and ability to stand } \\
\text { independently in upright } \\
\text { position for } 3 \text { secs and if } \\
\text { they had been without } \\
\text { steroid and } \\
\text { immunosuppressive } \\
\text { therapy } \\
\text { within the past } 4 \text { weeks }\end{array}$ & \begin{tabular}{|l|} 
Randomized: \\
45 \\
Analyzed: 33 \\
Attrition: $27 \%$ \\
(12/45) [Across \\
entire study - all \\
3 study arms]
\end{tabular} & $\begin{array}{l}\text { Turkey } \\
\text { Outpatient/home } \\
\text { RCT }\end{array}$ & NR \\
\hline $\begin{array}{l}\text { Calabro, } 2017 \\
\text { Aerobic Exercise } \\
\text { Robot-Assisted Gait } \\
\text { Training } \\
\text { Postintervention, } 8 \\
\text { weeks } \\
\text { Good }\end{array}$ & NA & $\begin{array}{l}\text { Multiple } \\
\text { sclerosis }\end{array}$ & $\begin{array}{l}\text { Inclusion Criteria: RRMS, } \\
\text { Age } 18-65, \text { moderate to } \\
\text { severe walking disability } \\
\text { EDSS } 4.0-5.5 \text {, Montreal } \\
\text { Cognitive Score }>24, \text { no } \\
\text { neurological or orthopedic } \\
\text { co-morbidities that } \\
\text { interfere with ambulation, } \\
\text { stable medications for } 6 \\
\text { months }\end{array}$ & $\begin{array}{l}\text { Randomized: } \\
\mathrm{N}=40 \\
\text { Analyzed: } 40 \\
\text { Attrition: } 0 \% \\
(0 / 40)\end{array}$ & \begin{tabular}{|l|} 
Italy \\
Outpatient \\
Randomized \\
Control Trial
\end{tabular} & None \\
\hline $\begin{array}{l}\text { Callesen, } 2019 \\
\text { Postural Control } \\
\text { Balance exercises } \\
\text { Strength interventions } \\
\text { Muscle Strength } \\
\text { Exercises } \\
\text { Postintervention,10 } \\
\text { weeks } \\
\text { Fair }\end{array}$ & $\begin{array}{l}\text { PRT group reported three falls but they } \\
\text { were not related to the intervention. }\end{array}$ & Multiple sclerosis & $\begin{array}{l}\text { Inclusion Criteria: People } \\
18 \text { years or older, EDSS } \\
\text { scale } 2.0 \text { to } 6.5\end{array}$ & $\begin{array}{l}\text { Randomized: } \\
71 \\
\text { Analyzed: } 71 \\
\text { Attrition: } 17 \% \\
(12 / 71)\end{array}$ & $\begin{array}{l}\text { Denmark } \\
\text { Outpatient clinic } \\
\text { RCT }\end{array}$ & $\begin{array}{l}\text { Danish foundation } \\
\text { TrygFonden }\end{array}$ \\
\hline
\end{tabular}




\begin{tabular}{|c|c|c|c|c|c|c|}
\hline $\begin{array}{l}\text { Author, Year } \\
\text { Intervention Type } \\
\text { Duration of } \\
\text { Postintervention } \\
\text { Followup } \\
\text { Quality } \\
\text { (See Appendix B for } \\
\text { Full Citation) }\end{array}$ & Harms & Condition & Inclusion Criteria & $\begin{array}{l}\text { Number } \\
\text { Randomized } \\
\text { Analyzed } \\
\text { Attrition }\end{array}$ & $\begin{array}{l}\text { Country } \\
\text { Setting } \\
\text { Study Design }\end{array}$ & Funding Source \\
\hline $\begin{array}{l}\text { Carling, } 2017 \\
\text { Postural Control } \\
\text { Balance } \\
\text { Postintervention, } 7 \\
\text { weeks } \\
\text { Fair }\end{array}$ & $\begin{array}{l}\text { Two adverse events (both falls) occurred } \\
\text { during intervention, neither fall was injurious } \\
\text { (cited from text). } \\
\text { Note: Figure } 1 \text { indicates ( } n=2 \text { ) Lost to } \\
\text { followup due to fall related fractures in the } \\
\text { early-intervention group } \\
\text { Prospectively reported falls: } \\
\text { The late-start group reported a total of } 245 \\
\text { falls and } 2220 \text { near falls during the study } \\
\text { period, giving a fall } \\
\text { rate of } 1.28 / p e r s o n / \text { month and a near fall } \\
\text { rate of } 11.64 / \\
\text { person/month }\end{array}$ & Multiple sclerosis & $\begin{array}{l}\text { Walking ability not } \\
\text { exceeding } 200 \mathrm{~m} \text { (with or } \\
\text { without a walking aid) }\end{array}$ & $\begin{array}{l}\text { Randomized: } \\
51 \\
\text { Analyzed: } 48 \\
\text { Attrition: } 6 \% \\
(3 / 51)\end{array}$ & $\begin{array}{l}\text { Sweden } \\
\text { Outpatient } \\
\text { RCT }\end{array}$ & $\begin{array}{l}\text { Mixed } \\
\text { Study was supported } \\
\text { by } \\
\text { grants from the } \\
\text { Uppsala-Örebro } \\
\text { Regional Research } \\
\text { Committé, the } \\
\text { research committee of } \\
\text { Örebro County } \\
\text { Council and the } \\
\text { Norrbacka-Eugenia } \\
\text { Foundation }\end{array}$ \\
\hline $\begin{array}{l}\text { Castro-Sanchez, } 2012 \\
\text { Aerobic Exercise } \\
\text { Aquatics } \\
\text { Postintervention, } 20 \\
\text { weeks, and } 30 \text { weeks } \\
\text { Good }\end{array}$ & NR & Multiple sclerosis & $\begin{array}{l}\text { Inclusion Criteria: People } \\
18 \text { to } 75 \text { years old, EDSS } \\
\text { scale } \leq 7.5 \text {, VAS }>4 \text {. }\end{array}$ & $\begin{array}{l}\text { Randomized: } \\
73 \\
\text { Analyzed: } 71 \\
\text { Attrition: } 2 \% \\
(2 / 73)\end{array}$ & $\begin{array}{l}\text { Spain } \\
\text { Outpatient therapy } \\
\text { clinic } \\
\text { RCT }\end{array}$ & NR \\
\hline $\begin{array}{l}\text { Chen, } 2016 \\
\text { Muscle Strength } \\
8 \text { weeks - mid } \\
\text { intervention } \\
16 \text { weeks - mid } \\
\text { intervention } \\
\text { Post-52-week } \\
\text { intervention } \\
4 \text { weeks } \\
\text { Fair }\end{array}$ & NR & Spinal cord injury & $\begin{array}{l}\text { 1. Injury located at C5-C7 } \\
\text { (C: cervical spinal nerve), } \\
\text { spinal injury of patients } \\
\text { conformed to International } \\
\text { Standards for } \\
\text { Neurological Classification } \\
\text { of Spinal Cord Injury } \\
\text { (ASIA, 4th Edition, 1992); } \\
\text { 2. Patients in stable } \\
\text { condition and could } \\
\text { cooperate to complete } \\
\text { pulmonary function test } \\
\text { and pulmonary } \\
\text { rehabilitation }\end{array}$ & $\begin{array}{l}\text { Randomized: } \\
98 \\
\text { Analyzed: } 98 \\
\text { Attrition: } 0 \% \\
(0 / 98)\end{array}$ & $\begin{array}{l}\text { China } \\
\text { Inpatient } \\
\text { RCT }\end{array}$ & NR \\
\hline
\end{tabular}




\begin{tabular}{|c|c|c|c|c|c|c|}
\hline $\begin{array}{l}\text { Author, Year } \\
\text { Intervention Type } \\
\text { Duration of } \\
\text { Postintervention } \\
\text { Followup } \\
\text { Quality } \\
\text { (See Appendix B for } \\
\text { Full Citation) }\end{array}$ & Harms & Condition & Inclusion Criteria & $\begin{array}{l}\text { Number } \\
\text { Randomized } \\
\text { Analyzed } \\
\text { Attrition }\end{array}$ & $\begin{array}{l}\text { Country } \\
\text { Setting } \\
\text { Study Design }\end{array}$ & Funding Source \\
\hline $\begin{array}{l}\text { Cho, } 2020 \\
\text { Muscle Strength } \\
\text { Immediately } \\
\text { Postintervention, } 6 \\
\text { weeks } \\
\text { Poor }\end{array}$ & $\begin{array}{l}\text { No adverse events reported, (one patient } \\
\text { dropped out "due to their health condition". }\end{array}$ & Cerebral palsy & $\begin{array}{l}\text { Inclusion Criteria: } \\
\text { Children between the } \\
\text { ages of } 6 \text { and } 13 \text { years } \\
\text { diagnosed with diplegic } \\
\text { CP, GMFCS level } \\
\text { between I and III. }\end{array}$ & $\begin{array}{l}\text { Randomized: } \\
25^{*} \\
\text { Analyzed: } 25 \\
\text { Attrition: } 0 \% \\
(0 / 25) \\
\\
\text { *Selected from } \\
28,10 \%(3 / 28)\end{array}$ & $\begin{array}{l}\text { Korea } \\
\text { Outpatient clinic } \\
\text { RCT }\end{array}$ & $\begin{array}{l}\text { Korean government } \\
\text { grant }\end{array}$ \\
\hline $\begin{array}{l}\text { Chrysagis } 2012 \\
\text { Aerobic Exercise } \\
\text { Treadmill } \\
\text { Postintervention, } 0 \\
\text { weeks } \\
\text { Fair }\end{array}$ & None reported & Cerebral palsy & 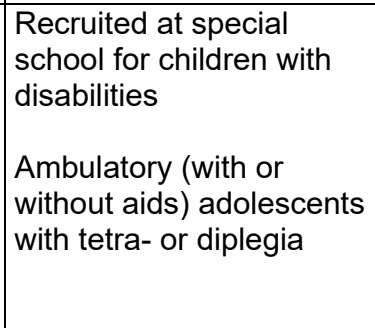 & $\begin{array}{l}\text { Randomized } 22 \\
\text { Analyzed } 22\end{array}$ & $\begin{array}{l}\text { Greece } \\
\text { RCT }\end{array}$ & NR \\
\hline $\begin{array}{l}\text { Claerbout, } 2012 \\
\text { Postural Control } \\
\text { Whole Body Vibration } \\
\text { Postintervention, } 0 \\
\text { weeks } \\
\text { Fair }\end{array}$ & NR & Multiple sclerosis & $\begin{array}{l}\text { Persons with clinically } \\
\text { definite MS and an EDSS } \\
\text { between } 3 \text { and } 7\end{array}$ & $\begin{array}{l}\text { Randomized: } \\
55 \\
\text { Analyzed: } 47 \\
\text { Attrition: } 14.5 \% \\
(8 / 55)\end{array}$ & $\begin{array}{l}\text { Belgium } \\
\text { Inpatient } \\
\text { RCT }\end{array}$ & $\begin{array}{l}\text { PF acknowledges the } \\
\text { FWO Flanders for } \\
\text { financial support } \\
\text { during the study } \\
\text { period. }\end{array}$ \\
\hline
\end{tabular}




\begin{tabular}{|c|c|c|c|c|c|c|}
\hline $\begin{array}{l}\text { Author, Year } \\
\text { Intervention Type } \\
\text { Duration of } \\
\text { Postintervention } \\
\text { Followup } \\
\text { Quality } \\
\text { (See Appendix B for } \\
\text { Full Citation) }\end{array}$ & Harms & Condition & Inclusion Criteria & $\begin{array}{l}\text { Number } \\
\text { Randomized } \\
\text { Analyzed } \\
\text { Attrition }\end{array}$ & $\begin{array}{l}\text { Country } \\
\text { Setting } \\
\text { Study Design }\end{array}$ & Funding Source \\
\hline $\begin{array}{l}\text { Collett, } 2010 \\
\text { Aerobic Exercise } \\
\text { Cycling } \\
\text { Postintervention, } 12 \\
\text { weeks } \\
\text { Poor }\end{array}$ & $\begin{array}{l}3 \text { participants from the combined } \\
\text { exercise group reported adverse events } \\
\text { during } \\
\text { the exercise intervention phase } \\
\text { (tachycardia, leg pain, and exacerbation of } \\
\text { a knee injury). } \\
\text { Intermittent group } 4 \text { participants } \\
\text { discontinued the intervention due to } \\
\text { adverse events } \\
\text { (two due to pain during cycling, one } \\
\text { because of an } \\
\text { exacerbation of MS symptoms and one due } \\
\text { to a loss } \\
\text { of consciousness during cycling). MS }\end{array}$ & Multiple sclerosis & $\begin{array}{l}\text { People with MS over } \\
18 \text { years of age identified } \\
\text { through local neurologists } \\
\text { or } \\
\text { self-referral. }\end{array}$ & $\begin{array}{l}\text { Randomized: } \\
\mathrm{N}=61 \\
\text { Analyzed:55 } \\
\text { Attrition: } 20 \% \\
(12 / 61)\end{array}$ & $\begin{array}{l}\text { United Kingdon } \\
\text { Gym } \\
\text { Randomized } \\
\text { comparator study }\end{array}$ & $\begin{array}{l}\text { Multiple } \\
\text { Sclerosis Society of } \\
\text { Great Britain and } \\
\text { Northern Ireland } \\
\text { and Oxfordshire } \\
\text { Primary Care Trust } \\
\text { (PCT) } \\
\text { extension to the MS } \\
\text { funding National } \\
\text { Institute of Health } \\
\text { Research }\end{array}$ \\
\hline $\begin{array}{l}\text { Curtis, } 2018 \\
\text { Postural Control } \\
\text { Balance } \\
\text { Postintervention, } 24 \\
\text { weeks } \\
\text { Fair }\end{array}$ & NR & Cerebral palsy & $\begin{array}{l}\text { Diagnosis of CP classified } \\
\text { as levels III-V of the } \\
\text { Gross Motor Function } \\
\text { Classification System, be } \\
\text { aged between } 2 \text { and } 15 \\
\text { years, and have trunk or } \\
\text { head postural control } \\
\text { deficits }\end{array}$ & $\begin{array}{l}\text { Randomized: } \\
28 \\
\text { Analyzed: } 23 \\
\text { Attrition: } 17.9 \% \\
(5 / 28)\end{array}$ & $\begin{array}{l}\text { NR } \\
\text { Outpatient clinic } \\
\text { and home } \\
\text { RCT }\end{array}$ & $\begin{array}{l}\text { Nonprofit } \\
\text { This trial was } \\
\text { supported financially } \\
\text { by grants from The } \\
\text { Association of } \\
\text { Danish } \\
\text { Physiotherapist's } \\
\text { Foundation for } \\
\text { Research, Education } \\
\text { and } \\
\text { Development of } \\
\text { Clinical Practice, Fund } \\
\text { for Physiotherapy in } \\
\text { Private } \\
\text { Practice and the Britta } \\
\text { Holles Fund. }\end{array}$ \\
\hline
\end{tabular}




\begin{tabular}{|c|c|c|c|c|c|c|}
\hline $\begin{array}{l}\text { Author, Year } \\
\text { Intervention Type } \\
\text { Duration of } \\
\text { Postintervention } \\
\text { Followup } \\
\text { Quality } \\
\text { (See Appendix B for } \\
\text { Full Citation) }\end{array}$ & Harms & Condition & Inclusion Criteria & $\begin{array}{l}\text { Number } \\
\text { Randomized } \\
\text { Analyzed } \\
\text { Attrition } \\
\end{array}$ & \begin{tabular}{|l} 
Country \\
Setting \\
Study Design \\
\end{tabular} & Funding Source \\
\hline $\begin{array}{l}\text { Dalgas, } 2009 \\
\text { Dalgas, } 2010 \\
\text { Muscle Strength } \\
\text { Immediately } \\
\text { postintervention } \\
\text { Dalgas, 2009: Fair to } \\
\text { Good } \\
\text { Dalgas, 2010: Poor }\end{array}$ & NR & Multiple sclerosis & \begin{tabular}{|l|} 
Patients with definite \\
relapsing-remitting MS \\
according to Mc- \\
Donald criteria, EDSS \\
score between 3.0 and 5.5 \\
with a pyramid function \\
score 2.0, ability to walk \\
$100 \mathrm{~m}$, no need for help \\
with transportation to \\
training facility, age 18 \\
years, and acceptance of \\
diagnosis and treatment.
\end{tabular} & $\begin{array}{l}\text { Randomized: } \\
38 \\
\text { Analyzed: } 31 \\
\text { Attrition: } 18.4 \% \\
(7 / 38)\end{array}$ & $\begin{array}{l}\text { Denmark } \\
\text { Outpatient clinic } \\
\text { RCT }\end{array}$ & $\begin{array}{l}\text { Supported by the } \\
\text { National Multiple } \\
\text { Sclerosis Society, The } \\
\text { Research Foundation } \\
\text { of the MS Clinic of } \\
\text { Southern Denmark } \\
\text { (Vejle, Esbjerg, and } \\
\text { Soenderborg), } \\
\text { Director Werner } \\
\text { Richter and Wife's } \\
\text { Grant, The } \\
\text { Augustinus- } \\
\text { Foundation, Engineer } \\
\text { Bent Boegh and Wife } \\
\text { Inge Boeghs } \\
\text { Foundation, Vilhelm } \\
\text { Bangs Foundation, } \\
\text { Manufacturer Mads } \\
\text { Clausen's Foundation, } \\
\text { The Toyota } \\
\text { Foundation, Mrs. } \\
\text { Benthine Lund's } \\
\text { Foundation, and AP } \\
\text { Moeller's Foundation. }\end{array}$ \\
\hline $\begin{array}{l}\text { Demuth, } 2012 \\
\text { Companion to: Fowler, } \\
2010 \\
\text { Aerobic Exercise } \\
\text { Cycling } \\
\text { Postintervention, } 0 \\
\text { weeks } \\
\text { Fair }\end{array}$ & NR & Cerebral palsy & $\begin{array}{l}\text { Inclusion criteria were: (1) } \\
\text { spastic diplegic CP; (2) } \\
\text { age between } 7 \text { and } 18 \\
\text { years; (3) the } \\
\text { ability to comply with } \\
\text { simple verbal directions; } \\
\text { (4) Gross } \\
\text { Motor Function } \\
\text { Classification System } \\
\text { (GMFCS) levels I to } \\
\text { III; and (5) selective motor } \\
\text { control rating of good or } \\
\text { fair for } \\
\text { at least one leg. }\end{array}$ & $\begin{array}{l}\text { Randomized: } \\
\mathrm{N}=64 \\
\text { Analyzed: } 58 \\
\text { Attrition:9 \% } \\
(6 / 64)\end{array}$ & $\begin{array}{l}\text { USA } \\
\text { Outpatient clinic } \\
\text { RCT }\end{array}$ & $\begin{array}{l}\text { Foundation for } \\
\text { Physical } \\
\text { Therapy }\end{array}$ \\
\hline
\end{tabular}




\begin{tabular}{|c|c|c|c|c|c|c|}
\hline $\begin{array}{l}\text { Author, Year } \\
\text { Intervention Type } \\
\text { Duration of } \\
\text { Postintervention } \\
\text { Followup } \\
\text { Quality } \\
\text { (See Appendix B for } \\
\text { Full Citation) }\end{array}$ & Harms & Condition & Inclusion Criteria & $\begin{array}{l}\text { Number } \\
\text { Randomized } \\
\text { Analyzed } \\
\text { Attrition } \\
\end{array}$ & \begin{tabular}{|l|} 
Country \\
Setting \\
Study Design \\
\end{tabular} & Funding Source \\
\hline $\begin{array}{l}\text { Deutz, } 2017 \\
\text { Postural Control } \\
\text { Hippotherapy } \\
\text { Middle of treatment } \\
\text { (after 8-week } \\
\text { observational phase } \\
\text { and 16- to 20-week } \\
\text { intervention) and end of } \\
\text { treatment (after 16- } \\
\text { week washout period, } \\
\text { 16- to 20-week } \\
\text { intervention, and 8- } \\
\text { week observational } \\
\text { phase) } \\
\text { Poor }\end{array}$ & $\begin{array}{l}1 \text { patient fell during hippotherapy and } \\
\text { fractured humerus }\end{array}$ & Cerebral palsy & $\begin{array}{l}\text { Inclusion Criteria: } \\
\text { bilateral spastic CP, age } 5 \\
\text { to } 16 \text { years, no HT and no } \\
\text { major surgery during the } \\
\text { preceding } 12 \text { months, no } \\
\text { allergy to horse hair, } \\
\text { informed consent of the } \\
\text { parents available, gross } \\
\text { motor function } \\
\text { classification level } \\
\text { (GMFCS) II to IV, and no } \\
\text { achillotenotomy performed } \\
\text { during the preceding } 6 \\
\text { months }\end{array}$ & $\begin{array}{l}\text { Randomized: } \\
73 \\
\text { Analyzed: } 66 \\
\text { (19 more did } \\
\text { not finish the } \\
\text { study, } 47 \\
\text { analyzed) } \\
\text { Attrition: } 10 \% \\
(7 / 73) \text { or } 36 \% \\
(26 / 73) \text { or } 29 \% \\
(19 / 66)\end{array}$ & $\begin{array}{l}\text { Germany } \\
\text { Outpatient clinic } \\
\text { Randomized } \\
\text { crossover trial }\end{array}$ & Nonprofit \\
\hline $\begin{array}{l}\text { Dodd, } 2011 \\
\text { Muscle Strength } \\
\text { Immediately } \\
\text { postintervention, } \\
12 \text { weeks } \\
\text { Good }\end{array}$ & $\begin{array}{l}\text { A vs. B } \\
\text { Increases in any sensory symptoms } \\
\text { characteristic of MS: } 0(0 \%) \text { vs. } 0(0 \%) \\
\text { Any injury that required participants to miss } \\
\text { a training session: } 0(0 \%) \text { vs. } 0(0 \%) \\
\text { Reductions in symptoms of muscle spasm: } \\
-2.8 \text { units ( } 95 \% \mathrm{Cl} 5.6 \text { to } 0.3) \text { vs. }-2.4 \text { units } \\
(95 \% \mathrm{Cl} 5.2 \text { to } 0.5), p>0.05 \\
\text { Short-term muscle soreness: } 69 \%(25) \text { vs. } \\
\text { NR }\end{array}$ & Multiple sclerosis & $\begin{array}{l}\text { Aged } 18 \text { years or more, } \\
\text { have } \\
\text { a confirmed diagnosis of } \\
\text { relapsing-remitting MS, } \\
\text { have an Ambulation Index } \\
\text { score of } 2,3 \text { or } 4 \text { (mild to } \\
\text { moderate walking } \\
\text { disabilities), and have } \\
\text { received medical } \\
\text { clearance to participate. }\end{array}$ & $\begin{array}{l}\text { Randomized: } \\
76 \\
\text { Analyzed: } 67 \\
\text { Attrition: } 11.8 \% \\
(9 / 76)\end{array}$ & $\begin{array}{l}\text { Australia } \\
\text { Community } \\
\text { gymnasiums } \\
\text { RCT }\end{array}$ & $\begin{array}{l}\text { Supported by Multiple } \\
\text { Sclerosis Research } \\
\text { Australia. }\end{array}$ \\
\hline
\end{tabular}




\begin{tabular}{|c|c|c|c|c|c|c|}
\hline $\begin{array}{l}\text { Author, Year } \\
\text { Intervention Type } \\
\text { Duration of } \\
\text { Postintervention } \\
\text { Followup } \\
\text { Quality } \\
\text { (See Appendix B for } \\
\text { Full Citation) }\end{array}$ & Harms & Condition & Inclusion Criteria & $\begin{array}{l}\text { Number } \\
\text { Randomized } \\
\text { Analyzed } \\
\text { Attrition }\end{array}$ & $\begin{array}{l}\text { Country } \\
\text { Setting } \\
\text { Study Design }\end{array}$ & Funding Source \\
\hline $\begin{array}{l}\text { Doulatabad, } 2013 \\
\text { Postural Control } \\
\text { Yoga } \\
\text { Postintervention, } 12 \\
\text { weeks } \\
\text { Poor }\end{array}$ & NR & Multiple sclerosis & $\begin{array}{l}\text { Women aged between 18- } \\
45 \text { with at least } 2 \text { year MS } \\
\text { history; and the ability to } \\
\text { participate in Yoga } \\
\text { exercise }\end{array}$ & $\begin{array}{l}\text { Randomized: } \\
60 \\
\text { Analyzed:60 } \\
\text { Attrition: } 0 \% \\
(0 / 60)\end{array}$ & $\begin{array}{l}\text { Iran } \\
\text { Group setting } \\
\text { RCT }\end{array}$ & $\begin{array}{l}\text { Nonprofit: Yasouj } \\
\text { University of Medical } \\
\text { Sciences }\end{array}$ \\
\hline $\begin{array}{l}\text { Duarte Nde, } 2014 \\
\text { Aerobic Exercise } \\
\text { Treadmill } \\
\text { Postintervention, } 3 \\
\text { weeks and } 5 \text { weeks } \\
\text { Fair } \\
\text { Note: May share } \\
\text { participants with } \\
\text { Grecco, } 2014\end{array}$ & NR & Cerebral palsy & $\begin{array}{l}\text { Inclusion Criteria: Spastic } \\
\text { cerebral palsy; GMFCS } \\
\text { levels I, ii or iii;;,between } 5 \\
\text { and } 10 \text { years old; } \\
\text { independent gate for at } \\
\text { least } 12 \text { months; able to } \\
\text { comprehend procedures }\end{array}$ & $\begin{array}{l}\text { Randomized: } \\
24 \\
\text { Analyzed: } 24 \\
\text { Attrition: } 0 \%\end{array}$ & $\begin{array}{l}\text { Brazil } \\
\text { Outpatient physical } \\
\text { therapy clinics } \\
\text { RCT }\end{array}$ & $\begin{array}{l}\text { Brazilian fostering } \\
\text { agencies CAPES and } \\
\text { FAPESP }\end{array}$ \\
\hline $\begin{array}{l}\text { Duff, } 2018 \\
\text { Muscle Strength } \\
\text { Postintervention, } 0 \\
\text { weeks } \\
\text { Fair }\end{array}$ & $\begin{array}{l}\text { No adverse events were reported in either } \\
\text { group during the intervention. However, } \\
\text { one participant experienced severe muscle } \\
\text { spasticity of the leg during the baseline } \\
\text { stimulation protocol. This person fully } \\
\text { recovered within } 2 \text { hours of the testing. }\end{array}$ & Multiple sclerosis & $\begin{array}{l}\text { Definite diagnosis of MS, } \\
\text { not restricted to a } \\
\text { wheelchair or scooter, and } \\
\text { the ability to travel to the } \\
\text { assessment and } \\
\text { intervention locations }\end{array}$ & $\begin{array}{l}\text { Randomized: } \\
30 \\
\text { Analyzed: } 27 \\
\text { Attrition: } 10 \% \\
(3 / 30)\end{array}$ & $\begin{array}{l}\text { Canada } \\
\text { Pilates studio } \\
\text { RCT }\end{array}$ & $\begin{array}{l}\text { This study was funded } \\
\text { by a Hermes Canada } \\
\text { MS Society of Canada } \\
\text { Wellness Research } \\
\text { Innovation grant. }\end{array}$ \\
\hline $\begin{array}{l}\text { Duffell, } 2014 \\
\text { Aerobic Exercise } \\
\text { Robot-Assisted Gait } \\
\text { Training } \\
\text { Postintervention, } 4 \\
\text { weeks } \\
\text { Poor }\end{array}$ & NR & Spinal cord injury & $\begin{array}{l}\text { Inclusion Criteria: subjects } \\
\text { with incomplete SCl }\end{array}$ & $\begin{array}{l}\text { Randomized: } \\
52 \\
\text { Analyzed: } 52 \\
\text { Attrition: } 0 \% \\
(0 / 52)\end{array}$ & $\begin{array}{l}\text { USA } \\
\text { Outpatient } \\
\text { Rehabilitation } \\
\text { Clinic } \\
\text { RCT }\end{array}$ & $\begin{array}{l}\mathrm{NIH} \text { and Craig } \mathrm{H} \\
\text { Nelson Foundation }\end{array}$ \\
\hline
\end{tabular}




\begin{tabular}{|c|c|c|c|c|c|c|}
\hline $\begin{array}{l}\text { Author, Year } \\
\text { Intervention Type } \\
\text { Duration of } \\
\text { Postintervention } \\
\text { Followup } \\
\text { Quality } \\
\text { (See Appendix B for } \\
\text { Full Citation) }\end{array}$ & Harms & Condition & Inclusion Criteria & $\begin{array}{l}\text { Number } \\
\text { Randomized } \\
\text { Analyzed } \\
\text { Attrition } \\
\end{array}$ & \begin{tabular}{|l|} 
Country \\
Setting \\
Study Design \\
\end{tabular} & Funding Source \\
\hline $\begin{array}{l}\text { Ebrahimi, } 2015 \\
\text { Multimodal } \\
\text { Postintervention, } 0 \\
\text { weeks } \\
\text { Poor }\end{array}$ & NR & Multiple sclerosis & $\begin{array}{l}\text { Definite MS according to } \\
\text { McDonald's criteria, with } \\
\text { relapsing-remitting form } \\
\text { of the disease and EDSS } \\
1.5 \text { to } 5.0\end{array}$ & $\begin{array}{l}\text { Randomized: } \\
34 \\
\text { Analyzed: } 30 \\
\text { Attrition: } 11.8 \% \\
(4 / 34)\end{array}$ & \begin{tabular}{|l} 
Iran \\
Outpatient \\
RCT
\end{tabular} & NR \\
\hline $\begin{array}{l}\text { Elnaggar } 2019 \\
\text { Strength } \\
\text { Plyometric training } \\
\text { Postintervention, } 8 \\
\text { weeks } \\
\text { Fair }\end{array}$ & NR & Cerebral palsy & $\begin{array}{l}\text { Inclusion Criteria: Spastic } \\
\text { unilateral CP as } \\
\text { determined by a pediatric } \\
\text { neurologist, 8-12 years of } \\
\text { age, independent } \\
\text { ambulators, categorized } \\
\text { as level I according to } \\
\text { Gross Motor Function } \\
\text { Classification System, } \\
\text { mild spastic (hypertonia } \\
\text { less than 1+ grade as } \\
\text { being measured by the } \\
\text { Modified Ashworth Scale), } \\
\text { ability to understand and } \\
\text { follow instructions. }\end{array}$ & $\begin{array}{l}\text { Randomized:44 } \\
\text { Analyzed: } 39 \\
\text { Attrition: } 11.4 \% \\
(5 / 44)\end{array}$ & $\begin{array}{l}\text { Saudi Arabia } \\
\text { Outpatient } \\
\text { RCT }\end{array}$ & NR \\
\hline $\begin{array}{l}\text { El-Shamy, } 2018 \\
\text { Postural Control } \\
\text { Motion gaming } \\
\text { Postintervention, } 12 \\
\text { weeks } \\
\text { Fair }\end{array}$ & NR & Cerebral palsy & $\begin{array}{l}\text { Inclusion Criteria: children } \\
\text { 6-8 years old with } \\
\text { hemiplegic CP, MACS I- } \\
\text { III, able to hear/see and } \\
\text { follow directions }\end{array}$ & $\begin{array}{l}\text { Randomized: } \\
30 \\
\text { Analyzed: } 30 \\
\text { Attrition: } 0 \% \\
(0 / 30)\end{array}$ & $\begin{array}{l}\text { Saudi Arabia } \\
\text { Children's Hospital } \\
\text { RCT }\end{array}$ & NR \\
\hline
\end{tabular}




\begin{tabular}{|c|c|c|c|c|c|c|}
\hline $\begin{array}{l}\text { Author, Year } \\
\text { Intervention Type } \\
\text { Duration of } \\
\text { Postintervention } \\
\text { Followup } \\
\text { Quality } \\
\text { (See Appendix B for } \\
\text { Full Citation) }\end{array}$ & Harms & Condition & Inclusion Criteria & $\begin{array}{l}\text { Number } \\
\text { Randomized } \\
\text { Analyzed } \\
\text { Attrition } \\
\end{array}$ & \begin{tabular}{|l} 
Country \\
Setting \\
Study Design \\
\end{tabular} & Funding Source \\
\hline $\begin{array}{l}\text { Emara, } 2016 \\
\text { Aerobic Exercise } \\
\text { Treadmill } \\
\text { Postintervention, } 12 \\
\text { weeks } \\
\text { Fair }\end{array}$ & NR & Cerebral palsy & $\begin{array}{l}\text { Inclusion Criteria: } \\
\text { Children } 6 \text { to } 8 \text { years old } \\
\text { with spastic diplegia, } \\
\text { gross motor function } \\
\text { classification system } \\
\text { (GMFCS) level III. }\end{array}$ & $\begin{array}{l}\text { Randomized: } \\
\mathrm{N}=22 \\
\text { Analyzed: } 20 \\
\text { Attrition: } .09 \% \\
(2 / 22)\end{array}$ & $\begin{array}{l}\text { Saudi Arabia } \\
\text { Outpatient clinic } \\
\text { RCT }\end{array}$ & $\begin{array}{l}\text { Nonprofit } \\
\text { Taibah University, Al } \\
\text { Madinah Al- } \\
\text { Munawara, Saudi } \\
\text { Arabia (Grant Number } \\
\text { 6093/1435). }\end{array}$ \\
\hline $\begin{array}{l}\text { Faramarzi, } 2020 \\
\text { Has companion: } \\
\text { Banitalebi, } 2020 \\
\text { Multimodal Exercise } \\
\text { Immediately } \\
\text { Postintervention, } 12 \\
\text { weeks } \\
\text { Fair }\end{array}$ & No adverse events were reported. & Multiple sclerosis & $\begin{array}{l}\text { Inclusion Criteria: Women } \\
\text { aged } 18 \text { to } 50 \text { with MS, } \\
\text { with no relapse or acute } \\
\text { exacerbation the past } 6 \\
\text { months. }\end{array}$ & $\begin{array}{l}\text { Randomized: } \\
94 \\
\text { Analyzed: } 89 \\
\text { Attrition: } 5 \% \\
(5 / 94)\end{array}$ & $\begin{array}{l}\text { Iran } \\
\text { Outpatient clinic } \\
\text { RCT }\end{array}$ & NR \\
\hline $\begin{array}{l}\text { Esclarin-Ruz, } 2014 \\
\text { Aerobic Exercise } \\
\text { Robot-Assisted Gait } \\
\text { Training } \\
\text { Postintervention, } 8 \\
\text { weeks } \\
\text { Fair }\end{array}$ & NA & $\begin{array}{l}\text { Spinal cord } \\
\text { injury }\end{array}$ & $\begin{array}{l}\text { Inclusion Criteria: C2 to L3 } \\
\text { SCI, ASIA C or D, onset } \\
<6 \text { months, ago } 16-70, \\
\text { able to stand with external } \\
\text { support }\end{array}$ & $\begin{array}{l}\text { Randomized: } \\
\mathrm{N}=88 \\
\text { Analyzed: } 81 \\
\text { Attrition: } 7.9 \%\end{array}$ & $\begin{array}{l}\text { Spain } \\
\text { Hospital } \\
\text { RCT }\end{array}$ & Research Grant \\
\hline
\end{tabular}




\begin{tabular}{|c|c|c|c|c|c|c|}
\hline $\begin{array}{l}\text { Author, Year } \\
\text { Intervention Type } \\
\text { Duration of } \\
\text { Postintervention } \\
\text { Followup } \\
\text { Quality } \\
\text { (See Appendix B for } \\
\text { Full Citation) }\end{array}$ & Harms & Condition & Inclusion Criteria & $\begin{array}{l}\text { Number } \\
\text { Randomized } \\
\text { Analyzed } \\
\text { Attrition }\end{array}$ & $\begin{array}{l}\text { Country } \\
\text { Setting } \\
\text { Study Design }\end{array}$ & Funding Source \\
\hline $\begin{array}{l}\text { Field-Fote, } 2011 \\
\text { Has companions: } \\
\text { Kressler, 2013; Sandler, } \\
2017 \\
\text { Aerobic Exercise } \\
\text { Robot-Assisted Gait } \\
\text { Training } \\
\text { Postintervention, } 12 \\
\text { weeks } \\
\text { Fair }\end{array}$ & NA & $\begin{array}{l}\text { Spinal cord } \\
\text { injury }\end{array}$ & $\begin{array}{l}\text { Inclusion Criteria: Asia } \\
\text { classification C or D } \\
\text { Spinal Cord Injury at T10 } \\
\text { or higher, able to take } 1 \\
\text { step with } 1 \text { leg, and ability } \\
\text { to rise to standing position } \\
\text { with at most moderate } \\
(50 \%) \text { assistance }\end{array}$ & $\begin{array}{l}\text { Randomized: } \\
\mathrm{N}=74 \\
\text { Analyzed: } 64 \\
\text { Attrition: } 14 \% \\
(10 / 74)\end{array}$ & $\begin{array}{l}\text { USA } \\
\text { Outpatient } \\
\text { Randomized } \\
\text { Control Trial }\end{array}$ & $\begin{array}{l}\text { Funding: National } \\
\text { Institutes of Health } \\
\text { and Miami Project to } \\
\text { Cure Paralysis }\end{array}$ \\
\hline $\begin{array}{l}\text { Forsberg, } 2016 \\
\text { Postural Control } \\
\text { Balance } \\
\text { Postintervention, } 8 \\
\text { weeks } \\
\text { Fair }\end{array}$ & $\begin{array}{l}\text { Two adverse events were reported: one } \\
\text { participant lost balance during challenging } \\
\text { tasks in standing and fell on a soft carpet, } \\
\text { and one fell while standing on his/her } \\
\text { knees. No injuries were reported. }\end{array}$ & Multiple sclerosis & $\begin{array}{l}\text { Patients with MS able to } \\
\text { walk } 100 \text { meters but } \\
\text { unable to maintain tandem } \\
\text { stance } \geq 30 \text { seconds }\end{array}$ & $\begin{array}{l}\text { Randomized: } \\
87 \\
\text { Analyzed:73 } \\
\text { (week 8) } \\
\text { Attrition: } 16.1 \% \\
\text { (14/87 - week } \\
\text { 8) } \\
\text { Analyzed: } 66 \\
\text { (week 24) } \\
\text { Attrition: } 24 \% \\
(21 / 87 \text { - week } \\
24)\end{array}$ & $\begin{array}{l}\text { Sweden } \\
\text { Hospital } \\
\text { RCT }\end{array}$ & $\begin{array}{l}\text { Government: } \\
\text { supported by the } \\
\text { Uppsala- "Orebro } \\
\text { Regional Research } \\
\text { Council (RFR- } \\
\text { 306241), the } \\
\text { Norrbacka-Eugenia } \\
\text { Foundation (Grant no. } \\
\text { 814/12), and the } \\
\text { Research Committee } \\
\text { of Region " Orebro } \\
\text { County (Grants } \\
\text { nos.OLL-216421 and } \\
\text { OLL- } \\
\text { 317511) }\end{array}$ \\
\hline $\begin{array}{l}\text { Fosdahl, 2019b } \\
\text { Multimodal Exercise } \\
\text { Postintervention, } 16 \\
\text { weeks and } 32 \text { weeks } \\
\text { Fair }\end{array}$ & NR & Cerebral palsy & $\begin{array}{l}\text { Inclusion Criteria: } \\
\text { Spastic bilateral CP } \\
\text { patients GMFCS levels I- } \\
\text { III }\end{array}$ & $\begin{array}{l}\text { Randomized: } \\
37 \\
\text { Analyzed: } 34 \\
\text { Attrition: } 9 \% \\
(34 / 37)\end{array}$ & \begin{tabular}{|l|} 
Norway \\
Pediatric outpatient \\
clinic \\
RCT
\end{tabular} & $\begin{array}{l}\text { Sophies Minde } \\
\text { Ortopedi AS, Oslo } \\
\text { University Hopsital }\end{array}$ \\
\hline
\end{tabular}




\begin{tabular}{|c|c|c|c|c|c|c|}
\hline $\begin{array}{l}\text { Author, Year } \\
\text { Intervention Type } \\
\text { Duration of } \\
\text { Postintervention } \\
\text { Followup } \\
\text { Quality } \\
\text { (See Appendix B for } \\
\text { Full Citation) }\end{array}$ & Harms & Condition & Inclusion Criteria & $\begin{array}{l}\text { Number } \\
\text { Randomized } \\
\text { Analyzed } \\
\text { Attrition }\end{array}$ & $\begin{array}{l}\text { Country } \\
\text { Setting } \\
\text { Study Design }\end{array}$ & Funding Source \\
\hline $\begin{array}{l}\text { Fowler, } 2010 \\
\text { Has companion: } \\
\text { Demuth, } 2012 \\
\text { Aerobic Exercise } \\
\text { Cycling } \\
\text { Postintervention, } 0 \\
\text { weeks } \\
\text { Fair }\end{array}$ & NR & Cerebral palsy & $\begin{array}{l}\text { Inclusion criteria were: (1) } \\
\text { between } 7 \text { and } 18 \text { years of } \\
\text { age; (2) ability to follow } \\
\text { simple verbal directions } \\
\text { (3) ability to walk } \\
\text { independently, with or } \\
\text { without an assistive } \\
\text { device, for short distances } \\
\text { (Gross Motor Function } \\
\text { Classification System } \\
\text { levels I-III); and (4) good } \\
\text { or fair selective voluntary } \\
\text { motor control for at least } \\
\text { one limb. }\end{array}$ & $\begin{array}{l}\text { Randomized: } \\
\mathrm{N}=64 \\
\text { Analyzed:58 } \\
\text { Attrition: } 9 \% \\
(6 / 64)\end{array}$ & $\begin{array}{l}\text { USA } \\
\text { Outpatient clinic } \\
\text { RCT }\end{array}$ & $\begin{array}{l}\text { Corporate donations } \\
\text { or discounts: Biodex } \\
\text { Inc, Freedom } \\
\text { Concepts, Helen's } \\
\text { Cycles, Santa Monica, } \\
\text { National AMBUCS } \\
\text { Inc, and Sam's Club. } \\
\text { Volunteers and } \\
\text { foundations: Caitlin } \\
\text { Fowler, Ernie } \\
\text { Meadows, Sidney } \\
\text { Stern Memorial Trust, } \\
\text { Steinmetz } \\
\text { Foundation, Sykes } \\
\text { Family Foundation, } \\
\text { and United Cerebral } \\
\text { Palsy Research and } \\
\text { Education } \\
\text { Foundation. }\end{array}$ \\
\hline $\begin{array}{l}\text { Fox, } 2016 \\
\text { Muscle Strength } \\
\text { Postintervention, } 4 \\
\text { weeks } \\
\text { Fair }\end{array}$ & $\begin{array}{l}\text { Four adverse events occurred: a } \\
\text { fractured ankle (Pilates group) and a } \\
\text { fractured humerus (standardized exercise } \\
\text { group) (both as a result of falls in the snow, } \\
\text { unrelated to the exercise sessions) and } \\
\text { pneumonia and pancreatitis (relaxation } \\
\text { group) (unrelated to the exercise sessions) }\end{array}$ & Multiple sclerosis & $\begin{array}{l}\text { Aged over } 18 \text { years, had a } \\
\text { definite diagnosis of MS } \\
\text { according to McDonald's } \\
\text { criteria, and had an EDSS } \\
\text { score of } 4.0 \text { to } 6.5 \text {, } \\
\text { meaning that, at best, they } \\
\text { were able to walk } \\
\text { independently without use } \\
\text { of an aid or rest for } 500 \text { m } \\
\text { (EDSS score } 4.0 \text { ) and, at } \\
\text { worst, they required } 2 \\
\text { walking aids (pair of } \\
\text { crutches or canes) to walk } \\
\text { about } 20 \text { m without } \\
\text { resting. }\end{array}$ & $\begin{array}{l}\text { Randomized: } \\
100 \\
\text { Analyzed: } 84 \\
\text { Attrition: } 16 \% \\
(16 / 100)\end{array}$ & $\begin{array}{l}\text { England } \\
\text { Outpatient clinic } \\
\text { RCT }\end{array}$ & NR \\
\hline
\end{tabular}




\begin{tabular}{|c|c|c|c|c|c|c|}
\hline $\begin{array}{l}\text { Author, Year } \\
\text { Intervention Type } \\
\text { Duration of } \\
\text { Postintervention } \\
\text { Followup } \\
\text { Quality } \\
\text { (See Appendix B for } \\
\text { Full Citation) }\end{array}$ & Harms & Condition & Inclusion Criteria & $\begin{array}{l}\text { Number } \\
\text { Randomized } \\
\text { Analyzed } \\
\text { Attrition }\end{array}$ & $\begin{array}{l}\text { Country } \\
\text { Setting } \\
\text { Study Design }\end{array}$ & Funding Source \\
\hline $\begin{array}{l}\text { Galea, } 2018 \\
\text { Multimodal Exercise } \\
\text { Postintervention, } 12 \\
\text { weeks } \\
\text { Fair }\end{array}$ & $\begin{array}{l}\text { A vs. B } \\
\text { Withdrawals due to AE or SAE: } 3.3 \%(2 / 60) \\
\text { vs. } 1.8 \%(1 / 56) \\
\text { Other AEs (only most common are } \\
\text { specifically called out) } \\
\text { Definitely related (n events) } \\
\text { All: } 85 \text { vs. } 28 \\
\text {-Skin abrasion/ bruising: } 25 \text { vs. } 2 \\
\text {-Autonomic dysreflexia: } 19 \text { vs. } 4 \\
\text {-Pain: } 17 \text { vs. } 19 \\
\text { Probably related (n events) } \\
\text { All: } 53 \text { vs. } 40 \\
\text {-Skin abrasion/ bruising: } 16 \text { vs. } 0 \\
\text {-Autonomic dysreflexia: } 7 \text { vs. } 3 \\
\text {-Pain: } 17 \text { vs. } 27 \\
\text {-Headache: } 0 \text { vs. } 3 \\
\text { Possibly related (n events) } \\
\text { All: } 56 \text { vs. } 64 \\
\text {-Skin abrasion/ bruising: } 0 \text { vs. } 4 \\
\text {-Pain: } 20 \text { vs. } 34 \\
\text {-Headache: } 0 \text { vs. } 12 \\
\text {-Dizziness/ nausea: } 5 \text { vs. } 0 \\
\text {-Bladder/bowel problems: } 5 \text { vs. } 0 \\
\text {-Fatigue: } 0 \text { vs. } 4\end{array}$ & Spinal cord injury & $\begin{array}{l}\text { More than } 18 \text { years of } \\
\text { age, had sustained a } \\
\text { motor complete or } \\
\text { incomplete traumatic SCl } \\
\text { above the level of } \mathrm{T} 12 \text { at } \\
\text { least } 6 \text { months prior to } \\
\text { consent, and had medical } \\
\text { clearance to participate. }\end{array}$ & $\begin{array}{l}\text { Randomized: } \\
116 \\
\text { Analyzed: } 86 \\
\text { Attrition: } 25.9 \% \\
(30 / 116)\end{array}$ & $\begin{array}{l}\text { Australia and New } \\
\text { Zealand } \\
\text { Outpatient clinic } \\
\text { RCT }\end{array}$ & $\begin{array}{l}\text { The study was funded } \\
\text { by the Transport } \\
\text { Accident Commission } \\
\text { (Victorian } \\
\text { Neurotrauma } \\
\text { Initiative), the Lifetime } \\
\text { Care and Support } \\
\text { Authority NSW, the } \\
\text { University of } \\
\text { Melbourne and The } \\
\text { University of Western } \\
\text { Australia. }\end{array}$ \\
\hline $\begin{array}{l}\text { Gandolfi, } 2015 \\
\text { Postural Control } \\
\text { Balance } \\
\text { Postintervention, } 5 \\
\text { weeks } \\
\text { Fair }\end{array}$ & $\begin{array}{l}7 \text { patients in the experimental group } 8.8 \% \\
(7 / 80) \text { withdrew for medical reasons or } \\
\text { because of difficulty arranging } \\
\text { transportation to the study site. No adverse } \\
\text { events were reported during the study } \\
\text { period }\end{array}$ & Multiple sclerosis & $\begin{array}{l}\text { Patients diagnosed with } \\
\text { MS aged } \leq 65 \text { years; } \\
\text { EDSS22 score } 1.5 \geq \times \\
\leq 6.0 \text {; Mini-Mental State } \\
\text { Evaluation score } \geq 24 ; \\
\text { subjective symptoms of } \\
\text { balance impairments; fear } \\
\text { of falling and/or history of } \\
\text { falls as defined by at least } \\
\text { one fall within the last year }\end{array}$ & $\begin{array}{l}\text { Randomized: } \\
80 \\
\text { Analyzed:80 } \\
\text { Attrition: } 0 \% \\
(0 / 80)\end{array}$ & $\begin{array}{l}\text { Italy } \\
\text { Outpatient clinic } \\
\text { RCT }\end{array}$ & $\begin{array}{l}\text { Nonprofit: Fondazione } \\
\text { Italiana Sclerosi } \\
\text { Multipla onlus (FISM) } \\
\text { grant no. FISM } \\
\text { 2009/R/27 }\end{array}$ \\
\hline
\end{tabular}




\begin{tabular}{|c|c|c|c|c|c|c|}
\hline $\begin{array}{l}\text { Author, Year } \\
\text { Intervention Type } \\
\text { Duration of } \\
\text { Postintervention } \\
\text { Followup } \\
\text { Quality } \\
\text { (See Appendix B for } \\
\text { Full Citation) }\end{array}$ & Harms & Condition & Inclusion Criteria & $\begin{array}{l}\text { Number } \\
\text { Randomized } \\
\text { Analyzed } \\
\text { Attrition }\end{array}$ & $\begin{array}{l}\text { Country } \\
\text { Setting } \\
\text { Study Design }\end{array}$ & Funding Source \\
\hline $\begin{array}{l}\text { Garrett, 2013a } \\
\text { Garrett, 2013b } \\
\text { (same author group a } \\
\text { as Hogan 2014) } \\
\text { Postural Control } \\
\text { Yoga } \\
\text { Postintervention, } 12 \\
\text { weeks }\end{array}$ & NR & Multiple sclerosis & $\begin{array}{l}\text { Over } 18 \text { years of age and } \\
\text { had a diagnosis of MS } \\
\text { that was confirmed by a } \\
\text { consultant physician or } \\
\text { neurologist. }\end{array}$ & $\begin{array}{l}\text { Garrett 2013a } \\
\text { (3 intervention } \\
\text { groups, 1 } \\
\text { control group, } \\
\text { postintervention } \\
\text { followup, ITT } \\
\text { analysis) } \\
\text { Randomized: } \\
372 \\
\text { Analyzed: } 242 \\
\text { Attrition: } 34.9 \% \\
\text { (130/372) } \\
\\
\text { Garrett 2013b } \\
\text { (3 intervention } \\
\text { groups, 12- } \\
\text { week followup, } \\
\text { no ITT } \\
\text { analysis) } \\
\text { Randomized: } \\
\text { 243 } \\
\text { Analyzed: } 121 \\
\text { Attrition: } 50 \% \\
\text { (122/243) } \\
\end{array}$ & $\begin{array}{l}\text { Ireland } \\
\text { Community gyms, } \\
\text { hotels, health } \\
\text { centers } \\
\text { RCT }\end{array}$ & $\begin{array}{l}\text { This work, designated } \\
\text { the 'Getting the } \\
\text { Balance Right project,' } \\
\text { was supported by the } \\
\text { Multiple Sclerosis } \\
\text { Society of Ireland } \\
\text { (MSI) through the } \\
\text { Tesco Charity of the } \\
\text { Year funding and the } \\
\text { Pobal, Dormant } \\
\text { Accounts Flagship } \\
\text { Fund. In addition, the } \\
\text { lead author was an } \\
\text { EMBARK PhD } \\
\text { Scholar who was } \\
\text { supported by the Irish } \\
\text { Research Council for } \\
\text { Science Engineering } \\
\text { and Technology. }\end{array}$ \\
\hline $\begin{array}{l}\text { Gervasoni } 2014 \\
\text { Aerobic Exercise } \\
\text { Treadmill } \\
\text { Postintervention, } 0 \\
\text { weeks } \\
\text { Fair }\end{array}$ & None reported & Multiple sclerosis & $\begin{array}{l}\text { Able to walk } 6 \text { meters with } \\
\text { or without assist device }\end{array}$ & $\begin{array}{l}30 \text { randomized } \\
30 \text { analyzed }\end{array}$ & $\begin{array}{l}\text { Iran } \\
\text { RCT }\end{array}$ & Government funded \\
\hline
\end{tabular}




\begin{tabular}{|c|c|c|c|c|c|c|}
\hline $\begin{array}{l}\text { Author, Year } \\
\text { Intervention Type } \\
\text { Duration of } \\
\text { Postintervention } \\
\text { Followup } \\
\text { Quality } \\
\text { (See Appendix B for } \\
\text { Full Citation) }\end{array}$ & Harms & Condition & Inclusion Criteria & $\begin{array}{l}\text { Number } \\
\text { Randomized } \\
\text { Analyzed } \\
\text { Attrition }\end{array}$ & $\begin{array}{l}\text { Country } \\
\text { Setting } \\
\text { Study Design }\end{array}$ & Funding Source \\
\hline $\begin{array}{l}\begin{array}{l}\text { Giangregorio } 2012 \\
\text { (Body composition) }\end{array} \\
\begin{array}{l}\text { Hitzig } 2013 \text { (quality of } \\
\text { life) }\end{array} \\
\text { Kapadia } 2014 \text { (walking } \\
\text { capacity) } \\
\text { Craven } 2017 \text { (bone } \\
\text { markers) } \\
\text { Aerobic Exercise } \\
\text { Treadmill } \\
\text { Postintervention, } 6 \\
\text { months } \\
\text { Fair }\end{array}$ & None reported & Spinal cord injury & $\begin{array}{l}\text { Incomplete injuries from } \\
\text { C2 to T12 } \\
\text { American Spinal Injury } \\
\text { Association Impairment } \\
\text { Scale C or D }\end{array}$ & $\begin{array}{l}34 \text { randomized } \\
28 \text { analyzed } \\
6 / 34=18 \% \\
\text { attrition }\end{array}$ & $\begin{array}{l}\text { Canada } \\
\text { Rehabilitation } \\
\text { hospital }\end{array}$ & $\begin{array}{l}\text { Ontario Neurotrauma } \\
\text { Foundation }\end{array}$ \\
\hline $\begin{array}{l}\text { Gibson, } 2018 \\
\text { Aerobic Exercise } \\
\text { Aerobics } \\
\text { Postintervention, } 12 \\
\text { weeks } \\
\text { Good }\end{array}$ & NR & Cerebral palsy & $\begin{array}{l}\text { Inclusion Criteria: CP } \\
\text { patients } 9 \text { to } 18 \text { years old } \\
\text { with GMFCS levels I-III }\end{array}$ & $\begin{array}{l}\text { Randomized: } \\
43 \\
\text { Analyzed: } 42 \\
\text { Attrition: } 2 \% \\
(1 / 43)\end{array}$ & $\begin{array}{l}\text { Australia } \\
\text { Outpatient therapy } \\
\text { clinic } \\
\text { RCT }\end{array}$ & $\begin{array}{l}\text { Non-government } \\
\text { Centre and Princess } \\
\text { Margaret Hospital } \\
\text { Foundation }\end{array}$ \\
\hline $\begin{array}{l}\text { Gorman, } 2019 \\
\text { Aerobic Exercise } \\
\text { Aquatics } \\
\text { Postintervention, } 12 \\
\text { weeks } \\
\text { Fair }\end{array}$ & $(\mathrm{N}=1$, treatment related $)$ & $\begin{array}{l}\text { Spinal cord } \\
\text { injury }\end{array}$ & $\begin{array}{l}\mathrm{SCI} \text { ASIA C or D, age 18- } \\
65 \text {, tolerate } 30 \text { minutes } \\
\text { standing frame }\end{array}$ & $\begin{array}{l}\text { Randomized: } \\
\mathrm{N}=37 \\
\text { Analyzed } 32 \\
\text { Attrition: } \\
13.5 \%)\end{array}$ & $\begin{array}{l}\text { USA } \\
\text { Outpatient } \\
\text { RCT }\end{array}$ & $\begin{array}{l}\text { Funding: } \\
\text { US Department of } \\
\text { Defense SCI } \\
\text { Research Program }\end{array}$ \\
\hline
\end{tabular}




\begin{tabular}{|c|c|c|c|c|c|c|}
\hline $\begin{array}{l}\text { Author, Year } \\
\text { Intervention Type } \\
\text { Duration of } \\
\text { Postintervention } \\
\text { Followup } \\
\text { Quality } \\
\text { (See Appendix B for } \\
\text { Full Citation) }\end{array}$ & Harms & Condition & Inclusion Criteria & $\begin{array}{l}\text { Number } \\
\text { Randomized } \\
\text { Analyzed } \\
\text { Attrition }\end{array}$ & $\begin{array}{l}\text { Country } \\
\text { Setting } \\
\text { Study Design }\end{array}$ & Funding Source \\
\hline $\begin{array}{l}\text { Grecco } 2013 \\
\text { Aerobic Exercise } \\
\text { Treadmill } \\
\text { RCT } \\
\text { Postintervention, } 3 \\
\text { weeks } \\
\text { Fair } \\
\end{array}$ & None reported & Cerebral palsy & $\begin{array}{l}\text { Recruited children from } \\
\text { specialized outpatient } \\
\text { clinics Children }\end{array}$ & $\begin{array}{l}24 \text { randomized } \\
24 \text { analyzed }\end{array}$ & Brazil & Government funded \\
\hline $\begin{array}{l}\text { Grecco } 2014 \\
\text { Aerobic Exercise } \\
\text { Treadmill } \\
\text { RCT } \\
\text { Postintervention, } 4 \\
\text { weeks } \\
\text { Fair }\end{array}$ & None reported & Cerebral palsy & $\begin{array}{l}\text { Recruited from outpatient } \\
\text { clinics } \\
\text { Children }\end{array}$ & $\begin{array}{l}35 \text { randomized } \\
35 \text { analyzed at } \\
\text { post } \\
33 \text { analyzed at } \\
1 \text {-month } \\
\text { followup } \\
2 / 35=6 \%\end{array}$ & $\begin{array}{l}\text { Brazil } \\
\text { RCT }\end{array}$ & Government funded \\
\hline $\begin{array}{l}\text { Harness, } 2008 \\
\text { Multimodal Exercise } \\
\text { Postintervention, } 0 \\
\text { weeks } \\
\text { Fair }\end{array}$ & NR & Spinal cord injury & $\begin{array}{l}\text { Age 18-70 years, SCI } \\
\text { greater than } 2 \text { months } \\
\text { prior that resulted in } \\
\text { paraplegia or quadriplegia } \\
\text { between } \mathrm{C} 2 \text { and T12, and } \\
\text { ASIA Impairment Scale A, } \\
\text { B, C, or D }\end{array}$ & $\begin{array}{l}\text { Randomized: } \\
\text { NA } \\
\text { Analyzed: } 29 \\
\text { Attrition: } 6.5 \% \\
(2 / 31)\end{array}$ & $\begin{array}{l}\text { USA } \\
\text { Outpatient clinic } \\
\text { Comparative } \\
\text { Cohort }\end{array}$ & $\begin{array}{l}\text { Funds provided by the } \\
\text { National Center of } \\
\text { Research Resources, } \\
5 \text { M011 RR- 00827-29, } \\
\text { US Public Health } \\
\text { Service. }\end{array}$ \\
\hline
\end{tabular}




\begin{tabular}{|c|c|c|c|c|c|c|}
\hline $\begin{array}{l}\text { Author, Year } \\
\text { Intervention Type } \\
\text { Duration of } \\
\text { Postintervention } \\
\text { Followup } \\
\text { Quality } \\
\text { (See Appendix B for } \\
\text { Full Citation) }\end{array}$ & Harms & Condition & Inclusion Criteria & $\begin{array}{l}\text { Number } \\
\text { Randomized } \\
\text { Analyzed } \\
\text { Attrition }\end{array}$ & $\begin{array}{l}\text { Country } \\
\text { Setting } \\
\text { Study Design }\end{array}$ & Funding Source \\
\hline $\begin{array}{l}\text { Hasanpour-Dehkordi, } \\
2014 \\
\text { "Comparison of regular } \\
\text { aerobic and yoga on the } \\
\text { quality of life in } \\
\text { patients" } \\
\text { Has companions: } \\
\text { Hasanpour-Dehkordi, } \\
\text { 2016; Hasanpour- } \\
\text { Dehkordi, } 2016 \text { (2) } \\
\text { Postural Control } \\
\text { Yoga } \\
\text { Postintervention, } 12 \\
\text { weeks } \\
\text { Poor }\end{array}$ & NR & Multiple sclerosis & $\begin{array}{l}\text { Diagnosis of MS and } \\
\text { ability to perform the } \\
\text { exercise program after the } \\
\text { medication therapy; } \\
\text { having no difficulty with } \\
\text { movement; having no } \\
\text { advanced heart failure }\end{array}$ & $\begin{array}{l}\text { Randomized: } \\
61 \\
\text { Analyzed: } 61 \\
\text { Attrition: } 0 \% \\
(0 / 61)\end{array}$ & $\begin{array}{l}\text { Iran } \\
\text { Hospital } \\
\text { RCT }\end{array}$ & $\begin{array}{l}\text { Nonprofit: Research } \\
\text { and Technology } \\
\text { Deputy of Shahrekord } \\
\text { University of Medical } \\
\text { Sciences } \\
\text { grant no. } 419\end{array}$ \\
\hline $\begin{array}{l}\text { Hasanpour-Dehkordi, } \\
2016 \text { (2) "Influence of } \\
\text { yoga and aerobics } \\
\text { exercise on fatigue, } \\
\text { pain and psychosocial } \\
\text { status..." } \\
\text { Postural Control } \\
\text { Yoga } \\
\text { Companion to: } \\
\text { Hasanpour-Dehkordi, } \\
2014 \\
\text { Postintervention, } 12 \\
\text { weeks } \\
\text { Poor }\end{array}$ & NR & Multiple sclerosis & $\begin{array}{l}\text { Diagnosis of MS; consent } \\
\text { to participate in the study; } \\
\text { and the ability to speak } \\
\text { and to move }\end{array}$ & $\begin{array}{l}\text { Randomized: } \\
61 \\
\text { Analyzed:61 } \\
\text { Attrition: } 0 \% \\
(0 / 61)\end{array}$ & $\begin{array}{l}\text { Iran } \\
\text { Gym } \\
\text { RCT }\end{array}$ & $\begin{array}{l}\text { Nonprofit: Research } \\
\text { and Technology } \\
\text { Deputy of Shahrekord } \\
\text { University of Medical } \\
\text { Sciences }\end{array}$ \\
\hline
\end{tabular}




\begin{tabular}{|c|c|c|c|c|c|c|}
\hline $\begin{array}{l}\text { Author, Year } \\
\text { Intervention Type } \\
\text { Duration of } \\
\text { Postintervention } \\
\text { Followup } \\
\text { Quality } \\
\text { (See Appendix B for } \\
\text { Full Citation) }\end{array}$ & Harms & Condition & Inclusion Criteria & $\begin{array}{l}\text { Number } \\
\text { Randomized } \\
\text { Analyzed } \\
\text { Attrition }\end{array}$ & $\begin{array}{l}\text { Country } \\
\text { Setting } \\
\text { Study Design }\end{array}$ & Funding Source \\
\hline $\begin{array}{l}\text { Hasanpour-Dehkordi, } \\
2016 \\
\text { "Effects of Yoga on } \\
\text { Physiological Indices, } \\
\text { Anxiety and Social } \\
\text { Functioning" } \\
\text { Postural Control } \\
\text { Yoga } \\
\text { Companion to: } \\
\text { Hasanpour-Dehkordi, } \\
2014 \\
\text { Poor }\end{array}$ & NR & Multiple sclerosis & $\begin{array}{l}\text { Diagnosis of MS; consent } \\
\text { to participate in the study; } \\
\text { and the ability to speak } \\
\text { and to move }\end{array}$ & $\begin{array}{l}\text { Randomized: } \\
60 \\
\text { Analyzed: } 60 \\
\text { Attrition: } 0 \% \\
(0 / 60) \\
\\
\text { *During the } \\
\text { study, } 10 \text { from } \\
\text { case group and } \\
10 \text { from control } \\
\text { group were } \\
\text { excluded }\end{array}$ & $\begin{array}{l}\text { Iran } \\
\text { Gym } \\
\text { RCT }\end{array}$ & $\begin{array}{l}\text { Nonprofit: Research } \\
\text { and Technology } \\
\text { Deputy of Shahrekord } \\
\text { University of Medical } \\
\text { Sciences }\end{array}$ \\
\hline $\begin{array}{l}\text { Hebert, } 2011 \\
\text { Aerobic Exercise } \\
\text { Cycling } \\
\text { Postintervention, } 4 \\
\text { weeks } \\
\text { Fair }\end{array}$ & $\begin{array}{l}\text { One patient in the exercise control group } \\
\text { had a minor ankle sprain }(1 / 13) \text {. }\end{array}$ & Multiple sclerosis & $\begin{array}{l}\text { MS patients } 18 \text { to } \\
65 \text { years old; able to walk } \\
100 \text { m with or without a } \\
\text { single-sided device; a } \\
\text { score of } 45 \text { out of } 84 \text { on } \\
\text { the Modified Fatigue } \\
\text { Impact Scale } \\
\text { questionnaire; composite } \\
\text { score of } 72 \text { on the } \\
\text { computerized SOT }\end{array}$ & $\begin{array}{l}\text { Randomized: } \\
38 \\
\text { Analyzed: } 38 * \\
\text { Attrition: } 0 \% \\
(0 / 38) \\
\text { *ITT }\end{array}$ & $\begin{array}{l}\text { US } \\
\text { Outpatient clinic }\end{array}$ & $\begin{array}{l}\text { National Multiple } \\
\text { Sclerosis Society, } \\
\text { Pilot } \\
\text { Project no. PP1501 }\end{array}$ \\
\hline $\begin{array}{l}\text { Hebert, } 2009 \\
\text { Companion to: Hebert, } \\
2011 \\
\text { Balance } \\
\text { Postinterventon, } 14 \\
\text { weeks } \\
\text { Fair }\end{array}$ & NR & Multiple sclerosis & $\begin{array}{l}\text { Ambulation of } 100 \mathrm{~m} \text { with } \\
\text { no greater than } \\
\text { intermittent or unilateral } \\
\text { constant use of an } \\
\text { assistive device, age } 18 \text { to } \\
60 \text { years }\end{array}$ & $\begin{array}{l}\text { Randomized: } \\
88 \\
\text { Analyzed: } 6 \\
\text { weeks: } 81 \\
\text { Analyzed: } 14 \\
\text { weeks: } 76 \\
\text { Attrition: } 15 \% \\
(13 / 88)\end{array}$ & $\begin{array}{l}\text { USA } \\
\text { Outpatient } \\
\text { RCT }\end{array}$ & Nonprofit \\
\hline
\end{tabular}




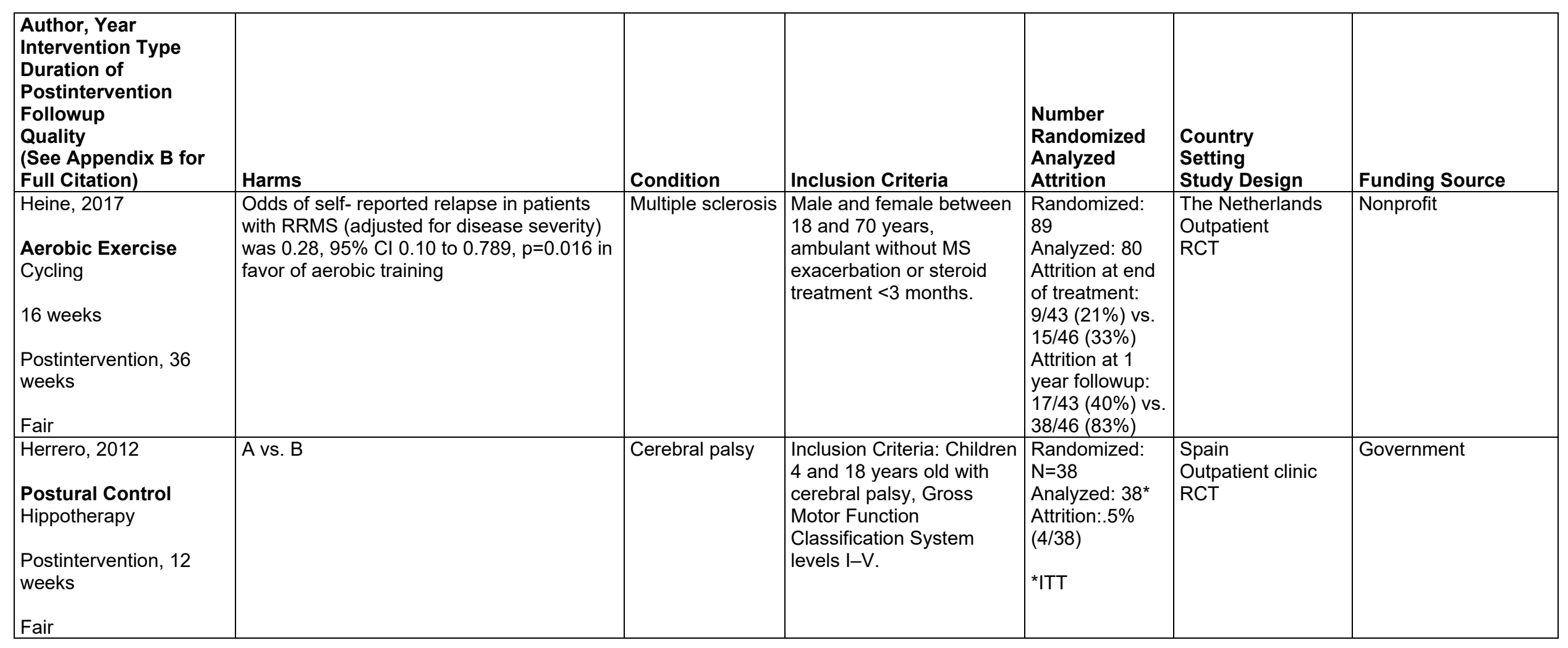




\begin{tabular}{|c|c|c|c|c|c|c|}
\hline $\begin{array}{l}\text { Author, Year } \\
\text { Intervention Type } \\
\text { Duration of } \\
\text { Postintervention } \\
\text { Followup } \\
\text { Quality } \\
\text { (See Appendix B for } \\
\text { Full Citation) }\end{array}$ & Harms & Condition & Inclusion Criteria & $\begin{array}{l}\text { Number } \\
\text { Randomized } \\
\text { Analyzed } \\
\text { Attrition }\end{array}$ & $\begin{array}{l}\text { Country } \\
\text { Setting } \\
\text { Study Design }\end{array}$ & Funding Source \\
\hline $\begin{array}{l}\text { Hochsprung, } 2017 \\
\text { Aerobic Exercise } \\
\text { Cycling } \\
\text { Postintervention, } 0 \\
\text { weeks } \\
\text { Poor }\end{array}$ & NR & Multiple sclerosis & $\begin{array}{l}\text { Inclusion criteria: (1) } \\
\text { referral by the neurologist } \\
\text { to our hospital's multiple } \\
\text { sclerosis unit; (2) } \\
\text { diagnosis of definite MS } \\
\text { according to the } \\
\text { McDonald criteria at least } \\
2 \text { years previously; (3) } \\
\text { EDSS score } 57 \\
\text { (established by a } \\
\text { neurologist); (4) age } \\
\text { between } 20 \text { and } 70 \text { years; } \\
\text { (5) clinical stability during } \\
\text { the } 3 \text { months previous to } \\
\text { recruitment; (6) no } \\
\text { cognitive impairment } \\
\text { according to the Mini- } \\
\text { Mental State Examination; } \\
\text { (7) willingness to sign an } \\
\text { informed consent form; } \\
\text { and (8) EDSS score } \\
\text { between } 2 \text { and } 6.5 \text {. }\end{array}$ & $\begin{array}{l}\text { Randomized: } \\
\mathrm{N}=61 \\
\text { Analyzed:61 } \\
\text { Attrition: } 0 \% \\
(0 / 61)\end{array}$ & $\begin{array}{l}\text { Spain } \\
\text { Outpatient clinic } \\
\text { RCT }\end{array}$ & NR \\
\hline
\end{tabular}




\begin{tabular}{|c|c|c|c|c|c|c|}
\hline $\begin{array}{l}\text { Author, Year } \\
\text { Intervention Type } \\
\text { Duration of } \\
\text { Postintervention } \\
\text { Followup } \\
\text { Quality } \\
\text { (See Appendix B for } \\
\text { Full Citation) }\end{array}$ & Harms & Condition & Inclusion Criteria & $\begin{array}{l}\text { Number } \\
\text { Randomized } \\
\text { Analyzed } \\
\text { Attrition }\end{array}$ & $\begin{array}{l}\text { Country } \\
\text { Setting } \\
\text { Study Design }\end{array}$ & Funding Source \\
\hline $\begin{array}{l}\text { Hogan, } 2014 \\
\text { (same author group and } \\
\text { protocol as Garrett } \\
2013 \text { a and } 2013 \mathrm{~b} \text { - see } \\
\text { notes) } \\
\text { Postural Control } \\
\text { Yoga } \\
\text { Postintervention, } 0 \\
\text { weeks }\end{array}$ & $\begin{array}{l}\text { Main problems reported by participants, } \mathrm{n} \\
\text {-Mobility/walking problems: } 49 \\
\text {-Fatigue: } 41 \\
\text {-Balance: } 36 \\
\text {-Weakness: } 34 \\
\text {-Bladder/bowel: } 29 \\
\text {-Pain: } 16 \\
\text {-Stiffness/spasms: } 16 \\
\text {-Vision: } 9 \\
\text {-Sensation: } 6 \\
\text {-Falls: } 3 \\
\text {-No problems: } 2\end{array}$ & Multiple sclerosis & $\begin{array}{l}\text { Over } 18 \text { years of age and } \\
\text { had a diagnosis of MS } \\
\text { that was confirmed by a } \\
\text { consultant physician or } \\
\text { neurologist. }\end{array}$ & $\begin{array}{l}\text { Randomized: } \\
146 \\
\text { Analyzed: } 111 \\
\text { Attrition: } 24 \% \\
(35 / 146)\end{array}$ & $\begin{array}{l}\text { Ireland } \\
\text { Community gyms, } \\
\text { hotels, health } \\
\text { centers } \\
\text { RCT }\end{array}$ & $\begin{array}{l}\text { This work, designated } \\
\text { the 'Getting the } \\
\text { Balance Right project,' } \\
\text { was supported by the } \\
\text { Multiple Sclerosis } \\
\text { Society of Ireland } \\
\text { (MSI) through the } \\
\text { Tesco Charity of the } \\
\text { Year funding and the } \\
\text { Pobal, Dormant } \\
\text { Accounts Flagship } \\
\text { Fund. In addition, the } \\
\text { lead author was an } \\
\text { EMBARK PhD } \\
\text { Scholar who was } \\
\text { supported by the Irish } \\
\text { Research Council for } \\
\text { Science Engineering } \\
\text { and Technology. }\end{array}$ \\
\hline $\begin{array}{l}\text { Hota, } 2020 \\
\text { Postural Control } \\
\text { Balance Exercises } \\
\text { Postintervention, } 4 \\
\text { weeks } \\
\text { Fair }\end{array}$ & None reported & $\begin{array}{l}\text { Spinal Cord } \\
\text { Injury }\end{array}$ & $\begin{array}{l}\text { Patients } 10 \text { years old or } \\
\text { more, admitted with } \\
\text { cervical injury, > } 30 \text { days } \\
\text { post injury }\end{array}$ & $\begin{array}{l}\text { Randomized: } \\
40 \\
\text { Analyzed: } 40 \\
\text { Attrition: } 0 \% \\
(0 / 40)\end{array}$ & $\begin{array}{l}\text { India } \\
\text { Spinal injury center } \\
\text { inpatient } \\
\text { rehabilitation } \\
\text { RCT }\end{array}$ & Funding NR \\
\hline $\begin{array}{l}\text { Hsieh, } 2018 \\
\text { Postural Control } \\
\text { Motion gaming } \\
\text { Postintervention, } 12 \\
\text { weeks } \\
\text { Fair }\end{array}$ & NR & Cerebral palsy & $\begin{array}{l}\text { Diagnosis of CP resulting } \\
\text { in hemiparesis, or a deficit } \\
\text { in movement and balance }\end{array}$ & $\begin{array}{l}\text { Randomized: } \\
40 \\
\text { Analyzed:40 } \\
\text { Attrition: } 0 \% \\
(0 / 40)\end{array}$ & $\begin{array}{l}\text { Taiwan } \\
\text { Outpatient clinic } \\
\text { RCT }\end{array}$ & NR \\
\hline
\end{tabular}




\begin{tabular}{|c|c|c|c|c|c|c|}
\hline $\begin{array}{l}\text { Author, Year } \\
\text { Intervention Type } \\
\text { Duration of } \\
\text { Postintervention } \\
\text { Followup } \\
\text { Quality } \\
\text { (See Appendix B for } \\
\text { Full Citation) }\end{array}$ & Harms & Condition & Inclusion Criteria & $\begin{array}{l}\text { Number } \\
\text { Randomized } \\
\text { Analyzed } \\
\text { Attrition }\end{array}$ & $\begin{array}{l}\text { Country } \\
\text { Setting } \\
\text { Study Design }\end{array}$ & Funding Source \\
\hline $\begin{array}{l}\text { Hsieh, } 2020 \\
\text { Postural Control } \\
\text { Motion Gaming } \\
\text { Postintervention, } 0 \\
\text { weeks } \\
\text { Fair }\end{array}$ & $\begin{array}{l}\text { No adverse effect } \\
\text { "was expected" but results NR. }\end{array}$ & Cerebral Palsy & $\begin{array}{l}\text { Children between } 6 \text { and } \\
10 \text { years old with cerebral } \\
\text { palsy and motor problems } \\
\text { GMFCS category level = } \\
\text { I-III }\end{array}$ & $\begin{array}{l}\text { Randomized: } \\
56 \\
\text { Analyzed: } 56 \\
\text { Attrition: } 0 \% \\
(0 / 56)\end{array}$ & $\begin{array}{l}\text { Taiwan } \\
\text { Pediatric } \\
\text { rehabilitation } \\
\text { RCT }\end{array}$ & Funding NR \\
\hline $\begin{array}{l}\text { Huang, } 2015 \\
\text { Aerobic Exercise } \\
\text { Robot-Assisted Gait } \\
\text { Training } \\
\text { Postintervention, } 4 \\
\text { weeks } \\
\text { Fair }\end{array}$ & none & Spinal cord injury & $\begin{array}{l}\text { Inclusion Criteria: } \\
\text { Incomplete SCl, T8 to L2, } \\
\text { injury within } 6 \text { months }\end{array}$ & $\begin{array}{l}\text { Randomized: } \\
\mathrm{N}=24 \\
\text { Analyzed: } 24 \\
\text { Attrition: } 0 \%\end{array}$ & $\begin{array}{l}\text { China } \\
\text { outpatient (?) } \\
\text { RCT }\end{array}$ & $\begin{array}{l}\text { Research on Design } \\
\text { Theory and Compliant } \\
\text { Control for } \\
\text { Underactuated Lower } \\
\text { Extremity } \\
\text { Rehabilitation Robotic } \\
\text { Systems }\end{array}$ \\
\hline $\begin{array}{l}\text { In, } 2018 \\
\text { Postural Control } \\
\text { Whole body vibration } \\
\text { Postintervention, } 0 \\
\text { weeks } \\
\text { Fair }\end{array}$ & None reported. & Spinal cord injury & $\begin{array}{l}\text { (1) diagnosed with } \\
\text { cervical level } 6 \text { or } 7 \\
\text { incomplete } \mathrm{SCl},(2) \text { onset } \\
\geq 6 \text { months, (3) American } \\
\text { Spinal Injury Association } \\
\text { Impairment Scale (AIS) } \\
\text { grade D motor and } \\
\text { sensory scores, (4) ability } \\
\text { to stand for at least } 5 \text { min, } \\
\text { (5) ability to understand } \\
\text { and follow verbal } \\
\text { commands, (6) medical } \\
\text { referral by a physician for } \\
\text { physical therapy, and (7) } \\
\text { ability to complete } \\
\text { designed WBV training } \\
\text { session. }\end{array}$ & $\begin{array}{l}\text { Randomized: } \\
32 \\
\text { Analyzed: } 28 \\
\text { Attrition: } 12.5 \% \\
(4 / 32)\end{array}$ & $\begin{array}{l}\text { South Korea } \\
\text { Outpatient } \\
\text { RCT }\end{array}$ & $\begin{array}{l}\text { This work was } \\
\text { supported by the } 2016 \\
\text { Gimcheon university } \\
\text { Research Grant. }\end{array}$ \\
\hline
\end{tabular}




\begin{tabular}{|c|c|c|c|c|c|c|}
\hline $\begin{array}{l}\text { Author, Year } \\
\text { Intervention Type } \\
\text { Duration of } \\
\text { Postintervention } \\
\text { Followup } \\
\text { Quality } \\
\text { (See Appendix B for } \\
\text { Full Citation) }\end{array}$ & Harms & Condition & Inclusion Criteria & $\begin{array}{l}\text { Number } \\
\text { Randomized } \\
\text { Analyzed } \\
\text { Attrition }\end{array}$ & $\begin{array}{l}\text { Country } \\
\text { Setting } \\
\text { Study Design }\end{array}$ & Funding Source \\
\hline $\begin{array}{l}\text { Johnston } 2011 \\
\text { Aerobic Exercise } \\
\text { Treadmill } \\
\text { Postintervention, } 4 \\
\text { weeks } \\
\text { Fair }\end{array}$ & None reported & Cerebral palsy & $\begin{array}{l}\text { Recruited from Shriners' } \\
\text { outpatient clinics children } \\
\text { with diplegia, triplegia or } \\
\text { quadriplegia } \\
\text { GMFCS III or IV } \\
\text { Able to take } 8 \text { steps } \\
6 \text { to } 13 \text { years old }\end{array}$ & $\begin{array}{l}34 \text { randomized } \\
26 \text { analyzed } \\
8 / 34=23.5 \% \\
\text { attrition }\end{array}$ & $\begin{array}{l}\text { USA } \\
\text { Physical therapists } \\
\text { and home setting } \\
\text { RCT }\end{array}$ & $\begin{array}{l}\text { funded by Shriners } \\
\text { (Foundation) }\end{array}$ \\
\hline $\begin{array}{l}\text { Jones, 2014a } \\
\text { Jones, 2014b } \\
\text { Multimodal Exercise } \\
\text { Postintervention, } \\
12 \text { weeks (for ALL } \\
\text { patients completing the } \\
\text { Activity Based Therapy } \\
\text { intervention) } \\
\text { Poor (for both) }\end{array}$ & $\begin{array}{l}\text { Withdrawals due to injuries related to } \\
\text { participation in intensive exercise: } 7.7 \% \\
(2 / 26) \text { vs. } 0 \%(0 / 22)\end{array}$ & Spinal cord injury & $\begin{array}{l}\text { AIS classification of } C \text { or } \\
D, \text { upper motor neuron } \\
\text { injury, preserved tendon } \\
\text { reflexes in the lower } \\
\text { extremities, at least } 1 \text { year } \\
\text { postinjury, and ages } 18 \text { to } \\
66 \text { years. }\end{array}$ & $\begin{array}{l}\text { Randomized: } \\
48 \\
\text { Analyzed: } 41 \\
\text { Attrition: } 14.6 \% \\
\text { (7/48) }\end{array}$ & $\begin{array}{l}\text { USA } \\
\text { Outpatient } \\
\text { RCT }\end{array}$ & $\begin{array}{l}\text { Supported in part by } \\
\text { the National Institute } \\
\text { on Disability and } \\
\text { Rehabilitation } \\
\text { Research (NIDRR), } \\
\text { U.S. Department of } \\
\text { Education (grant no. } \\
\text { H133G080031-10). }\end{array}$ \\
\hline $\begin{array}{l}\text { Jonnsdottir, } 2018 \\
\text { Aerobic Exercise } \\
\text { Treadmill } \\
\text { Postintervention, } 0 \\
\text { weeks } \\
\text { Fair }\end{array}$ & None reported & Multiple sclerosis & $\begin{array}{l}\text { Recruited inpatient rehab } \\
\text { service, able to walk } 10 \\
\text { meters }\end{array}$ & $\begin{array}{l}42 \text { randomized } \\
38 \text { analyzed } \\
4 / 42=9.5 \% \\
\text { attrition }\end{array}$ & $\begin{array}{l}\text { Italy } \\
\text { Rehabilitation } \\
\text { center } \\
\text { RCT }\end{array}$ & Government \\
\hline
\end{tabular}




\begin{tabular}{|c|c|c|c|c|c|c|}
\hline $\begin{array}{l}\text { Author, Year } \\
\text { Intervention Type } \\
\text { Duration of } \\
\text { Postintervention } \\
\text { Followup } \\
\text { Quality } \\
\text { (See Appendix B for } \\
\text { Full Citation) } \\
\end{array}$ & Harms & Condition & Inclusion Criteria & $\begin{array}{l}\text { Number } \\
\text { Randomized } \\
\text { Analyzed } \\
\text { Attrition } \\
\end{array}$ & \begin{tabular}{|l} 
Country \\
Setting \\
Study Design \\
\end{tabular} & Funding Source \\
\hline $\begin{array}{l}\text { Jung, } 2014 \\
\text { Aerobic Exercise } \\
\text { Aquatics } \\
\text { Postintervention, } 0 \\
\text { weeks } \\
\text { Fair }\end{array}$ & NR & Spinal cord injury & $\begin{array}{l}\text { Diagnosis of } \\
\text { American Spinal Injury } \\
\text { Association (ASIA) grade } \\
\text { B, C, or } \\
\text { D spinal cord injury at the } \\
\text { levels of C8 to L5. }\end{array}$ & $\begin{array}{l}\text { Randomized: } \\
\mathrm{N}=20 \\
\text { Analyzed: } 20 \\
\text { Attrition: } 0 \% \\
(0 / 20)\end{array}$ & $\begin{array}{l}\text { Korea } \\
\text { Inpatient } \\
\text { RCT }\end{array}$ & NR \\
\hline $\begin{array}{l}\text { Kalron, } 2016 \\
\text { Postural Control } \\
\text { Motion gaming } \\
\text { Postintervention, } 6 \\
\text { weeks } \\
\text { Fair }\end{array}$ & NR & Multiple sclerosis & $\begin{array}{l}\text { Diagnosis of definite } \\
\text { relapsing remitting MS; } \\
25-55 \text { years of age; } \\
\text { moderate neurological } \\
\text { disability as scored by the } \\
\text { EDSS; ranging from } 3.0 \text { to } \\
6.0 \text { inclusive with a } \\
\text { pyramidal functional score } \\
\text { of at least } 3\end{array}$ & $\begin{array}{l}\text { Randomized: } \\
32 \\
\text { Analyzed:30 } \\
\text { Attrition: } 6.3 \% \\
(2 / 32)\end{array}$ & $\begin{array}{l}\text { Israel } \\
\text { Outpatient clinic } \\
\text { RCT }\end{array}$ & $\begin{array}{l}\text { Nonprofit: supported } \\
\text { by a Pilot Research } \\
\text { Award from the } \\
\text { National Multiple } \\
\text { Sclerosis Society } \\
\text { (PP2208) }\end{array}$ \\
\hline $\begin{array}{l}\text { Kalron, } 2017 \\
\text { Muscle Strength } \\
\text { Postintervention, } 0 \\
\text { weeks } \\
\text { Fair }\end{array}$ & $\begin{array}{l}\text { No adverse or harmful events were } \\
\text { reported in both groups }\end{array}$ & Multiple sclerosis & $\begin{array}{l}\text { (1) diagnosis of definite } \\
\text { relapsing-remitting } \\
\text { multiple sclerosis } \\
\text { according to the revised } \\
\text { McDonald criteria (2) age } \\
\text { range from } 25-55 \text { years; } \\
\text { and (3) the EDSS score } \\
\text { ranging from } 3.0 \text { to } 6.0 \text {. } \\
\text { Additionally, only patients } \\
\text { receiving disease- } \\
\text { modifying drugs based on } \\
\text { interferon beta-1a for at } \\
\text { least } 3 \text { months. }\end{array}$ & $\begin{array}{l}\text { Randomized: } \\
50 \\
\text { Analyzed: } 45 \\
\text { Attrition: } 10 \% \\
(5 / 50)\end{array}$ & $\begin{array}{l}\text { Israel } \\
\text { Outpatient clinic } \\
\text { RCT }\end{array}$ & $\begin{array}{l}\text { This work was } \\
\text { supported by a grant } \\
\text { (EMR200136_642) } \\
\text { from the Merk KGaA, } \\
\text { Damstadt, Germany. }\end{array}$ \\
\hline
\end{tabular}




\begin{tabular}{|c|c|c|c|c|c|c|}
\hline $\begin{array}{l}\text { Author, Year } \\
\text { Intervention Type } \\
\text { Duration of } \\
\text { Postintervention } \\
\text { Followup } \\
\text { Quality } \\
\text { (See Appendix B for } \\
\text { Full Citation) }\end{array}$ & Harms & Condition & Inclusion Criteria & $\begin{array}{l}\text { Number } \\
\text { Randomized } \\
\text { Analyzed } \\
\text { Attrition } \\
\end{array}$ & $\begin{array}{l}\text { Country } \\
\text { Setting } \\
\text { Study Design }\end{array}$ & Funding Source \\
\hline $\begin{array}{l}\text { Kara, } 2017 \\
\text { Aerobic Exercise } \\
\text { Aerobics } \\
\text { Muscle Strength } \\
\text { Postintervention, } 0 \\
\text { weeks } \\
\text { Poor }\end{array}$ & NR & Multiple sclerosis & $\begin{array}{l}\text { Older than } 18 \text { years, } \\
\text { EDSS }<6 \\
\text { - Being diagnosed with } \\
\text { definite MS according to } \\
\text { McDonald criteria, an- } \\
\text { EDSS of } \geq 6 \\
\text { - Being older than } 18 \\
\text { years } \\
\text { - Not having an acute } \\
\text { attack. }\end{array}$ & $\begin{array}{l}\text { Randomized: } \\
\text { NA } \\
\text { Analyzed: } 35 \\
(64 \%) \\
\text { Attrition: } 22 \\
(40 \%)\end{array}$ & \begin{tabular}{|l|} 
Turkey \\
Outpatient \\
Quasiexperimental, \\
nonrandomized
\end{tabular} & No funding received \\
\hline $\begin{array}{l}\text { Kara, } 2020 \\
\text { Strength } \\
\text { Immediately } \\
\text { postintervention, } 12 \\
\text { weeks } \\
\text { Fair } \\
\end{array}$ & No adverse events occurred in either group. & Cerebral palsy & $\begin{array}{l}\text { (1) age between } 7 \text { and } 16 \\
\text { years; (2) classification as } \\
\text { levels I-III on the Manual } \\
\text { Ability Classification } \\
\text { System (MACS); and (3) } \\
\text { the ability to follow and } \\
\text { accept verbal } \\
\text { instructions. }\end{array}$ & $\begin{array}{l}\text { Randomized: } \\
34 \\
\text { Analyzed: } 30 \\
\text { Attrition: } 12 \% \\
(4 / 34)\end{array}$ & $\begin{array}{l}\text { Turkey } \\
\text { Outpatient } \\
\text { RCT }\end{array}$ & NR \\
\hline $\begin{array}{l}\text { Kargarfard, } 2017 \\
\text { Aerobic Exercise } \\
\text { Aquatics } \\
\text { Postintervention, } 0 \\
\text { weeks } \\
\text { Fair }\end{array}$ & NR & Multiple sclerosis & $\begin{array}{l}\text { Inclusion Criteria: } \\
\text { MS of a minimum of } 2 \\
\text { years, had no relapses in } \\
\text { the past month, and } \\
\text { were able to exercise } \\
\text { regularly. }\end{array}$ & $\begin{array}{l}\text { Randomized: } \\
\mathrm{N}=40 \\
\text { Analyzed: } 32 \\
\text { Attrition: } 20 \% \\
(8 / 40)\end{array}$ & $\begin{array}{l}\text { Iran } \\
\text { Outpatient clinic } \\
\text { RCT }\end{array}$ & NR \\
\hline $\begin{array}{l}\text { Kaya Kara, } 2019 \\
\text { Multimodal Exercise } \\
\text { Immediately } \\
\text { postintervention, } 12 \\
\text { weeks } \\
\text { Fair }\end{array}$ & $\begin{array}{l}\text { No adverse events reported, although one } \\
\text { person in the exercise group had ankle pain } \\
\text { following a fall while playing basketball. }\end{array}$ & Cerebral palsy & $\begin{array}{l}\text { Inclusion Criteria: GMFCS } \\
\text { levels I, ages } 7 \text { to } 16 \\
\text { years old }\end{array}$ & $\begin{array}{l}\text { Randomized: } \\
33 \\
\text { Analyzed: } 30 \\
\text { Attrition: } 9 \% \\
(3 / 33)\end{array}$ & $\begin{array}{l}\text { Turkey } \\
\text { Physical therapy } \\
\text { clinic } \\
\text { RCT }\end{array}$ & NR \\
\hline
\end{tabular}




\begin{tabular}{|c|c|c|c|c|c|c|}
\hline $\begin{array}{l}\text { Author, Year } \\
\text { Intervention Type } \\
\text { Duration of } \\
\text { Postintervention } \\
\text { Followup } \\
\text { Quality } \\
\text { (See Appendix B for } \\
\text { Full Citation) }\end{array}$ & Harms & Condition & Inclusion Criteria & $\begin{array}{l}\text { Number } \\
\text { Randomized } \\
\text { Analyzed } \\
\text { Attrition } \\
\end{array}$ & \begin{tabular}{|l|} 
Country \\
Setting \\
Study Design \\
\end{tabular} & Funding Source \\
\hline $\begin{array}{l}\text { Kerling, } 2015 \\
\text { Multimodal Exercise } \\
\text { Postintervention, } 0 \\
\text { weeks } \\
\text { Fair }\end{array}$ & NR & Multiple sclerosis & $\begin{array}{l}\text { Diagnosed MS, adult age } \\
\text { (18-65 years), and } \\
\text { mobility with a maximum } \\
\text { value of } 6 \text { (low to } \\
\text { moderate disability) on the } \\
\text { EDSS }\end{array}$ & $\begin{array}{l}\text { Randomized: } \\
60 \\
\text { Analyzed: } 37 \\
\text { Attrition: } 38.3 \% \\
(23 / 60)\end{array}$ & $\begin{array}{l}\text { Germany } \\
\text { Outpatient } \\
\text { RCT }\end{array}$ & $\begin{array}{l}\text { The study was } \\
\text { supported by Sanofi } \\
\text { Aventis. }\end{array}$ \\
\hline $\begin{array}{l}\text { Keser, } 2011 \\
\text { Aerobic Exercise } \\
\text { Aerobics } \\
\text { Postintervention, } 0 \\
\text { weeks } \\
\text { Poor }\end{array}$ & NR & Multiple sclerosis & EDSS between 1 and 5.5 & $\begin{array}{l}\text { Randomized: } \\
\text { NA } \\
\text { Analyzed: } 30 \\
\text { Attrition: } 0\end{array}$ & \begin{tabular}{|l|} 
Turkey \\
Outpatient \\
Quasiexperimental, \\
nonrandomized
\end{tabular} & NR \\
\hline $\begin{array}{l}\text { Khalil, } 2018 \\
\text { Postural Control } \\
\text { Motion gaming } \\
\text { Postintervention, } 6 \\
\text { weeks } \\
\text { Fair }\end{array}$ & NR & Multiple sclerosis & $\begin{array}{l}\text { Diagnosis of MS, } \\
\text { relapsing remitting type of } \\
\text { MS; age of above } 18 \\
\text { years, EDSS score of } 3 \text { to } \\
6.5 \text {; being relapse free for } \\
30 \text { days prior to } \\
\text { participation or to } \\
\text { completing testing }\end{array}$ & $\begin{array}{l}\text { Randomized:40 } \\
\text { Analyzed: } 32 \\
\text { Attrition: } 20 \% \\
(8 / 40)\end{array}$ & $\begin{array}{l}\text { Jordan } \\
\text { University } \\
\text { RCT }\end{array}$ & $\begin{array}{l}\text { Government: funding } \\
\text { support from EU } \\
\text { commission for } \\
\text { funding } \\
\text { support (grant } \\
\text { number: AR- 42). }\end{array}$ \\
\hline $\begin{array}{l}\text { Kim } 2015 \\
\text { Postural Control } \\
\text { Balance } \\
\text { Postintervention, } 0 \\
\text { weeks } \\
\text { Fair }\end{array}$ & None reported & Cerebral palsy & $\begin{array}{l}\text { Ambulatory adults (without } \\
\text { support) }\end{array}$ & $\begin{array}{l}\text { Randomized } 21 \\
\text { Analyzed } 21\end{array}$ & $\begin{array}{l}\text { Korean } \\
\text { RCT }\end{array}$ & Government funding \\
\hline
\end{tabular}




\begin{tabular}{|c|c|c|c|c|c|c|}
\hline $\begin{array}{l}\text { Author, Year } \\
\text { Intervention Type } \\
\text { Duration of } \\
\text { Postintervention } \\
\text { Followup } \\
\text { Quality } \\
\text { (See Appendix B for } \\
\text { Full Citation) }\end{array}$ & Harms & Condition & Inclusion Criteria & $\begin{array}{l}\text { Number } \\
\text { Randomized } \\
\text { Analyzed } \\
\text { Attrition }\end{array}$ & $\begin{array}{l}\text { Country } \\
\text { Setting } \\
\text { Study Design }\end{array}$ & Funding Source \\
\hline $\begin{array}{l}\text { Kim, } 2017 \\
\text { Postural Control } \\
\text { Balance } \\
\text { Social activity/exercise } \\
\text { (Boccia) } \\
\text { Postintervention, } 0 \\
\text { weeks } \\
\text { Poor }\end{array}$ & NR & Cerebral palsy & $\begin{array}{l}\text { Adults with CP in the age } \\
\text { bracket of } 18 \text { to } 30 \text { years } \\
\text { diagnosed with disability } \\
\text { of levels } 1 \text { to } 2 \\
\text { encephalopathy by } \\
\text { medical specialists in the } \\
\text { areas of rehabilitation } \\
\text { medicine and } \\
\text { neurosurgery. Those who } \\
\text { can sustain seated } \\
\text { posture on supplementary } \\
\text { wheelchair, those capable } \\
\text { of performing boccia } \\
\text { exercise, those who } \\
\text { achieved more than } 18 \\
\text { points in the Mini-Mental } \\
\text { Status Examination and } \\
\text { had no difficulties in } \\
\text { communicating and } \\
\text { interacting with the } \\
\text { researcher and } \\
\text { participants, those who } \\
\text { consented to voluntarily } \\
\text { participate in the } \\
\text { experiment on their own } \\
\text { will. }\end{array}$ & $\begin{array}{l}\text { Randomized: } \\
\text { N/A } \\
\text { Analyzed: } 23 \\
\text { Attrition: } 0 \% \\
(0 / 23)\end{array}$ & $\begin{array}{l}\text { South Korea } \\
\text { NR } \\
\text { Prospective } \\
\text { Comparative } \\
\text { Cohort }\end{array}$ & $\begin{array}{l}\text { This work was } \\
\text { supported by the } \\
\text { Research Fund of } \\
\text { Ulsan College in } \\
\text { Korea }\end{array}$ \\
\hline $\begin{array}{l}\text { Kirk, } 2016 \\
\text { Muscle Strength } \\
\text { Postintervention, } 0 \\
\text { weeks } \\
\text { Poor }\end{array}$ & $\begin{array}{l}\text { Most subjects that received the PRT } \\
\text { intervention reported delayed onset of } \\
\text { muscle soreness during the first couple of } \\
\text { training sessions, and } 3 \text { subjects reported } \\
\text { irritation in tendon tissue surrounding the } \\
\text { knee. }\end{array}$ & Cerebral palsy & $\begin{array}{l}\text { Diagnosed with CP, age } \\
18-65 \text { years, and gait } \\
\text { function with or without } \\
\text { walking aids }\end{array}$ & $\begin{array}{l}\text { Randomized: } \\
\text { N/A } \\
\text { Analyzed: } 32 \\
\text { Attrition: } 8.6 \% \\
(3 / 35)\end{array}$ & $\begin{array}{l}\text { Denmark } \\
\text { Gymnasium } \\
\text { Comparative } \\
\text { cohort }\end{array}$ & $\begin{array}{l}\text { The study was } \\
\text { supported by a grant } \\
\text { from the Ludvig and } \\
\text { Sara Elsass } \\
\text { Foundation and the } \\
\text { Association of Danish } \\
\text { Physiotherapists }\end{array}$ \\
\hline
\end{tabular}




\begin{tabular}{|c|c|c|c|c|c|c|}
\hline $\begin{array}{l}\text { Author, Year } \\
\text { Intervention Type } \\
\text { Duration of } \\
\text { Postintervention } \\
\text { Followup } \\
\text { Quality } \\
\text { (See Appendix B for } \\
\text { Full Citation) }\end{array}$ & Harms & Condition & Inclusion Criteria & $\begin{array}{l}\text { Number } \\
\text { Randomized } \\
\text { Analyzed } \\
\text { Attrition }\end{array}$ & $\begin{array}{l}\text { Country } \\
\text { Setting } \\
\text { Study Design }\end{array}$ & Funding Source \\
\hline $\begin{array}{l}\text { Kjolhede, } 2016 \\
\text { Muscle Strength } \\
\text { Postintervention, } 0 \\
\text { weeks } \\
\text { Fair }\end{array}$ & NR & Multiple sclerosis & $\begin{array}{l}\text { 18-60 years, a definite } \\
\text { relapsing-remitting MS } \\
\text { diagnosis according to the } \\
\text { McDonald criteria, EDSS } \\
2.0-5.5 \text { with a "pyramidal } \\
\text { functions" subscore } \geq 2 \\
\text { and receiving IFN- } \beta \text { 1a or } \\
1 \mathrm{~b} \text { (Rebif, Avonex, } \\
\text { Extavia, or Betaferon) for } \\
\text { at least } 3 \text { months (IFN- } \beta \\
\text { and Copaxone are the first } \\
\text { line of recommended } \\
\text { medication for relapsing- } \\
\text { remitting MS in Denmark). }\end{array}$ & $\begin{array}{l}\text { Randomized: } \\
35 \\
\text { Analyzed: } 30 \\
\text { Attrition: } 16.7 \% \\
(5 / 30)\end{array}$ & $\begin{array}{l}\text { Denmark } \\
\text { Outpatient clinic } \\
\text { RCT }\end{array}$ & $\begin{array}{l}\text { This study was } \\
\text { supported by The } \\
\text { Augustinus } \\
\text { Foundation, } \\
\text { Hestehandler Ole } \\
\text { Jacobsen Mindelegat, } \\
\text { and Biogen Idec. }\end{array}$ \\
\hline $\begin{array}{l}\text { Klobucka, } 2020 \\
\text { Aerobic Exercise } \\
\text { Robot-Assisted Gait } \\
\text { Training } \\
\text { Immediately } \\
\text { Postintervention, and } \\
\text { 12-16 weeks } \\
\text { Poor }\end{array}$ & No adverse events were reported. & Cerebral palsy & $\begin{array}{l}\text { Adolescent and adults } \\
\text { with bilateral spastic CP, } \\
\text { ages } 15 \text { years and older } \\
\text { with GMFCS levels I-IV. }\end{array}$ & $\begin{array}{l}\text { Randomized: } \\
47 \\
\text { Analyzed: } 47 \\
\text { and 45 } \\
\text { Attrition: } 10 \% \\
\text { (5/47) }\end{array}$ & $\begin{array}{l}\text { Slovakia } \\
\text { Outpatient } \\
\text { rehabilitation } \\
\text { Clinic } \\
\text { RCT }\end{array}$ & $\begin{array}{l}\text { This work was } \\
\text { supported by } \\
\text { KREATON } \\
\text { Project. }\end{array}$ \\
\hline $\begin{array}{l}\text { Kooshiar, } 2015 \\
\text { Aerobic Exercise } \\
\text { Aquatics }\end{array}$ & NR & Multiple sclerosis & $\begin{array}{l}\text { Inclusion Criteria: } \\
\text { Female patients affected } \\
\text { by MS, certified with a } \\
\text { medical documentation } \\
\text { and with a neurologist } \\
\text { approval; cognitive } \\
\text { competency to give } \\
\text { informed consent; citizen } \\
\text { of Iran and residing in } \\
\text { Mashhad; age ranging } \\
\text { from } 19 \text { to } 45 \text { years; and } \\
\text { Kurtzke EDSS } 9 \text { Score of } \\
\text { 1-5.5. }\end{array}$ & $\begin{array}{l}\text { Randomized: } \\
\mathrm{N}=40 \\
\text { Analyzed: } 37 \\
\text { Attrition: } 0.8 \% \\
(3 / 40)\end{array}$ & $\begin{array}{l}\text { Iran } \\
\text { Outpatient clinic } \\
\text { RCT }\end{array}$ & NR \\
\hline
\end{tabular}




\begin{tabular}{|c|c|c|c|c|c|c|}
\hline $\begin{array}{l}\text { Author, Year } \\
\text { Intervention Type } \\
\text { Duration of } \\
\text { Postintervention } \\
\text { Followup } \\
\text { Quality } \\
\text { (See Appendix B for } \\
\text { Full Citation) }\end{array}$ & Harms & Condition & Inclusion Criteria & $\begin{array}{l}\text { Number } \\
\text { Randomized } \\
\text { Analyzed } \\
\text { Attrition }\end{array}$ & $\begin{array}{l}\text { Country } \\
\text { Setting } \\
\text { Study Design }\end{array}$ & Funding Source \\
\hline $\begin{array}{l}\text { Kressler, } 2013 \\
\text { Companion to: Field- } \\
\text { Fote, } 2011 \\
\text { Aerobic Exercise } \\
\text { Robot-Assisted Gait } \\
\text { Training } \\
\text { Postintervention, } 12 \\
\text { weeks } \\
\text { Poor }\end{array}$ & None & $\begin{array}{l}\text { Spinal cord } \\
\text { injury }\end{array}$ & $\begin{array}{l}\text { Inclusion Criteria: chronic } \\
\text { motor-incomplete spinal } \\
\text { cord injury, minimal } \\
\text { walking ability }\end{array}$ & $\begin{array}{l}\text { Randomized: } \\
\mathrm{N}=74 \\
\text { Analyzed: } 64 \\
\text { Attrition: } 13.5 \%\end{array}$ & \begin{tabular}{|l|} 
US \\
Outpatient \\
(Academic medical \\
center research \\
lab) \\
RCT
\end{tabular} & NR \\
\hline $\begin{array}{l}\text { Kumru, } 2016 \\
\text { Aerobic Exercise } \\
\text { Robot-Assisted Gait } \\
\text { Training } \\
\text { Postintervention, } 8 \\
\text { weeks } \\
\text { Fair }\end{array}$ & $\begin{array}{l}9 \text { mild treatment-related adverse effects } \\
\text { (facial twitching, headache) }\end{array}$ & $\begin{array}{l}\text { Spinal cord } \\
\text { injury }\end{array}$ & $\begin{array}{l}\text { Inclusion Criteria: ASIA C } \\
\text { or D Cerivial or Thoracic } \\
\text { SCI, no limitation in } \\
\text { passive range of motion, } \\
\text { no changes in medical } \\
\text { treatment }\end{array}$ & $\begin{array}{l}\text { Randomized: } \\
\mathrm{N}=34 \\
\text { Analyzed: } 31 \\
\text { Attrition: } 9 \%\end{array}$ & $\begin{array}{l}\text { Spain } \\
\text { Inpatient } \\
\text { rehabilitation } \\
\text { hospital } \\
\text { RCT }\end{array}$ & $\begin{array}{l}\text { Foundation La Marato } \\
\text { and Instituto de Salud } \\
\text { Carlos }\end{array}$ \\
\hline $\begin{array}{l}\text { Kwon, } 2011 \\
\text { Postural Control } \\
\text { Hippotherapy } \\
\text { Postintervention, } 0 \\
\text { weeks } \\
\text { (End of treatment after } \\
\text { 8-week intervention) } \\
\text { Fair }\end{array}$ & NR & Cerebral palsy & $\begin{array}{l}\text { Inclusion Criteria: } \\
\text { diagnosis of bilateral } \\
\text { spastic cerebral palsy, } \\
\text { GMFCS level I or II, body } \\
\text { weight less than } 35 \mathrm{~kg} \text {, } \\
\text { and age of } 4 \text { to } 10 \text { years }\end{array}$ & $\begin{array}{l}\text { Randomized: } \\
\text { NA } \\
\text { Analyzed: } 32 \\
\text { Attrition: } 0 \% \\
(0 / 32)\end{array}$ & \begin{tabular}{|l|} 
Republic of Korea \\
Gym and \\
outpatient clinic \\
Quasiexperimental, \\
nonrandomized
\end{tabular} & Nonprofit \\
\hline
\end{tabular}




\begin{tabular}{|c|c|c|c|c|c|c|}
\hline $\begin{array}{l}\text { Author, Year } \\
\text { Intervention Type } \\
\text { Duration of } \\
\text { Postintervention } \\
\text { Followup } \\
\text { Quality } \\
\text { (See Appendix B for } \\
\text { Full Citation) }\end{array}$ & Harms & Condition & Inclusion Criteria & $\begin{array}{l}\text { Number } \\
\text { Randomized } \\
\text { Analyzed } \\
\text { Attrition }\end{array}$ & $\begin{array}{l}\text { Country } \\
\text { Setting } \\
\text { Study Design }\end{array}$ & Funding Source \\
\hline $\begin{array}{l}\text { Kwon, } 2015 \\
\text { Postural Control } \\
\text { Hippotherapy } \\
\text { Postintervention, } 0 \\
\text { weeks } \\
\text { (End of treatment after } \\
\text { 8-week intervention) } \\
\text { Good }\end{array}$ & $\begin{array}{l}2 \text { participants }(2 \%) \text { fell during the study } \\
\text { period. One participant returned to the } \\
\text { therapy, while the other dropped out. } \\
\text { A vs. B } \\
\text { Falls: } 2 \%(1 / 46) \text { vs. } 0 \%(0 / 46)\end{array}$ & Cerebral palsy & $\begin{array}{l}\text { Inclusion Criteria: } \\
\text { diagnosis of CP, body } \\
\text { weight less than } 35 \mathrm{~kg}, \\
\text { and age between } 4 \text { and } \\
10 \text { years }\end{array}$ & $\begin{array}{l}\text { Randomized: } \\
92 \\
\text { Analyzed: } 91 \\
\text { Attrition: } 1 \% \\
(1 / 92)\end{array}$ & $\begin{array}{l}\text { Republic of Korea } \\
\text { Home and } \\
\text { outpatient clinic } \\
\text { RCT }\end{array}$ & Nonprofit \\
\hline $\begin{array}{l}\text { Lai, } 2010 \\
\text { Aerobic Exercise } \\
\text { Cycling } \\
\text { Postintervention, } 12 \\
\text { weeks } \\
\text { Fair }\end{array}$ & NR & Spinal cord injury & $\begin{array}{l}\text { Having a neurologically } \\
\text { complete SCI motor lesion } \\
\text { (American Spinal Cord } \\
\text { Association (ASIA) } \\
\text { impairment scale (17) } \\
\text { grade A) between C5 } \\
\text { andT10; having muscle } \\
\text { responses to trial } \\
\text { electrical stimulation; and } \\
\text { never having undergone } \\
\text { FES therapy } \\
\end{array}$ & $\begin{array}{l}\text { Randomized: } \\
\mathrm{N}=24 \\
\text { Analyzed:24 } \\
\text { Attrition: } 0 \% \\
(0 / 24)\end{array}$ & $\begin{array}{l}\text { Taiwan } \\
\text { Inpatient } \\
\text { RCT }\end{array}$ & $\begin{array}{l}\text { National Science } \\
\text { Council }\end{array}$ \\
\hline $\begin{array}{l}\text { Lai, } 2015 \\
\text { Aerobic Exercise } \\
\text { Aquatics } \\
\text { Postintervention, } 0 \\
\text { weeks } \\
\text { Fair }\end{array}$ & None & Cerebral palsy & $\begin{array}{l}\text { Diagnosis of spastic } \\
\text { cerebral palsy; age of } 4 \text { to } \\
12 \text { years; Gross Motor } \\
\text { Function Classification } \\
\text { System levels of I to IV16; } \\
\text { and ability to follow } \\
\text { instructions }\end{array}$ & $\begin{array}{l}\text { Randomized: } \\
\mathrm{N}=27 \\
\text { Analyzed: } 24 \\
\text { Attrition: } 11 \% \\
(3 / 27)\end{array}$ & $\begin{array}{l}\text { Taiwan } \\
\text { Outpatient clinic } \\
\text { RCT }\end{array}$ & $\begin{array}{l}\text { National Science } \\
\text { Council and Chang } \\
\text { Gung Memorial } \\
\text { Hospital }\end{array}$ \\
\hline
\end{tabular}




\begin{tabular}{|c|c|c|c|c|c|c|}
\hline $\begin{array}{l}\text { Author, Year } \\
\text { Intervention Type } \\
\text { Duration of } \\
\text { Postintervention } \\
\text { Followup } \\
\text { Quality } \\
\text { (See Appendix B for } \\
\text { Full Citation) }\end{array}$ & Harms & Condition & Inclusion Criteria & $\begin{array}{l}\text { Number } \\
\text { Randomized } \\
\text { Analyzed } \\
\text { Attrition }\end{array}$ & $\begin{array}{l}\text { Country } \\
\text { Setting } \\
\text { Study Design }\end{array}$ & Funding Source \\
\hline $\begin{array}{l}\text { Lavado, } 2012 \\
\text { Aerobic Exercise } \\
\text { Hand cycling } \\
\text { Postintervention, } 0 \\
\text { weeks } \\
\text { Fair }\end{array}$ & NR & Spinal cord injury & $\begin{array}{l}\text { EDSS between } 1 \text { and } 5.5 \\
\text { Injury between C5 and L2, } \\
\text { excluded community } \\
\text { ambulators and those } \\
\text { unable to arm cycle }\end{array}$ & $\begin{array}{l}\text { Randomized: } \\
42 \\
\text { Analyzed: } 42 \\
(100 \%) \\
\text { Attrition: } 0\end{array}$ & $\begin{array}{l}\text { Brazil } \\
\text { Outpatient } \\
\text { RCT }\end{array}$ & No funding received \\
\hline $\begin{array}{l}\text { Lee, } 2013 \\
\text { Postural Control } \\
\text { Whole body vibration } \\
\text { Postintervention, } 0 \\
\text { weeks } \\
\text { Fair }\end{array}$ & NR & Cerebral palsy & $\begin{array}{l}\text { (1) cerebral palsy } \\
\text { diagnosed by both a } \\
\text { pediatric neurological } \\
\text { doctor and a physical } \\
\text { therapist; (2) no history of } \\
\text { serious surgery on the } \\
\text { spine; (3) diagnosis of } \\
\text { weak muscles in at least } \\
\text { one of the evaluated leg } \\
\text { muscles; muscle } \\
\text { weakness was determined } \\
\text { by symptoms of the } \\
\text { muscle's inability to } \\
\text { perform rising from a chair } \\
\text { (difficulty with movements) } \\
\text { - symptoms include: } \\
\text { fatigue, numbness in } \\
\text { muscles, inability to } \\
\text { support one's arms and } \\
\text { legs, drowsiness, } \\
\text { prolonged tiredness and } \\
\text { lethargy; (4) no drug being } \\
\text { taken for spasticity } \\
\text { control; (5) good vision; } \\
\text { (6) ability to comprehend } \\
\text { instructions; and (7) ability } \\
\text { to walk without the use of } \\
\text { walking aids. }\end{array}$ & $\begin{array}{l}\text { Randomized: } \\
30 \\
\text { Analyzed: } 30 \\
\text { Attrition: } 0 \% \\
(0 / 30)\end{array}$ & $\begin{array}{l}\text { South Korea } \\
\text { Outpatient } \\
\text { RCT }\end{array}$ & $\begin{array}{l}\text { This research } \\
\text { received no specific } \\
\text { grant from any } \\
\text { funding agency in the } \\
\text { public, commercial, or } \\
\text { not-for-profit sectors. }\end{array}$ \\
\hline
\end{tabular}




\begin{tabular}{|c|c|c|c|c|c|c|}
\hline $\begin{array}{l}\text { Author, Year } \\
\text { Intervention Type } \\
\text { Duration of } \\
\text { Postintervention } \\
\text { Followup } \\
\text { Quality } \\
\text { (See Appendix B for } \\
\text { Full Citation) }\end{array}$ & Harms & Condition & Inclusion Criteria & $\begin{array}{l}\text { Number } \\
\text { Randomized } \\
\text { Analyzed } \\
\text { Attrition }\end{array}$ & $\begin{array}{l}\text { Country } \\
\text { Setting } \\
\text { Study Design }\end{array}$ & Funding Source \\
\hline $\begin{array}{l}\text { Lee, } 2014 \\
\text { Postural Control } \\
\text { Hippotherapy } \\
\text { End of treatment (12- } \\
\text { week intervention) } \\
\text { Poor }\end{array}$ & NR & Cerebral palsy & $\begin{array}{l}\text { Inclusion Criteria: } \\
\text { MAS grade less than }+1 \text {, } \\
\text { perform more than } 10 \mathrm{~m} \\
\text { independent walking, } \\
\text { available for more than } \\
30 \text {-minute training per day }\end{array}$ & $\begin{array}{l}\text { Randomized: } \\
26 \\
\text { Analyzed: } 26 \\
\text { Attrition: } 0 \% \\
(0 / 26)\end{array}$ & $\begin{array}{l}\text { Republic of Korea } \\
\text { Outpatient clinic } \\
\text { RCT }\end{array}$ & NR \\
\hline $\begin{array}{l}\text { Liu, } 2019 \\
\text { Multimodal Exercise } \\
\text { Immediately } \\
\text { postintervention, } \\
12 \text { weeks } \\
\text { Fair }\end{array}$ & Reported no adverse events occurred. & Spinal cord injury & $\begin{array}{l}\text { Inclusion Criteria: } \\
\text { Ambulatory } \mathrm{SCl} \text { patients, } \\
18-50 \text { years old }\end{array}$ & $\begin{array}{l}\text { Randomized: } \\
40 \\
\text { Analyzed: } 29 \\
\text { Attrition: } 27 \% \\
(11 / 40)\end{array}$ & $\begin{array}{l}\text { China } \\
\text { Rehab center } \\
\text { RCT }\end{array}$ & $\begin{array}{l}\text { The Special Fund for } \\
\text { Basic Scientific } \\
\text { Research of Central } \\
\text { Public Institutes }\end{array}$ \\
\hline $\begin{array}{l}\text { Lorentzen, } 2015 \\
\text { Postural Control } \\
\text { Balance } \\
\text { Postintervention, } 20 \\
\text { weeks } \\
\text { Poor }\end{array}$ & NR & Cerebral palsy & $\begin{array}{l}\text { Diagnosis pf spastic } \\
\text { cerebral palsy (GMFCS I- } \\
\text { II; MACS I-II) based on } \\
\text { medical records and } \\
\text { classification by the } \\
\text { therapists participated in } \\
\text { the study }\end{array}$ & $\begin{array}{l}\text { Non } \\
\text { Randomized: } \\
34 \\
\text { Analyzed:34 } \\
\text { Attrition: } 0 \% \\
(0 / 34)\end{array}$ & \begin{tabular}{|l|} 
Denmark \\
Outpatient Clinic \\
Quasiexperimental, \\
nonrandomized
\end{tabular} & $\begin{array}{l}\text { Nonprofit: Ludvig and } \\
\text { Sara Elsass } \\
\text { foundation }\end{array}$ \\
\hline $\begin{array}{l}\text { Lucena-Anton, } 2018 \\
\text { Postural Control } \\
\text { Hippotherapy } \\
\text { Postintervention, } 1 \\
\text { week (13 weeks total } \\
\text { including 12-week } \\
\text { intervention) } \\
\text { Fair }\end{array}$ & No adverse effects were reported. & Cerebral palsy & $\begin{array}{l}\text { Inclusion Criteria: } \\
\text { prior diagnosis of spastic } \\
\text { CP, nonwalking children } \\
\text { (GMFCS levels: IV-V), } \\
\text { and children aged } 3 \text { to } 14 \\
\text { years }\end{array}$ & $\begin{array}{l}\text { Randomized: } \\
48 \\
\text { Analyzed: } 44 \\
\text { Attrition: } 4 \% \\
(2 / 48)\end{array}$ & $\begin{array}{l}\text { Spain } \\
\text { Outpatient clinic } \\
\text { RCT }\end{array}$ & NR \\
\hline
\end{tabular}




\begin{tabular}{|c|c|c|c|c|c|c|}
\hline $\begin{array}{l}\text { Author, Year } \\
\text { Intervention Type } \\
\text { Duration of } \\
\text { Postintervention } \\
\text { Followup } \\
\text { Quality } \\
\text { (See Appendix B for } \\
\text { Full Citation) }\end{array}$ & Harms & Condition & Inclusion Criteria & $\begin{array}{l}\text { Number } \\
\text { Randomized } \\
\text { Analyzed } \\
\text { Attrition }\end{array}$ & $\begin{array}{l}\text { Country } \\
\text { Setting } \\
\text { Study Design }\end{array}$ & Funding Source \\
\hline $\begin{array}{l}\text { Makhov, } 2018 \\
\text { Multimodal } \\
\text { exercise } \\
\text { Immediately } \\
\text { postintervention, } \\
15 \text { weeks } \\
\text { Poor }\end{array}$ & NR & Cerebral palsy & $\begin{array}{l}\text { Inclusion Criteria: CP } \\
\text { patients } 7 \text { to } 9 \text { years old } \\
\text { with spastic diplegia or } \\
\text { spastic tetra paresis }\end{array}$ & $\begin{array}{l}\text { Randomized: } \\
35 \\
\text { Analyzed: } 35 \\
\text { Attrition: } 0 \% \\
(0 / 35)\end{array}$ & $\begin{array}{l}\text { Russia } \\
\text { Setting Outpatient } \\
\text { clinic } \\
\text { RCT }\end{array}$ & NR \\
\hline $\begin{array}{l}\text { Marandi, 2013a } \\
\text { Aerobic Exercise } \\
\text { Aquatics } \\
\text { Has companion: } \\
\text { Marandi, 2013b } \\
\text { Postintervention, } 0 \\
\text { weeks } \\
\text { Poor } \\
\end{array}$ & NR & Multiple sclerosis & $\begin{array}{l}\text { Women with MS and a } \\
\text { EDSS score of less than } \\
4.5 \text { who visited Kashai } \\
\text { hospital in Esfahan }\end{array}$ & $\begin{array}{l}\text { Randomized: } \\
57 \\
\text { Analyzed: } 45 \\
\text { Attrition: } 21 \% \\
(12 / 57)\end{array}$ & $\begin{array}{l}\text { Iran } \\
\text { NR } \\
\text { RCT }\end{array}$ & $\begin{array}{l}\text { This study was } \\
\text { conducted as a thesis } \\
\text { funded by Isfahan } \\
\text { University, Isfahan, } \\
\text { Iran }\end{array}$ \\
\hline $\begin{array}{l}\text { Marandi, 2013b } \\
\text { Companion to: Marandi, } \\
2013 a \\
\text { Aerobic Exercise } \\
\text { Aquatics } \\
\text { Postintervention, } 0 \\
\text { weeks } \\
\text { Poor }\end{array}$ & NR & Multiple sclerosis & $\begin{array}{l}\text { Women with MS and a } \\
\text { EDSS score of less than } \\
4.5 \text { who visited Kashai } \\
\text { hospital inEsfahan }\end{array}$ & $\begin{array}{l}\text { Randomized: } \\
57 \\
\text { Analyzed: } 45 \\
\text { Attrition: } 21 \% \\
(12 / 57)\end{array}$ & $\begin{array}{l}\text { Iran } \\
\text { NR } \\
\text { RCT }\end{array}$ & $\begin{array}{l}\text { This study was } \\
\text { conducted as a thesis } \\
\text { funded by Isfahan } \\
\text { University, Isfahan, } \\
\text { Iran }\end{array}$ \\
\hline
\end{tabular}




\begin{tabular}{|c|c|c|c|c|c|c|}
\hline $\begin{array}{l}\text { Author, Year } \\
\text { Intervention Type } \\
\text { Duration of } \\
\text { Postintervention } \\
\text { Followup } \\
\text { Quality } \\
\text { (See Appendix B for } \\
\text { Full Citation) }\end{array}$ & Harms & Condition & Inclusion Criteria & $\begin{array}{l}\text { Number } \\
\text { Randomized } \\
\text { Analyzed } \\
\text { Attrition } \\
\end{array}$ & \begin{tabular}{|l|} 
Country \\
Setting \\
Study Design \\
\end{tabular} & Funding Source \\
\hline $\begin{array}{l}\text { Matusiak-Wieczorek, } \\
2016 \\
\text { Postural Control } \\
\text { Hippotherapy } \\
\\
\text { End of treatment (after } \\
\text { 12-week intervention) } \\
\text { Poor }\end{array}$ & NR & Cerebral palsy & $\begin{array}{l}\text { Inclusion criteria: } \\
\text { children aged } 6-12 \text { years } \\
\text { with spastic diplegia or } \\
\text { spastic hemiplegia CP, } \\
\text { GMFCS level } 1 \text { or } 2 \text {, able } \\
\text { to understand and follow } \\
\text { simple verbal instructions }\end{array}$ & $\begin{array}{l}\text { Randomized: } \\
\text { NA } \\
\text { Analyzed: } 39 \\
\text { Attrition: } 0 \% \\
(0 / 39)\end{array}$ & $\begin{array}{l}\text { Poland } \\
\text { Outpatient clinic } \\
\text { Quasiexperimental, } \\
\text { nonrandomized }\end{array}$ & NR \\
\hline $\begin{array}{l}\text { Matusiak-Wieczorek, } \\
2020 \\
\text { Postural Control } \\
\text { Hippotherapy } \\
\text { Immediately } \\
\text { Postintervention, } 12 \\
\text { weeks } \\
\text { Fair }\end{array}$ & Not reported & Cerebral Palsy & $\begin{array}{l}\text { Children with CP, aged 6- } \\
12 \text { years, classified as } \\
\text { Gross Motor Function } \\
\text { Classification System } \\
\text { (GMFCS) level I or II, with } \\
\text { spastic diplegia or } \\
\text { hemiplegia. }\end{array}$ & $\begin{array}{l}\text { Randomized: } \\
45 \\
\text { Analyzed: } 45 \\
\text { Attrition: } 0 \% \\
(0 / 45)\end{array}$ & $\begin{array}{l}\text { Poland } \\
\text { Outpatient } \\
\text { rehabilitation } \\
\text { (Indoor riding } \\
\text { arena) } \\
\text { RCT }\end{array}$ & None \\
\hline $\begin{array}{l}\text { Midik, } 2020 \\
\text { Aerobic Exercise } \\
\text { Robot-Assisted Gait } \\
\text { Training } \\
\text { Postintervention, and } 12 \\
\text { weeks } \\
\text { Fair }\end{array}$ & None reported & $\begin{array}{l}\text { Spinal Cord } \\
\text { Injury }\end{array}$ & $\begin{array}{l}\text { Male patients } 19 \text { to } 53 \\
\text { years old with traumatic } \\
\text { incomplete SCI for at least } \\
12 \text { weeks }\end{array}$ & $\begin{array}{l}\text { Randomized: } \\
30 \\
\text { Analyzed: } 30 \\
\text { Attrition: } 0 \% \\
(0 / 30)\end{array}$ & $\begin{array}{l}\text { Turkey } \\
\text { Inpatient } \\
\text { rehabilitation } \\
\text { RCT }\end{array}$ & None \\
\hline
\end{tabular}




\begin{tabular}{|c|c|c|c|c|c|c|}
\hline $\begin{array}{l}\text { Author, Year } \\
\text { Intervention Type } \\
\text { Duration of } \\
\text { Postintervention } \\
\text { Followup } \\
\text { Quality } \\
\text { (See Appendix B for } \\
\text { Full Citation) }\end{array}$ & Harms & Condition & Inclusion Criteria & $\begin{array}{l}\text { Number } \\
\text { Randomized } \\
\text { Analyzed } \\
\text { Attrition }\end{array}$ & $\begin{array}{l}\text { Country } \\
\text { Setting } \\
\text { Study Design }\end{array}$ & Funding Source \\
\hline $\begin{array}{l}\text { Mogharnasi, } 2018 \\
\text { Muscle Strength } \\
\text { exercise } \\
\text { Postintervention, } 0 \\
\text { weeks } \\
\text { Poor }\end{array}$ & NR & Spinal cord injury & $\begin{array}{l}\text { 1) All lesions were } \\
\text { complete and lesion levels } \\
\text { were T9-T12; 2) all lesions } \\
\text { were traumatic duo to } \\
\text { physical trauma; } 3 \text { ) all } \\
\text { subjects were physically } \\
\text { inactive in training after } \\
\text { occurrence of lower limb } \\
\text { paralysis; } 4 \text { ) all } \\
\text { participants were } \\
\text { examined by a physician } \\
\text { and received medical } \\
\text { approval for participation } \\
\text { in physical activities; } 5 \text { ) } \\
\text { they were able to sit down } \\
\text { while maintaining upper- } \\
\text { body balance; and } 6 \text { ) all } \\
\text { participants only used } \\
\text { wheelchairs without any } \\
\text { short leg braces } p \\
\text { crutches, long leg braces } \\
\text { p walker and crutches. } \\
\text { They were free from } \\
\text { pressure sores, bladder } \\
\text { infections, and potentially } \\
\text { damaging metabolic and } \\
\text { cardiovascular limitations. }\end{array}$ & $\begin{array}{l}\text { Randomized: } \\
20 \\
\text { Analyzed: } 20 \\
\text { Attrition: } 0 \% \\
(0 / 20)\end{array}$ & $\begin{array}{l}\text { Iran } \\
\text { NR } \\
\text { RCT }\end{array}$ & $\begin{array}{l}\text { This research } \\
\text { received no specific } \\
\text { grant from any } \\
\text { funding agency in the } \\
\text { public, commercial, or } \\
\text { not-for-profit sectors. }\end{array}$ \\
\hline $\begin{array}{l}\text { Moraes, } 2020 \\
\text { Postural Control } \\
\text { Hippotherapy } \\
\text { Postintervention, } 0 \\
\text { weeks } \\
\text { Fair }\end{array}$ & None reported & $\begin{array}{l}\text { Multiple } \\
\text { Sclerosis }\end{array}$ & $\begin{array}{l}\text { Diagnosis of relapsing- } \\
\text { remitting MS; } 18 \text { years or } \\
\text { older, able to walk with } \\
\text { an assistive device; have } \\
\text { EDSS } \leq 6.0 \text {; have PDDS } \\
\leq 5, \text { have not had a } \\
\text { relapse for more than } 6 \\
\text { mon }\end{array}$ & $\begin{array}{l}\text { Randomized: } \\
33 \\
\text { Analyzed: } 33 \\
\text { Attrition: } 0 \% \\
(0 / 33)\end{array}$ & $\begin{array}{l}\text { Brazil } \\
\text { Outpatient } \\
\text { rehabilitation } \\
\text { RCT }\end{array}$ & None \\
\hline
\end{tabular}




\begin{tabular}{|c|c|c|c|c|c|c|}
\hline $\begin{array}{l}\text { Author, Year } \\
\text { Intervention Type } \\
\text { Duration of } \\
\text { Postintervention } \\
\text { Followup } \\
\text { Quality } \\
\text { (See Appendix B for } \\
\text { Full Citation) }\end{array}$ & Harms & Condition & Inclusion Criteria & $\begin{array}{l}\text { Number } \\
\text { Randomized } \\
\text { Analyzed } \\
\text { Attrition }\end{array}$ & $\begin{array}{l}\text { Country } \\
\text { Setting } \\
\text { Study Design }\end{array}$ & Funding Source \\
\hline $\begin{array}{l}\text { Mutoh, } 2019 \\
\text { Postural Control } \\
\text { Hippotherapy } \\
\text { Postintervention, } 12 \\
\text { weeks (post } 48 \text { week } \\
\text { intervention) } \\
\text { Fair }\end{array}$ & NR & Cerebral palsy & $\begin{array}{l}\text { Inclusion Criteria: } \\
\text { CP patients } 4 \text { to } 14 \text { years } \\
\text { old with GMFCS levels II- } \\
\text { III }\end{array}$ & $\begin{array}{l}\text { Randomized: } \\
24 \\
\text { Analyzed: } 24 \\
\text { Attrition: } 0 \% \\
(0 / x 24)\end{array}$ & $\begin{array}{l}\text { United Kingdom } \\
\text { Outpatient } \\
\text { RCT }\end{array}$ & \begin{tabular}{|l|} 
Grants-in-Aid for \\
Scientific Research
\end{tabular} \\
\hline $\begin{array}{l}\text { Najafidoulataba, } 2014 \\
\text { Postural Control } \\
\text { Postintervention, } 12 \\
\text { weeks } \\
\text { Poor }\end{array}$ & NR & Multiple sclerosis & $\begin{array}{l}\text { Women aged } 18 \text { years } \\
\text { and older, diagnosed with } \\
\text { MS disease for the last } 2 \\
\text { years; no history of other } \\
\text { disabling diseases; } \\
\text { physically able to } \\
\text { participate in the study } \\
\text { and perform yoga } \\
\text { exercises }\end{array}$ & $\begin{array}{l}\text { Randomized: } \\
60 \\
\text { Analyzed:60 } \\
\text { Attrition: } 0 \% \\
(0 / 60)\end{array}$ & \begin{tabular}{|l} 
Iran \\
Unclear (not \\
specified) \\
RCT
\end{tabular} & $\begin{array}{l}\text { Nonprofit: financial } \\
\text { support from Yasuj } \\
\text { University of Medical } \\
\text { Sciences }\end{array}$ \\
\hline $\begin{array}{l}\text { Negaresh, } 2018 \\
\text { Aerobic Exercise } \\
\text { Cycling } \\
\text { Postintervention, } 0 \\
\text { weeks } \\
\text { Fair }\end{array}$ & NR & Multiple sclerosis & $\begin{array}{l}\text { Inclusion based on the } \\
\text { following criteria: (a) } \\
\text { RRMS type (revised } \\
\text { McDonald criteria19), (b) } \\
\text { general BMI ranging } \\
\text { between } 20 \text { to } 30 \mathrm{~kg} / \mathrm{m}^{2} \text {, } \\
\text { EDSS: } 4 \text {, and (d) age }>22 \\
\text { years. }\end{array}$ & $\begin{array}{l}\text { Randomized: } \\
\mathrm{N}=66 \\
\text { Analyzed:61 } \\
\text { Attrition: } 9 \% \\
(5 / 66)\end{array}$ & $\begin{array}{l}\text { Iran } \\
\text { Outpatient clinic } \\
\text { RCT }\end{array}$ & NR \\
\hline $\begin{array}{l}\text { Nilsagard, } 2012 \\
\text { Postural Control } \\
\text { Motion gaming } \\
\text { Postintervention, } 7 \\
\text { weeks } \\
\text { Fair }\end{array}$ & $\begin{array}{l}\text { At the final data collection, the balance } \\
\text { exercise group reported } 10 \text { falls during the } \\
\text { study period compared with } 14 \text { in the } \\
\text { nonexercise group. No falls occurred during } \\
\text { balance exercise, data collection or } \\
\text { travelling to or from the appointments. No } \\
\text { other adverse events were reported. }\end{array}$ & Multiple sclerosis & $\begin{array}{l}\text { Patients diagnosed with } \\
\text { MS in accordance with the } \\
\text { revised McDonald criteria; } \\
\text { subjectively perceived } \\
\text { impaired balance function } \\
\text { in standing or walking } \\
\text { activities; and the ability to } \\
\text { walk } 100 \text { m without resting }\end{array}$ & $\begin{array}{l}\text { Randomized: } \\
84 \\
\text { Analyzed:80 } \\
\text { Attrition: } 4.8 \% \\
(4 / 84)\end{array}$ & $\begin{array}{l}\text { Sweden } \\
\text { Unclear (Home or } \\
\text { Clinic) } \\
\text { RCT }\end{array}$ & $\begin{array}{l}\text { Nonprofit: funded by } \\
\text { the Uppsala-Örebro } \\
\text { Regional Research } \\
\text { Council, the Research } \\
\text { Committee of Örebro } \\
\text { County Council, and } \\
\text { the Norrbacka- } \\
\text { Eugenia Foundation }\end{array}$ \\
\hline
\end{tabular}




\begin{tabular}{|c|c|c|c|c|c|c|}
\hline $\begin{array}{l}\text { Author, Year } \\
\text { Intervention Type } \\
\text { Duration of } \\
\text { Postintervention } \\
\text { Followup } \\
\text { Quality } \\
\text { (See Appendix B for } \\
\text { Full Citation) }\end{array}$ & Harms & Condition & Inclusion Criteria & $\begin{array}{l}\text { Number } \\
\text { Randomized } \\
\text { Analyzed } \\
\text { Attrition }\end{array}$ & $\begin{array}{l}\text { Country } \\
\text { Setting } \\
\text { Study Design }\end{array}$ & Funding Source \\
\hline $\begin{array}{l}\text { Niwald, } 2017 \\
\text { Aerobic Exercise } \\
\text { Cycling } \\
\text { Postintervention, } 0 \\
\text { weeks } \\
\text { Fair }\end{array}$ & NR & Multiple sclerosis & $\begin{array}{l}\text { Diagnosed MS } \\
\text { Age }>18 \text { years } \\
\text { Informed consent to } \\
\text { participate in the study } \\
\text { Ability to perform aerobic } \\
\text { exercises }\end{array}$ & $\begin{array}{l}\text { Randomized: } \\
\mathrm{N}=53 \\
\text { Analyzed: NR } \\
\text { Attrition: NR }\end{array}$ & $\begin{array}{l}\text { Poland } \\
\text { Inpatient } \\
\text { Pre-post }\end{array}$ & $\begin{array}{l}\text { Young Scientists of } \\
\text { the Medical University } \\
\text { of Lodz }\end{array}$ \\
\hline $\begin{array}{l}\text { Norouzi, } 2019 \\
\text { Postural Control } \\
\text { Balance exercises } \\
\text { Immediately } \\
\text { postintervention, } \\
4 \text { weeks } \\
\text { Fair }\end{array}$ & NR & Spinal cord injury & $\begin{array}{l}\text { Inclusion Criteria: } \\
\text { Paraplegic veterans with } \\
\text { SCI at L3, L4 (ASIA B-D) }\end{array}$ & $\begin{array}{l}\text { Randomized: } \\
30 \\
\text { Analyzed: } 30 \\
\text { Attrition: } 0 \% \\
(0 / 30)\end{array}$ & $\begin{array}{l}\text { Iran } \\
\text { Setting Outpatient } \\
\text { clinic } \\
\text { RCT }\end{array}$ & NR \\
\hline $\begin{array}{l}\text { Nsenga, } 2013 \\
\text { Aerobic Exercise } \\
\text { Cycling } \\
\text { Postintervention, } 0 \\
\text { weeks } \\
\text { Fair }\end{array}$ & NR & Cerebral palsy & $\begin{array}{l}\text { CP (Gross Motor Function } \\
\text { Classification System } \\
\text { (GMFCS) levels I and II }\end{array}$ & $\begin{array}{l}\text { Randomized: } \\
\mathrm{N}=24 \\
\text { Analyzed: } 20 \\
\text { Attrition: } 17 \% \\
(4 / 24)\end{array}$ & $\begin{array}{l}\text { France } \\
\text { School } \\
\text { Pre-Post }\end{array}$ & NR \\
\hline $\begin{array}{l}\text { Nsenga-Leunkeu } 2012 \\
\text { Aerobic Exercise } \\
\text { Treadmill } \\
\text { Postintervention, } 0 \\
\text { weeks } \\
\text { Fair }\end{array}$ & None reported & Cerebral palsy & $\begin{array}{l}\text { Convenience sample } \\
\text { children from special } \\
\text { education school } \\
10 \text { to } 16 \text { years old }\end{array}$ & $\begin{array}{l}28 \text { randomized } \\
24 \text { analyzed } \\
4 / 28=14 \% \\
\text { attrition }\end{array}$ & $\begin{array}{l}\text { Canada } \\
\text { RCT }\end{array}$ & NR \\
\hline
\end{tabular}




\begin{tabular}{|c|c|c|c|c|c|c|}
\hline $\begin{array}{l}\text { Author, Year } \\
\text { Intervention Type } \\
\text { Duration of } \\
\text { Postintervention } \\
\text { Followup } \\
\text { Quality } \\
\text { (See Appendix B for } \\
\text { Full Citation) }\end{array}$ & Harms & Condition & Inclusion Criteria & $\begin{array}{l}\text { Number } \\
\text { Randomized } \\
\text { Analyzed } \\
\text { Attrition } \\
\end{array}$ & \begin{tabular}{|l|} 
Country \\
Setting \\
Study Design \\
\end{tabular} & Funding Source \\
\hline $\begin{array}{l}\text { Nsenga-Leunkeu, } 2012 \\
\text { Aerobic Exercise } \\
\text { Treadmill } \\
\text { Postintervention, } 0 \\
\text { weeks } \\
\text { Fair }\end{array}$ & $\begin{array}{l}\text { None of this group experienced any } \\
\text { limitation of walking because of pain. }\end{array}$ & Cerebral palsy & $\begin{array}{l}\text { Children and adolescents } \\
\text { with CP (GMFCS } 27 \text { levels } \\
\text { I or II; age range, } 10 \text { to } 16 \\
\text { years) }\end{array}$ & $\begin{array}{l}\text { Randomized: } \\
\text { N/A } \\
\text { Analyzed: } 24 \\
\text { Attrition: } 14.3 \% \\
(4 / 28)\end{array}$ & $\begin{array}{l}\text { NR } \\
\text { Outpatient clinic } \\
\text { Matched pairs } \\
\text { cohort }\end{array}$ & NR \\
\hline $\begin{array}{l}\text { Ortiz-Rubio, } 2016 \\
\text { Muscle Strength } \\
\text { Postintervention, } 0 \\
\text { weeks } \\
\text { Good }\end{array}$ & NR & Multiple sclerosis & $\begin{array}{l}\text { Diagnosis of relapsing- } \\
\text { remitting MS, secondary } \\
\text { progressive MS, or } \\
\text { primary progressive MS } \\
\text { according to the criteria } \\
\text { formulated by McDonald } \\
\text { et al; adults between the } \\
\text { ages of } 18 \text { and } 65 \text { years; } \\
\text { patients with an Expanded } \\
\text { Disability Status Scale } \\
<7.5 ; \text { and patients with a } \\
\text { Mini-Mental State } \\
\text { Examination >24. Patients } \\
\text { reported upper limb } \\
\text { impairment and had at } \\
\text { least on } 1 \text { hand a } \\
\text { pathological Nine Hole } \\
\text { Peg Test with } 2 \text { standard } \\
\text { deviations above the } \\
\text { mean normal values } \\
\text { published by Oxford Grice } \\
\text { et al at screening. }\end{array}$ & $\begin{array}{l}\text { Randomized: } \\
37 \\
\text { Analyzed: } 37 \\
\text { Attrition: } 0 \% \\
(0 / 37)\end{array}$ & $\begin{array}{l}\text { Spain } \\
\text { Home-based } \\
\text { RCT }\end{array}$ & NR \\
\hline
\end{tabular}




\begin{tabular}{|c|c|c|c|c|c|c|}
\hline $\begin{array}{l}\text { Author, Year } \\
\text { Intervention Type } \\
\text { Duration of } \\
\text { Postintervention } \\
\text { Followup } \\
\text { Quality } \\
\text { (See Appendix B for } \\
\text { Full Citation) }\end{array}$ & Harms & Condition & Inclusion Criteria & $\begin{array}{l}\text { Number } \\
\text { Randomized } \\
\text { Analyzed } \\
\text { Attrition }\end{array}$ & $\begin{array}{l}\text { Country } \\
\text { Setting } \\
\text { Study Design }\end{array}$ & Funding Source \\
\hline $\begin{array}{l}\text { Ozkul, } 2020 \\
\text { Postural Control } \\
\text { Balance Exercises } \\
\text { Motion Gaming } \\
\text { Postintervention, } 0 \\
\text { weeks } \\
\text { Fair }\end{array}$ & $\begin{array}{l}\text { None reported } \\
\text { (No adverse or harmful events in both } \\
\text { groups) }\end{array}$ & $\begin{array}{l}\text { Multiple } \\
\text { Sclerosis }\end{array}$ & $\begin{array}{l}\text { Diagnosis of MS; 18-65 } \\
\text { years old a, Expanded } \\
\text { Disability Status Scale } \\
\text { (EDSS) score under } 6\end{array}$ & \begin{tabular}{|l|} 
Randomized: \\
54 \\
(51 assigned to \\
intervention) \\
Analyzed: 39 \\
Attrition: $24 \%$ \\
$(13 / 54)$
\end{tabular} & $\begin{array}{l}\text { Turkey } \\
\text { Outpatient } \\
\text { rehabilitation } \\
\text { RCT }\end{array}$ & None \\
\hline $\begin{array}{l}\text { Ozkul, 2020b } \\
\text { Multimodal Exercise } \\
\text { Immediately } \\
\text { Postintervention, } 8 \\
\text { weeks } \\
\text { Fair }\end{array}$ & $\begin{array}{l}\text { Reported no adverse or } \\
\text { harmful events occurred. }\end{array}$ & Multiple sclerosis & $\begin{array}{l}\text { Diagnosis of relapsing- } \\
\text { remitting MS, adults age } \\
18-65 \text { with EDSS } \\
\text { score }<4 \text {, relapse-free for } \\
\text { the last } 3 \text { months, with } \\
\text { cognitive impairment } \\
\text { values that were below at } \\
\text { least one. }\end{array}$ & $\begin{array}{l}\text { Randomized: } \\
34 \\
\text { Analyzed: } 34 \\
\text { Attrition: } 0 \% \\
(0 / 34)\end{array}$ & $\begin{array}{l}\text { Turkey } \\
\text { Outpatient } \\
\text { Neurorehabilitation } \\
\text { Clinic } \\
\text { RCT }\end{array}$ & None \\
\hline $\begin{array}{l}\text { Park, } 2014 \\
\text { Postintervention, } 8 \\
\text { weeks (within } 2 \text { months } \\
\text { after 8-week } \\
\text { intervention) } \\
\text { Postural Control } \\
\text { Hippotherapy } \\
\text { Poor }\end{array}$ & NR & Cerebral palsy & $\begin{array}{l}\text { Inclusion Criteria: } \\
\text { age } 3 \text { to } 12 \text { years, body } \\
\text { weight less than } 40 \mathrm{~kg} \text {, } \\
\text { and gross motor function } \\
\text { classification system } \\
\text { (GMFCS) level I to IV }\end{array}$ & $\begin{array}{l}\text { Randomized: } \\
\text { NA } \\
\text { Analyzed: } 55 \\
\text { Attrition: } 17 \% \\
(11 / 66)\end{array}$ & $\begin{array}{l}\text { Republic of Korea } \\
\text { Outpatient clinic } \\
\text { Cohort }\end{array}$ & NR \\
\hline
\end{tabular}




\begin{tabular}{|c|c|c|c|c|c|c|}
\hline $\begin{array}{l}\text { Author, Year } \\
\text { Intervention Type } \\
\text { Duration of } \\
\text { Postintervention } \\
\text { Followup } \\
\text { Quality } \\
\text { (See Appendix B for } \\
\text { Full Citation) }\end{array}$ & Harms & Condition & Inclusion Criteria & $\begin{array}{l}\text { Number } \\
\text { Randomized } \\
\text { Analyzed } \\
\text { Attrition }\end{array}$ & $\begin{array}{l}\text { Country } \\
\text { Setting } \\
\text { Study Design }\end{array}$ & Funding Source \\
\hline $\begin{array}{l}\text { Peri, } 2017 \\
\text { Aerobic Exercise } \\
\text { Robot-Assisted Gait } \\
\text { Training } \\
\text { Postintervention, 4-10 } \\
\text { weeks } \\
\text { Poor }\end{array}$ & NR & Cerebral palsy & $\begin{array}{l}\text { Inclusion criteria: age 4- } \\
17 \text {, spastic bilateral } \mathrm{CP} \text {, } \\
\text { able to communicate, able } \\
\text { to walk independently, } \\
\text { femur length }>21 \mathrm{~cm}\end{array}$ & $\begin{array}{l}\text { Randomized: } \\
\mathrm{N}=44 \\
\text { Analyzed: } 44 \\
\text { Attrition: } 0 \%\end{array}$ & \begin{tabular}{|l|} 
Italy \\
Outpatient \\
Quasiexperimental, \\
nonrandomized
\end{tabular} & NR \\
\hline $\begin{array}{l}\text { Pourazar, } 2020 \\
\text { Postural Control } \\
\text { Motion Gaming } \\
\text { Postintervention, } 0 \\
\text { weeks } \\
\text { Fair }\end{array}$ & NR & Cerebral Palsy & $\begin{array}{l}\text { Girls with } 7 \text { to } 12 \text { years } \\
\text { with Spastic Hemiplegic } \\
\text { Cerebral Palsy, levels I } \\
\text { and II (MACS), GMFCS } \\
\text { score range } 1 \text { to } 3 \text { and } \\
\text { able to walk without an } \\
\text { assistive device }\end{array}$ & $\begin{array}{l}\text { Randomized: } \\
20 \\
\text { Analyzed: } 20 \\
\text { Attrition: } 0 \% \\
(0 / 20)\end{array}$ & $\begin{array}{l}\text { Iran } \\
\text { Outpatient } \\
\text { rehabilitation } \\
\text { RCT }\end{array}$ & None \\
\hline $\begin{array}{l}\text { Pompa, } 2017 \\
\text { Aerobic Exercise } \\
\text { Robot-Assisted Gait } \\
\text { Training } \\
\text { Postintervention, } 4 \\
\text { weeks } \\
\text { Fair }\end{array}$ & NR & $\begin{array}{l}\text { Multiple } \\
\text { sclerosis }\end{array}$ & $\begin{array}{l}\text { Diagnosis of MS, age 25- } \\
65, \text { EDSS } 6-7.5, \text { Mini } \\
\text { Mental State Exam >24 }\end{array}$ & $\begin{array}{l}\text { Randomized: } \\
\mathrm{N}=50 \\
\text { Analyzed: } 43 \\
\text { Attrition: } 14 \%\end{array}$ & \begin{tabular}{|l|} 
Italy \\
Inpatient \\
Rehabilitation \\
RCT
\end{tabular} & $\begin{array}{l}\text { Santa Lucia } \\
\text { Foundation and Italian } \\
\text { Ministry of Health }\end{array}$ \\
\hline
\end{tabular}




\begin{tabular}{|c|c|c|c|c|c|c|}
\hline $\begin{array}{l}\text { Author, Year } \\
\text { Intervention Type } \\
\text { Duration of } \\
\text { Postintervention } \\
\text { Followup } \\
\text { Quality } \\
\text { (See Appendix B for } \\
\text { Full Citation) }\end{array}$ & Harms & Condition & Inclusion Criteria & $\begin{array}{l}\text { Number } \\
\text { Randomized } \\
\text { Analyzed } \\
\text { Attrition }\end{array}$ & $\begin{array}{l}\text { Country } \\
\text { Setting } \\
\text { Study Design }\end{array}$ & Funding Source \\
\hline $\begin{array}{l}\text { Qi, } 2018 \\
\text { Postural Control } \\
\text { Tai Chi } \\
\text { Postintervention, } 6 \\
\text { weeks } \\
\text { Fair }\end{array}$ & NR & Spinal cord injury & $\begin{array}{l}\text { Right-handed SCl patients } \\
\text { who met the diagnostic } \\
\text { criteria for } \mathrm{SCl} \text { according } \\
\text { to the American Spinal } \\
\text { Injury Association; } \\
\text { between } 20 \text { and } 70 \text { years } \\
\text { old; able to communicate } \\
\text { and follow instructions, } \\
\text { and able to maintain a } \\
\text { sitting posture for more } \\
\text { than } 30 \text { min in a } \\
\text { wheelchair }\end{array}$ & $\begin{array}{l}\text { Randomized: } \\
40 \\
\text { Analyzed:40 } \\
\text { Attrition: } 0 \% \\
(0 / 40)\end{array}$ & $\begin{array}{l}\text { China } \\
\text { Unclear } \\
\text { RCT }\end{array}$ & $\begin{array}{l}\text { Government: } \\
\text { Financially supported } \\
\text { by Research Project } \\
\text { of Shanghai } \\
\text { Administration of } \\
\text { Sports (16Z015) }\end{array}$ \\
\hline $\begin{array}{l}\text { Razazian, } 2016 \\
\text { Aerobic Exercise } \\
\text { Aquatics } \\
\text { Postintervention, } 8 \\
\text { weeks } \\
\text { Poor }\end{array}$ & NR & Multiple sclerosis & $\begin{array}{l}\text { Women diagnosed with } \\
\text { primary-progressive } \\
\text { secondary-progressive } \\
\text { MS or relapsing-remitting } \\
\text { progressive-relapsing MS } \\
\text { as diagnosed by } \\
\text { neurologists, aged } \\
\text { between } 25 \text { and } 50 \text { years, } \\
\text { Expanded Disability } \\
\text { Status Scale e6, receiving } \\
\text { stable, regular, and } \\
\text { monitored } \\
\text { pharmacological treatment } \\
\text { of MS (immune } \\
\text { modulatory treatments) }\end{array}$ & $\begin{array}{l}\text { Randomized: } \\
54 \\
\text { Analyzed:54 } \\
\text { Attrition: } 0 \% \\
(0 / 54)\end{array}$ & $\begin{array}{l}\text { Iran } \\
\text { Gym, aquatic } \\
\text { rehab. center } \\
\text { RCT }\end{array}$ & Nonprofit \\
\hline
\end{tabular}




\begin{tabular}{|c|c|c|c|c|c|c|}
\hline $\begin{array}{l}\text { Author, Year } \\
\text { Intervention Type } \\
\text { Duration of } \\
\text { Postintervention } \\
\text { Followup } \\
\text { Quality } \\
\text { (See Appendix B for } \\
\text { Full Citation) }\end{array}$ & Harms & Condition & Inclusion Criteria & $\begin{array}{l}\text { Number } \\
\text { Randomized } \\
\text { Analyzed } \\
\text { Attrition }\end{array}$ & $\begin{array}{l}\text { Country } \\
\text { Setting } \\
\text { Study Design }\end{array}$ & Funding Source \\
\hline $\begin{array}{l}\text { Roppolo, } 2013 \\
\text { Multimodal Exercise } \\
\text { Immediately } \\
\text { postintervention, } \\
12 \text { weeks } \\
\text { Fair }\end{array}$ & NR & Multiple sclerosis & $\begin{array}{l}\text { Inclusion Criteria: Women } \\
\text { with relapsing MS mean } \\
\text { ages } 18 \text { to } 60 \text { years old, } \\
\text { and EDSS scores } 0 \text { to } 3\end{array}$ & $\begin{array}{l}\text { Randomized: } \\
35 \\
\text { Analyzed: } 35 \\
\text { Attrition: } 2 \% \\
(1 / 35)\end{array}$ & $\begin{array}{l}\text { Italy } \\
\text { Outpatient clinic } \\
\text { Quasiexperimental } \\
\text { study }\end{array}$ & NR \\
\hline $\begin{array}{l}\text { Russo, } 2018 \\
\text { Aerobic Exercise } \\
\text { Robot-Assisted Gait } \\
\text { Training } \\
\text { Postintervention, } 18 \\
\text { weeks } \\
\text { Fair }\end{array}$ & None & $\begin{array}{c}\text { Multiple } \\
\text { sclerosis }\end{array}$ & $\begin{array}{l}\text { Inclusion Criteria: } \\
\text { relapsing-remitting MS, } \\
\text { EDSS 3.0-5.5, Montreal } \\
\text { Cognitive Assessment } \\
\text { Score at least } 24, \text { no other } \\
\text { neurological or orthopedic } \\
\text { comorbidities, stable } \\
\text { medication for } 6 \text { months }\end{array}$ & $\begin{array}{l}\text { Randomized: } \\
\mathrm{N}=45 \\
\text { Analyzed: } 45 \\
\text { Attrition: } 0 \%\end{array}$ & \begin{tabular}{|l} 
Italy \\
Outpatient \\
RCT
\end{tabular} & NR \\
\hline $\begin{array}{l}\text { Sadeghi Bahmani, } 2019 \\
\text { Aerobic Exercise } \\
\text { Aerobics } \\
\text { Postural Control } \\
\text { Balance } \\
\text { Postintervention, } 8 \\
\text { weeks } \\
\text { Fair }\end{array}$ & NR & Multiple sclerosis & $\begin{array}{l}\text { Inclusion Criteria: Women } \\
\text { with MS } 18 \text { to } 65 \text { years } \\
\text { old, EDSS score }<6\end{array}$ & $\begin{array}{l}\text { Randomized: } \\
92 \\
\text { Analyzed: } 71 \\
\text { Attrition: } 23 \% \\
(21 / 92)\end{array}$ & $\begin{array}{l}\text { Iran } \\
\text { Outpatient clinic } \\
\text { RCT }\end{array}$ & NR \\
\hline
\end{tabular}




\begin{tabular}{|c|c|c|c|c|c|c|}
\hline $\begin{array}{l}\text { Author, Year } \\
\text { Intervention Type } \\
\text { Duration of } \\
\text { Postintervention } \\
\text { Followup } \\
\text { Quality } \\
\text { (See Appendix B for } \\
\text { Full Citation) }\end{array}$ & Harms & Condition & Inclusion Criteria & $\begin{array}{l}\text { Number } \\
\text { Randomized } \\
\text { Analyzed } \\
\text { Attrition }\end{array}$ & $\begin{array}{l}\text { Country } \\
\text { Setting } \\
\text { Study Design }\end{array}$ & Funding Source \\
\hline $\begin{array}{l}\text { Sadeghi Bahmani, } \\
\text { 2020a (aquatic) } \\
\text { Aerobic Exercise } \\
\text { Aquatic } \\
\text { Postintervention, } 5 \\
\text { weeks } \\
\text { Fair }\end{array}$ & NR & $\begin{array}{l}\text { Multiple } \\
\text { Sclerosis }\end{array}$ & $\begin{array}{l}\text { Diagnosis of MS; } 18 \text { to } 65 \\
\text { year old women, EDSS } \\
\text { score }<6\end{array}$ & $\begin{array}{l}\text { Randomized: } \\
62 \\
\text { Analyzed: } 39 \\
\text { Attrition: } 24 \% \\
(13 / 54)\end{array}$ & $\begin{array}{l}\text { Iran } \\
\text { Setting (outpatient, } \\
\text { rehabilitation) } \\
\text { RCT }\end{array}$ & Not reported \\
\hline $\begin{array}{l}\text { Sadowsky, } 2013 \\
\text { Aerobic Exercise } \\
\text { Cycling } \\
\text { Postintervention, } 0 \\
\text { weeks } \\
\text { Poor }\end{array}$ & NR & Spinal cord injury & $\begin{array}{l}\text { Diagnosed with chronic } \\
\text { SCl, defined as }>16 \\
\text { months following injury at } \\
\text { the time of initial } \\
\text { evaluation at the center. }\end{array}$ & $\begin{array}{l}\text { Randomized: } \\
\text { This was a } \\
\text { nonrandomized } \\
\text { study }\end{array}$ & $\begin{array}{l}\text { USA } \\
\text { Outpatient clinic } \\
\text { Retrospective } \\
\text { analysis }\end{array}$ & $\begin{array}{l}\text { Deans Fund at } \\
\text { Washington University } \\
\text { School of Medicine, } \\
\text { Barnes-Jewish } \\
\text { Hospital Foundation, } \\
\text { the Barnes-Jewish } \\
\text { Hospital Auxiliary } \\
\text { Foundation, } \\
\text { Christopher Reeve } \\
\text { Paralysis Foundation, } \\
\text { the Nextsteps } \\
\text { Foundation, the Sam } \\
\text { Schmidt Foundation, } \\
\text { Gateway to a Cure } \\
\text { Foundation, and the } \\
\text { Eric Westacott } \\
\text { Foundation and, in } \\
\text { part, by the Intramural } \\
\text { Research Program at } \\
\text { the NIH Clinical } \\
\text { Center. }\end{array}$ \\
\hline
\end{tabular}




\begin{tabular}{|c|c|c|c|c|c|c|}
\hline $\begin{array}{l}\text { Author, Year } \\
\text { Intervention Type } \\
\text { Duration of } \\
\text { Postintervention } \\
\text { Followup } \\
\text { Quality } \\
\text { (See Appendix B for } \\
\text { Full Citation) }\end{array}$ & Harms & Condition & Inclusion Criteria & $\begin{array}{l}\text { Number } \\
\text { Randomized } \\
\text { Analyzed } \\
\text { Attrition } \\
\end{array}$ & \begin{tabular}{|l} 
Country \\
Setting \\
Study Design \\
\end{tabular} & Funding Source \\
\hline $\begin{array}{l}\text { Salci, } 2016 \\
\text { Postural Control } \\
\text { Balance } \\
\text { Postintervention, } 6 \\
\text { weeks } \\
\text { Fair }\end{array}$ & $\begin{array}{l}4.2 \%(2 / 48) \text { discontinued intervention } \\
\text { reported acute exacerbation and femur } \\
\text { fracture after fall } \\
4.2 \%(2 / 48) \text { discontinued intervention } \\
\text { reported acute exacerbation and traffic } \\
\text { accident } \\
4.2 \%(2 / 48) \text { discontinued intervention } \\
\text { without any reason } \\
\begin{array}{l}\text { Study states: "No adverse effects of training } \\
\text { were reported } \\
\text { in the groups". }\end{array} \\
\end{array}$ & Multiple sclerosis & $\begin{array}{l}\text { Diagnosis of MS } \\
\text { (McDonald criteria); older } \\
\text { than } 18 \text { years, an EDSS } \\
\text { score between } 3 \text { and } 5 \\
\text { with prominently ataxic } \\
\text { problems; discontinuing } \\
\text { the use of corticosteroids } \\
\text { for } 3 \text { months prior to the } \\
\text { study; and having no } \\
\text { acute exacerbations and } \\
\text { no change in MS-specific } \\
\text { medications within } 3 \\
\text { months of the study }\end{array}$ & $\begin{array}{l}\text { Randomized: } \\
48 \\
\text { Analyzed: } 42^{*} \\
\text { Attrition: } 11 \% \\
(6 / 48) \\
\\
\text { *Studies states } \\
\text { that } n=0 \text { were } \\
\text { excluded from } \\
\text { the analysis yet } \\
\text { the n-analyzed } \\
\text { was } 42 / 48, \text { so } \\
\text { there was no } \\
\text { ITT. } \\
\end{array}$ & $\begin{array}{l}\text { Turkey } \\
\text { University } \\
\text { RCT }\end{array}$ & None \\
\hline $\begin{array}{l}\text { Samaei } \\
2014 \\
\text { Aerobic Exercise } \\
\text { Treadmill } \\
\text { Postintervention, } 0 \\
\text { weeks } \\
\text { Fair } \\
\end{array}$ & None reported & Multiple sclerosis & $\begin{array}{l}\text { Able to walk } 10 \text { meters in } \\
\text { less than } 10 \text { minutes } \\
\text { Score greater than } 3 \text { on } \\
\text { GNDS limb score }\end{array}$ & $\begin{array}{l}34 \text { randomized } \\
31 \text { analyzed } \\
3 / 34=9 \% \\
\text { attrition }\end{array}$ & Iran & Government/university \\
\hline
\end{tabular}




\begin{tabular}{|c|c|c|c|c|c|c|}
\hline $\begin{array}{l}\text { Author, Year } \\
\text { Intervention Type } \\
\text { Duration of } \\
\text { Postintervention } \\
\text { Followup } \\
\text { Quality } \\
\text { (See Appendix B for } \\
\text { Full Citation) }\end{array}$ & Harms & Condition & Inclusion Criteria & $\begin{array}{l}\text { Number } \\
\text { Randomized } \\
\text { Analyzed } \\
\text { Attrition }\end{array}$ & $\begin{array}{l}\text { Country } \\
\text { Setting } \\
\text { Study Design }\end{array}$ & Funding Source \\
\hline $\begin{array}{l}\text { Sandroff, } 2017 \\
\text { Multimodal Exercise } \\
\text { Mid-intervention (12 } \\
\text { weeks into intervention); } \\
\text { Immediately } \\
\text { postintervention } \\
\text { Fair }\end{array}$ & NR & Multiple sclerosis & $\begin{array}{l}\text { Between } 18 \text { and } 64 \text { years } \\
\text { of age; definite MS } \\
\text { diagnosis based on } \\
\text { neurologist's verification } \\
\text { using standard diagnostic } \\
\text { criteria; neurologist's } \\
\text { verification of EDSS score } \\
\text { between } 4.0 \text { and } 6.0 \\
\text { based on the participant's } \\
\text { most recent neurologist } \\
\text { administered score (i.e., } \\
\text { onset of substantial MS- } \\
\text { related mobility disability); } \\
\text { engaging in low levels of } \\
\text { physical activity (i.e., } \\
\text { participating in <2 days of } \\
\text { at least } 30 \text { min of aerobic } \\
\text { and/or resistance exercise } \\
\text { per week); being relapse- } \\
\text { free over the past } 30 \\
\text { days; and low risk for } \\
\text { contraindications for } \\
\text { exercise testing and } \\
\text { training based on no more } \\
\text { than one "yes" response } \\
\text { on all Physical Activity } \\
\text { Readiness Questionnaire } \\
\text { items. }\end{array}$ & $\begin{array}{l}\text { Randomized: } \\
83 \\
\text { Analyzed: } 62 \\
\text { Attrition: } 25.3 \% \\
(21 / 83)\end{array}$ & $\begin{array}{l}\text { USA } \\
\text { Outpatient clinic } \\
\text { RCT }\end{array}$ & $\begin{array}{l}\text { This paper was } \\
\text { supported by a grant } \\
\text { from the National } \\
\text { Multiple Sclerosis } \\
\text { Society (RG } \\
\text { 4991A3/1). }\end{array}$ \\
\hline $\begin{array}{l}\text { Sangelaji, } 2014 \\
\text { Multimodal Exercise } \\
\text { Postintervention, } 0 \\
\text { weeks } \\
\text { Poor }\end{array}$ & NR & Multiple sclerosis & $\begin{array}{l}\text { Suffering from recurrent } \\
\text { and improving type of MS, } \\
18 \text { to } 50 \text { years old, not } \\
\text { having had any MS attack } \\
\text { in the last } 3 \text { months and } \\
\text { consuming various types } \\
\text { of interferon for prevention } \\
\text { of MS attacks, EDSS } \\
\text { scores of } 0-4\end{array}$ & $\begin{array}{l}\text { Randomized: } \\
72 \\
\text { Analyzed: } 55 \\
\text { Attrition: } \\
23.6 \% \% \\
(17 / 72)\end{array}$ & $\begin{array}{l}\text { Iran } \\
\text { PT clinic } \\
\text { RCT }\end{array}$ & $\begin{array}{l}\text { The study is self- } \\
\text { funded }\end{array}$ \\
\hline
\end{tabular}




\begin{tabular}{|c|c|c|c|c|c|c|}
\hline $\begin{array}{l}\text { Author, Year } \\
\text { Intervention Type } \\
\text { Duration of } \\
\text { Postintervention } \\
\text { Followup } \\
\text { Quality } \\
\text { (See Appendix B for } \\
\text { Full Citation) }\end{array}$ & Harms & Condition & Inclusion Criteria & $\begin{array}{l}\text { Number } \\
\text { Randomized } \\
\text { Analyzed } \\
\text { Attrition } \\
\end{array}$ & \begin{tabular}{|l|} 
Country \\
Setting \\
Study Design \\
\end{tabular} & Funding Source \\
\hline $\begin{array}{l}\text { Sangelaji, } 2016 \\
\text { Multimodal Exercise } \\
\text { Postintervention, } 0 \\
\text { weeks } \\
\text { Fair }\end{array}$ & NR & Multiple sclerosis & $\begin{array}{l}\text { 1. Definite relapse- } \\
\text { remaining MS } \\
\text { 2. Adults between } 18 \text { and } \\
50 \text { years of age } \\
\text { 3. An EDSS level of } 0-5 \\
\text { 4. Right-handed } \\
\text { 5. No history of systemic } \\
\text { disease, concomitant } \\
\text { neurological disorders, } \\
\text { epilepsy, heart diseases, } \\
\text { anemia, or severe } \\
\text { depression. }\end{array}$ & $\begin{array}{l}\text { Randomized: } \\
40 \\
\text { Analyzed: } 40 \\
\text { Attrition: } 0 \% \\
(0 / 40)\end{array}$ & $\begin{array}{l}\text { Iran } \\
\text { PT clinic } \\
\text { RCT }\end{array}$ & $\begin{array}{l}\text { The study is funded } \\
\text { by Sport Science } \\
\text { Research Institute of } \\
\text { Iran. }\end{array}$ \\
\hline $\begin{array}{l}\text { Shin, } 2014 \\
\text { Aerobic Exercise } \\
\text { Robot-Assisted Gait } \\
\text { Training } \\
\text { Postintervention, } 4 \\
\text { weeks } \\
\text { Fair }\end{array}$ & NR & $\begin{array}{l}\text { Spinal cord } \\
\text { injury }\end{array}$ & $\begin{array}{l}\text { Inclusion Criteria: } \mathrm{SCl}<6 \\
\text { months, ASIA D, age 20- } \\
65\end{array}$ & $\begin{array}{l}\text { Randomized: } \\
\mathrm{N}=60 \\
\text { Analyzed: } 53 \\
\text { Attrition: } 11.7 \%\end{array}$ & $\begin{array}{l}\text { Korea } \\
\text { Inpatient } \\
\text { Rehabilitation } \\
\text { RCT }\end{array}$ & NR \\
\hline
\end{tabular}




\begin{tabular}{|c|c|c|c|c|c|c|}
\hline $\begin{array}{l}\text { Author, Year } \\
\text { Intervention Type } \\
\text { Duration of } \\
\text { Postintervention } \\
\text { Followup } \\
\text { Quality } \\
\text { (See Appendix B for } \\
\text { Full Citation) }\end{array}$ & Harms & Condition & Inclusion Criteria & $\begin{array}{l}\text { Number } \\
\text { Randomized } \\
\text { Analyzed } \\
\text { Attrition }\end{array}$ & $\begin{array}{l}\text { Country } \\
\text { Setting } \\
\text { Study Design }\end{array}$ & Funding Source \\
\hline $\begin{array}{l}\text { Silva e Borges, } 2011 \\
\text { Postintervention, } 0 \\
\text { weeks (after 6-week } \\
\text { intervention) } \\
\text { Postural Control } \\
\text { Hippotherapy } \\
\text { Fair }\end{array}$ & NR & Cerebral palsy & $\begin{array}{l}\text { Inclusion Criteria: } \\
\text { CP spastic diplegia }\end{array}$ & $\begin{array}{l}\text { Randomized: } \\
40 \\
\text { Analyzed: } 40 \\
\text { Attrition: } 0 \% \\
(0 / 40)\end{array}$ & $\begin{array}{l}\text { Brazil } \\
\text { Outpatient clinic } \\
\text { RCT }\end{array}$ & NR \\
\hline $\begin{array}{l}\text { Slaman, 2014a } \\
\text { Slaman, 2014b } \\
\text { Slaman, 2015 } \\
\text { Slaman, 2010 } \\
\text { (check QR study dates - } \\
\text { Slaman 2014, 2015a, } \\
\text { 2015b listed) } \\
\text { Multimodal Exercise } \\
\text { Mid-intervention (12 } \\
\text { weeks into intervention); } \\
\text { Immediately } \\
\text { postintervention, } \\
\text { 24 weeks } \\
\text { Fair }\end{array}$ & NR & Cerebral palsy & $\begin{array}{l}\text { Diagnosed with spastic } \\
\text { unilateral or bilateral CP; } \\
\text { age } 16 \text { to } 24 \text { years old; } \\
\text { and GMFCS levels I to IV. }\end{array}$ & $\begin{array}{l}\text { Randomized: } \\
57 \\
\text { Analyzed: } 42 \\
\text { Attrition: } 26.3 \% \\
(15 / 57)\end{array}$ & $\begin{array}{l}\text { Netherlands } \\
\text { outpatient clinic } \\
\text { RCT }\end{array}$ & NR \\
\hline $\begin{array}{l}\text { Straudi, } 2016 \\
\text { Aerobic Exercise } \\
\text { Robot-Assisted Gait } \\
\text { Training } \\
\text { Postintervention, } 6 \\
\text { weeks } \\
\text { Fair }\end{array}$ & none & $\begin{array}{l}\text { Multiple } \\
\text { sclerosis }\end{array}$ & $\begin{array}{l}\text { Primary or secondary } \\
\text { progressive MS, } 18 \text { or } \\
\text { older, and severe gait } \\
\text { impairment EDSS } 6.0-7.0\end{array}$ & $\begin{array}{l}\text { Randomized: } \\
\mathrm{N}=58 \\
\text { Analyzed: } 54 \\
\text { Attrition: } 6.9 \%\end{array}$ & \begin{tabular}{|l} 
Italy \\
2 outpatient \\
treatment centers \\
RCT
\end{tabular} & $\begin{array}{l}\text { Multiple Sclerosis } \\
\text { Italian Society }\end{array}$ \\
\hline
\end{tabular}




\begin{tabular}{|c|c|c|c|c|c|c|}
\hline $\begin{array}{l}\text { Author, Year } \\
\text { Intervention Type } \\
\text { Duration of } \\
\text { Postintervention } \\
\text { Followup } \\
\text { Quality } \\
\text { (See Appendix B for } \\
\text { Full Citation) }\end{array}$ & Harms & Condition & Inclusion Criteria & $\begin{array}{l}\text { Number } \\
\text { Randomized } \\
\text { Analyzed } \\
\text { Attrition }\end{array}$ & $\begin{array}{l}\text { Country } \\
\text { Setting } \\
\text { Study Design }\end{array}$ & Funding Source \\
\hline $\begin{array}{l}\text { Straudi, } 2019 \\
\text { Aerobic Exercise } \\
\text { Robot-Assisted Gait } \\
\text { Training } \\
\text { Postintervention, } 12 \\
\text { weeks } \\
\text { Good }\end{array}$ & NR & Multiple sclerosis & $\begin{array}{l}\text { Inclusion Criteria: People } \\
\text { with MS, } 18-65 \text { years old, } \\
\text { EDSS of } 6.0-7.0\end{array}$ & $\begin{array}{l}\text { Randomized: } \\
72 \\
\text { Analyzed: } 64 \\
\text { Attrition: } 12.5 \% \\
(8 / 64)\end{array}$ & $\begin{array}{l}\text { Italy } \\
\text { University Hospital } \\
\text { RCT }\end{array}$ & $\begin{array}{l}\text { Research Programme } \\
\text { of Emilia Romagna } \\
\text { Region }\end{array}$ \\
\hline $\begin{array}{l}\text { Swe } 2015 \\
\text { Aerobic Exercise } \\
\text { Treadmill } \\
\text { Pre to post only } \\
\text { Good }\end{array}$ & None reported & Cerebral palsy & $\begin{array}{l}\text { Recruited from } \\
\text { adolescents from schools } \\
\text { and clinics } \\
\text { Ability to walk with or } \\
\text { without assist device for at } \\
\text { least } 10 \text { meters }\end{array}$ & $\begin{array}{l}30 \text { randomized } \\
30 \text { analyzed }\end{array}$ & $\begin{array}{l}\text { Done in Singapore. } \\
\text { Author in Australia } \\
\text { RCT }\end{array}$ & Funding NR \\
\hline $\begin{array}{l}\text { Tak, } 2015 \\
\text { Postural Control } \\
\text { Motion gaming } \\
\text { Immediately } \\
\text { postintervention, } \\
6 \text { weeks } \\
\text { Fair }\end{array}$ & NR & Spinal cord injury & $\begin{array}{l}\text { Inclusion Criteria: } \\
\text { At least } 6 \text { months since } \\
\text { injury; able to sit } \\
\text { independently for at least } \\
30 \text { seconds and absence } \\
\text { pain sitting for } 2 \text { hours; } \\
\text { able to lift arms to head; } \\
\text { no musculoskeletal } \\
\text { deformities; less than } 5 \\
\text { points each leg on } \\
\text { American Spinal Cord } \\
\text { Injury Association Scale }\end{array}$ & $\begin{array}{l}\text { Randomized: } \\
26 \\
\text { Analyzed: } 26 \\
\text { Attrition: } 0 \%\end{array}$ & $\begin{array}{l}\text { South Korea } \\
\text { Rehabilitation } \\
\text { hospital } \\
\text { RCT }\end{array}$ & Sahmyook Univerisity \\
\hline
\end{tabular}




\begin{tabular}{|c|c|c|c|c|c|c|}
\hline $\begin{array}{l}\text { Author, Year } \\
\text { Intervention Type } \\
\text { Duration of } \\
\text { Postintervention } \\
\text { Followup } \\
\text { Quality } \\
\text { (See Appendix B for } \\
\text { Full Citation) }\end{array}$ & Harms & Condition & Inclusion Criteria & $\begin{array}{l}\text { Number } \\
\text { Randomized } \\
\text { Analyzed } \\
\text { Attrition } \\
\end{array}$ & \begin{tabular}{|l|} 
Country \\
Setting \\
Study Design \\
\end{tabular} & Funding Source \\
\hline $\begin{array}{l}\text { Tarakci, } 2013 \\
\text { Multimodal Exercise } \\
\text { Postintervention, } 0 \\
\text { weeks } \\
\text { Fair }\end{array}$ & $\begin{array}{l}\text { No adverse events occurred during the } \\
\text { training period of the current study. There } \\
\text { were no deleterious effects of the group } \\
\text { exercise training on balance, fatigue and } \\
\text { quality of life parameters. }\end{array}$ & Multiple sclerosis & $\begin{array}{l}\text { Diagnosis of definite MS } \\
\text { by McDonald criteria, } \\
\text { EDSS score between } 2.0 \\
\text { and } 6.5, \text { no relapse within } \\
30 \text { days, ability to adapt to } \\
\text { exercises, having stability } \\
\text { in medication and no } \\
\text { difficulty in the } \\
\text { transportation to the } \\
\text { hospital. }\end{array}$ & $\begin{array}{l}\text { Randomized: } \\
110 \\
\text { Analyzed: } 99 \\
\text { Attrition: } 10 \% \\
(11 / 110)\end{array}$ & $\begin{array}{l}\text { Turkey } \\
\text { Outpatient } \\
\text { RCT }\end{array}$ & $\begin{array}{l}\text { This research } \\
\text { received no specific } \\
\text { grant from any } \\
\text { funding agency in the } \\
\text { public, commercial, or } \\
\text { not-for-profit sectors. }\end{array}$ \\
\hline $\begin{array}{l}\text { Tarakci, } 2016 \\
\text { Postural Control } \\
\text { Motion gaming } \\
\text { Postintervention, } 12 \\
\text { weeks } \\
\text { Fair }\end{array}$ & NR & Cerebral palsy & $\begin{array}{l}\text { Diagnosis of CP (diplegic, } \\
\text { hemiplegic, dyskinetic } \\
\text { type); age 5-18 years of } \\
\text { age; GMFCS level 1, level } \\
2 \text { or level 3; no history of } \\
\text { epilepsy; no botulinum } \\
\text { toxin A treatment for the } \\
\text { lower extremities in the } \\
\text { previous } 6 \text { months; no } \\
\text { excessive spasticity in any } \\
\text { joint (score >2 on the } \\
\text { MAS); and confirmed } \\
\text { mental ability to be able } \\
\text { adapt to exercise }\end{array}$ & $\begin{array}{l}\text { Randomized: } \\
38 \\
\text { Analyzed:30 } \\
\text { Attrition: } 21 \% \\
(8 / 38)\end{array}$ & $\begin{array}{l}\text { Turkey } \\
\text { University rehab. } \\
\text { center } \\
\text { RCT }\end{array}$ & NR \\
\hline $\begin{array}{l}\text { Taylor, } 2013 \\
\text { Bania, } 2016 \\
\text { Muscle Strength } \\
\text { Immediately } \\
\text { postintervention, } \\
12 \text { weeks } \\
\text { Taylor, 2013: Good } \\
\text { Bania, 2016: Fair }\end{array}$ & $\begin{array}{l}\text { A vs. B } \\
\text { Short-term muscle soreness } \\
\text { was reported by most participants in group } \\
\text { A. } \\
\text { Minor calf strain: } 4.3 \%(1 / 23) \text { vs. } 0 \%(0 / 25) \\
\text { Minor discomfort due to plantar fascia: } \\
4.3 \%(1 / 23) \text { vs. } 0 \%(0 / 25)\end{array}$ & Cerebral palsy & $\begin{array}{l}\text { Patients with spastic } \\
\text { diplegic CP, aged } \\
\text { between } 14 \text { and } 22 \text { years, } \\
\text { be classified as level II or } \\
\text { III on the GMFCS, and be } \\
\text { able to follow simple } \\
\text { instructions. }\end{array}$ & $\begin{array}{l}\text { Randomized: } \\
49 \\
\text { Analyzed: } 48 \\
\text { Attrition: } 2 \% \\
(1 / 49)\end{array}$ & $\begin{array}{l}\text { Australia } \\
\text { Local gymnasiums } \\
\text { RCT }\end{array}$ & $\begin{array}{l}\text { This trial was } \\
\text { supported financially } \\
\text { by a grant from the } \\
\text { National Health and } \\
\text { Medical Research } \\
\text { Council of Australia } \\
\text { (ID 487321). }\end{array}$ \\
\hline
\end{tabular}




\begin{tabular}{|c|c|c|c|c|c|c|}
\hline $\begin{array}{l}\text { Author, Year } \\
\text { Intervention Type } \\
\text { Duration of } \\
\text { Postintervention } \\
\text { Followup } \\
\text { Quality } \\
\text { (See Appendix B for } \\
\text { Full Citation) } \\
\end{array}$ & Harms & Condition & Inclusion Criteria & $\begin{array}{l}\text { Number } \\
\text { Randomized } \\
\text { Analyzed } \\
\text { Attrition } \\
\end{array}$ & $\begin{array}{l}\text { Country } \\
\text { Setting } \\
\text { Study Design } \\
\end{array}$ & Funding Source \\
\hline $\begin{array}{l}\text { Tedla, } 2014 \\
\text { Strength interventions } \\
\text { Muscle Strength } \\
\text { Exercises } \\
\text { Immediately } \\
\text { postintervention, } \\
6 \text { weeks } \\
\text { Poor }\end{array}$ & $\begin{array}{l}\text { Strong pressure from hand held } \\
\text { dynameters was applied but reported to not } \\
\text { hurt the skin. }\end{array}$ & Cerebral palsy & $\begin{array}{l}\text { Inclusion Criteria: } \\
\text { CP patients } 5 \text { to } 14 \text { years } \\
\text { old with spastic diplegia, } \\
\text { GMFCS I - IV }\end{array}$ & $\begin{array}{l}\text { Randomized: } \\
60 \\
\text { Analyzed: } 60 \\
\text { Attrition: } 3 \% \\
(2 / 60)\end{array}$ & $\begin{array}{l}\text { Saudi Arabia } \\
\text { Rehabilitation } \\
\text { hospital } \\
\text { RCT }\end{array}$ & NR \\
\hline $\begin{array}{l}\text { Teixeira-Machado, } 2017 \\
\text { Aerobic Exercise } \\
\text { Aerobics (Dance) } \\
\text { Postintervention, } 12 \\
\text { weeks } \\
\text { Poor }\end{array}$ & NR & Cerebral palsy & $\begin{array}{l}\text { Patient } 15-29 \text { years old, } \\
\text { diagnosed with cerebral } \\
\text { palsy, increased muscle } \\
\text { tone and no cardiopathy } \\
\text { or neoplasy }\end{array}$ & $\begin{array}{l}\text { Randomized: } \\
26 \\
\text { Analyzed: } 26 \\
\text { Attrition: } 0 \%(0 / \\
26)\end{array}$ & $\begin{array}{l}\text { Italy } \\
\text { Outpatient clinic } \\
\text { RCT }\end{array}$ & NR \\
\hline $\begin{array}{l}\text { Tollar, } 2020 \\
\text { Aerobic Exercise } \\
\text { Cycling } \\
\text { Postural Control } \\
\text { Balance Exercises } \\
\text { Motion Gaming } \\
\text { Strength } \\
\text { proprioceptive } \\
\text { neuromuscular } \\
\text { facilitation (PNF) } \\
\text { Immediately } \\
\text { postintervention, } 5 \\
\text { weeks } \\
\text { Fair }\end{array}$ & NR & $\begin{array}{l}\text { Multiple } \\
\text { Sclerosis }\end{array}$ & $\begin{array}{l}\text { Diagnosis of MS; male or } \\
\text { female sex, age } \geq 30 \\
\text { years, EDSS score of } 4 \text { to } \\
6 \text {, a relapse frequency } \leq 1 \\
\text { per year over the past } 5 \\
\text { years to minimize a } \\
\text { change in medication, and } \\
\text { Mini-Mental State } \\
\text { Examination score } \geq 24\end{array}$ & $\begin{array}{l}\text { Randomized: } \\
70 \\
\text { Analyzed: } 68 \\
\text { Attrition: } 3 \% \\
(2 / 70)\end{array}$ & $\begin{array}{l}\text { Hungary and } \\
\text { The Netherlands } \\
\text { Outpatient clinic } \\
\text { RCT }\end{array}$ & None \\
\hline
\end{tabular}




\begin{tabular}{|c|c|c|c|c|c|c|}
\hline $\begin{array}{l}\text { Author, Year } \\
\text { Intervention Type } \\
\text { Duration of } \\
\text { Postintervention } \\
\text { Followup } \\
\text { Quality } \\
\text { (See Appendix B for } \\
\text { Full Citation) }\end{array}$ & Harms & Condition & Inclusion Criteria & $\begin{array}{l}\text { Number } \\
\text { Randomized } \\
\text { Analyzed } \\
\text { Attrition }\end{array}$ & $\begin{array}{l}\text { Country } \\
\text { Setting } \\
\text { Study Design }\end{array}$ & Funding Source \\
\hline $\begin{array}{l}\text { Totosy de Zepetnek, } \\
2015 \\
\text { Multimodal Exercise } \\
\text { Immediately } \\
\text { postintervention, } \\
16 \text { weeks } \\
\text { Fair }\end{array}$ & $\begin{array}{l}\text { NR, one adverse event not related to the } \\
\text { study reported. }\end{array}$ & Spinal cord injury & \begin{tabular}{|l|} 
Inclusion Criteria: People \\
with chronic SCI 1 year \\
post injury, 18 to 65 years \\
old
\end{tabular} & $\begin{array}{l}\text { Randomized: } \\
23 \\
\text { Analyzed: } 17 \\
\text { Attrition: } 7 \% \\
(2 / 23)\end{array}$ & $\begin{array}{l}\text { Canada Outpatient } \\
\text { clinic } \\
\text { RCT }\end{array}$ & $\begin{array}{l}\text { Ontario Neurotrauma } \\
\text { Foundation Grant }\end{array}$ \\
\hline $\begin{array}{l}\text { Valent, } 2010 \\
\text { Aerobic Exercise } \\
\text { Hand cycling } \\
\text { Postintervention, } 0 \\
\text { weeks } \\
\text { Fair }\end{array}$ & $\begin{array}{l}\text { NR } \\
\text { *this is questionable as one subject } \\
\text { dropped out due to elbow tendonitis }\end{array}$ & Spinal cord injury & $\begin{array}{l}\text { Included subjects met the } \\
\text { following criteria: (1) had } \\
\text { an acute SCl; (2) had a } \\
\text { prognosis of 'remaining } \\
\text { mainly wheelchair-bound'; } \\
\text { (3) had a lesion level of } \\
\text { C5 or lower (and } \\
\text { consequently were } \\
\text { expected to be able to } \\
\text { propel a hand cycle); (4) } \\
\text { were aged between 18 } \\
\text { and } 65 \text { years; (5) had } \\
\text { sufficient knowledge of the } \\
\text { Dutch language; (6) did } \\
\text { not have a progressive } \\
\text { disease or psychiatric } \\
\text { problem; (7) were free of } \\
\text { halo-frames or corset; (8) } \\
\text { were made familiar with } \\
\text { hand cycling and agreed } \\
\text { to participate according } \\
\text { the training protocol. }\end{array}$ & \begin{tabular}{|l|} 
Randomized: \\
Nonrandomized \\
study
\end{tabular} & $\begin{array}{l}\text { Netherlands } \\
\text { Inpatient } \\
\text { rehabilitation } \\
\text { Retrospective } \\
\text { analysis }\end{array}$ & $\begin{array}{l}\text { Netherlands } \\
\text { Organisation for } \\
\text { Health, } \\
\text { Research and } \\
\text { Development }\end{array}$ \\
\hline
\end{tabular}




\begin{tabular}{|c|c|c|c|c|c|c|}
\hline $\begin{array}{l}\text { Author, Year } \\
\text { Intervention Type } \\
\text { Duration of } \\
\text { Postintervention } \\
\text { Followup } \\
\text { Quality } \\
\text { (See Appendix B for } \\
\text { Full Citation) } \\
\end{array}$ & Harms & Condition & Inclusion Criteria & $\begin{array}{l}\text { Number } \\
\text { Randomized } \\
\text { Analyzed } \\
\text { Attrition } \\
\end{array}$ & $\begin{array}{l}\text { Country } \\
\text { Setting } \\
\text { Study Design }\end{array}$ & Funding Source \\
\hline $\begin{array}{l}\text { van der Scheer } 2016 \\
\text { Aerobic Exercise } \\
\text { Treadmill } \\
\text { 16-week intervention } \\
\text { Postintervention, } 0 \\
\text { weeks } \\
\text { Fair }\end{array}$ & None & Spinal cord injury & $\begin{array}{l}\text { Community dwelling } \\
\text { inactive manual } \\
\text { wheelchair users with } \mathrm{SCl}\end{array}$ & $\begin{array}{l}n=29 \\
\text { randomized } \\
27 \text { analyzed } \\
2 / 29 \text { attrition }\end{array}$ & $\begin{array}{l}\text { The Netherlands } \\
\text { RCT } \\
\text { Trained in } \\
\text { rehabilitation } \\
\text { center }\end{array}$ & Government \\
\hline $\begin{array}{l}\text { Van Wely, 2014a } \\
\text { Van Wely, 2014b } \\
\text { Van Wely, 2010 } \\
\text { Multimodal Exercise } \\
\text { Mid-intervention (16 } \\
\text { weeks into trial); } \\
\text { Immediately } \\
\text { postintervention, } \\
24 \text { weeks } \\
\text { Van Wely, 2014a: Good } \\
\text { Van Wely, 2014b: Fair }\end{array}$ & NR & Cerebral palsy & $\begin{array}{l}\text { Children with spastic } \\
\text { cerebral palsy, aged 7-13 } \\
\text { years who could walk, } \\
\text { classification in GMFCS } \\
\text { level I-III, understanding } \\
\text { of the Dutch language and } \\
\text { fulfilling at least one of the } \\
\text { following criteria as } \\
\text { determined in a telephone } \\
\text { interview: less active than } \\
\text { the international physical } \\
\text { activity norm of less than } \\
1 \text { hour daily at }>5 \\
\text { metabolic equivalents, } \\
\text { which is moderate or } \\
\text { vigorous intensity; no } \\
\text { regular participation in } \\
\text { sports or } \\
\text { (physiotherapeutic) fitness } \\
\text { program (i.e., less than } \\
\text { three times a week for at } \\
\text { least } 20 \text { minutes); and } \\
\text { experience of problems } \\
\text { related to mobility in daily } \\
\text { life or sports. }\end{array}$ & $\begin{array}{l}\text { Randomized: } \\
50 \\
\text { Analyzed: } 47 \\
\text { Attrition: } 6.0 \% \\
(3 / 50)\end{array}$ & $\begin{array}{l}\text { Netherlands } \\
\text { Outpatient clinic } \\
\text { and participants } \\
\text { home } \\
\text { RCT }\end{array}$ & $\begin{array}{l}\text { This project is part of } \\
\text { the Dutch } \\
\text { nationalLEARN } 2 \\
\text { MOVE research } \\
\text { program and is } \\
\text { supported financially } \\
\text { by ZonMw (grant } \\
\text { number } 89000002 \text { ), } \\
\text { Johanna Kinderfonds, } \\
\text { Stichting Rotterdams } \\
\text { Kinderrevalidatie } \\
\text { Fonds } \\
\text { Adriaanstichting, } \\
\text { Revalidatie-fonds, } \\
\text { Phelps Stichting, } \\
\text { Revalidatie } \\
\text { Nederland, and the } \\
\text { Nederlandse } \\
\text { Vereniging van } \\
\text { Revalidatieartsen. }\end{array}$ \\
\hline
\end{tabular}




\begin{tabular}{|c|c|c|c|c|c|c|}
\hline $\begin{array}{l}\text { Author, Year } \\
\text { Intervention Type } \\
\text { Duration of } \\
\text { Postintervention } \\
\text { Followup } \\
\text { Quality } \\
\text { (See Appendix B for } \\
\text { Full Citation) }\end{array}$ & Harms & Condition & Inclusion Criteria & $\begin{array}{l}\text { Number } \\
\text { Randomized } \\
\text { Analyzed } \\
\text { Attrition } \\
\end{array}$ & \begin{tabular}{|l|} 
Country \\
Setting \\
Study Design \\
\end{tabular} & Funding Source \\
\hline $\begin{array}{l}\text { Vermohlen, } 2018 \\
\text { Protocol: Wollenweber, } \\
2016 \\
\text { Postural Control } \\
\text { Hippotherapy } \\
\text { End of treatment (after } \\
\text { 12-week intervention) } \\
\text { Fair }\end{array}$ & $\begin{array}{l}\text { A ( }(n=30) \text { vs. } B(n=37) \\
\text { Patients with adverse events: } 13(43 \%) \text { vs. } \\
15(41 \%) \\
\text { Number of patients with adverse events } \\
\text { that are considered serious adverse events: } \\
1(3 \%) \text { vs. } 2(5 \%)\end{array}$ & Multiple sclerosis & $\begin{array}{l}\text { Inclusion Criteria: } \\
\text { confirmed multiple } \\
\text { sclerosis with spasticity of } \\
\text { the lower limbs, EDSS } \\
\text { score between } 4 \text { and } 6.5 \text {, } \\
\text { written informed consent } \\
\text { of the patient, approval of } \\
\text { the responsible study } \\
\text { physician, legal } \\
\text { competence, minimum } \\
\text { age of } 18 \text { years }\end{array}$ & $\begin{array}{l}\text { Randomized: } \\
70 \\
\text { Analyzed: } 41 \\
\text { (67 analyzed } \\
\text { for modified } \\
\text { ITT) } \\
\text { Attrition: } 41 \% \\
(29 / 70)\end{array}$ & $\begin{array}{l}\text { Germany } \\
\text { Outpatient clinic } \\
\text { RCT }\end{array}$ & Nonprofit \\
\hline $\begin{array}{l}\text { Wallard, } 2017 \\
\text { Wallard, } 2018 \\
\text { Aerobic Exercise } \\
\text { Robot-Assisted Gait } \\
\text { Training } \\
\text { Postintervention, } 4 \\
\text { weeks } \\
\text { Poor }\end{array}$ & NR & Cerebral palsy & $\begin{array}{l}\text { Inclusion Criteria: children } \\
\text { 8-10 years old with } \\
\text { bilateral spastic CP } \\
\text { GMFCS Level II, walk } \\
\text { 60m with or without } \\
\text { assistive device }\end{array}$ & $\begin{array}{l}\text { Randomized: } \\
\mathrm{N}=30 \\
\text { Analyzed: } 30 \\
\text { Attrition: } 0 \%\end{array}$ & $\begin{array}{l}\text { France } \\
\text { Outpatient } \\
\text { RCT }\end{array}$ & NR \\
\hline $\begin{array}{l}\text { Wens, 2015b } \\
\text { Multimodal Exercise } \\
\text { Postintervention, } 0 \\
\text { weeks } \\
\text { Fair }\end{array}$ & $\begin{array}{l}\text { No dropout or adverse events were } \\
\text { reported during the trial period }\end{array}$ & Multiple sclerosis & $\begin{array}{l}\text { MS patients diagnosed } \\
\text { according to McDonald } \\
\text { criteria (EDSS range } 1-5 \text { ), } \\
\text { aged }>18 \text { years }\end{array}$ & $\begin{array}{l}\text { Randomized: } \\
34 \\
\text { Analyzed: } 34 \\
\text { Attrition: } 0 \% \\
(0 / 34)\end{array}$ & $\begin{array}{l}\text { NR } \\
\text { NR } \\
\text { RCT }\end{array}$ & \begin{tabular}{|l|} 
Nonprofit \\
MS Fund, Limburg, \\
Flanders, Belgium
\end{tabular} \\
\hline $\begin{array}{l}\text { Williams, } 2020 \\
\text { Multimodal } \\
\text { Postintervention, } 0 \\
\text { weeks and } 8 \text { weeks } \\
\text { Fair }\end{array}$ & $\begin{array}{l}\text { No adverse events were reported as a } \\
\text { result of the intervention by either group. }\end{array}$ & Multiple sclerosis & $\begin{array}{l}\text { Diagnosis of MS made by } \\
\text { a neurologist, able to walk } \\
10 \text { meters with or without } \\
\text { an aid within } 2 \text { minutes, } \\
\text { no relapse of their MS in } \\
\text { the past } 4 \text { weeks, and no } \\
\text { other neurological or } \\
\text { orthopedic condition that } \\
\text { would affect their function. }\end{array}$ & $\begin{array}{l}\text { Randomized: } \\
50 \\
\text { Analyzed: } 44 \\
\text { Attrition: } 12 \% \\
(6 / 50)\end{array}$ & $\begin{array}{l}\text { Australia } \\
\text { Outpatient and } \\
\text { home } \\
\text { RCT }\end{array}$ & Nonprofit agency \\
\hline
\end{tabular}




\begin{tabular}{|c|c|c|c|c|c|c|}
\hline $\begin{array}{l}\text { Author, Year } \\
\text { Intervention Type } \\
\text { Duration of } \\
\text { Postintervention } \\
\text { Followup } \\
\text { Quality } \\
\text { (See Appendix B for } \\
\text { Full Citation) }\end{array}$ & Harms & Condition & Inclusion Criteria & $\begin{array}{l}\text { Number } \\
\text { Randomized } \\
\text { Analyzed } \\
\text { Attrition } \\
\end{array}$ & \begin{tabular}{|l|} 
Country \\
Setting \\
Study Design \\
\end{tabular} & Funding Source \\
\hline $\begin{array}{l}\text { Willoughby } 2010 \\
\text { Aerobic Exercise } \\
\text { Treadmill } \\
\text { Postintervention, } 10 \\
\text { weeks } \\
\text { Fair }\end{array}$ & One B child dropped out due to back pain & Cerebral palsy & $\begin{array}{l}\text { Recruited children from } \\
\text { school for children with } \\
\text { disabilities }\end{array}$ & $\begin{array}{l}33 \text { randomized } \\
26 \text { analyzed } \\
7 / 33=21 \% \\
\text { attrition for } 9- \\
\text { week study }\end{array}$ & $\begin{array}{l}\text { Done in Singapore, } \\
\text { Author Australia } \\
\text { Done at schools } \\
\text { RCT }\end{array}$ & NR \\
\hline $\begin{array}{l}\text { Wu, 2017a } \\
\text { "Robotic resistance } \\
\text { treadmill training } \\
\text { improves locomotor } \\
\text { function in children with } \\
\text { cerebral palsy: a } \\
\text { randomized controlled } \\
\text { pilot study" } \\
\text { Aerobic Exercise } \\
\text { Robot-Assisted Gait } \\
\text { Training } \\
\text { Postintervention,8 } \\
\text { weeks (after 6-week } \\
\text { intervention) } \\
\text { Fair }\end{array}$ & NR & Cerebral palsy & $\begin{array}{l}\text { Inclusion Criteria: bilateral } \\
\text { spastic CP, aged } 4 \text { to } 14 \\
\text { years, without botulinum } \\
\text { toxin treatment and } \\
\text { orthopedic surgery or } \\
\text { neurosurgery in the } 6 \\
\text { months before the onset } \\
\text { of training, GMFCS I to IV, } \\
\text { able to signal pain, fear, or } \\
\text { discomfort reliably, with } \\
\text { mild scoliosis (Cobb angle } \\
<20 \text { ), passive range of } \\
\text { motion within functional } \\
\text { limits, and able to follow } \\
\text { instructions on behavior } \\
\text { tests }\end{array}$ & $\begin{array}{l}\text { Randomized: } \\
\mathrm{N}=23 \\
\text { Analyzed: } 20 \\
\text { Attrition: } 13 \% \\
(3 / 23)\end{array}$ & $\begin{array}{l}\text { US } \\
\text { Outpatient clinic } \\
\text { RCT }\end{array}$ & Government \\
\hline
\end{tabular}




\begin{tabular}{|c|c|c|c|c|c|c|}
\hline $\begin{array}{l}\text { Author, Year } \\
\text { Intervention Type } \\
\text { Duration of } \\
\text { Postintervention } \\
\text { Followup } \\
\text { Quality } \\
\text { (See Appendix B for } \\
\text { Full Citation) }\end{array}$ & Harms & Condition & Inclusion Criteria & $\begin{array}{l}\text { Number } \\
\text { Randomized } \\
\text { Analyzed } \\
\text { Attrition }\end{array}$ & $\begin{array}{l}\text { Country } \\
\text { Setting } \\
\text { Study Design }\end{array}$ & Funding Source \\
\hline $\begin{array}{l}\text { Wu, } 2017 \mathrm{~b} \\
\text { "The effects of the } \\
\text { integration of dynamic } \\
\text { weight shifting training } \\
\text { into treadmill training on } \\
\text { walking function of } \\
\text { children with cerebral } \\
\text { palsy- a randomized } \\
\text { controlled study" } \\
\text { Aerobic Exercise } \\
\text { Robot-Assisted Gait } \\
\text { Training } \\
\text { Postintervention,8 } \\
\text { weeks (after 6-week } \\
\text { intervention) } \\
\text { Fair }\end{array}$ & NR & Cerebral palsy & $\begin{array}{l}\text { Inclusion Criteria: bilateral } \\
\text { spastic CP, age } 4 \text { to } 16 \\
\text { years, GMFCS I-IV, able } \\
\text { to signal pain, fear or } \\
\text { discomfort reliably, } \\
\text { passive range of motion } \\
\text { within functional limits } \\
(\text { ankle dorsiflexion }= \\
\text { neutral; knee flexion }=0- \\
120^{\circ} \text {; hip flexion }=0-90^{\circ} \text {; } \\
\text { and hip extension }=0- \\
\left.10^{\circ}\right) \text {, if scoliosis is } \\
\text { present, Cobb angle < } \\
20^{\circ}, \text { no Botulinum toxin } \\
\text { treatment within past } 3 \\
\text { months, no orthopedic } \\
\text { surgery or neurosurgery } \\
\text { within the past } 6 \text { months }\end{array}$ & $\begin{array}{l}\text { Randomized: } \\
\mathrm{N}=23 \\
\text { Analyzed: } 21 \\
\text { Attrition: } 8.7 \% \\
(2 / 23)\end{array}$ & $\begin{array}{l}\text { US } \\
\text { Outpatient } \\
\text { RCT }\end{array}$ & $\begin{array}{l}\text { NIDRR/RERC } \\
\text { Government }\end{array}$ \\
\hline $\begin{array}{l}\text { Yang } 2013 \\
\text { Aerobic Exercise } \\
\text { Treadmill } \\
\text { Pre to post, crossover } \\
\text { with } 2 \text { months rest } \\
\text { between } \\
8 \text { week intervention } \\
\text { Fair }\end{array}$ & $\begin{array}{l}\text { One drop out due to wrist pain worsening } \\
\text { during use of walker }\end{array}$ & Spinal cord injury & $\begin{array}{l}\text { SCI C1 to L1 > } 7 \text { months } \\
\text { ago } \\
\text { Able to walk > } 5 \text { meters } \\
\text { with walking aid or braces } \\
\text { Able to attend } 5 x / \text { week } \\
\text { training } \\
\text { Recruitment occurred over } \\
5 \text { years }\end{array}$ & \begin{tabular}{|l|}
$\mathrm{n}=22$ \\
randomized \\
and 20 \\
analyzed \\
$2 / 22$
\end{tabular} & $\begin{array}{l}\text { Canada } \\
\text { RCT, single blind, } \\
\text { cross over design } \\
\text { with } 2 \text { months rest } \\
\text { between }\end{array}$ & $\begin{array}{l}\text { Government and } \\
\text { Foundation }\end{array}$ \\
\hline
\end{tabular}




\begin{tabular}{|c|c|c|c|c|c|c|}
\hline $\begin{array}{l}\text { Author, Year } \\
\text { Intervention Type } \\
\text { Duration of } \\
\text { Postintervention } \\
\text { Followup } \\
\text { Quality } \\
\text { (See Appendix B for } \\
\text { Full Citation) }\end{array}$ & Harms & Condition & Inclusion Criteria & $\begin{array}{l}\text { Number } \\
\text { Randomized } \\
\text { Analyzed } \\
\text { Attrition }\end{array}$ & $\begin{array}{l}\text { Country } \\
\text { Setting } \\
\text { Study Design }\end{array}$ & Funding Source \\
\hline $\begin{array}{l}\text { Yazgan } 2020 \\
\text { Postural Control } \\
\text { Motion Gaming } \\
\text { Postintervention, } 0 \\
\text { weeks } \\
\text { Fair }\end{array}$ & None reported & $\begin{array}{l}\text { Multiple } \\
\text { Sclerosis }\end{array}$ & $\begin{array}{l}\text { Diagnosis of MS; } 25 \text { to } 60 \\
\text { years old, ambulatory } \\
\text { without relapses in } 3 \\
\text { months, EDSS between } \\
2.5 \text { and } 6\end{array}$ & $\begin{array}{l}\text { Randomized: } \\
47 \\
\text { Analyzed: } 42 \\
\text { Attrition: } 10 \% \\
(5 / 47)\end{array}$ & $\begin{array}{l}\text { Turkey } \\
\text { Setting (outpatient, } \\
\text { rehabilitation) } \\
\text { RCT }\end{array}$ & University \\
\hline $\begin{array}{l}\text { Yazici, } 2019 \\
\text { Aerobic Exercise } \\
\text { Robot-Assisted Gait } \\
\text { Training } \\
\text { Immediately } \\
\text { postintervention, } \\
12 \text { weeks } \\
\text { Poor }\end{array}$ & NR & Cerebral palsy & $\begin{array}{l}\text { Children with GFMS I-II } \\
\text { hemiplegic cerebral palsy, } \\
\text { no vision or hearing } \\
\text { impairment who were } \\
\text { attending a physiotherapy } \\
\text { rehabilitation program }\end{array}$ & $\begin{array}{l}\text { Randomized: } \\
24 \\
\text { Analyzed: } 22 \\
\text { Attrition: } 9.1 \% \\
(2 / 22)\end{array}$ & \begin{tabular}{|l} 
Turkey \\
University \\
Rehabilitation \\
Clinic \\
Cohort study
\end{tabular} & NR \\
\hline $\begin{array}{l}\text { Yildirim, } 2019 \\
\text { Aerobic Exercise } \\
\text { Robot-Assisted Gait } \\
\text { Training } \\
\text { Immediately } \\
\text { postintervention, } \\
8 \text { weeks } \\
\text { Fair }\end{array}$ & NR & Spinal cord injury & $\begin{array}{l}\text { 18-65 years olds with SCI } \\
\text { ASIA A-D complete or } \\
\text { incomplete, injury within } 6 \\
\text { months, ambulatory pre- } \\
\text { injury }\end{array}$ & $\begin{array}{l}\text { Randomized: } \\
88 \\
\text { Analyzed: } 88 \\
\text { Attrition: } 0 \% \\
(0 / 88)\end{array}$ & $\begin{array}{l}\text { Turkey } \\
\text { Rehbialita-tion } \\
\text { hospital } \\
\text { RCT }\end{array}$ & NR \\
\hline
\end{tabular}




\begin{tabular}{|c|c|c|c|c|c|c|}
\hline $\begin{array}{l}\text { Author, Year } \\
\text { Intervention Type } \\
\text { Duration of } \\
\text { Postintervention } \\
\text { Followup } \\
\text { Quality } \\
\text { (See Appendix B for } \\
\text { Full Citation) }\end{array}$ & Harms & Condition & Inclusion Criteria & $\begin{array}{l}\text { Number } \\
\text { Randomized } \\
\text { Analyzed } \\
\text { Attrition } \\
\end{array}$ & \begin{tabular}{|l|} 
Country \\
Setting \\
Study Design \\
\end{tabular} & Funding Source \\
\hline $\begin{array}{l}\text { Young, } 2019 \\
\text { Aerobic Exercise } \\
\text { Aerobics } \\
12 \text { weeks } \\
0 \text { weeks } \\
\text { Fair }\end{array}$ & $\begin{array}{l}\text { A vs. B vs. C } \\
\text { Total AEs: } \\
3 \text { vs. } 1 \text { vs. } 0 \\
\text { Falls: } \\
0 \text { vs. } 0 \text { vs. } 0 \\
\text { MSK-related: } \\
3 \text { vs. } 0 \text { vs. } 0 \\
\text { CV-related: } \\
0 \text { vs. } 1 \text { vs. } 0\end{array}$ & Multiple sclerosis & $\begin{array}{l}\text { PDSS between } 0 \text { to } 6 \\
\text { (8=bedridden) }\end{array}$ & $\begin{array}{l}\text { Randomized: } \\
81 \\
\text { Analyzed: } 81 \\
\text { presumed } \\
(100 \%) \\
\text { Attrition: } 20 \\
(25 \%)\end{array}$ & $\begin{array}{l}\text { USA } \\
\text { Outpatient } \\
\text { RCT }\end{array}$ & Government \\
\hline $\begin{array}{l}\text { Zoccolillo, } 2015 \\
\text { Postural Control } \\
\text { Motion gaming } \\
\text { Postintervention, } 8 \\
\text { weeks } \\
\text { Poor }\end{array}$ & $\begin{array}{l}1 \text { patient withdrew due to adverse event not } \\
\text { related to the intervention (external } \\
\text { exoskeleton for standing up in front of the } \\
\text { Kinect was broken, damage occurred } \\
\text { outside of the study. }\end{array}$ & Cerebral palsy & $\begin{array}{l}\text { Clinical diagnosis of CP; } \\
\text { age between } 4 \text { and } 14 \\
\text { years; level of GMFC } \\
\text { between I and IV }\end{array}$ & $\begin{array}{l}\text { Randomized: } \\
22 \\
\text { Analyzed: } 15 \\
\text { Attrition: } 31.8 \% \\
(7 / 22)\end{array}$ & $\begin{array}{l}\text { Italy } \\
\text { Outpatient clinic } \\
\text { Crossover RCT }\end{array}$ & $\begin{array}{l}\text { Government } \\
\text { Italian Ministry of } \\
\text { Health }\end{array}$ \\
\hline
\end{tabular}

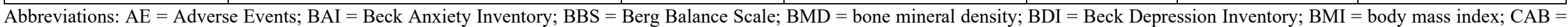

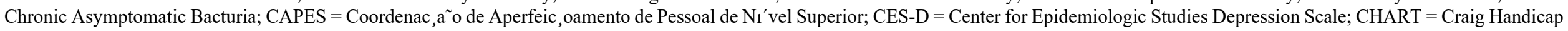

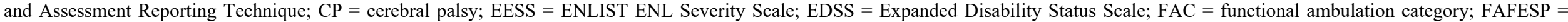

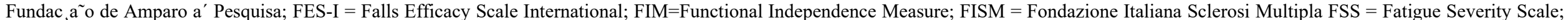

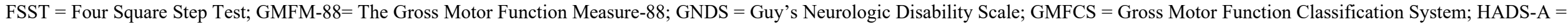

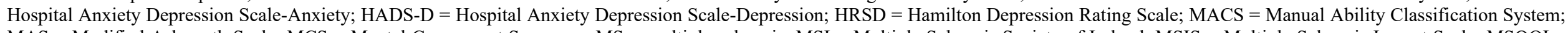

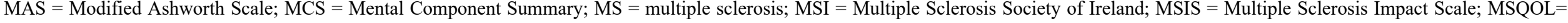

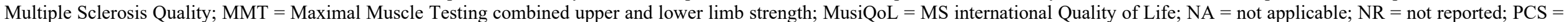

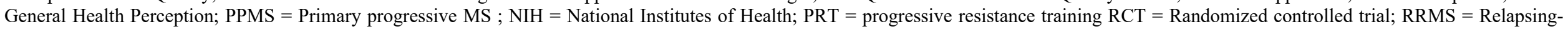

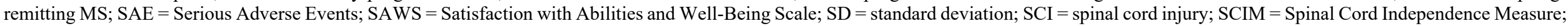

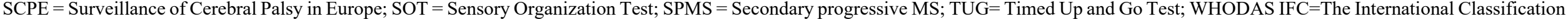
of Functioning, Disability and Health

See Appendix B. Included Studies for full study citation. 


\section{Appendix G. Quality Assessment}

Table G-1. Quality assessment of randomized controlled trials

\begin{tabular}{|c|c|c|c|c|c|c|c|c|c|}
\hline $\begin{array}{l}\text { Author, Year } \\
\text { (See Appendix B for } \\
\text { Full Citation) }\end{array}$ & $\begin{array}{l}\text { Randomization } \\
\text { Adequate }\end{array}$ & $\begin{array}{l}\text { Allocation } \\
\text { Concealment } \\
\text { Adequate }\end{array}$ & $\begin{array}{l}\text { Groups } \\
\text { Similar at } \\
\text { Baseline }(10 \% \\
\text { or Less } \\
\text { Difference) }\end{array}$ & $\begin{array}{l}\text { Outcome } \\
\text { Assessors } \\
\text { Masked }\end{array}$ & $\begin{array}{l}\text { Care Provider } \\
\text { Masked }\end{array}$ & $\begin{array}{l}\text { Patients } \\
\text { Masked }\end{array}$ & $\begin{array}{l}\text { Intent-to-Treat } \\
\text { Analysis (at } \\
\text { Least } 95 \% \\
\text { Analyzed) }\end{array}$ & $\begin{array}{l}\text { Overall Loss to } \\
\text { Followup } \\
\text { Acceptable/ } \\
\text { Differential Loss } \\
\text { to } \\
\text { Followup } \\
\text { Acceptable }\end{array}$ & Quality Rating \\
\hline Abbasi, 2019 & Yes & Unclear & Yes & Yes & No & No & Yes & Yes/Yes & Fair \\
\hline Acar, 2016 & Unclear & Unclear & No & Unclear & No & No & Yes & Yes/Yes & Poor \\
\hline Adar, 2017 & Unclear & Unclear & No & Yes & No & No & Yes & Yes/Yes & Fair \\
\hline Afrasiabifar, 2018 & Yes & Unclear & No & Yes & No & No & Yes & Yes/Yes & Good \\
\hline Ahmadi, 2013 & Unclear & Unclear & Yes & Unclear & No & No & Yes & Yes/Yes & Fair \\
\hline Ahmadizadeh, 2020 & Unclear & Unclear & Yes & Yes & Unclear & No & Yes & Yes/Yes & Fair \\
\hline Akkurt, 2017 & Unclear & Unclear & No & Yes & No & No & Yes & Yes/Yes & Fair \\
\hline Al-Sharman, 2019 & Unclear & Unclear & No & Yes & No & No & No & No/No & Poor \\
\hline Alexeeva, 2011 & Yes & Yes & No & Yes & No & No & Yes & Yes/No & Fair \\
\hline Amiri, 2019 & Unclear & Unclear & Yes & Unclear & No & No & Yes & Yes/Yes & Fair \\
\hline Aras, 2019 & Unclear & Unclear & Unclear & Unclear & No & No & Yes & Yes/Yes & Fair \\
\hline Arntzen, 2019 & Yes & Unclear & Yes & Yes & No & No & Yes & Yes/Yes & Good \\
\hline Azimzadeh, 2015 & No & \begin{tabular}{|l|} 
No; small \\
numbers
\end{tabular} & Yes & Unclear & No & No & Unclear & Yes & Poor \\
\hline Bahrami, 2019a & Unclear & Unclear & Yes & Unclear & No & No & No & Yes/Yes & Fair \\
\hline Baquet, 2018 & Yes & Unclear & No & Yes & No & No & Yes & Yes/Yes & Fair \\
\hline Brichetto, 2015 & Unclear & Yes & Yes & Yes & No & No & Yes & Yes & Good \\
\hline Bryant, 2013 & Unclear & Unclear & No & Yes & No & No & Yes & Yes/Yes & Fair \\
\hline Bulguroglu, 2017 & Unclear & Unclear & No & Yes & No & No & No & Yes/Unclear & Poor \\
\hline Cakit, 2010 & Yes & Unclear & No & Yes & No & No & Unclear & No/No & Poor \\
\hline Calabro, 2017 & Yes & Yes & Yes & Yes & No & No & Yes & Yes/Yes & Good \\
\hline Callesen, 2019 & $\begin{array}{l}\text { Unclear } \\
\text { Cluster } \\
\text { randomized }\end{array}$ & Unclear & Yes & Yes & No & No & No & $\begin{array}{l}\text { Overall, Yes (17\%) } \\
\text { Differential, No: } \\
\text { strength }(26 \%) \text { vs. } \\
\text { WL (10\%); } \\
\text { Yes: strength }(26 \%) \\
\text { vs. balance }(16 \%) \\
\text { and balance }(16 \%) \\
\text { vs. WL }(10 \%)\end{array}$ & Fair \\
\hline
\end{tabular}




\begin{tabular}{|c|c|c|c|c|c|c|c|c|c|}
\hline Chen, 2016 & Unclear & Unclear & $\begin{array}{l}\text { No for smoking } \\
\text { but had similar } \\
\text { baseline PFTs } \\
\text { and QoL } \\
\text { scores }\end{array}$ & Unclear & No & No & $\begin{array}{l}\text { Yes based on } \\
\text { SF-36 } \\
\text { answered } \\
\text { questionnaires }\end{array}$ & Yes/Yes & Fair \\
\hline Cho, 2020 & Unclear & Unclear & No & Unclear & Unclear & No & No & Yes/Yes & Poor \\
\hline Chrysagis, 2012 & Unclear & Yes & No & Yes & No & No & Yes & Yes/Yes & Fair \\
\hline Claerbout, 2012 & Unclear & No & Yes & Yes & No & Unclear & Yes & Yes/Yes & Fair \\
\hline Collett, 2011 & Yes & Unclear & $\begin{array}{l}\text { No (DMT, Leg } \\
\text { power) }\end{array}$ & Yes & No & No & Yes (imputed) & $\begin{array}{l}\text { No }(75 \% \text { completed } \\
12 \text { weeks) } \\
\text { No }(53 \%, 95 \% \text {, } \\
72 \%) \\
\end{array}$ & Poor \\
\hline Curtis, 2018 & Yes & Unclear & No - gender & Yes & No & No & No & No control lost more & Fair \\
\hline \begin{tabular}{|l|} 
Dalgas, 2009 \\
Dalgas, 2010 \\
\end{tabular} & Unclear & Unclear & Yes & No & No & No & No & Yes & Fair \\
\hline Demuth, 2012 & Unclear & Unclear & Yes & Yes & No & No & No & Yes & Fair \\
\hline Deutz, 2018 & Unclear & Unclear & Unclear & Unclear & No & No & Unclear & No $(29 \%)$ & Poor \\
\hline Dodd, 2011 & Yes & Yes & Yes & Yes & No & No & Yes LVCF & Yes & Good \\
\hline Doulatabad, 2012 & Unclear & Unclear & Unclear & Unclear & No & No & No & Unclear & Poor \\
\hline Duff, 2018 & Yes & Unclear & No & Yes & No & No & Yes & Yes & Fair \\
\hline Duffell, 2014 & Unclear & Unclear & Unclear & Unclear & No & No & Unclear & Unclear & Poor \\
\hline Ebrahimi, 2015 & Unclear & Unclear & No & Yes & No & No & No $(88 \%)$ & \begin{tabular}{|l} 
Yes/No (6\% vs. \\
$18 \%)$ \\
\end{tabular} & Poor \\
\hline Elnaggar, 2019 & Yes & Yes & No & Yes & No & No & No & Yes/Yes & Fair \\
\hline El-Shamy, 2018 & Yes & Yes & No & Yes & No & No & Yes & Yes/Yes & Fair \\
\hline Emara, 2016 & Yes & Unclear & Yes & Yes & No & No & No $(91 \%)$ & Yes & Fair \\
\hline Esclarin-Ruz, 2014 & Unclear & Unclear & No & Yes & No & No & Unclear & Yes/Yes & Fair \\
\hline $\begin{array}{l}\text { Faramarzi, } 2020 \\
\text { (Banitalebi, 2020) }\end{array}$ & Unclear & Unclear & $\begin{array}{l}\text { Unclear- } \\
\text { characteristics } \\
\text { based on } \\
\text { disability levels }\end{array}$ & No & No & No & Yes & Yes/Yes & Fair \\
\hline \begin{tabular}{|l|} 
Field-Fote, 2011 \\
(Sandler, 2017) \\
\end{tabular} & Unclear & Unclear & Yes & Yes & No & No & No & Yes & Fair \\
\hline Forsberg, 2016 & Yes & Yes & Yes & Yes & No & No & No & Yes & Fair \\
\hline Fosdahl, 2019b & Unclear & No & No & Yes & No & No & \begin{tabular}{|l} 
Yes \\
(imputation for \\
6MWT) \\
\end{tabular} & Yes & Fair \\
\hline Gandolfi, 2015 & Yes & Yes & Yes & Yes & No & No & No & Yes & Fair \\
\hline
\end{tabular}




\begin{tabular}{|c|c|c|c|c|c|c|c|c|c|}
\hline $\begin{array}{l}\text { Garrett, 2013a } \\
\text { "Exercise in the } \\
\text { community for people } \\
\text { with minimal gait..." } \\
\text { (Garrett, 2013b) }\end{array}$ & Yes & Yes & No & Yes & No & No & No & No & Poor \\
\hline Gervasoni, 2014 & Unclear & Unclear & Yes & NR & No & No & No & Yes & Fair \\
\hline $\begin{array}{l}\text { Giangregorio, } 2012 \\
\text { (Hitzig, 2013; Craven, } \\
\text { 2017;Kapadia, 2014) }\end{array}$ & Yes & Yes & Yes & Yes & No & No & Yes & No & Fair \\
\hline Gibson, 2018 & Yes & Yes & Yes & Yes & No & No & Yes & No & Good \\
\hline Gorman, 2019 & Yes & Yes & No & $\begin{array}{l}\text { No (not at both } \\
\text { sites) }\end{array}$ & No & No & Yes & Yes/Yes & Fair \\
\hline Grecco, 2013 & Unclear & Unclear & Yes & Yes & No & No & No & Yes & Fair \\
\hline Grecco, 2014 & Yes & Yes & Yes & Unclear & Unclear & No & Yes & Yes & Fair \\
\hline $\begin{array}{l}\text { Hasanpour Dehkordi, } \\
2016 \text { "Influence of..." }\end{array}$ & Yes & Unclear & Unclear & Unclear & No & No & Unclear & Unclear & Poor \\
\hline $\begin{array}{l}\text { Hasanpour-Dehkordi, } \\
2016 \text { "Effects of..." }\end{array}$ & No & No & Unclear & Yes & No & No & Unclear & Unclear & Poor \\
\hline $\begin{array}{l}\text { Hassanpour-Dehkordi, } \\
2014\end{array}$ & Unclear & Unclear & Unclear & Unclear & No & No & Unclear & Unclear & Poor \\
\hline $\begin{array}{l}\text { Hebert, } 2011 \text { (Hebert, } \\
\text { 2009) }\end{array}$ & Yes & Yes & Yes & $\begin{array}{l}\text { Yes (except } \\
\text { patient-reported) }\end{array}$ & Unclear & No & Yes & Yes & Fair \\
\hline Heine, 2017 & Yes & Yes & No & Yes & No & No & Unclear & $\begin{array}{l}2 \text { mos: Yes } \\
4 \text { mos: Yes } \\
6 \text { mos: Yes/No } \\
12 \text { mos: No } \\
\end{array}$ & Fair \\
\hline Herrero, 2012 & Yes & Yes & Yes & Yes & No & No & Yes & Yes/Yes & Fair \\
\hline Hochsprung, 2017 & Yes & Unclear & No & Yes & No & No & No & Yes/Unclear & Poor \\
\hline Hogan, 2014 & No & Unclear & No & Yes & No & No & No & No/No & Poor \\
\hline Hota, 2020 & Unclear & Unclear & No & Unclear & Unclear & No & Yes & Yes/Yes & Fair \\
\hline Hsieh, 2018 & Unclear & Unclear & Yes & Yes & No & No & Unclear & Yes/Yes & Fair \\
\hline Hsieh, 2020 & Yes & Unclear & \begin{tabular}{|l|} 
Unclear \\
\end{tabular} & Unclear & No & No & Yes & Yes/Yes & Fair \\
\hline Huang, 2015 & Unclear & Unclear & $\begin{array}{l}\text { Yes, but few } \\
\text { variables }\end{array}$ & No & No & No & Yes & Yes/Yes & Fair \\
\hline $\ln , 2018$ & Unclear & Unclear & Yes & Yes & No & No & No & Yes/Yes & Fair \\
\hline Johnston, 2011 & Yes & Unclear & $\begin{array}{l}\begin{array}{l}\text { Unclear (some } \\
\text { differences) }\end{array} \\
\end{array}$ & $\begin{array}{l}\text { Unclear (2 sites } \\
\text { yes, } 1 \text { site no) }\end{array}$ & No & No & No & No $(23 \%) /$ Yes & Fair \\
\hline $\begin{array}{l}\text { Jones, } 2014 a \text { "...results } \\
\text { from a randomized } \\
\text { clinical trial" }\end{array}$ & Unclear & Unclear & $\begin{array}{l}\text { No (several } \\
\text { differences) }\end{array}$ & No & No & No & Unclear & Unclear & Poor \\
\hline Jonsdottir, 2018 & Yes & Yes & No & Yes & No & No & Yes & Yes & Fair \\
\hline Jung, 2014 & Unclear & Unclear & $\begin{array}{l}\text { Unclear (some } \\
\text { differences) }\end{array}$ & No & No & No & Yes & Yes/Yes & Fair \\
\hline
\end{tabular}




\begin{tabular}{|c|c|c|c|c|c|c|c|c|c|}
\hline Kalron, 2016 & Unclear & Yes & Yes & Unclear & Unclear & No & $\begin{array}{l}\text { Yes }(6.6 \% \\
\text { missing })\end{array}$ & Yes/Yes & Fair \\
\hline Kalron, 2017 & Yes & Yes & Yes & $\begin{array}{l}\text { Yes (except } \\
\text { patient-reported) }\end{array}$ & Unclear & No & No & Yes & Fair \\
\hline \begin{tabular}{|l|} 
Kara, 2020 \\
\end{tabular} & Yes & Unclear & Yes & Yes & No & No & No & Yes/Yes & Fair \\
\hline Kargarfard, 2018 & Yes & Unclear & Yes & Unclear & No & No & Yes (LOCF) & Yes & Fair \\
\hline Kaya Kara, 2019 & Yes & Unclear & Yes & Yes & No & No & Yes & Yes & Fair \\
\hline Kerling, 2015 & Unclear & Yes & No & $\begin{array}{l}\text { Yes (primary } \\
\text { outcomes } \\
\text { objective) }\end{array}$ & No & No & Yes (LOCF) & No/Yes & Fair \\
\hline Khalil, 2018 & Unclear & Unclear & No & Yes & No & No & No & Yes & Fair \\
\hline Kim, 2015 & Yes & Yes & No & Unclear & No & No & Unclear & Unclear & Fair \\
\hline Kjolhede, 2016 & Unclear & Unclear & Yes & No & No & No & No & Yes & Fair \\
\hline Klobucka, 2020 & Unclear & Unclear & No & NR & No & No & \begin{tabular}{|l} 
Yes \\
(immediately \\
after \\
treatment); at \\
3-4 month LTF \\
only RAGT \\
reported \\
\end{tabular} & $\begin{array}{l}\text { Yes (immediately } \\
\text { post treatment); } \\
\text { No 3-4 month 24\% } \\
\text { LTF in RAGT }\end{array}$ & Poor \\
\hline Kooshiar, 2015 & No & No & Yes & Unclear & No & No & Yes & Yes/Yes & Fair \\
\hline Kressler, 2013 & Unclear & Unclear & $\begin{array}{l}\text { Unclear (no } \\
\text { demographics) }\end{array}$ & Unclear & No & No & Yes & Unclear & Fair \\
\hline Kumru, 2016 & Unclear & Unclear & Yes & Yes & No & Yes & Yes & Yes/Yes & Fair \\
\hline Kwon, 2015 & Yes & Yes & Yes & Yes & No & No & Yes & Yes/Yes & Good \\
\hline Lavado, 2013 & Yes & No & Unclear & Yes & No & No & Yes & Yes/Yes & Fair \\
\hline Lee, 2013 & Unclear & Unclear & Yes (limited) & Yes & No & No & Yes & Unclear & Fair \\
\hline Lee, 2014 & Unclear & Unclear & Yes (limited) & Unclear & No & No & Yes & Unclear & Poor \\
\hline Liu, 2019 & $\begin{array}{l}\text { Unclear } \\
\text { Unclear }\end{array}$ & $\begin{array}{l}\text { Unclear } \\
\text { Unclear } \\
\end{array}$ & \begin{tabular}{|l|} 
No \\
Yes \\
\end{tabular} & $\begin{array}{l}\text { Unclear } \\
\text { Unclear } \\
\end{array}$ & $\begin{array}{l}\text { No } \\
\text { No } \\
\end{array}$ & \begin{tabular}{|l|} 
No \\
No \\
\end{tabular} & $\begin{array}{l}\text { No } \\
\text { No } \\
\end{array}$ & \begin{tabular}{|l|} 
Overall: No \\
Differential: Yes No
\end{tabular} & Fair \\
\hline Lucena-Anton, 2018 & Yes & Unclear & Yes & Yes & No & No & Yes & Yes/Yes & Fair \\
\hline Makhov, 2018 & Unclear & Unclear & Unclear & Unclear & No & No & Unclear & Unclear & Poor \\
\hline $\begin{array}{l}\text { Marandi, 2013a "A } \\
\text { comparison of } 12 \\
\text { weeks..." } \\
\end{array}$ & Unclear & Unclear & No & Unclear & No & No & No $(79 \%)$ & No $(21 \%)$, Yes & Poor \\
\hline $\begin{array}{l}\text { Marandi, } 2013 \mathrm{~b} \text { "A } \\
\text { comparison between } \\
\text { pilates..." }\end{array}$ & Unclear & Unclear & No & Unclear & No & No & No & No & Poor \\
\hline \begin{tabular}{|l|} 
Mogharnasi, 2019 \\
\end{tabular} & Unclear & Unclear & Yes & Unclear & No & No & Yes & Yes & Poor \\
\hline Moraes, 2020 & Unclear & Unclear & Yes & Yes & No & No & Yes & Yes, Yes & Fair \\
\hline
\end{tabular}




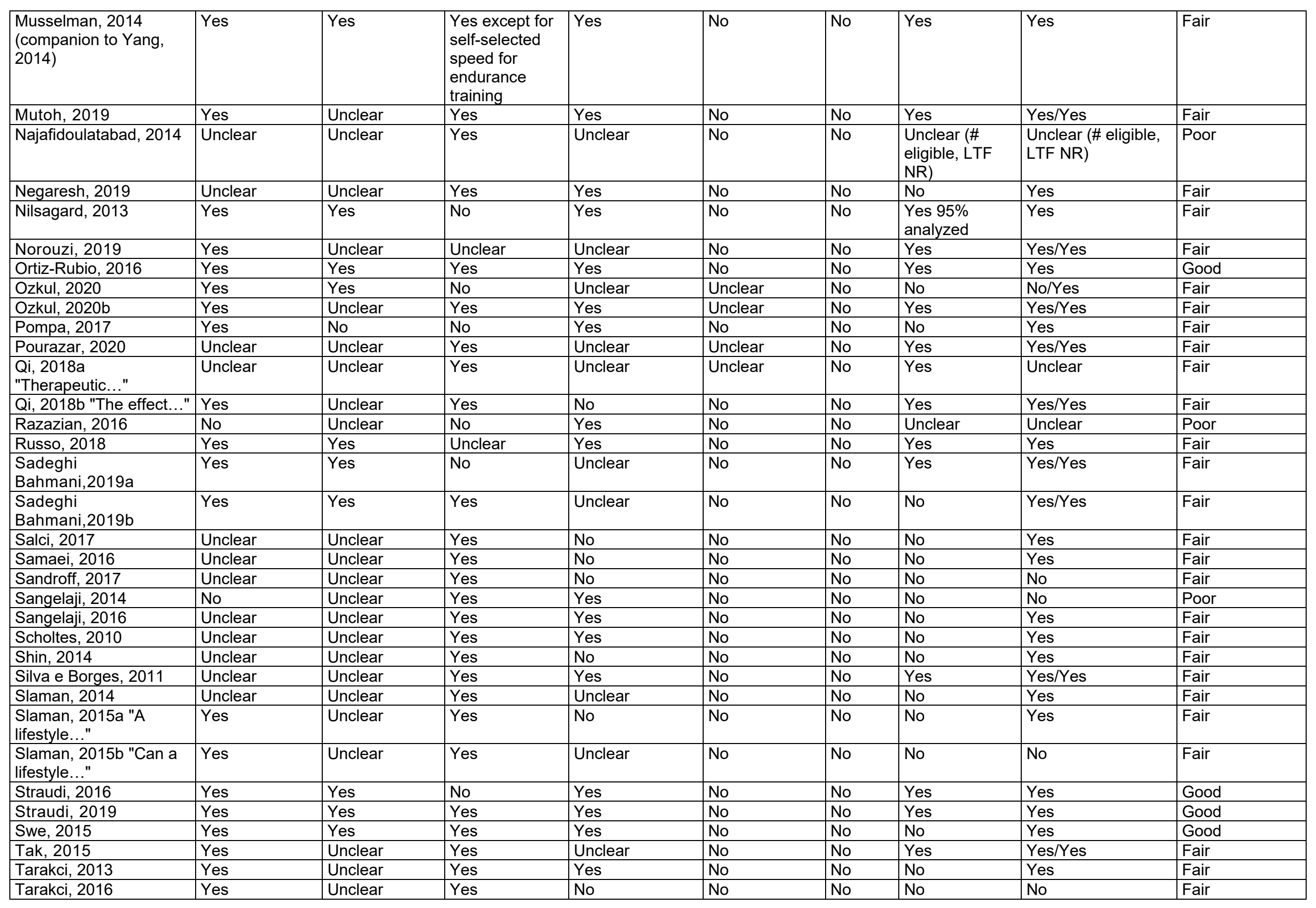




\begin{tabular}{|c|c|c|c|c|c|c|c|c|c|}
\hline $\begin{array}{l}\text { Taylor, } 2013 \text { and Bania, } \\
2016\end{array}$ & Yes & Yes & Yes & Yes & No & No & No & Yes & Good \\
\hline Tedla, 2014 & Unclear & Unclear & Unclear & No & No & No & Yes & Yes (97\%) & Poor \\
\hline Teixeira-Machado, 2017 & Yes & Unclear & Yes & Unclear & Unclear & No & Yes & Yes/Yes & Fair \\
\hline Tollar, 2020 & Yes & Unclear & No & Yes & Unclear & No & Yes & Yes/Yes & Fair \\
\hline $\begin{array}{l}\text { Totosy de Zepetnek, } \\
2015\end{array}$ & Yes & Unclear & No & Unclear & No & No & No & No/Yes & Fair \\
\hline van der Scheer, 2016 & Unclear & Unclear & $\begin{array}{l}\text { No (time since } \\
\text { injury) }\end{array}$ & No & No & No & Yes & Yes/Yes & Fair \\
\hline $\begin{array}{l}\text { Van Wely, 2014a, } \\
\text { 2014b }\end{array}$ & Yes & Yes & No for gender & Unclear & No & No & Yes & Yes/Yes & Fair \\
\hline Vermohlen, 2018 & Yes & Yes & Yes & \begin{tabular}{|l|} 
Yes (except \\
patient-reported)
\end{tabular} & Unclear & No & Yes & Yes & Fair \\
\hline Wallard, 2017 & Unclear & Unclear & Yes & Yes & No & No & Unclear & Unclear (NR) & Poor \\
\hline Wallard, 2018 & Unclear (NR) & Unclear & $\begin{array}{l}\text { Unclear (few } \\
\text { variables } \\
\text { reported) }\end{array}$ & Unclear & No & No & Unclear & Unclear (NR) & Poor \\
\hline $\begin{array}{l}\text { Wens, 2015a } \\
\text { "Impact..." }\end{array}$ & Unclear & Unclear & No & Yes & No & No & Unclear & Unclear & Poor \\
\hline Williams, 2020 & Yes & Yes & No & Yes & No & No & Yes & Yes/Yes & Fair \\
\hline Willoughby, 2010 & Yes & Yes & Yes & Yes & No & No & No & No $(24 \%) / Y e s$ & Fair \\
\hline Wu, 2017a "Robotic..." & Yes & Unclear & \begin{tabular}{|l|} 
Unclear \\
(numerous but \\
small \\
differences \\
favoring one \\
group) \\
\end{tabular} & Unclear & Unclear & No & $\begin{array}{l}\text { No }(13 \% \\
\text { missing) }\end{array}$ & Yes & Fair \\
\hline Wu, 2017b "Effects..." & Unclear & Yes & Yes & No & No & No & Yes & Yes/Yes & Fair \\
\hline Yang, 2014 & Unclear & Unclear & Yes & Yes & No & No & No & Yes/Yes & Fair \\
\hline Yazgan, 2020 & Yes & Unclear & No & No & No & No & No & Yes//No & Fair \\
\hline Yildirim, 2019 & No & No & Yes & Unclear & No & No & Yes & Yes & Fair \\
\hline Young, 2019 & Yes & Yes & Yes & Yes & No & No & Yes & No $(25 \%) /$ Yes & Fair \\
\hline Zoccolillo, 2015 & Unclear & Unclear & Unclear & No & No & No & No & No/No (41\%)/Yes & Poor \\
\hline
\end{tabular}


Table G-2. Quality assessment of quasiexperimental studies

\begin{tabular}{|c|c|c|c|c|c|c|c|c|c|}
\hline $\begin{array}{l}\text { Author, Year } \\
\text { (See Appendix B } \\
\text { for Full Citation) }\end{array}$ & $\begin{array}{l}\text { Did the Study } \\
\text { Attempt To Enroll a } \\
\text { Random Sample or } \\
\text { Consecutive } \\
\text { Patients Meeting } \\
\text { Inclusion Criteria } \\
\text { (Inception Cohort)? }\end{array}$ & $\begin{array}{l}\text { Were the } \\
\text { Groups } \\
\text { Comparable } \\
\text { at Baseline? }\end{array}$ & $\begin{array}{l}\text { Did the Study } \\
\text { Use Accurate } \\
\text { Methods for } \\
\text { Ascertaining } \\
\text { Exposures, } \\
\text { Potential } \\
\text { Confounders, } \\
\text { and Outcomes? }\end{array}$ & \begin{tabular}{|l} 
Were Outcome \\
Assessors \\
and/or Data \\
Analysts \\
Blinded to \\
Treatment? \\
\end{tabular} & \begin{tabular}{|l} 
Did the \\
Article \\
Report \\
Attrition? \\
\end{tabular} & $\begin{array}{l}\text { Did the Study } \\
\text { Perform } \\
\text { Appropriate } \\
\text { Statistical } \\
\text { Analyses on } \\
\text { Potential } \\
\text { Confounders? }\end{array}$ & $\begin{array}{l}\text { Overall Loss } \\
\text { to Followup } \\
\text { Acceptable? } \\
\text { Differential } \\
\text { Loss to } \\
\text { Followup } \\
\text { Acceptable? }\end{array}$ & $\begin{array}{l}\text { Were Outcomes } \\
\text { Prespecified and } \\
\text { Defined, and } \\
\text { Ascertained } \\
\text { Using Accurate } \\
\text { Methods? } \\
\end{array}$ & $\begin{array}{l}\text { Quality } \\
\text { Rating }\end{array}$ \\
\hline Aviram, 2017 & Unclear & No & Yes & No & Yes & No & Yes & Yes & Fair \\
\hline Bleyenheuft, 2017 & $\begin{array}{l}\text { No } \\
\text { (quasiexperimental) }\end{array}$ & No & Yes & $\begin{array}{l}\text { Yes (2 } \\
\text { outcomes) }\end{array}$ & No & No & Yes & Yes/Yes & Poor \\
\hline Burschka, 2014 & Unclear & Unclear & Yes & Unclear & Unclear & Unclear & No & Yes & Poor \\
\hline Kara, 2017 & Unclear & Unclear & Unclear & Yes & Yes & No & \begin{tabular}{|l|} 
No \\
$(36 \%) /(63 \%)$
\end{tabular} & Yes & Poor \\
\hline Keser, 2011 & Unclear & Yes & Yes & Unclear & No & No & Unclear & Yes & Poor \\
\hline Kirk, 2016 & No & unclear & Yes & No & No & No & Unclear & Yes & Poor \\
\hline Kwon, 2011 & No & $\begin{array}{l}\text { Yes (limited } \\
\text { variables) }\end{array}$ & Yes & Yes & No & \begin{tabular}{|l|} 
Unclear; at least \\
they did repeated \\
measures \\
analysis \\
\end{tabular} & Unclear & Yes & Fair \\
\hline Lai, 2010 & No & \begin{tabular}{|l|} 
No; small \\
numbers
\end{tabular} & Yes & Yes & Yes & Yes; ANCOVA & Yes & Yes & Fair \\
\hline Lorentzen, 2015 & No & $\begin{array}{l}\text { No; n's in Gr } \\
1=34, \mathrm{Gr} \\
2=12 ; \text { diff \% } \\
\text { but small \#s }\end{array}$ & Yes & Unclear & No & $\begin{array}{l}\text { Unclear; model } \\
\text { appears to focus } \\
\text { on interaction; } \\
\text { they do correct } \\
\text { for multiple } \\
\text { comparisons. }\end{array}$ & Unclear & Yes & Poor \\
\hline $\begin{array}{l}\text { Matusiak- } \\
\text { Wieczorek, } 2016\end{array}$ & No & Yes & No & No & Yes & No & Yes & Yes & Poor \\
\hline Niwald, 2017 & Unclear & Yes & Yes & No & $\begin{array}{l}\text { No, but } \\
\text { same } \\
\text { number } \\
\text { enrolled } \\
\text { reported } \\
\text { for } \\
\text { outcomes } \\
\end{array}$ & No & NR & Yes & Fair \\
\hline $\begin{array}{l}\text { Nsenga Leunkeu, } \\
2012\end{array}$ & $\begin{array}{l}\text { No (convenience } \\
\text { sample) }\end{array}$ & Unclear & Yes & No & Yes & No & Yes/Unclear & Yes & Fair \\
\hline Nsenga, 2013 & No & Yes & Yes & Unclear & Yes & Yes & Yes & Yes & Fair \\
\hline Peri, 2017 & Unclear & No & Unclear & Unclear & No & No & Unclear & Yes & Poor \\
\hline Roppolo, 2013 & Unclear & Yes & Yes & Unclear & Yes & Yes & Unclear & Yes & Fair \\
\hline
\end{tabular}


Table G-3. Quality assessment of cohort studies

\begin{tabular}{|c|c|c|c|c|c|c|c|c|c|}
\hline $\begin{array}{l}\text { Author, Year } \\
\text { (See Appendix B } \\
\text { for Full Citation) }\end{array}$ & $\begin{array}{l}\text { Did the Study } \\
\text { Attempt To Enroll a } \\
\text { Random Sample or } \\
\text { Consecutive } \\
\text { Patients Meeting } \\
\text { Inclusion Criteria } \\
\text { (Inception Cohort)? }\end{array}$ & $\begin{array}{l}\text { Were the } \\
\text { Groups } \\
\text { Comparable } \\
\text { at Baseline? }\end{array}$ & $\begin{array}{l}\text { Did the Study } \\
\text { Use Accurate } \\
\text { Methods for } \\
\text { Ascertaining } \\
\text { Exposures, } \\
\text { Potential } \\
\text { Confounders, } \\
\text { and Outcomes? }\end{array}$ & $\begin{array}{l}\text { Were Outcome } \\
\text { Assessors } \\
\text { and/or Data } \\
\text { Analysts } \\
\text { Blinded to } \\
\text { Treatment? } \\
\end{array}$ & \begin{tabular}{|l} 
Did the \\
Article \\
Report \\
Attrition?
\end{tabular} & $\begin{array}{l}\text { Did the Study } \\
\text { Perform } \\
\text { Appropriate } \\
\text { Statistical } \\
\text { Analyses on } \\
\text { Potential } \\
\text { Confounders? }\end{array}$ & $\begin{array}{l}\text { Overall Loss } \\
\text { to Followup } \\
\text { Acceptable? } \\
\text { Differential } \\
\text { Loss to } \\
\text { Followup } \\
\text { Acceptable? }\end{array}$ & $\begin{array}{l}\text { Were Outcomes } \\
\text { Prespecified and } \\
\text { Defined, and } \\
\text { Ascertained } \\
\text { Using Accurate } \\
\text { Methods? } \\
\end{array}$ & $\begin{array}{l}\text { Quality } \\
\text { Rating }\end{array}$ \\
\hline Harness, 2008 & Yes & Yes & Yes & No & Yes & No & Yes/Yes & Yes & Fair \\
\hline Kim, 2017 & Unclear & No & Yes & Unclear & No & No & Unclear & Yes & Poor \\
\hline Lai, 2015 & No & \begin{tabular}{|l|} 
No; small \\
numbers
\end{tabular} & Yes & Yes & Yes & Yes; ANCOVA & Yes & Yes & Fair \\
\hline Park, 2014 & No & No & Yes & Unclear & Yes & No & $\begin{array}{l}\text { Yes (17\%), No } \\
\text { (24\% vs. } 0 \%)\end{array}$ & Yes & Poor \\
\hline Sadowsky, 2013 & Unclear & No & Yes & Unclear & No & No & Unclear & Yes & Poor \\
\hline Yazici, 2019 & No & Unclear & Yes & Unclear & Yes & No & Yes/No & Yes & Poor \\
\hline Valent, 2010 & Unclear & Yes & Yes & Unclear & Yes & No & Yes/unclear & Yes & Fair \\
\hline
\end{tabular}




\section{Appendix H. Strength of Evidence}

Table H-1. Strength of evidence for Key Question 2: aerobic exercise for multiple sclerosis

\begin{tabular}{|c|c|c|c|c|c|c|c|c|c|}
\hline $\begin{array}{l}\text { Intervention } \\
\text { Category, } \\
\text { Intervention }\end{array}$ & Comparator & Outcome & $\begin{array}{l}\text { Number of Studies } \\
\text { (Participants) } \\
\text { Author Year } \\
\text { (See Appendix B for } \\
\text { Full Citation) }\end{array}$ & $\begin{array}{l}\text { Study } \\
\text { Limitations }\end{array}$ & Consistency & Precision & $\begin{array}{l}\text { Reporting } \\
\text { Bias }\end{array}$ & $\begin{array}{l}\text { Strength of } \\
\text { Evidence }\end{array}$ & $\begin{array}{l}\text { Findings, Direction and } \\
\text { Magnitude of Effect }\end{array}$ \\
\hline $\begin{array}{l}\text { Aerobic } \\
\text { Exercise } \\
\text { Aerobics }\end{array}$ & $\begin{array}{l}\text { Home } \\
\text { exercise } \\
\text { with DVD or } \\
\text { Attention } \\
\text { control }\end{array}$ & Sleep & $\begin{array}{l}2(\mathrm{~N}=77) \\
\text { Al-Sharman, } 2019 \\
\text { Sadeghi Bahmani, } \\
2019 \mathrm{~b}\end{array}$ & Moderate & Consistent & Imprecise & Undetected & $\begin{array}{l}\text { Low for } \\
\text { benefit }\end{array}$ & $\begin{array}{l}\text { PSQI: } 8.0(3.8) \text { to } 4.6(2.3) \\
\text { vs. } 8.9(4.3) \text { to } 7.1(3.2), \\
\text { p<0.001 } \\
\text { Pooled ISI: } 3.33,95 \% \mathrm{Cl} \\
\text { 1.03 to } 5.64, p=0.005 \\
\text { Total Sleep Time: } 333.38 \\
\text { (84.6) to } 372.4(59.4) \text { vs. } \\
325.9(84.5) \text { to } 320(54), \\
p=0.05\end{array}$ \\
\hline $\begin{array}{l}\text { Aerobic } \\
\text { Exercise } \\
\text { Aerobics }\end{array}$ & $\begin{array}{l}\text { Attention } \\
\text { control }\end{array}$ & Function & $\begin{array}{l}1(\mathrm{~N}=47) \\
\text { Sadeghi Bahmani, } \\
2019 \mathrm{~b}\end{array}$ & Moderate & Unknown & Imprecise & Undetected & Insufficient & $\begin{array}{l}\text { EDSS: } 2.46(1.50) \text { to } 2.27 \\
\text { (1.64) vs. } 2.02(1.84) \text { to } \\
1.98(1.70), p>0.05\end{array}$ \\
\hline $\begin{array}{l}\text { Aerobic } \\
\text { Exercise } \\
\text { Aerobics }\end{array}$ & Pilates & Function & $\begin{array}{l}1(\mathrm{~N}=55) \\
\text { Kara, 2017 }\end{array}$ & High & Unknown & Imprecise & Undetected & Insufficient & $\begin{array}{l}\text { TUG right: MD }-0.47,95 \% \\
\mathrm{Cl}-2.975 \text { to } 2.035, p=0.71 \\
\frac{\text { TUG left: }}{\mathrm{Cl}-6.341 \text { to }-3.07,95 \%}, \mathrm{p}=0.07\end{array}$ \\
\hline $\begin{array}{l}\text { Aerobic } \\
\text { Exercise } \\
\text { Aerobics } \\
\end{array}$ & Pilates & Balance & $\begin{array}{l}1(\mathrm{~N}=55) \\
\text { Kara, 2017 }\end{array}$ & High & Unknown & Imprecise & Undetected & Insufficient & $\begin{array}{l}\text { BBS: MD }-0.67,95 \% \mathrm{Cl}- \\
10.56 \text { to } 9.22, p=0.89\end{array}$ \\
\hline $\begin{array}{l}\text { Aerobic } \\
\text { Exercise } \\
\text { Aerobics } \\
\end{array}$ & $\begin{array}{l}\text { Neuro- } \\
\text { rehabilitation }\end{array}$ & Function & $\begin{array}{l}1(\mathrm{~N}=30) \\
\text { Keser, } 2011\end{array}$ & High & Unknown & Imprecise & Undetected & Insufficient & $\begin{array}{l}\text { MSFC: MD }-0.002(0.44) \\
\text { vs. } 0.02(0.23), p>0.05\end{array}$ \\
\hline $\begin{array}{l}\text { Aerobic } \\
\text { Exercise } \\
\text { Aerobics } \\
\end{array}$ & $\begin{array}{l}\text { Neuro- } \\
\text { rehabilitation }\end{array}$ & $\begin{array}{l}\text { Quality of } \\
\text { Life }\end{array}$ & $\begin{array}{l}1(\mathrm{~N}=30) \\
\text { Keser, } 2011\end{array}$ & High & Unknown & Imprecise & Undetected & Insufficient & $\begin{array}{l}\text { SF-36 total: MD } 0.20 \\
\text { (5.67) vs. } 1.73(7.75) \\
p>0.05\end{array}$ \\
\hline $\begin{array}{l}\text { Aerobic } \\
\text { Exercise } \\
\text { Aerobics } \\
\end{array}$ & $\begin{array}{l}\text { Neuro- } \\
\text { rehabilitation }\end{array}$ & Balance & $\begin{array}{l}1(\mathrm{~N}=30) \\
\text { Keser, } 2011\end{array}$ & High & Unknown & Imprecise & Undetected & Insufficient & $\begin{array}{l}\text { BBS: MD -1.73 (3.03) vs. - } \\
1.80(2.67), p>0.05\end{array}$ \\
\hline
\end{tabular}




\begin{tabular}{|c|c|c|c|c|c|c|c|c|c|}
\hline $\begin{array}{l}\text { Intervention } \\
\text { Category, } \\
\text { Intervention }\end{array}$ & Comparator & Outcome & $\begin{array}{l}\text { Number of Studies } \\
\text { (Participants) } \\
\text { Author Year } \\
\text { (See Appendix B for } \\
\text { Full Citation) }\end{array}$ & $\begin{array}{l}\text { Study } \\
\text { Limitations }\end{array}$ & Consistency & Precision & $\begin{array}{l}\text { Reporting } \\
\text { Bias }\end{array}$ & $\begin{array}{l}\text { Strength of } \\
\text { Evidence }\end{array}$ & $\begin{array}{l}\text { Findings, Direction and } \\
\text { Magnitude of Effect }\end{array}$ \\
\hline $\begin{array}{l}\text { Aerobic } \\
\text { Exercise } \\
\text { Aquatics }\end{array}$ & $\begin{array}{l}\text { Usual care, } \\
\text { previous } \\
\text { activity level } \\
\text { or attention } \\
\text { control }\end{array}$ & Function & $\begin{array}{l}1(\mathrm{~N}=32) \\
\text { Kargarfard, } 2018\end{array}$ & Moderate & Unknown & Imprecise & Undetected & Insufficient & $\begin{array}{l}\text { Mean (SD) } \\
\text { 6MWT: } \\
451(58) \text { vs. } 447(30) \\
\text { (baseline) } \\
503(57) \text { vs. } 418(29) \\
\text { (postintervention) } \\
\text { Between group difference } \\
\text { p<0.001 } \\
\text { Sit to Stand: } \\
21.0(5.7) \text { vs. } 21.4(4.7) \\
\text { (baseline) } \\
16.8(5.1) \text { vs. } 27.3(4.8) \\
\text { (postintervention) } \\
\text { Between group difference } \\
\text { p<0.001 }\end{array}$ \\
\hline $\begin{array}{l}\text { Aerobic } \\
\text { Exercise } \\
\text { Aquatics }\end{array}$ & $\begin{array}{l}\text { Ai-Chi } \\
\text { exercises on } \\
\text { mat }\end{array}$ & ADLs & $\begin{array}{l}1(\mathrm{~N}=73) \\
\text { Castro-Sanchez, } 2012\end{array}$ & Low & Unknown & Imprecise & Undetected & $\begin{array}{l}\text { Low for } \\
\text { benefit }\end{array}$ & $\begin{array}{l}\text { MSIS-29 Physical: } 48 \\
(15.91) \text { to } 41(12.37) \text { vs. } \\
46(18.34) \text { to } 45(17.14), \\
\text { p=0.014 } \\
\text { MSIS-29 Psychological: } \\
34(29.47) \text { to } 21(15.73) \\
\text { vs. } 30(23.53) \text { to } 25 \\
(19.36), p=0.023 \\
\text { Differences in MSIS-29 } \\
\text { maintained at } 30 \text { weeks } \\
\text { Barthel Index: } 91(7.12) \text { to } \\
86(9.23) \text { vs. } 87(10.34) \text { to } \\
88(8.92), p>0.05\end{array}$ \\
\hline
\end{tabular}




\begin{tabular}{|c|c|c|c|c|c|c|c|c|c|}
\hline $\begin{array}{l}\text { Intervention } \\
\text { Category, } \\
\text { Intervention }\end{array}$ & Comparator & Outcome & $\begin{array}{l}\text { Number of Studies } \\
\text { (Participants) } \\
\text { Author Year } \\
\text { (See Appendix B for } \\
\text { Full Citation) } \\
\end{array}$ & $\begin{array}{l}\text { Study } \\
\text { Limitations }\end{array}$ & Consistency & Precision & $\begin{array}{l}\text { Reporting } \\
\text { Bias }\end{array}$ & $\begin{array}{l}\text { Strength of } \\
\text { Evidence }\end{array}$ & $\begin{array}{l}\text { Findings, Direction and } \\
\text { Magnitude of Effect }\end{array}$ \\
\hline $\begin{array}{l}\text { Aerobic } \\
\text { Exercise } \\
\text { Aquatics }\end{array}$ & $\begin{array}{l}\text { Usual care, } \\
\text { previous } \\
\text { activity level } \\
\text { or attention } \\
\text { control }\end{array}$ & Balance & $\begin{array}{l}2(\mathrm{~N}=62) \\
\text { Kargarfard, } 2018 \\
\text { Marandi, } 2013\end{array}$ & Moderate & Consistent & Imprecise & Undetected & $\begin{array}{l}\text { Low for } \\
\text { benefit }\end{array}$ & $\begin{array}{l}\text { Six Spot Step Test, N=30: } \\
\text { Right leg dynamic } \\
\text { balance: } 8.57 \text { (3.64) vs. } \\
10.64(4.17) \text { (baseline) } \\
6.40(1.82) \text { vs. } 12.65 \\
\text { (6.05) (post-intervention) } \\
\text { Adjusted MD }-5.88 \text { (SE } \\
1.4), p<0.001 \\
\text { Left leg dynamic balance: } \\
9.12(4.31) \text { vs. } 10.16 \\
(3.76) \text { (baseline) } 6.26 \\
(1.95) \text { vs. } 12.49(4.63) \\
\text { (post-intervention) } \\
\text { Adjusted MD }-6.23 \text { (SE } \\
1.2), p<0.001 \\
\text { BBS, N=32 } \\
53.6(1.7) \text { vs. } 52.3(3.3) \\
\text { (baseline) } \\
55.2(1.2) \text { vs. } 50.2(4.6) \\
\text { (post-intervention) } \\
\text { Between group difference } \\
\text { p<0.001 }\end{array}$ \\
\hline $\begin{array}{l}\text { Aerobic } \\
\text { Exercise } \\
\text { Aquatics }\end{array}$ & $\begin{array}{l}\text { Usual care, } \\
\text { previous } \\
\text { activity level } \\
\text { or attention } \\
\text { control }\end{array}$ & $\begin{array}{l}\text { Female } \\
\text { Sexual } \\
\text { Function } \\
\text { Index }\end{array}$ & $\begin{array}{l}1(60) \\
\text { Bahmani, } 2020\end{array}$ & Moderate & Unknown & Imprecise & Undetected & $\begin{array}{l}\text { Low for } \\
\text { benefit }\end{array}$ & $\begin{array}{l}\text { Exercise } 2 \text { x/week vs. } \\
\text { 3x/week vs. active control: } \\
\text { FSFI: } 52.14 \text { vs. } 48.80 \text { vs. } \\
42.80, p<0.001\end{array}$ \\
\hline
\end{tabular}




\begin{tabular}{|c|c|c|c|c|c|c|c|c|c|}
\hline $\begin{array}{l}\text { Intervention } \\
\text { Category, } \\
\text { Intervention }\end{array}$ & Comparator & Outcome & $\begin{array}{l}\text { Number of Studies } \\
\text { (Participants) } \\
\text { Author Year } \\
\text { (See Appendix B for } \\
\text { Full Citation) }\end{array}$ & $\begin{array}{l}\text { Study } \\
\text { Limitations }\end{array}$ & Consistency & Precision & $\begin{array}{l}\text { Reporting } \\
\text { Bias }\end{array}$ & $\begin{array}{l}\text { Strength of } \\
\text { Evidence }\end{array}$ & $\begin{array}{l}\text { Findings, Direction and } \\
\text { Magnitude of Effect }\end{array}$ \\
\hline $\begin{array}{l}\text { Aerobic } \\
\text { Exercise } \\
\text { Cycling }\end{array}$ & $\begin{array}{l}\text { Usual care, } \\
\text { previous } \\
\text { activity level } \\
\text { or attention } \\
\text { control }\end{array}$ & Function & $\begin{array}{l}6(\mathrm{n}=277) \\
\text { Negaresh, } 2018 \\
\text { Hochsprung, } 2017 \\
\text { Baquet, 2018 } \\
\text { Hebert, 2011 } \\
\text { Tollar, 2020 } \\
\text { Heine, 2017 }\end{array}$ & Moderate & Consistent & Imprecise & Undetected & $\begin{array}{l}\text { Low-strength } \\
\text { evidence of } \\
\text { no clear } \\
\text { benefit }\end{array}$ & 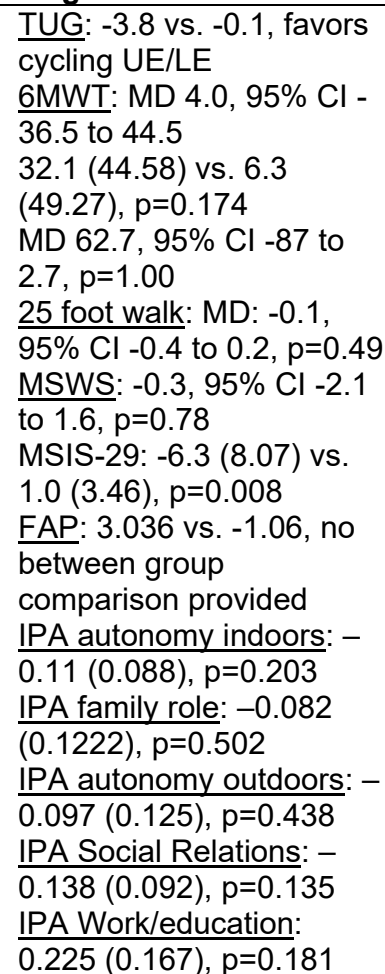 \\
\hline $\begin{array}{l}\text { Aerobic } \\
\text { Exercise } \\
\text { Cycling }\end{array}$ & $\begin{array}{l}\text { Usual care, } \\
\text { previous } \\
\text { activity level } \\
\text { or attention } \\
\text { control }\end{array}$ & $\begin{array}{l}\text { Quality of } \\
\text { Life }\end{array}$ & $\begin{array}{l}2(n=94) \\
\text { Baquet, } 2018 \\
\text { Tollar, } 2020\end{array}$ & Moderate & Inconsistent & Imprecise & Undetected & Insufficient & $\begin{array}{l}\text { HAQUAMS: }-0.4,95 \% \mathrm{Cl}- \\
4.5 \text { to } 3.7, p=0.84 \\
\text { EQ-5 Sum Score: }-1.4 \\
(1.7) \text { vs. } 0.0(1.13) \\
p=0.023\end{array}$ \\
\hline $\begin{array}{l}\text { Aerobic } \\
\text { Exercise } \\
\text { Cycling }\end{array}$ & $\begin{array}{l}\text { Usual care, } \\
\text { previous } \\
\text { activity level } \\
\text { or attention } \\
\text { control }\end{array}$ & ADLs & $\begin{array}{l}1(89) \\
\text { Heine, } 2017\end{array}$ & Moderate & Unknown & Imprecise & Undetected & Insufficient & $\begin{array}{l}\text { IPA: Autonomy indoors: - } \\
0.11(0.088), p=0.203 \\
\text { Family role: }-0.082 \\
(0.1222), p=0.502 \\
\text { Autonomy outdoors: }- \\
0.097(0.125), p=0.438 \\
\text { Social Relations: }-0.138 \\
(0.092), p=0.135 \\
\text { Work/education: } 0.225 \\
(0.167), p=0.181\end{array}$ \\
\hline
\end{tabular}




\begin{tabular}{|c|c|c|c|c|c|c|c|c|c|}
\hline $\begin{array}{l}\text { Intervention } \\
\text { Category, } \\
\text { Intervention }\end{array}$ & Comparator & Outcome & $\begin{array}{l}\text { Number of Studies } \\
\text { (Participants) } \\
\text { Author Year } \\
\text { (See Appendix B for } \\
\text { Full Citation) }\end{array}$ & $\begin{array}{l}\text { Study } \\
\text { Limitations }\end{array}$ & Consistency & Precision & $\begin{array}{l}\text { Reporting } \\
\text { Bias }\end{array}$ & $\begin{array}{l}\text { Strength of } \\
\text { Evidence }\end{array}$ & $\begin{array}{l}\text { Findings, Direction and } \\
\text { Magnitude of Effect }\end{array}$ \\
\hline $\begin{array}{l}\text { Aerobic } \\
\text { Exercise } \\
\text { Cycling }\end{array}$ & $\begin{array}{l}\text { Usual care, } \\
\text { previous } \\
\text { activity level } \\
\text { or attention } \\
\text { control }\end{array}$ & Balance & $\begin{array}{l}1(26) \\
\text { Tollar, } 2020\end{array}$ & Moderate & Unknown & Imprecise & Undetected & Insufficient & $\begin{array}{l}\text { BBS: } 2.5(2.62) \text { vs. }-0.2 \\
(2.62), p=0.015\end{array}$ \\
\hline $\begin{array}{l}\text { Aerobic } \\
\text { Exercise } \\
\text { Robot- } \\
\text { Assisted Gait } \\
\text { Training }\end{array}$ & $\begin{array}{l}\text { Usual care, } \\
\text { previous } \\
\text { activity level } \\
\text { or attention } \\
\text { control }\end{array}$ & Walking & $\begin{array}{l}2 \text { (95) } \\
\text { Straudi, } 2016 \\
\text { Pompa, } 2017\end{array}$ & Moderate & Inconsistent & Imprecise & Undetected & Insufficient & $\begin{array}{l}\text { 6MWT: } 23.22(32.23) \text { vs. } \\
-0.75(26.40), p=0.01 \\
\text { 10MWT: } 0.07(0.15) \text { vs. } \\
0.01(0.10), p=0.29 \\
\frac{2 M i n W T:}{p>0.05} 8.88 \text { vs. } 2.81 \\
\frac{\text { FAC (functional }}{\text { ambulation category): }} \\
6.86 \text { vs. } 0.00, p>0.05\end{array}$ \\
\hline $\begin{array}{l}\text { Aerobic } \\
\text { Exercise } \\
\text { Robot- } \\
\text { Assisted Gait } \\
\text { Training }\end{array}$ & $\begin{array}{l}\text { Usual care, } \\
\text { previous } \\
\text { activity level } \\
\text { or attention } \\
\text { control }\end{array}$ & $\begin{array}{l}\text { Quality of } \\
\text { Life }\end{array}$ & $\begin{array}{l}1(52) \\
\text { Straudi, } 2016\end{array}$ & Low & Unknown & Imprecise & Undetected & Insufficient & $\begin{array}{l}\text { SF 36-PCS: } 1.67(7.74) \\
\text { vs. 1.84 (6.77), } p=0.99 \\
\text { SF 36-MCS: } 5.37(9.58) \\
\text { vs. } 1.60(9.41), p=0.14\end{array}$ \\
\hline $\begin{array}{l}\text { Aerobic } \\
\text { Exercise } \\
\text { Robot- } \\
\text { Assisted Gait } \\
\text { Training }\end{array}$ & $\begin{array}{l}\text { Usual care, } \\
\text { previous } \\
\text { activity level } \\
\text { or attention } \\
\text { control }\end{array}$ & Balance & $\begin{array}{l}2(97) \\
\text { Straudi, } 2016 \\
\text { Russo, } 2018\end{array}$ & Moderate & Consistent & Imprecise & Undetected & $\begin{array}{l}\text { Low for } \\
\text { benefit }\end{array}$ & $\begin{array}{l}\text { TBS: } 0.48 \text { (SE } 0.22) \\
\text { p=0.04 } \\
\text { BBS: } 3.24(4.99) \text { vs. } 0.87 \\
(6.45), p=0.19\end{array}$ \\
\hline $\begin{array}{l}\text { Aerobic } \\
\text { Exercise } \\
\text { Robot- } \\
\text { Assisted Gait } \\
\text { Training }\end{array}$ & $\begin{array}{l}\text { Usual care, } \\
\text { previous } \\
\text { activity level } \\
\text { or attention } \\
\text { control }\end{array}$ & ADLs & $\begin{array}{l}\text { 1(43) } \\
\text { Pompa, } 2017\end{array}$ & Moderate & Unknown & Imprecise & Undetected & Insufficient & $\begin{array}{l}\text { Rivermean Mobility Index: } \\
\frac{0.73,95 \% \mathrm{Cl}-0.85 \text { to }}{2.31, \mathrm{p}=0.37}\end{array}$ \\
\hline $\begin{array}{l}\text { Aerobic } \\
\text { Exercise } \\
\text { Robot- } \\
\text { Assisted Gait } \\
\text { Training }\end{array}$ & $\begin{array}{l}\text { RAGT } \\
\text { without VR }\end{array}$ & Balance & $\begin{array}{l}1(40) \\
\text { Calabro, } 2017\end{array}$ & Low & Unknown & Imprecise & Undetected & Insufficient & $\begin{array}{l}\text { BBS: }-0.019,95 \% \mathrm{Cl}- \\
\underline{2.403 \text { to } 2.365, p=0.8}\end{array}$ \\
\hline
\end{tabular}




\begin{tabular}{|c|c|c|c|c|c|c|c|c|c|}
\hline $\begin{array}{l}\text { Intervention } \\
\text { Category, } \\
\text { Intervention }\end{array}$ & Comparator & Outcome & $\begin{array}{l}\text { Number of Studies } \\
\text { (Participants) } \\
\text { Author Year } \\
\text { (See Appendix B for } \\
\text { Full Citation) }\end{array}$ & $\begin{array}{l}\text { Study } \\
\text { Limitations }\end{array}$ & Consistency & Precision & $\begin{array}{l}\text { Reporting } \\
\text { Bias }\end{array}$ & $\begin{array}{l}\text { Strength of } \\
\text { Evidence }\end{array}$ & $\begin{array}{l}\text { Findings, Direction and } \\
\text { Magnitude of Effect }\end{array}$ \\
\hline $\begin{array}{l}\text { Aerobic } \\
\text { Exercise } \\
\text { Robot- } \\
\text { Assisted Gait } \\
\text { Training }\end{array}$ & $\begin{array}{l}\text { RAGT with } \\
\text { assistance }\end{array}$ & Function & $\begin{array}{l}1(23) \\
\text { Wu, 2017a }\end{array}$ & Moderate & Unknown & Imprecise & Undetected & Insufficient & $\begin{array}{l}\text { 6MWT: } 49.8,95 \% \mathrm{Cl}- \\
49.85 \text { to } 149.45, p=0.33 \\
\text { GMFM- } 66 \text { total: } 0.10,95 \% \\
\mathrm{Cl}-7.74 \text { to } 7.94, p=0.98 \\
\text { GMFM-66D: } 0.10,95 \% \mathrm{Cl} \\
-8.55 \text { to } 8.75, p=0.98 \\
\text { GMFM-66E: } 0.10,95 \% \mathrm{Cl} \\
-16.32 \text { to } 16.52, p=0.99\end{array}$ \\
\hline $\begin{array}{l}\text { Aerobic } \\
\text { Exercise } \\
\text { Robot- } \\
\text { Assisted Gait } \\
\text { Training }\end{array}$ & $\begin{array}{l}\text { Treadmill or } \\
\text { Overground } \\
\text { walking }\end{array}$ & Function & $\begin{array}{l}2(95) \\
\text { Wu, 2017b } \\
\text { Straudi, } 2019\end{array}$ & Low & Consistent & Imprecise & Undetected & $\begin{array}{l}\text { Low-strength } \\
\text { evidence of } \\
\text { no clear } \\
\text { benefit }\end{array}$ & $\begin{array}{l}\text { GMFM-66 total: }-5.1,95 \% \\
\text { Cl } 13.62 \text { to } 3.42, p=0.24 \\
\text { GMFM-66D: } 3.6,95 \% \mathrm{Cl}- \\
\text { 5.40 to } 12.60, p=0.43 \\
\text { GMFM-66E: } 0.2,95 \% \mathrm{Cl}- \\
\text { 17.79 to } 19.19, p=0.98 \\
\text { 6MWT: } M D 4,95 \% \mathrm{Cl}-10 \\
\text { to } 18, p=0.86 \\
\text { 25FWT: } M D \text { D } 0,95 \% \mathrm{Cl}- \\
\text { 0.06 to } 0.05, p=0.98 \\
\text { TUG: } M D \text { D } 7.8,95 \% \mathrm{Cl}-0.2 \\
\text { to } 15.8, p=0.25\end{array}$ \\
\hline $\begin{array}{l}\text { Aerobic } \\
\text { Exercise } \\
\text { Robot- } \\
\text { Assisted Gait } \\
\text { Training }\end{array}$ & $\begin{array}{l}\text { Overground } \\
\text { walking }\end{array}$ & QoL & $\begin{array}{l}1(72) \\
\text { Straudi, } 2019\end{array}$ & Low & Unknown & Imprecise & Undetected & $\begin{array}{l}\text { Low-strength } \\
\text { evidence of } \\
\text { no clear } \\
\text { benefit }\end{array}$ & $\begin{array}{l}\text { MSIS-29 motor: }-3,95 \% \\
\mathrm{Cl}-9 \text { to } 3, p=0.31 \\
\text { MSIS-29 psychological: - } \\
2,95 \% \mathrm{Cl}-5 \text { to } 1, p=0.22 \\
\text { SF-36 PCS: }-1,95 \% \mathrm{Cl}-4 \\
\text { to } 3, p=0.13 \\
\text { SF-36 MCS: } 1,95 \% \mathrm{Cl}-2 \\
\text { to } 4, p=0.94\end{array}$ \\
\hline $\begin{array}{l}\text { Aerobic } \\
\text { Exercise } \\
\text { Robot- } \\
\text { Assisted Gait } \\
\text { Training }\end{array}$ & $\begin{array}{l}\text { Overground } \\
\text { walking }\end{array}$ & Balance & $\begin{array}{l}1 \text { (72) } \\
\text { Straudi, } 2019\end{array}$ & Low & Unknown & Imprecise & Undetected & $\begin{array}{l}\text { Low-strength } \\
\text { evidence of } \\
\text { no clear } \\
\text { benefit }\end{array}$ & $\begin{array}{l}\text { BBS: } 0,95 \% \mathrm{Cl}-2 \text { to } 2, \\
p=0.91\end{array}$ \\
\hline
\end{tabular}




\begin{tabular}{|c|c|c|c|c|c|c|c|c|c|}
\hline $\begin{array}{l}\text { Intervention } \\
\text { Category, } \\
\text { Intervention }\end{array}$ & Comparator & Outcome & $\begin{array}{l}\text { Number of Studies } \\
\text { (Participants) } \\
\text { Author Year } \\
\text { (See Appendix B for } \\
\text { Full Citation) }\end{array}$ & $\begin{array}{l}\text { Study } \\
\text { Limitations }\end{array}$ & Consistency & Precision & $\begin{array}{l}\text { Reporting } \\
\text { Bias }\end{array}$ & $\begin{array}{l}\text { Strength of } \\
\text { Evidence }\end{array}$ & $\begin{array}{l}\text { Findings, Direction and } \\
\text { Magnitude of Effect }\end{array}$ \\
\hline $\begin{array}{l}\text { Aerobic } \\
\text { Exercise } \\
\text { Treadmill }\end{array}$ & $\begin{array}{l}\text { All } \\
\text { comparators }\end{array}$ & Function & $\begin{array}{l}4(119) \\
\text { Gervasoni, } 2014 \\
\text { Jonsdottir, } 2018 \\
\text { Samaei, } 2014 \\
\text { Ahmadi, } 2013\end{array}$ & Moderate & Consistent & Imprecise & Undetected & $\begin{array}{l}\text { Low for } \\
\text { benefit }\end{array}$ & $\begin{array}{l}\text { DGl: } 2.16 \text { vs. } 2.07, p=0.51 \\
\text { DG: } 0.2,95 \% \mathrm{Cl}-1.95 \text { to } \\
2.27, \mathrm{p}=0.87 \\
\text { TUG: }-2.83,95 \% \mathrm{Cl}-4.7 \\
\text { to }-0.9, \mathrm{p}=0.009 \\
\text { TUG: } 9.8(1.7) \text { to } 7.5(1.8) \\
\text { vs. } 9.4(2.3) \text { to } 8.9(0.9), \\
\text { p=0.041 } \\
\text { 10MWT: } 8.68(1.93) \text { to } \\
7.07(1.03) \text { vs. } 9.16(1.88) \\
\text { to } 9.47(1.92), \mathrm{p}=0.001 \\
\text { 2MWT: } 120.40(20.29) \text { to } \\
139.90(20.78) \text { vs. } 121.50 \\
(27.73) \text { to } 119.05(27.12), \\
\text { p=0.001 } \\
\text { 2MWT: } 28.3,95 \% \mathrm{Cl} \\
13.04 \text { to } 43.60, \mathrm{p}<0.001 \\
\text { 2MinWT: } 12.01(23.6) \text { to } \\
160.1(35.7) \text { vs. } 132.6 \\
(32.3) \text { to } 147.5(29.8), \\
\text { p<0.001 } \\
\text { 25-foot WT: } 8.7(2.4) \text { to } \\
6.1(1.8) \text { vs. } 7.9(1.1) \text { to } \\
7.0(1.6), p=0.001 \\
\text { Modified Riverman } \\
\text { Mobility Index: } 10.6(3.2) \\
\text { to } 14.3(2.7) \text { vs. } 10.5(2.3) \\
\text { to } 11.9(2.1), p=0.005\end{array}$ \\
\hline $\begin{array}{l}\text { Aerobic } \\
\text { Exercise } \\
\text { Treadmill }\end{array}$ & $\begin{array}{l}\text { Usual care } \\
\text { or waitlist }\end{array}$ & $\begin{array}{l}\text { Function/ } \\
\text { Walking }\end{array}$ & $\begin{array}{l}2 \text { (50) } \\
\text { Gervasoni, } 2014 \\
\text { Ahmadi, } 2013\end{array}$ & Moderate & Consistent & Imprecise & Undetected & $\begin{array}{l}\text { Low for } \\
\text { benefit }\end{array}$ & $\begin{array}{l}\text { DGI: } 0.2,95 \% \mathrm{Cl}-1.95 \text { to } \\
2.27, p=0.87 \\
\text { TUG: }-2.83,95 \% \mathrm{Cl}-4.7 \\
\text { to }-0.9, p=0.009 \\
\text { 10MWT: } 8.68(1.93) \text { to } \\
7.07(1.03) \text { vs. } 9.16(1.88) \\
\text { to } 9.47(1.92), p=0.001 \\
\text { 2MWT: } 120.40(20.29) \text { to } \\
139.90(20.78) \text { vs. } 121.50 \\
\text { (27.73) to } 119.05(27.12) \\
\text { p=0.001 } \\
\text { 2MWT: } 28.3,95 \% \mathrm{Cl} \\
\text { 13.04 to } 43.60, p<0.001\end{array}$ \\
\hline
\end{tabular}




\begin{tabular}{|c|c|c|c|c|c|c|c|c|c|}
\hline $\begin{array}{l}\text { Intervention } \\
\text { Category, } \\
\text { Intervention }\end{array}$ & Comparator & Outcome & $\begin{array}{l}\text { Number of Studies } \\
\text { (Participants) } \\
\text { Author Year } \\
\text { (See Appendix B for } \\
\text { Full Citation) } \\
\end{array}$ & $\begin{array}{l}\text { Study } \\
\text { Limitations }\end{array}$ & Consistency & Precision & $\begin{array}{l}\text { Reporting } \\
\text { Bias }\end{array}$ & $\begin{array}{l}\text { Strength of } \\
\text { Evidence }\end{array}$ & $\begin{array}{l}\text { Findings, Direction and } \\
\text { Magnitude of Effect }\end{array}$ \\
\hline $\begin{array}{l}\text { Aerobic } \\
\text { Exercise } \\
\text { Treadmill }\end{array}$ & $\begin{array}{l}\text { Strength } \\
\text { Training }\end{array}$ & Function & $\begin{array}{l}1(38) \\
\text { Jonsdottir, } 2018\end{array}$ & Moderate & Unknown & Imprecise & Undetected & Insufficient & $\begin{array}{l}\text { TUG: }-2.83,95 \% \mathrm{Cl}-4.7 \\
\text { to }-0.9, p=0.009 \\
\text { DGI: } 0.2,95 \% \mathrm{Cl}-1.95 \text { to } \\
2.27, p=0.87 \\
\frac{2 \mathrm{MWT}:}{13.04 \text { to } 43.60, p<0.001}\end{array}$ \\
\hline $\begin{array}{l}\text { Aerobic } \\
\text { Exercise } \\
\text { Treadmill }\end{array}$ & $\begin{array}{l}\text { Downhill vs. } \\
\text { Uphill } \\
\text { treadmill } \\
\text { training }\end{array}$ & Function & $\begin{array}{l}1(31) \\
\text { Samaei, } 2016\end{array}$ & Moderate & Unknown & Imprecise & Undetected & Insufficient & $\begin{array}{l}\text { 25-foot WT: } 8.7(2.4) \text { to } \\
6.1(1.8) \text { vs. } 7.9(1.1) \text { to } \\
7.0(1.6), p=0.001 \\
\text { 2MinWT: } 12.01(23.6) \text { to } \\
\text { 160.1 (35.7) vs. } 132.6 \\
(32.3) \text { to } 147.5(29.8), \\
\text { p<0.001 } \\
\text { TUG: } 9.8(1.7) \text { to } 7.5(1.8) \\
\text { vs. } 9.4(2.3) \text { to } 8.9(0.9), \\
\text { p=0.041 } \\
\text { Modified Riverman } \\
\text { Mobility Index: } 10.6(3.2) \\
\text { to } 14.3(2.7) \text { vs. } 10.5(2.3) \\
\text { to } 11.9(2.1), p=0.005\end{array}$ \\
\hline $\begin{array}{l}\text { Aerobic } \\
\text { Exercise } \\
\text { Treadmill }\end{array}$ & $\begin{array}{l}\text { Strength } \\
\text { Training }\end{array}$ & $\begin{array}{l}\text { Quality of } \\
\text { Life }\end{array}$ & $\begin{array}{l}1(38) \\
\text { Johsdottir, } 2018\end{array}$ & Moderate & Unknown & Imprecise & Undetected & Insufficient & $\begin{array}{l}\text { SF-12 mental: }-3.0,95 \% \\
\mathrm{Cl}-9.43 \text { to } 3.38, p=0.34 \\
\text { SF-12 physical: } 1.8,95 \% \\
\mathrm{Cl}-2.08 \text { to } 5.59, p=0.36\end{array}$ \\
\hline $\begin{array}{l}\text { Aerobic } \\
\text { Exercise } \\
\text { Treadmill }\end{array}$ & $\begin{array}{l}\text { Strength } \\
\text { Training }\end{array}$ & Balance & $\begin{array}{l}1(38) \\
\text { Jonsdottir, } 2018\end{array}$ & Moderate & Unknown & Imprecise & Undetected & Insufficient & $\begin{array}{l}\text { BBS: } 1.1,95 \% \mathrm{Cl}-1.4 \text { to } \\
3.7, p=0.39\end{array}$ \\
\hline $\begin{array}{l}\text { Aerobic } \\
\text { Exercise } \\
\text { Treadmill }\end{array}$ & $\begin{array}{l}\text { Usual care } \\
\text { or waitlist }\end{array}$ & Balance & $\begin{array}{l}2(50) \\
\text { Ahmadi, } 2013 \\
\text { Gervasoni, } 2014\end{array}$ & Moderate & Consistent & Imprecise & Undetected & $\begin{array}{l}\text { Low for } \\
\text { benefit }\end{array}$ & $\begin{array}{l}\text { BBS: } 4.01 \text { vs. } 3.15, \\
p=0.33 \\
\text { BBS: } 46.20(6.32) \text { to } 53.80 \\
\text { (2.34) vs. } 44.50(9.43) \text { to } \\
41.70(8.48), p=0.001\end{array}$ \\
\hline
\end{tabular}

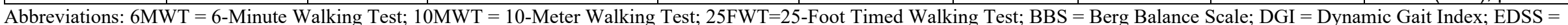

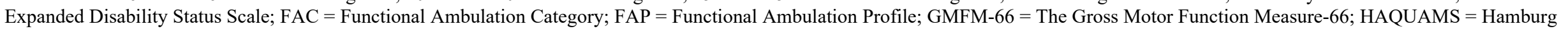

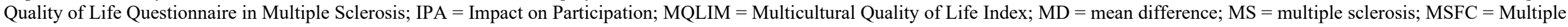

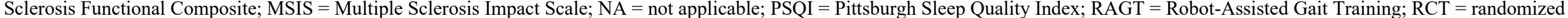

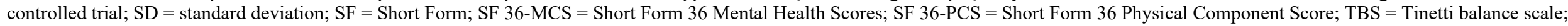
TUG $=$ Timed Up and Go Test 
Table H-2. Strength of evidence for Key Question 2: aerobic exercise for cerebral palsy

\begin{tabular}{|c|c|c|c|c|c|c|c|c|c|}
\hline $\begin{array}{l}\text { Intervention } \\
\text { Category, } \\
\text { Intervention }\end{array}$ & Comparator & Outcome & $\begin{array}{l}\text { Number of } \\
\text { Studies } \\
\text { (Participants) } \\
\text { Author Year } \\
\text { (See Appendix } \\
\text { B for Full } \\
\text { Citation) }\end{array}$ & $\begin{array}{l}\text { Study } \\
\text { Limitations }\end{array}$ & Consistency & Precision & $\begin{array}{l}\text { Reporting } \\
\text { Bias }\end{array}$ & $\begin{array}{l}\text { Strength of } \\
\text { Evidence }\end{array}$ & $\begin{array}{l}\text { Findings, Direction and } \\
\text { Magnitude of Effect }\end{array}$ \\
\hline $\begin{array}{l}\text { Aerobic } \\
\text { Exercise } \\
\text { Aerobics }\end{array}$ & $\begin{array}{l}\text { Usual care, } \\
\text { previous } \\
\text { activity level } \\
\text { or attention } \\
\text { control }\end{array}$ & ADLs & $\begin{array}{l}1(26) \\
\text { Teixeira- } \\
\text { Machado, } \\
2018\end{array}$ & Moderate & Unknown & Imprecise & Undetected & Insufficient & $\begin{array}{l}\text { ICF total change score: } \\
-44.56 \text { vs. } 14.90, p<0.001\end{array}$ \\
\hline $\begin{array}{l}\text { Aerobic } \\
\text { Exercise } \\
\text { Aerobics }\end{array}$ & Usual care & $\begin{array}{l}\text { Running/ } \\
\text { mobility }\end{array}$ & $\begin{array}{l}1(42) \\
\text { Gibson, } 2018\end{array}$ & Low & Unknown & Imprecise & Undetected & Insufficient & $\begin{array}{l}\text { Shuttle Run Test (min): } 0.9 \text {, } \\
95 \% \mathrm{Cl}-0.3 \text { to } 2.2, p=0.142 \\
\text { HiMat: } 0.8,95 \% \mathrm{Cl}-2.7 \text { to } \\
4.3, p=0.651 \\
10 \times 5 \text { sprint }(\mathrm{sec}):-1.3,95 \% \\
\mathrm{Cl}-5.4 \text { to } 2.8, p=0.535\end{array}$ \\
\hline $\begin{array}{l}\text { Aerobic } \\
\text { Exercise } \\
\text { Aquatics }\end{array}$ & $\begin{array}{l}\text { Land-based } \\
\text { exercise }\end{array}$ & Function & $\begin{array}{l}\mathrm{N}=32) \\
\text { Adar, } 2017\end{array}$ & Moderate & Unknown & Imprecise & Undetected & Insufficient & $\begin{array}{l}\text { Mean SD: } \\
\text { TUG: }-0.13(0.14) \text { vs. }-0.16 \\
(0.13), p=0.664 \text { GMGM-88: } \\
0.05(0.05) \text { vs. } 0.05(0.03) \\
p=0.451 \text { WeeFIM motor: } \\
0.04(0.04) \text { vs. } 0.06 \\
(0.06), p=0.860 \\
\text { WeeFIM total: }-0.13(0.14) \\
\text { vs. }-0.16(0.13), p=0.287 \\
N=24, \text { Mean }(S D)\end{array}$ \\
\hline $\begin{array}{l}\text { Aerobic } \\
\text { Exercise } \\
\text { Aquatics }\end{array}$ & $\begin{array}{l}\text { Rehabilitation } \\
\text { exercises }\end{array}$ & Function & $\begin{array}{l}1(24) \\
\text { Lai, } 2015\end{array}$ & Moderate & Unknown & Imprecise & Undetected & Insufficient & $\begin{array}{l}\text { GMFM-66: } 61.2(18.7) \text { vs. } \\
64.6(19.4) \text { (baseline) } 66.2 \\
\text { (18.2) vs. } 65.3(19.1) \\
\text { (postintervention) Difference } \\
\text { in change score between } \\
\text { groups: } p=0.007\end{array}$ \\
\hline $\begin{array}{l}\text { Aerobic } \\
\text { Exercise } \\
\text { Aquatics }\end{array}$ & $\begin{array}{l}\text { Rehabilitation } \\
\text { exercises }\end{array}$ & $\begin{array}{l}\text { Quality of } \\
\text { Life }\end{array}$ & $\begin{array}{l}1(24) \\
\text { Lai, } 2015\end{array}$ & Moderate & Unknown & Imprecise & Undetected & Insufficient & $\begin{array}{l}\text { Cerebral Palsy QoL Scale: } \\
\text { for Social, Functioning, } \\
\text { Participation, Emotional, } \\
\text { Access, Pain and Disability, } \\
\text { and Family Health: All NS }\end{array}$ \\
\hline
\end{tabular}




\begin{tabular}{|c|c|c|c|c|c|c|c|c|c|}
\hline $\begin{array}{l}\text { Intervention } \\
\text { Category, } \\
\text { Intervention }\end{array}$ & Comparator & Outcome & $\begin{array}{l}\text { Number of } \\
\text { Studies } \\
\text { (Participants) } \\
\text { Author Year } \\
\text { (See Appendix } \\
\text { B for Full } \\
\text { Citation) }\end{array}$ & $\begin{array}{l}\text { Study } \\
\text { Limitations }\end{array}$ & Consistency & Precision & $\begin{array}{l}\text { Reporting } \\
\text { Bias }\end{array}$ & $\begin{array}{l}\text { Strength of } \\
\text { Evidence }\end{array}$ & $\begin{array}{l}\text { Findings, Direction and } \\
\text { Magnitude of Effect }\end{array}$ \\
\hline $\begin{array}{l}\text { Aerobic } \\
\text { Exercise } \\
\text { Aquatics }\end{array}$ & $\begin{array}{l}\text { Rehabilitation } \\
\text { exercises }\end{array}$ & ADLs & $\begin{array}{l}1(24) \\
\text { Lai, 2015 }\end{array}$ & Moderate & Unknown & Imprecise & Undetected & Insufficient & $\begin{array}{l}\text { Vineline Adaptive Behavior } \\
\text { Scale for Daily Living: } 72.1 \\
\text { (48.5) vs. } 93.7(43.8) \\
\text { (baseline) } 76.5(7.6) \text { vs. } \\
76.4(10.8) \text { (post- } \\
\text { intervention) } \\
\text { Difference in change score } \\
\text { between groups: } p=0.393\end{array}$ \\
\hline $\begin{array}{l}\text { Aerobic } \\
\text { Exercise } \\
\text { Cycling }\end{array}$ & $\begin{array}{l}\text { Usual care, } \\
\text { previous } \\
\text { activity level } \\
\text { or attention } \\
\text { control }\end{array}$ & Function & $\begin{array}{l}2(85) \\
\text { Fowler } 2010 \\
\text { Bryant } 2013\end{array}$ & Moderate & Consistent & Imprecise & Undetected & Low for benefit & $\begin{array}{l}\text { GMFM-66 pooled: } 0.70, \\
95 \% \mathrm{Cl}-0.20 \text { to } 1.60, \\
\text { p=0.127 } \\
\text { GMFM-88D: } 5.4,95 \% \mathrm{Cl} \\
1.23 \text { to } 9.57, p=0.01 \\
\text { GMFM-88E: } 2.3,95 \% \mathrm{Cl} \\
0.20 \text { to } 4.40, p=0.03 \\
\text { 600-Yard Walk-Run Test: } \\
\text { Change from baseline: } 5.6 \text {, } \\
95 \% \mathrm{Cl} 1.6 \text { to } 9.5 \text { vs. } 2.5, \\
95 \% \mathrm{Cl}-1.1 \text { to } 6.0, p=0.24\end{array}$ \\
\hline $\begin{array}{l}\text { Aerobic } \\
\text { Exercise } \\
\text { Cycling }\end{array}$ & $\begin{array}{l}\text { Usual care, } \\
\text { previous } \\
\text { activity level } \\
\text { or attention } \\
\text { control }\end{array}$ & $\begin{array}{l}\text { Quality of } \\
\text { Life }\end{array}$ & $\begin{array}{l}1 \text { (62) } \\
\text { Demuth, } 2012\end{array}$ & Moderate & Unknown & Imprecise & Undetected & Insufficient & $\begin{array}{l}\text { Peds Quality of Life Total } \\
\text { Score: } 3.5,95 \% \mathrm{Cl}-2.0 \text { to } \\
8.8, p=0.21\end{array}$ \\
\hline
\end{tabular}




\begin{tabular}{|c|c|c|c|c|c|c|c|c|c|}
\hline $\begin{array}{l}\text { Intervention } \\
\text { Category, } \\
\text { Intervention }\end{array}$ & Comparator & Outcome & $\begin{array}{l}\text { Number of } \\
\text { Studies } \\
\text { (Participants) } \\
\text { Author Year } \\
\text { (See Appendix } \\
\text { B for Full } \\
\text { Citation) }\end{array}$ & $\begin{array}{l}\text { Study } \\
\text { Limitations }\end{array}$ & Consistency & Precision & $\begin{array}{l}\text { Reporting } \\
\text { Bias }\end{array}$ & $\begin{array}{l}\text { Strength of } \\
\text { Evidence }\end{array}$ & $\begin{array}{l}\text { Findings, Direction and } \\
\text { Magnitude of Effect }\end{array}$ \\
\hline $\begin{array}{l}\text { Aerobic } \\
\text { Exercise } \\
\text { Robot- } \\
\text { Assisted Gait } \\
\text { Training }\end{array}$ & $\begin{array}{l}\text { Usual care, } \\
\text { previous } \\
\text { activity level } \\
\text { or attention } \\
\text { control }\end{array}$ & Function & $\begin{array}{l}12 \text { RCTs }(77) ; \\
1 \text { cohort study } \\
(24) \\
\text { Wallard, } 2017 \\
\text { Wallard, } 2018 \\
\text { Klobucka, } \\
2020 \\
\text { Yazici, } 2019\end{array}$ & High & Consistent & Imprecise & Undetected & Insufficient & $\begin{array}{l}\text { GMFM-66D: } 4.73,95 \% \mathrm{Cl} \text { - } \\
\text { 6.14 to } 15.60, p=0.39 \\
\text { GMFM-66E: } 7.54,95 \% \mathrm{Cl} \text { - } \\
\text { 2.64 to } 17.42, p=0.15 \\
\text { GMFM-88: } 1.59,95 \% \mathrm{Cl} \text { - } \\
2.19 \text { to } 5.37, p=0.410 \\
\text { GMFM-88D: } 0.17,95 \% \mathrm{Cl} \text { - } \\
\text { 0.79 to } 1.13, p=0.729 \\
\text { GMFM-88E: } 1.14,95 \% \mathrm{Cl} \text { - } \\
\text { 1.69 to } 4.51, p=0.373 \\
\text { 6MWT: } 43.42,95 \% \mathrm{Cl} 19.64 \\
\text { to } 67.21, p<0.001 \\
\text { GMFM-88: MD } 9.43,95 \% \\
\text { Cl } 6.989 \text { to } 11.891 \text { vs. MD } \\
0.80,95 \% \mathrm{Cl} 0.154 \text { to } \\
1.446, p<0.001 \\
\text { GMFM-88D: MD } 8.30,95 \% \\
\text { Cl } 4.699 \text { to } 11.901 \text { vs. MD } \\
1.09,95 \% \mathrm{Cl}-0.438 \text { to } \\
2.619, p<0.001 \\
\text { GMFM-88E: MD } 9.32,95 \% \\
\text { Cl } 5.329 \text { to } 13.310 \text { vs. MD } \\
0.53,95 \% \text { Cl }-0.208 \text { to } \\
1.268, p<0.001\end{array}$ \\
\hline $\begin{array}{l}\text { Aerobic } \\
\text { Exercise } \\
\text { Robot-Assisted } \\
\text { Gait Training }\end{array}$ & $\begin{array}{l}\text { Usual care, } \\
\text { previous } \\
\text { activity level } \\
\text { or attention } \\
\text { control }\end{array}$ & Balance & $\begin{array}{l}1(24) \\
\text { Yazici, } 2019\end{array}$ & High & Unknown & Imprecise & Undetected & Insufficient & $\begin{array}{l}\text { BBS: } 1.25,95 \% \mathrm{Cl}-0.07 \text { to } \\
\underline{2.57, p=0.064}\end{array}$ \\
\hline
\end{tabular}




\begin{tabular}{|c|c|c|c|c|c|c|c|c|c|}
\hline $\begin{array}{l}\text { Intervention } \\
\text { Category, } \\
\text { Intervention }\end{array}$ & Comparator & Outcome & $\begin{array}{l}\text { Number of } \\
\text { Studies } \\
\text { (Participants) } \\
\text { Author Year } \\
\text { (See Appendix } \\
\text { B for Full } \\
\text { Citation) }\end{array}$ & $\begin{array}{l}\text { Study } \\
\text { Limitations }\end{array}$ & Consistency & Precision & $\begin{array}{l}\text { Reporting } \\
\text { Bias }\end{array}$ & $\begin{array}{l}\text { Strength of } \\
\text { Evidence }\end{array}$ & $\begin{array}{l}\text { Findings, Direction and } \\
\text { Magnitude of Effect }\end{array}$ \\
\hline $\begin{array}{l}\text { Aerobic } \\
\text { Exercise } \\
\text { Robot-Assisted } \\
\text { Gait Training }\end{array}$ & $\begin{array}{l}\text { Treadmill } \\
\text { training } \\
\text { (Partial body- } \\
\text { weight } \\
\text { supported; } \\
\text { Anti-gravity;) }\end{array}$ & Function & $\begin{array}{l}2(52) \\
\text { Aras, } 2019 \\
\text { Wu, 2017b }\end{array}$ & Moderate & Consistent & Imprecise & Undetected & Insufficient & 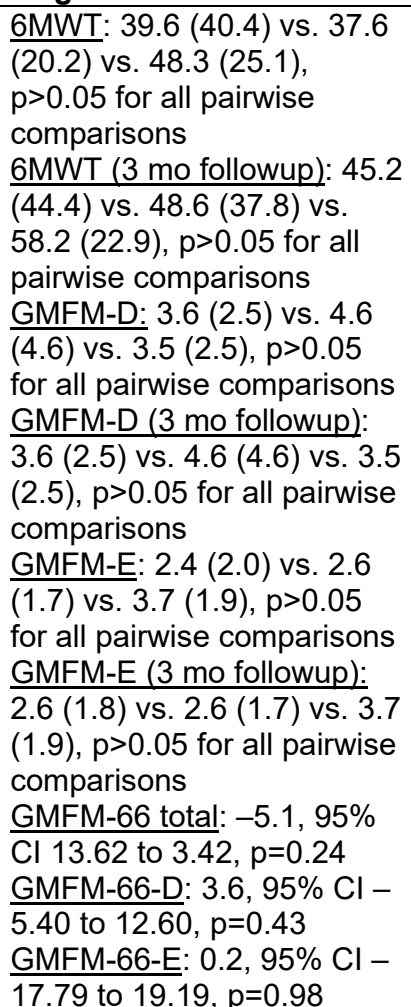 \\
\hline
\end{tabular}




\begin{tabular}{|c|c|c|c|c|c|c|c|c|c|}
\hline $\begin{array}{l}\text { Intervention } \\
\text { Category, } \\
\text { Intervention }\end{array}$ & Comparator & Outcome & $\begin{array}{l}\text { Number of } \\
\text { Studies } \\
\text { (Participants) } \\
\text { Author Year } \\
\text { (See Appendix } \\
\text { B for Full } \\
\text { Citation) }\end{array}$ & $\begin{array}{l}\text { Study } \\
\text { Limitations }\end{array}$ & Consistency & Precision & $\begin{array}{l}\text { Reporting } \\
\text { Bias }\end{array}$ & $\begin{array}{l}\text { Strength of } \\
\text { Evidence }\end{array}$ & $\begin{array}{l}\text { Findings, Direction and } \\
\text { Magnitude of Effect }\end{array}$ \\
\hline $\begin{array}{l}\text { Aerobic } \\
\text { Exercise } \\
\text { Treadmill }\end{array}$ & $\begin{array}{l}\text { Usual care, } \\
\text { previous } \\
\text { activity level } \\
\text { or attention } \\
\text { control }\end{array}$ & Function & $\begin{array}{l}2 \text { (53) } \\
\text { Chrysagis, } \\
2012 \\
\text { Bahrami, } \\
\text { 2019a }\end{array}$ & Moderate & Consistent & Imprecise & Undetected & Low for benefit & $\begin{array}{l}\text { GMFM-D+E: } 3.87 \text { vs. } 0.69, \\
\text { p=0.007 } \\
\text { Self-selected walking } \\
\text { speed: } 8.06 \text { vs. } 0.48, \\
\text { s=0.009 } \\
10 \text { MWT: } 1.080(0.47) \text { to } \\
1.22(0.50)[22.46 \% \text { change] } \\
\text { vs. } 0.99(0.56) \text { to } 1.02(0.61) \\
{[1.28 \% \text { change], } \% \text { change }} \\
\text { p<0.05 } \\
\text { 6MWT: } 291.13(160.28) \text { to } \\
\text { 342.63 (174.62) }[23.68 \% \\
\text { change] vs. } 276.10(167.19) \\
\text { to } 308.57(181.22)[16.54 \% \\
\text { change], } \% \text { change } p>0.05\end{array}$ \\
\hline $\begin{array}{l}\text { Aerobic } \\
\text { Exercise } \\
\text { Treadmill }\end{array}$ & Usual care & $\begin{array}{l}\text { Quality of } \\
\text { Life }\end{array}$ & $\begin{array}{l}1(30) \\
\text { Bahrami, } \\
2019 a\end{array}$ & Moderate & Unknown & Imprecise & Undetected & Insufficient & $\begin{array}{l}\text { WHOQOL-Brief: } 3.55(.55) \\
\text { to } 3.66(0.59)[3.83 \% \\
\text { change] vs. } 3.33(0.69) 3.57 \\
(0.67)[8.94 \% \text { change], } \% \\
\text { change p>0.05 }\end{array}$ \\
\hline $\begin{array}{l}\text { Aerobic } \\
\text { Exercise } \\
\text { Treadmill }\end{array}$ & $\begin{array}{l}\text { Overground } \\
\text { walking }\end{array}$ & Walking & $\begin{array}{l}5(130) \\
\text { Willoughby, } \\
2010 \\
\text { Swe, } 2015 \\
\text { Grecco, } 2013 \\
\text { Emara, } 2016 \\
\text { Kim, } 2015\end{array}$ & Moderate & Inconsistent & Imprecise & Undetected & $\begin{array}{l}\text { Low strength of } \\
\text { evidence for no } \\
\text { clear benefit }\end{array}$ & $\begin{array}{l}\text { 10MWT: } 0.4(0.04) \text { to } 0.5 \\
\text { (0.04) vs. } 0.4(0.03) \text { to } 0.6 \\
(0.04), p=0.12 \\
\text { 6MWT: } 149.7 \text { vs. } 44.8, \\
\text { p<0.001 } \\
\text { 6MWT: }-17.00,95 \% \mathrm{Cl}- \\
89.77 \text { to } 55.77, p=0.65 \\
\text { 10MWT: }-0.013,95 \% \mathrm{Cl} \text { - } \\
0.23,0.21, p=0.91 \\
\text { 10MWT: } 244.33(115.41) \text { to } \\
219.38(123.71) \text { vs. } 118.36 \\
(89.89) \text { to } 135.82(95.65), \\
\text { p=0.097 } \\
\text { 6MWT on treadmill: } 5.71, \\
95 \% \mathrm{Cl}-53.22 \text { to } 64.64, \\
\text { p=0.85 } \\
\text { 6MWT on overground } \\
\text { walking: } 24.07,95 \% \mathrm{Cl} \\
-46.80 \text { to } 94.94, p=0.51\end{array}$ \\
\hline
\end{tabular}




\begin{tabular}{|c|c|c|c|c|c|c|c|c|c|}
\hline $\begin{array}{l}\text { Intervention } \\
\text { Category, } \\
\text { Intervention }\end{array}$ & Comparator & Outcome & $\begin{array}{l}\text { Number of } \\
\text { Studies } \\
\text { (Participants) } \\
\text { Author Year } \\
\text { (See Appendix } \\
\text { B for Full } \\
\text { Citation) }\end{array}$ & $\begin{array}{l}\text { Study } \\
\text { Limitations }\end{array}$ & Consistency & Precision & $\begin{array}{l}\text { Reporting } \\
\text { Bias }\end{array}$ & $\begin{array}{l}\text { Strength of } \\
\text { Evidence }\end{array}$ & $\begin{array}{l}\text { Findings, Direction and } \\
\text { Magnitude of Effect }\end{array}$ \\
\hline $\begin{array}{l}\text { Aerobic } \\
\text { Exercise } \\
\text { Treadmill }\end{array}$ & $\begin{array}{l}\text { Overground } \\
\text { walking }\end{array}$ & Function & $\begin{array}{l}4 \text { (109) } \\
\text { Willoughby, } \\
2010 \\
\text { Swe, } 2015 \\
\text { Grecco, } 2013 \\
\text { Emara, } 2016\end{array}$ & Moderate & Inconsistent & Imprecise & Undetected & $\begin{array}{l}\text { Low strength of } \\
\text { evidence for no } \\
\text { clear benefit }\end{array}$ & $\begin{array}{l}\text { 5XSit-to-Stand: } 21.5(1.3) \text { to } \\
18.9(1.0) \text { vs. } 21.7(1.5) \text { to } \\
17.7(0.8), p=0.26 \\
\text { GMFM-88 D: } 12.5(1.6) \text { to } \\
15.8(1.5) \text { vs. } 12.0 \text { (0.7) to } \\
19.2(2.1), p=0.02, \text { favors } \\
\text { spider cage } \\
\text { GMFM-88 E: } 10.9(1.3) \text { to } \\
14.8(1.5) \text { vs. } 10.4(0.8) \text { to } \\
17.2(2.1), p=0.05, \text { favors } \\
\text { spider cage } \\
\text { TUG: }-6.4 \text { vs. }-2.0, p=0.004 \text {, } \\
\text { favors treadmill } \\
\text { GMFM-88D: } 23.9 \text { vs. } 8.1, \\
\text { p<0.001, favors treadmill } \\
\text { GMFM-88E: } 20.1 \text { vs. } 8.2, \\
\text { p<0.001, favors treadmill } \\
\text { GMFM-88D: }-2.94,95 \% \mathrm{Cl} \\
\text {-16.42 to } 10.64, p=0.67 \\
\text { GMFM-88E: }-2.8,95 \% \mathrm{Cl} \\
-20.02 \text { to } 14.42, p=0.75\end{array}$ \\
\hline $\begin{array}{l}\text { Aerobic } \\
\text { Exercise } \\
\text { Treadmill }\end{array}$ & $\begin{array}{l}\text { Treadmill } \\
\text { training with } \\
\text { TDC stim vs. } \\
\text { Treadmill } \\
\text { training with } \\
\text { sham TDC }\end{array}$ & Function & $\begin{array}{l}1(24) \\
\text { Grecco, } 2014\end{array}$ & Moderate & Unknown & Imprecise & Undetected & Insufficient & $\begin{array}{l}\text { 6MWT: } 102.4,95 \% \mathrm{Cl} 33.16 \\
\text { to } 171.64, p=0.004 \\
\text { GMFM-88D: } 7.8,95 \% \mathrm{Cl} \\
0.46 \text { to } 15.15, p=0.037 \\
\frac{\text { GMFM-88E: }}{p=0.251}-3.39 \text { to } 12.99 \text {, }\end{array}$ \\
\hline $\begin{array}{l}\text { Aerobic } \\
\text { Exercise } \\
\text { Treadmill }\end{array}$ & $\begin{array}{l}\text { Treadmill } \\
\text { training with } \\
\text { TDC stim vs. } \\
\text { Treadmill } \\
\text { training with } \\
\text { sham TDC }\end{array}$ & Balance & $\begin{array}{l}1(24) \\
\text { Duarte Nde, } \\
2014\end{array}$ & Moderate & Unknown & Imprecise & Undetected & Insufficient & $\begin{array}{l}\text { PBS: } 40.5(9.4) \text { to } 45.3(7.9) \\
\text { Vs.39.1 (9.8) to } 39.7(8.4) ; \\
\text { MD } 4.2,95 \% \mathrm{Cl}-2.88 \text { to } \\
11.28, p=0.245\end{array}$ \\
\hline
\end{tabular}




\begin{tabular}{|c|c|c|c|c|c|c|c|c|c|}
\hline $\begin{array}{l}\text { Intervention } \\
\text { Category, } \\
\text { Intervention }\end{array}$ & Comparator & Outcome & $\begin{array}{l}\text { Number of } \\
\text { Studies } \\
\text { (Participants) } \\
\text { Author Year } \\
\text { (See Appendix } \\
\text { B for Full } \\
\text { Citation) } \\
\end{array}$ & $\begin{array}{l}\text { Study } \\
\text { Limitations }\end{array}$ & Consistency & Precision & $\begin{array}{l}\text { Reporting } \\
\text { Bias }\end{array}$ & $\begin{array}{l}\text { Strength of } \\
\text { Evidence }\end{array}$ & $\begin{array}{l}\text { Findings, Direction and } \\
\text { Magnitude of Effect }\end{array}$ \\
\hline $\begin{array}{l}\text { Aerobic } \\
\text { Exercise } \\
\text { Treadmill }\end{array}$ & $\begin{array}{l}\text { Treadmill } \\
\text { training with } \\
\text { TDC stim vs. } \\
\text { Treadmill } \\
\text { training with } \\
\text { sham TDC }\end{array}$ & ADLs & $\begin{array}{l}1(24) \\
\text { Duarte Nde, } \\
2014\end{array}$ & Moderate & Unknown & Imprecise & Undetected & Insufficient & $\begin{array}{l}\text { PEDI self-care: } 46.1(10) \text { to } \\
48.0(9.5) \text { vs. } 45.0(9.2) \text { to } \\
45.5(9.3) ; M D 1.4,95 \% \mathrm{Cl} \\
-6.21 \text { to } 9.01, p=0.718 \\
\text { PEDI mobility: } 38.0(8.5) \text { to } \\
41.7(7.4) \text { vs. } 38.3(7.4) \text { to } \\
39.5(7.6) ; M D 2.5,95 \% \mathrm{Cl} \\
-3.71 \text { to } 8.71, p=0.430\end{array}$ \\
\hline $\begin{array}{l}\text { Aerobic } \\
\text { Exercise } \\
\text { Treadmill }\end{array}$ & $\begin{array}{l}\text { Individualized } \\
\text { strength- } \\
\text { based } \\
\text { physical } \\
\text { therapy }\end{array}$ & Function & $\begin{array}{l}1(26) \\
\text { Johnston, } \\
2011\end{array}$ & Moderate & Unknown & Imprecise & Undetected & Insufficient & $\begin{array}{l}\text { GMFM: } 62.7(17.5) \text { to } 63.3 \\
\text { (16.2) vs. } 58.4(26.9) \text { to } 60.1 \\
(25.1), p=0.66\end{array}$ \\
\hline
\end{tabular}




\begin{tabular}{|c|c|c|c|c|c|c|c|c|c|}
\hline $\begin{array}{l}\text { Intervention } \\
\text { Category, } \\
\text { Intervention }\end{array}$ & Comparator & Outcome & $\begin{array}{l}\text { Number of } \\
\text { Studies } \\
\text { (Participants) } \\
\text { Author Year } \\
\text { (See Appendix } \\
\text { B for Full } \\
\text { Citation) }\end{array}$ & $\begin{array}{l}\text { Study } \\
\text { Limitations }\end{array}$ & Consistency & Precision & $\begin{array}{l}\text { Reporting } \\
\text { Bias }\end{array}$ & $\begin{array}{l}\text { Strength of } \\
\text { Evidence }\end{array}$ & $\begin{array}{l}\text { Findings, Direction and } \\
\text { Magnitude of Effect }\end{array}$ \\
\hline $\begin{array}{l}\text { Aerobic } \\
\text { Exercise } \\
\text { Treadmill (for } \\
\text { adults with CP) }\end{array}$ & $\begin{array}{l}\text { Strength } \\
\text { Training or } \\
\text { Usual care }\end{array}$ & Function & $\begin{array}{l}2 \text { RCTs (51) } \\
\text { Kim, } 2015 \\
\text { Bahrami, } \\
2019 a \\
1 \text { quasiexperi- } \\
\text { mental trial } \\
\text { (95) } \\
\text { Aviram, } 2017\end{array}$ & Moderate & Inconsistent & Imprecise & Undetected & $\begin{array}{l}\text { Low-strength } \\
\text { evidence for no } \\
\text { clear benefit }\end{array}$ & 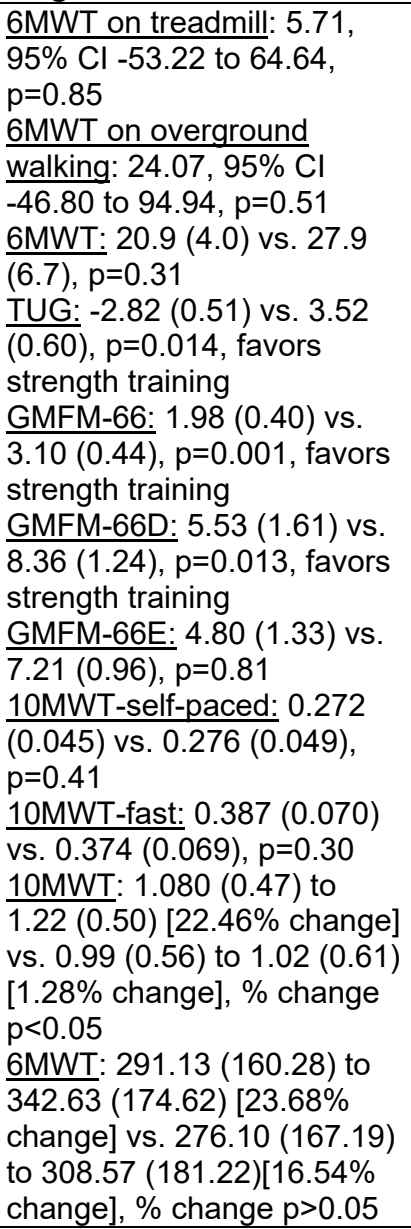 \\
\hline
\end{tabular}




\begin{tabular}{|c|c|c|c|c|c|c|c|c|c|}
\hline $\begin{array}{l}\text { Intervention } \\
\text { Category, } \\
\text { Intervention }\end{array}$ & Comparator & Outcome & $\begin{array}{l}\text { Number of } \\
\text { Studies } \\
\text { (Participants) } \\
\text { Author Year } \\
\text { (See Appendix } \\
\text { B for Full } \\
\text { Citation) }\end{array}$ & $\begin{array}{l}\text { Study } \\
\text { Limitations }\end{array}$ & Consistency & Precision & $\begin{array}{l}\text { Reporting } \\
\text { Bias }\end{array}$ & $\begin{array}{l}\text { Strength of } \\
\text { Evidence }\end{array}$ & $\begin{array}{l}\text { Findings, Direction and } \\
\text { Magnitude of Effect }\end{array}$ \\
\hline $\begin{array}{l}\text { Aerobic } \\
\text { Exercise } \\
\text { Treadmill (for } \\
\text { adolescents } \\
\text { with CP) }\end{array}$ & $\begin{array}{l}\text { Physical } \\
\text { Therapy or } \\
\text { Overground } \\
\text { Walking }\end{array}$ & Function & $\begin{array}{l}\text { 2 RCTs (56) } \\
\text { Chrysagis, } \\
2012 \\
\text { Swe, } 2015 \\
1 \text { Quasi- } \\
\text { experimental } \\
\text { study (24) } \\
\text { Nsenga- } \\
\text { Leunkau, } 2012\end{array}$ & Moderate & Consistent & Imprecise & Undetected & $\begin{array}{l}\text { Low-strength } \\
\text { evidence for no } \\
\text { clear benefit }\end{array}$ & $\begin{array}{l}\text { 6MWT: }-17.00,95 \% \mathrm{Cl} \\
\text {-89.77 to } 55.77, p=0.65 \\
\text { 10MWT: }-0.013,95 \% \mathrm{Cl} \\
-0.23,0.21, p=0.91 \\
\text { GMFM-88D: }-2.94,95 \% \mathrm{Cl} \\
-16.42 \text { to } 10.64, p=0.67 \\
\text { GMFM-88E: }-2.8,95 \% \mathrm{Cl} \\
-20.02 \text { to } 14.42, p=0.75 \\
\text { 10MWT: } 244.33(115.41) \text { to } \\
219.38 \text { (123.71) vs. } 118.36 \\
(89.89) \text { to } 135.82(95.65), \\
\text { p=0.097 } \\
\text { 6MWT: } 480 \text { to } 601 \text { vs. } 450 \\
\text { to } 450, \text { no difference in } \\
\text { baseline values, significant } \\
\text { difference in post- } \\
\text { intervention values favoring } \\
\text { treatment }\end{array}$ \\
\hline
\end{tabular}




\begin{tabular}{|c|c|c|c|c|c|c|c|c|c|}
\hline $\begin{array}{l}\text { Intervention } \\
\text { Category, } \\
\text { Intervention }\end{array}$ & Comparator & Outcome & $\begin{array}{l}\text { Number of } \\
\text { Studies } \\
\text { (Participants) } \\
\text { Author Year } \\
\text { (See Appendix } \\
\text { B for Full } \\
\text { Citation) }\end{array}$ & $\begin{array}{l}\text { Study } \\
\text { Limitations }\end{array}$ & Consistency & Precision & $\begin{array}{l}\text { Reporting } \\
\text { Bias }\end{array}$ & $\begin{array}{l}\text { Strength of } \\
\text { Evidence }\end{array}$ & $\begin{array}{l}\text { Findings, Direction and } \\
\text { Magnitude of Effect }\end{array}$ \\
\hline $\begin{array}{l}\text { Aerobic } \\
\text { Exercise } \\
\text { Treadmill (for } \\
\text { children with } \\
\text { CP) }\end{array}$ & $\begin{array}{l}\text { Overground } \\
\text { walking with } \\
\text { or without } \\
\text { spider cage, } \\
\text { treadmill } \\
\text { walking with } \\
\text { sham } \\
\text { transcranial } \\
\text { DC stim, } \\
\text { Individual } \\
\text { strength- } \\
\text { based PT }\end{array}$ & Function & $\begin{array}{l}4 \text { (103) } \\
\text { Johnston, } \\
2011 \\
\text { Emara, } 2016 \\
\text { Grecco, } 2013 \\
\text { Grecco, } 2014\end{array}$ & Moderate & Inconsistent & Imprecise & Undetected & $\begin{array}{l}\text { Low-strength } \\
\text { evidence for no } \\
\text { clear benefit }\end{array}$ & 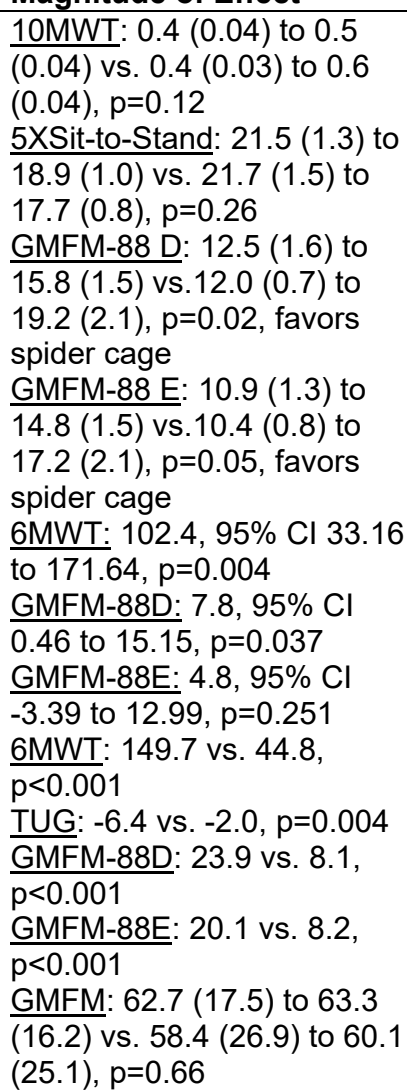 \\
\hline
\end{tabular}

Abbreviations: 6MWT = 6-Minute Walking Test; 10MWT=10-Minute Walking Test; BBS = Berg Balance Scale; $\mathrm{CI}=$ confidence interval; $\mathrm{CP}=$ cerebral palsy; HiMat = High Level Mobility

Assessment Tool; GMFM = The Gross Motor Function Measure; ICF = International Classification of Functioning, Disability and Health; NA = not applicable; PBS = Pediatric Balance Scale; PEDI

$=$ Pediatric Evaluation of Disability Inventory; RCT = randomized controlled trial; TUG= Timed Up and Go Test; WHOQOL = World Health Organization Quality of Life 
Table H-3. Strength of evidence for Key Question 2: aerobic exercise for cerebral palsy and multiple sclerosis

\begin{tabular}{|c|c|c|c|c|c|c|c|c|c|}
\hline $\begin{array}{l}\text { Intervention } \\
\text { Category, } \\
\text { Intervention }\end{array}$ & Comparator & Outcome & $\begin{array}{l}\text { Number of } \\
\text { Studies } \\
\text { (Participants) } \\
\text { Author Year } \\
\text { (See } \\
\text { Appendix B } \\
\text { for Full } \\
\text { Citation) }\end{array}$ & \begin{tabular}{|l} 
Study \\
Limitations
\end{tabular} & Consistency & Precision & $\begin{array}{l}\text { Reporting } \\
\text { Bias }\end{array}$ & $\begin{array}{l}\text { Strength of } \\
\text { Evidence }\end{array}$ & $\begin{array}{l}\text { Findings, Direction and } \\
\text { Magnitude of Effect }\end{array}$ \\
\hline $\begin{array}{l}\begin{array}{l}\text { Aerobic } \\
\text { Exercise }\end{array} \\
\text { Dance }\end{array}$ & $\begin{array}{l}\text { Usual care, } \\
\text { previous } \\
\text { activity level or } \\
\text { attention } \\
\text { control }\end{array}$ & Function & $\begin{array}{l}2(\mathrm{~N}=81) \\
\text { Teixera- } \\
\text { Machado, } \\
2017 ; \text { Young, } \\
2019\end{array}$ & Moderate & Consistent & Imprecise & Undetected & Low for benefit & $\begin{array}{l}\text { TUG: MD- } 1.89,95 \% \mathrm{Cl} \\
3.30 \text { to }-0.48, p=0.01 \\
\text { 6MWT: } \mathrm{MD} 40.98,95 \% \mathrm{Cl} \\
2.21 \text { to } 79.75, \mathrm{p}=0.04 \\
\text { 5xSit-to-Stand: } \mathrm{MD}-1.00, \\
95 \% \mathrm{Cl}-2.58 \text { to } 0.55, \mathrm{p}=0.38 \\
\frac{\text { FIM total change score: }}{\text { vs. } 0.03, \mathrm{p}<0.001}\end{array}$ \\
\hline
\end{tabular}

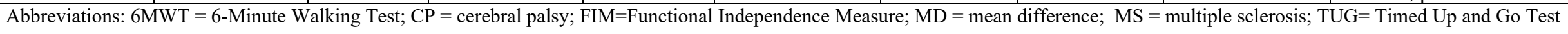


Table H-4. Strength of evidence for Key Question 2: aerobic exercise for spinal cord injury

\begin{tabular}{|c|c|c|c|c|c|c|c|c|c|}
\hline $\begin{array}{l}\text { Intervention } \\
\text { Category, } \\
\text { Intervention }\end{array}$ & Comparator & Outcome & $\begin{array}{l}\text { Number of } \\
\text { Studies } \\
\text { (Participants) } \\
\text { Author Year } \\
\text { (See Appendix } \\
\text { B for Full } \\
\text { Citation) }\end{array}$ & $\begin{array}{l}\text { Study } \\
\text { Limitations }\end{array}$ & Consistency & Precision & $\begin{array}{l}\text { Reporting } \\
\text { Bias }\end{array}$ & $\begin{array}{l}\text { Strength of } \\
\text { Evidence }\end{array}$ & $\begin{array}{l}\text { Findings, Direction and } \\
\text { Magnitude of Effect }\end{array}$ \\
\hline $\begin{array}{l}\text { Aerobic } \\
\text { Exercise } \\
\text { Aquatics }\end{array}$ & $\begin{array}{l}\text { Rehabilita- } \\
\text { tion } \\
\text { exercises }\end{array}$ & $\begin{array}{l}\text { Pulmonary } \\
\text { function }\end{array}$ & $\begin{array}{l}1(20) \\
\text { Jung, } 2014\end{array}$ & Moderate & Unknown & Imprecise & Undetected & Insufficient & $\begin{array}{l}\text { Difference in change scores } \\
\text { between groups: } \\
\text { FVC (L): MD }-1.8(1.3) \text { vs. - } \\
0.31(1.6), p=0.031 \\
\text { FEV1 (L): MD }-1.1(1.2) \text { vs. - } \\
0.21(0.3) ; p=0.038 \\
\text { FER (L/sec):-10.0 (9.7) vs. - } \\
5.4(7.0, p=0.238 \\
\text { FEV1/FVC: }-3.7(2.3) \text { vs. - } \\
2.1(3.4), p=0.234\end{array}$ \\
\hline $\begin{array}{l}\text { Aerobic } \\
\text { Exercise } \\
\text { Cycling (arm } \\
\text { and leg) }\end{array}$ & $\begin{array}{l}\text { Usual care, } \\
\text { previous } \\
\text { activity level } \\
\text { or attention } \\
\text { control }\end{array}$ & Function & $\begin{array}{l}1 \text { RCT (33) } \\
\text { Akkurt, 2017 } \\
1 \text { cohort (45) } \\
\text { Sadowsky, } \\
2013\end{array}$ & Moderate & Inconsistent & Imprecise & Undetected & Insufficient & $\begin{array}{l}\text { A vs. } B \text {, Mean change } \\
\text { scores: } \\
\text { FIM: } 0.5 \text { vs. }-0.5, p=1.00 \\
\text { FIM: } 80 \% \text { vs. } 60 \%, p<0.001\end{array}$ \\
\hline $\begin{array}{l}\text { Aerobic } \\
\text { Exercise } \\
\text { Cycling (arm } \\
\text { and leg) }\end{array}$ & $\begin{array}{l}\text { Usual care, } \\
\text { previous } \\
\text { activity level } \\
\text { or attention } \\
\text { control }\end{array}$ & $\begin{array}{l}\text { Quality of } \\
\text { Life }\end{array}$ & $\begin{array}{l}1 \text { RCT (33) } \\
\text { Akkurt, 2017 } \\
1 \text { cohort (45) } \\
\text { Sadowsky, } \\
2013\end{array}$ & Moderate & Inconsistent & Imprecise & Undetected & Insufficient & $\begin{array}{l}\text { WHOQOL-Bref, p>0.05 } \\
\text { SF-36: total and composite } \\
\text { scores NR; Significant } \\
\text { improvement in physical } \\
\text { function and role limit } \\
\text { physical with FES cycling, } \\
\text { no difference in mental } \\
\text { health subscales }\end{array}$ \\
\hline $\begin{array}{l}\text { Aerobic } \\
\text { Exercise } \\
\text { Cycling } \\
\text { (hand) }\end{array}$ & $\begin{array}{l}\text { Usual care, } \\
\text { previous } \\
\text { activity level } \\
\text { or attention } \\
\text { control }\end{array}$ & ADLs & $\begin{array}{l}1 \text { (33) } \\
\text { Akkurt, } 2017\end{array}$ & Moderate & Unknown & Imprecise & Undetected & Insufficient & CHART-sf, p>0.05 \\
\hline $\begin{array}{l}\text { Aerobic } \\
\text { Exercise } \\
\text { Robot- } \\
\text { Assisted Gait } \\
\text { Training }\end{array}$ & $\begin{array}{l}\text { Usual care } \\
\text { or } \\
\text { overground } \\
\text { walking } \\
\text { without } \\
\text { RAGT }\end{array}$ & ADLs & $\begin{array}{l}2(176) \\
\text { Yildirim, } 2019 \\
\text { Esclarin-Ruz, } \\
2014\end{array}$ & Moderate & Consistent & Imprecise & Undetected & Low for benefit & $\begin{array}{l}\text { FIM: } 69 \text { (31) to } 85(35) \text { vs. } \\
67(36) \text { to } 77(24), p=0.022 \\
\text { FIM/Motor: } p=0.09 \text {, favors } \\
\text { RAGT }\end{array}$ \\
\hline
\end{tabular}




\begin{tabular}{|c|c|c|c|c|c|c|c|c|c|}
\hline $\begin{array}{l}\text { Intervention } \\
\text { Category, } \\
\text { Intervention }\end{array}$ & Comparator & Outcome & $\begin{array}{l}\text { Number of } \\
\text { Studies } \\
\text { (Participants) } \\
\text { Author Year } \\
\text { (See Appendix } \\
\text { B for Full } \\
\text { Citation) }\end{array}$ & $\begin{array}{l}\text { Study } \\
\text { Limitations }\end{array}$ & Consistency & Precision & $\begin{array}{l}\text { Reporting } \\
\text { Bias }\end{array}$ & $\begin{array}{l}\text { Strength of } \\
\text { Evidence }\end{array}$ & $\begin{array}{l}\text { Findings, Direction and } \\
\text { Magnitude of Effect }\end{array}$ \\
\hline $\begin{array}{l}\text { Aerobic } \\
\text { Exercise } \\
\text { Robot- } \\
\text { Assisted Gait } \\
\text { Training }\end{array}$ & $\begin{array}{l}\text { Head-to- } \\
\text { head } \\
\text { comparison } \\
\text { (treadmill } \\
\text { training, } \\
\text { overground } \\
\text { walking) }\end{array}$ & Function & $\begin{array}{l}3(141) \\
\text { Esclarin-Ruz, } \\
2014 \\
\text { Kressler, } 2013 \\
\text { Shin, } 2014\end{array}$ & Moderate & Consistent & Imprecise & Undetected & Low for benefit & $\begin{array}{l}\text { 6MWT: } p=0.047 \text {, favors } \\
\text { RAGT } \\
\text { FIM/Motor: } p=0.09 \text {, favors } \\
\text { RAGT } \\
\text { WISC-II: } p=0.10 \text {, favors } \\
\text { RAGT } \\
\text { WISCI-II: } p=0.01 \\
\text {, favors RAGT } \\
\text { LEMS: } p=0.24 \\
\text { LEMS: } p<0.01 \text {, favors } \\
\text { RAGT } \\
\text { Velocity change: } p>0.05 \text {, } \\
\text { favors treadmill/ overground } \\
\text { walking } \\
\text { SCiM3-M: } 6 \text { vs. } 3, p=0.13\end{array}$ \\
\hline $\begin{array}{l}\text { Robot- } \\
\text { Assisted } \\
\text { Gait } \\
\text { Training }\end{array}$ & $\begin{array}{l}\text { Sham } \\
\text { transcranial } \\
\text { magnetic } \\
\text { stimulation }\end{array}$ & Function & $1(31)$ & Moderate & Unknown & Imprecise & Undetected & Insufficient & $\begin{array}{l}\text { 10MWT: } p=0.09, \text { favors } \\
\text { RAGT } \\
\text { LEMS: } p=0.001 \\
\text { UEMS: } p=0.02 \\
\text { WISCI-II: } p>0.05\end{array}$ \\
\hline
\end{tabular}




\begin{tabular}{|c|c|c|c|c|c|c|c|c|c|}
\hline $\begin{array}{l}\text { Intervention } \\
\text { Category, } \\
\text { Intervention }\end{array}$ & Comparator & Outcome & $\begin{array}{l}\text { Number of } \\
\text { Studies } \\
\text { (Participants) } \\
\text { Author Year } \\
\text { (See Appendix } \\
\text { B for Full } \\
\text { Citation) }\end{array}$ & $\begin{array}{l}\text { Study } \\
\text { Limitations }\end{array}$ & Consistency & Precision & $\begin{array}{l}\text { Reporting } \\
\text { Bias }\end{array}$ & $\begin{array}{l}\text { Strength of } \\
\text { Evidence }\end{array}$ & $\begin{array}{l}\text { Findings, Direction and } \\
\text { Magnitude of Effect }\end{array}$ \\
\hline $\begin{array}{l}\text { Aerobic } \\
\text { Exercise } \\
\text { Robot- } \\
\text { Assisted Gait } \\
\text { Training }\end{array}$ & $\begin{array}{l}\text { Usual } \\
\text { care/no } \\
\text { treatment }\end{array}$ & Function & $\begin{array}{l}3(170) \\
\text { Duffell, } 2014 \\
\text { Yildirim, } 2019 \\
\text { Midik, } 2020\end{array}$ & Moderate & Inconsistent & Imprecise & Undetected & $\begin{array}{l}\text { Low-strength } \\
\text { evidence for no } \\
\text { clear benefit }\end{array}$ & 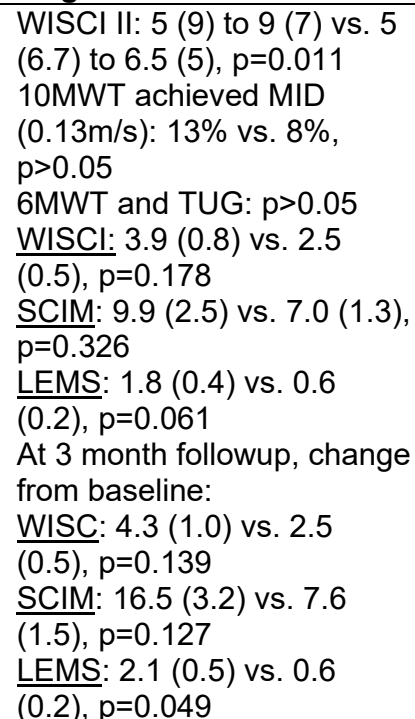 \\
\hline $\begin{array}{l}\text { Aerobic } \\
\text { Exercise } \\
\text { Treadmill }\end{array}$ & $\begin{array}{l}\text { Structured } \\
\text { PT, Aerobic } \\
\text { + Strength } \\
\text { Training }\end{array}$ & Function & $\begin{array}{l}2(55) \\
\text { Alexeeva, } 2011 \\
\text { Giangregorio, } \\
2012 \\
\text { Hitzig, } 2013 \\
\text { Kapadia, } 2014 \\
\text { Craven, } 2017\end{array}$ & Moderate & Consistent & Imprecise & Undetected & $\begin{array}{l}\text { Low-strength } \\
\text { evidence for no } \\
\text { clear benefit }\end{array}$ & $\begin{array}{l}\text { 10MWT (m/s): } 0.30(0.26) \text { to } \\
0.46(0.40) \text { vs. } 0.41(0.34) \text { to } \\
0.51(0.36) \\
\text { 10MWT: } 42.8(46.2) \text { to } 35.2 \\
\text { (40.8) to } 42.2(67.7) \text { vs. } 49.1 \\
(41.7) \text { to } 28.7(8.3) \text { to } 35.1 \\
(18.8), p=0.829 \\
\text { 6MWT: } 187.9(123.4) \text { to } \\
217.1(134.4) \text { to } 232.5 \\
(138.9) \text { vs. } 79.4(83.9) \text { to } \\
130(46.0) \text { to } 126.4(63.8), \\
\text { p=0.096 } \\
\text { TUG: } 43.6(25.5) \text { to } 33.0 \\
(15.7) \text { to } 32.2(19.1) \text { vs. } 61.6 \\
(36.2) \text { to } 49.5(21.9) \text { to } 51.3 \\
(19.6), p=0.138 \\
\text { FIM: } 4.7(1.82) \text { to } 5.19 \\
(1.80) \text { to } 5.19(1.83) \text { vs. } 4.18 \\
(2.14) \text { to } 4.82(1.66) \text { to } 5.09 \\
(2.98), p=0.115\end{array}$ \\
\hline
\end{tabular}




\begin{tabular}{|c|c|c|c|c|c|c|c|c|c|}
\hline $\begin{array}{l}\text { Intervention } \\
\text { Category, } \\
\text { Intervention }\end{array}$ & Comparator & Outcome & $\begin{array}{l}\text { Number of } \\
\text { Studies } \\
\text { (Participants) } \\
\text { Author Year } \\
\text { (See Appendix } \\
\text { B for Full } \\
\text { Citation) } \\
\end{array}$ & $\begin{array}{l}\text { Study } \\
\text { Limitations }\end{array}$ & Consistency & Precision & $\begin{array}{l}\text { Reporting } \\
\text { Bias }\end{array}$ & $\begin{array}{l}\text { Strength of } \\
\text { Evidence }\end{array}$ & $\begin{array}{l}\text { Findings, Direction and } \\
\text { Magnitude of Effect }\end{array}$ \\
\hline $\begin{array}{l}\text { Aerobic } \\
\text { Exercise } \\
\text { Treadmill }\end{array}$ & $\begin{array}{l}\text { Track } \\
\text { training, } \\
\text { Physical } \\
\text { therapy }\end{array}$ & $\begin{array}{l}\text { Quality of } \\
\text { Life }\end{array}$ & $\begin{array}{l}1(35) \\
\text { Alexeeva, } 2011\end{array}$ & Moderate & Unknown & Imprecise & Undetected & Insufficient & $\begin{array}{l}\text { SAWS (Satisfaction with } \\
\text { disabilities and well-being): } \\
39.3((8.3) \text { to } 35.2(8.7) \text { vs. } \\
35.9(6.9) \text { to } 32.4(7.6) \text { vs. } \\
36.6(9.9) \text { to } 29.0(7.9), \\
\text { p>0.05 }\end{array}$ \\
\hline $\begin{array}{l}\text { Aerobic } \\
\text { Exercise } \\
\text { Treadmill }\end{array}$ & $\begin{array}{l}\text { Track } \\
\text { training, } \\
\text { Physical } \\
\text { therapy }\end{array}$ & Balance & $\begin{array}{l}1(35) \\
\text { Alexeeva, } 2011\end{array}$ & Moderate & Unknown & Imprecise & Undetected & Insufficient & $\begin{array}{l}\text { Tinetti Balance Scale (TBS) } \\
9.8(5.4) \text { to } 19.4(5.0) \text { vs. } \\
10.5(3.4) \text { to } 11.9(2.5) \text { vs. } \\
10.1(3.6) \text { to } 12.9(2.7), \\
\text { p<0.05, Improvement from } \\
\text { baseline in track training } \\
\text { and physical therapy groups } \\
\text { but not treadmill group }\end{array}$ \\
\hline
\end{tabular}

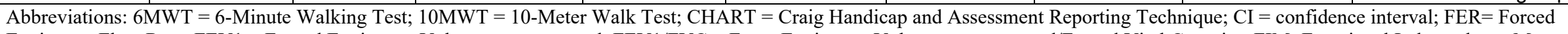

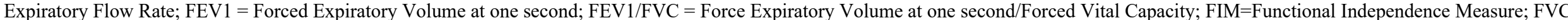

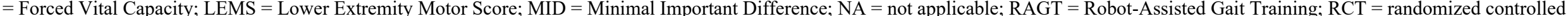

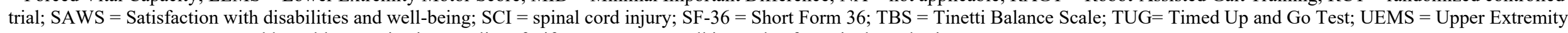
Motor Score; WHOQOL = World Health Organization Quality of Life; WISCI II = Walking Index for Spinal Cord Injury II 
Table H-5. Strength of evidence for Key Question 2: balance exercise for multiple sclerosis

\begin{tabular}{|c|c|c|c|c|c|c|c|c|c|}
\hline $\begin{array}{l}\text { Intervention } \\
\text { Category, } \\
\text { Intervention }\end{array}$ & Comparator & Outcome & $\begin{array}{l}\text { Number of } \\
\text { Studies } \\
\text { (Participants) } \\
\text { Author Year } \\
\text { (See Appendix } \\
\text { B for Full } \\
\text { Citation) }\end{array}$ & $\begin{array}{l}\text { Study } \\
\text { Limitations }\end{array}$ & Consistency & Precision & $\begin{array}{l}\text { Reporting } \\
\text { Bias }\end{array}$ & $\begin{array}{l}\text { Strength of } \\
\text { Evidence }\end{array}$ & $\begin{array}{l}\text { Findings, Direction and } \\
\text { Magnitude of Effect }\end{array}$ \\
\hline $\begin{array}{l}\text { Postural } \\
\text { Control } \\
\text { Balance } \\
\text { training }\end{array}$ & Usual care & $\begin{array}{l}\text { Quality of } \\
\text { life }\end{array}$ & $\begin{array}{l}2(\mathrm{~N}=106) \\
\text { Gandolfi, } 2015 \\
\text { Tollar, } 2020\end{array}$ & Moderate & Consistent & Imprecise & Undetected & Insufficient & $\begin{array}{l}\text { Mean between-group } \\
\text { difference: } \\
\text { MSQoL-54: } 5.02,95 \% \mathrm{Cl}-1.12 \\
\text { to } 9.92 \\
\text { EQ-5 Sum Score: }-0.6(1.15) \text { vs. } \\
0.0 \text { (1.13), } p=0.023\end{array}$ \\
\hline $\begin{array}{l}\text { Postural } \\
\text { Control } \\
\text { Balance } \\
\text { training }\end{array}$ & $\begin{array}{l}\text { Usual care or } \\
\text { waitlist/no } \\
\text { intervention }\end{array}$ & Function & $\begin{array}{l}7(\mathrm{~N}=369) \\
\text { Forsberg, } 2016 \\
\text { Callesen, } 2019 \\
\text { Carling, } 2017 \\
\text { Amiri, 2019 } \\
\text { Tollar, 2020 } \\
\text { Ozkul, 2020 } \\
\text { Arntzen, } 2020\end{array}$ & Moderate & Consistent & Imprecise & Undetected & $\begin{array}{l}\text { Low strength } \\
\text { of evidence } \\
\text { for benefit }\end{array}$ & $\begin{array}{l}\text { Pooled MSWS (4 studies): } \\
\text { 4.66, 95\% } \mathrm{Cl}-6.65 \text { to }-2.67 \\
\text { Pooled TUG ( } 3 \text { studies): } 0.45, \\
\text { 95\% Cl }-1.92 \text { to } 2.82 \\
\text { 2MWT: MD } 16.7,95 \% \mathrm{Cl} 8.15 \\
\text { to } 25.25 \\
\text { 10MWT: MD } 0.48,95 \% \mathrm{Cl} 0.11 \\
\text { to 0.85 } \\
\text { 25FWT (m/s): MD 0.10, } 95 \% \mathrm{Cl} \\
0.00 \text { to } 0.20, \mathrm{p}=0.04 \\
\text { FGA: } 2.1,95 \% \mathrm{Cl} 0.6 \text { to } 3.6, \\
\text { p=0.0079 } \\
\text { 2MWT: }-3.24(3.37), \mathrm{p}=0.34 \\
\text { Sit-to-Stand: } 0.24(2.12), \mathrm{p}=0.17 \\
\text { 10MWT: } 1.49 \text { (3.84), p=0.70 } \\
\text { Significant interaction between } \\
\text { time and group according to } \\
\text { baseline EDSS score for core } \\
\text { muscle endurance and strength, } \\
\text { p<0.05 } \\
\text { MSIS-29: }-6.3 \text { (4.23) vs. } 1.0 \\
\text { (3.46), p=0.008 }\end{array}$ \\
\hline
\end{tabular}




\begin{tabular}{|c|c|c|c|c|c|c|c|c|c|}
\hline $\begin{array}{l}\text { Intervention } \\
\text { Category, } \\
\text { Intervention }\end{array}$ & Comparator & Outcome & $\begin{array}{l}\text { Number of } \\
\text { Studies } \\
\text { (Participants) } \\
\text { Author Year } \\
\text { (See Appendix } \\
\text { B for Full } \\
\text { Citation) }\end{array}$ & $\begin{array}{l}\text { Study } \\
\text { Limitations }\end{array}$ & Consistency & Precision & $\begin{array}{l}\text { Reporting } \\
\text { Bias }\end{array}$ & $\begin{array}{l}\text { Strength of } \\
\text { Evidence }\end{array}$ & $\begin{array}{l}\text { Findings, Direction and } \\
\text { Magnitude of Effect }\end{array}$ \\
\hline $\begin{array}{l}\text { Postural } \\
\text { Control } \\
\text { Balance } \\
\text { training }\end{array}$ & $\begin{array}{l}\text { Usual care or } \\
\text { Waitlist }\end{array}$ & $\begin{array}{l}\text { Balance } \\
\text { BBS }\end{array}$ & $\begin{array}{l}10(\mathrm{~N}=553) \\
\text { Afrasiabifar, } \\
2018 \\
\text { Brichetto, } 2015 \\
\text { Gandolfi, } 2015 \\
\text { Carling, } 2017 \\
\text { Callesen, } 2019 \\
\text { Arntzen, } 2019 \\
\text { Forsberg, } 2016 \\
\text { Amiri, } 2019 \\
\text { Tollar, } 2020 \\
\text { Ozkul, } 2020\end{array}$ & Moderate & Consistent & Imprecise & Undetected & $\begin{array}{l}\text { Moderate for } \\
\text { benefit }\end{array}$ & $\begin{array}{l}\text { Pooled BBS ( } 7 \text { studies): MD - } \\
4.31495 \% \mathrm{Cl}-5.57 \text { to }-2.70 \\
\text { Pooled MiniBEST) } 2 \text { studies: } \\
2.40,95 \% \mathrm{Cl} 1.10 \text { to } 3.70 \\
1 \text { study: Significant interaction } \\
\text { between time and group } \\
\text { according to baseline EDSS } \\
\text { score for static and dynamic } \\
\text { stability p<0.05 }\end{array}$ \\
\hline $\begin{array}{l}\text { Postural } \\
\text { Control } \\
\text { Balance } \\
\text { training }\end{array}$ & $\begin{array}{l}\text { Usual care or } \\
\text { Waitlist }\end{array}$ & $\begin{array}{l}\text { Falls } \\
\text { Near falls }\end{array}$ & $\begin{array}{l}2 \text { (128) } \\
\text { Carling, } 2017 \\
\text { Gandolfi, } 2015\end{array}$ & Moderate & Consistent & Imprecise & Undetected & $\begin{array}{l}\text { Low for } \\
\text { benefit }\end{array}$ & $\begin{array}{l}\text { Falls: }-1.24(1.66), p<0.001 \\
\text { Near Falls: }-8.24(14.78), \\
p=0.002 \\
\text { \# of Falls: } 0.59(0.99) \text { to } 0.03 \\
\text { (0.16) vs. } 0.37(0.54) \text { to } 0.29 \\
(0.34), p=0.005 \text { (post- } \\
\text { intervention); } 0.59(0.99) \text { to } 0.08 \\
(0.27) \text { vs. } 0.37(0.54) \text { to } 0.27 \\
(0.55), p=0.53 \text { ( } 1 \text { month post } \\
\text { treatment) }\end{array}$ \\
\hline $\begin{array}{l}\text { Postural } \\
\text { Control } \\
\text { Balance } \\
\text { training }\end{array}$ & Attention control & Sleep & $\begin{array}{l}1(45) \\
\text { Sadeghi } \\
\text { Bahmani, 2019b }\end{array}$ & Moderate & Unknown & Imprecise & Undetected & Insufficient & $\begin{array}{l}\text { ISI: } 13.46(5.81) \text { to } 10.13(4.92) \\
\text { vs. } 1.71(5.43) \text { to } 11.14(5.39) \\
p>0.05\end{array}$ \\
\hline $\begin{array}{l}\text { Postural } \\
\text { Control } \\
\text { Balance } \\
\text { training }\end{array}$ & $\begin{array}{l}\text { Other active } \\
\text { interventions } \\
\text { (lumbar } \\
\text { stabilization and } \\
\text { task-oriented } \\
\text { training) }\end{array}$ & $\begin{array}{l}\text { Function } \\
\text { 2-Minute } \\
\text { Walk Test }\end{array}$ & $\begin{array}{l}1(\mathrm{~N}=42) \\
\text { Salci, } 2017\end{array}$ & Moderate & Unknown & Imprecise & Undetected & Insufficient & $\begin{array}{l}\text { Mean change from baseline: } \\
10.75 \mathrm{~m} \text { vs. } 25.55 \mathrm{~m} \text { vs. } 18.69 \\
\mathrm{~m} ; \mathrm{p}>0.05\end{array}$ \\
\hline $\begin{array}{l}\text { Postural } \\
\text { Control } \\
\text { Balance } \\
\text { training }\end{array}$ & $\begin{array}{l}\text { Other active } \\
\text { interventions } \\
\text { (lumbar } \\
\text { stabilization and } \\
\text { task-oriented } \\
\text { training) }\end{array}$ & $\begin{array}{l}\text { Balance } \\
\text { BBS }\end{array}$ & $\begin{array}{l}1(\mathrm{~N}=42) \\
\text { Salci, 2017 }\end{array}$ & Moderate & Unknown & Imprecise & Undetected & Insufficient & $\begin{array}{l}\text { Mean change from baseline: } \\
3.57 \text { vs. } 5.78 \text { vs. } 5.57 ; p=0.16\end{array}$ \\
\hline
\end{tabular}




\begin{tabular}{|c|c|c|c|c|c|c|c|c|c|}
\hline $\begin{array}{l}\text { Intervention } \\
\text { Category, } \\
\text { Intervention }\end{array}$ & Comparator & Outcome & $\begin{array}{l}\text { Number of } \\
\text { Studies } \\
\text { (Participants) } \\
\text { Author Year } \\
\text { (See Appendix } \\
\text { B for Full } \\
\text { Citation) }\end{array}$ & $\begin{array}{l}\text { Study } \\
\text { Limitations }\end{array}$ & Consistency & Precision & $\begin{array}{l}\text { Reporting } \\
\text { Bias }\end{array}$ & $\begin{array}{l}\text { Strength of } \\
\text { Evidence }\end{array}$ & $\begin{array}{l}\text { Findings, Direction and } \\
\text { Magnitude of Effect }\end{array}$ \\
\hline $\begin{array}{l}\text { Postural } \\
\text { Control } \\
\text { Hippotherapy }\end{array}$ & $\begin{array}{l}\text { Usual care, } \\
\text { previous activity } \\
\text { level or attention } \\
\text { control }\end{array}$ & $\begin{array}{l}\text { Quality of } \\
\text { life } \\
\text { MSQoL-54 }\end{array}$ & $\begin{array}{l}1(\mathrm{~N}=70) \\
\text { Vermohlen, } \\
2018\end{array}$ & Moderate & Unknown & Imprecise & Undetected & Insufficient & $\begin{array}{l}\text { Mental health score: mean } \\
\text { difference } 12.0,95 \% \mathrm{Cl} 6.2 \text { to } \\
17.7 \\
\text { Physical health score: } 14.4 \text {, } \\
95 \% \mathrm{Cl} 7.5 \text { to } 21.3\end{array}$ \\
\hline $\begin{array}{l}\text { Postural } \\
\text { Control } \\
\text { Hippotherapy }\end{array}$ & $\begin{array}{l}\text { Usual care, } \\
\text { previous activity } \\
\text { level or attention } \\
\text { control }\end{array}$ & $\begin{array}{l}\text { Balance } \\
\text { BBS }\end{array}$ & $\begin{array}{l}1(\mathrm{~N}=70) \\
\text { Vermohlen, } \\
2018\end{array}$ & Moderate & Unknown & Imprecise & Undetected & Insufficient & $\begin{array}{l}\text { Mean difference } 3.07,95 \% \mathrm{Cl} \\
1.00 \text { to } 5.14\end{array}$ \\
\hline $\begin{array}{l}\text { Postural } \\
\text { Control } \\
\text { Hippotherapy }\end{array}$ & $\begin{array}{l}\text { Usual care, } \\
\text { previous activity } \\
\text { level or attention } \\
\text { control }\end{array}$ & $\begin{array}{l}\text { Spasticity } \\
\text { NSR }\end{array}$ & $\begin{array}{l}1(\mathrm{~N}=70) \\
\text { Vermohlen, } \\
2018\end{array}$ & Moderate & Unknown & Imprecise & Undetected & Insufficient & $\begin{array}{l}\text { Mean difference }-0.9,95 \% \mathrm{Cl} \text { - } \\
1.9 \text { to }-0.1\end{array}$ \\
\hline $\begin{array}{l}\text { Postural } \\
\text { Control } \\
\text { Hippotherapy }\end{array}$ & $\begin{array}{l}\text { Usual care, } \\
\text { previous activity } \\
\text { level or attention } \\
\text { control }\end{array}$ & Walking & $\begin{array}{l}1(\mathrm{~N}=33) \\
\text { Moraes, } 2020\end{array}$ & Moderate & Unknown & Imprecise & Undetected & Insufficient & $\begin{array}{l}\text { 6MWT: } 459.06(118.34) \text { to } \\
503.59 \\
(126.38) \text { vs. } 513.00 \\
\text { p<01.97) to } 497.13(88.88), \\
\text { 25FWT: } 6.37(1.70) \text { to } 5.36 \\
\text { (1.43) vs. } 5.82(1.29) \text { to } 5.84 \\
(1.08), p<0.001\end{array}$ \\
\hline $\begin{array}{l}\text { Postural } \\
\text { Control } \\
\text { Tai Chi }\end{array}$ & Usual care & $\begin{array}{l}\text { Depression } \\
\text { Immediatel } \\
\text { y Post- } \\
\text { treatment }\end{array}$ & $\begin{array}{l}1 \text { QENR }(N=32) \\
\text { Burschka, } 2014\end{array}$ & High & Unknown & Imprecise & Undetected & Insufficient & $\begin{array}{l}\text { CES-D mean score } 7.67(5.12) \text {, } \\
p=0.007 \text { vs. } 16.13(11.99) \\
p=0.951 ; \text { favors Tai Chi, } \\
\text { interaction } p=<0.05\end{array}$ \\
\hline $\begin{array}{l}\text { Postural } \\
\text { Control } \\
\text { Tai Chi }\end{array}$ & Usual care & $\begin{array}{l}\text { Quality of } \\
\text { life } \\
\text { Immediatel } \\
\text { y Post- } \\
\text { treatment }\end{array}$ & $\begin{array}{l}\text { 1QENR }(\mathrm{N}=32) \\
\text { Burschka, } 2014\end{array}$ & High & Unknown & Imprecise & Undetected & Insufficient & $\begin{array}{l}\text { QLS mean score } 232.57 \\
(25.62), p=0.012 \text { vs. } 193.81 \\
(36.2), p=0.290, \text { Interaction } \\
p<0.01\end{array}$ \\
\hline $\begin{array}{l}\text { Postural } \\
\text { Control } \\
\text { Tai Chi }\end{array}$ & Usual care & $\begin{array}{l}\text { Balance } \\
\text { Immediatel } \\
\text { y Post- } \\
\text { treatment }\end{array}$ & $\begin{array}{l}\text { 1 QENR } \\
(\mathrm{N}=32) \\
\text { Burschka, } 2014\end{array}$ & High & Unknown & Imprecise & Undetected & Insufficient & $\begin{array}{l}\text { 14-task balance test: } 9.33 \\
(2.26), p=0.031, \text { for the } \\
\text { intervention vs. } 6.53(4.49) \\
p=0.439 \text {; interaction } p<0.05\end{array}$ \\
\hline $\begin{array}{l}\text { Postural } \\
\text { Control } \\
\text { Tai Chi }\end{array}$ & $\begin{array}{l}\text { Psychological } \\
\text { classes and } \\
\text { physical therapy) }\end{array}$ & $\begin{array}{l}\text { Balance } \\
\text { BBS } \\
\text { Immediate- } \\
\text { ly Post- } \\
\text { treatment }\end{array}$ & $\begin{array}{l}1(\mathrm{~N}=34) \\
\text { Azimzadeh, } \\
2015\end{array}$ & High & Unknown & Imprecise & Undetected & Insufficient & $\begin{array}{l}\text { BBS: } 52.25(3.39) \text { to } 53.94 \\
(2.23) \text { vs. } 53.22(2.23) \text { to } 53.61 \\
(2.14), p>0.05\end{array}$ \\
\hline
\end{tabular}




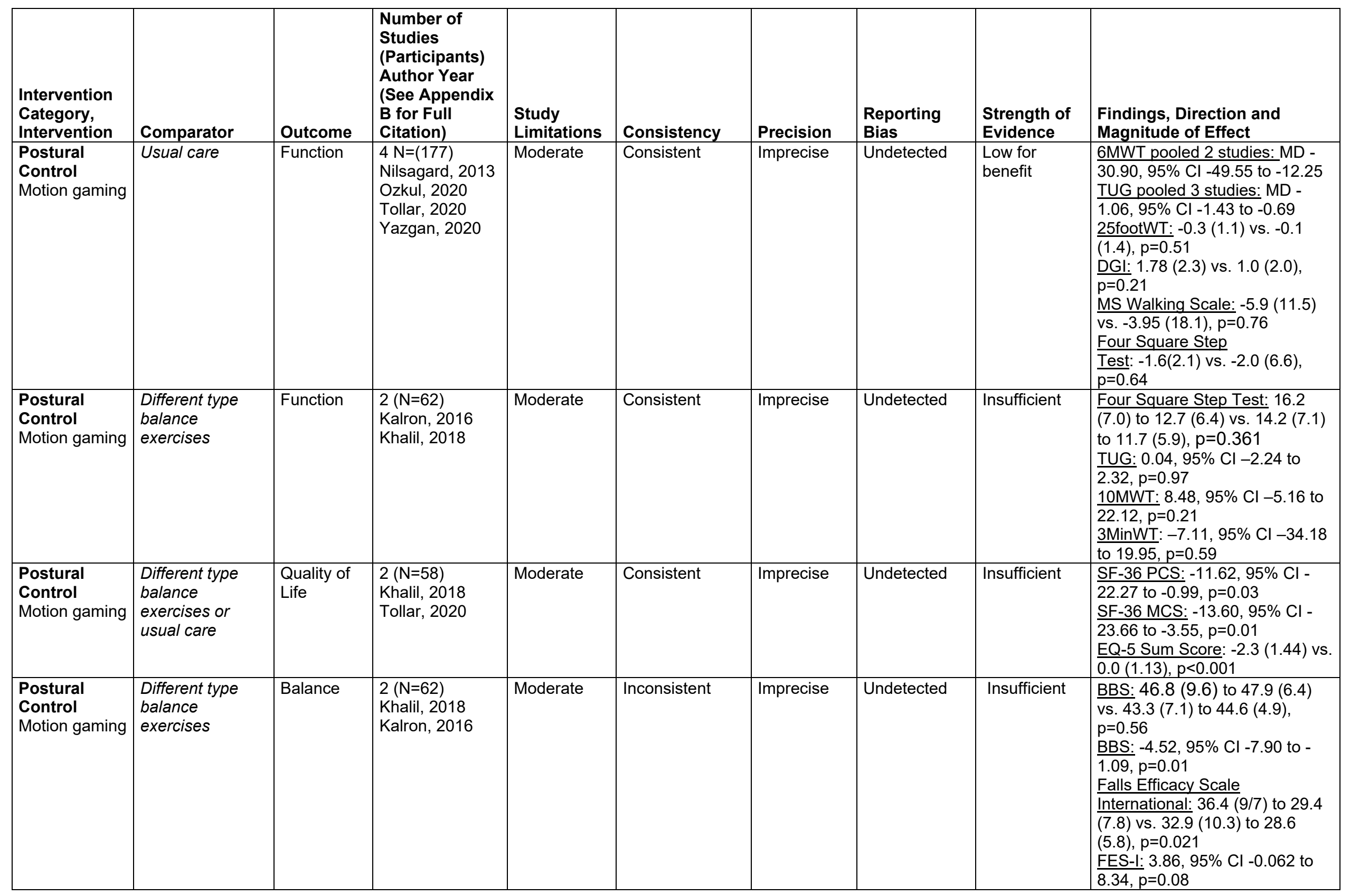




\begin{tabular}{|c|c|c|c|c|c|c|c|c|c|}
\hline $\begin{array}{l}\text { Intervention } \\
\text { Category, } \\
\text { Intervention }\end{array}$ & Comparator & Outcome & $\begin{array}{l}\text { Number of } \\
\text { Studies } \\
\text { (Participants) } \\
\text { Author Year } \\
\text { (See Appendix } \\
\text { B for Full } \\
\text { Citation) }\end{array}$ & $\begin{array}{l}\text { Study } \\
\text { Limitations }\end{array}$ & Consistency & Precision & $\begin{array}{l}\text { Reporting } \\
\text { Bias }\end{array}$ & $\begin{array}{l}\text { Strength of } \\
\text { Evidence }\end{array}$ & $\begin{array}{l}\text { Findings, Direction and } \\
\text { Magnitude of Effect }\end{array}$ \\
\hline \begin{tabular}{l|} 
Postural \\
Control \\
Motion gaming
\end{tabular} & Usual care & Balance & $\begin{array}{l}3(94) \\
\text { Tollar, } 2020 \\
\text { Ozkul, } 2020 \\
\text { Yazgan, } 2020\end{array}$ & Moderate & Consistent & Imprecise & Undetected & $\begin{array}{l}\text { Low for } \\
\text { benefit }\end{array}$ & $\frac{\text { BBS (pooled analysis } 3 \text { trials): }}{\text { MD }-3.43,95 \% \mathrm{Cl}-6.30 \text { to }-0.57}$ \\
\hline $\begin{array}{l}\text { Postural } \\
\text { Control } \\
\text { Whole body } \\
\text { vibration }\end{array}$ & Usual Care & Function & $\begin{array}{l}1(\mathrm{~N}=47) \\
\text { Claerbout, } 2012\end{array}$ & Moderate & Unknown & Imprecise & Undetected & Insufficient & $\begin{array}{l}\text { 3MinWT: } 45.0(42.6) \text { vs. } 20.4 \\
\text { (27.95), p>0.05 } \\
\text { TUG: } 11.32(5.21) \text { to } 11.16 \\
\text { (8.82) vs. } 14.43(3.20) \text { to } 14.57 \\
(4.02), p=0.05, N S\end{array}$ \\
\hline $\begin{array}{l}\text { Postural } \\
\text { Control } \\
\text { Whole body } \\
\text { vibration }\end{array}$ & Usual Care & Balance & $\begin{array}{l}1(\mathrm{~N}=47) \\
\text { Claerbout, } 2012\end{array}$ & Moderate & Unknown & Imprecise & Undetected & Insufficient & $\begin{array}{l}\text { BBS: } 3.9(4.4) \text { vs. } 4.2(6.1) \text { vs. } \\
0.2(7.5), p>0.05 \text { for all } \\
\text { comparisons }\end{array}$ \\
\hline $\begin{array}{l}\text { Postural } \\
\text { Control } \\
\text { Whole body } \\
\text { vibration }\end{array}$ & No treatment & QoL & $\begin{array}{l}1(46) \\
\text { Abbasi, } 2019\end{array}$ & Moderate & Unknown & Imprecise & Undetected & Insufficient & $\begin{array}{l}\text { QOL-54 (PCS): } 4.20(1.73 \\
8.40) \text { vs. }-1.26(-3.28,0) \\
\text { p<0.001 } \\
\text { QOL-54 (MCS): } 5.96(2.71 \\
\begin{array}{l}11.89) \text { vs. }-0.17 \\
\text { p<0.001 }\end{array}\end{array}$ \\
\hline $\begin{array}{l}\text { Postural } \\
\text { Control } \\
\text { Yoga }\end{array}$ & $\begin{array}{l}\text { Usual care, } \\
\text { previous activity } \\
\text { level or attention } \\
\text { control }\end{array}$ & Function & $\begin{array}{l}4(\mathrm{~N}=215) \\
\text { Garrett, 2013a/b } \\
\text { Hogan, } 2014 \\
\text { Young, } 2019 \\
\text { Ahmadi, } 2013\end{array}$ & High & Consistent & Imprecise & Undetected & $\begin{array}{l}\text { Low-strength } \\
\text { evidence for } \\
\text { no clear } \\
\text { benefit }\end{array}$ & 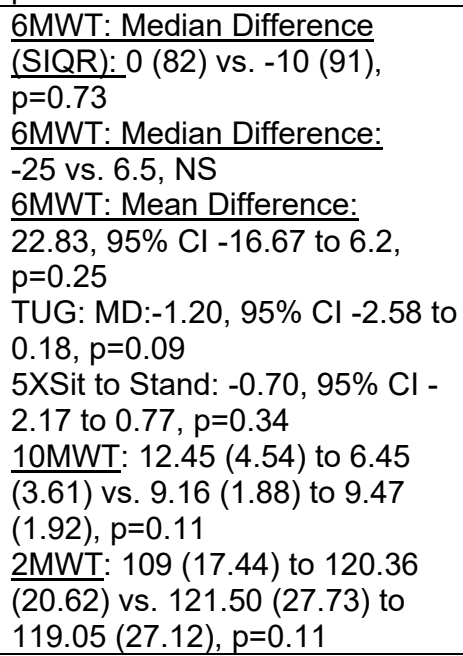 \\
\hline
\end{tabular}




\begin{tabular}{|c|c|c|c|c|c|c|c|c|c|}
\hline $\begin{array}{l}\text { Intervention } \\
\text { Category, } \\
\text { Intervention }\end{array}$ & Comparator & Outcome & $\begin{array}{l}\text { Number of } \\
\text { Studies } \\
\text { (Participants) } \\
\text { Author Year } \\
\text { (See Appendix } \\
\text { B for Full } \\
\text { Citation) }\end{array}$ & $\begin{array}{l}\text { Study } \\
\text { Limitations }\end{array}$ & Consistency & Precision & $\begin{array}{l}\text { Reporting } \\
\text { Bias }\end{array}$ & $\begin{array}{l}\text { Strength of } \\
\text { Evidence }\end{array}$ & $\begin{array}{l}\text { Findings, Direction and } \\
\text { Magnitude of Effect }\end{array}$ \\
\hline $\begin{array}{l}\text { Postural } \\
\text { Control } \\
\text { Yoga }\end{array}$ & $\begin{array}{l}\text { Usual care, } \\
\text { previous activity } \\
\text { level or attention } \\
\text { control }\end{array}$ & $\begin{array}{l}\text { Quality of } \\
\text { Life }\end{array}$ & $\begin{array}{l}4(\mathrm{~N}=241) \\
\text { Doulatabad, } \\
2013 \\
\text { Hasanpour- } \\
\text { Dehkordi, 2014 } \\
\text { Garrett, 2013a/b } \\
\text { Hogan, } 2014\end{array}$ & High & Inconsistent & Imprecise & Undetected & Insufficient & $\begin{array}{l}\text { Mean (SD) MSQoL-54: } \\
4.9 \pm 1.9 \text { vs.6.9 } \pm 1.5 \text { (baseline); } \\
7.4 \pm 2.16 \text { vs. } 6.8 \pm 1.9 \text { (post- } \\
\text { intervention), p=0.001 } \\
\text { Mean Difference SF-36: } \\
1106.41, p<0.001 \\
\text { Median Difference MSIS- } \\
\text { psychological: }-3.7(22.2) \text { vs. } 0 \\
\text { (16.7), p=0.04 } \\
\text { Median (SIQR) MSIS- } \\
\text { psychological: } 14(2.2) \text { baseline, } \\
15(4) \text { post intervention vs. } 17 \\
\text { (4) baseline, } 15 \text { (4.5) post- } \\
\text { intervention, NS } \\
\text { Mean Difference MSIS-physical: } \\
-4.0,95 \% \mathrm{Cl}-7.5 \text { to }-0.5 \text { vs. } \\
0.3,95 \% \mathrm{Cl}-4.0 \text { to } 4.6, \mathrm{p}=0.12 \\
\text { Mean Difference MSIS-physical: } \\
1.3,95 \% \mathrm{Cl}-4.7 \text { to } 7.3 \text { vs. }-4.8, \\
95 \% \mathrm{Cl}-10.4 \text { to }-0.60, \mathrm{NS}\end{array}$ \\
\hline $\begin{array}{l}\text { Postural } \\
\text { Control } \\
\text { Yoga }\end{array}$ & $\begin{array}{l}\text { Usual care, } \\
\text { previous activity } \\
\text { level or attention } \\
\text { control }\end{array}$ & Balance & $\begin{array}{l}2(\mathrm{~N}=49) \\
\text { Hogan, } 2014\end{array}$ & High & Unknown & Imprecise & Undetected & Insufficient & $\begin{array}{l}\text { BBS: MD: } 5.3,95 \% \mathrm{Cl}-3.1 \text { to } \\
7.5 \text { vs. }-3.1,95 \% \mathrm{Cl}-2.8 \text { to } 9.0 \text {, } \\
\text { NS } \\
\text { BBS: } 47.72(6.78) \text { to } 53.81 \\
\text { (3.40) vs. } 44.50(8.48) \text { to } 41.70 \\
(8.48), p=0.07\end{array}$ \\
\hline $\begin{array}{l}\text { Postural } \\
\text { Control } \\
\text { Yoga }\end{array}$ & $\begin{array}{l}\text { Movement to } \\
\text { Music (Dance) }\end{array}$ & Function & $\begin{array}{l}1(\mathrm{~N}=53) \\
\text { Young, } 2019\end{array}$ & Moderate & Unknown & Imprecise & Undetected & Insufficient & $\begin{array}{l}\text { 6MWT: MD: }-18.2,95 \% \mathrm{Cl}-56.4 \\
\text { to } 20.1, p=0.34 \\
\text { TUG: MD } 0.69,95 \% \mathrm{Cl}-0.71 \text { to } \\
2.08, p=0.33 \\
5 X S i t \text { to } S t a n d: \mathrm{MD} 0.30,95 \% \\
\mathrm{Cl}-1.21 \text { to } 1.82, p=0.69\end{array}$ \\
\hline $\begin{array}{l}\text { Postural } \\
\text { Control } \\
\text { Yoga }\end{array}$ & $\begin{array}{l}\text { Undescribed } \\
\text { control }\end{array}$ & $\begin{array}{l}\text { Quality of } \\
\text { Life: } \\
\text { Sexual } \\
\text { Satisfaction }\end{array}$ & $\begin{array}{l}1 \text { RCTs }(\mathrm{N}=60) \\
\text { Najafidoul- } \\
\text { atabad, } 2014\end{array}$ & Moderate & Unknown & Imprecise & Undetected & Insufficient & $\begin{array}{l}\text { Yoga baseline } 1.8 \text { (SD 2.0) to } \\
1.4 \text { (SD 1.5), } p=0.001 \text { versus } \\
\text { women in the control group } \\
\text { (baseline } 2.1 \text { (SD 1.2) to } 2.1 \\
\text { (SD 1.2), } p>0.05 \text {. }\end{array}$ \\
\hline $\begin{array}{l}\text { Postural } \\
\text { Control } \\
\text { Yoga }\end{array}$ & Aerobics & QoL & $\begin{array}{l}1(\mathrm{~N}=40) \\
\text { Hasanpour- } \\
\text { Dehkordi, } 2014\end{array}$ & High & Unknown & Imprecise & Undetected & Insufficient & $\begin{array}{l}\text { SF-36: MD between groups: } \\
\text { 229.32, } p=0.07\end{array}$ \\
\hline
\end{tabular}




\begin{tabular}{|c|c|c|c|c|c|c|c|c|c|}
\hline $\begin{array}{l}\text { Intervention } \\
\text { Category, } \\
\text { Intervention }\end{array}$ & Comparator & Outcome & $\begin{array}{l}\text { Number of } \\
\text { Studies } \\
\text { (Participants) } \\
\text { Author Year } \\
\text { (See Appendix } \\
\text { B for Full } \\
\text { Citation) }\end{array}$ & $\begin{array}{l}\text { Study } \\
\text { Limitations }\end{array}$ & Consistency & Precision & $\begin{array}{l}\text { Reporting } \\
\text { Bias }\end{array}$ & $\begin{array}{l}\text { Strength of } \\
\text { Evidence }\end{array}$ & $\begin{array}{l}\text { Findings, Direction and } \\
\text { Magnitude of Effect }\end{array}$ \\
\hline $\begin{array}{l}\text { Postural } \\
\text { Control } \\
\text { Yoga }\end{array}$ & $\begin{array}{l}\text { Physiotherapist- } \\
\text { led exercise }\end{array}$ & Function & $\begin{array}{l}1(\mathrm{~N}=126) \\
\text { Garrett, 2013a/b }\end{array}$ & High & Unknown & Imprecise & Undetected & Insufficient & $\begin{array}{l}\text { 6MWT: Median Difference } \\
\text { (SIQR): } 0 \text { (82) vs. } 10 \text { (52), NS }\end{array}$ \\
\hline $\begin{array}{l}\text { Postural } \\
\text { Control } \\
\text { Yoga }\end{array}$ & $\begin{array}{l}\text { Physiotherapist- } \\
\text { led exercise }\end{array}$ & $\begin{array}{l}\text { Quality of } \\
\text { Life }\end{array}$ & $\begin{array}{l}1(\mathrm{~N}=126) \\
\text { Garrett, 2013a/b }\end{array}$ & High & Unknown & Imprecise & Undetected & Insufficient & $\begin{array}{l}\text { MSIS (psychological): Median } \\
\text { Difference (SIQR): }-3.7 \text { (22.2) } \\
\text { vs. }-11.1(25.9), \text { NS } \\
\text { MSIS (physical): : MD }-4.0,95 \% \\
\mathrm{CI}-7.5 \text { to }-0.5 \text { vs. }-6.9,95 \% \mathrm{Cl}- \\
10.8 \text { to }-2.9, \mathrm{NS}\end{array}$ \\
\hline $\begin{array}{l}\text { Postural } \\
\text { Control } \\
\text { Yoga }\end{array}$ & Group exercise & Qol & $\begin{array}{l}1(\mathrm{~N}=61) \\
\text { Hogan, } 2014\end{array}$ & High & Unknown & Imprecise & Undetected & Insufficient & $\begin{array}{l}\text { MSIS-29 (psychological): } 18 \\
(5.38) \text { to } 17(4.8) \text { vs. } 8(5.5) \text { to } \\
15 \text { (5.7), p>0.05 } \\
\text { MS-29 (physical): } 54(11.5) \text { to } \\
49.4(12) \text { vs. } 50.5 \text { (9.5) to } 45.9 \\
(10.5) \text { vs, p=NR }\end{array}$ \\
\hline $\begin{array}{l}\text { Postural } \\
\text { Control } \\
\text { Yoga }\end{array}$ & Group exercise & Balance & $\begin{array}{l}1(\mathrm{~N}=61) \\
\text { Hogan, } 2014\end{array}$ & High & Unknown & Imprecise & Undetected & Insufficient & $\begin{array}{l}\text { BBS: } 30.4(11.6) \text { to } 34.2(9.8) \\
\text { vs. } 28.9(9.5) \text { to } 34.5(9.8) \\
\text { p<0.05 }\end{array}$ \\
\hline $\begin{array}{l}\text { Postural } \\
\text { Control } \\
\text { Yoga }\end{array}$ & Group exercise & Function & $\begin{array}{l}1(\mathrm{~N}=61) \\
\text { Hogan, } 2014\end{array}$ & High & Unknown & Imprecise & Undetected & Insufficient & $\begin{array}{l}6 \mathrm{MWT}: 83.9(39.8) \text { to } 100(55) \\
\text { vs. } 101(39.5) \text { to } 121.2(47.4) \\
p>0.05\end{array}$ \\
\hline $\begin{array}{l}\text { Postural } \\
\text { Control } \\
\text { Yoga }\end{array}$ & $\begin{array}{l}\text { One-on-one } \\
\text { exercise }\end{array}$ & Qol & $\begin{array}{l}1(\mathrm{~N}=48) \\
\text { Hogan, } 2014\end{array}$ & High & Unknown & Imprecise & Undetected & Insufficient & $\begin{array}{l}\text { MSIS-29 (psychological): } 18 \\
(5.38) \text { to } 17(4.8) \text { vs. } 14(2.2) \text { to } \\
15(4), p>0.05 \\
\text { MS-29 (physical): } 54(11.5) \text { to } \\
49.4(12) \text { vs. } 48.3(10.5) \text { to } 49.6 \\
(11.6), p=N R\end{array}$ \\
\hline $\begin{array}{l}\text { Postural } \\
\text { Control } \\
\text { Yoga }\end{array}$ & $\begin{array}{l}\text { One-on-one } \\
\text { exercise }\end{array}$ & Balance & $\begin{array}{l}1(\mathrm{~N}=48) \\
\text { Hogan, } 2014\end{array}$ & High & Unknown & Imprecise & Undetected & Insufficient & $\begin{array}{l}\text { BBS: } 30.4(11.6) \text { to } 34.2(9.8) \\
\text { vs. } 22.6(12.6) \text { to } 27.9(11.5) \\
p<0.05\end{array}$ \\
\hline $\begin{array}{l}\text { Postural } \\
\text { Control } \\
\text { Yoga } \\
\end{array}$ & $\begin{array}{l}\text { One-on-one } \\
\text { exercise }\end{array}$ & Function & $\begin{array}{l}1(\mathrm{~N}=48) \\
\text { Hogan, } 2014\end{array}$ & High & Unknown & Imprecise & Undetected & Insufficient & $\begin{array}{l}6 \mathrm{MWT}: 83.9(39.8) \text { to } 100(55) \\
\text { vs. } 70(30) \text { to } 45(54.5), p>0.05\end{array}$ \\
\hline $\begin{array}{l}\text { Postural } \\
\text { Control } \\
\text { Yoga }\end{array}$ & $\begin{array}{l}\text { Fitness } \\
\text { instructor-led } \\
\text { exercise }\end{array}$ & 6MWT & $\begin{array}{l}1(\mathrm{~N}=130) \\
\text { Garrett, 2013a/b }\end{array}$ & High & Unknown & Imprecise & Undetected & Insufficient & $\begin{array}{l}\text { Median Difference (SIQR): } 0 \\
\text { (82) vs. } 20 \text { (61), NS }\end{array}$ \\
\hline
\end{tabular}




\begin{tabular}{|c|c|c|c|c|c|c|c|c|c|}
\hline $\begin{array}{l}\text { Intervention } \\
\text { Category, } \\
\text { Intervention }\end{array}$ & Comparator & Outcome & \begin{tabular}{|l|} 
Number of \\
Studies \\
(Participants) \\
Author Year \\
(See Appendix \\
B for Full \\
Citation) \\
\end{tabular} & $\begin{array}{l}\text { Study } \\
\text { Limitations }\end{array}$ & Consistency & Precision & $\begin{array}{l}\text { Reporting } \\
\text { Bias }\end{array}$ & $\begin{array}{l}\text { Strength of } \\
\text { Evidence }\end{array}$ & $\begin{array}{l}\text { Findings, Direction and } \\
\text { Magnitude of Effect }\end{array}$ \\
\hline \begin{tabular}{|l|} 
Postural \\
Control \\
Yoga
\end{tabular} & \begin{tabular}{|l|} 
Fitness \\
instructor-led \\
exercise
\end{tabular} & $\begin{array}{l}\text { MSIS- } \\
\text { psychologi } \\
\text { cal }\end{array}$ & \begin{tabular}{|l|}
$1(\mathrm{~N}=130)$ \\
Garrett, 2013a/b
\end{tabular} & High & Unknown & Imprecise & Undetected & Insufficient & $\begin{array}{l}\text { MSIS (psychological): Median } \\
\text { Difference (SIQR): }-3.7(22.2) \\
\text { vs. }-3.7 \text { (22.2), NS } \\
\text { MSIS (physical): MD }-4.0,95 \% \\
\mathrm{Cl}-7.5 \text { to }-0.5 \text { vs. }-5.7,95 \% \mathrm{Cl}- \\
9.1 \text { to }-2.4, \mathrm{NS}\end{array}$ \\
\hline
\end{tabular}

Abbreviations: $2 \mathrm{MWT}=$ 2-Minute Walking Test; 3MinWT = 3-Minute Walking Test; 6MWT = 6-Minute Walking Test; 10MWT = 10-Meter Walking Test; 25-FWT=25-Foot Timed Walking Test; BBS=Berg Balance Scale; CES-D= Center for Epidemiological Studies Depression Scale, CI = confidence interval; ISI =Insomnia Severity Index; RCT=randomized controlled trial; MCS = Mental Component Summary; MD = mean difference; MS = multiple sclerosis; MSIS = Multiple Sclerosis Impact Scale; MSQOL= Multiple Sclerosis Quality of Life; NSR = nonsignificant risk;

QENR=quasiexperimental nonrandomized study; QLS=Questionnaire of Life Satisfaction; QOL = Quality of Life; SD = Standard Deviation; SF 36-MCS = Short Form 36 Mental Health Scores; SF 36-PCS = Short Form 36 Physical Component Score; SIQR = Symptom Impact Questionnaire; TUG= Timed Up and Go Test; WT = Walking Time 
Table H-6. Strength of evidence for Key Question 2: balance exercise for cerebral palsy

\begin{tabular}{|c|c|c|c|c|c|c|c|c|c|}
\hline $\begin{array}{l}\text { Intervention } \\
\text { Category, } \\
\text { Intervention }\end{array}$ & Comparator & Outcome & $\begin{array}{l}\text { Number of } \\
\text { Studies } \\
\text { (Participants) } \\
\text { Author Year } \\
\text { (See } \\
\text { Appendix B } \\
\text { for Full } \\
\text { Citation) }\end{array}$ & $\begin{array}{l}\text { Study } \\
\text { Limitations }\end{array}$ & Consistency & Precision & $\begin{array}{l}\text { Reporting } \\
\text { Bias }\end{array}$ & $\begin{array}{l}\text { Strength } \\
\text { of } \\
\text { Evidence }\end{array}$ & $\begin{array}{l}\text { Findings, Direction and Magnitude } \\
\text { of Effect }\end{array}$ \\
\hline $\begin{array}{l}\text { Postural } \\
\text { Control } \\
\text { Balance } \\
\text { Exercises }\end{array}$ & Usual care & Function & $\begin{array}{l}1(28) \\
\text { Curtis, 2018 } \\
2 \text { QENR (66) } \\
\text { Lorentzen, } \\
2015 \\
\text { Bleyenheuft, } \\
2017\end{array}$ & High & $\begin{array}{l}\text { Unclear as } \\
\text { not all } \\
\text { estimates of } \\
\text { effect were } \\
\text { reported }\end{array}$ & Imprecise & Undetected & Insufficient & 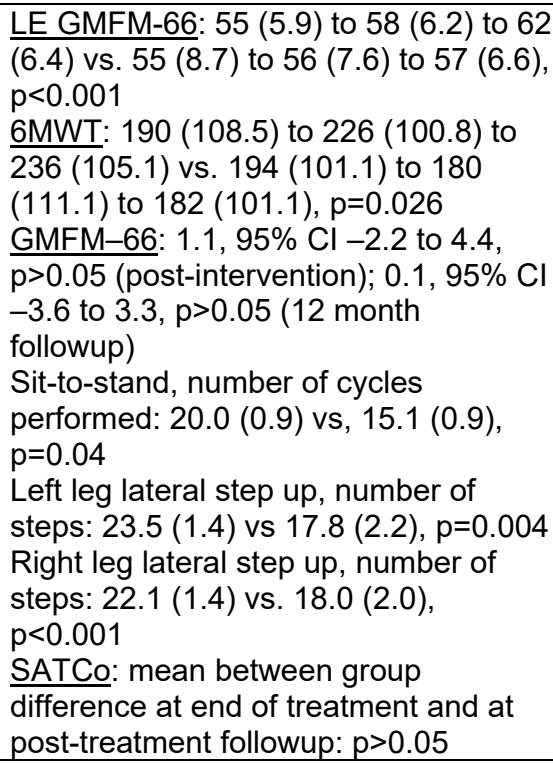 \\
\hline $\begin{array}{l}\text { Postural } \\
\text { Control } \\
\text { Balance } \\
\text { Exercises }\end{array}$ & Usual care & Balance & $\begin{array}{l}1 \text { (28) } \\
\text { Curtis, } 2018 \\
1 \text { QENR (46) } \\
\text { Lorentzen, } \\
2015\end{array}$ & High & Consistent & Imprecise & Undetected & Insufficient & $\begin{array}{l}\text { PBS: } 33(17.5) \text { to } 43(20.1) \text { to } 42(21.3) \\
\text { vs. } 30(23.9) \text { to } 27(22.2) \text { to } 26(23.2), \\
\text { p=0.002 } \\
\text { Romberg Balance Test center of } \\
\text { gravity maintenance area (mm2): } \\
462.2(62.5) \text { vs } 314.6(104.9), p=0.18\end{array}$ \\
\hline
\end{tabular}




\begin{tabular}{|c|c|c|c|c|c|c|c|c|c|}
\hline $\begin{array}{l}\text { Intervention } \\
\text { Category, } \\
\text { Intervention }\end{array}$ & Comparator & Outcome & $\begin{array}{l}\text { Number of } \\
\text { Studies } \\
\text { (Participants) } \\
\text { Author Year } \\
\text { (See } \\
\text { Appendix B } \\
\text { for Full } \\
\text { Citation) }\end{array}$ & $\begin{array}{l}\text { Study } \\
\text { Limitations }\end{array}$ & Consistency & Precision & $\begin{array}{l}\text { Reporting } \\
\text { Bias }\end{array}$ & $\begin{array}{l}\text { Strength } \\
\text { of } \\
\text { Evidence }\end{array}$ & $\begin{array}{l}\text { Findings, Direction and Magnitude } \\
\text { of Effect }\end{array}$ \\
\hline $\begin{array}{l}\text { Postural } \\
\text { Control } \\
\text { Balance } \\
\text { Exercises }\end{array}$ & Usual care & ADLs & $\begin{array}{l}1(28) \\
\text { Curtis, } 2018 \\
1 \text { QENR }(20) \\
\text { Bleyenheuft, } \\
2017 \\
1 \text { cohort (23) } \\
\text { Kim, } 2017\end{array}$ & High & $\begin{array}{l}\text { Unclear as } \\
\text { not all } \\
\text { estimates of } \\
\text { effect were } \\
\text { reported }\end{array}$ & Imprecise & Undetected & Insufficient & $\begin{array}{l}\text { PEDI: } 52(12.4) \text { to } 57 \text { (11.5) to } 60 \\
\text { (10.7) vs. } 51(14.6) \text { to } 51 \text { (15.3) to } 51 \\
\text { (15.8), } p=0.001 \\
\text { PEDI Self Care, PEDI Mobility, PEDI } \\
\text { Mobility Caregiver Assistance: mean } \\
\text { between group difference at end of } \\
\text { treatment and at post-treatment } \\
\text { followup: } p>0.05 \\
\text { Modified Barthel Index, mean change } \\
\text { from baseline: } 2.82(\mathrm{SD} 1.25) \text { vs } 1.58 \\
\text { (SD 1.38), } p<0.05 ; \text { MD } 1.24,95 \% \mathrm{Cl} \\
0.09 \text { to } 2.34, p=0.04\end{array}$ \\
\hline
\end{tabular}




\begin{tabular}{|c|c|c|c|c|c|c|c|c|c|}
\hline $\begin{array}{l}\text { Intervention } \\
\text { Category, } \\
\text { Intervention }\end{array}$ & Comparator & Outcome & $\begin{array}{l}\text { Number of } \\
\text { Studies } \\
\text { (Participants) } \\
\text { Author Year } \\
\text { (See } \\
\text { Appendix B } \\
\text { for Full } \\
\text { Citation) } \\
\end{array}$ & $\begin{array}{l}\text { Study } \\
\text { Limitations }\end{array}$ & Consistency & Precision & $\begin{array}{l}\text { Reporting } \\
\text { Bias }\end{array}$ & $\begin{array}{l}\text { Strength } \\
\text { of } \\
\text { Evidence }\end{array}$ & $\begin{array}{l}\text { Findings, Direction and Magnitude } \\
\text { of Effect }\end{array}$ \\
\hline $\begin{array}{l}\text { Postural } \\
\text { Control } \\
\text { Hippotherapy }\end{array}$ & $\begin{array}{l}\text { Usual care, } \\
\text { previous } \\
\text { activity level } \\
\text { or attention } \\
\text { control }\end{array}$ & Function & $\begin{array}{l}\text { 5 RCTs, } 2 \\
\text { QENRs } \\
\text { (N=333) } \\
\text { Deutz, 2018 } \\
\text { Herrero, } 2012 \\
\text { Kwon, 2015 } \\
\text { Silva e Borges, } \\
2011 \\
\text { Kwon, } 2011 \\
\text { Park, 2014 } \\
\text { Mutoh, 2019 }\end{array}$ & Moderate & Consistent & Imprecise & Undetected & $\begin{array}{l}\text { Low for } \\
\text { benefit }\end{array}$ & 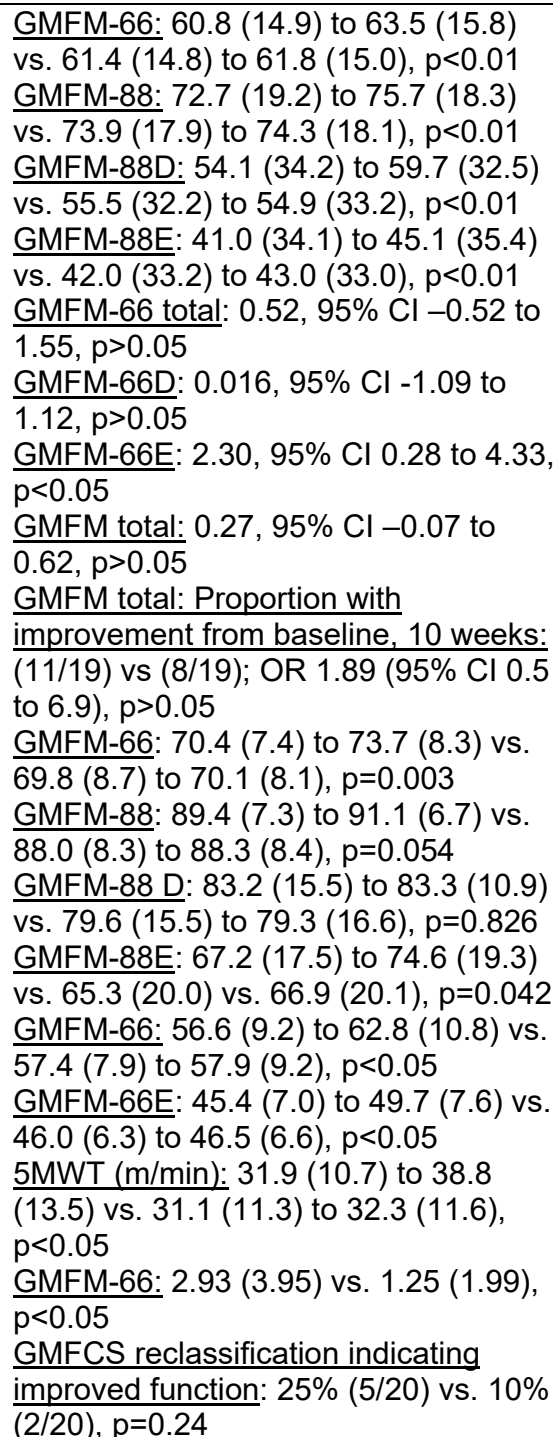 \\
\hline
\end{tabular}




\begin{tabular}{|c|c|c|c|c|c|c|c|c|c|}
\hline $\begin{array}{l}\text { Intervention } \\
\text { Category, } \\
\text { Intervention }\end{array}$ & Comparator & Outcome & $\begin{array}{l}\text { Number of } \\
\text { Studies } \\
\text { (Participants) } \\
\text { Author Year } \\
\text { (See } \\
\text { Appendix B } \\
\text { for Full } \\
\text { Citation) }\end{array}$ & $\begin{array}{l}\text { Study } \\
\text { Limitations }\end{array}$ & Consistency & Precision & $\begin{array}{l}\text { Reporting } \\
\text { Bias }\end{array}$ & $\begin{array}{l}\text { Strength } \\
\text { of } \\
\text { Evidence }\end{array}$ & $\begin{array}{l}\text { Findings, Direction and Magnitude } \\
\text { of Effect }\end{array}$ \\
\hline $\begin{array}{l}\text { Postural } \\
\text { Control } \\
\text { Hippotherapy }\end{array}$ & $\begin{array}{l}\text { Usual care, } \\
\text { previous } \\
\text { activity level } \\
\text { or attention } \\
\text { control }\end{array}$ & $\begin{array}{l}\text { Quality of } \\
\text { life }\end{array}$ & $\begin{array}{l}2 \text { RCT (97) } \\
\text { Deutz, } 2018 \\
\text { Mutoh, } 2019\end{array}$ & High & Consistent & Imprecise & Undetected & Insufficient & $\begin{array}{l}\text { No difference between groups in Child } \\
\text { Health Questionnaire-28 psychosocial } \\
\text { or physical subscale scores or on } \\
\text { KIDSCREEN-27 parental scale scores } \\
\text { WHOQOL (positive feelings): } 3.1(1) \text { to } \\
4.1(1) \text { vs. } 3.1(0.9) \text { to } 3.4(1), p<0.05 \\
\text { WHOQOL (self-esteem): } 2.9(1.2) \text { to } \\
4.0(0.7) \text { vs. } 3.3(1.1) \text { to } 3.7(0.7), \\
p<0.05 \\
\text { WHOQOL (negative feelings): } 2.9(0.8) \\
\text { to } 2.8(0.7) \text { vs. } 2.8\left(0.8^{*} \text { to } 2.8(0.8),\right. \\
p>0.05\end{array}$ \\
\hline $\begin{array}{l}\text { Postural } \\
\text { Control } \\
\text { Hippotherapy }\end{array}$ & $\begin{array}{l}\text { Usual care, } \\
\text { previous } \\
\text { activity level } \\
\text { or attention } \\
\text { control }\end{array}$ & $\begin{array}{l}\text { Spactiicity } \\
\text { MAS }\end{array}$ & $\begin{array}{l}1 \mathrm{RCT}(\mathrm{N}=44) \\
\text { Lucena-Anton, } \\
2018\end{array}$ & Moderate & Unknown & Imprecise & Undetected & Insufficient & $\begin{array}{l}\text { MAS, left adductors: } \\
2.50 \text { (SD 1.05) vs. } 2.54 \text { (SD 1.22), } \\
p=0.040 \\
\text { MAS, right adductors: } \\
1.77 \text { (SD 1.26) vs. } 2.31 \text { (SD 1.24), } \\
p=0.047\end{array}$ \\
\hline $\begin{array}{l}\text { Postural } \\
\text { Control } \\
\text { Hippotherapy }\end{array}$ & $\begin{array}{l}\text { Usual care, } \\
\text { previous } \\
\text { activity level } \\
\text { or attention } \\
\text { control }\end{array}$ & $\begin{array}{l}\text { Balance } \\
\text { PBS }\end{array}$ & $\begin{array}{l}\text { 1 RCT, } 2 \\
\text { QENRs } \\
(\mathrm{N}=150) \\
\text { Kwon, } 2015 \\
\text { Kwon, } 2011 \\
\text { Lee, } 2014 \\
\end{array}$ & Moderate & Consistent & Imprecise & Undetected & $\begin{array}{l}\text { Low for } \\
\text { benefit }\end{array}$ & $\begin{array}{l}\text { Pooled analysis ( } 3 \text { studies): MD } 3.14 \text {, } \\
95 \% \mathrm{Cl} 0.21 \text { to } 6.07, p=0.036\end{array}$ \\
\hline $\begin{array}{l}\text { Postural } \\
\text { Control } \\
\text { Hippotherapy }\end{array}$ & $\begin{array}{l}\text { Usual care, } \\
\text { previous } \\
\text { activity level } \\
\text { or attention } \\
\text { control }\end{array}$ & $\begin{array}{l}\text { Sitting } \\
\text { Balance } \\
\text { SAS }\end{array}$ & $\begin{array}{l}2 \text { RCT }(\mathrm{N}=83) \\
\text { Herrero, } 2012 \\
\text { Matusiak- } \\
\text { Wieczorek, } \\
2020 \\
1 \text { QENR } \\
\text { (N=39) } \\
\text { Matusiak- } \\
\text { Wieczorek, } \\
2016\end{array}$ & HModerate & Consistent & Imprecise & Undetected & Insufficient & $\begin{array}{l}\text { SAS: MD: Treatment effect: } 0.26(0.65) \\
\text { vs. }-0.21(0.92), p>0.05 \\
\text { SAS: } 14.42(4.39) \text { to } 15.63(3.65) \\
\text { vs. } 15.50(3.14) \text { to } 15.75(3.19), \\
p=0.010 \\
\text { SAS: } 10.93(3.97) \text { to } 13.13(3.46) \text { vs. } \\
\text { 14.87 (3.27) to } 15.13(3.36), p<0.001 \\
\text { (but worse disability in control group) }\end{array}$ \\
\hline
\end{tabular}




\begin{tabular}{|c|c|c|c|c|c|c|c|c|c|}
\hline $\begin{array}{l}\text { Intervention } \\
\text { Category, } \\
\text { Intervention }\end{array}$ & Comparator & Outcome & $\begin{array}{l}\text { Number of } \\
\text { Studies } \\
\text { (Participants) } \\
\text { Author Year } \\
\text { (See } \\
\text { Appendix B } \\
\text { for Full } \\
\text { Citation) }\end{array}$ & $\begin{array}{l}\text { Study } \\
\text { Limitations }\end{array}$ & Consistency & Precision & $\begin{array}{l}\text { Reporting } \\
\text { Bias }\end{array}$ & $\begin{array}{l}\text { Strength } \\
\text { of } \\
\text { Evidence }\end{array}$ & $\begin{array}{l}\text { Findings, Direction and Magnitude } \\
\text { of Effect }\end{array}$ \\
\hline $\begin{array}{l}\text { Postural } \\
\text { Control } \\
\text { Motion gaming }\end{array}$ & $\begin{array}{l}\text { Neurodeve- } \\
\text { lopmental } \\
\text { training }\end{array}$ & $\begin{array}{l}\text { Function } \\
\text { (QUEST) }\end{array}$ & $\begin{array}{l}2(\mathrm{~N}=61) \\
\text { Acar, 2016 } \\
\text { Zoccolillo, } \\
2015\end{array}$ & High & Consistent & Imprecise & Undetected & Insufficient & $\begin{array}{l}\text { QUEST (dissociated movement): } 80.1 \\
\text { (7.73) to } 85.6 \text { (8.54) vs. } 81.4 \text { (10.70) to } \\
86.4(8.78), p>0.05 \\
\text { QUEST (grasp): } 42.2(18.76) \text { to } 47.1 \\
\text { (16.64) vs. } 53.0 \text { (16.45) to } 55.7 \\
\text { (15.30), p>0.05 } \\
\text { QUEST (weight bearing): } 60.2 \text { to } 72.7 \\
\text { (19.60) vs. } 75.4 \text { (19.97) to } 77.3 \\
\text { (15.43), p>0.05 } \\
\text { QUEST (extension): } 72.9(14.78) \text { to } \\
77.0(12.05) \text { vs. } 71.0(23.53) \text { to } 74.0 \\
\text { (23.36), p>0.05 } \\
\text { QUEST: } 76(21) \text { to } 81 \text { (20) vs. } 74(20) \\
\text { to 78 (20), p>0.05 }\end{array}$ \\
\hline $\begin{array}{l}\text { Postural } \\
\text { Control } \\
\text { Motion gaming } \\
\text { (arm } \\
\text { exoskeleton) } \\
\end{array}$ & $\begin{array}{l}\text { Conventional } \\
\text { rehabilitation }\end{array}$ & $\begin{array}{l}\text { Function } \\
\text { (QUEST) }\end{array}$ & $\begin{array}{l}1(\mathrm{~N}=30) \\
\text { El-Shamy, } \\
2018\end{array}$ & Moderate & Unknown & Imprecise & Undetected & Insufficient & $\begin{array}{l}\text { QUEST total: } 61.9(2) \text { to } 84.6(2.7) \text { vs. } \\
62.3(1.8) \text { to } 79.1(2) ; \text { MD } 5.9,95 \% \mathrm{Cl} \\
3.7 \text { to } 7.3, p<0.05\end{array}$ \\
\hline $\begin{array}{l}\text { Postural } \\
\text { Control } \\
\text { Motion gaming }\end{array}$ & $\begin{array}{l}P C \text { gaming } \\
\text { using mouse } \\
\text { or traditional } \\
\text { balance } \\
\text { training }\end{array}$ & Function & $\begin{array}{l}3(\mathrm{~N}=126) \\
\text { Hsieh, } 2018 \\
\text { Tarakci, } 2016 \\
\text { Hsieh, } 2020\end{array}$ & Moderate & Inconsistent & Imprecise & Undetected & Insufficient & 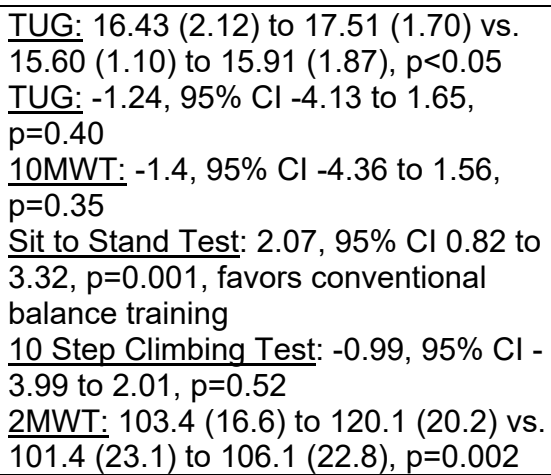 \\
\hline
\end{tabular}




\begin{tabular}{|c|c|c|c|c|c|c|c|c|c|}
\hline $\begin{array}{l}\text { Intervention } \\
\text { Category, } \\
\text { Intervention }\end{array}$ & Comparator & Outcome & $\begin{array}{l}\text { Number of } \\
\text { Studies } \\
\text { (Participants) } \\
\text { Author Year } \\
\text { (See } \\
\text { Appendix B } \\
\text { for Full } \\
\text { Citation) }\end{array}$ & $\begin{array}{l}\text { Study } \\
\text { Limitations }\end{array}$ & Consistency & Precision & $\begin{array}{l}\text { Reporting } \\
\text { Bias }\end{array}$ & $\begin{array}{l}\text { Strength } \\
\text { of } \\
\text { Evidence }\end{array}$ & $\begin{array}{l}\text { Findings, Direction and Magnitude } \\
\text { of Effect }\end{array}$ \\
\hline $\begin{array}{l}\text { Postural } \\
\text { Control } \\
\text { Motion gaming }\end{array}$ & $\begin{array}{l}\text { PC gaming } \\
\text { using mouse } \\
\text { or traditional } \\
\text { balance } \\
\text { training or } \\
\text { usual } \\
\text { physical } \\
\text { activity }\end{array}$ & Balance & $\begin{array}{l}4(\mathrm{~N}=146) \\
\text { Hsieh, 2018 } \\
\text { Tarakci, } 2016 \\
\text { Hsieh, } 2020 \\
\text { Pourazar, } \\
2020\end{array}$ & Moderate & Consistent & Imprecise & Undetected & $\begin{array}{l}\text { Low for } \\
\text { benefit }\end{array}$ & $\begin{array}{l}\text { BBS: } 44.74(2.75) \text { to } 48.81(4.74) \text { vs. } \\
44.39(2.33) \text { to } 45.37(2.68), p<0.05 \\
\frac{\text { Wiibalance: }}{p<0.001} .05,95 \% \mathrm{Cl} 0.64 \text { to } 1.46 \text {, } \\
\frac{\text { Tilt-table: } 11.00,95 \% \mathrm{Cl} 4.74 \text { to } 17.26 \text {, }}{\mathrm{p}=0.001} \\
\frac{\text { Tight-rope walking, heading in soccer, }}{\text { and ski slalom: } p<0.001} \\
\text { PBS-total: } 29.9(5.3) \text { to } 35.8(5.5) \text { vs. } \\
32.3(7.5) \text { to } 34.4(5.9), p=0.002 \\
\text { Dynamic balance was improved in the } \\
\text { anterior, posterolateral, and } \\
\text { posteromedial directions with virtual } \\
\text { reality dance game compare with the } \\
\text { control group, } p=0.001 \text { all comparisons }\end{array}$ \\
\hline $\begin{array}{l}\text { Postural } \\
\text { Control } \\
\text { Whole body } \\
\text { vibration }\end{array}$ & Usual care & Walking & $\begin{array}{l}2(\mathrm{~N}=50) \\
\text { Lee, } 2013 \\
\text { Ahmadizadeh, } \\
2020\end{array}$ & Moderate & Inconsistent & Imprecise & Undetected & Insufficient & $\begin{array}{l}\text { Walking speed (meters/second): } 0.37 \\
(0.04) \text { to } 0.48(0.06) \text { vs. } 0.39(0.05) \text { to } \\
0.40(0.05), p=0.001 \\
\text { 6MWT: } 158.8(100.24) \text { to } 189.45 \\
(115.47) \text { vs. } 194(78.82) \text { to } 271.5 \\
(60.81), p=0.04 \text { (favors control) }\end{array}$ \\
\hline
\end{tabular}

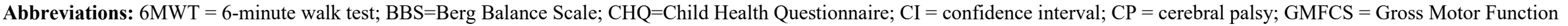

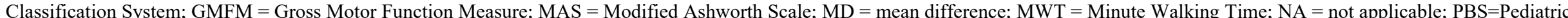

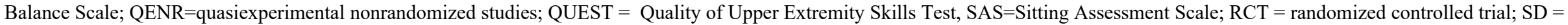
Standard Deviation; TUG= Timed Up and Go Test; WHOQOL = World Health Organization Quality of Life 
Table H-7. Strength of evidence for Key Question 2: balance exercise for spinal cord injury

\begin{tabular}{|c|c|c|c|c|c|c|c|c|c|}
\hline $\begin{array}{l}\text { Intervention } \\
\text { Category, } \\
\text { Intervention }\end{array}$ & Comparator & Outcome & $\begin{array}{l}\text { Number of } \\
\text { Studies } \\
\text { (Participants) } \\
\text { Author Year } \\
\text { (See Appendix } \\
\text { B for Full } \\
\text { Citation) }\end{array}$ & $\begin{array}{l}\text { Study } \\
\text { Limitations }\end{array}$ & Consistency & Precision & $\begin{array}{l}\text { Reporting } \\
\text { Bias }\end{array}$ & $\begin{array}{l}\text { Strength of } \\
\text { Evidence }\end{array}$ & $\begin{array}{l}\text { Findings, Direction and } \\
\text { Magnitude of Effect }\end{array}$ \\
\hline $\begin{array}{l}\text { Postural } \\
\text { Control } \\
\text { Balance } \\
\text { training }\end{array}$ & Usual care & Balance & $\begin{array}{l}2(\mathrm{~N}=60) \\
\text { Norouzi, } 2019 \\
\text { Hota, } 2020\end{array}$ & Moderate & Consistent & Imprecise & Undetected & Insufficient & $\begin{array}{l}\text { BBS pooled } 2 \text { studies: MD - } \\
4.53,95 \% \mathrm{Cl}-6.46,-2.61 \\
\text { (favors balance exercises) }\end{array}$ \\
\hline $\begin{array}{l}\text { Postural } \\
\text { Control } \\
\text { Tai Chi }\end{array}$ & Usual care & $\begin{array}{l}\text { Quality of life } \\
\text { Immediately } \\
\text { post- } \\
\text { treatment }\end{array}$ & $\begin{array}{l}1(\mathrm{~N}=40) \\
\mathrm{Qi}, 2018\end{array}$ & Moderate & Inconsistent & Imprecise & Undetected & Insufficient & $\begin{array}{l}\text { WHOQOL-BREF mean score: } \\
12.23(1.65) \text { vs. } 10.87(1.08) \\
p=0.04 \text {, favors Tai Chi }\end{array}$ \\
\hline $\begin{array}{l}\text { Postural } \\
\text { Control } \\
\text { Motion } \\
\text { Gaming }\end{array}$ & Usual care & $\begin{array}{l}\text { Dynamic } \\
\text { balance }\end{array}$ & $\begin{array}{l}1(26) \\
\text { Tak, } 2015\end{array}$ & Moderate & Unknown & Imprecise & Undetected & Insufficient & $\begin{array}{l}\text { T-shirt test (s): } 29.5(10.95) \text { to } \\
22.60(8.28) \text { vs. } 23.59(11.35) \\
\text { to } 22.15(12.28), p<0.05\end{array}$ \\
\hline $\begin{array}{l}\text { Postural } \\
\text { Control } \\
\text { Whole body } \\
\text { vibration }\end{array}$ & Usual care & Function & $\begin{array}{l}1(\mathrm{~N}=28) \\
\ln , 2018\end{array}$ & Moderate & Unknown & Imprecise & Undetected & Insufficient & $\begin{array}{l}\text { 10MWT: } 29.3(9.0) \text { to } 25.8 \\
\text { (8.1) vs. } 28.8(7.2) \text { to } 27.5 \\
(6.3), p=0.005 \\
\text { TUG: } 13.7(3.1) \text { to } 11.4(2.8) \\
\text { vs. } 14.7(4.5) \text { to } 13.7(4.1), \\
p=0.016\end{array}$ \\
\hline
\end{tabular}

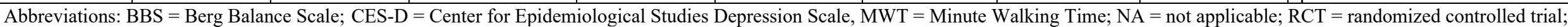

$\mathrm{SCI}=$ spinal cord injury; WHOQOL-BREF $=$ World Health Organization Quality of Life 
Table H-8. Strength of evidence for Key Question 2: muscle strength exercise for multiple sclerosis

\begin{tabular}{|c|c|c|c|c|c|c|c|c|c|}
\hline $\begin{array}{l}\text { Intervention } \\
\text { Category, } \\
\text { Intervention }\end{array}$ & Comparator & Outcome & $\begin{array}{l}\text { Number of } \\
\text { Studies } \\
\text { (Participants) } \\
\text { Author Year } \\
\text { (See Appendix } \\
\text { B for Full } \\
\text { Citation) } \\
\end{array}$ & $\begin{array}{l}\text { Study } \\
\text { Limitations }\end{array}$ & Consistency & Precision & $\begin{array}{l}\text { Reporting } \\
\text { Bias }\end{array}$ & $\begin{array}{l}\text { Strength of } \\
\text { Evidence }\end{array}$ & $\begin{array}{l}\text { Findings, Direction and } \\
\text { Magnitude of Effect }\end{array}$ \\
\hline $\begin{array}{l}\text { Strength } \\
\text { Interventions } \\
\text { Muscle } \\
\text { Strength } \\
\text { Exercise }\end{array}$ & $\begin{array}{l}\text { Usual care, } \\
\text { previous } \\
\text { activity level } \\
\text { or attention } \\
\text { control }\end{array}$ & $\begin{array}{l}\text { Walking } \\
\text { Immediately } \\
\text { Post- } \\
\text { treatment }\end{array}$ & $\begin{array}{l}\text { 6MWT } \\
5 \text { (N=161) } \\
\text { Kalron, 2017 } \\
\text { Duff, 2018 } \\
\text { Dalgas, } \\
\text { 2009/2010 } \\
\text { Callesen, } 2019 \\
\text { Tollar, 2020 } \\
\text { 2MWT } \\
3 \text { (N=153) } \\
\text { Kjolhede, 2016 } \\
\text { Kalron, 2017 } \\
\text { Dodd, 2011 } \\
\text { 10MWT } \\
\text { 2 (N=132) } \\
\text { Fox, 2016 } \\
\text { Dalgas, } \\
\text { 2009/2010 } \\
\text { MswS-12 } \\
3 \text { (N=165) } \\
\text { Kalron, 2017 } \\
\text { Fox, 2016 } \\
\text { Callesen, } 2019 \\
\\
\text { 25FWT } \\
2 \text { (N=65) } \\
\text { Kjolhede, 2016 } \\
\text { Callesen, 2019 }\end{array}$ & Moderate & Consistent & Imprecise & Undetected & $\begin{array}{l}\text { Low-strength } \\
\text { evidence for } \\
\text { no clear } \\
\text { benefit }\end{array}$ & $\begin{array}{l}\text { 6MWT } \\
\text { p5 trials, MD } \\
-12.69 \text { meters, } 95 \% \mathrm{Cl}-29.45 \\
\text { to } 4.07, \mathrm{I}^{2}=0 \% \\
\text { 2MWT } \\
\text { p3 trials, MD }-3.3 \text { meters, } 95 \% \\
\mathrm{Cl}-11.92 \text { to } 2.81, \mathrm{I}^{2}=0 \% \\
\text { 10MWT } \\
\text { p3 trials, MD }-1.04 \text { seconds, } \\
95 \% \mathrm{Cl}-2.48 \text { to } 0.69, \mathrm{I}^{2}=0 \% \\
\text { MSWS-12 }(0-100 \text { scale) } \\
-1.36,95 \% \mathrm{Cl}-4.83 \text { to } 2.10, \\
\mathrm{I}^{2}=26 \% \\
\mathbf{2 5 F W T} \\
\text { p2 trials, MD }-0.07 \mathrm{~m} / \mathrm{s}, 95 \% \\
\mathrm{Cl}-0.19 \text { to } 0.05, \mathrm{I}^{2}=47 \%\end{array}$ \\
\hline $\begin{array}{l}\text { Strength } \\
\text { Interventions } \\
\text { Muscle } \\
\text { Strength } \\
\text { Exercise }\end{array}$ & $\begin{array}{l}\text { Usual care, } \\
\text { previous } \\
\text { activity level } \\
\text { or attention } \\
\text { control }\end{array}$ & $\begin{array}{l}\text { Walking } \\
\text { Short term }\end{array}$ & $\begin{array}{l}\text { 10MWT } \\
2(\mathrm{~N}=132) \\
\text { Fox, 2016 } \\
\text { Dalgas, } \\
2009 / 2010\end{array}$ & Moderate & Consistent & Imprecise & Undetected & $\begin{array}{l}\text { Low-strength } \\
\text { evidence for } \\
\text { no clear } \\
\text { benefit }\end{array}$ & $\begin{array}{l}\text { 10MWT } \\
\text { p2 trials, MD }-1.27,95 \% \mathrm{Cl} \\
-2.75 \text { to } 0.22, I^{2}=0 \%\end{array}$ \\
\hline
\end{tabular}




\begin{tabular}{|c|c|c|c|c|c|c|c|c|c|}
\hline $\begin{array}{l}\text { Intervention } \\
\text { Category, } \\
\text { Intervention }\end{array}$ & Comparator & Outcome & $\begin{array}{l}\text { Number of } \\
\text { Studies } \\
\text { (Participants) } \\
\text { Author Year } \\
\text { (See Appendix } \\
\text { B for Full } \\
\text { Citation) }\end{array}$ & $\begin{array}{l}\text { Study } \\
\text { Limitations }\end{array}$ & Consistency & Precision & $\begin{array}{l}\text { Reporting } \\
\text { Bias }\end{array}$ & $\begin{array}{l}\text { Strength of } \\
\text { Evidence }\end{array}$ & $\begin{array}{l}\text { Findings, Direction and } \\
\text { Magnitude of Effect }\end{array}$ \\
\hline $\begin{array}{l}\text { Strength } \\
\text { Interventions } \\
\text { Muscle } \\
\text { Strength } \\
\text { Exercise }\end{array}$ & $\begin{array}{l}\text { Usual care, } \\
\text { previous } \\
\text { activity level } \\
\text { or attention } \\
\text { control }\end{array}$ & $\begin{array}{l}\text { Functional } \\
\text { capacity } \\
\text { Immediately } \\
\text { Post } \\
\text { treatment }\end{array}$ & $\begin{array}{l}\text { TUG } \\
3(\mathrm{~N}=113) \\
\text { Duff, 2018 } \\
\text { Bulguroglu, } 2017 \\
\text { Kalron, 2017 } \\
\text { SSST } \\
2(\mathrm{~N}=65) \\
\text { Marandi, 2013a/b } \\
\text { Callesen, 2019 }\end{array}$ & Moderate & Consistent & Imprecise & Undetected & $\begin{array}{l}\text { Low-strength } \\
\text { evidence for } \\
\text { no clear } \\
\text { benefit }\end{array}$ & $\begin{array}{l}\text { TUG } \\
\text { MD }-1.30 \text { seconds, } 95 \% \mathrm{Cl}- \\
4.38 \text { to }-1.78, \mathrm{I}^{2}=0 \% \\
\text { SSST } \\
\text { MD }-2.88,95 \% \mathrm{Cl}-7.51 \text { to } \\
1.74, \mathrm{I}^{2}=95 \%\end{array}$ \\
\hline $\begin{array}{l}\text { Strength } \\
\text { Interventions } \\
\text { Muscle } \\
\text { Strength } \\
\text { Exercise }\end{array}$ & $\begin{array}{l}\text { Usual care, } \\
\text { previous } \\
\text { activity level } \\
\text { or attention } \\
\text { control }\end{array}$ & $\begin{array}{l}\text { Quality of } \\
\text { Life } \\
\text { Immediately } \\
\text { Post- } \\
\text { treatment }\end{array}$ & $\begin{array}{l}\text { MSQol/SF36 } \\
\text { MCS } \\
3(\mathrm{~N}=100) \\
\text { Duff, 2018 } \\
\text { Bulguroglu, } 2017 \\
\text { Dalgas, 2010 }\end{array}$ & Moderate & Consistent & Imprecise & Undetected & $\begin{array}{l}\text { Low-strength } \\
\text { evidence for } \\
\text { no clear } \\
\text { benefit }\end{array}$ & $\begin{array}{l}\text { MSQol/SF36 MCS } \\
\text { (0-100 scale) } \\
\text { MD -3.48, 95\% Cl }-6.61 \text { to - } \\
0.27, \mathrm{I}^{2}=0 \%\end{array}$ \\
\hline $\begin{array}{l}\text { Strength } \\
\text { Interventions } \\
\text { Muscle } \\
\text { Strength } \\
\text { Exercise }\end{array}$ & $\begin{array}{l}\text { Usual care, } \\
\text { previous } \\
\text { activity level } \\
\text { or attention } \\
\text { control }\end{array}$ & $\begin{array}{l}\text { Quality of } \\
\text { Life } \\
\text { Immediately } \\
\text { Post- } \\
\text { treatment }\end{array}$ & $\begin{array}{l}\text { MSQol/SF36 } \\
\text { PCS } \\
3(\mathrm{~N}=100) \\
\text { Duff, 2018 } \\
\text { Bulguroglu, } 2017 \\
\text { Dalgas, 2010 }\end{array}$ & Moderate & Consistent & Imprecise & Undetected & $\begin{array}{l}\text { Low-strength } \\
\text { evidence for } \\
\text { no clear } \\
\text { benefit }\end{array}$ & $\begin{array}{l}\text { MSQol/SF36 PCS } \\
\text { (0-100 scale) } \\
\text { MD }-2.77,95 \% \mathrm{Cl}-6.88 \text { to } \\
3.12, \mathrm{I}^{2}=34 \%\end{array}$ \\
\hline $\begin{array}{l}\text { Strength } \\
\text { Interventions } \\
\text { Muscle } \\
\text { Strength } \\
\text { Exercise }\end{array}$ & $\begin{array}{l}\text { Usual care, } \\
\text { previous } \\
\text { activity level } \\
\text { or attention } \\
\text { control }\end{array}$ & $\begin{array}{l}\text { Quality of } \\
\text { Life } \\
\text { Immediately } \\
\text { Post- } \\
\text { treatment }\end{array}$ & $\begin{array}{l}\text { EQ5D total } \\
1(\mathrm{~N}=26) \\
\text { Tollar, } 2020\end{array}$ & Moderate & Consistent & Imprecise & Undetected & $\begin{array}{l}\text { Low-strength } \\
\text { evidence for } \\
\text { no clear } \\
\text { benefit }\end{array}$ & $\begin{array}{l}13.9(1.44) \text { vs. } 13.3(0.89) \\
\text { (baseline) } \\
-0.5(1.16) \text { vs. } 0.0(1.3) \\
\text { (followup) } \\
\text { Difference } \\
-0.5,95 \% \mathrm{Cl}-1.5 \text { to } 0.5\end{array}$ \\
\hline
\end{tabular}




\begin{tabular}{|c|c|c|c|c|c|c|c|c|c|}
\hline $\begin{array}{l}\text { Intervention } \\
\text { Category, } \\
\text { Intervention }\end{array}$ & Comparator & Outcome & $\begin{array}{l}\text { Number of } \\
\text { Studies } \\
\text { (Participants) } \\
\text { Author Year } \\
\text { (See Appendix } \\
\text { B for Full } \\
\text { Citation) } \\
\end{array}$ & $\begin{array}{l}\text { Study } \\
\text { Limitations }\end{array}$ & Consistency & Precision & $\begin{array}{l}\text { Reporting } \\
\text { Bias }\end{array}$ & $\begin{array}{l}\text { Strength of } \\
\text { Evidence }\end{array}$ & $\begin{array}{l}\text { Findings, Direction and } \\
\text { Magnitude of Effect }\end{array}$ \\
\hline $\begin{array}{l}\text { Strength } \\
\text { Interventions } \\
\text { Muscle } \\
\text { Strength } \\
\text { Exercise }\end{array}$ & $\begin{array}{l}\text { Usual care, } \\
\text { previous } \\
\text { activity level } \\
\text { or attention } \\
\text { control }\end{array}$ & Balance & $\begin{array}{l}\text { ABCS } \\
2(\mathrm{~N}=132) \\
\text { Bulguroglu, } 2017 \\
\text { Fox, 2016 } \\
\text { FABS } \\
1(\mathrm{~N}=30) \\
\text { Duff, 2018 } \\
\\
\text { BBS } \\
2(\mathrm{~N}=71) \\
\text { Kalron, 2017 } \\
\text { Tollar, 2020 } \\
\mathbf{6}(\mathrm{N}=319)\end{array}$ & Moderate & Consistent & Imprecise & Undetected & $\begin{array}{l}\text { Low-strength } \\
\text { evidence for } \\
\text { no clear } \\
\text { benefit }\end{array}$ & 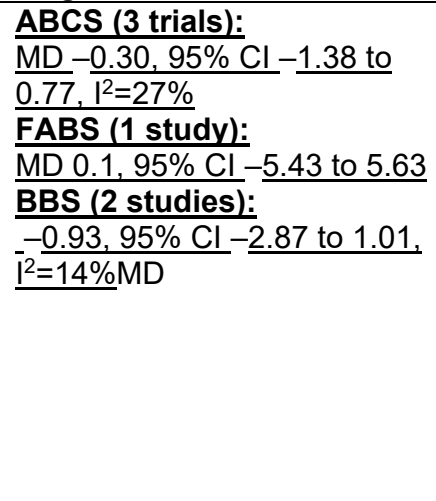 \\
\hline
\end{tabular}

Abbreviations: 6MWT = 6-Minute Walk Test; 10MWT = 10-Meter Walk Test; 25FWT = 25-Feet Walk Test; ABCS = Activities-Specific Balance Confidence Scale; BBB Berg Balance Scale; CI = confidence interval; EQ-5D = EuroQOL-5 Dimension Questionnaire; FABS = Fullerton Advanced Balance Scale; MD = mean difference; CI = confidence interval; MS = multiple sclerosis; MSQoLMCS = Multiple Sclerosis Quality of Life-54 instrument Mental Component Score; MSQoL-PCS = Multiple Sclerosis Quality of Life-54 instrument Physical Component Score; MSWS-12 =

Multiple Sclerosis Walking Scale; NA = not applicable; RCT = randomized controlled trial; SF-36 MCS = Short-Form 36 Mental Component Summary; SF-36 PCS = Short-Form 36 Physical Component Score; SSST = Six Spot Step Test; TUG = Timed Up and Go Test 
Table H-9. Strength of evidence for Key Question 2: muscle strength exercise for cerebral palsy

\begin{tabular}{|c|c|c|c|c|c|c|c|c|c|}
\hline $\begin{array}{l}\text { Intervention } \\
\text { Category, } \\
\text { Intervention }\end{array}$ & Comparator & Outcome & $\begin{array}{l}\text { Number of } \\
\text { Studies } \\
\text { (Participants) } \\
\text { Author Year } \\
\text { (See Appendix } \\
\text { B for Full } \\
\text { Citation) }\end{array}$ & $\begin{array}{l}\text { Study } \\
\text { Limitations }\end{array}$ & Consistency & Precision & $\begin{array}{l}\text { Reporting } \\
\text { Bias }\end{array}$ & $\begin{array}{l}\text { Strength of } \\
\text { Evidence }\end{array}$ & $\begin{array}{l}\text { Findings, Direction and } \\
\text { Magnitude of Effect }\end{array}$ \\
\hline $\begin{array}{l}\text { Strength } \\
\text { Interventions } \\
\text { Muscle } \\
\text { Strength } \\
\text { Exercise }\end{array}$ & Usual care & $\begin{array}{l}\text { Walking } \\
\text { Immediately } \\
\text { Post- } \\
\text { treatment }\end{array}$ & $\begin{array}{l}\text { 1MWT } \\
1 \text { (N=51) } \\
\text { Scholtes, 2008, } \\
2010,2011 \\
\\
\text { 6MWT } \\
1 \text { (N=49) } \\
\text { Taylor, } \\
\text { 2013/Bania, } 2016 \\
\text { 10MWT } \\
2 \text { (N=91) } \\
\text { Scholtes, 2008, } \\
2010,2011 \\
\text { Elnaggar, 2019 } \\
\text { Gait Profile } \\
\text { Score } \\
1 \text { (N=49) } \\
\text { Taylor, } \\
\text { 2013/Bania, } 2016\end{array}$ & Moderate & Consistent & Imprecise & Undetected & $\begin{array}{l}\text { Low-strength } \\
\text { evidence for } \\
\text { no clear } \\
\text { benefit }\end{array}$ & $\begin{array}{l}\text { 1MWT } \\
\text { difference } 0.7 \mathrm{~m} / \mathrm{s}, 95 \% \mathrm{Cl} \\
-0.23 \text { to } 0.9 \\
\text { 6MWT } \\
\text { difference } 0.0 \text { meters, } 95 \% \mathrm{Cl} \\
-41.6 \text { to } 41.6 \\
\text { 10MWT } \\
\text { MD }-0.26 \text { seconds, } 95 \% \mathrm{Cl}- \\
0.95 \text { to } 0.43,\left.\right|^{2}=44 \% \\
\text { Gait Profile Score } \\
\text { difference } 0.2 \text { degrees, } 95 \% \mathrm{Cl} \\
-0.86 \text { to } 1.26\end{array}$ \\
\hline $\begin{array}{l}\text { Strength } \\
\text { Interventions } \\
\text { Muscle } \\
\text { Strength } \\
\text { Exercise }\end{array}$ & Usual care & $\begin{array}{l}\text { Walking } \\
\text { Short term }\end{array}$ & $\begin{array}{l}\text { 1MWT } \\
1 \text { (N=51) } \\
\text { Scholtes, 2008, } \\
2010,2011 \\
\\
\text { 6MWT } \\
1 \text { (N=49) } \\
\text { Taylor, } \\
\text { 2013/Bania, } 2016 \\
\\
\text { 10MWT } \\
1(\mathrm{~N}=51) \\
\text { Scholtes, 2008, } \\
2010,2011\end{array}$ & Moderate & Consistent & Imprecise & Undetected & $\begin{array}{l}\text { Low-strength } \\
\text { evidence for } \\
\text { no clear } \\
\text { benefit }\end{array}$ & $\begin{array}{l}\text { 1MWT } \\
\text { difference } 0.0 \mathrm{~m} / \mathrm{s}, 95 \% \mathrm{Cl} \\
-0.15 \text { to } 0.15 \\
\text { 6MWT } \\
\text { difference } 10.7 \text { meters, } 95 \% \mathrm{Cl} \\
-32.3 \text { to } 53.7 \\
\text { 10MWT } \\
\text { difference }-0.06 \mathrm{~m} / \mathrm{s}, 95 \% \mathrm{Cl} \\
-0.17 \text { to } 0.05\end{array}$ \\
\hline
\end{tabular}




\begin{tabular}{|c|c|c|c|c|c|c|c|c|c|}
\hline $\begin{array}{l}\text { Intervention } \\
\text { Category, } \\
\text { Intervention }\end{array}$ & Comparator & Outcome & $\begin{array}{l}\text { Number of } \\
\text { Studies } \\
\text { (Participants) } \\
\text { Author Year } \\
\text { (See Appendix } \\
\text { B for Full } \\
\text { Citation) }\end{array}$ & $\begin{array}{l}\text { Study } \\
\text { Limitations }\end{array}$ & Consistency & Precision & $\begin{array}{l}\text { Reporting } \\
\text { Bias }\end{array}$ & $\begin{array}{l}\text { Strength of } \\
\text { Evidence }\end{array}$ & $\begin{array}{l}\text { Findings, Direction and } \\
\text { Magnitude of Effect }\end{array}$ \\
\hline $\begin{array}{l}\text { Strength } \\
\text { Interventions } \\
\text { Muscle } \\
\text { Strength } \\
\text { Exercise }\end{array}$ & Usual care & $\begin{array}{l}\text { Functional } \\
\text { Capacity } \\
\text { Immediately } \\
\text { Post- } \\
\text { treatment }\end{array}$ & $\begin{array}{l}\text { GMFM-66 } \\
1 \text { (N=51) } \\
\text { Scholtes, 2008, } \\
2010,2011 \\
\\
\text { GMFM-66(D) } \\
1 \text { ( }=49) \\
\text { Taylor, } \\
\text { 2013/Bania, } 2016 \\
\\
\text { GMFM-66(E) } \\
1 \text { ( }=49) \\
\text { Taylor, } \\
2013 / \text { Bania, } 2016 \\
\\
\text { GMFM-88 } \\
1 \text { (N=25) } \\
\text { Cho, 2020 } \\
\text { 30SEC LAT } \\
\text { STEP-UP } \\
1 \text { (N=51) } \\
\text { Scholtes, 2008, } \\
2010,2011 \\
\text { QUEST } \\
1(\mathrm{~N}=34) \\
\text { Kara, 2020 } \\
\text { COPM } \\
1(\mathrm{~N}=34) \\
\text { Kara, 2020 }\end{array}$ & Moderate & Consistent & Imprecise & Undetected & $\begin{array}{l}\text { Low-strength } \\
\text { evidence for } \\
\text { no clear } \\
\text { benefit }\end{array}$ & $\begin{array}{l}\text { GMFM-66 (0-100 scale) } \\
\text { difference } 1.3,95 \% \mathrm{Cl}-3.10 \text { to } \\
5.70 \\
\text { GMFM-66(D) }(0-100 \text { scale) } \\
\text { difference } 1.9,95 \% \mathrm{Cl}-2.58 \text { to } \\
6.38 \\
\text { GMFM-66 (E) }(0-100 \text { scale) } \\
\text { difference }-0.6,95 \% \mathrm{Cl}-8.29 \\
\text { to } 7.09 \\
\text { GMFM-88 (0-100 scale) } \\
71.78 \text { (21.1) vs. } 63.48(27.5) \\
\text { (postinervention), p=NR } \\
\\
\text { 30SEC LAT STEP-UP } \\
\text { difference } 0.5 \text { repetitions, } 95 \% \\
\text { CI -1.26 to } 2.26 \\
\text { QUEST total } \\
8.88 \text { (6.51) vs. } 2.22 \text { (4.74), MD } \\
6.65(95 \% \text { Cl } 2.4 \text { to } 10.9), \\
\text { p=0.001 } \\
\text { COPM total: } \\
6.12 \text { (2.33) vs. } 0.41 \text { (1.56), MD } \\
5.71 \text { (95\% Cl } 4.2 \text { to } 7.2), \\
\text { p<0.001 }\end{array}$ \\
\hline $\begin{array}{l}\text { Strength } \\
\text { Interventions } \\
\text { Muscle } \\
\text { Strength } \\
\text { Exercise }\end{array}$ & Usual care & $\begin{array}{l}\text { Functional } \\
\text { Capacity } \\
\text { Short term }\end{array}$ & $\begin{array}{l}\text { 30SEC LAT } \\
\text { STEP-UP } \\
1 \text { (N=51) } \\
\text { Scholtes, 2008, } \\
2010,2011\end{array}$ & Moderate & Unknown & Imprecise & Undetected & Insufficient & $\begin{array}{l}\text { 30SEC LAT STEP-UP } \\
\text { difference } 0.4 \text { repetitions, } 95 \% \\
\mathrm{Cl}-1.53 \text { to } 2.33\end{array}$ \\
\hline
\end{tabular}




\begin{tabular}{|c|c|c|c|c|c|c|c|c|c|}
\hline $\begin{array}{l}\text { Intervention } \\
\text { Category, } \\
\text { Intervention }\end{array}$ & Comparator & Outcome & $\begin{array}{l}\text { Number of } \\
\text { Studies } \\
\text { (Participants) } \\
\text { Author Year } \\
\text { (See Appendix } \\
\text { B for Full } \\
\text { Citation) }\end{array}$ & $\begin{array}{l}\text { Study } \\
\text { Limitations }\end{array}$ & Consistency & Precision & $\begin{array}{l}\text { Reporting } \\
\text { Bias }\end{array}$ & $\begin{array}{l}\text { Strength of } \\
\text { Evidence }\end{array}$ & $\begin{array}{l}\text { Findings, Direction and } \\
\text { Magnitude of Effect }\end{array}$ \\
\hline $\begin{array}{l}\text { Strength } \\
\text { Interventions } \\
\text { Muscle } \\
\text { Strength } \\
\text { Exercise } \\
\end{array}$ & $\begin{array}{l}\text { Neuromus- } \\
\text { cular } \\
\text { electrical } \\
\text { stimulation }\end{array}$ & $\begin{array}{l}\text { Functional } \\
\text { Capacity } \\
\text { Immediately } \\
\text { Post- } \\
\text { treatment }\end{array}$ & $\begin{array}{l}\text { GMFM-66 } \\
1(N=100) \\
\text { Qi, 2018a }\end{array}$ & Moderate & Unknown & Imprecise & Undetected & Insufficient & $\begin{array}{l}\text { GMFM-66 (0-100 scale) } \\
\text { difference }-13.4,95 \% \mathrm{Cl} \\
-16.90 \text { to }-9.90\end{array}$ \\
\hline $\begin{array}{l}\text { Strength } \\
\text { Interventions } \\
\text { Muscle } \\
\text { Strength } \\
\text { Exercise } \\
\end{array}$ & $\begin{array}{l}\text { Neuromus- } \\
\text { cular } \\
\text { electrical } \\
\text { stimulation }\end{array}$ & $\begin{array}{l}\text { Functional } \\
\text { Capacity } \\
\text { Short term }\end{array}$ & $\begin{array}{l}\text { GMFM-66 } \\
1(N=100) \\
\text { Qi, 2018a }\end{array}$ & Moderate & Unknown & Imprecise & Undetected & Insufficient & $\begin{array}{l}\text { GMFM-66 (0-100 scale) } \\
\text { difference and }-12.5,95 \% \mathrm{Cl} \\
-16.26 \text { to }-8.74\end{array}$ \\
\hline $\begin{array}{l}\text { Strength } \\
\text { Interventions } \\
\text { Muscle } \\
\text { Strength } \\
\text { Exercise }\end{array}$ & Usual care & Balance & $\begin{array}{l}\text { F-SFRT, } \\
\text { S-SFRT } \\
1(\mathrm{~N}=25) \\
\text { Cho 2020 } \\
\text { PBS } \\
1(\mathrm{~N}=62) \\
\text { Tedla 2014 }\end{array}$ & High & Consistent & Imprecise & Undetected & Insufficient & $\begin{array}{l}\text { F-SFRT: } 21.62 \text { (6.87) vs. } \\
28.17(14.49) \text { (baseline) } \\
26.65(7.92), p=0.000 \text { vs. } \\
25.37(10.20), p=0.261 \\
\text { (postintervention) } \\
\text { S-SFRT: } 11.57 \text { (5.72) vs. } \\
15.52(10.43) \text { (baseline) } \\
16.21(5.37), p=0.003 \text { vs. } \\
15.95(8.26), p=0.793 \\
\text { (postintervention) } \\
\text { PBS: } 7.23(3.350) \text { vs. } 1.87 \\
\text { (1.074), } p<0.001\end{array}$ \\
\hline
\end{tabular}

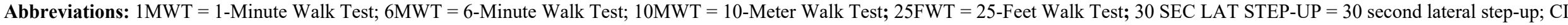

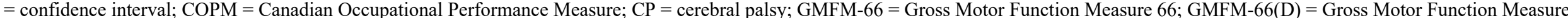

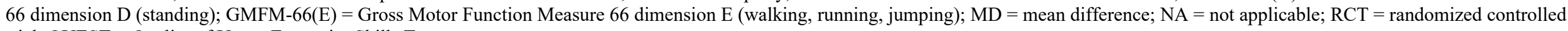
trial; QUEST = Quality of Upper Extremity Skills Test 
Table H-10. Strength of evidence for Key Question 2: muscle strength exercise for spinal cord injury

\begin{tabular}{|c|c|c|c|c|c|c|c|c|c|}
\hline $\begin{array}{l}\text { Intervention } \\
\text { Category, } \\
\text { Intervention }\end{array}$ & Comparator & Outcome & $\begin{array}{l}\text { Number of } \\
\text { Studies } \\
\text { (Participants) } \\
\text { Author Year } \\
\text { (See Appendix } \\
\text { B for Full } \\
\text { Citation) }\end{array}$ & $\begin{array}{l}\text { Study } \\
\text { Limitations }\end{array}$ & Consistency & Precision & $\begin{array}{l}\text { Reporting } \\
\text { Bias }\end{array}$ & $\begin{array}{l}\text { Strength of } \\
\text { Evidence }\end{array}$ & $\begin{array}{l}\text { Findings, Direction and } \\
\text { Magnitude of Effect }\end{array}$ \\
\hline $\begin{array}{l}\text { Strength } \\
\text { Interventions } \\
\text { Muscle } \\
\text { Strength } \\
\text { Exercise }\end{array}$ & Usual care & $\begin{array}{l}\text { Quality of } \\
\text { Life } \\
\text { Immediately } \\
\text { Post- } \\
\text { treatment }\end{array}$ & $\begin{array}{l}\text { SF-36 } \\
1(\mathrm{~N}=98) \\
\text { Chen, 2016 }\end{array}$ & Moderate & Unknown & Imprecise & Undetected & Insufficient & $\begin{array}{l}\text { SF-36 subscales } \\
\text { Physical function }(\mathbf{0}-100) \\
\text { difference } 26.7,95 \% \mathrm{Cl} 24.61 \\
\text { to } 28.79 \\
\text { Social function }(\mathbf{0}-100) \\
\text { difference } 28.9,95 \% \mathrm{Cl} 26.06 \\
\text { to } 31.74 \\
\text { Role emotional }(\mathbf{0}-100) \\
\text { difference } 22.0,95 \% \mathrm{Cl} 20.11 \\
\text { to } 23.89 \\
\text { Mental health }(\mathbf{0}-100) \\
\text { difference } 21.0,95 \% \mathrm{Cl} 19.10 \\
\text { to } 22.90 \\
\text { Body pain }(0-100) \\
\text { difference } 0.0,95 \% \mathrm{Cl}-2.74 \text { to } \\
2.74\end{array}$ \\
\hline
\end{tabular}

Abbreviations: $\mathrm{CI}=$ confidence interval; $\mathrm{NA}=$ not applicable; $\mathrm{RCT}$ = randomized controlled trial; SCI = spinal cord injury; SF-36 = Short-Form 36 questionnaire 
Table H-11. Strength of evidence for Key Question 2: multimodal exercise that includes strengthening for multiple sclerosis

\begin{tabular}{|c|c|c|c|c|c|c|c|c|c|}
\hline $\begin{array}{l}\text { Intervention } \\
\text { Category, } \\
\text { Intervention }\end{array}$ & Comparator & Outcome & $\begin{array}{l}\text { Number of } \\
\text { Studies } \\
\text { (Participants) } \\
\text { Author Year } \\
\text { (See Appendix B } \\
\text { for Full Citation) }\end{array}$ & $\begin{array}{l}\text { Study } \\
\text { Limitations }\end{array}$ & Consistency & Precision & $\begin{array}{l}\text { Reporting } \\
\text { Bias }\end{array}$ & $\begin{array}{l}\text { Strength of } \\
\text { Evidence }\end{array}$ & $\begin{array}{l}\text { Findings, Direction and } \\
\text { Magnitude of Effect }\end{array}$ \\
\hline $\begin{array}{l}\text { Multimodal } \\
\text { Exercise } \\
\text { Progressive } \\
\text { resistance or } \\
\text { strength } \\
\text { exercise } \\
\text { plus aerobic } \\
\text { or balance }\end{array}$ & $\begin{array}{l}\text { Usual care, } \\
\text { previous } \\
\text { activity level } \\
\text { or attention } \\
\text { control }\end{array}$ & $\begin{array}{l}\text { Walking } \\
\text { Immediately } \\
\text { Post- } \\
\text { treatment }\end{array}$ & $\begin{array}{l}\text { 6MWT } \\
35 \text { (N=276) } \\
\text { Sandroff, 2017 } \\
\text { Sangelaji, } 2016 \\
\text { Ebrahimi, } 2015 \\
\text { Faramazi, } 2020 \\
\text { Ozkul, } 2020 \\
\\
\text { 10MWT } \\
3 \text { (N=93) } \\
\text { Sangelaji, } 2016 \\
\text { Cakit, 2010 } \\
\text { Ebrahimi, } 2015 \\
\\
\text { Other } \\
\text { 1 (N=83) } \\
\text { Sandroff, } 2017\end{array}$ & Moderate & Consistent $^{a}$ & Imprecise & Undetected & Low for benefit & $\begin{array}{l}6 \mathrm{MWT} \\
4 \text { trials, MD }-64.92,95 \% \mathrm{Cl} \text { - } \\
73.5 \text { to }-56.2 \text {, } \\
\mathrm{I}^{2}=0 \% \text { excluding outlier trial } \\
\text { 10MWT } \\
3 \text { trials } \mathrm{MD}-1.99 \text { seconds, } \\
95 \% \mathrm{Cl}-2.8 \text { to }-1.2, \mathrm{I}^{2}=0 \% \text { : } \\
\text { excluding outlier trial }{ }^{\mathrm{c}} \\
\text { Other (no differences) } \\
\text { MSWS-12: difference }-3.30 \text {, } \\
95 \% \mathrm{Cl}-10.16 \text { to } 3.56(0- \\
100 \text { scale) } \\
\text { 25FWT: difference } 0.30 \\
\text { feet/second, } 95 \% \mathrm{Cl}-0.15 \\
\text { to } 0.75\end{array}$ \\
\hline $\begin{array}{l}\text { Multimodal } \\
\text { Exercise } \\
\text { Progressive } \\
\text { resistance or } \\
\text { strength } \\
\text { exercise } \\
\text { plus aerobic } \\
\text { or balance }\end{array}$ & $\begin{array}{l}\text { Usual care, } \\
\text { previous } \\
\text { activity level } \\
\text { or attention } \\
\text { control }\end{array}$ & $\begin{array}{l}\text { Walking } \\
\text { Short term }\end{array}$ & $\begin{array}{l}\text { 6MWT } \\
1(\mathrm{~N}=72) \\
\text { Sangelaji, } 2014\end{array}$ & High & Unknown & Precise & Undetected & Insufficient & $\begin{array}{l}\text { 6MWT } \\
\text { difference } 184.3 \pm 51.1 \\
\text { meters, } p=0.03\end{array}$ \\
\hline $\begin{array}{l}\text { Multimodal } \\
\text { Exercise } \\
\text { Progressive } \\
\text { resistance or } \\
\text { strength } \\
\text { exercise } \\
\text { plus aerobic } \\
\text { or balance }\end{array}$ & $\begin{array}{l}\text { Usual care, } \\
\text { previous } \\
\text { activity level } \\
\text { or attention } \\
\text { control }\end{array}$ & $\begin{array}{l}\text { Functional } \\
\text { capacity } \\
\text { Immediately } \\
\text { Post } \\
\text { treatment }\end{array}$ & $\begin{array}{l}23(\mathrm{~N}=142) \\
\text { Cakit, 2010 } \\
\text { Ebrahimi 2015 } \\
\text { Faramarzi, } 2020\end{array}$ & High & Inconsistent & Precise & Undetected & Insufficient & $\begin{array}{l}\text { TUG } \\
\text { MD }-2.15 \text { seconds, } 95 \% \mathrm{Cl} \\
-2.72 \text { to }-1.58, \mathrm{I}^{2}=0\end{array}$ \\
\hline
\end{tabular}




\begin{tabular}{|c|c|c|c|c|c|c|c|c|c|}
\hline $\begin{array}{l}\text { Intervention } \\
\text { Category, } \\
\text { Intervention } \\
\end{array}$ & Comparator & Outcome & $\begin{array}{l}\text { Number of } \\
\text { Studies } \\
\text { (Participants) } \\
\text { Author Year } \\
\text { (See Appendix B } \\
\text { for Full Citation) } \\
\end{array}$ & $\begin{array}{l}\text { Study } \\
\text { Limitations }\end{array}$ & Consistency & Precision & $\begin{array}{l}\text { Reporting } \\
\text { Bias }\end{array}$ & $\begin{array}{l}\text { Strength of } \\
\text { Evidence }\end{array}$ & $\begin{array}{l}\text { Findings, Direction and } \\
\text { Magnitude of Effect }\end{array}$ \\
\hline $\begin{array}{l}\text { Multimodal } \\
\text { Exercise } \\
\text { Progressive } \\
\text { resistance or } \\
\text { strength } \\
\text { exercise } \\
\text { plus aerobic } \\
\text { or balance }\end{array}$ & $\begin{array}{l}\text { Usual care, } \\
\text { previous } \\
\text { activity level } \\
\text { or attention } \\
\text { control }\end{array}$ & Balance & $\begin{array}{l}3(\mathrm{~N}=224) \\
\text { Sangelaji, } 2014 \\
\text { Sangelaji, } 2016 \\
\text { Ebrahimi, } 2015 \\
\text { Tarakci, } 2013\end{array}$ & Moderate & Consistent & Precise & Undetected & Low for benefit & $\begin{array}{l}\text { BBS: } \mathrm{MD}-3.37,95 \% \mathrm{Cl}- \\
3.76 \text { to }-3.14,12=38 \%\end{array}$ \\
\hline $\begin{array}{l}\text { Multimodal } \\
\text { Exercise } \\
\text { Progressive } \\
\text { resistance or } \\
\text { strength } \\
\text { exercise } \\
\text { plus aerobic } \\
\text { or balance }\end{array}$ & $\begin{array}{l}\text { Usual care, } \\
\text { previous } \\
\text { activity level } \\
\text { or attention } \\
\text { control }\end{array}$ & $\begin{array}{l}\text { MSQoL-54 } \\
\text { MCS } \\
\text { Immediately } \\
\text { Post- } \\
\text { treatment }\end{array}$ & $\begin{array}{l}23(\mathrm{~N}=119) \\
\text { Sangelaji, } 2014 \\
\text { Ebrahimi, 2105 } \\
\text { Ozkul, 2020b }\end{array}$ & High & Inconsistent & Imprecise & Undetected & Insufficient & $\begin{array}{l}\text { MSQoL-54 MCS }(0-100 \\
\text { scale): } 3 \text { trials MD }-10.7 \\
95 \% \mathrm{Cl}-22.6 \text { to } 1.24 \\
\mathrm{I}^{2}=91 \% \\
0\end{array}$ \\
\hline $\begin{array}{l}\text { Multimodal } \\
\text { Exercise } \\
\text { Progressive } \\
\text { resistance or } \\
\text { strength } \\
\text { exercise } \\
\text { plus aerobic } \\
\text { or balance }\end{array}$ & $\begin{array}{l}\text { Usual care, } \\
\text { previous } \\
\text { activity level } \\
\text { or attention } \\
\text { control }\end{array}$ & $\begin{array}{l}\text { MSQoL-54 } \\
\text { MCS } \\
\text { Long-term } \\
\text { (42 weeks) }\end{array}$ & $\begin{array}{l}1(\mathrm{~N}=51) \\
\text { Sangelaji, } 2014\end{array}$ & High & Unknown & Imprecise & Undetected & Insufficient & $\begin{array}{l}\text { MSQoL-54 MCS (0-100 } \\
\text { scale): } 13.54 \pm 5.37 \\
p=0.02\end{array}$ \\
\hline $\begin{array}{l}\text { Multimodal } \\
\text { Exercise } \\
\text { Progressive } \\
\text { resistance or } \\
\text { strength } \\
\text { exercise } \\
\text { plus aerobic } \\
\text { or balance }\end{array}$ & $\begin{array}{l}\text { Usual care, } \\
\text { previous } \\
\text { activity level } \\
\text { or attention } \\
\text { control }\end{array}$ & $\begin{array}{l}\text { MSQoL-54 } \\
\text { PCS } \\
\text { Immediately } \\
\text { Post- } \\
\text { treatment }\end{array}$ & $\begin{array}{l}23(\mathrm{~N}=119) \\
\text { Sangelaji, } 2014 \\
\text { Ebrahimi, } 2105 \\
\text { Ozkul. 2020b }\end{array}$ & High & Consistent $^{a}$ & Imprecise & Undetected & Insufficient & $\begin{array}{l}\text { MSQoL-54,PCS }(0-100 \\
\text { scale): } \mathrm{MD}-13.3,95 \% \mathrm{Cl}- \\
21.6 \text { to }-4.9, \mathrm{I}^{2}=75 \% \\
\text { After excluding outlier MD - } \\
12.0,95 \% \mathrm{Cl}-13.8 \text { to } 5.0, \\
\mathrm{I}^{2}=75 \%\end{array}$ \\
\hline
\end{tabular}




\begin{tabular}{|c|c|c|c|c|c|c|c|c|c|}
\hline $\begin{array}{l}\text { Intervention } \\
\text { Category, } \\
\text { Intervention }\end{array}$ & Comparator & Outcome & $\begin{array}{l}\text { Number of } \\
\text { Studies } \\
\text { (Participants) } \\
\text { Author Year } \\
\text { (See Appendix B } \\
\text { for Full Citation) }\end{array}$ & $\begin{array}{l}\text { Study } \\
\text { Limitations }\end{array}$ & Consistency & Precision & $\begin{array}{l}\text { Reporting } \\
\text { Bias }\end{array}$ & $\begin{array}{l}\text { Strength of } \\
\text { Evidence }\end{array}$ & $\begin{array}{l}\text { Findings, Direction and } \\
\text { Magnitude of Effect }\end{array}$ \\
\hline $\begin{array}{l}\text { Multimodal } \\
\text { Exercise } \\
\text { Progressive } \\
\text { resistance or } \\
\text { strength } \\
\text { exercise } \\
\text { plus aerobic } \\
\text { or balance }\end{array}$ & $\begin{array}{l}\text { Usual care, } \\
\text { previous } \\
\text { activity level } \\
\text { or attention } \\
\text { control }\end{array}$ & $\begin{array}{l}\text { MSQoL-54 } \\
\text { PCS } \\
\text { Long-term } \\
\text { (42 weeks) }\end{array}$ & $\begin{array}{l}1(\mathrm{~N}=51) \\
\text { Sangelaji, } 2014\end{array}$ & High & Unknown & Imprecise & Undetected & Insufficient & $\begin{array}{l}\text { MSQoL-54,PCS (0-100 } \\
\text { scale): difference } 10.9 \pm \\
4.55, p=0.02\end{array}$ \\
\hline $\begin{array}{l}\text { Multimodal } \\
\text { Exercise } \\
\text { Progressive } \\
\text { resistance or } \\
\text { strength } \\
\text { exercise } \\
\text { plus aerobic } \\
\text { or balance }\end{array}$ & $\begin{array}{l}\text { Multimodal } \\
\text { exercise vs. } \\
\text { Aerobic } \\
\text { exercise }\end{array}$ & $\begin{array}{l}\text { Quality of } \\
\text { Life; } \\
\text { SF-36 } \\
\text { MCS/PCS } \\
\text { Immediately } \\
\text { Post- } \\
\text { treatment } \\
\text { MusiQoL }\end{array}$ & $\begin{array}{l}1(\mathrm{~N}=60) \\
\text { Kerling, } 2015 \\
1(\mathrm{~N}=110)\end{array}$ & Moderate & Unknown & Imprecise & Undetected & Insufficient & $\begin{array}{l}\text { SF36 MCS (0-100 scale) } \\
\text { difference } 4.2,95 \% \mathrm{Cl} 0.2 \\
\text { to } 8.2 \text { (favors control) } \\
\text { SF36 PCS ( } 0-100 \text { scale) } \\
\text { difference }-0.7,95 \% \mathrm{Cl}- \\
3.9 \text { to } 2.2 \\
\text { MusiQOL }(0-100 \text { scale:) } \\
\text { difference }-2.38,95 \% \mathrm{Cl}- \\
4.68 \text { to }-0.08\end{array}$ \\
\hline $\begin{array}{l}\text { Multimodal } \\
\text { Exercise } \\
\text { Exercises to } \\
\text { improve } \\
\text { functional } \\
\text { strength, } \\
\text { balance, gait } \\
\text { speed and } \\
\text { endurance } \\
\text { plus } \\
\text { stretching } \\
\text { and core- } \\
\text { stability } \\
\text { work. }\end{array}$ & $\begin{array}{l}\text { Group } \\
\text { multimodal } \\
\text { exercise vs. } \\
\text { Home-based } \\
\text { multimodal } \\
\text { exercise }\end{array}$ & $\begin{array}{l}\text { Walking } \\
\text { Immediatetly } \\
\text { Post- } \\
\text { treatment } \\
\text { 6MWT, } \\
\text { 10MWT }\end{array}$ & $\begin{array}{l}1(\mathrm{~N}=44), \\
\text { Williams, } 2020\end{array}$ & Moderate & Unknown & Imprecise & Undetected & Insufficient & $\begin{array}{l}\text { 10MWT, } \\
\text { difference } 0.01(95 \% \mathrm{Cl} \\
-0.36 \text { to } 0.37 \mathrm{~m} / \mathrm{s}) \\
\text { 6MWT, } \\
\text { Difference } 18.67(95 \% \mathrm{Cl} \\
-78.22 \text { to } 115.56 \text { meters) }\end{array}$ \\
\hline
\end{tabular}




\begin{tabular}{|c|c|c|c|c|c|c|c|c|c|}
\hline $\begin{array}{l}\text { Intervention } \\
\text { Category, } \\
\text { Intervention }\end{array}$ & Comparator & Outcome & $\begin{array}{l}\text { Number of } \\
\text { Studies } \\
\text { (Participants) } \\
\text { Author Year } \\
\text { (See Appendix B } \\
\text { for Full Citation) }\end{array}$ & $\begin{array}{l}\text { Study } \\
\text { Limitations }\end{array}$ & Consistency & Precision & $\begin{array}{l}\text { Reporting } \\
\text { Bias }\end{array}$ & $\begin{array}{l}\text { Strength of } \\
\text { Evidence }\end{array}$ & $\begin{array}{l}\text { Findings, Direction and } \\
\text { Magnitude of Effect }\end{array}$ \\
\hline $\begin{array}{l}\text { Multimodal } \\
\text { Exercise } \\
\text { Exercises to } \\
\text { improve } \\
\text { functional } \\
\text { strength, } \\
\text { balance, gait } \\
\text { speed and } \\
\text { endurance } \\
\text { plus } \\
\text { stretching } \\
\text { and core- } \\
\text { stability } \\
\text { work. }\end{array}$ & $\begin{array}{l}\text { Group } \\
\text { multimodal } \\
\text { exercise vs. } \\
\text { Home-based } \\
\text { multimodal } \\
\text { exercise }\end{array}$ & $\begin{array}{l}\text { Walking } \\
\text { Short term (8 } \\
\text { weeks) } \\
\text { 6MWT, } \\
\text { 10MWT }\end{array}$ & $\begin{array}{l}1(\mathrm{~N}=44) \\
\text { Williams, } 2020\end{array}$ & Moderate & Unknown & Imprecise & Undetected & Insufficient & $\begin{array}{l}\text { 10MWT, } \\
\text { Difference }-0.19(95 \% \mathrm{Cl} \text { - } \\
0.41 \text { to } 0.03, \mathrm{~m} / \mathrm{s}) \\
\\
\text { 6MWT, } \\
\text { Difference }-20.5(95 \% \mathrm{Cl} \text { - } \\
60.21 \text { to } 19.21 \text { meters })\end{array}$ \\
\hline $\begin{array}{l}\text { Multimodal } \\
\text { Exercise } \\
\text { Exercises to } \\
\text { improve } \\
\text { functional } \\
\text { strength, } \\
\text { balance, gait } \\
\text { speed and } \\
\text { endurance } \\
\text { plus } \\
\text { stretching } \\
\text { and core- } \\
\text { stability } \\
\text { work. }\end{array}$ & $\begin{array}{l}\text { Group } \\
\text { multimodal } \\
\text { exercise vs. } \\
\text { Home-based } \\
\text { multimodal } \\
\text { exercise }\end{array}$ & $\begin{array}{l}\text { Balance } \\
\text { Immediatetly } \\
\text { Post- } \\
\text { treatment }\end{array}$ & $\begin{array}{l}1(\mathrm{~N}=44), \\
\text { Williams, } 2020\end{array}$ & Moderate & Unknown & Imprecise & Undetected & Insufficient & $\begin{array}{l}\text { BBS } \\
\text { Difference } 1.70(95 \% \mathrm{Cl} \\
-8.4 \text { to } 11.80)\end{array}$ \\
\hline
\end{tabular}




\begin{tabular}{|c|c|c|c|c|c|c|c|c|c|}
\hline $\begin{array}{l}\text { Intervention } \\
\text { Category, } \\
\text { Intervention }\end{array}$ & Comparator & Outcome & $\begin{array}{l}\text { Number of } \\
\text { Studies } \\
\text { (Participants) } \\
\text { Author Year } \\
\text { (See Appendix B } \\
\text { for Full Citation) }\end{array}$ & $\begin{array}{l}\text { Study } \\
\text { Limitations }\end{array}$ & Consistency & Precision & $\begin{array}{l}\text { Reporting } \\
\text { Bias }\end{array}$ & $\begin{array}{l}\text { Strength of } \\
\text { Evidence }\end{array}$ & $\begin{array}{l}\text { Findings, Direction and } \\
\text { Magnitude of Effect }\end{array}$ \\
\hline $\begin{array}{l}\text { Multimodal } \\
\text { Exercise } \\
\text { Exercises to } \\
\text { improve } \\
\text { functional } \\
\text { strength, } \\
\text { balance, gait } \\
\text { speed and } \\
\text { endurance } \\
\text { plus } \\
\text { stretching } \\
\text { and core- } \\
\text { stability } \\
\text { work. }\end{array}$ & $\begin{array}{l}\text { Group } \\
\text { multimodal } \\
\text { exercise vs. } \\
\text { Home-based } \\
\text { multimodal } \\
\text { exercise }\end{array}$ & $\begin{array}{l}\text { Balance short } \\
\text { term }(8 \\
\text { weeks }\end{array}$ & $\begin{array}{l}1(\mathrm{~N}=44), \\
\text { Williams, } 2020\end{array}$ & Moderate & Unknown & Imprecise & Undetected & Insufficient & $\begin{array}{l}\text { BBS } \\
\text { Difference }-1.9(-6.44 \text { to } \\
2.64)\end{array}$ \\
\hline
\end{tabular}

Abbreviations: BBS = Berg Balance Scale; $6 \mathrm{MWT}=6$-Minute Walk Test; $10 \mathrm{MWT}=10$-Meter Walk Test; $25 \mathrm{FWT}=2$-Feet Walk Test; $\mathrm{CI}=$ confidence interval; $\mathrm{MD}=$ mean difference; $\mathrm{MS}=$

multiple sclerosis; MSQoL-MCS = Multiple Sclerosis Quality of Life-54 instrument Mental Component Score; MSQoL-PCS = Multiple Sclerosis Quality of Life-54 instrument Physical Component Score; MSWS-12 = Multiple Sclerosis Walking Scale; NA = not applicable; RCT = randomized controlled trial; SF-36 MCS = Short-Form 36 Mental Component Summary; SF-36 PCS = Short-Form 36 Physical Component Score; TUG = Timed Up and Go Test.

${ }^{a}$ Effect estimates go in the same direction even though magnitude of effect may differ

${ }^{\text {b }}$ Outlier excluded, Sangelaji 2014

c Outlier excluded, Tarakci 2013 
Table H-12. Strength of evidence for Key Question 2: multimodal exercise that includes strengthening for cerebral palsy

\begin{tabular}{|c|c|c|c|c|c|c|c|c|c|}
\hline $\begin{array}{l}\text { Intervention } \\
\text { Category, } \\
\text { Intervention }\end{array}$ & Comparator & Outcome & $\begin{array}{l}\text { Number of } \\
\text { Studies } \\
\text { (Participants) } \\
\text { Author Year } \\
\text { (See Appendix } \\
\text { B for Full } \\
\text { Citation) } \\
\end{array}$ & $\begin{array}{l}\text { Study } \\
\text { Limitations }\end{array}$ & Consistency & Precision & $\begin{array}{l}\text { Reporting } \\
\text { Bias }\end{array}$ & $\begin{array}{l}\text { Strength of } \\
\text { Evidence }\end{array}$ & $\begin{array}{l}\text { Findings, Direction and } \\
\text { Magnitude of Effect }\end{array}$ \\
\hline $\begin{array}{l}\text { Multimodal } \\
\text { Exercise } \\
\text { Progressive } \\
\text { resistance or } \\
\text { strength } \\
\text { exercise plus } \\
\text { aerobic or } \\
\text { balance }\end{array}$ & Usual care & $\begin{array}{l}\text { Walking } \\
\text { Immediately } \\
\text { Post- } \\
\text { treatment }\end{array}$ & $\begin{array}{l}\text { 6MWT } \\
1(\mathrm{~N}=37) \\
\text { Fosdahl, 2019b } \\
\text { GDI } \\
1(\mathrm{~N}=37) \\
\text { Fosdahl, 2019b }\end{array}$ & Moderate & Unknown & Imprecise & Undetected & Insufficent & $\begin{array}{l}\text { 6MWT (meters) } \\
1 \text { trial: difference }-45.7(55.4) \\
\text { vs. }-55.4 \text { (55.5), adj. MD10.6 } \\
(95 \% \mathrm{Cl}-29.3 \text { to } 50.6), \\
\mathrm{p}=0.590 \text { (pre-post change) } \\
\text { GDI } \\
1 \text { trial: difference }-0.4(4.4) \text { vs. } \\
-0.8(7.14), \text { adj. MD }-1.0(95 \% \\
\mathrm{Cl}-5.3 \text { to } 3.3), p=0.65\end{array}$ \\
\hline $\begin{array}{l}\text { Multimodal } \\
\text { Exercise } \\
\text { Progressive } \\
\text { resistance or } \\
\text { strength } \\
\text { exercise plus } \\
\text { aerobic or } \\
\text { balance }\end{array}$ & Usual care & $\begin{array}{l}\text { Walking } \\
\text { Intermediate } \\
\text { term (16 } \\
\text { weeks) }\end{array}$ & $\begin{array}{l}6 M W T \\
1(N=37) \\
\text { Fosdahl, 2019b } \\
\text { GDI } \\
1(\mathrm{~N}=37) \\
\text { Fosdahl, 2019b } \\
\\
1 \mathrm{MWT} \\
2(\mathrm{~N}=80) \\
\text { Kaya Kara, 2019 } \\
\text { Van Wely, 2014a }\end{array}$ & Moderate & Inconsistent & Imprecise & Undetected & Insufficient & $\begin{array}{l}\text { 6MWT (meters) } \\
1 \text { trial: difference } \\
\text {-differences)vs. }-56.6 \text { (59.6), } \\
\text { adj. MD } 7.2(-43.3 \text { to } 57.7), \\
\text { p=0.772 (16 week change) } \\
\text { GDI } \\
1 \text { trial: difference }-0.7(6.0) \text { vs. } \\
1.01 \text { (5.9), adj. MD }-1.4(95 \% \\
\text { Cl }-5.6 \text { to } 2.8), p=0.504(16 \\
\text { week change) } \\
1 M W T: 2 \text { pooled trials: } \\
\text { MD }-5.28,95 \% \mathrm{Cl}-10.24 \text { to - } \\
0.33,\left.\right|^{2}=45 \%\end{array}$ \\
\hline
\end{tabular}




\begin{tabular}{|c|c|c|c|c|c|c|c|c|c|}
\hline $\begin{array}{l}\text { Intervention } \\
\text { Category, } \\
\text { Intervention }\end{array}$ & Comparator & Outcome & $\begin{array}{l}\text { Number of } \\
\text { Studies } \\
\text { (Participants) } \\
\text { Author Year } \\
\text { (See Appendix } \\
\text { B for Full } \\
\text { Citation) }\end{array}$ & $\begin{array}{l}\text { Study } \\
\text { Limitations }\end{array}$ & Consistency & Precision & $\begin{array}{l}\text { Reporting } \\
\text { Bias }\end{array}$ & $\begin{array}{l}\text { Strength of } \\
\text { Evidence }\end{array}$ & $\begin{array}{l}\text { Findings, Direction and } \\
\text { Magnitude of Effect }\end{array}$ \\
\hline $\begin{array}{l}\text { Multimodal } \\
\text { Exercise } \\
\text { Progressive } \\
\text { resistance or } \\
\text { strength } \\
\text { exercise plus } \\
\text { aerobic or } \\
\text { balance }\end{array}$ & Usual care & $\begin{array}{l}\text { Functional } \\
\text { Capacity } \\
\text { Immediately } \\
\text { Post- } \\
\text { treatment }\end{array}$ & $\begin{array}{l}\text { GMFM-66 } \\
2(N=105) \\
\text { Slaman, 2015a, } \\
2015 b, 2014, \\
2010 \\
\text { Van Wely, 2014a, } \\
2014 b, 2010 \\
\text { GMFM88-D/E } \\
1 \text { (N=30) } \\
\text { Kaya Kara, } 2019\end{array}$ & Moderate & $\begin{array}{l}\text { Inconsistent } \\
\text { (GMFM-66) } \\
\text { Unknown } \\
\text { (GMFM-88 } \\
\text { D/E) }\end{array}$ & Imprecise & Undetected & $\begin{array}{l}\text { Low-strength } \\
\text { evidence for } \\
\text { no clear } \\
\text { benefit }\end{array}$ & $\begin{array}{l}\text { GMFM-66 }(0-100 \text { scale) } \\
2 \text { trials, } \mathrm{MD}-1.5,95 \% \mathrm{Cl}-6.4 \\
\left.\text { to } 4.7, \mathrm{I}^{2}=71 \%\right) \text {. } \\
\text { No difference in one trial } \\
\text { (difference } 1.6,95 \% \mathrm{Cl}-2.7 \text { to } \\
5.9) \text { in one trial; the other trial } \\
\text { favored exercise over usual } \\
\text { care (difference }-3.1,95 \% \mathrm{Cl}- \\
5.7 \text { to }-0.6 \text { ) } \\
\text { GMFM-88-D } \\
1 \text { trial: difference }-0.2,95 \% \mathrm{Cl} \\
-0.9 \text { to } 0.6 \\
\text { GMFM-88-E } \\
1 \text { trial: difference } 2.7,95 \% \mathrm{Cl} \\
1.0 \text { to } 4.4\end{array}$ \\
\hline $\begin{array}{l}\text { Multimodal } \\
\text { Exercise } \\
\text { Progressive } \\
\text { resistance or } \\
\text { strength } \\
\text { exercise plus } \\
\text { aerobic or } \\
\text { balance }\end{array}$ & Usual care & $\begin{array}{l}\text { Functional } \\
\text { Capacity } \\
\text { Intermediate } \\
\text { term (16 } \\
\text { weeks) }\end{array}$ & $\begin{array}{l}\text { TUG } \\
1(\mathrm{~N}=37) \\
\text { Fosdahl, 2019b }\end{array}$ & Moderate & Unknown & Imprecise & Undetected & Insufficient & $\begin{array}{l}\text { TUG } \\
\text { difference }-1.1,95 \% \mathrm{Cl}-1.4 \text { to } \\
-0.78\end{array}$ \\
\hline $\begin{array}{l}\text { Multimodal } \\
\text { Exercise } \\
\text { Progressive } \\
\text { resistance or } \\
\text { strength } \\
\text { exercise plus } \\
\text { aerobic or } \\
\text { balance }\end{array}$ & Usual care & $\begin{array}{l}\text { Quality of } \\
\text { Life } \\
\text { Immediately } \\
\text { Post } \\
\text { treatment }\end{array}$ & $\begin{array}{l}\text { CP-QOL: } \\
1(\mathrm{~N}=50) \\
\text { Van Wely, 2014a, } \\
2014 b, 2010 \\
\text { SF-36: } \\
1(\mathrm{~N}=57) \\
\text { Slaman, 2015a, } \\
2015 b, 2014, \\
2010\end{array}$ & Moderate & Consistent & Unknown & Undetected & $\begin{array}{l}\text { Low-strength } \\
\text { evidence for } \\
\text { no clear } \\
\text { benefit }\end{array}$ & $\begin{array}{l}\text { No improvement in any domain } \\
\text { of either QOL measure was } \\
\text { seen in either study (please } \\
\text { see full report). }\end{array}$ \\
\hline
\end{tabular}

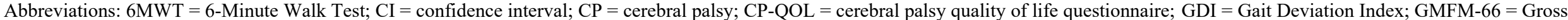

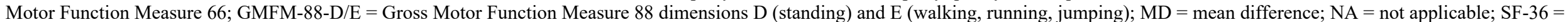
Short-Form 36 questionnaire; QoL = quality of life; RCT = randomized controlled trial; TUG $=$ Timed Up and Go Test 
Table H-13. Strength of evidence for Key Question 2: multimodal exercise that includes strengthening for spinal cord injury

\begin{tabular}{|c|c|c|c|c|c|c|c|c|c|}
\hline $\begin{array}{l}\text { Intervention } \\
\text { Category, } \\
\text { Intervention }\end{array}$ & Comparator & Outcome & $\begin{array}{l}\text { Number of } \\
\text { Studies } \\
\text { (Participants) } \\
\text { Author Year } \\
\text { (See Appendix } \\
\text { B for Full } \\
\text { Citation) } \\
\end{array}$ & $\begin{array}{l}\text { Study } \\
\text { Limitations }\end{array}$ & Consistency & Precision & $\begin{array}{l}\text { Reporting } \\
\text { Bias }\end{array}$ & $\begin{array}{l}\text { Strength of } \\
\text { Evidence }\end{array}$ & $\begin{array}{l}\text { Findings, Direction and } \\
\text { Magnitude of Effect }\end{array}$ \\
\hline $\begin{array}{l}\text { Multimodal } \\
\text { Exercise } \\
\text { Progressive } \\
\text { resistance or } \\
\text { strength } \\
\text { exercise plus } \\
\text { aerobic or } \\
\text { balance }\end{array}$ & Usual care & $\begin{array}{l}\text { Walking } \\
\text { ability } \\
\text { Immediately } \\
\text { Post- } \\
\text { treatment }\end{array}$ & $\begin{array}{l}1(\mathrm{~N}=48) \\
\text { Jones, 2014a, } \\
2014 \mathrm{~b}\end{array}$ & High & Unknown & Imprecise & Undetected & Insufficient & $\begin{array}{l}\text { Change scores } \\
6 \mathrm{MWT} \\
36.0 \pm 48.2 \text { vs. } 3.0 \pm 25.5 \\
\text { meters, } p=0.002 \\
10 \mathrm{MWT} \\
0.1 \pm 0.1 \text { vs. } 0.03 \pm 0.1 \text { meters } \\
\text { per second; } p=0.036 \\
\text { SCI-FAl (scale not provided) } \\
5.0 \pm 8.0 \text { vs. }-0.2 \pm 2.8, p=0.03\end{array}$ \\
\hline $\begin{array}{l}\text { Multimodal } \\
\text { Exercise } \\
\text { Progressive } \\
\text { resistance or } \\
\text { strength } \\
\text { exercise plus } \\
\text { aerobic or } \\
\text { balance }\end{array}$ & Usual care & $\begin{array}{l}\text { Functional } \\
\text { Capacity } \\
\text { Immediately } \\
\text { Post - } \\
\text { intervention }\end{array}$ & $\begin{array}{l}1(N=48) \\
\text { Jones, 2014a, } \\
2014 b\end{array}$ & High & Unknown & Imprecise & Undetected & Insufficient & $\begin{array}{l}\text { TUG } \\
\text { difference }-37.2 \pm 81.3 \text { vs. }- \\
6.2 \pm 18.1 \text { seconds; } p=0.267\end{array}$ \\
\hline $\begin{array}{l}\text { Multimodal } \\
\text { Exercise } \\
\text { Progressive } \\
\text { resistance or } \\
\text { strength } \\
\text { exercise plus } \\
\text { aerobic or } \\
\text { balance } \\
\text { (whole body) }\end{array}$ & $\begin{array}{l}\text { Multimodal } \\
\text { Exercise } \\
\text { (upper body } \\
\text { only) }\end{array}$ & $\begin{array}{l}\text { Walking } \\
\text { ability } \\
\text { Immediately } \\
\text { Post- } \\
\text { treatment }\end{array}$ & $\begin{array}{l}1(\mathrm{~N}=26) \\
\text { Galea, } 2018 \\
\text { (subset of } \\
\text { patients who } \\
\text { could walk) }\end{array}$ & Moderate & Unknown & Imprecise & Undetected & Insufficient & $\begin{array}{l}\text { 6MWT } \\
\text { difference }-12.30,95 \% \mathrm{Cl}- \\
68.01 \text { to } 43.41 \\
\text { 10MWT } \\
\text { difference }-0.10,95 \% \mathrm{Cl}-0.30 \\
\text { to } 0.10\end{array}$ \\
\hline $\begin{array}{l}\text { Multimodal } \\
\text { Exercise } \\
\text { Progressive } \\
\text { resistance or } \\
\text { strength } \\
\text { exercise plus } \\
\text { aerobic or } \\
\text { balance } \\
\text { (whole body) }\end{array}$ & $\begin{array}{l}\text { Multimodal } \\
\text { Exercise } \\
\text { (upper body } \\
\text { only) }\end{array}$ & $\begin{array}{l}\text { Walking } \\
\text { ability } \\
\text { Short-term } \\
\text { Followup (12 } \\
\text { weeks) }\end{array}$ & $\begin{array}{l}1(\mathrm{~N}=26) \\
\text { Galea, } 2018 \\
\text { (subset of } \\
\text { patients who } \\
\text { could walk) }\end{array}$ & Moderate & Unknown & Imprecise & Undetected & Insufficient & $\begin{array}{l}\text { 6MWT } \\
\text { difference }-88.0,95 \% \mathrm{Cl}- \\
143.71 \text { to }-32.29 \\
\text { 10MWT } \\
\text { difference }-0.80,95 \% \mathrm{Cl}-2.3 \\
\text { to } 0.70\end{array}$ \\
\hline
\end{tabular}

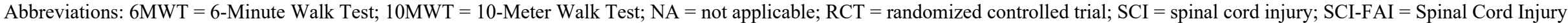

Function Ambulation Index; TUG $=$ Timed Up and Go Test. 
Table H-14. Strength of evidence for Key Question 2a clinical outcomes: mental health

\begin{tabular}{|c|c|c|c|c|c|c|c|c|c|}
\hline $\begin{array}{l}\text { Intervention } \\
\text { Category, } \\
\text { Intervention }\end{array}$ & Comparator & Outcome & $\begin{array}{l}\text { Number of } \\
\text { Studies } \\
\text { (Participants) } \\
\text { Author Year } \\
\text { (See Appendix } \\
\text { B for Full } \\
\text { Citation) }\end{array}$ & $\begin{array}{l}\text { Study } \\
\text { Limitations }\end{array}$ & Consistency & Precision & $\begin{array}{l}\text { Reporting } \\
\text { Bias }\end{array}$ & $\begin{array}{l}\text { Strength of } \\
\text { Evidence }\end{array}$ & $\begin{array}{l}\text { Findings, Direction and } \\
\text { Magnitude of Effect }\end{array}$ \\
\hline $\begin{array}{l}\begin{array}{l}\text { All-exercise } \\
\text { interventions }\end{array} \\
\text { All physical } \\
\text { exercise } \\
\text { interventions } \\
\text { in MS }\end{array}$ & $\begin{array}{l}\text { All } \\
\text { comparators }\end{array}$ & $\begin{array}{l}\text { Depression } \\
\text { scores }\end{array}$ & $\begin{array}{l}\text { 12 RCTs }(\mathrm{N}=564) \\
\text { Baquet, } 2018 \\
\text { Hebert, } 2011 \\
\text { Negaresh, } 2019 \\
\text { Russo, } 2018 \\
\text { Dalgas, } 2009 \\
\text { Cakit, 2010 } \\
\text { Razazian, } 2016 \\
\text { Ahmadi, } 2013 \\
\text { Sadeghi } \\
\text { Bahmani, } 2019 \\
\text { Tollar, 2020 } \\
\text { Ozkul, 2020b } \\
\text { Sadeghi } \\
\text { Bahmani, 2020 }\end{array}$ & Moderate & Consistent & Imprecise & Undetected & $\begin{array}{l}\text { Moderate for } \\
\text { benefit }\end{array}$ & $\begin{array}{l}\text { (SMD }-0.29,95 \% \mathrm{Cl}-0.50 \text { to } \\
\left.-0.03, \mathrm{I}^{2}=8 \%\right)\end{array}$ \\
\hline $\begin{array}{l}\text { All-exercise } \\
\text { interventions } \\
\text { All physical } \\
\text { exercise } \\
\text { interventions } \\
\text { in SCl }\end{array}$ & $\begin{array}{l}\text { All } \\
\text { comparators }\end{array}$ & $\begin{array}{l}\text { Depression } \\
\text { Scores }\end{array}$ & $\begin{array}{l}\text { 3 RCTs }(\mathrm{N}=171) \\
\text { Yang, } 2014 \\
\text { Akkurt, } 2017 \\
\text { Galea, } 2018\end{array}$ & Moderate & Consistent & Imprecise & Undetected & $\begin{array}{l}\text { Low-strength } \\
\text { evidence for } \\
\text { no clear } \\
\text { benefit }\end{array}$ & $\begin{array}{l}\text { CES-D: }-2.7 \text { vs. }-2.4, p>0.05 \\
\text { HADS change scores: } 0 \text { vs. } \\
0.5, p>0.05 \\
\text { CES-D change scores: }-3 \\
\text { vs. } 3, p>0.05 \\
\text { HADS-Depression } \\
10.5(2) \text { vs. } 10.4(2.1) \\
\text { (baseline) } \\
10(1.6) \text { vs. } 10.2(1.3) \text { (post- } \\
\text { intervention) } \\
\text { MD -0.28, } 95 \% \mathrm{Cl}-0.83 \text { to } \\
0.27, p=0.309 \text { (post- } \\
\text { intervention) } \\
10.1(1.5) \text { vs. } 10.2(1.4) \\
\text { MD }-0.23(95 \% \mathrm{Cl}-0.81 \text { to } \\
0.35), p=0.428(24 \text { weeks- } \\
12 \text { weeks post-intervention) }\end{array}$ \\
\hline
\end{tabular}




\begin{tabular}{|c|c|c|c|c|c|c|c|c|c|}
\hline $\begin{array}{l}\text { Intervention } \\
\text { Category, } \\
\text { Intervention }\end{array}$ & Comparator & Outcome & $\begin{array}{l}\text { Number of } \\
\text { Studies } \\
\text { (Participants) } \\
\text { Author Year } \\
\text { (See Appendix } \\
\text { B for Full } \\
\text { Citation) }\end{array}$ & $\begin{array}{l}\text { Study } \\
\text { Limitations }\end{array}$ & Consistency & Precision & $\begin{array}{l}\text { Reporting } \\
\text { Bias }\end{array}$ & $\begin{array}{l}\text { Strength of } \\
\text { Evidence }\end{array}$ & $\begin{array}{l}\text { Findings, Direction and } \\
\text { Magnitude of Effect }\end{array}$ \\
\hline $\begin{array}{l}\text { All-exercise } \\
\text { interventions } \\
\text { All physical } \\
\text { interventions } \\
\text { in MS and } \mathrm{SCl}\end{array}$ & $\begin{array}{l}\text { All } \\
\text { comparators }\end{array}$ & $\begin{array}{l}\text { Anxiety } \\
\text { scores }\end{array}$ & $\begin{array}{l}\text { 2 RCTs }(\mathrm{N}=146) \\
\text { Keser, } 2011 \\
\text { Galea, } 2018\end{array}$ & Moderate & Consistent & Imprecise & Undetected & Insufficient & $\begin{array}{l}1 \mathrm{MS} \text { : HAD Anxiety scores } \\
\text { not provided but no } \\
\text { difference between } \\
\text { calisthenics and neurorehab } \\
1 \mathrm{SCl} \text { : MD } 0.29,95 \% \mathrm{Cl} \text { - } \\
0.25 \text { to } 0.83, \mathrm{p}=0.291, \text { no } \\
\text { difference between whole } \\
\text { body strength training and } \\
\text { upper body strength on } \\
\text { depression scores between } \\
\text { groups with little change } \\
\text { from baseline in both groups }\end{array}$ \\
\hline
\end{tabular}

Abbreviations: MS = multiple sclerosis; $\mathrm{HAD}=$ Hospital and Depression (Scale); $\mathrm{MD}=$ mean difference; $\mathrm{NA}=$ not applicable; $\mathrm{RCT}=$ randomized controlled trial; $\mathrm{SCI}=$ spinal cor injury 
Table H-15. Strength of evidence for Key Question 2: general exercise effect across interventions and populations

\begin{tabular}{|c|c|c|c|c|c|c|c|c|c|}
\hline $\begin{array}{l}\text { Intervention } \\
\text { Category, } \\
\text { Intervention }\end{array}$ & Comparator & Outcome & $\begin{array}{l}\text { Number of RCTs } \\
\text { (Participants) } \\
\text { Author Year } \\
\text { (See Appendix B for } \\
\text { Full Citation) }\end{array}$ & $\begin{array}{l}\text { Study } \\
\text { Limitations }\end{array}$ & Consistency & Precision & $\begin{array}{l}\text { Reporting } \\
\text { Bias }\end{array}$ & $\begin{array}{l}\text { Strength of } \\
\text { Evidence }\end{array}$ & $\begin{array}{l}\text { Findings, Direction and } \\
\text { Magnitude of Effect }\end{array}$ \\
\hline $\begin{array}{l}\text { All-exercise } \\
\text { interventions } \\
\text { (General } \\
\text { exercise effect } \\
\text { across } \\
\text { interventions/ } \\
\text { populations) }\end{array}$ & Usual Care & 6MWT & \begin{tabular}{|l|}
$25(1196)$ \\
Baguet, 2018 \\
Hebert, 2011 \\
Kargarfard, 2018 \\
Young, 2019 \\
Kim, 2015 \\
Kalron, 2017 \\
Duff, 2018 \\
Dalgas, 2010 \\
Taylor, 2013 \\
Hogan, 2014 \\
Garrett, 2012ab \\
Sandroff, 2017 \\
Sangelaji, 2014 \\
Sangelaji, 2016 \\
Ebrahimi, 2015 \\
Jones, 2014a \\
Bahrami, 2019a \\
Callesen, 2019 \\
Fosdahl, 2019b \\
Tollar, 2020 \\
Yazgan, 2019 \\
Moraes, 2020 \\
Ahmadizadeh 2019
\end{tabular} & Moderate & Consistent & Precise & Undetected & $\begin{array}{l}\text { Moderate for } \\
\text { benefit }\end{array}$ & $\begin{array}{l}\text { 6MWT: Pooled analysis: MD - } \\
32.94,95 \% \mathrm{Cl}-46.07 \text { to - } \\
19.81, \mathrm{I}^{2}=78 \%\end{array}$ \\
\hline
\end{tabular}




\begin{tabular}{|c|c|c|c|c|c|c|c|c|c|}
\hline $\begin{array}{l}\text { Intervention } \\
\text { Category, } \\
\text { Intervention }\end{array}$ & Comparator & Outcome & $\begin{array}{l}\text { Number of RCTs } \\
\text { (Participants) } \\
\text { Author Year } \\
\text { (See Appendix B for } \\
\text { Full Citation) }\end{array}$ & $\begin{array}{l}\text { Study } \\
\text { Limitations }\end{array}$ & Consistency & Precision & $\begin{array}{l}\text { Reporting } \\
\text { Bias }\end{array}$ & $\begin{array}{l}\text { Strength of } \\
\text { Evidence }\end{array}$ & $\begin{array}{l}\text { Findings, Direction and } \\
\text { Magnitude of Effect }\end{array}$ \\
\hline All exercise & Usual Care & $\begin{array}{l}\text { Walking in } \\
\text { MS }\end{array}$ & $\begin{array}{l}25(1529) \text { Baguet, } \\
2018 \\
\text { Hebert, 2011 } \\
\text { Kargarfard, } 2018 \\
\text { Young, 2019 } \\
\text { Kalron, 2017 } \\
\text { Duff, 2018 } \\
\text { Dalgas, 2010 } \\
\text { Hogan, 2014 } \\
\text { Garrett, 2012a/b } \\
\text { Sandroff, 2017 } \\
\text { Sangelaji, 2014 } \\
\text { Sangelaji, 2016 } \\
\text { Ebrahimi, 2015 } \\
\text { Carling, 2017 } \\
\text { Cakit, 2010 } \\
\text { Tarakci, 2013 } \\
\text { Fox, 2016 } \\
\text { Forsberg, 2016 } \\
\text { Nilsagard, 2012 } \\
\text { Callesen, 2019 } \\
\text { Ahmadi, 213 } \\
\text { Arntzen, 2020 } \\
\text { Tollar, 2020 } \\
\text { Moraes, 2020 } \\
\text { Yazgan, 2019 } \\
\text { Faramarzi, 2020 } \\
\text { Ozkul, 2020b } \\
\end{array}$ & Moderate & Consistent & Precise & Not detected & High for benefit & $\begin{array}{l}\text { Pooled analysis ( } 19 \text { studies): } \\
\text { 6MWT: } \mathrm{MD}-42.70,95 \% \mathrm{Cl}- \\
57.05 \text { to }-28.35, \mathrm{I}^{2}=75 \% \\
\text { Pooled analysis (9 studies): } \\
\text { 10MWT: } \mathrm{MD}-1.44,95 \% \mathrm{Cl}- \\
2.74 \text { to }-0.13, \mathrm{I}^{2}=90 \% \\
\text { Pooled analysis ( } 9 \text { studies): MS } \\
\text { Walking Scale: } \mathrm{MD}-2.88,95 \% \\
\mathrm{Cl}-4.80 \text { to }-0.96, \mathrm{I}^{2}=33 \%\end{array}$ \\
\hline
\end{tabular}




\begin{tabular}{|c|c|c|c|c|c|c|c|c|c|}
\hline $\begin{array}{l}\text { Intervention } \\
\text { Category, } \\
\text { Intervention }\end{array}$ & Comparator & Outcome & $\begin{array}{l}\text { Number of RCTs } \\
\text { (Participants) } \\
\text { Author Year } \\
\text { (See Appendix B for } \\
\text { Full Citation) }\end{array}$ & $\begin{array}{l}\text { Study } \\
\text { Limitations }\end{array}$ & Consistency & Precision & $\begin{array}{l}\text { Reporting } \\
\text { Bias }\end{array}$ & $\begin{array}{l}\text { Strength of } \\
\text { Evidence }\end{array}$ & $\begin{array}{l}\text { Findings, Direction and } \\
\text { Magnitude of Effect }\end{array}$ \\
\hline All exercise & Usual Care & 10MWT & $\begin{array}{l}14 \text { (659) } \\
\text { Fox, 2016 } \\
\text { Dalgas, 2010 } \\
\text { Carling, 2017 } \\
\text { In, 2018 } \\
\text { Sangelaji, } 2016 \\
\text { Cakit, 2010 } \\
\text { Ebrahimi, 2015 } \\
\text { Tarakci, 2013 } \\
\text { Jones, 2014a } \\
\text { Bahrami, 2019 } \\
\text { Elnaggar, 2019 } \\
\text { Scholtes, 2012 } \\
\text { Ahmandi, 2013 } \\
\text { Arntzen, 2020 }\end{array}$ & Moderate & Consistent & Imprecise & Not detected & $\begin{array}{l}\text { Moderate for } \\
\text { benefit }\end{array}$ & MD $-1.24,95 \% \mathrm{Cl}-2.04$ to -0.44 \\
\hline All exercise & Usual Care & $\begin{array}{l}\text { Function: } \\
\text { GMFM-66 } \\
\text { in CP } \\
\\
\\
\text { GMFM-66D } \\
\text { in CP } \\
\text { GMFM-66E } \\
\text { in CP } \\
\text { TUG }\end{array}$ & $\begin{array}{l}7 \text { (353) } \\
\text { Fowler, } 2010 \\
\text { Bryant, 2012 } \\
\text { Scholtes, } 2010 \\
\text { Deutz, 2017 } \\
\text { Herrero, } 2012 \\
\text { Slaman, 2015 } \\
\text { Van Wely, } 2014 \\
\\
2 \text { (78) } \\
\text { Wallard, } 2018 \\
\text { Taylor, 2013 } \\
3 \text { (151) } \\
\text { Wallard, } 2018 \\
\text { Taylor, 2013 } \\
\text { Deutz, 2017 } \\
2 \text { (70) } \\
\text { Hsieh, 2018 } \\
\text { Kaya Kara, } 2019 \\
\end{array}$ & Moderate & Consistent & Imprecise & Not detected & $\begin{array}{l}\text { Low-strength } \\
\text { evidence for } \\
\text { benefit }\end{array}$ & $\begin{array}{l}\text { GMFM-66: MD }-0.58,95 \% \mathrm{Cl}- \\
\text { 1.62 to } 0.45,12=79 \% \\
\text { GMFM-66D: MD }-0.89,95 \% \mathrm{Cl} \\
-7.33 \text { to } 5.55,12=60 \% \\
\text { GMFM-66E: MD }-3.73,95 \% \mathrm{Cl} \\
-5.78 \text { to }-1.67,12=0 \% \\
\text { TUG: MD }-1.05,95 \% \mathrm{Cl}-1.35 \\
\text { to }-0.76)\end{array}$ \\
\hline
\end{tabular}




\begin{tabular}{|c|c|c|c|c|c|c|c|c|c|}
\hline $\begin{array}{l}\text { Intervention } \\
\text { Category, } \\
\text { Intervention }\end{array}$ & Comparator & Outcome & $\begin{array}{l}\text { Number of RCTs } \\
\text { (Participants) } \\
\text { Author Year } \\
\text { (See Appendix B for } \\
\text { Full Citation) } \\
\end{array}$ & $\begin{array}{l}\text { Study } \\
\text { Limitations }\end{array}$ & Consistency & Precision & $\begin{array}{l}\text { Reporting } \\
\text { Bias }\end{array}$ & $\begin{array}{l}\text { Strength of } \\
\text { Evidence }\end{array}$ & $\begin{array}{l}\text { Findings, Direction and } \\
\text { Magnitude of Effect }\end{array}$ \\
\hline All exercise & Usual Care & $\begin{array}{l}\text { Walking in } \\
\mathrm{CP}\end{array}$ & $\begin{array}{l}7(234) \\
\text { Kim, 2015 } \\
\text { Taylor, 2013 } \\
\text { Bahmani, 2019 } \\
\text { Fosdahl, 2019b } \\
\text { Ahmadizadeh, } 2019 \\
\text { Elnagger, } 2019 \\
\text { Scholtes, } 2012\end{array}$ & Moderate & Consistant & Imprecise & No detected & \begin{tabular}{|l|} 
Low for no clear \\
benefit
\end{tabular} & $\begin{array}{l}\frac{\text { Pooled analysis (4 trials) }}{6 \mathrm{MWT}: \mathrm{MD} 6.85,95 \% \mathrm{Cl}-} \\
\text { 13.39 to } 27.08, \mathrm{I}^{2}=0 \% \\
\frac{\text { Pooled analysis ( } 3 \text { trials) }}{10 \mathrm{MWT}: \mathrm{MD}-0.46,95 \% \mathrm{Cl}} \text { - } \\
\text { 1.55 to } 0.63, \mathrm{I}^{2}=44 \%\end{array}$ \\
\hline All exercise & Usual Care & BBS & $\begin{array}{l}19(1006) \\
\text { Gervasoni, } 2014 \\
\text { Kargarfard, } 2018 \\
\text { Afrasiabifar, } 2018 \\
\text { Forsberg, } 2016 \\
\text { Carling, } 2017 \\
\text { Gandolfi, } 2015 \\
\text { Hsieh, 2018 } \\
\text { Vermohlen, } 2018 \\
\text { Sangelaji, } 2014 \\
\text { Sangelaji, } 2016 \\
\text { Ebrahimi, } 2015 \\
\text { Tarakci, 2013 } \\
\text { Brichetto, } 2015 \\
\text { Tollar, 2020 } \\
\text { Ozkul, 2020 } \\
\text { Yazgan, } 2019 \\
\text { Hota, 2020 } \\
\text { Ahmandi, } 2013 \\
\text { Kalron, } 2017 \\
\end{array}$ & Moderate & Consistent & Precise & Not detected & $\begin{array}{l}\text { Moderate for } \\
\text { benefit }\end{array}$ & $\begin{array}{l}\text { MD }-3.64,95 \% \mathrm{Cl}-4.23 \text { to }- \\
3.04, \mathrm{I}^{2}=68 \%\end{array}$ \\
\hline
\end{tabular}




\begin{tabular}{|c|c|c|c|c|c|c|c|c|c|}
\hline $\begin{array}{l}\text { Intervention } \\
\text { Category, } \\
\text { Intervention }\end{array}$ & Comparator & Outcome & $\begin{array}{l}\text { Number of RCTs } \\
\text { (Participants) } \\
\text { Author Year } \\
\text { (See Appendix B for } \\
\text { Full Citation) } \\
\end{array}$ & $\begin{array}{l}\text { Study } \\
\text { Limitations }\end{array}$ & Consistency & Precision & $\begin{array}{l}\text { Reporting } \\
\text { Bias }\end{array}$ & $\begin{array}{l}\text { Strength of } \\
\text { Evidence }\end{array}$ & $\begin{array}{l}\text { Findings, Direction and } \\
\text { Magnitude of Effect }\end{array}$ \\
\hline All exercise & Usual Care & TUG & $\begin{array}{l}19(\mathrm{~N}=882) \\
\text { Negaresh, } 2018 \\
\text { Russo, 2018 } \\
\text { Young, 2019 } \\
\text { Duff, 2018 } \\
\text { Bulguroglu, } 2017 \\
\text { Kalron, 2017 } \\
\text { Carling, 2017 } \\
\text { Forsberg, } 2016 \\
\text { Claerbout, } 2012 \\
\text { Nilsagard, } 2012 \\
\text { Hsieh, 2018 } \\
\text { In, 2018 } \\
\text { Cakit, 2010 } \\
\text { Ebrahimi, 2015 } \\
\text { Jones, 2014a } \\
\text { Kaya Kara, 2019 } \\
\text { Ozkul, 2020 } \\
\text { Yazgan, 2019 } \\
\text { Faramarzi, } 2020 \\
\end{array}$ & Moderate & Consistent & Imprecise & Undetected & $\begin{array}{l}\text { Low-strength } \\
\text { evidence for } \\
\text { benefit }\end{array}$ & $\begin{array}{l}\text { TUG: MD }-0.66,-1.28 \text { to }-0.04 \text {, } \\
\mathrm{I}^{2}=85 \%\end{array}$ \\
\hline All exercise & Usual Care & TUG in MS & $\begin{array}{l}15 \text { (N=743) } \\
\text { Negaresh, } 2018 \\
\text { Russo, } 2018 \\
\text { Young, } 2019 \\
\text { Duff, 2018 } \\
\text { Bulguroglu, } 2017 \\
\text { Kalron, 2017 } \\
\text { Carling, 2017 } \\
\text { Forsberg, } 2016 \\
\text { Claerbout, } 2012 \\
\text { Nilsagard, } 2012 \\
\text { Cakit, 2010 } \\
\text { Ebrahimi, } 2015 \\
\text { Ozkul, 2020 } \\
\text { Yazgan, 2019 }\end{array}$ & Moderate & Consistent & Precise & Undetected & $\begin{array}{l}\text { Moderate- } \\
\text { strength } \\
\text { evidence for no } \\
\text { clear benefit }\end{array}$ & $\begin{array}{l}\text { TUG: MD }-0.30,95 \% \mathrm{Cl}-1.18 \\
\text { to } 0.59, \mathrm{I}^{2}=89 \%\end{array}$ \\
\hline
\end{tabular}




\begin{tabular}{|c|c|c|c|c|c|c|c|c|c|}
\hline $\begin{array}{l}\text { Intervention } \\
\text { Category, } \\
\text { Intervention }\end{array}$ & Comparator & Outcome & $\begin{array}{l}\text { Number of RCTs } \\
\text { (Participants) } \\
\text { Author Year } \\
\text { (See Appendix B for } \\
\text { Full Citation) }\end{array}$ & $\begin{array}{l}\text { Study } \\
\text { Limitations }\end{array}$ & Consistency & Precision & $\begin{array}{l}\text { Reporting } \\
\text { Bias }\end{array}$ & $\begin{array}{l}\text { Strength of } \\
\text { Evidence }\end{array}$ & $\begin{array}{l}\text { Findings, Direction and } \\
\text { Magnitude of Effect }\end{array}$ \\
\hline All exercise & Usual Care & BBS in MS & $\begin{array}{l}17 \text { (906) } \\
\text { Gervasoni, } 2014 \\
\text { Kargarfard, } 2018 \\
\text { Afrasiabifar, } 2018 \\
\text { Forsberg, } 2016 \\
\text { Carling, 2017 } \\
\text { Gandolfi, } \\
\text { 2015Vermohlen, } 2018 \\
\text { Sangelaji, 2014 } \\
\text { Sangelaji, } 2016 \\
\text { Ebrahimi, } 2015 \\
\text { Tarakci, 2013 } \\
\text { Ahmadi, 2013 } \\
\text { Tollar, 2020 } \\
\text { Kalron, 2017 } \\
\text { Brichetto, } 2015 \\
\text { Ozkul, 2020 } \\
\text { Yazgan, } 2019 \\
\end{array}$ & Moderate & Consistent & Precise & Not detected & $\begin{array}{l}\text { Moderate for } \\
\text { benefit }\end{array}$ & $\begin{array}{l}\text { BBS: MD }-3.56,95 \% \mathrm{Cl}-4.58 \text { to } \\
-2.54, I^{2}=77 \%\end{array}$ \\
\hline All exercise & Usual Care & $\begin{array}{l}\text { Function in } \\
\mathrm{CP}\end{array}$ & \begin{tabular}{|l}
$11(500)$ \\
Hsieh, 2018 \\
Kaya Kara, 2019 \\
Fowler, 2010 \\
Bryant, 2012 \\
Schlotes, 2010 \\
Deutz, 2017 \\
Herrero, 2012 \\
Mutoh, 2019 \\
Slaman, 2015 \\
Van Wely, 2014
\end{tabular} & Moderate & Consistent & Imprecise & Not detected & Low for benefit & $\begin{array}{l}\text { BBS: MD }-3.09,95 \% \mathrm{Cl}-4.60 \text { to } \\
\text {-1.58 } \\
\text { Pooled TUG: }-1.05,95 \% \mathrm{Cl}- \\
1.35 \text { to }-0.76, \mathrm{I}^{2}=0 \% \\
\text { Pooled GMFM-66: } \mathrm{MD}-0.58 \\
95 \% \mathrm{Cl}-1.62 \text { to } 0.45, \mathrm{I}^{2}=79 \%\end{array}$ \\
\hline All exercise & Usual Care & $\begin{array}{l}\text { Function in } \\
\mathrm{SCl}\end{array}$ & $\begin{array}{l}4(129) \\
\text { Norouzi, } 2019 \\
\text { Hota, } 2020 \\
\text { Jones, } 2014 \\
\text { In, } 2018\end{array}$ & Moderate & Consistent & Imprecise & Not detected & Low for benefit & $\begin{array}{l}\text { Pooled BBS: MD }-4.53,95 \% \mathrm{Cl} \\
\text {-6.46 to }-2.61, \mathrm{I}^{2}=0 \% \\
\text { 6MWT: MD }-32.97,95 \% \mathrm{Cl}- \\
\text { 68.17 to } 2.23 \\
\frac{\text { Pooled analysis ( } 2 \text { trials) }}{10 \mathrm{MWT}: \mathrm{MD}-5.06,95 \% \mathrm{Cl}-} \\
13.29 \text { to } 3.15, \mathrm{I}^{2}=55 \% \\
\text { Pooled analysis (2 trials) TUG: - } \\
10.33,95 \% \mathrm{Cl}-37.10 \text { to } 16.45, \\
\mathrm{I}^{2}=61 \%\end{array}$ \\
\hline
\end{tabular}

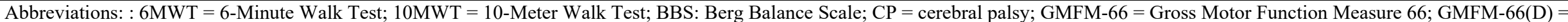

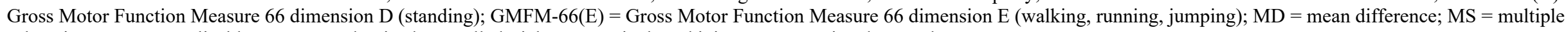
sclerosis; NA = not applicable; RCT = randomized controlled trial; $\mathrm{SCI}=$ spinal cord injury; $\mathrm{TUG}=\mathrm{Timed}$ Up and Go Test 
Table H-16. Strength of evidence for Key Question $2 \mathrm{~b}$ intermediate outcomes: effect of physical activity interventions on $\mathrm{VO}_{2}$ peak and $\mathrm{VO}_{2}$ max

\begin{tabular}{|c|c|c|c|c|c|c|c|c|c|}
\hline $\begin{array}{l}\text { Intervention } \\
\text { Category, } \\
\text { Intervention }\end{array}$ & Comparator & Outcome & $\begin{array}{l}\text { Number of } \\
\text { Studies } \\
\text { (Participants) } \\
\text { Author Year } \\
\text { (See Appendix B } \\
\text { for Full Citation) }\end{array}$ & $\begin{array}{l}\text { Study } \\
\text { Limitations }\end{array}$ & Consistency & Precision & $\begin{array}{l}\text { Reporting } \\
\text { Bias }\end{array}$ & $\begin{array}{l}\text { Strength of } \\
\text { Evidence }\end{array}$ & $\begin{array}{l}\text { Findings, Direction and } \\
\text { Magnitude of Effect }\end{array}$ \\
\hline $\begin{array}{l}\text { Aerobic } \\
\text { Exercise } \\
\text { in } \mathrm{CP}\end{array}$ & Usual care & $\mathrm{VO}_{2}$ peak & $\begin{array}{l}2 \\
\text { quasiexperimental } \\
(54) \\
\text { Nsenga, } 2013 \\
\text { Nsenga Leunkeu, } \\
2012\end{array}$ & Moderate & Consistent & Imprecise & Undetected & $\begin{array}{l}\text { Low for } \\
\text { benefit }\end{array}$ & $\begin{array}{l}\text { Both studies show significant } \\
\text { increase in VO2 Peak } \mathrm{ml} / \mathrm{min} \\
\text { from baseline with training, not } \\
\text { in usual care. (estimates from } \\
\text { graphs): } \\
\text { VO2 Peak: } 32.5 \text { to } 39.0 \\
\text { ( }<<0.05 \text { ) vs. } 32.5 \text { to } 32.5 \\
\text { (P>0.05) } \\
\text { VO2 Peak (ml } / \mathrm{kg} / \mathrm{min}: 7.00 \\
95 \% \text { Cl } 1.93 \text { to } 12.07, p=0.007\end{array}$ \\
\hline $\begin{array}{l}\text { Aerobic } \\
\text { Exercise } \\
\text { in MS }\end{array}$ & Usual care & $\mathrm{VO}_{2}$ peak & $\begin{array}{l}4(251) \\
\text { Baquet, 2018, } \\
\text { Heine, } 2017 \\
\text { Negaresh, } 2018\end{array}$ & Moderate & Inconsistent & Imprecise & Undetected & Insufficient & $\begin{array}{l}\text { Mean difference between } \\
\text { groups: } \\
\text { Study } 1 \text { : VO2 Peak (ml/min): - } \\
51.4,95 \% \mathrm{Cl}-165.2 \text { to } 62.5, \\
p=0.37 \\
\text { VO2 Peak (ml } / \mathrm{min} / \mathrm{kg}):-0.9, \\
95 \% \mathrm{Cl}-2.5 \text { to } 0.6, p=0.24 \\
\text { Study } 2: \text { A vs. B, difference } \\
\text { between groups (SD), } \\
\text { VO2 Peak (L/min): MD } 0.048 \\
\text { (0.082), } p=0.561 \\
\text { VO2 Peak (mL } / \mathrm{kg} / \mathrm{min}): \text { MD } \\
0.979(1.075), p=0.364 \\
\text { Study 3: VO2 Peak (change } \\
\text { from baseline, estimated from } \\
\text { graph): } 2.7 \text { vs. } 0 \text { vs. } 1.9 \text { vs. } \\
0.6, p=0.001\end{array}$ \\
\hline $\begin{array}{l}\text { Aerobic } \\
\text { Exercise in } \\
\text { SCl }\end{array}$ & Usual care & $\mathrm{VO}_{2}$ peak & $\begin{array}{l}2(71) \\
\text { van der Scheer } \\
2016 \\
\text { Lavado, } 2012 \\
1 \text { cohort study } \\
(\mathrm{N}=17) \\
\text { Valent, } 2010\end{array}$ & Moderate & Consistent & Imprecise & Undetected & $\begin{array}{l}\text { Low for } \\
\text { benefit }\end{array}$ & $\begin{array}{l}\text { Median change } \\
\text { RCT 1: VO2 Peak (L/min): } 0.05 \\
\text { to }-0.07, p=0.01 \\
\text { RCT 2: VO2 Peak (mL/min): } \\
939 \text { to } 1154(p=0.009) \text { vs. } 896 \\
\text { to } 834, p=0.906 ; \text { Post- } \\
\text { intervention comparison, } \\
\text { p<0.001 } \\
\text { A vs. B, Mean change } \\
\text { Cohort: VO2peak }(\mathrm{ml} / \mathrm{min}): \\
0.21 \text { vs. } 0.13, p=0.356 \\
\text { VO2 Peak }(\mathrm{ml} / \mathrm{kg} / \mathrm{min}): 2.9 \text { vs. } \\
1.5, p=0.274\end{array}$ \\
\hline
\end{tabular}




\begin{tabular}{|c|c|c|c|c|c|c|c|c|c|}
\hline $\begin{array}{l}\text { Intervention } \\
\text { Category, } \\
\text { Intervention }\end{array}$ & Comparator & Outcome & $\begin{array}{l}\text { Number of } \\
\text { Studies } \\
\text { (Participants) } \\
\text { Author Year } \\
\text { (See Appendix B } \\
\text { for Full Citation) }\end{array}$ & $\begin{array}{l}\text { Study } \\
\text { Limitations }\end{array}$ & Consistency & Precision & $\begin{array}{l}\text { Reporting } \\
\text { Bias }\end{array}$ & $\begin{array}{l}\text { Strength of } \\
\text { Evidence }\end{array}$ & $\begin{array}{l}\text { Findings, Direction and } \\
\text { Magnitude of Effect }\end{array}$ \\
\hline $\begin{array}{l}\text { Aerobic } \\
\text { Exercise in } \\
\text { SCl }\end{array}$ & $\begin{array}{l}\text { Head to head } \\
\text { comparison of } \\
\text { aerobic } \\
\text { programs }\end{array}$ & $\mathrm{VO}_{2}$ peak & $\begin{array}{l}3(100) \\
\text { Gorman, } 2019 \\
\text { Alexeeva, } 2011 \\
\text { Akkurt, } 2017\end{array}$ & Moderate & Inconsistent & Imprecise & Undetected & Insufficient & $\begin{array}{l}\text { Median change: } \\
\text { VO2Peak (ml/kg/min): } 4.30 \text { vs. } \\
1.35, p=0.02 \\
\text { A vs. B, Mean (SD) } \\
\text { VO2Peak (ml/kg/min): } 16.48 \\
(5.39) \text { to } 16.18(5.11) \text { vs.13.33 } \\
\text { (3.06) to } 14.31(3.88), p=0.063 \\
\text { A vs. B vs. C, Mean (SD) } \\
\text { VO2Peak (ml/km/min): } 12 \% \\
\text { nonsignificant increase within } \\
\text { groups, but no differences } \\
\text { between groups, } p>0.05\end{array}$ \\
\hline $\begin{array}{l}\text { Multimodal } \\
\text { Exercise in CP }\end{array}$ & Usual care, & $\mathrm{VO}_{2}$ peak & $\begin{array}{l}1(57) \\
\text { Slaman, 2014a, } \\
2014 b, 2018\end{array}$ & Moderate & Unknown & Imprecise & Undetected & Insufficient & $\begin{array}{l}\text { Mean difference between } \\
\text { groups: } \\
\text { VO2 Peak ( } \mathrm{mL} / \mathrm{min}): \mathrm{MD} \\
195.2,95 \% \mathrm{Cl} 57.3 \text { to } 333.1 \text {, } \\
\text { p<0.01 }\end{array}$ \\
\hline $\begin{array}{l}\text { Multimodal } \\
\text { Exercise in } \\
\text { MS }\end{array}$ & Usual care & $\begin{array}{l}\mathrm{VO}_{2} \\
\mathrm{max} / \text { peak }\end{array}$ & $\begin{array}{l}12 \text { (123) } \\
\text { Wens, 2015b (high } \\
\text { intensity) } \\
\text { Banitalebi, } 2020\end{array}$ & Moderate & UConsistent & Imprecise & Undetected & $\begin{array}{l}\text { ILow for } \\
\text { benefit }\end{array}$ & $\begin{array}{l}\text { Mean (SD) } \\
\text { Study 1: } \\
\text { VO2 Max (ml/min): } 17.8 \% \\
(4.6 \%) \text { vs. } 2.5 \%(4.1 \%), p<0.01 \\
\text { VO2 Max }(\mathrm{ml} / \mathrm{min} / \mathrm{kg}): 17.8 \% \\
(4.6 \%) \text { vs. } 2.5 \%(4.1 \%), p<0.01 \\
\% \text { Body fat: } \\
\text { Study } 2: \\
\text { VO2 peak: } p=0.004, \text { effect of } \\
\text { disability*exercise } p=0.097\end{array}$ \\
\hline
\end{tabular}




\begin{tabular}{|c|c|c|c|c|c|c|c|c|c|}
\hline $\begin{array}{l}\text { Intervention } \\
\text { Category, } \\
\text { Intervention }\end{array}$ & Comparator & Outcome & $\begin{array}{l}\text { Number of } \\
\text { Studies } \\
\text { (Participants) } \\
\text { Author Year } \\
\text { (See Appendix B } \\
\text { for Full Citation) }\end{array}$ & $\begin{array}{l}\text { Study } \\
\text { Limitations }\end{array}$ & Consistency & Precision & $\begin{array}{l}\text { Reporting } \\
\text { Bias }\end{array}$ & $\begin{array}{l}\text { Strength of } \\
\text { Evidence }\end{array}$ & $\begin{array}{l}\text { Findings, Direction and } \\
\text { Magnitude of Effect }\end{array}$ \\
\hline $\begin{array}{l}\text { Multimodal } \\
\text { Exercise in } \\
\text { MS }\end{array}$ & $\begin{array}{l}\text { Head-to-head } \\
\text { comparison }\end{array}$ & $\begin{array}{l}\mathrm{VO}_{2} \\
\text { peak/max }\end{array}$ & $\begin{array}{l}2 \text { (96) } \\
\text { Wens, 2015b (high } \\
\text { intensity); } \\
\text { Sandroff, } 2017\end{array}$ & Moderate & Unknown & Imprecise & Undetected & Insufficient & $\begin{array}{l}\text { Mean (SD) } \\
\text { VO2 Peak (ml/kg/min): } \\
16.5(6.5) \text { vs. } 15.4(6.2), p=N R \\
\text { (baseline) } \\
17.1(5.9) \text { vs. } 15.9 \text { (5.5), p=NR } \\
\text { (post-intervention) } \\
\text { Time X Group interaction } \\
\text { p>0.20 } \\
\text { Mean (SD) of \% change } \\
\text { A vs. Control } \\
\text { VO2 Max (ml/min): } 17.8 \% \\
(4.6 \%) \text { vs. } 2.5 \%(4.1 \%), p<0.01 \\
\text { VO2 Max (ml/min/kg):17.8\% } \\
(4.6 \%) \text { vs. } 2.5 \% \\
\text { B vs. Control } \\
\text { VO2 Max (ml } / \mathrm{min}): 7.5 \% \\
(5.8 \%) \text { vs. } 2.5 \%(4.1 \%), p>0.05 \\
\text { VO2 Max (ml } / \mathrm{min} / \mathrm{kg}): 7.5 \% \\
(5.8 \%) \text { vs. } 2.5 \%(4.1 \%), p>0.05\end{array}$ \\
\hline
\end{tabular}

Abbreviations: $\mathrm{CI}=$ confidence interval; $\mathrm{NA}=$ not applicable; $\mathrm{RCT}=$ randomized controlled trial. $\mathrm{SD}=$ standard deviation 
Table H-17. Strength of evidence for Key Question 2b intermediate outcomes: effect of physical activity interventions on pulmonary function tests

\begin{tabular}{|c|c|c|c|c|c|c|c|c|c|}
\hline $\begin{array}{l}\text { Intervention } \\
\text { Category, } \\
\text { Intervention }\end{array}$ & Comparator & Outcome & $\begin{array}{l}\text { Number of } \\
\text { Studies } \\
\text { (Participants) } \\
\text { Author Year } \\
\text { (See Appendix B } \\
\text { for Full Citation) }\end{array}$ & $\begin{array}{l}\text { Study } \\
\text { Limitations }\end{array}$ & Consistency & Precision & $\begin{array}{l}\text { Reporting } \\
\text { Bias }\end{array}$ & $\begin{array}{l}\text { Strength } \\
\text { of } \\
\text { Evidence }\end{array}$ & $\begin{array}{l}\text { Findings, Direction and } \\
\text { Magnitude of Effect }\end{array}$ \\
\hline $\begin{array}{l}\text { Aerobic } \\
\text { Exercise in } \\
\text { SCl }\end{array}$ & Usual care & $\begin{array}{l}\text { Pulmonary } \\
\text { function }\end{array}$ & $\begin{array}{l}1 \text { cohort study } \\
(n=17) \\
\text { Valent, } 2010\end{array}$ & Moderate & Unknown & Imprecise & Undetected & Insufficient & $\begin{array}{l}\text { Mean change scores, } p=\text { between } \\
\text { groups: } \\
\text { FVC\%: }-9.4 \text { vs. }-7.8, p=0.619 \\
\text { PEF\%: }-12.6 \text { vs. }-10.0, p=0.722\end{array}$ \\
\hline $\begin{array}{l}\text { Aerobic } \\
\text { Exercise in } \\
\text { SCl }\end{array}$ & $\begin{array}{l}\text { Head-to- } \\
\text { head } \\
\text { comparison } \\
\text { of aerobic } \\
\text { programs }\end{array}$ & $\begin{array}{l}\text { Pulmonary } \\
\text { function }\end{array}$ & $\begin{array}{l}\text { 1 RCT }(n=33) \\
\text { Akkurt, } 2017\end{array}$ & Moderate & Unknown & Imprecise & Undetected & Insufficient & $\begin{array}{l}\text { Median change } \\
\text { VO2Peak }(\mathrm{ml} / \mathrm{kg} / \mathrm{min}): 4.30 \text { vs. } \\
1.35, p=0.02 \\
\text { FEV1 (ml): }-0.14 \text { vs. } 0.17, p>0.05 \\
\text { FEV1 \%: } 1 \text { vs. } 5, p>0.05 \\
\text { FVC (ml): }-0.31 \text { vs. }-0.20, p>0.05 \\
\text { FVC \%: } 1.5 \text { vs. } 1.5, p>0.05 \\
\text { FEV1/FVC, } 3.51-0.50, p>0.05\end{array}$ \\
\hline $\begin{array}{l}\text { Aerobic } \\
\text { Exercise in } \\
\text { SCl }\end{array}$ & $\begin{array}{l}\text { Head-to- } \\
\text { head } \\
\text { comparison } \\
\text { of aerobic } \\
\text { programs }\end{array}$ & $\begin{array}{l}\text { Pulmonary } \\
\text { function }\end{array}$ & $\begin{array}{l}\text { 1 RCT }(n=20) \\
\text { Jung, } 2014\end{array}$ & Moderate & Unknown & Imprecise & Undetected & Insufficient & $\begin{array}{l}\text { Mean change scores, } p=\text { between } \\
\text { groups: } \\
\frac{\text { FVC }(L):}{p=0.031} 1.8(1.3) \text { vs. } 0.31(1.6), \\
\frac{\text { FEV1(L): } 1.1(1.2) \text { vs. } 0.21(0.3) ;}{p=0.038} \\
\frac{\text { FER(L/sec): } 10.0(9.7) \text { vs. } 5.4}{(7.0), p=0.238} \\
\frac{\text { FEV1/FVC: } 3.7(2.3) \text { vs. } 2.1(3.4),}{p=0.243}\end{array}$ \\
\hline $\begin{array}{l}\text { Strength } \\
\text { Exercise } \\
\text { in } \mathrm{SCl}\end{array}$ & Usual Care & $\begin{array}{l}\text { Pulmonary } \\
\text { function }\end{array}$ & $\begin{array}{l}1 \text { RCT }(n=98) \\
\text { Chen, } 2016\end{array}$ & Moderate & Unknown & Imprecise & Undetected & Insufficient & $\begin{array}{l}\text { Mean (SD), } p=\text { post-intervention: } \\
\text { FEV1: } 1.17(0.25) \text { to } 2.20(0.45) \\
\text { vs. } 1.17(0.45) \text { to } 1.14(0.44), \\
\text { p<0.05 } \\
\text { FVC: } 2.16(0.36) \text { to } 2.98(0.54) \text { vs } \\
2.16(0.42) \text { to } 2.17(0.42), p<0.05 \\
\text { MVV: } 50.5(11.8) \text { to } 75.2(6.8) \text { vs. } \\
50.5(11.8) \text { to } 51.5(10.6), p<0.05 \\
\text { FEV1/FVC: } 0.53(0.17 \text { to } 0.75 \\
(0.08) \text { vs. } 0.53(0.17) \text { to } 0.52 \\
(0.15), p<0.05\end{array}$ \\
\hline
\end{tabular}

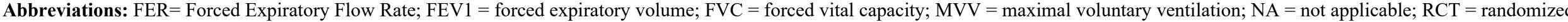
controlled trial; SCI = spinal cord injury 
Table H-18. Strength of evidence for Key Question 2c: reduction of harms of immobility

\begin{tabular}{|c|c|c|c|c|c|c|c|c|c|}
\hline $\begin{array}{l}\text { Intervention } \\
\text { Category, } \\
\text { Intervention }\end{array}$ & Comparator & Outcome & $\begin{array}{l}\text { Number of } \\
\text { Studies } \\
\text { (Participants) } \\
\text { Author Year } \\
\text { (See Appendix } \\
\text { B for Full } \\
\text { Citation) }\end{array}$ & $\begin{array}{l}\text { Study } \\
\text { Limitations }\end{array}$ & Consistency & Precision & $\begin{array}{l}\text { Reporting } \\
\text { Bias }\end{array}$ & $\begin{array}{l}\text { Strength of } \\
\text { Evidence }\end{array}$ & $\begin{array}{l}\text { Findings, Direction and } \\
\text { Magnitude of Effect }\end{array}$ \\
\hline--- & --- & $\begin{array}{l}\text { Decubitus } \\
\text { Ulcer }\end{array}$ & No studies & --- & --- & --- & --- & --- & No studies \\
\hline $\begin{array}{l}\text { Arm cycling + } \\
\text { strength + } \\
\text { stretching }\end{array}$ & $\begin{array}{l}\text { Usual } \\
\text { physical } \\
\text { therapy }\end{array}$ & $\begin{array}{l}\text { Asymptoma } \\
\text { tic bacturia }\end{array}$ & $\begin{array}{l}1(42) \\
\text { Lavado, } 2012\end{array}$ & Moderate & Unknown & Imprecise & Not detected & Insufficient & $\begin{array}{l}\text { RR } 0.20,95 \% \text { Cl } 0.07 \text { to } \\
0.54, p<0.001\end{array}$ \\
\hline RAGT & $\begin{array}{l}\text { Treadmill } \\
\text { training }\end{array}$ & $\begin{array}{l}\text { Enema } \\
\text { dose } \\
\text { needed }\end{array}$ & $\begin{array}{l}1(24) \text { Huang, } \\
2015\end{array}$ & Moderate & Unknown & Imprecise & Not detected & Insufficient & $-29 \mathrm{ml}$ vs. $-11 \mathrm{~mL}, \mathrm{p}<0.05$ \\
\hline RAGT & $\begin{array}{l}\text { Treadmill } \\
\text { training }\end{array}$ & $\begin{array}{l}\text { Defecation } \\
\text { time }\end{array}$ & $\begin{array}{l}1(24) \text { Huang, } \\
2015\end{array}$ & Moderate & Unknown & Imprecise & Not detected & Insufficient & $-29 \min$ vs. $-15 \min , p<0.05$ \\
\hline--- & --- & $\begin{array}{l}\text { Autonomic } \\
\text { dysreflexia }\end{array}$ & No studies & --- & --- & --- & --- & --- & No studies \\
\hline
\end{tabular}


Table H-19. Strength of evidence for Key Question 2d, decreased risk of adverse outcomes of mobility devices: effect of physical activity interventions on spasticity

\begin{tabular}{|c|c|c|c|c|c|c|c|c|c|}
\hline $\begin{array}{l}\text { Intervention } \\
\text { Category, } \\
\text { Intervention }\end{array}$ & Comparator & Outcome & $\begin{array}{l}\text { Number of } \\
\text { Studies } \\
\text { (Participants) } \\
\text { Author Year } \\
\text { (See } \\
\text { Appendix B } \\
\text { for Full } \\
\text { Citation) } \\
\end{array}$ & $\begin{array}{l}\text { Study } \\
\text { Limitations }\end{array}$ & Consistency & Precision & $\begin{array}{l}\text { Reporting } \\
\text { Bias } \\
\end{array}$ & $\begin{array}{l}\text { Strength } \\
\text { of } \\
\text { Evidence }\end{array}$ & $\begin{array}{l}\text { Findings, Direction and } \\
\text { Magnitude of Effect }\end{array}$ \\
\hline $\begin{array}{l}\text { Aerobic Exercise } \\
\text { (Treadmill, } \\
\text { Aquatic) in } \mathrm{CP}\end{array}$ & Usual care & Spasticity & $\begin{array}{l}\text { 1 RCT }(\mathrm{N}=11) \\
1 \text { Cohort } \\
(\mathrm{N}=11) \\
\text { Chrysagis, } \\
2012 \\
\text { Lai, } 2015\end{array}$ & Moderate & Inconsistent & Imprecise & Undetected & Insufficient & $\begin{array}{l}\text { RCT: Mean change, } p=\text { value } \\
\text { Modified Ashworth Scale: } \\
\text { Knee extensors: } 0.32 \text { vs. } 0.18 \text {, } \\
p=0.827 \\
\text { Knee flexors: } 0.31 \text { vs. } 0.22 \\
p=0.632 \\
\text { Foot plantar flexors: } 0.32 \text { vs. } \\
0.17, p=0.460 \\
\text { Cohort: } \\
\text { A vs B (ANCOVA p-values) } \\
\text { Modified Ashworth Scale: } \\
\text { Ankle: } 0.614 \\
\text { Knee: } 1.000 \\
\text { Wrist: } 1.000 \\
\text { Elbow: } 1.000\end{array}$ \\
\hline $\begin{array}{l}\text { Aerobic Exercise } \\
\text { (Treadmill) in CP }\end{array}$ & $\begin{array}{l}\text { RAGT vs } \\
\text { Treadmill }\end{array}$ & Spasticity & $\begin{array}{l}\text { 1 RCT }(\mathrm{N}=21) \\
\text { Wu, 2017a } \\
\text { (pilot study) }\end{array}$ & Moderate & Unknown & Imprecise & Undetected & Insufficient & $\begin{array}{l}\text { Modified Ashworth Scale } \\
\text { (Baseline vs } 6 \text { weeks vs } 8 \\
\text { weeks f/u) } \\
0.62(0.46) \text { to } 0.67(0.60) \text { to } \\
0.41(0.38), p=0.18, \text { vs. } \\
0.65(0.36) \text { to } 0.48(0.47) \text { to } \\
0.58(0.44), p=0.19\end{array}$ \\
\hline $\begin{array}{l}\text { Aerobic Exercise } \\
\text { in } \mathrm{CP}\end{array}$ & $\begin{array}{l}\text { Partial body- } \\
\text { weight } \\
\text { supported } \\
\text { treadmill vs } \\
\text { individualized } \\
\text { strength } \\
\text { training }\end{array}$ & Spasticity & $\begin{array}{l}\text { 1 RCT }(\mathrm{N}=26) \\
\text { Johnston, } \\
2011\end{array}$ & Moderate & Unknown & Imprecise & Undetected & Insufficient & $\begin{array}{l}\text { Mean difference between } \\
\text { groups, } p=\text { between groups } \\
\text { KinCom computerized } \\
\text { dynamometer: } \\
\text { Plantar Flexor Spasticity }(\mathrm{J} / \mathrm{O} / \mathrm{s}) \text { : } \\
-0.0003, \mathrm{p}=0.75 \\
\text { Knee flexor spasticity }(\mathrm{J} / \mathrm{O} / \mathrm{s}) \text { : } \\
-0.0026, \mathrm{p}=0.59\end{array}$ \\
\hline
\end{tabular}




\begin{tabular}{|c|c|c|c|c|c|c|c|c|c|}
\hline $\begin{array}{l}\text { Intervention } \\
\text { Category, } \\
\text { Intervention }\end{array}$ & Comparator & Outcome & $\begin{array}{l}\text { Number of } \\
\text { Studies } \\
\text { (Participants) } \\
\text { Author Year } \\
\text { (See } \\
\text { Appendix B } \\
\text { for Full } \\
\text { Citation) }\end{array}$ & $\begin{array}{l}\text { Study } \\
\text { Limitations }\end{array}$ & Consistency & Precision & $\begin{array}{l}\text { Reporting } \\
\text { Bias }\end{array}$ & $\begin{array}{l}\text { Strength } \\
\text { of } \\
\text { Evidence }\end{array}$ & $\begin{array}{l}\text { Findings, Direction and } \\
\text { Magnitude of Effect }\end{array}$ \\
\hline $\begin{array}{l}\text { Aerobic Exercise } \\
\text { in } C P\end{array}$ & $\begin{array}{l}\text { Aquatic vs } \\
\text { land-based } \\
\text { exercise }\end{array}$ & Spasticity & $\begin{array}{l}1 \mathrm{RCT}(\mathrm{N}=32) \\
\text { Adar, } 2017\end{array}$ & Moderate & Unknown & Imprecise & Undetected & Insufficient & $\begin{array}{l}\text { Median pre-post } p \text {-values on } \\
\text { MAS } \\
\text { Location Aquatics } \quad \text { Land } \\
\text { RKneeFlexors } 0.039 \\
0.008 \\
\text { LKneeFlexors } 0.003 \\
0.003 \\
\text { RAnkleFlexors } 0.005 \\
0.001 \\
\text { LAnkleFlexors } 0.046 \\
0.046 \\
\text { RHipAdductors } 0.025 \\
0.083 \\
\text { LHipAdductors } 0.003 \\
0.013\end{array}$ \\
\hline $\begin{array}{l}\text { Aerobic Exercise } \\
\text { in MS }\end{array}$ & $\begin{array}{l}\text { Neuromuscul } \\
\text { ar electrical } \\
\text { stimulation+ } \\
\text { Strength } \\
\text { exercises vs } \\
\text { NMS alone }\end{array}$ & Spasticity & $\begin{array}{l}\text { RCT } \\
(\mathrm{N}=100) \\
\text { Qi, 2018a }\end{array}$ & Moderate & Unknown & Imprecise & Undetected & Insufficient & $\begin{array}{l}\text { Mean difference between } \\
\text { groups: } \\
\text { Comprehensive Spasticity Scale } \\
\frac{(\mathrm{CSS}): 1.6,95 \% \text { Cl } 0.33 \text { to } 2.87,}{\mathrm{p}=0.01}\end{array}$ \\
\hline $\begin{array}{l}\text { Aerobic Exercise } \\
\text { in MS }\end{array}$ & $\begin{array}{l}\text { Lokomat-Pros } \\
\text { (RAGT+VIRT } \\
\text { UAL } \\
\text { REALITY) VS } \\
\text { Lokomat- } \\
\text { Nanos } \\
\text { (RAGT alone) }\end{array}$ & Spasticity & $\begin{array}{l}\text { 1 RCT (N=40) } \\
\text { Calabro, } 2017\end{array}$ & Low & Unknown & Imprecise & Undetected & Insufficient & $\begin{array}{l}\text { Effect size, } p \text {-value is between } \\
\text { groups: } \\
\text { MAS: }-0.01,95 \% \mathrm{Cl}-0.539 \text { to } \\
0.539, p=0.40\end{array}$ \\
\hline $\begin{array}{l}\text { Aerobic Exercise } \\
\text { in MS }\end{array}$ & $\begin{array}{l}\text { RAGT vs } \\
\text { Conventional } \\
\text { walking } \\
\text { training }\end{array}$ & Spasticity & $\begin{array}{l}\text { 1 RCT (N=23) } \\
\text { Pompa, } 2017\end{array}$ & Moderate & Unknown & Imprecise & Undetected & Insufficient & $\begin{array}{l}\text { Mean SD, } p=\text { between groups: } \\
\text { Spasticity VAS } 100 \mathrm{~mm} \text { ranged } \\
\text { from "no problem" to "very bad": } \\
5.05 \text { to } 3.40 \text { vs. } 5.31 \text { to } 5.23, \\
p=0.048\end{array}$ \\
\hline $\begin{array}{l}\text { Aerobic Exercise } \\
\text { in MS }\end{array}$ & $\begin{array}{l}\text { Aquatics vs. } \\
\text { land-based } \\
\text { relaxation } \\
\text { exercises }\end{array}$ & Spasticity & $1 \mathrm{RCT}(\mathrm{N}=73)$ & Low & Unknown & Imprecise & Undetected & $\begin{array}{l}\text { Low for } \\
\text { benefit }\end{array}$ & $\begin{array}{l}\text { Spasm VAS: } 5(2.8) \text { to } 2(4.3) \\
\text { vs. } 6(3.1) \text { to } 4(4.5), 91 \% \\
\text { improvement vs. } 10 \% \\
\text { improvement, } p<0.05\end{array}$ \\
\hline
\end{tabular}




\begin{tabular}{|c|c|c|c|c|c|c|c|c|c|}
\hline $\begin{array}{l}\text { Intervention } \\
\text { Category, } \\
\text { Intervention }\end{array}$ & Comparator & Outcome & $\begin{array}{l}\text { Number of } \\
\text { Studies } \\
\text { (Participants) } \\
\text { Author Year } \\
\text { (See } \\
\text { Appendix B } \\
\text { for Full } \\
\text { Citation) }\end{array}$ & $\begin{array}{l}\text { Study } \\
\text { Limitations }\end{array}$ & Consistency & Precision & $\begin{array}{l}\text { Reporting } \\
\text { Bias }\end{array}$ & $\begin{array}{l}\text { Strength } \\
\text { of } \\
\text { Evidence }\end{array}$ & $\begin{array}{l}\text { Findings, Direction and } \\
\text { Magnitude of Effect }\end{array}$ \\
\hline $\begin{array}{l}\text { Aerobic Exercise } \\
\text { in SCl }\end{array}$ & $\begin{array}{l}\text { Body weight } \\
\text { support } \\
\text { treadmill with } \\
\text { FES vs } \\
\text { Aerobic and } \\
\text { resistance } \\
\text { training }\end{array}$ & Spasticity & $\begin{array}{l}1 \mathrm{RCT}(\mathrm{N}=34) \\
\text { Kapadia, } 2014\end{array}$ & Moderate & Unknown & Imprecise & Undetected & Insufficient & $\begin{array}{l}\text { MAS: No between group } \\
\text { differences in MAS involving the } \\
\text { hip, knee, and ankle } \\
\text { joints.(data/results not reported) }\end{array}$ \\
\hline $\begin{array}{l}\text { Aerobic Exercise } \\
\text { in } \mathrm{SCl}\end{array}$ & $\begin{array}{l}\text { RAGT+rTMS } \\
\text { vs } \\
\text { RAGT+sham } \\
\text { rTMS }\end{array}$ & Spasticity & $\begin{array}{l}\text { 1 RCT }(\mathrm{N}=31) \\
\text { Kumru, } 2016\end{array}$ & Moderate & Unknown & Imprecise & Undetected & Insufficient & $\begin{array}{l}\text { Mean Difference between } \\
\text { groups: } \\
\frac{\text { MAS: }}{0.54, p=0.59}, 95 \% \mathrm{Cl}-0.94 \text { to }\end{array}$ \\
\hline $\begin{array}{l}\text { Strength } \\
\text { Exercise } \\
\text { (progressive } \\
\text { resistance) in CP }\end{array}$ & Usual care & Spasticity & $\begin{array}{l}1(N=49) \\
\text { Scholtes, } 2010\end{array}$ & Moderate & Unknown & Imprecise & Undetected & Insufficient & $\begin{array}{l}\text { Effect Size: } 0.46,95 \% \mathrm{Cl}-0.34 \\
\text { to } 1.26, p=0.26\end{array}$ \\
\hline $\begin{array}{l}\text { Strength } \\
\text { Exercise } \\
\text { (progressive } \\
\text { resistance) in MS }\end{array}$ & $\begin{array}{l}\text { Attention } \\
\text { control (social } \\
\text { program) }\end{array}$ & Spasticity & $\begin{array}{l}1(\mathrm{~N}=71) \\
\text { Dodd, } 2011\end{array}$ & Low & Unknown & Imprecise & Undetected & $\begin{array}{l}\text { Low for no } \\
\text { clear } \\
\text { benefit }\end{array}$ & $\begin{array}{l}\text { Mean Difference between } \\
\text { groups: } \\
\text { MSIS-88 stiffness: }-2.4,95 \% \mathrm{Cl} \\
-0.52 \text { to } 0.5 \\
\text { MSIS-88 muscle spasms: }-2.8 \text {, } \\
95 \% \mathrm{Cl}-5.6 \text { to } 0.03\end{array}$ \\
\hline $\begin{array}{l}\text { Balance Exercise } \\
\text { Hippotherapy in } \\
\text { CP }\end{array}$ & $\begin{array}{l}\text { Usual care } \\
\text { (physical } \\
\text { therapy) }\end{array}$ & Spasticity & $\begin{array}{l}1(\mathrm{~N}=44) \\
\text { Lucena-Anton, } \\
2018\end{array}$ & Moderate & Unknown & Imprecise & Undetected & Insufficient & $\begin{array}{l}\text { Mean (baseline to post- } \\
\text { treatment), } p=\text { between groups } \\
\text { Modified Ashworth Scale: } \\
\text { Left Abductors: } 2.77 \text { to } 2.50 \text { vs. } \\
2.59 \text { to } 2.54, p=0.040 \\
\text { Right Abductors: } 2.22 \text { to } 1.77 \\
\text { vs. } 2.40 \text { to } 2.31, p=0.047\end{array}$ \\
\hline $\begin{array}{l}\text { Balance Exercise } \\
\text { Hippotherapy in } \\
\text { MS }\end{array}$ & $\begin{array}{l}\text { Usual care } \\
\text { (physical } \\
\text { therapy) }\end{array}$ & Spasticity & $\begin{array}{l}1(\mathrm{~N}=70) \\
\text { Vermohlen, } \\
2018\end{array}$ & Moderate & Unknown & Imprecise & Undetected & Insufficient & $\begin{array}{l}\text { Mean Difference between } \\
\text { groups: } \\
\text { Spasticity NRS: }-0.9(95 \% \mathrm{Cl} \\
-1.9 \text { to }-0.1), p=0.031\end{array}$ \\
\hline
\end{tabular}

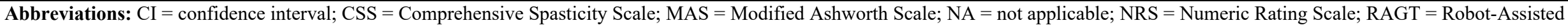
Gait Training; $\mathrm{SD}=$ standard deviation; $\mathrm{RCT}=$ randomized controlled trial. 
Table H-20. Strength of evidence for Key Question 2e: harms of physical activity

\begin{tabular}{|c|c|c|c|c|c|c|c|c|c|}
\hline Intervention & Comparator & Outcome & $\begin{array}{l}\text { Number of RCTs } \\
\text { (Participants) } \\
\text { Author Year } \\
\text { (See Appendix B } \\
\text { for Full Citation) }\end{array}$ & $\begin{array}{l}\text { Study } \\
\text { Limitations }\end{array}$ & Consistency & Precision & $\begin{array}{l}\text { Reporting } \\
\text { Bias }\end{array}$ & $\begin{array}{l}\text { Strength of } \\
\text { Evidence }\end{array}$ & $\begin{array}{l}\text { Findings, Direction and } \\
\text { Magnitude of Effect }\end{array}$ \\
\hline $\begin{array}{l}\text { Intensive } \\
\text { whole body } \\
\text { exercises }\end{array}$ & $\begin{array}{l}\text { Intensive } \\
\text { upper body } \\
\text { exercises }\end{array}$ & $\begin{array}{l}\text { Autonomic } \\
\text { dysreflexia }\end{array}$ & $\begin{array}{l}1(116) \\
\text { Galea, } 2018\end{array}$ & Moderate & Unknown & $\begin{array}{l}\text { Unknown } \\
\text { as number } \\
\text { of events } \\
\text { rather } \\
\text { than } \\
\text { number of } \\
\text { persons } \\
\text { with event }\end{array}$ & $\begin{array}{l}\text { Not } \\
\text { detected }\end{array}$ & $\begin{array}{l}\text { Moderate- } \\
\text { strength } \\
\text { evidence that } \\
\text { AD can occur } \\
\text { with exercise in } \\
\text { SCl; Low- } \\
\text { strength } \\
\text { evidence that } \\
\text { risk is increased } \\
\text { with whole body } \\
\text { vs. upper body } \\
\text { exercise in SCl }\end{array}$ & $\begin{array}{l}26 \text { episodes of AD ( } 3 \\
\text { serious) with intensive } \\
\text { exercise along with } 5 \\
\text { episodes of dizziness/ } \\
\text { nausea vs. } 7 \text { episodes in } \\
\text { upper body exercise along } \\
\text { with } 15 \text { episodes of } \\
\text { headache ( } n=60 \text { whole body } \\
\text { exercises vs. } n=56 \text { in upper } \\
\text { body exercises group) }\end{array}$ \\
\hline $\begin{array}{l}\text { Hippotherapy } \\
\text { Pilates } \\
\text { Balance } \\
\text { exercises } \\
\text { Usual physio- } \\
\text { therapy }\end{array}$ & $\begin{array}{l}\text { Usual care, } \\
\text { no treatment }\end{array}$ & Falls & $\begin{array}{l}6(456) \\
\text { Kwon, } 2015 \\
\text { Deutz, } 2017 \\
\text { Vermohlen, } 2018 \\
\text { Fox, 2016 } \\
\text { Carling, } 2017 \\
\text { Gandolfi, } 2015 \\
\end{array}$ & Moderate & Consistent & Imprecise & $\begin{array}{l}\text { Not } \\
\text { detected }\end{array}$ & Insufficient & $\begin{array}{l}\text { RR } 3.74,95 \% \text { Cl } 0.80 \text { to } \\
17.45, p=0.093 \\
\text { Gandolfi } p<0.005 \\
\text { Kwon } 2 \text { falls, groups not } \\
\text { specified }\end{array}$ \\
\hline $\begin{array}{l}\text { Multiple } \\
\text { interventions }\end{array}$ & $\begin{array}{l}\text { Usual care, } \\
\text { no treatment, } \\
\text { other } \\
\text { interventions }\end{array}$ & $\begin{array}{l}\text { Most } \\
\text { frequently } \\
\text { reported } \\
\text { AEs: Joint } \\
\text { pain, joint } \\
\text { swelling, } \\
\text { muscle } \\
\text { soreness, } \\
\text { muscle } \\
\text { cramps, } \\
\text { sprain, } \\
\text { strains, arm } \\
\text { pain, leg } \\
\text { pain }\end{array}$ & Multiple studies & Moderate & Unknown & Unknown & $\begin{array}{l}\text { Potential } \\
\text { reporting } \\
\text { bias as } \\
\text { many trials } \\
\text { did not } \\
\text { address } \\
\text { harms }\end{array}$ & Insufficient & $\begin{array}{l}\text { Not possible to determine } \\
\text { among outcomes which are } \\
\text { due specifically to an } \\
\text { overuse; no intervention was } \\
\text { more likely to lead to muscle } \\
\text { and joint pains than another } \\
\text { based on current evidence; } \\
\text { pains were also frequent in } \\
\text { control groups } \\
\text { All population subgroups } \\
\text { also had insufficient } \\
\text { evidence for AEs due to } \\
\text { inadequate reporting } \\
\text { All intervention subgroups } \\
\text { also had insuffient evidence } \\
\text { for AEs due to inadequate } \\
\text { reporting }\end{array}$ \\
\hline
\end{tabular}

Abbreviations: $\mathrm{AE}=$ adverse event; $\mathrm{RR}=$ risk ratio; $\mathrm{SCI}=$ spinal cord injury 